\title{
$86^{\text {th }}$ Annual Meeting
}

of the International Association of Applied Mathematics and Mechanics

\section{March 23-27, 2015 Lecce, Italy}

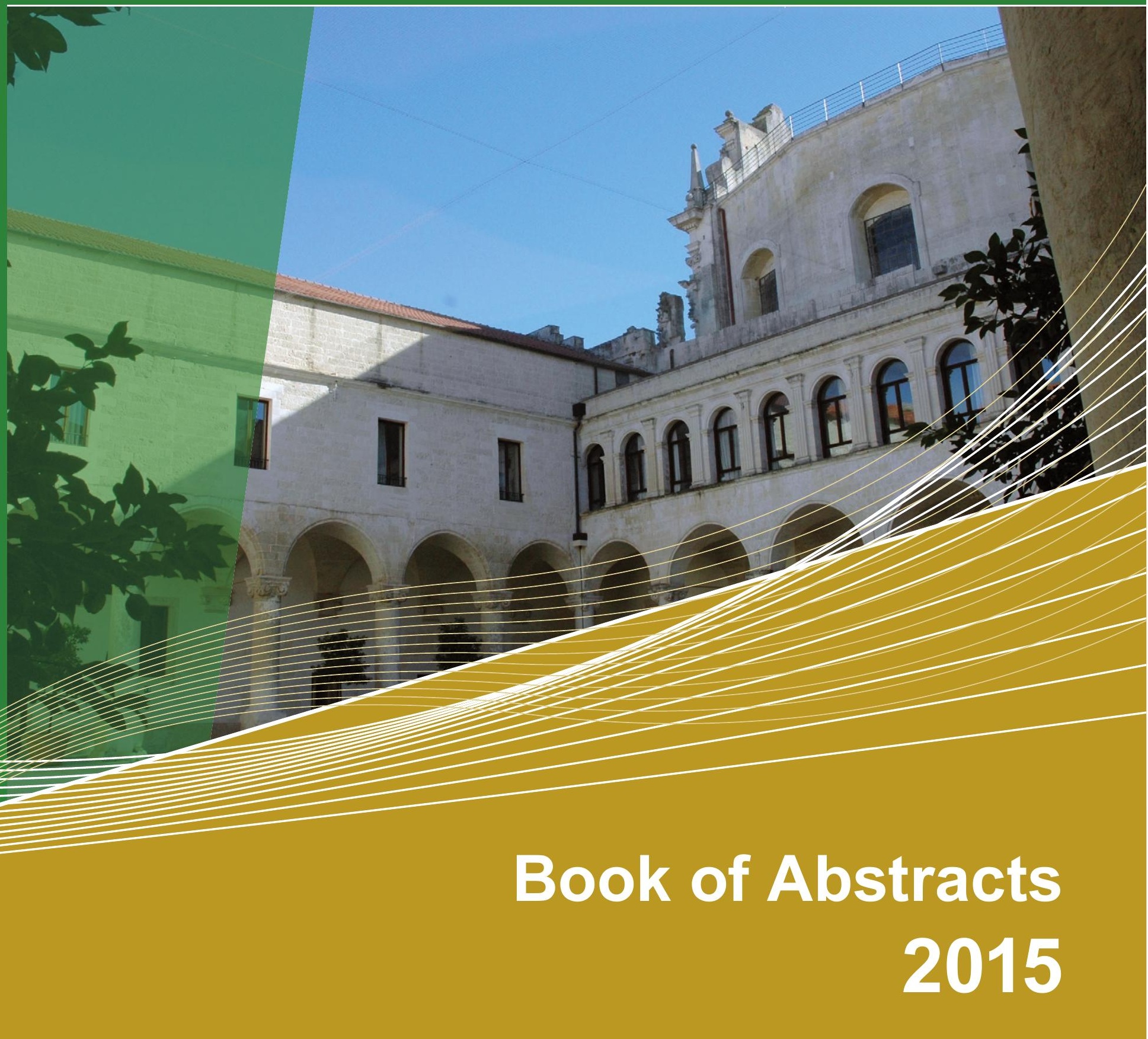




\section{Scientific Program - Timetable}

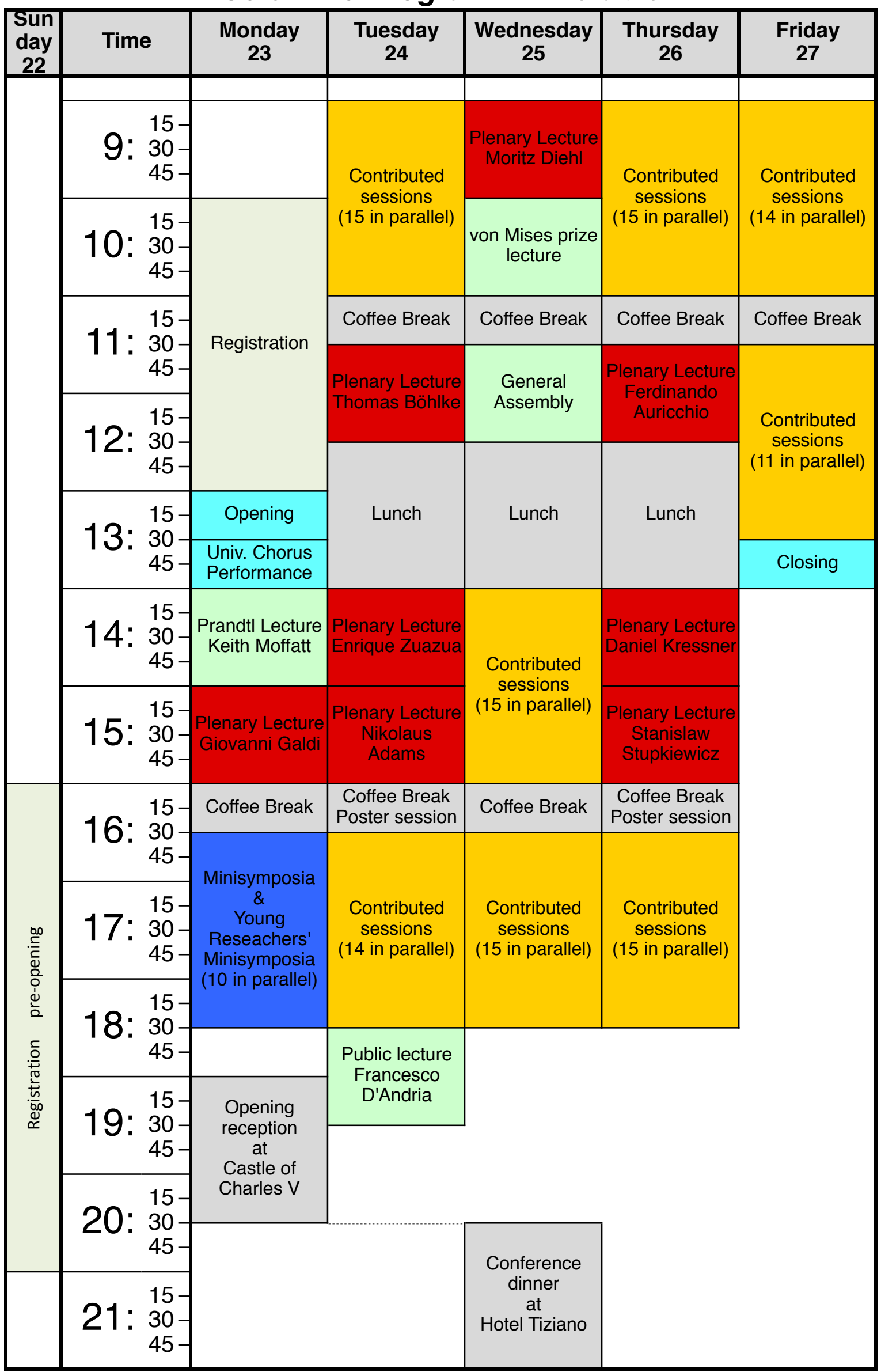




\title{
GAMM 2015
}

\author{
Università del Salento
}

\section{Scientific Program}

PRL: Prandtl Lecture

Discontinuities and topological jumps in slowly evolving vortical flows

Moffatt . . . . . . . . . . . . . . . . . . . . . . 40

PBL: Public Lecture

Do the Gates to the Hell really exist?

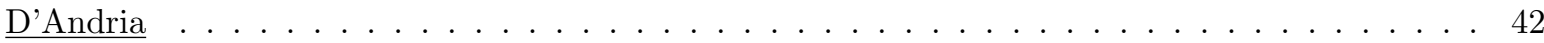

$\begin{array}{ll}\text { PLL: Plenary Lectures } & 43\end{array}$

On the Motion of a Rigid Body with a Liquid-filled Cavity

Galdi . . . . . . . . . . . . . . . . . . . . . . . . . . . . . 44

Micromechanics based modeling of applied materials

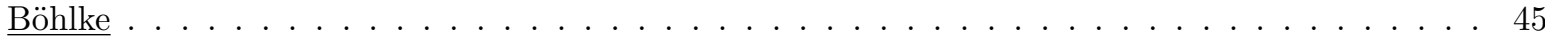

Optimal placement of sensors and actuators for waves in homogeneous and heterogeneous media

Zuazua . . . . . . . . . . . . . . . . . . . . . . . . 46

Particle methods for complex flows of complex fluids

Adams $-\mathrm{Hu}-$ Adami . . . . . . . . . . . . . . . . . . . . . . . 47

Nonlinear optimal control for airborne wind energy systems

Diehl - Horn - Zanon . . . . . . . . . . . . . . . . . . . . . . . . 48

Shape memory alloys: from recent modeling proposals to cardiovascular device simulations

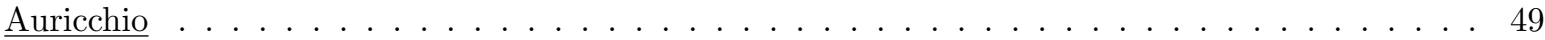

Low-rank techniques for high-dimensional problems in engineering and data-analysis

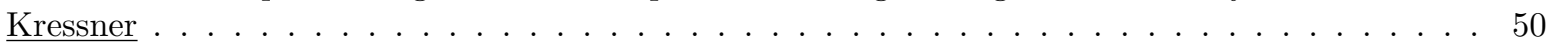

Interfacial energy and size effects in evolving martensitic microstructures

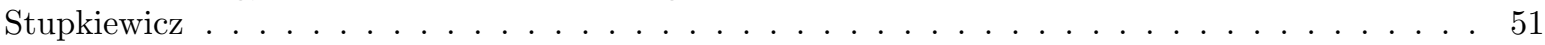

MS1: Multi-Scale Modeling of Ferroic Functional Materials $\quad 52$

Multiscale mechanical behaviour of nematic elastomers

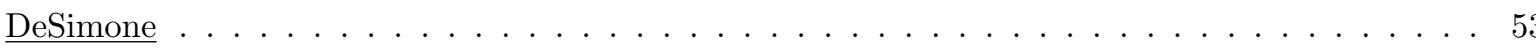

A laminate-based modelling approach for rate-dependent switching in ferroelectric materials

Menzel - Dusthakar - Svendsen . . . . . . . . . . . . . . . . . . . . . . 54

Variational Structural and Material Stability Analysis in Finite Electro-Magneto-Mechanics of Active Materials

Miehe - Vallicotti

Simulation of Size Effects in Ferroelectric Materials using a Phase Field Model

Müller - Gross - Schrade . . . . . . . . . . . . . . . . . . . . . . . . . . . . 56 
On molecular statics simulations of ferroelectric functional materials

Steinmann - Endres . . . . . . . . . . . . . . . . . . . . . . . . 57

MS2: Applications of the Virtual Element Method $\quad 58$

Virtual Element Methods: an overview

Brezzi - Beirao da Veiga - Marini - Russo . . . . . . . . . . . . . . . . . . . . . . . . . . 59

The Virtual Element Method for large scale Discrete Fracture Network simulations: fractureindependent mesh generation

Berrone - Benedetto - Borio - Pieraccini - Scialò . . . . . . . . . . . . . . . . . . . . . . . . . . 60

The Plane Wave Virtual Element Method for the Helmholtz Problem

Pietra - Perugia - Russo . . . . . . . . . . . . . . . . . . . . . . 61

Virtual Element Methods for parabolic problems on polygonal meshes

Vacca . . . . . . . . . . . . . . . . . . . . . . . . 62

A $C^{1}$ virtual element method for the Cahn-Hilliard problem

Verani - Antonietti - Beirao Da Veiga - Scacchi . . . . . . . . . . . . . . . . 63

MS3: Topological Defects in Solids

Discrete to continuum analysis of magnetic systems with continuous symmetries

Cicalese - Ruf - Solombrino . . . . . . . . . . . . . . . . . . . . . . . . . 65

Derivation of the line tension energy for dislocations in 3D

Garroni - Conti - Ortiz . . . . . . . . . . . . . . . . . . . . . . 66

Quantization effects in dislocation energies

Luckhaus ...................................667

Energy scaling law for the regular cone

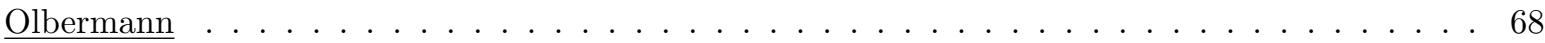

MS4: Optimal Control and Hybrid Systems 69

Sequential Action Control for Nonlinear and Hybrid Systems

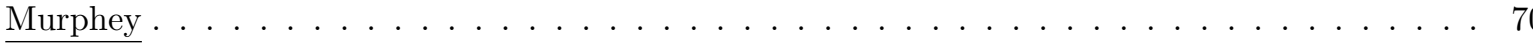

Output Regulation in Differential Variational Inequalities using Internal Model Principle and Passivity-Based Approach

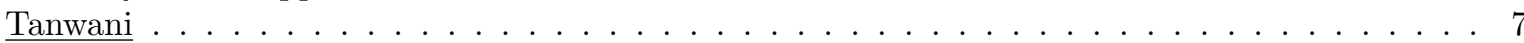

Gene regulatory networks: equivalence between Utkin's and sigmoidal approach

Elia - Del Buono - Lopez . . . . . . . . . . . . . . . . . . . . . . . . . . . . . . . 72

On the value function of mixed-integer optimal control problems

Hante-Gugat . . . . . . . . . . . . . . . . . . . . . . . . . 73

Relaxing mixed integer optimal control problems using a time transformation

Leyendecker - Ringkamp - Ober-Blöbaum . . . . . . . . . . . . . . . . . . . . . . . 74

Optimal Energy-Based Control of Hybrid Systems with Applications to Robotic Walking

Sinnet - Ames . . . . . . . . . . . . . . . . . . . . . . . . 75

MS5: Mechanics in an Inter., Multiphysics Env., Transforming Materials Sciences and Biology 77 
Mechanics of biological interfaces under stretch and across scales: lipid bilayer membranes and epithelia

Arroyo

The fiber reorientation problem revisited in the context of Eshelbian micromechanics: theory and computations

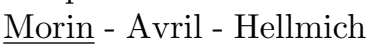

Multiscale hierarchical mechanics in soft tissues

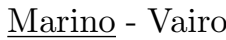

The theory of mechanobiological stability: on the theoretical foundations of mechanobiology in soft tissue

Cyron - Humphrey

Coupling X-ray physics and engineering mechanics, for enhanced analysis of Computer Tompgraphic images

Hellmich - Dejaco - Blanchard

Look different, better understand: computational multphysics enhanced imaging and measuring in biomedicine

Wall - Schoeder - Roth - Kronbichler

YRMS1: Analysis, Applications and Approximation of Constrained PDEs

Stable and efficient simulation of hyperbolic PDAEs describing flow networks

Huck - Jansen - Tischendorf . . . . . . . . . . . . . . . . . . . . . . . .

Stochastic Modeling and Regularity of the Nonlinear Elliptic-Parabolic Magnetoquasistatic Equation

Römer - Schöps . . . . . . . . . . . . . . . . . . . . . . . 86

Hydrodynamic force elements: A PDAE approach

Fiedler - Arnold . . . . . . . . . . . . . . . . . . . . . . . . . . . 87

The Pressure Manifold in the Unsteady Navier-Stokes Equation and in Semi-Discretizations Heiland . . . . . . . . . . . . . . . . . . . . . . . . 88

YRMS2: Phase Field Modeling in Mechanics and Applied Mathematics

On numerical schemes for phase-field models for electrowetting with electrolyte solutions

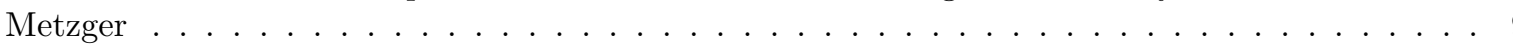

On elastic Cahn-Hilliard systems coupled with evolution inclusions for damage processes

Heinemann - Kraus . . . . . . . . . . . . . . . . . . . . . . . . . 91

Phase field modeling of ferroelectric materials with defects

$\mathrm{Xu}-\mathrm{Zuo}-\mathrm{Ma} \ldots \ldots \ldots \ldots \ldots$

Simulation of Atomic Force Microscopy for investigating $\mathrm{BaTiO}_{3}$ and $\mathrm{LiMn}_{2} \mathrm{O}_{4}$ nanostructures based on Phase Field Approach

Thai - Keip - Schröder - Amanieu - Rosato . . . . . . . . . . . . . . . . . . . . . . . . . 93

YRMS3: Discretization Aspects in PDE Constrained Optimization 94

Shape optimization by pursuing diffeomorphisms

Paganini - Hiptmair . . . . . . . . . . . . . . . . . . . . . . . . . 9

Fast Iterative Solvers for Discretizations of PDE-Constrained Optimization Problems

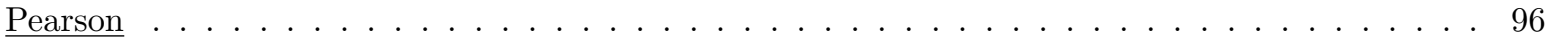


Finite element error estimates for Dirichlet boundary control problems on polygonal domains Pfefferer - Apel - Mateos - Rösch . . . . . . . . . . . . . . . . . . . . . . . . . 97

Scaling Limits in Computational Bayesian Inversion

Schillings - Schwab . . . . . . . . . . . . . . . . . . . . . . . 98

A Posteriori Error Estimation for State-Constrained Optimal Control Problems

Steinig - Siebert - Rösch . . . . . . . . . . . . . . . . . . . . . . . . 999

Optimal convergence order for control constrained optimal control problems

Wachsmuth - Schneider . . . . . . . . . . . . . . . . . . . . 100

YRMS4: Co-/Sparsity, Inverse Problems and Compressive Imaging

Recovering overcomplete sparse representations from structured sensing

Needell - Krahmer - Ward

Computational Aspects of Sparse Recovery

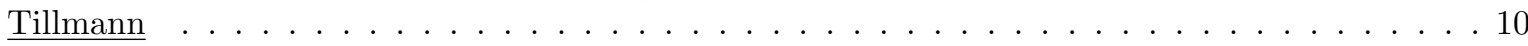

Joint reconstruction and segmentation from sparse Radon data

Frikel - Storath - Weinmann - Unser . . . . . . . . . . . . . . . . . . . . . . . . . . . . 104

Empirical phase transitions in sparsity-regularized X-ray CT

Jørgensen . . . . . . . . . . . . . . . . . . . . . . . . . . . . . . 105

Cosparse models and recovery algorithms for inverse problems in acoustics and electro-encephalography

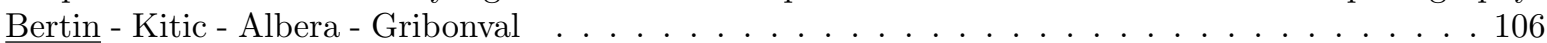

YRMS5: Topics in Low-rank Tensor Approximation

Finding low-rank bases of matrix subspaces

Uschmajew - Nakatsukasa - Soma . . . . . . . . . . . . . . . . . . . . . 108

Decoupling multivariate functions using tensor decompositions

Dreesen - Ishteva - Schoukens ． . . . . . . . . . . . . . . . . . . . . . . . . . . . 109

Soft Thresholding of Hierarchical Tensors and Its Application in Iterative Methods

Bachmayr - Schneider . . . . . . . . . . . . . . . . . . . . . . . 110

Tensor-structured approximation for the solution of differential equations

Kazeev . . . . . . . . . . . . . . . . . . . . . . . . . . . 111

Riemannian BFGS on the Tensor Train component space using an inherited tensor metric

Pfeffer - Schneider . . . . . . . . . . . . . . . . . . . . . . . . . . . 113

Preconditioned Riemannian optimization for low-rank tensor equations

Vandereycken - Kressner - Steinlechner . . . . . . . . . . . . . . . . . . . . . . . . . . . . 114

S01: Multi-body dynamics

Impulse-based control of simple oscillators within the nonsmooth mechanics approach

Schindler - Mayet - Seiwald . . . . . . . . . . . . . . . . . . . . . 116

Learning Robot Force/Position Control for Repetitive High Speed Applications with Unknown Non-Linear Contact Stiffness

Parzer - Gattringer - Müller . . . . . . . . . . . . . . . . . . . . . . . . . . . . . . 117

An Optimal Control Approach to the Simulation of Problems with Servo Constraints

Altmann - Heiland . . . . . . . . . . . . . . . . . . . . . . . . 118 
An explicit approach for time-optimal trajectory planning for kinematically redundant robots

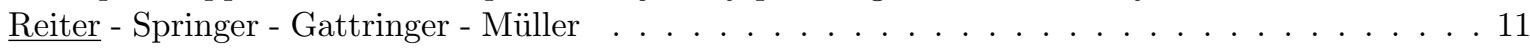

A numerical method for the servo constraint problem of underactuated mechanical systems

Yang - Betsch - Altmann . . . . . . . . . . . . . . . . . . . . . . . . 120

Active damping control for an underactuated multibody system

Burkhardt - Morlock - Seifried - Eberhard . . . . . . . . . . . . . . . . . . . . . . 121

Guideway based damping control of vehicle suspensions

Schiehlen . . . . . . . . . . . . . . . . . . . . . . . . 123

Modelling a pushbelt variator

Grundl - Schindler - Rixen - Ulbrich - van der Velde - Yildiz . . . . . . . . . . . . . . . . . . . . . 124

Modal Analysis of Vehicle Power Trains

Haslinger - Offner - Sopouch . . . . . . . . . . . . . . . . . . . . . . . . 125

Study on Real-Time Simulation of Elastic Multibody Systems with Application in Vehicle Dynamics

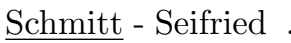

A mechanical model of a passive dynamic walker with unilateral viscoelastic contact formulation

Deppler - Lüdke - Fidlin . . . . . . . . . . . . . . . . . . . . . . . . . . . . . . . . 128

Investigation of optimal bipedal walking gaits subject to different energy-based objective functions

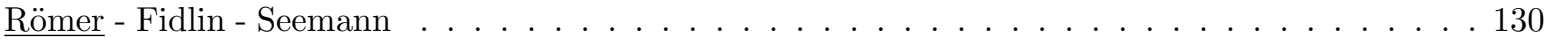

Coupling Elastic Bodies with an Enhanced Craig-Bampton-like Scheme

Eberhard - Holzwarth . . . . . . . . . . . . . . . . . . . . . . . . . 131

Back-Transformation into Physical Configuration Space after Model Order Reduction onto a General Subspace

Beitelschmidt - Lein

Topology Optimization of Flexible Bodies in Multibody Systems using the Floating Frame of Reference Approach

Seifried - Held - Moghadasi

Nonexpansivity of the Newton's Cradle Impact Law

Leine - Winandy . . . . . . . . . . . . . . . . . . . . . . . . . . 134

An annular Kirchhoff plate model tailored for rotating and non-rotating external loads

Heckmann - Kaiser . . . . . . . . . . . . . . . . . . . . . . . . . . 135

Performance assessment of a tracjectory-tracking approach for a manipulator with uncertainties using inverse fuzzy arithmetic

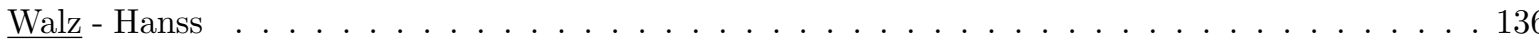

Variational integrators for thermo-viscoelastic discrete systems

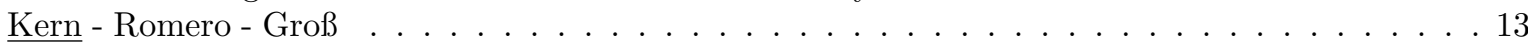

Variational integrators of higher order for flexible multibody systems

Bartelt - Groß . . . . . . . . . . . . . . . . . . . . . . . . 138

Error estimation approach for controlling the comunication step size for semi-implicit co-simulation methods

Meyer - Schweizer . 
A Reproducible Excitation Mechanism for Analyzing Electric Guitars

Hanss - Bestle - Eberhard . . . . . . . . . . . . . . . . . . . . . . . . . . . . . . 140

Transient amplification of maximum vibration amplitudes

Bonhage - Hentschel - Panning-v. Scheidt - Wallaschek . . . . . . . . . . . . . . . . . . . . . 141

Prox formulation of the cavitation problem in elastohydrodynamic lubrication contact

Krinner - Rixen . . . . . . . . . . . . . . . . . . . . . . . . . . . . . . . 142

Rigid body motion in a medium: data preparation for execution of experiments

Shamolin . . . . . . . . . . . . . . . . . . . . . . . . . 143

S02: Biomechanics

A new fully explicit algorithmic strategy for the simulation of bone healing directly on computed tomography data

Diebels - Roland - Tjardes - Bouillon . . . . . . . . . . . . . . . . . . . . . . . . . . . . . 145

Evolution of Mechanical Properties in Tissues Undergoing Deformation-Related Fiber Remodeling Processes

Topol - Demirkoparan - Pence - Wineman

Numerical Calculation of Fiber Orientation in Three-Dimensional Arterial Walls

Fausten - Balzani - Schröder

Towards an accurate mechanical characterisation of human's aortic leaflets during the heart cycle Morales Ortuno - Röhrle . . . . . . . . . . . . . . . . . . . . . . . . . . . . . . . . 148

Towards effective properties of active muscle tissue via homogenisation

Bleiler - Ponte Castañeda - Rohrle . . . . . . . . . . . . . . . . . . . . . . . . . . . 1

Modeling of skin anisotropy directions for realistic finite element simulations of the female breast $\mathrm{Li}$ - Jabareen - Raith - Itskov . . . . . . . . . . . . . . . . . . . . . . . . . . . 150

Experimental testing of transversely isotropic biological tissues - interaction between fibre and matrix material

$\underline{B o ̈ l}$ - Ehret - Leichsenring - Ernst

A porous media approach for plantar tissue during gait

Boso - Sciumè - Schrefler

A multi-scale time-dependent constitutive model of soft collagenous tissue

Hillgärtner - Linka - Itskov

Extremal loading of soft fibrous tissues: multi-scale mechanics and constitutive modeling

Linka - Khiem - Itskov . . . . . . . . . . . . . . . . . . . . . . .

A Numerical Study of Fluid Flow in Articular Cartilage Based on the Darcy-Forchheimer Law Carfagna - Grillo

Forchheimer's Correction in Modelling Flow in Poroelastic Materials with Statistical Fibre-

Reinforcement

Grillo - Carfagna - Federico

Relaxed incremental Variational Approach for Damage in Arteries

Balzani - Schmidt

Computation of residual stress distributions and opening angles of 3D patient-specific arterial walls

von Hoegen - Schröder . . . . . . . . . . . . . . . . . . . . . . . . . . . 15 
Phase-field modeling of fracture in biological tissues

Raina - Miehe . . . . . . . . . . . . . . . . . . . . . . . . . . 160

Fluid structure interaction in hemodynamics

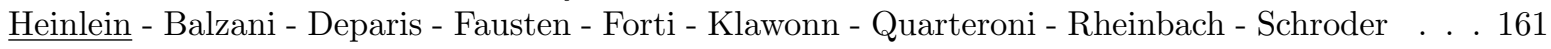

Linking structural dynamics to the nonlinear viscoelasticity and fatigue mechanics in fibrin biopolymers

Kurniawan - Koenderink

Mechanics of growing tumors: impact of modeling assumptions and boundary conditions on reliability of numerical results

Sciumè - Schrefler - Santagiuliana - Zavarise . . . . . . . . . . . . . . . . . . . . . . . . 163

Tumor growth in agarose and collagen-agarose co-gels

Mills - Rudraraju - Garikipati - Kemkemer . . . . . . . . . . . . . . . . . . . . . . . . . . . . . . 164

The role of the microvascular tortuosity in tumor transport phenomena

Penta - Ambrosi . . . . . . . . . . . . . . . . . . . . . . 165

Towards the continuum-mechanical modelling of metastatic tumour growth in the brain

Schröder - Wagner - Ehlers . . . . . . . . . . . . . . . . . . . . . . . . 166

Theoretical and numerical aspects in the multiphasic modelling of human brain tissue

Wagner - Ehlers . . . . . . . . . . . . . . . . . . . . . 167

On a Multi Scale and Multi Phase Model for the Description of Drug Uptake by the Human Liver

Werner - Ricken - Holzhütter - König - Dahmen - Dirsch ． . . . . . . . . . . . . . . . . . 168

Mechanics of cell-division: A new continuum model for growth inhomogeneities

Bolea Albero - Böl . . . . . . . . . . . . . . . . . . . . . . . . . . . . . 169

A geometric approach to characterize rigidity in proteins

Budday - Leyendecker - van den Bedem

Computational simulation of piezoelectric coating surrounding activated tooth implant

Shirazi Beheshtiha - Nackenhorst . . . . . . . . . . . . . . . . . . . . . . . . 171

Coupling an active middle ear implant to the round window of the cochlea

Ziegler - Wahl - Eiber . . . . . . . . . . . . . . . . . . . . . . . . . . 172

Eigenfrequencies of the reconstructed middle ear after tympanoplasty and stapedotomy corresponding to the case when the prosthesis is fixed

Slavashevich . . . . . . . . . . . . . . . . . . . . . . . . 173

S03: Damage and fracture mechanics

Stabilizing the XFEM for static and dynamic crack simulations

Löhnert . . . . . . . . . . . . . . . . . . . . . . . . . . . . . . 175

Thermo-mechanical modeling of crack propagation in dynamically loaded elastomer specimens using a scaled boundary finite element approach

Behnke - Kaliske . . . . . . . . . . . . . . . . . . . . . . . 176

Fitting stress intensity factors from crack opening displacements in 2D and 3D XFEM

Schätzer - Fries . . . . . . . . . . . . . . . . . . . . . . . . . 177

On corner singularities in Reissner's theory of elastic plates

Felger - Becker 
Crack propagation at bi-material interfaces

Parmigiani - Alam

Global approaches for an accurate loading analysis at multiple cracks systems

Judt - Ricoeur . . . . . . . . . . . . . . . . . . . . . . . . . . . . . . . . . . . 180

Crack initiation in elliptically notched plates

Weißgraeber - Felger - Becker . . . . . . . . . . . . . . . . . . . . . . 181

Phase-Field Modeling of Fracture in Anisotropic Media

Teichtmeister - Miehe . . . . . . . . . . . . . . . . . . . . . . . . 182

A non-isothermal phase-field model for damage in two-phase materials

Radszuweit - Kraus . . . . . . . . . . . . . . . . . . . . . . . . . . . . . . . 183

A novel treatment of crack boundary conditions in phase field models of fracture

Strobl - Seelig . . . . . . . . . . . . . . . . . . . . . . . . . . . . . . . . . . 184

Phase-Field Modeling of Hydraulic Fracture

Mauthe - Miehe . . . . . . . . . . . . . . . . . . . . . . . . . . . . . 185

3D ductile crack propagation with the XFEM

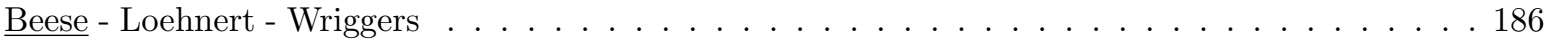

Numerical methods for crack loading analyses in quasicrystals

Wang-Ricoeur . . . . . . . . . . . . . . . . . . . . . . . 187

Application of a cohesive zone element for prediction of damages in laminated structures

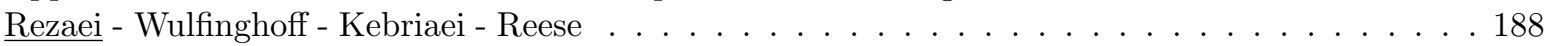

Macroscopic Damage modeling for Silicon Nitride

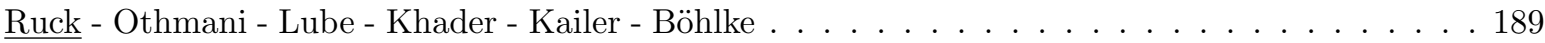

A regularization approach for damage models based on a displacement gradient

Schwarz - Junker - Hackl . . . . . . . . . . . . . . . . . . . . . . . . . . . . . . . . 190

Investigation of an elastoplastic material model coupled to nonlocal damage in an implicitgradient framework

Brepols - Wulfinghoff - Reese

A three-dimensional progressive damage model for fiber reinforced composites with an implicitexplicit integration scheme

Maaß - Gruttmann . . . . . . . . . . . . . . . . . . . . . . . . 193

Effects of a circular defect size and position on the damage progression in hybrid laminates under compressive loading

Tala-Ighil - Mokhtari .

3D modeling of crack percolation due to alkali-silica reaction in concrete RVEs

Cuba Ramos - Dunant - Molinari . . . . . . . . . . . . . . . . . . . . . . . . . 195

Numerical simulation of the fracturing processes in masonry arches

Invernizzi - Carpinteri - Accornero - Testone . . . . . . . . . . . . . . . . . . . . . . . . . 196

A damage-plasticity model to simulate the mode-II fatigue behavior of interfaces between thin adherends

Carrara - De Lorenzis

Predicting delamination in multilayered CFRP laminates

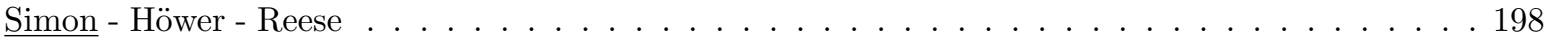


Fatigue mechanisms of cord-rubber composites

Jha - Nackenhorst

Determination of the fracture properties from size effect in ceramic-fibers-reinforced PMMA

Díaz - Mosler . . . . . . . . . . . . . . . . . . . . . . . . . . 200

On Anisotropic and Nonlocal Damage Models

Wulfinghoff - Reese - Brepols - Fassin . . . . . . . . . . . . . . . . . . . . . . . . 201

Model Reduction and Clustering Techniques for Crash Simulations

Fehr - Grunert . . . . . . . . . . . . . . . . . . . . . . . . . . 202

Mechanical properties of cold sprayed Ti- and Ni-based composites coatings

Silvello - Cavaliere . . . . . . . . . . . . . . . . . . . . . . . . . . . . . 204

Finite Element Method for the Vibration of cracked Beams with Varying Cross Section

Haskul - Kisa . . . . . . . . . . . . . . . . . . . . . . . . . . . . 205

Growth of a substrate material damage as a result of waves localization

Abramian - Vakulenko . . . . . . . . . . . . . . . . . . . . . 206

$\begin{array}{ll}\text { S04: Structural mechanics } & 207\end{array}$

T-splines discretizations for large deformation contact problems

Dimitri - Zavarise . . . . . . . . . . . . . . . . . . . . . . . . . . 208

Following forces as an inverse contact algorithm

Konyukhov - Schweizerhof - Izi . . . . . . . . . . . . . . . . . . . . . . . . . . 210

Application of the virtual element method to non-conforming contact interfaces

Rust - Wriggers - Reddy . . . . . . . . . . . . . . . . . . . . . . . . . . 211

Non-unique Equilibria of a Statically Indeterminate System with Coulomb Friction

Steiner . . . . . . . . . . . . . . . . . . . . . . . . . . 212

Efficient computation of surface-dominated structures using isogeometric elements

Zimmermann - Sauer . . . . . . . . . . . . . . . . . . . . . . . 213

Evaluation of a Finite Element Approach for Damping Determination

Hentschel - Bonhage - Panning-von Scheidt - Wallaschek . . . . . . . . . . . . . . . . . . 214

Modeling of non-stationary vibration signals based on the modified Kronecker sequences

Girip - Munteanu . . . . . . . . . . . . . . . . . . . . . . . . . . . 215

A homotopy method for the eigenvalue analysis of circular saw blade with inner slits

Luo - Schmidt - Gaul . . . . . . . . . . . . . . . . . . . . . . . . 216

Meta-structures for Cloaking Bending Waves

Gei - Colquitt - Brun - Movchan - Jones - Movchan . . . . . . . . . . . . . . . . . . . . . . . 217

Shrink fit with FGM-hub subject to heating and rotation

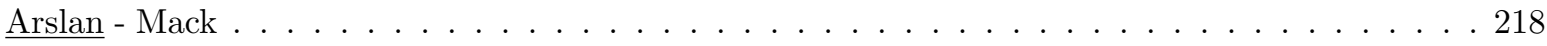

A projection approach to optimal control of elastic beam dynamics

Kostin . . . . . . . . . . . . . . . . . . . . . . . . . . . 219

Energy-momentum conserving discretization of mixed shell elements for large deformation problems

Janz - Betsch . . . . . . . . . . . . . . . . . . . . . . . . . . . 220 
The Strong Formulation Finite Element Method Applied to Structural Mechanics Problems

Tornabene - Fantuzzi - Bacciocchi - Viola . . . . . . . . . . . . . . . . . . . . . . . . . . . . . . 221

Weighted overconstrained least-squares mixed finite elements for hyperelasticity

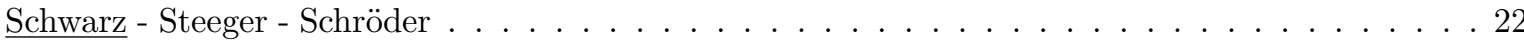

A stress-velocity least-squares mixed finite element formulation for incompressible elastodynamics.

Nisters - Schwarz - Schröder

Comparison of a mixed least-squares formulation using different approximation spaces

Steeger - Schröder - Schwarz . . . . . . . . . . . . . . . . . . . . . . 225

A surface oriented solid formulation based on a hybrid Galerkin-collocation method

Klinkel - Chen - Dornisch . . . . . . . . . . . . . . . . . . . . . 226

Application of Discontinuous Galerkin Finite Element Method for Discontinuities in Small Deformation Regimes

Bayat - Reese - Wulfinghoff

A New Mixed Finite Element for the Analysis of Structures with Material and Geometric Nonlinearities

Nodargi - Bisegna.

Discussion of the Particle Finite Element Method in the Context of Strength of Materials

York Duran - Sator - Müller - Sabel . . . . . . . . . . . . . . . . . . . . . . 229

Nonlinear SFEM with fluctacting input parameters

Caylak - Dridger - Mahnken . . . . . . . . . . . . . . . . . . . . . . 230

A high-order enrichment strategy for the finite cell method

Joulaian - Düster . . . . . . . . . . . . . . . . . . . . . . . . . . . . . . . 231

An efficient and robust Reissner-Mindlin shell formulation for isogeometric analysis

Dornisch - Müller - Klinkel . . . . . . . . . . . . . . . . . . . . . . . . . . . . . . 232

Corotational flat triangular elements for the nonlinear analysis of thin shell structures

Caselli - Bisegna . . . . . . . . . . . . . . . . . . . . . . . . . 233

Flexure Hinge Mechanisms Modeled by Nonlinear Euler-Bernoulli-Beams

Friedrich - Lammering . . . . . . . . . . . . . . . . . . . . . . . . . . . . . . . . . . . 234

A layer-wise theory for the structural analysis of glass and photovoltaic laminates

Naumenko - Altenbach - Eremeyev . . . . . . . . . . . . . . . . . . . . . 235

A user-defined element based on a layer-wise theory for laminated glasses and photovoltaic panels Eisenträger - Naumenko - Altenbach . . . . . . . . . . . . . . . . . . 236

On a mathematical problem of cusped double-layered plates

Chinchaladze . . . . . . . . . . . . . . . . . . . . . . . 237

A membrane finite element formulation for woven fabrics using the generalized polyconvex hyperelastic model

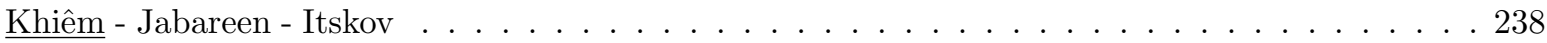

On the Mechanics of Ultralight Hollow Microlattices

Eidel . . . . . . . . . . . . . . . . . . . . . . . . . . . . . . . 239 
Mechanical Analysis of Metallic SLM-Lattices on Small Scales: Finite Element Simulations versus

Experiments

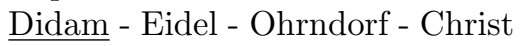

Investigation of elastoplastic effects of cables under large spatial deformation

Dörlich - Diebels - Linn . . . . . . . . . . . . . . . . . . . . . . . . . . . . . . . . . . . . 241

Determination of a Constitutive Friction Law Using an Elastic-Plastic Half-Space Model

Beyer - Willner . . . . . . . . . . . . . . . . . . . . . 242

Application of fibre Bragg grating sensors for residual stress analysis

Hannusch - Stockmann - Ihlemann . . . . . . . . . . . . . . . . . . . . . . . 243

Kink banding in laminated composite structures

Völlmecke . . . . . . . . . . . . . . . . . . . . . . . . . . . . . . 244

Interlaminar stress recovery for arbitrarily curved laminated shells

Winkler - Haller - Gerstmayr . . . . . . . . . . . . . . . . . . . . . . . . . . . . 245

Optimization of two-layered steel/aluminum hollow cylinders under combined load

Apatay - Mack . . . . . . . . . . . . . . . . . . . . . . . . 246

Experimental investigations on PP-PE foil specimens

Sguazzo - Hartmann . . . . . . . . . . . . . . . . . . . . . . . . . . 247

Stress concentration control in the problem of plane elasticity theory

Odishelidze - Criado Aldeanueva - Criado . . . . . . . . . . . . . . . . . . . . . . 248

Stress analyses of multi-layered composite pipes subjected to internal pressure

Sülü - Temiz . . . . . . . . . . . . . . . . . . . . . . . . . . . . . 249

A model reduction approach for hyperelastic materials based on Proper Orthogonal Decomposition

Hürkamp - Kaliske

XFEM for Deformation Theory of Plasticity

Omerović - Fries . . . . . . . . . . . . . . . . . . . . . . . . 251

Advanced FE-analysis of metal-to-metal seals considering fluid pressure penetration at two scales

Gorash - Dempster - Nicholls - Hamilton . . . . . . . . . . . . . . . . . . . . . . . . . . . . 252

Investigations on clamping effects with Die-Less-Hydroforming-Structures

Metzger - Ruff - Ummenhofer . . . . . . . . . . . . . . . . . . . . . . . . . 253

On one contact problem of plane elasticity theory with partially unknown boundary

Tsintsadze - Odishelidze . . . . . . . . . . . . . . . . . . . . . . . . . 254

Frequency veering and mode degeneration of a rectangular disc

Brouet - Twiefel - Wallaschek . . . . . . . . . . . . . . . . . . . . . . 255

S05: Nonlinear oscillations

Rotordynamics of Two-Pole Turbo Generators with Refined Modelling of the Unbalanced Magnetic Pull

Boy - Hetzler

Stabilization of a rotating shaft by electromagnetic actuators

Przybylowicz 
Stability and bifurcation behaviour of a Laval-Rotor considering fluid forces in compliant liquid seals

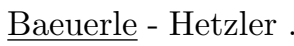

Dynamic stability of composite rotating shafts with Brazier's nonlinearity

Tylikowski - Starczewski

Synchronization effects in rotors partly filled with fluid

Keisenberg - Ostermeyer . . . . . . . . . . . . . . . . . . . . 261

On the self-balancing of the planetary rotor

Drozdetskaya - Fidlin .

Control of Nonlinearly Coupled Oscillators Using a Method of Averaging

Radisch - Kreuzer .

On some aspects of the dynamic behavior of the softening Duffing oscillator under harmonic excitation

von Wagner - Lentz

1:3-Resonance in a Hopf-Hopf bifurcation

Steindl . . . . . . . . . . . . . . . . . . . . . . . . . . . 265

On the chaotic behavior of the non-ideal vibrating systems

Chiroiu - Dumitriu . . . . . . . . . . . . . . . . . . . . . 266

Modeling and vibration analysis of rotors with hydrodynamic MF-film bearings

Perek - Kurnik . . . . . . . . . . . . . . . . . . . . . . . . 267

A DAE formulation for geared rotor dynamics including frictional contact between the teeth Jehle - Fidlin . . . . . . . . . . . . . . . . . . . . . . . . . . . . . 268

Adaptive Fuzzy Sliding Mode Controller and Observer for a Dive Cell

Pick - Bessa - Kreuzer - Krumm - Solowjow

The Vibrational Behavior of Coupled Bladed Disks with Variable Rotional Speed

Kaptan - Panning-von Scheidt - Wallaschek . . . . . . . . . . . . . . . . . . . . . . 270

Multi-mode model of a piezomagnetoelastic energy harvester under random excitation

Lentz - von Wagner . . . . . . . . . . . . . . . . . . . . . . . . . . 271

Optimal impedance load of a bistable energy harvester

Heymanns - Hagedorn . . . . . . . . . . . . . . . . . . . . . . . . 272

On vibrations in non linear, forced, friction-excited systems

Stender - Tiedemann - Hoffmann . . . . . . . . . . . . . . . . . . . . . . . . . 273

A finite element model for a soft robot equipped with a flexible limb

de Payrebrune - O'Reilly . . . . . . . . . . . . . . . . . . . . . . . . 274

On the influence of vibrations on macroscopic frictional contacts

Hammerschmidt - von Wagner . . . . . . . . . . . . . . . . . . . . . . . . . . 275

On the Effect of Contact Compliance on Vibrational Smoothing of Dry Friction

Kapelke - Seemann . . . . . . . . . . . . . . . . . . . . 276

S06: Material modelling in solid mechanics

A novel stabilization technique for X-FEM like enriched formulations

Ventura - Rabino . . . . . . . . . . . . . . . . . . . . . . 278 
Automatic Implementation of Elasto-plastic Incremental Formulations at Finite Strains using Hyper-Dual Numbers

Tanaka - Balzani - Schroeder .

Efficient time integration in multiplicative inelasticity

Landgraf - Shutov - Ihlemann

Towards a finite element simulation of coating by means of thermal spraying

Berthelsen - Denzer - Menzel

Modeling the moisture and temperature dependant material behavior of adhesive bonds

Goldschmidt - Diebels

Determination of material parameters corresponding to viscoelastic curing polymers

Klinge - Steinmann . . . . . . . . . . . . . . . . . . . . . . . 283

Towards modelling the curing process in particle-filled electro-active polymers

Hossain - Steinmann . . . . . . . . . . . . . . . . . . . . . . . . . . . . . . . . . 284

FE-simulation of spatially graded gelation during adhesive's curing

Rudolph - Ihlemann . . . . . . . . . . . . . . . . . . . . . . . 285

Characterisation of filled rubber with a pronounced nonlinear viscoelasticity

Scheffer - Goldschmidt - Diebels . . . . . . . . . . . . . . . . . . . . . . . . . . . . 286

A study on the influence of mechanical preconditioning on the fatigue behavior of rubber materials

Krause - Juhre . . . . . . . . . . . . . . . . . . . . . . . . . . . 287

On the generation of soft magneto-electric effects through Maxwell interactions

Keip . . . . . . . . . . . . . . . . . . . . . . . . . . . . . . . 288

Numerical Aspects of Energy Relaxation-Based Magnetostriction Modeling

Buckmann - Kiefer - Bartel - Menzel . . . . . . . . . . . . . . . . . . . . . . . . . 289

Computational homogenization in micromagnetics

Sridhar - Keip - Miehe . . . . . . . . . . . . . . . . . . . . . . . . . . . . . . 290

An atomistic scale analysis of ferroelectric nanodomain interfaces

Endres - Steinmann . . . . . . . . . . . . . . . . . . . . . . . . . . . . 291

Analysis of Micro- and Macro-Instability Phenomena in Computational Homogenization of Finite Electro-Statics

Vallicotti - Miehe

Modeling of Carbon Fiber Reinforced Plastics for Wave Propagation Analysis in Plates

Lammering - Hennings . . . . . . . . . . . . . . . . . . . . . . . . . .

Strain determination in full-field measurements using DIC

Hartmann - Sguazzo . . . . . . . . . . . . . . . . . . . . . . . . . . . 294

Inverse Parameter Identification for Orthotropic Elasto-Plastic Sheet-Steel

Soehngen - Willner . . . . . . . . . . . . . . . . . . . . . . 295

The Inversion of Hutchinson's Flow Rule

Kalisch - Bertram . . . . . . . . . . . . . . . . . . . . . . . . . . . 296

Parameter Identification by Inverse Modelling of Biaxial Tensile Tests of Discontinous Fiber Reinforced Polymers

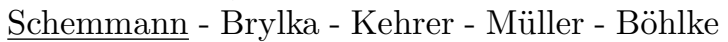


Shape control of piezolaminated thin-walled composite structures using nonlinear piezoelectric actuators

$\underline{\text { Rao }}$ - Schröder - Tarun

On the sonic composites with scatterers made from auxetic material

Mosnegutu - Donescu . . . . . . . . . . . . . . . . . . . . . . . . . 299

The influence of a damping coefficient on a non-invasive strategy for inverse form-finding

Landkammer - Steinmann . . . . . . . . . . . . . . . . . . . . . . 300

Modeling of imperfect contact interfaces in sonic composites

Munteanu - Brisan . . . . . . . . . . . . . . . . . . . . . . . . 301

Direct and Inverse Identification of Composites with Microstructure

Yanakieva - Nikolov - Baltov . . . . . . . . . . . . . . . . . . . . . . . 302

Optimal bounds from below of the critical load for elastic solids subject to uniaxial compression

Foti - Castellano - Fraddosio - Marzano - Piccioni . . . . . . . . . . . . . . . . . . . . . . . . . 303

Constitutive modeling of fiber-reinforced aerogels

Rege - Dargazany - Itskov . . . . . . . . . . . . . . . . . . . . . . . . . 305

Characterization of short fiber reinforced polymers

Röhrig - Diebels . . . . . . . . . . . . . . . . . . . . . . . 306

Formation of microstructure in the plates under compression

Nguyen - Le - Koster . . . . . . . . . . . . . . . . . . . . . . . . . 307

On fast and accurate modelling of distributed bre directions in composites

Goldberg - Ihlemann . . . . . . . . . . . . . . . . . . . . . . . . . . . 308

Meso-macro modelling of fiber-reinforced composites exhibiting elastoplastic deformation

Bedzra - Simon - Reese . . . . . . . . . . . . . . . . . . . . . . . . . 309

A micromechanical model for the transformation induced plasticity in poly-crystalline steels

Waimann - Junker - Hackl . . . . . . . . . . . . . . . . . . . . . . . . . . . 310

A novel contribution to the relaxation of non-convex energy density functionals in the context of martensitic phase transformations

Bartel - Kiefer - Buckmann - Menzel

A simplified approach to calculate adhesive joints in multi-material structures

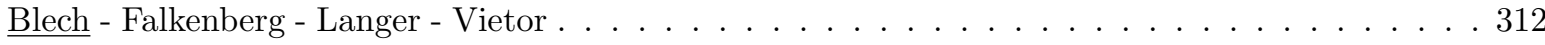

On the natural Fiber (Maize) Composite material

Ilie - Chiroiu . . . . . . . . . . . . . . . . . . . . . . . . . . . . . . 313

Numerical Modeling of Functional Fatigue in NiTi Wires

Osman - Bartel - Menzel . . . . . . . . . . . . . . . . . . . . . . . . . . . . 314

On the variational formulation and implementation of Allen-Cahn- and Cahn-Hilliard-type phase field theories

Bartels - Mosler

A coupled phase-field - Cahn-Hilliard model for lower bainitic transformation

Düsing - Mahnken . . . . . . . . . . . . . . . . . . . . . . . 316

Martensitic Transformations and Damage: A Combined Phase Field Approach

Schmitt - Kuhn - Mueller . . . . . . . . . . . . . . . . . . . . . . . . . 317 
Phase-field modeling of martensitic phase transformations in polycrystals coupled with crystal plasticity

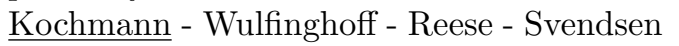

Phase Field Modeling of Ductile Fracture

Aldakheel - Raina - Miehe

Simulation of micro cutting considering crystal plastic deformations

Lohkamp - Schneider - Aurich - Kuhn - Müller . . . . . . . . . . . . . . . . . . . . . 320

Finite Gradient Elastoplasticity with Internal Constraints

Bertram - Glüge . . . . . . . . . . . . . . . . . . . . . . . . . . 321

A link between the molecular statistical theory and Ogden's model of rubber elasticity

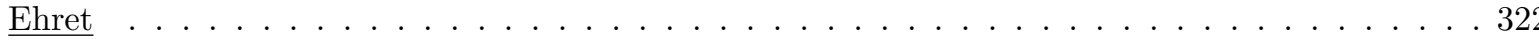

Equivalent Plastic Strain Gradient Theory with a Grain Boundary Yield Condition

Prahs - Bayerschen - Böhlke . . . . . . . . . . . . . . . . . . . . . . . . . . . . . 323

Primary and secondary instabilities in soft bilayered systems

Budday - Andres - Steinmann - Kuhl . . . . . . . . . . . . . . . . . . . . . . . . 324

A physically motivated model for filled elastomers including strain rate and amplitude dependency in finite viscoelasticity

Juhre - Raghunath - Klüppel

A Hardening Model based on a Finite-Deformation Gradient Crystal Plasticity Description: Formulation and Numerical Implementation

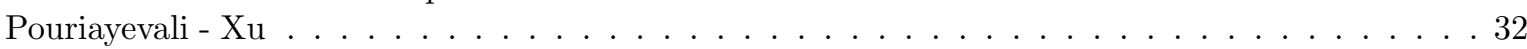

Phenomenological modelling for viscohyperelasticity: How to find suitable evolution laws in order to extend hyperelastic models?

Kröger - Juhre . . . . . . . . . . . . . . . . . . . . . . . . . . 327

Comparison of micromorphic, micropolar and microstrain continua

Leismann - Mahnken . . . . . . . . . . . . . . . . . . . . . . . . . 328

A variational viscosity-limit approach to the evolution of microstructures in finite crystal plasticity

Günther - Junker - Hackl . . . . . . . . . . . . . . . . . . . . . . . . . . . . . . . 329

Thermoplastics under Long-Term Loading: Experiments and Viscoelastic-Viscoplastic Modeling

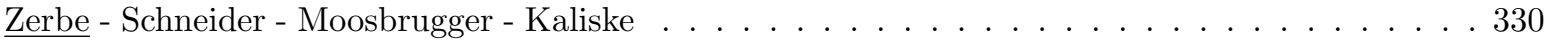

Formation of grain boundaries in ductile single crystals at simple shear

Koster - Le - Nguyen . . . . . . . . . . . . . . . . . . . . . . . . . . . . . . . . . . . 331

Modeling Dynamic Recrystallization in Polycrystalline Materials via Probability Distribution Functions

Nguyen - Isfahani - Hackl - Renner . . . . . . . . . . . . . . . . . . . . . . . . 332

Chiral acoustic metamaterials: dispersive waves and low frequency band-gaps

Gambarotta - Bacigalupo . . . . . . . . . . . . . . . . . . . . . . . 33

Finite Element Simulation of the creep behavior of directionally solidified NiAl-9Mo

Albiez - Böhlke . . . . . . . . . . . . . . . . . . . . . . . . . 334

Direct connection of rheological elements at large strains: Application to multiplicative viscoplasticity

Kießling - Landgraf - Ihlemann _. . . . . . . . . . . . . . . . . . . . . . 335 
Modelling Thermoplastic Material Behaviour of Dual-Phase Steels on a Microscopic Length Scale

Zeller - Löhnert - Wriggers . . . . . . . . . . . . . . . . . . . . . . . . . 336

On the thermodynamics of pseudo-elastic material models which reproduce stress softening effects

Naumann - Ihlemann . . . . . . . . . . . . . . . . . . . . . . . . . 337

Experimental and numerical characterization of the mechanical properties of Ni/Al hybrid metal foams from the atomic to the microscale

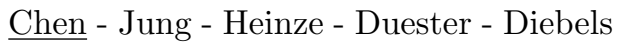

An Intrinsic Geometric Formulation of the Equilibrium Equations in Continuum Mechanics

Eugster

Numerical analysis of $\mathrm{Ni} / \mathrm{Al}$ hybrid metal foams using the finite cell method

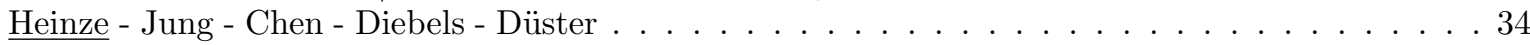

The perturbation method applied to tube drawing with floating plug

Ioan - Ioan . . . . . . . . . . . . . . . . . . . . . . . . . . . . . . . . . . . 341

Mathematical problems in the theory of elasticity for materials with double porosity

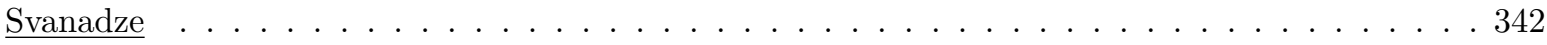

S07: Coupled problems

Thermo-mechanical modelling of cellular ceramic composites by a multiphase approach of porous media

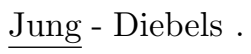

Thermo-mechanical computation of inelastic pavement structures under rolling tire loads

Wollny - Hartung - Kaliske . . . . . . . . . . . . . . . . . . . . . . . . 34

Shock structure for macroscopic multi-temperature model of binary mixtures: comparison with kinetic models

Madjarevic

Efficient Simulation of the Heat Flux Input in Moving Contacts

Partzsch - Beitelschmidt . . . . . . . . . . . . . . . . . . . . . . . . . . 348

Simulation of ice under mechanical and thermal load

Urban - Beitelschmidt . . . . . . . . . . . . . . . . . . . . . . . . . . 349

Reduction Approaches for Thermogasdynamic Lubrication Problems

Mahner - Lehn - Schweizer . . . . . . . . . . . . . . . . . . . . . . . . . . 350

A phase-field theory for fracture in porous media

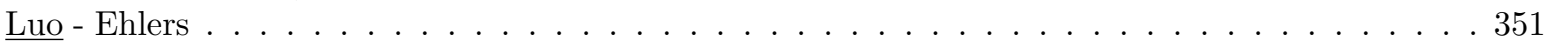

Isogeometric Analysis of Mechanically Coupled Phase Segregation in Li-Ion Battery Electrode Particles

$\underline{\text { Zhao }}$ - Stein - Xu

Eulerian large deformation formulation coupled with phase-field

Borukhovich - Steinbach . . . . . . . . . . . . . . . . . . . . . . . 353

A continuummechanical, bi-phasic, two-scale model for thermal driven phase transition during solidification

Moj - Ricken - Steinbach 
Formability enhancement in deformed AZ31 magnesium sheets via texture evolution: experiments and phase-field study

Darvishi Kamachali - Kim - Steinbach

Isogeometric Analysis of size-dependent behavior of Li-ion battery electrode particles

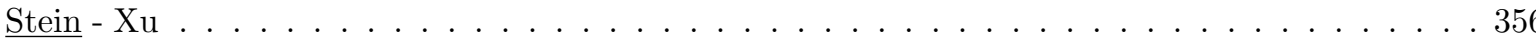

Variational Principles and Stability of Diffusion in Hydrogels

Nateghi - Miehe - Mauthe . . . . . . . . . . . . . . . . . . . . . . 357

Modeling of electrodynamic-mechanical coupling phenomena in smart magnetoelectroelastic materials

Merkel - Ricoeur . . . . . . . . . . . . . . . . . . . . . . . . . . 358

Modeling of the Crosstalk Phenomenon by the Non-Stationary Maxwell Equations Coupled with the Circuit Equations

Niroomand Rad - Steinbrecher

Velocity vector field optimization in bioventing

Notarnicola . . . . . . . . . . . . . . . . . . . . . . . . . 360

A constitutive and damage model for high cycle fatigue of tetragonal ferroelectrics

Lange - Ricoeur . . . . . . . . . . . . . . . . . . . . . . . . . . . . . 361

Phase field simulation on mechanically coupled switching dynamics in nanomagnets

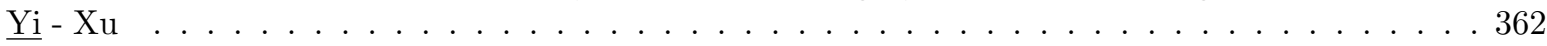

The electrocaloric effect in ferroelectrics: nonlinear modeling and simulation

Wingen - Ricoeur . . . . . . . . . . . . . . . . . . . . . . . . 363

Nonlinear numerical simulation of ferroelectric-ferromagnetic multifunctional composites

Avakian - Ricoeur . . . . . . . . . . . . . . . . . . . . . . . . . 364

Intrinsic symmetries in constitutive modeling of magneto-elastic materials

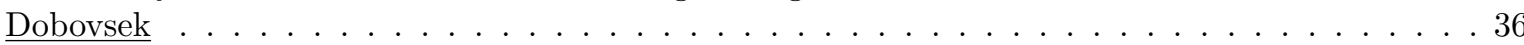

Correction of Characteristics of Subsystems of Torsionally Vibrating Complex Mechatronic Systems as Introduction to Solution of Their Inverse Task

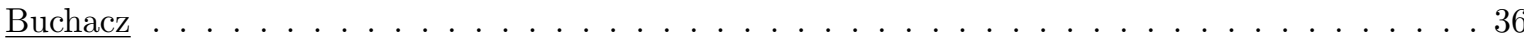

Modeling a Halfspace with Tunnel using a Coupled Integral Transform Method - Finite Element Method Approach

Hackenberg - Müller

Fluid-Porous-Media Interaction: A Decoupled Solution Algorithm via Localised Lagrange Multipliers

Zinatbakhsh - Koch - Ehlers .

Simulation of vortex-induced oscillations within a shear-thinning liquid

Gundlach - Schlosser - Wünsch . . . . . . . . . . . . . . . . . . . 369

A numerical approach to the dynamics analysis of mooring lines

Stabile - Borri - Matthies . . . . . . . . . . . . . . . . . . . . . . 370

Critical velocities for flow induced vibrations of a U-shaped belt

Strecha - Steinrück . . . . . . . . . . . . . . . . . . . . . . . . . 371

Hydroelastic stability of multi-plate structures interacting with flowing fluid

Lekomtsev - Bochkarev - Matveenko . . . . . . . . . . . . . . . . . . . . 372 
Validation with Numerical Simulations of a Simplified Model of a Hybrid Rocket Motor

Frunzulica -Stoia-Djeska . . . . . . . . . . . . . . . . . . . . . . . 373

Thermodynamically consistent description of mass transfer in a porous medium by a singular surface

Häberle - Ehlers .

A coupled multiphasic description of biological methane oxidation in landfill cover layers

Thom - Ricken - Bluhm - Gehrke - Denecke

Modeling, Simulation and Parameter Identification for Rate-Dependent Magnetoactive Polymer Response

Kiefer - Haldar - Menzel . . . . . . . . . . . . . . . . . . . . . . . . . . 376

On electrostatic-viscoelastic simulation of dielectric actuators

Schlögl - Leyendecker . . . . . . . . . . . . . . . . . . . . . . . . . . . . . . 377

Microscopic modeling and finite element simulation of magnetorheological elastomers

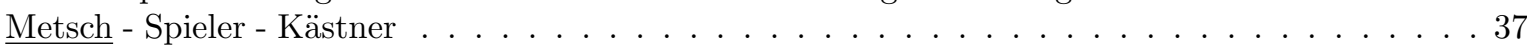

Harvesting energy with a cone-type dielectric elastomer generator

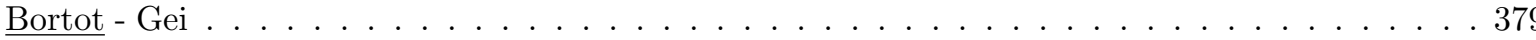

Modeling of thermo-electro-mechanical coupling effects in ionic electroactive polymers within the Theory of Porous Media

Serdas - Bluhm - Schröder

Deformation and interaction of surface energy driven systems

Sauer - Duong . . . . . . . . . . . . . . . . . . . . . . . . . . . . . . 381

Parallel solution of volumetrically coupled multi-field problems using an Abaqus-PANDAS software interface

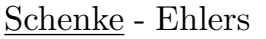

Application and modification of the POD Method and the POD-DEIM for model reduction in porous-media simulations

Fink - Ehlers . . . . . . . . . . . . . . . . . . . . . . . . 38

NURBS-based Approaches in Fluid Flow Simulations

Elgeti - Stavrev - Hosters - Behr . . . . . . . . . . . . . . . . . . . . . . . . . . . . . 384

Numerical Approaches towards Plasticity

Schröder - Kuhl . . . . . . . . . . . . . . . . . . . . . . . . . . . . . . . 385

S08: Multiscales and homogenization

A two scale phase field model for elastic shape optimization

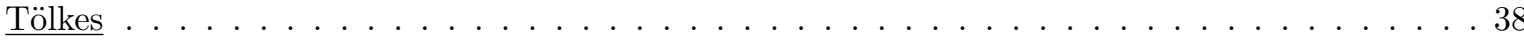

Study on Statistically Similar RVEs for real microstructures based on different statistical descriptors

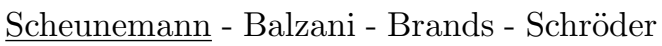

Polynomial shape functions on the logarithmic space: the LogFE method

Schröppel - Wackerfuß

Convergence Properties of GMRES for FFT-based Galerkin Homogenization of Periodic Media

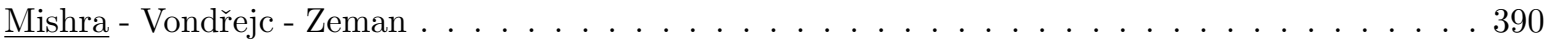


A two-scale homogenisation approach for fluid saturated porous media

Bartel - Ricken - Schröder . . . . . . . . . . . . . . . . . . . . . . . . . . . 391

An Extended Cascade Micromechanics Model for the Effective Diffusivity of Porous Materials accounting for Pore-Size Distribution

Timothy - Meschke

Grain-scale-based simulation of granular material

Bidier - Ehlers .

Materials design of elastic properties of multiphase polycrystalline composites using model functions

Lobos - Yuzbasioglu - Böhlke

A coupled two-scale shell model for comb-like sandwich structures

Heller - Gruttmann . . . . . . . . . . . . . . . . . . . . . . . . . . 395

Dual-Phase Steel Simulations Based on Representative Three-Dimensional Microstructures

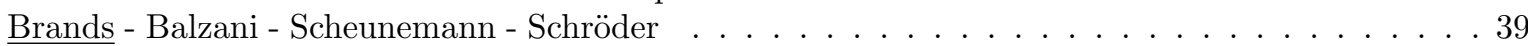

Computational characterization of magneto-electric composites: the role of ferroelectric prepolarization

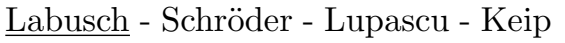

Electro- and Magneto-active Soft Composites with Periodic and Random Microstructures

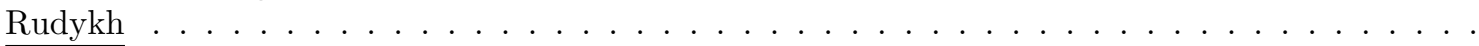

Multi-scale modeling of beam-like structures: A new boundary condition concept for the RVE

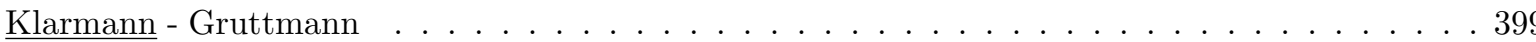

Micromechanical modeling of textile materials by means of 1-D structural elements

Mehnert - Fillep - Mergheim - Steinmann . . . . . . . . . . . . . . . . . . .

Experimental investigation and approximation of the temperature-dependent stiffness of shortfibre reinforced polymers

Kehrer - Müller - Brylka - Böhlke

Coupling atomistic and continuum models with nodes having translational and rotational degrees of freedom

Niederhöfer - Wackerfuß . . . . . . . . . . . . . . . . . . . . . . 4 4

Representative Volume Element Size Convergence for a Parallel Fiber Bundle Micro-Model

Stapleton - Appel - Reese . . . . . . . . . . . . . . . . . . . . . . . . . . . 4403

Comparison of the Interface Orientation Distribution Average to RVE simulations

Glüge - Kalisch . . . . . . . . . . . . . . . . . . . . . . .

Continuum modeling of material interfaces and surfaces based on molecular statics computations

Sievers - Mosler . . . . . . . . . . . . . . . . . . . . . . . 4 4

A multiscale contact homogenization approach for hysteresis friction of rubber on rough surfaces Wagner - Wriggers - Klapproth - Prange . . . . . . . . . . . . . . . . . . . . . . . . 406

Reduced order nonlinear homogenization of composites with cohesive interfaces

Fritzen - Leuschner . . . . . . . . . . . . . . . . . . . . . . . . 4407

Use of Gurtin-Murdoch model in predicting effective properties of nano-composites with application to nanoporous gold

Nazarenko - Bargmann - Stolarski . . . . . . . . . . . . . . . . . . . . . . . . 408 
Stability analysis of the flow past miniature vortex generators in a Blasius boundary layer Siconolfi - Camarri - Fransson . . . . . . . . . . . . . . . . . . . . . . .

P-norm optimal 3D perturbations in the Poiseuille flow

Farano - Cherubini - Robinet - De Palma . . . . . . . . . . . . . . . . . . . . . . . 411

Unified description of bifurcation processes associated with laminar boundary-layer separation Kluwick - Braun . . . . . . . . . . . . . . . . . . . . . . . . . . . . . . . 412

Investigation of the roughness-induced transition: linear and non-linear optimal perturbations

Cherubini - Loiseau - De Palma - Robinet . . . . . . . . . . . . . . . . . . . . . . . . . . . . . . 413

Extreme event detection in near-wall turbulence using reflection-encoded readout of micropillar arrays

Bruecker - Hegner - Mikulich - Axtmann - Rist . . . . . . . . . . . . . . . . . . . . . . . . . . . 414

Spreading of Linear Disturbances in Boundary Layers at Mach Number Five and the Effect of Wall Cooling

Jocksch - Kleiser

Receptivity and non-uniqueness of turbulent boundary layer flows

Scheichl

Axisymmetric flow over a sudden expansion in an annular pipe

Beladi - Kuhlmann . . . . . . . . . . . . . . . . . . . . . . . . . . . . 417

The Clebsch transformation and its capabilities towards fluid and solid mechanics

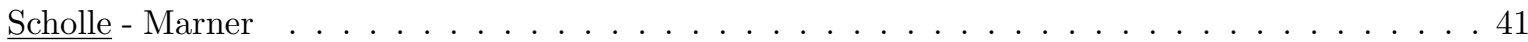

Potential theory application to superfluidic multiphase flows

Brazaluk . . . . . . . . . . . . . . . . . . . . . . . . . . . . . . . 419

S10: Turbulence and reactive flows

On the universality of small-scale statistics in turbulence

Schumacher . . . . . . . . . . . . . . . . . . . . . . . 421

Scaling in turbulent canonical flows - a ubiquitous property of all moments?

Oberlack - Rosteck - Avsarkisov - Mehdizadeh - Hoyas - Waclawczyk

NOx formation in premixed flames: a direct numerical simulation study

Pitsch - Trisjono - Gibelhaus . . . . . . . . . . . . . . . . . . . . . . . . . . . .423

High-resolution LES of the Cambridge Stratified Flame

Kempf - Proch - Domingo - Vervisch . . . . . . . . . . . . . . . . . . . . . . . . 424

A Flamelet Progress Variable model for compressible reacting flows

Coclite - Pascazio - De Palma

Assessment of subgrid-scale models for large-eddy simulation of complex flows

Nicoud - Baya Toda - Truffin - Bruneaux - Cabrit . . . . . . . . . . . . . . . . . . . . . . . .426

Assessment of non-linear $k-\epsilon$ turbulence models for the prediction of wind flow around isolated buildings and multiple obstacles

Parente - Moonens

Simulation of wake effects in windfarms using an Actuator Disk implementation

Hornung - Class - Viellieber 
Numerical Simulation of Continuous and Pulsed Film Cooling at the leading edge of a symmetrical turbine blade

Nemdili - Azzi - Dellil

Coarse-Grid-CFD for the Thermal Hydraulic Investigation of Rod-Bundles

Viellieber - Class . . . . . . . . . . . . . . . . . . . . . . . 430

Particles in Turbulence: Macro- Consequences from Micro- Interactions

Soldati . . . . . . . . . . . . . . . . . . . . . . . . . 4

An efficient numerical method for fully resolved particle simulations on high-performance computers

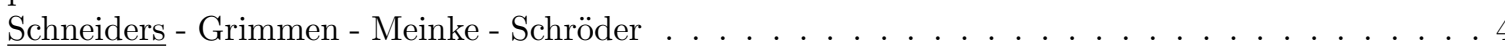

A stochastic SGS model for Lagrangian particle tracking in large-eddy simulation velocity fields

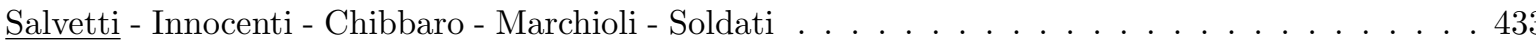

Budget analysis of the kinetic energy for bubbly flows

Santarelli - Roussel - Fröhlich . . . . . . . . . . . . . . . . . . . . . . 4

Asymptotic solutions of an extended Korteweg-de Vries equation describing solitary waves with weak and strong downstream decay in turbulent open-channel flow

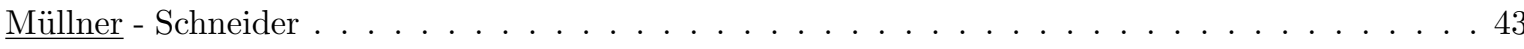

Recent results in isotropic turbulence decay theory

Sagaut - Meldi - Mons . . . . . . . . . . . . . . . . . . . . . . . . . 436

Numerical simulation of dense gas compressible homogeneous isotropic turbulence

Sciacovelli - Cinnella . . . . . . . . . . . . . . . . . . . . . . . . 437

High Reynolds number effects on turbulent scalings in compressible channel flow

Modesti - Pirozzoli - Bernardini . . . . . . . . . . . . . . . . . . . . . . . . . 439

Effect of outer stratification inside the inner region of a convective boundary layer

Mellado - Garcia - van Heerwaarden . . . . . . . . . . . . . . . . . . . . . . . . . . . . 440

Reynolds-analogy factor and new formulations for the temperature defect law for turbulent boundary layers on a plate

$\underline{\text { Vigdorovich }}$

Experimental and numerical studies of active and passive control of combustion instabilities

Poinsot - Bauerheim - Staffelbach - Gicquel . . . . . . . . . . . . . . . . . . . . . . . . 442

LES of high-frequency transverse combustion instabilities in complex combustion chambers

Ghani - Poinsot - Maestro - Gicquel . . . . . . . . . . . . . . . . . . . . . . . . . . . 443

Ignition and mixing in shock-bubble interaction with chemical reactions

Diegelmann - Tritschler - Hickel - Adams . . . . . . . . . . . . . . . . . . . . . . . . . . . . 444

Large-eddy simulations of controlled wake flows using a penalty model of synthetic micro-jet

Peres - Pasquetti . . . . . . . . . . . . . . . . . . . . . . . 446

Computational Aeroacoustics of Subsonic and Supersonic Impinging Jets

Sesterhenn - Wilke . . . . . . . . . . . . . . . . . . . . . . . . . . . . 447

S11: Interfacial flows

Simulating Free-Surface Flows with Moving Boundaries: A Combination of an Interface-Tracking and an Interface-Capturing Approach

Frings - Behr - Elgeti . . . . . . . . . . . . . . . . . . . . . . . . . . . 449 
Towards higher-order XFEM for interfacial flows

Fries

Numerical Modelling of Laser-Induced Cavitation Bubbles with a Finite Volume Method

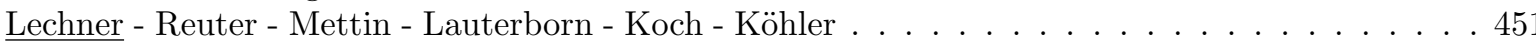

A comparison of viscoelastic and empirical rheological models in context of squeeze flows

Descher - Wünsch . . . . . . . . . . . . . . . . . . . . . . . . 452

On the implementation of free-slip interfaces for the immersed boundary method

Kempe - Fröhlich . . . . . . . . . . . . . . . . . . . . . . . . . 453

Linear stability of a thin non-isothermal droplet spreading on a rotating disk

Boettcher - Externbrink . . . . . . . . . . . . . . . . . . . . . . 45

A circulating gravity wave in a cylindrical tank

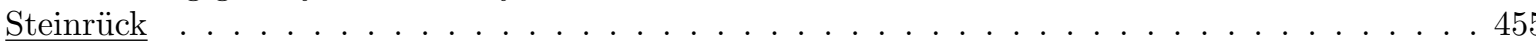

Experiments beyond the limits of Nusselt theory: The linear stability of gravity-driven films over undulated inclines

Schörner - Reck - Aksel . . . . . . . . . . . . . . . . . . . . . . . . . . 456

Continuous size-dependent separation of blood components from human whole blood samples in microfluidic spirals

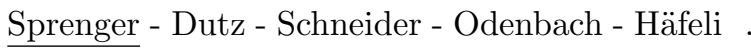

Modeling and simulation of mobile jet agitator for a biogas plant

Alimi - Wünsch . . . . . . . . . . . . . . . . . . . . . . . . . . . 458

Interaction of a finite-size particle with a wall

Romanò - Kuhlmann . . . . . . . . . . . . . . . . . . . . . . . . . . . . . . . . 459

The magnetoviscous effect of a biocompatible ferrofluid diluted in sheep blood

Nowak - Odenbach . . . . . . . . . . . . . . . . . . . . . . . . 460

The anisotropy of the magnetoviscous effect in a magnetite ferrofluid with weak interparticle interactions

Linke - Odenbach

An innovative phase transition modeling for reproducing cavitation through a five-equation and seven-equation models and complex equation of state

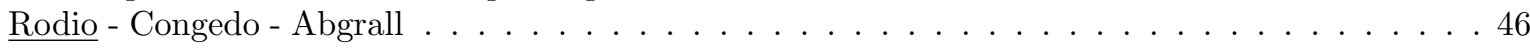

Numerical investigation of a liquid displacing a gas in thin porous layers

Neumann - Boettcher - Gödeke - Ehrhard . . . . . . . . . . . . . . . . . . . . . . . . 464

Mechanisms for wave generation in countercurrent air-water turbulent flows

Zonta - Onorato - Soldati . . . . . . . . . . . . . . . . . . . . 465

Diffuse interface models for locally inextensible vesicles

Aland - Lowengrub - Voigt . . . . . . . . . . . . . . . . . . . . . . . . . . . . 466

Polymer Devolatilization in a Rotating Apparatus

Hirschfeld - Hermann - Wünsch . . . . . . . . . . . . . . . . . . . . . 467

Gas Bubbles in Micro-Capillaries - Hydrodynamics and Mass Transfer

Lakshmanan - Ehrhard . . . . . . . . . . . . . . . . . . . . . . . . . . 468

Simulation of mass transfer at free liquid/liquid interfaces

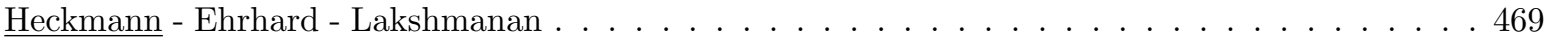


Three-phase contact line pinning at structured surfaces by molecular dynamics simulation

Horsch - Becker - Kohns - Hasse . . . . . . . . . . . . . . . . . . . . . . . . . . 470

New density based OpenFOAM solver with a stochastic fields approach for two phase flow

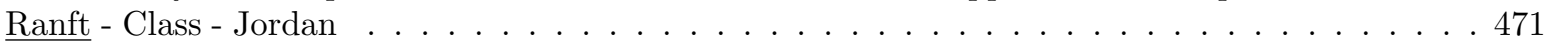

S12: Waves and acoustics

Propagation of acoustic waves in a turbulent boundary layer

Gloerfelt . . . . . . . . . . . . . . . . . . . . . . . . . . 473

Non-destructive testing of porous media by means of sound wave analysis

Albers . . . . . . . . . . . . . . . . . . . . . . . . 474

Calculation of the effective speed of sound in corrugated pipes by multiple scales

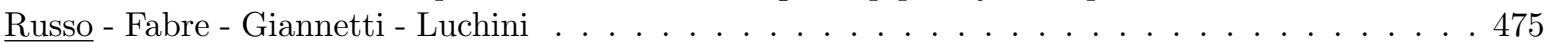

Numerical Calculation of Acoustic Sources for the Landing Gear of Aeroplane during Take-off and Landing

Rasuo - Jazarevic . . . . . . . . . . . . . . . . . . . . . . . . 476

Forward and Inverse Viscoacoustic Modelling in a Tunnel Environment

Musayev - Hackl - Baitsch . . . . . . . . . . . . . . . . . . . . . . . . . 477

On numerical simulation of tsunami run-up on shore

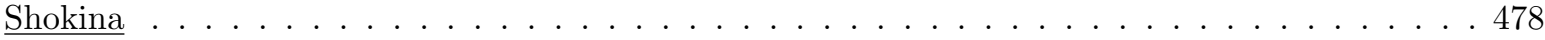

Potential flow simulations of Peregrine-type deep water surface gravity wave packets

Wenzel - Bünte - Hoffmann . . . . . . . . . . . . . . . . . . . . . . . . . . .479

Application of variational-asymptotic method to modulation theory

Nguyen - Le . . . . . . . . . . . . . . . . . . . . . . . . . . . . . . . . . . . 480

A novel approach to mode - tracing in SBFEM - Simulations: Higher-order Taylor- and Padeapproximation

Krome - Gravenkamp . . . . . . . . . . . . . . . . . . . . . . . . . . . . . . 481

Numerical modeling of a sub-sonic roll-over load along a rods skin

Weber - Zastrau - Löpitz - Balzani . . . . . . . . . . . . . . . . . . . . . . . . . . . . . . . . . 482

S14: Applied analysis

Weak solution to certain problem in fluid mechanics

Feireisl . . . . . . . . . . . . . . . . . . . . . . . . . . . . . . . . . . .484

A low volume-fraction limit for martensic microstructures in shape-memory alloys

Diermeier . . . . . . . . . . . . . . . . . . . . . . . . . . . 485

Homogenization of layered structures with rigid components in single-slip finite elastoplasticity

Christowiak - Kreisbeck . . . . . . . . . . . . . . . . . . . . . . 486

Homogenization for dislocation based gradient visco-plasticity

Nesenenko . . . . . . . . . . . . . . . . . . . . . . . . . . . . . . . . 487

On the use of potential fields in fluid mechanics

Marner - Scholle . . . . . . . . . . . . . . . . . . . . . . . . . . . . . . . . .488

Well-Posedness and Stability for some Volume-Preserving Curvature Flows with Boundary Contact or Triple Junctions

Abels - Arab - Garcke - Müller . 
Nonlocal Cahn-Hilliard equation with a reaction term

Melchionna - Rocca . . . . . . . . . . . . . . . . . . . . . . . . 449

Well-posedness and optimal control of Allen-Cahn type equations with singular potentials and dynamic boundary conditions

Calatroni - Colli .

Relaxation in nonlinear elasticity with constraints on the determinant

Dolzmann - Conti . . . . . . . . . . . . . . . . . . . . . . . 492

A Linear Scale-Space Theory for Continuous Nonlocal Evolutions

Cárdenas - Weickert - Schaeffer . . . . . . . . . . . . . . . . . . . . . . . . . . . 493

Rate-independent systems with viscosity and inertia - Evolutionary Gamma-convergence

Thomas - Rossi . . . . . . . . . . . . . . . . . . . . . . . . . 494

Analysis of a gradient enhanced damage model

Susu - Meyer

Mixed-growth plasticity and generalized rigidity

Ginster . . . . . . . . . . . . . . . . . . . . . . . . 496

New results on Cahn-Hilliard-Navier-Stokes systems with nonlocal interactions

Frigeri . . . . . . . . . . . . . . . . . . . . . . . 497

Wulff shape and isoperimetric characterization of crystals

Piovano . . . . . . . . . . . . . . . . . . . . . . . . . . . . . 498

Effective Maxwell equations in a geometry with flat rings of arbitrary shape

Lamacz - Schweizer . . . . . . . . . . . . . . . . . . . . . . . . . . . . . . . 499

A line-tension energy for dislocation networks on several slip planes

Gladbach . . . . . . . . . . . . . . . . . . . . . . . . 500

S15: Applied stochastics

Efficient Bayesian analysis of rare events in numerical models

Straub - Betz - Papaioannou . . . . . . . . . . . . . . . . . . . . . . . . . . . . .502

Polynomial chaos approach for tuned bladed rotors with geometric modification

Koebelé-Cousquer - Proppe . . . . . . . . . . . . . . . . . . . . . . . . 503

Damage models described by uncertain parameters

Sarfaraz - Matthies . . . . . . . . . . . . . . . . . . . . . . . . . . . . . 504

Uncertainty quantification for linear elastic bodies with fluctuating input parameters

Dridger - Caylak - Mahnken . . . . . . . . . . . . . . . . . . . . . . 505

Probabilistic Sensitivities for Fatigue Using Adjoint Methods

Saadi . . . . . . . . . . . . . . . . . . . . . . . . . . 506

Irreversible material behaviour under presence of uncertainty

Rosic - Matthies . . . . . . . . . . . . . . . . . . . . . . . . . . 507

Jump Phenomena and Bifurcations in Stochastic Vehicle-Road Dynamics

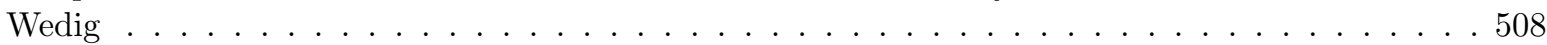

Regional Frequency Analysis of extreme rainfall events in Tuscany (Italy)

Chiarello - Caporali - Matthies 
On Gaussian approximation of the strength of Daniels' bundle with brittle Weibull fibers

Sadilek - Vorechovsky . . . . . . . . . . . . . . . . . . . . . . . . .510

Best practice in metamodeling for data derived from civil engineering applications

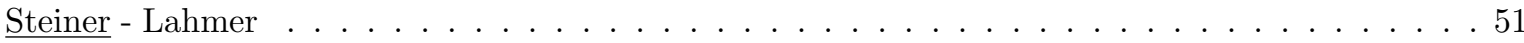

Asymptotic sampling - a tool for efficient reliability computation in high dimensions

Bucher . . . . . . . . . . . . . . . . . . . . . . . . . . . 513

Polynomial Chaos and the Heave Motion of a Cylinder in Random Seas

Solowjow - Kreuzer . . . . . . . . . . . . . . . . . . . . . . . . . . . 514

Stochastic quantification of the impact of uncertainty in inlet conditions on the aerodynamics of a $5: 1$ rectangular cylinder

Mariotti - Salvetti - Shoeibi Omrani - Witteveen . . . . . . . . . . . . . . . . . . . 515

A Bayesian approach for the quantification of parametric and model-form uncertainties in densegas flow models

Merle - Cinnella . . . . . . . . . . . . . . . . . . . . . . . . . . . . . . . . . . . .

Investigation of cavitation and turbulence models uncertainties for cavitating flows

Congedo - Rodio - Goncalves . . . . . . . . . . . . . . . . . . . . . . . . . 517

Influence of Uncertainties of the Aerodynamical Parameters on the Simulation Model for a Civil Aircraft with Active High Lift System

Friedman - Diekmann - Matthies . . . . . . . . . . . . . . . . . . . . . 518

Stochastic quantification of the effects of inlet velocity conditions on the dynamics of spatially evolving mixing layers

Meldi - Sagaut - Salvetti

Solution of the First passage problem by Path Integration for normal, Poissonian, and alphastable white noise

Di Paola - Pirrotta - Bucher . . . . . . . . . . . . . . . . . . . . . . . . .520

Adaptation and Enhancement of Generalized Polynomial Chaos for Industrial Applications

Glaser - Petridis - Heuveline . . . . . . . . . . . . . . . . . . . . . . . . . . . . . 522

S16: Optimization

Construction of a Magnet as an Absolute Positioning Scale

Mojsic - Sokol - Ludszuweit - Fügenschuh . . . . . . . . . . . . . . . . . . . . . . 524

Control of water reservoirs aeration process

Abdelwahed . . . . . . . . . . . . . . . . . . . . . . . . .526

Virtual Process Design in Combined Electromagnetic-Classical Forming Processes: Optimization of Current Parameters

Rozgic - Kiliclar - Stiemer - Reese . . . . . . . . . . . . . . . . . . . . . . . . . . 527

Methods with Successive and Parallel Approximations of Inverse Operators for the Nonlinear Least Squares Problem

Iakymchuk - Shakhno

Geometry Optimization of Branched Sheet Metal Structures with a Globalization Strategy by Adaptive Cubic Regularization

Göllner - Ulbrich . . . . . . . . . . . . . . . . . . . . . . . . . . . 529 
Multidimensional parametrization of microcells in two scale optimization with sparse grid interpolation

Hübner - Stingl

A staggered approach to structural shape and topology optimization

Riehl - Steinmann . . . . . . . . . . . . . . . . . . . . . . . . . . 531

On curvature control in node-based shape optimization

Schmitt - Steinmann . . . . . . . . . . . . . . . . . . . . . . . . . 532

Optimizing Extrusion Dies with Profile Shape as an Objective Function

Siegbert - Behr - Elgeti . . . . . . . . . . . . . . . . . . . . 5 533

Robust optimization of trusses under dynamic loads via nonlinear semidefinite programming

Kuttich - Ulbrich . . . . . . . . . . . . . . . . . . . . . . . . . . 534

About the design of morphing airfoils under uncertainties

Fusi - Quaranta - Guardone - Congedo . . . . . . . . . . . . . . . . . . . . . . . . . 535

Robust Design using classical optimization

Erschen - Zimmermann . . . . . . . . . . . . . . . . . . . . . . 537

Optimization of the double sided spiral groove thrust bearing: A comparison to approximate analytical solutions

$\underline{\text { Lehn }}$ - Schweizer

Multi-criteria optimisation of the vibro-isolation properties

Krzyżyński - Maciejewski . . . . . . . . . . . . . . . . . . . . 539

S17: Applied and numerical linear algebra

Designing rational filter functions for solving eigenvalue problems by contour integration

Van Barel . . . . . . . . . . . . . . . . . . . . . . . . . . . . . . . 541

Parallel Bidiagonal SVD via the Method of Multiple Relatively Robust Representations

Winkelmann - Bientinesi . . . . . . . . . . . . . . . . . . . . . . 543

A Hessenberg reduction algorithm for diagonal plus low rank matrices

Robol - Bini . . . . . . . . . . . . . . . . . . . . . . . . . . . . . . . . . . . . . 544

An extended Hessenberg form for Hamiltonian matrices

Ferranti - Iannazzo - Mach - Vandebril . . . . . . . . . . . . . . . . . . . . . . . . . . 545

Rank-revealing decomposition via block anti-triangular factorization

Mastronardi - Van Dooren . . . . . . . . . . . . . . . . . . . . . . . . . . 546

On complex $J$-symmetric eigenproblems

Fassbender - Yang - Benner . . . . . . . . . . . . . . . . . . . . . . . . . . 547

Nonlinear eigenvalue problem expressed in Hermite basis

Shayanfar - Fassbender . . . . . . . . . . . . . . . . . . . . . . . . . . . 548

Backward error of polynomial eigenvalue problems solved by linearization

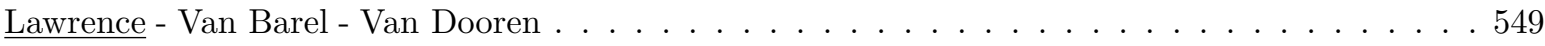

IgA vs. FEA in the Spectral Approximation: Symbol-Based Analysis

Garoni - Hughes - Manni - Reali - Serra-Capizzano - Speleers . . . . . . . . . . . . . . . . . . . . . 550

How to compute efficiently the Markovian Joint Spectral Radius?

Cicone - Guglielmi - Protasov . . . . . . . . . . . . . . . . . . . . . . . . . 552 
On Krylov subspace methods for the time-fractional Schrödinger equation

Stability-Preserving Parametric Model Reduction by Matrix Interpolation using Invariance Properties of Krylov Subspaces

Barthlen - Lang .

Comparison of polynomial Krylov methods with limited memory consumption for approximating Stieltjes matrix functions

Schweitzer - Güttel

Orthogonal projection vs. Oblique projection in Krylov subspace recycling

Bozovic - Bolten - Frommer . . . . . . . . . . . . . . . . . . . . . . 556

A new approach for preconditioning discontinuous Galerkin discretizations

Hajian - Gander . . . . . . . . . . . . . . . . . . . . . . . . . . . 557

Rational least squares approximation via RKFIT

Güttel - Berljafa . . . . . . . . . . . . . . . . . . . . . . . . . . . . . . . 558

A fast nonstationary preconditioning strategy for ill-posed problems, with application to image deblurring

$\underline{\text { Donatelli }}$

Iterated fractional Tikhonov regularization

Bianchi - Buccini - Donatelli - Serra-Capizzano

Spectral behavior of preconditioned non-Hermitian multilevel block Toeplitz matrices with matrixvalued symbol

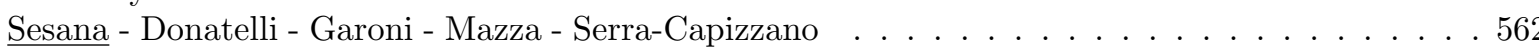

Local Fourier Analysis of Pattern Structured Operators

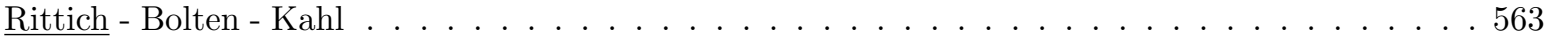

Fast Recovery and Approximation of Hidden Cauchy Structure

Luce - Liesen . . . . . . . . . . . . . . . . . . . . . . . . . . . . . . . 564

Parallel Tensor Sampling

Grasedyck - Loebbert

Multigrid methods for tensor structured problems

Sokolovic - Bolten - Kahl . . . . . . . . . . . . . . . . . . . . . . . . 566

Hierarchical tensor approximation of parameter-dependent PDEs

Ballani . . . . . . . . . . . . . . . . . . . . . . . . . . 567

On the block and global methods for linear systems with multiple right hand sides

Rashedi - Frommer - Ebadi . . . . . . . . . . . . . . . . . . . . . 568

S18: Numerical methods for differential equations

Well-Balanced, Entropy Stable Discontinuous Galerkin Spectral Element Method for the Shallow Water Equations

Winters - Gassner .

An Enhanced Time-Discontinuous Galerkin Method for Rotating Geometries such as Artificial Blood Pumps

Hassler - Pauli - Behr . . . . . . . . . . . . . . . . . . . . . . . . . . . . . 571 
A posteriori error estimates for IMEX schemes

Giesselmann . . . . . . . . . . . . . . . . . . . . . . . . . . . . . . 572

Well balanced ALE: on time dependent mesh adaptation for Shallow Water flow

Arpaia - Ricchiuto . . . . . . . . . . . . . . . . . . . . . 573

Computation of state variables of one-dimensional non-stationary pipe flow at orifices on pipe ends

Niessner

An adaptive space-time discontinuous Galerkin method for Maxwell's equations

Wieners - Findeisen - Dörfler

Tracking-Type Finite-Volume Schemes for Phase Transition Problems

Wiebe . . . . . . . . . . . . . . . . . . . . . . . 576

Coupling Surface and Subsurface Flows

Magiera

Multigrid preconditioning for time-periodic Navier-Stokes problems

Hupp - Obrist - Arbenz . . . . . . . . . . . . . . . . . . . . . . 578

Online Parameter Identification for Traffic Simulation via Lagrangian Sensing

Thonhofer - Jakubek . . . . . . . . . . . . . . . . . . . . . . . 579

Robust Identification of Parametric Radiation Force Models via Impulse Response Fitting

Hatecke - Krüger . . . . . . . . . . . . . . . . . . . . . . . . . . . . . . . . . 580

Numerical simulation for wine fermentation based on IDEs

Schenk - Schulz . . . . . . . . . . . . . . . . . . . . . . . . . . . 581

Improving real-time capability of linearly-implicit solvers using structural information

Loderer - Bertsch - Heuveline . . . . . . . . . . . . . . . . . . . . . . . . . . . . 582

Interpolatory Model Reduction for Quadratic Bilinear Descriptor Systems

Ahmad - Feng - Benner . . . . . . . . . . . . . . . . . . . . . . . . 583

A joint IMEX-MOR approach for Water Networks

Jansen - Grundel . . . . . . . . . . . . . . . . . . . . . . . . . . . . . . . . . . 584

Upwind Based Parameter Uniform Convergence Analysis for Two Parametric Parabolic Convection Diffusion Problems by Moving Mesh Methods

Das - Mehrmann . . . . . . . . . . . . . . . . . . . . . . . . 586

Adaptive FEM with goal-oriented error estimation and an approximation of the dual problem for inelastic problems

Widany - Mahnken

An Adaptive Local Basis for Elliptic Problems with Complicated Discontinuous Coefficients

Weymuth - Sauter . . . . . . . . . . . . . . . . . . . . . 588

Error measurement and enhanced FEM for phase field modeling

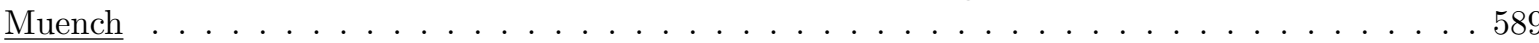

Interior penalty finite element methods for high-order local boundary conditions

Schmidt - Diaz - Heier . . . . . . . . . . . . . . . . . . . . . . . . . . . 590

Generalized approximated regular boundary element method

Yevdokymov 
Boundary element application to non-linear boundary integral equations

Poliakov . . . . . . . . . . . . . . . . . . . . . . . . . . 592

Boltzmann collision operator: How to model rotational invariance

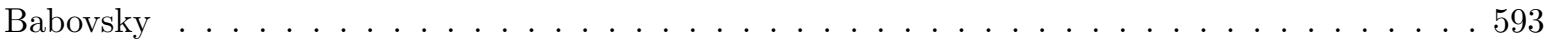

Fully-Implicit Log-Conformation Formulation of Viscoelastic Constitutive Laws

Knechtges - Behr - Elgeti . . . . . . . . . . . . . . . . . . . . . . . . 594

Approximate exponential time integration for diffusion problems

Sun - Manhart . . . . . . . . . . . . . . . . . . . . . . . . . . . 595

On multi-dimensional discrete equations of convolution type

Vasilyev - Vasilyev . . . . . . . . . . . . . . . . . . . . . . . 596

\section{S19: Optimization of differential equations}

Two Types of Globally Convergent Numerical Methods for Coefficient Inverse Problems

Klibanov . . . . . . . . . . . . . . . . . . . . . . . . . . 598

A Riemannian SQP method for PDE constrained shape optimization

Welker - Schulz - Siebenborn

A second order convergent trial method for free boundary problems in three dimensions

Harbrecht - Bugeanu . . . . . . . . . . . . . . . . . . . . . . . . . . . . 601

Primal-Dual Active Set Strategy for Singular Optimal Control Problems

Fischer - Bechmann . . . . . . . . . . . . . . . . . . . . . . . . . . 602

Adaptive Optimal Control of the Obstacle Problem

Wollner - Meyer - Rademacher . . . . . . . . . . . . . . . . . . . . . . 603

Optimal Control of a linear unsteady Fluid-Structure Interaction Problem

Failer - Meidner - Vexler . . . . . . . . . . . . . . . . . . . . . . . . . . . . . . . 604

Parabolic optimal control problems with pointwise controls

Leykekhman - Vexler . . . . . . . . . . . . . . . . . . . . . . . . 605

Optimal Control of Signorini Contact Problems

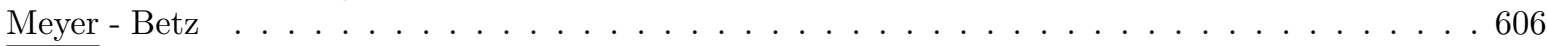

Frequency-sparse Control of Bilinear Quantum Systems

Henneke - Frisecke - Kunisch . . . . . . . . . . . . . . . . . . . . . . . 607

Looking for strictly positive solutions of elliptic PDEs

Schiela . . . . . . . . . . . . . . . . . . . . . . . . 608

Time optimal control for the monodomain equations - a monolithic approach

Pieper - Kunisch - Rund

The optimal shape of a pipe

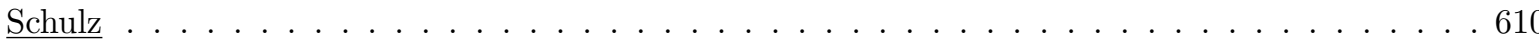

Topological derivative for nonlinear magnetostatics

Gangl - Amstutz - Langer . . . . . . . . . . . . . . . . . . . . . . . . . . . . .611

Multiple state optimal design problems with random perturbation

Vrdoljak . . . . . . . . . . . . . . . . . . . . . . . 612 
Robust methods and adaptivity for the numerical solution of variational inequalities for phasefield-based fracture problems

Wick .

A Bridge from State-constrained ODE Optimal Control to State-constrained elliptic PDE Optimal Control: New Necessary Condtions, New Optimization Problems, and New Numerical Methods

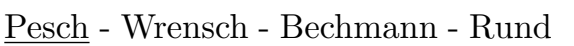

Optimal control for lithium-ion batteries

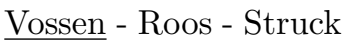

Numerical approximation of a quasistatic evolution problem in cohesive fracture

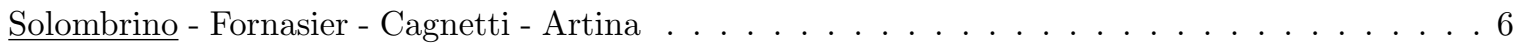

Sparse optimal control of the KDV equation

Boulanger - Trautmann - Vexler

Traffic flow control: avoiding shocks via variable speed limit

Delle Monache - Piccoli - Rossi

Luré dynamical systems - existence and stability results

Gwinner . . . . . . . . . . . . . . . . . . . . . . . . . . . . . 619

A smooth and localized version of the Hughes model for pedestrian flow

Martin - Carrillo - Wolfram . . . . . . . . . . . . . . . . . . . 620

On Modelling, Simulation and Optimisation of Solar Updraft Towers with Sloped Collectors

Gasser - Kamboh . . . . . . . . . . . . . . . . . . . . . . . . . . 621

The identifiability approach for time-dependent full waveform inversion

Kourounis - Schenk . . . . . . . . . . . . . . . . . . . . . . . . . . . .622

Local minimization algorithms for dynamic programming equations

Kröner - Kalise - Kunisch . . . . . . . . . . . . . . . . . . . . . . . . . . . . . . . 623

Optimization of Multirate Partial Differential Algebraic Equations

Pulch - Kugelmann . . . . . . . . . . . . . . . . . . . . . . . . . . . . . 624

A priori error estimates for nonstationary optimal control problems with gradient state constraints

Ludovici - Neitzel - Wollner . . . . . . . . . . . . . . . . . . . . . . . . . . . . 625

Direct and indirect multiple shooting for parabolic optimal control problems

Carraro - Geiger . . . . . . . . . . . . . . . . . . . . . . 626

Functional a posteriori estimates for cost functionals of elliptic optimal control problems

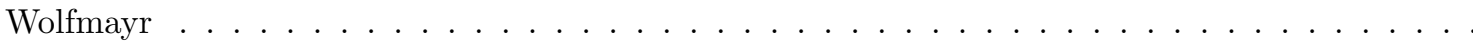

Multiobjective Optimization of the Flow Around a Cylinder Using Model Order Reduction

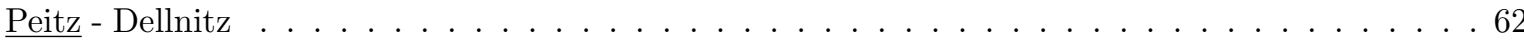

A Certified Reduced Basis Approach for Parametrized Linear-Quadratic Optimal Control Problems with Control Constraints

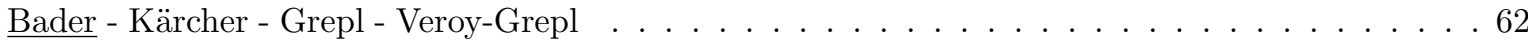

Wavelet-based lossy trajectory compression for optimal control of parabolic PDEs

Goetschel - Weiser . . . . . . . . . . . . . . . . . . . . . . . . 631

S20: Dynamics and control 
Control design of the vibration reduction systems

Maciejewski - Krzyzynski

Coordination of guidance and stabilization tasks for bicycle rider modelling

Edelmann - Haudum - Plöchl . . . . . . . . . . . . . . . . . . . . . . . . 634

Parameter identification of a scaled experimental running gear

Keck - Schwarz . . . . . . . . . . . . . . . . . . . . . . . . . 635

Nonlinear Control of a Variable Displacement Vane Pump

Koester - Fidlin . . . . . . . . . . . . . . . . . . . . . . 636

A kinematic approach based on an equivalent track for a skid-steering robot

Galati - Giannoccaro - Messina - Reina . . . . . . . . . . . . . . . . . . . . . 637

Harmonic Mistuning of Blisks

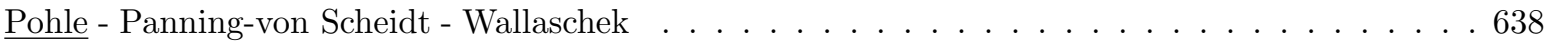

Stability analysis of implicit difference equations

Mehrmann - Thuan . . . . . . . . . . . . . . . . . . . . . . 639

Controllability characterization for switched DAEs

Trenn - Küsters . . . . . . . . . . . . . . . . . . . . . . . . . . . . . . . . . . 640

The Kalman-Yakubovich-Popov Inequality for Differential-Algebraic Equations

Voigt - Reis - Rendel . . . . . . . . . . . . . . . . . . . . . . . . . . 641

Controlled invariance for DAEs

Berger . . . . . . . . . . . . . . . . . . . . . . . . 642

Control of underactuated systems with coupling input forces

Korczak . . . . . . . . . . . . . . . . . . . . . . . . . . . . . 643

Stabilization using discounted optimal control problems

Gruene - Gaitsgory - Thatcher . . . . . . . . . . . . . . . . . . . . . . . . . . . . . . . . . . . 644

Economic Model Predictive Control under Bounded Disturbances

Bayer - Allgöwer . . . . . . . . . . . . . . . . . . . . . . . . . . . 645

Approximate linear programming for optimal control design: a solution based on function approximation and randomization

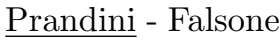

Multi-objective optimal control of fluid mixing

Ober-Blöbaum - Padberg-Gehle

An optimal junction solver for traffic flow

Garavello - Ancona - Cesaroni - Coclite

Vanishing dielectric constant regime for the Navier Stokes Maxwell equations

Spirito - Donatelli . . . . . . . . . . . . . . . . . . . . . . . . . 650

Schaeffer's regularity theorem and the case of systems

Caravenna - Spinolo . . . . . . . . . . . . . . . . . . . . . . 651

Minimal data rates and entropy in digitally networked systems

Kawan . . . . . . . . . . . . . . . . . . . . . . . . . . . . 652 
Reachable states of a quasilinear hyperbolic control system: an application to particulate processes

Zuyev

Automated generation of a dynamic feedforward control law using local model networks with disturbance inputs

Euler-Rolle - Hametner - Jakubek . . . . . . . . . . . . . . . . . . . . . . . . . . . . 654

Structure-preserving discrete-time LQR problems

Flaßkamp - Murphey . . . . . . . . . . . . . . . . . . . . . . . . 655

Reconstruction of independent sub-domains for a class of Hamilton-Jacobi equations and application to parallel computing

Festa . . . . . . . . . . . . . . . . . . . . . . . . . 65

S21: Mathematical image processing

On learning reaction diffusion models

Pock - Chen . . . . . . . . . . . . . . . . . . . . . . . . . . . . 658

Adaptive Total Variation Regularization

Lenzen - Lellmann - Becker - Petra - Schnoerr . . . . . . . . . . . . . . . . . . . . . . . . . . . . .659

Joint Motion Estimation and Image Reconstruction

Dirks - Burger - Schönlieb . . . . . . . . . . . . . . . . . . . . . . . . . . . 660

Regularization methods for flow fields with smooth transitions and sharp edges

Frerking - Burger - Vestweber - Brune . . . . . . . . . . . . . . . . . . .661

Regularisation by Circular Hough Transform

Grah - Burger - Schoenlieb . . . . . . . . . . . . . . . . . . . . . 662

Artifact-free variational MPEG decompression

Holler - Bredies . . . . . . . . . . . . . . . . . . . . . . . . . . . . 663

Cartoon-Texture-Noise Decomposition with Transport Norms

Brauer - Lorenz . . . . . . . . . . . . . . . . . . . . . . . . . . . . . . . . . . . . . . . 664

Asymptotics of Spatial Sparsity Priors

Heins - Burger . . . . . . . . . . . . . . . . . . . . . . . 665

Image Warping via Optimal Transport with Sources

Simon - Maas - Rumpf . . . . . . . . . . . . . . . . . . . . . . . . . .666

Non-rigid registration for pre-operative $3 \mathrm{D}$ surfaces and intra-operative $2.5 \mathrm{D}$ surfaces

Tatano - Berkels . . . . . . . . . . . . . . . . . . . . . . . 667

Active-contours for image segmentation relying on non-stationary subdivision schemes

Romani - Novara - Schmitter - Uhlmann - Unser . . . . . . . . . . . . . . . . . . . . . . 668

Deterministic Sparse FFT Algorithms

Plonka - Wannenwetsch . . . . . . . . . . . . . . . . . . . . . 669

Reconstruction of Multivariate Exponential Functions from Measurements

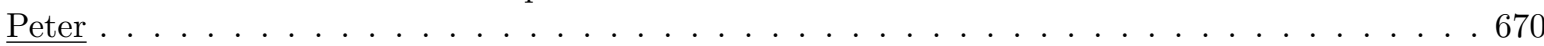

Ambiguities in one-dimensional discrete phase retrieval from Fourier magnitudes

Beinert - Plonka . . . . . . . . . . . . . . . . . . . . . . . 671 
Optimal mollifiers for the reconstruction of spherical images from circular means

Hielscher - Quellmalz . . . . . . . . . . . . . . . . . . . . . . . . . . . . . 672

A robust estimation method for camera calibration with known rotation.

Egozi - Maass - Sagiv . . . . . . . . . . . . . . . . . . . . . . . 673

Harmonic analysis of projectors

Ehler - Graef . . . . . . . . . . . . . . . . . . . . . . . . . . . 674

Perturbations of frame sequences and the effect on their duals

Philipp - Kutyniok - Paternostro . . . . . . . . . . . . . . . . . . . . 675

Construction of Multichannel Wavelets via Full Rank Subdivision Schemes

Conti - Cotronei - Sauer . . . . . . . . . . . . . . . . . . . . . . . . . 676

Shearlet-Based Edge Detection: Flame Fronts and Tidal Flats

King - Reisenhofer - Kiefer - Li - Heygster - Lim . . . . . . . . . . . . . . . . . . . . . . 677

Classification of Edges Using Compactly Supported Shearlets

Petersen - Kutyniok . . . . . . . . . . . . . . . . . . . . . . . 678

Approximation in variation for nonlinear Mellin integral operators

Angeloni - Vinti . . . . . . . . . . . . . . . . . . . . . . . . . . . . 679

Multivariate sampling Kantorovich operators: from the theory to the Digital Image Processing algorithm

Costarelli - Vinti

Stockwell Transform and Applications to Image Analysis

Battisti - Pirro - Riba - Sambuelli . . . . . . . . . . . . . . . . . . . . . . . . . . . 683

Applications of Approximation Theory to thermographic images in earthquake engineering

Minotti - Cluni - Costarelli - Vinti . . . . . . . . . . . . . . . . . . . . . . . . . 684

A generalization of the Zak transform with applications in sampling theory and physics Jüstel . . . . . . . . . . . . . . . . . . . . . . . . . . . . . . . . . 685

Multivariate Generalized Sampling Type Series: estimates of pointwise convergence

Bardaro - Mantellini . . . . . . . . . . . . . . . . . . . . . . 686

A New Blind Source Separation Numerical Technique

Gerace - Scognamiglio . . . . . . . . . . . . . . . . . . . . . . 687

Geometric Means of Toeplitz Matrices by Positive Parametrizations

Iannazzo - Bini - Serra Capizzano . . . . . . . . . . . . . . . . . . . . . . . . . 688

A unifying theory for convergence of linear sampling operators in Orlicz spaces

Zampogni - Vinti . . . . . . . . . . . . . . . . . . . . 689

Digital image processing algorithms for diagnosis in arterial diseases

Seracini - Costarelli - Vinti . . . . . . . . . . . . . . . . . . . . . . . . . . . . . 691

Nonnegative Tensor Grid Decomposition

Jeuris - Iannazzo - Pompili . . . . . . . . . . . . . . . . . . . . . . . . . . . . . . . . . . . . 693

Poisson Noise Removal from High-Resolution Electron Micrographs based on periodic Blockmatching

Mevenkamp - Binev - Dahmen - Voyles - Yankovich - Berkels 
Application of learning algorithms for colour recognition on underwater images

Hoth - Kowalczyk . . . . . . . . . . . . . . . . . . . . . . . 695

Edge detection based on fractional order differentiation and its application to railway track images

Telke - Beitelschmidt . . . . . . . . . . . . . . . . . . . . . 697

S22: Scientific computing

Micro-macro parareal methods for multiscale problems

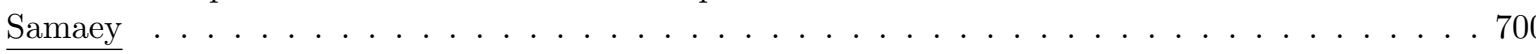

Adaptive space time finite element methods for dynamic nonlinear thermomechanical coupled problems

Rademacher . . . . . . . . . . . . . . . . . . . . . . . 701

Parallel simulation of large scale multibody systems

Kloeppel - Naumann - Waurich - Walther - Wensch . . . . . . . . . . . . . . . . . . . 702

Simulation of coupled machine components on long time scales

Naumann - Klöppel - Wensch . . . . . . . . . . . . . . . . . . . . . . . . 703

Boris-SDC: A high-order Boris integrator

Speck - Winkel - Ruprecht . . . . . . . . . . . . . . . . . . . . . . . . . 704

Efficient Nested Chebyshev Smoothing

Sanan - Schenk . . . . . . . . . . . . . . . . . . . . . . . . 705

Rayleigh Quotient Inverse Iteration with Adaptive Algebraic Multigrid for Lattice QCD

Rottmann . . . . . . . . . . . . . . . . . . . . 706

An Auxiliary Space Type Preconditioner for Simulations in Lattice QCD

Frommer - Brannick - Kahl - Leder - Rottmann - Strebel . . . . . . . . . . . . . . . . . . . . 707

A GPU implementation of the Factored Sparse Approximate Inverse preconditioner for the iterative solution of SPD linear systems

Janna - Bernaschi - Bisson - Fantozzi . . . . . . . . . . . . . . . . . . . . . . 708

Finite Volume Methods for Sound-Advection-Buoyancy Systems

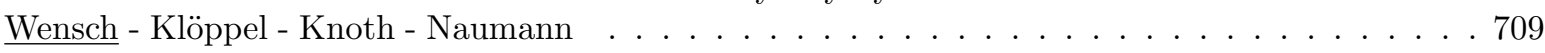

HPC methods for structured inverse modeling in diffusive processes

Siebenborn - Schulz . . . . . . . . . . . . . . . . . . . . . . . . . . 710

Using Automatic Differentiation to Create Sparse Jacobians for the Solution of Nonlinear Partial Differential Equations

Zwicke - Knechtges - Behr - Elgeti . . . . . . . . . . . . . . . . . . . . . . . . 711

Multiple-output variable fidelity modeling of vehicle aerodynamics under geometric shape variations

Sauerbrei - Zimmermann . . . . . . . . . . . . . . . . . . . . . . . . . . 712

Sensitivities calculations for unsteady flow problems

Stoia-Djeska - Frunzulica . . . . . . . . . . . . . . . . . . . . . . . . . . . 713

EWE - A coupled electro-mechanical heart model in the general purpose FEM framework MOOSE

Ruprecht - Winkel - Krause . . . . . . . . . . . . . . . . . . . . . . . . . . . . 714

Enriching Finite Elements with meshless nodes in structural mechanics

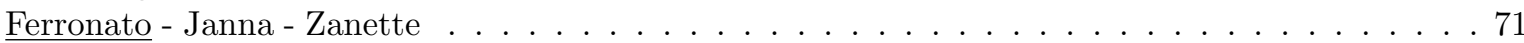


Simulations of turbulent Rayleigh-Bénard convection with a spectral element method

Kooij - Botchev - Geurts . . . . . . . . . . . . . . . . . . . . . . . 716

A fully meshless method for 'gas - evaporating droplet' flow modelling

Rybdylova - Osiptsov - Sazhin - Begg - Heikal . . . . . . . . . . . . . . . . . . . 717

The Qualitative Analysis and the Critical Hypersurfaces of Stationary Elliptic PDEs

Nastase . . . . . . . . . . . . . . . . . . . . . . . . . . 718

S23: Applied operator theory

Squeezing of arbitrary order

Szafraniec . . . . . . . . . . . . . . . . . . . . . . . . 720

Lebesgue type decompositions and Radon-Nikodym derivates for unbounded linear operators and relations

de Snoo

Remarks on the convergence of pseudospectra

Siegl - Bögli . . . . . . . . . . . . . . . . . . . . . . . . . . . . . . 722

Recent results on functional calculus for Tadmor-Ritt operators

Schwenninger - Zwart . . . . . . . . . . . . . . . . . . . . . . . . . . . . 723

A functional analytic look upon Remling's oracle theorem

Vogt - Seifert . . . . . . . . . . . . . . . . . . . . . . . . . 724

On Abstract grad-div Systems

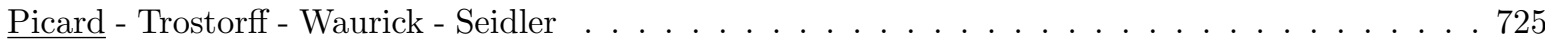

Eigenfunction expansions associated with the one-dimensional Schrödinger operator

Gilbert . . . . . . . . . . . . . . . . . . . . . . . . . . 726

Collocation-quadrature methods and fast summation for Cauchy singular integral equations with fixed singularities

Kaiser - Junghanns - Potts . . . . . . . . . . . . . . . . . . . . . . . . . . . 727

Exponential stability of a second order integro-differential equation with delay

Trostorff . . . . . . . . . . . . . . . . . . . . . . . . . . . . . 728

The div M grad-operator without ellipticity

Kostrykin - Hussein - Krejcirik - Makarov - Schmitz . . . . . . . . . . . . . . . . . . . . . . . . . 729

Rational matrix solutions of a Bezout type equation on the half plane

Ran - Frazho - Kaashoek . . . . . . . . . . . . . . . . . . . . . . . . . . .730

On the trace class property of the resolvent regularization of a Dirac-type operator on $R^{3}$

Waurick - Gesztesy . . . . . . . . . . . . . . . . . . . . . . . 731

On the spectrum of non-selfadjoint operators over dynamical systems

Seifert . . . . . . . . . . . . . . . . . . . . . . . . 732

Uniform mean ergodicity of C0-semigroups in a class of Frechet spaces

Albanese - Bonet - Ricker . . . . . . . . . . . . . . . . . . . . . . . . . . 733

On deformations of classical Jacobi matrices

Wojtylak . . . . . . . . . . . . . . . . . . . . . . . 734

S24: History of mechanics 
The change of perspective with the advent of Quantum Mechanics

Esposito . . . . . . . . . . . . . . . . . . . . . . 736

Walter Noll and the Bourbakization of Mechanics

Del Piero . . . . . . . . . . . . . . . . . . . . . . . 737

Beltrami and mathematical physics in non-Euclidean spaces

Capecchi - Ruta . . . . . . . . . . . . . . . . . . . . . . 738

Gustav R. Kirchhoff and the dynamics of tapered beams

Cazzani . . . . . . . . . . . . . . . . . . . . . . . 739

On the Derivation of the Equations of Hydrodynamics 65 years after Irving\&Kirkwood

Podio-Guidugli . . . . . . . . . . . . . . . . . . . . . . . . . . . . . 740

How does dynamic complexity contribute to the advancement of mechanics

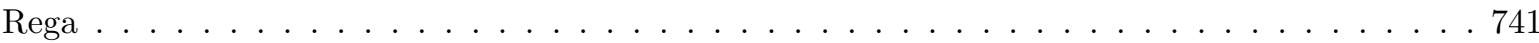

On the role of virtual work in Levi-Civita's parallel transport

Ruta - Iurato . . . . . . . . . . . . . . . . . . . . . . . . . . . 743

Nineteenth century molecular models with a glance at modern discrete-continuum theories

Trovalusci . . . . . . . . . . . . . . . . . . . . . . . . . . . . . . . 744

The discovery of the vector representation of moments and angular velocity $(1750-1830)$

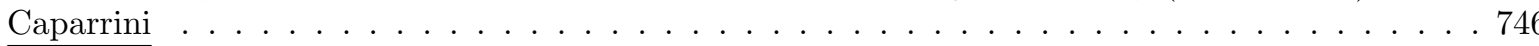

Domenico Fontana and the entrance of mechanics in architecture

Tocci - Masiani . . . . . . . . . . . . . . . . . . . . . . . . . . . 747

On the graphic statics for the analysis of masonry domes

Cavalagli - Gusella . . . . . . . . . . . . . . . . . . . . . . . . . . 748

G. W. Leibniz's "Machina Deciphratoria", the first described cypher machine from the 17th century, constructed and built in 2013/14 for the Leibniz Exhibition of the Leibniz Universitat Hannover

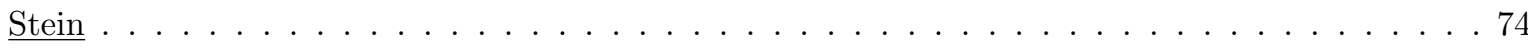


PRL

PRL: Prandtl Lecture

Prandtl Lecture 


\title{
Discontinuities and topological jumps in slowly evolving vortical flows
}

\author{
$\underline{\text { Keith Moffatt }}$ \\ University of Cambridge, Dept of Applied Mathematics and Theoretical Physics \\ h.k.moffatt@damtp.cam.ac.uk
}

The idea that "vortex lines are frozen" in the flow of an ideal fluid goes back to Helmholtz (1858). However, singular structures such as vortex sheets or filaments are a characteristic feature of such flows, and viscous reconnection of vortex lines can occur in the neighbourhood of such singularities. A model for viscous reconnection suggested by the recent experimental observations of knotted vortex tubes of Kleckner \& Irvine (2013) [Nature Phys. 9, 253-258] will be described. A parallel investigation of the response of a soap-film to slow boundary deformation shows that rapid topological jumps can occur here also. The jump of a Möbius-strip soap film from one-sided to two-sided topology provides an intriguing illustration of what is involved in such transitions.

\section{References}

[1] Kimura, Y. \& Moffatt, H. K. Reconnection of skewed vortices. J. Fluid Mech. 751, 329-345, (2014).

[2] Goldstein, R. E., McTavish, J., Moffatt, H. K. \& Pesci, A. I. Boundary singularities produced by the motion of soap films. Proc. Natl. Acad. Sci. 111 (23), 8339-8344, (2014). 
PBL

PBL: Public Lecture

Public Lecture 


\section{Do the Gates to the Hell really exist?}

$\underline{\text { Francesco D'Andria }}$

Università del Salento, Dept of Cultural Heritage - francesco.dandria@unisalento.it

For human beings, natural phenomena such as earthquakes, volcanoes, thermal springs and gas emissions are a powerful engine for the creation of myths, arousing wonder and raising important questions. Together with the recent archaeological discoveries, the disciplines of geology, seismology and organic chemistry can today provide an answer to the question of whether there really is an entrance to the Underworld. 
PLL

PLL: Plenary Lectures

Plenary Lectures 


\title{
On the Motion of a Rigid Body with a Liquid-filled Cavity
}

\author{
Giovanni P. Galdi \\ Department of Mechanical Engineering and Materials Science, University of Pittsburgh, USA
}

Let $\mathcal{S}$ be the coupled system constituted by a rigid body with an interior cavity completely filled with a viscous liquid. In this talk I shall present a number of analytical results concerning the motion of $\mathcal{S}$ around its center of mass, with or without the action of external forces. In particular, I show that the presence or absence of the liquid in the cavity can dramatically affect the ultimate dynamics of $\mathcal{S}$. I will also present suitable numerical tests that, on the one hand, corroborate the mathematical analysis and, on the other hand, pose new questions that lay the foundations for future analytic work.

\section{References}

[1] G.P. Galdi, G. Mazzone, P. Zunino, Inertial Motions of a Rigid Body with a Cavity Filled with a Viscous Liquid, Comptes Rendus Mécanique, 341 (2013) 760-765.

[2] G.P. Galdi, G. Mazzone, On the Motion of a Pendulum with a Cavity Entirely Filled by a Viscous Liquid., submitted 


\title{
Micromechanics based modeling of applied materials
}

\author{
Thomas Böhlke \\ Chair for Continuum Mechanics, Karlsruhe Institute of Technology (KIT)
}

Nowadays, micromechanical approaches and homogenization techniques are standard research methods in mechanical engineering and materials science. This is due to the increased computer capabilities and the better characterization techniques for both the material microstructures and the micro-constitutive behavior. In contrast to phenomenological approaches, homogenization techniques allow to take into account the material microstructures and the resulting fluctuations in the stress and deformation fields. From the perspective of applied mechanics, crucial issues are the ability of the scale bridging methods to reproduce experimental data and to be applied in fully coupled two-scale simulations. Additionally, the accessibility of necessary microstructure and material data and the applicability of standard software tools are of importance. In the talk, different types of materials are considered, and the aforementioned issues are addressed.

In the first part of the presentation, fiber reinforced polymers (FRP) are considered. These structures are increasingly used for different kinds of applications. A robust dimensioning of light-weight structures made of these materials is still a challenging task. FRP show heterogeneities with respect to micro-structural properties like the fiber volume fraction and the fiber length as well as the orientation distributions. As an example, the microstructure of injection-molded specimens made of polypropylene reinforced with $30 \mathrm{wt} \%$ of short glass fibers (PPFG30) is modeled based on micro-computer tomography data. The elastic and the thermoelastic properties are predicted based on different mean field approaches. The estimates are evaluated with macroscopic testing data. Additionally, the mechanical properties of long fiber reinforced thermoplastics which depend on the heterogeneous fiber orientation distribution induced through manufacturing on a direct LFT line, are predicted for compression molded rectangular plates. Fiber orientation tensors based on measured microcomputed tomography data of selected samples as well as on filling simulations are used for the determination of mechanical properties. The results are evaluated with dynamic mechanical analysis measurements.

In the second part of the presentation micromechanics is applied to metals. As an example, automotive dualphase steels are modeled. Within the Hashin-Shtrikman mean-field approach, the load-transfer from ferrite to martensite is captured based on simple microstructural characteristics in a physically motivated manner. The employed Estrin-Kocks-Mecking constituent models are built on a combination of the work of Ashby on geometrically-necessary dislocations, and the Mughrabi theory on long-range stresses. The proposed model is able to quantitatively predict the average macroscopic and microscopic stress-strain behavior as well as the long-range stress magnitude for the measured dual-phase steel. Finally, a gradient crystal plasticity model is presented which incorporates some of the recent developments in gradient plasticity, such as grain boundary yield conditions which are of significant importance to reproduce size effects of grain structures. Additionally, a gradient crystal plasticity framework is extended by taking into account the continuous dislocation density and curvature fields obtained by homogenization of discrete dislocation structures. Thereby, a dislocation based gradient plasticity framework is established that takes into account dislocation transport. 


\title{
Optimal placement of sensors and actuators for waves in homogeneous and heterogeneous media
}

\author{
Enrique Zuazua \\ BCAM \& Ikerbasque - Bilbao - Basque Country - Spain
}

In this lecture we discuss some problems related to wave control, observation and numerical approximation.

We shall mainly focus on the problem of the optimal placement of sensors and actuators for wave propagation, that will be recast as spectral optimal design problems.

We show that, depending on the complexity of the data to be observed/controlled, several scenarios have to be distinguished and, in particular, those in which the solution is a classical set constituted by a finite number of simply connected subdomains, Cantor type sets and those leading to relaxation phenomena.

We also explain how closely this topic is related to the fine properties of the high frequency behavior of the eigenfunctions of the laplacian, which is intimately linked to the ergodicity properties of the dynamical system generated by the corresponding billiard.

We then discuss numerical approximation issues, emphasizing the unexpected spurious effects that heterogenous grids may have on the propagation properties of numerical waves.

This talk will be inspired in recent joint work with, in particular, Y. Privat and E. Tr ÃClat (LJLL-Paris VI), S. Ervedoza (Toulouse) and A. Marica (Bucharest). 


\title{
Particle methods for complex flows of complex fluids
}

\author{
Nikolaus A. Adams, Xiangyu Hu, Stefan Adami \\ Technische Universität München \\ Institute of Aerodynamics and Fluid Mechanics \\ Boltzmannstrasse 15 \\ 85748 Garching \\ Germany
}

In this contribution we will review the state of the art of Lagrangian particle discretizations of the NavierStokes equations. We will focus on low-Mach-number flows and consider mainly the Smoothed-ParticleHydrodynamics (SPH) approach. We will show that the weakly-compressible formulation of SPH under certain constraints provides a reliable approach for the approximate solution of incompressible single- and multi-phase flows, and how it can be extended for monolithic fluid-structure interaction and modeling of free-surface flows. Limitations of standard formulations of SPH will be discussed, and we will derive conditions under which highorder convergence of the formally zero-order consistent conservative SPH discretizations can be achieved. The recently derived transport-velocity formulation will be motivated and explained, and related to the conditions for effective high-order consistency. We will demonstrate that with the transport-velocity formulation SPH delivers reliable results for classical flow problems of similar quality as can be obtained by grid-based methods. Unlike grid-based methods, however, SPH can deal with physically very complex multi-phase flows as it maintains inherently discrete mass and momentum conservation. We will conclude in showing how SPH can be converted to a mesoscopic model for microfluidic simulations, a variant which is called Smoothed Dissipative Particle Dynamics (SDPD) and what kind of numerical issues are faced with this approach. Finally we will demonstrate that state-of-the-art SPH has the capability to serve as reliable tool for industrial applications.

\section{References}

[1] J.J. Monaghan. Smoothed particle hydrodynamics. Rep. Prog. Phys. 68:1703-1759, 2005.

[2] P. Espanol, M. Revenga. Smoothed dissipative particle dynamcis. Phys. Rev. E 67:026705, 2003.

[3] S. Adami, X.Y. Hu, N.A. Adams. A transport-velocity formulation for smoothed particle hydrodynamics, J. Copmput. Phys. 241:292-307, 2013.

[4] S. Adami, X.Y. Hu, N.A. Adams. A conservative SPH method for surfactant dynamics. J. Copmput. Phys. 229:1909-1926, 2010.

[5] S. Litvinov, X.Y. Hu, N.A. Adams. Numerical simultion of tethered DNA in shear flow. J. Phys. Cond. Matter. 23:184118, 2011.

[6] S. Litvinov, X.Y. Hu, N.A. Adams. A splitting scheme for highly dissipative smoothed particle dynamics. J. Comp. Phys. 229:5457-5464, 2010.

[7] S. X.Y. Hu, N.A. Adams. A multi-phase SPH method for macroscopic and mesoscopic flows . J. Comp. Phys. 213:844-861, 2006. 


\title{
Nonlinear optimal control for airborne wind energy systems
}

\author{
Moritz Diehl, Greg Horn, and Mario Zanon \\ Systems Control and Optimization Laboratory \\ IMTEK, University of Freiburg and \\ ESAT-STADIUS, University of Leuven
}

\begin{abstract}
Airborne wind energy (AWE) [1], is an emerging technology to harvest power from the strong and consistent winds in altitudes between 200 and 600 meters. AWE systems consist of fast flying wings that are anchored to the ground by a tether, and an electrical generator converting the wing's motion into usable power. One can distinguish two ways to generate electricity, one using a ground-based generator and a periodic reel-out and reel-in of the tether, the other using small wind turbines on-board the flying wing. In both cases, the motion of the wing in the windfield is a strongly nonlinear and unstable process that requires advanced control and estimation technology. In this talk, we first outline the physical background of the technology and then discuss the differential-algebraic equation (DAE) models describing the dynamics of AWE systems. We then show how state-of-the-art direct methods for nonlinear optimal control - that are available in the open-source software packages CasADi [2] and ACADO [3] - can be used to answer relevant questions such as which periodic orbits are power optimal or which system topology is best [6], and help to generate model-based predictive controllers that use fast online optimization in order to generate an approximation to optimal feedback controls $[4,5]$.
\end{abstract}

\section{References}

[1] U. Ahrens, M. Diehl, and R. Schmehl, editors. Airborne Wind Energy. Springer, 2013.

[2] J. Andersson, J. Åkesson, and M. Diehl. CasADi - A symbolic package for automatic differentiation and optimal control. In S. Forth, P. Hovland, E. Phipps, J. Utke, and A. Walther, editors, Recent Advances in Algorithmic Differentiation, Lecture Notes in Computational Science and Engineering, Berlin, 2012. Springer.

[3] B. Houska, H.J. Ferreau, and M. Diehl. An Auto-Generated Real-Time Iteration Algorithm for Nonlinear MPC in the Microsecond Range. Automatica, 47(10):2279-2285, 2011.

[4] A. Ilzhoefer, B. Houska, and M. Diehl. Nonlinear MPC of kites under varying wind conditions for a new class of large scale wind power generators. International Journal of Robust and Nonlinear Control, 17(17):15901599, 2007.

[5] M. Zanon, G. Horn, S. Gros, and M. Diehl. Control of Dual-Airfoil Airborne Wind Energy Systems Based on Nonlinear MPC and MHE. In European Control Conference, 2014.

[6] Mario Zanon, Sébastien Gros, Joel Andersson, and Moritz Diehl. Airborne Wind Energy Based on Dual Airfoils. IEEE Transactions on Control Systems Technology, 21, July 2013. 


\title{
Shape memory alloys: from recent modeling proposals to cardiovascular device simulations
}

\author{
$\underline{\text { F. Auricchio }}$ \\ Dipartimento di Ingegneria Civile e Architettura, Università di Pavia, Italy
}

The present contribution wishes to address several aspects related to shape-memory alloy (SMA) materials and structures, with a final emphasis on the use of such tools for the simulations of stent cardiovascular devices. In particular, we plan to:

- review some recent 3D constitutive model developments (with models taking into account with different level of accuracy several physical phenomena, e.g., martensite reorientation, different kinetics between forward/reverse phase transformations, smooth thermo-mechanical response, low- stress phase transformations, transformation-dependent elastic properties) and their numerical treatment (for the more complex models through the use of Fischer-Burmeister complementarity conditions) to predict experimental results on spring-like actuators [1]

- explore the use of simpler constitutive models to capture the response of geometrically complex biomedical devices like self-expandable cardiovascular stents in the large deformation regime, with particular attention to a correct modeling of buckling phenomena [2]

- investigate the extension of Dang Van high cycle fatigue criterion to SMAs and its application to uniaxial experimental data taken from the literature with the final goal of approaching the development of a general multiaxial SMA failure criterion [3]

\section{References}

[1] F. Auricchio, E. Bonetti, G. Scalet, F. Ubertini. Theoretical and numerical modeling of shape memory alloys accounting for multiple phase transformations and martensite reorientation. International Journal of Plasticity, 59 (2014) $30-54$.

[2] F. Auricchio, M. Conti, M. Ferraro, S. Morganti, A. Reali, R.L. Taylor. Innovative and efficient stent flexibility simulations based on isogeometric analysis. Submitted for publication (2015)

[3] F. Auricchio, A. Constantinescu, C. Menna, G. Scalet. A shakedown analysis of high cycle fatigue of shape memory alloys. Submitted for publication (2015) 


\title{
Low-rank techniques for high-dimensional problems in engineering and data-analysis
}

\author{
Daniel Kressner \\ EPF Lausanne, Switzerland
}

The last few years have seen a quite some activity in the development of low-rank matrix and tensor approximation techniques for addressing high-dimensional computational tasks arising from engineering and data analysis. These developments have resulted in new algorithms capable of solving linear algebra problems whose sheer size renders the use of traditional approaches infeasible. The aim of this talk is to give a survey of this exciting research direction and point out the many challenging questions that remain.

The basic idea of low-rank techniques is to formally consider a regular discretization, for example of a $d$ dimensional partial differential equations. As $d$ increases, the number of degrees of freedom grow exponentially, which implies an excessive cost of standard methods for computing the solution. To address this issue, the solution is approximated on a manifold of much lower dimension. Low-rank matrix and tensor decompositions constitute such manifolds that appear to be very well suited for this purpose, often resulting in highly accurate approximations at a much lower cost. This will be illustrated for a variety of high-dimensional situations, including high-dimensional partial differential equations, parameter-dependent and stochastic equations, as well as applications in data analysis. 


\title{
Interfacial energy and size effects in evolving martensitic microstructures
}

\author{
Stanisław Stupkiewicz \\ Institute of Fundamental Technological Research (IPPT), Warsaw, Poland
}

Shape memory alloys (SMA) exhibit unusual effects, such as pseudoelasticity and shape memory effect, which are utilized in many advanced practical applications. These effects are associated with martensitic phase transformation that can be induced by temperature changes or mechanical loading. At the microscale, the transformation proceeds by formation and evolution of complex martensitic microstructures at multiple length scales. Understanding these multiscale phenomena is crucial for development of reliable predictive models of SMA behaviour. Significant research efforts have thus been spent in the last two decades on micromechanical modelling of shape memory alloys, martensitic microstructures, and related phenomena.

Evolution of martensitic microstructures is associated with formation and propagation of interfaces at multiple scales. It is well known that size effects are governed by size-dependent interfacial energy contributions. Modelling of size effects in SMA requires thus consideration of the interfacial energy contributions at various scales of martensitic microstructures. For that purpose, an incremental energy minimization framework has been recently developed $[1,2]$ in which interfacial energy is assumed to contribute both to the free energy and to the dissipated energy. Related applications include modelling of grain-size effects in pseudoelastic polycrystalline SMA [2,3] and a study of stability of equilibrium of evolving laminates [4]. In the latter work, it has been shown that a homogenized phase-transforming laminate with no length scale exhibits a localization instability. However, for a laminate of finite spacing, its evolution is stabilized by the elastic micro-strain energy at the boundary of the localization zone.

Interfacial energy of austenite-twinned martensite interfaces constitutes an essential input to the modelling of size effects such as that discussed above. An interface between austenite and twinned martensite is a typical example of a microstructured interface where the local incompatibility of transformation strains is accommodated by elastic micro-strains in a thin transition layer along the interface. The associated interfacial energy of elastic micro-strains is a bulk energy at a suitably fine scale, and it is interpreted as an interfacial energy at a higher scale. This corresponds to a kind of scale transition. Importantly, this interfacial energy cannot be measured directly so that theoretical predictions seem to be the only alternative. A suitable micromechanical sharp-interface framework has been developed in $[5,6,7]$. Currently, we are studying a related class of problems using the phase-field (diffuse-interface) approach. Preliminary results of the phase-field modelling confirm the main findings of our earlier sharp-interface modelling.

\section{References}

[1] H. Petryk and S. Stupkiewicz. Interfacial energy and dissipation in martensitic phase transformations. Part I: Theory. J. Mech. Phys. Solids, 58:390-408, 2010.

[2] H. Petryk, S. Stupkiewicz, and G. Maciejewski. Interfacial energy and dissipation in martensitic phase transformations. Part II: Size effects in pseudoelasticity. J. Mech. Phys. Solids, 58:373-389, 2010.

[3] S. Stupkiewicz and H. Petryk. Grain-size effect in micromechanical modelling of hysteresis in shape memory alloys. ZAMM, 90:783-795, 2010.

[4] H. Petryk and S. Stupkiewicz. Instability of equilibrium of evolving laminates in pseudo-elastic solids. Int. J. Non-Linear Mech., 47:317-330, 2012.

[5] G. Maciejewski, S. Stupkiewicz, and H. Petryk. Elastic micro-strain energy at the austenite-twinned martensite interface. Arch. Mech., 57:277-297, 2005.

[6] S. Stupkiewicz, G. Maciejewski, and H. Petryk. Low-energy morphology of the interface layer between austenite and twinned martensite. Acta Mater., 55:6292-6306, 2007.

[7] S. Stupkiewicz, G. Maciejewski, and H. Petryk. Elastic micro-strain energy of austenite-martensite interface in NiTi. Modelling Simul. Mater. Sci. Eng., 20:035001, 2012. 


\section{MS1: Multi-Scale Modeling of Ferroic Functional Materials}

In the minisymposium, latest developments related to two central aspects of multi-scale modeling of ferroic functional materials will be discussed, namely techniques for the modeling at different scales, e.g. discrete atomistic (MD) simulations, phase-field approaches, energy relaxation techniques, and scale-bridging techniques, e.g. micro-mechanics and direct micro- macro transitions through numerical homogenization. Such tools allow the computation of effective properties for multi-functional composites, as well as advanced multi-scale analyses of critical constitutive mechanisms related, for instance, to micro- and domain structure evolution, fracture, damage or fatigue, in a physically well-motivated manner. 


\title{
Multiscale mechanical behaviour of nematic elastomers
}

\author{
Antonio DeSimone \\ SISSA-International School for Advanced Studies
}

Phase transforming materials have been used as actuator materials for some time already. They are typically made of hard materials but, more recently, interest has grown on soft materials, which are capable of responding to external stimuli with large deformations. Nematic elastomers provide one example in which spontaneous deformations can be induced by temperature changes, applied electric fields, or irradiation with light.

We will discuss the mechanical multiscale behaviour of nematic elastomers and speculate on their possible applications in the field of soft robotics. 


\title{
A laminate-based modelling approach for rate-dependent switching in ferroelectric materials
}

\author{
D.K. Dusthakar ${ }^{1}$, A. Menzel $^{1,2}$, B. Svendsen ${ }^{3,4}$ \\ ${ }^{1}$ Institute for Mechanics, TU Dortmund, Dortmund, Germany \\ ${ }^{2}$ Division of Solid Mechanics, Lund University, Lund, Sweden \\ ${ }^{3}$ Chair of Material Mechanics, RWTH Aachen, Aachen, Germany \\ ${ }^{4}$ Microstructure Physics and Alloy Design, \\ Max Planck Institute for Iron Research, Düsseldorf, Germany
}

Ferroelectric materials, both single- and polycrystals, exhibit characteristic hysteric behaviour when subjected to a sufficiently high macroscopic cyclic electric field. Moreover, related butterfly curves and hysteresis loops depend on the rate of electrical loading; cf. Zhou et al. [1]. Experimental studies show that the shape of such hysteresis curves additionally depends on the magnitude of external compressive stresses acting along the direction of the applied electrical field; cf. Li et al. [2], Zhou et al. [3], Shieh et al. [4].

This work focuses on the modelling and simulation of ferroelectric single- and polycrystalline materials based on a laminate-based mixture-type theory, wherein several phases or rather variants are considered; cf. Yen et al. [6] and references cited therein. By considering compatibility between ferroelectric domains, this formulation rests upon the framework of sequential lamination to generate so-called engineered domain microstructures in ferroelectric materials. Furthermore, the formulation is governed by an energy-enthalpy function in terms of the total strains, the electric field and a set of internal variables, namely the multi-rank laminate volume fractions. The evolution of these volume fractions is determined by the dissipation potential introduced. Based on a previously established micromechanically motivated and rate-dependent laminate-based approach for the modelling of single crystal ferroelectrics, see Dusthakar et al. [5], this work extends that formulation to computationally capture polycrystalline ferroelectric material behaviour under the influence of external stresses and at different loading rates. The proposed laminate-based approach accounts for a limited number of material parameters and, at the same time, remains computationally efficient.

\section{References}

[1] D. Zhou, M. Kamlah, and D. Munz. Rate dependence of soft PZT ceramics under electric field loading. Proc. SPIE 4333 (2001), 64-70.

[2] F. Li, D. Fang, J.-J. Lee, and H. C. Kim. Effects of compressive stress on the nonlinear electromechanical behavior of ferroelectric ceramics. Science in China, Series E: Technological Sciences, 49 (2006), 29-37.

[3] D. Zhou, M. Kamlah, and D. Munz. Effects of uniaxial prestress on the ferroelectric hysteretic response of soft PZT. Journal of the European Ceramic Society, 25 (2005), 425-432.

[4] J. Shieh, J. H. Yeh, Y. C. Shu, and J. H. Yen. Operation of multiple $90^{\circ}$ switching systems in barium titanate single crystals under electromechanical loading. Applied Physics Letters, 91 (2007), 0629011-0629013.

[5] D.K. Dusthakar, A. Menzel, and B. Svendsen. Comparison of phenomenological and laminate-based models for rate-dependent switching in ferroelectric continua. GAMM-Mitteilungen, 38 (2015), in press.

[6] J. H. Yen, Y. C. Shu, J. Shieh, and J. H. Yeh. A study of electromechanical switching in ferroelectric single crystals. J. Mech. Phys. Solids, 56 (2008), 2117-2135. 


\title{
Variational Structural and Material Stability Analysis in Finite Electro-Magneto-Mechanics of Active Materials
}

\author{
C. Miehe \& D. Vallicotti \\ Institute of Applied Mechanics (CE) \\ University of Stuttgart \\ Pfaffenwaldring 7, 70569 Stuttgart, Germany \\ christian.miehe@mechbau.uni-stuttgart.de
}

\begin{abstract}
Materials like electro-active polymers (EAPs) and magneto-rheological elastomers (MREs) exhibit coupled electro- and magneto-mechanical behavior at large strains. They respond by a deformation to an applied electric or magnetic field and are used in advanced industrial environments as sensors and actuators, for example in robotics, biomimetics and smart structures. In field-activated or electronic EAPs, the electric activation is driven by Coulomb-type electrostatic forces, resulting in Maxwell stresses. MREs are composites consisting of a polymer matrix with embedded magnetizable iron particles, which allow a change of mechanical properties by application of magnetic fields. Polymer-based magneto-electric composites (MECs) are a new class of tailormade materials with promising future in industrial applications. The ME effect is the phenomenon of inducing electric polarization by applying an external magnetic field or vice versa.

However, these types of composite materials suffer from different types of instabilities. This concerns structural instabilities, such as buckling and wrinkling of EAP and MRE devices, as well as material instabilities, such as limit- and bifurcation-points in the constitutive response which induce snap-through and fine scale localization of local states.

In this work, we outline variational-based definitions for structural and material stability of MRCs, and design algorithms for accompanying stability checks in typical finite element computations of composites. The formulation starts from stability criteria for a canonical energy minimization principle of electro-magneto-elastostatics, and then shifts them over to representations related to an enthalpy-based saddle point principle that is considered as the most convenient setting for numerical implementation. Here, global structural stability is analysed based on a perturbation of the total electro-magneto-mechanical energy, and related to statements of positive definiteness of incremental finite element tangent arrays. We base the local material stability on an incremental quasi-convexity condition of the electro-magneto-mechanical energy, inducing the positive definiteness of both the incremental electro-magneto-mechanical moduli as well as a generalized acoustic tensor. It is shown that the incremental arrays to be analysed in the stability criteria appear within the enthalpy-based setting in a distinct diagonal form, with mechanical, electric and magnetic partitions. Applications of accompanying stability analyses in finite element computations are demonstrated by means of representative model problems.
\end{abstract}

\section{References}

[1] Ethiraj, G. and Miehe, C., Multiplicative Magneto-Elasticity of Magnetosensitive Polymers Incorporating Micromechanically-Based Network Kernels. Submitted to Proceedings of the Royal Society A, 2014.

[2] Miehe, C., Rosato, D. and Kiefer, B., Variational Principles in Dissipative Electro-Magneto-Mechanics: A Framework for the Macro-Modeling of Functional Materials. International Journal for Numerical Methods in Engineering 86: 1225-1276, 2011.

[3] Miehe, C., Vallicotti, D. and Zäh, D., Computational Structural and Material Stability Analysis in Finite Electro-Elasto-Statics of Electro-Active Materials. International Journal for Numerical Methods in Engineering, in press, 2014.

[4] Zäh, D. and Miehe, C., Multiplicative Electro-Elasticity of Electroactive Polymers Accounting for Micromechanically-Based Network Models. Computer Methods in Applied Mechanics and Engineering, 2014.

[5] Rosato, D. and Miehe, C., Dissipative ferroelectricity at finite strains. Variational principles, constitutive assumptions and algorithms. International Journal of Engineering Science 74: 163-189, 2014. 


\title{
Simulation of Size Effects in Ferroelectric Materials using a Phase Field Model
}

\author{
Dietmar Gross $^{1}$, Ralf Müller ${ }^{2}$, David Schrade ${ }^{2}$ \\ ${ }^{1}$ Division of Solid Mechanics, TU Darmstadt, Darmstadt, Germany \\ ${ }^{2}$ Institute of Applied Mechanics, University of Kaiserslautern, Kaiserslautern, Germany
}

Ferroelectric materials couple mechanical and electrical fields. Crucial for the coupling is the material microstructure which consists of different domains separated by domain walls. A domain is an area of almost constant polarization. The application of mechanical or electrical fields causes a temporal change of the domain structure which establishes a time dependent material behavior. In this presentation a phase field model based on the remanent polarization as order parameter is proposed, see [1]. The evolving anisotropy is considered by using a formulation of the electric enthalpy based on invariant theory, see [2]. This approach can capture the polarization dependent material properties quite well, as is demonstrated by the example of barium titanate. The invariant formulation allows for a straight forward application of the model in two and three dimensions. In order to analyse size effects the boundary conditions on the order parameter (polarization) are discussed and recast into a Robin-type boundary condition. Numerical simulations using finite elements demonstrate size effects in nano dots and thin films, see [3]. The presentation also addresses the evolution of volume fractions of different domain orientations within a polycrystalline aggregate. In polycrystalline settings the grain boundaries require a special treatment of the order parameter. As additional jump conditions for the order parameter are required from a mathematical point of view, which cannot be derived from physical arguments based on balance relations, two limit cases are analyzed by virtue of numerical simulations. In the first case, a continuity of the order parameter across the grain boundary is assumed, resulting into a strong interaction of the grains. In a second approach, the order parameters are decoupled on the grain boundary, resulting in a communication of different grains only via the electrical and mechanical jump conditions on the grain boundary and thus a weak coupling. Again the size effect is studied by simulation of hysteresis loops for different grain sizes of a polycrystalline microstructure.

\section{References}

[1] D. Schrade, R. Müller, D. Gross. On the physical interpretation of material parameters in phase field models for ferroelectrics. Arch. Appl. Mech. 83 (2013), 1393 -1413.

[2] D. Schrade, R. Müller, D. Gross, M.-A. Keip, H. Thai, J. Schröder. An invariant formulation for phase field models in ferroelectrics. Int. J. Solids Struct. 51 (2014), 2144-2156.

[3] D. Schrade, R. Müller, D. Gross, P. Steinmann. Phase field simulations of the poling behavior of $\mathrm{BaTiO}_{3}$ nano-scale thin films with $\mathrm{SrRuO}_{3}$ and Au electrodes. Eur. J. Mech. A/Solids 49 (2015), 455-466. 


\title{
On molecular statics simulations of ferroelectric functional materials
}

\author{
$\underline{\text { Paul Steinmann }}^{1}$ and Florian Endres ${ }^{1}$ \\ ${ }^{1}$ Chair of Applied Mechanics \\ University of Erlangen - Nuremberg \\ Egerlandstrasse 5 \\ 91058 Erlangen, Germany
}

\begin{abstract}
Smart materials, such as ferroelectric functional materials are of great interest for technological and scientific reasons and various material models and simulation algorithms are available in order to predict material behaviour on different length scales. On the macroscopic, mesoscopic and sub-monocrystal length scale continuum mechanics models, e.g. phase field models can accurately calculate phenomena like polarization switching or the change of polarization due to mechanical stress. However, most material models used for continuum mechanics do not fit the material behaviour of ferroelectric materials on the length scale of a few nanometers whereas simulation models and algorithms on the atomistic level are required.

Today it is possible to fabricate ferroelectric nanocomponents like ferroelectric nanofilms with less than 5 nanometers in thickness due to rapid developments in manufacturing technologies [1]. Therefore, also simulations on the atomistic length scale are becoming more important to predict and understand material behaviour.

Methods like the density functional theory can accurately calculate the ground state of atomistic systems [2]. However, the system sizes of quantum energy models and methods are restricted due to the significant computational costs of such approaches. In order to analyze size effects of ferroelectric nanofilms or nanowires several thousand atoms have to be considered. Molecular dynamics (MD) methods are able to calculate large ferroelectric atomistic systems using the core-shell model [3]. Nevertheless the computational costs for the simulation of electromechanical problems are still significant due to long range Coulomb interactions. Furthermore the coupling of MD approaches with continuum mechanics is a challenging task.

To overcome the disadvantages of the mentioned methods an extended molecular statics algorithm has been developed which efficiently calculates the ferroelectric behaviour of large discrete particle systems [4]. The proposed algorithm is able to compute efficiently the continuum deformation of a discrete particle system caused by an external electrical field in analogy to the Parrinello-Rahman method [5]. Therefore, the proposed algorithm is able to not only calculate the piezoelectric effect but also the converse piezoelectric effect which is still a limitation for other atomistic simulation algorithms of ferroelectric materials.

The aim of this talk is to present the developed extended molecular statics algorithm and the applied coreshell model for the simulation of ferroelectric materials and discuss the possibilities of the coupling of molecular statics and continuum mechanics.
\end{abstract}

\section{References}

[1] N. Setter, D. Damjanovic, L. Eng, G. Fox, S. Gevorgian, S. Hong, A. Kingon, H. Kohlstedt, N. Y. Park, G. B. Stephenson, I. Stolitchnov, A. K. Taganstev, D. V. Taylor, T. Yamada, S. Streiffer. Ferroelectric thin films: Review of materials, properties, and applications. J. Appl. Phys. 100.5 (2006), 051606.

[2] S. F. Matar, I. Baraille, M. A. Subramanian First principles studies of $\mathrm{SnTiO}_{3}$ perovskite as potential environmentally benign ferroelectric material. Chem. Phys. 355.1 (2009), 43-49.

[3] S. Tinte, MG Stachiotti, M Sepliarsky, R.L. Migoni, C.O. Rodriguez. Atomistic modelling of $\mathrm{BaTiO}_{3}$ based on first-principles calculations. J. Phys.: Condens. Matter. 11.48 (1999), 9679.

[4] F. Endres, P. Steinmann. An extended molecular statics algorithm simulating the electromechanical continuum response of ferroelectric materials. Comp. Mech. 54.6 (2014), 1515-1527.

[5] M. Parrinello, A. Rahman. Strain fluctuations and elastic constants. J. Chem. Phys. 76.5 (1982), 2662-2666. 


\section{MS2: Applications of the Virtual Element Method}

The Virtual Element Method is a brand new methodology, related to Mimetic Finite Differences (MFD), to XFEMS, and more generally to the extension of Finite Elements to polygonal and polyhedral decompositions. Unlike MFD, they are a natural Galerkin method, and unlike other extensions of FEM they provide the exact satisfaction of the Patch Test in a number of relevant problems.

The minisymposium would deal with the latest applications of the method, including mixed formulations, time dependent problems, convection or reaction dominated flows, topology optimization, fractured domains and possibly several others. The method is quite new, since the first paper appeared in 2013, and rapidly developing, so that we can expect several important novelties. 


\title{
Virtual Element Methods: an overview
}

\author{
L. Beirão da Veiga, F. Brezzi , L.D. Marini, A. Russo \\ Università di Milano Statale \\ IUSS Pavia \\ Università di Pavia \\ Università di Milano Bicocca
}

The talk will present a short overview of the Virtual Element Method (VEM) and of the results obtained by the authors in the last year. The first part of the talk will consist of a simple presentation of the general ideas that are at the basis of VEM's, especially suited for those, in the audience, that are not familiar with them. Taking the (simplest) Poisson problem in two dimensions, we will see the main features of Virtual Elements, and the general philosophy behind them. Then, in the second part, we shall move, at a swifter pace, through more advanced features, including three-dimensional problems, variable coefficients, mixed formulations, and some basic applications to simple problems like linear elasticity and Kirchhoff plates.

A particular effort will be done in trying to underline the strong points of VEM's as they stand now, and the aspects that still require an additional work. More generally we will present what are, in our opinion, the actual limits of the method and what are, always in our opinion, the next achievable goals.

\section{References}

[1] B. Ahmed, A. Alsaedi, F. Brezzi, L.D. Marini, A. Russo. Equivalent Projectors for Virtual Element Methods. Comput. Math. Appl. 66 (2013), 376-391.

[2] P. F. Antonietti, L. Beirão da Veiga, D. Mora, M. Verani: A Stream Virtual Element Formulation of the Stokes Problem on Polygonal Meshes. SIAM J. Numerical Analysis 52 (2014) 386-404.

[3] L. Beirão da Veiga, F. Brezzi, A. Cangiani, G. Manzini, L.D. Marini, A. Russo. Basic Principles of Virtual Element Methods. Math. Models Methods Appl. Sci. 23 (2013), 199-214.

[4] L. Beirão da Veiga, F. Brezzi, L.D. Marini. Virtual Elements for linear elasticity problems. SIAM J. Num. Anal. 51 (2013), 794-812.

[5] L. Beirão da Veiga, F. Brezzi, L.D. Marini, A. Russo. The Hitchhiker's Guide to the Virtual Element Method. Math. Models Methods Appl. Sci. 24 (2014) 1541-1573.

[6] L. Beirão da Veiga, F. Brezzi, L.D. Marini, A. Russo. Virtual Element Methods for general second order elliptic problems on polygonal meshes, submitted.

[7] F. Brezzi, R. S. Falk, L. D. Marini. Basic principles of mixed virtual element methods. ESAIM Math. Model. Numer. Anal. 48 (2014), 1227-1240.

[8] F. Brezzi, L.D. Marini. Virtual elements for plate bending problems. Comput. Methods Appl. Mech. Engrg. 253 (2013) 455-462.

[9] A. Cangiani, G. Manzini, A. Russo, N. Sukumar. Hourglass stabilization and the virtual element method. Internat. J. Numer. Methods Engrg. (2015), to appear. 


\title{
The Virtual Element Method for large scale Discrete Fracture Network simulations: fracture-independent mesh generation
}

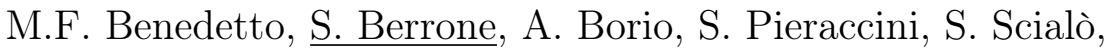 \\ Dipartimento di Scienze Matematiche, Politecnico di Torino
}

\begin{abstract}
Subsurface fluid flow has applications in a wide range of fields, including e.g. oil/gas recovery, gas storage, pollutant percolation, water resources monitoring. Underground fluid flow in fractured media is a heterogeneous multi-scale phenomenon that involves complex geological configurations; a possible approach for modeling the phenomenon is given by Discrete Fracture Networks (DFNs), which are complex sets of polygonal intersecting fractures. We focus on the resolution of the steady-state flow in large fracture networks. The quantity of interest is the hydraulic head in the whole network, which is the sum of pressure and elevation, and is evaluated by means of the Darcy law. We consider impervious rock matrix and fluid can only flow through fractures and through fracture intersections (called traces), but no longitudinal flow along the traces is allowed. Matching conditions need to be added in order to preserve hydraulic head continuity and flux balance at fracture intersections.

Geological fractured media are therefore characterized by a challenging geometrical complexity. This talk concerns the application of the Virtual Element Method [1,2] to simulations in DFNs [3], in order to tackle this geometrical complexity. Indeed, a crucial issue in DFN flow simulations is the need to provide on each fracture a good quality mesh $[4,5]$. Namely, if the mesh on the fractures are required to be conforming to the traces, and also conforming each other, the meshing process for each fracture is not independent of the others, thus yielding in practice a quite demanding computational effort for the mesh generation process. In some cases, the meshing process may even result infeasible.

Here, the VEM will be used in conjunction with a newly conceived PDE-constrained optimization approach for dealing with DFN simulations without the need of mesh conformity $[6,7,8]$, as well in conjunction with a mortar approach. Indeed, taking advantage from the great flexibility of VEM in allowing the use of rather general polygonal mesh elements, a suitable mesh for representing the solution can be easily obtained starting from an arbitrary triangular mesh independently built on each fracture, and independent of the trace disposition.
\end{abstract}

\section{References}

[1] L. Beirão da Veiga, F. Brezzi, A. Cangiani, G. Manzini, L. D. Marini, A. Russo, Basic Principles of Virtual Element Methods, Mathematical Models and Methods in Applied Sciences, Vol. 23, No. 1 (2013) 199-214.

[2] F. Brezzi, R. S. Falk, L. D. Marini, Basic principles of mixed Virtual Element Methods ESAIM: Mathematical Modelling and Numerical Analysis, Vol. 48, No. 4, (2014), 1227-1240.

[3] F.M. Benedetto, S. Berrone, S. Pieraccini, S. Scialò, The Virtual Element Method for Discrete Fracture Network simulations, Comput. Methods Appl. Mech. Engrg. Vol. 280, No. 1, (2014), 135-156.

[4] G. Pichot, J. Erhel, J.R. de Dreuzy A Generalized Mixed Hybrid Mortar Method for Solving Flow in Stochastic Discrete Fracture Networks, SIAM Journal on Scientific Computing, Vol. 34, No. 1, (2012), B86-B105.

[5] J.D. Hyman, C.W Gable, S.L. Painter, N. Makedonska, Conforming Delaunay Triangulation of Stochastically Generated Three Dimensional Discrete Fracture Networks: A Feature Rejection Algorithm for Meshing Strategy SIAM Journal on Scientific Computing Vol. 36, No. 4 (2014), A1871-A1894.

[6] S. Berrone, S. Pieraccini, S. Scialò, A PDE-constrained optimization formulation for discrete fracture network flows, SIAM Journal on Scientific Computing, Vol. 35 n. 2, (2013), B487-B510.

[7] S. Berrone, S. Pieraccini, S. Scialò, On simulations of discrete fracture network flows with an optimizationbased extended finite element method, SIAM Journal on Scientific Computing, Vol. 35 n. 2, (2013), A908A935.

[8] S. Berrone, S. Pieraccini, S. Scialò, An optimization approach for large scale simulations of discrete fracture network flows, Journal of Computational Physics, Vol. 256, (2014), 838-853. 


\title{
The Plane Wave Virtual Element Method for the Helmholtz Problem
}

\author{
Ilaria Perugia, Paola Pietra, Alessandro Russo \\ Faculty of Mathematics, University of Vienna, Vienna, Austria \\ Istituto di Matematica Applicata e Tecnologie Informatiche "E. Magenes", CNR, Pavia, Italy \\ Dipartimento di Matematica e Applicazioni, Università di Milano-Bicocca, Milano, Italy
}

Concerned with the time-harmonic wave propagation governed by the Helmholtz equation, we present a novel Galerkin approximation that can deal with general polygonal partitions. Virtual element methods have been recently introduced $[1,2]$ as extension of finite elements to general polygonal decompositions for different classes of definite and semidefinite problems. Here we design and analyse a method for an indefinite problem.

Because of the oscillatory behavior of solutions to the Helmholtz equation, methods that incorporate information about the solution in the form of plane waves have received attention in the last years. Our virtual element method for the Helmholtz problem in two dimensions introduces modulated plane wave basis functions.

\section{References}

[1] L. Beirão da Veiga, F. Brezzi, A. Cangiani, G. Manzini, L.D. Marini, A. Russo: Basic principles of Virtual Element Methods. Math. Models Methods Appl. Sci. 23, (2013), 199-214.

[2] L. Beirão da Veiga, F. Brezzi, L.D. Marini, A. Russo: The hitchhiker guide to the Virtual Element Method. Math. Models Methods Appl. Sci. 24 (2014), 1541-1573. 


\title{
Virtual Element Methods for parabolic problems on polygonal meshes
}

\author{
Giuseppe Vacca \\ Dipartimento di Matematica, Università degli Studi di Bari
}

The Virtual Element Method (in short, VEM) is a recent technology introduced in [2] as a generalization of the finite element method, that can make use of very general polygonal/polyhedral meshes without the need to integrate complex non-polynomial functions on the elements and preserving an optimal order of convergence. Making use of polygonal meshes brings forth a range of advantages, including for instance better domain meshing capabilities, automatic use of nonconforming grids, more efficient approximation of geometric data features, more efficient and easier adaptivity, more robustness to mesh deformation.

The main idea behind VEM is to use approximated discrete bilinear forms that require only integration of polynomials on the (polygonal) element in order to be computed. The ensuing discrete solution is conforming and the accuracy granted by such discrete bilinear forms turns out to be sufficient to recover the correct order of convergence. Following such approach, VEM is able to make use of very general polygonal/polyhedral meshes without the need to integrate complex non-polynomial functions on the elements and without loss of accuracy. As a consequence, VEM is not restricted to low order converge and can be easily applied to three dimensions and use non convex (even non simply connected) elements.

The talk describes the Virtual Element Methods for parabolic problems. It is considered as a model problem the classical time-dependent diffusion equation. The discretization of the problem requires the introduction of two discrete bilinear forms, one being the approximated grad-grad form of the stationary case [2] and the other being a discrete counterpart of the $L^{2}$ scalar product. The latter is built making use of the the enhancements techniques of [3]. In the talk we develop a full theoretical analysis, first focusing on the error among the semidiscrete and the continuous problems and later giving an example of error analysis involving the fully discrete case. A large range of numerical tests in accordance with the theoretical derivations is presented. In particular, we study also the possibility to use a simpler but non-coercive $L^{2}$ discrete bilinear form. Since the low modes are expected to dominate the problem, this choice leads to good results in a lot of situations, as shown by the numerical tests.

This is a joint work with Lourenço Beirão da Veiga from the University of Milano.

\section{References}

[1] G. Vacca, L. Beirão da Veiga. Virtual Element Methods for parabolic problems on polygonal meshes. Submitted.

[2] L. Beirão da Veiga, F. Brezzi, A. Cangiani, G. Manzini, L. D. Marini, A. Russo. Basic principles of virtual element methods. Math. Models Methods Appl. Sci. 23 (2013), 199-214.

[3] B. Ahmad, A. Alsaedi, F. Brezzi, L. D. Marini, A. Russo. Equivalent projectors for virtual element methods. Comput. Math. Appl. 66 (2013), 376-391. 


\title{
A $C^{1}$ virtual element method for the Cahn-Hilliard problem
}

\author{
Paola F. Antonietti ${ }^{1}$, Lourenco Beirão da Veiga ${ }^{2}$, Simone Scacchi $^{2}, \underline{\text { Marco Verani }}^{1}$ \\ ${ }^{1}$ MOX-Dipartimento di Matematica, Politecnico di Milano \\ ${ }^{2}$ Dipartimento di Matematica, Università degli Studi di Milano
}

Due to the wide spectrum of applications (e.g. phase separation in binary alloys, tumor growth, galaxy formation, foam formation, solidification processes and image processing) the study of efficient numerical methods for the approximate solution of Cahn-Hilliard equations has been the object of an intensive research activity. As the Cahn-Hilliard problem is a fourth order nonlinear equation, a natural numerical approach is to resort to the use of $C^{1}$ finite elements. However, the well known difficulties related to the practical implementation of $C^{1}$ finite elements have represented so far an important obstruction that has drastically limited their use in practical applications, thus paving the road to the use of mixed methods (with an increase of the numbers of degrees of freedom, and thus of the computational cost).

In this talk we introduce and analyze a $C^{1}$ virtual element method (VEM) for the approximate solution of the following Cahn-Hilliard problem: find $u: \Omega \times[0, T] \rightarrow \mathbb{R}$ such that:

$$
\left\{\begin{array}{lr}
\partial_{t} u-\Delta\left(\phi(u)-\gamma^{2} \Delta u(t)\right)=0 & \text { in } \Omega \times[0, T] \\
u(\cdot, 0)=u_{0}(\cdot) & \text { in } \Omega \\
\partial_{\mathbf{n}} u=\partial_{n}\left(\phi(u)-\gamma^{2} \Delta u(t)\right)=0 & \text { on } \partial \Omega \times[0, T],
\end{array}\right.
$$

where $\partial_{\mathbf{n}}$ denotes the (outward) normal derivative, the function $\phi(x)=\psi^{\prime}(x)$ with $\psi(x)=\left(1-x^{2}\right)^{2} / 4$ and $\gamma \in \mathbb{R}^{+}, 0<\gamma<<1$, represents the interface parameter. The virtual element method (see, e.g., [2] for an introduction to the method and [3] for the details of its practical implementation) is characterized by the capability of dealing with very general polygonal/polyedral meshes and the possibility of easily implementing highly regular discrete spaces [5,4]. In the present contribution we develop an evolution of the $C^{1}$ virtual elements (of minimal degree) of [4] for the approximation of the Cahn-Hilliard equations. Also taking inspiration from the enhancement techniques of [1], we define the virtual space in order to be able to compute three different projection operators, that are used for the construction of the discrete scheme. Afterwards, we show convergence of the approximation scheme and investigate its performance numerically. We underline that, on our knowledge, this is the first application of the newborn virtual element technology to a nonlinear problem.

\section{References}

[1] B. Ahmed, A. Alsaedi, F. Brezzi, L.D. Marini, A. Russo. Equivalent Projectors for Virtual Element Methods. Comput. Math. Appl. 66(2013), 376-391.

[2] L. Beirão da Veiga, F. Brezzi, A. Cangiani, G. Manzini, L.D. Marini, A. Russo. Basic Principles of Virtual Element Methods. Math. Models Methods Appl. Sci. 23(2013), 199-214.

[3] L. Beirão da Veiga, F. Brezzi, L.D. Marini, A. Russo. The Hitchhiker's Guide to the Virtual Element Method Math. Models Methods Appl. Sci. 24(2014), 1541-1573.

[4] L. Beirão da Veiga, G. Manzini. A virtual element method with arbitrary regularity. IMA J. Numer. Anal., $34(2014), 759-78$.

[5] F. Brezzi, L.D. Marini. Virtual Element Method for plate bending problems, Comput. Methods Appl. Mech. Engrg., 253 (2012), $455-462$. 


\section{MS3: Topological Defects in Solids}

Topological defects have been an active field of study since the pioneering work of Volterra on the mathematical theory of dislocations a century ago, and are very important also in mechanics, for example in the study of the plastic behavior of solids. Relevant connections to topological defects in Ginzburg-Landau models, used to describe for example superconducting or magnetic materials, have meanwhile become apparent. Recent progress in the mathematical analysis of some models generated a renewed interest, which extends from the energetic (variational) treatment to evolution problems.

In this minisymposium we intend to address some of the most interesting recent results on models for topological defects in solids, focusing on the common mathematical structure and spanning over different applications, which include in particular dislocations and plasticity, singularities in elastic plates, as well as Ginzburg-Landau models. 


\title{
Discrete to continuum analysis of magnetic systems with continuous symmetries
}

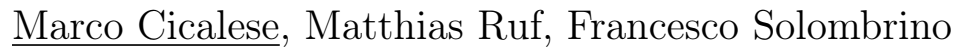 \\ Technische Universität München
}

\begin{abstract}
We review some recent results regarding the behaviour of energy-driven classical spin systems with continuous symmetries through the construction of approximate continuous problems. In particular we discuss the multiscale behaviour of the so called xy-helical model in dimension 1 and 2 in the case of $S^{1}$ or $S^{2}$-valued spins. The emergence of concentration phenomena related to the formation of topological vortex-type singularities as well as of phase changes due to chirality transitions will be emphasized.
\end{abstract}

\section{References}

[1] M. Cicalese, F. Solombrino. Frustrated ferromagnetic spin chains: a variational approach to chirality transitions. Journal of Nonlinear Science, to appear.

[2] M. Cicalese, M. Ruf, F. Solombrino. A variational analysis of the xy-helical spin system. In progress. 


\title{
Derivation of the line tension energy for dislocations in 3D
}

\author{
Sergio Conti, Adriana Garroni, Michael Ortiz \\ Universität Bonn, 53115 Bonn, Germany \\ Sapienza, Università di Roma, 00185 Roma, Italy \\ California Institute of Technology Pasadena, CA 91125, USA
}

\begin{abstract}
Dislocations are line defects in crystals which are fundamental to understand plastic deformation. I will present a three dimensional model of dilute dislocations based on linear elasticity and determine its asymptotic behavior in terms of Gamma convergence in the limit of small lattice spacing. As a result one obtains a line-tension model, which is an energy on matrix-valued divergence-free measures concentrated on lines. The line-tension energy can be determine by the leading term of the elastic energy of infinite straight dislocations after a relaxation process that may require microstructure.
\end{abstract}




\title{
Quantization effects in dislocation energies
}

\author{
Stephan Luckhaus

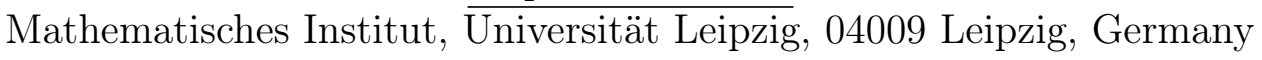

In small angle grain boundaries the stored energy in principle could depend on the cutoff of the elastic energy to the core, the core energy itself or the size of the Burgers vector. Here we give an argument - up to now with rigorous upper, heuristic lower bounds - that the leading order term depends on the size of the Burgers vector. 


\title{
Energy scaling law for the regular cone
}

\author{
Heiner Olbermann \\ Hausdorff Center, Bonn
}

Consider a thin elastic sheet in the shape of a disk whose reference metric is that of a singular cone. I.e., the reference metric is flat away from the center and has a defect there. This setting is called the regular cone, since one expects that minimizers of the elastic energy are approximately conical. We want to find upper and lower bounds for the minimum elastic energy that have the same scaling with respect to the thickness of the sheet $h$. Establishing a suitable lower bound is supposed to be hard, the main difficulty lying in the vast amount of potential candidates for energy minimization supplied by the constructions by Nash and Kuiper of $C^{1}$-isometric immersions. Isometric immersions have zero stretching energy - hence one needs a general principle that shows that the maps occurring in the constructions by Kuiper are associated to high bending energy. This is also the main problem in proving lower bounds for the so-called confinement or crumpling problem, which consists in fitting a thin elastic sheet into a container whose size is smaller than the diameter of the sheet. Here we suggest an ansatz for proving such lower bounds. We apply it to the regular cone under a technical assumption on the shape of the sheet. Under this assumption, we show that the elastic energy per unit thickness of the regular cone in the leading order of $h$ is given by $C h^{2}|\log h|$, where the value of $\mathrm{C}$ is given explicitly. 


\section{MS4: Optimal Control and Hybrid Systems}

On the one hand, optimal control of real physical processes is of crucial importance in all modern technological sciences. On the other hand, extensive research has been made in the field of hybrid systems in the last decades, since it is well known that the behavior of modern technical systems can only be modeled appropriately by a combination of continuous dynamics and discrete events models. Relevant applications are for example mechanical systems with impacts, power and electronic systems with switches, or complex networks whose coupling structure changes at discrete events. A great interest lies in the optimal control of hybrid systems since this includes not only the computation of optimal control trajectories for the continuous parts but also an optimization of the discrete variables.

The topics in this minisymposium are the analysis and control of hybrid and switched systems, switchingtime optimization, mixed-integer optimization for ODE and PDE systems as well as applications in engineering systems and robotics such as bipedal walking. 


\title{
Sequential Action Control for Nonlinear and Hybrid Systems
}

\author{
T.D. Murphey \\ Northwestern University
}

Sequential Action Control (SAC) [1] is a control technique based on ideas from hybrid control theory. The idea behind SAC is that one wants to optimize the next control action (defined by a control vector $u$ at a specified time $t$ for a specified duration $\lambda$ ) that improves some cost $J$ by a specified amount $\alpha \in \mathcal{R}$. These actions can then be sequenced to form a discrete-time feedback law, applicable to any nonlinear control system. Surprisingly, for any smooth objective function $J=\int_{t_{0}}^{t_{f}} \ell(x, t) d t+m\left(x\left(t_{f}\right)\right)$ and dynamic constraint $\dot{x}=g(x)+h(x) u$ (almost everywhere differentiable with respect to $x$ ), optimizing $J_{2}=\int_{t_{0}}^{t_{f}} \frac{1}{2}\left[\frac{d J}{d \lambda}-\alpha\right]^{2}+\|u\|^{2} d t$ with respect to $u$ has a unique solution that depends only on the evaluation of $x(t)$ over the time horizon and evaluation of the adjoint variable $\rho(t)$ over the same time horizon. In $J_{2}, \frac{d J}{d \lambda}$ is the needle variation that arises in optimal control and hybrid control, so optimizing this expression essentially sets the needle variation equal to $\alpha$ (regularized by the norm on $u$ ). Interestingly, the optimal choice of $u$ is a Tikonov regularization; as a consequence it scales nicely with respect to state. The resulting control is a new type of "local" controller, in the sense that $J_{2}$ is a local model of $J$ that one optimizes to obtain a control value. Typically local controllers are local with respect to a trajectory $x(t)$ (e.g., linear quadratic regulators), whereas SAC is local both with respect to the state and the duration of application, but it is global with respect to the time horizon $t_{f}$ and does not locally approximate the dynamics (in contrast to linear quadratic controllers). To obtain a control signal, one may compute these control actions in a sequence, updating as the state updates, so the control calculation is naturally of the form of a feedback law. Moreover, one may optimize over when the next control action is taken, since the next control action is well defined for all time $t$ up to $t_{f}$. Lastly, since the control is optimal for a infinitesimal duration $\lambda$, a backtracking line search may be used to determine the finite duration of the action.

Sequential Action Control is effective across a relatively wide variety of nonlinear and nonsmooth problems. It solves a variety of benchmark problems, including cart-pendulum swing up [2], acrobot swing up [3], pendubot swing up [4], and the minimum time parking problem. In these examples, SAC outperforms best-case solutions in the literature. Compared to direct optimization techniques based on sequential dynamic programming (SQP), SAC finds a solution to the acrobot swing up problem $10^{7}$ times faster than SQP. A change of seven orders of magnitude in computational requirement changes what types of control problems are tractable, particularly as the dimension of an optimal control problem increases.

Other typical requirements for optimal control calculations are also easier to compute using SAC than in many methods for nonlinear and hybrid control. For instance, SAC natively admits control saturation, and incorporates unilateral state constraint easily. Moreover, SAC is guaranteed to be linear near equilibria, so local stability can be assessed using standard techniques (e.g., using sum of squares (SOS) techniques).

Lastly, SAC is as easy to compute for hybrid systems as it is for smooth systems. This is because the adjoint variable $\rho(t)$ can be computed for a given trajectory so long as there are only a finite number of discontinuities in the dynamics. Applying SAC to hopping/running control for SLIP models of human and animal locomotion [5] leads to reliable motion control including nonlinear dynamics and challenging terrain that includes both nonlinearities and nonsmooth characteristics.

\section{References}

[1] A. Ansari and T.D. Murphey. Sequential Action Control: Closed-Form Optimal Control for Nonlinear Systems. IEEE Transactions on Robotics (Submitted).

[2] K.J. Astrom and K. Furuta. Swinging up a pendulum by energy control. Automatica 36(2) (2000), 287-295.

[3] M.W Spong. The swing up control problem for the acrobot. IEEE Control Systems 15(1) (1995), 49-55.

[4] M.W Spong and D.J Block. A mechatronic system for control research and education. In IEEE Conference on Decision and Control (1995), 555-556.

[5] P. Holmes, R. J. Full, D. Koditschek, and J. Guckenheimer. The Dynamics of Legged Locomotion: Models, Analyses, and Challenges. SIAM Review 48(2) (2006), 207-304. 


\title{
Output Regulation in Differential Variational Inequalities using Internal Model Principle and Passivity-Based Approach
}

\author{
Aneel Tanwani \\ Department of Mathematics, University of Kaiserslautern, Germany
}

In this talk, we consider the problem of designing feedback control laws for output regulation in systems with differential variational inequalities (DVIs). In addition to ordinary differential equations, DVIs comprise variational inequalities (VIs) which describe algebraic constraints and relations on the state trajectories [1]. The class of DVIs considered in this paper are described as follows:

$$
\begin{aligned}
& \dot{x}(t)=f(t, x)+G \lambda(t) \\
& v(t)=H x(t)+J \lambda(t), \quad v(t) \in \mathcal{S}(t), \\
& \left\langle v^{\prime}-v(t), \lambda(t)\right\rangle \geq 0, \quad \forall v^{\prime} \in \mathcal{S}(t) .
\end{aligned}
$$

where $\mathcal{S}:[0, \infty) \rightrightarrows \mathbb{R}^{d_{s}}$ is a set-valued mapping, and it is assumed that $\mathcal{S}(t)$ is closed, convex, and nonempty, for each $t \geq 0$. The variational inequalities expressed in (1c) find utility across many applications [2] and may be used to express optimality conditions, mechanical systems with impacts, and electrical circuits with diodes. While VIs can also be written using the notion of (set-valued) normal cone operators so that the differential inclusion resulting from (1) has maximal monotone operators, the introduction of matrices $G, H, J$ and the vector field $f(\cdot, \cdot)$ may not preserve monotonicity which makes it difficult to study the solution of such systems. From control point of view, the discontinuities in the description of such systems are state-dependent which introduces several complexities in designing feedback control laws.

To address these issues [3], we first derive conditions to study the existence and uniqueness of solutions in such systems. It is shown that under certain conditions on the system data, the given system could be equivalently written as a differential inclusion where the right-hand side is the sum of a multivalued maximal monotone operator and a Lipschitz-continuous function. Such inclusions are then shown to possess unique solutions. For the output regulation problem, we let $f(t, x):=A x+B u(t)+F x_{r}(t)$, where $x_{r}$ is an exogenous signal generated by an exosystem, and our objective is to design feedback control $u$ such that the resulting closed-loop system is well-posed and the state $x$ asymptotically tracks $x_{r}$. The derivation of control laws is based on the use of internal model principle, and two cases are treated: first, a static feedback control law is derived when full state measurements are available; In the second case, only the error to be regulated is assumed to be available for measurement and a dynamic compensator is designed. Under the condition that the closedloop system can be rendered passive, we show that the overall system is indeed well-posed and the desired error variable indeed converges to the origin. As applications, we demonstrate how control input resulting from the solution of a variational inequality results in regulating the output of the system while maintaining polyhedral state constraints. Another application is seen in designing switching signals for regulation in power converters.

\section{References}

[1] J.-S. Pang and D.E. Stewart. Differential variational inequalities. Math. Prog., Ser. A, 113:345 - 424, 2008.

[2] D. Kinderlehrer and G. Stampacchia. An Introduction to Variational Inequalities and Their Applications. Ser. Classics in Applied Mathematics, SIAM, 2000.

[3] A. Tanwani, B. Brogliato and C. Prieur. On output regulation in systems with differential variational inequalities. In Proc. $53^{\text {rd }}$ IEEE Conf. Decision $\&$ Control, 2014. 


\title{
Gene regulatory networks: equivalence between Utkin's and sigmoidal approach
}

\author{
Del Buono N., Elia C., Lopez L. \\ University of Bari
}

\begin{abstract}
Mathematical models of gene regulatory networks have received increasing attention in the literature in the past decades. One of the pioneering approaches, due to Glass and Kauffman ([1]), proposes piecewise linear control systems to model gene networks. However, a major concern with such models is that the vector field is not defined on (the intersection of) discontinuity hyperplanes. To overcome this issue, Utkin's and Filippov's approaches have been employed in literature, even though they are in general both ambiguous on the intersection of discontinuity hyperplanes. An alternative continuous model of gene networks, based on steep sigmoidal functions, has been given more recently by Plahte and Kjoglum in [2]. Our purpose in this talk is twofold: show that Utkin's approach gives a unique vector field on the intersection $\Sigma$ of two discontinuity hyperplanes (under assumptions of attractivity), and investigate when Utkin's approach and the steep sigmoidal approach are equivalent, i.e., when the corresponding solutions on $\Sigma$ are the same. This allows one to study the piecewise dynamical system, and hence the gene regulatory network it models, with no ambiguity.
\end{abstract}

\section{References}

[1] Glass, L., Kauffman, S. A. The logical analysis of continuous, non-linear biochemical control networks. Journal of Theoretical Biology 39. (1973), 103-129.

[2] Plahte, E., Kjóglum, S. Analysis and generic properties of gene regulatory networks with graded response functions. Physica D 201 (1). (2005), 150-176.

[3] Del Buono N., Elia C., Lopez L., On the equivalence between the sigmoidal approach and Utkin's approach for models of gene regulatory networks. Siam Journal on Applied Dynamical Systems 13 (3). (2014). 12701292 . 


\title{
On the value function of mixed-integer optimal control problems
}

\author{
Martin Gugat, Falk M. Hante
}

We present sensitivity analysis for the optimal value $\nu=\inf \Phi(y)$ with $y$ given by a control problem that involves both continuous and discrete control decisions $u$ and $v$, respectively:

$$
\dot{y}=A y+f(y, u, v), u \in \mathcal{U}(v) \text { on }\left(t_{0}, t_{f}\right), \quad y\left(t_{0}\right)=y_{0},
$$

where the pair $(A, f)$ represents an abstract semilinear evolution system on a Banach space and the sets $\mathcal{U}(v)$ represents control constraints.

For parametric initial data $y_{0}=y_{0}(\lambda)$, we obtain local Lipschitz continuity of the optimal value function $\nu(\lambda)$ for rather mild regularity assumptions on the pair $(A, f)$. The result ist based on a characterization of the optimal value function using a reduction to operational differential inclusions using the theory of [1]. For parametric control constraints $\mathcal{U}(v)=\mathcal{U}(v, \lambda)$ given in terms of inequalities, we obtain the same regularity for convex programs satisfying a Slater-type constraint qualification. In this case, we can give an explicit representation of the one-sided derivatives of the optimal value function $\nu(\lambda)$. The result is based on a strong duality result for parametric disjunctive programming [2].

Our analysis can be applied to assess the robustness of mixed-integer optimal controls even for certain control problems involving partial differential equations and being generated for example by relaxation methods [3].

\section{References}

[1] H. Frankowska. A priori estimates for operational differential inclusions. J. Differential Equations, 84(1):100$128,1990$.

[2] M. Gugat. Parametric disjunctive programming: one-sided differentiability of the value function. In Journal of Optimization Theory and Applications, 92(2):285-310, 1997.

[3] F. M. Hante, S. Sager. Relaxation Methods for Mixed-Integer Optimal Control of Partial Differential Equations. Computational Optimization and Applications, Vol. 55, Nr. 1, pp 197-225, 2013. 


\title{
Relaxing mixed integer optimal control problems using a time transformation
}

\author{
M. Ringkamp*, S. Leyendecker*, S. Ober-Blöbaum\# \\ * Chair of Applied Dynamics, University of Erlangen-Nuremberg \\ \# Computational Dynamics and Optimal Control, University of Paderborn
}

Nonlinear control systems with instantly changing dynamic behavior can be described by differential equations $\dot{x}=F(x, u, v)$ that depend on an integer valued control function $v \in \mathcal{L}^{\infty}(I, \mathcal{V})$, mapping the time interval $I=\left[t_{0}, t_{f}\right]$ to the integer values $\mathcal{V}=\left\{1, \ldots, n_{\mathcal{V}}\right\}$. Such systems occur for example in the optimal control of a driving car with different gears [1], or a subway ride with different operation modes [2], leading to a mixed integer optimal control problem (MIOCP). A discretize-then-optimize approach leads to a mixed integer optimization problem that is not differentiable with respect to the integer variables, such that gradient based optimization methods can not be applied. Differentiability with respect to all optimization variables can be achieved by reformulating the MIOCP, e.g. by using a relaxed binary control function [2], or by introducing a fixed integer control function $\bar{v}_{N, n} \in \mathcal{L}^{\infty}(I, \mathcal{V})$ and a time transformation $t_{w} \in \mathcal{W}^{1, \infty}(I, I)$ that allows to partially deactivate the fixed integer control function [1]. The latter approach is presented here, while the main focus lies on new theoretical and numerical results that take different functions $\bar{v}_{N, n}$ into account.

The time interval $I$ is partitioned into $N$ major intervals $I_{j}$, each $I_{j}$ into $n$ minor intervals $I_{j}^{i}$ and $\bar{v}_{N, n}$ is defined to be constant on each of the minor intervals $I_{j}^{i}$. The combination of $\bar{v}_{N, n}$ and a time control $w \in \mathcal{L}^{\infty}(I, \mathbb{R})$ with $w(\tau)=\frac{d t_{w}}{d \tau}(\tau)=t_{w}^{\prime}(\tau)$ for a.e. $\tau \in I$ results in the following time transformed MIOCP (TMIOCP):

$$
\begin{aligned}
& \min _{x, u, w} \quad J^{*}(x, u, w)=\int_{I} w(\tau) B\left(x(\tau), u(\tau), \bar{v}_{N, n}(\tau)\right) d \tau \\
& \begin{array}{ll}
\text { s. t. } & \dot{x}(\tau)=w(\tau) F\left(x(\tau), u(\tau), \bar{v}_{N, n}(\tau)\right) \quad \text { for a.e. } \tau \in I
\end{array} \\
& g_{0}(x(\tau), u(\tau)) \leq 0 \quad \text { for a.e. } \tau \in I \\
& w(\tau) g\left(x(\tau), u(\tau), \bar{v}_{N, n}(\tau)\right) \leq 0 \quad \text { for a.e. } \tau \in I \\
& r\left(x\left(t_{0}\right), x\left(t_{N}\right)\right)=0 \\
& w(\tau) \geq 0 \quad \text { for a.e. } \tau \in I \\
& \Delta I_{j}=\int_{I_{j}} w(s) d s
\end{aligned}
$$

Here, $J$ is the objective functional, $g_{0}$ and $g$ are inequality constraints and $r$ represents the boundary conditions. A fixed integer control function $\bar{v}_{N, n}$ is called control consistent (CC), if a switch of $v$ at any time in a major interval $I_{j}$ from a value $l_{1} \in \mathcal{V}$ to a value $l_{2} \in \mathcal{V}$ can be achieved with $\bar{v}_{N, n}$ by scaling the minor intervals $I_{j}^{i}$, in particular some minor intervals can be deactivated by scaling to zero. The optimal trajectory resulting from the TMIOCP using a CC fixed integer function $\bar{v}_{N, n}$ can coincide with the optimal trajectory of the MIOCP. In contrast to that, the optimal trajectory resulting from the TMIOCP in the case that $\bar{v}_{N, n}$ is not CC (NCC) can result in a higher objective value. Examples demonstrate this and furthermore that solving a TMIOCP using a CC fixed integer control function $\bar{v}_{N, n}$ can lead to a lower number of discretization variables as a TMIOCP that utilizes a fixed integer function that is NCC. The number of necessary discretization variables depends on the total number of used minor intervals and it is shown that the total number can be unbounded in the NCC case and is bounded in the $\mathrm{CC}$ case, even though the number of minor intervals for each major interval can be lower in the NCC case. An extension to bipedal walking models is planed in future works.

\section{References}

[1] M. Gerdts. A variable time transformation method for mixed-integer optimal control problems. Optimal Control Applications and Methods, Vol. 27, No. 3, pp. 169-182, 2006.

[2] S. Sager, H.G. Bock, G. Reinelt. Direct methods with maximal lower bound for mixed-integer optimal control problems. Mathematical Programming, Vol. 118, No. 1, pp. 109-149, 2009. 


\title{
Optimal Energy-Based Control of Hybrid Systems with Applications to Robotic Walking
}

\author{
Ryan W. Sinnet and Aaron D. Ames \\ Texas A\&M University
}

Over the last fifty years, researchers have addressed the problem of bipedal robotic locomotion using a range of approaches with varying degrees of formality [3]. In this presentation, we are interested in one particular approach known as energy shaping. The main idea behind energy shaping approaches involves using the structure of energy to create stabilizing controllers for periodic behaviors in dynamical systems. The primary focus is on recent results [5] which demonstrate an optimal controller for stabilizing the energy dynamics of periodic behaviors in hybrid mechanical system while maintaining exponential stability of the overall hybrid systems.

The development of energy shaping approaches spans the past few decades although energy-based approaches to analytical mechanics date back over 200 years to the work of Lagrange and the principles of stability involved were first presented by Lyapunov over 100 years ago. The ideas herein also build on more recent results spread throughout the literature. Over the last few decades, researchers have presented control schemes which seek to achieve periodic behaviors in dynamical systems and bipedal walking in particular by formulating their control objectives in terms of the energy of a system. Based on McGeer's observation that compass-gait bipeds ${ }^{1}$ with appropriate mass distributions can walk down shallow slopes without actuation [4], Spong presented controlled symmetries [8] as a method for obtaining walking on a compass-gait biped on flat ground by injecting energy in a such a way that the shaped potential energy of the robot's gait on flat ground mimicked the potential energy of a passive biped walking down a slope. Based on this idea, Spong later provided a controller which could shape the total energy of a compass-gait biped and showed that the controller would guarantee asymptotic stability of the energy dynamics to a reference level, $E_{\text {ref }}$, through the continuous dynamics although nothing was formally established with regards to stability of the overall system. He later demonstrated how the ideas could be extended to non-conservative systems [8] by considering energy storage functions. While the compassgait biped is of interest as a low-dimensional example of walking, the same method of controlled symmetries has been used as a basis for achieving walking in more complex models with knees and non-trivial foot action [6] and has been extended to three-dimensional bipeds using functional Routhian reduction [7].

The energy shaping problem, as formulated herein, falls under a class of problems involving stability of systems with zero dynamics. In [1], a similar problem was considered in which a stabilizing control law was constructed using control Lyapunov functions to stabilize to a zero dynamics which exhibited hybrid invariance; that is, for initial conditions on the intersection of the switching surface and the hybrid zero dynamics manifold, application of the reset map will result in a state which is still on the hybrid zero dynamics. This was a key assumption underlying [1] but this assumption does not hold for energy shaping as energy is generally not invariant through impact, though there may be pathological examples which demonstrate this property. In fact, for certain conservative systems like the compass-gait biped, which exhibits local exponential stability, energy change can only occur through discrete transitions and so impacts actually act as a stabilizing influence.

In order to apply energy shaping, one begins with an autonomous hybrid mechanical system with position and velocity coordinates $(q, \dot{q})$ which contains a locally exponentially stable periodic orbit, possibly induced by some feedback control law. For systems of this type, a conserved energy quantity exists through the continuous dynamics and it comprises kinetic and potential energy as well as an additional term. This additional term can be treated with a storage function which tracks the energy flowing out of the system; such energy flow occurs due to non-conservative forcing. For periodic behaviors, this conserved quantity, expressed as

$$
E_{c}=T(q, \dot{q})+U(q)-\int_{0}^{t} F_{n c}(q(\tau), \dot{q}(\tau)) \cdot \frac{d q(\tau)}{d \tau} d \tau
$$

with the terms respectively representing kinetic energy, potential energy, and stored energy, is also periodic and, by resetting the storage function appropriately through the discrete dynamics, can be made to be a constant. Using the conserved quantity, which is a function of the system state, and its constant reference level, one can design an energy shaping controller to drive the conserved energy of the system to the reference level, thereby stabilizing the system's energy dynamics. The particular approach discussed achieves this energy stabilization through the use of a rapidly exponentially stabilizing control Lyapunov function (RES-CLF) [1, 2]. Roughly

\footnotetext{
${ }^{1}$ Video of a compass-gait biped available online http://youtu.be/mwugDGGhPmI.
} 
speaking, one must choose control inputs such that the energy of the system is exponentially stable through the continuous dynamics. This is done by constructing the Lyapunov candidate function $V(q, \dot{q})=\left(E_{c}(q, \dot{q})-E_{r e f}\right)^{2}$. Because $\dot{V}(q, \dot{q}, u)=L_{f} V(q, \dot{q})+L_{g} V(q, \dot{q}) u$ is affine with respect to the control input, the problem can be posed as a quadratic program operating on a convex set:

$$
\begin{aligned}
\mu_{\varepsilon}(q, \dot{q})=\underset{u \in \mathbb{R}^{m}}{\arg \min } & u^{T} u \\
\text { s.t. } & L_{f} V(q, \dot{q})+L_{g} V(q, \dot{q}) u+\frac{c_{3}}{\varepsilon} V(q, \dot{q}) \leq 0 .
\end{aligned}
$$

This results in an optimal controller that can be evaluated with relatively low computational cost. In addition to being easy to solve, this formulation confers the added benefit of allowing torque limitations as well as constraints on friction and on ground reaction forces, although doing so may destroy feasibility. With no constraints, the solution is feasible and is known in closed form, often referred to as the pointwise min-norm controller [2].

In contrast to its predecessors, it has been formally shown that the presented controller maintains exponential stability of the hybrid system to which it is applied for small enough control gains. Although formal results regarding the robustness of the shaped system to perturbations in initial conditions have not yet been established, simulations show that the method can result in a larger domain of attraction and shorter stabilization times for limit cycles of certain systems. Indeed simulation results are provided for a cart-spring system to demonstrate improved convergence properties and for a compass-gait biped to demonstrate the robustness improvement that can be conferred by energy shaping. Despite the lack of formal claims with respect to global properties, the local stability properties are practically useful when using energy shaping as a stabilizing controller in operating regions around the desired behavior. Ultimately, the goal will be to apply these methods to complex biped and humanoid robots with a view toward robotic-assistive devices.

\section{References}

[1] A. D. Ames, K. Galloway, K. Sreenath, and J. W. Grizzle. Rapidly exponentially stabilizing control Lyapunov functions and hybrid zero dynamics. IEEE T. Automat. Contr., 59(4):876-891, 2014.

[2] R. A. Freeman and P. Kokotović. Robust Nonlinear Control Design. Birkhäuser, Boston, MA, 1996.

[3] J. W. Grizzle, C. Chevallereau, R. W. Sinnet, and A. D. Ames. Models, feedback control, and open problems of 3D bipedal robotic walking. Automatica, 50(8):1955-1988, 2014.

[4] T. McGeer. Passive dynamic walking. Int. J. Robot. Research, 9(2):62-82, 1990.

[5] R. W. Sinnet and A. D. Ames. A Lyapunov approach to energy shaping in non-conservative hybrid systems. Submitted.

[6] R. W. Sinnet and A. D. Ames. 2D bipedal walking with knees and feet: A hybrid control approach. In Proc. 48th IEEE Conf. Decision Contr./28th Chinese Contr. Conf., pages 3200-3207, Shanghai, China, December 2009.

[7] R. W. Sinnet and A. D. Ames. 3D bipedal walking with knees and feet: A hybrid geometric approach. In Proc. 48th IEEE Conf. Decision Contr./28th Chinese Contr. Conf., pages 3208-3213, Shanghai, China, December 2009.

[8] M. W. Spong, J. K. Holm, and D. Lee. Passivity-based control of bipedal locomotion. IEEE T. Robotic. Autom., 14(2):30-40, 2007. 


\section{MS5: Mechanics in an Inter., Multiphysics Env., Transforming Ma- terials Sciences and Biology}

Mechanics belongs to the oldest branches of physics, being characterized by an exceptional degree of maturity, and having fostered innumerable technological advancements, pervading daily life as well as breathtaking engineering deeds. In this context, mechanics sometimes appears as a closed, somehow "completed" discipline, in contrast to emerging branches of science, such as life sciences or biotechnology.

This symposium is to show concrete examples that the aforementioned appearance may be regrettable, but more importantly, that it is actually not supported by reality: In fact, engineering mechanics has turned out as a game changer in topics where it would not be expected in the first place: examples at the mini symposium will cover bio-nano-technology, systems biology, molecular chemistry, and bio-physics, to name just a few. 


\title{
Mechanics of biological interfaces under stretch and across scales: lipid bilayer membranes and epithelia
}

\author{
Marino Arroyo \\ Universitat Politècnica de Catalunya, Barcelona, Spain
}

\begin{abstract}
Biological interfaces hierarchically partition our body at different scales. In cells and within cells, these interfaces are molecularly thin biomembranes. At the tissue scale, epithelia are cohesive two-dimensional sheets of cells, which line free surfaces and cavities. From the point of view of mechanics of materials, these two biological interfaces are multifunctional active materials, which are capable of dealing with conflicting mechanical requirements. On the one hand, they must be malleable to adopt functional shapes, or to self-repair. On the other hand, they must be resilient to provide structural integrity in a mechanically active environment. I will present recent work trying to understand how these material interfaces perform these mechanical functions when adhered to a substrate and subject to stretch. In all these studies, continuum models and simulations of membrane [1] and hydrogel [2] mechanics have been very important.

I will first describe how synthetic bilayers passively regulate stress and shape in response to lateral and osmotic stresses [3]. The mechanoadaptation of biomembranes in living cells is commonly though to involve active cell processes like endocytosis and exocytosis or the formation and flattening of membrane invaginations/evaginations. Strikingly, in recent work we have shown that the passive, purely mechanical mechanism identified on synthetic membranes are also exploited by biological membranes to cope with sudden stresses [4]. I will then describe how structural integrity of epithelia is challenged under stretch, creating interfacial cracks at cell-cell junctions [5].
\end{abstract}

\section{References}

[1] M. Rahimi, M. Arroyo. Shape dynamics, lipid hydrodynamics and the complex viscoelasticty of bilayer membranes. Phys. Rev. E 86 (2012), 11932-11947.

[2] W. Hong, X. Zhao, J. Zhou, Z. Suo. A theory of coupled diffusion and large deformation in polymeric gels. J. Mech. Phys. Solids 56 (2008), 1779-1793.

[3] M. Staykova, M. Arroyo, M. Rahimi, H. A. Stone. Confined Bilayers Passively Regulate Shape and Stress. Phys. Rev. Lett. 110 (2013), 028101.

[4] A.J. Kosmalska, L. Casares, A. Elosegui-Artola, J.J. Thottacherry, R. Moreno-Vicente, M.A. del Pozo, S. Mayor, M. Arroyo, D. Navajas, X. Trepat, N.C. Gauthier, P. Roca- Cusachs. Physical principles of membrane remodelling during cell mechanoadaptation. Under review (2014).

[5] L. Casares, R. Vincent, D. Zalvidea, N. Campillo, D. Navajas, M. Arroyo, X. Trepat. Hydraulic fracture during epithelial stretching. Nature Materials, accepted (2015). 


\title{
The fiber reorientation problem revisited in the context of Eshelbian micromechanics: theory and computations
}

\author{
Claire Morin, Stéphane Avril, Christian Hellmich \\ Ecole Nationale Supérieure des Mines, CIS-EMSE, CNRS:UMR5307, LGF \\ F-42023 Saint-Etienne, France; claire.morin@emse.fr; avril@emse.fr \\ Institute for Mechanics of Materials and Structures, \\ Vienna University of Technology (TU Wien) 1040 Vienna, Austria; \\ christian.hellmich@tuwien.ac.at
}

\begin{abstract}
Constitutive modeling of soft biological tissues has been the topic of an abundant literature, trying to capture the complex mechanical behavior of tissues, made of an assembly of loose collagenous fibrils, which are stretched by application of a mechanical loading. This complex, non-linear, mechanical behavior originates in geometrical changes in the tissue microstructure, the initially crimped and variously oriented collagenous fibrils being progressively decrimped and reoriented so as to reinforce the tissue in the direction of the applied load [1].

We here propose to model the fibrillar decrimping and reorientation through extension of the classical framework of continuum micromechanics [2], exploiting the famous Eshelby inhomogeneity problem [3]. A Representative Volume Element of a collagenous soft tissue contains an arrangement of variously oriented, infinitely long, linear elastic inclusions embedded in a linear elastic, soft matrix. The rotations of the inclusions resulting from the application of a macroscopic strain field are deduced from use of the aforementioned inhomogeneity problem: in more detail, we employ the antimetric part of this problem's displacement gradient so as to compute the second-order spin tensor. In this context, we first derive an analytical expression for what we call "Eshelby rotation operator" (considered as the equivalent to the Hill tensor for the microscopic strain tensors), which relates the microscopic spin tensor to the inclusion eigenstress. On this basis, we can relate the spin-tensor to remote strains subjected to the infinite solid matrix, which we then combined with the strain average rule, as to arrive at a micro-spin-to-macro-RVE-strain relation. The latter quantifies load-induced micro-configurational changes [4], which completes our new random homogenization schemes.

The resulting multiscale model for fibrillar tissue allows to qualitatively reproducing the macroscopic response of soft tissues, as well as the evolution of the fibrillar inclination during loading, opening a new framework for the development of 3D multiscale constitutive laws for soft tissues, able to quantify the specific contribution of each constituent on the overall response of the tissue.
\end{abstract}

The long-term application of such models, coupled to proper failure mechanisms is foreseen for the failure risk-assessment of vascular diseases, such as aneurysms.

\section{References}

[1] R.H. Cox. Regional variation of series elasticity in canine arterial smooth muscles. Am. J. Physiol. Heart \& Circulatory Physiol. 234 (1978) 542-551.

[2] A. Zaoui. Continuum micromechanics: Survey. J. Eng. Mech. 128 (2002) 808-816.

[3] J.D. Eshelby. The Determination of the Elastic Field of an Ellipsoidal Inclusion, and Related Problems. Proc. R. Soc. London. Ser. A 241 (1957) 376-396.

[4] A. Kailasam, P. Ponte Castañeda. A general constitutive theory for linear and nonlinear particulate media with microstructure evolution. J. Mech. Phys. Solids 46 (1998) 427-465. 


\title{
Multiscale hierarchical mechanics in soft tissues
}

\author{
$\underline{\text { Michele Marino }}^{a}$, Giuseppe Vairo ${ }^{b}$ \\ ${ }^{a}$ Institute for Continuum Mechanics \\ G. W. Leibniz University of Hannover - 30167 Hannover, Germany \\ ${ }^{b}$ Department of Civil Engineering and Computer Science Engineering \\ University of Rome "Tor Vergata" - 00133 Rome, Italy
}

Soft tissues are throughout the whole human body and they include tendons, ligaments, skin, fibrous tissues, muscles, blood vessels. They link, support, and are part of other bio-structures and organs, playing a key role in the biomechanics of many body systems (e.g., musculo-skeletal, respiratory, cardiovascular). Soft tissues are generally fibrous connective tissues which can be either dense or loose, depending on the collagen amount. They consist primarily of elastin, amorphous ground substance, cells and collagen fibers. As confirmed by well-established studies [1], the highly nonlinear constitutive response of soft tissues at the macroscale is strictly related to the hierarchical organization of collagen from nano (namely, molecular arrangement) up to the microscale (fibrils and fibers). Accordingly, different collagen patterns and amounts induce different mechanical responsesvat the macroscale, in terms of stiffness and strength features. As a result, functional values of compliance for arterial walls in cardio-vascular system, of stiffness for pulmonary tissues in respiratory system, and of extensibility for tendons in musculo-skeletal system, are experienced. Moreover, altered tissue response in disease (e.g., aneurism, keratoconus, arthofibrosis) arises from pathological tissue remodeling and arrangement alterations at the nano and microscale, inducing unphysiological histology and biochemical composition.

In this scenario, the development of theoretical results and computational methods for effectively correlating mechano-regulated physio-pathological processes occurring at very different length scales, as well as to identify relationships among alterations and diseases, represents an open challenge at the cutting edge of modern biomechanics.

In this paper, mechanics of soft tissues is modeled by describing tissue structured hierarchical arrangement, reducing model complexity by means of multiscale homogenization techniques. Such an approach, employed for example in [2-6], is referred to as a structural multiscale method. It consists in regarding the tissue at the macroscale as a fiber-reinforced composite material, wherein properties of reinforcement phase are recovered by mechanical models at smaller (than the macro one) length scales, coupled each other by means of consistent inter-scale relationships. Accordingly, the equivalent responses of tissue substructures at different scales, including possible damage and inelastic mechanisms [7, 8], are analytically derived and consistently integrated and upscaled, allowing to include at the macroscale the dominant mechanisms occurring at smaller scales.

\section{References}

[1] P. Fratzl. Collagen: Structure and mechanics. Springer-Verlag (2008), New York.

[2] H. Tang, M.J. Buehler, B. Moran. A constitutive model of soft tissue: from nanoscale collagen to tis- sue continuum. Ann. Biomed. Eng. 37 (2009), 1117-1130

[3] F. Maceri, M. Marino, G. Vairo. A unified multiscale mechanical model for soft collagenous tissues with regular fiber arrangement. J. Biomech. 43 (2010), 355-363.

[4] F. Maceri , M. Marino, G. Vairo. Age-dependent arterial mechanics via a multiscale elastic approach. Int. J. Comput. Methods Eng. Sci. Mech. 14 (2013), 141-151.

[5] M. Marino, G. Vairo. Multiscale elastic models of collagen bio-structures: from cross-linked molecules to soft tissues. Stud. Mechanobiol. Tissue Eng. Biomater. 14 (2013), 73-102.

[6] M. Marino, G. Vairo. Stress and strain localization in stretched collagenous tissues via a multiscale modelling approach. Comput. Methods Biomech. Biomed. Eng. 17 (2014), 11-30.

[7] F. Maceri, M. Marino, G. Vairo. Elasto-damage modeling of biopolymer molecules response. CMES 87 (2012), 461-481.

[8] M. Marino, G. Vairo. Influence of inter-molecular interactions on the elasto-damage mechanics of collagen fibrils: A bottom-up approach towards macroscopic tissue modeling. J. Mech. Phys. Solids 73 (2014), 38-54. 


\title{
The theory of mechanobiological stability: on the theoretical foundations of mechanobiology in soft tissue
}

\author{
Christian J. Cyron, Jay D. Humphrey \\ Yale University, New Haven, CT, USA
}

In living tissue, the relation between load and deformation can be understood on short time scales (up to minutes) largely from a handful of well-known and simple principles such as Newton's axioms or Hooke's law (including its generalizations). On long time scales, however, it is often governed by mechanobiolgy, that is, mechano-regulated, cell-driven growth and remodeling. Although recent experimental findings have underlined more and more the overwhelming importance of mechanobiology in biomechanics, its mathematical foundations remain poorly understood to date.

In this presentation, we introduce the theory of mechanobiological stability $[1,2]$ as a simple conceptual framework to understand continuum mechanobiology in soft tissue. Though mainly based on but two simple assumptions, the presence of mass turnover and the existence of a state of tensional homeostasis, it provides natural explanations for a host of salient features of living soft tissue such as the virtual omnipresence of prestress and mechanoregulated growth and remodeling, as well as the often observed creeping loss of geometric integrity (e.g., in aneurysms).

The presentation will be concluded by a number of examples demonstrating the excellent agreement between the theory of mechanobiological stability and well-known clinical and experimental observations.

\section{References}

[1] C.J. Cyron, J.D. Humphrey. Vascular homeostasis and the concept of mechanobiological stability. Int. J. Eng. Sci. 85 (2014), 203-223.

[2] C.J. Cyron, John S. Wilson, J.D. Humphrey. Mechanobiological stability: a new paradigm to understand the enlargement of aneurysms?. J. R. Soc. Interface. (2014), 20140680. 


\title{
Coupling X-ray physics and engineering mechanics, for enhanced analysis of Computer Tomographic images
}

\author{
Christian Hellmich, Alexander Dejaco, Romane Blanchard \\ Vienna University of Technology (TU Wien), Austria
}

\begin{abstract}
Since its invention in the 1960s, Computed Tomography has become one of the most powerful and versatile non-destructive imaging tools, with applications ranging from biomedicine to concrete technology. For about two decades, it is also common to use CT images as the basis for Finite Element modeling of the scanned objects. Thereby, the main focus has been classically laid upon the accurate representation of geometrical details, while particularly for solids made up of natural non-homogeneous materials, the question of material property assignment has remained an open challenge over the years.

Since 2008 [1], our group, in cooperation with colleagues from Germany, Italy, Russia, Poland, Belgium, and Iceland $[1,2,3,4,5,6]$, has been deeply involved in overcoming this challenge, by more deeply studying the X-ray physics underlying Computed Tomography: we developed increasingly mature methods to retrieve, from the grey value-defined voxel characteristics given in CT images, the actually underlying physical property, called X-ray attenuation coefficient. The latter contains information on the chemical composition of the material making up the considered voxel, and combining this information with known chemical characteristics of the material class making up the scanned object, gives access to important microstructural information inside the voxel, such as microporosity, or contents of known chemical substances. The latter then enter, as input values, experimentally validated micromechanical formulations representing the material inside the voxel, so as to reliably determine the voxel's mechanical properties. Corresponding CT-to-mechanics conversion schemes will be presented in appropriate detail, with applications ranging from various ceramics $[2,3,6]$ and polymer-ceramic composites [4] used in tissue engineering, to organs made up of the natural material bone $[1,5]$.
\end{abstract}

\section{References}

[1] Ch. Hellmich, C. Kober, B. Erdmann. Micromechanics-based conversion of CT data into anisotropic elasticity tensors, applied to FE simulations of a mandible. Ann. Biomed. Eng. 36 (2008), 108 -122.

[2] S. Scheiner, R. Sinibaldi, B. Pichler, V. Komlev, C. Renghini, C. Vitale-Brovarone, F. Rustichelli, Ch. Hellmich. Micromechanics of bone tissue-engineering scaffolds, based on resolution error-cleared computer tomography, Biomat. 30 (2009), $2411-2419$.

[3] A. Dejaco, V. Komlev, J. Jaroszewicz, W. Swieszkowski, Ch. Hellmich. Micro CT-based multiscale elasticity of double-porous (pre-cracked) hydroxyapatite granules for regenerative medicine. J. Biomech. 45 (2012), $1068-1075$.

[4] K. Luczynski, A. Dejaco, O. Lahayne, J. Jaroszewicz, W. Swieszkowski, Ch. Hellmich. MicroCT/Micromechanics-Based Finite Element Models and Quasi-Static Unloading Tests Deliver Consistent Values for Young's Modulus of Rapid-Prototyped Polymer-Ceramic Tissue Engineering Scaffold. CMES 87 (2012), 505-528.

[5] R. Blanchard, A. Dejaco, E. Bongaers, Ch. Hellmich. Intravoxel bone micromechanics of microCT-based finite element simulations. J. Biomech. 40 (2013), $2710-2721$.

[6] A. Czenek, R. Blanchard, A. Dejaco, O. Sigurjonsson, G. Örlygsson, P. Gargiulo, Ch. Hellmich. Quantitative intravoxel analysis of microCT-scanned resorbing ceramic biomaterials - Perspectives for computer-aided biomaterial design. J. Mat. Res. 29 (2014), $2757-2772$. 


\title{
Look different, better understand: computational multphysics enhanced imaging and measuring in biomedicine
}

\author{
Svenja Schoeder, Christian Roth, Martin Kronbichler, Wolfgang A. Wall \\ Institute for Computational Mechanics, Technical University Munich \\ Boltzmannstr. 15, 85747 Garching, Germany, http://www.lnm.mw.tum.de/
}

Computational modeling can obviously not only be used to analyze or design new technical systems. It can also be used to allow unprecedented scientific insight in cases when experimental or theoretical approaches reach their limits. Just one example from our recent work is sub-cellular biophysics, where systems are far too complex to be studied theoretically but also experimental methods are limited in their ability to create well defined configurations or measure every detail $[1,2]$. But the same is also true in modern, highly sophisticated imaging and measurement techniques in medicine. New and advanced (multiphysics) models and respective computational methods can boost insight and value of these methods, while both exposure of the patients as well as financial investments are unaltered. In this talk we will give two examples we are currently working on: photoacoustic tomography and electrical impedance tomography.

Photoacoustic tomography is a medical imaging technique which combines diffuse optical tomography and ultrasonography. An object of interest is illuminated by a short time laser pulse and the light propagates within the object according to its optical properties. At areas of high absorption, the optical energy is transformed into heat followed by thermal expansion. This expansion initiates pressure waves which are eventually measured by acoustical detectors. The recorded acoustical signal allows for conclusions on optical and mechanical properties of the underlying material. The explanatory power of the recorded signals depends among others on the accuracy of the used reconstruction algorithm. We have developed and will report on a novel approach for this purpose that is based upon an efficient simulation approach for wave propagation in heterogeneous media via the hybridizable discontinuous Galerkin (HDG) method [3] and the formulation of the inverse problem by use of the adjoint method for the discretized photoacoustic problem.

It is extremely difficult to obtain insight into important physiological quantities and states of the lung during breathing or ventilation. This hampers medical therapy but also the development and evaluation of lung models that in turn could be used for improved therapies. One key parameter in respiratory imaging is the magnitude of regional lung aeration which is optimized during mechanical ventilation trials. One non-invasive and radiationfree approach is electrical impedance tomography (EIT). It is based on the measurement of potential differences between electrodes on the surface of a body when small alternating currents are sent into the studied subject at high frequency. While current injections take place at adjacent pairs of 16 self-adhesive surface electrodes placed around the patient's chest, an image showing local tissue bioimpedance can be reconstructed by solving the inverse electrical problem for $\mathrm{N}=208$ voltage measurements. EIT performs especially well in lung imaging as tissue bioimpedance considerably changes with local aeration due to the insulating properties of the enclosed air in the alveoli. Based on the alveolar microstructure, we have derived a link between air content and measured bioimpedance [4] and by solving the highly ill-posed EIT reconstruction problem, new insights into the lung's local structure and function are provided. We believe this information will result in more protective ventilation protocols, especially in such pathophysiology where patient-specific trial-and-error methods are at high risk for respiratory failure.

\section{References}

[1] Müller K.W., Bruinsma R.F., Lieleg O., Bausch A.R., Wall W.A., Levine A.J.: Rheology of semiflexible bundle networks with transient linkers. Physical Review Letters, 112, 238102, 2014.

[2] Cyron C.J., Müller K.W., Schmoller K.M., Bausch A.R., Wall W.A., Bruinsma R. F.: Equilibrium phase diagram of semi-flexible polymer networks with linkers. Europhysics Letters, 102, 38003, 2013.

[3] Kronbichler M., Schoeder S., Müller C., Wall W.A.: Comparison of implicit and explicit hybridizable discontinuous Galerkin methods for the acoustic wave equation. International Jorunal for Numerical Methods in Engineering, submitted 2015.

[4] Roth C., Ehrl A., Becher T., Frerichs I., Weiler N., Wall W.A.: Correlation between alveolar ventilation and electrical properties of lung parenchyma. Physiological Measurement, submitted 2014. 


\section{YRMS1: Analysis, Applications and Approximation of Constrained PDEs}

A common and convenient way to model multi-component phenomena is to model the components separately and to glue them together via coupling the variables at the interfaces. This approach, however, leads to constrained PDEs that are more often referred to as abstract DAEs or PDAEs and that require sophisticated methods for their numerical and analytical treatment.

The speakers of our minisymposium reflect the broad application area of constrained PDEs and discuss difficulties in the application side and recent advances in the analysis and the numerical approximation. The particular talks will cover general theoretical aspects and applications in the modeling of elastodynamics, electromagnetics, flow networks, and fluid dynamics. 


\title{
Stable and efficient simulation of hyperbolic PDAEs describing flow networks
}

\author{
Christoph Huck ${ }^{1}$, Lennart Jansen ${ }^{2}$, Caren Tischendorf ${ }^{1}$ \\ ${ }^{1}$ Humboldt-University of Berlin, \\ ${ }^{2}$ Heinrich-Heine-University Düsseldorf
}

We consider partial differential algebraic equation systems (PDAEs) that consist of hyperbolic PDEs of the type

$$
\begin{aligned}
p_{t}+A q_{x} & =0 \\
q_{t}+B p_{x}+G(q) q+H & =0
\end{aligned}
$$

and are coupled via algebraic boundary conditions. Such systems appear in the modeling of flow networks as e.g. water or gas supplying networks [1]. For our simulation approach we use the method of lines, yielding a differential algebraic equation (DAE) which is adaptively discretized in time.

We present a perturbation analysis for a simple prototype for different variants of space discretizations. In particular we show that the index of the resulting DAEs may depend on the chosen space discretization.

Additionally, we present a network topology dependent space discretization guaranteeing DAEs of index 1. Furthermore we study a network topological procedure to reduce the resulting DAEs into semi-explicit systems of the form

$$
\begin{aligned}
x^{\prime} & =f(x, t) \\
y & =M x+r(t) .
\end{aligned}
$$

that can be exploited for more efficient simulations, e.g. by use of model order reduction or exponential integrators.

\section{References}

[1] L. Jansen, C. Tischendorf. A unified (P)DAE modeling approach for flow networks. In Progress in Differential-Algebraic Equations. Differential-Algebraic Equations Forum, 127-151. Springer Berlin Heidelberg, 2014. 


\title{
Stochastic Modeling and Regularity of the Nonlinear Elliptic-Parabolic Magnetoquasistatic Equation
}

\author{
Ulrich Römer, Sebastian Schöps \\ Institut für Theorie Elektromagnetischer Felder, Technische Universitaet Darmstadt \\ Graduate School of Computational Engineering, Technische Universitaet Darmstadt
}

Simulation predictions with increased reliability can be realized by taking into account uncertainties in the inputs of mathematical models. In this contribution we are concerned with the nonlinear elliptic-parabolic magnetoquasistatic equation

$$
\sigma \partial_{t} \vec{A}+\nabla \times(\nu(|\nabla \times \vec{A}|) \nabla \times \vec{A})=\vec{J}
$$

with uncertainties, where $\vec{A}$ and $\vec{J}$ represent the magnetic vector potential and source current density, respectively. This model, which is important for magnetic devices such as electrical machines or magnets, is elliptic in the non-conducting air part and parabolic in the conducting iron part. Its numerical approximation by the finite element method leads to a system of differential-algebraic equations [1] of index one or possibly higher for a coupling to external circuits. Here, we are concerned with uncertainties in the material properties $\nu$ expressing the magnetic iron properties. To this end a stochastic/parametric model is proposed and analyzed. In particular we discuss the modeling of randomness in $\nu$ in the presence of a monotonicity constraint. Also an efficient spline-based discretization of the random input by the Karhunen-Loève expansion is presented. The stochastic model has a high-dimensional deterministic counter-part

$$
\sigma \partial_{t} \vec{A}(\vec{y})+\nabla \times(\nu(\vec{y},|\nabla \times \vec{A}(\vec{y})|) \nabla \times \vec{A}(\vec{y}))=\vec{J},
$$

with parameter vector $\vec{y} \in \Gamma \subset \mathbb{R}^{M}$. Its efficient numerical approximation is a challenging task. A particularly appealing method is based on stochastic collocation [2] as it features a rapid convergence rate and results in the repetitive solution of deterministic problems. Although this technique is well-established in practice, a priori estimates of the collocation error of system (1) are the subject of ongoing work. Starting from a regularity analysis for the uncoupled elliptic system [3] yielding an algebraic convergence rate of $p^{-k}$ for tensor product collocation of polynomial degree $p$, possible extensions to the case of a field-circuit coupling and to the timetransient case will be addressed. The result is confirmed by academic and engineering benchmark examples.

\section{References}

[1] A. Nicolet, F. Delincé. Implicit Runge-Kutta Methods for Transient Magnetic Field Computation. IEEE Transactions on Magnetics 32 (1996), 1405-1408.

[2] I. Babuška, F. Nobile, and R. Tempone. A stochastic collocation method for elliptic partial differential equations with random input data. SIAM review 52 (2010), 317-355.

[3] U. Römer, S. Schöps, and T. Weiland. Stochastic Modeling and Regularity of the Nonlinear Elliptic curl-curl Equation. Submitted manuscript. 


\title{
Hydrodynamic force elements: A PDAE approach
}

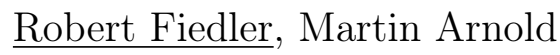 \\ Martin-Luther-University Halle-Wittenberg
}

The simulation of mechanical systems results often in multi-component phenomena with different time scales or different solution strategies which influence each others problem characteristics. In our case we have a flexible multibody system coupled with special force elements. The modelling of elastohydrodynamic bearings in combustion engines leads to a coupled system of partial differential algebraic equations, which is represented by a flexible multibody system model of crankshaft and bearing and by the Reynolds equation that describes the non-linear effects in the fluid film. The hydrodynamic forces depend strongly on the position and the elastic deformation of crankshaft and bearing shell therefore a fine spatial discretisation is needed.

The influence of the spatial discretisation on accuracy and numerical effort will be discussed. Since a fine one substantially slows down the numerical solution, we propose an asymptotic analysis with methods from singular perturbation theory to speed-up time integration. The interplay of this semi-analytical approach with index reduction techniques for the multibody part is studied for the fourbar test problem.

Numerical tests for a realistic benchmark problem illustrate the advantages of this approach. 


\title{
The Pressure Manifold in the Unsteady Navier-Stokes Equation and in Semi-Discretizations
}

\author{
Jan Heiland \\ Max-Planck Institute for Complex Dynamical Systems Magdeburg, Germany
}

The right treatment of the pressure $p$ is key in stable approximation schemes for time dependent NavierStokes equations.

$$
\begin{aligned}
\dot{v}+(v \cdot \nabla) v+\nabla p-\nu \Delta v & =f, \\
\operatorname{div} v & =0, \quad \text { in } \Omega \times(0, T) .
\end{aligned}
$$

A discrete approximation to (1) is typically given as

$$
\begin{aligned}
M \dot{v}_{k}-A\left(v_{k}\right)-J_{k}^{\top} p_{k} & =f_{k}, \\
J_{k} v_{k} & =0, \quad \text { in }(0, T),
\end{aligned}
$$

i.e. the spatial component in (1) is discretized by approximating $v(t)$ and $p(t)$ via finite-dimensional vectors $v_{k}(t)$ and $p_{k}(t)$

Commonly used methods like projection or pressure correction schemes for the time discretization of (2) base on the repeated solution of the so called Pressure Poisson Equation:

$$
-J_{k} M^{-1} J_{k}^{\top} p_{k}=J_{k} M^{-1} f+J_{k} M^{-1} A\left(v_{k}\right) .
$$

For stable discretization schemes, the discrete Pressure Poisson Equation is well defined for (2), which may not be the case for the corresponding continuous equation (1).

In my talk, I will discuss a decoupling of the Navier-Stokes equations by means of a continuous Pressure Poisson Equation that is in line with the schemes for the semi-discrete approximations. 


\section{YRMS2: Phase Field Modeling in Mechanics and Applied Mathe- matics}

The coupling of phase field equations to solid or fluid mechanics offers a wide range of possibilities in the modeling of e.g. ferroelectric solids. The mechanically inclined talks focus on the modeling and numerical simulation of phase field models for piezoelectric and ferroelectric materials. Theoretical aspects like the well-posedness of the coupled PDEs of phase field models, stability issues, and conservation properties of the numerical schemes are addressed in the mathematically inclined contributions. 


\title{
On numerical schemes for phase-field models for electrowetting with electrolyte solutions
}

\author{
Stefan Metzger \\ Friedrich-Alexander-Universität Erlangen-Nürnberg
}

We present an energy-stable, decoupled discrete scheme for a recent model (see [1]) supposed to describe electrokinetic phenomena in two-phase flow with general mass densities. This model couples momentum and Cahn-Hilliard type phase-field equations with Nernst-Planck equations for ion density evolution and an elliptic transmission problem for the electrostatic potential.

The transport velocities in our scheme are based on the old velocity field updated via a discrete time integration of the force densities. This allows to split the equations into three blocks which can be treated sequentially: The phase-field equation, the equations for ion transport and electrostatic potential, and the Navier-Stokes type equations. By establishing a discrete counterpart of the continuous energy estimate, we are able to prove the existence of discrete solutions. Finally, we shall present numerical simulations of lab-on-a-chip applications to underline the full practicality of this approach.

\section{References}

[1] E. Campillo-Funolet, G. Grün, F. Klingbeil. On Modeling and Simulation of Electrokinetic Phenomena in Two-Phase Flow with General Mass Densities. SIAM Journal on Applied Mathematics, Society for Industrial and Applied Mathematics 72 (2012), 1899-1925. 


\title{
On elastic Cahn-Hilliard systems coupled with evolution inclusions for damage processes
}

\author{
Christian Heinemann, Christiane Kraus \\ Weierstrass Institute
}

In elastically stressed alloys different physical processes such as separation of chemical substances and propagation of micro-cracks/micro-voids are shaping the micro-structure simultaneously. These processes are usually modeled independently by adapting phase-field approaches in context of Cahn-Hilliard systems and gradientof-damage models. However, especially in modern materials, the interaction of damage and phase separation cannot be neglected for a realistic description. For instance, as indicated by experiments and numerical simulations, the long-term behavior of solder joints significantly depends on the interplay of both processes.

The aim of this talk is to present a system of partial differential equations coupling two phase field models in order to describe damage phenomena and phase separation in elastic media in one unifying model. The system reads as

$$
\begin{aligned}
c_{t}= & \operatorname{div}(m(c, z) \nabla \mu) \\
\mu= & -\Delta c+\Psi^{\prime}(c)-\mathbf{C}(c, z)\left(\varepsilon(\mathbf{u})-\varepsilon^{*}(c)\right):\left(\varepsilon^{*}\right)^{\prime}(c) \\
& +\frac{1}{2} \mathbf{C}_{, c}(c, z)\left(\varepsilon(\mathbf{u})-\varepsilon^{*}(c)\right):\left(\varepsilon(\mathbf{u})-\varepsilon^{*}(c)\right) \\
\lambda \mathbf{u}_{t t} & -\operatorname{div}\left(\mathbf{C}(c, z)\left(\varepsilon(\mathbf{u})-\varepsilon^{*}(c)\right)\right)=\ell \\
z_{t}- & \operatorname{div}\left(|\nabla z|^{p-2} \nabla z\right)+f^{\prime}(z)+\xi+\varphi \\
& +\frac{1}{2} \mathbf{C}_{, z}(c, z)\left(\varepsilon(\mathbf{u})-\varepsilon^{*}(c)\right):\left(\varepsilon(\mathbf{u})-\varepsilon^{*}(c)\right)=0 \\
\xi \in \partial I_{(-\infty, 0]}\left(z_{t}\right) & \\
\varphi \in & \partial I_{[0,1]}(z)
\end{aligned}
$$

The order parameter $z$ modeling the damage process is not only constrained to the unit interval but also has to obey an irreversibility condition. Because of this highly nonsmooth evolution, the possible nonsmoothness of the underlying domain and the mixed boundary condition for the elastic deformation field, a new notion of weak solutions had to be devised.

To this end, a notion which reformulates the double inclusion for these kind of systems by a variational approach in combination with a total energy-dissipation inequality was introduced in [2] and extended to further cases in [1,3]. Global-in-time existence of weak solutions for various types of system (1) are provided. Our contribution includes cases where the mechanical forces are assumed to be in a quasi-static equilibrium $(\lambda=0)$ and cases where inertia terms are taken into account $(\lambda>0)$.

\section{References}

[1] C. Heinemann, C. Kraus. Existence of weak solutions for a PDE system describing phase separation and damage processes including inertial effects. Discrete Contin. Dyn. Syst. Ser. A (to appear)

[2] C. Heinemann, C. Kraus. Existence of weak solutions for Cahn-Hilliard systems coupled with elasticity and damage. Adv. Math. Sci. Appl. 21 (2011), 321-359.

[3] C. Heinemann, E. Rocca. Damage processes in thermoviscoelastic materials with damage-dependent thermal expansion coefficients. Math. Methods Appl. Sci. (to appear) 


\title{
Phase field modeling of ferroelectric materials with defects
}

\author{
Bai-Xiang Xu, Yinan Zuo, Yangbin Ma \\ Division Mechanics of Functional Materials, Institute of Materials Science, TU Darmstadt, \\ Alarich-Weiss-Strasse 2, Darmstadt, 64287, Germany
}

The phase field method is known as a powerful tool for the simulation of microstructure evolution in various materials, as it was revealed in the review article [1]. Recently it has become also successful for the study of polarization switching in ferroelectric materials, e.g. [2, 3]. Piezoelectrics/ferroelectrics are widely used materials for sensor and actuator applications, and are known to suffer from aging and fatigue. The degradation mechanism is closely related to defects in the materials. Therefore, it is of importance to study the domain structure in the presence of defects. In this work the phase field approach is applied to study the influence of defects on the domain structures in ferroelectrics.

By a continuum phase field model implemented with the finite element method various effects of the defects in ferroelectrics, such as space charge contribution and defect dipole, are taken into account [4, 5]. The evolution of the phase field variables is governed by the Ginzburg-Landau type evolution equation. The space charge contribution of defects are considered by formulating a volume charge density according to the semiconducting theory, while the defect dipole was treated through a static eigen-polarization. Energetically unfavorable headto-head domain structures can be stabilized by space charges induced by defects.

In addition, the abnormal behavior of relaxor ferroelectrics is simulated by applying the phase field potential to Lattice-based Monte Carlo simulations. Results show that random local electric fields can be used to explain the characteristic behavior of relaxor ferroelectrics, such as the double loop dielectric hysteresis and diffusive phase transitions.

\section{References}

[1] I. Steinbach, Phase-field models in materials science. Modelling Simul. Mater. Sci. Eng. 17 (2009) 073001.

[2] L.Q. Chen, Phase-field method of phase transitions/domain structures in ferroelectric thin films: a review J. Am. Ceram. Soc. 91 (2008) 1835-1844.

[3] W. Zhang and K. Bhattacharya, Acta Mater. 53, 185 (2005).

[4] Y. Zuo, Y.A. Genenko, B.X. Xu, Charge compensation of head-to-head and tail-to-tail domain walls in barium titanate and its influence on conductivity. J. Appl. Phys. 116 (2014) 044109

[5] Y. Zuo, Y.A. Genenko, A. Klein, P. Stein, B.X. Xu, Domain wall stability in ferroelectrics with space charges. J. Appl. Phys. 115 (2014) 084110. 


\title{
Simulation of Atomic Force Microscopy for investigating $\mathrm{BaTiO}_{3}$ and $\mathrm{LiMn}_{2} \mathrm{O}_{4}$ nanostructures based on Phase Field Approach
}

\author{
Huy Thai ${ }^{1}$, Marc-André Keip ${ }^{2}$, Jörg Schröder ${ }^{1}$, \\ Hugues-Yanis Amanieu ${ }^{3}$ and Daniele Rosato ${ }^{3}$ \\ ${ }^{1}$ Institute of Mechanics, Department of Civil Engineering, University of Duisburg-Essen \\ ${ }^{2}$ Institute of Applied Mechanics (CE), Chair I, University of Stuttgart \\ ${ }^{3}$ Applied Research 1, Advanced Functional and Sintered Materials, \\ Material Characterization and Component Design (CR/ARM1), Robert Bosch GmbH
}

Atomic force microscopy (AFM) probes the surface features of specimens using an extremely sharp tip scanning the sample surface, AFM is widely used for investigating the electrically non-conductive materials by applying an electric potential on the tip [1]. Piezoresponse Force Microscopy (PFM) and Electrochemical Strain Microscopy (ESM) are variants of AFM for different materials. Both PFM and ESM signals are obtained by observing the displacement of the tip when applying electric fields during the scanning process. The PFM technique is based on converse piezoelectric effect of ferroelectrics [2] and the ESM technique is based on electrochemical coupling in solid ionic conductors [3]. In this talk, two continuum-mechanical formulations for simulation of PFM and ESM are discussed.

In the first model, for PFM simulation, a phase field approach based on the Allen-Cahn equation for nonconserved order parameters is employed for ferroelectrics [4]. Here, the polarization vector is chosen as order parameter. Since ferroelectrics have highly anisotropic properties, this model accounts for transversely isotropic symmetry using an invariant formulation. The polarization switching behavior under the applicant of the electric field will be discussed with some numerical examples.

In the simulation of ESM, we employ a constitutive model based on the work of Bohn et. al. [5] for the modeling Lithium manganese dioxide $\mathrm{LiMn}_{2} \mathrm{O}_{4}$ (LMO). The model is similar to phase field approach based on Cahn-Hilliard equation where the concentration of Lithium is order parameter. It simulates the deformation of the LMO particle according to the applying voltage and the evolution of Lithium concentration after removing an DC pulse. The modeling results are compared to experimental data of ESM.

\section{References}

[1] S. V. Kalinin and A. Gruverman. Scanning probe microscopy of functional materials. Nanoscale imaging and spectroscopy. Springer (2010).

[2] E. Soergel. Piezoresponse force microscopy (PFM). J. Phys. D: Appl. Phys. 44 (2011), 464003.

[3] A. Morozovska, E. Eliseev, N. Balke, and S.V. Kalinin. Local probing of ionic diffusion by electrochemical strain microscopy: spatial resolution and signal formation mechanisms. J. Appl. Phys. 108 (2010), 053712.

[4] D. Schrade, R. Müller, D. Gross, M.-A. Keip, H. Thai, and J. Schröder. An invariant formulation for phase field models in ferroelectrics. Int. J. Solids. Struct. 51(11-12) (2014), 2144-2156.

[5] E. Bohn, T. Eckl, M. Kamlah and R. McMeeking. A model for lithium diffusion and stress generation in an intercalation storage particle with phase change. J. Electrochem. Soc. 160(10) (2013), A1638-A1652. 


\section{YRMS3: Discretization Aspects in PDE Constrained Optimization}

Optimization problems with partial differential equations (PDEs) as constraint have been of tremendous research interest over the past decades. Due to the PDE, these problems are of infinite dimension and can rarely be tackled directly. Instead, the PDE needs to be discretized.

This important step in the numerical consideration of PDE constrained optimization problems gives rise to a multitude of research questions, going from the choice of a suitable discretization for a given application, convergence analysis for the discrete approximations to the limit as the mesh is refined, to the design of appropriate (finite dimensional) solvers for the corresponding discrete problems. 


\title{
Shape optimization by pursuing diffeomorphisms
}

\author{
Ralf Hiptmair, Alberto Paganini \\ Seminar for Applied Mathematics, ETHZ
}

We consider PDE constrained shape optimization in the framework of finite element discretization of the underlying boundary value problem. We present an algorithm tailored to preserve and exploit the approximation properties of the finite element method, and that allows for arbitrarily high resolution of shapes. It is based on the method of mappings and it employs (i) B-spline based representations of the deformation diffeomorphism, and (ii) superconvergent domain integral expressions for the shape gradient. We provide numerical evidence of the performance of this method both on prototypical well-posed and ill-posed shape optimization problems.

\section{References}

[1] R. Hiptmair, A. Paganini and S. Sargheini. Comparison of approximate shape gradients. BIT Numerical Mathematics (2014).

[2] R. Hiptmair, A. Paganini. Shape optimization by pursuing diffeomorphisms. SAM-Report 2014-27. 


\title{
Fast Iterative Solvers for Discretizations of PDE-Constrained Optimization Problems
}

\author{
John W. Pearson \\ University of Edinburgh
}

\begin{abstract}
A key consideration when discretizing PDE-constrained optimization problems is that of devising fast and effective methods for solving the resulting discretized systems. In this talk we focus on constructing preconditioned iterative methods for such problems, by exploiting the saddle point structure of the matrix systems that arise.

Using strategies that we have developed for more fundamental problems of time-independent [2] and timedependent [4] form, we consider fast solvers for time-dependent PDE-constrained optimization problems. We focus on two specific such problems, from different application areas. Firstly we examine the time-dependent Stokes control problem [3], and build solvers for the sparse matrix systems involved using effective approximations of the (1,1)-block and Schur complement. Secondly we examine dense matrix systems that arise from the optimal control of fractional differential equations [1], and discover whether our approaches may also be applied to such problems.

For each problem presented in this talk, we consider the likely theoretical convergence properties of our methods, the practical rate of convergence observed in numerical experiments, and whether our methods may lead to parallelizable solution strategies.
\end{abstract}

\section{References}

[1] S. Dolgov, J. W. Pearson, D. V. Savostyanov and M. Stoll, Fast Tensor Product Solvers for Optimization Problems with Fractional Differential Equations as Constraints, to be submitted.

[2] J. W. Pearson and A. J. Wathen, A New Approximation of the Schur Complement in Preconditioners for PDE-Constrained Optimization, Numerical Linear Algebra with Applications, 19(5), pp.294-310, 2012.

[3] J. W. Pearson, Fast Iterative Solvers for Large Matrix Systems Arising from Time-Dependent Stokes Control Problems, submitted, 2014.

[4] J. W. Pearson, M. Stoll and A. J. Wathen, Regularization-Robust Preconditioners for Time-Dependent PDEConstrained Optimization Problems, SIAM Journal on Matrix Analysis and Applications, 33(4), pp.1126$1152,2012$. 


\title{
Finite element error estimates for Dirichlet boundary control problems on polygonal domains
}

\author{
Th. Apel ${ }^{1}$, M. Mateos ${ }^{2}$, J. Pfefferer $^{1}$, and A. Rösch ${ }^{3}$ \\ ${ }^{1}$ Universität der Bundeswehr München \\ ${ }^{2}$ Universidad de Oviedo \\ ${ }^{3}$ Universtät Duisburg-Essen
}

In this talk we study the control constrained Dirichlet boundary control problem

$$
\begin{aligned}
& \min J(u)=\frac{1}{2} \int_{\Omega}\left(S u(x)-y_{\Omega}(x)\right)^{2} d x+\frac{\nu}{2} \int_{\Gamma} u^{2}(x) d \sigma(x) \\
& \text { subject to }(S u, u) \in H^{1 / 2}(\Omega) \times L^{2}(\Gamma), \\
& u \in U_{a d}=\left\{u \in L^{2}(\Gamma): a \leq u(x) \leq b \quad \text { for a.a. } \quad x \in \Gamma\right\},
\end{aligned}
$$

where $S u$ is the solution $y$ of the state equation

$$
-\Delta y=0 \text { in } \Omega, y=u \text { on } \Gamma .
$$

The underlying domain $\Omega$ is assumed to be polygonal but not necessarily convex. Since for $u \in U_{a d}$ the state equation does not possess a variational solution, the state equation is understood in the transposition sense.

In the first part of this talk, we investigate the regularity of the solution of the optimal control problem. It is well known that in polygonal domains the solution of an elliptic partial differential equation generally contains singular terms which depend on the size of the interior angles of the domain. By analyzing these terms in detail we are able to improve existing regularity results for the solution of the optimal control problem in convex domains. Moreover, completely new regularity results are presented for problems posed in non-convex domains. For example, we show that the optimal control is a continuous function although the normal derivative of the adjoint state may be unbounded.

In the second part, we discuss error estimates for the discretized optimal control problem where we apply a full discretization with piecewise linear and continuous functions for both the state and the control. The error estimates which we obtain mainly depend on the size of the interior angles but also on the presence of control constraints.

Finally, different numerical examples are presented in order to illustrate the theoretical results. 


\title{
Scaling Limits in Computational Bayesian Inversion
}

\author{
Claudia Schillings, Christoph Schwab \\ University of Warwick \\ SAM - ETHZ
}

In this talk, preconditioning strategies for sparse, adaptive quadrature methods for computational Bayesian inversion of operator equations with distributed uncertain input parameters will be presented. Based on sparsity results of the posterior, error bounds and convergence rates of dimension-adaptive Smolyak quadratures can be shown to be independent of the parameter dimension, but the error bounds depend exponentially on the inverse of the covariance of the additive, Gaussian observation noise. We will discuss asymptotic expansions of the Bayesian estimates, which can be used to construct quadrature methods combined with a curvature-based reparametrization of the parametric Bayesian posterior density near the (assumed unique) global maximum of the posterior density leading to convergence with rates independent of the number of parameters as well as of the observation noise variance. 


\title{
A Posteriori Error Estimation for State-Constrained Optimal Control Problems
}

\author{
K.G. Siebert ${ }^{1}$, A. Roesch ${ }^{2}$, S. Steinig ${ }^{3}$ \\ ${ }^{1}$ University of Stuttgart \\ ${ }^{2}$ University Duisburg-Essen \\ ${ }^{3} \mathrm{TU}$ Dortmund
}

In this contribution we will examine a finite element discretisation of a state-constrained elliptic optimal control problem. We will present a reliable a posteriori error estimator giving an upper bound for the error between the true solution and the discrete one up to data-dependent constants which also takes into account the regularisation error naturally coming into play when tackling these kind of problems numerically. Together with a basic convergence result for a sequence of finite element discretisations we are thus able to build a convergent adaptive scheme for this type of problem mirroring the results derived for PDEs and control-constrained optimal control problems in [1] and [2] respectively.

\section{References}

[1] P. Morin, K.G. Siebert, A. Veeser. A basic convergence result for conforming adaptive finite elements. Mathematical Models and Methods in Applied Sciences. 18 (2008), 707-737.

[2] K. Kohls, A. Roesch, K.G. Siebert. A posteriori error analysis of optimal control problems with control constraints. SIAM Journal on Control and Optimization. 52 (2014), 1832-1861. 


\title{
Optimal convergence order for control constrained optimal control problems
}

\author{
René Schneider, Gerd Wachsmuth
}

In this talk we consider the numerical solution of control constrained optimal control problems. We are interested in obtaining the optimal convergence rate for the $L^{2}$-error w.r.t. the number of degrees of freedom. Due to the control constraint, the optimal control possesses a kink at the interface between the active and inactive set w.r.t. the control constraint. This kink limits the convergence order of a uniform discretization to $h^{3 / 2}$.

We compare some approaches from the literature. Moreover, we provide a new, efficient and robust error estimator which is used for an adaptive refinement of the mesh.

We also present a new method for solving control constrained problems. In this method, we move the nodes of the mesh at the interface between the active and inactive set. This yields optimal order of convergence. 


\section{YRMS4: Co-/Sparsity, Inverse Problems and Compressive Imaging}

At the core of many inverse and imaging problems are signal models which in addition to stabilizing the solutions of these problems are also capable of dealing with undersampling. In this context, a very popular model is the sparse synthesis model. Here, the assumption is that the signals can be expressed as a linear combination of a few signal components from a dictionary. In the last decade, there has been a lot of theoretical and practical work dealing with this model in the field of compressive sensing. Despite their success, practical applications often do not meet the theoretical conditions that compressive sensing relies on. Hence, their success is yet to be mathematically grounded. The goal of this mini-symposium is to draw attention to the theoretical gap between theory and practice, and to give young researchers working in inverse problems, imaging and compressed sensing the opportunity to present their new results. 


\title{
Recovering overcomplete sparse representations from structured sensing
}

\author{
Felix Krahmer ${ }^{1}$, Deanna Needell ${ }^{2}$, Rachel Ward ${ }^{3}$ \\ ${ }^{1}$ Inst. Numerische und Angewandte Mathematik, Universität Göttingen \\ ${ }^{2}$ Claremont McKenna College \\ ${ }^{3}$ University of Texas at Austin
}

In many signal processing applications, one wishes to acquire images that are approximately sparse in transform domains such as wavelets using frequency domain samples. Often the quality of the sparsity based model significantly improves when one considers redundant representation systems such as wavelet frames. To date, compressed sensing with redundant representation systems has, however, only been studied for measurement systems that have certain concentration properties, which is not the case for frequency domain samples. In this talk, we close this gap, providing more general reconstruction guarantees for signals that are sparse with respect to redundant systems. 


\title{
Computational Aspects of Sparse Recovery
}

\author{
Andreas M. Tillmann \\ Technische Universität Braunschweig
}

The fundamental problem of sparse recovery from underdetermined linear measurements reads

$$
\min \|x\|_{0} \quad \text { such that }\|A x-b\| \leq \delta,
$$

where $\delta \geq 0$ is an estimate of measurement noise, $\|x\|_{0}$ denotes the number of nonzeros in $x$ (i.e., the support size) and $\|\cdot\|$ is some norm (often the $\ell_{2}$-norm). This problem is well-known to be NP-hard in the strong sense and also difficult to approximate, even in the noiseless case $\delta=0$.

In this talk, we discuss a selection of aspects from the sparse recovery context, with a focus on computational matters:

Replacing the discrete objective by the $\ell_{1}$-norm has become a standard approach to obtain sparse solutions, backed by empirical success as well as theoretical guarantees for when this strategy in fact solves (1). For the noiseless basis pursuit model

$$
\min \|x\|_{1} \quad \text { such that } A x=b,
$$

in the presence of a sparse solution, a simple "heuristic optimality check" (HOC) procedure can improve both solution speed and quality of many different problem-specific algorithms [1]. We extend the HOC idea to several noise-aware $\ell_{1}$-minimization models (for both the synthesis and analysis approach) and provide preliminary numerical results that demonstrate the potential effectiveness of HOC schemes in this context.

It is well-known that basis pursuit (2) can be written as a linear program. We show that the converse result is also true (i.e., every LP can be transformed into a basis pursuit problem via a polynomial reduction) and sketch possible applications and complexity-theoretical implications.

In general, verifying that a relaxation or heuristic such as basis pursuit indeed provides the sparsest possible solution is often as hard as solving (1) itself, since evaluating the strongest known conditions that guarantee recovery success for heuristics is also NP-hard $[2,3]$. On the one hand, this gives rise to the question whether there are other polynomially solvable special cases of (1) besides those identified by the known recovery conditions. On the other, it motivates tackling (1) directly by exact methods from discrete and/or combinatorial optimization.

In fact, there seem to be only very few attempts to solve (1) directly. Continuing the work from [4], we investigate branch-and-cut methods for binary set-covering integer program reformulations of the sparse recovery task. In this context, to make use of the observation that (measurement) equations with few nonzero coefficients may provide stronger information regarding the optimal support, we encounter another interesting combinatorial problem named matrix sparsification, which can also be seen as a special dictionary learning problem [5]. We briefly discuss work-in-progress on the branch-and-cut approach and the connection to matrix sparsification.

\section{References}

[1] D. A. Lorenz, M. E. Pfetsch, A. M. Tillmann. Solving basis pursuit: Subgradient algorithm, heuristic optimality check, and solver comparison. ACM T. Math. Software, to appear (2014).

[2] A. M. Tillmann. Computational aspects of compressed sensing. Doctoral dissertation, TU Darmstadt, 2013.

[3] A. M. Tillmann, M. E. Pfetsch. The computational complexity of the restricted isometry property, the nullspace property, and related concepts in compressed sensing. IEEE Trans. Inf. Theory 60(2) (2014), $1248-1259$.

[4] S. Jokar, M. E. Pfetsch. Exact and approximate sparse solutions of underdetermined linear equations. SIAM J. Sci. Comput. 31(1) (2008), 23-44.

[5] A. M. Tillmann. On the computational intractability of exact and approximate dictionary learning. IEEE Signal Process. Lett. 22(1) (2015), 45-49. 


\title{
Joint reconstruction and segmentation from sparse Radon data
}

\author{
Martin Storath ${ }^{1}$, Andreas Weinmann ${ }^{2}$, Jürgen Frikel ${ }^{2}$, and Michael Unser ${ }^{1}$ \\ ${ }^{1}$ Biomedical Imaging Group, EPFL Lausanne, Switzerland \\ ${ }^{2}$ Helmholtz Center Munich, Germany
}

In many tomographic imaging setups the reconstruction and segmentation are classically performed in two separate steps. In such cases, the performance of the segmentation step greatly depends on the quality of the reconstruction and, hence, often yields unsatisfactory results. In particular, if only a few noisy projections are available, such strategies lead to particularly poor performance. To overcome this, combined reconstruction and segmentation (one-step) strategies have shown to yield promising results.

In this talk, we present an approach for joint reconstruction and segmentation using the Potts model, cf. [1]. More specifically, we propose a new algorithmic approach to the non-smooth and nonconvex Potts problem (also called piecewise-constant Mumford-Shah problem) for inverse imaging problems. We derive a suitable splitting into specific subproblems that can all be solved efficiently. Our method does not require a priori knowledge on the gray levels nor on the number of segments of the reconstruction. Further, it avoids anisotropic artifacts such as geometric staircasing. We demonstrate the suitability of our method for joint image reconstruction and segmentation. We focus on Radon data, where we in particular consider sparse angle data situations. For instance, our method is able to recover all segments of the Shepp-Logan phantom from 7 angular views only. As further applications, we also consider reconstructions from spherical Radon data which arises in photoacoustic tomography.

\section{References}

[1] M. Storath, A. Weinmann, J. Frikel, and M. Unser. Joint image reconstruction and segmentation using the Potts model. To appear in Inverse Problems, Preprint at arXiv:1405.5850. 


\title{
Empirical phase transitions in sparsity-regularized X-ray CT
}

\author{
Jakob S. Jørgensen \\ Technical University of Denmark, Denmark
}

Sparsity regularization in X-ray computed tomography (CT) has shown large potential for accurate reconstruction from reduced data, see e.g. [1, 2], leading to substantially reduced patient x-ray exposure in medical imaging and shorter scan times in materials science. One driving factor for research in sparsity-regularized methods has been developments in compressed sensing (CS), connecting the possible undersampling level to the image sparsity level. CS offers guarantees of accurate reconstruction of sparse images from reduced data under suitable assumptions on the measurement matrix.

However, existing CS guarantees, e.g. based on the restricted isometry property (RIP) do not apply to the structured and sparse measurement matrices of X-ray CT, while other coherence-based guarantees lead to essentially useless bounds. The empirical success of sparsity regularization for X-ray CT therefore remains unexplained from a theoretical viewpoint.

Using empirical phase diagrams we study image recoverability from X-ray CT data as function of image sparsity and undersampling level. We consider sparsity in the image and gradient domains and reconstruction by 1-norm and TV regularization. We demonstrate empirically that X-ray CT exhibits a pronounced relation between image sparsity and the possible undersampling level as well as sharp phase transitions for certain image classes. We demonstrate that recovery performance of X-ray CT is almost comparable with the standard CS Gaussian matrix ensemble, which suggests a similarity with CS. However, we also highlight some important differences, for example, that structure in the non-zero locations of the image and not just sparsity level affects recoverability in X-ray CT.

Furthermore we investigate the phase-transition behavior theoretically for a simple class of images consisting of sparse superpositions of radial basis functions (RBFs). RBFs are an often-used alternative to pixels and voxels for image representation in X-ray CT and commonly referred to as "blobs". The use of RBFs in X-ray CT image reconstruction is motivated by a reduction of model errors arising from anisotropies of the rectangular pixels and voxels.

The rotational symmetry of RBFs implies a regularity that can be exploited to analyze the setup from a CS perspective. The analysis exploits non-negativity of the sampling matrix and the signal and utilizes new mathematical tools from CS theory in the form of expander graphs. This approach has recently been used to derive average-case recovery guarantees for certain restricted discrete tomography setups [6]. Here, we address how to generalize the approach to the more complicated scanning setups used in standard (non-discrete) tomography, for example parallel and fan-beam geometries. We compare the theoretical recovery guarantees with empirical phase diagrams.

\section{References}

[1] E.Y. Sidky, X. Pan. Image reconstruction in circular cone-beam computed tomography by constrained, total-variation minimization. Phys. Med. Biol. 53 (2008), 4777-4807.

[2] J. Bian, J.H. Siewerdsen, X. Han, E.Y. Sidky, J.L. Prince, C.A. Pelizzari, X. Pan. Evaluation of sparse-view reconstruction from flat-panel-detector cone-beam CT. Phys. Med. Biol. 55 (2010), 6575-6599.

[3] J.S. Jørgensen, E.Y. Sidky. How little data is enough? Phase-diagram analysis of sparsity-regularized X-ray CT. Submitted (2014).

[4] J.S. Jørgensen, C. Kruschel, D.A. Lorenz. Testable uniqueness conditions for empirical assessment of undersampling levels in total variation-regularized X-ray CT. Inverse Probl. Sci. Eng. (2014), 23 pp. DOI: 10.1080/17415977.2014.986724.

[5] J.S. Jørgensen, E.Y. Sidky, P.C. Hansen, X. Pan. Empirical average-case relation between undersampling and sparsity in X-ray CT. Submitted (2014). Available from: http://arxiv.org/abs/1211.5658

[6] S. Petra, C. Schnörr: Average case recovery analysis of tomographic compressed sensing. Linear Algebra Appl. 441 (2014), 168-198. 


\title{
Cosparse models and recovery algorithms for inverse problems in acoustics and electro-encephalography
}

\author{
Srđan Kitić ${ }^{1}$, Laurent Albera ${ }^{1,2,3}$, Nancy Bertin ${ }^{4,1}$, Rémi Gribonval ${ }^{1}$ \\ ${ }^{1}$ Inria, Centre Inria Rennes Bretagne Atlantique \\ ${ }^{2}$ Inserm UMR 642 \\ ${ }^{3}$ LTSI - Université Rennes 1 \\ ${ }^{4}$ IRISA - CNRS UMR 6074
}

Sparse data models are powerful tools for solving ill-posed inverse problems. In their most general formulation, linear inverse problems can be expressed as the problem of recovering a signal $\mathbf{x} \in \mathbb{R}^{n}$ from measurements $\mathbf{y} \in \mathbb{R}^{m}:$

$$
\mathbf{y} \approx \mathrm{Mx}
$$

where $\mathbf{M} \in \mathbb{R}^{m \times n}$ is a transfer matrix modeling the signal acquisition process. In most cases, this problem is ill-posed, and solving it implies the use of additional knowledge in the problem formulation, in order to regularize it. Sparse data models have emerged as a pervasive tool to perform such regularization. The now ubiquitous sparse synthesis model states that the signal $\left(\mathbf{x} \in \mathbb{R}^{n}\right)$ is constructed by a linear combination of a few column vectors (atoms) taken from a dictionary $\mathbf{D} \in \mathbb{R}^{n \times d}$, such that

$$
\mathbf{x}=\mathbf{D s},
$$

where the coefficient vector $\mathbf{s}$ contains very few non-zero elements and is called a sparse representation of $\mathbf{x}$.

A counterpart of sparse synthesis is the sparse analysis or cosparse data model, which has gained attraction more recently [1]. This model assumes that there exist an analysis operator $\mathbf{A} \in \mathbb{R}^{b \times n}(\mathrm{~b} \geq \mathrm{n})$, such that the following analysis representation:

$$
\mathbf{z}=\mathbf{A x}
$$

of the signal $\mathbf{x}$ is sparse. A signal $\mathbf{x}$ whose analysis representation $\mathbf{z}=\mathbf{A x} \in \mathbb{R}^{b}$ contains $\ell$ zero elements is said to be $\ell$-cosparse. The analysis and the synthesis sparse models are nominally equivalent in only one special case: when $\mathbf{A}=\mathbf{D}^{-1}$, where the analysis operator and the dictionary are square, non-singular matrices [2].

In this presentation, we will show two settings where knowledge at hand is encoded through known Partial Differential Equations (PDEs), governing the underlying physical phenomena, which can be used to design the dictionary $\mathbf{D}$ (in the sparse synthesis case) or the analysis operator $\mathbf{A}$ (in the cosparse analysis model). We will present the resulting regularization framework based on the sparse analysis models for two problems governed by linear partial differential equations: acoustic pressure source localization [4], governed by the wave equation, and brain electrical current source localization [3], which can be described by the Poisson equation. The cosparse model can very naturally incorporate this physical knowledge in an analysis operator $\mathbf{A}$ resulting from a direct discretization of the underlying PDE. When solved by specially tailored optimization algorithm based on Alternating Direction Method of Multipliers, the underlying inverse problems can be solved efficiently and with much better scalability than their synthesis-based counterpart formulations.

\section{References}

[1] S. Nam, M.E. Davies, M. Elad, R. Gribonval. The cosparse analysis model and algorithms. Applied and Computational Harmonic Analysis 34 (2013).

[2] M. Elad, P. Milanfar, R. Rubinstein. Analysis versus synthesis in signal priors. Inverse problems 23 (2007).

[3] L. Albera, S. Kitić, N. Bertin, G. Puy, R. Gribonval. Brain source localization using a physics-driven structured cosparse representation of EEG signals. MLSP (2014).

[4] S. Kitić, N. Bertin, R. Gribonval. Hearing behind walls: localizing sources in the room next door with cosparsity. ICASSP (2014). 


\section{YRMS5: Topics in Low-rank Tensor Approximation}

The recent years showed an emerging interest in low-rank tensor techniques in scientific computing for solving "high-dimensional" tensor structured problems to overcome the curse of dimension by using separation of variables. This includes eigenvalues problems, linear equations, stochastic and parametric PDEs, dynamical systems, or ground state calculations in quantum spin systems. At the same time, low-rank tensor techniques are successfully used in exploratory data analysis, signal processing, and statistics. Ideally, at least three aspects need to be addressed: justification of the low-rank approach by a-priori approximability results, design and efficient implementation of algorithms avoiding "high-dimensional" objects at any stage, and convergence analysis of the typically nonlinear methods. These and other challenging tasks make low-rank tensor techniques an interesting and many-sided field. This Young Researchers Symposium will highlight some of these aspects at the hand of the speaker's latest research results. 


\title{
Finding low-rank bases of matrix subspaces
}

\author{
Yuji Nakatsukasa ${ }^{1}$, Tasuku Soma ${ }^{1}$, André Uschmajew ${ }^{2}$ \\ ${ }^{1}$ University of Tokyo, Japan \\ ${ }^{2}$ University of Bonn, Germany
}

Given a $d$-dimensional matrix subspace spanned by matrices $M_{1}, M_{2}, \ldots, M_{d} \in \mathbb{R}^{m \times n}$, one can ask whether this subspace contains a basis of low-rank matrices. Having such a basis, it is easy to imagine useful applications, for instance a compressed storage of the initial matrices $M_{1}, M_{2}, \ldots, M_{d}$. It is interesting to note that the specific question of whether a basis of rank-one matrices exists is equivalent to the question, whether the $m \times n \times d$ tensor with slices $M_{1}, M_{2}, \ldots, M_{d}$ has canonical tensor rank $d$. In general, the task is twofold: if one knows the smallest ranks for which a basis exists, one can design many optimization procedures to hopefully find such a basis. For instance, if a rank-one basis exists, one may use tensor decomposition algorithms like alternating least squares. For the case of higher ranks, we propose a simple alternating projection strategy with hard singular value thresholding, and investigate its convergence to some extent. The hard task, however, is to estimate the smallest possible ranks a basis can have. We present a heuristic based on nuclear norm minimization (soft thresholding) and, again, alternating projections, which works astonishingly well. 


\title{
Decoupling multivariate functions using tensor decompositions
}

\author{
Philippe Dreesen, Mariya Ishteva, Johan Schoukens \\ Vrije Universiteit Brussel, Dept. VUB-ELEC, Brussels, Belgium
}

We present a method to decompose a set of multivariate real functions into linear combinations of univariate functions in linear forms of the input variables [3]. The procedure collects first-order information by evaluating the Jacobian matrix of the function in a set of points. A tensor decomposition of the tensor constructed from the Jacobian matrices provides the decoupled representation.

Let $\mathbf{f}: \mathbb{R}^{m} \rightarrow \mathbb{R}^{n}$ be a given multivariate real function that allows a parameterization

$$
\mathbf{f}(\mathbf{u})=\mathbf{W g}\left(\mathbf{V}^{T} \mathbf{u}\right)
$$

where $\mathbf{W} \in \mathbb{R}^{n \times r}$ and $\mathbf{V} \in \mathbb{R}^{m \times r}$ are unknown linear transformations, and $\mathbf{g}: \mathbb{R}^{r} \rightarrow \mathbb{R}^{r}$ is an unknown vector function consisting of univariate functions $g_{i}\left(x_{i}\right)$, i.e., every component of $\mathbf{g}$ only depends on a single variable $x_{i}$, which is the $i$-th component of $\mathbf{V}^{T} \mathbf{u}$, or $x_{i}=\mathbf{v}_{i}^{T} \mathbf{u}$.

The decoupling method proceeds by considering the first-order information of the functions $f_{i}(\mathbf{u})$, which is captured by the $n \times m$ Jacobian matrix $\mathbf{J}(\mathbf{u})=\left[\partial f_{i}(\mathbf{u}) / \partial u_{j}\right]$. By using the parameterization (1), the Jacobian matrix $\mathbf{J}(\mathbf{u})$ can be written as

$$
\mathbf{J}(\mathbf{u})=\mathbf{W} \operatorname{diag}\left(g_{i}^{\prime}\left(\mathbf{v}_{i}^{T} \mathbf{u}\right)\right) \mathbf{V}^{T} .
$$

The Jacobian is evaluated in the points $\mathbf{u}^{(1)}, \ldots, \mathbf{u}^{(N)}$, resulting in the Jacobian matrices $\mathbf{J}\left(\mathbf{u}^{(1)}\right), \ldots, \mathbf{J}\left(\mathbf{u}^{(N)}\right)$, which we stack into a three-way tensor $\mathcal{J}$ having dimensions $n \times m \times N$. Finding the unknowns in the parameterization (1) then amounts to solving a simultaneous matrix diagonalization problem, which is computed using the canonical polyadic decomposition [5]. Indeed, we can write the tensor of Jacobians as

$$
\mathcal{J}=\sum_{i=1}^{r} \mathbf{w}_{i} \circ \mathbf{v}_{i} \circ \mathbf{h}_{i}
$$

where $\circ$ denotes the outer product. Decomposition (2) directly returns the unknowns $\mathbf{W}$ and $\mathbf{V}$, as well as the necessary information to reconstruct the univariate functions $g_{i}\left(x_{i}\right)$.

A variation of the decoupling method employs the block-term tensor decomposition $[1,2]$ instead of the canonical polyadic decomposition, making it possible to achieve partial decoupling. In a similar fashion, now a number of internal multivariate functions acts between smaller sets of internal partially coupled variables, rather than one-to-one univariate functions.

We will highlight applications in block-oriented system identification [4] where the above procedure is employed to decouple multiple-input-multiple-output static nonlinearities in order to recover physical interpretability and to reduce the number of parameters.

\section{References}

[1] L. De Lathauwer. Decompositions of a higher-order tensor in block terms - Part I: Lemmas for partitioned matrices, SIAM J. Matrix Anal. Appl., 30 (2008), pp. 1022-1032.

[2] L. De Lathauwer. Decompositions of a higher-order tensor in block terms - Part II: Definitions and uniqueness, SIAM J. Matrix Anal. Appl., 30 (2008), pp. 1033-1066.

[3] P. Dreesen, M. Ishteva and J. Schoukens. Decoupling Multivariate Polynomials Using First-Order Information, 2014; arXiv:1410.4060.

[4] P. Dreesen, M. Schoukens, K. Tiels, and J. Schoukens. Decoupling static nonlinearities in a parallel WienerHammerstein system: A first-order approach. Technical report, Department ELEC, Vrije Universiteit Brussel, 2014. Available from http://homepages.vub.ac.be/ pdreesen/decpWH.pdf.

[5] T.G. Kolda and B.W. Bader. Tensor decompositions and applications. SIAM Rev., 51(3):455-500, September 2009. 


\title{
Soft Thresholding of Hierarchical Tensors and Its Application in Iterative Methods
}

\author{
Markus Bachmayr, Reinhold Schneider \\ UPMC Paris 06 \\ TU Berlin
}

A widespread approach for solving high-dimensional problems using tensor representations is to modify a convergent standard iterative method by an additional rank reduction in each step. In the context of the hierarchical tensor format, this reduction is usually achieved by truncation of a higher-order singular value decomposition. In this talk, we consider an alternative type of rank reduction based on soft thresholding of tensors.

Whereas hard thresholding produces sparse approximations of a sequences by removing entries of absolute value below a certain threshold, soft thresholding also modifies all remaining entries. Applying soft thresholding to a sequence amounts to applying the function $s_{\alpha}(x):=\operatorname{sign}(x) \max \{|x|-\alpha, 0\}$ to each entry, and in contrast to the analogous function applied in hard thresholding, $s_{\alpha}$ is non-expansive, that is, Lipschitz continuous with constant one. It is well-known that non-expansiveness also holds for the resulting thresholding operation on sequences (with respect to the $\ell_{2}$-norm) and on matrices (where it is applied to the singular values, and one has non-expansiveness with respect to the Frobenius norm).

In this talk, we describe a soft thresholding operation for hierarchical tensor representations that also preserves this property and which can serve as a substitute of truncated higher-order singular value decompositions (which would correspond to hard thresholding) for rank reduction in iterative methods. We give an analysis of the approximation properties of the thresholding for different types of hierarchical singular value decay and consider in detail its combination with convergent fixed point iterations, where non-expansiveness turns out to be surprisingly useful. We then focus on the treatment of linear elliptic operator equations based on a fixed discretization. We propose a convergent method with a posteriori choice of an appropriate sequence of thresholding parameters that only requires bounds on the spectrum of the operator. The favorable properties of this scheme, especially concerning the resulting hierarchical ranks of iterates, are also demonstrated in numerical tests. 


\title{
Tensor-structured approximation for the solution of differential equations
}

\author{
Vladimir Kazeev \\ Seminar for Applied Mathematics, ETH Zurich
}

In $d$ dimensions, we consider problems with linear second-order elliptic differential operators of the form

$$
\mathcal{L}=-\nabla^{\top} A \nabla+b^{\top} \nabla+c,
$$

where the coefficients $A, b$ and $c$ are sufficiently smooth. A textbook approach based on the low-order finitedifference or finite-element approximations constructed on a uniform, tensor-product grid of size $n \times \ldots \times n$ seems infeasible due to the rapid growth of the complexity of both the representation of the discretizations and the whole solution algorithm, which are at least $\mathcal{O}\left(n^{d}\right)$. This very approach, however, turns out to be highly efficient when the vectors and matrices involved in the numerical algorithm are parametrized using tensor decompositions [1, 2], of which we consider here the tensor train (TT) [3, 4] and quantized tensor train (QTT) $[5,6]$ representations of tensors.

If a $d$-dimensional $n \times \ldots \times n$-vector $\boldsymbol{u}$ satisfies the equation

$$
\boldsymbol{u}_{j_{1}, \ldots, j_{d}}=\sum_{\alpha_{1}=1}^{r_{1}} \ldots \sum_{\alpha_{d-1}=1}^{r_{d-1}} U_{1}\left(j_{1}, \alpha_{1}\right) \cdot U_{2}\left(\alpha_{1}, j_{2}, \alpha_{2}\right) \cdot \ldots \cdot U_{d-1}\left(\alpha_{d-2}, j_{d-1}, \alpha_{d-1}\right) \cdot U_{d}\left(\alpha_{d-1}, j_{d}\right)
$$

for $1 \leq j_{k} \leq n$ and $1 \leq k \leq d$ with two- and three-dimensional arrays $U_{1}, U_{2}, \ldots, U_{d}$, then $\boldsymbol{u}$ is said to be represented in the TT decomposition in terms of the core tensors $U_{1}, U_{2}, \ldots, U_{d}$. The summation limits $r_{1}, \ldots, r_{d-1}$ are called ranks of the TT representation. The quantization of the $k$ th dimension relies on the positional representation of the "physical" indices in terms of "virtual" indices, e.g., for $n=2^{l}$, on the binary encoding:

$$
j_{k} \mapsto \overline{j_{k, 1}, \ldots, j_{k, l}}=1+\sum_{\mu=1}^{l_{k}} 2^{l-\mu}\left(j_{k, \mu}-1\right) \quad \text { for } \quad 1 \leq k \leq d .
$$

The QTT representation is then a combination of the quantization of the "physical" dimensions with the TT decomposition, in which the latter separates the "virtual" dimensions produced by the former. The number of parameters TT and QTT representations involve is bounded from above by $d n R_{\mathrm{TT}}^{2}$ and $2 d l R_{\mathrm{QTT}}^{2}$ respectively, where $R_{\mathrm{TT}}$ and $R_{\mathrm{QTT}}$ are upper bounds on the TT and QTT ranks. These complexity bounds may be dramatically smaller than $n^{d}$, the formal number of degrees of freedom, provided that the dependence of the rank bounds $R_{\mathrm{TT}}$ and $R_{\mathrm{QTT}}$ on $n$ and $d$ is moderate. This is often observed experimentally for the data involved in the numerical solution of PDEs, see $[2,7,8,9,10]$ and references therein. The talk will present recent theoretical results on the rank bounds obtained jointly with Ch. Schwab (SAM ETH Zurich).

\section{References}

[1] W. Hackbusch. Tensor Spaces and Numerical Tensor Calculus. Springer, Berlin Heidelberg (2012).

[2] L. Grasedyck, D. Kressner and Ch. Tobler. A literature survey of low-rank tensor approximation techniques. GAMM-Mitt. 36 (2013), 53-78.

[3] I. V. Oseledets and E. E. Tyrtyshnikov. Breaking the curse of dimensionality, or how to use SVD in many dimensions. SIAM J. Sci. Comput. 31 (2009), 3744-3759.

[4] I. V. Oseledets. Tensor train decomposition. SIAM J. Sci. Comput. 33 (2011), 2295-2317.

[5] I. V. Oseledets. Approximation of $2^{d} \times 2^{d}$ matrices using tensor decomposition. SIAM J. Matrix Anal. Appl. 31 (2010), 2130-2145.

[6] B. N. Khoromskij. $\mathcal{O}(d \log N)$-quantics approximation of $N$ - $d$ tensors in high-dimensional numerical modeling. Constr. Approx. 34 (2011), 257-280. 
[7] V. A. Kazeev, O. Reichmann and Ch. Schwab. hp-DG-QTT solution of high-dimensional degenerate diffusion equations. Technical Reports of SAM ETHZ, No. 13 (2012).

[8] V. A. Kazeev, O. Reichmann and Ch. Schwab. Low-rank tensor structure of linear diffusion operators in the TT and QTT formats. Linear Algebra Appl. 438 (2013), 4204-4221.

[9] I. Oseledets and S. Dolgov. Solution of linear systems and matrix inversion in the TT format. SIAM J. Sci. Comput. 34 (2012), A2718-A2739.

[10] S. Dolgov and D. Savostyanov. Alternating minimal energy methods for linear systems in higher dimensions. SIAM J. Sci. Comput. 36 (2014), A2248-A2271. 


\title{
Riemannian BFGS on the Tensor Train component space using an inherited tensor metric
}

\author{
Max Pfeffer, Reinhold Schneider \\ TU Berlin
}

We apply existing concepts of Riemannian geometry to the Tensor Train (TT) component space, which is a quotient space of the full component space

$$
\mathcal{C}=\prod_{i=1}^{d} \mathbb{R}_{*}^{r_{i-1} \times n_{i} \times r_{i}}
$$

It has been established that the TT component space is a smooth manifold that is related to a product of Grassmannians [1]. The according tangent space is obtained by posing a gauge condition. We use a noncanonical Riemannian metric and introduce projections, retractions and vector transport in order to be able to perform second order optimization [2]. The metric simulates the TT manifold of tensors of fixed TT rank,

$$
\mathcal{M}_{\mathbf{r}}=\left\{\mathcal{U} \in \mathbb{R}^{n_{1} \times \cdots \times n_{d}}: \operatorname{rank}_{\mathrm{TT}}(\mathcal{U})=\mathbf{r}\right\}
$$

and as such, it allows us to break down minimization problems on this space to the component space. The Riemannian BFGS is defined and performed in local coordinates, which ensures the optimality of the BFGS update. The possibility of a limited memory method is explored [3].

\section{References}

[1] A. Uschmajew, B. Vandereycken. The geometry of algorithms using hierarchical tensors. Linear Algebra Appl. 439 (2013), 133-166.

[2] P.-A. Absil, R. Mahony, R. Sepulchre. Optimization Algorithms on Matrix Manifolds. Princeton University Press. Princeton, NJ (2008).

[3] B. Savas, L.-H. Lim. Quasi-Newton methods on Grassmannians and multilinear approximations of tensors. SIAM J. Sci. Comput. 32 (2010), 3352-3393. 


\title{
Preconditioned Riemannian optimization for low-rank tensor equations
}

\author{
Daniel Kressner ${ }^{1}$, Michael Steinlechner ${ }^{1}$, Bart Vandereycken ${ }^{2}$ \\ ${ }^{1}$ EPF Lausanne, Switzerland \\ ${ }^{2}$ Université de Genève, Switzerland
}

The solution of very large linear systems is a challenging task often encountered as a core ingredient when solving partial differential equations on high-dimensional domains. In these cases, the degrees of freedom in the linear system grow exponentially with the number of dimensions, making classic approaches unfeasible. Approximation of the solution by low-rank tensor formats often allows us to avoid this curse of dimensionality by exploiting the underlying structure of the linear operator. We propose a new algorithm that performs a preconditioned gradient method on the manifold of tensors of fixed rank. In particular, we focus on tensors represented in the Tensor Train (TT) / Matrix Product States (MPS) format. We demonstrate the flexibility of our algorithm by comparing different approximations of the Riemannian Hessian as preconditioners for the gradient directions. Finally, we compare the efficiency of our algorithm with other tensor-based approaches such as the Alternating Linear Scheme (ALS). 


\section{S01: Multi-body dynamics}

Multibody dynamics enables the simulation of a wide variety of systems, all characterized by having multiple parts in relative motion with one another. Applications span from biological to engineering systems, requiring diverse capabilities which range from real-time simulation to high fidelity modeling of complex multidisciplinary systems. Goal of this mini-symposium is to present a view on the latest developments in models and advanced numerical methods in multibody dynamics. Focus is on techniques that enable applications to complex real-life problems. 


\title{
Impulse-based control of simple oscillators within the nonsmooth mechanics approach
}

\author{
Thorsten Schindler, Johannes Mayet, Philipp Seiwald \\ Institute of Applied Mechanics, TU München, 85747 Garching, Germany
}

In this contribution, we present an impulse-based control scheme for a simple spring-mass oscillator subject to set-valued friction forces. In contrast to PD or PID control laws, the proposed control law prevents nonzero steady state errors and limit cycles. Motivated by Wouw and Leine [1], it is shown that impulse-based control laws can lead to satisfactory and in particular robust behavior under the account of uncertain system parameters, i.e., mass or static friction, if the oscillator starts sticking. An overview on frictional oscillators for which stick-slip phenomena occur and an introduction to standard control laws indicate the need of problemspecific extensions. In contrast to reference [1], the equations of motion are extended by a simple model of the actor dynamics allowing for a practical realization. By using the framework of nonsmooth mechanical systems and applying timestepping schemes, it is shown how to overcome the drawbacks of ordinary control techniques. Apart from a continuous state feedback, we apply an impulsive feedforward in the case of sticking which prevents permanent sticking and resolves unwanted behavior like steady state errors or limit cycles.

\section{References}

[1] Nathan van de Wouw and Remco Leine. Robust impulsive control of motion systems with uncertain friction. Int J Robust Nonlinear Control, 22:369-397, 2012. 


\title{
Learning Robot Force/Position Control for Repetitive High Speed Applications with Unknown Non-Linear Contact Stiffness
}

\author{
Herbert Parzer, Hubert Gattringer, Andreas Müller \\ Institute of Robotics, Johannes Kepler University Linz
}

In many industrial applications manipulators are used to perform repetitive force controlled tasks. Typically such tasks are polishing, grinding, assembly as well as endurance testing of machine parts. The repetitive nature of such tasks allow for using iterative learning control (ILC) methods [1] or adaptive learning feed-forward control [2]. For such a controller it is necessary that the feed-back controlled system is stable, so that a learning feed-forward control, for example, minimizes a resulting error from one repetition to the next.

Considering a task, where a robot processes the same kind of workpiece in a recurring manner, the endeffector of the robot has to provide a predefined contact force while following a trajectory along the workpiece. To achieve this goal, a parallel force/position robot control, as suggested in [3], is best suited. Thereby, the force control manipulates a desired end-effector trajectory ${ }_{I} \mathbf{r}_{d}$, of a position controlled system, in such a way, that the desired force ${ }_{I} \mathbf{f}_{d}$ is reached. The force control law is described by the differential equation $\mathbf{K}_{A, p I} \ddot{\mathbf{r}}_{c}+\mathbf{K}_{V, p I} \dot{\mathbf{r}}_{c}={ }_{I} \mathbf{f}_{d}-{ }_{I} \mathbf{f}$, where $\mathbf{K}_{A, p}$ and $\mathbf{K}_{V, p}$ denote positive definite controller parameters and the vector ${ }_{I} \mathbf{f}$ represents the measured force in the inertial frame $(I)$. Using the parallel composition ${ }_{I} \mathbf{r}_{r}={ }_{I} \mathbf{r}_{d}+{ }_{I} \mathbf{r}_{c}$, with the solution ${ }_{I} \mathbf{r}_{c}$ of the above differential equation, the reference trajectory ${ }_{I} \mathbf{r}_{r}$ is calculated. This reference trajectory serves as input of the position controlled system.

Consequently following a fast desired force trajectory leads to various difficulties. First of, all the exact contact position as well as the contact stiffness is not always known. Further, the stiffness may vary from one point on the surface to another. These problems can be addressed by a separate identification of the stiffness and measuring the contact point position of different kinds of workpieces. To avoid this time consuming work, the idea, inspired by ILC and learning feed-forward control, is to divide the task into an on-line task, where the robot is actually moving, and an off-line task, where the computation and trajectory correction is done. Different to classical ILC methods, the previous mentioned issues are overcome by controlling the task with stretched time by a factor $t_{\text {scale, }}$, depending on the task. In this time stretched on-line task the robot is able to follow the desired position and force trajectory. By replacing the desired trajectory ${ }_{I} \mathbf{r}_{d, j}$ in the next iteration step $(j+1)$ with the stored reference trajectory ${ }_{I} \mathbf{r}_{r, j}$ from the actual step $(j)$ the learning law ${ }_{I} \mathbf{r}_{d, j+1}={ }_{I} \mathbf{r}_{d, j}+{ }_{I} \mathbf{r}_{c, j}$, depending on the force error, is developed. To improve performance, the trajectory from the start to the contact point (and back from the last contact point to the start point) is calculated off-line using splines with continuous transition conditions at start and end. However, if a condition for the force error, for example $\Delta \mathbf{F}_{\max }>\sup _{0 \leq \tau \leq T}\left\|_{I} \mathbf{f}_{d}(\tau)-{ }_{I} \mathbf{f}(\tau)\right\|$, where $\Delta \mathbf{F}_{\text {max }}$ is an upper error limit and $T$ is the cycle time of one repetition, is satisfied, the time scale factor $t_{\text {scale }}$ is reduced and a next iteration is started. Now the force controller only acts on small force errors, arising from contact damping and system dynamics. This iterations are done till $t_{\text {scale }}=1$, so that the normal process time is reached. Thereby the desired trajectory ${ }_{I} \mathbf{r}_{d, j}$ is trained for this group of workpieces and the learning process is stopped. For all subsequent workpieces, the process can be started with the original process time and force errors, arising from a slightly misaligned workpiece or other influences, are corrected again by the training process.

Summarizing, this paper proposes a learning robot force/position control for high speed force trajectory following. Experimental results, where a test object with non-linear stiffness is mounted at the end-effector of the industrial gantry robot and a repetitive disturbance acting at the contact point are presented.

\section{References}

[1] D.A. Bristow, M. Tharayil, A.G. Alleyne. A Survey of Iterative Learning Control. IEEE Control Systems Magazine, (2006), 96-114

[2] W.J.R. Velthuis, T.J.A. de Vries, J. van Amerongen. Design Procedure for a Learning Feed-Forward Controller. Proc. 1st IFAC Conf. on Mechatronic Systems, (2000)

[3] B. Siciliano, L. Villani. Robot Force Control. Kluwer Academic Publishers, Dordrecht (1999) 


\title{
An Optimal Control Approach to the Simulation of Problems with Servo Constraints
}

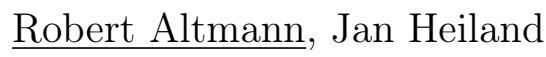 \\ TU Berlin \\ MPI Magdeburg
}

Mechanical systems with servo (or control) constraints appear in several crane models. A typical example is a robot for which we like to prescribe the trajectory of the end effector by a given number of input variables. Such models lead to DAEs of high index, often we deal with index-5 problems.

The simulation of such high-index DAEs is a challenging problem because of the involved stability issues known from DAEs. Due to the weak coupling of input and output, small changes in the desired trajectory may lead to high peaks in the input variables. Hence, index reduction techniques are inevitable for numerical simulations.

We consider the example of two mass points which are coupled by a spring. The task is then to control the movement of the second point whereas we may only actuate a force at the first [1]. This is a minimal example of a mechanical system with a servo constraint and gives a DAE of index 5 .

In this talk, we relax the constraint of following the given trajectory exactly and consider an optimal control problem instead. For this, we consider the dynamics of the mechanical system and introduce a cost functional which penalizes deviations from the trajectory as well as the input and its derivatives. Of course, the DAE problems are not simply gone, as it becomes visible within variations of the penalization parameters. However, we can achieve good performances at lower costs and for trajectories that would not be allowed in the direct approach. We illustrate this fact in several numerical experiments.

\section{References}

[1] W. Blajer and K. Kołodziejczyk. A geometric approach to solving problems of control constraints: theory and a DAE framework. Multibody Syst. Dyn., 11(4):343-364, 2004. 


\title{
An explicit approach for time-optimal trajectory planning for kinematically redundant robots
}

\author{
Alexander Reiter, Klemens Springer, Hubert Gattringer, Andreas Müller \\ Institute of Robotics, Johannes Kepler University Linz
}

Kinematic redundancy describes a manipulator's morphological property of featuring more joints $\mathbf{q} \in \mathbb{R}^{n}$ than necessary to assume any configuration in its task space $\mathbf{z}_{\mathrm{E}}^{\top}=\left(\begin{array}{cc}\mathbf{r}_{\mathrm{E}}^{\top} & \boldsymbol{\varphi}_{\mathrm{E}}^{\top}\end{array}\right) \in \mathbb{R}^{m}$ of given dimension, i.e. $m<n$. During the last years, the importance of kinematically redundant serial robots has risen due to striking advantages such as their improved flexibility and adaptiveness in structured workspaces. Their inherent capability of null space motion results in remarkable performance compared to conventional, non-redundant serial robots. Attempting to increase the cost-effectiveness of industrial processes, introducing time-optimal trajectories may yield economical advantages due to reduced motion cycle times.

While for non-redundant serial robots this problem has been addressed exhaustively, it has not been sufficiently covered for redundant manipulators. Well-known methods for obtaining minimum-time trajectories for non-redundant setups are not applicable to their full extent since for redundant robots not only the physical construction, but also the mathematical structure of the resulting equations differs greatly, e.g. the existence of a null space, i.e. joint velocities $\dot{\mathbf{q}}_{0}$ exist that have no influence on the current task space velocity of the end-effector such that $\dot{\mathbf{z}}_{\mathrm{E}}=\mathbf{J}(\mathbf{q}) \dot{\mathbf{q}}_{0}=\mathbf{0}$ where $\mathbf{J}$ denotes the geometric Jacobian. Additionally, no analytic inverse kinematics can be obtained since the direct kinematics equation system $\mathbf{z}_{\mathrm{E}}=\mathbf{f}(\mathbf{q})$ is under-determined.

In [1] a method for obtaining minimum-time trajectories along predefined, parameterized task space paths $\mathbf{z}_{\mathrm{E}}(s)$ of serial robots with one redundant degree of freedom is presented where $s$ denote as scalar path coordinate. The robot joints are divided in a non-redundant set of joints $\mathbf{q}_{\mathrm{nr}} \in \mathbb{R}^{m}$ and a redundant part $\mathbf{q}_{\mathrm{r}} \in \mathbb{R}^{n-m}$. Timeoptimal trajectories are obtained for the path parameter $s(t) \in[0,1]$ where $s(0)=0, s\left(t_{\mathrm{E}}\right)=1$, and the redundant joints $\mathbf{q}_{\mathrm{r}}(t)$ by means of an optimal control problem initiating null space motion in order to reduce the trajectory end time $t_{\mathrm{E}}$. The resulting trajectories are only $\mathcal{C}^{1}$ continuous, which limits the method's range of possible applications.

The present contribution adapts the separation approach from [1] and is applied to serial robots with one redundant degree of freedom. Instead of finding time-optimal trajectories by means of an optimal control problem, time-dependent multi-interval B-spline curves of degree $d$ for the path parameter $s=s(t)$ and the redundant joint $q_{\mathrm{r}}=q_{\mathrm{r}}(t)$ are assumed whose control points $\mathbf{c}_{\mathrm{nr}}$ and $\mathbf{c}_{\mathrm{r}}$ and the common end time $t_{\mathrm{E}}$ are optimization variables $\mathbf{x}$ of an optimization problem, i.e. $\mathbf{x}^{\top}=\left(\begin{array}{lll}t_{\mathrm{E}} & \mathbf{c}_{\mathrm{nr}}^{\top} & \mathbf{c}_{\mathrm{r}}^{\top}\end{array}\right)$. The non-redundant joint positions are then found using analytic inverse kinematics for the end-effector position computed by means of the parameterized path and the position of the redundant joint, i.e. $\mathbf{q}_{\mathrm{nr}}=\mathbf{q}_{\mathrm{nr}}\left(\mathbf{z}_{\mathrm{E}}(s), q_{\mathrm{r}}\right)$. The optimization problem is subjected to the dynamics of the robot, and physical and technological constraints such as limitations in the joint velocities $\dot{\mathbf{q}}$, the joint accelerations $\ddot{\mathbf{q}}$, or the joint torques $\mathbf{Q}$. While changing the position of the control point influences the general shape of the B-spline curves, their property of local approximation yields advantageous local adaptiveness. A solution for finding the initial values for the optimization problem is to obtain them by means of a least-squares B-spline curve approximation of the resulting trajectory of a numerical first-order inverse kinematics approach for a generic task space trajectory along the predefined path.

With an appropriate selection of the redundant degree of freedom, the manipulator's kinematic redundancy can be explicitly exploited. The resulting joint trajectories can be expressed as piecewise polynomial functions that are continuously differentiable $d$ times w.r.t. time $t$. Unlike most conventional methods trajectory data can be stored in a minimalistic way since only general parameters of the B-spline curves are required to generate the trajectory functions.

The presented approach was applied to the example of a planar three-link SCARA with straight lines as its given end-effector paths. Kinematic redundancy is introduced by only considering the Cartesian position of the end-effector in the plane but not its orientation as task space coordinates, i.e. $\mathbf{z}_{\mathrm{E}}=\mathbf{r}_{\mathrm{E}} \in \mathbb{R}^{2}$. As the motion is subject to joint torque constraints, continuous feed-forward torques are required to be computed yielding $d \geq 3$. Simulation results demonstrate time-optimality of the resulting trajectories.

\section{References}

[1] S. Ma, M. Watanabe. Time Optimal Path-Tracking Control of Kinematically Redundant Manipulators. JSME International Journal, Series C, Vol. 47, No. 2 (2004), 582-590. 


\title{
A numerical method for the servo constraint problem of underactuated mechanical systems
}

\author{
Yinping Yang*, Peter Betsch*, Robert Altmann \\ *Institut für Mechanik, Karlsruher Institut für Technologie \\ Kaiserstraße 12, 76131 Karlsruhe, Germany \\ Institut für Mathematik, Technische Universität Berlin \\ Straße des 17. Juni 136, 10623 Berlin, Germany
}

Servo constraints are used in the inverse dynamics simulation of discrete mechanical systems, especially in trajectory tracking control problems [1]. The desired system outputs described in terms of system states are treated as servo constraints [2]. The governing equations take the form of differential algebraic equations (DAEs), in which servo constraints are algebraic equations. In fully actuated multibody systems, the control inputs are solved from the equations of motion by model inversion, as the input distribution matrix is nonsingular and invertible. For underactuated multibody systems the number of degrees of freedom is greater than the number of control inputs and the input distribution matrix can not be inverted anymore. In contrast to passive constraints, the realization of servo constraints with the use of control forces can range from orthogonal to tangential [3]. Therefore the determination of control inputs that force the underactuated system to realize the partly specified motion, is difficult. For example the (differentiation) index can exceed three for differentially flat underactuated systems. Thus index reduction techniques are necessarily applied to reduce the index, such as Blajer's projection approach [3], [4]. In the present work we apply index reduction by minimal extension [5] to differentially flat underactuated systems, such as overhead and rotary cranes. We show that the index can be reduced from five to three and even to one. We consider as well nonflat underactuated multibody systems where the stability of the internal dynamics is also of paramount importance and ensures the controllability of the system [6], [7], [8].

\section{References}

[1] V.I. Kirgetov. The motion of controlled mechanical systems with prescribed constraints (servoconstraints). J. Appl. Maths. Mechs., 31(3):465-477, 1967.

[2] W. Blajer. Dynamics and control of mechanical systems in partly specified motion. J. Franklin Inst. B, 334(3):407-426, 1997.

[3] W. Blajer and K. Kolodziejczyk. A geometric approach to solving problems of control constraints: theory and a DAE framework. Multiody Syst. Dyn., 11(4):343-364, 2004.

[4] Y. Yang and P. Betsch. Alternative approaches to the incorporation of control constraints in multibody dynamics. Proc. of the ECCOMAS Thematic Conference on Multibody Dynamics, Zagreb, Croatia, July $1-4,2013$

[5] P. Kunkel and V. Mehrmann. Index reduction for differential-algebraic equations by minimal extension. Z. Angew. Math. Mech. (ZAMM), 84(9):579-597, 2004.

[6] R. Seifried and W. Blajer. Analysis of servo-constraint problems for underactuated multibody systems. Mechanical Sciences, 4(1):113-129, 2013.

[7] W. Blajer. The use of servo-constraints in the inverse dynamics analysis of underactuated multibody systems. J. Comput. Nonlinear Dynam., 9(4):041008/1-11, 2014.

[8] W. Blajer, R. Seifried, and K. Kolodziejczyk. Diversity of servo-constraints problems for underactuated mechanical systems: A case study illustration. Solid State phenomena, 198:473-482, 2013. 


\title{
Active damping control for an underactuated multibody system
}

\author{
Markus Burkhardt*, Merlin Morlock ${ }^{\dagger}$, Robert Seifried ${ }^{\dagger}$, Peter Eberhard* \\ *Institute of Engineering and Computational Mechanics, University of Stuttgart \\ ${ }^{\dagger}$ Institute of Mechanics and Ocean Engineering, Hamburg University of Technology
}

Light-weight robots and manipulators stand out due to their very good weight-to-load ratio and a low energy consumption. Unfortunately, the light-weight design yields a lower stiffness, which results in undesired elastic deformations, especially during high-speed working motion. One way to limit these unwanted oscillations can be implemented with modifications of the command signals, e.g. by input shaping or pre-computed feed-forward control [1]. However, a feedback control concept has the ability to provide a fast compensation of elastic deformations due to high-speed working motions, in case of unwanted environment contact or in the presence of other external disturbances.

In this contribution, an active damping control (ADC) for fast moving manipulators with significant structural elasticities is presented. This approach does not require additional actuators and it is suitable for a large variety of manipulators that exhibit structural vibrations, as long as they possess as many control inputs as rigid degrees of freedom. The ADC requires a robust and accurate position control for the actuated joints or bearings, which can be achieved by cascaded position, velocity, and current controllers together with a friction compensation. Then, the active damping control can be implemented by superimposing the position and velocity trajectories of the cascaded controllers with a motion that stabilizes the elastic deformation. Because of the underlying position control, this approach is applicable even in the presence of dry friction in the actuated joints or bearings.

The design of the ADC is based on a flexible multibody model of the system. The equations of motion can be stated as

$$
\left[\begin{array}{ll}
\boldsymbol{M}_{\mathrm{rr}}\left(\boldsymbol{q}_{\mathrm{r}}, \boldsymbol{q}_{\mathrm{e}}\right) & \boldsymbol{M}_{\mathrm{re}}\left(\boldsymbol{q}_{\mathrm{r}}, \boldsymbol{q}_{\mathrm{e}}\right) \\
\boldsymbol{M}_{\mathrm{er}}\left(\boldsymbol{q}_{\mathrm{r}}, \boldsymbol{q}_{\mathrm{e}}\right) & \boldsymbol{M}_{\mathrm{ee}}\left(\boldsymbol{q}_{\mathrm{r}}, \boldsymbol{q}_{\mathrm{e}}\right)
\end{array}\right]\left[\begin{array}{c}
\ddot{\boldsymbol{q}}_{\mathrm{r}} \\
\ddot{\boldsymbol{q}}_{\mathrm{e}}
\end{array}\right]=\left[\begin{array}{c}
\boldsymbol{f}_{\mathrm{r}}\left(\boldsymbol{q}_{\mathrm{r}}, \boldsymbol{q}_{\mathrm{e}}, \dot{\boldsymbol{q}}_{\mathrm{r}}, \dot{\boldsymbol{q}}_{\mathrm{e}}\right) \\
\boldsymbol{f}_{\mathrm{e}}\left(\boldsymbol{q}_{\mathrm{r}}, \boldsymbol{q}_{\mathrm{e}}, \dot{\boldsymbol{q}}_{\mathrm{r}}, \dot{\boldsymbol{q}}_{\mathrm{e}}\right)
\end{array}\right]+\left[\begin{array}{c}
\boldsymbol{B}_{\mathrm{r}}\left(\boldsymbol{q}_{\mathrm{r}}, \boldsymbol{q}_{\mathrm{e}}\right) \\
\boldsymbol{B}_{\mathrm{e}}\left(\boldsymbol{q}_{\mathrm{r}}, \boldsymbol{q}_{\mathrm{e}}\right)
\end{array}\right] \boldsymbol{u},
$$

in which $\boldsymbol{M}$ is the mass matrix, $\boldsymbol{q}$ is the vector of the generalized coordinates and $\boldsymbol{f}$ is the vector of generalized forces. The product of the input matrix $\boldsymbol{B}$ and the control inputs $\boldsymbol{u}$ account for the forces of the linear direct drives. The subscripts $\mathrm{r}$ and e distinguish the rigid and the elastic part, respectively. For perfect feedback controllers, the dynamics of the elastic part can be approximated by

$$
\boldsymbol{M}_{\mathrm{ee}}\left(\boldsymbol{q}_{\mathrm{r}, \mathrm{d}}, \boldsymbol{q}_{\mathrm{e}}\right) \ddot{\boldsymbol{q}}_{\mathrm{e}}=\boldsymbol{f}_{\mathrm{e}}\left(\boldsymbol{q}_{\mathrm{r}, \mathrm{d}}, \boldsymbol{q}_{\mathrm{e}}, \dot{\boldsymbol{q}}_{\mathrm{r}, \mathrm{d}}, \dot{\boldsymbol{q}}_{\mathrm{e}}\right)-\boldsymbol{M}_{\mathrm{er}}\left(\boldsymbol{q}_{\mathrm{r}, \mathrm{d}}, \boldsymbol{q}_{\mathrm{e}}\right) \ddot{\boldsymbol{q}}_{\mathrm{r}, \mathrm{d}}
$$

The linearization of Eq. (2) with respect to the elastic coordinates and their derivatives about a perturbed working motion $\ddot{\boldsymbol{q}}_{\mathrm{r}, \mathrm{d}}=\ddot{\overline{\boldsymbol{q}}}_{\mathrm{r}, \mathrm{d}}+\Delta \ddot{\boldsymbol{q}}_{\mathrm{r}, \mathrm{d}}$ yields the linear dynamics

$$
\overline{\boldsymbol{M}}_{\mathrm{ee}}\left(\boldsymbol{q}_{\mathrm{r}, \mathrm{d}}\right) \Delta \ddot{\boldsymbol{q}}_{\mathrm{e}}+\overline{\boldsymbol{D}}_{\mathrm{ee}}\left(\boldsymbol{q}_{\mathrm{r}, \mathrm{d}}\right) \Delta \dot{\boldsymbol{q}}_{\mathrm{e}}+\overline{\boldsymbol{K}}_{\mathrm{ee}}\left(\boldsymbol{q}_{\mathrm{r}, \mathrm{d}}\right) \Delta \boldsymbol{q}_{\mathrm{e}}=\overline{\boldsymbol{H}}\left(\boldsymbol{q}_{\mathrm{r}, \mathrm{d}}\right) \Delta \ddot{\boldsymbol{q}}_{\mathrm{r}, \mathrm{d}}+\overline{\boldsymbol{d}}\left(\boldsymbol{q}_{\mathrm{r}, \mathrm{d}}, \dot{\boldsymbol{q}}_{\mathrm{r}, \mathrm{d}}, \ddot{\overline{\boldsymbol{q}}}_{\mathrm{r}, \mathrm{d}}\right)
$$

in which $\overline{\boldsymbol{M}}_{\text {ee }}$ is mass matrix, $\overline{\boldsymbol{D}}_{\text {ee }}$ is the damping matrix, and $\overline{\boldsymbol{K}}_{\text {ee }}$ is the stiffness matrix. The input matrix $\overline{\boldsymbol{H}}$ equals the linearized matrix $-\boldsymbol{M}_{\mathrm{er}}$. The linearized elastic dynamics is driven by the disturbance $\overline{\boldsymbol{d}}$ caused by the working motion. The goal of the ADC is the stabilization of the disturbed dynamics with the perturbation of the acceleration $\Delta \ddot{\boldsymbol{q}}_{\mathrm{r}, \mathrm{d}}$, or more precisely, to increase the damping of the dominant mode of oscillation. Therefore, a modal decomposition of the model is performed and the dominant mode is isolated. The transformed equations of motion are evaluated at different operating points in order to provide a state-dependent distribution of the compensation term via a spline interpolation. For systems with multiple inputs, dominant directions of the input matrices at different working points are obtained by QR-decompositions. For the implementation of the feedback controller, it is necessary to obtain the elastic deformations of the system, e.g., with strain gauges. The output of the strain gauge $y_{\mathrm{c}}$ is processed by a band-pass filter in order to isolate the dominant mode of oscillation, differentiated, multiplied with a gain $G$ and used as feedback yielding

$$
m_{\mathrm{ee}} \ddot{q}_{\mathrm{dom}}+d_{\mathrm{ee}} \dot{q}_{\mathrm{dom}}+h G \dot{y}_{\mathrm{c}, \mathrm{filt}}+k_{\mathrm{ee}} q_{\mathrm{dom}}=\bar{d} .
$$

Due to the fact that the output is processed by a band-pass filter, the influence of the feedback to the other modes of oscillations is tolerable. In addition, there is no offset in the working motion. 
The capabilities of the proposed ADC are demonstrated in simulation and in experiments with the help of a parallel manipulator with highly flexible links [3]. The experimental platform consists of two identical linear direct drives which are mounted on a granite plate. The positions of the sliders $s_{i}, i=1,2$ are used to describe the rigid-body motion of the system. Two revolute joints, each located on top of one slider, define the rotational axes of two links with different length. Another revolute joint connects the middle of the long link with the end of the short link, i.e. these links are arranged similar to the Greek letter $\lambda$. An additional mass, which is attached to the free end of the long link, defines the end-effector point. Hence, this parallel manipulator permits a non-redundant movement of the end effector in the horizontal plane. The currents of the direct drives, which are proportional to the forces that act on the sliders, are used as the control inputs $u_{i}$. The elastic deformations of the long link are measured with strain gauges. Due to the high stiffness of the short link, it is assumed to be rigid and no strain gauges are applied to it.

For rest-to-rest maneuvers, the desired trajectories of the slider positions $s_{\mathrm{d}, i}$ and velocities $\dot{s}_{\mathrm{d}, i}$ are obtained from the step response of two identical fourth-order low-pass filters, whose cutoff frequencies define the dynamics of the rest-to-rest maneuver. The desired trajectories are fed forward to two distinct P-PI-cascade controllers for the slider positions. The underlying control law, composed of a feed-forward and a feedback part, can be stated as

$$
u_{i}(t)=u_{\mathrm{ff}, i}(t)+K_{\mathrm{P}, \mathrm{v}} e_{\mathrm{i}}+K_{\mathrm{I}, \mathrm{v}} \int_{0}^{t} e_{\mathrm{i}} \mathrm{d} \tau,
$$

in which $e_{\mathrm{i}}$ is the augmented velocity error according to

$$
e_{\mathrm{i}}=\dot{s}_{\mathrm{d}, i}(t)-\dot{s}_{i}(t)+K_{\mathrm{P}, \mathrm{p}}\left(s_{\mathrm{d}, i}(t)-s_{i}(t)\right) .
$$

The controller parameters are set to $K_{\mathrm{P}, \mathrm{p}}=80 \mathrm{~s}^{-1}, K_{\mathrm{P}, \mathrm{v}}=20 \mathrm{Cm}^{-1}$ and $K_{\mathrm{I}, \mathrm{v}}=400 \mathrm{Am}^{-1}$. The feed-forward currents $u_{\mathrm{ff}, i}$ in Eq. (5) are obtained from an online friction compensation. Based on this setup, the performance of the ADC is demonstrated on rest-to-rest maneuvers as well as on trajectory-tracking tasks. For trajectorytracking tasks, it is possible to feed-forward precomputed trajectories of the motor currents as well.

\section{References}

[1] W. Höbarth, H. Gattringer, H. Bremer. Modelling and Control of an Articulated Robot with Flexible Links/Joints. Proceedings of the the 9th International Conference on Motion and Vibration Control (MOVIC). (2008).

[2] A.A. Shabana. Dynamics of Multibody Systems. Cambridge University Press, New York. (2005).

[3] M. Burkhardt, R. Seifried, P. Eberhard. Experimental Studies of Control Concepts for a Parallel Manipulator with Flexible Links. Journal of Mechanical Science and Technology. (submitted for publication). 


\title{
Guideway based damping control of vehicle suspensions
}

\author{
$\underline{\text { Werner Schiehlen }}$ \\ Institute of Engineering and Computational Mechanics \\ University of Stuttgart \\ Pfaffenwaldring 9, 70550 Stuttgart, Germany
}

Vehicle systems are usually modelled by multibody systems of different complexity depending on the engineering task. The most simple quarter car model describes already all the required basic dynamic phenomena. This model features five design parameters representing body mass, body spring, shock absorber or damper, respectively, wheel mass and tire spring. The stochastic process of the guideway unevenness can be approximated by white velocity noise and added to the vehicle's equations of motion, resulting in the state equations of the vehicle guideway system as shown, e.g., in [1].

Road vehicle suspensions are generating vertical forces in the contact patches of the tires on the road providing horizontal contact forces for vehicle propulsion and guidance. The static contact forces are complemented by the dynamic loads due to suspension vibrations excited by the guideway unevenness. In addition, the shock absorber represents a non-restricted parameter for the free proposal of the suspension designer.

From the main tasks of a road vehicle suspension system follow as assessment criteria the driving comfort and the driving safety both mathematically defined by state variables. Due to the random excitation of the vehicle, both criteria are also random variables characterized by their standard deviations or variances, respectively. The covariance analysis is introduced and explicit algebraical equations for the criteria are found depending nonlinearly on the suspension parameters.

The assessment criteria comfort and safety are with respect to the shock absorber in conflict with each other requiring a multicriteria optimization resulting in a pareto-optimal set of parameters only [2, 3]. Therefore, in some of the latest passenger cars the damping parameter is adjustable by the driver with the options COMFORT, NORMAL, SPORT or SPORT $+[4,5,6]$. Thus, the driver is left to solve the pareto-optimal problem. This means an additional task for the driver requiring at least some knowledge on the course of the guideway ahead.

In this paper it is shown how data from a navigation system can be used to adapt the shock absorber to either maximal comfort or maximal safety depending on the course of the guideway. In particular, maximal safety on a curvaceous road results in lower comfort. Thus, decreasing comfort means a warning to the driver to reduce the speed. The methods applied in this paper are originating from multibody dynamics and they are extended by the stochastic road unevenness and the guideway curvature accessed from a navigation system using GPS data.

\section{References}

[1] K. Popp, W. Schiehlen. Ground vehicle dynamics, Springer, Berlin, 2010.

[2] M. Mitschke, H. Wallentowitz. Dynamik der Kraftfahrzeuge (in German), Springer, Berlin, 2004.

[3] W. Schiehlen, I. Iroz. Uncertainties in road vehicle suspensions, Procedia IUTAM, 2015, to appear.

[4] http://www.thyssenkrupp-bilstein.de/en/produkte/print.php?link=elektronisch-geregeltedaempfer.php (accessed 2014-12-08).

[5] http://www.bmw.com/com/en/insights/technology/technology_guide/articles/electronic_ damper_control.html (accessed 2014-12-08).

[6] http://media.chrysler.com/print.do; jsessionid=94C9E9ACAD607AE41782DC75C259A41B?\&id=13707 $\gg$ Outstanding ride, handling and capability (accessed 2014-12-08). 


\title{
Modelling a pushbelt variator
}

\author{
$\underline{\text { Kilian Grundl* }}$, Thorsten Schindler*, Daniel J. Rixen*, Heinz Ulbrich*, \\ Arie v. d. Velde ${ }^{\dagger}$, Semih Yildiz \\ * Institute of Applied Mechanics \\ Technische Universität München \\ Boltzmannstraße 15, 85748 Garching bei München, Germany \\ [kilian.grundl, thorsten.schinder, rixen, ulbrich]@tum.de \\ ${ }^{\dagger}$ Bosch Transmission Technology \\ Dr. Hub van Doorneweg 120, 5026 RA Tilburg, Netherlands \\ [Arie.vanderVelde, Semih.Yildiz]@nl.bosch.com
}

A pushbelt continuously variable transmission (CVT) consists of about 400 steel elements guided by two loopsets and transmitting torque from a primary to a secondary pulley due to ring-tension and element-push forces. About 1500 degrees of freedom and about 3500 contacts in a spatial model of the system combined with nonlinear effects and coupled deformations of the single parts lead to a high complexity and not fully understood dynamic effects.

In the recent years, a joint research project between the Institute of Applied Mechanics of the Technische Universität München and Bosch Transmission Technology has dealt with the detailed modelling of CVTs ([1], [2] and [3]). The model is implemented in the general framework for nonsmooth multibody systems, MBSIM [4]. Besides standard elements, i.e. rigid bodies and unilateral spring-dampers, specific elements for the pushbelt CVT have been derived and implemented including e.g. a coupled force law to represent the pulley-sheave deformation or different nonlinear beam models for the representation of the loop-sets.

This work summarizes the most important findings including a detailed validation of the results comparing stationary situations in local output curves, e.g. element axial forces, element push forces or spiral running, and in global output curves, e.g. thrust ratio or transmission torques. Furthermore different modelling techniques are compared concerning physical influence (i.e. forces and kinematics), but also numerical output (e.g. smooth vs. nonsmooth dynamics).

\section{References}

[1] Geier, Thomas: Dynamics of Push Belt CVTs. München, Technische Universität München, Dissertation, 2007

[2] Schindler, Thorsten: Spatial Dynamics of Pushbelt CVTs. München, Technische Universität München, Dissertation, 2010

[3] Cebulla, Thomas: Spatial Dynamics of Pushbelt CVTs: Model Enhancements to a Non-smooth Flexible Multibody System. München, Technische Universität München, Dissertation, 2014

[4] MBSim - Multi-Body Simulation Software. GNU Lesser General Public License http://code.google.com/p/mbsim-env/. 


\title{
Modal Analysis of Vehicle Power Trains
}

\author{
Haslinger Josef*, Offner Günter\#, Sopouch Martin\# \\ *Radon Institute for Computational and Applied Mathematics, \\ Austrian Academy of Sciences and MathConsult GmbH, \\ Altenberger Str. 69, 4040 Linz, Austria \\ \#Advanced Simulation Technologies, AVL List GmbH, \\ Hans-List-Platz 1, 8020 Graz, Austria
}

Modal analysis - the determination of natural frequencies and mode shapes - provides essential insight into the dynamical behavior of mechanical systems. The mechanical systems under consideration consist of rigid and flexible bodies. These bodies are interconnected by nonlinear force elements. The time-dependent description of the system is based on the floating frame of reference approach. This approach leads to the standard system of second order differential-algebraic equations as used in the multi-body simulation software AVL-EXCITE [2]. The differential equation of this system is given as:

$$
\boldsymbol{M} \boldsymbol{q}^{\prime \prime}+\boldsymbol{D} \boldsymbol{q}^{\prime}+\boldsymbol{K} \boldsymbol{q}=\boldsymbol{f}^{\text {inertia }}\left(\boldsymbol{y}, \boldsymbol{y}^{\prime}, \boldsymbol{y}^{\prime \prime}\right)+\boldsymbol{f}^{\text {external }}\left(\tilde{\boldsymbol{y}}, \tilde{\boldsymbol{y}}^{\prime}\right)
$$

The vector of displacement variables of a single body consists of the global translation variables $\boldsymbol{x}$, the global rotational variables $\boldsymbol{\theta}$, and the local variables $\boldsymbol{q}$. They constitute the displacement vector $\boldsymbol{y}=\left(\boldsymbol{x}^{T}, \boldsymbol{\theta}^{T}, \boldsymbol{q}^{T}\right)^{T}$ for a single body. $\boldsymbol{M}, \boldsymbol{D}$ and $\boldsymbol{K}$ denote the mass, damping and stiffness matrix, resulting from spatial finite element discretization. Nonlinear inertia forces - like Coriolis and gyroscopic forces and torques - are collected in $f^{\text {inertia }}$.

External forces and moments applied at a single body are covered by $\boldsymbol{f}^{\text {external }}$. This vector includes the forces and moments resulting from externally applied loads and torques, e.g. gravity or gas pressures. It also includes the forces and moments resulting from coupling between the bodies. Hence $\boldsymbol{f}^{\text {external }}$ does not only depend on coordinates of the body itself, but also on possibly all states of other bodies, which are summarized in the vector $\tilde{\boldsymbol{y}}$. Interaction between different bodies is modeled by force-elements (joints). These force-elements are preferred, as they often model radial or axial slider bearings, where clearance gaps, wear and lubrication are of importance. Beneath non-linear spring-damper force laws, more advanced hydrodynamic models like the Reynolds equations may be used for these force laws.

The differential equation is completed by algebraic equations called reference conditons, which seperate the global motion and elastic deformations in a unique manner [2]. The rotational motion of the global frame is parameterized by the four parameter family of quaternions [4]. The normalization condition of the quaternions gives another algebraic constraint on the single body level, in addition to the reference conditions.

In order to apply modal analysis, the highly nonlinear equations of motion are linearized at specified points in time. Inertia and connections forces yield a contribution to the mass, damping, and stiffness matrix of the multi-body system, like the force equilibrium approach in [3]. The linearization is dependent on the set of coordinates selected to describe the motion of the system, especially the coordinates used to describe global rotational motion of rigid bodies.

In this contribution different sets of large orientation parameters lead to different forms of the linearized equations of motion and to different natural frequencies and eigenmodes, similar to [5]. The resulting system of linearized ordinary differential equations has constant coefficients, as soon as a common reference frame can be chosen that renders the relative motion of the bodies independent of time. A standard eigenvalue analysis such as the characteristic exponent method [1] is applied to extract natural frequencies and eigenmodes.

The approach is evaluated by computing eigenvalues and eigenmodes of a vehicle power train. The vehicle power train consists of components like cranktrain/crankshaft, transmission with internal shafts as well as engine/transmission housing supported by mounts. Some of the bodies are flexible, some are rigid, some are rotating. The resulting eigenvalue problem is of medium size. The approach is validated against ANSYS and MSC ADAMS. 


\section{References}

[1] O.A. Bauchau, J. Wang. Stability analysis of complex multibody systems. Journal of Computational and Nonlinear Dynamics. 1 (2005), 71-80.

[2] C.B. Drab, H.W. Engl, J.R. Haslinger, G. Offner, R.U. Pfau, W. Zulehner. Dynamic simulation of crankshaft multibody systems. Multibody System Dynamics. 22 (2009), 133-144.

[3] J.S. Kang, S. Bae, J.M. Lee, T.O. Tak. Force equilibrium approach for linearization of constrained mechanical system dynamics. Journal of Mechanical Design. 125 (2003), 143-149.

[4] J.B. Kuipers. Quaternions and Rotation Sequences. Princeton University Press, Princeton, New Jersey. (1999).

[5] A.A. Shabana, M.H. Zaher, A.M. Recuero, R. Cheta. Study of nonlinear system stability using eigenvalue analysis: gyroscopic motion. Journal of Sound and Vibration. 330 (2011), 6006-6022. 


\title{
Study on Real-Time Simulation of Elastic Multibody Systems with Application in Vehicle Dynamics
}

\author{
Prof. Dr.-Ing. Robert Seifried, Dipl.-Ing. Alexander Schmitt \\ Institute of Mechanics and Ocean Engineering, Hamburg University of Technology (TUHH)
}

Modern vehicle development largely uses simulation technology of various vehicle states to reduce costintensive tests of prototype vehicles and to perform component tests prior to application in cars. Real-time simulation models are used for virtual prototyping in Driver-in-the-Loop-environments, in Hardware-in-the-Loop tests of electronic control units and in tests of mechanical components. Also in modern model-based control concepts real-time modeling might be necessary [1]. These models have to be both accurate and calculable in real-time.

The majority of the current simulation models are built as rigid body models equipped with empirical components and measurement data. However, since one of the main goals of current vehicle development is weight reduction, deformation of vehicle components might not any more be negligible and may have significant influence on vehicle behavior. Flexible components such as suspension struts or the car body itself may be modeled with the finite element (FE) method, but implementing a full FE model of a car body in a vehicle dynamics simulation will largely exceed real-time capability. One method to reduce the simulation time is to use a reduced elastic body within the multibody simulation.

This contribution presents the implementation of a reduced flexible body in a real-time vehicle simulation environment. The research code Neweul- $\mathrm{M}^{2}$ [2] has been used to build up the symbolic formulation of suspension kinematics as well as the movement of the car body itself. Tire behavior and tire-road contact have been realized with the popular MF-Tire model [3] combined with the OpenCRG road surface model [4]. Both models have been proven to be reliable and time-efficient for a real-time simulation environment.

Simulations have been performed with the real-time simulation model, including handling tests and performance on uneven road. The results show that the combination of a vehicle model with symbolic formulation and reduced flexible bodies provide an efficient combination to cover structural elasticity in real-time simulation. Further post-processing steps of the real-time simulation may include visualization of the flexible bodies and stress calculation with stress recovery methods for reduced elastic multibody systems.

\section{References}

[1] R. Seifried: Dynamics of Underactuated Multibody Systems: Modeling, Control and Optimal Design. Springer, Berlin (2014)

[2] T. Kurz, P. Eberhard: Symbolic modeling and analysis of elastic multibody systems. International Symposium on Coupled Methods in Numerical Dynamics, Brusseles, Belgium (2009), 187-201.

[3] H. B. Pacejka: Tire and Vehicle Dynamics. Elsevier/BH, Amsterdam, Boston, 3rd edition (2012), 165-211

[4] http://www.opencrg.org: VIRES Simulationstechnologie GmbH, project website (2014). 


\title{
A mechanical model of a passive dynamic walker with unilateral viscoelastic contact formulation
}

\author{
Jens Deppler, Simon Lüdke, Alexander Fidlin \\ Institut für Technische Mechanik - Karlsruhe Institute of Technology \\ Kaiserstraße 10 \\ 76131 Karlsruhe \\ jens.deppler@kit.edu
}

Energy efficient locomotion is a demanding and important topic in lots of different research branches like for instance mechanical engineering, mathematics, computer science or medicine. The most simple model describing bipedal locomotion consists of a passive dynamic walker, a machine walking down an inclined plane with a slight slope with no external energy source other than gravity. In this contribution a mechanical multibody model of a passive dynamic walker with seven degrees of freedom is presented. It has been show in [1], that the energy efficiency of a dynamic walker can be improved by elastic couplings between its legs. This effect can also be observed in experiments. If the legs are coupled by a linear spring in a suitable fashion, the velocity of the walker increases significantly. Simulations are performed in order to confirm these results numerically using a detailed unilateral contact model. In order to describe the foot ground contact, a detailed contact model is needed, since the contact shows quite an interesting behavior. If the walker is standing on a plane the contact is in a sticking state. While walking one foot rolls over the ground until the edge of the second foot hits the ground. Since the relative velocity between the second foot and the ground usually doesn't vanish at the beginning of the impact, a silding motion occurs. This implies the necessity for a detailed contact model that is capable of describing sliding, sticking and rolling motions in a general unified way. The foot to ground contact of the walker is modelled by a bounded toroidal surface, describing the walkers foot and an inclined plane. The contact surface itself is discretized by a grid of point contacts, moving relative to the body. The kinetic contact model of this unilateral contact is capable of transmitting both sliding and sticking forces, where rolling is considered as a special form of sticking. Resistance against rolling and drilling friction are taken into account as well, in order to obtain the energy dissipation needed to attain a stable limit cycle solution. The contact formulation in normal and tangential direction is based on a viscoelastic description proposed in [2]. The ideally rigid nonholonomic constraint equations are approximated by an extension of the contact model given in [2] to viscoelastic nonholonomic motions. A theorem that proves the convergency of the solution of the viscoelastic constraint description to an ideally rigid constraint formulation is given [3]. Usually a general multibody system with nonholonomic constraint equations can be described by a differential algebraic equation in the following form

$$
\begin{aligned}
M\left(q_{d}\right) \ddot{q}_{d} & =F\left(q_{d}, \dot{q}_{d}, t\right)-G^{\top}\left(q_{d}\right) \lambda_{d}, \\
0 & =G\left(q_{d}\right) \dot{q}_{d} .
\end{aligned}
$$

where $M$ denotes the mass matrix, $F$ the column matrix of gyroscopic and external forces, $G$ the constraint matrix and $\lambda$ the Lagrange multiplier. The corresponding viscoelastic description of the constraints is given by

$$
\begin{aligned}
M\left(q_{v}\right) \ddot{q}_{v} & =F\left(q_{v}, \dot{q}_{v}, t\right)-G^{\boldsymbol{\top}}\left(q_{v}\right)\left(\frac{c}{\varepsilon} z+\frac{d}{\sqrt{\varepsilon}} \dot{z}\right), \\
\dot{z} & =G\left(q_{v}\right) \dot{q}_{v} .
\end{aligned}
$$

with the internal variable $z$. The distance of the solutions can be estimated above according to:

$$
\left\|q_{d}-q_{v}\right\| \leq K \sqrt{\varepsilon} .
$$

Thus the proposed contact model provides a suitable approximation of an ideally rigid nonoholonomic constraint equation, which can be used to simulate a three dimensional motion of a passive dynamic walker.

\section{References}

[1] F. Bauer. Optimierung der Energieeffizienz zweibeiniger Roboter durch elastische Kopplungen. KIT Scientific Publishing. (2014). 
[2] W. Stamm. Modellierung und Simulation von Mehrkörpersystemen mit flächigen Reibkontakten. KIT Scientific Publishing. (2011).

[3] J. Deppler, A. Fidlin, B. Braun. Regularization of nonholonomic constraints in multibody systems. 11th World Congress on Computational Mechanics (WCCM XI). July 20-25, 2014, Barcelona, Spain. http://www.wccm-eccm-ecfd2014.org/admin/files/fileabstract/a733.pdf

[4] A. Kuo, Stabilization of Lateral Motion in Passive Dynamic Walking. The International Journal of Robotics Research. Vol. 18, No. 9

[5] T. McGear, Passive Dynamic Walking. The International Journal of Robotics Research. Vol. 9, No. 2 


\title{
Investigation of optimal bipedal walking gaits subject to different energy-based objective functions
}

\author{
Römer U. J., Fidlin A., Seemann W. \\ Institute of Engineering Mechanics, Karlsruhe Institute of Technology
}

This contribution compares walking gaits of a bipedal robot model generated for different energy-based objective functions. There is a variety of optimality criteria for the analysis and generation of bipedal as well as quadrupedal gaits in the literature [1-3]. While there are some individual exceptions (e.g. maximum walking speed [3]) most of those criteria optimize either energy efficiency or stability. A combination of different criteria - based on energy and/or stability - is employed in inverse optimal control approaches trying to identify the objective criterion underlying gaits measured via motion capturing [4]. Furthermore, energy efficiency is frequently used as criterion for changes in the locomotion pattern, like transition from walking to running in bipedal locomotion [5] or transition between walking, trotting and galloping in quadruped locomotion [6].

Frequently encountered energy-based criteria are torques squared, the mechanical work or the absolute value of the mechanical work [2]. Those works are often normalized by weight and step length resulting in the cost of transport to compare different models. While some of those criteria are motivated by physical properties of the actuating motors or muscles, others, especially torques squared, are often used because of mathematical properties like convexity and global differentiability.

To investigate how different objective functions influence the generated gaits in bipedal walking, a simple planar rigid body model of a bipedal robot is simulated. The actual energy input for actuation by electric motors in all joints is derived and used as a benchmark for the energy-based criteria from the literature.

The hybrid zero dynamics (HZD) concept from [7] is used to derive a feedback controller for the robot model. The controlled model has one degree of underactuation which means it cannot transmit any torque to the ground (no flat foot). This allows for, rather than suppresses, the evolution of the model's natural dynamics. Sequential quadratic programming (SQP) is employed to generate optimal gaits for the different objective functions. The results are compared to the benchmark gait for actual energy efficiency.

\section{References}

[1] D.F. Hoyt, C.R. Taylor. Gait and the energetics of locomotion in horses. Nature (1981).

[2] P.H. Channon, S.H. Hopkins, D.T. Pham. Simulation and optimization of gait for a bipedal robot. Mathematical and Computer Modelling 14 (1990), 463-467.

[3] K. H. Koch, K. D. Mombaur, P. Souères. Studying the effect of different optimization criteria on humanoid walking motions. In Simulation, Modeling, and Programming for Autonomous Robots (2012). Springer Berlin Heidelberg. 221-236

[4] K. Mombaur, A. Truong, J. Laumond. From human to humanoid locomotion?an inverse optimal control approach. Autonomous robots 28.3 (2010). 369-383.

[5] U. Römer, F. Bauer, A. Fidlin. Transition from walking to running of a bipedal robot to optimize energy efficiency. Proc. of the 2014 Conf. on Climbing and Walking Robots (Clawar 2014), Poznan, Poland (2014)

[6] R.M. Alexander. The gaits of bipedal and quadrupedal animals. The International Journal of Robotics Research 3.2 (1984). 49-59.

[7] E.R. Westerveld et al. Feedback control of dynamic bipedal robot locomotion. Boca Raton: CRC press, 2007. 


\title{
Coupling Elastic Bodies with an Enhanced Craig-Bampton-like Scheme
}

\author{
Peter Eberhard, Philip Holzwarth \\ Institute of Engineering and Computational Mechanics, University of Stuttgart \\ Pfaffenwaldring 9, 70569 Stuttgart, Germany \\ \{peter.eberhard,philip.holzwarth\}@itm.uni-stuttgart.de, www.itm.uni-stuttgart.de
}

Elastic multibody systems (EMBS) are widely used as description of mechanical systems that consist of separable components. These components are represented by elastic bodies that interact by the means of force and damping elements as well as boundary conditions. The floating frame of reference approach allows for large, nonlinear rigid body motion. Deformations of the elastic bodies are defined relative to the reference frame. The overall motion of the bodies consists of the superposition of the nonlinear rigid body motion and the linear elastic deformation, which delivers valid results for small deformations.

Linear elastic models are usually obtained from the linear Finite Element method. The spatial discretization leads to second order ordinary differential equations of large dimension for describing only the deformations with respect to the large reference motion,

$$
\boldsymbol{M} \ddot{\boldsymbol{x}}(t)+\boldsymbol{K} \boldsymbol{x}(t)=\boldsymbol{f}(t),
$$

with mass matrix $\boldsymbol{M}$, stiffness matrix $\boldsymbol{K}$ and force excitation $\boldsymbol{f}$. A reduction of the elastic quantities is required in order to reduce the huge numerical burden in the EMBS environment. The typical procedure relies on a projection of the nodal coordinates $\boldsymbol{x}$ onto a suited subspace $\mathcal{V}=\operatorname{colspan}(\boldsymbol{V})$ of small dimension. The Galerkin projection leads to a differential equation in terms of reduced generalized coordinates $\boldsymbol{q}_{\mathrm{r}}$, such that $\boldsymbol{x} \approx \boldsymbol{V} \boldsymbol{q}_{\mathrm{r}}$, structurally equivalent to Eq. (1).

The current standard reduction technique was introduced by Craig and Bampton [1], which bases on a splitting of the degrees of freedom into boundary and internal quantities. At boundary degrees of freedom, interactions with the environment are assumed to take place, e. g. force elements or constraints connecting the body with other bodies or the inertial system. The Craig-Bampton scheme combines static condensation [2] modes with fixed-interface eigenmodes, the solution of the eigenvalue problem with fixed interface coordinates. A major drawback of this scheme is that the eigenvalue problem, which is solved for the description of the internal deformation, does not consider any excitation of the system. However, if the boundary degrees of freedom move, the internal dynamics are indeed excited. There exist several methods, such as Krylov subspace methods and Balanced Truncation, that focus on the input-output behavior of systems, thus taking into account how the system is excited and where measurements are taken. In terms of approximation of the transfer behavior, these methods show approximation errors that are much smaller in comparison to plain modal truncation, [3]. This enables faster calculations. Also, automated variants and error estimators, respectively bounds, are available.

If the elastic body is connected to the environment, the input-output behavior also changes. In order to maintain the compatibility properties that are ensured with static condensation, a modified algorithm for the input-output based methods can be used. The fixed-interface eigenmodes in the Craig-Bampton scheme are replaced with ansatzfunctions from alternative model order reduction schemes, based on the excitation by inertial forces due to boundary movement. In this setup, arbitrary input-output based model reduction can be used and possibly time-varying boundary conditions can still be met.

The reduction scheme that is presented in this contribution is at least as reliable as and shows a faster error decay than the standard technique by Craig and Bampton.

\section{References}

[1] Craig, R.; Bampton, M.: Coupling of Substructures for Dynamic Analyses. AIAA Journal, Vol. 6, No. 7, pp. 1313-1319, 1968.

[2] Guyan, R.J.: Reduction of Stiffness and Mass Matrices. AIAA Journal, Vol. 3, No. 2, p. 380, 1965.

[3] Fehr, J.: Automated and Error-Controlled Model Reduction in Elastic Multibody Systems, Vol. 21 of Schriften aus dem Institut für Technische und Numerische Mechanik der Universität Stuttgart. Aachen: Shaker Verlag, 2011. 


\title{
Back-Transformation into Physical Configuration Space after Model Order Reduction onto a General Subspace
}

\author{
Michael Beitelschmidt, Claudius Lein \\ Technische Universität Dresden, Faculty of Mechanical Science and Engineering, \\ Department of Solid Mechanics, Chair of Dynamics and Mechanism Design \\ 01062 Dresden, Germany \\ e-mail: Claudius.Lein@tu-dresden.de, Michael.Beitelschmidt@tu-dresden.de
}

Model order reduction (MOR) techniques are state of the art and well established in control theory and likewise in structural mechanics. Methods that project onto a general subspace like KRYLOV-subspace-based reduction techniques are common practise, e.g. [1], [2]. In a general subspace $\mathcal{K}$ the coordinates of the reduced order model (ROM) have no physical meaning anymore, since they are not defined on the physical configuration space $\mathbb{R}$. Although being acceptable when applying a RITz-approach, e.g. in Elastic Multi-Body-Dynamics (EMBS), any further utilization - where a physical interpretation is required - is not feasible, e.g. the coupling of single coordinates or the determination of mass and inertia properties.

To overcome this drawback, a novel back-projection approach was introduced by KouTSOvASILIS [2], where the information is projected back onto the physical configuration space. By applying a linear and orthogonal projection onto a KRYLOV-subspace, the projection matrix $\mathbf{V}^{\mathcal{K}} \in \mathcal{K}^{N \times n}$ is gained. For a physical meaning, the system is partitioned into master and slave coordinates. The back-projection approach requires the inversion of the master-partition of the projection matrix $\mathbf{V}_{m m}^{\mathcal{K}}$, which finally yields to the back-projection matrix $\mathbf{V}^{\mathcal{K} \rightarrow \mathbb{R}}$. The reduced system matrices in physical configuration space, e.g. $\overline{\mathbf{M}}^{\mathbb{R}} \in \mathbb{R}^{n \times n}$, are derived.

$$
\mathbf{x}_{m}^{\mathbb{R}}=\underbrace{\mathbf{V}_{m m}^{\mathcal{K}}}_{\mathbf{V}^{\mathbb{R} \rightarrow \mathcal{K}}} \overline{\mathbf{x}}^{\mathcal{K}}, \quad \mathbf{V}^{\mathcal{K} \rightarrow \mathbb{R}}=\left(\mathbf{V}_{m m}^{\mathcal{K}}\right)^{-1}, \quad \overline{\mathbf{M}}^{\mathbb{R}}=\left(\mathbf{V}^{\mathcal{K} \rightarrow \mathbb{R}}\right)^{T} \underbrace{\left(\mathbf{V}^{\mathcal{K}}\right)^{T} \mathbf{M}^{\mathbb{R}} \mathbf{V}^{\mathcal{K}}}_{\overline{\mathbf{M}^{\mathcal{K}} \in \mathcal{K}^{n \times n}}} \mathbf{V}^{\mathcal{K} \rightarrow \mathbb{R}} \quad \text { with } \quad \mathbf{M}^{\mathbb{R}} \in \mathbb{R}^{N \times N}
$$

Due to the dimension of $\mathbf{V}_{m m}^{\mathcal{K}} \in \mathcal{K}^{n \times n}$ we call this approach back-transformation deviating from KouTSOVASILIS [2]. By the back-transformation, the eigenvalues of the system are not affected. Unfortunately, the inversion works not sufficiently for an arbitrary reduced model, since the submatrix $\mathbf{V}_{m m}^{\mathcal{K}}$ may be ill-conditioned, see [2]. This is mainly caused due to the spatial distribution of interface coordinates, which is often unfavourable in mechanical models because of cluttered interfaces.

In the novel approach, the inversion is stabilized by introducing a rank criterion. If the product $\mathbf{V}_{m m}^{T} \mathbf{V}_{m m}$ has full rank $n$, the evaluation of the pseudo-inverse given by $\mathbf{V}_{m m}^{+}=\left(\mathbf{V}_{m m}^{T} \mathbf{V}_{m m}\right)^{-1} \mathbf{V}_{m m}$ is always possible. The rank increase is achieved by selecting additional master coordinates, by the help of sensor placement methods, e.g. the Effective Independence (EfI) [3] or the MoGeSeC-Algorithm [4].

The novel back-transformation approach is demonstrated at the example of a wheel-set axle FE-model and the complex model of a gear box housing. Due to the additional coordinates, the ROM dimension slightly increases, but with the benefit that the ROM is defined in physical configuration space. The quality of the recent $\mathrm{ROM}$ is verified by using correlation methods and the computational effort is evaluated.

\section{References}

[1] Salimbahrami, B. Structure Preserving Order Reduction of Large Scale Second Order Models. PhD thesis, Lehrstuhl für Regelungstechnik, Technische Universität München, Germany, 2005.

[2] Koutsovasilis, P. Model Order Reduction in Structural Mechanics - Coupling the Rigid and Elastic Multi Body Dynamics. PhD thesis, Professur für Fahrzeugmodellierung und -simulation, Technische Universität Dresden, Germany, 2009.

[3] Kammer, D. C. Sensor Placement for On-Orbit Modal Identification and Correlation of Large Space Structures. In: AIAA Journal of Guidance, Control, and Dynamics, Vol. 14, No. 9, pp. 251-259, 1991.

[4] Bonisoli, E.; Delprete, C.; Rosso, C. Proposal of a modal-geometrical-based master nodes selection criterion in modal analysis. In: Mechanical Systems and Signal Processing, Vol. 23, No. 3, Elsevier, pp. 606-620, 2009 . 


\title{
Topology Optimization of Flexible Bodies in Multibody Systems using the Floating Frame of Reference Approach
}

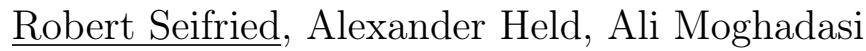 \\ Institute of Mechanics and Ocean Engineering, Hamburg University of Technology
}

Energy efficiency is a permanent issue in modern design of active multibody systems. In order to reduce the energy consumption, lightweight design techniques are applied to lower the moving masses and improve the mass to payload ratio. However, as a result the stiffness of the bodies decreases and nonnegligible structural deformations might appear during the working motion. For example in lightweight manipulators structural flexibility might lead to large unacceptable end-effector tracking errors. To decrease the structural deformation one might either use modern nonlinear control approaches or one might use optimization methods to design the members of the flexible system appropriately. In this research the second approach is taken, since it allows the use of well established basic control methods which are rather simple to implement. In order to obtain an optimized structural design, simulations of flexible multibody systems using the floating frame of reference approach are coupled with topology optimization for flexible members of the multibody system to improve their tracking behavior.

Topology optimization is a powerful tool for designing lightweight structures. This method tries to find the best distribution of material in a fixed design space. Therewith, this method allows for any formation of material inside the specified domain. The optimization problem is formulated using the solid isotropic material with penalization (SIMP) approach, see [2]. Such optimizations are often performed using finite element models. Thereby the objective functions are usually defined with respect to the compliance, displacements or stresses of the structure obtained from a set of static load cases. However, in order to improve system performance of dynamical systems the actual dynamic loads must be taken into account in the design of the system's components. First promising results of such an optimization philosophy are discussed in [1], [3], [4] for various types of active flexible multibody systems. However, many open questions remain, especially concerning, parameterizations, modeling and computational choices during such optimization procedures.

In this research the objective functions are computed from fully dynamical simulations of a flexible multibody system. Thus, all relevant loads on the flexible members of the system are captured. Consequently, all steps including the finite element modeling, the model reduction, the derivation of the equations of motion, establishing of feedback control and the transient simulation must be performed in each optimization loop. Thereby in each step a variety of possibilities exists. This talk will address some of these possibilities. Examples are the choice of the SIMP parameterizations, choice of global shape functions to approximate the elastic deformation field, computation of the gradients and type of objective functions.

\section{References}

[1] A. Albers, J. Ottnad, H. Weiler, P. Haeussler. Methods for Lightweight Design of Mechanical Components in Humanoid Robots. IEEE-RAS International Conference on Humanoid Robots, Pittsburg, Pennsylvania, USA (2007).

[2] Bendsøe, O. Sigmund. Topology Optimization - Theory, Methods and Applications. Berlin: Springer (2003).

[3] O. Brüls, E. Lemaire, P. Duysinx, P. Eberhard. Optimization of Multibody Systems and heir Structural Components. Multibody Dynamics: Computational Methods and Applications, K. Arczewski, W. Blajer, J. Fraczek, M. Wojtyra (Eds.), Computational Methods in Applied Sciences, Vol. 23, Springer (2011) 49-68.

[4] A. Held. On Structural Optimization of Flexible Multibody Systems. Schriften aus dem Institut für Technische und Numerische Mechanik der Universität Stuttgart, Band 33, Aachen: Shaker Verlag (2014). 


\title{
Nonexpansivity of the Newton's Cradle Impact Law
}

\author{
Remco I. Leine, Tom Winandy \\ Institute for Nonlinear Mechanics \\ University of Stuttgart \\ Pfaffenwaldring 9, D-70569 Stuttgart, Germany \\ remco.leine@inm.uni-stuttgart.de, tom.winandy@iam.uni-stuttgart.de
}

In this paper, we present an impact law for Newton's Cradle with 3 balls which is kinematically and kinetically consistent and enjoys the maximal monotonicity property. Our aim is to divulge the structure of impact laws in order to be able to formulate maximal monotone impact laws for rigid multi-body systems that do not have the problems of existing impact laws such as kinematic, kinetic, and energetic inconsistency [1]. It is interesting to consider Newton's Cradle because its phenomena cannot be described by the classical Newton's or Poisson's impact laws.

Kinematic consistency means that the impenetrability of unilateral constraints requires the post-impact contact velocities $\gamma_{i}^{+}$to be non-negative. In view of numerical integration, an impact-law should guarantee that arbitrary (also kinematically inconsistent) pre-impact contact velocities are mapped to kinematically consistent post-impact contact velocities.

The interest in the maximal monotonicity property stems from stability analysis and control of mechanical systems with unilateral constraints [3]. Since the maximal monotonicity property implies dissipativity it seems to be a physically reasonable property of an impact law.

We consider Newton's Cradle that consists of three balls of equal mass $m$ that are aligned along the same axis. The velocities of the balls are given by $\mathbf{u}=\left(\begin{array}{lll}u_{1} & u_{2} & u_{3}\end{array}\right)^{\mathrm{T}}$. The contact velocities are given by the relative velocities between the balls $\gamma=\left(\begin{array}{ll}\gamma_{1} & \gamma_{2}\end{array}\right)=\left(u_{2}-u_{1} u_{3}-u_{2}\right)^{\mathrm{T}}$. The pre- and post-impact velocities are designated by $\mathbf{u}^{-}$and $\mathbf{u}^{+}$respectively. Analogously, $\gamma^{-}$and $\gamma^{+}$designate the pre- and post-impact relative velocities.

In this work, we analyze the classical outcomes of the 3-ball Newton's cradle and complement these with thought-experiments for kinematic inconsistent cases. Six different pre-impact configurations can be distinguished of which the outcomes are described by a piece-wise linear relation for the impact mapping $S$

$$
\boldsymbol{\gamma}^{+}=S\left(\boldsymbol{\gamma}^{-}\right)=\mathbf{Q}_{i} \boldsymbol{\gamma}^{-} \text {for } \quad \mathbf{Q}_{i} \boldsymbol{\gamma}^{-} \geq 0 \quad i=1, \ldots, 6
$$

that maps the pre-impact relative velocities to the post-impact relative velocities.

A major result of the paper is that we can rigorously prove that the derived mapping (1) is non-expansive in the metric $\mathbf{G}^{-1}$, i.e.

$$
\left\|\gamma_{1}^{+}-\gamma_{2}^{+}\right\|_{\mathbf{G}^{-1}} \leq\left\|\gamma_{1}^{-}-\gamma_{2}^{-}\right\|_{\mathbf{G}^{-1}},
$$

where $\mathbf{G}=\mathbf{W}^{\mathrm{T}} \mathbf{M}^{-1} \mathbf{W}$ is the Delassus matrix. Therefore, it follows from [2] that the corresponding impact law is maximal monotone. Therefore, the impact mapping (1) that we propose guarantees kinematic, kinetic, and energetic consistency. The corresponding impact law has the maximal monotonicity property and it may allow to reveal further details about the general structure of set-valued impact laws.

\section{Acknowledgement}

This research is supported by the Fonds National de la Recherche, Luxembourg (Project Reference 8864427).

\section{References}

[1] Ch. Glocker. An Introduction to Impacts. In Nonsmooth Mechanics of Solids, CISM Courses and Lectures Vol. 485, Springer Verlag, Wien, New York, pp. 45-101, 2006.

[2] R. I. Leine, M. Baumann. Variational analysis of inequality impact laws. In Proceedings of the ENOC 2014 Conference, Vienna, 2014.

[3] R. I. Leine, N. van de Wouw. Stability and Convergence of Mechanical Systems with Unilateral Constraints. Springer Verlag, Berlin, 2008. 


\title{
An annular Kirchhoff plate model tailored for rotating and non-rotating external loads
}

\author{
Andreas Heckmann*, Ingo Kaiser* \\ Institute of System Dynamics and Control, German Aerospace Center (DLR), \\ Oberpfaffenhofen, 82234 Wessling, Germany
}

The bending deformation of rotating annular plates and the associated vibration behaviour is important in engineering applications which range from automotive or railway brake systems to discs that form essential components in turbomachinery.

However the modeling of rotating discs often has to cope with the difficulty to describe non-rotating forces acting on the disc such as the normal and friction forces at a disc brake.

In the floating frame of reference approach commonly used in flexible multibody dynamics the deformation field is formulated in terms of material fixed Lagrangian coordinates. As a consequence it is easy to introduce external forces that rotate synchronically with the disc, since here the Lagrangian coordinate of the force attachment point is constant in time. On the contrary for non-rotating forces the Lagrangian coordinate of the force attachment point has to be evaluated at each considered point in time which requires a contact formulation.

Due to the rotational symmetry properties of an annular plate it is possible to specifically adopt the so-called Arbitrary Langrangian-Eulerian (ALE) representation [1] and formulate the bending deformation of a Kirchhoff plate in an elegant way. An additional sliding frame of reference is introduced that does not perform the rotating motion. Since the transformation between the floating and the sliding frame of reference can be given, both rotating and non-rotating external forces can conveniently introduced into the equations of motion.

The contribution will give an overview on the theoretical background of this approach that has been implemented in the object-oriented modeling language Modelica [2]. The discussion of some simulation examples will conclude the presentation.

\section{References}

[1] I. Kaiser, A. Heckmann and F. van der Linden: On an ALE-Approach for Rotating Elastic Structures. In: Proceedings of the Multibody Dynamics 2007. ECCOMAS Thematic Conference.

[2] A. Heckmann, M. Otter, S. Dietz, Stefan and J.D. López: The DLR FlexibleBody library to model large motions of beams and of flexible bodies exported from finite element programs. In: Proceedings of the 5 th International Modelica Conference, 2006. 


\title{
Performance assessment of a tracjectory-tracking approach for a manipulator with uncertainties using inverse fuzzy arithmetic
}

\author{
Nico-Philipp Walz, Michael Hanss \\ Institute of Engineering and Computational Mechanics, Stuttgart, Germany
}

Uncertainty analysis is concerned with the effects of model uncertainties on the prediction of the model response. Thereby, for instance, the reliability of a model and the conclusions drawn from the calculated results may be rated or be put into perspective with the associated real system. Furthermore, the behavior and the change of behavior of the system for the possible conditions may be investigated, providing a better understanding of the system.

On the one hand, this is possible in a direct approach by assigning uncertainties to the model - derived from assumptions, expert knowledge or identification procedures - and propagating those uncertain quantities through the model in order to obtain the range of responses for the feasible realizations of the model. On the other hand, an inverse approach can be applied, being capable of identifying uncertainties in a model in order to provide uncertainty bounds for a parameter estimation and to perform a model validation on that basis [1].

The uncertainties may stem from variability in the model parameters, i.e. from randomness. There are various methodologies for handling uncertainties of that type in the different analyses described above. Uncertainties due to a lack of knowledge or due to simplifications made during modelling are commonly termed epistemic uncertainties and are not characterized by randomness. Instead, the actually deterministic real system and the model differ due to the imperfect knowledge and the effects neglected during modeling. Therefore, fuzzy numbers are commonly used to model those epistemic uncertainties, since they comply with the possibilistic nature of this class of uncertainties. Hence, fuzzy arithmetical methods have to be employed for the analysis of systems with epistemic uncertainties [2].

In this contribution, the fuzzy arithmetical approach is applied to an inverse problem in multibody dynamics in order to assess the system performance considering intrinsic uncertainties. A major issue during the design phase is indeed characterized by the imperfect knowledge of the model parameters and the actual operational configuration. For instance, the controller design is performed for a nominal model that is assumed to resemble most of the system dynamics.

In particular, a trajectory tracking problem of a manipulator with highly flexible links will be addressed that possesses unstable internal dynamics. A feed-forward control based on stable inversion is applied which provides an exact tracking of the end-effector motion for the nominal system without disturbances. As usual, in order to account for the inherent uncertainties and disturbances, the system is supplemented by an additional feedback control. Since the feed-forward control provides a trajectory planning with desired drive positions and velocities, a collocated feedback can be employed for the drives [3]. Hence, stability of the control scheme can be achieved despite the non-minimun phase characteristic of the system.

The effect of the model uncertainties are then to be identified, as the control scheme only affects the drive positions and thus does not unconditionally ensure that the end effector tracks the desired trajectory with satisfactory accuracy, especially in the case of significant elastic deformation. Hence, the performance of the system shall be assessed by investigating the robustness of the tracking approach with respect to inexact matching of initial conditions and uncertain model parameters. Therefore, a concept for estimating the robustness of a system with respect to epistemic uncertainties within a model is presented using inverse fuzzy arithmetic.

\section{References}

[1] T. Haag and M. Hanss. Model assessment using inverse fuzzy arithmetic. In Eyke Hüllermeier, Rudolf Kruse, and Frank Hoffmann, editors, Information Processing and Management of Uncertainty in Knowledge-Based Systems. Applications, volume 81 of Communications in Computer and Information Science, pages 461-470. Springer Berlin Heidelberg, 2010.

[2] M. Hanss. Applied Fuzzy Arithmetic - An Introduction with Engineering Applications. Springer, Berlin, 2005.

[3] M. Burkhardt, R. Seifried and P. Eberhard. Experimental Studies ofControl Concepts for a Parallel Manipulator with Flexible Links Journal of Mechanical Science and Technology, (submitted for publication). 


\title{
Variational integrators for thermo-viscoelastic discrete systems
}

\author{
Dominik Kern ${ }^{1}$, Ignacio Romero ${ }^{2}$, Michael Groß ${ }^{1}$ \\ ${ }^{1}$ Chair of Applied Mechanics and Dynamics, TU Chemnitz, Germany \\ ${ }^{2}$ Computational Mechanics Group, E.T.S.I.I., UP Madrid, Spain
}

Variational integrators are modern time-integration schemes based on a discretization of the underlying variational principle. They thus skip the direct formulation and time discretization of differential equations. In this paper, Hamilton's principle is approximated by an action sum, whose vanishing variation results in discrete Euler-Lagrange equations or, equivalently, in discrete evolution equations for the position and momentum. Variational integrators are, by design, structure preserving (symplecticity and momentum) and show excellent long-time behavior in total energy.

In this work, heat transfer is accounted for after the dissipationless type II model of Green and Naghdi. We will show that, in this way, thermal effects enter the variational principle both via the free energy, and Fourier's law of heat conduction accounting for thermal dissipation. This formulation requires the notion of thermacy, a quantity also called "thermal displacement" whose time derivative corresponds to the temperature. It results in a natural definition of the entropy as "thermal momentum" and entropy flux as "thermal force". The viscoelastic effects are also introduced via the free energy by an internal variable formulation. In order to include the corresponding viscous and thermal virtual work (mechanical and thermal virtual dissipation), Hamilton's principle is extended by D'Alembert terms, which account for the time evolution equation of the internal variable and Fourier's law.

From this variational formulation, variational integrators using different orders of approximation of the state variables as well as of the quadrature of the action integral are constructed and compared. A thermo-viscoelastic double pendulum comprised of two discrete masses connected by thermoelastic springs and dash pots (Poyntingelements), and subject to heat conduction both between the two springs and between each of the springs and the environment, serves as a discrete model problem. 


\title{
Variational integrators of higher order for flexible multibody systems
}

\author{
Michael Groß, Matthias Bartelt \\ Chair of Applied Mechanics and Dynamics, TU Chemnitz, Germany
}

Variational integrators are structure-preserving time stepping schemes for dynamical systems which are derived from a discrete variational principle. This is in contrast to the usual discretization of EULER-LAGRANGE equations derived from a continuous variational principle. Therefore, a variational integrator always takes the form of discrete EULER-LAGRANGE equations or the equivalent position-momentum equations.

In this presentation, we consider the motion of a flexible rope subject to different boundary conditions as model problem for a distributed parameter system. One considered boundary condition is to consider the rope as flexible connection between two mass points, which represent a physical model of a tethered satellite system. Furthermore, we simulate the motion of the rope subject to time-dependet DIRICHLET boundary conditions, as a fixed or moved suspension point, for instance. The introduction of non-holonomic constraints by the LAGRANGE multiplier technique makes this possible.

The corresponding variational integrators are derived from a space-time discretization of HAMILTON's principle. The space discretization is based on one-dimensional linear LAGRANGE polynomials and a two-point GAUSSIAN quadrature, whereas the time discretization is based on higher-order polynomials and higher-order quadrature rules. In this way, we obtain higher-order accurate variational integrators.

As numerical examples, we consider the flexible rope system subject to different combinations of DIRICHLET and Neumann boundary conditions, and investigate the convergence behaviour and computional costs of the variational integrator in comparison to standard time stepping schemes. Further, we also compare different polynomial bases with equidistant or other special interpolation points in the time approximation. 


\title{
Error estimation approach for controlling the comunication step size for semi-implicit co-simulation methods
}

\author{
Tobias Meyer, Bernhard Schweizer \\ Department of Mechanical Engineering \\ Institute of Applied Dynamics \\ Technical University Darmstadt, Germany
}

This contribution presents an approach for controlling the macro-step size in connection with co-simulation methods [1, 3]. The investigated step-size controller is tailored for semi-implicit co-simulation techniques. Concretely, we consider predictor/corrector co-simulation approaches [2]. By comparing variables from the predictor and the corrector step, an error estimator for the local error can be constructed. Making use of the estimated local error, a step-size controller for the macro-step size can be implemented. Different numerical examples are presented, which show on the one hand the applicability of the method and on the other hand the benefit of a variable macro-grid with respect to simulation time.

\section{References}

[1] M. Busch, B. Schweizer. An explizit approach for controlling the macro-step size of co-simulation methods. ENOC (2011), 24-29.

[2] B. Schweizer, D. Lu. Predictor/corrector co-simulation approches for solver coupling with algebraic constraints. ZAMM, Journal of Applied Mathemtics and Mechanics, DOI: 10.1002/zamm.201300191 (2014).

[3] Verhoeven, A., Tasic, B., Beelen, T.G.J., ter Maten, E.J.W., Mattheij, R.M.M.: BDF compound-fast multirate transient analysis with adaptive stepsize control. J. Numer. Anal. Ind. Appl.Math. 3(3-4), 275-297 (2008). 


\title{
A Reproducible Excitation Mechanism for Analysing Electric Guitars
}

\author{
Pascal Bestle, Michael Hanss, Peter Eberhard \\ Institute of Engineering and Computational Mechanics \\ University of Stuttgart \\ Pfaffenwaldring 9, 70550 Stuttgart, Germany
}

The history of musical instruments dates back nearly as long as the humanity itself. During the last centuries, the development of the quality as well as of the play of musical instruments was pushed empirically and experimentally by instrument makers and players. This development can hardly be surpassed. Therefore, at first sight, the scientific study of instruments does not seem to allow any further significant improvement. It is, however, very important to gain insight into these complex systems and to achieve an understanding of the generated sound and of what makes it agreeable for the listener.

A very popular instrument is the electric guitar, whose sound is commonly assumed to originate mainly from the plugged electronics. This assumption, however, is not compatible to the large variety of commercially available models of electric guitars, featuring a significant spread in material and construction, and also in quality and price. In fact, there is reason to believe that there is a not to be underestimated influence of the used materials, which is also motivated by investigations of acoustic guitars. However, the sound of acoustic guitars is mainly formed in there hollow body [1], strongly influenced by the structural behavior of the body and the neck. The body of an electrical guitar, instead, is made of solid wood, and a sound hole does not exist.

The goal of this investigation is to gain insight into the sound generation of electric guitars, the interaction between string excitation and structural response as well as the influence of material and geometry parameters. This contribution presents an experimental set-up for measuring the structural behavior of the guitar body using Laser-Doppler-Vibrometers, to be compared to the generated sound. To ensure a well-defined and reproducible excitation of the strings, a sophisticated mechanism is constructed, making use of the methods of multibody dynamics and optimization. With this mechanism, it is possible to determine the influence of different parameters on the induced vibrations and the generated sound, which can be assessed by specific criteria [2]. Exemplarily, the influence of the adjustable settings of the electronics and the pickups are presented.

\section{References}

[1] French, R.M.: Engineering the Guitar: Theory and Practice. Springer Heidelberg, 2008.

[2] Bestle, P.: Experimental and Numerical Analysis of the Musical Behavior of Triangle Instruments. Proceedings of the 5th European Conference of Computational Mechanics (ECCM V), 2014, 3104-3114. 


\title{
Transient amplification of maximum vibration amplitudes
}

\author{
Marius Bonhage, Olaf Hentschel, Lars Panning-v. Scheidt, Jörg Wallaschek \\ Institute of Dynamics and Vibration Research
}

Many low damped structures as turbine blades or drill strings are exposed to high dynamical loads causing high vibration amplitudes. These applications comprise sub-critical eigenfrequencies. Hereby, the lower eigenfrequencies have to be passed before reaching the operating point. Most investigations of vibration amplitudes caused by a resonance passage deal with the computation of one degree of freedom systems. Thereby, it has been shown that the stationary vibration response provides the highest possible amplitude. Further it can be stated that the maximum vibration response of the resonance passage decreases with an increasing sweep velocity, see [1].

To describe linear systems with isolated modes it is straight forward to investigate only one-degree-offreedom systems. Since the system can be decoupled it is sufficient to regard the separated modes individually. Subsequently the mode shape can be described by the multiplication of the amplification function of the mode and the belonging eigenvector. There are only some recent works that deal with resonance passages of vicinal modes, e. g. [2] and [3]. But no revised statements are provided concerning the behavior of the maximum amplitudes. In this paper a three dimensional system comprising nearby modes is studied to investigate the amplitude of a resonance passage. To calculate the transient vibration response an analytical approach is used. It will be shown that the maximum amplitude of the stationary vibration response is not the least upper bound for the maximum amplitude of the resonance passage and that the maximum amplitude may rise while the sweep velocity increases. Hence, regarding a multi degree of freedom system the maximum amplitude of the resonance passage can exaggerate the maximum amplitude of the stationary vibration response. This is statement extends the known statements about transient vibrations.

\section{References}

[1] Goloskokov, E. G. und Filippov, A. Instationäre Schwingungen mechanischer Systeme. Akademie-Verlag. (1971)

[2] Ayers, J. P., Feiner, D. M., und Griffin, J. H. A reduced-order model for transient analysis of bladed disk forced response. Journal of Turbomachinery, 466 - 473. (2006).

[3] Kaneko, Y. Study on transient vibration of mistuned bladed disk passing through resonance. In Proceedings of ASME Turbo Expo 2013, San Antonio. (2013). 


\title{
Prox formulation of the cavitation problem in elastohydrodynamic lubrication contact
}

\author{
Andreas Krinner, Daniel J. Rixen \\ Technische Universität München \\ Faculty of Mechanical Engineering \\ Institute of Applied Mechanics
}

\begin{abstract}
This contribution presents an alternative method, how to find the cavitation region in elastohydrodynamic (EHD) lubrication using an augmented lagragian approach. A theoretical framework for the use of the prox formulation instead of a Linear Complementary Problem (LCP) is given and the application for multibody systems with EHD contacts is shown. The prox formulation is solved by the Newton-Raphson method. As an example, the number of iteration for the solution using the prox formulation is compared with the number obtained by the pivot based Murty algorithm during time integration of a rotor with unbalance in an elastic

In EHD lubrication theory, the pressure distribution in the fluid film between two contacting rigid or elastic bodies is usually calculated by a numerical solution of the Reynolds equation. In zones with negative pressure, the fluid is said to cavitate and therefore, the negative pressure is set to the cavitation pressure. In order to ensure mass conservation at the boundary between fluid zone and cavitation zone, the pressure and the pressure gradient have to vanish, which is known as the Reynolds boundary condition. The so called cavitation problem can be formulated as a LCP. The aim is to find a fluid region with positive pressure $p$ and vanishing flux $q$ and a cavitation region with a zero cavitation pressure and a negative flux:
\end{abstract} bearing.

$$
\begin{gathered}
b-A \cdot p=q \\
0 \leq p \perp q \geq 0
\end{gathered}
$$

A widely spread cavitation algorithm for this problem is the pivot based Murty algorithm, which was applied first by Goenka 1985 in a blockwise form [1].

Here, a prox formulation of the cavitation problem is presented as an competitive alternative. The prox formulation was first stated in the beginnings of the 1990s by Alart and Curnier for frictional contact problems [2]. In [3] the prox formulation and the well known LCP are compared for simulation of multibody systems with contact. Hence, for the treatment of EHD contacts in multibody systems, a prox formulation of the cavitation problem can be a useful alternative.

\section{References}

[1] K. P. Oh, P. K. Goenka. The elastohydrodynamic solution of journal bearings under dynamic loading. Journal of Tribology 107 (1985), 389-394.

[2] P. Alart, A. Curnier. A mixed formulation for frictional contact problems prone to Newton like solution methods. Computer methods in applied mechanics and engineering 92 (1991), 353-375.

[3] T. Schindler, B. Nguyen, and J. Trinkle. Understanding the difference between prox and complementarity formulations for simulation of systems with contact. Intelligent Robots and Systems (IROS), 2011 IEEE/RSJ International Conference on. IEEE, 2011. 


\title{
Rigid body motion in a medium: data preparation for execution of experiments
}

\author{
Maxim V. Shamolin \\ Lomonosov Moscow State University
}

\begin{abstract}
Proposed work presents next stage of the study of the problem of the plane-parallel motion of a rigid body interacting with a resistant medium through the frontal plane part of its external surface. Under constructing of the force acting of medium, we use the information on the properties of medium streamline flow around in quasi-stationarity conditions (for instance, on the homogeneous circular cylinder input into the water). The medium motion is not studied, and we consider such problem in which the characteristic time of the body motion with respect to its center of masses is comparable with the characteristic time of motion of the center of masses itself.
\end{abstract}

If in [1], we represent the asymptotical stability conditions of the rectilinear translational deceleration (drag), and in $[2,3]$, we obtained the new multi-parametric family of phase patterns in the space of quasi-velocities, then in this work, we prepare the qualitative material for the preparation of further natural experiments on the motion of the hollow circular cylinders in a medium.

Let give the brief summary to the previous stages of studying. Also, by the reason of complexity of nonlinear analysis, the initial stage of such a study is the neglecting of the dependence of the medium interaction force moment on the angular velocity and use of such dependence on the angle of attack only [1]).

From the practical view point it is important the problem of studying of stability of so-called unperturbed (rectilinear translational) motion under which the velocities of body points are perpendicular to the plate (cavitator).

The whole spectrum of results found under the simplest assumption on the absence of the medium damping action on a rigid body allowed the author to make the conclusion that it is impossible to find those conditions under which there exist the solutions corresponding to the angular body oscillations of a finite amplitude.

The experiment in the motion of homogeneous circular cylinders in the water (see [2]) justified that in modelling the medium action on the rigid body, it is also necessary to take account of an dependence of the medium interaction force moment on the angular velocity of the body. Herewith, there arise the additional members that brings a dissipation to the system.

In studying the class of body motions with the finite angles of attack, the principal problem is finding those conditions under which there exist the finite amplitude oscillations in a neighborhood of the unperturbed motion. Therefore, there arises the necessity of a complete nonlinear study.

In earlier author's works, one has succeeded to use the instability of the rectilinear translational body motion for the methodological purposes (see [3]), i. e., in determination of unknown parameters of the medium action on the body in quasi-stationarity conditions.

The account of the medium damping action on the rigid body leads to an affirmative answer to the principal question of the nonlinear analysis: under the body motion in a medium with finite angles of attack, in principle, there can arise stable auto-oscillations which can be explained by the account of an additional dependence of the medium action on the body angular velocity that brings an additional dissipation to the system.

Furthermore, under the applying of methodology of studying of the dissipative dynamical systems of certain type, we obtain the new multi-parametric family of phase patterns on the two-dimesional cylinder; this family consists of the infinite set of topologically non-equivalent phase patterns changing its topological types under the variation of the system parameters by the degenerate way (see also $[1,2,3])$.

\section{References}

[1] V.A. Eroshin, V.A. Samsonov, and M.V. Shamolin. Model problem of body grag in a resisting medium under streamline flow around. Izv. Ross. Akad. Nauk, Mekhanika Zhidkosti i Gaza. 3 (1995), 23-27.

[2] M.V. Shamolin. Rigid body motion in a resisting medium. Matem. Mod. 23, No. 12 (2011), 79-104.

[3] M.V. Shamolin. A multiparameter family of phase portraits in the dynamics of a rigid body interacting with a medium. Vestn. MGU, Ser. 1, Mat., Mekh. 3 (2011), 24-30. 


\section{S02: Biomechanics}

The section will be focused on numerical and experimental models for the study of structure, function and evolution of biological systems at a broad spectrum of scales: from cells to tissues and from organs to the entire body and its interaction with the environment. The discussed topics will be: gait analysis and foot biomechanics, musculoskeletal and orthopedic biomechanics, remodeling, cardiovascular biomechanics, multiphase modeling of biological tissues, tumor growth modeling, transport oncophysics, nanomechanics of biological materials, nanomedicine, modeling of drugs delivery. 


\title{
A new fully explicit algorithmic strategy for the simulation of bone healing directly on computed tomography data
}

\author{
Michael Roland, Thorsten Tjardes, Bertil Bouillon, Stefan Diebels \\ Chair of Applied Mechanics, Saarland University, Campus A4 2, 66123 Saarbrücken \\ Department of Trauma and Orthopedic Surgery, University of Witten/Herdecke, \\ Faculty of Health - School of Medicine, Cologne Merheim Medical Center, \\ Ostmerheimerstr. 200, 51109 Cologne, Germany
}

Starting with a routinely acquired tomographic data set of a real patient with a fractured tibia, a segmentation procedure is used to generate a mesh and to assign the material properties. This mesh is the initial point for a diffusion-based algorithm that generates a realistic callus shape for the individual fracture of the patient by a finite element simulation taking the influence of the mechanobiological stimulus on the early callus formation into account [4].

In the next step, the computed callus shape is the basis for the definition of the bone healing area. After an appropriate meshing of this area, the bone healing is simulated with a fully explicit finite difference scheme of an elastic material model combined with a mechanoregulation algorithm to describe the cellular processes involved in the healing process, e.g. proliferation, migration, apoptosis, and differentiation of cells [3].

The main part of the algorithmic healing strategy is based on the ideas presented in $[1,2]$ namely an iterative procedure working directly on the mesh cells initialized from the resolution of the computed tomogram. The healing process itself is realized with a dynamic and competitive model describing the influence of mechanical parameters and criteria.

The boundary conditions of the simulations are assumed from an OpenSim simulation of a gait cycle with respect to the size and the weight of the considered patient. This allows the implementation of realistic muscle loading forces into this patient-specific simulations.

\section{References}

[1] M. Roland, T. Tjardes, R. Otchwemah, S. Diebels, B. Bouillon. An optimization algorithm for individualized biomechanical analysis and simulation of tibia fractures. J. Biomech. (accepted).

[2] T. Tjardes, M. Roland, R. Otchwemah, T. Dahmen, S. Diebels, B. Bouillon. Less than full circumferential fusion of a tibial nonunion is sufficient to achieve mechanically valid fusion - Proof of concept using a finite element modeling approach. BMC Musculoskel. Dis. (2014), 15:434, doi:10.1186/1471-2474-15-434.

[3] D. P. Byrne, D. Lacroix, P. J. Prendergast. Simulation of Fracture Healing in the Tibia: Mechanoregulation of Cell Activity Using a Lattice Modeling Approach. Inc. J. Orthop. Res. 29 (2011), 1496-1503.

[4] F. O. Ribeiroa, J. Folgadoa, J. M. Garcia-Aznarb, M. J. Gomez-Benitob, P. R. Fernandesa. Is the callus shape an optimal response to a mechanobiological stimulus? Med. Eng. Phys. 36 (2014), 1508-1514 


\title{
Evolution of Mechanical Properties in Tissues Undergoing Deformation-Related Fiber Remodeling Processes
}

\author{
Heiko Topol*, Hasan Demirkoparan*, Thomas J. Pence**, Alan Wineman*** $^{* *}$ \\ ${ }^{*}$ Carnegie Mellon University in Qatar \\ ** Department of Mechanical Engineering, Michigan State University \\ *** Department of Mechanical Engineering, University of Michigan
}

This research deals with biological soft tissues containing collagen fibers embedded in an isotropic matrix. The mechanical properties of the material is described by the additive strain energy density function

$$
W=W_{m}+W_{f} .
$$

The mechanical properties of the matrix are considered to be time independent, and represented by the strain energy density $W_{m}$. The fibers undergo a remodeling process which depends on the deformation of the fibers. The strain energy density of the fibers $W_{f}$ is modeled in terms of the strain energy density function $\psi$ of the protofibers, and a factor denoted as the survival kernel

$$
\zeta=\chi_{c} \exp \left(-\int_{\tau}^{t} \eta \mathrm{d} s\right)
$$

with a constant fiber creation rate $\chi_{c}$ and a deformation dependent fiber dissolution rate $\eta$. This dissolution rate is a function of the fiber stretch and is modeled to approximate experimental results such as [1]. The survival kernel describes the changes of the mechanical properties of fibers created at time $\tau, \tau \leq t$, so that the mechanical properties of the material not only depend on the current deformation but also on the entire deformation history. While the matrix deforms from a reference configuration $\kappa_{0}$ into a deformed configuration $\kappa$, fibers created at time $\tau$ might have a different natural configuration $\kappa_{f}$. Topol et al. [2] investigate the development of the fiber density for different uniaxial deformation histories when the fiber network is considered to be unstructured and the fibers undergo the deformation-related remodeling process. Based on these results, Topol et al. [3] describe the uniaxial load-deformation relation for such a material model. This research seeks to generalize the results obtained in $[2,3]$ by investigating multiaxial deformation processes.

Acknowledgement: This work was made possible by NPRP grant No. 4-1138-1-178 from the Qatar National Research Fund (a member of the Qatar Foundation). The statements made herein are solely the responsibility of the authors.

\section{References}

[1] A. P. Bhole, B. P. Flynn, M. Liles, N. Saeidi, C. Dimarzio, J. Ruberti. Mechanical strain enhances survivability of collagen micronetworks in the presence of collagenase: Implications for load-bearing matrix growth and stability, Philos. T. R. Soc. A 367 (2009), 3339-3362

[2] H. Topol, H. Demirkoparan, T. J. Pence, A. Wineman. A theory for deformation dependent evolution of continuous fibre distribution applicable to collagen remodelling. IMA J. Appl. Math. 79 (2014), 947-977

[3] H. Topol, H. Demirkoparan, T. J. Pence, A. Wineman. Uniaxial load analysis under stretch dependent fiber remodeling applicable to collageneous tissue. J. Eng. Math. (2014), accepted. 


\title{
Numerical Calculation of Fiber Orientation in Three-Dimensional Arterial Walls
}

\author{
$\underline{\text { Simon Fausten }}^{1}$, Daniel Balzani², Jörg Schröder ${ }^{1}$ \\ ${ }^{1}$ Institut für Mechanik, Abteilung Bauwissenschaften, Fakultät für Ingenieurwissenschaften, \\ Universität Duisburg-Essen, Universitätsstr. 15, 45141 Essen, Germany \\ ${ }^{2}$ Institut für Mechanik und Flächentragwerke, Fakultät Bauingenieurwesen, \\ Technische Universität Dresden, 01062 Dresden, Germany \\ simon.fausten@uni-due.de, daniel.balzani@tu-dresden.de, \\ j.schroeder@uni-due.de
}

In the field of biomechanics soft biological tissues are usually described by quasi-incompressible nonlinear anisotropic material models. In the case of arterial walls multilayered fiber reinforced materials, characterized by two fiber families which are embedded in a soft matrix material and which are arranged crosswise helically around the artery in axial direction, are a suitable choice for a proper material description. Due to their living nature biological tissues continuously change and adapt according to their mechanobiological environment. Hence, the consideration of a biomechanically motivated fiber orientation approach will yield a more realistic description of arterial tissues that goes beyond the assumption of a constant fiber orientation in each layer of the tissue. Such an approach is motivated by the fact that the embedded collagen fibers have a major impact on the overall mechanical behavior inside arterial walls, i.e. their orientation strongly influences the passive response and transmural stress distribution of arterial tissues. Especially with respect to atherosclerotic arteries a biomechanically motivated fiber orientation is advantageous since the experimental determination of fiber orientations in the individual components is difficult. Suitable approaches for the calculation of the fiber directions are given in [1] and [2], based on the assumption that the fiber orientation is mainly governed by the principal stress directions, in order to maximize the load-bearing capacities of the tissue. Based on this assumption, in this contribution an approach is presented, which focusses on volume-averaged principal stresses. Then, an effective iterative fiber reorientation algorithm is proposed and applied to the polyconvex nonlinear anisotropic material model presented in [3]. Furthermore, the presented fiber reorientation approach is investigated for patient-specific arterial geometries and the resulting fiber distributions are studied. For this purpose, the so-called virtual histology is taken into account, which provides information regarding a stack of axially distributed two-dimensional cross-section images from which the individual components of the arterial wall such as the media, adventitia and plaque can be segmented, cf. [4].

\section{References}

[1] I. Hariton, G. deBotton, T.C. Gasser and G.A. Holzapfel, Stress-driven collagen fiber remodeling in arterial walls. Biomechan Model Mechanobiol, 6(2007),163-175.

[2] E. Kuhl and G. A. Holzapfel. A continuum model for remodeling in living structures. Journal of Materials Science, 42(2007),8811â8823.

[3] D. Balzani, P. Neff, J. Schröder and G.A. Holzapfel. A polyconvex framework for soft biological tissues. adjustment to experimental data. International Journal of Solids and Structures, 43(2006), 6052-6070.

[4] D. Balzani, D. Böse, D. Brands, R. Erbel, A. Klawonn, O. Rheinbach and J. Schröder. Parallel Simulation of Patient-specific Atherosclerotic Arteries for the Enhancement of Intravascular Ultrasound Diagnostics. Engineering Computations, 29(2012). 


\title{
Towards an accurate mechanical characterisation of human's aortic leaflets during the heart cycle
}

\author{
Morales Ortuno Sergio $^{1,2}$, Röhrle Oliver ${ }^{1,2}$ \\ ${ }^{1}$ Institute of Applied Mechanics (Chair II), University of Stuttgart \\ ${ }^{2}$ Stuttgart Research Center for Simulation Technology
}

While the available, conventional prosthetic valves satisfy most of the needs of the valvular replacement market, they do not represent a satisfactory solution for a specific part of the population. This specific group of the population is composed by the pedriatic population, women of child-bearing age, patients with infective endocarditis, small aortic roots and patients intolerant to anticoagulants [1],[2]. The specific cases mentioned above can be treated by the use of human allografts or tissue-engineered valves. Since the tissue-engineered valves are considered to be in an experimental stage, the use of human allografts is more widespread but restricted by availability. An important source of human allograft valves is the explanted hearts from patients receiving a transplant as treatment for ischaemic (ICM) or dilated cardiomyopathy (DCM) [1].

The construction of a mechanically oriented 3-D Finite Element Model of an aortic leaflet is presented in detail. In order to investigate the stress distribution in the tissue and have a better insight of the mechanical response of the leaflet under physiological conditions, we aim to build an accurate, reliable model which incorporates a more realistic geometry of the leaflet and also the fibrous tissue network, composed mainly by collagen and elastin. Being collagen the principal load-bearing element in such soft tissues. The main challenge relies on finding the correct mathematical model able to account for the nonlinear, anisotropic behaviour of the leaflets. We describe the process of fitting existing strain-energy functions to available data and analyse their applicability in the model. The latter includes the results of implementing each adapted mathematical model and the direct consequences of them on the modelling assumptions, and features, like geometry, fibre distribution and boundary conditions used, for example.

\section{References}

[1] Schenke-Layland K. et. al. Cardiomyopathy is associated with structural remodelling of heart valve extracellular matrix. 2009.

[2] Vesely I. Heart Valve Tissue Engineering. 2005. 


\title{
Towards effective properties of active muscle tissue via homogenisation
}

\author{
$\underline{\text { Christian Bleiler }}^{1,2}$, Pedro Ponte Castañeda ${ }^{3}$, Oliver Röhrle ${ }^{1,2}$ \\ ${ }^{1}$ Institute of Applied Mechanics (Chair II), University of Stuttgart \\ ${ }^{2}$ Stuttgart Research Center for Simulation Technology \\ ${ }^{3}$ Department of Mechanical Engineering and Applied Mechanics, \\ University of Pennsylvania, Philadelphia
}

The constitutive modelling of skeletal muscle tissue in a continuum-mechanical framework demands the consideration of many complex material characteristics. This complexity stems from the microscopic arrangement of different structural elements, such as, for example, sarcomeres and connective tissue. The former are responsible for the active contractile behaviour while the latter are mainly responsible for the passive stiffness of the muscle tissue. Thus, it is highly challenging to construct appropriate macroscopic models based on phenomenological descriptions in order to take into account most of the complex material behaviour. As a result, strain energy functions are adapted for every significant material phenomena coming from experimental testing of muscle tissue.

Another way to treat the modelling of active muscle tissue is to describe important parts of the heterogeneous microstructure and use homogenisation techniques to derive effective overall properties. From a continuummechanical point of view, the primary goal is to obtain a homogenised strain energy function. To do so, various techniques exist in this field. This contribution will present the initial steps towards the homogenisation of skeletal muscle tissue including the active behaviour. 


\title{
Modeling of skin anisotropy directions for realistic finite element simulations of the female breast
}

\author{
$\underline{\text { Xuhui Li }^{1}}$, Stefan Raith ${ }^{2}$, Mahmood Jabareen ${ }^{3}$, Mikhail Itskov ${ }^{1}$ \\ ${ }^{1}$ Department of Continuum Mechanics, RWTH Aachen University, Germany \\ ${ }^{2}$ Department of Prosthodontics and Dental Materials, RWTH Aachen University, Germany \\ ${ }^{3}$ Faculty of Civil and Environmental Engineering, Technion \\ Israel Institute of Technology, Israel
}

\begin{abstract}
Biomechanical simulations of the female breast are important for surgical applications such as implants augmentation, tumorectomy and reconstruction after the tumor removal. One of the main challenges for such breast simulations is to define its reference configuration which can be considered as a stress free state. Indeed, MRI scans of the breast can be obtained only under gravitational load which introduces a considerable stress and strains level for any position of the patient. Moreover, realistic material properties especially anisotropy of skin should be taken into account. This anisotropy can play an important role but have not so far been considered in biomechanical simulations of the breast.

In the current contribution, we implement an iterative method to define the reference configuration of the breast model according to MRI data of certain individuals in the prone position. In order to automatically generate principal material directions of skin, a numerical procedure based on the Laplacian smoothing method of user-drawn strokes [1] is proposed. A polyconvex model [2] and a new type mixed element [3] are exploited to simulate large deformations of breast tissues. Finally, the accuracy of the proposed method is validated by comparing FE breast simulations to 3-D surface imaging data in the standing position.
\end{abstract}

\section{References}

[1] Li X, Raith S, Itskov M. Numerical simulation of anisotropy directions in soft tissues and in particular in skin.PAMM 2013; 13(1):57-58.

[2] Ehret AE, Itskov M. A polyconvex hyperelastic model for fiber-reinforced materials in application to soft tissues. Journal of Materials Science 2007; 42(21):8853-8863.

[3] Schröder J, Wriggers P, Balzani D. A new mixed finite element based on different approximations of the minors of deformation tensors. Computer Methods in Applied Mechanics and Engineering 2011; 200(49):35833600 . 


\title{
Experimental testing of transversely isotropic biological tissues - interaction between fibre and matrix material
}

\author{
Markus Böl ${ }^{1}$, Alexander E. Ehret ${ }^{2}$, Kay Leichsenring ${ }^{1}$, Michael Ernst ${ }^{1}$ \\ ${ }^{1}$ Institute of Solid Mechanics, Technische Universität Braunschweig, Braunschweig, Germany \\ Institute of Mechanical Systems, ETH Zurich, Zurich, Switzerland
}

Fibre-reinforced materials are used in many fields of engineering. They combine favourable characteristics of the fibres and a matrix material in which the fibres are embedded. The basic idea of fibre-reinforced materials also applies to a large variety of soft biological tissues, where fibres are surrounded by an extracellular matrix. On the one side, this includes skeletal muscle tissue, the striking feature of which is the ability of force generation based on a large number of contractile muscle fibres embedded in a composition of connective tissues forming the extracellular matrix, blood vessels and nerves. On the other side, also tendon tissue, to be a specialised tissue that usually connects muscles and bones, consists of collagen fibres embedded into a non-collagenous matrix. Aforementioned types of soft biological tissues are often tested using various experimental protocols. However, the load transfer between fibre and matrix materials is far from well-understood. Therefore, this paper deals with the role of the fibres and matrix material in muscle as well as tendon tissue when it is subjected to compressive loads $[1,2]$. To this end, dissected tissue samples were tested in compression modes which induced states of fibres in compression (I), in tension (II) or at constant length (III), respectively. A comparison of the stress responses indicated that the tissue behaviour is significantly different for these modes, including differences between the modes (I) and (III). This contradicts the paradigm of many constitutive models that the stress response can be decomposed into an isotropic part relating to the extracellular matrix and an anisotropic fibre part the contribution of which can be neglected under compression. Conversely, the results provide experimental evidence that there is an anisotropic contribution of the fibre direction to the compressive stress. Interpreting these results in terms of recent microscopical studies, potential connections between the observed behaviour and the structure of muscle extracellular matrix are established.

\section{References}

[1] M. Böl, A. E. Ehret, K. Leichsenring, C. Weichert, R. Kruse. On the anisotropy of skeletal muscle tissue under compression. Acta Biomater. 10 (2014), 3225-3234.

[2] M. Böl, A. E. Ehret, K. Leichsenring, M. Ernst. Tissue-scale anisotropy and compressibility of tendon in semi-confined compression tests. J. Biomech. (2014), submitted. 


\title{
A porous media approach for plantar tissue during gait
}

\author{
Daniela Boso, Giuseppe Sciumè, Bernhard Schrefler \\ Department of Civil, Environmental and Architectural Engineering, University Padua \\ Department of Innovation Engineering, University of Salento, Lecce \\ International Centre for Mechanical Sciences, Udine
}

\begin{abstract}
Recently a new computational model, based on the thermodynamically constrained averaging theory [1], has been proposed to predict tumor initiation and proliferation [2-4] and afterwards to study plantar tissue mechanics [5]. The foot tissue is modeled as an elastic porous medium, in large strain regime and completely filled by a fluid phase. In detail, the tissue cells and their extracellular matrix form the solid skeleton with pores saturated by the interstitial fluid. The primary variables of the model are: the interstitial fluid pressure $p^{f}$, the displacement vector of the solid phase $\mathbf{u}^{s}$, and the mass fraction of oxygen dissolved in the interstitial fluid, $\omega^{\overline{o f}}$. With respect to these primary variables, the governing equations are discretized in space by the finite element method, in time by using the $\vartheta$-Wilson method and then solved numerically. By considering the interstitial fluid, it is possible to mimic the viscoelastic behavior of the plantar tissue observed experimentally by Gefen [6]. This is shown in the simulated cases, where a foot during stance and some gait cycles are modeled. The presented examples integrate experimental data at different scales (patient specific foot geometry, tissue elasticity and permeability, possible tissue vasculopathy, global forces measured during gait, etc.) and allow validating the developed modeling procedure by comparisons between numerical and measured plantar pressures. Being the global response of the bi-phase system viscoelastic, it is shown that the duration of stance as well as of each of gait cycle has an influence on tissue strain and stress fields.
\end{abstract}

\section{References}

[1] W.G. Gray, C.T. Miller. Thermodynamically constrained averaging theory approach for modelling flow and transport phenomena in porous medium systems: 1. Motivation and overview. Advances in Water Resources, 28 (2005) 161-180

[2] G. Sciumè, S. Shelton, W.G. Gray, C.T. Miller, F. Hussain, M. Ferrari, P. Decuzzi, B.A. Schrefler. A multiphase model for three-dimensional tumor growth. New Journal of Physics, 15 (2013) 015005

[3] G. Sciumè, S. Shelton, W.G. Gray, C.T. Miller, F. Hussain, M. Ferrari, P. Decuzzi, B.A. Schrefler. Tumor growth modelling from the perspective of porous media mechanics. Cellular and Molecular Biomechanics, $9(3)$ (2012) 193-212

[4] G. Sciumè, W.G. Gray, F. Hussain, M. Ferrari, P. Decuzzi and, B.A. Schrefler. Three phase flow dynamics in tumor growth. Computational Mechanics, 53(3) (2014) 465-484.

[5] G. Sciumè, D.P. Boso, W.G. Gray and B.A. Schrefler. A two-phase model of plantar tissue: a step toward prediction of diabetic foot ulceration, Int. J. Numer. Meth. Biomed. Engng. 30 (2014) 1153-1169.

[6] A. Gefen, M. Megido-Ravid, Y. Itzchak. In vivo biomechanical behavior of the human heel pad during the stance phase of gait. Journal of Biomechanics. 34 (2001) 1661-1665 


\title{
A multi-scale time-dependent constitutive model of soft collagenous tissue
}

\author{
Markus Hillgärtner, Kevin Linka, Mikhail Itskov \\ Department of Continuum Mechanics, RWTH Aachen, Kackertstr. 9, Aachen, Germany
}

This contribution presents a micro-mechanically motivated, time-dependent constitutive model of soft biological tissues. It considers seperate contributions of the matrix material, collagen fibrils, proteoglycans (PGs) as well as their interactions. PG bridges facilitate sliding between fibrils (Rigozzi et al. [1]), described in the proposed model by the Coulomb law in zones where the fibrils are not separated by PGs.

The initial overlapping lengths of the PG bridges are statistically distributed and decrease due to slippage.. A linear-elastic force response of a PG bridge is assumed, where the stiffness depends on the overlapping length. Damage in PG bridges is reversible and decays over time (cf. Gupta et al. [2]). This behaviour is taken into account by a healing model based on the evolution of the overlapping length.

The damage in the PG bridges decreases the PG density and in turn increases the contact area of the fibril contact, leading to fibril stretch. The strain energy function of fibrils is based on the response of a single tropocollagen and takes both, an entropic and an energetic regime into account. At higher strains, fibrils can additionally undergo damage, which in contrast to the PG damage is irreversible.

The so obtained constitutive model is capable to predict several mechanical phenomena of soft tissues, such as non-linearity, Mullins effect, hysteresis and permanent set. Finally the model is compared against experimental data available in the literature.

\section{References}

[1] S. Rigozzi, R. Müller, A. Stemmer, J.G. Snedeker. Tendon glycosaminoglycan proteoglycan sidechains promote collagen fibril sliding-AFM observations at the nanoscale. Journal of biomechanics 46 (2013), 813-818.

[2] H.S. Gupta, J. Seto, S. Krauss, P. Boesecke, H.R.C. Screen. In situ multi-level analysis of viscoelastic deformation mechanisms in tendon collagen. Journal of Structural Biology 169 (2010), 183-191. 


\title{
Extremal loading of soft fibrous tissues: multi-scale mechanics and constitutive modeling
}

\author{
Kevin Linka, Vu Ngoc Khiêm, and Mikhail Itskov \\ Department of Continuum Mechanics, RWTH Aachen University \\ Kackertstr. 9, 52072 Aachen, Germany \\ linka@km.rwth-aachen.de
}

In the current contribution, we present a multi-scale constitutive model capturing macroscopic inelastic effects (like stress softening and permanent set) in soft tissues under cyclic loading. To this end, the mechanics of single soft tissue components and their mutual interactions are described and taken into account.

Soft biological tissues can be described as a biological composite material. The extracellular matrix is hereby reinforced by collagen fibers which themself are an assembly of collagen fibrils embedded in a proteoglycan (PG) rich matrix. Micro-damage induced by cyclic loading is treated by an interaction scenario between the fibrils and the PGs. At the low strain regime PGs promote sliding between fibrils $[1,2]$ until yielding of statistically distributed overlapping segments starts. The breakage of the PG-bridges is defined by a decreasing PG-density. The damage accumulated in PG-connections increases strains in the collagen fibrils. This finally drives the overstretching of the fibrils, associated with a permanent rupture of the hydrogen bonds inside of the tropocollagen molecules [3]. The so obtained model is in line with recent experimental findings available in literature [3, 2].

\section{References}

[1] S. Rigozzi, R. Müller and J.G. Snedeker, Local strain measurement reveals a varied regional dependence of tensile tendon mechanics on glycosaminoglycan content. Journal of Biomechanics. 42 (2009), 1547-1552.

[2] S. Rigozzi, R. Müller, A. Stemmer and J.G. Snedeker, Tendon glycosaminoglycan proteoglycan sidechains promote collagen fibril sliding-AFM observations at the nanoscale. Journal of Biomechanics. 46 (2013), 813-818.

[3] S.P. Veres, and J.M. Lee, Designed to Fail : A Novel Mode of Collagen Fibril Disruption and Its Relevance to Tissue Toughness. Biophysical Journal. 102 (2012), 2876-2884. 


\title{
A Numerical Study of Fluid Flow in Articular Cartilage Based on the Darcy-Forchheimer Law
}

\author{
Melania Carfagna, Alfio Grillo \\ DISMA "G. L. Lagrange", Politecnico di Torino. \\ E-mail: \{melania.carfagna alfio.grillo\}@polito.it
}

The study of the flow of a fluid in a binary system, comprising the fluid itself and a deformable porous medium, is usually performed by relating, through the permeability tensor $k$ of the porous matrix, the average (or macroscopic) velocity of the fluid with the pressure drop inside the system.

In most of the cases, Darcy's law, which states a linear relationship between the pressure gradient $\nabla p$ and the fluid filtration velocity $\boldsymbol{q}$, is employed to model such physical systems. However, in some cases, the Darcy-Forchheimer law provides a more accurate description for the microscopic interactions between the solid and the fluid. This is particularly true for those cases in which a strongly heterogeneous solid structure, high flow rates, or critical pressures for low permeabilities have to be considered. For instance, such an improved model of the relation between pressure gradient and fluid filtration velocity should be adopted when dealing with multifaceted materials, which form the porous matrix, or fluids that have to be described by means of non-standard rheological laws [1]. This is the case of human cartilage.

Human cartilage can be viewed as a mixture, which comprises a porous matrix with a depth dependent permeability, collagen fibres, which strongly contribute to the fluid behaviour, and the synovial fluid, which has non-Newtonian properties [2].

In this work, we present some numerical results regarding both confined and unconfined compression tests on a sample of human cartilage. In the numerical solution of the coupled and non-linear equations governing the system, the permeability tensor has been expressed as a function of the deformation of the solid matrix, and the distribution of collagen fibres as done in [3, 4]. Moreover, also the role played by the fibres in the elastic properties of the solid has been taken into account. For what concerns the Darcy-Forchheimer law, the following relation is assumed to hold in the spatial framework $[5,6]$

$$
-k \nabla p=\left(1+\rho \beta k_{\mathrm{eq}}\|\boldsymbol{q}\|\right) \boldsymbol{q},
$$

with $\rho, \beta$ and $k_{\text {eq }}$ being the fluid density, the factor accounting for the inertial effects related to the fluid motion at the pore scale, and an equivalent permeability, respectively. Many authors have supplied semi-empirical correlations for $\beta$ as a function of porosity, permeability and, in some cases, of the tortuosity of the porous matrix [7]. Some of these correlations have been considered in our simulations and adapted to the anisotropic case in order to show the main variations induced by the Forchheimer correction to the pure Darcian case.

\section{References}

[1] T. Tosco, D. L. Marchisio, F. Lince, R. Sethi. Extension of the Darcy-Forchheimer Law for Shear-Thinning Fluids and Validation via Pore-Scale Flow Simulations. Transp. Porous Med. 96:1-20 (2013).

[2] V. Tirtaatmadja, D. V. Boger, J. R. E. Fraser. The dynamic and steady shear properties of synovial fluid and of the components making up synovial fluid. Rheol Acta 23: 311 - 321 (1984).

[3] A. Tomic, A. Grillo, S. Federico. Numerical Implementation of a Permeability Model for Fibre-Reinforced Materials with an Application to Articular Cartilage. IMA Journal of Applied Mathematics 1-30 (2005).

[4] G. A. Ateshian, J.A. Weiss. Anisotropic Hydraulic Permeability Under Finite Deformation. Journal of Biomechanical Engineering 132 (2010).

[5] S. M. Hassanizadeh, A. Leijnse. A non-linear theory of the high-concentration-gradient dispersion in porous media. Advances in Water Resources 18(4):203-215 (1995).

[6] A. Grillo, D. Logashenko, S. Stichel, G. Wittum. Forchheimer's correction in modelling flow and transport in fractured porous media. Comput. Visual Sci. 15:169-190 (2012).

[7] F. Thauvin, K. K. Mohanty. Network Modeling of Non-Darcy Flow Through Porous Media. Transport in Porous Media 31: 19-37 (1998). 


\title{
Forchheimer's Correction in Modelling Flow in Poroelastic Materials with Statistical Fibre-Reinforcement
}

\author{
$\underline{\text { Alfio Grillo }}^{1}$, Melania Carfagna ${ }^{1}$, Salvatore Federico ${ }^{2}$ \\ ${ }^{1}$ DISMA "G. L. Lagrange", Politecnico di Torino. \\ E-mail: \{alfio.grillo melania.carfagna\}@polito.it \\ ${ }^{2}$ Dept of Mechanical and Manufacturing Engineering, The University of Calgary. \\ E-mail: salvatore.federico@ucalgary.ca
}

Soft biological tissues are often macroscopically described as biphasic media, comprising a fluid and a solid phase. In the case of articular cartilage, the fluid phase is in osmotic equilibrium with the synovial fluid, and hosts ions, hyaluronic acid, nutrients for the tissue's cells, and byproducts of the cellular metabolism, while the solid phase is identified with a porous medium whose main constituents are cells, collagen fibres and extracellular matrix. Due to the arrangement of the collagen fibres, the solid phase is modelled in many circumstances as a fibre-reinforced, poroelastic material with statistical distribution of reinforcing fibres [1]. The saturation condition is usually assumed to hold, which means that the volumetric fractions of the solid and fluid phase, denoted by $\phi_{\mathrm{f}}$ and $\phi_{\mathrm{s}}$, respectively, are required to respect the constraint $\phi_{\mathrm{f}}+\phi_{\mathrm{s}}=1$ at all times and at all points of the tissue.

A widely accepted hypothesis in the biomechanics of articular cartilage is that the motion of the fluid phase follows Darcy's Law [2,3]. Accordingly, the specific discharge $\boldsymbol{q}=\phi_{\mathrm{f}} \boldsymbol{v}_{\mathrm{fs}}\left(\boldsymbol{v}_{\mathrm{fs}}\right.$ is the velocity of the fluid relative to that of the solid) is prescribed to be driven by the pressure gradient in the tissue, $\operatorname{grad}(p)$, through the expression $\boldsymbol{q}=-(\boldsymbol{k} / \mu) \operatorname{grad}(p) \equiv \boldsymbol{q}_{\mathrm{D}}[4]$. Here, $\boldsymbol{k}$, which is referred to as the permeability the of the porous medium, is a second-order tensor function depending on the deformation of the solid phase and, in the case of anisotropic materials, on the orientation and volumetric fraction of the fibres [1, 3], while $\mu$ is interpreted as the dynamic viscosity of the interstitial fluid.

When Darcy's Law is used, the following hypotheses are accepted: (i) negligibility of the inertial forces acting on the fluid phase at the macroscale (these inertial forces involve only the volume-average of the fluid mass density and the mass-average of the fluid velocity); (ii) no body forces act on the fluid phase other than the momentum exchange with the solid phase; (iii) the dissipative part of the momentum exchange rate between the fluid and the solid phase is linear in the specific discharge; (iv) the fluid phase is macroscopically inviscid (i.e., the only stress bearable by the fluid is hydrostatic and given by $-\phi_{\mathrm{f}} p \boldsymbol{g}^{-1}$, where $\boldsymbol{g}$ is the metric tensor). While the first two hypotheses are a priori statements on the dynamic regime of the fluid phase and on its interactions with the environment external to the tissue, the third and the fourth hypotheses are constitutive prescriptions that relegate the information about the pore scale behaviour of the fluid to $k$ and $\mu$. Although, on the one hand, these approximations reduce considerably the computational effort required to perform realistic simulations of articular cartilage, on the other hand, they do not require any knowledge about the pore scale dynamics and constitutive behaviour of the fluid. In particular, if $\mu$ is given from the outset as a model parameter, or as a function of the composition of the fluid phase, but is left uncorrelated with the fluid velocity, there is no way to model the non-Newtonian behaviour of the fluid phase, which can be inferred from the non-Newtonian behaviour of the synovial fluid that has been observed by some authors. Indeed, Barnett [5] (cf. also references therein) pointed out that the synovial fluid shows non-Newtonian behaviour, except when it is quite dilute, while Tirtaatmadja et al. [6] found out that the synovial fluid can be modelled by means of a rheological model of Carreau type.

In this contribution, the Forchheimer's correction is incorporated into the equations describing the mechanical response of articular cartilage to the unconfined compression test. Following [7, 8], when the Forchheimer's correction is introduced, the specific discharge can be written as

$$
\boldsymbol{q}=f\left(A,\left\|\boldsymbol{q}_{\mathrm{D}}\right\|\right) \boldsymbol{q}_{\mathrm{D}}, \quad f\left(A,\left\|\boldsymbol{q}_{\mathrm{D}}\right\|\right)=\frac{2}{1+\sqrt{1+4 A\left\|\boldsymbol{q}_{\mathrm{D}}\right\|}},
$$

where $\boldsymbol{q}_{\mathrm{D}}$ is the specific discharge predicted by Darcy's Law, and $A$ is referred to as Forchheimer's coefficient. The motivation for using the Forchheimer's correction is threefold: (a) there can be situations in which the fluid phase cannot be regarded as dilute; (b) the inertial forces acting on the fluid at the fibre scale may not be a priori negligible (for example, due to the combined effect of high pressure gradients and the anisotropy of the permeability, which is typically higher along the direction of local fibre alignment $[9,10,11]$ ), and (c) the 
Forchheimer's correction permits to introduce an effective viscosity, $\mu_{\mathrm{eff}}=\mu(1+A\|\boldsymbol{q}\|)$, which, depending on the specific discharge, may be considered as a macroscopic representation of the non-Newtonian behaviour of the fluid.

The purpose of this contribution is to discuss the main differences between the standard model of fluid flow in articular cartilage, which is based on Darcy's Law, and the theoretical framework employing the Forchheimer's correction. Some of these differences shall be visualised with the aid of numerical results. A detailed explanation of the numerical simulations performed to achieve these tasks has been presented in [12].

\section{References}

[1] A. Tomic, A. Grillo, S. Federico. Poroelastic materials reinforced by statistically oriented fibres - numerical implementation and application to articular cartilage. IMA Journal of Applied Mathematics 1-33 (2014) doi:10.1093/imamat/hxu039.

[2] M. H. Holmes, V. C. Mow. The nonlinear characteristics of soft gels and hydrated connective tissues in ultrafiltration. Journal of Biomechanics 23 (1990).

[3] G. A. Ateshian, J. A. Weiss. Anisotropic hydraulic permeability under finite deformation. Journal of Biomechanical Engineering 132 (2010).

[4] J. Bear, Y. Bachmat. Introduction to Modeling of Transport Phenomena in Porous Media. Kluwer, Dordrecht, Boston, London, 1990.

[5] C. H. Barnett. Measurement and interpretation of synovial fluid viscosities. Ann. Rheum. Dis. 17, 229-233 (1958).

[6] V. Tirtaatmadja, D. V. Boger, J. R. E. Fraser. The dynamic and steady shear properties of synovial fluid and of the components making up synovial fluid. Rheol Acta 23: 311 - 321 (1984).

[7] S. M. Hassanizadeh, A. Leijnse. A non-linear theory of the high-concentration-gradient dispersion in porous media. Advances in Water Resources 18(4):203-215 (1995).

[8] A. Grillo, D. Logashenko, S. Stichel, G. Wittum. Forchheimer's correction in modelling flow and transport in fractured porous media. Comput. Visual. Sci. 15:169-190 (2012).

[9] S. Federico, W. Herzog. On the anisotropy and inhomogeneity of permeability in articular cartilage. Biomech. Model. Mechanobiol. 7:367-378 (2008).

[10] S. Federico, W. Herzog. On the permeability of fibre-reinforced porous materials. Int. J. Solids Struct. 45:2160-2172 (2008).

[11] S. Federico, A. Grillo. Elasticity and permeability of porous fibre-reinforced materials under large deformations. Mech. Mater. 44:58-71 (2012).

[12] M. Carfagna, A. Grillo. A numerical study of fluid flow in articular cartilage based on the Darcy-Forchheimer Law. Contribution submitted to GAMM 2015. 


\title{
Relaxed incremental Variational Approach for Damage in Arteries
}

\author{
Daniel Balzani ${ }^{1}$, Thomas Schmidt ${ }^{2}$ \\ ${ }^{1}$ Dresden University of Technology, Institute of Mechanics and Shell Structures, \\ 01062 Dresden, Germany \\ ${ }^{2}$ University of Duisburg-Essen, Institute of Mechanics, 45141 Essen, Germany
}

\begin{abstract}
A variety of damage models is proposed in the literature for arterial walls. This is because microscopic damage, mainly occurring in embedded collagen fibers, is believed to be the reason for macroscopic tissue softening as observed during balloon angioplasty. Thus, the modeling of this effect in predictive finite element simulations may help to better understand the tissue mechanics during the aforementioned clinical intervention and to improve the methods of treatment. Most of the available damage models so far are formulated in a (standard) continuum damage mechanics (CDM) framework, which in principle enables numerical damage calculations in arterial geometries by means of the finite element method. However, the drawback of CDM approaches is, that at certain deformations a loss of convexity of the underlying formulation occurs and meshdependent solutions may be obtained. A way to avoid this problem is the consideration of relaxed incremental incremental variational formulations for damage, see e.g. [2, 4] for small strains. In [1] an extension to large strains was derived for fiber-reinforced materials. Within this contribution we provide further extensions to the latter approach in order to appropriately account for the description of arterial tissues. In particular, hysteresis behavior is included by the construction of hyperelastic unloading and reloading paths and fiber dispersion is reflected by consideration of a microsphere-like model in the sense of [3]. Thereby, different amounts of dispersion are accounted for in tangential and in radial direction of the arterial wall. Moreover, a surrogate model is proposed, which enables the efficient adjustment of the above relaxed formulation to real experimental data of arterial tissue. The applicability of the newly introduced formulation to numerical simulations of overstretched arteries in a finite element framework is demonstrated.
\end{abstract}

\section{References}

[1] D. Balzani, M. Ortiz. Relaxed incremental variational formulation for damage at large strains with application to fiber-reinforced materials and materials with truss-like microstructures. Int. J. Numer. Methods Eng. 92 (2012), 551-570.

[2] G.A. Francfort, A. Garroni. A variational view of partial brittle damage evolution. Arch. Ration. Mech. Anal. 182 (2006), 125-152.

[3] S. Göktepe, C. Miehe. A micro-macro approach to rubber-like materials. Part III: the micro-sphere model of anisotropic Mullins-type damage. J. Mech. Phys. Solids 53 (2005), 2259-2283.

[4] E. Gürses, C. Miehe. On evolving deformation microstructures in non-convex partially damaged solids. J. Mech. Phys. Solids 59 (2011), 1268-1290. 


\title{
Computation of residual stress distributions and opening angles of 3D patient-specific arterial walls
}

\author{
Jörg Schröder, Markus von Hoegen \\ Institute of Mechanics, Faculty of Engineering Dept. Civil Engineering, \\ University of Duisburg-Essen, Germany
}

The stress distribution of a cylindrical segment over the radius is believed to have a sustainable influence on the arterial health. In order to improve simulations of all kind of mechano-biological processes, residual stresses should be taken into account. Classical computations, for example by [1], concerning the analytical opening angle method are based on the assumption of plane strain conditions. Theses models, based on only one parameter, are not able to incorporate axial residual stresses sufficiently.

Residual stresses are commonly accepted to be compressive on the innermost and tensile on the outermost layer. As a result the in vivo stresses are smoothed due to residual stresses and become more equally distributed, i.e. the radial stress gradient decreases. Based on the latter assumption, this contribution presents a model which is capable to predict the amount of residual stresses for 3D patient-specific arteries. The arterial model is generated based on VH IVUS images (Virtual Histology Intrvascular Ultrasound), for details see [2]. Because of the strong anisotropy displayed by soft tissues, suitable stress invariants have to be derived for this purpose. Building on those achievements the smoothing procedure is applied on the individual material layers independently. The algorithmic incrementation is explained in detail and numerical results of diseased arteries suffering from atherosclerosis under physiological conditions will be presented. The calculated residual stresses are applied on unloaded three-dimensional arterial segments and the so called opening angle is approximated by numerical simulations. This is motivated by several experimental studies, see e.g. [3] or [4]. The proposed numerical tool provides the possibility of adjustment to experimental data.

\section{References}

[1] C.J. Chuong, Y.C. Fung. On Residual Stress in Arteries. J. Biomech. Eng. 108 (1986), 189-191.

[2] D. Balzani, D. Böse, D. Brands, R. Erbel, A. Klawonn, O. Rheinbach and J. Schröder. Parallel simulation of patient-specific atherosclerotic arteries for the enhancement of intravascular ultrasound diagnostics. Eng. Computation. 29 (2012), 888-906.

[3] S.Q. Liu, Y.C. Fung. Relationship between Hypertension, Hypertrophy, and Opening Angle of Zero-Stress State of Arteries Following Aortic Constriction. J. Biomech. Eng. 111 (1989), 325-335.

[4] G.A. Holzapfel, G. Sommer, M. Auer, P. Regitnig, and R.W. Ogden. Layer-Specific 3D Residual Deformations of Human Aortas with Non-Atherosclerotic Intimal Thickening. Ann. Biomed. Eng. 35 (2007), 530-545. 


\title{
Phase-field modeling of fracture in biological tissues
}

\author{
Arun Raina, Christian Miehe \\ Institute of Applied Mechanics (CE) Chair I, \\ University of Stuttgart, 70550 Stuttgart, Pfaffenwaldring 7, Germany
}

\begin{abstract}
This work presents the modeling of fracture phenomena in anisotropic soft biological tissues at finite deformations with the phase-field method. These tissues consist of an extra-cellular matrix which is composed of networks of elastin and collagen fibers surrounded by smooth muscle cells and fluids. Such a microstructure endows an incompressible, anisotropic and highly nonlinear macroscopic bulk response to these materials. To model fracture in these complex soft materials, recently developed phase-field methods $[1,2]$ are equipped with an anisotropic hyperelastic bulk energy and a Hill-type anisotropic failure criterion. The phase-field models present an innovative approach to thermodynamically consistent modeling of fracture, which is applicable to both rate-dependent or rate-independent, brittle and ductile failure modes. A regularized crack surface functional is introduced that $\Gamma$-converges to sharp crack topology for vanishing length scale parameter $\ell$. A crack driving stress-based state function, derived from Hill-type failure criterion, governs the anisotropic crack phasefield evolution in a modular format. This allows flexibility to introduce alternative failure criteria, e.g. energetic or strain-based, whose maximum value from the deformation history drives the irreversible crack phase field. The resulting multi-field problem is solved numerically by a robust operator split scheme that successively updates the history field, the crack phase field and finally the displacement field in a typical incremental time step. The representative numerical simulations of fracture in human iliac arteries [3] are performed and a quantitative comparison with experimental data is provided for verification of the proposed methodology.
\end{abstract}

\section{References}

[1] C. Miehe, F. Welschinger, M. Hofacker. Thermodynamically consistent phase-field models of fracture: Variational principles and multi-field fe implementations. Int. J Numer. Methods Eng. 83 (2010), 1273-1311.

[2] C. Miehe, L. Schänzel, H. Ulmer. Phase field modeling of fracture in multi-physics problems. Part I. Balance of crack surface and failure criteria for brittle crack propagation in thermo-elastic solids. (2014) Submitted to Comput. Methods Appl. Mech. Engrg.

[3] G. Holzapfel, G. Sommer, P. Regitnig. Anisotropic mechanical properties of tissue components in human atherosclerotic plaques. J. Biomech. Eng. 126 (2004), 657-665. 


\title{
Fluid structure interaction in hemodynamics
}

\author{
Daniel Balzani ${ }^{1}$, Simone Deparis ${ }^{2}$, Simon Fausten ${ }^{3}$, Davide Forti ${ }^{2}$, Alexander Heinlein ${ }^{4}$, \\ Axel Klawonn ${ }^{4}$, Alfio Quarteroni ${ }^{2}$, Oliver Rheinbach ${ }^{5}$, Jörg Schröder ${ }^{3}$ \\ ${ }^{1}$ Institute of Mechanics and Shell Structures, Technical University \\ Dresden, 01062 Dresden, Germany \\ ${ }^{2}$ Chair of Modeling and Scientific Computing, MATHICSE - EPFL, Station 8, \\ 1015 Lausanne, Switzerland \\ ${ }^{3}$ Institute of Mechanics, University of Duisburg-Essen, 45141 Essen, Germany \\ ${ }^{4}$ Mathematical Institute, University of Cologne, Weyertal 86-90, 50931 Köln, Germany \\ ${ }^{5}$ Institute of Numerical Mathematics and Optimization, TU Bergakademie Freiberg, \\ Akademiestraße 6, 9599 Freiberg, Germany
}

\begin{abstract}
We consider the fluid-structure-interaction problem in a blood vessel using a monolithic coupling approach, first using a Convective Explicit approach for the fluid. We believe that the prediction of transmural stresses requires the use of sophisticated nonlinear material models for the vessel wall. Fortunately, such models have been developed in the past and their parameters have been adapted to experimental data. Here, we use an anisotropic, polyconvex hyperelastic material model for the structure. The coupled simulations build on the LifeV software library and FEAP. Absorbing boundary conditions on the outflow are imposed to reduce reflections.
\end{abstract}




\title{
Linking structural dynamics to the nonlinear viscoelasticity and fatigue mechanics in fibrin biopolymers
}

\author{
Nicholas A. Kurniawan, Gijsje H. Koenderink \\ FOM Institute AMOLF, Amsterdam, The Netherlands
}

Fibrin is a fibrous biopolymer network that forms when our blood clots. Recent works have shown that fibrin can be stretched up to 5 times its original length and can stiffen more than 100 fold in the process. These extraordinary material properties are important in preserving clot mechanical integrity against shear-induced damage due to blood flow. Unraveling the physical mechanisms behind these phenomena not only can help us better understand how our body maintains haemostasis, but also can provide useful design principles for (bio)materials. In this study, we show that the viscoelastic properties of fibrin results in network weakening during cyclic shear loading. However, fibrin is observed to retain mechanical memory of the deformations that it has previously been subjected to, reminiscent of Mullins effect that is prevalent in elastomers and shape-memory alloys. Moreover, at large deformations, fibrin exhibits unexpected gradual yet reversible rupture weakening, suggesting a progressive crack propagation. We systematically examine the microstructural origins of these behaviors, and the results indicate a structural rearrangement that underlies the viscoelastic stiffening and viscoplastic response of fibrin network. Macroscopically, this rearrangement results in a material with large extensibility that remembers its deformation history, but forgets its shape history. 


\title{
Mechanics of growing tumors: impact of modeling assumptions and boundary conditions on reliability of numerical results
}

\author{
Giuseppe Sciumè $^{1}$, Bernhard Schrefler ${ }^{2}$, Raffaella Santagiuliana ${ }^{3}$, Giorgio Zavarise $^{1}$ \\ ${ }^{1}$ Department of Innovation Engineering, University of Salento, Lecce \\ ${ }^{2}$ International Centre for Mechanical Sciences, Udine \\ ${ }^{3}$ Department of Civil, Environmental and Architectural Engineering, University of Padua
}

A new computational model based on porous media mechanics has been recently developed for prediction of tumor growth [1]. The tumor mass is modeled as a four-phase system consisting of a solid phase, the extracellular matrix (ECM), and three immiscible fluid phases: the interstitial fluid (IF); the tumor cells (TC) and the healthy cells (HC) (TC and $\mathrm{HC}$ are modeled as adhesive fluids). Being the tumor growth strongly influenced by nutrients availability, the diffusion of oxygen coming from the nearby existing vessels is also considered. The mathematical model - governed by mass balance equations of phases and species and by the linear momentum balance equation of the solid scaffold (ECM) - has been discretized in space by finite elements and in the time domain by finite differences, and implemented in Cast3M (FE code of the French Atomic Agency).

When in 2011 we started working on this model we introduced two simplifying assumptions: i) a unique pressure was considered for both cell populations $\left(p^{T C}=p^{H C}\right)$ and ii) the ECM was assumed rigid. Then, the introduction of relevant constitutive relationships for the pressure difference among each pair of fluid phases (these are based on relative wettability of fluids and fluid-fluid interfacial tensions, see [2]) has allowed for relaxation of the first hypothesis and for a more realistic modeling of cell adhesion and invasion [3]. More recently also the second hypothesis has been relaxed and ECM deformability and its impact on tumor growth can be properly taken into account [4].

Our final aim is to develop a numerical tool which can be a complement for in vivo and in vitro experimental tests and help scientists in better understanding physical interaction between tumor and its surrounding. Hence, we will present our recent efforts to extensively validate the model, the impact of modeling assumptions and boundary conditions on reliability of numerical results, and some perspectives of enhancement of the model.

\section{References}

[1] G. Sciumè, S.E. Shelton , W.G. Gray, C.T. Miller, F. Hussain, M. Ferrari, P. Decuzzi, B.A. Schrefler. A multiphase model for three dimensional tumor growth. New Journal of Physics, 15 (2013) 015005.

[2] G. Sciumè, M. Ferrari, B.A. Schrefler. Saturation-pressure relationships for two-and three-phase flow analogies for soft matter. Mechanics Research Communications 62 (2014) 132-137

[3] G. Sciumè, W.G. Gray, F. Hussain, M. Ferrari, P. Decuzzi and, B.A. Schrefler. Three phase flow dynamics in tumor growth. Computational Mechanics, 53(3) (2014) 465-484.

[4] G. Sciumè, R. Santagiuliana, M. Ferrari, P. Decuzzi, B.A. Schrefler. A tumor growth model with deformable ECM. Physical Biology 11(6) (2014) 065004 


\title{
Tumor growth in agarose and collagen-agarose co-gels
}

\author{
$\underline{\text { K.L. Mills }}^{1}$, R. Kemkemer ${ }^{2}$, S. Rudraraju ${ }^{3}$, K. Garikipati ${ }^{3}$ \\ ${ }^{1}$ Rensselaer Polytechnic Institute, Troy, USA \\ ${ }^{2}$ Reutlingen University, Reutlingen, Germany \\ ${ }^{3}$ University of Michigan, Ann Arbor, USA
}

The biomechanical environment of a solid tumor is in a transformed state in which tumor-associated cells stiffen the environment through increased deposition and rearrangements of matrix fibers. Additionally, the uncontrolled proliferation of tumor cells means that excessive stresses are built up due to tumor expansion [1]. The effects of such an altered mechanical environment are not completely understood, but it is becoming obvious that it plays a role in the progression of the disease. In order to extrapolate the significance of the mechanical interactions between a growing tumor and its environment, we use an in vitro tumor growth model whose mechanical and adhesive properties can be tuned.

The in vitro tumor growth model is based on hydrogels that vary in agarose and collagen I concentrations. Agarose provides a bioinert matrix whose stiffness can be varied over the range of healthy and diseased tissues (100s of $\mathrm{Pa}$ to $10 \mathrm{~s}$ of $\mathrm{kPa}$ ). Cell adhesion sites are introduced with the addition of collagen, whose stiffness is much lower than the most compliant agarose. Without collagen, tumors inside agarose gels grow to ellipsoidal shapes with very well defined boundaries [2]. This is remarkable because tumors with defined boundaries in vivo are described as ellipsoidal in shape [3]. Using the theory of elasticity we show how certain ellipsoidal shapes minimize the relevant free energy. Furthermore, tumor size distribution is dependent on many experimental factors including agarose mechanical properties, tumor seeding density, and time of growth. In the presence of collagen, however, invasive outgrowths and cell migration away from the tumor were observed.

\section{References}

[1] A.C. Shieh. Biomechanical forces shape the tumor microenvironment. Ann. Biomed. Eng. 39 (2011), 1379-1389.

[2] K.L. Mills, R. Kemkemer, S. Rudraraju, K. Garikipati. Elastic free energy drives the shape of prevascular solid tumors. PLoS ONE 9 (2014), e103245.

[3] U. Güth, D. Brenckle, D.J. Huang, A. Schözau, C.T. Viehl, H. Dieterich, W. Holzgreve, E. Wight, and G. Singer. Three-dimensional pathological size assessment in primary breast carcinoma. Breast Cancer Res. Tr. 116 (2009), 257-262. 


\title{
The role of the microvascular tortuosity in tumor transport phenomena
}

\author{
Raimondo Penta, Davide Ambrosi \\ AG Numerik, FB Mathematik, TU Darmstadt, Germany \\ MOX, Dipartimento di Matematica, Politecnico di Milano, Italy
}

The role of the microvascular network geometry on transport phenomena in solid tumors and its interplay with the leakage and pressure drop across the vessels is qualitatively and quantitatively discussed. Our starting point are the asymptotic homogenization models developed in [1], [2], which are derived exploiting the sharp length scale separation that exists between the characteristic vessels and tumor tissue spatial scales, referred to as the microscale and the macroscale, respectively. The coupling between interstitial and capillary compartment is described by a double Darcy model on the macroscale, whereas the geometric information on the microvascular structure is encoded in the effective hydraulic conductivities, which are numerically computed solving classical differential problems on the microscale representative cell. Then, microscale information is injected into the macroscopic model, which is analytically solved in a prototypical geometry and compared with previous experimentally validated, phenomenological models, [3], [4]. In this way, we are able to capture the role of the standard blood flow determinants in the tumor, such as the tumor radius, tissue hydraulic conductivity and vessels permeability, as well as the influence of the vascular tortuosity on fluid convection. The results quantitatively confirm that transport of blood (and, as a consequence, of any advected anti-cancer drug) can be dramatically impaired by increasing the geometrical complexity of the microvasculature. Hence, our quantitative analysis supports the argument that geometric regularization of the capillary network improve blood transport and drug delivery in the tumor mass.

\section{References}

[1] R. J. Shipley, J. Chapman. Multiscale modelling of fluid and drug transport in vascular tumors. Bulletin of Mathematical Biology, 72 (2010), 1464-1491.

[2] R. Penta, D. Ambrosi, A. Quarteroni. Multiscale homogenization for fluid and drug transport in vascularized malignant tissues. Mathematical Models and Methods in Applied Sciences (M3AS) 25 (2015), 79-108.

[3] R. K. Jain, L. T. Baxter. Mechanisms of heterogeneous distribution of monoclonal antibodies and other macromolecules in tumors: significance of elevated interstitial pressure. Cancer Research 48 (1988), $7022-7032$.

[4] Y. Boucher, L. T. Baxter, R. K. Jain. Interstitial pressure gradiennts in tissue-isolated and subcutaneous tumors: implications for therapy. Cancer Research 50 (1990), 4478-4484. 


\title{
Towards the continuum-mechanical modelling of metastatic tumour growth in the brain
}

\author{
Patrick Schröder, Arndt Wagner, Wolfgang Ehlers \\ Institute of Applied Mechanics (CE), Chair of Continuum Mechanics \\ University of Stuttgart, Germany
}

The spreading of malignant tumour cells to distant organs is a severe problem in cancer treatment. Extra hazardous are metastases in the brain. Unfortunately, lung cancer, one of the most common cancer types, often spreads to the brain and requires a therapy. The growth of the metastases increase the stress in the brain, create leaky blood vessels and disrupt regular brain cells. Malfunctions, respectively death, are the consequences. A promising treatment of the metastases in the brain can be applied via the direct infusion of a therapeutic solution into the extra-vascular space.

The liquid-saturated brain tissue is considered as a porous material. Therefore, the framework of the Theory of Porous Media (TPM) provides an excellent tool for its description. In particular, the TPM relates a homogenised macroscopic model to the processes occuring on the microscale. For this purpose, the microscopic structure of a representative elementary volume is volumetrically homogenised, resulting in superimposed and interacting continua for each constituent. The introduced model is composed of a solid skeleton and two immiscible pore liquids. The solid part is given by the brain cells and the metastases. The pore liquids are the interstitial fluid and the blood. These pore liquids consist of real mixtures of components including liquid solvents and several solutes. The specific components of the interstitial fluid are the solvent, nutrients, metastatic tumour cells and a therapeutic agent. The blood includes a solvent and metastatic tumour cells.

The mechanisms concerned in the model are migration of cells and substances, nutrient-induced growth, blood vessel growth (angiogenesis), cell death owing to a lack of nutrients (necrosis) and cell death due to the therapeutic agent (apoptosis). The mass exchanges and the transport of the involved components are realised within the concept of the TPM. Moreover, the proposed model represents a closed system, which only permits interactions between the constituents.

Additional information of the microscopic structure and tumour-specific data are incorporated through parameters into the model. Measurements of the apparent water diffusion tensor obtained by Diffusion-weighted Magnetic Resonance Imaging (DTI) are taken as a basis for the permeability and diffusion tensors of the respective constituents. Thus, the anisotropic structure of the brain is considered.

In conclusion, the goal of this study is to develop a multicomponent metastases-growth model to improve tumour-growth predictions and treatment options. 


\title{
Theoretical and numerical aspects in the multiphasic modelling of human brain tissue
}

\author{
Arndt Wagner, Wolfgang Ehlers \\ Institute of Applied Mechanics (CE), Chair of Continuum Mechanics, University of Stuttgart
}

A surgical intervention is often required if the functionality of the sensitive human brain tissue is seriously compromised, e.g., due to the occurrence of malignant brain tumours. A promising method for an effective tumour-treatment procedure is provided by the so-called convection-enhanced drug delivery. Therein, a direct extra-vascular infusion of a therapeutic solution is applied via catheters within the brain-tissue aggregate. By this means, the aim of this contribution is to simulate the expected effects as well as coupled impacts of a scheduled surgical procedure with numerical computations based on a sophisticated multicomponent and multiphysical theoretical modelling strategy for human brain tissue.

The enormous microscopical complexity of the multicomponent brain-tissue aggregate motivates the application of the well-known Theory of Porous Media (TPM). Certainly, this represents a well-suited way to model the brain tissue in a compact and elegant manner. Using the TPM, a volumetrical homogenisation procedure (smearing) of the underlying microscopical structure over a representative elementary volume (REV) leads to an idealised macroscopical model of superimposed and mutually interacting constituents. In this particular case, the modelling approach contains immiscible and miscible constituents, which are treated in the framework of the so-called extended TPM. More precisely, a quaternary model is proposed, which is strongly related to the drug-delivery problem within the brain's tissue. Therein, the model proceeds from an anisotropically deformable solid skeleton, provided by tissue cells and vascular walls. This solid skeleton is perfused by two mobile liquids, the blood and the interstitial fluid. Moreover, the latter is furthermore treated as a real mixture of two components, the liquid solvent and the dissolved therapeutic solute. Moreover, the anisotropic permeability properties of the brain tissue are considered by a spatial diversification of the permeability tensor coefficients obtained from diffusion-weighted Magnetic Resonance Imaging (DTI).

The computational setting of the proposed model is based on four primary variables. These are the solid deformation, the effective pore pressures of the interstitial fluid and the blood as well as the molar concentration of the therapeutic agent (uppc formulation). For the numerical solution of the arising coupled partial differential equations, the system is discretised in space by the Finite-Element (FE) Method and in time by an implicit (Euler) time-integration scheme. Finally, the system is solved monolithically via an implementation within the in-house FE tool PANDAS. Numerical examples demonstrate the applicability of the presented model for selected problems. In particular, the anisotropic therapeutic spreading of the infused drug, using a single catheter or multiple infusion catheters, and accompanying coupled effects (such as local deformation or pressure states) are shown. 


\title{
On a Multi Scale and Multi Phase Model for the Description of Drug Uptake by the Human Liver
}

\author{
$\underline{\text { D. Werner }}^{1}$, T. Ricken ${ }^{1}$, H.G. Holzhütter ${ }^{2}$, M. König ${ }^{2}$, U. Dahmen ${ }^{3}$, O. Dirsch ${ }^{4}$ \\ ${ }^{1}$ Institute of Mechanics - Structural Analysis - Dynamics, TU Dortmund University, \\ ${ }^{2}$ Computational Systems Biochemistry Group, Charité Berlin, \\ ${ }^{3}$ Department of General, Visceral and Transplantation Surgery, University Hospital Jena, \\ ${ }^{4}$ Institute of Pathology, University Hospital Jena
}

Metabolism in the liver is a complex time depending and non-linear coupled function-perfusion-mechanism. The function of the liver, e.g. nutrient storage and uptake of drugs, is directly coupled to its blood perfusion. In this talk we present a computational model that describes both mechanisms in a coupled manner influenced by a change of perfusion due to lipid inclusions.

The metabolism happens in the liver cells, the hepatocytes, which are arranged in hexagonal functional segments, the liver lobules. Nutrients, oxygen, and other substances are transported to the hepatocytes via a delicate system of capillaries, so called sinusoids. The inhomogeneous distribution of the sinusoidal network leads to an anisotropic blood flow in the liver lobules. Due to this highly complex inner structure of the lobules it is impracticable to give an accurate geometrical description in a continuum mechanical manner. Therefore, a multiphase mixture theory based on the theory of porous media (TPM) is used; see [1].

In case of the presented liver model is considering a porous solid body $\varphi^{\mathrm{S}}$ and a fluid $\varphi^{\mathrm{L}}$. Both phases are considered as immiscible, heterogeneously composed materials. Each phase contains one carrier phase and $\nu-1$ miscible concentrations $\varphi^{\alpha \beta}$, whereas the solid carrier phase $\varphi^{\mathrm{S}}$ contains a set of internal concentrations $\varphi^{\mathrm{S} \beta}$, such as glycogen and triglyceride, and the fluid carrier phase $\varphi^{\mathrm{L}}$ a set of external concentrations $\varphi^{\mathrm{L} \beta}$, such as glucose and lactate. For the microscopic cell level use of an embedded set of ordinary differential equations (ODE) is used. The ODE model mimics a simplified metabolism that takes place in the hepatocytes converting nutrients into vital products, such as glucose. Knowledge of glucose production, utilization, and storage is a necessity for the description of fat accumulations within the liver; see [2].

\section{References}

[1] R. de Boer. Theory of Porous Media - highlights in the historical development and current state. SpringerVerlag (2000)

[2] T. Ricken, D. Werner, H.G. Holzhütter, U. Dahmen, O. Dirsch. Modeling function-perfusion behavior in liver lobules including tissue, blood, glucose, lactate and glycogen by use of a coupled two-scale PDE-ODE approach. Biomech. Model. Mechanobiol. 9 (2010), 435-450 


\title{
Mechanics of cell-division: A new continuum model for growth inhomogeneities
}

\author{
Antonio Bolea Albero, Markus Böl \\ TU Braunschweig, Institute of Solid Mechanics
}

The self-reproduction ability of mitotic cells results in an increase of the number of cells with the same characteristics in living bodies. While cells grow in volume and divide themselves, the living body consequently increases in volume and changes its mechanical properties depending on the internal distribution. When the factors which regulate the growth process are inhomogeneously distributed, growth takes place at different rates and directions.

If loads are applied to the living tissue, cell-division is reorientated following the main direction of the stresses [1]. The new cells will be created in the direction of the stresses while relaxing the elastic deformations in the body at the same time. However, the amount of cells which change their cell-division orientation will increase depending on several factors and the orientation in the body is usually not complete [2,3]. Further, the material relaxation can be seen as a viscoelastic flow of the new material contribution although the material behaviour is typical of a solid [3]. Considering extreme volume expansion of the body, cells become quiescent when volume restrictions appear.

In this work, we present a modelling approach for bodies which grow in volume and are able to change their growth direction. We use the multiplicative split of the total deformation gradient into growth and elastic contributions. The growth part is computed by considering the orientation of cell-division which depends on the stresses. The elastic part is responsible for the stresses and for the compatibility between the particles of the body. If the hydrostatic pressure increases critically due to volume restrictions, the growth process is expected to stop. The growth rate will change depending on this volume availability by decreasing its value in a smooth way [4]. Three-dimensional examples for different growth cases are shown and discussed in order to illustrate the behaviour of the new modelling approach.

\section{References}

[1] M. Uyttewaal, A. Burian, K. Alim, B. Landrein, D. Borowska-Wykret, A. Dedieu, A. Peaucelle, M. Ludynia, J. Traas, A. Boudaoud, D. Kwiatkowska, O. Hamant. Mechanical stress acts via katanin to amplify differences in growth rate between adjacent cells in Arabidopsis. Cell. 149 (2012), 439-451.

[2] T.M. Lynch and P.M. Lintilhac. Mechanical signals in plant development: A new method for single cell studies. Dev. Biol. 181 (1997), 246-256.

[3] M. Böl, A. Bolea Albero. On a new model for inhomogeneous volume growth of elastic bodies. J. Mech. Behav. Biomed. Mater. 29 (2014), 582-593.

[4] A. Bolea Albero, A.E. Ehret, M. Böl. A new approach to the simulation of microbial biofilms by a theory of fluid-like pressure-restricted finite growth. Comput. Methods in Appl. Mech. Eng. 272 (2014), $271-289$. 


\title{
A geometric approach to characterize rigidity in proteins
}

\author{
Dominik Budday*, Sigrid Leyendecker*, Henry van den Bedem ${ }^{\dagger}$ \\ ${ }^{*}$ Chair of Applied Dynamics, Friedrich-Alexander University Erlangen-Nuremberg \\ ${ }^{\dagger}$ Stanford Synchrotron Radiation Lightsource, Stanford University
}

Proteins operate and interact with partners by changing between conformational substates on a wide range of spatiotemporal scales. Structurally characterizing these changes is challenging, both experimentally and computationally. Experimental methods such as X-ray crystallography yield highly detailed structural data, but are mostly limited to a low-energy ground state of the molecule. While molecular dynamics (MD) simulations can provide atomically detailed insight to dynamics, computational demands to adequately sample conformational ensembles of large biomolecules and their complexes often require tremendous resources. By contrast, time-independent or non-deterministic sampling-based algorithms together with simplified biomolecular representations can efficiently explore the conformational landscape, which have lead to new biological insights [1]. Here, we model a protein as a kinematic linkage, with groups of atoms as rigid bodies and covalent, rotatable bonds as links with a torsional degree of freedom. Hydrogen bonds are encoded as additional constraints, which lead to nested, interdependent cycles that require coordinated changes of the torsional angles [2]. Admissible velocities of the torsional angles that maintain cycle closure lie in the tangent space $T_{q} Q$ to the constraint manifold $Q$ at the current configuration $q$ which coincides with the nullspace of the constraint Jacobian matrix [3]. We characterize biomolecular rigidity directly from detailed analysis of the nullspace. The velocity equation

$$
\dot{\mathbf{q}}_{\mathrm{N}}=\mathbf{N} \dot{\mathbf{u}},
$$

relates generalized velocities of the internal degrees of freedom $\mathbf{u}$ to admissible angular velocities $\dot{\mathbf{q}}_{\mathrm{N}}$ of the torsional angles through the nullspace matrix $\mathbf{N}$. We identify the set of rigid clusters in the biomolecule and validate our method on a small set of proteins. In contrast to methods based on explicit, combinatorial constraint counting [4], we obtain valid results for both regular and singular configurations. In addition, our geometric approach provides an explicit basis for motions along floppy modes, resulting in an efficient procedure to probe conformational space. We show how singularities can affect molecular rigidity and identify singular motions that remain undetected with combinatorial approaches. Our kinematic analysis can provide high-level insights into dynamic processes beyond the reach of MD simulations, with broad implications for drug design, protein engineering and human health.

\section{References}

[1] R. Fonseca, D.V. Pachov, J. Bernauer, H. van den Bedem. Characterizing RNA ensembles from NMR data with kinematic models. Nucleic acids research, Oxford Univ Press, 2014, 42 (15), 9562-9572

[2] P. Yao, L. Zhang, J.-C. Latombe. Sampling-based exploration of folded state of a protein under kinematic and geometric constraints. Proteins: Structure, Function, and Bioinformatics, Wiley Online Library, 2012, $80,25-43$

[3] P. Betsch, S. Leyendecker. The discrete null space method for the energy consistent integration of constrained mechanical systems. Part II: Multibody dynamics. International journal for numerical methods in engineering, Wiley Online Library, 2006, 67, 499-552

[4] N. Fox, F. Jagodzinski, Y. Li, I. Streinu. KINARI-Web: A Server for Protein Rigidity Analysis. Nucleic Acids Research, 2011, 39, W177-W183 


\title{
Computational simulation of piezoelectric coating surrounding activated tooth implant
}

\author{
A. Shirazi Beheshtiha, Udo Nackenhorst \\ Institute of Mechanics and Computational Mechanics, \\ Leibniz Universität Hannover, Hannover, Germany
}

Since the natural ligament responsible for the fixation of teeth in jawbone is destroyed when artificial replacements are implanted, the mechanical stimulation of the bone is reversed. Idea of this research project is the development of active implants which provide additional electrical stimulation for bone adaption.

The electric properties of bone material have been widely investigated, when Fukada and Yasuda in 1957 [1] discovered that bone exhibits piezoelectric behavior. A clinical study demonstrated that a local electromagnetic field accelerates the healing process after bone fracture [2]. Ramtani [2] presented a mathematical model relating to the benefit of the electric field in the repair and maintenance of the solid matrix of bone. Garzon-Alvardo [3] has developed a new electro-mechanical bone remodeling model using the remodeling model of Nackenhorst[4] as a starting point. The electro-mechanical model of bone remodeling that involves mechanical and electrical stimuli can be written, hypothetically, as follows

$$
\frac{d \rho}{d t}=g_{\text {mech }}\left(\rho, W_{\text {mech }}(\rho)\right)+g_{\text {elect }}\left(\rho, W_{\text {elect }}(\epsilon(\rho))\right)
$$

Where $W_{\text {mech }}$ and $W_{\text {elect }}$ are the mechanical strain energy and the electrical field potentioal, respectively. The bone mass density variation over time depends on the electrical and mechanical stimulus that exists at every spatial point of the bone.

In this research project, 3D-finite element model of lower mandible has been reconstructed from a CT data set of a 63 years old male patient using segmentation techniques. Furthermore, the implant used in this project is a conical endosseous implant and is placed as a incisor tooth at the ventral part of the mandible. The implant, consists of a conical part with a screw thread, an abutment and a crown. In addition, to investigate the effect of electrical field with in the bone matrix, a thin piezoelectric layer of $3 \mathrm{D}$ contact elements is inserted between implant and bone. This FEM model totally consist of about 20.000 nodes and 100.000 elements. As Dirichlet boundary conditions the models are fixed at the medial and dorsal ends. The crown is loaded with about $45 \mathrm{~N}$ which this load was calculated for a single tooth according to biting force statistics of people in this age. The results indicate the distribution of stress, electric potentioal and electrical field in each elements of the interface layer, the implant and the jawbone.

\section{References}

[1] Fukada E, Yasuda I. On the piezoelectric effect of bone. J. Phys. Soc. Japan 12 (1957), 1158-1162.

[2] Ramtani S. Electro-mechanics of bone remodelling. Int. J. Eng. Sci. 46 (2008), 1173-1182.

[3] Garzon-Alvardo et al. Numerical test concerning bone mass apposition under electrical and mechanical stimulus. Theor. Biol. Med. Model. (2012), 9-14

[4] Nackenhorst U. Numerical simulation of stress stimulated bone remodeling. Tech. Mech. 17 (1997), 31-40. 


\title{
Coupling an active middle ear implant to the round window of the cochlea
}

\author{
Philipp Wahl, Pascal Ziegler and Albrecht Eiber \\ Institute of Engineering and Computational Mechanics \\ University of Stuttgart \\ Pfaffenwaldring 9 \\ 70569 Stuttgart, Germany
}

\begin{abstract}
The inner ear or cochlea is composed of a bone structure of spiral shape which is separated into two chambers by a soft membrane, the basilar membrane. These chambers or scalae are closed by the stapes footplate and the round window membrane. In a normal ear, sound is received by the eardrum and is transmitted through the middle ear ossicular chain and excites the inner ear fluid through the vibration of the stapes footplate. The pressure changes in the inner ear fluid excite the basilar membrane and the displacement is the major phenomenon eventually resulting in hearing impression. The function of the round window is mainly pressure compensation in the scalae.
\end{abstract}

Pathological changes can result in conductive hearing loss. This is most often caused by a reduced elasticity of the stapedial annular ligament and thus a reduced amplitude of the stapes footplate. In contrast to classical hearing aids or passive protheses, one way to reconstruct hearing is to couple an active implant to the round window membrane to drive the basilar membrane to compensate the conductive hearing loss. The transfer behavior of the coupled system not only depends on the dynamics of the actuator and the mechanical characteristics of the round window membrane, but also on the interface between middle ear implant and round window membrane. To transfer the vibrations of the implant to the round window membrane with a minimum of distortions, several requirements, such as preload, transversal guidance, and an elastic suspension are necessary. However, these parameters have a significant impact on the transfer behavior of the entire coupled system, investigated in this study using an active middle ear implant, the Floating Mass Transducer (FMT).

In order to address these requirements, laboratory experiments were performed to analyze the dynamic behavior of the separated actuator and the coupled system. To reduce experiments on real human temporal bones, laboratory experiments on a technical inner ear model have been performed. The technical model consists of a fluid column enclosed by two silicone membranes. The vibration of the active implant and the membranes were measured by Laser Doppler Vibrometers. First, it will be shown that special membranes had to be developed to describe the mechanical characteristics of the round window membrane and the stapedial annular ligament. Then, the coupled system will be investigated with respect to preload, transversal guidance and distortion. Also, the experimental results from the technical model will be compared to measurements performed on a real temporal bone as well as with simple mechanical models. 


\title{
Eigenfrequencies of the reconstructed middle ear after tympanoplasty and stapedotomy corresponding to the case when the prosthesis is fixed
}

\author{
Iryna Slavashevich \\ Belarusian State University, Minsk, Belarus
}

\begin{abstract}
The normal middle ear consists of the tympanic membrane (TM) and three hinged bones: the malleus, the incus, and the steps, which are connected to each other by small joints and suspended by ligaments and muscles in the middle ear cavity. The ossicular chain begins at the malleus, which is attached to the eardrum, and ends at the stapes footplate, which vibrates with a small amplitude through the oval window into the inner ear vestibule. The vestibule is filled with the perilymphatic fluid. The vibration of the stapes footplate transmits pressure waves into the cochlea to activate the hearing nerves.

Mechanical injuries of the middle ear elements, pathologic changes and different diseases of the middleear structures can result in a hearing loss. Common treatment for the pathological diseases is the surgical reconstruction of the middle. The most rare and worst case of similar procedure consists in replacement of all elements of the middle ear. This surgery is performed in two steps: at the first stage, a surgeon performs tympanoplasty using cartilage transplants; and at the second step, he inserts the total ossicular replacement prosthesis (TORP) bridging a gap in the ossicular chain [1]. If the stapes footplate is immobilized by bone growth around the oval window, a small hole is drilled in the footplate (stapedotomy). Then the piston-like total cylindrical prosthesis is glued by its plate on the restored TM, and inserted into the vestibule through the drilled hole.

The negative consequence of the stapedotomy surgery is a reduction in the total stiffness of the middle-ear structure and, as a result, a drop in the natural frequencies of the biomechanical oscillating system [2]. For these type of reconstructions, including tympanoplasty and stapedectomy, the outcome is influenced by the cartilage transplant thickness, the prosthesis mass and geometrical design [2], and its position with respect to the TM [1].

This study relates to the case when the middle ear is subjected to both tympanoplasty and stapedotomy. The main goal is to study the natural frequencies of the eardrum, which do not stimulate oscillations of the adjoint prosthesis. These modes of the reconstructed TM was called the dead ones in [3]. And also to study the dependence of the plate vibration forms on a position of the prosthesis at the eardrum.

The reconstructed TM will be considered as a plate close to an ellipse. The prosthesis consists of the pliable shaft and the plate, which can be positioned according to the plane of the reconstructed TM. The prosthesis plate is treated as a perfectly rigid thin circular plate. We assume also rigid coupling between the prosthesis plate and the reconstructed TM.
\end{abstract}

\section{References}

[1] K.-B. Huttenbrink.Mechanical aspects of middle ear reconstruction..Dept. of Oto-Rhino-Laringology. Univ. of Technology, Dresden (1997), 165-168.

[2] G.I. Mikhasev, I.L. Slavashevich. Eigenfrequencies of the middle ear oscillating systems after total reconstruction. Vestnik St. Petersburg University. Ser. 1. 3 (2012), 107-116.

[3] G.I. Mikhasev, I. Slavashevich, K. Yurkevich. Prediction of Eigenfrequencies of the Middle Ear Oscillating System after Tympanoplasty and Stapedotomy. Advanced structured Materials. Vol. 45. Springe. DOI 10.1007/978-3-319-02535-3-14 (2015), 243-265. 


\section{S03: Damage and fracture mechanics}

The section focuses on damage mechanisms and fracture mechanics for all kinds of solid materials and structures. It aims at bringing together related research including experimental observations, modeling approaches and numerical techniques. In general, material failure is a complex process which may be considered on different length scales ranging from the atomistic up to the component, or even to the structural, level. Besides the material behavior of course also aspects of loading situations are crucial to describe failure. Thus, contributions focusing on static failure, dynamic failure or cyclic fatigue are equally welcome. 


\title{
Stabilizing the XFEM for static and dynamic crack simulations
}

\author{
Stefan Loehnert \\ Institute of Continuum Mechanics \\ Leibniz Universität Hannover
}

The eXtended Finite Element Method (XFEM) is a widely used technique for the simulation of cracks, heterogeneities and other problems in solid and fluid mechanics showing discontinuities in the field variables or their derivatives or localization phenomena of any type. It has shown to be very efficient and attractive for two and three dimensional simulations. However, the geometry of the discontinuity in conjunction with the mesh geometry and the applied XFEM enrichment scheme might in certain cases lead to very badly conditioned or even singular coefficient matrices, in general due to (near) linear dependencies between standard and enriched degrees of freedom. During the last years several stabilization techniques have been proposed in literature based on modifications of the enrichment functions, special preconditioning techniques, modifications of the mesh geometry or enrichment scheme or direct modifications of the resulting equation system.

In this contribution advantages and disadvantages of some of the stabilization techniques are presented. Additionally a simple but very efficient regularization method presented in [1] is combined with other stabilization techniques and extended to dynamic crack simulation problems. The technique is based on an eigenvalue decomposition of the element stiffness matrices of selected fully enriched elements and a stabilization of the subspace that causes ill-conditioning. This regularization technique has very little effect on the solution of the resulting equation system but significantly improves the condition number and is thus ideal especially for large three dimensional eXtended Finite Element problems. This is demonstrated with a number of numerical examples for static and dynamic crack simulations.

\section{References}

[1] S. Loehnert. A stabilization technique for the regularization of nearly singular extended finite elements. Computational Mechanics. 54 (2014), 523-533 


\title{
Thermo-mechanical modeling of crack propagation in dynamically loaded elastomer specimens using a scaled boundary finite element approach
}

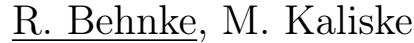 \\ Institute for Structural Analysis, Technische Universität Dresden
}

Recently, a scaled boundary finite element (SBFE) formulation for geometrically and physically nonlinear materials has been developed [2] using the scaled boundary finite element method (SBFEM) [3]. The SBFE formulation has been employed to describe plane stress problems of notched and unnotched hyperelastic elastomer specimens. However, elastomers show an inelastic nonlinear mechanical behavior at large deformations which is temperature-dependent in general.

In this contribution, the derived SBFE formulation is extended to nonlinear time- and temperature-dependent material behavior. Subsequently, the SBFE formulation is incorporated into a crack propagation scheme to model crack propagation in cyclically loaded elastomer specimens. Crack propagation is supposed to start at an initial notch. As benchmark example, a pure shear specimen of the so-called tear fatigue analyzer (TFA) is addressed. A plane and initially notched elastomer specimen is subjected to force-controlled cyclic loading at different excitation frequencies. The simulation concept is characterized by A) a multigrid finite element computation for displacement and temperature field, B) treatment of the thermo-mechanically coupled problem in the form of subproblems to account for different time scales resulting from high excitation frequencies and slowacting temperature increase, C) the evaluation of crack growth criteria and crack propagation direction criteria in the near field of the crack tip, D) a nonlinear finite viscoelasticity description of the elastomer material [1] for a wide frequency and temperature range, and E) a mesh modification procedure to represent crack growth. A curved crack path is tracked by mesh modification and remapping of the solution fields. The influence of the crack tip near field (heterogeneously distributed material properties, dynamic loading, viscous process zone, temperature state) on the resulting crack path and potential crack path deflections is numerically examined. In addition, the temperature increase at the crack tip and the rest of the elastomer specimen due to mechanical energy dissipation is evaluated and compared.

\section{References}

[1] R. Behnke, H. Dal, M. Kaliske. An extended tube model for thermo-viscoelasticity of rubberlike materials: theory and numerical implementation. In: S. Jerrams, N. Murphy (eds.): Constitutive Models for Rubber VII, CRC Press, Taylor \& Francis Group (2011), 87-92.

[2] R. Behnke, M. Mundil, C. Birk, M. Kaliske. A physically and geometrically nonlinear scaled-boundary-based finite element formulation for fracture in elastomers. Int. J. Numer. Meth. Engng 99 (2014), 966-999.

[3] J.P. Wolf, C. Song. Finite-Element Modelling of Unbounded Media. Wiley \& Sons, Chichester, 1996. 


\title{
Fitting stress intensity factors from crack opening displacements in $2 \mathrm{D}$ and $3 \mathrm{D}$ XFEM
}

\author{
Markus Schätzer, Thomas-Peter Fries \\ Institute of Structural Analysis, Graz University of Technology
}

In the literature, a large number of methods exists which deal with the computation of stress intensity factors (SIFs). Many of these approaches are energy-based such as those based on the J- or interaction integral. A disadvantage of these methods is that the extension to $3 \mathrm{D}$ is difficult. An easier access is possible by techniques which observe the displacement or stress fields at the crack tip, e.g. displacement extrapolation or fitting approaches. These methods are intuitive and the extension from $2 \mathrm{D}$ to $3 \mathrm{D}$ is often straightforward. A comparison of these approaches is given by Muthu [4].

We propose the computation of SIFs by crack opening displacements (CODs) in linear elastic fracture mechanics (LEFM) with stressfree crack surfaces, in the context of the extended finite element method (XFEM) and a hybrid explicit-implicit crack description [1, 2, 3]. An explicit crack description by means of straight line segments in two dimensions or flat triangles in three dimensions has the advantage that the update of the crack geometry during crack propagation is quite simple. For calculations with the XFEM, an implicit crack description is used by means of level-set functions, which are derived from the explicit master configuration.

To compute SIFs by CODs, points have to be found on the implicitly described crack surface. As the displacement field is discontinuous on the crack surface the points have to be split in two opposite but infinitisimally close points. The global displacements of these points are extracted from the XFEM approximation. The difference of these two points describes the CODs in the global coordinate system. This CODs is now compared with the expected opening for a pure mode $I, I I$ and $I I I$. Therefore, a reference coordinate system has to be found, in which the analytical modes are evaluated. This reference system is aligned with the (curved) crack surface and extracted from the implicit level-set functions. With this special description of the local coordinate system no differences exist whether the crack is planar or not. The CODs for pure mode $I, I I$ and $I I I$ are compared to the approximated CODs in order to fit SIFs. Another advantage of this approach is that mode $I I I$ is consistently treated as the other modes.

The numerical studies show how to specify the fitting regions on the crack surface and confirm that accurate SIFs are robustly obtained with the proposed approach.

\section{References}

[1] T.P. Fries, M. Baydoun. Crack propagation with the extended finite element method and a hybrid explicitimplicit crack description. Numerical Methods in Engineering 89 (2012), 1527-1558

[2] T.P. Fries, T. Belytschko. The extended/generalized finite element method: An overview of the method and its applications. Numerical Methods in Engineering 84 (2000), 253-304

[3] N. Moës, J. Dolbow, T. Belytschko. A finite element method for crack growth without remeshing. Numerical Methods in Engineering 46 (1999), 131-150

[4] N. Muthu, S.K. Maiti, B.G.Falzon, I. Guiamatsia. A comparison of stress intensity factors obtained through crack closure integral and other approaches using eXtended element-free Galerkin method. Comput. Mech. 52 (2013), 587-605 


\title{
On corner singularities in Reissner's theory of elastic plates
}

\author{
J. Felger, W. Becker \\ Technische Universität Darmstadt, Fachgebiet Strukturmechanik, Franziska-Braun-Strasse 7, 64289 Darmstadt, Germany
}

In the framework of linear elasticity, stress singularities may occur in domains with non-smooth boundary like corners or sharp notches. In applications of fracture mechanics, the local stress field in the vicinity of stress concentrations is of particular interest. Therefore, methods of asymptotic analysis can be applied advantageously. The singularity analysis of elastic plates goes back to Williams [4] using the classical Kirchhoff-Love plate theory and a series expansion of the containing stress function. Extending the classical theory by taking shear deformations into account leads to Reissner's theory. The analysis of singularities for Reissner's plate theory has been examined by some authors using a stress potential approach and a Williams-type-expansion $[1,5]$ or a separation of variable ansatz and a series expansion for the displacements [2]. The solution obtained by a mathematical elaborate formulation based on weak solution theory in [3] indicates that the former results are incomplete.

In the present work, an asymptotic solution of the governing system of partial differential equation of Reissner's plate theory is obtained by using a complex potential approach. Within an asymptotic analysis, a reduced PDE-System of Reissner's theory is obtained, which can be solved analytically by introducing three arbitrary holomorphic potentials, which determine the deformations as well as the resultant forces and moments. This formulation allows for describing the singular behaviour of the stresses near a wedge vertex. Within a singularity analysis, the singularity exponent $\lambda$ is calculated for different boundary conditions on the edges. To solve the boundary value problem, the three complex potentials have to be in a form so that the prescribed boundary conditions are fulfilled. By choosing an adequate ansatz for the holomorphic functions, this requirement leads to a characteristic equation, whose roots represent the singularity exponent. The obtained results coincide with those in [3] but the present approach can be applied with less mathematical effort.

\section{References}

[1] WS Burton and GB Sinclair. On the singularities in Reissner's theory for the bending of elastic plates. Journal of Applied Mechanics, 53(1):220-222, 1986.

[2] CS Huang. Stress singularities at angular corners in first-order shear deformation plate theory. International Journal of Mechanical Sciences, 45(1):1-20, 2003.

[3] A Rössle and AM Sändig. Corner singularities and regularity results for the Reissner/Mindlin plate model. Journal of Elasticity, 103(2):113-135, 2011.

[4] ML Williams. Surface stress singularities resulting from various boundary conditions in angular corners of plates under bending. In Journal of Applied Mechanics, 18:325-328, 1951

[5] DHY Yen and M Zhou. On the corner singularities in Reissner's theory for the bending of elastic plates. Journal of Applied Mechanics, 56(3):726-729, 1989. 


\title{
Crack propagation at bi-material interfaces
}

\author{
Mahabub Alam, John P. Parmigiani \\ Oregon State University
}

The manner in which a crack propagates through a composite material significantly affects its macroscopic toughness. Consider, for example, a simple composite consisting of two materials bonded to each other. The first material contains a crack through its entire thickness and incident on the interface between the materials (the bond line). The crack can thus propagate in one of two ways; either along the interface (referred to as deflection) or across the interface and into the second material (referred to as penetration). Deflection leads to crack blunting and macroscopic toughening of the composite while penetration consists of direct propagation of the initial crack and macroscopic embrittlement.

Historically, the work of He and Hutchinson [1], based on linear elastic fracture mechanics (LEFM), has been widely used to determine deflection versus penetration behavior. Using this approach, a critical ratio of material toughness to interface toughness indicates when crack propagation transitions between deflection and penetration. However, more recently, the finite element work of Parmigiani and Thouless [2] has shown that the LEFM approach with its corresponding metric of only a critical toughness ratio is generally insufficient and that a combined strength and toughness approach is required. Using this combined approach, propagation by deflection versus penetration is determined by a critical ratio of material strength to interface strength, a critical ratio of material toughness to interface toughness, and several other dimensionless parameters. The finite element work of Strom and Parmigiani [3] provided further clarification by showing that the LEFM approach is only valid for conditions distant from those corresponding to the transition between deflection and penetration. Accurate predictions of penetration versus deflection behavior near transition conditions (which is typically the region of greatest interest) require the strength-and-toughness approach.

Currently published work using the strength-and-toughness approach focuses on comparisons to the LEFM approach, trends in toughness and strength ratios at transition, and applied loads at transition. The focus of the work presented here is to discuss how transition conditions vary for different specific materials. This is new information not included in prior published work. The results are applicable to a variety of material systems but are presented in terms of intergranular (i.e. deflection) versus transgranular (i.e. penetration) fracture of grains of a number of materials. Results show that delfection is more likely to occur as grain toughness increases , grain stiffness decreases, and grain size decreases. The phase angle (relative measure of mode I and mode II contributions to fracture) of the propagating crack is also considered and is shown to increase as grain toughness decreases, grain stiffness increases, and grain size increases. These results are significant in that they provide additional insight into how a crack incident on an interface will propagate in specific materials.

\section{References}

[1] M.Y. He, J.W. Hutchinson. Crack deflection at an interface between two orthotropic media. J. Appl. Mech. - Trans ASME. 59 (1992), 79-87.

[2] J.P. Parmigiani, M.D. Thouless. The roles of toughness and cohesive strength on crack deflection at interfaces. J. Mech. Phys. Solids 54(2) (2006), 266-287.

[3] J.L. Strom, J.P. Parmigiani. A study of transition of crack path at bi-material interfaces. Eng. Fract. Mech. 115 (2014), 13-21 


\title{
Global approaches for an accurate loading analysis at multiple cracks systems
}

\author{
Paul Judt, Andreas Ricoeur \\ Institute of Mechanics, University of Kassel, 34125 Kassel, Germany
}

Based on the work by Eshelby [1, 2], the path-independent $J_{k}$-integral $[3,4], M$ - and $L$-integrals $[4,5]$ and the interaction-integral [6] or $I_{k}$-integral were introduced and applied to cracks for the accurate calculation of crack tip loading quantities. Applying the FE-method to solve boundary value problems with cracks, numerically inaccurate values are observed within the crack tip region affecting the accuracy of local approaches. Simulating crack paths, local approaches face further problems as cracks are running towards interfaces, internal boundaries or other crack faces. Within global approaches, the path-independent integrals are calculated along remote contours far from the crack tip, essentially exploiting numerically reliable data requiring special treatment only for the near-tip crack faces $[7,8]$. To provide path independence, additional integrals along interfaces, internal boundaries and crack faces are necessary.

$J_{k^{-}}, I_{k^{-}}, M$ - and $L$-Integrals are akin to each other but bare different features which may be utilized for the development of global approaches considering multiple defects. A global approach of the $I_{k}$-integral can be used for the loading analysis of multiple cracks, by using appropriate auxiliary fields [9]. The selective choice of local coordinate systems qualifies $M$ - and $L$-integrals to be used to distinguish between the material forces of two defects [10].

In this paper, new global approaches of path-independent integrals are presented and applied to the calculation of crack paths at interacting cracks systems. The resulting paths are compared to standard methods being in very good agreement.

\section{References}

[1] J.D. Eshelby. The continuum theory of lattice defects. Sol. Stat. Phys. 3 (1956), 79-144.

[2] J.D. Eshelby. The elastic energy-momentum tensor. J. Elast. 5 (1975), 321-335.

[3] J.R. Rice. A path independent integral and the approximate analysis of strain concentration by notches and cracks. J. Appl. Mech. 35 (1968), 379-386.

[4] B. Budiansky, J.R. Rice. Conservation laws and energy-release rates. J. Appl. Mech. 40(1) (1973), 201-203.

[5] J.K. Knowles, E. Sternberg. On a class of conservation laws in linearized and finite elastostatics. Arch. Rat. Mech. Analysis. 44 (1972), 187-211.

[6] M. Stern, E.B. Becker, R.S. Dunham. A contour integral computation of mixed-mode stress intensity factors. Int. J. Fract. 12(3) (1976), 359-368.

[7] P.O. Judt, A. Ricoeur. Accurate loading analyses of curved cracks under mixed-mode conditions applying the J-integral. Int. J. Fract. 182 (2013), 53-66.

[8] P.O. Judt, A. Ricoeur. Consistent application of path-independent interaction integrals to arbitrary curved crack faces. Arch. Appl. Mech. (2014), DOI 10.1007/s00419-014-0897-z

[9] P.O. Judt, A. Ricoeur. Crack growth simulation of multiple cracks systems applying remote contour interaction integrals. Theor. Appl. Fract. Mech. (2014), DOI 10.1016/j.tafmec.2014.11.001

[10] P.O. Judt, A. Ricoeur. Accurate loading analyses and crack path predictions applying path-independent integrals to multiple cracks problems. Proceedings of the First World Conference on Fracture and Damage Mechanics, Kottayam, India, (2014), published on CD 


\title{
Crack initiation in elliptically notched plates
}

\author{
P. Weißgraeber, J. Felger, W. Becker \\ Technische Universität Darmstadt, Fachgebiet Strukturmechanik, Franziska-Braun-Straße 7, 64287 Darmstadt, Germany
}

Notches or flaws are ever-present in engineering structures. Fracture analyses are required to assess the criticality of these stress concentrators under the expected loading conditions. In this work, the general case of an elliptical notch in a plate under uniaxial tensile loading is considered. Crack initiation at the stress concentration is studied by using a Finite Fracture Mechanics approach [4]. It is shown that the analysis comprises the limit cases of a vertical and a horizontal crack. In these cases the present analysis reverts to a strength of material approach or to Linear Elastic Fracture Mechanics. When intermediate aspect ratios of the ellipse are considered, a smooth transition of a stress-governed failure to an energy-governed failure is observed. Size effects that show a dependence on the size of the hole, which does not change the actual stress fields, are covered by this approach.

To implement the Finite Fracture Mechanics approach, the stress field in the notched plate and the stress intensity factor of cracks emanating from the notch are required. Whereas the former can be obtained in closedform $[2,1]$, the latter cannot be calculated analytically. In literature, approximate solutions, generally expressed as

$$
K_{I}=F \sigma \sqrt{\pi a}
$$

can be found [5, 3]. Detailed numerical analyses were performed to study the accuracy of the available form functions $F$ and it was found that they are only valid for very short cracks and limited aspect ratios. A more general form function $F$ has been developed that yields good approximate solutions for a very wide range of aspect ratios and crack lengths. With explicit expression of the stress field and the stress intensity factor, the Finite Fracture Mechanics criterion can be evaluated very advantageously.

\section{References}

[1] C.E. Inglis. Stresses in a plate due to the presence of cracks and sharp corners. Transactions of the Institute of Naval Architects, 55:219-230, 1913.

[2] G. Kirsch. Die Theorie der Elastizität und die Bedürfnisse der Festigkeitslehre. Zeitschrift des Vereines Deutscher Ingenieure, 42:727-807, 1898.

[3] D. Kujawski. Estimations of stress intensity factors for small cracks at notches. Fatigue E6 Fracture of Engineering Materials \&5 Structures, 14(10):953-965, 1991.

[4] D. Leguillon. Strength or toughness? A criterion for crack onset at a notch. European Journal of MechanicsA/Solids, 21(1):61-72, 2002.

[5] P. Lukas. Stress intensity factor for small notch-emanated cracks. Engineering Fracture Mechanics, 26(3):471-473, 1987. 


\title{
Phase-Field Modeling of Fracture in Anisotropic Media
}

\author{
Stephan Teichtmeister, Christian Miehe \\ Insitute of Applied Mechanics (CE) \\ University of Stuttgart \\ Pfaffenwaldring 7, 70569 Stuttgart, Germany \\ teichtmeister@mechbau.uni-stuttgart.de
}

In the recent years phase-field modeling of fracture has become of high importance to describe complex crack patterns in all kinds of solid materials. Many of the models assume an isotropic material behavior, which of course is not a meaningful assumption for e.g. biological tissues such as arterial walls. The incorporation of anisotropy in phase-field models is also motivated by the emersion of sawtooth crack patterns. Since the phase-field approach introduces an additional (smeared) phase describing the evolution of the crack, this method is well suited to be extended for anisotropic materials without thinking about an adaption of the discretization techniques.

Anisotropy can be incorporated in several ways, like by an extension of the surface energy, i.e. by making the energy release rate orientation dependent, as considered in LI ET AL. [1]. Our ansatz is based on a pure geometrical approach, namely on an anisotropic formulation of the crack surface itself by using the theory of tensor invariants. This gives through the minimization of the crack surface a physically reasonable meaning, see Miene ET AL. [2]. The proposed formulation also opens a good starting point for more complex phase-field models such as coupling with other physical fields, e.g. fracture behavior in porous media, or the incorporation of higher order terms.

Some representative numerical examples show the applicability of our approach by using a robust algorithm based on operator splits, see MiEHE ET AL. [3] for more details on this.

\section{References}

[1] B. Li, C. Peco, D. Millán, I. Arias, and M. Arroyo. Phase-field modeling and simulation of fracture in brittle materials with strongly anisotropic surface energy. International Journal for Numerical Methods in Engineering, in Press, 2014.

[2] C. Miehe, F. Welschinger, and M. Hofacker. Thermodynamically consistent phase-field models of fracture: Variational principles and multi-field FE implementations. International Journal for Numerical Methods in Engineering, 83:1273-1311, 2010.

[3] C. Miehe, M. Hofacker, and F. Welschinger. A phase field model for rate-independent crack propagation: Robust algorithmic implementation based on operator splits. Computer Methods in Applied Mechanics and Engineering, 199:2765-2778, 2010. 


\title{
A non-isothermal phase-field model for damage in two-phase materials
}

\author{
Markus Radszuweit, Christiane Kraus \\ Weierstrass Intitute for Applied Analysis and Stochastics \\ Mohrenstrasse 39, 10117 Berlin, Germany
}

The understanding of stability and lifetime of multi-phase materials such as, for instance, alloys requires knowledge about the interplay of the different mechanical properties of each phase under external load or thermally induced internal stresses. A widely used model for spinodal decomposotion that accounts for elastic deformation due to lattice mismatch of the phases is the Cahn-Larché system [1]. As a consequence of these internal stresses microcracks form. A phase-field model that introduces a macroscopic damage variable is used, which yields a subdifferential evolution equation [2]. The resulting rate-dependent damage evolution [3] obeys a Griffith-like criterium when a crack is formed. During heating/cooling processes or through energy dissipation by crack growth [4] large temperature gradients can occur in the material. In order to account for this effect, we extend the model to the non-isothermal case by considering entropy balance. We investigate the presented model using two-dimensional Finite Element numerical simulations. In particular, the interaction of the path with the phase boundaries is examined. Since multiple spatiotemporal scales are involved, the application of adaptive discretization is advantageous.

\section{References}

[1] H. Garcke. On a Cahn-Hilliard model for phase separation with elastic misfit. Ann. I. H. Poincaré - AN 22 (2005), 165-185.

[2] M. Frémond, B. Nedjar. Damage, gradient and principle of virtual power. Int. J. Solid structures 33 (1996), 1083-1103.

[3] C. Heinemann, C. Kraus. Existence of Weak Solutions for Cahn-Hilliard Systems Coupled with Elasticity and Damage. Adv. Math. Sci. Appl. 21 (2011), 321-359

[4] A.T. Zehnder, A.J. Rosakis. On the Temperature Distribution at the Vicinityof Dynamically Propagating Cracks in 4340 Steel. J. Mech. Phys. Solids 39 (1991), 385-415. 


\title{
A novel treatment of crack boundary conditions in phase field models of fracture
}

\author{
Michael Strobl, Thomas Seelig \\ Institute of Mechanics, Karlsruhe Institute of Technology, Kaiserstraße 12, 76131, Karlsruhe
}

Computation of complex crack patterns is a challenging task in fracture mechanics and the phase field approach thereto shows excellent capabilities. A scalar phase field variable $\mathcal{S}$ degrades the elastic energy density in a regularized representation of cracks in isotropic materials [1]. Different methods are used so far to model the property of a crack to weaken a material only in tension and shear. One approach is the decomposition of strain in negative spherical, positive spherical and deviatoric parts, in which only the energy density of the latter two is degraded by the phase field [2]. Another method is based on the spectral decomposition of strain [3] and the split into positive and negative principal strains. However, both models avoid to take an explicit representation of the crack direction into account so that particular situations lead to stress states which violate the crack boundary conditions.

This contribution presents a new approach which takes the direction of the crack into account. For an exact representation of cracks, the stress component normal to the crack surface should only be non-zero in case of compression and shear stresses acting on a frictionless crack surface should vanish. In order to satisfy these conditions stresses and strains are represented with respect to a coordinate system oriented with the crack. The crack normal direction is computed from the gradient of the phase field

$$
n=\frac{\nabla \mathcal{S}}{|\nabla \mathcal{S}|},
$$

which is already available from the description of the smeared surface energy. The degrading function of the phase field acts only on some parts of the elastic energy density

$$
\psi^{\mathrm{el}}=\tilde{\psi}(\tilde{\varepsilon}, \mathcal{s})+\hat{\psi}(\hat{\varepsilon}) .
$$

Only the terms using $\tilde{\varepsilon}$ are degraded by the phase field. The degradation of shear stress requires special attention in case there is no unique gradient. The derivation of the stress tensor leads to an elasticity tensor displaying transverse isotropy with symmetry about the crack normal. The five parameters for transversely isotropic material are replaced by the two elastic constants of the undamaged isotropic material and parameters including the phase field variable $\mathcal{S}$.

The new model is able to treat crack opening and closure in a correct manner. In addition no extra numerical effort has to be done to compute stresses and strains. The performance of the proposed model is demonstrated by means of numerical simulations, including representative tests for stationary cracks as well as simulations for dynamically propagating cracks.

\section{References}

[1] C. Kuhn, R. Müller. A continuum phase field model for fracture. Engineering Fracture Mechanics (2010), vol. 77 , issue 18:3625-3634

[2] H. Amor, J.-J. Marigo, C. Maurini. Regularized formulation of the variational brittle fracture with unilateral contact: Numerical experiments. Journal of the Mechanics and Physics of Solids (2009) vol. 57, issue 8:12091229

[3] C. Miehe, F. Welschinger, M. Hofacker. Thermodynamically consistent phase-field models of fracture: Variational principles and multi-field FE implementations. Int. J. Numer. Meth. Engng. (2010) vol. 83, issue 10:1273-1311 


\title{
Phase-Field Modeling of Hydraulic Fracture
}

\author{
Steffen Mauthe, Christian Miehe \\ Institute of Applied Mechanics (CE) \\ University of Stuttgart \\ Pfaffenwaldring 7, 70569 Stuttgart, Germany \\ steffen.mauthe@mechbau.uni-stuttgart.de
}

Hydraulically driven fracture has gained more and more research activity in the last few years, especially due to the growing interest of the petroleum industry. Hydraulic fracturing, commonly known as fracking, is a technique to increase hydrocarbon production from the subsurface system of reservoirs. Key challenge for a powerful simulation of this scenario is an effective modeling and numerical implementation of the behavior of the solid skeleton and the fluid phase, the mechanical coupling between the two phases as well as the incorporation of the fracture process.

For this purpose we propose a new compact model structure for the Biot-type fluid transport in porous media at finite strains based on only two constitutive functions, that is the free energy function $\hat{\psi}$ and a dissipation potential $\hat{\phi}$. This formulation is then coupled to a phase-field approach for fracture which characterizes an intuitive and descriptive regularization of a crack surface that converges for vanishing length-scale parameter to a sharp crack. In contrast to formulations with a sharp-crack discontinuities, the proposed regularized approach has the main advantage of a straight-forward modeling of complex crack patterns including branching. In this work, we extend these concepts to the coupled problem of fluid transport in porous media at fracture.

We apply a rigorous geometric approach to the diffusive crack surface, governed by the crack phase field. The irreversibility of the evolution of the crack phase field is modeled through a critical stress based growth function. A modular concept is outlined for linking of the diffusive crack phase field to the poroelastic response of the bulk material. This includes a transition from an isotropic Darcy-type fluid flow in the unbroken bulk material to an anisotropic Poiseuille-type fluid flow within a crack. The modular structure is exploited in the numerical implementation of the proposed formulation by constructing a robust finite element method, based on an algorithmic decoupling of updates for the crack phase field and the state variables of the poroelastic bulk response. We demonstrate the model capabilities and performance by means of representative numerical examples of complex fracturing scenarios in two and three dimensions. 


\title{
3D ductile crack propagation with the XFEM
}

\author{
M.Sc. Steffen Beese \\ Dr.-Ing. Stefan Loehnert \\ Prof. Dr.-Ing. habil. Dr. h.c. mult. Peter Wriggers \\ Institute of Continuum Mechanics, Leibniz University Hannover
}

In industrial forming processes metals are subjected to large plastic deformations. Related to the plastic deformation pores will start to grow, merge and finally lead to micro cracks within the material. Macroscopically this is recognized as a loss of stiffness of the structural behavior. This effect is usually modeled in the context of damage mechanics, where in the last fifteen years several theories were established to circumvent the mesh dependent localization. Most of them are based on the introduction of a new degree of freedom for the thermodynamic driving force of the damage. This new degree of freedom is then computed by solving an additional scalar balance equation of Helmholtz type. Nevertheless, if damage evolves and is used to predict macro cracks the global stiffness matrix becomes ill conditioned. Furthermore cracks are just represented in a smeared way and their dimension is related to the mesh size and some artificial internal length parameter. To overcome this drawback the damage is transfered to discrete cracks if it exceeds a material depended threshold value. The representation of fracture is modeled with the eXtended Finite Element Method (XFEM). This numerical tool enables a nearly mesh independent crack representation. The strong discontinuities are modeled by level set functions and the spatial discretization of the displacement field becomes enriched. The enrichment functions are chosen in a way to enable discontinuities in the displacement and even singularities or nearly singularities in derived quantities. Within the XFEM only a local neighborhood of the crack becomes enriched and the overall number of unknowns increases only moderately.

Since classical crack propagation criteria loose their validity in a finite deformation context and inelastic material behavior one has to think about new ones. The argumentation for the chosen damage based criterion is that once the crack is initiated it will propagate if the pores around the crack front will continue to coalesce. So the mechanism for crack initiation and propagation is the same.

The problem of evolving the front is treated with a purely geometric approach, which preserves the property of perpendicular level sets. After the propagation no remeshing is needed, but the enrichment scheme has to be updated in the vicinity of the crack front. There is also a need for adapting the history variables in these elements. This becomes necessary because the integration points in the new crack front elements are replaced in a way to consider the discontinuous character of the displacements within these elements.

The load displacement path of cracking structures is highly challenging. That's the reason why neither an entirely force driven nor a displacement driven simulation is able to follow the path and an arc length approach is used.

Keywords: XFEM,crack propagation, non local damage 


\title{
Numerical methods for crack loading analyses in quasicrystals
}

\author{
Zhibin Wang, Andreas Ricoeur \\ Institute of Mechanics, University of Kassel, D-34125 Kassel
}

The quasicrystals (QC), short for quasiperiodic crystals, have long-range orientational order but without translational symmetry in particular direction. With such special atom arrangement the quasicrystal is a new material class besides crystals and non-crystals (amorphous solids). Crystallographically, quasicrystals can be categorised into some subclasses $(1 \mathrm{D}, 2 \mathrm{D}$ or $3 \mathrm{D})$ which depends on how many quasiperiodic directions there are, e.g. one dimensional quasicrystals have two periodic atom arrangement directions and one quasiperiodic direction [1]. Since the first discovery of quasicrystals in a man made Al-Mn alloy about thirty years ago, people made great effort to research this kind of outstanding material. The investigation of physical properties of quasicrystals, such as the fracture behavior, electronic properties, hardness etc., is essential extending their fields of application. Today, QC are known to exhibit e.g. a very good wear resistance, low friction coefficients and to possess a very low porosity.

This work focuses on macroscopic cracks in 1D quasicrystalline plates, where the material is governed by constitutive equations under the consideration of quasicrystal linear elasticity theory. Analytically, the explicit solutions for the coupled phonon-phason fields of quasicrystals are derived applying a generalized Stroh formalism [2]. The numerical model and simulation for arbitrary cracks are established in a FE environment, where the constitutive behavior of coupled phonon and phason fields is included in special user elements. Different numerical techniques like a displacement interpretation method or modified crack closure integral are applied in a generalized form to calculate stress intensity factors and energy release rates. The validity of conventional fracture criteria and the influence of coupled fields on the mechanical and fracture behavior of quasicrystals are finally investigated.

\section{References}

[1] T.Y. Fan and Y.W. Mai. Elasticity theory, fracture mechanics, and some relevant thermal properties of quasi-crystalline materials. Appl. Mech. Rev. 57(5), (2004), 325-343.

[2] Y. Gao, A. Ricoeur, L.L. Zhang, L.Z. Yang. Crack solutions and weight functions for plane problems in three-dimensional quasicrystals. Arch. Appl. Mech. 84(8), (2014), 1103-1115. 


\title{
Application of a cohesive zone element for prediction of damages in laminated structures
}

\author{
S. Rezaei, S. Wulfinghoff, R. Kebriaei, S. Reese \\ Institute of Applied Mechanics, RWTH Aachen University, Germany
}

In the last decades applications of the repetition tools in production and manufacturing processes have widely increased. These tools have to be coated with the materials which have satisfactory lifetime and degradation resistance. Therefore, the main goal of this study is to investigate antiadhesive and wear resistant coatings made of ceramics, plastics and metals produced by High Power Puls Magnetron Sputtering (HPPMS) technique [1]. A cohesive zone element technique $(\mathrm{CZ})$ is applied to realize the interactions of the coatings and the substrate surfaces (see [2]). This goes along with the investigations of the delamination and failure behavior of the involved surfaces. To see the applicability of the model, several structural simulations are peformed. The developed CZ element model is capable of modeling the separation, the contact and also the irreversible reloading conditions in both normal and tangential directions [3]. The model is further developed to be applicable for different structures including different bonding behaviors, with a higher stability. The talk concludes with a detailed discussion of the numerical results of different material and interface properties.

\section{References}

[1] K. Bobzin, R. Nickel, N. Bagcivan, F. D. Manz, PVD - Coatings in Injection Molding Machines for Processing Optical Polymers. Plasma Processes and Polymers. 4 (2007), S144-S149.

[2] R. Kebriaei, I. N. Vladimirov, S. Reese, Joining of the alloys AA1050 and AA5754 - Experimental characterization and multiscale modeling based on a cohesive zone element technique. J. Mater. Process. Technol. 214 (2014), 2146-2155.

[3] R. Kebriaei, A. Mikloweit, I. N. Vladimirov, M. Bambach, S. Reese, G. Hirt, A cohesive zone model for simulation of the bonding and debonding in metallic composite structures - experimental validations. Key Engineering Materials 622 (2014), 443-452. 


\title{
Macroscopic damage modeling for silicon nitride
}

\author{
Ruck, J., Othmani, Y., Lube, T. ${ }^{a}$, Khader,I. ${ }^{b}$, Kailer, A. ${ }^{b}$, Böhlke, T. \\ Institute of Engineering Mechanics, Karlsruhe Institue of Technology \\ ${ }^{a}$ Institute of Structural and Functional Ceramics, University of Leoben \\ ${ }^{b}$ Fraunhofer Institute for Mechanics of Materials IWM
}

\begin{abstract}
Modeling the damage of brittle materials is of great importance considering a variety of structural components. Prominent examples are high strength engineering ceramics. The present work is concerned with silicon nitride, a material with increasing importance in industrial applications. Using EBSD data allows to map the microstructure of polycrystallines and provides a tool to examine the grains, grain boundaries and their respective orientation. Based on EBSD data, micromechanical simulations of silicon nitride was performed using a micromechanical thermoelastic model by Wippler (2012). The micromechanical model includes damage for the different constituents of silicon nitride and the respective interface. As a result, the effective material properties of silicon nitride were determined. In the sense of a hierarchical model structure the effective properties were applied to macromechanical, phenomenological damage models for monotonous and cyclic loading. It is, thereby, essential to capture the general anisotropic nature of damage. For this purpose, an anisotropic smeared crack model has been adopted which was originally formulated by Govindjee et al. (1995). This model is extended to include crack-closure based on a geometrical approximation proposed by Ortiz (1985). The monotonous damage model, hereby, uses a damage evolution equation proposed by Govindjee et al. (1995). The cyclic damage model is based on an effective crack evolution for slow crack growth in silicon nitride (Lube et al., 2007) which was reinterpreted in terms of damage. The effect of damage on the effective material properties is shown by making use of the directional dependent Young's modulus. Furthermore the application of the cyclic damage model to a four point bending test of silicon nitride as well as the application of the monotonous model to a threed thrust bearing are discussed.
\end{abstract}

\section{References}

[1] Govindjee, S., Gregory, J.K., and Simo, J. Anisotropic modelling and numerical simulation of brittle damage in concrete. International Journal for Numerical Methods in Engineering 38 (1995), 3611-3633.

[2] Ortiz, M. A constitutive theory for the inelastic behavior of concrete. Mechanics of materials 4 (1985), 67-93.

[3] Wippler, J. Micromechanical Finite Element Simulations of Crack Propagation in Silicon Nitride. Dissertation (2012).

[4] Lube, T., Dusza, J. A silicon nitride reference material - A testing program of ESIS TC6. Journal of the European Ceramic Society 27 (2007), 1203-1209. 


\title{
A regularization approach for damage models based on a displacement gradient
}

\author{
Stephan Schwarz, Philipp Junker, Klaus Hackl \\ Institute of Mechanics of Materials, Ruhr-University Bochum, Germany
}

Common material models that take into account softening effects due to damage have the problem of illposed boundary value problems if no regularization is applied. This condition leads to a non-unique solution for the resulting algebraic system and a strong mesh dependence of the numerical results. A possible solution approach to prevent this problem is to apply regularization techniques that take into account the non-local behavior of the damage [1]. For this purpose a field function is often used to couple the local damage parameter to a non-local level, in which differences between the local and non-local parameter as well as the gradient of the non-local parameter can be penalized.

In contrast, we present a novel approach to regularization in which no field function is needed [2]. For that purpose, the regularization directly affects the displacements. The idea is that with an increasing value of the damage the element's volume will increase as well, which is a result of the softening due to the occurring damage. The increasing volume can then be measured by the divergence of the displacements, on which the gradient is now applied. The lack of any field function and the direct application of the gradient to the divergence of the displacements entails several advantages: the numerical effort is considerably reduced and the convergence behavior is improved as well. Naturally, the numerical results are mesh independent due to the regularization.

\section{References}

[1] B. Dimitrijevic, K. Hackl. A method for gradient enhancement of continuum damage models. Tech. Mech., 28(1):43-52 (2008)

[2] P. Junker, S. Schwarz, J. Makowski, K, Hackl. A gradient-based regularization approach without field functions. Submitted (2014) 


\title{
Investigation of an elastoplastic material model coupled to nonlocal damage in an implicit-gradient framework
}

\author{
T. Brepols ${ }^{\dagger}$, S. Wulfinghoff, S. Reese \\ Institute of Applied Mechanics, RWTH Aachen University \\ Mies-van-der-Rohe-Straße 1, 52074 Aachen, Germany \\ †tim.brepols@rwth-aachen.de
}

Since the pioneering works by Kachanov [1] and Rabotnov [2] in the 50's and 60's of the past century and especially during the last three decades, continuum damage mechanics (CDM) has continuously proven to be a suitable framework for describing degradation processes in materials from a macroscopic viewpoint. By introducing additional internal variables into the formulation in order to capture essential microscopic material defects in an averaged sense, CDM becomes especially attractive for e. g. finite element simulations of large-scale problems involving damage and elastoplasticity. Although different approaches exist on how exactly damage is incorporated into a CDM model, most formulations have in common that the material exhibits strain-softening at a certain stage of the loading history (see e.g. [3]).

Unfortunately, the application of conventional (i. e. 'local') softening models in numerical procedures as e. g. the finite element method may lead to serious mesh-dependent results if the damage becomes sufficiently high and the strains localize into a narrow band (see e. g. [4], [5]). This problem is related to the conceptual simplicity of the material model itself which possesses no length scale entering its constitutive description. Therefore, several solution strategies were proposed in the past to restore the well-posedness of the formulation and ensure meshobjective results. One specific class of models abandones a classical assumption made in continuum mechanics and exploits the principle of 'nonlocal action' (see e.g. [6]). Physical arguments supporting the hypothesis of the nonlocal character of damage were given e. g. by Baẑant [7].

Among the latter type of formulations, the so-called implicit gradient-enhanced damage models have gained attention in more recent years (see e.g. [8], [9]). The main ingredient of these models is an additional partial differential equation for a selected nonlocal quantity which has to be solved in addition to the normal balance equations and which becomes a further unknown of the problem. The damage variable itself is then considered to be functionally dependent on this quantity and becomes therefore 'nonlocal' by its own nature. It can be shown that this type of model is equivalent to a specific nonlocal integral-type formulation [10], but its mathematical description remains strictly local which makes its implementation comparably easy from an algorithmic point of view.

The goal of the current study is to examine the behavior and capabilities of an elastoplastic material model which is coupled to damage in an implicit gradient-enhanced setting. Its suitability to describe the crack development phase in case of ductile materials shall be carefully investigated. Various benchmark problems are considered and corresponding finite element computations are carried out in order to estimate the model's capabilities to produce mesh-independent simulation results.

\section{References}

[1] L. M. Kachanov. Time of the rupture process under creep conditions. Izvestiya Akademii Nauk SSSR, Otdelenie Tekhnicheskikh Nauk 8 (1958), 26-31.

[2] Y. N. Rabotnov. On the equations of state for creep. Progress in Applied Mechanics - The Prager Anniversary Volume (1963), 307-315.

[3] Z. P. Baẑant, T. Belytschko, T. Chang. Continuum theory for strain-softening. Journal of Engineering Mechanics 110 (1984), 1666-1692.

[4] R. de Borst, L. J. Sluys, H.-B. Mühlhaus, J. Pamin. Fundamental issues in finite element analyses of localization of deformation. Engineering computations 10 (1993), 99-121.

[5] D. Lasry, T. Belytschko. Localization limiters in transient problems. International Journal of Solids and Structures 24 (1988), 581-597. 
[6] G. Pijaudier-Cabot, Z. P. Baẑant. Nonlocal damage theory. Journal of Engineering Mechanics 113 (1987), 1512-1533.

[7] Z. P. Baẑant. Why continuum damage is nonlocal: Micromechanics arguments. Journal of Engineering Mechanics 117 (1991), 1070-1087.

[8] R. A. B. Engelen, M. G. D. Geers, F. P. T. Baaijens. Nonlocal implicit gradient-enhanced elasto-plasticity for the modelling of softening behaviour. International Journal of Plasticity 19 (2003), 403-433.

[9] R. H. J. Peerlings, R. de Borst, W. A. M. Brekelmans, J. H. P. de Vree. Gradient enhanced damage for quasi-brittle materials. International Journal for Numerical Methods in Engineering 39 (1996), 3391-3403.

[10] R. H. J. Peerlings, M. G. D. Geers, R. de Borst, W. A. M. Brekelmans. A critical comparison of nonlocal and gradient-enhanced softening continua. International Journal of solids and Structures 38 (2001), 7723-7746. 


\title{
A three-dimensional progressive damage model for fiber reinforced composites with an implicit-explicit integration scheme
}

\author{
Grischa Maaß, Friedrich Gruttmann \\ Technische Universität Darmstadt
}

\begin{abstract}
This contribution proposes a fully three dimensional continuum damage model to describe the interlaminar and intralaminar failure mechanisms of transversely isotropic elastic-brittle materials under static loading. The constitutive model is derived from an energy function with independent damage variables for fiber, matrix and shear failure, i.e. five different failure modes are considered: fiber tension, fiber compression, matrix tension, matrix compression and shear.

The evolution law is based on energy dissipation within the damage process and the increase of damage is controlled by equivalent displacements for each damage mode. The damage model is implemented in a finite element program (FEAP), taking into account the critical energy release rate to weaken the effect of mesh dependend outcome.

It is a well-known fact that the lack of numerical robustness in nonlinear solid mechanics (such as continuum damage mechanics) using standard iterative Newton-Raphson schemes leads to numerical convergence inconveniences and thus in some cases no useable results can be obtained. An implicit-explicit integration scheme, first proposed by Oliver [3] in isotropic damage models, is used to increase the stability and robustness of numerical simulations and to decrease the computational cost of material failure analyses.
\end{abstract}

\section{References}

[1] R.G. Cuntze, A. Freund. The predictive capability of failure mode concept-based strength criteria for multidirectional laminates. Composites Science and Technology Volume 64, Issues 3-4 (2004), 343-377

[2] Ireneusz Lapczyk, Juan A. Hurtado. Progressive damage modeling in fiber-reinforces materials. Compos Part A, 38 (2007), 2333-2341

[3] J. Oliver, A.E. Huespe, J.C. Cante An implicit/explicit integration scheme to increase computability of nonlinear material and contact/friction problems Comput. Methods Appl. Mech. Eng., 197 (2008), 1865-1889 


\title{
Effects of a circular defect size and position on the damage progression in hybrid laminates under compressive loading
}

\author{
Ahcene Mokhtari 1, Nacer Tala-Ighil 1 \\ Welding and NDT Research Center (CSC). BP 64 CHERAGA - ALGERIA 1
}

\begin{abstract}
The composite structures made from glass fiber reinforced with polymer matrices have resistance to compression considerably lower when holes (defects) are present. The hardness of the polymer matrix, the properties of the interface matrix/fiber and fiber stability are the main factors that affect the resistance to compression of notched laminates. The importance of these factors results in complex damage modes which involve the matrix cracking, delamination, local buckling and shear failure.The importance of the damage tolerance analysis of composite materials was shown by many authors [1]. An approach was presented by Wang [2] related to the delamination problem of the composite laminates edges. Davis and Jones [3] have examined the effect of manufacturing defects on the fracture of composite structures. An experimental and numerical study on the residual stresses in the composite laminates subjected to an impact loading was done by the authors [4]. The progressive evolution of the damage in the laminates under compressive loading containing a circular hole has been studied experimentally [5], it was observed that the rupture was initiated at the instability localized at the surface of the hole edge.

In this paper, the effects of a circular defect size and position on the post-damage response of the FMLs laminates under compressive loading has been studied. Compression tests are performed on composite plates comprising a hole having different diameters $(8 \mathrm{~mm}, 12 \mathrm{~mm}$ and $18 \mathrm{~mm})$ and located at different positions (25\%, $50 \%$ and $75 \%$ ) in the loading direction. A numerical analysis using the finite element method FEM has permits the identification of the initiation zones and the description of the damage evolution in these laminates. The damage has been Modelled by the combined use of the Cohesive Zone Model [6] (CZM) and the Linde mechanical model [7]. The results of the numerical simulation are in good agreement with the experimental results. The FEM has well predicted the critical damage zones. The failure modes are instantaneous in the plates having a defect on the side, this is explained by the high instability of the hybrid laminate (local buckling) once past to the deformation of the second order and the rupture occurs suddenly. However, for the plates having a defect in the center, a lateral deformation of the first order (global buckling) is observed and the rupture is gradual because the aluminum layers tend to stop the crack growth and a stresses redistribution occurs across the plate.
\end{abstract}

\section{References}

[1] J.W. Mar. Fracture, longevity, and damage tolerance of graphite/epoxy filamentary composite material. J. Aircraft. 21 (1) (1984), 77-83.

[2] S.S. Wang. Fracture mechanics for delamination problems in composite materials. J. Composite Materials. 17 (1983), 210-223.

[3] M.J. Davis and R. Jones. Damage tolerance of fiber composite laminates. G.C. Sih etal., Fracture Mechanics Technology Applied to Material Evaluation and Structure Design, Martinus Nijhoff, Leiden (1978).

[4] G.E. Husman, J.M. Whitney and J.C. Halpin. Residual strength characterization of laminated composites subjected to impact loading. Foreign Object Impact Damage to Composites, ASTM STP 568, American Society for Testing and Materials (1975), 92-113.

[5] A. M. Waas, C.D. Jr. Babcock and W. G. Knauss. Damage Progression in Compressively Loaded Laminates Containing a Circular Cutout. AIAA Journal. 29 (3) March (1991), 436-443.

[6] M. Benzeggagh and M. Kenane. Measurement of Mixed-Mode Delamination Fracture Toughness of Unidirectional Glass/Epoxy Composites with Mixed-Mode Bending Apparatus. Composite Science and Technology 56 (1996), 439-449.

[7] P. Linde, J. Pleitner, H. De Boer and C. Carmone. Modelling and Simulation of Fiber Metal Laminates. ABAQUS Users Conference (2004). 


\title{
3D modeling of crack percolation due to alkali-silica reaction in concrete RVEs
}

\author{
A. Cuba Ramos ${ }^{1,2}$, C. Dunant ${ }^{2}$, J.-F. Molinari ${ }^{1,2}$ \\ ${ }^{1}$ Civil Engineering Institute, \\ École polytechnique fédérale de Lausanne (EPFL), \\ Station 18, CH-1015 Lausanne, Switzerland \\ ${ }^{2}$ Materials Science and Engineering Institute, \\ École polytechnique fédérale de Lausanne (EPFL), \\ Station 18, CH-1015 Lausanne, Switzerland
}

The alkali-silica reaction (ASR) is a reaction in concrete, which occurs between the alkalis in the cement paste and reactive silica within the aggregates. This type of concrete deterioration has been observed in structures all over the world, notably in mass concrete. Since the reaction is slow, ASR is often detected only after many service years. During the reaction, part of the aggregate material is transformed into silica gel that starts swelling as it adsorbs water. The gel expansion is constrained by the surrounding aggregate or paste material and consequently internal stresses in the structure arise. These stresses cause a macroscopic expansion of the structure, cracking and eventually spalling. From a mechanical point of view this internal degradation can be characterized by a loss of strength and elastic modulus. Studying the evolution and the effects of ASR in massive concrete structures such as dams is of particular importance as they are designed for a long service life and repairs are expensive.

The reaction rate of ASR, i.e. the rate at which the silica gel forms, depends on various physical and chemical parameters such as the aggregate type, temperature and relative humidity. However, it has been shown in [1] that the macroscopic expansion of unrestrained concrete and the damage are uniquely related to the amount of gel present in the structure. This observation makes it possible to model the mechanical effects of ASR by using the amount of gel present in structure as an input parameter, without having to take into account the speed at which the gel has formed, if creep is neglected. A 2D model based on this conclusion, in which the cement matrix, the aggregates and the swelling gel are modeled as separate phases, was developed by Dunant in [2]. The author used an isotropic continuum damage approach to model the formation and propagation of cracks in ASR samples. The results obtained with this model show the same qualitative trends observed in experiments of ASR samples under uniaxial load [3]. The simulations furthermore revealed that microcracks are aligned with the loading direction and that external loads accelerate the damage evolution.

In this work Dunant's model is extended to 3D in order to simulate full 3D concrete samples. This allows also to make more accurate predictions for the onset of crack percolation. Massively parallel finite-element simulations are carried out to study the mechanical consequences of ASR in 3D representative volume elements (RVEs). For the first set of simulations an isotropic damage formulation, like in the original 2D model, is used. However, since the loading conditions in the case of ASR are complex, the damage evolution in real ASR samples is anisotropic. In order to capture this effect in the simulations the scalar damage variable has to be replaced by a higher order damage tensor [4]. Consequently, a second set of simulations is run using an anisotropic damage formulation. The results for both sets are compared in order to analyze how the initial damage zone around the gel sites changes with the damage formulation.

\section{References}

[1] M. Ben Haha, E. Gallucci, A. Guidoum, K. Scrivener. Relation of expansion due to alkali-silica reaction to the degree of reaction measured by sem image analysis. Cem. Conr. Res. 37(8) (2007), 1206-1214.

[2] C. Dunant. Experimental and modelling study of the alkali-silica reaction in concrete. PhD thesis, Ecole polytechnique fédérale de Lausanne (2009).

[3] C. Dunant, K. Scrivener. Effects of uniaxial stress on alkali-silica reaction induced expansion of concrete. Cem. Conr. Res. 42(3) (2012), 567-576.

[4] J. Lemaitre. A course on damage mechanics. Springer-Verlag (1996). 


\title{
Numerical simulation of the fracturing processes in masonry arches
}

\author{
Alberto Carpinteri, Stefano Invernizzi, \\ Federico Accornero, Luca Testone \\ Department of Structural, Geotechnical and Building Engineering, \\ Politecnico di Torino, Corso Duca degli Abruzzi 24, \\ 10129 Torino, Italy
}

Masonry arch structures, and, more generally, vaulted structures, are traditionally assessed using a wellestablished approach, such as linear elasticity or limit analysis, whereby system behavior at the intermediate stage -which occurs when the material's tensile strength has been exceeded but the collapse mechanism has not yet formed -is disregarded. A more accurate interpretation requires a thorough analysis that can take into account the intermediate cracking stage and uses a constitutive law providing a closer approximation to the actual behavior of the material [1]. In this paper, an evolutionary fracturing process analysis for the stability assessment of masonry arches is presented. This method makes it possible to capture the damaging process that takes place when the conditions evaluated by means of linear elastic analysis no longer apply and before the conditions assessed through limit analysis set in. Furthermore, the way the thrust line is affected by the opening of cracks and the redistribution of internal stresses can be checked numerically [2]. The results obtained with the described approach are compared with a numerical simulation performed with the finite element code Diana (TNO, The Netherlands) adopting discrete cracking with nonlinear cohesive laws. Finally, the case study of the arch of the Mosca Bridge over the Dora River in Turin, Italy, is described.

\section{References}

[1] A. Carpinteri, An. Carpinteri. Softening and fracturing process in masonry arches. Proc., $6^{\text {th }}$ Int. Brick Masonry Conf. (1982), 502-510.

[2] A. Carpinteri, G. Lacidogna, F. Evolution of the fracturing process in masonry arches. ASCE Journal of Structural Engineering. (2014), DOI: 10.1061/(ASCE)ST.1943-541X.0001071. 


\title{
A damage-plasticity model to simulate the mode-II fatigue behavior of interfaces between thin adherends
}

\author{
Pietro Carrara, Laura De Lorenzis \\ Technical University of Braunschweig, Institute of Applied Mechanics, Germany
}

Interfacial strength deterioration due to fatigue is still a major open issue relevant for a broad range of applications [1], such as delamination of composite materials [2] or more generally fatigue life prediction in bonded joints [3]. In particular, fatigue may induce the accumulation of plastic dislocations and damage at the interface level which, in turn, may lead to the nucleation of micro-cracks. Their gradual coalescence may result in a macro-crack, whose propagation is responsible for interface failure [2] even for applied stresses smaller than the maximum attained in monotonic quasi-static conditions [4].

Numerical methods based on the definition of suitable constitutive cohesive interface laws are one of the most versatile and used approach to simulate the behavior of bonded joints. However, some models employ a large number of parameters leading to a complicate calibration and to a very demanding implementation (e.g. [2]). Moreover, these parameters sometimes do not have a straightforward physical meaning (e.g. [1]). On the other hand, simple models based on simplifying assumptions may not fulfill thermodynamic laws (e.g. [5]).

The present work proposes a novel thermodynamically consistent model for the behavior of interfaces under shear (i.e. mode-II) fatigue loading conditions. The interface behavior is defined coupling damage and associated isotropic-kinematic plasticity. Friction and interlocking at the interface level are neglected. The fulfillment of the second law of thermodynamics, in the form of the Clausius-Planck inequality, is ensured by the definition of a dissipative potential following [6]. This potential rules also the coupling between damage and plasticity. The admissible states domain is formulated restricting the tangential interface stress to non-negative values, which makes the model suitable for interfaces with thin adherends. Linear softening is assumed so as to reproduce, under monotonic conditions, a bilinear mode-II interface law. Two different damage variables govern respectively the loss of strength and of stiffness of the interface. The proposed model introduces only four parameters, i.e. three defining the monotonic mode-II interface law, and one ruling the fatigue behavior. This limited number of parameters and their clear physical meaning facilitate experimental calibration. Model predictions are compared with experimental results on fiber reinforced polymer sheets externally bonded to concrete since this case meets the basic assumptions of the model. Different loading histories are accounted for, and an excellent agreement is obtained.

While more analyses are needed to confirm the obtained results, the approach presented appears to be a very promising tool to study the fatigue life of bonded joints. A simple calibration of the fatigue parameter using a relative small number of experimental tests would enable the extension of the prediction of the fatigue behavior of a bonded joint to conditions different from the tested ones.

\section{References}

[1] K. Roe, T. Siegmund, An irreversible cohesive zone model for interface fatigue crack growth simulation, Engineering Fracture Mechanics 70 (2) (2003) 209-232.

[2] A. Turon, J. Costa, P. Camanho, J. Mayugo, Simulation of delamination propagation in composites under high-cycle fatigue by means of cohesive zone models, NASA Technical report (December).

[3] A. J. Kinloch, S. O. Osiyemi, Predicting the Fatigue Life of Adhesively-Bonded Joints, The Journal of Adhesion 43 (1-2) (1993) 79-90.

[4] C. Carloni, K. V. Subramaniam, Investigation of sub-critical fatigue crack growth in FRP/concrete cohesive interface using digital image analysis, Composites Part B: Engineering 51 (2013) 35-43.

[5] E. Martinelli, A. Caggiano, A Unified Theoretical Model for the Monotonic and Cyclic Response of FRP Strips Glued to Concrete, Polymers 6 (2) (2014) 370-381.

[6] J. Lemaitre, R. Desmorat, Engineering Damage Mechanics, Springer-Verlag, Berlin/Heidelberg, 2005. 


\title{
Predicting delamination in multilayered CFRP laminates
}

\author{
Jaan-Willem Simon, Daniel Höwer, Stefanie Reese \\ Institute of Applied Mechanics, RWTH Aachen University, Aachen, Germany
}

In many technical applications, carbon fiber reinforced plastics (CFRP) are gaining importance due to their very high Young's modulus and low density. These are particularly leveraged in thin shell-like structures of lightweight constructions. The composites examined in this paper consist of multiple layers, each of which is composed of carbon fibers embedded in an epoxy matrix material. Two different kinds of layers are considered: (1) unidirectional CFRP with one family of fibers, and (2) woven CFRP with two families of fibers. In order to describe realistically the material behavior of such composites, a slightly modified version of the mesomechanically motivated material model proposed by Reese [1] is used, which has been shown to be well suitable for such applications [2].

Structural collapse in fiber composite structures can possibly be caused by different damage modes: matrix transverse cracking, fiber fracture, or delamination. From these, the delamination is particularly important, because it drastically reduces the bending stiffness of the structure and promotes local buckling in case of compressive loads. Including delamination into the computation of composite structures requires the definition of an appropriate criterion for its onset as well as the prediction of its growth after an initial crack has evolved. In the present work, the delamination onset allows the consideration of different modes as well as mixed mode situations. Once the delamination onset criterion is met, the propagation of the delamination is predicted by a damage formulation which acts like a cohesive zone model.

The use of a fully three-dimensional material model strongly suggests using solid elements. On the other hand, due to the application in thin shell-like structures, shell elements should usually be preferred. Therefore, a solid-shell element is applied here, which has been presented by Schwarze and Reese $[2,3]$ combining the advantages of both solid elements and shell elements at the same time. This solid-shell formulation utilizes a reduced integration scheme within the shell plane using one integration point, whereas a full integration is used in thickness direction. Thus, an arbitrary number of integration points can be chosen over the shell thickness. Thereby, different fiber orientations of the layers can be taken into account easily, since the material parameters can be defined for each integration point separately.

\section{References}

[1] S. Reese. Meso-macro modelling of fiber-reinforced rubber-like composites exhibiting large elastoplastic deformation. Int J Solids \& Struct 40 (2003), 951-980.

[2] J.-W. Simon, B. Stier, S. Reese. Numerical Analysis of Layered Fiber Composites Accounting for the Onset of Delamination. Adv Eng Soft 80 (2015), 4-11.

[2] M. Schwarze, I. Vladimirov, S. Reese. Sheet metal forming and springback simulation by means of a new reduced integration solid-shell finite element technology. Comput Methods Appl Mech Engrg 200 (2011), $454-476$.

[3] M. Schwarze, S. Reese. A reduced integration solid-shell finite element based on the EAS and the ANS concept â large deformation problems. Int J Numer Methods Engng 85 (2011), 289-329. 


\title{
Fatigue mechanisms of cord-rubber composites
}

\author{
M.Sc. Niraj Kumar Jha and Prof. Dr.-Ing. Udo Nackenhorst \\ Institute of Mechanics and Computational Mechanics, Leibniz Universität Hannover
}

This article presents a multiscale continuum damage mechanics approach to characterize the fatigue mechanisms of cord-rubber composites. The performance of cord-rubber composite is highly influenced by the properties of interface between them which plays a significant role in stress transfer between cord and rubber matrix. An airspring bellow which consists of layers of rubber and reinforcing cords is considered for this work. The phenomenological material model of the rubber is formulated for the purpose of analyzing the rate dependent behavior under cyclic loading. The rate dependency and hysteretic behavior are characterized by using the concept of internal variables [1]. The implementation of the constitutive formulation for rubber material is done in Abaqus via UMAT.

A representative volume element (RVE) of a cord-rubber composite is employed to analyze microscale damage mechanisms i.e. matrix cracking and interfacial debonding. At macroscale, homogenized damage model is applied to predict the stress-strain response. Within the frame of finite element cohesive zone modeling, interfacial debonding is studied. Furthermore, the relationship between two failure mechanisms of fiber-matrix interfacial debonding and matrix cracking are also discussed [2].

\section{References}

[1] A. Suwannachit, U. Nackenhorst. On the constitutive modeling of reinforced rubber in a broad frequency domain. ZAMM (2010), Z. Angew. Math. Mech.90, No. 5, 418-435

[2] R. Talreja, C.V.Singh. Damage and Failure of Composite Materials.Cambridge University Press (2012) 


\title{
Determination of the fracture properties from size effect in ceramic-fibers-reinforced PMMA
}

\author{
Guillermo Díaz, Jörn Mosler \\ Institute of Materials Research, Materials Mechanics, Helmholtz-Zentrum Geesthacht \\ Institute of Mechanics, Departament of Mechanical Engineering, TU Dortmund
}

This talk deals with numerical analyses of size effects in composite materials. The considered composite material is a PolyMethylMethAcrylate (PMMA) matrix reinforced by ceramic fibers. While the bulk's response of the fibre and that of the matrix are modeled by means of standard finite hyperelastoplasticity, material failure in the form of fibre cracking and debonding between the fibre and the surrounding matrix is captured by the strong discontinuity approach. Since the mechanical model parameters characterizing debonding failure are not known, they are computed through an inverse analysis. This inverse analysis confirms a pronounced size effects of the failure modes.

\section{References}

[1] I. Scheider, T. Xiao, N. Huber, and J. Mosler. Computational Material Scince 80, 35-42 (2013).

[2] I. Scheider, Y. Chen, A. Hinz, N. Huber, and J. Mosler. Eng. Frac. Mech. 100, 17-27 (2013). 


\title{
On Anisotropic and Nonlocal Damage Models
}

\author{
Stephan Wulfinghoff, Stefanie Reese, Tim Brepols, Marek Fassin \\ Institute of Applied Mechanics, RWTH Aachen University, D-52074 Aachen, Germany
}

The talk investigates the modeling of anisotropic damage based on second and forth order damage tensors. Different approaches like the strain or the energy equivalence principle are compared concerning their similarities and differences. The central difference of these individual approaches are different definitions of effective stress tensors. Advantages and open issues of the individual formulations are discussed from a thermodynamical perspective. In addition, the question is raised to what extend the thermodynamical point of view should influence the model formulation. Also addressed are related restrictions on damage models like thermodynamical consistency and the requirement of monotonically increasing damage.

Possible approaches to couple damage with plasticity models and to regularize mesh-dependency are investigated. The findings are illustrated by means of simple simulation results. 


\title{
Model Reduction and Clustering Techniques for Crash Simulations
}

\author{
Dennis Grunert, Jörg Fehr \\ Institute of Engineering and Computational Mechanics, University of Stuttgart \\ Pfaffenwaldring 9, 70569 Stuttgart, Germany \\ \{dennis.grunert,joerg.fehr\}@itm.uni-stuttgart.de
}

\begin{abstract}
Nowadays, crash simulation is a core area of vehicle development. Recently, regulations and standards also allow the application of numerical simulation (or Virtual Testing) results instead of hardware tests. In the future, hardware tests are expected to be stint in favor of virtual tests conducted to evaluate the safety of a vehicle. Commercial Finite Element (FE) solvers are used to simulate recurring crash scenarios. In addition, with the promotion of active safety techniques, the so-called pre-crash phase is taken into account, which is over 20 times longer than the actual crash phase. Bearing in mind that crash simulation is one of the most (calculating) timeconsuming task in car design, this leads to tremendous simulation times. In order to allow for parallelization on computer clusters, explicit instead of implicit methods are used to solve the occurring differential equations in crash simulation. This already reduces the response time but not the overall computational cost. Therefore, the deployment of model reduction for speed-up, data reduction and energy saving in the computing centers is a logical consequence.

But unfortunately, crash simulations possess all three basic sources of nonlinearities in mechanics: Large deformation, nonlinear material behavior and multiple contact scenarios. Using linear reduction with a nonlinear model would result in an unfeasible error. Therefore, nonlinear techniques like POD-DEIM from [1] are investigated for practicability in crash simulations. It uses the POD-Galerkin projection, which finds $N \ll n$ orthogonal vectors $\mathbf{v}_{i}$ that approximate a given subspace $\operatorname{span}\left\{\mathbf{y}_{1}, \ldots, \mathbf{y}_{s}\right\} \subseteq \mathbb{R}^{n}$ of $s$ snapshots (samples of
\end{abstract} trajectories) best in the sense that

$$
\sum_{i=1}^{s}\left\|\mathbf{y}_{i}-\sum_{j=1}^{N}\left\langle\mathbf{y}_{i}, \mathbf{v}_{j}\right\rangle \mathbf{v}_{j}\right\|_{2}^{2}
$$

is minimal. These $\mathbf{v}_{i}$ can be calculated as the columns of the left projection matrix $\mathbf{V}$ of the singular value decomposition of $\left(\mathbf{y}_{1}, \ldots, \mathbf{y}_{s}\right)$. Substitution of $\mathbf{y}(t)$ with $\mathbf{V} \tilde{\mathbf{y}}(t), \tilde{\mathbf{y}}(t) \in \mathbb{R}^{N}$, in a system of nonlinear ODEs

$$
\frac{d}{d t} \mathbf{y}(t)=\mathbf{L y}(t)+\mathbf{F}(\mathbf{y}(t))
$$

separated into a linear part $\mathbf{L} \in \mathbb{R}^{n \times n}$ and a nonlinear function $\mathbf{F}: \mathbb{R}^{n} \rightarrow \mathbb{R}^{n}$ leads to

$$
\frac{d}{d t} \tilde{\mathbf{y}}(t)=\mathbf{V}^{T} \mathbf{L V} \tilde{\mathbf{y}}(t)+\mathbf{V}^{T} \mathbf{F}(\mathbf{V} \tilde{\mathbf{y}}(t))
$$

Since the nonlinear part $\mathbf{f}(t):=\mathbf{F}(\mathbf{V} \tilde{\mathbf{y}}(t))$ of the system (3) still scales with the high-dimensional model after applying POD, it is essential to apply e.g. the Discrete Empirical Interpolation Method (DEIM) to overcome this problem. The POD-DEIM algorithm from [1] selects only a few, say $m$, indices from the vector-valued function $\mathbf{f}$ with a projection matrix $\mathbf{P}$ in such a way that a certain error bound can be established. The projection is then given as

$$
\hat{\mathbf{f}}(t)=\mathbf{U}\left(\mathbf{P}^{T} \mathbf{U}\right)^{-1} \mathbf{P}^{T} \mathbf{f}(t)
$$

with $\mathbf{U}$ the matrix containing the projection basis constructed from snapshots of the full-order function. The reduced system

$$
\frac{d}{d t} \tilde{\mathbf{y}}(t)=\mathbf{V}^{T} \mathbf{L V} \tilde{\mathbf{y}}(t)+\mathbf{V}^{T} \mathbf{U}\left(\mathbf{P}^{T} \mathbf{U}\right)^{-1} \mathbf{F}\left(\mathbf{P}^{T} \mathbf{V} \tilde{\mathbf{y}}(t)\right)
$$

has e.g. the computational complexity $\mathcal{O}\left(N^{2}+m N\right)$ if the evaluation of $\mathbf{F}$ and its derivative at $p$ components scales linear in $p$.

It is unnecessary and computationally too expensive to reduce the complete model with nonlinear techniques. Therefore, in a first step, parts with linear and nonlinear characteristics are identified. The identification could either be performed by an experienced user or by the application of methods from machine learning. First the 
crash will be simulated with small parameter variations multiple times and the result data is exported from the LS-DYNA binary database to Matlab with a self-written converter. Now clustering methods like the k-means algorithm, PCA or spectral clustering can be used to identify parts of the model that behave similar, c.f. [2] and [3]. This will allow the application of nonlinear methods like the aforementioned POD-DEIM to parts with large deformations and linear methods based on Krylov subspaces and Gramian matrices, which are implemented in the software MOREMBS [4] developed at our institute, to the remaining parts. The size of the local reduced basis can also be decreased since similar behavior often implies a smaller solution space.

Whereas the well-known k-means algorithm has some restrictions, e.g. each cluster is always located in a convex subset of the underlying space, spectral clustering is more flexible. The algorithm summarized in [5] works as follows: First similarities between all pairs of nodes with the position data at each time step and simulation run for different parameters need to be defined, c.f. [3] for details. These will be converted to a $l$-nearest neighbor graph with $l$ chosen around the logarithm of the number of nodes. As a next step, the first $k$ eigenvectors (with $k$ the number of desired clusters) of the normalized graph Laplacian are calculated and collected in a matrix, whose rows are clustered with the k-means algorithm. This procedure returns clusters of nodes with similar deformation and is justified by heuristic comparisons with graph cutting problems and spectral perturbation theory.

A high-detailed FE model of a 2001 Ford Taurus by the National Crash Analysis Center serves as a validation model. However, the usage of the complete Finite Element model for developing new methods is too complex. Therefore, only a high deformed part like a longitudinal chassis beam is used in place of the complete model to test different clustering algorithms.

\section{References}

[1] S. Chaturantabut, D. Sorensen. Nonlinear model reduction via discrete empirical interpolation. SIAM Journal on Scientific Computing 32 (2010). 2737-2764.

[2] B. Bohn, J. Garcke, R. Iza-Teran, A. Paprotny, B. Peherstorfer, U. Schepsmeier, C. A. Thole. Analysis of Car Crash Simulation Data with Nonlinear Machine Learning Methods. Procedia Computer Science 18 (2013). 621-630.

[3] L. Mei, C. Thole. Data analysis for parallel car-crash simulation results and model optimization. Simulation Modelling Practice and Theory 16 (2008). 329-337.

[4] J. Fehr, P. Eberhard. Simulation process of flexible multibody systems with non-modal model order reduction techniques. Multibody System Dynamics 25 (2011). 313-334.

[5] U. von Luxburg. A tutorial on spectral clustering. Stat. Comput. 17 (2007). 395-416. 


\title{
Mechanical properties of cold sprayed Ti- and Ni-based composites coatings
}

\author{
P. Cavaliere, A. Silvello \\ Università del Salento
}

Cold spray is a safe and innovative coating technology characterized by high efficiency and low cost if compared to the traditional technologies. Very fine particles are accelerated into a De Laval nozzle and sprayed on a substrate. The severe plastic deformation, experienced by the sprayed particles, occurs at temperature well below the melting point leading to the unique mechanical properties experienced by such kinds of coatings. It has potentials to be applied in aerospace, automobile, chemical industry. Cold spray appears very promising in the production of nanostructured composites with enhanced microstructural and mechanical properties. In the present paper the microstructural and mechanical behavior of Ti- and Ni-based nanocomposites coatings produced via cold spray are presented. The results are analyzed as a function of spray velocity and they are compared to those belonging to the unreinforced base materials. 


\title{
Finite Element Method for the Vibration of Cracked Beams with Varying Cross Section
}

\author{
Mehmet Haskul, Murat Kisa, \\ Inonu University, Departman of Mechanical \\ Harran University, Departman of Mechanical
}

This contribution presents ideas, the vibration analysis of the non-uniform cracked beams has been investigated. A computer program using the finite element method has been written to find the dynamical characteristics (natural frequencies and mode shapes) of the beam. The cracked section in the beam has been modeled by a massless spring whose flexibility depens on the local flexibility induced by the crack. The stiffness of spring has been derived from the linear elastic fracture mechanics theory as the inverse of the compliance matrix calculated the using stress intensity factors and strain energy release rate expression. Some examples has been given to explain the proposed method and investigate the effects of the depth and location of cracks on the natural frequencies and mode shapes. The results of current study and those in the literature are compared and good agreements have been found. Consequently it is showed that proposed method is reliable and simple. 


\title{
Growth of a substrate material damage as a result of waves localization
}

\author{
Abramian A.K., Vakulenko S.A. \\ Institute for Problems in Mechanical Engineering RAS, St. Petersburg,199178, Russia
}

Some types of a laminated safety glass consists of an interlayer surrounded by two thin films (see in [1]). An optimal level of adhesion is then required for the safety glass to absorb enough of the impact energy to prevent projectile penetration and delamination. Such considerations have motivated us to study the behavior of such structures undergoing shock like loading and introduce the mathematical model of the structure behavior.The structure under consideration is modeled as a string, an elastic foundation of which is assumed to be imperfect, and which coefficient depends on a damage function of a substrate.The imperfection of an elastic foundation is modeled by a damage function for which the evolution equation is introduced.The governing equation is as follows:

$$
\gamma u_{x x}-K(n) u-\rho_{0} u_{t t}=Q(x, t, \epsilon), \quad t>0
$$

where $u$ is a displacement, $Q$ is an external force.

$$
Q(x, t, \epsilon)=\delta_{\epsilon}\left(x-x_{0}\right) \sum_{j=0}^{M} \delta(t-j \Delta t), \quad x \in(-\infty,+\infty), \quad t>0 .
$$

Here $\Delta t$ is a time step for the strikes and $\delta_{\epsilon}$ is a smoothed $\delta$, function. Here $K[n]$ is a complicated functional of $u$ that involves a damage function $n$. The quantity $K$ depends on $n$ via the following relations

$$
K(n)=\mu(n) G(n), \quad \mu(n)=\frac{k_{0}}{k_{0}+G(n)}
$$

where $k_{0}>0$ is a constant, $G(n)=G_{0}(1-n), \quad 0 \leq n \leq 1 ; G(n)=0, \quad n>1$, Here $G_{0}$ is a constant. The time evolution of the damage function $n(x, t)$ is defined by the differential equation

$$
\frac{\partial n}{\partial t}=\beta H(\mu(n)|u|-\Delta)(1-n)
$$

where $\beta, \Delta>0$ are positive constants, and $H$ is the Heaviside step function. We assume that $Q$ is a fast decreasing in $|x|$ smooth function and we set the boundary and initial conditions $u(x, t) \rightarrow 0 \quad(|x| \rightarrow \infty) ; u(x, 0)=$ $0, \quad u_{t}(x, 0)=0, \quad x \in(-\infty,+\infty) ; n(x, 0)=n_{0}(x), \quad x \in(-\infty,+\infty)$ where $n_{0}$ also is a fast decreasing in $|x|$ smooth function.If $x \in[-h, h]$, we set the Dirichlet boundary condition $u(h, t)=u(-h, t)=0$. If $\beta$ is small, then $d n / d t<<1$ and the coefficient $K$ is a function of the slow time $\tau=\beta t$. Therefore, we can use a quasi-stationary approximation by the two-time scales perturbation method. We assume that $\beta<<1$ and $K(n)$ is a function of the slow time $\tau=\beta t$. For each fixed $\tau$, there occurs a Schrodinger operator associated with this problem,

$$
\mathcal{H} \Psi=\frac{d^{2} \Psi}{d x^{2}}-\Phi(x, \tau) \Psi=E \Psi, \quad \Phi=\gamma^{-1}\left(K(n(x, \tau))-\bar{k}_{0}\right)
$$

The eigenfunctions $\Psi$ may be localized ones, when $\Psi=\Psi_{j}(x, \tau), E=\gamma^{-1}\left(\bar{k}_{0}-\rho_{0}^{-1} \omega_{j}^{2}\right)$, where $j=1, \ldots, N(\tau)$, and may belong to the continuous spectrum. The analysis of the obtained asymptotic solution shows that for all times there always exists a localized eigenfunction and, thus, $N(\tau) \geq 1$. Also a possibility of resonances between the time periodic external strike load and localized modes has been found.The obtained solution consist of two terms: an oscillating term and a wave term. Main effects are as follows: the damage zone in substrate can cause the non-stationary wave localization in it, and there are possible transitions between different types of the front destruction dynamics. Three scenario of $n(x, t)$ behavior are possible: a monotone growth of $n$; a piecewise like constant growth of $n$ (when $n$ increases during some intervals and $n$ is constant between these growth intervals); no growth of $n$.

\section{References}

[1] Lu Nanshu Lu et al.,Failure by simultaneous grain growth, strain localization, and interface debonding in metal films on polymer substrates,J.Mater.Res., 24 (2009) , 2, 379-385. 


\section{S04: Structural mechanics}

The section will focus on advanced theoretical, numerical and experimental models for the evaluation of the behavior of structures. The diffusion of innovative materials characterized by high strength, anisotropy and unconventional mechanical responses (metamaterials) pose new challenges to the design and the performance of various structural elements like beams, plates and shells. In particular, structural issues may appear at different scales when materials with an internal architecture are employed. Particularly welcome are models and algorithms for structures that address nonlinear material behaviors and investigate structural stability at different scales. 


\title{
T-splines discretizations for large deformation contact problems
}

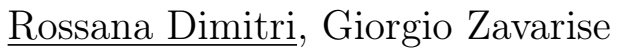 \\ Dipartimento di Ingegneria dell'Innovazione, Università del Salento, ITALY
}

The isogeometric analysis (IGA) represents a new method of computational analysis that merges design and analysis into one model by using a unified geometric representation. NURBS (Non-Uniform Rational B-Splines) and T-Splines are the most widespread technologies in today's CAD modelling tools and therefore are adopted as basis functions for analyses. In this work the isogeometric concept [1] is applied to study the large deformation multi-body contact problems, which still represent a significant challenge for the analysts in terms of robustness and stability of solutions. For this reason, the development of more efficient, fast and stable finite element contact discretizations is still a hot topic, especially due to the fact that engineering applications become more and more complex. Among the most important challenges that have to be met with respect to finite element discretization is the sensitivity of contact problem to the geometry accuracy.

Non-smooth, $\mathrm{C}^{0}$-continuous finite element basis functions lead to convergence problems in the analysis of sliding contact and to highly oscillatory contact interactions even when convergence is achieved. Various contact smoothing techniques have been proposed in the literature to address this issue [2-6] which consider the smoothing of the master and slave surfaces as achieved by high-order finite element interpolation based on Lagrange, hierarchic, spline or NURBS interpolations. Within the isogeometric framework, a contact surface possessing $\mathrm{C}^{1}$ or higher continuity is easily achieved and significant advantages over conventional finite element descriptions have been demonstrated in the last years by applying NURBS based isogeometric discretizations [4-7] to frictionless and/or frictional multi-patch contact problems.

A key problem of multivariate NURBS basis functions, in any case, is their rigid tensor product structure, which implies that refinement is a global process propagating throughout the domain. A possible way to improve the quality of contact results in terms of local pressures and global time-history curves with limited increase in the computational effort is represented by local refinement. This has been recently considered in [8] for frictionless contact applications by using analyis-suitable T-splines discretizations and here extended to large deformation Coulomb frictional contact problems. A Gauss-point-to-surface (GPTS) formulation is combined with the penalty method to treat the contact constraints in the discretized setting, as done in [9]. Using the Bèzier extraction, the suitable T-splines isogeometric discretizations are automatically generated for any analysis-suitable CAD geometry and easily incorporated into the finite element framework [10]. Some numerical examples show that the proposed contact formulation deliver accurate and robust predictions and demonstrate the potential of T-spline-based IGA to solve challenging contact problems in $2 \mathrm{D}$ and 3D.

\section{References}

[1] T.J.R. Hughes, J.A. Cottrell, Y. Bazilevs, Isogeometric analysis: CAD, finite elements, NURBS, exact geometry and mesh refinement, Comput. Methods Appl. Mech. Eng., 194(2005) 4135-4195.

[2] M.A. Puso, T.A. Laursen, J. Solberg, A segment-to-segment mortar contact method for quadratic elements and large deformations, Comput Methods Appl. Mech. Eng., 197 (2008) 555-566.

[3] D. Franke, A. Düster, V. Nübel, E. Rank, A comparison of the h-, p-, hp- and rp-version of the FEM for the solution of the 2D Hertzian contact problem, Comput. Mech., 45 (2010) 513-522.

[4] L. De Lorenzis, I. Temizer, P. Wriggers, G. Zavarise. A large deformation frictional contact formulation using NURBS-based isogeometric analysis, Int. J. Numer. Meth. Eng. 87(13) (2011) 1278-1300.

[5] L. De Lorenzis, P. Wriggers, G. Zavarise, A mortar formulation for 3D large deformation contact using NURBS-based isogeometric analysis and the augmented Lagrangian method, Comput. Mech. 49(1) (2012) $1-20$.

[6] I. Temizer, P. Wriggers, T.J.R. Hughes, Contact treatment in isogeometric analysis with NURBS, Comput. Methods Appl. Mech. Eng. 200 (2011) 1100-1112.

[7] I. Temizer, P. Wriggers, T.J.R. Hughes, Three-dimensional mortar-based frictional contact treatment in isogeometric analysis with NURBS, Comput. Methods Appl. Mech. Eng. 209 (2012) 115-128. 
[8] R. Dimitri, L. De Lorenzis, M. Scott, P.Wriggers, R.L. Taylor, G. Zavarise, Isogeometric large deformation frictionless contact using T-splines, Comput. Methods Appl. Mech. Eng. 269 (2014) 394-414.

[9] KA. Fischer, P. Wriggers, Frictionless 2D contact formulations for finite deformations based on the mortar method, Comput. Mech. 36 (2005) 226-244.

[10] M.A. Scott, X. Li, T.W. Sederberg, T.J.R. Hughes, Local refinement of analysis-suitable T-splines, Comput. Methods Appl. Mech. Eng. 213-216 (2012) 206-222. 


\title{
Following forces as an inverse contact algorithm
}

\author{
A. Konyukhov, R. Izi, K. Schweizerhof \\ Karlsruhe Institute of Technology
}

On of the essential finding withing the the geometrically exact theory for contact interactions [1], is that the theory can be understood and implemented in a very simple fashion starting with 2D examples, [2]. The geometrically exact theory for the computational contact mechanics is described in step-by-step manner, using examples of strict derivation from a mathematical point of view. The final goal of the theory is to construct in the independent of approximation form /so-called covariant form/ including application to high-order and isogeometric finite elements.

The essential findings within this reduction are several cases, which allowing to verify all computational algorithms step-by-step as well as to solve another famous computational mechanics.

One of this is representation of the algorithm for the following forces as an inverse contact algorithm. This computational algorithm is constructed as contact algorithm in a covariant geometrically exact form, in which the contact force is assumed as an external following force, while the corresponding tangent matrices are just selected from the already derived in closed form matrices. Such a method is independent on the approximation and is easily applied to the isogeometric finite elements.

Another example is FE modeling numerical modeling of the generalized Euler-Eytelwein problem, the analytically solution is recently obtained in [3].

\section{References}

[1] A. Konyukhov, K. Schweizerhof. Computational contact mechanics: geometrically theory for arbitrary shaped bodies, Springer, Heildelberg, New York, Dordrecht, London. (2013), 443 P.

[2] A.Konyukhov, R. Izi Introduction to Computational Contact Mechanics: A Geometrical Approach, Wiley, (2015), $304 \mathrm{P}$.

[3] A. Konyukhov. Contact of ropes and orthotropic rough surfaces. ZAMM Z. Angew. Math. Mech., 1-18 (2013). 


\title{
Application of the virtual element method to non-conforming contact interfaces
}

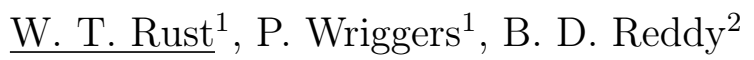 \\ ${ }^{1}$ G. W. Leibniz Universität Hannover \\ Institute of Continuum Mechanics \\ Appelstraße 11, 30167 Hannover, Germany \\ Email: rust@ikm.uni-hannover.de \\ ${ }^{2}$ University of Cape Town \\ Center of Computational Mechanics \\ Cape-Town
}

In the literature numerous formulations for classical contact and different discretization methods for the contact zone are available. Widely used are the penalty and Lagrange multiplier method to enforce contact constraints in the finite element environment. But especially non-conforming contact interfaces require a high effort in discretizing the contact surface in order to properly link the degrees of freedom. In this work we adapt the standard contact approaches penalty and Lagrange multiplier method to be used with virtual elements in the contact zone. This allows for an easy and robust contact algorithm for non-conforming meshes with a nodal enforcement of the contact constraint.

The main idea of the recently developed virtual element method is to find a single function that can project the nodal values on the element area while being compatible with the interpolated values on the boundary. This gives the advantage over classical finite elements that it is easily possible to discretize a geometry using convex or non-convex polygons with arbitrary number of vertices. Additionally it offers a simple formulation, easy integration and the possibility to achieve higher continuity.

For this work the virtual elements are implemented for the case of linear elasticity. Therefore a suitable polynomial decomposition has to be chosen. Furthermore, the elements in a contact problem generally can be subject to different loading states along the surface. Because the pure virtual ansatz function only represents an interpolation of the nodal values, the method has to be stabilized with an additional term to give good results. It will be shown in which way this was done in the present work in order to prevent effects such as locking.

The actual contact element consists mainly of a nodal projection algorithm. Here the feature of the virtual elements is used that arbitrary nodes can be inserted along the element boundary. Since this can be done without the need to recompute or change the element ansatz, an easy and robust algorithm is created that can actually transform a non-conforming mesh in a conforming mesh during the computation by projecting and inserting nodes on the opposing surface where needed. After matching the meshes the well known node-to-node contact procedures can easily be applied. In this talk the used methods are described and numerical examples are presented that show the behaviour in small deformation problems with and without friction. 


\title{
Non-unique Equilibria of a Statically Indeterminate System with Coulomb Friction
}

\author{
W. Steiner \\ University of Applied Sciences Upper Austria \\ Stelzhamerstrasse 23, 4600 Wels, Austria
}

The goal of this contribution is to show that the Coulomb friction model in a statically indeterminate system can result in ambiguous states of equilibrium. We demonstrate that a simple framework under specific external loading is in equilibrium and satisfies all conditions of Coulomb friction in the support points for several states of stress and deformation. The presented problem is motivated by the example of a two dimensional elastic body discussed in [1] where non-unique static solutions were found, too. In our presentation, a new method [2] which extends Castigliano's theorem to problems with Coulomb friction is applied to efficiently analyze numerous states of sticking and sliding of the framework's supports.

\section{References}

[1] P. Hild, An example of nonuniqueness for the continuous static unilateral contact model with Coulomb friction, C. R. Acad. Sci. Paris, Ser. I 337 (2003).

[2] W. Steiner, The use of Castigliano's theorem in Coulomb friction problems, Acta Mechanica, September 2014, Volume 225, Issue 9, pp 2471-2483. 


\title{
Efficient computation of surface-dominated problems using isogeometric finite elements
}

\author{
Christopher Zimmermann, Roger A. Sauer \\ Aachen Institute for Advanced Study in Computational Engineering Science (AICES) \\ RWTH Aachen University \\ Schinkelstr. 2, 52062 Aachen, Germany
}

\begin{abstract}
Physical problems are often dominated by local surface effects that determine the entire problem behaviour. Geometrically exact membrane and shell formulations can be used to model a wide range of mechanical applications [1]. An example is a water droplet coming into contact with a rigid substrate, where the contact boundary varies due to the deformation of the droplet. Another example is rough surface contact, where at very small asperities local stress peaks can appear. The global behaviour of the entire system is in both examples governed by the behaviour of local, critical surface domains. The accuracy of the computational results strongly depend on the surface discretization especially in local contact regions. Refinement of the entire mesh leads to high computational costs due to a great number of degrees of freedom located at surface domains of minor interest.

In this work, a technique for efficient computation of surface-dominated problems using isogeometric finite elements is presented. The tensor product structure of standard NURBS-based isogeometric analysis lacks local refinement [2]. To overcome this drawback, and to achieve highly locally refined meshes, the Locally Refined B-splines (LR B-splines) were introduced [3]. This refinement is directly performed in the parameter space that is represented locally. The LR B-splines still achieve the high accuracy of isogeometric analysis due to the smoothness of the basis functions that describe the geometry and approximate the solution field. An adaptive refinement indicator is used to determine isogeometric finite elements for refinement which are located at critical surface domains. By the use of the Bézier extraction operator [4], the isogeometric elements are included into an existing finite element simulation framework conveniently.

Numerical results are presented for computations of surface-dominated problems, involving frictional contact and multiple contact interactions. The technique of adaptive local refinement exhibits a decrease of the computational costs, while still achieving high accuracy in comparison to uniformly refined models.
\end{abstract}

\section{References}

[1] R. A. Sauer, T. X. Duong, and C. J. Corbett. A computational formulation for constrained solid and liquid membranes considering isogeometric finite elements. Computer Methods in Applied Mechanics and Engineering, 271(0) (2014), 48-68.

[2] T.J.R. Hughes, J. Cottrell, and Y. Bazilevs. Isogeometric analysis: CAD, finite elements, NURBS, exact geometry and mesh refinement. Comp. Meth. Appl. Mech. Engrg. 194 (2005), 4135-4195.

[3] T. Dokken, T. Lyche, and K. F. Pettersen. Polynomial splines over locally refined box-partitions. Computer Aided Geometric Design, 30(3) (2013), 331-356.

[4] M.J. Borden, M.A. Scott, J.A. Evans, and T.J.R. Hughes. Isogeometric finite element data structure based on Bézier extraction of NURBS. Int. J. Numer. Meth. Engng. 87 (2011), 15-41. 


\title{
Evaluation of a Finite Element Approach for Damping Determination
}

\author{
Olaf Peter Hentschel, Marius Bonhage, \\ Lars Panning-von Scheidt, Jörg Wallaschek \\ Institute of Dynamics and Vibration Research, \\ Leibniz University Hannover, \\ Appelstrasse 11, Hannover, 30167, Germany
}

Due to irreversible processes within the material, mechanical energy in vibratory systems is partly converted into thermal energy. This energy dissipation is known as damping. In freely oscillating systems, damping is characterized by a decreasing vibration amplitude, while in externally excited systems, the vibration amplitude is limited by the damping process. One of the primary objectives in many fields of mechanical engineering is the prediction and reduction of vibration amplitudes to reduce the risk of HCF. In this context, mechanical damping is of essential relevance for the dynamic behavior. Nonlinear calculation tools are used to calculate vibration amplitudes. To enable such a calculation, the specification of structural damping due to dissipations within the material is necessary.

In the full length paper, a well-known law for material damping description (see [1], [2] and [3]) is verified. Thereby, material damping is characterized as a function of local stress amplitudes. To identify the resulting structural damping, a calculation of local stresses is necessary. In a first step, an analytical approach is used to calculate the damping on the basis of the stress distribution. In a second step, the local stress distribution of the described structure is calculated using a finite element approach. In this context, damping is calculated using the identified discrete stress values through the structure. Hereby, an equivalent tensile stress theory is applied. Varying the mesh density, the resulting damping is compared to the calculated damping using the analytical results. Finally it can be seen that the quality of the calculated damping value depends on the quality of the calculated stress value and therefore on the mesh density.

\section{References}

[1] B. J. Lazan. Damping of Materials and Members in Structural Mechanics. Pergamon Press (1968). New York.

[2] Y. Kume, F. Hashimoto. Material damping of cantilever beams. Journal of Sound and Vibration 80(1) (1982). $1-10$.

[3] G. D. Gounaris, N. K. Anifantis. Structural damping determination by fnite element approach. Computers and Structures (1999). $445-452$. 


\title{
Modeling of non-stationary vibration signals based on the modified Kronecker sequences
}

\author{
Iulian Girip and Ligia Munteanu \\ Institute of Solid Mechanics, Romanian Academy, Bucharest
}

A vehicle is a multi degree of freedom system and its vibration behaviour (ride comfort) is often random with highly non-stationary characteristics. The vibration behaviour depends on the natural frequencies and mode shape of the vehicle. The paper proposes a model to represent non-stationary random vibration signals based on the modified Kronecker sequences implemented into quasi-Monte Carlo method. The modified Kronecker sequence constructed via generalizing golden ratio is one of the special types of low discrepancy sequences which have better dimensional projections [1]. The actual modeling and simulation of non-stationary random data is more suitable for seismological signals but not proper for the vehicle vibrations [2], [3]. Under these circumstances, this paper presents a new algorithm for finding the modified Kronecker sequences in order to generate non-stationary vehicle vibration signals which mostly withhold the amplitude-frequency-time distribution of the sample signal. The simulated signals hold the similar waveform and their fluctuations of energy as those of the sample signal [4]. Some examples to prove the effectiveness of the model conclude the paper.

\section{References}

[1] H.Chi. Generation of parallel modified Kronecker seequences. Monte Carlo Methods Appl., 19 (2013), 261271.

[2] L.Munteanu, V.Chiroiu, C.Brişan, D.Dumitriu, T.Sireteanu, S.Petre. On the 3D normal tire/off-road vibrocontact problem with friction. Mechanical Systems and Signal Processing (in press) (2014).

[3] C.Brişan, R.V.Vasiu, L.Munteanu. A modular road auto-generating algorithm for developing the road models for driving simulators. Transportation Research part C: Emerging Technologies, 26 (2013), 269-284.

[4] I.Girip. Computational and experimental Monte Carlo algorithms for modeling the systems with hysteretic characteristics. PhD thesis Institute of Solid Mechanics, September 2014. 


\title{
A homotopy method for the eigenvalue analysis of circular saw blade with inner slits
}

\author{
Junjie Luo, André Schmidt, Lothar Gaul \\ University of Stuttgart
}

Vibration reduction is one of the main concerns in the design of circular saw blades because it causes such problems as the deterioration of surface quality and the inaccuracy of sizing. Some studies indicate that the inclusion of inner slits with some viscoelastic media can reduce vibration and noise. Therefore, in this contribution we investigate how inner slits influence the structural behaviour of the saw blades under consideration, particularly the critical rotational speed, above which buckling occurs. Furthermore, inner slits filled with viscoelastic media cause internal damping, when relative deformation of the saw blade occurs. As internal damping can destabilize a system, stability needs to be examined.

This work is focused on how the stability of circular saw blades is influenced by different parameters, such as the rotational speed and the damping properties. This question leads to solving a parameter-dependent eigenvalue problem. Since bifurcation may occur in the spectrum of the eigenform, it is necessary to follow the paths of eigenvalues with respect to some specific parameter in both stable and unstable regions. Because the Newton-Raphson method is extremely sensitive to initial conditions, an algorithm based on homotopy is used to solve this problem [1]. The homotopy method, which is sometimes called the numerical continuation method, in principle, is to construct a homotopy from a trivial function to the one of interest. Smooth curves starting from the trivial solutions will then lead to the desired solutions under some circumstances. The method is applied to a perfect circular saw blade model and then is compared to the one with inner slits, which enables us to study the effects of different geometries. The results can contribute to the further optimization in the design of circular saw blades.

\section{References}

[1] J. Hadian, A.H. Nayfeh. Modal interaction in circular plates. Journal of Sound and Vibration (1990), 142(2), 279-292. 


\title{
Meta-structures for Cloaking Bending Waves
}

\author{
$\underline{\text { M. Gei }}^{1}$, D.J. Colquitt ${ }^{2}$, M. Brun ${ }^{3}$, A.B. Movchan ${ }^{4}$, N.V. Movchan ${ }^{4}$, I.S. Jones ${ }^{5}$ \\ ${ }^{1}$ University of Trento, Trento, Italy \\ ${ }^{2}$ Imperial College, London, U.K. \\ ${ }^{3}$ University of Cagliari, Cagliari, Italy \\ ${ }^{4}$ University of Liverpool, Liverpool, U.K. \\ ${ }^{5}$ Liverpool John Moores University, Liverpool, U.K.
}

The talk addresses an important issue of cloaking transformations for fourth-order partial differential equations representing flexural waves in thin elastic plates [1]. It is shown that, in contrast with the Helmholtz equation, the general form of the partial differential equation is not invariant with respect to the cloaking transformation. The significant result of this paper is the analysis of the transformed equation and its interpretation in the framework of the linear theory of pre-stressed plates. The paper provides a formal framework for transformation elastodynamics as applied to elastic plates. Furthermore, an algorithm is proposed for designing a broadband square cloak for flexural waves, which employs a regularised push-out transformation. Illustrative numerical examples show high accuracy and efficiency of the proposed cloaking algorithm. In particular, a physical configuration involving a perturbation of an interference pattern generated by two coherent sources is presented. It is demonstrated that the perturbation produced by a cloaked defect is negligibly small even for such a delicate interference pattern.

\section{References}

[1] D.J. Colquitt, M. Brun, M. Gei, A.B. Movchan, N.V. Movchan, I.S. Jones. Transformation elastodynamics and cloaking for flexural waves. J. Mech. Phys. Solids 72 (2014), 131-143 . 


\title{
Shrink fit with FGM-hub subject to heating and rotation
}

\author{
Eray Arslan, Werner Mack, \\ Department of Mechanical Engineering, Inonu University, Malatya, Turkey \\ Institute of Mechanics and Mechatronics, Vienna University of Technology, Vienna, Austria
}

Shrink fits find wide-spread use in mechanical engineering as an efficient means of transfer of moment; examples are shrunk-on rings, armature bandages in rotating machines, or tires of railway wheels [1]. Since the transferable moment essentially depends on the interface pressure between inclusion and hub, it should be as large as possible. This may be facilitated by a partially plastic design, which however also has some drawbacks like a possible permanent redistribution of the stresses after operating at high angular speeds and temperatures [2]. Hence, an interesting alternative (or at least supplement) to admitting partial plasticization is the use of a functionally graded material (FGM), particularly for the hub. As is well known, in a machine part of FGM the material properties vary continuously and can - to a certain extent - be tailored in an appropriate way [3]. Therefore, the aim of the present study is to investigate the essential features of a purely elastically designed shrink fit under plane stress conditions with solid homogeneous inclusion and functionally graded hub, taking both rotation and an elevated temperature into account. The material properties are presupposed to vary according to a power law in the radial direction. The basic grading law is however not postulated for the volume fractions of the constituents, but for the modulus of elasticity, and the dependence of the other physical quantities on the radius then is derived by the rule of mixture. This gives rise to a unique grading index for all the physical quantities (except for Poisson's ratio), and a closed-form solution of the differential equations can be found. Thus, a purely analytical discussion of the problem is possible.

It is shown that in case of radially decreasing density for a sufficiently large ratio of outer surface radius of the hub to interface radius a considerably better performance at rotation may be achieved, accompanied by a substantial saving of weight as compared to a homogeneous hub [4]. These two significant advantages must be weighted against the fact that a marginally worse evolution of the interface pressure with increasing temperature may occur. To discuss these features, a comprehensive comparison of the interface pressure in a shrink fit with homogeneous hub and with FGM-hub for arbitrary combinations of loading by rotation and by heating is provided.

\section{References}

[1] I. Dolezel, V. Kotlan, B. Ulrych. Design of joint between disk and shaft based on induction shrink fit. J. Comp. Appl. Math. 270 (2014), 52-62.

[2] W. Mack, M. Plöchl. Transient heating of a rotating elastic-plastic shrink fit. Int. J. Engng. Sci. 38 (2000), 921-938.

[3] J.J. Sobczak, L. Drenchev. Metallic functionally graded materials: a specific class of advanced composites. J. Mater. Sci. Technol. 29 (2013), 297-316.

[4] E. Arslan, W. Mack. Shrink fit with solid inclusion and functionally graded hub. Comp. Struct. 121 (2015), $217-224$. 


\title{
A projection approach to optimal control of elastic beam dynamics
}

\author{
Georgy Kostin \\ Institute for Problems in Mechanics RAS, Moscow, Russia
}

A projection approach is presented to model and optimize the controlled lateral motions of an elastic beam. The time $T$ of control process is fixed and the beam has the shape of a long rectangular prism which height $2 a_{1}$ is sufficiently greater than the sizes $2 a_{2}$ and $2 a_{3}$ of its cross section so that

$$
\{t, \boldsymbol{x}\} \in \Omega=(0, T) \times V, \quad \boldsymbol{x}=\left\{x_{1}, x_{2}, x_{3}\right\}^{\mathrm{T}} \in \mathbb{R}^{3}, \quad V=\left\{\boldsymbol{x}: x_{i}<a_{i}, i=1,2,3\right\} .
$$

The approach is based on an integro-differential statement [1] of the original initial-boundary value problem in linear elasticity with the velocity-momentum and stress-strain relations generalized according to

$$
\begin{gathered}
\int_{\Omega} \boldsymbol{v} \cdot \boldsymbol{q} \mathrm{d} \Omega=0 \quad \text { for } \forall \boldsymbol{q} \in L^{2}(\Omega) \text { and } \int_{\Omega} \xi: \tau \mathrm{d} \Omega=0 \quad \text { for } \forall \tau \in L^{2}(\Omega) ; \\
\boldsymbol{p}_{t}=\nabla \cdot \sigma, \quad \varepsilon=\frac{1}{2}\left(\nabla \boldsymbol{w}+\nabla \boldsymbol{w}^{\mathrm{T}}\right), \quad \boldsymbol{v}=\boldsymbol{w}_{t}-\rho^{-1} \boldsymbol{p}, \quad \xi=\varepsilon-\mathrm{C}^{-1}: \sigma \quad \text { for } \quad\{t, x\} \in \Omega ; \\
\sigma \cdot \boldsymbol{n}=\mathbf{0} \text { for } x_{1}=a_{1} \text { and } \quad x_{i}= \pm a_{i}, \quad i=2,3 ; \quad \boldsymbol{w}=\{0,0, u(t)\}^{\mathrm{T}} \text { for } x_{1}=-a_{1} ; \\
\boldsymbol{w}=\boldsymbol{p}=\mathbf{0} \text { for } t=0 .
\end{gathered}
$$

Here, the volume density $\rho$ and the elastic modulus tensor $C$ are mechanical parameters for the beam of homogeneous isotropic material, $\boldsymbol{n}$ is the output normal to the beam boundary. The displacement vector $\boldsymbol{w}(t, x)$, the momentum density vector $\boldsymbol{p}(t, x)$, and the stress tensor $\sigma(t, x)$ are unknown variables, whereas $\boldsymbol{q}(t, x)$ is a vector of virtual momentum density and $\tau(t, x)$ is a tensor of virtual stresses. The function $u(t) \in \mathbb{R}$ is a boundary control input.

On the basis of piecewise polynomial approximations of both the trial variables $\boldsymbol{w}, \boldsymbol{p}, \sigma$ and the test functions $\boldsymbol{v}, \tau$, a semi-discretization scheme is worked out by taking into account the properties of the beam symmetry [2]. An explicit energy criterion of solution quality is given by

$$
\Delta=\Phi \Psi^{-1}<\delta \ll 1 \quad \text { with } \Phi=\frac{1}{2} \int_{\Omega}(\rho \boldsymbol{v} \cdot \boldsymbol{v}+\xi: C: \xi) \mathrm{d} \Omega \quad \text { and } \quad \Psi=\frac{1}{2} \int_{\Omega}\left(\rho^{-1} \boldsymbol{p} \cdot \boldsymbol{p}+\varepsilon: \mathrm{C}: \varepsilon\right) \mathrm{d} \Omega
$$

where $\Phi[u]$ defines the global energy error of approximate solutions and $\Psi[u]$ is the time integral of the total mechanical energy stored by the beam during the control process.

The resulting ODE system is used to design the optimal control $u^{*}(t)$ that minimizes the mean energy of the elastic beam [3] as follows:

$$
J\left[u^{*}\right]=\min _{u \in H^{1}(0, T)} J[u] \quad \text { with } \quad J=T^{-1} \Psi
$$

subject to the terminal constraints

$$
\boldsymbol{w}(T, x)=\left\{0,0, w_{T}\right\}^{\mathrm{T}} \text { and } \boldsymbol{p}(T, x)=\mathbf{0} \quad \text { for } \quad \boldsymbol{x} \in V .
$$

The numerical results obtained for the spatial case are analyzed and compared with the conventional Bernoulli beam model.

\section{References}

[1] G.V. Kostin, V.V. Saurin. Integrodifferential Relations in Linear Elasticity. De Gruyter, Berlin, 2012.

[2] G.V. Kostin, V.V. Saurin. Modelling and analysis of the natural vibrations of a prismatic elastic beam based on a projection approach. J. Appl. Math. Mech. 75 (2011), 700-710.

[3] G.V. Kostin. The construction of optimal control for the motion of elastic bodies by using the method of integro-differential relations. J. Comp. Sys. Sci. Int. 46 (2007), 532-542. 


\title{
Energy-momentum conserving discretization of mixed shell elements for large deformation problems
}

\author{
Alexander Janz, Peter Betsch \\ Institute of Mechanics \\ Karlsruhe Institute of Technology \\ Otto-Ammann-Platz 9, 76131 Karlsruhe, Germany \\ peter.betsch@kit.edu, alexander.janz@kit.edu \\ www.ifm.kit.edu
}

In the present work we consider structure-preserving integration methods in the context of mixed finite elements. Low-order mixed finite elements such as the shell element proposed in [1] typically exhibit improved coarse mesh accuracy. On the other hand energy-momentum (EM) consistent time-stepping schemes have been developed in the realm of nonlinear structural dynamics to enhance the numerical stability properties. EM schemes typically exhibit superior robustness and thus offer the possibility to use large time steps while still producing physically meaningful results. Accordingly, combining mixed finite element discretizations in space with EM consistent discretizations in time shows great promise for the design of numerical methods with superior coarse mesh accuracy in space and time.

Starting with a general Hu-Washizu-type variational formulation we develop a second-order accurate structurepreserving integration scheme. The present approach is applicable to a large number of mixed finite element formulations. As sample application we will deal with the mixed shell element [1]. The resulting method can be viewed as mixed extension of the EM method proposed in [2]. Numerical examples dealing with large deformations will show the improved coarse mesh accuracy in space and time of the advocated approach.

\section{References}

[1] W. Wagner and F. Gruttmann. A robust non-linear mixed hybrid quadrilateral shell element. Int. J. Numer. Meth. Engng, Vol. 64, 635-666, 2005.

[2] P. Betsch and N. Sänger. On the use of geometrically exact shells in a conserving framework for flexible multibody dynamics. Comput. Methods Appl. Mech. Engrg., Vol. 198, 1609-1630, 2009. 


\title{
The Strong Formulation Finite Element Method Applied to Structural Mechanics Problems
}

\author{
Francesco Tornabene, Nicholas Fantuzzi, Michele Bacciocchi, Erasmo Viola \\ DICAM Department, School of Engineering and Architecture, University of Bologna, ITALY
}

The Strong Formulation Finite Element Method (SFEM) [1] is a numerical approach that can be used for solving civil, environmental, mechanical, aerospace and naval engineering problems. Generally, practical engineering problems are complex due to geometry, material and load discontinuities. For solving them, it is necessary to divide the whole domain into finite elements of arbitrary shape. The mapping technique is introduced at this level to transform an arbitrarily shaped element to a parent element (computational element). The classic Finite Element Method (FEM) uses the above procedure and the problem at the parent element level is solved by means of weak (variational) formulation. On the contrary, the SFEM summarises a class of methods that is able to approximate total and partial derivatives at discrete points, thus the solution is found in its strong form. The SFEM has its own roots in the Differential Quadrature Method (DQM), which was introduced in the early 1970s. Nevertheless, DQM does not allow to treat arbitrarily shaped domains and problems where discontinuities are present. These features are proper of finite element approaches, in which the global domain is divided into several smaller elements, and after the assembly procedure they solve the complete system. Therefore the SFEM is an hybrid scheme given by the DQM and the FEM. The most significant difference between these two methodologies lays on the formulation used for solving the parent element. In order to clarify the idea about the fact that the SFEM comprehends several numerical techniques, the reader can review a class of methods in the article [2], where it has been clarified that the most important and wide-spread numerical approaches are a sub-class of the method of weighted residuals. Moreover a former review article about DQM can be found in [3] where a state of the art of that time was given. Unfortunately the authors limited their analysis to DQM and they did not focus their attention on the generalization of the DQM concepts to a wider prospective. As far as the authors are concerned, the first paper regarding the present topic was presented in [4]. The discussion was extended in a survey paper published recently [5], where a significant historical review about strong and weak numerical tools was carried out. The authors provided stability and accuracy of one-dimensional and two-dimensional problems when compared to classic exact solutions related to structural problems, such as rods, beams, membranes and plates. The first application of the SFEM regarding one-dimensional in-plane multi-stepped and multi-damaged arches was published in [6]. The authors investigated the vibration of thin membranes in a review paper [7], where several well-known numerical applications were compared to the literature. Some other applications were presented concerning the behavior of elastostatic and elastodynamic plane structures in $[8,9,10,11]$. Later the authors presented the SFEM applied to the modal analysis of Reissner-Mindlin plates [12, 13]. The SFEM based on DQM and Radial Basis Function (RBF) method has been presented in the work [14]. Finally in the works [15, 16] a particular emphasis has been given to the stress recovery procedure for the evaluation of the three dimensional strain and stresses at all the physical points of the problem. As a definition the SFEM is a numerical procedure that decomposes the physical domain or problem in finite elements and used the strong formulation inside each element mapped on the parent (or computational) element. When in the above procedure the weak formulation is used (instead of the strong form), the WFEM is defined. The latter is well-known in literature as FEM. This paper aims to investigate the application of the SFEM to structural mechanics problems. Since its numerical solutions depend on the number of collocation points, the basis functions used, the location of the points and the number of domain divisions, the authors report in graphical form the stability, accuracy and reliability of the present technique. In this way several aspects are raised and remarks are given as closure.

\section{References}

[1] F. Tornabene, N. Fantuzzi (2014) - Mechanics of Laminated Composite Doubly-Curved Shell Structures. The Generalized Differential Quadrature Method and the Strong Formulation Finite Element Method, Esculapio, Bologna. ISBN: 978-88-7488-687-6.

[2] B.A. Finlayson, L.E. Scriven, The method of weighted residual: a review, Appl. Mech. Rev., 19 (1966) 735-748. 
[3] C.W. Bert, M. Malik, Differential quadrature method in computational mechanics, Appl. Mech. Rev., 49 (1996) 1-27.

[4] F. Tornabene, N. Fantuzzi, M. Bacciocchi, The Strong Formulation Finite Element Method: Stability and Accuracy, Fract. Struct. Integr., 29 (2014) 251-265.

[5] F. Tornabene, N. Fantuzzi, F. Ubertini, E. Viola. Strong Formulation Finite Element Method Based on Differential Quadrature: A Survey, Appl. Mech. Rev., (2014) In Press. doi:10.1115/1.4028859.

[6] E. Viola, M. Dilena, F. Tornabene, Analytical and Numerical Results for Vibration Analysis of MultiStepped and Multi-Damaged Circular Arches, J. Sound Vib. 299 (2007) 143-163.

[7] N. Fantuzzi, F. Tornabene, E. Viola, Generalized Differential Quadrature Finite Element Method for Vibration Analysis of Arbitrarily Shaped Membranes, Int. J. Mech. Sci. 79 (2014) 216-251.

[8] E. Viola, F. Tornabene, E. Ferretti, N. Fantuzzi, On Static Analysis of Composite Plane State Structures via GDQFEM and Cell Method, CMES 94 (2013) 421-458.

[9] E. Viola, F. Tornabene, E. Ferretti, N. Fantuzzi, Soft Core Plane State Structures Under Static Loads Using GDQFEM and Cell Method, CMES 94 (2013) 301-329.

[10] E. Viola, F. Tornabene, E. Ferretti, N. Fantuzzi, GDQFEM Numerical Simulations of Continuous Media with Cracks and Discontinuities, CMES 94 (2013) 331-369.

[11] N. Fantuzzi, New insights into the strong formulation finite element method for solving elastostatic and elastodynamic problems, Curved Layer. Struct. 1 (2014) 94-127.

[12] E. Viola, F. Tornabene, N. Fantuzzi, Generalized Differential Quadrature Finite Element Method for Cracked Composite Structures of Arbitrary Shape, Compos. Struct. 106 (2013) 815-834.

[13] N. Fantuzzi, F. Tornabene, E. Viola, Four-Parameter Functionally Graded Cracked Plates of Arbitrary Shape: a GDQFEM Solution for Free Vibrations, Mech. Adv. Mat. Struct. (2014) In Press. doi: 10.1080/15376494.2014.933992.

[14] N. Fantuzzi, F. Tornabene, E. Viola, A.J.M. Ferreira, A Strong Formulation Finite Element Method (SFEM) Based on RBF and GDQ Techniques for the Static and Dynamic Analyses of Laminated Plates of Arbitrary Shape, Meccanica 49 (2014) 2503-2542.

[15] N. Fantuzzi, F. Tornabene, Strong Formulation Finite Element Method for Arbitrarily Shaped Laminated Plates - I. Theoretical Analysis, Adv. Aircraft Space. Sci. 1 (2014) 124-142.

[16] N. Fantuzzi, F. Tornabene, Strong Formulation Finite Element Method for Arbitrarily Shaped Laminated Plates - II. Numerical Analysis, Adv. Aircraft Space. Sci. 1 (2014) 143-173. 


\title{
Weighted overconstrained least-squares mixed finite elements for hyperelasticity
}

\author{
Alexander Schwarz, Karl Steeger, Jörg Schröder \\ Institute of Mechanics, Faculty of Engineering, \\ University Duisburg-Essen, Universitätsstr. 15, 45141 Essen, GERMANY \\ alexander.schwarz@uni-due.de, karl.steeger@uni-due.de, j.schroeder@uni-due.de
}

The main goal of this contribution is the improvement of the approximation quality of geometrically nonlinear elastic problems solved by the least-squares finite element method (LSFEM). Compared with other variational approaches as for example the Galerkin method, a general drawback of least-squares formulations is the unsatisfying approximation quality especially for lower-order elements, see e.g. [1]. In the current work we present a mixed element based on a first-order stress-displacement formulation resulting from a classical least-squares method. By including the stress symmetry in an explicit form, similar to [2], an overconstrained system is derived. We consider different weights for this additional constraint and use a mixed least-squares formulation with a maximal cubic polynomial interpolation order. For the continuous approximation of the stresses Raviart-Thomas elements are used, while for the displacements standard conforming elements are employed. We provide some benchmark problems with the main focus on bending-dominated hyperelastic problems. The proposed formulation is compared to recently developed classical LSFEMs and Galerkin FEMs, in order to show the improvement of performance and accuracy.

\section{References}

[1] A. Schwarz, J. Schröder, G. Starke, A modified least-squares mixed finite element with improved momentum balance, Int. J. Numer. Meth. Eng. 81 (2010) 286-306.

[2] A. Schwarz, K. Steeger and J. Schröder. Weighted overconstrained least-squares mixed finite elements for static and dynamic problems in quasi-incompressible elasticity, Comp. Mech. 54 (2014) 603-612. 


\title{
A stress-velocity least-squares mixed finite element formulation for incompressible elastodynamics.
}

\author{
Carina Nisters $^{1}$, Alexander Schwarz ${ }^{1}$, Jörg Schröder ${ }^{1}$ \\ ${ }^{1}$ Institute for Mechanics, Faculty of Engineering, \\ University Duisburg-Essen, Universitätsstr. 15, 45141 Essen, GERMANY \\ carina.nisters@uni-due.de, alexander.schwarz@uni-due.de, j.schroeder@uni-due.de
}

The purpose of the presented work is the development and implementation of a stress-velocity least-squares mixed finite element formulation under the assumptions of small strain elastodynamics. The idea of implementing a stress-velocity formulation is well-known in fluid-dynamics and is the basic motivation of the $\sigma-v$ formulation in solid dynamics. The formulation of the stress-velocity approach in fluid dynamics is found in [1]. The $L_{2}$-norm minimization of the time-discretized residuals of the given first-order system of partial differential equations leads to a functional depending on the stresses $\boldsymbol{\sigma}$ and the velocities $\mathbf{v}$

$$
\mathcal{F}(\boldsymbol{\sigma}, \mathbf{v})=\frac{1}{2}\left(\left\|\alpha_{1}(\operatorname{div} \boldsymbol{\sigma}+\mathbf{f}-\rho \mathbf{a})\right\|_{0}^{2}+\left\|\alpha_{2}\left(\mathbb{C}^{-1} \boldsymbol{\sigma}-\nabla^{s} \mathbf{u}\right)\right\|_{0}^{2}\right)
$$

with $\alpha_{i}(i=1,2)$ denoting weighting factors, wherein $\mathbf{f}$ describes the body forces, $\rho$ is the density of the solid and $\mathbb{C}$ is the fourth-order material tensor. The accelerations are denoted with $\mathbf{a}=\mathbf{a}(\mathbf{v})$ and the displacements with $\mathbf{u}=\mathbf{u}(\mathbf{v})$, both values are depending on the velocities $\mathbf{v}$ due to the applied time discretization. The stresses are interpolated with vector-valued Raviart-Thomas interpolation functions, while for the approximation of the velocities a standard polynomial interpolation is chosen, see e.g. [2]. First, we examine the influence of different time integration schemes and secondly investigate weighting factors $\alpha_{i}$ for each residual, which are tested for the spectrum of numerical functionality, compared with the stress-displacement formulation in [3]. In the numerical examples, the proposed stress-velocity mixed least-squares finite element formulation is tested and the results are compared to alternative standard approaches.

\section{References}

[1] Z. Cai, B.Lee, and P.Wang, Least-squares methods for incompressible Newtonian fluid flow: Linear stationary problems, SIAM J. NUMER. ANAL. Vol. 42, No. 2 (2004) 843-859.

[2] A. Schwarz, J. Schröder, and G. Starke, A modified least-squares mixed finite element with improved momentum balance, Int. J. Numer. Meth. Eng. 81 (2010) 286-306.

[3] K. Steeger, A. Schwarz, and J. Schröder. A weighted least-squares mixed finite element formulation for quasi incompressible elastodynamics. PAMM. Vol. 11 (2011) 287-288. 


\title{
Comparison of a mixed least-squares formulation using different approximation spaces.
}

\author{
Karl Steeger ${ }^{1}$, Jörg Schröder ${ }^{1}$, Alexander Schwarz ${ }^{1}$ \\ ${ }^{1}$ Institute of Mechanics, Faculty of Engineering, \\ University Duisburg-Essen, Universitätsstr. 15, 45141 Essen, GERMANY \\ karl.steeger@uni-due.de, j.schroeder@uni-due.de, alexander.schwarz@uni-due.de
}

The main goal of the present work is the comparison of the performance of a least-squares mixed finite element formulation where the solution variables (displacements and stresses) are interpolated using different approximation spaces. Basis for the formulation is a weak form resulting from the minimization of a least-squares functional, compare e.g. [1]. The stresses are approximated in $H^{1}(\mathcal{B})$ or in $H(\operatorname{div}, \mathcal{B})$, whereas the displacements are always interpolated in $H^{1}(\mathcal{B})$. As suitable functions for $H^{1}(\mathcal{B})$ standard interpolation polynomials of Lagrangian type are chosen. For the conforming discretization of the Sobolev space $H(\operatorname{div}, \mathcal{B})$ vector-valued Raviart-Thomas interpolation functions, see also [2], are used. The resulting elements are named as $P_{m} P_{k}$ and $R T_{m} P_{k}$. Here $m$ (stresses) and $k$ (displacements) denote the approximation order of the particular interpolation function. For the comparison we consider two-dimensional structural mechanical problems under plain strain conditions and small strain assumptions.

\section{References}

[1] Cai, Z. and Starke, G., [2004], 'Least-squares methods for linear elasticity", SIAM J. Numer. Anal., Vol. 42, pp. 826-842.

[2] Raviart, P. A. and Thomas, J. M., [1977], "A mixed finite element method for second order elliptic problems", Lectures Notes in Math., Vol. 606, Springer Verlag. 


\title{
A surface oriented solid formulation based on a hybrid Galerkin-collocation method
}

\author{
$\underline{\text { Sven Klinkel }}^{1}$, Lin Chen $^{1}$, Wolfgang Dornisch ${ }^{2}$ \\ ${ }^{1}$ Institute of Structural Analysis and Dynamics, RWTH Aachen University \\ ${ }^{2}$ Institute of Applied Mechanics, University of Kaiserslautern
}

The contribution is concerned with a numerical method to analyze the mechanical behavior of 3D solids. The method employs directly the geometry defined by the boundary representation modeling technique, which is frequently used in CAD to define solids. It combines the benefits of the isogeometric analysis methodology [1] with the scaled boundary finite element method [2]. In the present approach, only the boundary surfaces of the solid is discretized. No tensor-product structure of three-dimensional objects is exploited to parameterize the physical domain. The weak form is applied only on the boundary surfaces. The governing partial differential equations of elasticity are transformed to an ordinary differential equation (ODE) of Euler type. For the numerical analysis the isogeometric Galerkin approach is employed to approximate the displacement response at the boundary surfaces. It exploits the two-dimensional NURBS objects to parameterize the boundary surfaces. To solve the Euler type ODE, the NURBS based collocation approach is applied, see [3]. The accuracy of the method is validated against the analytical solutions. The presented method is able to analyze solids, which are bounded by an arbitrary number of surfaces. Numerical examples will show the capabilities of the presented method.

\section{References}

[1] T. J. R. Hughes, J. A. Cottrell, Y. Bazilevs. Isogeometric analysis: CAD, finite elements, NURBS, exact geometry and mesh refinement. Comput. Meth. Appl. Mech. Engrg. 194 (2005), 4135-4195.

[2] C. Song, J. P. Wolf. The scaled boundary finite-element method-alias consistent infinitesimal finite-element cell method-for elastodynamics. Comput. Methods Appl. Mech. Engrg. 147 (1997), 329-355.

[3] S. Klinkel, L. Chen, W. Dornisch. A NURBS based hybrid collocation-Galerkin method for the analysis of boundary represented solids. Comp. Meth. Appl. Mech. Engrg. 284 (2015), 689-711. 


\title{
Application of Discontinuous Galerkin Finite Element Method for Discontinuities in Small Deformation Regimes
}

\author{
H. R. Bayat, S. Reese, S. Wulfinghof \\ Institute of Applied Mechanics (IFAM) RWTH Aachen University, Germany
}

In this paper, finite element formulation is defined in the frame work of discontinuous Galerkin method. Discontinuous Galerkin methods $(\mathrm{dGm})$ are classically used in fluid mechanics, however recently their application in solid mechanics has become more vivid among scientists. Of special interest is their application in problems tackling with cracks [1], in elliptic problems with constraints such as incompressibility which leads to volumetric locking phenomenon and also in some structural models of shells, plates and beams with constraints for compatibility relation between the degrees of freedom which brings about shear locking [2].

While classical continuous Galerkin methods must be piecewise affine conforming (continuous), dG methods can be applied for discontinuities across element boundaries by application of piecewise constant approximates within each element and use of slope limiters to ensure stability for higher order schemes [2]. In the present work, we implement the Q1SP [3] element in dG formulation and investigate its behaviour for small deformation regimes.

\section{References}

[1] J. Mergheim, E. Kuhl, P. Steinmann. A hybrid discontinuous Galerkin/interface method for the computational modelling of failure. Communications in Numerical Methods in Engineering (2004), 511-519.

[2] A. Lew, A.T. Eyck, R. Rangarajan. Some Applications of Discontinuous Galerkin Methods in Solid Mechanics. IUTAM Symposium on Theoretical, Computational and Modelling Aspects of Inelastic Media. (2008), 167-189.

[3] S. Reese, P. Wriggers, B.D. Reddy. A new locking-free brick element technique for large deformation problems in elasticity. Computers and Structures (2000), 291-304. 


\title{
A New Mixed Finite Element for the Analysis of Structures with Material and Geometric Nonlinearities
}

\author{
Nicola A. Nodargi and P. Bisegna \\ Department of Civil Engineering and Computer Science \\ University of Rome "Tor Vergata", via del Politecnico 1, 00133 Rome, Italy \\ E-mail: nodargi@ing.uniroma2.it, URL:www.dicii.uniroma2.it
}

\begin{abstract}
The analysis of structural behavior in engineering applications often requires to take into account material and geometric nonlinearities. Aim of the present work is the formulation of a finite element which allows an accurate and effective treatment of such problems.

In the recent literature, mixed finite elements have been proved to be especially appropriate to overcome wellknown limits of the classical displacement-based formulation (for an overview on mixed elements, see [1]). In a nonlinear material framework, usually involving direct strain-stress relationships, the natural derivation of such elements is based on the Hu-Washizu functional (e.g., see [2]). A modified version of such functional, in which internal equilibrium is a priori enforced in strong form (for instance, see [3]), is here adopted. Accordingly, the displacement field interpolation is only required on the element boundary and square-integrable interpolations can be selected for stresses and strains. In particular, an interpolation which is continuous only within the individual element is selected for the self-equilibrated stress field (for instance, see $[4,5]$ ), while the strain field is expanded by a linear combination of Dirac delta measures centered on element Gauss points. As a consequence, stress and strain interpolation parameters can be condensed at element level by solving the local compatibility and constitutive equations, whereas the equilibrium is imposed at structural level with respect to the unknown nodal displacement degrees of freedom.

In the present work, an original procedure to carry out the element state determination is proposed. More specifically, the local equations are regarded as the stationarity conditions associated to a linearly constrained minimization problem involving an appropriate incremental element energy function. An efficient minimization algorithm, based on a Newton-Raphson iterative scheme in conjunction with a suitable line-search method, is then discussed.

Structural scale applications are carried out for assessing the performances of the proposed finite element. Geometric nonlinearities are treated by means of the the polar decomposition based corotational formulation proposed in [6]. Besides elastic case studies, applications involving elasto-plastic materials or shape memory alloys are considered. In particular, numerical results show that the present element is free from locking and spurious zero-energy modes, and exhibits a good response in presence of mesh distortion.
\end{abstract}

\section{References}

[1] D. Boffi, F. Brezzi, M. Fortin. Mixed finite element methods and applications. Springer-Verlag, Berlin, (2013).

[2] P. Wriggers, J. Korelc. On enhanced strain methods for small and finite deformations of solids. Comput. Mech. 18(1996) 413-428.

[3] R.L. Taylor, F.C. Filippou, A. Saritas, F. Auricchio. A mixed finite element method for beam and frame problems. Comput. Mech. 31(2003), 192-203.

[4] A. Madeo, G. Zagari, R. Casciaro. An isostatic quadrilateral membrane finite element with drilling rotations and no spurious modes. Finite Elem. Anal. Des. 50(2012), 21-32.

[5] R. Grimaldi, D. Addessi, V. Ciampi. Localization and regularization behavior of mixed finite elements for 2D structural problem with damaging material. Comput. Methods Appl. Mech. Engrg. 197(2007), 255-264.

[6] F. Caselli, P. Bisegna. Polar decomposition based corotational framework for triangular shell elements with distributed load. Int. J. Numer. Methods Eng. 95(2013), 499-528. 


\title{
Discussion of the Particle Finite Element Method in the Context of Strength of Materials
}

\author{
Matthias Sabel, José York Duran, Christian Sator, Ralf Müller \\ Institute of Applied Mechanics \\ University of Kaiserslautern, \\ Gottlieb-Daimler-Str., \\ D-67663, Kaiserslautern, Germany
}

\begin{abstract}
The particle finite element method (PFEM) combines the benefits of discrete modeling techniques and continuum based methods. While discrete models are well suited for tracking large changes in the topology, they are computationally expensive for problems with large length- and time scales. Standard finite element methods (FEM) are a common way to solve problems with macroscopic length and time scales. However, simulations with large configurational changes like a separation of material require frequent remeshing. The PFEM algorithm used for this work first determines the boundary of a set of particles which is accomplished with the so called $\alpha$-shape method. This method originates from the field of computer graphics and is named after its crucial parameter $\alpha$. After detecting the boundary, the region can be meshed with finite elements. Subsequently, a FEM problem can be solved, where the deformation data of previous load steps have to be included. Finally, the resulting displacements are used to update the particle coordinates, and the history variables are stored for the following load step. A central part in the analysis is the determination of the boundary. Although the PFEM has been applied to a number of complex applications with free boundaries (liquids), so far only experience based values for $\alpha$ are provided in literature. The numerical examples in this contribution show that the choice of $\alpha$ affects the material behavior. A simple tensile test shows the influence of $\alpha$ on the material response. High values represent tough materials, while a low $\alpha$ is associated with brittle behavior. Other examples studied in this work include machining simulations. Especially the formation of chips leads to large deformations and topological changes, which is why the machining simulations are well suited to demonstrate the advantages of the PFEM. In these machining simulations, the cutting force is studied for a varying $\alpha$ and again a dependency of the material response on $\alpha$ is observed.
\end{abstract}




\title{
Nonlinear SFEM with fluctacting input parameters
}

\author{
I. Caylak, Alex Dridger, Rolf Mahnken \\ University of Paderborn, Chair of Engineering Mechanics
}

In many engineering applications the materials, e.g. adhesives, are heterogeneous. This heterogeneity leads often to uncertainty in the material properties and to uncertainty in the mechanical response. Therefore, macroscopically heterogeneous adhesives should be modelled by a stochastic approach instead a deterministic approach. This contribution presents a nonlinear stochastic finite element method (SFEM). Mostly used SFEMs are Monte Carlo (MC), Galerkin and polynomial chaos expansion (PCE) [1, 2].

The key idea of our contribution is to consider the uncertainity by random material parameters, which are modeled as stochastic fields. Then, from experimental data the distribution of the random variables, i.e. elastic and inelastic material parameters, are known. Consequently, elastic and inelastic material parameters are expanded with the PCE. Furthermore, in the context of material nonlinear problems the stresses and the material tangent, respectively, are functions of stochastic variables. The collacation method is used in order to calculate the PC coefficients of functions. In addition, during the local Newton algorithm in order to calculate the plastic corrector $\Delta \lambda$ also a stochastic Newton method is considered.

As a numerical example we consider the static problem for uniaxial tension of the rectangular plate. This problem is investigated under plane strain conditions with suitable boundary conditions and some stochastic material parameters. For the material description the non-linear elasto-plastic material model of adhesives as developed in Mahnken and Schlimmer [3] is used. Results of the deterministic solution and the influence of the distribution of the material parameters are presented.

\section{References}

[1] H. G. Matthies and A. Keese. Galerkin methods for linear and nonlinear elliptic stochastic partial differential equations. Comput. Methods Appl. Mech. Engrg., 194:1295-1331, (2005), 491-503.

[2] R. G. Ghanem and P. D. Spanos. Stochastic Finite Elements: A Spectral Approach. Springer-Verlag, New York, (1991)

[2] R. Mahnken; M. Schlimmer: Simulation of strength difference in elasto-plasticity for adhesive materials. Int. J. Numer Met Eng 63, Nr. 10, 1461-1477, (2005) 


\title{
A high-order enrichment strategy for the finite cell method
}

\author{
Meysam Joualain, Alexander Düster \\ Numerical Structural Analysis with Application in Ship Technology (M-10) \\ Hamburg University of Technology, Schwarzenbergstr. 95C, 21073 Hamburg, Germany
}

The modeling and the computation of structures made of heterogeneous materials is very challenging. Developing a suitable finite element model of such structures involves a lot of efforts and is generally very time consuming. Moreover, the resulting numerical models are usually very large and thus require considerable computational resources. The finite cell method (FCM), which is an immersed boundary method based on finite elements, is considered as a possible method for numerically handling these kinds of problems, especially for homogenization applications and multiscale problems $[1,2,3]$. The main characteristic of the FCM is that thanks to the application of the immersed boundary method - the mesh can be defined independently from the geometry. This allows to employ Cartesian grids for the purpose of meshing, for instance. In addition, the employment of high-order shape functions together with the FCM makes it possible to achieve high-order convergence rates and, in consequence, to save computational resources.

Although the nonconforming meshes in the FCM lead to a significant simplification in the modeling part, they might cause several difficulties on the solution part. One of the possible problems will occur if the solution exhibits a kink inside an element, for instance at material interfaces. In such a case, the regularity of the solution is lost and so the optimal convergence rate might be deteriorated. In this presentation we will address this issue and show some examples where this can be problematic. We will also explore different remedies by considering the partition of unity method as well as domain decomposition techniques [4]. The proposed solution is based on defining high-order enrichment functions with the help of a high-order implicit representation of the material interface. To this end, we will apply Lagrange shape functions defined on Babuška-Chen points together with the level-set function. Several numerical examples will serve to illustrate the performance of the proposed method.

\section{References}

[1] J. Parvizian, A. Düster, E. Rank. Finite cell method - h- and p-extension for embedded domain problems in solid mechanics. Comput. Mech. 41 (2007), 121-133

[2] A. Düster, J. Parvizian, Z. Yang, E. Rank. The finite cell method for three-dimensional problems of solid mechanics. Comput. Methods in Appl. Mech. Eng. 197 (2008), 3768-3782

[3] A. Düster, H.-G. Sehlhorst, E. Rank. Numerical homogenization of heterogeneous and cellular materials utilizing the finite cell method. Comput. Mech. 50 (2012), 413-431

[4] M. Joulaian, A. Düster. Local enrichment of the finite cell method for problems with material interfaces. Comput. Mech. 52 (2013), 741-762 


\title{
An efficient and robust Reissner-Mindlin shell formulation for isogeometric analysis
}

\author{
Wolfgang Dornisch $^{1}$, Ralf Müller ${ }^{1}$, Sven Klinkel ${ }^{2}$ \\ ${ }^{1}$ Institute of Applied Mechanics, University of Kaiserslautern \\ ${ }^{2}$ Chair of Structural Analysis and Dynamics, RWTH Aachen University
}

Isogeometric analysis was introduced in 2005 by Hughes et al. [4]. The novel idea is to use the basis functions of the geometry description of the design model also for the analysis. Thus, the geometry is represented exactly on element level. A closer integration of design and analysis is fostered by the usage of one common geometry model for design and analysis. A prevalent choice for the geometry description in isogeometric shell analysis is Non-Uniform Rational B-spline (NURBS) surfaces, which are commonly used in industrial design software to model thin structures. In order to directly compute structures defined by NURBS surfaces, an efficient isogeometric shell formulation is required. One of the main advantages of NURBS-based isogeometric analysis is the higher continuity between elements, which occurs if $k$-refinement is used for order elevation. The higher continuity requires special efforts to arrive at an efficient rotational-based shell formulation. In [1] it is shown that concepts which are adapted from common Lagrange-based Reissner-Mindlin shell formulations potentially yield divergent deformation convergence behavior for order elevation. A new rotation formulation adapted to isogeometric analysis is presented in [1]. This formulation makes high-order computations competitive in comparison to low-order computations. An extension to geometries with kinks and sharp interfolds is presented in [2]. However, this formulation lacks efficiency in comparison to standard Lagrange-based shell formulation.

In this contribution an isogeometric Reissner-Mindlin shell formulation derived from the continuum theory is presented. The shell body is described by a shell reference surface, which is defined by NURBS surfaces, and a director vector. The director vector in the reference configuration is interpolated from nodal values, which are determined in a pre-process by a patch-wise $L_{2}$-projection. The director vector in the current configuration is computed by an orthogonal rotation using Rodrigues' tensor in every integration point. The interpolation of the axial vector of the rotation is performed according to an idea mentioned in [5] and applied in [3]. In the framework of $C^{0}$-continuous shell formulations the higher numerical effort does not pay off. But the more accurate interpolation of the rotations entails significantly more precise results for NURBS-based isogeometric analysis with high continuity. A multiplicative update formulation for the rotations accounts for finite rotations. Several benchmark examples show the superior accuracy of the presented shell formulation for linear and nonlinear computations. The convergence behavior is shown to be correct for $k$-refinement. The computational cost of the new formulation are compared to standard formulations, assessing both costs for a given mesh size and costs to fall below a predefined error bound.

\section{References}

[1] W. Dornisch, S. Klinkel, B. Simeon. Isogeometric Reissner-Mindlin shell analysis with exactly calculated director vectors. Comput. Meth. Appl. Mech. Engrg. 253 (2013), 491-504.

[2] W. Dornisch, S. Klinkel. Treatment of Reissner-Mindlin shells with kinks without the need for drilling rotation stabilization in an isogeometric framework. Comput. Meth. Appl. Mech. Engrg. 276 (2014), 35-66.

[3] F. Gruttmann, S. Klinkel, W. Wagner. A finite rotation shell theory with application to composite structures. Rev. Européenne Élém. 4 (1995), 597-631.

[4] T.J.R. Hughes, J.A. Cottrell, Y. Bazilevs. Isogeometric analysis: CAD, finite elements, NURBS, exact geometry and mesh refinement. Comput. Meth. Appl. Mech. Engrg. 194 (2005), 4135-4195.

[5] J.C. Simo, D.D. Fox. On a stress resultant geometrically exact shell model. Part III: Computational aspects of the nonlinear theory. Comput. Meth. Appl. Mech. Engrg. 79 (1990), 21-70. 


\title{
Corotational flat triangular elements for the nonlinear analysis of thin shell structures
}

\author{
Federica Caselli, Paolo Bisegna \\ Department of Civil Engineering and Computer Science, \\ University of Rome "Tor Vergata"
}

This contribution shows the effectiveness and versatility of the corotational formulation in the development of shell finite elements for geometric and material nonlinear analysis of thin structures $[1,2,3]$.

The corotational approach is based on the idea of separating rigid body motions from strain producing ones. The fundamental idea is to find a transformation that operates like a filter on the global displacements, removing the rigid-body contribution before the finite element kernels use them. The advantage is that most of the geometric nonlinearity of the problem is transferred to the transformation relating the filtered displacements to the total displacements. The strain energy thus obtained proves to be objective with respect to rigid body motions. In addition, the corotational approach leads to a separation of the material and geometrical nonlinearities.

For problems with arbitrarily large displacements and rotations but small strain response, existing elements formulated in the small strain regime can be reused as core elements in the geometrically nonlinear context, after large rigid body motions have been filtered out. This feature has been exploited in [3] in order to develop a new Shape Memory Alloy (SMA) shell finite element. SMA-based devices typically undergo significant configuration changes in their operation, however large rotations rather than large strains are most often involved. By resorting to the corotational formulation, it is possible to adopt constitutive models formulated in the small strain regime, which are simpler and computationally less expensive with respect to finite strain approaches. In particular, a small strain plane-stress SMA model based on the thermodynamically consistent formulation proposed in [4] and able to account for the pseudo-elastic as well as the shape memory effect was considered in [3].

Although the hypothesis of large displacements and rotations but small strains is often suitable, there are many practical situations where shell structures undergo large strains, like in metal forming and applications involving rubber-like or biological materials. The restriction to small strain has represented a long standing limitation for the corotational approach. One of the main reason is that, in order to allow the extension to large strain problems, the correct identification of the element rigid motion plays a crucial role. In particular, as first pointed out by Crisfield [5], a polar decomposition based corotational approach is required. In [2], a corotational flat triangular element for large strain analysis of thin shells with applications to soft biological tissues has been presented, which is based on the polar decomposition based corotational framework developed in [1], combined with an original kinematic description of the core-element displacement field, based on the multiplicative (instead of additive) superposition of membrane and bending actions.

\section{References}

[1] F. Caselli, P. Bisegna. Polar decomposition based corotational framework for triangular shell elements with distributed loads. Int. J. Numer. Methods Eng. 95(6) (2013), 499-528.

[2] F. Caselli, P. Bisegna. A corotational flat triangular element for large strain analysis of thin shells with applications to soft biological tissues. Comput. Mech. 54(3) (2014), 847-864.

[3] P. Bisegna, F. Caselli, S. Marfia, E. Sacco. A new SMA shell element based on the corotational formulation. Comput. Mech. 54(5) (2014), 1315-1329.

[4] V. Evangelista, S. Marfia, E. Sacco. Phenomenological 3-D and 1-D consistent models for SMA materials. Comput. Mech. 44(3) (2009), 405-421.

[5] G. F. Moita, M. A. Crisfield. A finite element formulation for 3-D continua using the co-rotational technique. Int. J. Numer. Methods Eng. 39(22) (1996), 3775-3792. 


\title{
Flexure Hinge Mechanisms Modeled by Nonlinear Euler-Bernoulli-Beams
}

\author{
Robert Friedrich, Rolf Lammering \\ Helmut-Schmidt-University/University of the Federal Armed Forces, \\ Institute of Mechanics, Holstenhofweg 85, 22043 Hamburg, Germany
}

A flexure hinge is an innovative engineering solution for providing relative motion between two adjacent stiff members by the elastic deformation of a flexible connector. Compliant mechanisms based on flexure hinges find application in aeronautics, positioning systems and medical-technical devices. Major drawback of these mechanisms is their limited motion range. Also modeling and optimization are highly demanding steps in the design process. This study aims for a simplification of the design process by means of optimization methods.

Regarding the modeling of flexure hinges, mostly linear approaches have been investigated. A recent study demonstrates that higher order Bernoulli beam elements of variable cross section can be employed to model the linear static and dynamic behavior of flexure hinges accurately [1, 2]. In this study the element is applied with regard to the geometric nonlinear nature of deformation. Axial displacement and transverse deflection are approximated by higher order shape functions. The element stiffness matrix and element tangential stiffness matrix are established in accordance with [3]. The integrals are solved numerically taking the variable cross section of the flexure hinge into account. The solution of nonlinear equations requires an iterative NewtonRaphson procedure where the unknown state $\mathbf{x}^{(r)}$ is computed from the known state $\mathbf{x}^{(r-1)}$ and its increment $\delta \mathbf{x}^{(r-1)}$. After assembly of the stiffness matrix $\mathbf{K}(\mathbf{x})^{(r-1)}$ and the tangential stiffness matrix $\mathbf{T}(\mathbf{x})^{(r-1)}$ and for a given load vector $\mathbf{F}$ the nonlinear system of equations is solved. The solution procedure is implemented in Matlab. This modeling approach captures the geometric nonlinear behavior of flexure hinges, which is demonstrated by selected benchmarks where the convergence and accuracy of the solution is regarded. Compared to commonly applied finite element methods, this approach reduces the model's degrees of freedom enormously.

Size and computational time privilege the application of the model to geometric design parameter optimization. The maximum output motion serves as objective function, which is constrained by the mechanical stress in the flexure hinge region while geometric design parameters are optimization variables. It is shown that the proposed design process is simplified, accelerated and even more reliable than established methods based on continuum models.

\section{References}

[1] R. Friedrich, R. Lammering, M. Rösner. On the modeling of flexure hinge mechanisms with finite beam elements of variable cross section. Prec. Eng. 38 (2014), 915-920.

[2] R. Friedrich, R. Lammering. An improved modeling approach for circular flexure hinges with application to geometry optimization. Proc. Appl. Math. Mech. 13 (2013), 95-96.

[3] J.N. Reddy. An introduction to nonlinear finite element analysis. Oxford Univ. Press (2010). 


\title{
A layer-wise theory for the structural analysis of glass and photovoltaic laminates
}

\author{
K. Naumenko, H. Altenbach, V. A. Eremeyev \\ Otto-von-Guericke-University Magdeburg, Institute of Mechanics, \\ PF 4120, D-39016 Magdeburg, Germany
}

Laminated plates with glass skin layers and a core layer from soft polymers are widely used in civil engineering. Photovoltaic panels currently available on the market are composed from stiff front and back layers and a core layer. The core layer comprises the solar cells in a soft polymeric encapsulant.

This contribution presents a layer-wise theory for the structural analysis of glass and photovoltaic laminates. Governing equations for the individiual layers, kinematical constraints, and appropriate interaction forces represent the starting point to deduce a twelfth order system of partial differential equations. The Airy stress function, the deflection function, and the vector of relative in-plane displacements of the skin layers are the primary variables in this theory.

A system of differential equations with respect to scalar potentials is presented for symmetric laminates. Three of these differential equations correspond to the first order shear deformation plate theory. The additional second order differential equation represents a correction for laminates with soft core layer. In order to demonstrate the importance of this correction, closed form analytical solutions for a plate strip are derived. Moreover, the significance of additional boundary conditions is shown, using two examples of free and framed plate edges. Since the edges of photovoltaic laminates are usually fixed by frames to restrict the relative sliding of skin layers, the boundary conditions for framed edges are of importance for practical applications. 


\title{
A user-defined element based on a layer-wise theory for laminated glasses and photovoltaic panels
}

\author{
J. Eisenträger, K. Naumenko, H. Altenbach \\ Otto-von-Guericke-University Magdeburg, Institute of Mechanics, \\ PF 4120, D-39016 Magdeburg, Germany
}

Laminated plates and solar modules are composed of three layers, whereas the core layer, comprising the solar cells and their encapsulation, is much more shear-compliant than the skin layers. If the difference in stiffnesses becomes too large, first-order shear deformation theories cannot be applied to these laminated plates. For this reason, a layer-wise theory for plates has been introduced in [1].

This contribution presents a user-defined quadrilateral Serendipity element with quadratic shape functions, based on the layer-wise theory. In order to determine the element stiffness relation, the principle of virtual work is deduced starting with the governing equations of the layer-wise theory. The element possesses nine degrees of freedom including two components of the in-plane displacement vector of the laminate, two components of the relative displacement vector of the skin layers, the deflection, two components of the cross-section rotation vector of the laminate, and two components of the relative rotation vector of the skin layers. It is implemented into the Abaqus FE code using the subroutine "User Element". Closed form analytical solutions are referred to the layer-wise theory in order to verify the results of the user-defined element. To assess the deformation state of a photovoltaic panel in practice, two different kinds of boundary conditions are taken into account: free supports and a rigid frame.

\section{References}

[1] K. Naumenko, V. A. Eremeyev. A layer-wise theory for laminated glass and photovoltaic panels. Composite Structures 112 (2014), 283-291. 


\title{
On a mathematical problem of cusped double-layered plates
}

\author{
Natalia Chinchaladze \\ I. Vekua Institute of Applied Mathematics \& Faculty of Exact and natural Sciences \\ I. Javakhishvili Tbilisi State University
}

Investigations of cusped elastic prismatic shells actually takes its origin from the fifties of the last century, namely, in 1955 I.Vekua raised the problem of investigation of elastic cusped prismatic shells, whose thickness on the prismatic shell entire boundary or on its part vanishes ([1], [2], [3]). The survey of the elastic shells and plates is given in [4]. Using I. Vekua's dimension reduction method hierarchical models for elastic layered prismatic shells are constructed in [5], [6]. In the symmetric case of the prismatic shells we have to do with plates of variable thickness (see, e.g. [4]). The present talk is devoted to the system of degenerate partial differential equations arising in the zero approximation of hierarchical models of layered plates. The wellposedness of boundary value problems under the reasonable boundary conditions at the cusped edge and given displacements at the non-cusped edge is studied. Some numerical resalts are also given.

Acknowledgement. The work was supported by the Shota Rustaveli National Science Foundation (SRNSF) grant No. $30 / 28$.

\section{References}

[1] I. Vekua, On a method of computing prismatic shells, Akad. Nauk Gruzin. SSR. Trudy Tbiliss. Mat. Inst. Razmadze, 21 (1955), 191-259, in Russian.

[2] I. Vekua, Theory of thin shallow shells of variable thickness, Akad. Nauk Gruzin.SSR Trudy Tbiliss. Mat. Inst. Razmadze, 30 (1965), 3-103, in Russian.

[3] I. Vekua, Shell Theory: General Methods of Construction, Monographs, AdvancedTexts and Surveys in Pure and Applied Mathematics, 25. Pitman (Advanced Pub-lishing Program), Boston, MA. 1985.

[4] G.V. Jaiani, Cusped shell-like structures, Springer Briefs in Applied Science and Technology, Springer, Heidelberg, 2011.

[5] G. Jaiani, On a model of layered prismatic shells, Proceedings of I. Vekua Institute of Applied Mathematics, 63 (2013), 13-24, for electronic version see: http://www.viam.science.tsu.ge/publish/proceed.html.

[6] N. Chinchaladze, On a cusped double-layered prismatic shell, Proceedings of I. Vekua Institute of Applied Mathematics, 64 (2014, in print), for electronic version see: http://www.viam.science.tsu.ge/publish/proceed.html. 


\title{
A membrane finite element formulation for woven fabrics using the generalized polyconvex hyperelastic model
}

\author{
Vu Ngoc Khiêm ${ }^{1}$, Mahmood Jabareen ${ }^{2}$, and Mikhail Itskov ${ }^{1}$ \\ ${ }^{1}$ Department of Continuum Mechanics, RWTH Aachen University, \\ Kackertstr. 9, 52072 Aachen, Germany \\ ${ }^{2}$ Faculty of Civil and Environmental Engineering Technion, Israel Institute of Technology, \\ Haifa 32000, Israel \\ vu@km.rwth-aachen.de
}

Due to the light weight and the high degree of drapability, textile composite has become a prominent replacement for metals in automotive and aeronautic industry. In this contribution, we propose a membrane finite element formulation for woven fabrics, and implement it into the commercial FEM software package ABAQUS via user subroutine UEL. The constitutive law for woven fabrics is based on the generalized polyconvex hyperelastic model [1], and is formulated with respect to a geodesic basis. In contrast to the usual approach via the user-defined material in commercial FEM softwares, no Green-Naghdi stress rate formulation is needed and the components of the deformation gradient always lie in the element plane. Thus, important features of textile composites such as deformed fiber orientations can be efficiently captured. Robustness and convergence properties of the proposed membrane element formulation are demonstrated by various numerical examples. Predictions of the constitutive model and finite element formulation are comparable to experimental data of picture frame and double dome test.

\section{References}

[1] A. E. Ehret, M. Itskov. A polyconvex hyperelastic model for fiber-reinforced materials in application to soft tissues. Journal of Materials Science. 42 (2007), 8853-8863. 


\title{
On the Mechanics of Ultralight Hollow Microlattices
}

\author{
Bernhard Eidel \\ Lehrstuhl für Numerische Mechanik, Universität Siegen, Germany \\ Institut für Mechanik, Universität Duisburg-Essen, Germany
}

Recent experimental work on the fabrication and mechanical testing of hollow-tube microlattices [1, 2] has attracted considerable interest in industry and academia for several outstanding mechanical properties of these structural materials; a lattice with a unit cell of bcc type is (i) ultralight with $\rho \approx 0.9 \mathrm{mg} / \mathrm{cm}^{3}$, (ii) shows considerable energy dissipation and (iii) exhibits almost full recovery of the initial lattice shape even after compressions up of $50 \%$. Hollow-tube microlattices are a novel class of cellular materials, which (iv) allow by their periodic, quasi-deterministic composition for a highly effective topology optimization in contrast to foams, which are stochastic in nature.

The objective of the present work is a thorough understanding of the mechanics of microlattices by finite element analysis. For that aim, we establish a thin-shell model along with an isotropic material law of elastoplasticity. Already the reduced unit-cell model representing the total lattice captures significant characteristics of compression experiments very well and reveals the interplay of geometrical features such as buckling and folding with inelastic phenomena of plasticity and damage. We show in terms of Ashby-diagrams, that the simulation results follow universal scaling laws for effective stiffness as well as for strength as a function of density, which is in excellent agreement with the experiments. We propose a modified, bcc-derived unit cell that exhibits an increase in elastic stiffness and strength by 1-2 orders of magnitude compared to the original bcc lattice of the experiments in [1,2], thus making a step towards ultralight and ultratight properties of tailored structural materials.

\section{References}

[1] T.A. Schaedler, A.J. Jacobsen, A. Torrents, A.E. Sorensen, J.Lian, J.R. Greer, L. Valdevit, W.B. Carter. Ultralight metallic microlattices. Science 334 (2011), 962-965.

[2] A. Torrents, T.A. Schaedler, A.J. Jacobsen, W.B. Carter, L. Valdevit. Characterization of nickel-based microlattice materials with structural hierarchy from the nanometer to the millimeter scale. Acta Mater. 60 (2012), 3511-3522. 


\title{
Mechanical Analysis of Metallic SLM-Lattices on Small Scales: Finite Element Simulations versus Experiments
}

\author{
$\underline{\text { S. Didam }}^{1}$, B. Eidel ${ }^{1}$, A. Ohrndorf ${ }^{2}$, H.-J. Christ ${ }^{2}$ \\ ${ }^{1}$ Lehrstuhl für Numerische Mechanik, Universität Siegen, Germany \\ ${ }^{2}$ Lehrstuhl für Materialkunde und Werkstoffprüfung, Universität Siegen, Germany
}

There is an ever more increasing need for lightweight materials in mechanical structures on different length scales. Foam materials are inherently stochastic in nature, which limits the effectivity of optimizing their composition. Quite recently, high-resolution selective laser melting (SLM) has enabled the fabrication of metallic lattices on small scales. As a consequence, lightweight cellular materials with tailored properties with respect to stiffness, strength, and ductility can be fabricated with high fidelity and applied in promising applications as e.g. for bone replacement in orthopedic surgery.

The present contribution deals with the mechanics of metallic SLM-micro-lattices made of stainless steel as a prototype material. Finite element analyses with material parameters identified in experiments investigate the structural load bearing behavior of different unit cell topologies as well as global deformation of entire lattices. Typical failure modes like local buckling as well as global localization in shear bands are analyzed in simulations of compressive, shear and mixed loading conditions and are compared to corresponding results of mechanical tests. Ashby diagrams [1] for the scaling behavior of stiffness and strength at various densities are determined from the simulations and experiments, respectively. Characterization by scanning electron microscopy accompany the deformation analyses and provide detailed insights into laser-melting microstructure in single struts and their nodal connections. Perspectives of future topology optimization of SLM-microlattices are addressed with respect to product-specific properties.

\section{References}

[1] L.J. Gibson, M.F. Ashby. Cellular Solids. Cambridge University Press (1999). 


\title{
Investigation of elastoplastic effects of cables under large spatial deformation
}

\author{
$\underline{\text { Vanessa Dörlich }}^{1,2}$, Stefan Diebels ${ }^{1}$, Joachim Linn ${ }^{2}$ \\ ${ }^{1}$ Lehrstuhl für Technische Mechanik, Universität des Saarlandes \\ ${ }^{2}$ Department Mathematical Methods in Dynamics and Durability, \\ Fraunhofer Institute for Industrial Mathematics
}

Cables are flexible, slender components with a complex multi-layer structure. They can be described physically correct by the Cosserat rod theory [1] which basically consists of three parts: geometrically exact kinematics relating configuration variables and objective strain measures, balance equations that govern the dynamic equilibrium of the sectional kinetic quantities and constitutive equations which yield the sectional forces and moments in terms of the deformation.

Finding an appropriate constitutive model is necessary to enable a realistic simulation of a structure under load. While a standard linear elastic description may be sufficient to represent small deformations in academic test examples, every day experience shows that for example electric cables behave quite differently under large deformations. Since they are composed of various layers, effects like friction, delamination and pull-out can occur in practice. Additionally, the particular layers consist of different classes of materials including metallic wires which may reach the yield stress under large spatial deformations. Consequently, inelastic material behavior cannot be neglected in the model. A viscoelastic constitutive model formulated for the sectional force and moment quantities and objective strain measures of the Cosserat rod theory was already presented in [2] and [3]. Our work aims at a similar approach for plasticity. Coupling between the single stiffnesses can be expected for complex, multiaxial loading and should eventually be included in the description extending the approach of Simo et al. [4].

Furthermore, it is important that the model parameters are accessible. Our contribution focuses on the design of suitable experiments. Classical standardized tests to measure bending, tensile and torsional stiffnesses cannot cover the mentioned effects and are only sufficient to determine the parameters for elastic models. Consequently, finite deformation experiments have to be developed which provide access to parameters in the inelastic range. Information about the coupling between the single stiffnesses can be provided by multiaxial experiments combining bending, torsion and tension of cables.

\section{References}

[1] J.C. Simo. A finite strain beam formulation: the three dimensional dynamic problem - Part I. Comp. Method. Appl. M. 49 (1985), 55-70.

[2] H. Lang, J. Linn, A. Tuganov. Geometrically exact Cosserat rods with Kelvin-Voigt type viscous damping. Mech. Sci. 4 (2013), 79-96.

[3] O.A. Bauchau, Z. Lao, M. Lyu, S. Brändle, J. Linn. Formulation of viscoelastic constitutive laws for beams in flexible multibody dynamics. Proc. IMSD, Busan, Korea (2014).

[4] J.C. Simo, K.D. Hjelmstad, R.L. Taylor. Numerical formulations for finite deformation problems of beams accounting for the effect of transverse shear. Comp. Method. Appl. M. 42 (1984), 301-330. 


\title{
Determination of a Constitutive Friction Law Using an Elastic-Plastic Half-Space Model
}

\author{
F. Beyer, K. Willner \\ Chair of Applied Mechanics, University of Erlangen-Nuremberg, Egerlandstraße 5, \\ 91058 Erlangen, Germany
}

This contribution presents a procedure, how contact interaction of rough surfaces are examined with a halfspace approximation for the deduction of a constitutive friction law. Roughness is composed of fine random irregularities of a surface structure which affect the functionality of surfaces. Due to this fact, technical surfaces get into contact only with their peaks for low to moderate contact load. The resulting real contact area is smaller than the apparent contact area. Furthermore, the contact load is distributed locally on the surface peaks which are exposed to plastic deformation. For the investigation of friction of surfaces it is necessary to determine this deformation as frictional shear stress is transferred in the real contact.

In addition, the mutli-scale character coming with roughness demands on the one hand a very fine resolution for the accurate reproduction of such irregularities and surface peaks. On the other hand, the contact region has to be large enough to be representative to show waviness or the surface structure itself.

The usage of the Finite-Element-Method is a broadly used possibility to treat such a complex matter numerically due to the continuously rising computing capacity. However, computing power is still a limiting factor. Therefore, a canny alternative is taken into account with the application of a half-space approximation. It only depends on the two-dimensional surface boundary which consumes less computing effort than FEM with its 3-dimensional volume approach for the same surface resolution and area.

The elastic-plastic half-space model based on a variational principle of the minimization of the complementary energy is validated and calibrated with experiments. The simulations of several contact configurations are combined for the determination of a constitutional friction law consisting of two equations. The law is implemented into the framework of the commerical FEM-software Simufact.forming to see the impact in a metal forming process which is commonly simulated with Tresca's law of friction. 


\title{
Application of fibre Bragg grating sensors for residual stress analysis
}

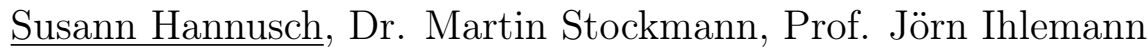 \\ Professorship of Solid Mechanics, Faculty of Mechanical Engineering, \\ Technische Universität Chemnitz, Chemnitz, Germany
}

All components exhibit residual stresses which might be caused by the manufacturing process. Especially casting is a procedure in which influences of residual stresses, like the warpage of components after being removed from the mould, become visible. Obviously these effects are related to metals, plastics or reinforced plastics. Additionally, the residual stresses can influence life expectancy or the capacity to withstand stresses of the component in a positive or negative way.

In order to profit from the positive or to minimize the negative influences of the residual stresses it is important to know which kind of residual stresses are emerged. Therefore, the measurement of residual stresses is necessary although they can only be measured indirectly. Moreover, most common methods have been developed for metals and not all of them can be applied to plastics. One well-investigated method is the hole-drilling method which was developed by Josef Mathar [1]. This method varies the stress state by drilling a hole into the specimen. The resulting deformations are measured and converted into the desired residual stresses by employing a mathematical model. At the present state the hole-drilling method is standardised in ASTM E837-08 [2] for the application on metal materials in which the strains are measured by strain-gauge rosettes. This method measures the strains solely on the surface while changing the stress state which is one main disadvantage. In consequence the method provides only less information on the distribution of the residual stresses in the material.

The present work describes a new approach which allows the measurement of strains in several plains while the stress state is changed. Therefore, a measurement sensor is necessary which can be embedded into the specimen. In order to investigate reinforced or pure plastics, fibre Bragg grating sensors are most suitable because these are glass fibres which are also embedded as reinforcing fibres. By the application of fibre Bragg grating sensors the experimental set-up has to be changed in a way that strains are measured in tangential and not radial direction as typically. This alternation of the set-up is necessary because of technological aspects. The usage of the new approach requires an adapted experimental set-up as well as a new method to calculate the residual stresses based on the tangential strains of different plains.

\section{References}

[1] J. Mathar. Determination of initial stresses by measuring the deformation around drilled holes. Trans. ASME 56 (1934), 249-259.

[2] ASTM E 827-08. Standard Test Method for Determing Residual Stresses by the Hole-Drilling Strain-Gage Method (2008). 


\title{
Kink banding in laminated composite structures
}

\author{
Christina Völlmecke \\ Technische Universität Berlin, Institut für Mechanik, \\ Stabilität und Versagen funktionsoptimierter Strukturen
}

The formation of kink bands in fibre-reinforced composite components can yield a significant reduction of the stiffness of a structure. Subsequently it may also lead to further failure modes such as matrix damage, delamination etc. [1].

Thus herein an analytical model is proposed for kink band deformations occurring in laminated composites whilst the underlying formulations are based on geometric and potential energy principles. Therefore the total potential energy $V\left(Q_{i}, \Delta\right)$ of the system is derived from the strain energy $U\left(Q_{i}\right)$ minus the work done of the load $P \Delta\left(Q_{i}\right)$. The equilibrium paths are subsequently derived by minimizing the energy with respect to the generalized coordinates $Q_{i}$.

The model originates from a previous pilot study on kink banding in unidirectional laminae [2]. Earlier formulations are adapted and enhanced such that nonlinear material behaviour and imperfections stemming from initial fibre waviness can be allowed for [3, 4].

Furthermore the model is significantly extended to simulate a symmetric, multi-directional laminate lay-up whilst the kink band is assumed to occur in the $0^{\circ}$ laminae on the neutral axis only [5]. The surrounding, outer layers are modelled with homogenized material properties derived using Classical Laminate Theory and a Rayleigh-Ritz approach is utilized to approximate the displacement of the remaining layers.

Parametric investigations are undertaken and the results are in very good agreement with the literature. The proposed analytical approach thus encourages further studies on systematic, phenomenological investigations of kink banding in composite structures using energy minimization principles.

\section{References}

[1] Prabhakar, P., Waas, A. M. Interaction between kinking and splitting in the compressive failure of unidirectional fiber reinforced laminated composites. Compos. Struct. 98 (2013), 85-92.

[2] Wadee, M. A., Völlmecke, C., Haley, J. F., Yiatros, S. Geometric modeling of kink banding in laminated structures. Philos. Trans. R. Soc. Lond., A 370 (2012), 1827-1849.

[3] Zidek, R. A. E., Völlmecke, C. On the influence of material non-linearities in geometric modeling of kink band instabilities in unidirectional fiber composites. Int. J. Nonlinear Mech. 62 (2014), 23-32.

[4] Zidek, R. A. E., Völlmecke, C. Analytical studies on the imperfection sensitivity and on the kink band inclination angle of unidirectional fiber composites. Compos. Part A-Appl. S. 64 (2014), 177-184.

[5] Völlmecke, C., Zidek, R. A. E. Geometric modelling of kink banding in multidirectional composites. J. Eng. Math. (2014), accepted manuscript. 


\title{
Interlaminar stress recovery for arbitrarily curved laminated shells
}

\author{
$\underline{\text { Robert Winkler }}^{1}$, Herbert Haller ${ }^{2}$, Johannes Gerstmayr ${ }^{1}$ \\ ${ }^{1}$ Institute of Mechatronics, Leopold-Franzens-Universität Innsbruck, Austria \\ ${ }^{2}$ Intales GmbH, Natters, Austria
}

Interlaminar shear (ILS) and normal (ILN) stress components play an important role in turn of strength analyses of laminated composite structures, in particular, if they involve strongly curved components such as stiffening members like channel section beams. Conventional shell theories and corresponding finite element formulations do not provide accurate distributions of stress components along the thickness of laminated composites. Therefore, post processing techniques have been developed to calculate the stress distribution from equilibrium and boundary conditions based on the membrane strains obtained from a conventional finite element analysis, see [1], [2], and [3], for plate bending problems. A recovery technique for ILN strains of curved shells has been presented in Ref. [4].

Here a recovery technique for both, ILN and ILS strains, valid for arbitrarily curved shells is described. Therefore, the shell reference surface is parametrized by curvilinear coordinates $\left(\xi^{\alpha}\right), \alpha=1,2$. To parametrize the shell body, a third coordinate, $\xi^{3}$, is introduced which measures the distance perpendicular to the reference surface. Provided that the in-plane stress components $\sigma^{\alpha \beta}\left(\xi^{3}\right)$ are known, the ILS and INS components $\sigma^{\alpha 3}\left(\xi^{3}\right)$ and $\sigma^{33}\left(\xi^{3}\right)$, respectively, can be obtained by numerical integration from Cauchy's equilibrium conditions. For this purpose the equilibrium conditions are expressed in curvilinear coordinates $\left(\xi^{\alpha}, \xi^{3}\right)$. Consequently, they involve the covariant derivatives of stress components and the stress components themselves. Projecting these vector equations to the reference surface, two equations are obtained determining $\sigma^{\alpha 3}\left(\xi^{3}\right)$. Projection onto the transverse $\left(\xi^{3}\right)$ direction yields the governing equation for $\sigma^{33}\left(\xi^{3}\right)$. If the required functions $\sigma^{\alpha \beta}\left(\xi^{3}\right)$ are obtained from a conventional shell finite element (FE) model, special attention has to be payed to the determination of the covariant in-plane derivatives $\left.\sigma^{\alpha \beta}\right|_{\gamma}$. Under certain assumptions, a well-known procedure allows to determine the latter approximately from the shear forces. For plates, this procedure is elaborated in Ref. [1], e.g. Here we adopt this approach for curved shells and, in particular, if normal stress components become relevant. Further it is demonstrated how the zero-stress condition at the lower and at the upper boundary of the laminate can be fulfilled using a certain freedom in the choice of unspecified terms involving the in-plane derivatives $\sigma^{33}, \alpha$ and $\left.\sigma^{\beta 3}\right|_{\alpha}$. Due to the application of general curvilinear coordinates, no restrictions are imposed on how the curvature information is provided to the algorithm. In particular, there is no need to determine principal directions and curvatures even if the finite elements are arbitrarily oriented or distorted.

The procedure has been verified numerically comparing the results obtained from a conventional shell FE analysis via our postprocessing technique with a reference solution obtained from a three-dimensional (3D) FE analysis performed with Abaqus. For the shell analysis we apply a four node curved shell element. The curvature information is provided by the normal directions at the nodes. For the reference solution the laminate is modeled with the 27 node hexahedral continuum element C3D27. Thereby each ply is represented by a layer of elements. The example problems range from a curved beam bending test to a doubly curved shell model representing a curved channel section beam. Thereby an excellent coincidence of the results is observed for $\sigma^{33}$ in general (including very low curvature radius-to-thickness ratios) and a good one for $\sigma^{\alpha 3}$ in the case of dominating shear forces. In the case of dominating bending moments the ILS stresses are relatively small and the results of the 3D analyses do no longer serve as a proper reference since they strongly depend on the exact type of the load introduction.

\section{References}

[1] R. Rolfes, K. Rohwer, Improved transverse shear stresses in composite finite elements based on first order shear deformation theory. Int. J. Numer. Meth. Engng. 40 (1997), 51-60.

[2] R. Rolfes, K. Rohwer, M. Ballerstaedt, Efficient linear transverse normal stress analysis of layered composite plates. Computers and Structures. 68 (1998), 643-652.

[3] M. Schürg, W. Wagner, F. Gruttmann. An enhanced FSDT model for the calculation of interlaminar shear stresses in composite plate structures. Computational Mechanics. 44 (2009), 765-776.

[4] R. Roos, G. Kress, P. Ermanni, A post-processing method for interlaminar normal stresses in doubly curved laminates. Composite Structures 81 (2007), 463-470. 


\title{
Optimization of two-layered steel/aluminum hollow cylinders under combined load
}

\author{
Tunc Apatay, Werner Mack \\ Department of Mechanical Engineering, Gazi University, Ankara, Turkey \\ Institute of Mechanics and Mechatronics, Vienna University of Technology, Vienna, Austria
}

Thick-walled long hollow cylinders are structural elements found frequently in various industrial fields like power engineering and chemical engineering, where pressure vessels, fluid conveying tubes or centrifuges, e.g., are widely used devices. Typically, these elements are loaded by a radial temperature gradient, by internal pressure or by rotation, and often also by a combination thereof. In some cases, it is advantageous not to use a homogeneous tube, but a layered one consisting of two or more materials; reasons for this may be, amongst others, different chemical and/or thermal requirements at the inner and outer surface, improved strength or the demand for a reduction of the weight of the device. While there exist many investigations of the stress fields in thermally loaded layered hollow cylinders for given material data and geometry (e.g.[1]-[3]), in particular for given ratios of the thicknesses of the layers, the problem of finding the optimum composition for given (maximum) combined load was rarely addressed, however (compare [4] and the related study of shrink-fitted vessels without rotation [5]).

Hence, subject of the present study is a two-layered tube under generalized plane strain subject to combinations of rotation, internal pressure, and elevated temperature at the inner surface. Since centrifugal forces are proportional to material density and radius, it is presupposed that the inner cylindrical layer consists of the heavier material, whereas the outer layer is made of a material with lower density. As criterion for the maximum allowable stresses the yield criterion by von Mises is applied, and the device is optimized with respect to its weight. The present investigation not only gives a comprehensive overview of the elastic limits of composite tubes of the above type (where plasticization may start at different radii) but also provides a straightforward procedure for determining the optimum composition. While particularly steel/aluminum composite hollow cylinders are discussed, the results nevertheless are applicable to any material combinations with similar ratios of the material properties.

\section{References}

[1] Y.-C. Yang, C.-K. Chen. Thermoelastic transient response of an infinitely long annular cylinder composed of two different materials. Int. J. Engng. Sci. 24 (1986), 569-581.

[2] Z.-Y. Lee, C.K. Chen, C.-I. Hung. Transient thermal stress analysis of multilayered hollow cylinder.Acta Mech. 151 (2001), 75-88.

[3] A.N. Eraslan. Thermally induced deformations of composite tubes subjected to a nonuniform heat source. J. Thermal Stresses 26 (2003), 167-193.

[4] J. Huang, Y. Lu, C. Shen. Thermal elastic-plastic limit analysis and optimal design for composite cylinders of ceramic/metal functionally graded materials. Mater. Sci. Forum 423-425 (2003), 681-686.

[5] G. Yuan, H. Liu, Z. Wang. Optimum design for shrink-fit multi-layer vessels under ultrahigh pressure using different materials. Chin. J. Mech. Eng. 23 (2010), 582-589. 


\title{
Experimental investigations on PP-PE foil specimens
}

\author{
Carmen Sguazzo, Stefan Hartmann \\ Institute of Applied Mechanics, Clausthal University of Technology, \\ Clausthal-Zellerfeld, Germany
}

\begin{abstract}
Multi-layered composite are adopted in numerous structures related to different engineering fields. When they are employed into the automotive field, characteristics as lightweight together with good formability are very important. Consequently, it becomes essential to investigate how each material contributes with its own mechanical characteristics to the behaviour of the final product. In the three layered composite, object of the present investigation, an internal polymer core is used in order to enhance the damping characteristics, while external steel layers improve its ductility. Particularly, the three-layered sandwich is a combination of external layers of non-alloy low carbon mild steel and a polymer core of Polypropylene and Polyethylene (PP-PE). In the present work, the experimental characterization of the PP-PE core is presented. The experiments, have been carried out within the large deformation range and have been monitored by means of a Digital Image Correlation system. The rate-dependence and the temperature-dependence have been investigated. The experimental work has been conducted in order to interpret the resulting physical evidence by means of the Continuum Mechanics approach. This will give the possibility to model the behaviour of the polymer core in an appropriate way with the respect to the process in which the material is used.
\end{abstract}




\title{
Stress concentration control in the problem of plane elasticity theory
}

\author{
Nana Odishelidze, Francisco Criado Aldeanueva, Francisco Criado, \\ Iv. Javakhishvili Tbilisi State University, Faculty of Exact and Natural Sciences \\ Malaga University,Campus El Ejido, Department of Applied Physics II \\ Malaga University,Campus Teatinos, Department of Statistics and Operational Research
}

In engineering practice, one of the important problem is the problem of stresses concentration investigation near the hole-contour. In the plate with a hole, at some point the tangential-normal stresses (in case of plane elasticity theory) and the tangential-normal moments (in case of bending of thin plates), can reach such values that cause destruction of plates or the formation of plastic zones near the hole.

It is proven that in case of infinite domains the minimum of maximum values of tangential-normal stresses (tangential-normal moments) will be obtainedon such holes, where these values maintain constant. Such holes are called the full-strength ones. These problems are considered in the [1], [2].

For finite domains the axis-symmetric and cycle symmetric problems of the plane theory of elasticity and plate bending with partially unknown boundaries are studied in [3], [4], [6], [7].

The paper addresses a problem of plane elasticity theory for a doubly connected domain $S$ on the plane $z=x+i y$, which external boundary is a rhombus boundary, whose diagonals lie at the coordinate axes $O X$ and $O Y$, the internal boundary required full-strength hole, whose symmetric axes are the rhombus diagonals.

Let to every link of the broken line (outer boundary of the given body) apply the absolutely smooth rigid stamps with rectilinear bases which is displaced under the action of concentrated normally compressive forces $P$, applied to the stamp midpoints. There is no friction between the given elastic body and stamps.

Under the above assumptions, the tangential stresses $\tau_{n s}=0$ are equal to zero along the entire boundary of the domain $S$ and the normal displacements of every link of external boundary $v_{n}=v=$ const are the piecewise constant functions.

The most effective methods used for such problem investigation are the methods of analytical functions theory. On the basis of the well-known Kolosov-Muskelishvili's formulas [5], the shape of the unknown full-strength hole and the stress state of the body are determined. Using numerical analysis method the corresponding graphs are constructed.

\section{References}

[1] N. B. Banichuk. Optimization of elastic solids. (Russian) Nauka, Moscow, 1980.

[2] G. Cherepanov, Inverse problem of plane theory of elastisity. (Russian) Prikl. Mat. Mekh. No. 6, 38(1974), $963-980$.

[3] R. Bantsuri., On one mixed problem of the plane theory of elasticity with a partially unknown boundary , Proc. A. Razmadze Math. Inst., Vol. 140, (2006), 9 -16.

[4] R. Bantsuri., Solution of the mixed problem of plate bending for a multi-connected domain with partially unknown boundary in the presence of cyclic symmetr, Proc. A. Razmadze Math. Inst.,Vol. 145, (2007), 9 -22 .

[5] N. I. Muskhelishvili., Some Basic problems of mathematical theory of elasticity, (Russian), Nauka, Moscow 1966.

[6] N. Odishelidze, F. Criado-Aldeanueva, F. Criado, J. M. Sanchez. On one contact problem of plane elasticity for a doubly connected domain: application to a hexagon. Zeitschrift fïœr Angewandte Mathematik und Physik, Vol. 64, No.1,(2013), $193-200$.

[7] N. Odishelidze, F. Criado-Aldeanueva, J.M. Sanchez . A mixed problem of plate bending for a regular octagon weakened with a required full-strength hole. Acta Mechanica, vol. 224, No. 1, (2013), 183 -192. 


\title{
Stress analyses of multi-layered composite pipes subjected to internal pressure
}

\author{
$\underline{\text { İsmail Yasin Sülü }}^{1}$, Şemsettin Temiz ${ }^{2}$ \\ ${ }^{1}$ Inonu University, Department of Mechanical Engineering, Turkey \\ ${ }^{2}$ Batman University, Department of Mechanical Engineering, Turkey
}

The stress analyses of four, six and eight layered composite pipes with different orientation angles, under internal pressure, was investigated. The code of a numerical model was created in ANSYS software for numerical analyses, and the numerical results of four layered composite pipes were confirmed by experimental results with different orientation angles. Each layer of composite pipes was modeled with the same characteristics. The problems were studied using a computational tool based on the Finite Element Method (FEM). Each layer of the composite pipes was examined with different orientation angles. Failure loads of four layered composite pipes were obtained from experimental tests and compared with the numerical results. The hoop and shear stresses were obtained numerically for each layer. Radial, tangential and axial stresses were determined in the radial direction of the composite pipes. The shear extension coupling was considered because the lay-up angles with $+\theta$ and $-\theta$ layers were in the different radii 


\title{
A model reduction approach for hyperelastic materials based on Proper Orthogonal Decomposition
}

\author{
André Hürkamp, Michael Kaliske \\ Institute for Structural Analysis, TU Dresden
}

Computational models in all fields of engineering applications tend to get more detailed and complex. The usage of nonlinear Finite Element (FE) methods is well established in engineering problems and it has been proven that it provides reliable results. Nevertheless, for complex models a lot of time and computational resources are required. Especially when parameter studies are carried out, similar problems are solved repetitively. For solving such kind of problems more efficiently, sophisticated model reduction techniques are needed. Those techniques are even essential, when we deal with high dimensional problems, as in the field of uncertainty quantification, or with real time simulations in the field of surgery training and support.

By the Proper Orthogonal Decomposition (POD), the most relevant information of a set of data is extracted. For example, with the aid of the POD we obtain singular values and corresponding base vectors from a matrix of snapshots, e.g. the FE solution of the displacement field calculated at several instants of time. Hence, using only base vectors corresponding to the largest singular values leads to a subspace of the problem with a lower dimension than the original one. This procedure is similar to a principal component analysis or KarhunenLoève decomposition. Once the subspace for a certain type of problem is identified, it is possible to solve similar problems with a reduced number of degrees of freedom much smaller than for the original problem. Furthermore, we are able to compute time and space functions from the subspace, so that e.g. the displacement over a certain time domain can be approximated by a series expansion of a few functions depending only on time and functions depending only on space. Hence, it is possible to reproduce complete FE solutions only by matrix multiplication.

In this contribution, we present a novel model reduction approach for hyperelastic materials based on the separation of time and space dependent functions. Several computational examples involving large deformations, complex geometries and different constitutive models are investigated and the results obtained from the reduced computation are compared with detailed FE computations. 


\title{
XFEM for Deformation Theory of Plasticity
}

\author{
Samir Omerović, Thomas-Peter Fries \\ Graz University of Technology
}

In plasticity theory, in general two different kinds of discontinuities in the displacement field appear. There are weak discontinuities in the transition zone between elastic and elastoplastic behaviour (plastic interface), as shown in [1]. For perfectly plastic behaviour, even strong discontinuities can occur, therefore corresponding to a jump in the displacement field [2]. The location of the interface is not known a priori, but constitues an additional unknown for the boundary value problem. This introduces additional difficulties when discretizing the weak form as the plastic interface is within elements. This poses problems in terms of the convergence properties, the convergece rate changes drastically, and also the absolute error increases.

Up to now different strategies were applied to improve the performance of the numerical methods. This includes e.g. adaption of the element size ( $h$-refinement), increasing the approximation space over each element ( $p$-refinement) [3], reallocation of the nodes ( $r$-refinement) and also combinations of the preceding (e.g. $h p$ and $r p$-extensions [1]). However, these strategies improve the accuracy by using extensive remeshing procedures, that are numerically expensive and/or time consuming.

We propose to enrich the approximation space in the presence of weak discontinuities at the plastic interface with modified abs functions [4]. As commonly done within the framework of the Extended Finite Element Method (XFEM), the plastic interface is tracked using the level set method [5]. It is stressed that no remeshing whatsoever is necessary. An other approach is also shown using the Heaviside enrichment and enforcing continuity using Lagrange multipliers. The numerical examples confirm that with minimal effort, the absolute error is significantly reduced. Using the same enrichment, it is also possible to model strong discontinuities (occuring for example in slip lines) within the same framework.

\section{References}

[1] V. Nübel, A. Düster, E. Rank. An $r p$-adaptive finite element method for the deformation theory of plasticity. Computational Mechanics 39(5) (2007), 557-574.

[2] C. Johnson, R. Scott. A Finite Element Method for Problems in Perfect Plasticity Using Discontinous Trial Functions. Nonlinear Finite Element Analysis in Structural Mechanics (1981), 307-324.

[3] A. Düster, Rank E. The $p$-Version of the Finite Element Method Compared to an Adaptive $h$-Version for the Deformation Theory of Plasticity. Int. J. Numer. Methods Eng. 52 (2001), 673-703.

[4] N. Moës, M. Cloirec, P. Cartraud, J.-F. Remacle. A computational approach to handle complex microstructure geometries. Comput. Method Appl. M. 192 (2003), 3163-3177.

[5] T.-P. Fries, T. Belytschko The extended/generalized finite element method: An overview of the method and its applications. Int. J. Numer. Meth. Engng. 84(3) (2010), 253-304. 


\title{
Advanced FE-analysis of metal-to-metal seals considering fluid pressure penetration at two scales
}

\author{
Yevgen Gorash, William Dempster, William D. Nicholls and Robert Hamilton \\ Department of Mechanical and Aerospace Engineering, University of Strathclyde, \\ 75 Montrose Street, Glasgow G1 1XJ, Scotland, UK
}

This numerical study investigates the behaviour of the contact faces in the metal-to-metal seal of a typical pressure relief valve [1] in the commercial FE-package ANSYS. The valve geometry is simplified to an axisymmetric problem, which comprises a simple representative geometry consisting of only three components. A cylindrical nozzle, which has a valve seat on top, contacts with a disk, which is preloaded by a compressed linear spring. All the components are made of the steel AISI type 316N(L) [2] defined using the multilinear kinematic hardening material model based on monotonic and cyclic tests at $20^{\circ} \mathrm{C}$. Analysis considerations include the effects of the Fluid Pressure Penetration (FPP) across the valve seat which exists at two different scales. In-service observations show that there is certain limited fluid leakage through the valve seat at operational pressures about $90 \%$ of the set pressure [3], which is caused by the fluid penetrating into surface asperities at the microscale [4]. At the macroscale, non-linear FE-analysis using the FPP technique available in ANSYS revealed that there is also a limited amount of fluid penetrating into gap, which is caused primarily by the global plastic deformation of the valve seat. Plastic strain is assessed using multilinear kinematic hardening model based on the monotonic stress-strain curve obtained from a monotonic tensile test and the cyclic stressstrain curve obtained from a number of tests with stabilised cyclic response [5]. Accurate prediction of the fluid pressure profile over the valve seat is addressed in this study by considering the FPP interaction on both scales. The shape of this pressure profile introduces an additional component of the spring force, which needs to be considered to provide a reliable sealing. Moreover, the analysis showed that the evolution of the profile, which is caused by the isotropic softening of the material, is significant during the cyclic operation of the valve. It was also demonstrated that the type of working fluid affects the shape of the pressure profile in the multiscale. Finally, suggestions to improve the leakage tightness of the metal-to-metal seals are formulated.

\section{References}

[1] Malek, M.A., Pressure Relief Devices: ASME and API Code Simplified. McGraw-Hill: New York, USA, 2006.

[2] Gorash, Y., Altenbach, H. \& Lvov, G., Modelling of high-temperature inelastic behaviour of the austenitic steel AISI type 316 using a continuum damage mechanics approach. Journal of Strain Analysis, 47(4), pp. 229-243, 2012.

[3] Hellemans, M., The Safety Relief Valve Handbook: Design and Use of Process Safety Valves to ASME and International Codes and Standards. Butterworth-Heinemann: Oxford, UK, 2009.

[4] Müller, H.K. \& Nau, B.S., Fluid Sealing Technology: Principles and Applications. Marcel Dekker, Inc.: New York, USA, 1998.

[5] Chaboche, J.-L., Dang Van, K. \& Cordier, G., Modelization of the strain memory effect on the cyclic hardening of 316 stainless steel. Trans. 5th Int. Conf. on Structural Mechanics in Reactor Technology, IASMiRT: Berlin, Germany, number L11/3 in SMiRT5, pp. 1-10, 1979. 


\title{
Investigations on clamping effects with Die-Less-Hydroforming-Structures
}

\author{
Andreas Metzger, Daniel C. Ruff, Thomas Ummenhofer \\ Karlsruhe Institute of Technology, KIT Steel \& Lightweight Structures, Research Center \\ for Steel, Timber \& Masonry
}

The development of objects, items and structures produced by a special forming technology called "Die-Less Hydroforming" is currently very popular among many designers as well as artists and is also focused by some researchers (e.g. see [1]). Within the scope of "Die-Less Hydroforming", two or more CNC-cut thin flat 2-D steel blanks with arbitrary geometry are seal-welded at their edges to generate a closed initial chamber for inflation by a medium such as air, water or oil. When inflating the initial structure, a transformation into a 3 -D object is performed showing plastic deformations combined with local high plastic strain. No auxiliary die or punch is used, i.e. only the initial geometry, combined with the internal pressure, determines the shape of the resulting body, which also shows stability phenomena like wrinkling and buckling.

When using a special blank geometry with openings or cutouts ("pie slices") at the edges, it is possible to generate a clamping effect due to the deformation of the flat blanks during inflation because of the multidimensional forming process of the cutouts at the edge. The openings or cutouts at the edges transform to a 3-D shape and will close like a clamp.

As an example, we want to refer to a nice stool called "Puff", designed and built by the Israeli designer Moran Barmaper. The seating of this stool initially consists of two thin flat seal-welded blanks with special openings at the edge. Before inflating, wooden chair legs are inserted in the openings. By inflating, the sealwelded blanks transform into something like a 3-D-pillow and at the same time, the openings are closing and fixing the stool legs and a bearing hybrid stool is generated (c.f. [2]).

In this contribution, we want to present numerical and experimental results of a study of these clamping effects, investigated on tests with special Die-Less-Hydroforming-samples. We will focus on a special geometry that looks like a "Pac-Man". We investigate the closing and clamping effect of the "mouth" of the "Pac-Man" in practical inflating tests and compare the results with numerical forming simulations using LS-DYNA.

After finishing the ongoing investigations, we might be able to present some first technical application for the use of clamping effects of Die-Less-Hydroforming-Structures.

\section{References}

[1] A. Metzger, D.C. Ruff and T. Ummenhofer. FEM-Simulation of "Die-Less Hydroforming". Conference Contribution (2014), Presented at the 85th Annual Meeting of GAMM, Erlangen-Nürnberg, PAMM Publication in process

[2] "metal furniture inflated using a bike pump puff by moran barmaper", Andrea Chin, www.designboom.com, Oct 5, 2012 retrieved on Dec 19, 2014 from

http://www.designboom.com/design/metal-furniture-inFLated-using-a-bike-pump-puFF-by-moran-barmaper 


\title{
On one contact problem of plane elasticity theory with partially unknown boundary
}

\author{
Magda Tsintsadze, Nana Odishelidze \\ Iv. Javakhishvili Tbilisi State University, Faculty of Exact and Natural Sciences, Georgia
}

\begin{abstract}
Applications of elastic plates weakened with full-strength holes are of a great interest in several mechanical constructions (building practice, mechanical engineering, shipbuilding, aircraft construction, etc).

It's proven that in case of infinite domains the minimum of tangential normal stresses (tangential normal moments ) maximal values will be obtained on such contours, where these values maintain constant. These holes are named full-strength holes. The solvability of these problems allow to control stress optimal distribution at the hole boundary via appropriate hole shape selection. These problems are considered in the [1],[2]. It has been proved that in frames of the same load, the weight of a plate weakened by a hole with full-strength contours is $40 \%$ less than the weight of the one weakened by a circular hole. [2].

For finite domains the above mentioned problems are studied in [3], [4],[5],[6]. The paper addresses a problem of plane elasticity theory for a doubly connected domain $S$ on the plane $z=x+i y$, which external boundary is an isosceles trapezoid boundary; the internal boundary is required full-strength hole including the origin of coordinates. The trapezoid axis coincides with the $O Y$ axis. To every link of the given body broken line, the absolutely smooth rigid stamps with rectilinear bases are applied and they are under the action of $P$ normallycompressive forces. There is no friction between the surface of given elastic body and stamps. Uniformly distributed normal stress is applied to the unknown full-strength contour. Tangential stresses along the entire boundary $\tau_{n s}=0$ are equal to zero and normal displacements are the piecewise constants $v_{n}=v=$ const. Linear segments are endowed with the boundary conditions of the third problem. The most effective methods for studying these problems are the methods of analytical function theory. These problems are both mechanical and geometrical character since the shape of hole is required and the conformal mapping is used to define it. The formulas analogous to Kolosov-Muskhelishvili [7]. are used for investigation of these problems. The unknown full-strength contour and stressed state of the body are determined.
\end{abstract}

\section{References}

[1] N. B. Banichuk, Optimization of elastic solids. (Russian) Nauka, Moscow, 1980;

[2] G. Cherepanov, Inverse problem of plane theory of elastisity. (Russian) Prikl. Mat. Mekh. No. 6, 38(1974),963-980.

[3] R. Bantsuri., On one mixed problem of the plane theory of elasticity with a partially unknown boundary, Proc. A. Razmadze Math. Inst., Vol. 140, (2006),9-16.

[4] N. Odishelidze and F. Criado-Aldeanueva., A mixed problem of plane elasticity for a domain with a partially unknown boundary,International Applied Mechanics, Vol. 42, No. 3, (2006),342 -349.

[5] N. Odishelidze, F. Criado-Aldeanueva., Some axially symmetric problems of the theory of plane elasticity with partially unknown boundaries, Acta Mech., Vol. 199, (2008),227 -240.

[6] N. Odishelidze, F. Criado-Aldeanueva, F. Criado, J. M. Sanchez. On one contact problem of plane elasticity for a doubly connected domain: application to a hexagon. Zeitschrift fïœer Angewandte Mathematik und Physik, (2013), Vol. 64, No.1, $193-200$.

[7] N. I. Muskhelishvili., Some Basic problems of mathematical theory of elasticity, (Russian), Nauka, Moscow 1966. 


\title{
Frequency veering and mode degeneration of a rectangular disc
}

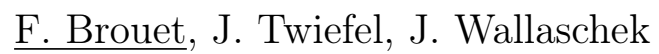 \\ Institut für Dynamik und Schwingungen, Leibniz Universität Hannover, Hannover, Germany
}

The modal properties of an elementary rectangular disc with four slots, aimed to be driven in the longitudinal mode, are investigated. The eigenfrequencies are plotted as a function of the geometrical aspect ratio heightwidth of the disc. Curve veering effects occur in some regions of close frequencies and the modal properties are strongly perturbed.

The veering phenomenon in dynamic systems is well known for many applications like turbine blades, bridges with aeroelastic effects or rotating cantilever beams. In some regions the frequency loci approach each other closely and suddenly veer away again, each one taking on the trajectory of the other. Then the mode shapes are swapped in a smooth transition.

By deriving the eigenvalue sensitivity with respect to a variation parameter, Fox AND KAPOOR [1] calculated the eigenvector sensitivity for the undamped dynamic eigenvalue problem. J.L. DU BoIs, S. AdHIKARI, N.A.J. LIEVEN [2] introduced indices and criterions to quantify the veering intensity.

The eigenvalue veering phenomenon is often associated with irregularities or mistuning because it occurs when this mistuning is of the same order or greater than the coupling, see AfolABI [3]. YANG AND GRIFFIN [4] projected the mode shapes in a new basis of nominal modes by defining a coefficient matrix of participation factors.

In this contribution, one veering zone affecting the longitudinal mode and one spurious mode is investigated. The coupling between both modes is estimated at one critical aspect ratio and the longitudinal mode can be retrieved after tuning the disc geometry.

The spurious mode can be eliminated by coupling both end edges of the disc with a rigid spring in the simulation and the longitudinal mode is retrieved in an artificial way. The new pseudo-cylic state defines the basis of nominal modes and the mistuning of the system can be seen as the removal of the coupling spring. Then the shapes of both localized longitudinal and spurious modes can be spanned by the new created basis containing the unperturbed longitudinal mode. Since the nominal modes and the mistuning matrices are now determined, the modal coupling can be calculated.

The modal coupling between the eigenvectors of the nominal basis can be reduced thanks to several methods like linearized sensitivity studies or minimizing functions, by adapting the disc design. The resulting modes (without the rigid coupling spring) remain uncoupled and the longitudinal mode is retrieved in the critical region.

\section{References}

[1] R.L. Fox, M.P. Kapoor. Rates of changes of eigenvalues and eigenvectors. AIAA Journal (1968), Vol. 6(12) $2426-2429$.

[2] J.L. du Bois, S. Adhikari, N.A.J. Lieven. On the quantification of eigenvalue curve veering: a veering index. Journal of Applied Mechanics (2011), Vol. 78.

[3] D. Afolabi. Modal interaction in linear dynamic systems near degenerates modes. National Aeronautics and Space Administration (1991).

[4] M.T. Yang, J.H. Griffin. A normalized modal eigenvalues approach for resolving modal interaction (1997), Vol. 119 647-650. 


\section{S05: Nonlinear oscillations}

The section covers all fields of vibrational problems in solid mechanics or mechatronics including nonlinear effects. Submissions may address, for example, systems with nonlinear material behavior, nonlinearities in joints, mathematical solution methods (analytical or numerical), control or description of nonlinear behavior like bifurcations or chaos, or experimental idendification of nonlinearities. 


\title{
Rotordynamics of Two-Pole Turbo Generators with Refined Modelling of the Unbalanced Magnetic Pull
}

\author{
Felix Boy, Hartmut Hetzler \\ Engineering Dynamics Group, Institute of Mechanics, University of Kassel, Germany
}

Within this contribution the rotordynamic behaviour of two-pole turbo generators, which are typically used in power plants in combination with gas or steam turbines, is discussed. In view of the fact that a large part of the electric power is provided by these generators, understanding their dynamics is quite relevant. To this end a basic model originally proposed by Kellenberger [1] is reviewed and extended in this analysis.

For the rotordynamics of induction or synchronous machines one of the most important electromagnetic forces is the well known unbalanced magnetic pull (UMP). It results from the asymmetric magnetic field which is mainly caused by the excentricity of the rotor. In classic approaches the UMP acts radially outwards in direction of the smallest air-gap and against the shaft's restoring force [3]. Kellenberger [1] experimentally found that the direction and magnitude of this electromagnetic force may also depend on the rotor's orientation relative to the position of the smallest air-gap. It's influence becomes especially relevant, when large generators are regarded. This dependency has also been reported by Belmans et al. [2].

Kellenberger regarded this instance by introducing a small additional force which varies in direction twice along the circumference, considering the circumstance that the UMP changes due to the orientation of the magnetic poles. After nondimensional scaling the governing equations for the lateral movement of a classical Laval-Rotor (Jeffcott-Rotor) enhanced by a refined model of the electromagnetic forces read

$$
\begin{aligned}
& Y^{\prime \prime}+2 D Y^{\prime}+(1-\lambda \cos (2 \eta \tau)) Y-\lambda \sin (2 \eta \tau) Z=\eta^{2} \cos (\eta \tau-\Phi), \\
& Z^{\prime \prime}+2 D Z^{\prime}-\lambda \sin (2 \eta \tau) Y+(1+\lambda \cos (2 \eta \tau)) Z=\eta^{2} \sin (\eta \tau-\Phi) .
\end{aligned}
$$

Here $Y$ and $Z$ represent the specific horizontal and vertical position of the elastic center, $D$ is the damping ratio, $\lambda$ defines the ratio of additional electromagnetic force to the effective elastic force, $\eta$ represents the ratio of angular rotation frequency to angular eigenfrequency and $\Phi$ is the orientation of the excentricity-axis of the centre of mass towards the rotor's pole axis.

For stationary motions Kellenberger found that the additional force can cause a resonance catastrophe even in presence of damping. In this contribution the analysis is extended by a stability analysis. It turns out that the parametric excitation caused by the additional force may influence the stability of stationary motions. Furthermore an excentric point of application for the electromagnetic forces is introduced and instationary motions are regarded. In this context the systems start-up behaviour is investigated and compared to that of the classic Laval-Rotor's as discussed in [5]. It is found, that the magnetic excentricity may alter the system's characteristics considerably and thus must be considered.

\section{References}

[1] W. Kellenberger. Der magnetische Zug in Turbogenerator-Rotoren als Ursache einer Instabilität des mechanischen Laufes. Archiv für Elektrotechnik. 50 (1966), 253-265.

[2] R. Belmans, A. Vandenput, W.Geysen. Calculation of the flux density and the unbalanced pull in two pole induction machines. Archiv für Elektrotechnik. 70 (1987), 151-161.

[3] G. Müller, K.Vogt, B.Ponick. Berechnung elektrischer Maschinen. John Wiley \& Sons, 2012.

[4] U. Werner. Mathematical rotordynamic model for lateral vibration analysis of induction motors with dynamic eccentricities regarding start-up. Forschung im Ingenieurwesen 78.1-2 (2014), 27-43.

[5] R. Gasch. Biegeschwingungsverhalten rotierender Wellen beim Durchlaufen der kritischen Drehzahlen. Konstruktion 29.9 (1977), 355-365. 


\title{
Stabilization of a rotating shaft by electromagnetic actuators
}

\author{
Piotr M. Przybylowicz \\ Warsaw University of Technology
}

It is known that imperfect (e.g. visco-elastic) supporting of a rotating shaft or presence of internal friction in the material it is made of may destabilize static equilibrium position during its operation [1], [2]. At a certain angular velocity, called the critical speed, the static equilibrium bifurcates into a new oscillatory state manifested by transverse vibrations of the rotating shaft. To prevent the system from such a situation, sometimes dangerous due to large amplitudes, passive and active methods are being incorporated. In this paper, an active approach employing electromagnetic actuators [3] is proposed and discussed. The electromagnetic resistant force developed by the actuators introduces additional external damping into the system. Moreover, the attractive forces depending on the gap between the shaft and electromagnets bring some additional stiffness. Both mechanical effects induced by electromagnetic phenomena, i.e. the enlarged damping and stiffness, change conditions in which the rotor loses its stability.

The paper presents fundamentals of dynamics of rotating shafts with internal friction and describes the principle of working of the electromagnetic actuators. The governing equation of motion of such a coupled mechano-electromagnetic structure is as following

$$
\frac{\partial^{2} \tilde{w}}{\partial t^{2}}+a^{2}\left[1+\beta\left(\frac{\partial}{\partial t}-\mathrm{i} \Omega\right)\right] \frac{\partial^{4} \tilde{w}}{\partial x^{4}}+\tilde{F}_{M w} \delta\left(x-x_{a}\right)=0
$$

where the complex parameters marked by tildes denote: $\tilde{w}=y+\mathrm{i} z, \tilde{F}_{M w}=F_{M y}+\mathrm{i} F_{M z}$ and $\mathrm{i}=\sqrt{-1}$. In the above equations $t$ is time, $x$ is the axial coordinate, $y$ and $z$-transversal displacements of the shaft, $a^{2}=Y J / \rho A$ - parameter related to the material and geometrical data of the shaft ( $Y$ stands for Young's modulus, $J$ - cross-sectional moment of inertia, $\rho$ - volume density, $A$ - area of the cross-section), $\beta$ is the retardation time of the assumed Kelvin-Voigt model of the shaft material, $\Omega$ - angular velocity, $F_{M y}$ and $F_{M z}$ - respective components of the force exerted by the electromagnetic actuators, $\delta($.$) - Dirac's delta function, x_{a}$ - coordinate of the actuators placement. The electromagnetic force $F_{M w}$ generated by a pair of each actuator in the $y$ or $z$ direction is

$$
F_{M w}=\mu_{0} S N^{2}\left[\left(\frac{i_{w 2}}{2\left[\Delta-w\left(x_{a}\right)\right]+l / \mu}\right)^{2}-\left(\frac{i_{w 1}}{2\left[\Delta+w\left(x_{a}\right)\right]+l / \mu}\right)^{2}\right]
$$

where $w$ denotes either $y$ or $z$ transverse direction, indices 1 and 2 correspond to the respective part of each actuator pair, $\mu_{0}$ describes magnetic permeability of vacuum, $\mu$ - relative permeability of the magnetic core of the actuator, $S$ - cross-section area of the core, $N$ - number of wire coils wound around the core, $l$ - core length, $\Delta$ - nominal gap (in the equilibrium position) between the shaft and the actuator core, $i$ - dynamic current developed in core 1 or 2 of the actuator acting in the $w=y$ or $w=z$ direction, $w\left(x_{a}\right)$-actual (dynamic) distance between the shaft and the actuator.

In the analysis, Galerkin's method has been used to discretize the system and find an approximating set of ordinary differential equations of motion. The carried out numerical simulations prove that the electromagnetic actuators may increase the critical rotation speed by even 50 percent. Additionally, highly nonlinear shape of the magnetization curve strongly contributes to near-critical behavior of the shaft and newly born self-excited vibration. It also determines the character of the bifurcating response of the entire system. The problem of efficiency of the applied method of stabilization as well as nonlinear effects brought about by the electromagnetic actuators are highlighted and addressed in the paper.

\section{References}

[1] F.M. Dimentberg. Flexural Vibrations of Rotating Shafts. London: Butterworth 1961.

[2] W. Kurnik. Stability and bifurcation analysis of a nonlinear transversally loaded rotating shaft. Nonlin. Dyn. 5 (1994) 39-52.

[3] T. Szmidt, P.M. Przybylowicz. Critical load and non-linear dynamics of Beck's column with electromagnetic actuators . Int. J. Nonlin. Mech. 67 (2014) 63-73. 


\title{
Stability and bifurcation behaviour of a Laval-Rotor considering fluid forces in compliant liquid seals
}

\author{
Simon Baeuerle, Hartmut Hetzler \\ Engineering Dynamics Group, Institute of Mechanics, University of Kassel, Germany
}

\begin{abstract}
This contribution discusses the influence of fluid forces from compliant liquid seals on the steady state stability and bifurcation behaviour of a Laval-Rotor. In rotating machinery like pumps, annular seals are extensively used to reduce leakage flows between volumes of different pressure. Compliant seals offer the possibility to reduce the sealing gap and at the same time minimize the risk of the shaft rubbing against the outer sealing shell. Therefore, leakage flow is decreased and efficiency increased.

To analyse the fluid flow in sealings, full CFD-simulations or reduced models (like Bulk-Flow-models) are used. In order to incorporate the fluid forces resulting from the pressure field into rotor dynamic investigations, a non-linear semi-empirical model by Muszynska [1] is often applied. The turbulent flow through compliant foil seals in particular has been discussed in [2] and the advantages of compliant metal seals have recently been reviewed on the basis of experimental investigations in [3].
\end{abstract}

The model used in this work for the steady state stability and bifurcation analysis consists of a Laval-Rotor (Jeffcott-Rotor) where the disc runs in a liquid sealing. While the seal shell itself is assumed to be rigid, it is elastically connected to the environment. This elasticity is used to model the compliance of the sealing itself or of the surrounding structure. The Muszynska model is applied to account for the forces from turbulent, incompressible flow through the seal. The whole model is described by the equations

$$
\begin{gathered}
\boldsymbol{M}_{R} \ddot{\boldsymbol{r}}_{R}+\boldsymbol{B}_{R} \dot{\boldsymbol{r}}_{R}+\boldsymbol{K}_{R} \boldsymbol{r}_{R}=\boldsymbol{F}\left(\boldsymbol{r}_{R}, \boldsymbol{r}_{S}\right) \quad \boldsymbol{M}_{S} \ddot{\boldsymbol{r}}_{S}+\boldsymbol{B}_{S} \dot{\boldsymbol{r}}_{S}+\boldsymbol{K}_{S} \boldsymbol{r}_{S}=-\boldsymbol{F}\left(\boldsymbol{r}_{R}, \boldsymbol{r}_{S}\right) \\
\text { where } \\
\boldsymbol{F}\left(\boldsymbol{r}_{R}, \boldsymbol{r}_{S}\right)=-\left(\begin{array}{cc}
m_{f} & 0 \\
0 & m_{f}
\end{array}\right)\left(\ddot{\boldsymbol{r}}_{R}-\ddot{\boldsymbol{r}}_{S}\right)-\left(\begin{array}{cc}
\bar{D} & 2 \bar{\tau} \Omega m_{f} \\
-2 \bar{\tau} \Omega m_{f} & \bar{D}
\end{array}\right)\left(\dot{\boldsymbol{r}}_{R}-\dot{\boldsymbol{r}}_{S}\right)-\left(\begin{array}{cc}
\bar{K}-m_{f} \bar{\tau}^{2} \Omega^{2} & \bar{\tau} \Omega \bar{D} \\
-\bar{\tau} \Omega \bar{D} & \bar{K}-m_{f} \bar{\tau}^{2} \Omega^{2}
\end{array}\right)\left(\boldsymbol{r}_{R}-\boldsymbol{r}_{S}\right)
\end{gathered}
$$

where $\boldsymbol{M}, \boldsymbol{D}, \boldsymbol{K}$ and $\boldsymbol{r}$ are the mass-, damping-, stiffness-matrices and the position vectors respectively. The subscripted $R$ indicates rotor-related and the subscripted $S$ seal-related variables and parameters. $\boldsymbol{F}\left(\boldsymbol{r}_{R}, \boldsymbol{r}_{S}\right)$ is the fluid force. Furthermore $m_{f}$ is the coefficient of the fluid inertia, $\bar{D}$ is the coefficient of the fluid damping, $\bar{K}$ is the coefficient of the fluid radial stiffness, $\Omega$ is the rotating speed of the rotor and $\bar{\tau}$ is the fluid average circumferential velocity ratio.

The objectives of the analysis are to investigate the stability of the steady state and the related bifurcation behaviour. The dependencies of the critical eigenvalue on the parameters is investigated. In particular, the influence of the compliance of the sealing is discussed.

Further steps in this investigation comprise the discussion of anisotropic seal flexibilities, damping and unbalance.

\section{References}

[1] A. Muszynska. Improvements in Lightly Loaded Rotor/ Bearing and Rotor/ Seal Models. J. Vib. Acoust. 110 (1988), 129-136.

[2] M. Salehi, H. Heshmat, J.F. Walton, S. Cruszen. The Application of Foil Seals to a Gas Turbine Engine. $35^{\text {th }}$ AIAA/ASME/SAE/ASEE joint Propulsion Conference and Exhibit 20-24 June, 1999, Los Angeles.

[3] L. San Andrés, A. Anderson, An All-Metal Compliant Seal Versus a Labyrinth Seal. J. Eng. Gas Turb. Power 137 (2015), 1-8. 


\title{
Dynamic stability of composite rotating shafts with Brazier's nonlinearity
}

\author{
Z. Starczewski, A. Tylikowski \\ Warsaw University of Technology, Institute of Machine Design Fundamentals
}

Thinwalled rotating shafts reveal a considerable deformation of cross-section contour during bending. The ovalizing phenomenon is named Brasier's effect [1]. This results in a specific degressive geometric nonlinearity.

$$
M / E J=\kappa\left(1-\gamma \kappa^{2}\right)
$$

where: $M$ - bending moment, $\kappa$ - curvature, $E J$ - bending stiffness, and $\gamma$ - constant depending on the geometric, strength parameters and the internal pressure $q$. It is assumed that the rotating shaft is loaded by uniformly distributed time-independent centrifugal forces. Another important problem of this paper is the description of the global damping of a laminated shaft. Despite the fact that in case of viscoelastic orthotropic plies the resulting constitutive equation is of higher order, the simple Voigt-Kelvin model is assumed. In our dynamics study the rotating symmetrically laminated hybrid circular cylindrical shell will be treated as a beam-like structure. It contains both the conventional (e.g.) graphite or glass) fibers oriented at $+\theta$ and $-\theta$ to the shaft axis and the thermoactive shape memory alloy fibers parallel to the shaft axis. The reduction is justified by a symmetric plies arrangement and negligible circumferrential stresses in the shaft. The shaft movement is described in immovable coordinate system $y, z$ The shaft is assumed to be subjected to an axial stochastic force, which can be expresseed in terms of the Wiener process and the dynamics of shaft is described by the Itô differential equation

$$
\begin{gathered}
d u=u_{, t} d t \\
d u_{, t}=-\left\{e\left[u_{, x x}\left(1-\gamma w_{, x x}^{2}\right)\right]_{, x x}+\beta e\left(u_{, x x x x t}+\omega v_{, x x x x}\right)\right\} d t-\varsigma u_{, x x} d \mathcal{W} \\
d v_{, t}=v_{, t} d t \\
d v_{, t}=-\left\{e\left[v_{, x x}\left(1-\gamma w_{, x x}^{2}\right)\right]_{, x x}+\beta e\left(v_{, x x x x t}-\omega u_{, x x x x}\right)\right\} d t-\varsigma v_{, x x} d \mathcal{W}
\end{gathered}
$$

where $\beta$ - damping coefficient of shaft material, $\varsigma$ - intensity of wide-band Gaussian process. The shaft is assumed to be simply supported at its ends. The time dependent axial force destabilizes the rectilinear shape of the shaft. The direct Liapunov method is used to analyse the uniform stochastic stability of the equilibrium state. Using a measure of distance between solutions $\|.,$.$\| the trivial solution is uniformly stochastically stable$ if the following logic sentence is true

$$
\bigwedge_{\epsilon \geq 0} \bigwedge_{\delta \geq 0} \bigvee_{r \geq 0}\|u(., 0), v(., t)\| \leq r \Rightarrow P\left(\sup _{t \geq 0}\|u(., t), v(., t)\| \geq \epsilon\right) \leq \delta
$$

Since the dynamic equations are strongly nonlinear, special attention is paid to a positive-definiteness of the appropriate energy-like Liapunov functional

$$
V=\frac{1}{2} \int_{0}^{\ell}\left\{u_{, t}^{2}+\left(u_{, t}+\beta e u_{, x x x x}\right)^{2}+v_{, t}^{2}+\left(v_{, t}+\beta e v_{, x x x x}\right)^{2}+2 e\left(u_{, x x}^{2}+v_{, x x}^{2}\right)\left[1-\frac{\gamma}{2}\left(u_{, x x}^{2}+v_{, x x}^{2}\right)\right]\right\} d x \leq V_{*}
$$

where the functional $V^{*}$ corresponds to the linearized problem $\gamma=0$. Analysing the local positive-definiteness and the supermartingale property of functional $V$ along the dynamics equations lead to sufficient stability conditions, expressed in terms of the rotation speed, the damping coefficients, the bending stiffness and the axial force intensity. The influence of SMA fiber activation on increase of stability region is analysed.

\section{References}

[1] E. Reissner. On finite bending of pressured tubes. ASME J. Appl. Mechs. 26 (1959), 366-392. 


\title{
Synchronization effects in rotors partly filled with fluid
}

\author{
Tobias Keisenberg, Georg-Peter Ostermeyer
}

TU Braunschweig, Institut für Dynamik und Schwingungen

In fluid-filled rotors self-excited vibrations occur induced by a surface wave of the fluid. A characteristic property is the instability over the full range of angular velocity above the natural frequency of the system. A possible explanation is the occurrence of synchronization effects between fluid and rotor.

The behaviour of rotors partly filled with fluid was mostly studied under the aspect of stability in steady-state conditions. For non-steady-state investigations, discrete models with reduced number of degrees of freedom and reasonable ability to model the system behaviour are desirable due to the complexity of fluid modelling.

This talk analyses several models and shows synchronization effects between fluid and rotor model. 


\title{
On the self-balancing of the planetary rotor
}

\author{
Olga Drozdetskaya, Alexander Fidlin \\ Institute for Engineering Mechanics, Karlsruhe University of Technology, \\ Kaiserstraße 10, D- 76131 Karlsruhe, Germany
}

The phenomenon of self-balancing of rigid rotors is well known and investigated for rotors with fixed bearings [1], [3]. However, in some technical devices the rotor performs complex motions. An example of such system is a computed tomography scanner. Its anode rotates very fast in the housing of the X-ray tube. At the same time the X-ray tube itself rotates rather slowly around the patient's body. It is very important for CT scanner to keep the minimal possible level of vibrations in order to obtain good image quality. The objective of this paper is to investigate how and to which extent the self-balancing devices can be used for reducing vibrations in a planetary moving rotor.

Consider the system representing a rotating rotor on a rigid carrier. The rotor of mass $M$ is fixed on the end of the carrier which length is $R$. The other end of the carrier is elastically suspended with two spring-dampers of a certain stiffness $c$ and damping $\beta$. The carrier rotates around it's point of suspension with a constant velocity $\Omega$. At the same time the rotor rotates around its symmetry axis with a given velocity $\omega$. Its centre of mass has an offset $\gamma$ relative to the rotation axis. Two pendulum balancers of mass $m$, moment of inertia $J$ and length $r$ are placed on the rotation axis of the rotor.

$$
\begin{array}{r}
(M+2 m) \ddot{x}+\beta \dot{x}+x=(M+2 m) R \Omega^{2} \cos \Omega t+M \gamma \omega^{2} \cos \omega t+m r \sum_{i=1}^{2}\left(\dot{\varphi}_{i}{ }^{2} \cos \varphi_{i}+\ddot{\varphi}_{i} \sin \varphi_{i}\right) \\
(M+2 m) \ddot{y}+\beta \dot{y}+y=(M+2 m) R \Omega^{2} \sin \Omega t+M \gamma \omega^{2} \sin \omega t+m r \sum_{i=1}^{2}\left(\dot{\varphi}_{i}{ }^{2} \sin \varphi_{i}-\ddot{\varphi}_{i} \cos \varphi_{i}\right) \\
\left(J+m r^{2}\right) \ddot{\varphi}_{i}+\beta_{\varphi}(\dot{\varphi}-\omega)=m r R \Omega^{2} \sin \left(\omega t-\varphi_{i}\right)+m r\left(\ddot{x} \sin \varphi_{i}-\ddot{y} \cos \varphi_{i}\right) ; i=1,2
\end{array}
$$

The equations of motion of the whole system (1) - (3) can be split into two groups. The first two equations describe the radial vibrations of the carrier system. The last two equations describe the phases of the pendulums. Herewith it has been assumed that the damping both in the oscillation subsystem $\beta$ and in the rotation degrees of freedom for pendulums $\beta_{\varphi}$ is not small.

The approximate asymptotic solution has been obtained using averaging technique for the strongly damped systems [2]. There are four stationary solutions in this system. Only one of them provides the compensation of the unbalance. The stability investigation has shown that the compensating configuration is stable only in the overcritical domain of the rotation speeds of the rotor. The improved first order approximation has been calculated for the compensating configuration of the system in order to estimate the intensity of the remaining oscillations.

Hence the self-balancing of pendulum type is effective for planetary rotor in the overcritical speed domain. However the balancing is limited by the centrifugal forces causing small vibrations of pendulums. The expressions for the residual amplitudes of vibrations are obtained in dependency on the velocity of the planetary motion. Analytic results match very well with numeric simulations when velocity of planetary motion is sufficiently small.

\section{References}

[1] I.I. Blekhman. Synchronization of Dynamical Systems. Nauka, Moskow, (1971).

[2] A. Fidlin, J.J. Thomsen. Non-trivial effects of high-frequency excitation for strongly damped mechanical systems. Int. J. of Non-Linear Mechanics 43 (2008), 569-578.

[3] L. Sperling, B. Ryzhik, H. Duckstein. Single-Plane Auto-Balancing of Rigid Rotors. Technische Mechanik, 24 (2004) 1. 


\title{
Control of Nonlinearly Coupled Oscillators Using a Method of Averaging
}

\author{
Edwin Kreuzer, Christian Radisch \\ Institute of Mechanics and Ocean Engineering, \\ Hamburg University of Technology
}

Container cranes belong to the class of underactuated systems for which the design of control laws is a challenging task. We study the stabilization of certain modes of a crane system using the method of averaging [1]. Based on the local approximation to the system dynamics by a second order Taylor series expansion, the effects of nonlinear couplings are exploited to steer degrees of freedom, for which no linear actuation is available. The nonlinearity is assumed to be weak, which is shown to be a valid assumption in the proximity of certain operating points of the crane.

The Institute of Mechanics an Ocean Engineering runs a container crane test bench in the scale 1:6. It consists of a trolley and a load connected by four ropes which can be individually controlled. Furthermore, the experiment features measurements of all rope forces, the servo motion parameters for rope lengths and trolley position as well as a basic inertial measurement unit in the load itself. For verification of state estimation and control algorithms, a calibrated multi camera system is available.

For this crane system, there is no direct actuation of the rotation about the vertical axis of the load (in the literature, this motion is sometimes referred to as skew or yaw oscillation [2]). However, we enhance damping for this mode of load oscillation and provide information about potential excitation of this degree of freedom when other controllers - neglecting rotations - are applied. The synthesis model with a minimal number of degrees of freedom has been presented in [3]. This model includes two dimensional load swing as well as rotational load oscillations with inextensible ropes and individually variable rope lengths.

The quadratic coupling terms are analyzed using the method of averaging. Therefore, the solution of the linear system without quadratic coupling is used as a reference. Amplitude and phase of the linear solution vary slowly due to the nonlinear system dynamics. First, a pole placement strategy modifies the system to have specific resonant frequencies. Then, employing the variation of parameters for all degrees of freedom, amplitudes and phases are determined. Further, by means of averaging over one system period, the mean rates of change of the amplitudes and phases are determined.

From the mean rate of change, which is a function of the amplitudes and phases of the degrees of freedom involved, a design rule for control laws is deduced: A phase relation guarantees an average fastest decrease of the concerned amplitude. As a reference for the actuated degrees of freedom, a function of the states is derived which satisfies the phase condition. The state feedback control law is implemented as a variable structure reference tracking controller which is known to have good robustness properties [4]. Experimental results from the container crane test bench show the effectiveness of the approach.

\section{References}

[1] A.H. Nayfeh. Introduction to Perturbation Techniques (Vol. 3). John Wiley \& Sons. (2011).

[2] U. Schaper, C. Dittrich, E. Arnold, K. Schneider, O. Sawodny. 2-DOF skew control of boom cranes including state estimation and reference trajectory generation. Control Engineering Practice, 33 (2014), 63-75.

[3] E. Kreuzer, C. Radisch. Active damping of rotational load oscillations at container cranes by individual rope length actuation. PAMM, 13(1) (2013), 461-462.

[4] B. Song, J.K. Hedrick. Dynamic Surface Control of Uncertain Nonlinear Systems. London: Springer. (2011). 


\title{
On some aspects of the dynamic behavior of the softening Duffing oscillator under harmonic excitation
}

\author{
Lukas Lentz, Utz von Wagner \\ Chair of Mechatronics and Machine Dynamics, \\ Department of Applied Mechanics, \\ Technische Universität Berlin
}

The Duffing oscillator is a classic example of nonlinear dynamics and therefore taught as a standard in nonlinear vibration classes $[1,2]$. The presentation deals with some problems arising, when a softening Duffing oscillator with very weak damping and harmonic excitation is under consideration and classical semi-analytical methods as Harmonic Balance, Perturbation Analysis or Multiple Scales are used. Those solutions are evaluated by several criteria and comparison with numerical analysis is performed. Additionally attractors in the parameter region of multiple stable solutions are considered.

\section{References}

[1] K. Klotter, Technische Schwingungslehre, Band 1, Einfache Schwinger, Teil B, Nichtlineare Schwingungen (Springer Verlag, 1980)

[2] I. Kovacic and M. J. Brennan (eds.), The Duffing Equation, Nonlinear Oscillators and their Behaviour (John Wiley and Sons Ltd, 2011) 


\title{
$1: 3$-Resonance in a Hopf-Hopf bifurcation
}

\author{
Alois Steindl \\ Vienna University of Technology
}

In this talk the bifurcations of stationary and periodic solutions of the reduced system close to the simultaneous occurence of two Hopf bifurcations with 1:3-resonance are investigated. The unfolded Normal Form equations on the Center Manifold are given by

$$
\begin{aligned}
& \dot{z}_{1}=\left(\lambda+i \omega+A_{1}\left|z_{1}\right|^{2}+A_{2}\left|z_{2}\right|^{2}\right) z_{1}+A_{3} \bar{z}_{1}^{2} z_{2}, \\
& \dot{z}_{2}=\left(\mu+3 i \omega+i \delta+A_{4}\left|z_{1}\right|^{2}+A_{5}\left|z_{2}\right|^{2}\right) z_{2}+A_{6} z_{1}^{3},
\end{aligned}
$$

where $\lambda, \mu$ and $\delta$ denote the unfolding parameters and the coefficients $A_{j} \in \mathbb{C}$ of the cubic terms are obtained from the nonlinearities of the underlying model. In the absence of the resonance the last terms in both equations could be eliminated and after introducing polar coordinates $z_{j}=r_{j} \exp \left(i \varphi_{j}\right)$ the amplitude and phase equations would decouple. Due to the resonance the resonance angle $\psi=3 \varphi_{1}-\varphi_{2}$ cannot be eliminated and the reduced dynamics takes place in a 3 -dimensional space.

In the non-resonant case there exist two single-mode periodic solutions as primary branches and a mixedmode quasiperiodic solution, which bifurcates from the periodic solutions in a pitchfork bifurcation. For the resonant system the term $A_{6} z_{1}^{3}$ precludes the existence of a pure mode-1 solution, instead a Duffing-like szenario for the fast oscillation is observed. The pure mode- 2 solution is still possible, but its loss of stability is governed by a nonlinear Mathieu equation.

Numerical investigation of the bifurcation equations shows, that also a Shilnikov szenario occurs, which leads to very chaotic behaviour. 


\title{
On the chaotic behavior of the non-ideal vibrating systems
}

\author{
Veturia Chiroiu, Dan Dumitriu \\ Institute of Solid Mechanics, Romanian Academy, Bucharest \\ E-mail: veturiachiroiu@yahoo.com \\ E-mail: dumitri04@yahoo.com
}

The paper discusses the chaotic behavior of the non-ideal vibrating systems composed from structures to which an electrical motor is connected. The structural response of such systems may act like energy sink under certain conditions so that a part of the energy supplied by the source is spend to vibrate the structure rather than increasing the drive speed, according to the Sommerfeld effect [1]. According to this effect, the energy source is influenced by the response of the system and consequently, the system exhibits jumps at critical values of the energy source. The system mimics a disappearance of the energy in the resonance regions which can affect the stability of the system, sending it to chaos [2]-[4]. The chaotic behavior of non-ideal vibrating systems is characterized by an infinite number of unstable periodic orbits which becomes unstable in the least two directions in the vecinity of a transition point. The transition chaos-hyperchaos and the formation of hyperchaotic attractor are described in this paper for a structure equipped with an electromechanical vibration absorber. The growing of the higher-dimensional attractor is explained by bursting along new unstable directions. The appearance of the first unstable orbit with more than one unstable direction represents the beginning of the riddling of the basin of attraction and bubbling of the chaotic attractor. This phenomenon is a typical way by which higher-dimensional attractors grow by bursting along the new unstable direction.

\section{References}

[1] A.Sommerfeld. Beitrage Zum Dynamischen Ausban Der Festigkeitslehe. Physikal Zeitschr 3 (1902), $266-286$.

[2] J.L.P.Felix, J.M.Balthazar. Comments on a nonlinear and nonideal electromechanical damping vibration absorber. Sommerfeld effect and energy transfer. Nonlinear Dynamics, 55 (2009), 1-11.

[3] L.Munteanu, V.Chiroiu, T.Sireteanu. On the response of small buildings to vibrations. Nonlinear Dynamics, 73(3) (2013) 1527-1543.

[4] L.Munteanu, C.Brişan, V.Chiroiu, D.Dumitriu, R.Ioan. Chaos-hyperchaos transition in a class of models governed by Sommerfeld effect. Nonlinear Dynamics, 78(1) (2014). 


\title{
Modeling and vibration analysis of rotors with hydrodynamic MF-film bearings
}

\author{
Wlodzimierz Kurnik, Anna Perek \\ Institute of Machine Design Fundamentals, Warsaw University of Technology, Poland
}

The dynamic behaviour of rigid rotors in usual passive hydrodynamical bearings is quite well recognized with the basic phenomenon of flutter-type instability under increasing rotation speed [1]. It is known that modal parameters of such two-degree of freedom system, i.e. eigen-frequencies and damping coefficients strongly depend on the rotation speed and one of the two dampings decreases and decays at the critical speed what leads to bifurcation of a rotation-dependent equilibrium and a near-critical self-excited transverse vibration with sub- or postcritical amplitude evolution [1]. On the other hand, a thin oil film provides viscoelastic rotor support that can reduce transverse vibrations caused by external excitations like unbalance and bearing housing movement.

In this paper the slide bearing serves as a semi-active viscoelastic support due to application of a magnetic or magnetorheologic fluids (MF or MRF) [2]. Both types of adaptive lubricant show changes of dynamic viscosity when exposed to magnetic field. The shear MRF effect is applied and the shear rate is proportional to the angular speed $(\omega)$ of the rotor, even with the basic for MF/MRF Bingham fluid model, the effective lubricant viscosity depends on both - magnetic field $(H)$ and rotation speed. Thus, under increasing angular speed the rotor behaves like a smart structure, providing quickly a necessary uplift force [3], increasing flutter critical speed and reducing viscous resistance due to drop of the viscosity. The MF/MRF properties are adopted from the literature $[4,5]$. The dynamic analyses of stability, bifurcation and resonant behaviour are made for a rigid rotor supported in one rolling and one slide bearing, thus performing transverse vibrations under both nominally constant load $(Q)$ and additionally harmonic forcing, with the giroscopic effect taken into account. The equation of the rotor transverse motion can be expressed as follows:

$$
\frac{d \mathbf{u}}{d t}=f(\mathbf{u}, \omega, Q, H, t ; p)
$$

where the notations not defined above are: $\mathbf{u}$ - 4-dimensional vector of displacements and velocities around nontrivial equilibrium within the bearing clearance, $f(\mathbf{u}, \omega, Q, H, t ; p)$ - vector function derived from the rotor angular momentum law, including nominal transverse load, fluid-film forces resulting from the Reynolds equation for the fluid flow and possible harmonic excitation in case of resonant behaviour and $p$ - 7-dimensional quantity representing all the mass and geometric parameters of the rotor.

The results are presented in form of a 3D dimensionless rotor stability map, modal parameters as functions of rotor speed and magnetic field, resonant amplitude behaviour under unbalanced and kinematic excitations affected by the magnetic field.

\section{References}

[1] W. Kurnik. Hopf Bifurcation in the Dynamics of a Rotor Bearing System. W. Schiehlen (Ed.), Nonlinear Dynamics in Engineering Systems. Springer Verlag. (1990), 181-188.

[2] N. Tipei. Theory of lubrication with ferrofluid: application to short bearings. Transactions of the ASME. (1982), 104, 510-515.

[3] A. Perek. Stability and Resonant Behaviour of a Rotor in Slide Bearings with Bingham Fluid As a Lubricant. Machine Dynamics Research. (2012), Vol. 36, No 2, 107-118.

[4] S. Odenbach, S. Thurm. Magnetoviscous effects in ferrofluids. Springer-Verlag, Berlin, Heidelberg. (2002), 594, 185-201.

[5] R. Patzwald. Magnetische Fluessigkeiten als Schmierstoff in hydrodynamischen Gleitlagern. Disertation. (2001). 


\title{
A DAE formulation for geared rotor dynamics including frictional contact between the teeth
}

\author{
Georg Jehle, Alexander Fidlin \\ Institut für Technische Mechanik, Karlsruher Institut für Technologie
}

A DAE approach is presented for geared rotor dynamics simulations with rigid helical evolvent gears. It includes the normal contact force between the teeth as well as tangential components.

Given the evolvent tooth flank geometry of gear 1 and gear $2\left(\boldsymbol{x}^{(1)}\right.$ and $\left.\boldsymbol{x}^{(2)}\right)$ [1], the contact line $\boldsymbol{x}_{c}$ and the velocity difference in the contact $\dot{\boldsymbol{x}}^{(2)}\left(\boldsymbol{x}_{c}\right)-\dot{\boldsymbol{x}}^{(1)}\left(\boldsymbol{x}_{c}\right)$ are found. The requirement of no penetration of the teeth yields a non-holonomic constraint and the contact normal force [2]. The tangential force is obtained by applying Coulomb's friction model [3], even though in experiments all situations between dry friction and hydrodynamic lubrication are observed $[4,5,6]$. The friction force is the integral tangential force acting on all flanks. In addition, a friction caused torque acts on the center of rotation of the gears.

The approach is used to investigate the dynamics of two rotors which are connected by gears. Both of them have three translational DoFs. The driving rotor has a given angular speed, while the driven rotates unrestrainedly and is connected to a rotational damper.

Two different solutions of the problem are compared. The first solution is produced by directly integrating the index-2 DAE using a modified Runge-Kutta algorithm. Because of the periodicity of the geometry, it is obvious that the stationary solution is periodic. For this reason, a harmonic ansatz is applied to calculate the variables' amplitudes as a second solution.

The two solutions are compared and the evolution of the amplitudes with respect to the angular speed is studied.

\section{References}

[1] K. Zirpke. Zahnräder. VEB-Fachbuchverlag (1978).

[2] G. Jehle, A. Fidlin. Friction induced vibrations in shift gearboxes. Z. Angew. Math. Mech. 94 (2014), 911-916.

[3] K.L. Johnson. Contact mechanics. Cambridge university press (1987).

[4] K.F. Martin. A Review of Friction Predictions in Gear Teeth. Wear 49 (1978), 201-238.

[5] G.H. Benedict, B.W. Kelley. Instantaneous Coefficients of Gear Tooth Friction. ASLE transactions 4(1) (1961), 59-70.

[6] S. He, S. Cho, R. Singh. Prediction of Dynamic Friction Forces in Spur Gears using Alternate Sliding Friction Formulations. J. Sound Vib. 309 (2008), 843-851. 


\title{
Adaptive Fuzzy Sliding Mode Controller and Observer for a Dive Cell
}

\author{
Wallace M. Bessa ${ }^{1}$, Edwin Kreuzer ${ }^{2}$, Ludwig Krumm², Marc-André Pick $^{2}$, Eugen Solowjow ${ }^{2}$ \\ ${ }^{1}$ Federal University of Rio Grande do Norte, Natal, Brazil \\ ${ }^{2}$ Hamburg University of Technology, Hamburg, Germany
}

Water particles move on orbitals under the influence of surface gravity waves. These orbitals are usually visualized by optical methods, such as the PIV method. We develop a dive cell which is supposed to reach and stay on an isobar.

Since for that case the average density of the dive cell equals the density of the surrounding water, the dive cell describes the same trajectories as the water particles. The dive cell is composed of a pressure sensor, a micro controller and an actuator to change the volume of the dive cell. Furthermore, an acceleration sensor and a mounted SD card provide the possibility to analyze the kinematics of the dive cell, hence of the water particle trajectories. In order to reach and stay on a certain isobar with the dive cell an adaptive fuzzy sliding mode controller including an observer is developed and compared to a plain vanilla sliding mode controller.

In a first step the equations of motion are derived under consideration of quadratic viscous damping as well as the actuator dynamics (single integrator dynamics):

$$
\begin{aligned}
\ddot{z} & =\frac{1}{m}\left[\rho_{w} g\left(V_{0}-V_{w}\right)-m g-k \dot{z}|\dot{z}|\right] \quad, \\
V_{w} & =b \cdot u,
\end{aligned}
$$

with the variable $z$ as the dive depth, the mass of the dive cell $m$, water density $\rho_{w}$, gravitational acceleration $g$ and a viscous damping coefficient $k$. The value of $V_{w}$ is the volume of water in the piston of the dive cell, while $V_{0}$ represents the volume corresponding to zero buoyancy which in turn should reasonably correspond to the middle piston position. Due to the nonlinear damping and the uncertain parameters, such as the hydrodynamic added mass, a sliding mode approach is chosen for the design of controller and observer, which has proven to be a well-established methodology for robust nonlinear controller synthesis in the presence of modeling imprecisions and external disturbances. After the derivation of the plain vanilla sliding mode controller, we enhance the controller by an adaptive term, which is computed via an adaptive fuzzy algorithm according to [1]. Since the controller requires access to all of the system states while the noisy pressure measurement provides the position state only, we further augment the system by a sliding mode observer. The adaptive fuzzy sliding mode controller shows superior performance in comparison to the plain vanilla one which is demonstrated by means of numerical simulations.

\section{References}

[1] Wallace M. Bessa, Max S. Dutra, Edwin Kreuzer. Depth control of remotely operated underwater vehicles using an adaptive fuzzy sliding mode controller. Robotics and Autonomous Systems. 56 (2008), 670-677. 


\title{
The Vibrational Behavior of Coupled Bladed Disks with Variable Rotational Speed
}

\author{
Ferhat Kaptan ${ }^{1}$, Lars Panning-von Scheidt ${ }^{1}$, Jörg Wallaschek ${ }^{1}$ \\ ${ }^{1}$ Institute of Dynamics and Vibration Research, \\ Appelstrasse 11, D-30167 Hannover, Germany
}

Low pressure steam turbine blades are subjected to high static and dynamic loads during operation. These loads strongly depend on the turbine's rotational speed, leading to entirely new load conditions. To avoid high dynamic stresses due to the forced vibrations, a coupling of the blades, such as shrouds or snubber coupling, is applied to reinforce the structure. In this work the influence of the rotational speed on the vibration behavior of shrouded blades is investigated. Two fundamental phenomena are considered: the stress stiffening and the spin softening effect. Both effects are caused by centrifugal forces and affect the structural mechanical properties, i.e. the stiffness matrix $\mathbf{K}$, of the rotating system. Since the rotational speed $\Omega$ appears quadratically, it is possible to derive the stiffness matrix as a second order matrix polynomial in $\Omega^{2}[3]$.

In the case of shrouded blades, contact forces between neighboring blades must be taken into account. The contact status and the pressure distribution in particular is strongly influenced by the rotational speed, respectively, centrifugal forces, caused by the untwisting and radial deformation of the blades. For the calculation, a three dimensional structural mechanical model including a spatial contact model is considered. The solution of the nonlinear equations of motion is based on the well known Multiharmonic Balance Method [2]. Here, the nonlinear forces are computed in the time domain and transferred in the frequency domain by the use of the Fast Fourier Transformation (FFT), also known as the Alternating Frequency Time method (AFT) [1].

\section{References}

[1] T.M. Cameron, J.H. Griffin. An Alternating Frequency/Time Domain Method for Calculating the SteadyState Response of Nonlinear Dynamic Systems. Journal of Applied Mechanics (1989), Vol. 56, pp. $149-154$.

[2] C. Siewert, L. Panning-von Scheidt, J. Wallaschek, C. Richter. Multiharmonic forced response analysis of a turbine blading coupled by nonlinear contact forces. Journal of Engineering for Gas Turbines and Power 132.8 (2010): 082501.

[3] A. Sternchüss, E. Balmès. On the reduction of quasi-cyclic disk models with variable rotation speeds. Proceedings ISMA (2006). 


\title{
Multi-mode model of a piezomagnetoelastic energy harvester under random excitation
}

\author{
Lukas Lentz, Utz von Wagner \\ Chair of Mechatronics and Machine Dynamics, TU Berlin
}

\begin{abstract}
The transformation of ambient vibrational energy into electric energy through the use of piezoelectric energy harvesting devices has been the subject of numerous investigations [1]. A commonly studied energy harvesting device which performs especially well under broadband excitation is the piezomagnetoelastic energy harvester investigated by Erturk et al. [2] which is usually discretized for the fundamental vibration mode resulting in a single-mode model.

This contribution presents the study of a multi-mode model of the piezomagnetoelastic energy harvester under random excitation. The probabilty density function (PDF) is computed as the solution of the corresponding FOKKER-PLANCK equation using a GALERKIN type method [3, 4]. Based on the PDF, the resulting voltage variance is computed as a measurement for the expected power output as demonstrated in [5]. The results of the multi-mode model are then compared with the results of the single-mode model.
\end{abstract}

\section{References}

[1] R.L. Harne, K.W. Wang. A review of the recent research on vibration energy harvesting via bistable systems. Smart Mater. Struct. 22 (2013).

[2] A. Erturk, J.Hoffmann, D.J.Inman. A piezomagnetoelastic structure for broadband vibration energy harvesting. Appl. Phys. Lett. 94 (2009).

[3] U. von Wagner, W.V. Wedig. On the calculation of stationary solutions of multi-dimensional Fokker-Planck equations by orthogonal functions. Nonlinear Dyn. 21 (2000), 289-306.

[4] W. Martens. On the solution of the Fokker-Planck-Equation for multi-dimensional nonlinear mechanical systems (Shaker Verlag, Aachen, 2014).

[5] W. Martens, U. von Wagner, G. Litak. Stationary response of nonlinear magneto-piezoelectric energy harvester systems under stochastic excitation. Eur. Phys. J. S. T. 222 (2013), 1665-1673. 


\title{
Optimal impedance load of a bistable energy harvester
}

\author{
Matthias Heymanns, Peter Hagedorn \\ TU Darmstadt, fnb, Dynamics and Vibrations Group
}

Within this contribution we investigate a bistable energy harvester regarding its optimal impedance load. A bistable energy harvester exhibits different type of oscillations: Intra-well (about a stable equilibrium), crosswell (between the wells) and inter-well (about the unstable equilibrium). The occurring oscillation type depends for instance on the excitation parameters or the initial conditions. It already has been observed ([1]) that the optimal impedance, which allows to maximize the power output, varies for each occurring oscillation type. In our investigations we complement these findings with analytical and numerical calculations. For our analysis we examine the non-dimensionalized coupled equations of a bistable energy harvester

$$
\begin{aligned}
x^{\prime \prime}(t)+\mu x^{\prime}(t)-\delta v(t)-\alpha x(t)+\beta x(t)^{3} & =y(t) \\
\phi v(t)+\eta x^{\prime}(t)+v^{\prime}(t) & =0
\end{aligned}
$$

whereby

$$
\begin{aligned}
& y(t)=\hat{f} \sin (\Omega t) \\
& \mu, \delta, \beta, \phi, \eta>0 .
\end{aligned}
$$

As indicated we will limit our analysis to harmonic excitations.

The results show that for intra-well and inter-well oscillations more than one optimal impedance load can be found, at which the power output exhibits a maximum. These impedance loads can strongly deviate compared with the ones of the linearized system. The optimal impedance loads also show strong sensitivity to changes in the excitation amplitude $\hat{f}$.

\section{References}

[1] S. C. Stanton, B. A.M. Owens, B. P. Mann. Harmonic balance analysis of the bistable piezoelectric inertial generator. Journal of Sound and Vibration 331 (2012), 3617-3627. 


\title{
On vibrations in non linear, forced, friction-excited systems
}

\author{
Merten Tiedemann ${ }^{1}, \underline{\text { Merten Stender }}^{1}$, Norbert Hoffmann ${ }^{2}$ \\ ${ }^{1}$ Hamburg University of Technology, Germany \\ ${ }^{2}$ Imperial College London, UK
}

In development of friction brakes the trend is towards new experimental methods which include external excitation of brakes systems during dynamometer testing [1]. The underlying idea is to be able to identify in a more efficient and reliable manner parameter regions in which limit cycle oscillations can occur. Ideally, testing could be conducted in parameter regions where the steady sliding state of the non linear, non forced, friction-excited systems is still stable. In this context, this contribution discusses vibrations in non linear, forced, friction-excited systems of the form

$$
\mathbf{M} \ddot{\mathbf{q}}+\mathbf{D} \dot{\mathbf{q}}+(\mathbf{K}+\mathbf{N}) \mathbf{q}+\mathbf{f}_{\mathrm{NL}}(\dot{\mathbf{q}}, \mathbf{q})=\mathbf{f}_{\mathrm{ext}}(t),
$$

where $\mathbf{q}$ is the vector of generalized coordinates. $\mathbf{M}, \mathbf{D}, \mathbf{K}$ and $\mathbf{N}$ are the mass matrix, the damping matrix, the stiffness matrix and the circulatory matrix, respectively. $\mathbf{f}_{\mathrm{NL}}(\dot{\mathbf{q}}, \mathbf{q})$ and $\mathbf{f}_{\text {ext }}(t)$ are the vectors of non linear restoring forces and external excitation. A minimal model (see [2] for more details) is employed to numerically reproduce the test procedures, i.e. stepped sinusoidal excitation of the system in the frequency range of interest. In pre-flutter studies, i.e. studies in parameter regions where the steady sliding state is stable, frequency response functions for different excitation directions and amplitudes are calculated. Based on these results we try to challenge and derive criteria which allow for the estimation of stability borders. Energy terms are used as supporting measures. In post-flutter studies, i.e. studies in parameter regions where limit cycle solutions exist, transient and steady-state solutions are determined. Regions of entrainment are estimated.

The results reveal that non linear, forced, friction-excited systems are rich in phenomena. The examined criteria for the estimation of stability borders work reliably in vicinity of the Hopf point. A further enhancement of the methods is therefore necessary to ensure proper interpretation of test results also in larger distance from the bifurcation point which is a priori unknown.

\section{References}

[1] P. Stegmann, S. Kruse, K. Augsburg. Comparison of Different Excitation Concepts for the Characterization of Brake Systems Robustness regarding Squeal. In: Proceedings of Eurobrake 2014, Lille, France, 2014.

[2] S. Kruse, M. Tiedemann, B. Zeumer, P. Reuss, N. Hoffmann, H. Hetzler. The influence of joints on friction induced vibrations in brake squeal. J. Sound Vibr., in press. 


\title{
A finite element model for a soft robot equipped with a flexible limb
}

\author{
Kristin M. de Payrebrune, Oliver M. O'Reilly \\ Department of Mechanical Engineering; UC Berkeley; 6112 Etcheverry Hall; Berkeley, CA 94720-1740; USA
}

In many industrial and biomedical fields, robots and automata are used to reduce dangerous or repeatable tasks people do not wish to perform, to overcome human limitations in strength and speed, or to operate in hostile environments where humans are unable to work [1,2]. Recently, a new kind of robot, known as a soft robot, has been the object of extensive research. This research has been motivated by the potential benefits of soft robots in applications to healthcare, cooperative human assistance, service robots and biomechanically compatible interactions $[2,3]$. With the absence of a skeleton-like structure and the use of soft materials, complex locomotion or movements of the soft robot are possible. However new control strategies and mathematical models are required to advance the field of soft robots. The development of accurate yet feasible models is a particularly challenging problem.

In the presented research, a dynamic model for a soft robot that features in a new locomotion scheme proposed by Zhou et al. [4] is developed and analyzed. The soft robot studied consists of a single flexible limb attached to a rigid mass. By carefully controlling the intrinsic curvature of the limb and exploiting friction forces, locomotion, in principle, is possible. Here, we develop a rich model for the robot by using a finite element based model for the soft limb. The feasibility of the proposed locomotion scheme is then assessed and conclusions generated for expanding the locomotion scheme to more complex soft robot designs.

\section{References}

[1] M. Hägele, K. Wegener. Service-Roboter-Visionen. Hanser Verlag, (2004).

[2] C. Majidi. Soft robotics: A perspective - current trends and prospects for the future. Soft Robotics SoRo, 1 (2013), $5-11$.

[3] S. Kim, C. Laschi, B. Trimmer. Soft robotics: a bioinspired evolution in robotics. Trends in Biotechnology, 31 (2013), $287-294$.

[4] X. Zhou, C. Majidi, O.M. O'Reilly. Flexing into Motion: A Locomotion Mechanism for Soft Robots. Flexing into Motion: A Locomotion Mechanism for Soft Robots, Submitted for publication (2014) 


\title{
On the influence of vibrations on macroscopic frictional contacts
}

\author{
Antonia Hammerschmidt, Utz von Wagner \\ Chair of Mechatronics and Machine Dynamics, TU Berlin
}

Ultrasonic vibrations in the micrometer amplitude range have been proven to be capable to influence frictional contacts with respect to decreasing friction coefficients in experiments on small test set-ups solely loaded by their small weight $[1,2]$. In this presentation experimental results on applying this effect to large objects are presented. To get large vibration amplitudes and velocities detailed pre-investigation on the excitation of the structures in contact have been performed. These structures are investigated in a roller rig designed for these purposes.

\section{References}

[1] V. L. Popov, J. Starcevic, and A. E. Filippov. Influence of Ultrasonic In-Plane Oscillations on Static and Sliding Friction and Intrinsic Length Scale of Dry Friction Processes. Tribology Letters 39 (2009), 25-30.

[2] V. L. Popov, J. Starcevic, and E. Teidelt. Influence of In-Plane and Out-Of-Plane Ultrasonic Oscillations on Sliding Friction. Energy and Environmental Aspects of Tribology. (2010), 128-136. 


\title{
On the Effect of Contact Compliance on Vibrational Smoothing of Dry Friction
}

\author{
Simon Kapelke, Wolfgang Seemann \\ Institute of Engineering Mechanics (ITM), Karlsruhe Institute of Technology
}

High-frequency vibrations may be utilized in order to smooth the characteristics of dry friction at low sliding velocities and, consequently, quench undesired friction induced phenomena such as stick-slip motion [1]. Many studies have been published so far, most of them using classical Coulomb friction models and yielding conpact results. Unfortunately, the agreement with related experimental results is insufficient. As the Coulomb model overestimates the smoothing effect, improved modelling seems to be necessary [2].

In order to overcome one of the main disadvantages of the Coulomb friction model, contact compliance is considered here. Based on the friction model suggested by Dahl, the effect of longitudinal and transverse highfrequency vibrations on a 1-DoF friction oscillator is investigated.

Due to some shortcomings of Dahl's friction model, further improvement of the model is discussed. The LuGremodel can be regarded as an extension of Dahl's model capturing velocity dependencies. The model proposed by Dupont et al. additionally accounts for stiction regimes and is therefore the most evolved friction model used within this contribution [3].

It is shown, that the qualitative agreement between measurements and simulations is highly improved when accounting for contact compliance. Using Dahl's model, compact analytical results can be derived from approximation, while the extended models have to be evaluated numerically. Quantitative results can only be obtained after experimental determination of model parameters.

\section{References}

[1] Thomsen, J. J.: Using fast vibrations to quench friction-induced oscillations. Journal of Sound and Vibration 228 (1999), 1079-1102.

[2] Storck, H. et al.: The effect of friction reduction in presence of ultrasonic vibrations and its relevance to travelling wave ultrasonic motors. Ultrasonics 40 (2002), 379-383.

[3] Dupont, P. et al.: Single state elastoplastic friction models. IEEE Transactions on Automatic Control 47 (2002), 787-792. 


\section{S06: Material modelling in solid mechanics}

The section focuses on constitutive modeling of natural and artificial materials subjected to elastic and inelastic deformation processes. The aim is to compare new constitutive models formulated on phenomenological and micromechanics basis to determine their validity limits also by means of the simulation of the experimental data. A wide range of open problems will be considered in the Section, from multi-scale modeling of heterogeneous materials, to the implementation of constitutive models in numerical applications until to the virtual testing of structural systems. 


\title{
A novel stabilization technique for X-FEM like enriched formulations
}

\author{
Giulio Ventura, Irene Rabino \\ Department of Structural, Geotechnical and Building Engineering \\ Politecnico di Torino, Italy
}

The extended Finite Element Method, introduced by Ted Belytschko and coworkers and reviewed in [1], is considered nowadays an almost mature technology, so that various commercial implementations exist. Nonetheless, there are several fundamental aspects normally overlooked, that can be considerably improved in view of large scale application of the method. Typical examples are blending to standard finite elements, where effective solutions have been found, and efficient quadrature in enriched elements that is still an open problem although many contributions exist.

Among these fundamental aspects, a major one is played by the so-called "stabilization", i.e. the fact that, in some circumstances, the X-FEM formulation turns out into an indeterminate system of equations. This is evidenced by the blow up of the condition number in linear equations solving with the consequent ill-conditioning. Typically, the problem arises when a discontinuity comes close to enrichment nodes, and an element is split into two parts, one of which is very small.

Although this problem arises frequently in practical applications, it is surprisingly faced by few publications. Proposed techniques for its solution include modification of the enrichment structure [2], use of system preconditioners, eigenvalue decomposition of the element stiffness matrix [3]. While the proposed literature approaches are based on the idea of correcting the enrichment space to solve the problem, here a markedly different approach is presented. An analytic study of the problem has been done to see how the structure of the problem changes in these degenerate cases. Then, to ensure optimal conditioning, computational speed in large scale applications and to keep the lightweight structure of the original X-FEM enrichment, the standard and enrichment variables are constrained by small penalty terms in the global variational problem. This contribution presents the development of this new stabilization approach as well as the results on some benchmark problems.

\section{References}

[1] T. Belytschko, R. Gracie, G. Ventura. A review of extended/generalized finite element methods for material modeling. Modelling Simul. Mater. Sci. Eng. 17, 043001.

[2] I. Babuška, U. Banerjee. Stable Generalized Finite Element Method (SGFEM). Comput. Methods Appl. Mech. Engrg. $201-204$ (2012) $91-111$.

[3] S. Loehnert. A stabilization technique for the regularization of nearly singular extended finite elements. Comput. Mech. 54 (2014), $523-533$. 


\title{
Automatic Implementation of Elasto-plastic Incremental Formulations at Finite Strains using Hyper-Dual Numbers
}

\author{
Masato Tanaka, Daniel Balzani, Jörg Schröder \\ Toyota Central R\&D Labs. Inc. \\ Institute of Mechanics and Shell Structures, Technical University Dresden \\ Institute of Mechanics, University of Duisburg-Essen
}

\begin{abstract}
Numerical simulation of standard dissipative materials undergoing finite strains remains an important and challenging topic in computational mechanics. For their implicit finite element implementation, the stresses and consistent tangent moduli are required. Whereas the precision quality of the stress calculation determines the physical accuracy of the numerical simulation, the accuracy of the algorithmic tangent moduli influences the convergence behavior in an iterative solution scheme as well as the detection of material instabilities in localization analysis, cf. e.g., [1]. For conventional material formulations, evolution equations are defined for the variables associated with the dissipative material behavior. Ortiz et al.[2] recasted the inelasticity theory as an equivalent optimization problem where the incremental stress potential within time interval $\left[t_{n}, t_{n+1}\right]$ is minimized with respect to the internal variables. The scheme is referred to as incremental variational formulation (IVF). The IVF provides a general numerical framework which is suitable for the implementation of a broad range of constitutive models and their thermodynamical consistency is a priori guaranteed, cf. e.g., [3]. One of the important advantages of IVFs is their quasi-hyperelastic potential structure which allows to investigate the existence of minimizers by analyzing generalized convexity conditions, cf. e.g., [1]. On the other hand, the main disadvantage is that their implementation requires more effort than classical formulations. This is partially due to higher-order derivatives e.g. of the incremental stress potential with respect to the internal varialbes which are additionally required for solving the inner minimization problem in terms of the NewtonRaphson method. Therefore, numerical approximations of the derivatives may be a useful alternative reducing the implementation time in particular for scientific development purposes. However, often-used numerical differentiation schemes, such as the finite difference method, will end up in a comparably poor accuracy being also sensitive with respect to perturbation values, especially for the higher-order derivatives. Fike [4] developed a method for exact and automatic first- and second-order derivative calculations independent on the choice of perturbation values using hyper dual numbers (HDNs). Tanaka et al. [5] exploited this for a numerical scheme to implement hyperelastic materials. There, it is shown that the numerical calculation of stresses and tangent moduli is almost identical to the implementation of the analytic derivatives in the sense of computer accuracy. In this contribution, a novel implementation of IVFs using HDNs is presented to arrive at a fully automatic and robust scheme with computer accuracy. This implementation scheme provides a compact model-independent framework, which means that once the framework is implemented, any other standard dissipative material model can be incorporated by solely modifying the energy functions, i.e., the Helmholtz free energy function, the dissipation potential function and additional side constraints such as e.g. the yield function in the case of plasticity. Its uncomplicated implementation for associative finite strain elasto-plasticity and performance is illustrated by some representative numerical examples.
\end{abstract}

\section{References}

[1] J. Schröder. Plasticity and Beyond - Microstructures, Chrystal-Plasticity and Phase Tran- sitions (CISM Lecture Notes), chapter A numerical two-scale homogenization scheme: the FE ${ }^{2}$-method. Springer, (2013).

[2] M. Ortiz, L. Stainier. The variational formulation of viscoplastic constitutive updates. Comput. Methods Appl. Mech. Eng. 171 (1999), 419-444.

[3] L. Stainier, M. Ortiz. Study and validation of a variational theory of thermomechanical coupling in finite visco-plasticity. Int. J. Solids Struct. 47 (2010), 705-715.

[4] J.A. Fike. Multi-objective optimization using hyper-dual numbers. PhD thesis (2013).

[5] M. Tanaka, T. Sasagawa, R. Omote, M. Fujikawa, D. Balzani, J. Schröder. A highly accurate 1st- and 2nd-order differentiation scheme for hyperelastic material models based on hyper-dual numbers. Comput. Methods Appl. Mech. Eng. 283 (2015), 22-45. 


\title{
Efficient time integration in multiplicative inelasticity
}

\author{
Ralf Landgraf, Alexey Shutov, Jörn Ihelmann \\ Professorship of Solid Mechanics, Faculty of Mechanical Engineering, \\ Technische Universität Chemnitz, Chemnitz, Germany
}

Models of multiplicative inelasticity are widely used to simulate the phenomenological behaviour of, for example, polymeric or metallic materials. Typical specific examples are models of multiplicative viscoelasticity, elastoplasticity or viscoplasticity. Within numerical simulations, a time stepping scheme of the constitutive equations is needed. Beside stability and accuracy of the numerical algorithm, its efficiency is one crucial aspect.

In Shutov et. al. [1], a famous model of Maxwell fluid, which is based on the multiplicative decomposition of the deformation gradient and includes a Neo-Hookean hyperelasticity relation as well as an incompressible viscous flow rule, has been considered. More precisely, a time stepping algorithm for implicit time integration of the inelastic flow rule has been presented. The integration algorithm is based on a backward Euler time discretisation, is iteration free and prevents error accumulation.

In this contribution, the basic idea of Shutov et. al. [1] is applied to more general models of multiplicative inelasticity. Here, models of multiplicative viscoelasticity, elastoplasticity and viscoplasticity are considered. Thereby, efficient time stepping algorithms are derived, where only one scalar equation for one scalar unknown has to be solved within every time step. Some properties of the algorithms will be discussed, numerical test will be presented and examples of the application within the finite element method will be demonstrated.

\section{References}

[1] A.V. Shutov, R. Landgraf, J. Ihlemann. An explicit solution for implicit time stepping in multiplicative finite strain viscoelasticity. Comput. Methods in Appl. Mech. Eng. 265 (2013), 213-225. 


\title{
Towards a finite element simulation of coating by means of thermal spraying
}

\author{
$\underline{\text { Rolf Berthelsen }}^{1}$, Ralf Denzer ${ }^{1,2}$, Andreas Menzel $^{1,2}$ \\ ${ }^{1}$ Institute of Mechanics, TU Dortmund, Leonhard-Euler-Straße 5, 44227 Dortmund, Germany \\ ${ }^{2}$ Division of Solid Mechanics, Lund University, Ole Römers väg, 22363 Lund, Sweden
}

In the automotive industry, metal sheet forming processes like deep drawing are applied in order to produce carriage parts in mass production. Therefore, forming tool surfaces are required that are well protected against wear. This ensures a long tool life cycle with a constant high product quality. Such surfaces are, amongst other techniques, produced by thermal spraying of hard material coatings. These coatings considerably improve the wear resistance of the coated surfaces. The thermal spraying process itself is a highly transient thermomechanical process. In order to obtain a better understanding of the heat input and transfer during thermal spraying, a simulation framework for thermal spraying processes is presented.

A finite-element-based software tool for the simulation of heat transfer during thermal spraying was recently proposed, [1]. This continuum thermodynamical framework, based on [2, 3] and references cited therein, is now extended by the application of hot particles on the surface of a workpiece. A time dependent configuration of a rigid heat conductor is considered. The configuration can change from one time step to another by the addition of hot coating particles that model the mass application of a considered thermal spray gun. The heat transfer between the considered workpiece and the environment is captured by Robin type boundary conditions. Several numerical examples of the underlying framework are presented.

\section{References}

[1] R. Berthelsen, T. Wiederkehr, R. Denzer, A. Menzel, H. Müller. Efficient simulation of nonlinear heat transfer during thermal spraying of complex workpieces. World Journal of Mechanics 4 (2014), 289-301

[2] I-S. Liu. Continuum mechanics. Springer, Berlin; New York (2002)

[3] K. Hutter. Continuum methods of physical modeling. Springer, Berlin; New York (2004) 


\title{
Modeling the moisture and temperature dependant material behavior of adhesive bonds
}

\author{
Florian Goldschmidt, Stefan Diebels \\ Universität des Saarlandes, Lehrstuhl für Technische Mechanik
}

As adhesive bonding becomes more and more relevant in joining technology, a precise description of the mechanical material behavior has to be developed. This work presents a three-dimensional continuum mechanical model to respect environmental influences like changes in temperature or a moist environment that can cause changes in this behavior.

Due to the fact, that adhesive layers are, in the majority of cases, made of cross-linked polymers, the concept of modeling such materials is the starting point of modeling adhesives.

For cross-linked polymers, it is usual to behave viscoelastic close to the glass transition temperature. This rate dependent behavior vanishes if the material is tested at significantly higher or lower temperatures, because intermolecular movement is enhanced or inhibited. In general, this can be assumed to be the case for adhesives as well. For Polymers this effect has be described before $[1,3,4]$. During application, adhesives are subjected to further environmental influences like moisture. Experience has shown, that viscoelastic properties are reduced by increasing moisture inside the specimen. This effect has been taken into account as well [2, 5].

In the past each and every effect that influences the mechanical material behavior of polymers has been investigated separately. In the presented contribution a model taking all of these effects into account is presented. To this the viscoelastic parameters $\mu_{e}^{i}$ are formulated as functions

$$
\mu_{e}^{i}(\chi, \Theta)=\mu_{0}^{i} f^{i}(\chi) \cdot f^{i}(\Theta)
$$

of the additional moisture $\chi$ and temperature $\Theta$ fields. The used interpolation functions $f^{i}(\bullet)$ are chosen to fit for interpolation between experimental results and to provide physically reasonable values for extrapolation.

\section{References}

[1] Dippel, B., M. Johlitz, A. Lion [2014]. Thermo-mechanical couplings in elastomers - experiments and modelling. ZAMM - Journal of Applied Mathematics and Mechanics / Zeitschrift f ür Angewandte u Mathematik und Mechanik .

[2] Goldschmidt F., S. Diebels. Modelling and numerical investigations of the mechanical behavior of polyurethane under the influence of moisture. Archive of Applied Mechanics. (2014), 1-8.

[3] Johlitz, M., S. Diebels, W. Possart. Investigation of the thermoviscoelastic material behaviour of adhesive bonds close to the glass transition temperature. Archive of Applied Mechanics. (2012), 1089-1102.

[4] Johlitz, M., A. Lion Chemo-thermomechanical ageing of elastomers based on multiphase continuum mechanics. Continuum Mechanics and Thermodynamics. (2013), 605-624.

[5] Ranz, T. Elementare Materialmodelle der Linearen Viskoelastizität im Zeitbereich. Beiträge zur Materialtheorie. (2007) 


\title{
Determination of material parameters corresponding to viscoelastic curing polymers
}

\author{
Sandra Klinge, Paul Steinmann \\ Technical University Dortmund, 44227 Dortmund, Germany \\ University of Erlangen-Nuremberg, 91058 Erlangen, Germany
}

Two goals characterize the present contribution: First, the development of a numerical approach for determining the properties of the material microstructure, and second, the shift of the focus of the inverse analysis from investigating a purely elastic material toward the parameter identification related to heterogeneous inelastic materials. As a rule, the constitutive laws in this case involve a greater number of material parameters the determination of which requires different kinds of tests.

The numerical approach proposed uses the combination of the Levenberg-Marquardt method and the multiscale FEM [1, 2]. The former is a gradient-based method coupling the advantages of the steepest descent method and of the minimization of the Taylor approximation of a function. On the other hand, the multiscale FEM is a numerical homogenization method such that the coupling of the macroscopic and microscopic scales is realized through the Hill macro-homogeneity condition. Here, the macroscopic scale corresponds to the structural level while the microscale is related to the RVE response. The proposed scenario is advantageous as it is easily applicable for different microheterogenous materials. For this purpose, the global algorithm has to be retained whereas the material subroutines have to be exchanged at the microlevel.

The application of the method is demonstrated on the basis of an example studying a curing polymer combined with a nonlinear elastic material. The mechanical model related to the curing material includes an equilibrium and a non-equilibrium part and depends on 11 material constants [3]. The potential of the elastic inclusion corresponds to the neo-Hooke material and includes two material parameters. The identification procedure proposed comprises three phases: The final elastic parameters of the curing material and the elastic parameters of the inclusion are determined at the first stage. The second stage deals with the evaluation of the final value of the relaxation time and the elastic parameters related to the curing material. Finally, the last stage determines the constants related to the curing process.

The simulations in the first phase show that the procedure is highly sensitive on the quality of measured values and that their error leads to the inaccuracy and to the non-uniqueness of the solution. This shortcoming opens many interesting issues which can be studied in the future.

\section{References}

[1] S. Klinge. Inverse analysis for multiphase nonlinear composites with random microstructure. Int. J. Multiscale Comp. Eng. 10 (2012), 361-373.

[2] S. Klinge, P. Steinmann. Inverse analysis for heterogeneous materials and its application to viscoelastic curing polymers. Comput. Mech. (Submitted.)

[3] S. Klinge, A. Bartels, P Steinmann. The multiscale approach to the curing of polymers incorporating viscous and shrinkage effects. Int. J. Solid. Struct. 49 (2012), 3883-3900. 


\title{
Towards modelling the curing process in particle-filled electro-active polymers
}

\author{
Mokarram Hossain $^{\dagger}$, Paul Steinmann ${ }^{\dagger}$ \\ ${ }^{\dagger}$ Chair of Applied Mechanics, University of Erlangen-Nuremberg, Germany \\ mokarram.hossain@ltm.uni-erlangen.de, paul.steinmann@ltm.uni-erlangen.de
}

In dielectric elastomers, a large actuation voltage is required to produce a desired mechanical deformation. To reduce the amount of the actuation voltage, several mechanisms can be applied and the inclusion of high dielectric permittivity fillers in the matrix material in the uncured stage is one of them. Moreover, to obtain a maximum advantage from the high dielectric permittivity fillers, an electric field is applied during the curing process which helps the particles to align in a preferred direction. The polymer curing process is a complex viscoelastic phenomenon where a liquid polymer gradually transforms into a solid due to cross-linking of the initially short polymer chains. This phase transition comes along with an increase in material stiffness and a volume shrinkage. Such stiffness gaining is modelled by an appropriate constitutive relation where the temporal evolution of the material parameters is considered. We present a phenomenologically-inspired large strain framework for simulating the curing process of polymers that can work under the use of an electro-mechanically coupled load. The application of the proposed approach is demonstrated via some numerical examples. These illustrate that the model can predict common features in particle-filled electro-active polymers undergoing curing processes in the presence of an electro-mechanically coupled load [1,2].

\section{References}

[1] M. Hossain, P. Saxena, P. Steinmann, Modelling the mechanical aspects of the curing process of magneto-sensitive elastomeric materials, Submitted

[2] M.R. Kashani, S. Javadi, N. Gharavi, Dielectric properties of silicone rubber-titanium dioxide composites prepared by dielectrophoretic assembly of filler particles, Smart Materials and Structures, 19, 2010, 1-7 


\title{
FE-simulation of spatially graded gelation during adhesive's curing
}

\author{
Martin Rudolph, Jörn Ihlemann \\ Professorship of Solid Mechanics, Faculty of Mechanical Engineering, \\ Technische Universität Chemnitz, Chemnitz, Germany
}

Against the backdrop of increasing demands on energy efficiency and the integration of functions, the Collaborative Research Center/Transregio 39 PT-PIESA of the German Research Foundation (DFG) conducts research on lightweight components with inherent actuator and sensor functionality [1]. One process chain studied within this research project is the manufacturing of layered metal compounds with integrated piezo electric modules by sheet metal forming. Former works present a special layered setup with an adhesive layer on the inside, which protects the piezo from critical loads during the manufacturing process, and demonstrate the fundamental feasibility of this approach [2]. Since the forming is carried out while the adhesive is still in liquid state, spacers surrounding the adhesive's volume are required. The subsequent curing reaction of the already formed compound assures the material closure.

The present work contributes to the further development of this manufacturing process in order to accomplish its series quality. This is to be achieved by employing a different adhesive system as well as a temporally and spatially distinct temperature control which allows for a graded gelation. Thus, the adhesive represents a volume with two different phases and hence fulfills both: protection of the piezo by a liquid core and the fixation as a solid spacer at the outside. In order to evaluate the feasibility of the new process, a simulation based process design is strived. Within this contribution, we focus on processes within the adhesive layer. Several measurement strategies for process dependent mechanical and thermal material properties as well as a material modelling approach based on the work in [2] are shown. Moreover, a finite element based simulation of the partial curing process due to the selected temperature regime and under consideration of the exothermic reaction heat is presented. By the help of this simulation tool, parameter variations as well as the derivation of important information on the process design are enabled.

\section{References}

[1] R. Neugebauer, L. Lachmann, W.G. Drossel, M. Nestler, S. Hensel. Smart Semi-finished Parts for the Application in Sheet-Metal Structures. Future Trends in Production Engineering (2013), 121-130. doi: 10.1007/978-3-642-24491-9_13

[2] R. Landgraf, M. Rudolph, R. Scherzer, J. Ihlemann. Modelling and simulation of adhesive curing processes in bonded piezo metal composites. Comput. Mech. 54 No. 2 (2014), 547-565. doi: 10.1007/s00466-014-1005-5 


\title{
Characterisation of filled rubber with a pronounced nonlinear viscoelasticity
}

\author{
Tobias Scheffer $^{1}$, Florian Goldschmidt ${ }^{1}$, Stefan Diebels ${ }^{1}$ \\ ${ }^{1}$ Lehrstuhl für Technische Mechanik, Universität des Saarlandes
}

\begin{abstract}
This contribution presents the characterisation of an incompressible carbon black-filled elastomer as one characteristical example for highly filled rubber. It shows a strongly pronounced nonlinear viscoelastic behaviour and the most important characteristic is the extremely long relaxation time which has to be taken into account. The material model is developed with respect to uniaxial tension data. In this work, a step by step development of a phenomenological model is presented starting with the basic elasticiy. For this evaluation the long term relaxation behaviour results in a complex experimental procedure. Therefore, special attention has to be paid according to an optimised experimental process in order to get the necessary reference data in an adequate and reproduceable way [1].

With this model basis further investigations are taken into account concerning the time-dependent viscoelasticity. Therefore, cyclic deformation from zero up to a maximum of deformation are considered for different strain rates. Furthermore, the relaxation behaviour is investigated for multiple strain levels. The phenomena which are observed in the experimental results yield a purely viscoelastic model, based on a rheological analogous model consisting of an equilibrium spring and several Maxwell-elements which contain nonlinear relations for the relaxation times of the dashpot elements [1, 2].

The material model's numerical realisation is accomplished in two ways. Because of its numerical simplicity especially according to the parameter identification the model is restricted only to the simple case of uniaxial tension as the first solution. A second, alternative implementation is executed providing the benefit that more complex deformation conditions can also be taken into account. Therefore, the three-dimensional finite model is implemented in an open-source Finite Element library [3].
\end{abstract}

\section{References}

[1] T. Scheffer, H. Seibert, S. Diebels. Optimisation of a pretreatment method to reach the basic elasticity of filled rubber materials. Arch. Appl. Mech. 83 (11) (2013) 1659-1678.

[2] N. Koprowski-Theiß, M. Johlitz, S. Diebels. Characterizing the time dependence of filled EPDM. Rubber Chem. Technol. 84 (2) (2011) 147-165.

[3] T. Scheffer, F. Goldschmidt, S. Diebels. Strongly pronounced nonlinear viscoelasticity of an incompressible filled rubber. Arch. Appl. Mech (2015), submitted. 


\title{
A study on the influence of mechanical preconditioning on the fatigue behavior of rubber materials
}

\author{
$\underline{\text { Maria Krause }}^{1}$, Daniel Juhre ${ }^{1,2}$ \\ ${ }^{1}$ Deutsches Institut für Kautschuktechnologie e.V. (DIK), Hanover, Germany \\ 2 Institute of Mechanics, Otto-von-Guericke University, Magdeburg, Germany
}

Rubber is increasingly engaged to many industrial applications like tires, dampers, seals and medical equipment. High demands are set to these technical devices and parts. For instance, the durability in service conditions must be ensured. Hence, it is important to predict the fatigue behavior of technical rubber parts, already during their design phase.

Approaches that assess the lifetime of technical rubber components are intensively elaborated and documented, see e.g. [1, 2]. Most approaches are experimentally motivated by lifetime investigations that base on fatigue tests under uniaxial tension conditions [3]. Usually, these tests are performed with respect to varying stress amplitudes. Any pretreatment of these test samples is generally not considered. Nonetheless, it is well known that diverse preconditioning procedures can lead to markable different degrees of material softening caused by the inelastic behavior of filled elastomers. This means the material softening can be controlled by the preconditioning of the test samples. This leads to the question if the preconditioning influences the lifetime noticable. In practical applications, it is hard to avoid a preconditioning of the rubber components which is simply triggered by prestretching or -loading these parts during installation. In experimental investigations, the samples are supposed to be barely touched before analyzing the fatigue behavior. It is not clear, if any preconditioning would lead to a reduced or extended lifetime. However, to come closer to a realistic lifetime prediction of filled elastomers, the preconditioning cannot be neglected.

In this contribution we will present a study of investigating the influence of preconditioning on rubber samples. Conventional uniaxial tension tests are extended with different preloading and prestretching conditions. The experimental tests will show that the measured lifetime can depend on the assigned preconditioning. The kind of loading and the type of observed rubber are decisive. Using the Model of Rubber Phenomenology (MORPH) [4], which enables the numerical modelling of softening behavior for large deformed filled elastomers, simulations are performed. The simulations consider the strain-history during loading cycles and allow a more realistic prediction of lifetime. A final comparison of experimental and simulation results with and without preconditioning concludes this study.

\section{References}

[1] N. Saintier, N. Andre, G. Cailletaud and R. Piques. Multiaxial fatigue life prediction for a natural rubber. International Journal of Fatigue 28,530-539, 2006

[2] A. Andriyana, N. Saintier and E. Verron. Configurational Mechanics and Critical Plane Approach: Concept and application to fatigue failure analysis of rubberlike materials. International Journal of Fatigue. 32: 1627-1638, 2010

[3] M. Flamm, T. Steinweger, U. Weltin. Schadensakkumulation bei Elastomeren. Kautschuk Gummi Kunststoffe 55. Jahrgang, Nr. 12/2002

[4] J. Ihlemann. Kontinuumsmechnische Nachbildung hochbelasteter technischer Gummiwerkstoffe. Fortschr.Ber. VDI Reihe 18 Nr. 288. Düsseldorf, 2003 


\title{
On the generation of soft magneto-electric effects through Maxwell interactions
}

\author{
Marc-André Keip \\ Institute of Applied Mechanics (CE), Chair I \\ University of Stuttgart, 70569 Stuttgart, Pfaffenwaldring 7, Germany
}

Materials with strong magneto-electric (ME) coupling are of particular interest for the development of new functional materials with multi-field properties. Since in single-phase materials the ME coupling is extremely weak at room temperature, there has been a steady increase in attention to the generation of ME composites. Classically, such ME composites are built from magnetostrictive and piezoelectric phases, whose mechanical interaction gives rise to effective ME coupling [1, 2]. Due to the important notion of the interphase strain, ME composites obtain their optimal performance when built from constituent phases with "giant" magnetostriction and piezoelectric coupling (like, for example, in the case of Barium-Titanate-Cobalt-Ferrite or Lead-ZirconateTitanate-Terfenol-D composite systems).

As an attractive alternative to that, this talk dicusses a new way for the generation of ME coupling based on the construction of soft composites. The main idea of these composites is to produce the effective ME coupling through large-strain Maxwell interactions, whose key drive is given by electrically and magnetically induced Maxwell stresses [3]. Such soft composites may have several advantages compared to multiferroic composites: Since they are soft, they are less fragile than their crystalline counterparts. Furthermore, they are easy to process, have a long life time, and are cheap. This talk will outline the basic working principle of soft magneto-electric composites as well as possible routes to the generation of maximum coupling coefficients. For the determination and the design of the overall properties, a computational homogenization scheme will be employed $[4,5]$.

\section{References}

[1] M. Fiebig. Revival of the magnetoelectric effect. J. Phys. D: Appl. Phys. 38 (2005), R123-R152.

[2] M. Labusch, M. Etier, D. C. Lupascu, J. Schröder \& M.-A. Keip. Product properties of a two-phase magnetoelectric composite: Synthesis and numerical modeling. Comput. Mech. 54 (2014), 71-83.

[3] L. Liu, P. Sharma. Giant and universal magnetoelectric coupling in soft materials and concomitant ramifications for materials science and biology. Phys. Rev. E 88 (2013), 040601.

[4] J. Schröder, M.-A. Keip. Two-scale homogenization of electromechanically coupled boundary value problems. Comput. Mech. 50 (2012), 229-244.

[5] M.-A. Keip, P. Steinmann, J. Schröder. Two-scale computational homogenization of electro-elastity at finite strains. Comput. Methods Appl. Mech. Engrg. 278 (2014), 62-79. 


\title{
Numerical Aspects of Energy Relaxation-Based Magnetostriction Modeling
}

\author{
$\underline{\text { Karsten Buckmann }}^{1}$, Björn Kiefer ${ }^{1}$, Thorsten Bartel ${ }^{1}$, Andreas Menzel ${ }^{1,2}$ \\ ${ }^{1}$ Institute of Mechanics, Department of Mechanical Engineering, TU Dortmund \\ ${ }^{2}$ Division of Solid Mechanics, Lund University
}

\begin{abstract}
Among the broad spectrum of active and multifunctional materials, the subclass of multiferroic materials are particularly interesting candidates to potentially enable novel applications in, e.g., actuation, sensing, transducing, and data storage applications, due to their unique response properties triggered by the complex multiscale interplay of ferromagnetic (spontaneous magnetization), ferroelectric (spontaneous polarization), or ferroelastic (spontaneous straining) mechanisms. Magnetic shape memory alloys (MSMA) are intrinsically multi-ferroic materials, exhibiting strong magnetomechanical coupling behavior in addition to the conventional shape memory effect. The strain and magnetization response is nonlinear, anisotropic, hysteretic, and highly stress level-dependent. The presented energy relaxation model [1] builds on the foundation of the constrained theory of magnetoelasticity by DeSimone and James [2], which combines the Ball and James theory of microstructure formation [3] with classical micromagnetics approaches [4] to describe the formation of fine-scale microstructures in magnetostrictive materials. Their approach is based on the relaxation of non-convex energy densities, wherein the state variables are constrained to take values in the energy wells. Although the constrained theory is able to predict many important features of the magnetic shape memory effect, other essential features, e.g. hysteretic behavior and the stress-level dependence of the maximum magnetic field induced strain, are not captured. The presented approach aims to overcome some of its limitations, particularly that (i) elastic strains are omitted, (ii) the magnetizations are rigidly attached to easy axes, and (iii) dissipation is not considered.

In the first step of formulating the extended model we allow for "non-energy-well"-states with respect to elastic deformations, necessitating assumptions about the total strains in each martensitic variant, and magnetization orientations, captured by a finite magnetocrystalline anisotropy energy contribution. Secondly, the hysteretic nature of the response is accounted for by introducing an appropriate dissipation functional in an incremental variational setting. The strains and local magnetization orientations represent additional microstructural degrees of freedom, which are determined via minimization of an incremental potential. In this manner, a relaxed energy density is obtained which serves as an approximation of the mathematically and physically desired quasiconvex hull. Two numerical relaxation approaches for the elastic strain energy are considered. The convexification approach offers the possibility of accounting for the full spectrum of martensitic phases in a straightforward manner and yields a lower energy bound. The rank-one relaxation approach on the other hand enforces interface compatibility requirements and produces an upper energy bound of the quasiconvex hull. The presented MSMA response predictions with the extended model show excellent qualitative and very reasonable quantitative agreement with experiments. We also report on first results in the endeavor of embedding the developed energy relaxation-based magnetostriction models into a micromagnetics-inspired finite element framework solving the magnetomechanically coupled field equations. This approach allows response simulations for single crystalline samples and devices of essentially arbitrary geometry as well as meso-scale analyses of polycrystalline aggregates, and will eventually make the discussed models accessible to numerical homogenization based scale-bridging techniques. On-going work is concerned with the extension of the presented approaches to the fully three-dimensional setting and their application to other magnetostrictive material systems.
\end{abstract}

\section{References}

[1] B. Kiefer, K. Buckmann, T. Bartel. Numerical Energy Relaxation to Model Microstructure Evolution in Functional Magnetic Materials. GAMM-Mitt. 38(1) (2015), accepted for publication.

[2] A. DeSimone, R. D. James. A constrained theory of magnetoelasticity. J. Mech. Phys. Solids 50 (2002), 283-320.

[3] J. M. Ball, R. D. James. Fine phase mixtures as minimizers of energy. Arch. Ration. Mech. Anal. 100 (1987), $13-52$.

[4] W. F. Brown Jr. Micromagnetics (1963), John Wiley \& Sons. 


\title{
Computational homogenization in micromagnetics
}

\author{
Ashish Sridhar, Marc-André Keip, Christian Miehe \\ Institute of Applied Mechanics (CE), Chair I \\ University of Stuttgart, 70569 Stuttgart, Pfaffenwaldring 7, Germany
}

Ferromagnetic solids are characterized by a complex microstructure that consists of magnetic domains separated by domain walls. The evolution of the microstructure can be driven by external magnetic and mechanical stimuli and gives rise to the overall hysteretic response of the material. In order to directly link the microstructure evolution with the macroscopic response we propose a computational homogenization framework for dissipative magnetostriction. The formulation is based on a rigorous exploitation of rate-type and incremental variational principles bridging the two scales. These principles serve as canonical ingredients for the micro-to-macro transition and determine macroscopic variables in terms of their microscopic counterparts. The scale transition is performed between a gradient-extended dissipative micromagnetic model and a standard Boltzmann continuum on the macroscale. On the microscale, we use a phase field model of micromagnetics coupled with projection based methods in order to satisfy the unity constraint on the magnetization. An important aspect for the design of such a computational homogenization method is the definition of suitable boundary conditions at the micro-level. These are derived from an extension of a Hill-Mandel-type micro-macro compatibility condition to the micromagnetic scenario. In order to incorporate these boundary conditions into the proposed variational homogenization principles we use Lagrange multiplier and penalty methods conceptually similar to the purely mechanical treatments. Two- and three-dimensional numerical simulations will analyze the micromagnetic domain evolution as well as the associated overall hysteretic response of ferromagnetic materials for macroscopic strain- and magnetic-field-driven scenarios.

\section{References}

[1] C. Miehe, G. Ethiraj. A geometrically consistent incremental variational formulation for phase field models in micromagnetics. Comput. Methods Appl. Mech. Engrg. 245-246 (2012), 331-347.

[2] D. Zäh, C. Miehe. Computational homogenization in dissipative electro-mechanics of functional materials. Comput. Methods Appl. Mech. Engrg. 267 (2013), 487-510.

[3] M.-A. Keip, P. Steinmann, J. Schröder. Two-scale computational homogenization of electro-elastity at finite strains. Comput. Methods Appl. Mech. Engrg. 278 (2014), 62-79. 


\title{
An atomistic scale analysis of ferroelectric nanodomain interfaces
}

\author{
Florian Endres $^{1}$ and Paul Steinmann ${ }^{1}$ \\ ${ }^{1}$ Chair of Applied Mechanics \\ University of Erlangen - Nuremberg \\ Egerlandstrasse 5 \\ 91058 Erlangen, Germany
}

Ferroelectric functional materials are of gaining importance for modern sensing and actuation applications. Today it is possible to fabricate ferroelectric components on length scales of only several nanometers [1]. Therefore, atomistic simulations are indispensable to predict and understand material behaviour of ferroelectric nanocomponents. Especially nanodomain interfaces are of great interest. Atomistic simulations help to understand the physical properties of domainwalls and interfaces. Furthermore, atomistic simulations also increase the accuracy of constitutive models, e.g. phase field modelling by providing model parameters such as domain wall energies and thicknesses.

The aim of this talk is to present the latest numerical results of nanodomain interfaces using a new extended molecular statics algorithm for ferroelectric materials [2]. The new algorithm is able to not only calculate the change of the polarization behaviour caused by strain but also the influence on the polarization behaviour due to mechanical stress. The size effects of $180^{\circ}$ head to head and tail to tail nanodomains have already been investigated [3]. This study also considers $90^{\circ}$ domain walls and discusses the impact of mechanical stress on polarization patterns and system energies of nanodomain interfaces.

\section{References}

[1] P. Sc. H. Ghosez, X. Gonze, J.P. Michenaud. Ab initio phonon dispersion curves and interatomic force constants of barium titanate. Ferroelectrics. 90.21 (1998), 205-217.

[2] F. Endres, P. Steinmann. An extended molecular statics algorithm simulating the electromechanical continuum response of ferroelectric materials. Comp. Mech. 54.6 (2014), 1515-1527.

[3] F. Endres, P. Steinmann. Molecular statics simulations of head to head and tail to tail nanodomains of rhombohedral barium titanate. Comp. Mat. Sci. 97 (2015), 20-25. 


\title{
Analysis of Micro- and Macro-Instability Phenomena in Computational Homogenization of Finite Electro-Statics
}

\author{
D. Vallicotti, C. Miehe \\ Institute of Applied Mechanics (CE) \\ University of Stuttgart \\ Pfaffenwaldring 7, 70569 Stuttgart, Germany \\ daniel.vallicotti@mechbau.uni-stuttgart.de
}

Dielectric materials such as electro-active polymers (EAPs) belong to the class of functional materials which are used in advanced industrial environments as sensors or actuators and in other innovative fields of research. Driven by Coulomb-type electrostatic forces EAPs are theoretically able to withstand deformations of several hundred percents. These devices become practical as soon as the material is robust, reliable and long lived. However, large actuation fields and different types of instabilities prohibit the ascend of these materials. One distinguishes between global structural instabilities such as buckling and wrinkling of EAP devices, and local material instabilities such as limit- and bifurcation-points in the constitutive response. Numerically predicting global and local instabilities is a necessary, however demanding task for designing optimal devices.

We outline variational-based stability criteria in finite electro-elastostatics and design algorithms for accompanying stability checks in typical finite element computations. We start from a canonical energy minimization principle which is then shifted to an enthalpy-based saddle-point principle for convenience of numerical treatment. Here, global structural stability is based on a perturbation of the total electro-mechanical energy and related to statements of positive definiteness of incremental finite element tangent arrays. Local material stability is based on an incremental quasi-convexity condition inducing the positive definiteness of both the incremental electro-mechanical moduli as well as a generalized acoustic tensor. It is shown that the incremental arrays to be analysed in the stability criteria appear within the enthalpy-based setting in a distinct diagonal form, with pure mechanical and pure electrical partitions.

These accompanying stability checks are embedded into a computational homogenization framework to predict the macroscopic overall response and onset of local material instability of particle filled composite materials. Application and validation of the suggested method is demonstrated by representative model problems. 


\title{
Modeling of Carbon Fiber Reinforced Plastics for Wave Propagation Analysis in Plates
}

\author{
Bianca Hennings, Rolf Lammering \\ Institute of Mechanics \\ Helmut-Schmidt-University / University of the Federal Armed Forces Hamburg
}

The increasing use of carbon fiber reinforced plastic, e.g. in aerospace structures, requires advanced techniques for the monitoring of components from this material. Actually, guided waves (LAMB-waves), cf. [1], are considered as an appropriate means for the detection of structural defects like delaminations. After the excitation of guided waves, e.g. by a thin adhered piezoelectric actuator, at least two wave modes are propagating, i.e. the symmetric $S_{0}$-mode and the antisymmetric $A_{0}$-mode. Due to the higher group velocity, the $S_{0}$-mode is faster than the $A_{0}$-mode and thus these wave modes separate with time.

In non-damaged plates the optical observation of propagating waves through a scanning laser vibrometer shows a regular pattern, which, however, is disturbed by structural defects. At defect localizations, mode conversions take place which become clearly visible by optical measurements. Moreover, a detailed experimental observation of the wave propagation shows an additional effect between the moving $S_{0^{-}}$and $A_{0^{-}}$waves which has the appearance of an $A_{0}$-wave. At this location, the occurrence of an $A_{0}$-wave cannot be explained by the originally excited $A_{0}$-wave, since the group velocity is too low. For this reason, a "quasi-continuous" mode conversion is assumed, cf. [3].

First, this work presents mode conversion effects in carbon fiber reinforced plastic plates. A single layer has to be considered as transversely isotropic. Furthermore, the material is inhomogeneous by nature since it consists of stiff carbon fibers in a comparatively weak epoxy matrix. The optically observed pattern of wave propagation, which include these effects and which contain additionally the information of eventually existing defects, will be discussed.

Secondly, the modeling of the carbon fiber reinforced plastic for subsequent finite element analysis of wave propagation in plates from this material will be presented. Microscopic observations show, that the distribution of the fibers in the matrix material is not periodical, but clusters of fibers and matrix material occur. Therefore, a random distribution of stiffness and mass is present. This local inhomogeneity has to be considered in a microscale model if the above mentioned quasi-continuous mode conversion shall be captured properly, cf. [2]. Finally, a numerical example will show the influence of the material model on the developing wave modes.

\section{References}

[1] K. F. Graff. Wave motion in elastic solids. Oxford University Press, 1975.

[2] B. Hennings. Elastische Wellen in faserverstärkten Kunststoffplatten - Modellierung und Berechnung mit spektralen finiten Elementen im Zeitbereich. Dissertation, Institut für Mechanik, Helmut-SchmidtUniversität / Universität der Bundeswehr Hamburg, 2014.

[3] M.N. Neumann, B. Hennings, R. Lammering. Quasi-continuous mode conversion of LAMB-waves in CFRPplates due to inhomogeneity on micro- and mesoscale. 7th European Workshop on Structural Health Monitoring, July 8-11, 2014, Nantes, France. 


\title{
Strain determination in full-field measurements using DIC
}

\author{
Stefan Hartmann, Carmen Sguazzo \\ Institute of Applied Mechanics, Clausthal University of Technology, \\ Adolph-Roemer-Str. 2A, 38678 Clausthal-Zellerfeld, Germany, \\ stefan.hartmann@tu-clausthal.de
}

\begin{abstract}
Digital image correlation (DIC) provides the experimental possibility to obtain the distributed deformation at the surface of a specimen or an entire mechanical part, see, for example, [1]. Similar to commercial finite elements, the concrete implementation is not known to users. Moreover, different approaches to determine the strain distribution are proposed in the literature. In view of the concept of verification and validation, see [2], these codes must be verified. Thus, verification examples must be provided.

In this contribution the strain determination at the surface of a material body is discussed leading to strains in a manifold. In this respect, the surface description is one essential ingredient in the geometrical representation. Moreover, verification examples are developed which make clear that there might be large errors, in particular, in metal forming processes, where large curvatures occur. These are simple shear, bending of a thin plate, torsion and a deep drawing problem. Finally, it is shown that the strain determination procedure is also applicable for comparison purposes with finite elements.
\end{abstract}

\section{References}

[1] Sutton, M. A., Orteu, J.-J. and Schreier, H. W. Image correlation for shape, motion and deformation measurements. Springer, New York (2009)

[2] The American Society of Mechanical Engineers. Guide for Verification and Validation in Computational Solid Mechanics. New York (2006) 


\title{
Inverse Parameter Identification for Orthotropic Elasto-Plastic Sheet-Steel
}

\author{
Benjamin Soehngen, Kai Willner \\ University of Erlangen-Nuremberg
}

Reducing the need for experimental testing and therewith development time and costs, simulating manufacturing processes and real world usage has established itself as a standard. The obtained predictions of simulation however can only be as good as the modelling. This includes the choice of an appropriate material model, as well as suitable input parameters.

In this contribution the Finite Element Model Updating (FEMU) approach is utilized to determine the material parameters of sheet-steel. From experimental testing it is observed that the considered cold-rolled steel exhibits orthotropic behaviour. To regard this in the simulation, a user-implemented material model based on Hill's yield function [1] is used. Via the method of digital image correlation (DIC), the displacement field of a biaxially loaded specimen is measured from images taken at different stages of loading. Using full-field optical methods, the obtained resolution is very high and the measurement has the advantage of being contactless. The experimentally determined displacements are interpolated to the according nodes of the FEM mesh and compared to those obtained by simulation. This way an error measure is defined which can be minimized by optimization algorithms. Starting with initial values for the orthotropic elasto-plastic material parameters, the FEM model is thus updated consecutively until a specified error margin is reached.

This work is supported by the German Research Foundation (DFG) within the Collaborative Research Center SFB/TR73: "Manufacturing of complex functional components with variants by using a new sheet metal forming process - Sheet-Bulk Metal Forming".

\section{References}

[1] Hill, R. A Theory of the Yielding and Plastic Flow of Anisotropic Metals. Proceedings of the Royal Society A: Mathematical, Physical and Engineering Sciences, Vol. 193, 1033, (1948) 281-297 


\title{
The Inversion of Hutchinson's Flow Rule
}

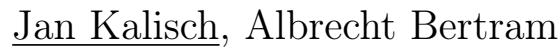 \\ Otto von Guericke University, Magdeburg, Germany
}

An important problem in the application of crystal plasticity is the inversion of Hutchinson's flow rule [1], i. e., determining the stress deviator $\mathbf{T}^{\prime}$ for a given strain rate deviator $\mathbf{D}^{\prime}$

$$
\frac{1}{\dot{\gamma}_{0}} \mathbf{D}^{\prime}=\sum_{\alpha} \operatorname{sign} x_{\alpha}\left|x_{\alpha}\right|^{p} \mathbf{S}_{\alpha} \quad x_{\alpha}:=\frac{1}{\tau_{C}} \mathbf{T}^{\prime} \cdot \mathbf{S}_{\alpha}
$$

where $\dot{\gamma}_{0}, \tau_{C}$ and $p$ are material parameters (shear rate, critical resolved shear stress, and strain-rate sensitivity, respectively) and $\mathbf{S}_{\alpha}$ is the symmetric part of the Schmid tensor of slip system $\alpha$.

While this flow rule is bijective and has been suggested in order to circumvent the problem of selecting active slip systems [2], it still proves numerically challenging due to the highly nonlinear $(p \gg 1)$ function involved. This function maps quite different stress states to very similar strain rates. Though in principle this problem can be overcome by means of a sufficiently large data base [3], even browsing that data base can prove prohibitive in terms of computing time.

We start with the analysis of the mathematical problem and establish a representation of the inverse flow rule. This is followed different strategies for the numerical solution. For moderate strain-rate sensitivity $p \sim 25$, a newly designed algorithm requires on average 5-6 Newton iterations to reduce the relative error to $10^{-8}$. Key to this algorithm is the selection of an appropriate starting point for the subsequent iterative procedure. Some two-dimensional examples are used to elucidate the problem, but the results refer to the three-dimensional case.

\section{References}

[1] J. Hutchinson. Bounds and self-consistent estimates for creep of polycrystalline materials. Proc. R. Soc. Lond. A 348 (1976), 101-127

[2] A. Bertram. Elasticity and Plasticity of Large Deformations. Springer (2012).

[3] M. Knezevic, H.F. Al-Harbi, S.R. Kalidindi. Crystal plasticity simulations using discrete Fourier transforms. Acta Materialia 57 (2009), 1777-1784 


\title{
Parameter Identification by Inverse Modelling of Biaxial Tensile Tests of Discontinous Fiber Reinforced Polymers
}

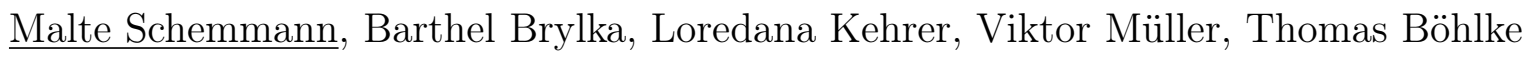 \\ Karlsruhe Institute of Technology \\ Institute of Engineering Mechanics, Chair for Continuum Mechanics
}

Due to their high lightweight potential, economical mass-production, and excellent formability long fiber reinforced polymers are used in nonstructural components in the automotive sector. However, the application of this class of materials is hindered by a lack of a understanding for robust modeling the whole process chain as well the process related thmero-mechanical properties. The material classes under consideration are LFTD (long fiber reinforced thermoplastics manufactured in a direct in-line process) and SMC (sheet molding compounds). The fiber orientation and length distribution of both material types is highly heterogenous and determined by the moldfilling flow of the plastificate. As a result the material behavior is spatially heterogeneous and anisotropic in a process sensitive way.

To gain a better insight into the material behavior, biaxial tensile tests on cruciform specimen are performed. The electro-mechanical biaxial testing machine used for the experiments allows for a maximum load of $150 \mathrm{kN}$ on each axis and is equipped with an integrated strain measurement system, which is also used for adaptive midpoint control. The full 3D strain field is measured via gray scale correlation.

The observed results including inelastic effects are discussed in detail for uniaxial and biaxial loading paths. The anisotropic material properties are identified through inverse modeling and digital image correlation (DIC) by comparison of the heterogeneous experimental and simulated strain fields. A Gauss-Newton type algorithm is used to identify the optimal parameter set [1].

The identified material parameters are compared to homogenized material properties based on fiber orientation distribution obtained by micro-computer-tomography and fiber orientation analysis of moldfilling simulations. Meanfield approaches such as the three phase model interaction direct derivative estimate [2] and a Mori-Tanaka scheme are used to determine the macroscopic material properties.

\section{References}

[1] D. Lecompte, A. Smits, H. Sol, J. Vantomme, D. Van Hemelrijck. Mixed numerical-experimental technicque for orthotropic parameter identification using biaxial tensile tests on cruciform specimen. Int. J. Solids Struct. 44 (2007), 1643-1656

[2] P. Ponte-Castaneda, P. Suquet. Nonlinear composites. Adv. Appl. Mech. 34 (1998) 171-302

[3] Z.-S. Zeng, D.-X. Du. An explicit and universally applicable estimate for the effective properties of multiphase composites wich account for inclusion distribution. J. Mech. Phys. Solids 49 (2001), 2765-2788 


\title{
Shape control of piezolaminated thin-walled composite structures using nonlinear piezoelectric actuators
}

\author{
M.N. Rao, K.-U. Schröder, S. Tarun \\ Institute of Structural Mechanics and Lightweight Design \\ RWTH Aachen University \\ Aachen, Germany
}

Smart piezolaminated thin-walled composite structures gained significant research interest in recent decades. Many of the available nonlinear models deal with geometrically nonlinear strain-displacement relations with linear electromechanical coupling. A very few authors considered the material nonlinearities by considering the rate-independent hysteresis in ferroelectricity. However, the literature on the behavior of piezolaminated structures under strong electric fields using nonlinear constitutive relations is very scarce. Nevertheless, all the numerical models available in literature except [1] neglect the two-way electromechanical coupling which results inaccurate solutions. Furthermore the literature $[1,3]$ is restricted to beam or plate finite elements only.

Therefore, the present study is focused on the nonlinear FE-simulation of smart piezolaminated composite shells using nonlinear constitutive relations in piezo-mechanical coupling. The present shell element is based on the assumptions of small strains and large electric fields. The large electric field characteristic is considered by applying rotationally invariant nonlinear constitutive relations of Tiersten [2]. A numerical model is developed and validated with the available experimental and numerical results in literature. Additionally, static behavior of the piezolaminated composite structures under strong applied electric fields is demonstrated by deploying developed numerical FE-model in some numerical examples.

\section{References}

[1] S. Kapuria, M.Y. Yasin. A nonlinear efficient layerwise finite element model for smart piezolaminated composites under strong applied electric field. Smart Mater. Struct. 13 (2013), 055021.

[2] H.F. Tiersten. Electroelastic equations of electroded thin plates subject to large deriving voltages. J. Appl. Phys. 74 (1993), 3389-93.

[3] L.Q. Yao, J.G. Zhang, L. Lu, M.O. Lai. Nonlinear extension and bending of of piezoelectric laminated plate under large applied field actuation. Smart Mater. Struct. 13 (2004), 404-414. 


\title{
On the sonic composites with scatterers made from auxetic material
}

\author{
Valerica Moşneguţu, Ştefania Donescu \\ Institute of Solid Mechanics, Romanian Academy, Bucharest \\ Technical University of Civil Engineering from Bucharest \\ E-mail: valeriam732000@yahoo.com, E-mail: stefania.donescu@yahoo.com
}

\begin{abstract}
A finite size periodic array of resonators made from auxetic material, embedded into an epoxy matrix is analyzed in this paper. By definition, an auxetic material (with negative Poisson's ratio) expands in all directions when pulled in only one, giving an opposite deformation to that of conventional foams. The classical mechanics fail in describing the mechanical behavior of the auxetic material under deformation. The Cosserat, the micropolar and classical theories of elasticity are continuum theories, which make no reference to atoms or other structural features of the material. Elasticity theory represents more than an analytical description of the phenomenological behavior since it can be derived as a first approximation of the interaction between atoms in the solid [1], [2]. According to the Bragg's theory, the sound attenuation band is due to the superposition of multiple reflected waves inverse proportional to the central distance between resonators. The sound attenuation in such composites is studied using a new method that combines the features of the cnoidal method and the genetic algorithm [3], [4]. The sound transmission through a single finite panel of this composite is characterized with the transfer matrix method coupled with the spatial window technique. The finite size of the panel is taken into account by applying a double spatial window to the incident waves and the vibration velocities of the infinite panel before calculating the radiated field. The method seems to give a correct description of the sound transmission through the acoustic panel and the transmission factor is calculated for every incidence angle with high accuracy.
\end{abstract}

\section{References}

[1] Cosserat, E. and F. Theorie des Corps Deformables. Hermann et Fils, Paris (1909).

[2] E.Kröner. On the physical reality of torque stresses in continuum mechanics. Int. J. Engng. Sci., 1(1963), 261-278.

[3] L.Munteanu, V.Chiroiu. On the dynamics of locally resonant sonic composites, European Journal of Mechanics-A/Solids, 29(5) (2002), 871-878.

[4] V.Chiroiu, C.Brişan, M.A.Popescu, I.Girip, L.Munteanu. On the sonic composites without/with defects. Journal of Applied Physics, 114 (2013), 164909. 


\title{
The influence of a damping coefficient on a non-invasive strategy for inverse form-finding
}

\author{
Philipp Landkammer, Paul Steinmann \\ Friedrich-Alexander-Universität Erlangen-Nürnberg
}

Inverse form finding aims in finding the optimal blank design (material configuration) of a workpiece, whereby the desired spatial configuration, that is obtaining after a forming process, is known. Inputting the optimal material configuration, a subsequent finite element computation results in exactly the nodal (spatial) coordinates of the desired deformed workpiece.

To determine the optimal blank design, [1] recently proposed an iterative algorithm, which is purely based on geometrical considerations. As a benefit this geometrical approach is not depend on a constitutive model and can be easily coupled non-invasively via subroutines to an arbitrary finite element programm. The applicability of this strategy to metal forming problems with friction, contact and large plastic strains is examplified by [2] and [3] for the case of coupling the form finding algorithm, which is implemented in Matlab, with the commercial simulation software MSC.MarcMentat.

Applying a damping coefficient within the update step of the material coordinates avoids mesh distortions and also improves the convergency rate for finding the optimal blank design. The damping coefficient is herein automatically controlled by analyzing the mesh quality.

Acknowledgements This work is part of the collaborative research project Manufacturing of complex functional components with variants by using a new metal forming process - Sheet-Bulk metal forming (SFB/TR-73).

\section{References}

[1] P. Landkammer, P. Steinmann. A non-invasive approach to inverse form finding. submitted.

[2] P. Landkammer, P. Steinmann. Application of a non-invasive form finding algorithm to the ring compression test with varying friction coefficients. Material Key Enigineering, Proceedings of the 18th International Conference of Metal Forming, ESAFORM 2015, submitted.

[3] P. Landkammer, B. Söhngen, A. Loderer, E. Krebs, P. Steinmann, K. Willner, T. Hausotte, P. Kersting, D. Biermann. Experimentell Verifizierung eines Benchmark-Umformprozesses. Tagungsband zum 3. Industriekolloqium Blechmassivumformung 2015. submitted. 


\title{
Modeling of imperfect contact interfaces in sonic composites
}

\author{
Ligia Munteanu ${ }^{1}$, Cornel Brişan ${ }^{2}$ \\ ${ }^{1}$ Institute of Solid Mechanics, Romanian Academy, Bucharest \\ ${ }^{2}$ Technical University of Cluj-Napoca, Cluj-Napoca, Romania
}

A sonic composite is a finite size periodic array composed of scatterers embedded in a homogeneous material. It exhibits the full band-gaps, where the sound is not allowed to propagate due to complete reflections. The band-gaps or the well-known Bragg reflections occur at different frequencies inverse proportional to the central distance between two scaterers. If the band-gaps are not wide enough, their frequency ranges do not overlap. These band-gaps can overlap due to reflections on the surface of thick scatterers, as well as due to wave propagation inside them. Then, any wave is reflected completely from this periodic array of acoustic scatterers in the frequency range where all the band-gaps for the different periodical directions overlap [1], [2]. Sonic composites are characterized by imperfect interface conditions defined in terms of linear relations between interface tractions and displacement jumps. These kinds of interfaces can be evaluated on the basis of the Eshebly's [3] and the Hashin models [4], [5]. In this paper, the tangential and normal discontinuities at the interface between the scatterer and the matrix are independently modeled, and these relative displacements are directly proportional to the corresponding components of tractions at the interface. The effective elastic moduli are obtained on the basis of the derived imperfect interface conditions for a sonic plate with an array of acoustic piezoceramic hollow spheres scatterers embedded in an epoxy matrix. The scatterers are made from functionally graded materials with radial polarization (the Reddy and cosine laws). Numerical evaluation has shown that imperfect interface may have a significant effect on the generation of full band-gaps.

\section{References}

[1] L.Munteanu, V.Chiroiu. On the dynamics of locally resonant sonic composites, European Journal of Mechanics-A/Solids, 29(5) (2002).

[2] V.Chiroiu, C.Brişan, M.A.Popescu, I.Girip, L.Munteanu. On the sonic composites without/with defects. Journal of Applied Physics, 114 (2013).

[3] J.D.Eshelby. The the elastic field outside an ellipsoidal inclusion. Proc. Roy. Soc., A252 (1959), 561-569.

[4] Z.Hashin. Thermoelastic properties of particulate composites with imperfect interface, J. Mech. Phys. Solids, 39(6) (1991), 745-762.

[5] Z.Hashin. The spherical inclusion with imperfect interface. Journal of Applied Mechanics, Transactions of ASME, 58 (1991), 444-449. 


\title{
Direct and Inverse Identification of Composites with Microstructure
}

\author{
Aguel Baltov, Ana Yanakieva, Nikola Nikolov \\ Institute of Mechanics, Bulgarian Academy of Sciences
}

The study treats two level modeling of composites, namely: (i) micromodeling considering a multi-component microstructure, and (ii) macromodeling considering a homogeneous macrostructure. The direct identification supposes knowing the mechanical characteristics of the microstructure components. An approximate method of finding the characteristics of the homogeneous macromaterial can be proposed on that basis. The approach is applied to thin plates undergoing plain stress. A rectangular representative element is considered for the purpose of study, applying various combinations of uniform macroloads at its boundaries. Load combinations are selected following a cubic plan of the numerical experiments. Regarding specific conditions, one can find the macrostrains, performing an averaging procedure along the macroelement boundaries. As a result, an approximate homogenization is realized. On the other hand, the inverse identification is performed applying an iterative procedure which involves direct solutions. Linear elastic materials are objects of study, most of all, but nonlinear materials can also be modeled via a step-wise linearization. The procedure developed is applied to real composite materials.

ACKNOWLEDGEMENT: The study has been supported by the National Fund "Scientific Research", Project DFNI E02/10 121214.

\section{References}

[1] Hurang Hua, Landon Onyebuekea, Ayo Abatanb. Characterizing and Modeling Mechanical Properties of Nanocomposites - Review and Evaluation, Journal of Minerals and Materials Characterization and Engineering, 9(4). (2010), 275-319.

[2] Baltov A., Yanakieva A., Continuum approach in Nanomechanics.Mechanics of Nanomaterials and Nanotechnology, Thematic collection. IMech, BAS. (2012), 133-151.

[3] A.A. Griffith. The phenomena of rupture and flow in solids. Philos. Trans. Roy. Soc. London 221 (1921), 163-198.

[4] Geoffrey H. Campbell et al. Multi-scale modeling of polycrystal plasticity: a workshop report, Materials Science and Engineering A251. (1998), 1-22.

[5] W. Szczepinski. Experimental methods in solid mechanics, Technical Mechanics, Vol. X, PWN, Warsaw. (1984)

[6] Nikolov, N. Mathematical models of Tg and Tx, and search for maximal supercooled liquid regions Tx for Zr-Al-Cu-Ni glassy alloys, Technical Ideas (4). (2004), 67-79.

[7] Mori T., K.Tanaka, Average stress in matrix and average elastic energy of materials with missfitting inclusions, Acta Metallurgica (21). (1973), 571-574 


\title{
Optimal bounds from below of the critical load for elastic solids subject to uniaxial compression
}

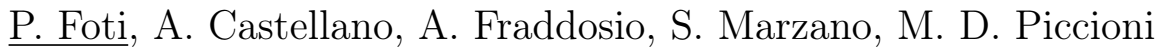 \\ Dipartimento di Scienze dell'Ingegneria Civile e dell'Architettura \\ Politecnico di Bari- Italy, \\ e mail: pilade.foti@poliba.it, aguinaldo.fraddosio@poliba.it, \\ salvatore.marzano@poliba.it, mariodaniele.piccioni@poliba.it
}

Bifurcation and stability issues are of considerable importance in the framework of the non-linear theory of elasticity, since they provide a setting for studying various phenomena relevant for engineering applications.

Bifurcation and stability theories deal with different general issues at the fundamental level: bifurcation theory basically concerns the non-uniqueness of solutions, while stability theory regards the behaviour of deformed bodies under disturbances. Nevertheless, it is possible to find examples which show a close relationship between bifurcation and stability, for instance when studying small on large problems through the classical method of adjacent equilibria, i.e., when one checks if there exist other equilibrium solutions close to a given primary equilibrium state through the analysis of the equilibrium equations linearized around the deformed configuration.

In this vein, the Hadamard energetic criterion of infinitesimal stability (cf. [1]) is a classical and widely used tool for testing the stability of an equilibrium state, since the violation of the so-called Hadamard stability condition plays the role of an indicator of possible bifurcations from the primary equilibrium configuration. Indeed, in a monotonic loading process governed by a loading parameter it is usually assumed that the body remains in the fundamental equilibrium state (no bifurcation allowed) until the Hadamard stability condition ceases to hold. Then, possible bifurcation modes may arise at the critical load, defined as the value of the load parameter which first renders the Hadamard functional zero.

These considerations show that stability in the Hadamard sense requires the determination of the sign of the Hadamard functional but, unfortunately, this is not a simple task. A strategy for avoiding the direct study of the sign of the Hadamard functional may be represented by the evaluation of lower bound estimates of the Hadamard functional, with the aim of seeking a lower bound estimate of the critical load, below which the Hadamard stability condition is definitely satisfied.

In the literature, one my find lower bound estimates for the critical load both for compressible or incompressible elastic bodies (see, e.g., [2-4]). In particular, we proposed in [5] a new procedure (based on the Korn inequality) for determining a lower bound estimate of the critical load for compressible elastic solids which improves other proposals in literature.

Here, on the basis of [6], we first determine new sufficient conditions for the Hadamard stability, and then apply our method to the determination of an optimal lower bound estimate of the critical load for incompressible elastic solids. The effectiveness of our procedure, which is expandable to general cases of hyperelastic materials subject to inhomogeneous deformations, is here discussed with reference to the homogeneous uniaxial compression a homogeneous, incompressible, isotropic Mooney-Rivlin circular cylinder. In particular, we show that our approach improves the estimate proposed in [4] and it is also advantageous for applications, since it yields a very simple method for numerical applications.

This work has been supported by the MIUR PRIN project entitled Dynamics, stability, and control of flexible structures and by the INDAM GNFM Progetto Giovani 2014 entitled Bifurcation and stability Issues with Applications to Nonlinear Elastic Problems.

\section{References}

[1] C. Truesdell and W. Noll. The Non-Linear Field Theories of Mechanics. Handbuch der Physik III/3, Springer, Berlin (1965).

[2] J. T. Holden. Estimation of critical loads in elastic stability theory. Arch. Ration. Mech. Anal. 17 (1964), 171-183.

[3] G. Del Piero. Lower bounds for the critical loads of elastic bodies. J. of Elasticity 10 (1980), 135-143. 
[4] G. Del Piero and R. Rizzoni. Weak local minimizers in finite elasticity. J. of Elasticity 93 (2008), 203-244.

[5] R. Fosdick, P. Foti, A. Fraddosio, M. D. Piccioni. A lower bound estimate of the critical load for compressible elastic solids. Continuum Mech. Thermodyn. 22 (2010) 77-97.

[6] R. Fosdick, P. Foti, A. Fraddosio, S. Marzano, M. D. Piccioni. A lower bound estimate of the critical load in bifurcation analysis for incompressible elastic solids. Mathematics and Mechanics of Solids 20 - Part 1 (2015), 53-79. 


\title{
Constitutive modeling of fiber-reinforced aerogels
}

\author{
Ameya Rege ${ }^{1}$, Roozbeh Dargazany ${ }^{2}$, Mikhail Itskov $^{1}$ \\ ${ }^{1}$ Department of Continuum Mechanics, RWTH Aachen University \\ ${ }^{2}$ Department of Civil and Environmental Engineering, Michigan State University
}

Aerogels are open-cell highly porous solids having exclusive properties such as lowest bulk density, lowest thermal conductivity and lowest acoustic velocity. These properties make them useful over a large spectrum of applications. However, their brittle and hydrophilic natures result in a road block which is overcome by polymer or fiber reinforcement [1]. In this study, we are focussing on fiber-reinforced aerogels which retain the exclusive properties of aerogels mentioned above while giving them a good load bearing strength at the same time. These fiber-reinforced aerogels show many complex phenomena under loading such as, a strong nonlinearity, cyclic stress softening, and permanent set. However, there have been very few studies on fiberreinforced aerogels oriented towards their mechanical characterization $[2,3]$. To the best of our knowledge, there also is no micromechanical model that can capture the constitutive response of these fiber-reinforced aerogels.

In this contribution, we propose a micro-mechanically motivated constitutive model of fiber reinforced aerogels. The prime source of elasticity and damage in these aerogels is the effect of bending and breakage of fibers in the material network. Accordingly, we consider non-linear bending of fibers supported by an elastic foundation of particles. Based on this concept, the strain energy function is analytically derived for a single fiber in a particular direction. The strain energy of the whole network is then obtained by integration over an unit sphere. The model shows good agreement with experimental data.

\section{References}

[1] H. Maleki, L. Durães, A. Portugal. An overview on silica aerogels synthesis and different mechanical reinforcing strategies. Journal of Non-Crystalline Solids 385 (2014), 55-74.

[2] X. Yang, Y. Sun, D. Shi, J. Liu. Experimental investigation on mechanical properties of a fiber-reinforced silica aerogel composite. Materials Science and Engineering A 528 (2011), 4830-4836.

[3] H. Wu, D. LI, S. Zhu, F. Huang. Studies on Dynamic Mechanical Properties of Glass Fiber Reinforced Silica Aerogel. Advances in Mechanical Engineering. 2(4) (2012), 24. 


\title{
Characterization of short fiber reinforced polymers
}

\author{
C. Röhrig ${ }^{1}$, S. Diebels ${ }^{1}$ \\ ${ }^{1}$ Lehrstuhl für Technische Mechanik, Universität des Saarlandes
}

This contribution presents ideas, how composite materials can be characterized with respect to experimental testing. Composite materials, like short fiber reinforced polymers, are of great interest because of their good properties for industrial applications. In this work a polybutylene therephthalate (PBT) reinforced by short glass fibers is characterized. The matrix material PBT is chosen due to its heat stability over a large range and the high resistance against outer influences as water. The stiffness and the geometrical stability of the polymer are increased by adding short fibers during injection molding process. The benefit in comparison to metals, like alumina or other metal-composites for applications in automotive industries, is its lightweight.

In this work the material properties of the investigated material are obtained by providing results from the experiment in order to seperate different material effects such as elasticity, plasticity, damage, viscoelasticity, compressibililty and anisotropy. The material under study shows a pronounced strain rate dependency, which has to be taken into account. Therefore, cyclic uniaxial tensile tests with different rates are accomplished. The application of the material in this work is the machining by a three-dimensional forming process. Hence, multiaxial loadings have to be additionally taken into account matching these conditions. At first, bending experiments are added. In order to provide more information, biaxial tensile tests are realized using a testing device supplying the additional necessary experimental data $[1,2]$. The difference according to the uniaxial tensile test is, that the multiaxial deformation information is not provided directly by the device output. In order to examine the complete strain information, an optical areal analysis with a digital image correlation (DIC) software is applied. For the biaxial tensile test, the testing force and the deformation field cannot be correlated directly. Therefore, an inverse method comparing the information of the complete deformation field as well as the force data of the experiment and the model is executed [3].

A final aim of this work is to realize a verification experiment representing the three-dimensional forming process as realistically as possible, e.g. a Nakajima test [4]. For this case, a three-dimensional optical analysis in order to get the necessary measurement data, is indispensable.

\section{References}

[1] H. Seibert, T. Scheffer, S. Diebels. Biaxial testing of elastomers - Experimental setup, measurement and experimental optimisation of specimen's shape. Technische Mechanik 34(2) (2014), 72-89.

[2] M. Johlitz, S. Diebels. Characterisation of a polymer using biaxial tension tests. Part I: Hyperelasticity. Archive of Applied Mechanics 81(10) (2011), 1333-1349.

[3] E. Tyulyukovskiy, N. Huber. Identification of viscoplastic material parameters from spherical indentation data: Part I. Neural networks. Journal of Materials Research 21(3) (2006), 664-676.

[4] P.F. Bariani, S. Bruschi, A. Ghiotti, A. Turetta. Testing formability in the hot stamping of HSS. CIRP Annals-Manufacturing Technology 57 (2008), 265-268. 


\title{
Formation of microstructure in the plates under compression
}

\author{
M. Koster, K. C. Le, B. D. Nguyen \\ Lehrstuhl für Mechanik - Materialtheorie, Ruhr-Universität Bochum, \\ D-44780 Bochum, Germany
}

Deformation of the plates under compression is investigated by the theory of formation of grain boundaries in ductile single crystals within the nonlinear continuum dislocation theory (CDT) that leads to the minimization problem of a non-convex energy functional. By constructing an energy minimizing sequence having piecewise constant plastic slip it is shown that the formation of shear bands in these plates under compression is energetically more preferable. The number of shear bands is estimated by comparing the energy of shear banding plates including low angle tilt boundaries with that of the uniformly compressed plates. 


\title{
On fast and accurate modelling of distributed fibre directions in composites
}

\author{
Niels Goldberg, Jörn Ihlemann \\ Professorship of Solid Mechanics, Faculty of Mechanical Engineering, \\ Technische Universität Chemnitz, Chemnitz, Germany
}

Composites like connective tissues with collagen fibres or short fibre reinforced thermoplastics contain fibres whose arrangement deviates from unidirectionality. There are several approaches to describe the mechanical behaviour of such materials. All of them employ a distribution function, which describes the probability of every possible fibre direction. The integration of the stress combined with the distribution function over the unit sphere, i.e. the solution of angular integrals (AI), is supposed to yield the exact solution. However, those integrals require numerous calculations as they usually cannot be solved analytically. In order to shorten the calculation time, the concept of generalised structural tensors (GST) makes use of averaged tensor representations of the fibre distribution. Thus, only a one-time integration is necessary. Recently this concept was extended to higher order tensors (cf. [1]). However, a known disadvantage of the GST is their tendency to respond softer to strain than the AI.

This contribution presents an alternative approach, which is almost as accurate as the AI method but considerably faster. It makes use of representative directions according to an Angular Central Gaussian distribution function and does not require the solution of angular integrals. As the choice of the representative directions bears a significant impact on the accuracy of the method, a study on the integration error is presented. Furthermore, the results of homogeneous deformations using the AI, the GST as well as the new approach are compared to each other, pointing out the advantages and disadvantages of each method.

\section{References}

[1] D.H. Cortes , D.M. Elliott. Accurate prediction of stress in fibers with distributed orientations using generalized high-order structure tensors. Mechanics of Materials 75 (2014), 73-83. 


\title{
Meso-macro modelling of fiber-reinforced composites exhibiting elastoplastic deformation
}

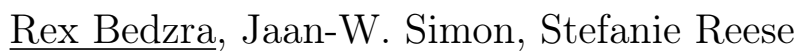 \\ Institute of Applied Mechanics, RWTH Aachen University, Aachen, Germany
}

Fiber composite materials such as carbon fiber-reinforced composites are typically characterized by their high Young's modulus and low density making them particularly attractive in lightweight constructions. In addition, the architecture of these materials means that the correct modelling of their orthotropy is very important.

Anisotropic elastoplasticity has been investigated by various authors over the years, especially in the context of crystal plasticity where the structural tensors in the intermediate configuration are fixed and the yield condition is formulated in terms of the Schmidt stress. In the present work, the situation is different in that the fiber orientations represent so called material line elements. This kind of material is described in the framework of phenomenological anisotropic modelling. Such a model has been proposed in [1] and [2] for the large deformation behaviour of pneumatic membranes with the former restricted to purely elastic behaviour and the latter taking into account the inelastic behaviour of the fibers. In recent times, the model in [1] restricted to purely elastic behaviour has been adopted in [3,4] through a slight modification to model carbon fiber-reinforced composites.

The present model is also meso-mechanically motivated and it is an extension to the model proposed in [3]. It is based on structural tensors as well as the linearized strain energy function proposed in [1]. Additionally, the inelastic nature of the matrix material is taken into account whilst still preserving the linear elastic nature of the fibers. The model is divided into two main sections. The first section deals with virtual experiments to generate stress-strain curves upon which the phenomenological model is fitted. The reason for this is to mainly understand the effect of the material inhomogeneities on the overall material response. The second step deals with implementation of the derived model into a finite element software as a user material subroutine.

\section{References}

[1] S. Reese, P. Wriggers, B.D. Reddy. Finite element modelling of orthotropic material behaviour in pneumatic membranes. International Journal of Solids and Structures 38 (2001), 9525-9544.

[2] S. Reese. Meso-macro modelling of fiber-reinforced rubber-like composites exhibiting large elastoplastic deformation. International Journal of Solids and Structures 40 (2003), 951-980.

[3] B. Stier, J.-W. Simon, S. Reese. Finite Element Analysis of Layered Fiber Composite Structures Accounting for the Material's Microstructure. Applied Composite Material 21 (2014), 1-17.

[4] J.-W. Simon, B. Stier, S. Reese. Numerical Analysis of Layered Fiber Composites Accounting for the Onset of Delamination. Advances in Engineering Software 80 (2015), 4-11. 


\title{
A micromechanical model for the transformation induced plasticity in poly-crystalline steels
}

\author{
Johanna Waimann, Philipp Junker, Klaus Hackl \\ Institute of Mechanics of Materials, Ruhr-University Bochum, Germany
}

The effect of transformation induced plasticity (TRIP) results in an improvement of the steels' ductility [1]. Therefore, TRIP-steels are very promising materials, e.g. for the automobile industry. The material behavior is characterized by very complex inner processes, namely phase transformation coupled with plastic deformation and kinematic hardening. To describe the change in microstructure, we will use the volume fractions of the single phases, the plastic strain and the hardening parameter in every grain of the poly-crystalline material as internal variables. Furthermore, we will use the Principle of the Minimum of the Dissipation Potential to model the material behavior. The use of a coupled dissipation functional and a combined Voigt/Reuss bound will directly result in coupled evolution equations for the internal variables and in one combined yield function. Additionally, we will show some numerical results that prove our model's ability to give a first prediction of the TRIP-steels' complex material behavior.

\section{References}

[1] W.J. Dan, S.H. Li, W.G. Zhang, Z.Q. Lin. The effect of strain-induced martensitic transformation on mechanical properties of TRIP steel. Mater. Des. 29(3) (2008), 604-612. 


\title{
A novel contribution to the relaxation of non-convex energy density functionals in the context of martensitic phase transformations
}

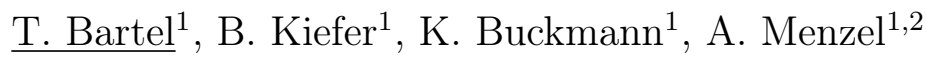 \\ ${ }^{1}$ Institute of Mechanics, TU Dortmund, Leonhard-Euler-Strasse 5, D-44227 Dortmund \\ ${ }^{2}$ Division of Solid Mechanics, Lund University, P.O. Box 118, SE-221 00 Lund
}

Energy relaxation methods are known to yield reliable frameworks in the context of material modelling providing both physical and mathematical plausibility. The most frequently applied relaxation scheme is rank-one convexification with respect to laminates of different order. This concept has successfully been utilised for, e.g., the modelling of large strain crystal plasticity and solid-to-solid phase transformations in shape memory alloys. In the latter case, energy relaxation based on laminated microstructures in particular allows the micromechanically well-motivated modelling of single crystalline behavior in terms of explicitly taking into account a parent phase (austenite) and several crystallographic variants (of martensite). While standard lamination approaches facilitate to consider exactly $2^{n}$ different phases, where $n$ indicates the order of the laminates, the ansatz established in [1] allows the modelling of a parent phase together with an arbitrary number of martensite variants, the crystallographic orientations of which are kept static. However, experimental observations reveal that rather mixtures of austenite and twinned martensite, i.e. two martensite variants with a compatible interface, occur in reality. In this regard, this contribution focusses on the derivation of a laminate-based material model which takes into account only two martensite phases. In order to avoid cumbersome and inefficient combinatorical treatments for the determination of the energetically favourable composition of phases, rotational degrees of freedom for the martensite crystal structures are introduced. These serve as additional minimisers and improve the relaxation of the underlying non-convex energy landscape with regard to the approximation of the quasiconvex hull. The proposed framework thus provides a continuous modelling and simualtion of twin formation.

\section{References}

[1] T. Bartel, K. Hackl. A micromechanical model for martensitic phase-transformations in shape-memory alloys based on energy-relaxation. Z. Angew. Math. Mech. (2009), 792-809. 


\title{
A simplified approach to calculate adhesive joints in multi-material structures
}

\author{
Christopher Blech, Paul Falkenberg, Sabine C. Langer, Thomas Vietor \\ Institut für Konstruktionstechnik \\ TU Braunschweig
}

With the requirement of reducing energy consumption of mobile applications, lightweight design significantly gained in importance. One way to develop lightweight vehicles is the utilisation of multi-material structures. In such components like hybrid car bodies dissimilar materials are applied which require a reliable junction. For most material combinations, adhesive joints are particularly suited and widely used. Another opportunity of lightweight design is the application of topology optimization to reduce the material requirement. Our current development is the combination of both aspects. To get realistic results, the adhesive joints should be taken into account during the topology optimization process. The optimization process requires a mesh consisting of equilateral hexaeder elements and modifies the structure based on stress components. Hence, the stress states of the adhesive joints are crucial for the optimization process.

The challenge is to calculate the maximum normal and shear stresses in the adhesive layer, since dissimilar materials lead to stress singularities at boundaries. Therefore, a non-linear material model and geometrically non-linear, special finite elements are commonly required. This conflicts with the requirement of a minimum of computational costs for the topology optimization. In order to solve this problem and obtain an optimized structure including adhesive joints in reasonable time, the modelling of the adhesive layer should not exceed the minimal necessary complexity.

In this paper, the possibility of a simplified modelling approach using linear continuum elements with virtual zero thickness is examined. The in-house finite element code elPaSo is used as a numerical tool. The first step is to calculate the relevant stress components of simple structures such as a single lap joint. The stress state is compared to complex calculations in academic literature. Finally, the usability of the simplified approach for the multi-material topology optimization and the transferability to complex structures are evaluated. 


\title{
On the natural Fiber (Maize) Composite material
}

\author{
Ruxandra Ilie, Veturia Chiroiu \\ Technical University of Civil Engineering from Bucharest \\ Institute of Solid Mechanics, Romanian Academy, Bucharest \\ E-mail: rux_i@yahoo.com, E-mail: veturiachiroiu@yahoo.com
}

This work is presented the properties of a composite material made of stalk-based fiber of maize and unsaturated polyester resin polymer as matrix with methyl ethyl ketone peroxide as a catalyst and Cobalt Octoate as a promoter [1]. The potential of this material for noise enclosures is investigated by using a coupled method cnoidal - Extended Finite Element Method (XFEM). XFEM enables the accurate approximation of solutions with jumps, discontinuities or general high gradients across interfaces [2], [3]. The dissipation of the sound power into a plate/cavity system shows the efficiency of this composite to achieve noise reduction better to that obtained at low and higher frequencies with traditional foams. The interesting features of the natural fiber composite combine both sound absorption and enhanced insulation. At low frequencies up to $200 \mathrm{~Hz}$, the power radiated by system is governed by the in vacuo plate modes, and in this case the composite is laid against the cavity rigid wall. At higher frequencies up to $500 \mathrm{~Hz}$, the noise radiation is driven by the rigid walled cavity modes, and in this case the composite is applied in front of the plate. In both cases, the composite material increases the sound absorption and reduces the sound transmitted by the plate outside the system.

\section{References}

[1] D.Saravana Bavan, G.C.Mohan Kumar. Finite Element Analysis of a natural Fiber (Maize) Composite Beam. Hindawi Publishing Corporation, Journal of Engn., article ID 450381 (2013).

[2] K.W.Cheng, T.P.Fries. Higher-order XFEM for curved strong and weak discontinuities. Internat. J. Numer. Methods Engrg., 82 (2010), 564-590.

[3] T.P.Fries, T.Belytschko. The intrinsic XFEM: A method for arbitrary discontinuities without additional unknowns. Internat. J. Numer. Methods Engrg., 68 (2006), 1358-1385. 


\title{
Numerical Modeling of Functional Fatigue in NiTi Wires
}

\author{
M. Osman, T. Bartel and A. Menzel \\ Institute of Mechanics, Technical University of Dortmund
}

\begin{abstract}
Shape memory alloys (SMA) have a high potential for a wide variety of industrial applications due to their remarkable ability to recover their original shape at certain characteristic temperatures under large applied strains. One of the challenging problems connected to such a process is the so called functional fatigue, which describes the deterioration of the beneficial features of SMA under cyclic loading. This phenomenon is strongly related to the phase transformation which mainly governs the distinguished SMA effect. A numerical treatment of such challenging problems is therefore necessary in order to improve the characteristic features of SMA. In this work, we introduce a thermodynamically consistent model describing martensitic phase transformations in SMA. It is based on an explicit geometrical resolution of phase fronts obtained by an extended Ritz-type ansatz within a one-dimensional setting. To be more precise, the position of the phase front-which is treated as a global variable - coincides with the position of a weak discontinuity captured by a $\mathrm{C}^{0}$-smooth displacement field. The associated global variables are determined by incremental minimization of the total energy potential. Functional fatigue is introduced via a phenomenological model which incorporates the decreasing stress plateau during cyclic loading and the occurrence of the "characteristic point" in the stress-strain diagram, together with increasing loading amplitudes and accumulating irreversible strains showing up even under pseudoelastic conditions. Diverse numerical examples are provided to clarify the theory and underline the key conclusions.
\end{abstract}




\title{
On the variational formulation and implementation of Allen-Cahn- and Cahn-Hilliard-type phase field theories
}

\author{
Alexander Bartels* ${ }^{*}$ Jörn Mosler* \\ *TU Dortmund, Institute of Mechanics, Leonhard-Euler-Str. 5, 44227 Dortmund, Germany
}

Phase field theory is a promising framework for analyzing evolving microstructures in existing materials and for designing new materials. Phenomena like those related to microstructures in Ni-based superalloys, twin structures in martensites or precipitation in Al-alloys can be predicted by phase field theory. Due to different elastic properties in the phases, interfacial energies, crystallographic eigen strains and chemical diffusion processes, the constitutive modeling is indeed very challenging. Typically, phase transformations such as those characterizing twinning are captured by an Allan-Cahn-type approach, while a Cahn-Hilliard-type formulation is used, if the respective interface motion is driven by the concentration of the species. Although the AllenCahn and the Cahn-Hilliard formulation are indeed different, they do share some similarities. To be more precise, a Cahn-Hilliard model is obtained by enforcing balance of mass in the Allen-Cahn approach. Within an energy-based formulation this can be implemented by adding additional energy terms to the underlying CahnHilliard energy. Such a universal energy-based framework is elaborated in this presentation. Furthermore, implementation aspects concerning the enforcement of natural constraints are also discussed within this work. 


\title{
A coupled phase-field - Cahn-Hilliard model for lower bainitic transformation
}

\author{
M. Düsing, R. Mahnken \\ Chair of Engineering Mechanics (LTM), University of Paderborn, Paderborn, Germany
}

The phase-field method is a widely used tool to model the material behavior on a mesoscale [1]. Especially for steel there are many approaches describing the different transformations using this method [2]. Yet there are few phase-field models for the bainitic transformation [3], because it is one of the most complex transformations in steel. Bainite consists of carbides, bainitic ferrite and may have residual austenite. In recent reports the formation of carbides has not been considered.

A phase field model to simulate the transformation of lower bainite including carbon diffusion und carbide formation has been developed. To model the evolution of the carbides it is necessary to simulate the diffusion of the carbon. Therefore the model which is based on a classical phase-field approach is coupled with a viscous Cahn-Hilliard equation to simulate the typical coarsening of the carbon. During the isothermal process a sheaf of bainitic ferrite grows. The carbon starts coarsening because the bainitic ferrite can only contain a fraction of the carbon which was stored in the austenite. At the peaks of the carbon concentration carbides are formed. The simulations successfully show the described growth characteristics of the lower bainite transformation including carbide formation.

\section{References}

[1] I. Steinbach. Phase-field models in materials science. Modelling Simul. Mater. Sci. Eng. 17 (2009).

[2] A. Yamanaka, T. Takaki, Y. Tomita. Phase-Field Simulation of Austenite to Ferrite Transformation and Widmanstätten Ferrite Formation in Fe-C Alloy. Materials Transactions. 47 (11) (2006), 2725-2731.

[3] T.T. Arif, R.S. Qin. A phase-field model for bainitic transformation. Computational Materials Science. 77 (2013), 230-235. 


\title{
Martensitic Transformations and Damage: A Combined Phase Field Approach
}

\author{
Regina Schmitt ${ }^{1}$, Charlotte Kuhn², Ralf Müller ${ }^{1}$ \\ ${ }^{1}$ Institute of Applied Mechanics, University of Kaiserslautern, Germany \\ ${ }^{2}$ Computional Mechanics, University of Kaiserslautern, Germany
}

There are different structures with various mechanical properties on the microlevel of metastable austenitic steels. During the martensitic transformation the crystal lattice changes from the $\gamma$-austenitic to the $\alpha$ '-martensitic phase which is accompanied by a volume change plus a lattice shear, leading to an eigenstrain within the martensitic phase. The focus of this investigation is on the complex interactions of the phase transformation with propagating microcracks. A phase field model for martensitic transformations which considers the transformation induced eigenstrain is combined with a phase field model for fracture. Since the eigenstrain leads to both tensile and compressive loads, the model accounts for the sign of the local volume change. With aid of this model the interactions between microcrack propagation and the formation of the martensitic phase are studied in two dimensions. Within the phase field approach an order parameter indicates the present phase while a damage variable is related to the fracture field. The evolutions of both, order parameter and crack field, are governed by time dependent Ginzburg-Landau type equations. The coupled field equations are solved using finite elements together with an implicit time integration scheme. Illustrative numerical examples demonstrate the interactions of microcrack propagation and formation of martensitic phase. 


\title{
Phase-field modeling of martensitic phase transformations in polycrystals coupled with crystal plasticity
}

\author{
J. Kochmann, S. Wulfinghoff, S. Reese, B. Svendsen \\ Institute of Applied Mechanics, RWTH Aachen University, Germany \\ Chair of Material Mechanics, RWTH Aachen University, Germany
}

The purpose of this work is the modeling of fcc to bcc martensitic phase transformations in polycrystals, the coupling with crystal plasticity and the incorporation of inheritence of plastic deformation during the phase transformation. Assuming periodic fields (e.g. stress, strain), Green function and fast Fourier transform (FFT) methods (e.g. [1], [2],[3]) are used to compute the micromechanical fields of heterogeneous microstructures. Following [3], an elasto-viscoplastic model of crystal plasticity is coupled to the phase-field modeling of martensitic phase transformations (e.g. [4]) in polycrystals. The non-conserved order parameters are updated by using a semi-implicit time integration scheme in Fourier space [5]. Two dimensional results of fcc to bcc martensitic phase transformations are presented and the interplay between phase-field evolution and plastic slip is discussed at different stages of the deformation.

\section{References}

[1] H. Moulinec, P. Suquet. A fast numerical method for computing the linear and nonlinear mechanical properties of composites. Comptes Rendus de l'Academie des Sciences. 318 (1994), 1417-1423.

[2] J. C. Michel, H. Moulinec, P. Suquet. A computational method based on augmented Lagrangians and fast Fourier transforms for composites with high contrast. Computer Modeling in Engineering Sciences. 1 (2000), 79-88.

[3] R. A. Lebensohn, A. K. Kanjarla, P. Eisenlohr. An elasto-viscoplastic formulation based on fast Fourier transforms for the prediction of micromechanical fields in polycrystalline materials. International Journal of Plasticity. 32-33 (2012), 59-69.

[4] A. Yamanaka, T. Takaki, Y. Tomita. Elastoplastic phase-field simulation of martensitic transformations with plastic deformation in polycrystal. International Journal of Mechanical Sciences. 52 (2010), 245-250.

[5] L. Q. Chen and J. Shen. Application of semi-implicit Fourier-spectral method to phase-field equations. Computer Physics Communications. 108 (1998), 147-158. 


\title{
Phase Field Modeling of Ductile Fracture
}

\author{
Fadi Aldakheel, Arun Raina, Christian Miehe \\ Insitute of Applied Mechanics (CE) \\ University of Stuttgart \\ Pfaffenwaldring 7, 70569 Stuttgart, Germany \\ fadi.aldakheel@mechbau.uni-stuttgart.de
}

Most metals fail in a ductile fashion, i.e, fracture is preceded by significant plastic deformation. The modeling of failure in ductile metals must account for complex phenomena at micro-scale, such as nucleation, growth and coalescence of micro-voids, as rooted in the work of GURSON [1]. In this work, we start with a simple model based on standard von-Mises plasticity without considering void generation. The modeling of macroscopic cracks can be achieved in a convenient way by recently developed continuum phase field approaches to fracture, which are based on the regularization of sharp crack discontinuities [2]. This avoids the use of complex discretization methods for crack discontinuities, and can account for complex crack patterns. The key aspect of this work is the extension of the energetic and the stress-based phase field driving force function in brittle fracture to account for a coupled elasto-plastic response in line with our recent work [3]. We develop a new theoretical and computational framework for the phase field modeling of ductile fracture in elastic-plastic solids. To account for large strains, the constitutive model is constructed in the logarithmic strain space, which simplify the model equations and results in a formulation similar to small strains. We start our investigation from a homogeneous one dimensional model problem to illustrate the response of the coupled elasto-plastic phase field driving force. Later we advance the model to account for multi-dimensional cases. We demonstrate the modeling capabilities and algorithmic performance of the proposed formulation by representative simulations of ductile failure mechanisms in metals.

\section{References}

[1] A. L. Gurson. Continuum theory of ductile rupture by void nucleation and growth, Part I - Yield criteria and flow rules for porous ductile media. Journal of Engineering Materials and Technology-Transactions of the ASME, 99:2-15, 1977.

[2] C. Miehe, M. Hofacker and F. Welschinger. A phase field model for rate-independent crack propagation: Robust algorithmic implementation based on operator splits Computer Methods in Applied Mechanics and Engineering, 199:2765-2778, 2010.

[3] C. Miehe, M. Hofacker, L.-M. Schänzel and F. Aldakheel. Phase Field Modeling of Fracture in Multi-Physics Problems. Part II. Brittle-to-Ductile Failure Mode Transition and Crack Propagation in Thermo-ElasticPlastic Solids. Computer Methods in Applied Mechanics and Engineering, in Press, 2015. 


\title{
Simulation of micro cutting considering crystal plastic deformations
}

\author{
$\underline{\text { Richard Lohkamp }}^{1}$, Frank Schneider ${ }^{2}$, Jan Aurich $^{2}$, Charlotte Kuhn $^{3}$, Ralf Müller ${ }^{1}$ \\ ${ }^{1}$ Institute of Applied Mechanics, University of Kaiserslautern \\ ${ }^{2}$ Institute for Manufacturing Technology and Production Systems, University of Kaiserslautern \\ ${ }^{3}$ Computational Mechanics, University of Kaiserslautern
}

In micro machining processes the heterogeneous microstructure influences the cutting forces as well as the surface properties. As the characteristic size of the grain structure of the material is comparable to the size of the micro cutting tool a crystal plastic material model for cp-titanium is employed. It considers basal and prismatic slip systems of a hcp crystal and latent hardening. In a small strain setting the interaction of a crack tip with a grain boundary is studied by means of configurational forces, which can be interpreted as driving forces on material inhomogeneities such as crack tips [1]. Assuming a standard dissipative medium the calculation of the elastic contribution of the configurational force is straightforward. The part due to the dissipative processes in the material, is more complex, because the gradients, treated as internal history variables, are to be computed [2]. This analysis gives some basic understanding of the deformation mechanisms encountered in machining situations. This model is then extended to large deformations according to the method proposed in [3] in order to simulate cutting processes. The examples demonstrate the dependency of surface properties on the crystal orientation.

\section{References}

[1] C. Kuhn, R. Müller, D. Gross. Configurational Forces in Crystal Plasticity - An Analysis of the Influence of Grain Boundaries on Crack Driving Forces. AAA. 84. (2014)

[2] C. Kuhn, R. Lohkamp, R. Müller, F. Schneider, and J. C. Aurich.Finite Element Computation of Discrete Configurational Forces in Crystal Plasticity. IJSS. Accepted.

[3] A. Cuitino and M.Ortiz. A material-independent method for extending stress update algorithms from smallstrain plasticity to finite plasticity with multiplicative kinematics. Engng Comp. 9, (1992). 


\title{
Finite Gradient Elastoplasticity with Internal Constraints
}

\author{
Albrecht Bertram, Rainer Glüge \\ University of Magdeburg
}

The intention of this contribution is to give a mechanical framework for the modelling of gradient effects in elasticity and plasticity. It is an extension of the approaches in Refs. [1] and [2] to finite deformations. The theory fulfils Euclidean invariance and is based on the concept of material isomorphisms to describe the thermoelastic behaviour in all elastic ranges. This concept has been used in Ref. [3] in the context of classical finite plasticity.

Presently, the general form of a finite constitutive gradient theory is given, including the notion of symmetry transformations, anisotropy and isotropy, etc. This is then extended to materials with non-classical internal constraints. Such constraints not only restrict the deformations of the material, but also the distribution of the deformations within the body. It turns out that a natural introduction of internal constraints can be given in the same way for simple materials (see [4]) and for second gradient materials. This opens the door for interesting applications in continuum mechanics.

\section{References}

[1] A. Bertram and S. Forest. The thermodynamics of gradient elastoplasticity. Continuum Mechanics and Thermodynamics 26 (2014), 269-286.

[2] S. Forest and R. Sievert. Elastoviscoplastic frameworks for generalized continua. Acta Mechanica 160 (2003), $71-111$.

[3] A. Bertram. Elasticity and Plasticity of Large Deformations - an Introduction. Springer-Verlag, 2005, 2008, 2012.

[4] A. Bertram. An introduction of internal constraints in a natural way. Zeitschrift für Angewandte Mathematik und Mechanik 60 (1980), T100-T101. 


\title{
A link between the molecular statistical theory and Ogden's model of rubber elasticity
}

\author{
Alexander E. Ehret \\ Institute of Mechanical Systems, ETH Zürich, 8092 Zürich, Switzerland
}

It has been shown in the past by several authors that many phenomenological non-linear constitutive models that are formulated in terms of the principal strain invariants can be reconciled with the molecular statistical theory of rubber elasticity, see e.g. $[1,2,3]$. In the present contribution, this discussion is extended to strainenergy functions which are given in terms of the principal stretches. In particular, a link is established between Ogden's model [5], in a general form with an arbitrary number of terms, and the non-Gaussian statistical theory.

The reconciliation uses similar steps as those applied for invariant-based strain-energy functions before, however, accomplished in an alternative manner. Particularly three representative chains are considered that undergo non-affine deformations and whose motion is subjected to topological constraints. Together with an alternative approximation of the inverse Langevin function in terms of a generalised power series, this leads to a representation of Ogden's material in terms of physical constants that characterise the polymer chain and network, and a few additional parameters that account for the non-affine deformation of topologically constrained polymer chains [4].

The reformulated model provides good agreement with different sets of experimental data at a reduced number of fitting constants. Moreover, the parameters of the statistical and the usual phenomenological representations are connected and it is shown that the parameter set obtained by Ogden [5] for Treloar's data [6] entails a good approximation of the inverse Langevin function in the statistical model. The results of this work suggest that the success and excellent performance of Ogden's model is due to a strong link with the physical basis of rubber elasticity [4].

\section{References}

[1] M.C. Boyce. Direct comparison of the Gent and the Arruda-Boyce constitutive models of rubber elasticity. Rubber Chem. Technol. 69 (1996), 781-785.

[2] M.C. Boyce, E.M. Arruda. Constitutive models of rubber elasticity: A review. Rubber Chem. Technol. 73 (2000), 504-523.

[3] C.O. Horgan, G. Saccomandi. A molecular-statistical basis for the Gent constitutive model of rubber elasticity. J. Elast. 68 (2002), 167-176.

[4] A.E. Ehret. On a molecular statistical basis for Ogden's model of rubber elasticity. Submitted to J. Mech. Phys. Solids (2014).

[5] R.W. Ogden. Large deformation isotropic elasticity - on the correlation of theory and experiment for incompressible rubberlike solids. Proc. R. Soc. Lond. A 326 (1972), 565-584.

[6] L.R.G. Treloar. Stress-strain data for vulcanised rubber under various types of deformation. Trans. Faraday Soc. 40 (1944), 59-70. 


\title{
Equivalent Plastic Strain Gradient Theory with a Grain Boundary Yield Condition
}

\author{
Andreas Prahs, Eric Bayerschen, Thomas Böhlke \\ Chair for Continuum Mechanics, Institute of Engineering Mechanics, \\ Karlsruhe Institute of Technology (KIT) \\ Karlsruhe, Germany
}

In the context of microstructured materials it is essential to discuss non-local mechanical behavior of micro specimens. Strain gradient plasticity theories introduce an internal length scale and thus can be used to investigate size-dependent effects, e.g. the Hall-Petch effect, cf. e.g. [1]. One major influence on the non-local behavior of crystals with a few grains is the presence of grain boundaries and their resistance against the transmission of dislocations through the grain boundary. This resistance, in general, may depend on a broad variety of micro-material properties like, e.g., the crystallographic orientation of the adjacent grains.

An equivalent plastic strain gradient plasticity model for small deformations, cf. [2], is extended by the dependency of the grain boundary resistance against plastic flow on the geometric mismatch between grains. For this purpose, the grain boundary dislocation density (GBD), cf. [3], is used as a geometrical criterion to express the total mismatch between all glide systems of two adjacent grains. The applicability of the GBD for tilt grain boundaries is investigated by numerical calculations of a bicrystal. Twist grain boundaries are discussed by analytical means. Finite-Element simulations are performed under different loading conditions. An application of the continuum model regarding discrete dislocation dynamics is discussed, cf. [4]. Further possibilities of a refinement of the theory with respect to the incorporated defect energy are outlined.

\section{References}

[1] R. Armstrong, I. Codd, R. Douthwaite, N. Petch. The plastic deformation of polycrystalline aggregates. Philosoph. Mag. 73 (1962), 45-58.

[2] S. Wulfinghoff, E. Bayerschen, T. Böhlke. A gradient plasticity grain boundary yield theory. Intern. J. of Plast. 51 (2013), 33-46.

[3] L.P. Evers, W. Brekelmans, M. Geers. Scale dependent crystal plasticity framework with dislocation density and grain boundary effects. Intern. J. of Sol. and Struct. 41 (2004), 5209-5230

[4] E. Bayerschen, M. Stricker, S. Wulfinghoff, D. Weygand, T. Böhlke. Equivalent Plastic Strain Gradient plasticity with Grain Boundary Hardening. submitted. 


\title{
Primary and secondary instabilities in soft bilayered systems
}

\author{
Silvia Budday ${ }^{1}$, Sebastian Andres ${ }^{1}$, Paul Steinmann ${ }^{1}$, Ellen Kuhl ${ }^{2}$ \\ ${ }^{1}$ Chair of Applied Mechanics, University of Erlangen-Nuremberg, \\ 91058 Erlangen, Germany \\ ${ }^{2}$ Departments of Mechanical Engineering and Bioengineering, Stanford University, \\ Stanford, CA 94305, USA
}

Wrinkling phenomena emerging from mechanical instabilities in inhomogeneously compressed soft bilayered systems can evoke a wide variety of surface morphologies. The cause of compression can be of different nature: external impacts like pressure but also internal effects like constrained growth can evoke surface wrinkling. Applications range from undesired instabilities in engineering structures such as sandwich panels, via fabricating surfaces with controlled buckling patterns of unique properties, to wrinkling phenomena in biological systems such as lungs, mucosas, and brain convolutions [1]. While moderate compression generates a regular pattern of sinusoidal wrinkles with a relatively homogeneous energy distribution, higher compression induces secondary instabilities - the surface bifurcates into increasingly complex morphologies. Weakly nonlinear theoretical approaches have provided first insights into secondary instabilities [2], but the highly nonlinear post-buckling regime remains underinvestigated. Here, we establish a computational model of differential growth to explore the evolving buckling pattern beyond the first instability point [3]. With continuing growth, the energy progressively localizes and new complex morphologies emerge. Our model provides a mechanistic understanding of growth-induced primary and secondary instabilities and reveals the effect of layer thickness, stiffness, and growth on the evolving buckling pattern. We show that amongst all possible secondary bifurcations, the mode of period-doubling is energetically favorable. Yet, we can numerically suppress the emergence of period-doubling, by choosing the simulation domain such that periodicity favors alternative modes, e.g., period-tripling. We experimentally validate our simulation results by examining buckling of a mechanically compressed polymer film on a soft foundation. The polymer experiments qualitatively and quantitatively confirm the numerical predictions. Our computational studies not only open new paths in the microfabrication design of unique multiperiodic patterns but have broad applications in the morphogenesis of living systems, where growth is progressive and the formation of structural instabilities is critical to biological function.

\section{References}

[1] S. Budday, P. Steinmann, E. Kuhl. The role of mechanics during brain development. J. Mech. Phys. Solids 72 (2014), 75-92.

[2] F. Brau, H. Vandeparre, A. Sabbah, C. Poulard, A. Boudaoud, P. Damman. Multiple-length-scale elastic instability mimics parametric resonance of nonlinear oscillators. Nat. Phys. 7 (2011), 56-60.

[3] S. Budday, E. Kuhl, J.W. Hutchinson. Period-doubling and period-tripling in growing bilayered systems. submitted for publication. 


\title{
A physically motivated model for filled elastomers including strain rate and amplitude dependency in finite viscoelasticity
}

\author{
Daniel Juhre $^{1,2}$, Rathan Raghunath ${ }^{2}$, Manfred Klüppel ${ }^{2}$ \\ ${ }^{1}$ Institute of Mechanics, Otto-von-Guericke University Magdeburg, Germany \\ ${ }^{2}$ Deutsches Institut für Kautschuktechnologie e.V. (DIK), Hanover, Germany
}

A microstructure-based model of rubber reinforcement, the so-called dynamic flocculation model (DFM), is presented describing filler-induced stress softening and hysteresis by the breakdown and reaggregation of strained filler clusters [1]. An extension of this model allows to consider incomplete deformation cycles that occur in the simulation of arbitrary deformation histories [2]. Good agreement between measurement and the model is obtained for CB-filled elastomers like NR, SBR or EPDM, loaded along various deformation histories. One very important aspect is that the model parameters can be directly referred to the physical properties.

This benefit is used to extend the model to further essential effects like time- and rate-dependent material behavior. In the limit range above the glass transition temperature these viscoelastic effects originate mainly from the filler-filler interactions. In the material model these interactions are characterized by two material parameters $s_{v}$ and $s_{d}$, respectively. The parameter $s_{v}$ defines the strength of the virgin filler cluster, whereas $s_{d}$ represents the strength according to the broken or damaged filler clusters. Both parameters can be defined as functions of time $s_{v, d}=\hat{s}_{v, d}(t)$, which can be motivated by physical meaning [3]. Due to this extension it is possible to capture the very complex strain rate and amplitude dependency during loading and relaxation.

The implementation of the model into the finite element method is realized by using the concept of representative directions [4]. In this framework we are able to validate the model also for arbitrary loading histories on complex structures.

\section{References}

[1] H. Lorenz, M. Freund, D. Juhre, J. Ihlemann, \& M. Klüppel. Constitutive generalization of a microstructurebased model for filled elastomers. Macromolecular Theory and Simulations. (2011), 20, 110- 123.

[2] H. Lorenz, M. Klüppel. Microstructurebased modeling of arbitrary deformation histories of filler-reinforced elastomers. Journal of the Mechanics and Physics of Solids. (2012), 60, 1842-1861.

[3] D. Juhre, R. Raghunath, M. Klüppel and H. Lorenz. A microstructure-based model for filled elastomers including time-dependent effects. Constitutive Models for Rubber VIII. (2013), 297-302.

[4] M. Freund, H. Lorenz, D. Juhre, J. Ihlemann, \& M. Klüppel. Finite element implementation of a microstructure-based model for filled elastomers. International Journal of Plasticity. (2011), 27, 902-919. 


\title{
A Hardening Model based on a Finite-Deformation Gradient Crystal Plasticity Description: Formulation and Numerical Implementation
}

\author{
Habib Pouriayevali, Baixiang Xu \\ Mechanics of functional materials Division, Institute of Material Science, \\ Technische Universitat Darmstadt, Petersenstrasse 32, D-64287 Germany
}

Intrinsic size-dependence response of materials along with inhomogeneous plastic flow on the micro-scale level are widely observed in the experimental results [1]. Prediction of such a size-dependence response requires incorporation of atomistic slip systems, gradient description and length scale parameters into the conventional plasticity models. In this study, a well-defined finite-deformation gradient crystal plasticity is employed to study size-dependence strengthening behavior of a single crystal under simple shear loading [2-5]. The constitutive description is an extended crystal plasticity model based on the microscopic force balance combined with consistency of thermodynamic laws. Free energy comprises two parts: a hyperelastic description for largedeformation compressible material and a function of dislocation densities via Peach-Koehler forces conjugate to corresponding glide directions. A non-local plastic flow rule in the form of partial differential equation is introduced, while incorporates energetic and dissipative gradient strengthening as well as latent hardening in a multi slip-system crystal.

In terms of numerical solution, an implementation method which has been recently introduced in [6] is adopted. In this method, the proposed constitutive model is implemented in the FEM software ABAQUS via a user-defined element subroutine (UEL). A plain-strain quadratic-element (8-node element with 9 integration points) in which displacement components and dislocation densities were treated as nodal degrees of freedom is defined (Global variable). The flow rule is applied to the integration points and solved to obtain the plastic flow in each slip system via Newton-Raphson scheme (Local variable).

As examples, effect of gradient strengthening, latent hardening and scale-variation in mechanically plastic response of a single crystal subjected to isothermal quasi-static loading is studied and discussed.

\section{References}

[1] Hutchinson, J.W. Plasticity at the micron scale. International Journal of Solids and Structures. 37(1-2) (2000), 225-238.

[2] Lele, S.P., L. Anand. A large-deformation strain-gradient theory for isotropic viscoplastic materials. International Journal of Plasticity. 25(3) (2009), 420-453.

[3] Gurtin, M. E. A finite-deformation, gradient theory of single-crystal plasticity with free energy dependent on densities of geometrically necessary dislocations. International Journal of Plasticity. 24(2) (2008), 702-725.

[4] Gurtin, M. E., L. Anand. A theory of strain-gradient plasticity for isotropic, plastically irrotational materials. Part II: Finite deformations. International Journal of Plasticity. 21(12) (2005), 2297-2318.

[5] Gurtin, M. E., et al. Gradient single-crystal plasticity with free energy dependent on dislocation densities. Journal of the Mechanics and Physics of Solids. 55(9) (2007), 1853-1878.

[6] Klusemann, B., B. Svendsen, S. Bargmann. Analysis and comparison of two finite element algorithms for dislocation density based crystal plasticity. GAMM-Mitteilungen.36(2)(2013), 219-238. 


\title{
Phenomenological modelling for viscohyperelasticity: How to find suitable evolution laws in order to extend hyperelastic models?
}

\author{
Nils Hendrik Kröger ${ }^{1}$, Daniel Juhre ${ }^{2}$ \\ ${ }^{1}$ Deutsches Institut für Kautschuktechnologie e.V. (DIK), Hannover, Germany \\ ${ }^{2}$ Institute of Mechanics, Otto-von-Guericke University, Magdeburg, Germany
}

The expansion of the utilization of modern adhesive tapes, for example to install glas windows into building structures, calls for long-term prediction of the material behaviour of the tapes itself. The used polymers to produce those adhesive tapes undergo large deformations during application, and additionally, show timedependent and strain-rate-dependent effects in their stress-strain-time response in experimental testing.

In order to predicted the long-term behaviour suitable material models are required. For our reference polymer the quasi-static experimental testing suggests a material behaviour which can be modelled via models for hyperelasticity. Deformation at different strain rates and recording stress relaxation at high hold strains indicate that the use of viscohyperelastic material models is needed to cover the observed effects. A possibility to extend existing hyperelastic models for viscous effects is via the Prony series approach, see [1].

In this paper we present an alternative modelling approach for viscohyperelasticity based on pure phenomenological results directly derived by the experiments. Separating in the loading and relaxation parts, we are able to develop a suitable evolution law for the shear modulus of a classic hyperelastic Arruda-Boyce model to capture the viscous effect during the relaxation. Identifying the parameter sets for different loadings during tension, we suggest an extension of the model using the response surface methodology.

Based on the ideal shear modulus, the Arruda-Boyce model, see [2], is extended by stating an evolution equation for the shear modulus. Capturing phenomenological dependencies on equivalent strain rate and additional dependency on the first strain invariant, this differential equation approach in its simplicity allows us to cover and to predict the visco-hyperelatisic material behaviour of the reference material for a variety of tension tests with different strain rates, and influences to the relaxation behaviour.

\section{References}

[1] M.A.P. Mohammed. Visco-Hyperelastic Model for Soft Rubber-like Materials. Sains Malaysiana. 43(3)(2014), $451-457$.

[2] E. Arruda, M. Boyce. A three-dimensional constitutive model for the large stretch behavior of rubber elastic materials. Journal of the Mechanics and Physics of Solids, 41(1993), 389 - 412. 


\title{
Comparison of micromorphic, micropolar and microstrain continua
}

\author{
T. Leismann, R. Mahnken \\ Chair of Engineering Mechanics (LTM), University of Paderborn, Paderborn, Germany
}

Micromorphic continua are equipped with additional degrees of freedom in comparison to the classical continuum, representing micro deformations of the material points of a body, see [1]. Secondary they are provided with a higher order gradient. Therefore, they are able to account for material size-effects and to regularize the boundary value problem, when localization phenomena arise. Arbitrary micro deformations are allowed for in the micromorphic continuum, while the special cases micropolar continuum and microstrain continuum merely allow for micro rotation and micro strain respectively. Only the micropolar case has been covered extensively in the literature, e.g. [2]. The microstrain continuum was introduced in [3].

One goal of this presentation is to make the transition from a full micromorphic continuum, as presented in [4], to a micropolar or microstrain continuum, by varying the constitutive equations. Another goal is to present a constitutive model encompassing the micromorphic, micropolar and microstrain continuum as special cases and arbitrary mixtures of micropolar and microstrain parts, enabling the representation of versatile material behaviour. The results are illustrated by numerical examples.

\section{References}

[1] A. C. Eringen. Microcontinuum Field Theories. Springer (1999).

[2] W. Ehlers, W. Volk. On theoretical and numerical methods in the theory of porous media based on polar and non-polar elasto-plastic solid materials. Int. J. Solids Structures 35 (1998), 4597-4617.

[3] S. Forest, R. Sievert. Nonlinear microstrain theories. Int. J. Solids Structures 43 (2006), 7224-7245.

[4] C. B. Hirschberger, E. Kuhl, P. Steinmann. On deformational and configurational mechanics of micromorphic hyperelasticity - Theory and computation. Comput. Methods Appl. Mech. Engrg. 196 (2007), 4027-4044. 


\title{
A variational viscosity-limit approach to the evolution of microstructures in finite crystal plasticity
}

\author{
Christina Günther, Philipp Junker, Klaus Hackl \\ Ruhr-Universität Bochum
}

Kochmann and Hackl introduced in [1] a micromechanical model for finite single crystal plasticity. Based on thermodynamic variational principles this model leads to a non-convex variational problem. Employing the Lagrange functional, an incremental strategy was outlined to model the time-continuous evolution of a first order laminate microstructure. Although this model provides interesting results on the material point level, due to the global minimization in the evolution equations, the calculation time and numerical instabilities may cause problems when applying this model to macroscopic specimens.

In order to avoid these problems, a smooth transition zone between the laminates is introduced to avoid global minimization, which makes the numerical calculations cumbersome compared to the model in [1]. By introducing a smooth viscous transition zone, the dissipation potential and its numerical treatment have to be adapted. We outline rate-dependent time-evolution equations for the internal variables based on variational techniques and show as first examples single slip shear and tension/compression tests.

\section{References}

[1] Kochmann, D. and Hackl, K. The evolution of laminates in finite crystal plasticity: a variational approach, Continuum Mechanics and Thermodynamics 23 (2011), pp. 63-85. 


\title{
Thermoplastics under Long-Term Loading: Experiments and Viscoelastic-Viscoplastic Modeling
}

\author{
$\underline{\text { Patrick Zerbe }}^{1,2}$, Benjamin Schneider ${ }^{1}$, Egon Moosbrugger ${ }^{1}$, Michael Kaliske $^{2}$ \\ ${ }^{1}$ Corporate Sector Research and Advance Engineering, Robert Bosch GmbH \\ ${ }^{2}$ Institute for Structural Analysis, Technische Universität Dresden
}

Thermoplastics subjected to long-term quasi-static loading generally show increasing deformation over time, so-called creep. During periods of partial or complete unloading, the deformation recovers at least partially. To further analyze this phenomenon and more accurately describe the long-term time-dependent deformation behavior of a semicrystalline thermoplastic, uniaxial tensile experiments are carried out and a suitable material model is formulated.

For the initial experiments, the load applied is held constant to determine creep deformations without recovery. During following experiments, the specimens are unloaded periodically such that distinct alternating creep and recovery phases are observed. The recovery phases allow for a distinction between recoverable and irrecoverable deformation during creep.

Based on identified characteristic features of the deformation behavior, a viscoelastic-viscoplastic model using an additive split of deformations is formulated. The viscoelastic part is represented by a generalized Maxwell model whereas the viscoplastic part derives from typical nonlinear creep evolution equations without incorporating a yield surface. An additional damage model interacts with both parts to represent further degradation of the material.

Numerical uniaxial simulations based on the experimental loading profiles are carried out and compared with the experimental results. The overall fit of the model towards the experimental results is demonstrated. 


\title{
Formation of grain boundaries in ductile single crystals at simple shear
}

\author{
M. Koster, K.C. Le, B.D. Nguyen \\ Institute of Mechanics of Materials, Ruhr University Bochum \\ D-44780 Bochum, Germany
}

This presentation is concerned with the formation of grain boundaries in single crystals. From experiments producing large deformations, like equal-channel angular pressing (ECAP) the formation of grain boundaries in crystals can be observed [1]. Our aim is to propose a model, which is capable of predicting this phenomenon.

Plastic deformation of crystals is possible due to the movement and generation of dislocations in the material's crystal lattice. The kinematics related to these deformations is based on the nonlinear continuum dislocation theory (CDT) [2]. To model the formation of sub-grain boundaries all necessary equilibrium conditions are derived for a single crystal containing a surface of discontinuity, which is interpreted as a grain boundary, using the variational principle of minimum of the energy. Two novel equations are presented, namely

$$
w_{\nabla \beta} \cdot \mathbf{n}=0,
$$

where $w_{\nabla \beta}$ is the partial derivative of the free energy per unit volume of the single crystal with respect to the gradient of plastic slip $\beta$ and $\mathbf{n}$ is the unit normal vector of the surface of discontinuity. This equation can be interpreted as the equilibrium of micro-forces acting on dislocations. The second equation represents a thermodynamic condition that ensures that the driving force $f$ acting on grain boundaries vanishes

$$
f=-\mathbf{q} \cdot \mathbf{P} \cdot \mathbf{n}+\llbracket w \rrbracket+2 \zeta \eta=0,
$$

preventing movement of grain boundaries in the crystal. Here $\mathbf{q}$ is a constant vector taken from Hadamard's compatibility condition for the total deformation, $\mathbf{P}$ is the first Piola-Kirchhoff stress tensor, $\llbracket w \rrbracket$ denotes the jump of the free energy density at the surface of discontinuity and $\zeta$ and $\eta$ are the energy per unit area of the surface of discontinuity and its mean curvature, respectively.

To illustrate how the derived set of equations can be used to model the formation of grain boundaries, the case of plane-strain simple shear deformation of a single crystal is investigated. It is shown that the proposed energy density function of the material exhibits non-convex behavior, indicating that there are states that allow for a laminate structure formation in the material in order to lower the energy of the crystal. This is accomplished by constructing an energy minimizing sequence mixing two energy states [3].

\section{References}

[1] T.G. Langdon. The principles of grain refinement in equal-channel angular pressing. Mater. Sci. Eng., A. $462(2007), 3-11$.

[2] K.C. Le, C. Günther. Nonlinear continuum dislocation theory revisited. Int. J. Plast. 53 (2014), 164-178.

[3] C. Carstensen, K. Hackl, A. Mielke. Non-convex potentials and microstructures in finite strain plasticity. Proc. R. Soc. London, Ser. A. 458 (2002), 299-317. 


\title{
Modeling Dynamic Recrystallization in Polycrystalline Materials via Probability Distribution Functions
}

\author{
Hai Van Nguyen ${ }^{1}$, Vahid Ebrahimzade Isfahani ${ }^{2}$, Klaus Hackl ${ }^{1}$, Jörg Renner ${ }^{3}$ \\ ${ }^{1}$ Lehrstuhl für Mechanik-Materialtheorie, \\ Ruhr-Universität Bochum, D-44780 Bochum, Germany \\ ${ }^{2}$ Forschungszentrum Jülich GmbH, Leo-Brandt-Strasse, 52428 Jülich, Germany \\ ${ }^{3}$ Institut für Geologie, Mineralogie und Geophysik, \\ Ruhr-Universität Bochum, D-44780 Bochum, Germany
}

\begin{abstract}
A model of dynamic recrystallization in polycrystalline materials is investigated in this work. Within this model a probability distribution function representing a polycrystalline aggregate is introduced. This function characterizes the state of individual grains by grain size and dislocation density. By specifying free energy and dissipation within the polycrystalline aggregate an evolution equation for the probability density function is derived via a thermodynamic extremum principle. For distribution functions describing a state of dynamic equilibrium we obtain a partial differential equation in parameter space. To facilitate numerical treatment of this equation, the equation is further modified by introducing an appropriately rescaled variable. In this the source term is considered to account for nucleation of grains. Then the differential equation is solved by an implicit time-integration scheme based on a marching algorithm [3]. From the obtained distribution function macroscopic quantities like average strain and stress can be calculated. Numerical results of the theory are subsequently presented. The model is compared to an existing implementation in Abaqus as well.
\end{abstract}

\section{References}

[1] K. Hackl, J. Renner. High-temperature deformation and recrystallization: A variational analysis and its application to olivine aggregates. J. Geophys. Res. Solid Earth. 118 (2013), 1-10.

[2] K. Hackl, F.D. Fischer. On the relation between the principle of maximum dissipation and inelastic evolution given by dissipation potentials. Proc. R. Soc. A. 464 (2008), 117-132.

[3] H.V. Nguyen, K. Hackl, J. Renner. A Variational Approach to Dynamic Recrystallization Using Probability Distributions. PAMM. Proc. Appl. Math. Mech. 13 (2013), 275-276. 


\title{
Chiral acoustic metamaterials: dispersive waves and low frequency band-gaps
}

\author{
A. Bacigalupo, L. Gambarotta \\ IMT Institute for Advanced Studies, Lucca \\ Department of Civil, Chemical and Environmental Engineering, University of Genoa
}

Periodic acoustic metamaterials designed to get complete sound attenuation for a certain frequency range, namely acoustic wave spectral gap, pose interesting issues concerning their mechanical modelling [1], [2], [3]. The present analysis is focused on chiral lattices made up of an array of circular rings connected by ligaments and is devoted to understand the influence of resonators located inside the rings on the band gap structure. To get the essential aspects of the system some simplifying assumptions are put forward. The ligaments are assumed massless, while both the rings and the internal resonators are modelled as rigid. A discrete Lagrangian model having six dofs per node is derived and by a Hamiltonian approach the equations of motion are obtained. Dispersive waves in the periodic micro-structure are analysed in terms of dispersive functions and polarization vectors. The exact acoustic and optical branches are derived for harmonic waves in the discrete model. A further simplified description to obtain a synthetic representation of waves propagation is obtained by assuming an expansion of the generalized displacement field around the node of interest. This allows to get an enriched micropolar equivalent continuum whose equations of motion (partially relies on that obtained in [4], [5]) involves six degree of freedom. To assess the validity limits of the enriched micropolar continuum model the dispersive functions in terms of the fundamental parameters of the mass-in-mass dynamic system are compared with those obtained from the discrete Lagrangian model.

\section{References}

[1] H.H. Huang, C.T. Sun. Wave attenuation mechanism in an acoustic metamaterial with negative effective mass density. New Journal of Physiscs, 11 (2009), 013003-1.

[2] D. Bigoni, S. Guenneau, A.B. Movchan, M. Brun. Elastic metamaterials with inertial locally resonant structures: Application to lensing and localization. Physical Review, B 87 (2013), 174303.

[3] K.F. Tee, A. Spadoni, F. Scarpa, M. Ruzzene. Wave propagation in auxetic tetrachiral honeycombs. J. of Vibration and Acoustics ASME, 132 (2012), 031007-1/8.

[4] A. Bacigalupo, L. Gambarotta. Homogenization of periodic hexa- and tetra-chiral cellular solids. Composite Structures, 116 (2014), 461-476.

[5] A. Bacigalupo, L. Gambarotta. A micropolar model for the analysis of dispersive waves in chiral mass-in-mass lattices. Fracture and Structural Integrity, 29 (2014), 1-8. 


\title{
Finite Element Simulation of the creep behavior of directionally solidified NiAl-9Mo
}

\author{
J. Albiez, T. Böhlke \\ Chair for Continuum Mechanics, Institute of Engineering Mechanics, \\ Karlsruhe Institute of Technology (KIT), \\ Karlsruhe, Germany
}

For high temperature structural applications, the B2-ordered intermetallic phase NiAl has promising material properties, such as high oxidation resistance, high melting temperature, high thermal conductivity and relatively low density [1]. However, the use of NiAl for structural applications suffers from its low creep resistance and its weak room temperature fracture toughness [2]. To improve these two material properties at the same time, directional solidification of eutectic alloys leads to a formation of stoichiometric $\mathrm{NiAl}$ and a reinforcing phase of a refactory metal e.g. Cr, Mo, W, Re [1]. A directional solidified NiAl-9Mo (at. \%) eutectic consists of wellaligned single-crystal molybdenum-rich fibers embedded in a Ni-50Al matrix. The steady-state creep rate of the directional solidified NiAl-9Mo can be more than five orders of magnitude lower compared to the intermetallic $\mathrm{NiAl}[3]$. It has been shown that these as-grown molybdenum-rich fibers are dislocation free and plastic flow begins, when the fibers' stress approaches the theoretical strength [4]. After start of yielding, the flow stress reduces due to strain softening of the fibers [4]. An additional advantage of using the directional solidification of eutectic alloys is that the phases are thermodynamically stable even up to the melting point [1]. To be able to predict the directional solidified NiAl-9Mo material behavior under several conditions, material models describing each phase are necessary. Our single crystal plasticity model for large deformation is based on a phenomenological approach with a state variable describing the average dislocation density of the glide systems. We use an overstress type power law flow rule to model the slip in each slip system and to consider the elastic range of the fibers. The two phases are modeled by an elasto-viscoplastic approach with a softening behavior for the fibers and a perfect plasticity behavior for the matrix. The softening behavior is motivated by the observation of the strain softening of the fibers [4]. This elasto-viscoplastic approach shows for a one-dimensional model reasonable agreement with experimental results. A creep curve simulation of a NiAl-9Mo representative volume element with periodic boundary conditions is compared to experimental results. The simulation results show that the molybdenum-rich fibers carry the load and reduce the stress in the NiAl matrix. This load partitioning leads to a reduction of the steady-state creep rate compared to the NiAl intermetallic phase.

\section{References}

[1] D. R. Johnson. Processing and mechanical properties of in-situ composites from the NiAl-Cr and the NiAl(Cr,Mo) eutectic systems. Intermetallics 3 (1995), 99-113.

[2] R. D. Noebe. Physical and mechanical properties of the B2 compound NiAl. International Materials Reviews 38 (1993), 193-232.

[3] M. Dudová. Creep in directionally solidified NiAl-Mo eutectics. Scripta Materialia 65 (2011), 699-702.

[4] H. Bei. Effects of pre-strain on the compressive stress-strain response of Mo-alloy single-crystal micropillars. Acta Materialia 56 (2008), 4762-4770. 


\title{
Direct connection of rheological elements at large strains: Application to multiplicative viscoplasticity
}

\author{
Robert Kießling, Ralf Landgraf, Jörn Ihlemann \\ Professorship of Solid Mechanics, Faculty of Mechanical Engineering, \\ Technische Universität Chemnitz, Chemnitz, Germany
}

In the field of nonlinear continuum mechanics, rheological models are often used to exemplify the structure of complex material models at large strains. For this purpose, different rheological elements are combined in series and parallel connections. One approach to deduce the according constitutive equations consists of a multiplicative decomposition of the deformation gradient, a formulation of a free energy function and the evaluation of the Clausius-Duhem inequality. Subsequently, inelastic material behaviour is defined by appropriate evolution equations. Contrary to this, Ihlemann [1] developed an innovative concept, which enables material modelling at large strains based on the direct connection of rheological elements. In this contribution, we present the application of this approach to multiplicative viscoplasticity.

Starting from the additive decomposition of the stress power and demanding the stress power equivalence of the rheological connections, we first formulate constitutive equations within the framework of the multiplicative decomposition of the deformation gradient. These relations were already applied in the development of nonlinear viscoelasticity of Maxwell type by Landgraf and Ihlemann [2]. Next, we modify the derived connection relations with respect to the limitation on near-incompressibility. Then, individual material models, i.e. the rheological elements, are introduced. Among others, we define elements of hyperelasticity, Newtonian and non-Newtonian viscous flow as well as deformation rate independent plastic flow.

The regarded concept is employed to a model of multiplicative viscoplasticity. On the one hand, the connection relations are analytically evaluated to derive the constitutive equations typically used in the literature. On the other hand, we propose a numerical procedure, which evaluates the connection relations rather than solving the evolution equations for inelastic strains. Thereby, a predictor-corrector strategy is applied to consider the element of plastic flow. This alternative numerical procedure is tested and compared to the commonly used numerical algorithms. Due to a demonstrable accordance with the analytical and numerical solutions from the literature, the potential of the examined concept in the context of material modelling at large strains is shown.

\section{References}

[1] J. Ihlemann. Beobachterkonzepte und Darstellungsformen der nichtlinearen Kontinuumsmechanik. VDIVerlag (2014)

[2] R. Landgraf, J. Ihlemann, On the direct connection of rheological elements in nonlinear continuum mechanics, Proceedings in Applied Mathematics and Mechanics 12 (2012), 307-308. 


\title{
Modelling Thermoplastic Material Behaviour of Dual-Phase Steels on a Microscopic Length Scale
}

\author{
Sebastian Zeller, Stefan Löhnert, Peter Wriggers \\ G. W. Leibniz Universität Hannover \\ Institute of Continuum Mechanics \\ Appelstraße 11, 30167 Hannover, Germany \\ Email: zeller@ikm.uni-hannover.de
}

\begin{abstract}
To support and physically motivate the development of a thermomechanical material model for the macroscopic material behaviour, the polycrystalline microstructure of the material is considered necessitating the development of a material model for the single crystal behaviour. In this work focus is especially placed on the behaviour of the ferritic matrix of dual-phase steels. Since the driving mechanisms for plastic deformation here are the formation, movement and pile-up of dislocations on preferred planes in preferred directions, many crystal plasticity models for the purely mechanical single crystal behaviour motivated by these mechanisms were proposed in the past. In this work these ideas are extended to thermomechanical material behaviour and a thermomechanical crystal plasticity model is developed for the single crystal behaviour. Attention is paid to the description of the evolution of the microstructure and its dependence on the history of the temperature of the processes under consideration. Elementary processes of multiplication and annihilation of dislocations are analysed in terms of temperature and deformation rate dependence. Thermal activation is used to describe these dependences and evolution equations are postulated for the state variables describing the current dislocation structure and by this the influence of the deformation and temperature history on current material behaviour. As the underlying dislocation structure determines the yield stress and the energy stored in the material, these evolution equations and their temperature and rate dependence are crucial for the formulation of the model. Estimations of the critical resolved shear stress necessary to move dislocations through the crystal resulting from the interaction of dislocations on different slip systems and the resistance of the atomic lattice and the energy associated with a line element of a dislocation allow to formulate a thermoplastic constitutive material model for the single crystal behaviour of the ferritic phase.

The developed ideas and the resulting model are transferred to the macroscopic behaviour of sheet metal wrought material consisting of dual-phase steels. Focus is placed on the consistency between experimental observations on a macroscopic length scale, the belonging constitutive thermoplastic material model on a macroscopic length scale and the developed material model for the single crystal behaviour. Possible forms and dependences on temperature and state variables for the macroscopic thermomechanical material model are deduced. Especially a thermodynamically consistent form for the energy stored in the material is presented.
\end{abstract}




\title{
On the thermodynamics of pseudo-elastic material models which reproduce stress softening effects
}

\author{
Christoph Naumann, Jörn Ihlemann \\ Professorship of Solid Mechanics, Faculty of Mechanical Engineering, \\ Technische Universität Chemnitz, Chemnitz, Germany
}

The mechanical material behavior of rubber is primarily affected by a stress softening effect called the Mullins-effect. Due to this effect, the stress depends strongly on the maximum load which occurred in the history of the material. Especially in inhomogeneously loaded components this effect has to be considered, as different regions with varying stiffness evolve. Due to this resulting inhomogeneous distribution of the material properties the overall behavior of the component is affected considerably. Therefore the Mullins-effect has to be taken into account to simulate the behavior of elastomeric components.

A very popular material model which allows for the Mullins-effect was proposed by Ogden and Roxburgh [1]. They introduced so-called pseudo-elastic material models, which are based on an arbitrary hyperelastic stress. After reaching a maximum load which is measured by the free energy of the basic hyperelastic model, the stress on unloading and successive reloading is decreased by a so-called softening function. A similar approach was used by Elías-Zúñiga and Beatty [2]. There, the norm of the right Cauchy-Green tensor is used as a measure for the load in the material.

In [1] and [2] only a few thermodynamical considerations are incorporated. Thus, the two mentioned models are analyzed theoretically and their thermomechanical properties are derived. These findings are used to deduce an alternative approach to deviate pseudo-elasticity. This is achieved by defining a suitable free energy which yields conditional equations for the stress tensor and the dissipation after exploiting the Clausius-Duhem inequality. It is shown, that under weak demands on the properties of the softening function the dissipation is always non-negative.

The concept of pseudo-elasticity is then generalized to extend arbitrary thermomechanical, even inelastic, material models to allow for softening effects. Under weak assumptions on the softening function the thermomechanical consistency is shown [3].

\section{References}

[1] R. Ogden, D. Roxburgh. A pseudo-elastic model for the Mullins effect in filled rubber. Proceedings of the Royal Society of London. Series A: Mathematical, Physical and Engineering Sciences 455 (1999), 2861-2877.

[2] A. Elías-Zúñiga, M.F. Beatty. A new phenomenological model for stress-softening in elastomers. Zeitschrift für angewandte Mathematik und Physik 53 (2002), 794 - 814.

[3] C. Naumann, J. Ihlemann. On the thermodynamics of pseudo-elastic material models which reproduce stress softening effects. arXiv:1408.0671 [cond-mat.mtrl-sci] (2014). 


\title{
Experimental and numerical characterization of the mechanical properties of $\mathrm{Ni} / \mathrm{Al}$ hybrid metal foams from the atomic to the microscale
}

\author{
Zhaoyu Chen ${ }^{1}$, Anne Jung ${ }^{1}$, Stephan Heinze ${ }^{2}$, Alexander Düster ${ }^{2}$, Stefan Diebels ${ }^{1}$ \\ ${ }^{1}$ Chair of Applied Mechanics, Saarland University, D-66123 Saarbrücken, Germany \\ ${ }^{2}$ Numerical Structural Analysis with Application in Ship Technology (M-10) \\ Hamburg University of Technology, D-21073 Hamburg, Germany
}

The increasing demand for lightweight and energy absorbing materials leads to popular design of foams and other cellular materials which combine many interesting physical and mechanical properties [1]. In this contribution, nano-Ni/Al hybrid foams were investigated. This hybrid foams consist of an open-cell aluminum alloy $\left(\mathrm{AlSi}_{7} \mathrm{Mg}_{0.3}\right)$ foam strengthened with a nanocrystalline nickel coating [2]. The enhanced mechanical properties are characterized by experiments and numerical modeling at different scales. Nanoindentation is a well-known technique to measure the mechanical behavior of thin coatings with an extremely high resolution at nano-scale. Hence, the local mechanical properties of both the nanocrystalline nickel coating and the aluminum alloy strut are measured from nanoindentation with a very sharp Berkovich tip. The hardness and elastic modulus are determined through the Oliver \& Pharr method. An elastic-plastic model without hard! ening is considered to describe the force-displacement behavior in nanoindentation. The local values of the elastic modulus and the yield stress are identified with an inverse computation based on the FEM simulation and the experimental measurements [3]. The local properties obtained from nanoindentation strongly depend on the distance of the indentation position to the $\mathrm{Ni} / \mathrm{Al}$ interface, which is believed to be related with the microstructure [4]. Therefore, nanoindentation is coupled with EBSD to reliably explain the local measurement and to identify the relationship between the local microstructure and local properties.

Besides, the results obtained above are verified with molecular dynamic simulation of nanoindentation on different structured nickel atoms. The determined hardness and elastic modulus at the atomic scale will be compared with the values obtained at the nano-scale.

Furthermore, micro-tensile tests are performed on a wire made of! the same matrix material and coating as the nano-Ni/Al hybrid foams. These were done to get a more homogeneous elastic-plastic behavior of this $\mathrm{Ni} / \mathrm{Al}$ composite. So it is able to compare the local properties of Ni coating and Al strut at nano-scale with the global stress-strain behavior of the $\mathrm{Ni} / \mathrm{Al}$ composite at micro-scale.

\section{References}

[1] Ashby MF, Evans A, Fleck NA, Gibson LJ, Hutchinson JW, Wadley HNG . Metal Foams: A Design Guide. (2000), Butterworth-Heinemann, Oxford

[2] Jung A, Natter H, Diebels S, Lach E, Hempelmann R. Nanonickel coated aluminum foam for enhanced impact energy absorption. Advanced Engineering Materials (2011), 13: 23-28, DOI10.1002/adem.201000190

[3] Chen Z.. Nanoindentation testing of soft polymers: Computation, experiments and parameters identification. (2014), Dissertation, Schaker Verlag

[4] Jung A, Diebels S, Koblischka-Veneva A, Schmauch J, Barnoush A, Koblischka M. Microstructural analysis of electrochemical coated open-cell metal foams by EBSD and nanoindentation. Advanced Engineering Materials (2014) 16:15-20 


\title{
An Intrinsic Geometric Formulation of the Equilibrium Equations in Continuum Mechanics
}

\author{
Simon R. Eugster \\ Institute for Nonlinear Mechanics \\ University of Stuttgart \\ Pfaffenwaldring 9, D-70569 Stuttgart, Germany \\ simon.eugster@inm.uni-stuttgart.de
}

In this paper an intrinsic differential geometric formulation of the equilibrium equations in continuum mechanics is presented in its weak and strong variational form. Invariant continuum mechanics as proposed by Segev [1], considers the body $\mathcal{B}$ and the physical space $\mathcal{S}$ to be three-dimensional smooth manifolds with and without boundary, respectively. Furthermore the physical space is equipped with an affine connection. To respect the impenetrability condition the configuration $\kappa: \mathcal{B} \rightarrow \mathcal{S}$ has to be a $C^{1}$-continuous embedding. Thus, the the set of all $C^{1}$-continuous embeddings constitutes the infinite dimensional configuration manifold $\mathcal{Q}:=\operatorname{Emb}(\mathcal{B}, \mathcal{S})$. As in analytical mechanics, the virtual displacement field $\delta \kappa$ is an element of the tangent space at the current configuration $T_{\kappa} \mathcal{Q}$ which is isomorphic to the space of $C^{1}$-continuous sections of the pull-back bundle $C^{1}\left(\kappa^{*} T \mathcal{S}\right)$. By duality, the forces of the continuum can be represented in the domain of the body $\mathcal{B}$ by smooth covector- and tensor-valued volume forms. On the boundary, the forces are represented by covectorvalued surface forms. For the classical theory, [2] postulates the principle of virtual work in the sense of Germain $[3]$ as the fundamental principle of invariant continuum mechanics, stating that

$$
\delta W(\delta \kappa)=-\int_{\mathcal{B}} \nabla \delta \kappa: \pi+\int_{\mathcal{B}} \delta \kappa \cdot \beta+\int_{\partial \mathcal{B}} \delta \kappa \cdot \tau=0 \quad \forall \delta \kappa \in C^{1}\left(\kappa^{*} T \mathcal{S}\right) .
$$

Herein, the covector-valued volume form $\beta \in \Gamma\left(\kappa^{*} T^{*} \mathcal{S} \otimes \Lambda^{3} T^{*} \mathcal{B}\right)$ describes the external body forces, the covectorvalued surface form $\tau \in \Gamma\left(\kappa^{*} T^{*} \mathcal{S} \otimes \Lambda^{2} T^{*} \partial \mathcal{B}\right)$ the external traction forces and the tensor-valued volume form $\pi \in \Gamma\left(\kappa^{*} T^{*} \mathcal{S} \otimes T \mathcal{B} \otimes \Lambda^{3} T^{*} \mathcal{B}\right)$ is called the variational stress describing the internal forces. The dot and the colon denote the contraction and double contraction of the adjacent tensor slots. The covariant derivative of a pull-back section of $C^{1}\left(\kappa^{*} T \mathcal{S}\right)$ is denoted by $\nabla$. The principle of virtual work as the weak variational form of the equilibrium equations defines insofar the interaction between internal and external forces independent of any constitutive laws.

With the definition of a contraction map $p_{\sigma}: \Gamma\left(\kappa^{*} T^{*} \mathcal{S} \otimes T \mathcal{B} \otimes \Lambda^{3} T^{*} \mathcal{B}\right) \rightarrow \Gamma\left(\kappa^{*} T^{*} \mathcal{S} \otimes \Lambda^{2} T^{*} \mathcal{B}\right)$ a new object, a covector-valued area form $\sigma=p_{\sigma}(\pi)$, called traction stress [4], is introduced. Using a telescopic expansion and defining the divergence of the variational stress by the relation $\delta \kappa \cdot \operatorname{Div} \pi=-\nabla \delta \kappa: \pi+\mathrm{d}\left(\delta \kappa \cdot p_{\sigma}(\pi)\right)$, where $\mathrm{d}$ denotes the exterior derivative, the strong variational form

$$
\delta W(\delta \kappa)=\int_{\mathcal{B}} \delta \kappa \cdot(\operatorname{Div} \pi+\beta)+\int_{\partial \mathcal{B}} \delta \kappa \cdot\left(\tau-\iota^{*} \sigma\right)=0 \quad \forall \delta \kappa \in C^{1}\left(\kappa^{*} T \mathcal{S}\right)
$$

is obtained by Stokes' Theorem. The pullback $\iota^{*}$ by the inclusion map $\iota: \mathcal{B} \rightarrow \partial \mathcal{B}$ restricts the covector-valued area form $\sigma$ to the oriented boundary $\partial \mathcal{B}$ and induces a traction force. By the arguments of the Fundamental Lemma of Calculus of Variations, the local equilibrium equations and the traction boundary conditions follow directly.

\section{References}

[1] R. Segev. Forces and the existence of stresses in invariant continuum mechanics. J. Math. Phys. 27(1) (1986), 163-170.

[2] S.R. Eugster. On the foundations of continuum mechanics and its application to beam theories. PhD thesis, ETH Zurich (2014).

[3] P. Germain. The method of virtual power in continuum mechanics. Part 2: Microstructure. SIAM J. Appl. Math., 25 (1973), 556-575.

[4] R. Segev. Notes on stresses for manifolds. Rend. Sem. Univ. Pol. Torino. 58(2) (2000), 199-205. 


\title{
Numerical analysis of $\mathrm{Ni} / \mathrm{Al}$ hybrid metal foams using the finite cell method
}

\author{
Stephan Heinze $^{1}$, Anne Jung ${ }^{2}$, Zhaoyu Chen ${ }^{2}$, Stefan Diebels ${ }^{2}$, Alexander Düster ${ }^{1}$ \\ ${ }^{1}$ Numerical Structural Analysis with Application in Ship Technology (M-10) \\ Hamburg University of Technology, D-21073 Hamburg, Germany \\ ${ }^{2}$ Chair of Applied Mechanics, Saarland University, D-66123 Saarbrücken, Germany
}

Light-weight structures are used more and more in many engineering disciplines in order to save weight and to preserve natural resources. Metal foams represent one important class of materials that are used to manufacture such structures. Beside their low density they also have a good acoustic absorption and mechanical damping behavior which also make them attractive for many other applications. A new method to further improve the properties of aluminum metal foams is to coat them by electrodeposition with a thin nickel layer [3]. In order to find a macro-mechanical material law for such hybrid metal foams a lot of mechanical experiments are necessary. These experiments can be supported or replaced to some extend by a numerical homogenization procedure [2] which allows for a precise and systematic variation of the quantities of interest and help to understand the complex deformation mechanism. One of the main obstacles for such simulations is the immense labor effort to generate the mesh for a suitable discretization. In case of aluminum foams coated by nickel this problem becomes even more severe due to the thin nickel layer. In order to avoid this problem we employ the finite cell method (FCM) [1]. The FCM is a combination of a fictitious domain method and a high order finite element method. Due to the fictitious domain approach the surface of the foam and the material interface between the aluminum and the nickel do not need to be taken into account during meshing. This simplifies the meshing process and allows to automatically generate a simple Cartesian grid using the voxel model which comes out of a computed tomography scan. Due to the high order shape functions each element - or cell as referred to in terms of the FCM - can represent a set of voxel. As a consequence the number of degrees of freedom can be reduced a lot as compared to a voxel FEM simulation. Different issues of the finite cell method and of the numerical homogenization procedure will be discussed in order to carry out an efficient computation of the elastic properties of nickel coated aluminum foams.

\section{References}

[1] J. Parvizian, A. Düster, E. Rank. Finite cell method - h- and p-extension for embedded domain problems in solid mechanics. Comput. Mech. 41 (2007), 121-133

[2] A. Düster, H.-G. Sehlhorst, E. Rank, Numerical homogenization of heterogeneous and cellular materials utilizing the finite cell method, Computational Mechanics 50 (2012) 413-431.

[3] A. Jung, H. Natter, R. Hempelmann, E. Lach, Nano nickel strengthend open cell metal foams under quasistatic and dynamic loading. DYMAT 2009 - 9th International Conference on the Mechanical and Physical Behaviour of Materials under Dynamic Loading, Brussels, Belgium, 2009, 717-723. 


\title{
The perturbation method applied to tube drawing with floating plug
}

\author{
Rodica Ioan, Stefan Ioan \\ Institute of Solid Mechanics, Romanian Academy, \\ Spiru Haret University, Bucharest \\ SC Delta A.C.M. 93 SRL, Bucharest \\ E-mail: rodicaioan08@yahoo.com, stefan.ioan@deltaacm.ro
}

The determination of the load on the machine tool is crucial in the mechanics of metal forming processes as it has a critical influence over the deformation of the material into a new shape and the optimal conditions for the process. The problems of strip, wire, tube drawing without plug, tube drawing with floating plug [1]-[4] viscoplastic deformation has no exact solutions, but this paper addresses them using an approximate method. The influence of speed given by Bingham parameter and the influence of acceleration given by Reynolds parameter in the tube drawing with floating plug, is considered. The problem is solved using a viscoplastic constitutive equation of Bingham type. The friction between the rigid tool and the sliding viscoplastic continuum was considered in a local form, $t_{r \theta}=m \sqrt{I I_{t^{\prime}}}$, where $I I_{t^{\prime}}$ is the second invariant of the deviatoric part of the stress tensor and $m \in[0,1]$ is the friction factor. An exact functional relationship between friction and variables such as: normal stress, lubrication, sliding speed etc. cannot be easily formulated, even though the description of the friction resistance between die and material in plastic forming of metals has been much studied. The shape of the floating plug, the drawing force are determined involving various parameters of the problem (the plug angle, the die angle, friction coefficients, the Bingham and Reynolds numbers), which allow the optimization of the drawing process.

\section{References}

[1] G. Camenschi, N. Cristescu, N. Sandru. High Speed Wire Drawing. Archives of Mechanics, 31(1979), 5.

[2] St. Ioan. A Mathematical Model of the Theory of Tube Drawing with Floating Plug. Influence of Inertia. Rev. Roum. Sci. Tehn. - Mec. App. 39(3) (1994), 263-294.

[3] R. Ioan. A Mathematical Model of Tube Drawing without Plug. Proceedings - Modern Technologies in XXI century, Technical Military Academy, Bucharest (2001).

[4] R. Ioan. Mathematical Modelling of Viscoplastic Flow in some Technological processes. Roumanian Academy Publishing House (2008). 


\title{
Mathematical problems in the theory of elasticity for materials with double porosity
}

\author{
Merab Svanadze \\ Ilia State University, Tbilisi, Georgia
}

The theories of elasticity and thermoelasticity of double porosity media, as originally developed for the mechanics of naturally fractured reservoirs, has found applications in civil engineering, geotechnical engineering, technology and biomechanics. Indeed, the double porosity model would consider the bone fluid pressure in the vascular porosity and the bone fluid pressure in the lacunar-canalicular porosity [18].

The theory of consolidation for elastic materials with double porosity was presented in [19]. This theory unifies the earlier proposed models of Barenblatt for porous media with double porosity [3] and Biot for porous media with single porosity [4]. The theory of elasticity for double porosity materials [19] is constructed with the help of Darcy's law and the basic equations involve the displacement vector field, a pressure associated with the pores, and a pressure associated with the fissures.

The double porosity concept was extended to media with multiple porosity in [1], [11]. A great attention has been paid to the theories of poroelasticity taking into account the thermal effects. The basic equations of the one and two-temperatures thermo-hydro-mechanical coupling theories for elastic materials with double porosity are presented in [2], [7], [9], [10]. The basis properties of the plane harmonic waves and the boundary value problems (BVPs) of the theories of elasticity and thermoelasticity for double porosity materials are investigated by using the potential method (boundary integral method) and the theory of singular integral equations in [5], [12]-[18].

Recently, the theories of elasticity and thermoelasticity for materials with a double porosity structure are presented in [8]. In this paper the theory of materials with voids [6] is generalized to derive a theory of thermoelastic solids, which have a double porosity structure. The new theory is not based on Darcy's law. The basic equations for elastic materials with a double porosity structure involve the displacement vector field and the volume fraction fields associated with the pores and the fissures.

In the first part of this talk, a review of the basic results in the theories of elasticity and thermoelasticity for materials with double porosity are presented. In the second part, the linear theory of elasticity for materials with a double porosity structure [8] is considered and a wide class of the internal and external BVPs of steady vibrations are investigated. Indeed, the Green's formulas and Somigliana-type integral representation of regular vector and classical solution of equations of steady vibrations are obtained. The radiation conditions for regular vector are established. The uniqueness theorems of the internal and external BVPs of steady vibrations are proved. The basic properties of surface (single-layer and double-layer) and volume potentials are established. The singular integral operators of the linear theory of elasticity for materials with a double porosity structure are studied. The existence of regular (classical) solutions of the internal and external BVPs by means of the potential method and the theory of singular integral equations are proved.

\section{References}

[1] M. Bai, D. Elsworth, J.C. Roegiers. Multiporosity/multipermeability approach to the simulation of naturally fractured reservoirs. Water Resour. Res. 29 (1993), 1621-1633.

[2] M. Bai, J.C. Roegiers. Fluid flow and heat flow in deformable fractured porous media. Int. J. Eng. Sci. 32 (1994) 1615-1633.

[3] G.I. Barenblatt, I.P. Zheltov, I.N. Kochina. Basic concept in the theory of seepage of homogeneous liquids in fissured rocks (strata). J. Appl. Math. Mech. 24 (1960), 1286-1303.

[4] M.A. Biot. General theory of three-dimensional consolidation. J. Appl. Phys. 12 (1941), 155-164.

[5] M. Ciarletta, F. Passarella, M. Svanadze. Plane waves and uniqueness theorems in the coupled linear theory of elasticity for solids with double porosity. J. Elasticity 114 (2014), 55-68.

[6] S.C. Cowin, J.W Nunziato. Linear elastic materials with voids. J. Elasticity 13 (1983), 125-147. 
[7] R. Gelet, B. Loret, N. Khalili. Borehole stability analysis in a thermoporoelastic dual-porosity medium. Int. J. Rock Mech. Mining Sci. 50 (2012), 65-76.

[8] D. Iesan, R. Quintanilla. On a theory of thermoelastic materials with a double porosity structure. J. Thermal Stresses 37 (2014), 1017-1036.

[9] N. Khalili, A.P.S. Selvadurai. A fully coupled constitutive model for thermo-hydro-mechanical analysis in elastic media with double porosity. Geophys. Res. Lett. 30 (2003), 2268.

[10] N. Khalili, A.P.S. Selvadurai. On the constitutive modelling of thermo-hydro-mechanical coupling in elastic media with double porosity. Elsevier Geo-Eng. Book Series 2 (2004), 559-564.

[11] K.N. Moutsopoulos, A.A. Konstantinidis, I. Meladiotis, Ch.D. Tzimopoulos, E.C. Aifantis. Hydraulic behavior and contaminant transport in multiple porosity media. Trans Porous Media 42 (2001), 265-292.

[12] E. Scarpetta, M. Svanadze. Uniqueness theorems in the quasi-static theory of thermoelasticity for solids with double porosity. J. Elasticity (2015), DOI 10.1007/s10659-014-9505-2 (in press).

[13] E. Scarpetta, M. Svanadze, V. Zampoli. Fundamental solutions in the theory of thermoelasticity for solids with double porosity. J. Thermal Stresses 37 (2014), 727-748.

[14] M. Svanadze. Plane waves and boundary value problems in the theory of elasticity for solids with double porosity. Acta Appl. Math. 122 (2012), 461-471.

[15] M. Svanadze. The boundary value problems of the full coupled theory of poroelasticity for materials with double porosity. PAMM 12 (2012), 279-282.

[16] M. Svanadze. Uniqueness theorems in the theory of thermoelasticity for solids with double porosity. Mecanicca 49 (2014), 2099-2108.

[17] M. Svanadze. Boundary value problems in the theory of thermoporoelasticity for materials with double porosity. PAMM 14 (2014) (in press).

[18] M. Svanadze, A. Scalia. Mathematical problems in the coupled linear theory of bone poroelasticity. Comp. Math. Appl. 66 (2013), 1554-1566.

[19] R.K. Wilson, E.C. Aifantis. On the theory of consolidation with double porosity - I. Int. J. Eng. Sci. 20 (1982), 1009-1035. 


\section{S07: Coupled problems}

Coupled problems arise in several applications. From a general point of view each problem containing more than one primary field is called a coupled one. Usually the class of coupled problems is subdivided into volumetrically coupled problems and problems with surface coupling. The class of volumetrically coupled problems contains e.g. the fluid flow in porous solids described by mixture theory, thermo-mechanically coupled problems, chemo-mechanically coupled problems and electro- or magneto-mechanically coupled problems while in the second class problems like the fluid-solid interaction via an interface are included.

All problems is in common that the presence of different fields in the numerical treatment requires special attention with respect to the multi-field formulation and the solution strategy. The session on coupled problems deals with all aspects mentioned above, i.e. ranging from modelling aspects to numerical solution strategies. 


\title{
Thermo-mechanical modelling of cellular ceramic composites by a multiphase approach of porous media
}

\author{
Anne Jung ${ }^{1}$, Stefan Diebels ${ }^{1}$ \\ ${ }^{1}$ Chair of Applied Mechanics, Saarland University, D-66123 Saarbrücken, Germany
}

Refractory materials have a wide range of applications in steel-making industry as lining of furnaces, oxygen converters or for ladles. Often, magnesia carbon bricks (MgO-C) are used. These consist of a periclase phase $(\mathrm{MgO})$ with inclusions of carbon and gas filled pores. In their applications, refractories are subjected to thermal and mechanical loads causing damage. In recent studies, the thermo-mechanical properties of MgO-C composites could significantly be improved using cellular MgO-C composites based on carbon foams [1, 2]. Modelling of $\mathrm{MgO}-\mathrm{C}$ composite foams is not only a multi-phase, but also a multi-physics problem, in which the displacement field and the temperature field have to be taken into account. Cellular materials, as foams, are micro heterogeneous materials consisting of three different hierarchical levels reaching from the macro scale, dealing with whole components, over the meso scale, dealing with several pores, up to the micro level, comprising single struts. Hence, in experiments and modelling as well, cellular materials can be described on these different scales. In previous work, a mesoscopic elastic thermo-mechanically coupled model was used to investigate the structure-property relationship of $\mathrm{MgO}-\mathrm{C}$ hybrid foams, in order to reduce thermally induced stresses and accompanying damage $[3,4,5]$.

In the present contribution, based on the optimized micro structures of the meso scale model, a fully coupled phenomenological thermo-mechanical continuum model was developed. The theory of porous media (TPM) with a kinematic coupling of the displacement and temperature fields of all constituents was used. It was assumed, that there is no interdiffusion of the three constituents $(\mathrm{MgO}, \mathrm{C}$ and pore gas) with the result that the motion functions and hence the deformation gradients of all phases are equal. Linear thermoelasticity with a multiplicative decomposition of the deformation gradient into an elastic and a thermal part for isotropic materials according to the Duhamel-Neumann relation was extended to the mixture of $\mathrm{MgO}$ and $\mathrm{C}$ phase. The difference in the coefficient of thermal expansion (CTE) leads to different thermal induced stresses in both phases. For the pore gas, the ideal gas law was used as equation of state for the evolution of pressure as function of the density and the temperature. The total macroscopic stress was calculated using the theory of mixture, inclusing the contributions from the pore pressure and the two solid phases.

\section{References}

[1] Falk G, Jung A, Da Silveira W, Diebels S. Experimental characterization and thermo-mechanical modelling of micro structure interactions in cellular carbon magnesia refractories. Journal of Ceramic Science and Technology, Vol. 5(2) (2014) 101-114.

[3] Jung A, Falk G, Petri D, Diebels S. Thermal shock resistivity of hybrid carbon foam materials: Experiments and model predictions. Mechanics of Materials (accepted)

[4] Jung A, Diebels S. Thermo-mechanical modelling of cellular hybrid refractories. Proceedings in Applied Mathematics and Mechanics (accepted)

[2] Falk G, Petri D, Jung A, Diebels S. Refractory MgO-C composites with a cellular carbon structure, Part 1: Experimental characterization. Keramische Zeitschrift Vol. 4 (2014) 221-226.

[5] Jung A, Falk G, Petri D, Diebels S. Refractory MgO-C composites with a cellular carbon structure, Part 2: Microstructural optimization by thermo-mechanical simulation. Keramische Zeitschrift Vol. 5-6 (2014) 295301. 


\title{
Thermo-mechanical computation of inelastic pavement structures under rolling tire loads
}

\author{
I. Wollny, F. Hartung, M. Kaliske \\ Institute for Structural Analysis, TU Dresden
}

Pavements, as one main part of the world wide infrastructure, are exposed to increasing traffic loads. Especially, in case of asphalt pavement structures, the formation of ruts depends beside the tire loads also on the temperature dependent behavior of asphalt. In order to predict the long term behavior of pavements after a multitude of tire overruns, first the structural behavior of pavement structures at single tire overruns has to be investigated.

Therefore, this contribution presents, a coupled tire-pavement-model based on Finite Element Method (FEM) and an Arbitrary Langrangian Eulerian (ALE) formulation. Within this ALE formulation, the reference configuration is no more fixed in space (like in Lagrangian formulation) but moves with the rigid body motion of the tire through the space. In case of a steady state rolling tire, the deformation, stress and strain state of the tire and the pavement are then time independent with respect to this moved reference frame, what allows for a numerical efficient computation compared to a transient Lagrangian one [1]. Nevertheless, this model implies a material flow through the FE mesh of tire and pavement, so that the material history has to be followed through the mesh in case of inelastic materials. The presented ALE formulation of the pavement includes an unsplit solution technique for the time derivative of internal variables at inelastic material models, e.g. for asphalt, that utilizes the material history of the previous integration point in case of a regular FE mesh [2].

The staggered tire-pavement-iteration is organized by a program interface that is required, since, the pavement model is included in an inhouse FE code while the tire and contact formulation is computed by a commercial FE code. The program interface manages the transfer of the contact forces and the deformed shape of the contact surface between the two FE codes. In order to avoid an oscillating and slow convergence in case of large pavement deformations (e.g. at soft soil materials), a stabilization scheme is included.

For the investigation of the temperature dependent structural pavement behavior, first, the temperature field induced by climatic influences of a pavement cross-section is computed by a transient thermal FE model. Then, the FE ALE simulation of the coupled tire-pavement-model can be conducted at different points in time, by transferring the associated temperature field of the pavement cross-section to the ALE pavement model. Further, an inelastic material model for asphalt [3] is utilized with temperature dependent material parameter functions, that are fitted based on experimental test results. The numerical results show a significant influence of the temperature field on the structural behavior of pavements under rolling tire loads.

\section{References}

[1] U. Nackenhorst. The ALE-formulation of bodies in rolling contact - theoretical foundations and finite element approach. Computer Methods in Applied Mechanics and Engineering 193 (2004), 4299-4322.

[2] I. Wollny, M. Kaliske. Numerical simulation of pavement structures with inelastic material behavior under rolling tyres based on an arbitrary Lagrangian Eulerian (ALE) formulation. Road Materials and Pavement Design 14 (2013), 71-89.

[3] C. Zopf, M.A. Garcia, M. Kaliske. A Continuum Mechanical Approach to Model Asphalt. International Journal of Pavement Engineering 16 (2015), 105-125. 


\title{
Shock structure for macroscopic multi-temperature model of binary mixtures: comparison with kinetic models
}

\author{
Damir Madjarević \\ Faculty of Technical Sciences, University of Novi Sad, Serbia
}

The present study deals with the shock wave profiles in the macroscopic multi-temperature (MT) model of binary gaseous mixtures. For that purpose we have adopted the hyperbolic model developed within the framework of extended thermodynamics [4]. Recently, we used this model restricted to the simplest case of non-viscous and non-heat-conducting inert gases to compare our results with experimental data in the case of helium and argon mixture [2]. With diffusion as only dissipative mechanism the structure of the source terms is determined using the general principles of extended thermodynamics - invariance of equations with respect to the Galilean transformation and the entropy principle. Diffusivity and relaxation times are taken from kinetic theory for mixture of monatomic gases. Excellent agreement was obtained but with restriction on the shock strength, i.e. Mach number.

In the present study we include viscosity and thermal conductivity to eliminate restriction on parameters. This allows as to compare our results with more sophisticated kinetic solutions which were computed for hypothetic mixtures of gases [1]. Numerical implementation of the MT model is considerably simpler than the one for Boltzmann equations for mixtures or the direct simulation Monte Carlo method (DSMC). Analysis of results is carried out with special regard to the temperature overshoot (TO) of the heavier constituent using a large set of values for parameters. The presence of the extra dissipation (viscosity and thermal conductivity) implicitly introduced atomic diameters of the components in the model, which are not present in inviscid approximation. By varying the parameters it has been found that the temperature overshoot changes non-monotonically with the mass ratio of the constituents, like in the inviscid case in [3]. Also, the dependence of the shock thickness parameter, Mach number and equilibrium concentration on mass ratio is analyzed.

\section{References}

[1] S. Kosuge, K. Aoki, S. Takata. Shock-wave structure for a binary gas mixture: finite-difference analysis of the Boltzmann equation for hard sphere molecules. Eur. J. Mech. B Fluids 17 (2001), 87-126.

[2] D. Madjarević, S. Simić. Shock structure in helium-argon mixture - a comparison of hyperbolic multitemperature model with experiment. EPL (Europhysics Letters) 102(4), (2013), 44002.

[3] D. Madjarević, T. Ruggeri, S. Simić. Shock structure and temperature overshoot in macroscopic multitemperature model of mixtures. Physics of Fluids (1994-present) 26(10), (2014), 106102.

[4] T. Ruggeri, S. Simić. On the hyperbolic system of a mixture of eulerian fluids: a comparison between singleand multi-temperature models. Mathematical Methods in the Applied Sciences 30(7), (2007), 827-849. 


\title{
Efficient Simulation of the Heat Flux Input in Moving Contacts
}

\author{
Marian Partzsch, Michael Beitelschmidt \\ Technische Universität Dresden, Faculty of Mechanical Science and Engineering, \\ Department of Solid Mechanics, Chair of Dynamics and Mechanism Design \\ 01062 Dresden, Germany \\ e-mail: Marian.Partzsch@tu-dresden.de, Michael.Beitelschmidt@tu-dresden.de
}

One approach for increasing the quality of thermal analyses of machine tools is the additional consideration of their structural movement abilities including the related impacts on the temperature field by frictional heating. Therefore, discretely adjusting the position of the moving parts in transient analyses is a very intuitive but computationally expensive way, especially when aiming for the time convergent respectively continuous solution (see [1]). One strategy to reduce the necessary effort of this approach is the usage of a coarse time step size while countering the consequential defects in result quality with computational low cost methods. Based on formerly published work which dealt with the kinematic depended portion of this loss in quality (see [2]), this time we consider the effect of rarely updating a time depended frictional heat flux (NEUMANN-BC) in the contacts of the analyzed, structural variable systems.

We present a method for applying contact loads which are corrected under the consideration of the actual time discretization. This method uses a maximal error heat flux which is pre-determined under the condition of conserving the supplied energy between the continuous and the discrete solution. To ensure this, it is also necessary to appropriately considerate the simulated motion as well as the operating principle of the later used time integration scheme (in our case: backward EULER). For conclusion, we present the performance of the correction for an exemplary motion, executed with ANSYS. It is stated explicitly, that result defects stemming from a coarse discretization in the spatial domain are not considered.

The presented work is embedded within the research activities conducted by the CRC/Transregio 96 .

\section{References}

[1] Partzsch, M. Beitelschmidt, M. Simulation of Pose-and Process-Dependent Machine Tool Models. In Thermoenergetic Design of Machine Tools, Springer, (2015).

[2] Partzsch, M. Beitelschmidt, M. An arithmetic correction for avoiding non-uniform heat input distribution caused by translational motions within time-discrete thermal analyses. In: Proceedings of the ASME 2014 12th Biennial Conference on Engineering Systems Design and Analysis, ASME, (2014). 


\title{
Simulation of ice under mechanical and thermal load
}

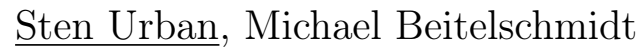 \\ Technische Universität Dresden, Faculty of Mechanical Science and Engineering, \\ Department of Solid Mechanics, Chair of Dynamics and Mechanism Design \\ 01062 Dresden, Germany \\ e-mail: Sten.Urban@tu-dresden.de, Michael.Beitelschmidt@tu-dresden.de
}

\begin{abstract}
The contact physics of the wheel-rail contact of a railway vehicle under presence of water and ice at low temperatures is still not completely understood. Especially for locomotives in cargo operations with a high traction load per wheel and under extreme winter conditions, a significantly higher wheel wear is observed in praxis. For the development of measures to reduce the re-profiling costs exact knowledge about the local contact conditions and the processes in the wheel-rail contact zone are necessary. To investigate these processes a simulation is needed, which is able to calculate the normal and tangential contact, the temperature field and the fluid-structural interaction between wheel and rail at very low temperatures under presence of snow and ice. The effects on the process zone by the behavior of the ice under the contact conditions are a major issue. The typical pressure conditions within the wheel-rail contact are in the range of $0 \mathrm{MPa}$ up to $1500 \mathrm{MPa}$. At this loads and in the range between $-40^{\circ} \mathrm{C}$ and $20^{\circ} \mathrm{C}$, ice can exist in several phases (water (w), ice Ih (1), ice III (3), ice $\mathrm{V}(5)$, ice $\mathrm{VI}(6))$. At a vehicle speed of $100 \mathrm{~km} / \mathrm{h}$ the contact time for a material point traveling through the contact zone is approximately $500 \mu \mathrm{s}$. Publications [1], [2] show that the ice can make rapid phase transitions under similar shock loads. The density anomaly between ice Ih and the other phases leads to volume changes that may be responsible for an additional load on the wheel materials. A numerical simulation of phase changes in the ice under typical conditions at the wheel-rail contact, is presented in this paper. Based on the theory of TshiJov [1], a dimensionless ice probe is loaded by typical wheel-rail contact pressure curves. In a first approximation the ice sample is assumed as an adiabatic system.
\end{abstract}

$$
\begin{aligned}
& E(p, T)=\sum_{i \in \Psi} x_{i} E_{i}(p, T), \quad V(p, T)=\sum_{i \in \Psi} x_{i} V_{i}(p, T), \quad p=p(t) \\
& \frac{\mathrm{d} E}{\mathrm{~d} t}+p \frac{\mathrm{d} V}{\mathrm{~d} t}=0, \quad \frac{\mathrm{d} x_{i}}{\mathrm{~d} t}=F_{i}(p, T) \quad(i=w, 1,3,5,6)
\end{aligned}
$$

The data of thermodynamic properties which are necessary for the calculation, such as the specific volume $V_{i}$ or the specific internal energy $E_{i}$, are determined from the individual equations of phase states. The description of the water phase is based on the specific HeLmHOLTz free energy formulated in IAPWS95 [4]. The ice-phase is described with the aid of the specific GIBBS energy according to [3]. The remaining ice phases are formulated via state equations $V_{i}(p, T)$ by [1].

\section{References}

[1] V. Tchijov, J. Keller, and S. Rodriguez-Romo. Kinetics of Phase Transitions Induced by Shock-Wave Loading in Ice. J. Phys. Chem. B 101 (1997), 6215-6218.

[2] V.E. Chizhov. Investigation of the dynamiv loading of ice. Journal of Applied Mechanics and Technical Physics Vol. 36 No. 6 (1995), 933-938.

[3] R. Feistel, W. Wagner. A New Equation of State for $\mathrm{H}_{2} \mathrm{O}$ Ice Ih. J. Phys. Chem. Ref. Data 35 (2006), 1021-1047.

[4] W. Wagner, A. Pruß. The IAPWS Formultion 95 for the thermodynamic properties of ordinary water substance for general and scientific use. J. Phys. Chem. Ref. Data 31 (2002), 387-535. 


\title{
Reduction Approaches for Thermogasdynamic Lubrication Problems
}

\author{
Marcel Mahner, Andreas Lehn and Bernhard Schweizer \\ Technical University Darmstadt, Department of Mechanical Engineering, \\ Institute of Structural Dynamics
}

In many technical applications with rotating devices (gas turbines, turbochargers, etc.), oil thrust bearings are used to support a shaft in axial direction. To describe the pressure and temperature distribution in the fluid film of oil thrust bearings, the geometry of thrust bearing pads is frequently simplified and the finite slider bearing is used to analyze and optimize thrust bearing pads.

Instead of oil thrust bearings, gas thrust bearings are often used in order to reduce costs, weight and maintenance effort. Gas thrust bearings may advantageously be applied in low-load applications, e.g. in air cycle machines, in gas turbines or in rotor systems for fuel cell applications [1].

The pressure and temperature distribution in gas thrust and oil slider bearings is described by the generalized Reynolds equation according to Dowson [2] and the 3D energy equation. The numerical solution of the generalized Reynolds equation in combination with the 3D energy equation yields a system of nonlinear integrodifferential equations. Solving this system with a finite element approach is related with a high computational effort. Due to the nonlinearity of the problem, also convergence problems are frequently observed.

In this paper, two different approaches are presented in order to reduce the dimension of the governing integro-differential equations system and in order to stabilize the solution process. The approaches are discussed for the thermohydrodynamic and the thermogasdynamic case.

In the first reduction approach, see [3], the temperature in the fluid is averaged across the fluid film yielding an averaged 2D temperature field. Viscosity and density are calculated with the 2D temperature field using appropriate constitutive equations. Due to the averaging approach, the generalized Reynolds equation can be replaced by the classical Reynolds equation. Both, the classical Reynolds equation and the 3D energy equation are discretized by a finite element approach.

In the second approach, Legendre polynomials of different order are used to approximate temperature, density and fluidity across the fluid film, see $[4,5,6]$. The integrals in the generalized Reynolds equation can be calculated approximately using Lobatto point quadrature, see [4]. For calculating the temperature at the Lobatto points, two different weighted residual methods are applied, namely a point collocation approach, see $[5,6]$, and a general Galerkin approach. The reduction of the 3D energy equation and the consistent formulation of the boundary conditions is discussed in detail for the point collocation and the Galerkin method.

The different reduction approaches are compared with respect to numerical efficiency, accuracy and convergence behaviour for gas thrust and oil slider bearings.

\section{References}

[1] K. Radil, S. Howard, B. Dykas, The role of radial clearance on the performance of foil air bearings, Tribology Transactions 45:4 (2002) 485-490.

[2] D. Dowson, A generalized Reynolds equation for fluid-film lubrication, International Journal of Mechanical Sciences 4 (1961) 150-170.

[3] D. Lee, D. Kim, Three-dimensional thermohydrodynamic analysis of Rayleigh step air foil thrust bearings with radial arranged bump foils, Tribology Transactions 54:3 (2011) 432-448.

[4] H. G. Elrod, D. E. Brewe, Thermohydrodynamic analysis for laminar lubricating films, Tech. rep., NASA Technical Memorandum 88845 (1986).

[5] H. G. Elrod, Efficient numerical method for computation of thermohydrodynamics of laminar lubricating films, Tech. rep., NASA Contractor Report 185136 (1989).

[6] L. E. Moraru, Numerical prediction and measurements in the lubrication of aeronautical engine and transmission components, Ph.D. thesis, University of Toledo (2005). 


\title{
A phase-field theory for fracture in porous media
}

\author{
Chenyi Luo, Wolfgang Ehlers \\ Institute of Applied Mechanics (CE), Chair of Continuum Mechanics, University of Stuttgart
}

The investigation of crack phenomena has been an elaborate topic for several decades. From a numerical point of view, discontinuities in structures and the generation of internal boundaries coming along with crack propagation complicate the problem. Since the 1920s, pioneering work has been done by Griffith, Irwin and Barenblatt to depict crack phenomena theoretically. In the last twenty years, a new attempt based on the phase-field theory has been exploited by Bourdin, Ralph Müller and Miehe, whose work proves the feasibility and potential of this method. In 2001, Ingo Müller conceptually extended the phase-field theory to multi-phasic material, such as porous media. However, his work is devoted to a general description of phase-field models and their interpretation in a theoretical mixture approach.

The present contribution proposes a new way to describe crack propagation in porous media. It addresses a biphasic material composed of solid and pore fluid by integrating the phase-field theory into the Theory of Porous Media (TPM). The resulting model is advantageous in terms of providing a detailed description of the evolution of cracks in porous solids and the interaction of cracks with the pore-fluid flow.

The realisation of the transfer starts from assigning the motion function to each material and the formulations of the corresponding balance equations. In order to maintain the thermodynamical consistency, proper constitutive equations and evolution equations are postulated to fulfil the Clausius-Planck inequality for both constituents. Here, a new variable $\phi$ together with its gradient, originated from the phase-field theory, is introduced into the TPM. $\phi$ depicts the accumulation of cracks in the micro-structure of the solid constituent and therefore measures the current state of material whether the solid constituent is intact or not. Accordingly, a new form of the momentum production due to interaction as well as the Helmholtz free energy is suggested in this paper. Manifestly, the new form must be dependent not only on the motion of solid and fluid but also $\phi$ and $\operatorname{grad} \phi$. Finally, a simulation based on a tensile test is performed, where a dramatic change of the flow velocity in the crack zone is observed. 


\title{
Isogeometric Analysis of Mechanically Coupled Phase Segregation in Li-Ion Battery Electrode Particles
}

\author{
Ying Zhao ${ }^{1,2}$, Peter Stein ${ }^{2}$, Bai-Xiang $\mathrm{Xu}^{2}$ \\ ${ }^{1}$ Graduate School of Computational Engineering, TU Darmstadt, Germany \\ ${ }^{2}$ Mechanics of Functional Materials, Department of Materials Science, \\ TU Darmstadt, Germany
}

In pursuit of Lithium-ion batteries with larger capacity, electrode materials such as Silicon, Tin-Oxide have be introduced. However, those electrodes experience irreversible mechanical degradation even after limited charging/discharging cycles due to phase segregation induced high stresses.

In this contribution a mechanically coupled Cahn-Hilliard phase field model in the framework of finite deformation theory is proposed and implemented with isogeometric nonlinear finite element method. In particular, the mechanical properties are assumed to be hyper-elastic and phase-dependent. In the literature $[1,2,3]$ the drifting effect due to hydrostatic stress gradient has been considered. The present model includes also the coupling effect due to phase-dependent mechanical properties. In addition, the influence of the volumeric change due to concentration will be fully considered. The influence of these additional coupling effects on phase segregation behavior such as spinodal points and equilibrium concentrations will be demonstrated. The Isogeometric Analysis is employed for the treatment of high-order problem and the smooth boundary geometry. Firstly, the fourth-order spatial derivative of the concentration is required to account for the diffusive phase interface, and the third-order differential appears in the chemo-mechanical coupling term [4]. The smooth shape functions in IGA allow us to straightforwardly treat the high-order differential equations. Secondly, in spinodal region the whole system is sensitive to infinitesimal composition fluctuation, either due to compositional inhomogeneity or computational error. Approximation failing to give a solution with high precision will lead to incorrect results on phase segregation. Compared to traditional FEM, IGA has its advantages in modeling the curved boundary geometry precisely, e.g. spheres and ellipsoids, even with coarse meshes. Thus the numerical effects of the boundary on phase segregation can be avoided.

This work is supported by the "Excellence Initiative" of the German Federal and State Governments and the Graduate School of Computational Engineering at Technische Universität Darmstadt.

\section{References}

[1] C. Miehe, S. Mauthe and H. Ulmer. Formulation and numerical exploitation of mixed variational principles for coupled problems of Cahn-Hilliard-type and standard diffusion in elastic solids. Int. J. Numer. Meth. Engng. 99 (2014), 737-762

[2] L. Anand. A Cahn-Hilliard-type theory for species diffusion coupled with large elastic-plastic deformations. J. Mech. Phys. Solids. 60 (2012), 1983-2002.

[3] C. V. Di Leo, E. Rejovitzky, L. Anand. A Cahn-Hilliard-type phase-field theory for species diffusion coupled with large elastic deformations: Application to phase-separating Li-ion electrode materials. J. Mech. Phys. Solids. 70 (2014), 1-29.

[4] P. Stein and B.-X. Xu. 3D Isogeometric Analysis of intercalation-induced stresses in Li-ion battery electrode particles. Comput. Methods Appl. Mech. Eng. 268 (2014), 225-244 


\title{
Eulerian large deformation formulation coupled with phase-field
}

\author{
Efim Borukhovich, Ingo Steinbach \\ Interdisciplinary Centre for Advanced Materials Simulation (ICAMS), \\ Ruhr-Universität Bochum, \\ Universitätsstr. 90a, 44789, Bochum, Germany
}

\begin{abstract}
The presented work deals with large deformation modelling using the Eulerian frame of reference. The authors present a scheme that combines a fast Fourier small strain solver [1] with an explicit consideration of geometrical effects as translation, rotation and stretch. While the translation is applied by advection of the system variables, the stretch and rotation are considered by recalculating anisotropic and density like variables by operators derived from the displacement gradient.

The benefits of the presented approach are the straight forward implementation due to the use of space fixed coordinates, that eliminates the need of the remeshing procedure typically employed in the Lagrangian FEM frameworks like Simulia Abaqus [2] and the possibility of combination with other Eulerian modelling frameworks. The developed model is combined with the phase-field framework OpenPhase [3]. In the diffuse interface the problem of the appropriate interpolation procedure of the orientation arises. Therefore, the rotation tensors are transformed to quaternions, that can be linearly interpolated. With that procedure at hands, determination of the effective orientation within the diffuse interface becomes possible. Moreover, the linear interpolation simplifies the advection procedure for the orientation information.
\end{abstract}

\section{References}

[1] S. Y. Hu, L. Q. Chen. A phase-field model for evolving microstructures with strong elastic inhomogeneity. Acta Materialia 49 (2001) 1879-1890.

[2] Dassault Systèmes Simulia Corp. Abaqus 6.11. Providence, RI, USA (2011)

[3] Interdisciplinary Centre for Advanced Materials Simulation (ICAMS); Ruhr University Bochum, Germany. OpenPhase - the open source phase-field simulation package. www.openphase.de 


\title{
A continuummechanical, bi-phasic, two-scale model for thermal driven phase transition during solidification
}

\author{
${ }^{*}$ Lukas Moj, ${ }^{*}$ Tim Ricken, ${ }^{* *}$ Ingo Steinbach \\ ${ }^{*}$ Institute of Mechanics - Structural Analysis - Dynamics, TU Dortmund University \\ ** Materials Research Department, University of Bochum
}

\begin{abstract}
Numerical simulations of hot working processes has gained significant importance for steel making industries in order to improve manufacturing. Hence, a continuum-mechanical, bi-phasic, two-scale model is developed to predict thermally driven phase transition during solidification processes. The solid and liquid physical states are formulated in the framework of enhanced theory of porous media (TPM) [1], by transition rate terms and thermal coupling [2], respectively. Furthermore, finite plasticity superimposed by secondary creep law has been considered to describe realistic material behavior. A linear viscoelastic material law and Darcy's permeability were chosen for the liquid phase material description. The phase transition is formulated by a two-scale approach considering the phase-field model on the micro-scale [3]. Here, a double-well potential consisting of two local minima at completely solid and liquid physical states is utilized. The finite element method and the finite difference method are employed to solve the macroscopic and the microscopic boundary value problem.
\end{abstract}

\section{References}

[1] De Boer, R.: Theory of Porous Media, Springer, New York, 2008.

[2] Bluhm, J.: Modelling of thermoelastic porous solid with different. In: Ehlers, W. and Bluhm J. (Hrsg.): Porous Media: Theory, experiments and numerical applications, Springer, New York, 2002.

[3] Kobayashi, R.: Modeling and numerical simulation of dendritic crystal growth. Physica D: Nonlinear Phenomena, 410-423, 1993. 


\title{
Formability enhancement in deformed AZ31 magnesium sheets via texture evolution: experiments and phase-field study
}

\author{
Reza Darvishi Kamachali, Se-Jong Kim and Ingo Steinbach \\ ICAMS, Ruhr-University Bochum, 44801 Bochum, Germany \\ KIMS, 66 Sangnam-dong, Changwon-si, Gyeongnam 641-831, South Korea
}

Experimental and phase-field studies are conducted to investigate preferential texture modification in AZ31 $\mathrm{Mg}$ sheets. The texture and stress states of materials after initial deformation are studied via electron back scattered diffraction (EBSD) technique before and after annealing. Using the EBSD analysis, a phase-field model is constructed to simulate the texture evolution after initial deformation. Current results suggest that the residual-stresses induced by in-plane compression is the main driving force for recrystallization and grain growth. The inhomogeneous stress distribution conducts preferential growth of $\left\{\begin{array}{llll}2 & \overline{1} & \overline{1} & 0\end{array}\right\}$ texture, which are at lowest stress state, at the expense of initial basal texture. Limited mobility of twin boundaries changes the mixture of textures but the non-basal textures are still preferred. The formability tests confirm a significant enhancement of the final product compared to as-received sheets. 


\title{
Isogeometric Analysis of size-dependent behavior of Li-ion battery electrode particles
}

\author{
Peter Stein, Bai-Xiang Xu \\ Mechanics of functional materials Division, Technische Universität Darmstadt, \\ Jovanka-Bontschits-Str. 2, D-64287 Darmstadt, Germany
}

Lithium-ion batteries find wide application as power source for mobile devices such as cellphones and tablet PCs. Unfortunately, they suffer from gradual capacity fade over their lifetime. Besides electrochemical effects, mechanical degradation has been identified as one of the sources of this deterioration. The repeated intercalation and extraction of Li ions results in high stress levels in the electrodes, leading to delamination of the electrodes' structure and to fracture of individual particles. However, these particles show a distinct size effect and it has been shown that nanostructured electrodes exhibit a higher resilience against diffusion-induced stresses and mechanical degradation.

We have recently developed a coupled three-dimensional constitutive model for bulk diffusion [1], aiming to gain a better understanding of the mechanical processes taking place in the electrodes. In this model, the diffusion is enhanced by a drift term based on the gradient of the stress field. By virtue of the drift term, the model produces enhanced diffusion rates due to mechanical effects which, in turn, induce a stress relaxation effect and lower stress levels in the electrode material. This term requires basis functions of $C^{1}$-continuity and is often neglected in Finite Element-implementations which provide only a $C^{0}$-continuous basis. Instead of using a mixed-variational formulation, our Finite Element-implementation is based on the concept of Isogeometric Analysis (IGA) [2] which allows for a straightforward and stable discretization of the weak form.

This talk is concerned with the representation of size effects in our model. We achieve this through two approaches, namely gradient elasticity [3] and surface stresses. In particular the latter have been shown to allow for a faithful representation of nanoscale materials [4]. We describe the mathematical models and their implementation by means of IGA. Using a single electrode particle, we discuss the influence of particle size, charge rates, material parameters, and shape parameters on the stress levels in a single electrode particle.

\section{References}

[1] P. Stein and B. Xu. 3D Isogeometric Analysis of intercalation-induced stresses in Li-ion battery electrode particles. Comput. Methods Appl. Mech. Engrg. 268 (2014), 225-244.

[2] T. J. R. Hughes, J. A. Cottrell, and Y. Bazilevs. Isogeometric analysis: CAD, finite elements, NURBS, exact geometry and mesh refinement. Comput. Methods Appl. Mech. Engrg. 194 (2005), 4135-4195.

[3] R. D. Mindlin. Micro-structure in linear elasticity. Arch. Rational Mech. Anal. 16(1) (1964), 51-78.

[4] Dietmar Gross, Ralf Müller, Michael Müller, Bai-Xiang Xu, Karsten Albe. On the origin of inhomogeneous stress and strain distributions in single-crystalline metallic nanoparticles. Int. J. Mater. Res. 102(6) (2011), $743-747$. 


\title{
Variational Principles and Stability of Diffusion in Hydrogels
}

\author{
Aref Nateghi, Christian Miehe, Steffen Mauthe \\ Institute of Applied Mechanics (CE) \\ University of Stuttgart \\ Pfaffenwaldring 7, 70569 Stuttgart, Germany \\ aref.nateghi@mechbau.uni-stuttgart.de
}

Due to their wide range of applications, a precise knowledge of the mechanical behavior of hydrogels is essential. The most important mechanical property attributed to hydrogels is swelling due to diffusion of fluid molecules into the polymer network. Large deformations caused by swelling can lead to instabilities. This stability issue underlines the importance of a robust numerical treatment of the coupled diffusion-deformation problem.

This work shows that the diffusion-deformation problem can be based upon a variational principle, which determines the coupled chemo-mechanical response of hydrogels. The proposed formulation requires only two constitutive functions, the free energy function and a dissipation potential function and thus provides a new, canonically compact modeling framework. For this purpose we start by constructing rate-type potentials for the coupled chemo-mechanical evolution problem. These potentials can be exploited in the numerical implementation by construction of time-discrete incremental potentials, which fully determine the update equations given by typical time stepping procedures. For the space discretization of the problem finite element method is used. This provides the most fundamental approach to the chemo-mechanical problem under consideration, leading to symmetric algebraic systems of equations for iterative update procedures.

A well known issue in the treatment of coupled problems is the numerical stability due to the saddlepoint structure of the variational principle, which is connected to the Babuška-Brezzi or LBB condition. We propose a new minimization principle for the coupled diffusion-deformation problem by applying a Legendre transformation on the introduced potential. With this pure minimization problem, no requirements have to be fulfilled for the numerical ansatz spaces, leading to a unconditionally stable formulation. Furthermore, when dealing with instabilities, using pure minimization principles is more favorable. We demonstrate the capabilities of our model and its performance by means of representative numerical examples with complex instability issues in two and three dimensions. 


\title{
Modeling of electrodynamic-mechanical coupling phenomena in smart magnetoelectroelastic materials
}

\author{
Eugen Merkel, Andreas Ricoeur \\ Institute of Mechanics, University of Kassel, 34125 Kassel, Germany
}

The coupling of electric, magnetic and mechanical phenomena may have various reasons. The famous Maxwell equations of electrodynamics describe the interaction of transient magnetic and electric fields. On the constitutive level of dielectric materials, coupling mechanisms are manyfold comprising piezoelectric, magnetostrictive or magnetoelectric effects. Electromagnetically induced specific forces acting at the boundary and within the domain of a dielectric body are, within a continuum mechanics framework, commonly denoted as Maxwell stresses. In transient electromagnetic fields, the Poynting vector gives another contribution to mechanical stresses.

First, a system of transient partial differential equations is presented, comprehensively describing all coupling phenomena. Introducing scalar and vector potentials for the electromagnetic fields and representing the mechanical strain by displacement fields, seven coupled differential equations govern the boundary value problem, accounting for linear constitutive equations of magnetoelectroelasticity. To reduce the effort of numerical solution, the system of equations is partly decoupled applying generalized forms of Coulomb and Lorenz gauge transformations [1, 2], introducing appropriate scalar gauge functions [3]. A weak formulation is given to establish a basis for a finite element solution. The influence of constitutive magnetoelectric coupling on electromagnetic wave propagation is finally demonstrated with a simple one-dimensional example.

\section{References}

[1] L. Landau, E. Lifshitz. The classical theory of fields. Elsevier Butterworth Heinemann (2007).

[2] J.D. Jackson. From Lorenz to Coulomb and other explicit gauge transformations. Am. J. Physics 70 (2002), 917-928.

[3] E. Merkel. Schwache Formulierung gekoppelter elektrodynamisch-elastischer Randwertaufgaben in multifunktionalen Festkörpern. Masterarbeit Institut für Mechanik, Universität Kassel (2014). 


\title{
Modeling of the Crosstalk Phenomenon by the Non-Stationary Maxwell Equations Coupled with the Circuit Equations
}

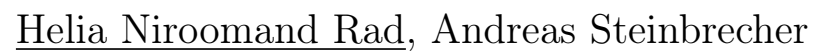 \\ Technical University of Berlin
}

In this work, we propose a new modeling approach in a general framework in order to describe the crosstalk phenomenon in an extended way. We investigate the phenomenon within electro-magnetic systems, and in particular, where electrical elements such as inductors, capacitors, and resistors are tightly located within electrical circuits. Crosstalk in electro-magnetic systems, loosely speaking, refers to the undesirable signal coupling where the electrical elements are communicating with each other. We model the crosstalk phenomenon by bilateral coupling of two sets of differential equations where the main set is the set of non-stationary Maxwell equations, and the second set contain the circuit equations formulated in the framework of modified nodal analysis.

For the purpose of modeling the electro-magnetic phenomena where the dynamical behavior of the electrical circuit is also involved, virtually the non-stationary Maxwell equations cannot fully explain the phenomena. Therefore, as a remedy to model the crosstalk we consider the circuit equations in addition to the Maxwell equations. Considering these two sets of equations as input-output systems, the bilateral coupling approach connects the output of one system to the input of the other system and conversely, via coupling and re-coupling relations. These relations, which are in principle physical constitutive relations, are introduced in our model by suitable operator structures. The coupling of these two sets lead to a set of partial differential-algebraic equations modeling the crosstalk phenomenon. In addition to the modeling, a numerical example is also simulated where the crosstalk is considered only between two inductors within a simple electrical circuit .

\section{References}

[1] J. D. Jackson. Classical electrodynamics, Wiley, 1998.

[2] P. Monk. Finite element methods for Maxwell's Equations, Oxford University Press, New York, 2003.

[3] A. Steinbrecher, T. Stykel. Model order reduction of nonlinear circuit equations, Int. J. Circ. Theor. App., 2013. 


\title{
Velocity vector field optimization in bioventing
}

\author{
Filippo Notarnicola \\ Istituto per le Applicazioni del Calcolo, I.A.C. - CNR \\ via Amendola 122-d, 70126 Bari (Italy) \\ e-mail: f.notarnicola@ba.iac.cnr.it
}

Bioventing is a technology used to remove some kinds of pollutants from subsoil and it is based on the capability of some bacteria species to biodegrade contaminants. The biochemical reaction requires, among other things, oxygen and, therefore, oxygen is inflated in the subsoil by wells.

The mathematical model describes the movement of the different fluids which are present in the subsoil - air, water, pollutants, oxygen and so on - and the bacteria population dynamics. The presence of source reactive terms in the continuity equations allows the contaminant biodegradation to be described.

The design of a subsoil decontamination intervention concerns bioavailability problems and, in particular, the oxygen concentration. The optimization of the subsoil oxygen velocity field is, therefore, required in the polluted area in order to enhance the biodegradation phenomenon. The goal is obtained by an appropriate choice of the well positions and of the well air or oxygen inflating rates.

In mathematical terms, the design problem consists of obtaining the decontamination of the subsoil in correspondence of a minimal value of an objective function, such as decontamination time or costs, by acting on some control variables which, in our case, are the well positions and inflating rates. The control variables are subject to some physical limitations: the number of the wells and the total air or oxygen flow rate are limited resources.

The state variables of the system are subject to be a solution of the mathematical model, that is they are solutions of the partial differential equations describing the decontamination phenomenon.

\section{References}

[1] Z. Chen, G. Huan, Y. Ma. Computational Methods for Multiphase Flows in Porous Media. SIAM, Philadelphia (PA) USA, (2006).

[2] J. T. Cookson. Bioremediation Engineering: design and application. McGraw-Hill, New York, (1995).

[3] H. H. Gerke, U. Hornung, Y. Kelanemer, M. Slodicka, S. Schumacher. Optimal Control of Soil Venting: Mathematical Modeling and Applications. Birkhauser, Basel, (1999).

[4] F. Notarnicola. Mathematical Formulation of Bioventing Optimal Design Strategies. Manuscript submitted for publication, (2014).

[5] J.A. Tindall, J.R. Kunkel, D.E. Anderson. Unsaturated Zone Hydrology. Prentice Hall, Upper Side River (NJ) USA, (1999). 


\title{
A consitutive and damage model for high cycle fatigue of tetragonal ferroelectrics
}

\author{
Stephan Lange, Andreas Ricoeur \\ Institute of Mechanics, Chair of Engineering Mechanics / Continuum Mechanics, \\ University of Kassel, Moenchebergstr. 7, 34109 Kassel, Germany
}

Ferroelectric materials, such as barium titanate, are widely used in smart structures and devices as actuators, sensors etc. To investigate the material behavior, a condensed model for ferroelectric solids with tetragonal unit cells is presented. The approach is microelectromechanically and physically motivated, considering discrete switching processes on the level of unit cells and quasi-continuous evolution of inelastic fields on the domain wall level. To calculate multiple grain interactions, an averaging technique is applied [1, 2]. Hysteresis loops are simulated for a pure electric and an electromechanical loading to demonstrate the influence of a compression preload on the poling and stress-strain behavior. Further, residual stresses are calculated as a result of switching processes and interaction between crystallits. To study the high cycle fatigue damage and to predict lifetime of ferroelectric devices, an accumulation model is proposed based on the growth of microcracks [2]. Here, the Paris law [3] is applied to calculate fatigue crack growth rates. The lifetime is calculated considering different parameters, e.g. initial micro crack lengths, material parameters, loading cases etc. The simulations agree with experimental findings, where actuation efficiency and structural integrity come out to be opposing properties.

\section{References}

[1] S. Lange, A. Ricoeur. A condensed microelectromechanical approach for modeling tetragonal ferroelectrics. Int. J. Solids. Struct. (2014), http://dx.doi.org/10.1016/j.ijsolstr.2014.11.004.

[2] S. Lange, A. Ricoeur. A condensed microelectromechanical constitutive and damage model for tetragonal ferroelectrics. Proc. Appl. Math. Mech. (2014), in press.

[3] P. Paris, F. Erdogan. A critical analysis of crack propagation laws. J, Basic Eng. 85 (1963), 528-534. 


\title{
Phase field simulation on mechanically coupled switching dynamics in nanomagnets
}

\author{
$\underline{\text { Min } \mathrm{Yi}^{1}}{ }^{1,2}$, Bai-Xiang $\mathrm{Xu}^{1}$ \\ ${ }^{1}$ Mechanics of Functional Materials Division, Institute of Materials Science, \\ Technische Universität Darmstadt, Darmstadt 64287, Germany \\ 2 School of Aeronautic Science and Engineering, \\ Beihang University (BUAA), Beijing 100191, China
}

Miniaturization of the magnetic devices is highly recommended for achieving large areal density up to 1 Tbit/in ${ }^{2}$ in magnetic data storage. Recently, based on the bit patterned media (BPM) concept, nanomagnets have show huge prospect in this aspect. For the BPM concept, the nanomagnets-based logic and memory applications require a deterministic $180^{\circ}$ switching of the magnetization, i.e. the nanomagnet changes its bit state from 0 to 1 , or vice versa. Thus, the strategy for switching the magnetization state in nanomagnets is indispensable. Presently, the magnetic field and spin-polarized current are two available ways. In this contribution, we will propose a mechanical strategy, i.e. controlling the switching dynamics in nanomagnets by the magneto-mechanical coupling. This strategy is demonstrated by a constraint-free and mechanically coupled phase field model which was initially developed for simulating domain evolution in ferromagnetics [1].

Taking single-crystal Cobalt nanomagnet as the model material, we find that due to the magneto-mechanical coupling, the mechanically induced switching dynamics in nanomagnets has two regimes, i.e. coherent switching with homogeneous magnetization distribution and incoherent switching with inhomogeneous magnetization distribution. The transition boundary of these two regimes can be identified in terms of the geometry of the nanomagnets. Coherent switching happens in the small nanomagnets while incoherent switching in the large nanomagnets. In the coherent switching which is very interesting for the BPM concept, only a deterministic $90^{\circ}$ can be achieved by a constant mechanical loading. But we show that by using the dynamic nature and overrun behavior of the magnetization, a deterministic $180^{\circ}$ switching can happen if the mechanical loading is removed once the magnetization rotates to the achievable largest angle. The dependence of the $180^{\circ}$ switching time on the mechanical loading magnitude is furthermore investigated. We also studied the switching dynamics under the combination of magnetic filed and mechanical loading. These results have shed light on the design of an optimum mechanically driven nanomagnets-based logic and memory devices.

\section{References}

[1] M. Yi, B.-X. Xu. A constraint-free phase field model for ferromagnetic domain evolution. Proc. R. Soc. A-Math. Phys. Eng. Sci. 470 (2014), 20140517(1-19). 


\title{
The electrocaloric effect in ferroelectrics: nonlinear modeling and simulation
}

\author{
Marius Wingen, Andreas Ricoeur \\ Institute of Mechanics, Chair of Engineering Mechanics / Continuum Mechanics, \\ University of Kassel, Moenchebergstr. 7, 34109 Kassel, Germany
}

Due to their special electromechanical properties, nowadays ferroelectric materials are widely used in many technical applications, mostly as actuators or sensors. Advantages compared to other smart devices are the extremely fast reaction times in a range of $\mu m-m s$ and large actuation forces. Influences of temperature and heat flux due to electrocaloric and thermomechanical effects are mostly neglected in models, although they may have a non-negligible impact on issues like phase transitions, domain wall motion or reliability and lifetime. In particular, the cooling due to domain reorientation in ferroelectrics is under current investigation mostly by physicists, who observed partly high temperature changes due to electric fields [1].

In this paper, the theoretical background of a micromechanically and physically based constitutive model is presented. In addition to the nonlinear ferroelectric behavior, the model considers the mutual nonlinear coupling of thermal and electromechanical fields. Results are presented in terms of analytical solutions for one-dimensional problems and single domain configurations. First steps of a finite element implementation are also demonstrated by implementing a linear mutual coupling between temperature and electromechanical fields into an ABAQUS user element.

\section{References}

[1] Yang Liu, I.C. Infantel, Xiaojie Lou, D.C. Lupascu, B. Dkhil. Giant mechanically-mediated electrocaloric effect in ultrathin ferroelectric capacitors at room temperature. Applied Physics Letters 104, 012907 (2014). 


\title{
Nonlinear numerical simulation of ferroelectric-ferromagnetic multifunctional composites
}

\author{
Artjom Avakian and Andreas Ricoeur \\ University of Kassel
}

The coupling of magnetic and electric fields due to the constitutive behavior of a material is commonly denoted as magnetoelectric (ME) effect. The latter is only observed in a few crystal classes exhibiting a very weak coupling, mostly at low temperatures, which can hardly be exploited for technical applications. Much larger coupling coefficients are obtained at room temperature in composite materials with ferroelectric and ferromagnetic constituents. The ME-effect is then induced by the strain of matrix and inclusions converting electrical and magnetic energies based on the piezoelectric and magnetostrictive effects.

In this paper, the theoretical background of nonlinear constitutive multifield behavior as well as the Finite Element (FE) implementation are presented. Nonlinear material models describing the magneto-ferroelectric or electro-ferromagnetic behaviors are presented. Both physically and phenomenologically motivated constitutive models have been developed for the numerical calculation of the nonlinear magnetostrictive and ferroelectric behaviors. On this basis, the polarization in the ferroelectric and magnetization in the ferromagnetic respectively magnetostrictive phases are simulated and the resulting effects analyzed. Numerical simulations focus on the prediction of local domain orientations and residual stress going along with the poling process, thus supplying information on favorable electric-magnetic loading sequences. Goals are to improve the efficiency of ME coupling and to reduce damage associated with the poling process. Further, the developed tools enable the prediction of the electromagnetomechanical properties of smart multiferroic composites and supply useful means for their optimization. The resulting final state of a poling simulation can be implemented as a starting condition for approximate linear simulations and multifield homogenization procedures. 


\title{
Intrinsic symmetries in constitutive modeling of magneto-elastic materials
}

\author{
Igor Dobovšek \\ University of Ljubljana \\ Faculty of Mathematics and Physics \\ Jadranska 19, Ljubljana 1000, Slovenia \\ e-mail: igor.dobovsek@fmf.uni-lj.si
}

We consider a class of non-dissipative materials whose constitutive equations are derived from a suitably constructed thermodynamic potential function. First, we establish an appropriate Helmholtz energy relation in terms of temperature and magnetization. Then by invoking the Legendre transformation we introduce the Gibbs energy relation as a function of stress, strain, magnetic field, magnetization, and temperature, and by minimization with respect to the chosen subset of independent variables seek the condition for local minimum in strains and magnetization. Derivation is essentially three dimensional based on truncated polynomial energy relations. The chosen form of the free energy function leads to the linear elastic and nonlinear ferromagnetic coupling where non-linearity emerges in terms associated with the strength of magnetization. In principle, the adopted order of non-linearity can accommodate nonlinear behavior of ferromagnet in the region of hysteresis with sufficient level of accuracy in qualitative and quantitative sense. 


\title{
Correction of Characteristics of Subsystems of Torsionally Vibrating Complex Mechatronic Systems as Introduction to Solution of Their Inverse Task
}

\author{
Andrzej Buchacz \\ Silesian University of Technology, Faculty of Mechanical Engineering, \\ Institute of Engineering Processes Automation and Integrated Manufacturing Systems
}

\begin{abstract}
The mechatronic system composed from many mechanical subsystems having the same length and variable cross section, loaded by the focused moment was analysed e.g. $[1,2,3,4,5]$. The main subject of deliberation was to determine the flexibility of the mechanic system with constant cross section using the exact and approximate methods. Next the method comparison and the correction of approximate methods where made. As far as the mechatronic system flexibility determinations concerned the approximate methods has been chosen. Two cases of attachment the systems were considered. The research of subsystems establishes the foundation to complex systems analysis with cascade structure. Analysing the diagrams of characteristics of confirmed system it has been determined that in case of approximate method the resonance frequencies cover with those which have been determined with exact method. However the values of the characteristic in other areas are different. Therefore there is the mistake of approximate method, which in case of studying the single systems does not have any influence because in resonance areas the characteristic values of the system approach to the infinity. However the difference in values of flexibility within two methods has the great influence on the result of complex systems. That is why it was necessary to correct the results of approximate method.
\end{abstract}

Acknowledgements: This work has been conducted as a part of research project PBS2/A6/17/2013 supported by the National Centre for Research and Development in 2013-2016.

\section{References}

[1] A. Buchacz. Influence of a Piezolectric on Characteristics of Vibrating Mechatronical System. Journal of Achievements in Materials and Manufacturing Engineering. Vol. 17, Issue 1-2. International OCOSCO World Press. July-August 2006, 229-232.

[2] A. Buchacz. Calculation of Characterisics of Torsionally Vibrating Mechatronic System. Journal of Achievements in Materials and Manufacturing Engineering, Vol. 20, Issue 1-2, International OCOSCO World Press. January-February (2007), 327-330.

[3] A. Buchacz. Characteristics of Discrete-Continuous Flexibly Vibrating Mechatronic System. Journal of Achievements in Materials and Manufacturing Engineering, International OCOSCO World Press. Vol. 28. Issue 1. May (2008), 43-46.

[4] A. Buchacz. Orthogonalization Method of Analysis of Mechanical and Mechatronic Systems. LES PROBLĖMES DU TECHNOSHĖRE ET DE LA FORMATION DES CADRES D'INGÉNIEURS CONTEMPORAINS. Recueil des exposés des participants du III Séminaire international scientifique et méthodique. Novembre 2009 à Sousse (Tunisie), Donetsk (2009), 85-88.

[5] A. Buchacz, Comparision of Characteristics of Subsystems and Systems as introduction to Synthesis of Torsionally Vibrating Mechatronic Systems. LES PROBLÈMES DU TECHNOSHÈRE ET DE LA FORMATION DES CADRES D'INGÉNIEURS CONTEMPORAINS. Recueil des exposés des participants du IV Séminaire international scientifique et méthodique, Novembre 2010 à Hammamet (Tunisie), Donetsk (2010), $10-13$. 


\title{
Modeling a Halfspace with Tunnel using a Coupled Integral Transform Method - Finite Element Method Approach
}

\author{
Manuela Hackenberg ${ }^{1}$, Gerhard Müller ${ }^{1}$ \\ ${ }^{1}$ Chair of Structural Mechanics, Technische Universität München, \\ Arcisstr. 21, 80333 München, Germany
}

Moving loads in an underground tunnel lead to oscillations inside the soil which can cause annoyances or health problems for humans. Moreover, sensitive machines can be disturbed by these oscillations. Due to urbanization and permanent advancements in machine construction, the effects are still increasing. Therefore, an accurate modeling of the system halfspace with tunnel is required.

In this contribution, a coupled Integral Transform Method (ITM) - Finite Element Method (FEM) approach is presented. The soil as first substructure is described analytically using ITM which is appropriate for the description of the infinite extension of the medium. A solution for a halfspace with cylindrical cavity is derived as a superposition of two fundamental systems. The degrees of freedom on the surface of the cavity inside the halfspace are coupled to the degrees of freedom of a finite element mesh. Thus, the complex geometry of the emission system of the tunnel as second substructure can be modeled using FEM.

The solution of a halfspace with cylindrical cavity is obtained superposing the fundamental systems of a halfspace and a fullspace with cylindrical cavity. Both fundamental systems are solved in a threefold Fourier transformed domain. The Lamé equation as basic equation in elastodynamics consists of three coupled, partial differential equations. First, a decoupling is performed using a Helmholtz approach. Afterward, a transformation from partial into ordinary differential equations is done applying a threefold Fourier transformation [2]. Depending on the coordinate system, different Fourier transformations are carried out. The halfspace which is modeled in Cartesian coordinates, is transformed from time into frequency domain and from the spatial coordinates in the plane of the halfspace $x$ and $y$ into the respective wavenumbers $k_{x}$ and $k_{y}$. The fullspace with cylindrical cavity with its cylindrical coordinates is also transformed into the frequency domain and into the wavenumber domain regarding the longitudinal coordinate of the cavity $x$. As a third transformation, a Fourier series expansion regarding the circumferential angle is applied. The two fundamental systems are superposed with the boundary conditions on the external surfaces [1]. As both calculations are carried out in dependency on the frequency $\omega$ and wavenumber $k_{x}$, a quasi-static, two-dimensional calculation is done for each combination of $\omega$ and $k_{x}$.

The finite element mesh for the modeling of the tunnel geometry is also defined in the wavenumber-frequency domain. Therefore, also quasi-static, two-dimensional computations are carried out. A two-dimensional mesh with four-node elements is modeled with three degrees of freedom at each node. The FEM formulation is adapted to the wavenumber-frequency domain and the stiffness matrix is transformed on the same basis as the cylindrical coupling surface of the ITM.

The two substructures are coupled using the substructure technique and the coupling conditions on the cylindrical surface. Thus, the complete system of halfspace with tunnel can be modeled and the response due to loads on the halfspace surface or inside the finite element mesh can be calculated. The effects of mitigation measures e. g. of a mass-spring system inside the tunnel can be assessed and different installation situations can be compared.

\section{References}

[1] G. Frühe. Überlagerung von Grundlösungen in der Elastodynamik zur Behandlung der dynamischen TunnelBoden-Bauwerk-Interaktion. Shaker-Verlag. (2010), 60-65.

[2] G. Müller. Ein Verfahren zur Erfassung der Fundament-Boden-Wechselwirkungen unter Einwirkung periodischer Lasten. Mitteilungen aus dem Institut für Bauingenieurwesen. (1989), 14-17. 


\title{
Fluid-Porous-Media Interaction:
}

\section{A Decoupled Solution Algorithm via Localised Lagrange Multipliers}

\author{
Seyedmohammad Zinatbakhsh, David Koch, Wolfgang Ehlers \\ Institute of Applied Mechanics (CE), Chair of Continuum Mechanics \\ University of Stuttgart, Germany
}

The fluid-porous-media interaction (FPMI) refers to a surface interaction among several non-overlapping subsystems, composed of either a bulk fluid or a porous medium. This could be the interaction of blood with a blood vessel wall, a body of water with an earth-dam structure, or acoustic waves with acoustic panels used in soundproofing, etc. These are highly coupled phenomena inheriting different coupling mechanisms which take place within the interacting subdomains as well as across common boundaries. Consequently, the mathematical models of such phenomena also consist of sets of coupled differential equations, which are commonly solved numerically, following a monolithic or a decoupled approach. Here, the focus is on the latter.

The design of an algorithm for the decoupled solution of the fluid-porous-media-interaction problem can be advantageous in several ways. First and foremost, the process of decoupling breaks down the problem into several subproblems, which can be solved efficiently employing specialised fluid and porous-media solvers. Furthermore, solution of several decoupled subproblems instead of one highly coupled problem may be more economical in terms of computational costs. This is especially the case if the scheme permits parallel treatment of the produced subproblems.

Here, motivated by the idea of the method of localised Lagrange multipliers, a partitioned solution algorithm for the problem of surface interaction between an incompressible and inviscid fluid with a saturated biphasic porous medium with intrinsically incompressible and inert constituents is proposed. This method facilitates spatial partitioning of the problem and a parallel solution of the interacting components, and allows for using tailored solvers optimised for each subproblem. Moreover, using the method of perturbed Lagrange multipliers within the subsystems, the pressure fields are eliminated. It reduces in the size of the problem, simplifies the formulation of the interface constraints and also removes the burden of using mixed finite elements. It is known that the decoupled solution of a coupled problem may lead to conditional stability of the produced numerical results. In this regard, considering the 1-dimensional (1-d) version of the equations, a stability analysis of the proposed solution method is performed, and the unconditional stability of the partitioned solution scheme is shown. Solving 1- and 2-d numerical benchmark examples, the applicability of the proposed scheme is demonstrated. 


\title{
Simulation of vortex-induced oscillations within a shear-thinning liquid
}

\author{
Janto Gundlach, Alexander Schlosser and Olaf Wünsch \\ Institute of Mechanics, Chair of Fluid Mechanics, University of Kassel, Germany.
}

The present study discusses the influence of a viscous shear-thinning fluid flow on the vibration behavior of an elastic bar which is attached behind a rigid obstacle. The problem geometry is defined on the basis of the well-known FSI benchmark described in [1]. Owing to the asymmetric position of the obstacle within a laminar channel flow, a periodical Kármán vortex street occurs and the continuous eddy shedding stimulates the bar to the observed oscillations. In contrast to [1], in this work the fluid corresponds to a model of the Carreau-Yasuda type (cf. [2]). The model represents an explicit equation to determine the local shear viscosity in dependance of the deformation rate. It is well-suited to reproduce the drawdown of viscosity, for instance, taking place in polymer melts. All parameters of the model are chosen according to a test liquid that has been specified in simulations of vortex streets in [3] with non-Newtonian matter.

In the current investigation above illustrated setting is discretized and calculated in the framework of OpenFOAM@: foam-extend-3.1. In order to accomplish the coupling between the liquid and solid region, a partitioned FSI approach is used with the IQN-ILS algorithm (e.g. [4]) serving as coupling scheme. From the numerical solutions different characteristical quantities, which are suitable to describe the interaction, such as the periodic time and the oscillation amplitude, are determined. In a parameter study the level of shearthinning and the shape of the obstacle are changed. The solutions show remarkable deviations compared to results obtained with a Newtonian fluid. These differences embody the additional nonlinearity introduced by the material model.

\section{References}

[1] S. Turek, J. Hron. Proposal for numerical benchmarking of fluid-structure interaction between an elastic object and laminar incompressible flow. Ergebnisberichte des Instituts für Angewandte Mathematik, Fakultät für Mathematik, TU Dortmund, 312 (2006).

[2] G. Böhme. Strömungsmechanik nichtnewtonscher Fluide. Leitfäden der angewandten Mathematik und Mechanik, Teubner-Verlag, 2nd ed. (2000).

[3] C. Lund. Ein Verfahren zur numerischen Simulation instationärer Strömungen mit nichtlinear-viskosen Fließeigenschaften. Fortschrittberichte VDI 7:344 (1998).

[4] J. Degroote et al. Partitioned simulation of the interaction between an elastic structure and free surface flow. Comput. Methods Appl. Mech. Engrg. 199 (2010), 2085-2098. 


\title{
A numerical approach to the dynamics analysis of mooring lines
}

\author{
$\underline{\text { Giovanni Stabile }}^{1}$, Claudio Borri ${ }^{1}$, and Hermann G. Matthies ${ }^{2}$ \\ ${ }^{1}$ Dep. of Civil and Environmental Engineering, University of Florence, Via di Santa Marta 3, \\ 50139 Florence, Italy \\ ${ }^{2}$ Institute of Scientific Computing, Technische Universität Braunschweig, Hans-Sommer-Str. \\ 65, D-38092 Braunschweig, Germany
}

\begin{abstract}
Mooring lines in deep water are exposed to the hydrodynamic forces due to the relative motion between the water and the cables. The response may involve complex phenomena such as vortex induced vibration, unsteady lock-in, dual resonance and travelling waves response [1]. This contribution present a novel numerical approach to capture the non-linear behaviour of mooring lines in deep water. A possible way to capture the complex behaviour of a marine cable is using a fluid structure interaction (FSI) solver coupling together a computational fluid dynamic solver (CFD) with a computational structural dynamic (CSD) solver. This approach, with nowadays computational resources, is feasible only for a limited size of the domain. The present work aims to study the local behaviour of the cable using a small scale FSI model in order to gather computational data that may be used to create a reduced order model suitable to solver the full scale problem. The FSI problem is solved with a partitioned approach coupling together a finite volume method (FVM) solver with a finite element method (FEM) solver through the approach of software components [2]. The importance of several parameters, such as the size of the small scale problem, degrees of freedom taken into account, and the characteristics of the imposed motion in terms of amplitude and frequency, is assessed.
\end{abstract}

\section{References}

[1] Wu, Xiaodong, Fei Ge, and Youshi Hong. A review of recent studies on vortex-induced vibrations of long slender cylinders. Journal of Fluids and Structures 28 (2012), 292-308

[2] Kassiotis, Christophe, et al. Nonlinear fluid-structure interaction problem. Part I: implicit partitioned algorithm, nonlinear stability proof and validation examples. Computational Mechanics 47.3 (2011), 305-323. 


\title{
Critical velocities for flow induced vibrations of a U-shaped belt
}

\author{
Johannes Strecha, Herbert Steinrück \\ Institute of Fluid Mechanics and Heat Transfer, Vienna University of Technology
}

A prism with U-shaped cross-section in cross flow offers interesting features to study, in particular for intermediate aspect ratios: frontal height of the $\mathrm{U}$ over its length, $B / H \approx 4 \ldots 5$. In engineering applications, slender structures with U-shaped cross section are sometimes encountered in bridge decks of long-span suspension bridges or conveyor belts for bulk materials. Considered from a scholarly point of view, the U-profile has aspects of the rectangular prism and the H-prism as well. It has an unbroken side (rectangular prism) as well as a cavity (H-prism) but is asymmetric and therefore presents an extension to these extensively researched configurations.

In the present study we investigate the flow around a stationary U-profile and excitation mechanisms for flow induced two-degree of freedom (2DOF) vibrations - vertical motion (heave) and rotation about the long axis of the belt (pitch). We consider the case where the respective eigenfrequencies $f_{\mathrm{y}}$ and $f_{\varphi}$ are equal, $f_{\mathrm{y}} / f_{\varphi}=1$. The flow field is studied with the help of unsteady RANS simulations using the commercial CFD solver ANSYS Fluent, where the belt is modelled as a two-dimensional U-profile. Wind tunnel results for validation comprise Particle Image Velocimetry (PIV) experiments investigating the flow around a stationary model, free 2DOF vibration tests with a rigid section model, and free vibration tests with a tensioned belt.

The presentation will focus on two excitation mechanisms for flow induced vibrations, occurring at two different dimensionless reduced velocities $U^{*}=u_{\infty} / H f_{0}$, i.e. the far-field flow velocity $u_{\infty}$ made dimensionless by the product of frontal height $H$ and eigenfrequency $f_{0}$.

The most interesting vibrations occur at low reduced velocities, $U^{*} \approx 14$ due to vortex formation patterns inherent to the U-profile. Normally, vortex induced vibrations would be expected at even lower reduced velocities, $U^{*}=7 \ldots 10$ [1]. Naturally, vortices form in the wake of the U-profile, similar to the von Kármán vortices. Additional vortices can form right behind the windward flange of the U. They were observed in the simulations and were confirmed with good agreement by PIV wind tunnel experiments. These vortices can excite torsional vibrations with a very large amplitude. This was mainly observed by means of simulation, and suggested by free vibration experiments with a tensioned belt.

Flutter vibrations at large reduced velocities $\left(U^{*}>30\right)$ were observed in simulations and confirmed experimentally. They occur at the so-called flutter frequency which - generally - does not coincide with the eigenfrequency of the structure. The excitation is not due to von Kármán or other vortices but rather due to the response of the flow-field to a profile motion at a frequency much lower than the vortex shedding frequency. It can be reasoned that the torsional vibration mode first becomes unstable and gives rise to flutter vibrations. An attempt to determine aeroelastic stability by the so-called aerodynamic derivatives was made. They describe the dependency of the aerodynamic forces on a small profile motion [2] and were obtained by forced profile motion simulations. Good agreement with respect to the predicted critical reduced velocity was achieved.

\section{References}

[1] M. Matsumoto, T. Yagi, H. Tamakic, T. Tsubota. Vortex-induced vibration and its effect on torsional flutter instability in the case of B/D $=4$ rectangular cylinder. J. Wind Eng. Ind. Aerodyn. 96 (2008), 971-983.

[2] R.H. Scanlan, J.J. Tomko. Airfoil and Bridge Deck Flutter Derivatives. J. Eng. Mech. Div. 97 (1971), $1717-1737$ 


\title{
Hydroelastic stability of multi-plate structures interacting with flowing fluid
}

\author{
Sergey A. Bochkarev, Sergey V. Lekomtsev, Valery P. Matveenko \\ Institute of Continuous Media Mechanics UB RAS
}

Plate structures consisting of a single or multiple parallel plates partially or totally immersed into a fluid flow are used extensively in modern engineering applications. High flow velocities and server size and weight restrictions necessitate exclusive safety standards and preliminary estimation of the effect of the hydrodynamic pressure in the case of two-way interaction of the elastic stricture-fluid system. In this case it is equally important to investigate the dynamic response of a thin-walled plates subjected to hydrodynamic loading and to estimate the critical flow velocity, exceeding of which leads to the loss of plane stability. The study of this phenomenon is represented mainly by numerical-analytical works. Most of them are usually restricted by either stability analysis of plates having an infinite extent in one of the directions or by two-dimensional model of the flowing fluid [1-2]. Only in [3] proposed a hybrid finite-element method for the analysis of hydroelastic stability of finite length plates placed in three-dimensional channel with rigid walls, containing incompressible flowing fluid. In practice, the structures are often subjected to 3D loads and have complicated clamping, which lead to the onset of different types of spatial vibrations and instability. In this paper we propose a finite element algorithm for calculating the spatial frequencies and vibration modes and also the boundaries of hydrodynamic stability of a multi-plate structure interacting with compressible fluid.

Equations of fluid motion written in the framework of potential theory, as well as the impermeability condition and corresponding boundary conditions are transformed using the Bubnov-Galerkin method. The deformation of elastic plates is determined either in the framework of the elasticity theory, which is based on the Kirchhoff-Love hypotheses, or with the use of Timoshenko's hypothesis. A mathematical formulation of the dynamic problem for thin-walled structures is developed using the variational principle of virtual displacements, which takes into account the work done by the inertial forces and hydrodynamic pressure exerted on the wetted surfaces of the plates. Numerical solution of the three-dimensional problem is carried out by means of the finite element method. The criterion of stability of the system is based on the analysis of the sign of imaginary part of eigenvalues of a coupled system of equations obtained for different values of flow velocity. The eigenvalue problem of asymmetric matrix is solved with the use of algorithm, which is based on the implicitly restarted Arnoldi method for sparse matrices.

The implementation of numerical experiments allowed us to estimate the influence of linear dimensions, the thickness of the gap between the plates and the type of the flow past the plates on the spectrum of eigenfrequencies and the boundaries of hydrodynamic stability of the structure. The analysis of the structure behavior at various combinations of kinematic boundary conditions imposed on the plate ends has revealed the existence of different instability modes. The effect of the walls rigidity of the three-dimensional channel on critical velocities of instability has been estimated.

The study was supported by the Russian Foundation for Basic Research grant (project 15-01-05254) and by the grant of the President of the Russian Federation for young scientists and leading scientific schools NSh2590.2014.1.

\section{References}

[1] C.Q. Guo, M.P. Paidoussis. Analysis of hydroelastic instabilities of rectangular parallel-plate assemblies. J. Pressure Vessel Technol. 122 (2000), 502-508.

[2] C.Q. Guo, M.P. Paidoussis. Stability of rectangular plates with free side-edges in two-dimensional inviscid channel flow. J. Appl. Mech. 67 (1999), 171-176.

[3] Y. Kerboua, A.A. Lakis, M. Thomas, L. Marcouiller. Modeling of plates subjected to a flowing fluid under various boundary conditions. Eng. Appl. Comp. Fluid Mech. 2 (2008), 525-539. 


\title{
Validation with numerical simulations of a simplified model of a hybrid rocket motor
}

\author{
Florin Frunzulica, Marius Stoia-Djeska \\ Politechnica University of Bucharest, Faculty of Aerospace Engineering, Bucharest, Romania
}

The hybrid propulsion offers some remarkable advantages like high safety and high specific impulse and thus it is considered a promising technology for the next generation launchers and space systems. The mechanism characterizing hybrid rockets is the boundary layer combustion. The complex interaction between (turbulent) flow and combustion in hybrid rockets arise some scientific challenges like regression rate modeling, the increase of combustion efficiency and instabilities mitigation by active control. Furthermore, the pressure sensitivity of the combustion process, the coupling between the motor and the oxidizer feed system, the vortex shedding in the aft mixing chamber makes indispensable a study of the mutual coupling between the main flow stream, the fuel gasification and combustion and the thermal conduction into the solid fuel. The purpose of this work is to validate through complex numerical simulations a design tool for hybrid rocket motors (HRM). A simpler model is required for performing overall analysis and parametric studies which are necessary for optimisation and for the development of control techniques for instabilities mitigation. In the present work, we use first a simplified HRM model which is based on the coupling of the hybrid combustion process with the complete unsteady flow. The flow model is based on the one-dimensional Euler equations. The flow equations and the fuel regression rate law are solved in a coupled manner. Each propellant combination has its unique regression rate formula and thus such a model includes some key operational parameters that are known and used by engineers in the design process. The platform of the numerical simulations is an implicit fourth-order Runge-Kutta high order cell-centred finite volume method. The FLUENT commercial code is after then used for the numerical simulation of the mutually interacting three-dimensional flow, combustion and heat conduction processes in a very rigorous manner. In order to take into account the modification of the geometry of the combustion port due to the consumption of the solid fuel, one uses an UDF to implement the effects of the regression rate on the moving boundary. The numerical results allow the comparison of the pressure and temperature in the combustion chamber and of the overall thrust with those obtained with the simpler model. The numerical results obtained with both models show a good agreement between them and with published experimental and numerical results. 


\title{
Thermodynamically consistent description of mass transfer in a porous medium by a singular surface
}

\author{
Kai Häberle, Wolfgang Ehlers \\ Institute of Applied Mechanics (CE), Chair of Continuum Mechanics \\ University of Stuttgart, Germany
}

Phase transition processes are important and ubiquitous physical phenomena. These processes are not limited to systems like batch reactors or evaporation from open fluid surfaces, e. g. lakes, but can also appear inside porous solids, such as in $\mathrm{CO}_{2}$ sequestration, drying of paper during production, or in the food industry. In these processes, mainly first-order transitions between the liquid and the gaseous phases of a certain fluid occur and are characterized by a jump in density and the coexistence of both phases during phase change. Second-order transitions, which do not show this behaviour, are not part of this work. The jump at the interface between the two fluid phases is numerically handled by introducing a singular surface. This allows for a thermodynamically consistent description of the mass transfer during phase transition. The mass transfer is driven by the change in internal energy and couples the two mass balance relations of the two fluid phases.

Obviously, the formulation of a model for a multiphasic porous aggregate in a non-isothermal environment, while accounting for the thermodynamics of the fluids and the phase transition, calls for a potent theory for its description. Here, the Theory of Porous Media (TPM) comes into play, since it provides a well-founded, continuum-mechanical basis to model deformable, fluid-saturated porous media. In this particular case, a threephasic model is proposed, consisting of the thermoelastic solid phase, which is percolated by the compressible gaseous and liquid fluid phases. The thermodynamical behaviour, i. e. the dependency of the fluid densities on temperature and pressure, is governed by the Redlich-Kwong-Soave equation of state and the Antoine equation for the vaporisation/condensation line. Moreover, the representation of the interface between the fluid phases by a singular surface results in additional terms in the balance relations. The evaluation of these terms leads to a consistent formulation of the mass transfer, which basically compares the energy added to the system with the latent heat of the phase transition (energy needed for the phase change of a certain amount of substance). This mass-transfer term is furthermore dependent on the interfacial area, a factor defined by porosity and saturation. Thus, geometrical and fluid-flow-dependent parameters are included into the phase change process.

Finally, the proposed model for phase transition inside a porous medium is based on the mixture momentum balance, the volume balances of the fluid phases and the energy balance. Consequently, the four primary variables are the solid deformation, the effective pore pressures of the two fluid phases and the temperature. This strongly coupled system of partial differential equations is solved monolithically using the finite element program PANDAS with an implicit time-integration scheme. The numerical simulation of condensation or evaporation of $\mathrm{CO}_{2}$ in a porous solid rock, which can be either caused by changes in temperature or in pressure, allows for the demonstration of possible application areas for the presented model. 


\title{
A coupled multiphasic description of biological methane oxidation in landfill cover layers
}

\author{
${ }^{*}$ Andrea Thom, ${ }^{*}$ Tim Ricken, ${ }^{* *}$ Joachim Bluhm, ${ }^{\dagger}$ Tobias Gehrke, ${ }^{\dagger}$ Martin Denecke \\ *Institute of Mechanics - Structural Analysis - Dynamics, TU Dortmund University \\ **Institute of Mechanics, University of Duisburg-Essen \\ $\dagger$ Insitute of Waste Management, University of Duisburg-Essen
}

Under aerobic conditions methanotrophic bacteria are able to convert methane $\left(\mathrm{CH}_{4}\right)$ into carbon dioxide $\left(\mathrm{CO}_{2}\right)$ and water. This exothermic reaction leads to a significant reduction of the climate impact. Over a period of 100 years, the global warming potential of methane is 25 times higher than that of carbon dioxide.

Since methanotrophic bacteria are situated within the landfill cover layer and therefore can convert the harmful methane emissions arising from the degradation of organic waste to the less harmful carbon dioxide, the biological oxidation of methane can be considered as a method of passive aftercare for landfills to reduce climate-impact.

The application of methanotrophic treatment is limited by the low forecast ability of the biological processes in the landfill cover. These dynamic processes are influenced by a variety of environmental factors. For a full scale implementation a model with high reliability is needed to simulate the behavior of methanotrophic layers on a landfill site.

To model the coupled processes during methane oxidation, the well-known Theory of Porous Media has been used in order to develop a multiphasic FE-calculation concept, see [1]. The thermodynamic consistent model analyzes the relevant gas productions of methane, carbon dioxide and oxygen. The model also accounts for the driving phenomena of production, diffusion and advection. A multiphasic continuum approach is presented, focusing on the constitutive modelling of the gas phase as well as the energy production. For validation, selected numerical results are compared to experimental results.

Acknowledgement: This work is supported by the DFG (Grant No. RI 1202/3-1,2).

\section{References}

[1] T. Ricken, A. Sindern, J. Bluhm, R. Widmann, M. Denecke, T. Gehrke and T. C. Schmidt. Concentration driven phase transitions in multiphase porous media with application to methane oxidation in landfill cover layers. J. Appl. Math. Mech. 94, 7-8 (2014), 609-622. 


\title{
Modeling, Simulation and Parameter Identification for Rate-Dependent Magnetoactive Polymer Response
}

\author{
Björn Kiefer ${ }^{1}$, Krishnendu Haldar ${ }^{1}$, Andreas Menzel $^{1,2}$ \\ ${ }^{1}$ Institute of Mechanics, Department of Mechanical Engineering, TU Dortmund \\ 2 Division of Solid Mechanics, Lund University
}

Magneto-active polymers (MAP), which are composite materials consisting of a polymeric matrix and embedded magnetizable particles, have recently attracted much attention due to their unique magneto-mechanical coupling properties that enable a contactless, kinematically reversible, and continuous tunability of stiffness and viscoelastic (damping) properties through magnetic field application. Another attractive feature is the possibility to equip this active composite material with tailored anisotropies by positioning particles through proper field application during the curing phase of the matrix material. This unique combination of magneto(thermo)-mechanical coupling, non-linearity, rate-dependence, hysteresis and anisotropy poses new challenges for constitutive modeling. While there has been significant activity in the area of electro- and magnetoactive polymer modeling throughout the past decade, such efforts, particularly for MAP, have been impeded by the lack of sufficiently detailed and reliable experimental data.

In the present work, the magneto-viscoelastic behavior of MAPs is studied by means of a thermodynamicallyconsistent constitutive model. A finite deformation based framework of nonlinear magneto-viscoelastic coupling is introduced with a multiplicative decomposition of the deformation gradient. Viscous effects, which are entirely attributed to the properties of the polymeric matrix material, are captured by appropriate evolution equations for the internal state variables. A Gent-type model is adopted to describe the hyperelastic energy storage. Another important contribution is the proposition of an energy function that consistently captures the saturation effects in both the magnetization as well as the magnetostrictive behavior. The modeling efforts were strongly supported by the availability of detailed experimental characterization data provided by a collaborating partner as basis for rigorous, nonlinear optimization-based parameter identification. In addition to the model development at the material point level, a numerical finite element-based code for the analysis of magneto-mechanically coupled finite deformation boundary value problems was developed, into which the newly formulated, calibrated and validated MAP constitutive model has been implemented. This tool can for instance be used to predict the behavior of smart MAP structures under complex loading conditions, and some technologically relevant numerical examples are discussed. It is further demonstrated, that FE-analysis is in fact indispensable to ensure material model calibrations that are independent of the sample geometry, so that the model parameters reflect the true constitutive behavior and not the system response which is typically recorded in experiments due to the so-called demagnetization effect.

\section{Acknowledgment}

The authors would like to thank Prof. Z. Major (Institute of Polymer Product Engineering, Johannes Kepler University Linz, Austria) for providing the experimental characterization data for a broad spectrum of MAP types and testing conditions. 


\title{
On electrostatic-viscoelastic simulation of dielectric actuators
}

\author{
Tristan Schlögl, Sigrid Leyendecker \\ Bionicum, Chair of Applied Dynamics, University of Erlangen-Nuremberg
}

Modern robotic systems suffer some severe dynamic limitations. The rigid coupling between electrical drives and joints does not allow for dynamic motions like they occur in nature, where muscles act as an energy buffer and store energy. However, this elastic behaviour plays an important role when considering humanoid systems in terms of safety, energy efficiency and robustness. Stacked dielectric elastomer actuators are composed of a series of elastic capacitors. Each capacitor consists of two conductive layers separated by an insulating material with small Young's modulus and large permittivity [1]. As with capacitors, when an external voltage is applied to the conductive layers, an electric field is established. The electric field leads to polarisation. Attracting bound charges then induce a contraction of the silicone. Due to its similarity with real muscles, dielectric actuators are also knows as artificial muscles.

The functional principle can be described by the Maxwell equations, the balance of momentum and constitutive material laws. It is assumed that the electric field $\mathbf{E}$ can be regarded as quasi-static, no external magnetic fields are applied and no free currents and electric charges are present. The mechanical behaviour is covered by the balance of momentum with the deformation gradient $\mathbf{F}$ and its determinant $J$. In analogy to the Kelvin-Voigt model where the total force is split into the sum of an elastic and a viscous part, the mechanical stress tensor can be split into the sum of a conservative elastic stress tensor and a time dependent viscous part to allow for viscoelastic material behaviour. Coupled by the electrostatic body force [3], these equations set up the strong form of the electrostatic-viscoelastic simulation model.

The coupled strain energy density of the dielectric actuator is given by

$$
\Omega(\mathbf{F}, \mathbf{E})=\underbrace{\frac{\mu}{2}[\mathbf{C}: \mathbf{1}-3]-\mu \ln (J)+\frac{\lambda}{2}[\ln (J)]^{2}}_{\text {Neo-Hooke }}+\underbrace{c_{1} \mathbf{E} \cdot \mathbf{E}}_{\text {electric }}+\underbrace{c_{2} \mathbf{C}:[\mathbf{E} \otimes \mathbf{E}]}_{\text {coupling }}-\underbrace{\frac{1}{2} \varepsilon_{0} J \mathbf{C}^{-1}:[\mathbf{E} \otimes \mathbf{E}]}_{\text {free space }},
$$

with the Lamé parameters $\mu$ and $\lambda$, the Cauchy-Green tensor $\mathbf{C}=\mathbf{F}^{T} \cdot \mathbf{F}$, the identity matrix $\mathbf{1}$, two electrical parameters $c_{1}$ and $c_{2}$ and the vacuum permittivity $\varepsilon_{0}$ [4]. Together with the kinetic energy, the Lagrange function $L$ can be evaluated. The Lagrange d'Alembert principle $\delta S+\int_{0}^{T} \delta W^{\text {ext }} d t=0$, with the action $S=\int_{0}^{T} L d t$ for a time interval $[0, T]$ and non-conservative contributions $W^{\text {ext }}$ (that include viscous terms) then leads to a variational setting of the coupled problem.

The action integral is approximated using quadrature rules. Hexahedral finite elements with linear shape functions are used for the spatial discretisation and finite differences are used for the temporal discretisation. Then, the Lagrange d'Alembert principle is applied to the discrete set of equations, resulting in a variational integration scheme. Variational integration guarantees that important characteristics of the system are preserved exactly [2].

The implicit integration scheme is implemented as C++ code using the library deal.II. Simulation examples illustrate the use of the coupled model for the simulation of dielectric elastomers. In future work, the artificial muscles will be used to actuate humanoid kinematics. The model is then used for the optimal control of motions that are driven by dielectric actuators.

\section{References}

[1] Y. Bar-Cohen. Electroactive polymers as artificial muscles - capabilities, potentials and challenges. Handbook on biomimetics 11:8 (2000).

[2] S. Leyendecker, J.E. Marsden, M. Ortiz. Variational integrators for constrained dynamical systems. Z. Angew. Math. Mech. 88 (2008), 677-708.

[3] Y.H. Pao. Electromagnetic forces in deformable continua. Mechanics Today 4 (1978), 209-306.

[4] D.K. Vu, P. Steinmann, G. Possart. Numerical modelling of non-linear electroelasticity. Int. J. Numer. Meth. Engng 70 (2006), 685-704. 


\title{
Microscopic modeling and finite element simulation of magnetorheological elastomers
}

\author{
Philipp Metsch, Christian Spieler, Markus Kästner \\ Institute of Solid Mechanics, Technische Universität Dresden, 01062 Dresden, Germany
}

\begin{abstract}
Magnetorheological elastomers (MRE) feature mechanical moduli that become enhanced by an applied external magnetic field as well as the ability to generate magnetically induced deformations and mechanical actuation stresses. Typically, these materials represent a two-component system, in which micron-sized magnetizable particles are embedded in a cross-linked polymer network. The spatial distribution of these particles can be either isotropic or anisotropic depending on whether the particles have been aligned by an applied magnetic field before the cross-linking of the polymer. Since the effective material behavior of MRE is essentially determined by the constitutive properties of the individual components and their geometrical arrangement in the composite, this contribution will apply a homogenization approach [1] for coupled magneto-mechanical problems. Based on a microscopically motivated model for the electrodynamics of continua $[2,3]$ the balance of momentum is expressed in terms of the total stress tensor which is split into a mechanical and a magnetic part.

The governing equations are solved using the extended finite element method [4] that allows for the use of nonconforming, structured meshes which do not have to be adapted to the particle-matrix interfaces. This is advantageous if complex systems representing stochastic and structured particle distributions are considered. Based on the simulation of the magneto-mechanical interactions in a microscopic representative volume element, the effective coupled material behavior has been predicted using periodic boundary conditions for both the magnetic potential and the displacements. Results obtained from computations are comparable to experiments as well as the analytic findings of Ponte Castañeda et al. [5] and are outlined in more detail in $[1,6]$.

Moreover, a large deformation framework for the magneto-mechanical boundary value problem is derived and compared to the small deformation model. For the nonmagnetic polymeric matrix of the MRE a compressible neo-Hookean material model is used. Since the enclosed particles exhibit a nonlinear magnetization behavior with saturation at higher values of the magnetic field but negligible hysteresis effects, a phenomenological model is used to describe the magnetic nonlinearity. One crucial task is the formulation of thermodynamically consistent constitutive equations for large deformations [6] which, in the limit case, should coincide with the small deformation model used in $[1,4]$.
\end{abstract}

\section{References}

[1] C. Spieler, M. Kästner, J. Goldmann, J. Brummund, V. Ulbricht. XFEM modeling and homogenization of magnetoactive composites. Acta Mechanica 224 (2013), 2453-2469.

[2] S. R. de Groot, L. G. Suttorp. Foundations of Electrodynamics. North-Holland Publishing Company, Amsterdam, 1972.

[3] A. C. Eringen, G. A. Maugin. Electrodynamics of Continua I: Foundations and Solid Media. Springer-Verlag, New York, 1990.

[4] M. Kästner, S. Müller, J. Goldmann, C. Spieler, J. Brummund, V. Ulbricht. Higher-order extended FEM for weak discontinuities - level set representation, quadrature and application to magneto-mechanical problems. International Journal for Numerical Methods in Engineering 93 (2013), 1403-1424.

[5] P. Ponte Castañeda, E. Galipeau. Homogenization-based constitutive models for magnetorheological elastomers at finite strain. Journal of the Mechanics and Physics of Solids. 59 (2011), 194-215.

[6] C. Spieler, P. Metsch, M. Kästner, V. Ulbricht. Microscale Modeling of Magnetoactive Composites Undergoing Large Deformations. Technische Mechanik 34 (2014), 39-50. 


\title{
Harvesting energy with a cone-type dielectric elastomer generator
}

\author{
Eliana Bortot, Massimiliano Gei \\ DICAM - University of Trento
}

The realization of large and medium scale dielectric elastomer generators (DEGs) for harvesting energy from sea waves is one of the current challenges in energy-conversion engineering: a feasible and adaptable suitable device is represented by a generator deforming out-of-plane. This note concerns the numerical analysis of the performance, in terms of gained energy and efficiency, of a cone-type DEG deforming non-homogeneously out-of-plane.

A thin circular membrane of dielectric elastomer is constrained at the boundary by a rigid ring and at the centre by a movable rigid plate, where an external load is applied. The generator undergoes an electromechanical cycle where the external load and the charge are alternately held constant. The membrane is first stretched, then charged and afterwards released, so that the charge is finally harvested at high voltage. In order to avoid loss of the tensile stress state, electric breakdown and electromechanical instability, we control the applied voltage limiting in this way the maximum voltage and keeping the maximum stretch in an admissible range.

The prestretch of the membrane is crucial for an improved behaviour of the device. We evaluate its positive effects on the performance employing an hyperelastic formulation. Moreover, fixing the external radius, we compare the behaviour of two generators characterized by different values of the radius ratio $R_{e} / R_{i}$, namely 2 and 4. Numerical results show that the generator characterized by the smaller ratio has a better performance. Even if the membrane volume is smaller, this configuration allows to leverage the material to obtain a better performance in a wider range of prestretch values.

In the final part, a comparison of the capabilities of the cone-type generator with respect to those associated with other explored configurations and some considerations on viscoelastic effects will be provided. 


\title{
Modeling of thermo-electro-mechanical coupling effects in ionic electroactive polymers within the Theory of Porous Media
}

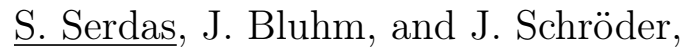 \\ Faculty of Engineering, Department of Civil Engineering \\ Institue of Mechanics, University of Duisburg-Essen, 45141 Essen, Germany
}

In this contribution we focus on the numerical simulation of ionic electroactive polymers (EAPs) by using the Theory of Porous Media (TPM). For the description of these EAPs, we investigate the material behavior by considering the thermo-electro-mechanical coupling. The presented model consists of a charged polymer (Solid) saturated by an electrolyte solvent (Fluid). The matrix is plated between two deformable electrodes. In the present talk a thermodynamically consistent model based on the TPM will be discussed in consideration of the electrostatic forces and the osmotic and fluid pressure. Representative simulation of a cantilever beam will be demonstrated. The ability of the model in view of the distribution of the concentration of the mobile cations and the volume fractions of the solvent will be analyzed.

\section{References}

[1] Chen, X.H., Pao, W. \& Li, X. Coupled thermo-hydro-mechanical model with consideration of thermalosmosis based on modified mixture theory. Inter. J. Engrg. Sc. 64 (2013), 1-13.

[2] A.Y. Acartürk. Simulation of Charged Hydrated Porous Materials. PhD-Thesis, 2009.

[3] G. Del Bufalo, L. Placidi, \& M. Profiri. A mixture theory framework for modeling the mechanical actuation of ionic polymer metal composites. Smart Mater. Struct. 17:045010, 2008. 


\title{
Deformation and interaction of surface energy driven systems
}

\author{
Roger A. Sauer, Thang X. Duong \\ Aachen Institute for Advanced Study in Computational Engineering Science (AICES), \\ RWTH Aachen University, Templergraben 55, 52056 Aachen, Germany
}

This contribution presents a general formulation for mechanical bodies governed by surface energy. Both solid and liquid bodies are considered. The surface energy can characterize both membrane and shell-like surface behavior. It leads to a coupling between the surface and bulk fields. Further coupling is introduced by the interaction between different bodies. As an example, a liquid droplet in contact with a deformable solid substrate is considered [1]. This setup can be easily modified to two liquids or two solids in contact. The surface kinematics, essential to the modeling of surface energy driven systems, are described here in curvilinear coordinates [2]. In particular modeling focus are the contact conditions at the interface between the bodies. It is shown that in the case of quasi-statics and hyperelasticity, the governing equations can be derived from a global potential that accounts for contact as well as the energy storage within the bulk and surface domains. Altogether, 21 coupled Euler-Lagrange equation are derived in this manner. Apart from these strong form equations, the governing weak form as well as its complete linearization, which are required for computational methods, are also discussed. It is further shown that in some cases the governing equations can be further simplified into a reduced set of equations that are then suitable for efficient computational implementations of such systems $[3,4]$. The presented formulation is illustrated by several numerical examples.

\section{References}

[1] R.A. Sauer. A contact theory for surface tension driven systems. Math. Mech. Solids (2014), DOI: $10.1177 / 1081286514521230$.

[2] D.J. Steigmann. On the relationship between the Cosserat and Kirchhoff-Love theories of elastic shells. Math. Mech. Solids 4 (1999), 275-288.

[3] R.A. Sauer, X.T. Duong, C.J. Corbett. A computational formulation for constrained solid and liquid membranes considering isogeometric finite elements. Comput. Methods Appl. Mech. Engrg. 271 (2014), 48-68.

[4] R.A. Sauer. Stabilized finite element formulations for liquid membranes and their application to droplet contact. Int. J. Numer. Meth. Fluids 75 (2014), 519-545. 


\title{
Parallel solution of volumetrically coupled multi-field problems using an Abaqus-PANDAS software interface
}

\author{
Maik Schenke, Wolfgang Ehlers \\ Institute of Applied Mechanics (CE), Chair of Continuum Mechanics \\ University of Stuttgart, Germany
}

The appearance of coupled multi-field problems in nature is manifold and can, thus, be assigned to different engineering disciplines, such as mechanical, civil, environmental and biomechanical engineering. In general, coupled problems can be split into so-called surface- and volume-coupled problems. In surface-coupled problems, adjoining subdomains with different physical properties are linked together via common interfaces, for instance, in fluid-structure-interaction problems. In contrast, in case of volume-coupled problems, distinct domain-coupling interfaces cannot be identified. Instead, the coexisting physical fields exhibit intrinsic interacting properties, for instance, in thermomechanical problems. However, the type of physical coupling may depend on the considered length scale. For instance, the micro-structural surface-coupled problem in porous materials (pore-fluid-solid-skeleton interaction) can be recast into a macroscopically volume-coupled problem by employing a suitable homogenisation technique. The present contribution addresses the simulation of macroscopic volume-coupled problems. The reliability of these simulated processes, apart from computational issues, such as numerical stability, strongly depends on the governing material model, especially, when strongly coupled multi-field problems come into play. Basically, material models are developed or improved either during academic or during industrial research projects and are then implemented into existing software packages. However, it often appears that these packages are either not well suited, or it requires considerable efforts to use them for the solution of complex initial-boundary-value problems (IBVP) in order to prove their capability in practically relevant scenarios.

The present work introduces a general interface between the research code PANDAS, which is a multi-field finite-element solver based on a monolithic solution strategy, and the commercial finite-element package Abaqus. The coupling is based on the user-defined element subroutine (UEL) of Abaqus. This procedure allows, on the one hand, a straight-forward embedding of all material models of PANDAS into Abaqus. On the other hand, it provides, in comparison to the native UEL subroutine of Abaqus, a user-friendly programming environment for user-defined material models with an arbitrary number of degrees of freedom. Furthermore, the coupling exhibits minimal-invasive properties with respect to the IBVP definition process in Abaqus and allows for the parallel analysis of large-scale problems on high-performance computing clusters.

The Abaqus-PANDAS linkage can be applied to various coupled multi-field problems, such as partially or fully saturated soils, vacuum-assisted resin injections (VARI) of dry fibre fabrics, or chemically or electrochemically driven swelling phenomena as they appear, for example, within hydrogels. Additionally, discontinuities, such as cracks, can be described for instance via phase-field models or by the extended finite-element method (XFEM). 


\title{
Application and modification of the POD Method and the POD-DEIM for model reduction in porous-media simulations
}

\author{
Davina Fink, Wolfgang Ehlers \\ Institute of Applied Mechanics (CE), Chair of Continuum Mechanics, University of Stuttgart
}

Researchers with a continuum-mechanical background typically use a multi-phasic and multi-component modelling approach for materials with a saturated porous microstructure. Therefore, the mechanical behaviour of porous media is considered in a continuum-mechanical manner and solved using the Finite-Element (FE) Method. The constitutive models for the subsequent FE simulations are based on the well-founded Theory of Porous Media (TPM), leading to macroscopic models of superimposed and interacting constituents.

The developed models need to be, on the one hand, as simple as possible and, on the other hand, complex enough to capture the relevant properties of the investigated materials. In most cases, this requirement results in simulations with a very large number of degrees of freedom (DOF) which are associated with long computing times. The aim of the present contribution is to reduce the computing time and the numerical effort of these simulations through model-reduction methods, while the accuracy of the solution needs to be maintained. Therefore, the method of Proper-Orthogonal Decomposition (POD) for linear problems and the Discrete-Empirical-Interpolation Method (DEIM) in combination with the POD Method (POD-DEIM) for nonlinear problems are investigated as model-reduction techniques for FE simulations of porous media.

Using the POD Method, a given data set is approximated with a low-dimensional subspace which ensures a high flexibility in application. To generate this data set, the vector of unknowns of the FE simulation, which contains the values of the primary variables at each node of the FE grid, is stored in a pre-computation in the full (unreduced) system in each time-step. The generated vectors are the so-called "snapshots" of the system.

Dealing with porous-media problems, the primary variables are the solid displacement, the pore pressure and, depending on the particular problem, other primary variables such as the solid velocity and the seepage velocity for dynamic problems or the molar concentration of a therapeutic agent in biomechanical applications. Following this, the primary variables have entries with very huge differences in their absolute values. As a result, rounding errors may occur when applying the POD Method, which are not negligible. To overcome this problems, modifications of the classical POD Method and the POD-DEIM need to be performed for such problems. The present contribution discusses this issue and presents results for the reduced simulations of porous media with different material behaviour. 


\title{
NURBS-based Approaches in Fluid Flow Simulations
}

\author{
Stefanie Elgeti ${ }^{\mathbf{1}}$, Atanas Stavrev ${ }^{\mathbf{2}}$, Norbert Hosters ${ }^{\mathbf{1}}$ and Marek Behr ${ }^{\mathbf{1}}$ \\ ${ }^{1}$ Chair for Computational Analysis of Technical Systems, CCES, RWTH Aachen University, Germany \\ ${ }^{2}$ Aachen Institute for Advanced Study in Computational Engineering Science, RWTH Aachen University, Germany
}

Fluid flow applications can involve a number of coupled problems. One is the simulation of free-surface flows, which require the solution of a free-boundary problem. Within this problem, the governing equations of fluid flow are coupled with a domain deformation approach. These domain deformation approaches can either fall into the category of interface capturing, such as level-set or volume-of-fluid methods, or interface tracking, where, at least in portions of the mesh, a Lagrangian view point is adopted and the mesh nodes are displaced with the fluid flow. Our solution approach is based on the Deforming-Spatial Domain/Stabilized Space-Time (DSD/SST) finite element method in combination with a boundary conforming interface tracking scheme. In DSD/SST, the variational form is written over the complete space-time domain, thus easily incorporating deforming domains into the formulation. The stabilization is performed using a Galerkin/Least-Squares technique. In order to enhance the aspect of boundary conformation, the scheme employs Non-Uniform Rational B-Splines (NURBS) as a support of the standard finite element representation of geometry and flow solution. As the basis of CAD systems, NURBS are closely connected to any engineering application, particularly since the concept of Isogeometric Analysis (IGA) [1] has introduced NURBS to the numerical analysis. However, the generation of complex three-dimensional grids suited for IGA is still a challenge, restricting its use in the area of fluid mechanics. Nevertheless, methods for fluid simulation can profit immensely from the use of NURBS as a boundary description. Several approaches (here, all connected to FEM) are possible:

1. Certain information needed for the computation (e.g., curvature or normals) is computed from a NURBS representing the boundary [2].

2. The computational domain is represented exactly using NURBS, but the solution is still interpolated using polynomials. This idea is, for example, realized in the NURBS-enhanced finite element method [3].

3. The NURBS represent the geometry and in addition represent the solution at the boundaries.

Such approaches can have a variety of advantages, stemming from both the exact boundary description and the superior approximation properties of NURBS. They involve the computational accuracy reached with a specific computational cost as well as the efficiency and accuracy of coupling schemes. The advantages of the discussed approaches are demonstrated on two numerical examples of fluid flow, namely two-phase flow with drops and sloshing tanks. The case of drops requires an accurate geometry description particularly to evaluate surface tension effects, which rely on the curvature of the boundary. The curvature, and therefore second derivatives of the geometry representation, are often hard to obtain from standard finite element meshes. This information is however provided by NURBS. In the sloshing tank case, we concentrate on representing tank walls of arbitrary shape in an interface tracking context.

\section{References}

[1] T. J. R. Hughes, J. A. Cottrell,Y. Bazilevs. Isogeometric analysis: CAD, finite elements, NURBS, exact geometry and mesh refinement. Computer Methods in Applied Mechanics and Engineering 194 (2005), $4135-4195$.

[2] S. Elgeti, H. Sauerland, L. Pauli, M. Behr. On the usage of NURBS as interface representation in free-surface flows. International Journal for Numerical Methods in Fluids 69 (2012), 73-87.

[3] R. Sevilla, S. Fernandez-Mendez, A. Huerta. NURBS-Enhanced Finite Element Method (NEFEM): A Seamless Bridge Between CAD and FEM. Archives of Computational Methods in Engineering 18 (2011), $441-484$. 


\title{
Numerical Approaches towards Plasticity
}

\author{
Schröder, B., Kuhl, D. \\ Institute of Mechanics and Dynamics, University of Kassel
}

Integrated thermomechanical forming processes are developed to optimize mass products' properties application oriented. As an example for this kind of procedures the fabrication of a metal flange shaft can be considered. At first the workpiece is heated inductively creating a heterogeneous temperature field, forged thermomechanically due to the contact with the forming die and in the last step a local cooling using a high pressured air stream is carried out, cf. [1]. One of the main topics characterizing this integrated manufacturing process is the second part where finite thermoviscoplastic deformations take place. Thus, adequate material models as well as proper numerical schemes have to be established.

The first step towards creating and analyzing numerical schemes for finite thermoviscoplasticy, is to generate a series of model problems considering different aspects. As a starting point for numerical investigation a classical small strain plasticity model of the vON MISES typeand its numerical implementation is examined, cf. [2]. Therein distinct time discretization schemes, like Runge-KutTa and GalERKin, are applied to solve the evolution equations on the GaUss point level as well as the balance of linear momentum on the element level, cf. $[3,4,5]$.

In contrast a consistent variational approach is presented. Therein a dissipation potential is assumed and the principle of JOURDAIN is exploited to generate a multifield formulation of plasticity enabling the usage of one single time discretization scheme on the element level, cf. $[6,7,8]$. An active set strategy is developed to judge whether elastic or plastic phenomena prevail at distinct element nodes.

Hence, a comparison between the conventional and the variational procedure taking into account the different time discretization schemes will be presented.

\section{References}

[1] K. Steinhoff, U. Weidig and N. Saba. Investigation of Plastic Forming Under the Influence of Locally and Temporally Variable Temperature and Stress States. In: Functionally Graded Materials in Industrial Mass Production, Verlag Wissenschaftliche Scripten, 2009.

[2] J. C. Simo and T. J. R Hughes. Computational Inelasticity, Springer-Verlag, 1997.

[3] P. Ellsiepen and S. Hartmann. Remarks on the interpretation of current non-linear finite element analyses as differential-algebraic equations. International Journal for Numerical Methods in Engineering, Vol. 51, 679-707, 2001.

[4] J. C. Simo. Numerical Analysis and Simulation of Plasticity, Elsevier Science, 1998.

[5] T. Gleim and D. Kuhl. Higher order accurate discontinuous and continuous p-Galerkin methods for linear elastodynamics. Zeitschrift für angewandte Mathematik und Mechanik, Vol. 96, 177-194, 2013.

[6] P. Germain. The method of virtual power in continuum mechanics. Part 2: Microstructure. SIAM Journal on Applied Mathematics, Vol. 25, 556-576, 1973.

[7] K. Hackl. Generalized standard media and variational principles in classical and finite strain elastoplasticity. Journal of the Mechanics and Physics of Solids, Vol. 45, 667-688, 1997.

[8] C. Miehe. A multi-field incremental variational framework for gradient-extended standard dissipative solids. Journal of Mechanics and Physics of Solids, Vo. 59, 898-923, 2011. 


\section{S08: Multiscales and homogenization}

This section is dedicated to discuss recent advances in multiscale and homogenization techniques. Topics of particular interest are nonlinear homogenization techniques, multiscale modelling of failure processes and localization phenomena, FE2 methods, multiphysics phenomena, atomistic to continuum coupling, contact homogenization, numerical techniques for bridging the gap between scales, coarse graining methods, model reduction techniques and multiscale techniques for dynamic problems. 


\title{
A two scale phase field model for elastic shape optimization
}

\author{
$\underline{\text { Sascha Tölkes }}$ \\ Institute for Numerical Simulation \\ University of Bonn
}

In a two scale model for elastic deformations on perforated domains the macroscopic material properties are determined by a microscopic geometry. This talk discusses the optimization of a cost functional with respect to the microscopic geometry, which is described by phase field functions (compare [1]). The cost functional decomposes into a compliance type cost, a material cost, and a rescaled perimeter of the microscopic perforation selecting a particular microscopic cell size. The approach is discretized using piecewise affine finite elements both on the micro and the macro scale. For the optimization scheme Newton's method is applied to the associated Lagrangian. The relation to microstructures described by sequential laminates [2] is exploited. Further numerical experiments allow material optimization in more complex scenarios with macroscopically non-homogeneous two-scale materials.

This is joint work with M. Rumpf.

\section{References}

[1] P. Penzler, M. Rumpf, and B. Wirth. A phase-field model for compliance shape optimization in nonlinear elasticity. ESAIM: Control, Optimisation and Calculus of Variations, 18(1):229-258, 2012.

[2] Allaire, G. Shape optimization by the homogenization method Springer-Verlag, 2002, 146 


\title{
Study on Statistically Similar RVEs for real microstructures based on different statistical descriptors
}

\author{
Lisa Scheunemann $^{1}$, Daniel Balzani ${ }^{2}$, Dominik Brands ${ }^{1}$, Jörg Schröder ${ }^{1}$ \\ ${ }^{1}$ Institut für Mechanik, Abteilung Bauwissenschaften, Fakultät für Ingenieurwissenschaften, \\ Universität Duisburg-Essen, Universitätsstr. 15, 45141 Essen, Germany \\ ${ }^{2}$ Institut für Mechanik und Flächentragwerke, Fakultät Bauingenieurwesen, \\ Technische Universität Dresden, 01062 Dresden, Germany \\ lisa.scheunemann@uni-due.de, daniel.balzani@tu-dresden.de, \\ dominik.brands@uni-due.de,j.schroeder@uni-due.de
}

In the field of microheterogeneous materials, the internal microstructure is important for the simulation of the macroscopic behavior of the material. For example, in a dual-phase (DP) steel, the interaction of its two phases, martensite and ferrite, are important contributors to the advantageous properties of the material, such as high strength and good formability. The consideration of the microstructure in the form of a representative volume element (RVE) can be incorporated into the modeling using computational homogenization approaches, such as the $\mathrm{FE}^{2}$ method, see e. g. [1]. Therein, a microscopic boundary value problem considering an RVE is evaluated at every macroscopic gauss-point and the macroscopic quantities are computed using suitable averages of the microscopic counterparts. The incorporation of an RVE based on a real microstructure raises the issue of high computational costs, since an expensive discretization is necessary to account for the complex microstructure morphology. This drawback can be circumvented using statistically similar RVEs (SSRVEs), as proposed in [2], which are governed by a similarity with respect to specific statistical measures and the mechanical behavior, but which are much smaller in size and contain a lower level of complexity in their morphology. SSRVEs are constructed by minimizing a least-square functional which takes into account the differences of statistical measures computed for an underlying real microstructure and the SSRVE. In [3], a staggered optimization approach was proposed, which consists of an inner optimization problem comparing the statistical measures and an outer optimization problem evaluating the similarities in the overall mechanical response to obtain a suitable SSRVE. The statistical measures used in the construction of SSRVEs plays a crucial role. Different measures capture different aspects of the morphology of a microstructure; it was shown in [3] that hybrid approaches comparing a set of multiple statistical measures are beneficial, as well as the consideration of statistical descriptors of higher order. The spectral density and lineal-path function have proven to be suitable measures for the construction of SSRVEs. In this contribution measures based on certain Minkowski functionals, cf. [5], are proposed and applied in the construction process of SSRVEs. The SSRVEs obtained using different sets of statistical descriptors are evaluated regarding their mechanical response compared with the real microstructure and further considering the efficiency of the optimization process.

\section{References}

[1] C. Miehe, J. Schröder, J. Schotte. Computational homogenization analysis in finite plasticity. Simulation of texture development in polycrystalline materials. Computer Methods in Applied Mechanics and Engineering, 171 (1999), 69-94.

[2] J. Schröder, D. Balzani, D. Brands. Approximation of random microstructures microstructures by periodic statistically similar representative volume elements based on lineal-path functions. Archive of Applied Mechanics, 81 (2011), 975-997.

[3] D. Balzani, L. Scheunemann, D. Brands, J. Schröder. Construction of Two- and Three-Dimensional Statistically Similar RVEs for Coupled Micro-Macro Simulations. Computational Mechanics (2014), doi: $10.1007 / \mathrm{s} 00466-014.1057-6$.

[4] C.L.Y Yeong and S. Torquato. Reconstructing random media. Physical Review E. 57 (1998), 495-506.

[5] G.E. Schröder-Turk, W. Mickel, S. Kapfer, M. Klatt, F. Schaller, M. Hoffmann, N. Kleppmann, P. Armstrong, A. Inayat, D. Hug, M. Reichelsdorfer, W. Peukert, W. Schwieger, K. Mecke. Minkowski Tensor Shape Analysis of Cellular, Granular and Porous Structures. Advanced Materials, 23 (2011), 2535-2553. 


\title{
Polynomial shape functions on the logarithmic space: the LogFE method
}

\author{
Christian Schröppel, Jens Wackerfuß \\ Emmy Noether Research Group MISMO, \\ Institute of Structural Analysis, University of Kassel
}

In order to obtain robust and rapid convergence in multi-grid methods, it is desirable that the approximation algorithms operating on the meshes of different coarseness, including the prolongation and restriction steps, work primarily on their respective length scales, thus achieving a separation of the shape functions in the (spatial) frequency domain. At the same time, the space of deformations in the low-frequency domain must be rich enough so as to avoid locking phenomena.

To realize these objectives, we propose the "Logarithmic finite element" (LogFE) method, a finite element approach that focuses on the approximation of the low-frequency part of a deformation, using a small number of degrees of freedom. In contrast to the standard Ritz-Galerkin approach, the (internal and external) degrees of freedom are given as coefficients of shape functions on a Lie algebra, allowing to reduce the number of degrees of freedom without incurring the locking phenomena associated with linear shape functions.

Equation (1) shows the transformation of a point at position $\mathbf{x}(\xi)$ by the deformation function $g(\mathbf{u})$, resulting from a set of shape functions associated with a given node at the initial position $\mathbf{v} . N_{i}(\xi)$ and $\bar{N}_{j}(\xi)$ denote scalar-valued shape functions, $u_{i}$ and $\bar{u}_{j}$ are the respective degrees of freedom. The bases $\mathbf{e}_{i}$ and $\mathbf{B}_{j}$ generate linear subspaces of the larger vector space $\mathbf{V}:=\left\langle\mathbf{e}_{i}\right\rangle \oplus\left\langle\mathbf{B}_{j}\right\rangle$ of the Lie algebra. For $\bar{N}_{j}(\xi) \equiv 0$, the proposed LogFE method collapses to the special case of the commonly used displacement-based finite element method.

$$
\mathbf{x}(\xi) \hookrightarrow(\mathbf{x}(\xi), \mathbf{v}) \stackrel{g(\mathbf{u})}{\longmapsto}\left(\mathrm{e}^{\sum_{j} \bar{u}_{j} \bar{N}_{j}(\xi) \mathbf{B}_{j}}(\mathbf{x}(\xi)-\mathbf{v})+\mathbf{v}+\sum_{i} u_{i} N_{i}(\xi) \mathbf{e}_{i}, \mathbf{v}+\sum_{i} u_{i} N_{i}(\xi) \mathbf{e}_{i}\right)
$$

The shape functions induce a tight coupling of translations, rotations and dilatations within a single finite element. Choosing appropriate basis vectors on the Lie algebra and suitable shape functions is of crucial importance for the performance of the model.

We will present models based on the LogFE method for 2D beams as well as for 3D beams, and provide comparisons of the results with reference solutions obtained from displacement-based FE models, including commercially available finite element programs. 


\title{
Convergence Properties of GMRES for FFT-based Galerkin Homogenization of Periodic Media
}

\author{
$\underline{\text { Nachiketa Mishra }}^{1}$, Jaroslav Vondřejc ${ }^{2,1}$, Jan Zeman ${ }^{1,3}$ \\ ${ }^{1}$ Faculty of Civil Engineering, \\ Czech Technical University in Prague, \\ Thakurova 7, 16629 Prague, Czech Republic \\ e-mail: nachiketa.mishra@fsv.cvut.cz \\ ${ }^{2}$ Faculty of Applied Sciences, \\ University of West Bohemia in Pilsen, \\ Univerzitni 22, 30614 Pilsen, Czech Republic \\ e-mail: vondrejc@kme.zcu.cz \\ ${ }^{3}$ Centre of Excellence IT4Innovations, \\ Technical University in Ostrava, \\ 17. listopadu 15/2172, 70833 Ostrava-Poruba, Czech Republic \\ e-mail: zemanj@cml.fsv.cvut.cz
}

The central result of the homogenization theory for linear elliptic partial differential equations (PDEs) with periodic oscillating coefficients states that the limit problem attains the form of the homogenized elliptic PDE with constant coefficients determined from the periodic corrector (or cell) problem. In the present contribution, we are concerned with FFT-based methods for the solution of the cell problem, originally based on an iterative solution to an integral equation of the Lippman-Schwinger type [1]. Recently, it has been demonstrated by Vondřejc et al. [2,3] that this approach is equivalent to a Fourier-Galerkin discretization of the cell problem, resulting in a linear system of the form:

$$
F^{-1} \widehat{G} F A x=F^{-1} \widehat{G} F A b \text { with } x \in \mathbb{E} .
$$

Here, $\mathbb{E}$ is a subspace of $\mathbb{R}^{n}, F^{-1}$ and $F \in \mathbb{C}^{n \times n}$ are the inverse and forward Fourier transform operators, $\widehat{G} \in \mathbb{R}^{n \times n}$ is a sparse projection matrix from $\mathbb{R}^{n}$ to $\mathbb{E}$ in the Fourier domain, sparse $A \in \mathbb{R}^{n \times n}$ collects the coefficients at the cell level, and $b \notin \mathbb{E}$ is a given vector. Our goal is to solve the system using GMRES, but its standard application leads to the failure of the Arnoldi orthogonalization step because of the singularity of the system matrix. In the talk, we will outline possibilities how to overcome these difficulties in GMRES and compare its convergence properties with other Krylov subspace-based solvers of (1).

AMS Subject Classification: 74Q99, 65M12, 65F10

\section{References}

[1] H. Moulinec, P. Suquet, A fast numerical method for computing the linear and nonlinear mechanical properties of composites, Comptes rendus de l'Académie des sciences. Série II, Mécanique, physique, chimie, astronomie, 1994, 318(11):1417-1423.

[2] J. Vondřejc, J. Zeman, I. Marek, An FFT-based Galerkin Method for Homogenization of Periodic Media, Computers \& Mathematics with Applications, 2014; 68(3):156-173

[3] J. Vondřejc, J. Zeman \& I. Marek (2014), arXiv:1404.3614

\section{Acknowledgments.}

This work was supported by the European Union under the project No. CZ.1.07/2.3.00/30.0034. 


\title{
A two-scale homogenisation approach for fluid saturated porous media
}

\author{
B.Sc. Florian Bartel ${ }^{1}$, Prof. Dr.-Ing. Tim Ricken ${ }^{1}$, Prof. Dr.-Ing. Jörg Schröder ${ }^{2}$ \\ ${ }^{1}$ TU Dortmund University, Germany \\ ${ }^{2}$ Duisburg-Essen University, Germany
}

Thinking about the description of biomaterials, e.g. human tissue, plants or sponges, we always have to take into account a global design composed of various substructures with different characteristics on a lower level. Examples of such substructures are pores which can be saturated with fluids or gases, fibres with different orientations or cells which can be influenced by chemical reactions. For the theoretical description of the behaviour, enhanced continuum mechanical models give promising approaches. Up to now, due to the high complexity, it has not been possible to simulate a biological system with only one design model. Hence, it is necessary to think about techniques which simplify the model but still consider the essential characteristics. This contribution will present a two-scale homogenisation approach for fluid saturated porous media with a reduced two-phase material model, which covers the behaviour of large poro-elastic deformation. The main aspects of theoretical derivation for the weak form, the lower level boundary conditions and the averaged macroscopic tangent moduli will be pointed out and a numerical example will be shown. 


\title{
An Extended Cascade Micromechanics Model for the Effective Diffusivity of Porous Materials accounting for Pore-Size Distribution
}

\author{
J. J. Timothy, G. Meschke \\ Ruhr University Bochum, Germany
}

The effective molecular diffusivity $D_{\text {eff }}$ of porous materials such as cementitious or geological materials is strongly affected by the complexity of the pore-space which may span across multiple scales from the nanometer to the sub-millimeter range. Recently, a semi-analytical Cascade Continuum Micromechanics (CCM) Model $[1,2]$ was presented, which allows to compute estimates of $D_{\text {eff }}$ given the porosity $\phi$, the intrinsic diffusivity of molecules in the pore-fluid $D$ and a complexity parameter $n$. In contrast to existing micromechanics models, the CCM model is able to predict a physically consistent percolation threshold.

The CCM model recursively solves for the effective diffusivity based on the EsHELBY Matrix-Inclusion morphology characterized by the complexity parameter $n$ that is also the recursion index. However, in order to incorporate a realistic pore-size distribution it is necessary to have explicit access to the volume fraction of pores that are homogenized at a particular cascade level. To this end, an extension of the CCM model is proposed to account for a particular volume fraction of porosity characteristic of a particular size at a particular recursion index $n$. The recursion index $n$ now characterizes the scale of a particular REV. At a specified scale $n$, a particular volume fraction $\psi^{(n)}$ of the homogeneous REV (representative of a porous material with smaller pores $\left.r^{(n-1)}<<r^{(n)}\right)$ material is de-homogenized into impermeable solid spherical particles and spherical pores. The de-homogenization process transforms the original homogeneous REV into a heterogeneous REV which is now re-homogenized using the EsHELBY Matrix-Inclusion technique.

The unknown $\psi^{(n)}$ at each level can be specified given a particular pore-size distribution by using an inverse transformation. For porous materials with the same porosity, but different pore size distributions, the model predicts a higher effective diffusivity for materials with dominating volume fraction of large pores in comparison to a porous material with a larger volume fraction of smaller pores. The predictions from the semi-analytical extended CCM model are compared to numerical pore-network simulations.

\section{References}

[1] J. J. Timothy, G. Meschke. Micromechanics model for tortuosity and homogenized diffusion properties of porous materials with distributed micro-cracks. Proc. Apl. Math. Mec. 11 (2011), 555-556.

[2] J. J. Timothy, G. Meschke. Diffusion in Fracturing Porous Materials: Characterizing Topological Effects using Cascade Micromechanics and Phase-Field Models. Poromechanics V (2013), 2250-2259. 


\title{
Grain-scale-based simulation of granular material
}

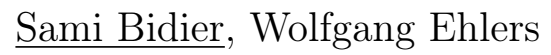 \\ Institute of Applied Mechanics (CE), Chair of Continuum Mechanics, University of Stuttgart
}

In recent years, new developments in experimental mechanics, e.g., particle-tracking methods using X-ray micro-tomography, have provided a new insight into the material behaviour of granular media. The possibility to capture the individual grain displacement and rotation of all particles within a sample provides a new fundament for a reliable numerical simulation of granulates. This is, in particular, the case for deformation localisation in shear zones as they are observed in bi- and triaxial compression tests, where the exact thickness of the localised zone as well as the amount of accompanying grain displacements and rotations are measurable in a precise and direct way.

In this context, the Discrete-Element (DE) Method has become a promising and frequently used modelling technique for granular materials. In contrast to macroscopically based continuum-mechanical approaches, the DE approach treats each particle as a rigid and uncrushably body and is, therefore, defined on a microscopic level of description. This consequently allows a direct comparison with experimental data from grain-scale tracking methods. In the present contribution, the DE method is applied in order to solve problems of localisation in granular media. Therefore, constitutive inter-particle contact laws are formulated for forces and torques which are transferred between interacting particles. The formulations are based on the assumption of an inter-particle contact area and a subsequent idealisation towards a point contact, which can be effectively implemented within a DE approach. This again allows the application of a simplified model based on spherical particles but still incorporating the characteristic effects due to particle shape and distribution. The corresponding set of material parameters needs to be calibrated with respect to experimental data in order to overcome the gap between a qualitative and a quantitative description of the material behaviour.

With a given solution of initial-boundary-value problems on the microscale, a computational homogenisation procedure leads to macroscopic quantities purely stemming from microscopic information. Herein, a particlecentre-based volume averaging technique leads to macroscopic quantities which correspond to quantities known from micropolar continuum theories. The contribution presents a framework for the homogenisation in all time steps in a fully 3-dimensional context and discusses the activation of micropolar effects in the localised zone. Furthermore, the admissible size of the chosen representative elementary volume is addressed. 


\title{
Materials design of elastic properties of multiphase polycrystalline composites using model functions
}

\author{
M. Lobos, T. Yuzbasioglu, T. Böhlke \\ Institute of Engineering Mechanics, Karlsruhe Institute of Technology (KIT) \\ Karlsruhe 76131, Germany
}

The present work presents a method for the materials design of elastic properties of multiphase polycrystalline composites. The method follows inverse design methodologies, as presented, e.g., in [1] for general materials and in [2] and [3] specialized for cubic materials. The method takes into consideration the material constants and the orientation distribution of each arbitrarily anisotropic phase. Based on the material constants of each phase, zeroth-order bounds are used in order to decide which combination of materials are physically suitable for prescribed effective properties. This is an approach which can be easily used also within large local or online data bases in order to reflect design possibilities of anisotropic materials and composites based on energetic principles. For the chosen combination of materials, a crystallite orientation distribution function for each phase is formulated as a superposition of analytic central model functions, as the ones presented, e.g., in [4]. It is shown that the chosen central model functions allow the analytic integration of orientation averages of arbitrarily anisotropic fourth-order tensors. Based on this result, the first-order bounds and the geometric average of the stiffness can be expressed explicitly in closed forms for arbitrarily anisotropic polycrystalline materials and number of phases depending on the volume fractions and the influence of the crystallographic texture of each constituent. These expressions are then used in design examples for the determination of volume fractions and orientation distribution of selected materials for prescribed properties.

\section{References}

[1] B.L. Adams, S.R. Kalidindi, D.T. Fullwood. Microstructure Sensitive Design for Performance Optimization. Butterworth-Heinemann, Waltham, MA. (2013).

[2] T. Böhlke, M. Lobos. Representation of Hashin-Shtrikman bounds of cubic crystal aggregates in terms of texture coefficients with application in materials design. Acta. Mater. 67 (2014), 324-334.

[3] M. Lobos, T. Böhlke. Materials design for the anisotropic linear elastic properties of textured cubic crystal aggregates using zeroth-, first- and second-order bounds. Int. J. Mech. Mater. Des. 10(3) (2014), 1-20.

[4] S. Matthies, J. Müller, G.W. Vinel. On the Normal Distribution in the Orientation Space. Texture Microstruct. 10 (1988), 77-96. 


\title{
A coupled two-scale shell model for comb-like sandwich structures
}

\author{
D. Heller, F. Gruttmann \\ Technische Universität Darmstadt
}

In the present contribution a coupled two-scale sandwich shell model is proposed, where 4-node quadrilaterals are employed both on the global and the local scale. The coupled global-local boundary value problem is derived by means of a variational formulation and ensuing linearization. Several numerical simulations are carried out for linear elastic and elasto-plastic material behavior with small strains, as well as geometrically nonlinear large displacement calculations assuming finite rotations.

On the local scale, shell elements with 6 degrees of freedom and intersections [2] based on a Hu-Washizu functional [3] are employed to model comb-like microstructures as a representative volume element. In-plane displacements are prescribed on the lateral surfaces of the RVE, determined by the shell strains on the global scale in a specific integration point. Additionally, link conditions are applied for the out-of-plane displacements to allow for unrestricted torsional strains [1]. At the top and bottom face layers, stress boundary conditions are imposed. For simple configurations some components of the matrix of linearized stress resultants are validated using analytical expressions. Constant membrane and bending stiffness components are obtained for different feasible choices of RVEs describing the same microstructure.

The resulting coupled nonlinear boundary value problems are solved simultaneously in a Newton iteration with incremental load steps. Various types of sandwich models are investigated in the form of uni- and bidirectionally stiffened structures as well as a hexagonal honeycomb structure. For the unidirectionally stiffened beam, an analytical reference solution is present by means of classical beam theory. In addition, the numerical results of all coupled calculations are compared to full scale shell models, showing very good agreement while significantly reducing the size of occurring system matrices.

\section{References}

[1] F. Gruttmann, W. Wagner. A coupled two-scale shell model with applications to layered structures. Int. J. Numer. Meth. Engng. 94 (2013), 1233-1254.

[2] J.C. Simo. On a stress resultant geometrically exact shell model. Part VII: shell intersections with 5/6-DOF finite element formulations. Comput. Methods Appl. Mech. Engrg. 108 (1993), 319-339.

[3] W. Wagner, F. Gruttmann. A robust non-linear mixed hybrid quadrilateral shell element. Int. J. Numer. Meth. Engng. 64 (2005), 635-666. 


\title{
Dual-Phase Steel Simulations Based on Representative Three-Dimensional Microstructures
}

\author{
$\underline{\text { D. Brands }}^{1}$, D. Balzani ${ }^{2}$, L. Scheunemann ${ }^{1}$, J. Schröder ${ }^{1}$ \\ ${ }^{1}$ Institut für Mechanik, Abteilung Bauwissenschaften, Fakultät für Ingenieurwissenschaften, \\ Universität Duisburg-Essen, Germany \\ ${ }^{2}$ Institut für Mechanik und Flächentragwerke, Fakultät für Bauingenieurwesen, \\ TU Dresden, Germany
}

In many fields of steel applications, e.g. automotive engineering or manufacturing, the optimization of the material properties has become one of the main challenges. The reduction of weight as well as an increasing stability and crash safety are main driving forces. The properties of dual-phase (DP) steels are in good accordance with these aspects and, consequently, they are often applied in the aforementioned fields of applications. It has been shown that these properties mainly depend on microstructural factors such as volume fraction, shape, size and spatial distribution of inclusions. Consequently, for a reliable computational modeling a consideration of the microstructure should be included and for that reason we present a strategy to obtain a representative volume element (RVE) for multiscale simulations like the $\mathrm{FE}^{2}$-method, see e.g. [1, 2]. These RVEs will be constructed by a set of tomographic measurements and mechanical tests. In order to arrive at more efficient numerical schemes we also construct statistically similar RVEs (SSRVEs), which are characterized by a lower complexity compared to the real microstructure but which represent the overall material behavior accurately, see e.g. [3].

In addition to the morphology of the microstructure, the austenite-martensite transformation during the steel production has a relevant influence on the mechanical properties and is considered in this contribution. This transformation induces a volume expansion of the martensite phase. A further effect is determined in nano indentation tests, where it turns out that the hardness in the ferrite phase increases exponentially when approaching the martensitic inclusion. To capture these gradient properties in the computational model, the volumetric expansion is applied to the martensite phase and the arising equivalent plastic strain distribution in the ferrite phase serves as a basis for a locally graded modification of the ferritic yield curve, cf. [4]. Good accordance of the model considering the gradient yield behavior in the ferrite phase is observed in the numerical simulations with corresponding data.

\section{References}

[1] C., Miehe, J. Schröder and J. Schotte. Computational homogenization analysis in finite plasticity. Simulation of texture development in polycrystalline materials. Comp. Meth. Appl. Mech. Eng. 171 (1999), 387-418.

[2] J. Schröder. A numerical two-scale homogenization scheme: the FE²-method. Plasticity and Beyond - Microstructures, Crystal-Plasticity and Phase Transitions. CISM Lecture Notes 550, Eds. J. Schröder, K. Hackl. Springer, 2014.

[3] D. Balzani, L. Scheunemann, D. Brands, and J. Schröder. Construction of two- and three-dimensional statistically similar RVEs for coupled micro-macro simulations. Comp. Mech. 54(5) (2014), 1269-1284.

[4] J. Schröder, D. Balzani, H. Richter, P. H. Schmitz, and L. Kessler. Simulation of microheterogeneous steels based on a discrete multiscale approach. Numisheet 2008: Proceedings of the 7th international conference and workshop on numerical simulation of $3 \mathrm{~d}$ sheet metal forming processes, 2008. 


\title{
Computational characterization of magneto-electric composites: the role of ferroelectric pre-polarization
}

\author{
$\underline{\text { M. Labusch }}^{1}$, J. Schröder ${ }^{1}$, M.-A. Keip ${ }^{3}$, D.C. Lupascu ${ }^{2}$ \\ ${ }^{1}$ Institute of Mechanics, University of Duisburg-Essen, \\ Universitätsstraße 15, 45141 Essen, Germany \\ ${ }^{2}$ Institute for Materials Science, University of Duisburg-Essen, \\ Universitätsstraße 15, 45141 Essen, Germany \\ ${ }^{3}$ Institute of Applied Mechanics (CE), Chair I, University of Stuttgart \\ Pfaffenring 7, 70569 Stuttgart, Germany
}

\begin{abstract}
The interaction between electric and magnetic fields enables smart devices, like the measurement of magnetic brain activities and their conversion into electric signals, and may find applications in sensor technology and data storage [1]. Materials showing magneto-electric (ME) coupling combine two or more ferroic characteristics and are known as multiferroics. Since natural single-phase materials show an interaction between polarization and magnetization mostly at very low temperature and at the best a too small ME coefficient at room temperature for technical applications, composite materials becomes important. These ME composites consist of ferromagnetic and ferroelectric phases and generate the ME coupling as a strain-induced product property, where we distinguish between the direct and converse ME effect. The direct effect characterizes magnetically induced polarization, where an applied magnetic field yields a deformation of the magneto-active phase which is transferred to the electric phase. As a result, a strain-induced polarization is observed. On the other hand, the converse effect characterizes electrically activated magnetization. Several experiments on composite multiferroics showed remarkable ME coefficients at room temperature that are orders of magnitudes higher than those of singlephase materials. Due to the large influence of the microstructure on the ME effect, we have derived a two-scale homogenization framework, which allows for the consideration of microscopic morphologies [2,3]. A further major influence on the overall ME properties is the polarization state of the ferroelectric phase. In order to account for different polarization states, a material model is implemented that considers the switching behavior of the spontaneous polarization [4] and enables a more exact comparison to experimental measurements [5].
\end{abstract}

\section{References}

[1] N.A. Spalding, M. Fiebig. The renaissance of magnetoelectric multiferroics. Materials Science 309 (2005), 391-392

[2] J. Schröder, M.-A. Keip. Two-scale homogenization of electro-mechanically coupled boundary value problems. Computional Mechanics 50 (2012), 229-244

[3] M. Labusch, M. Etier, D.C. Lupascu, J. Schröder, M.-A. Keip. Product properties of a two-phase magnetoelectric composite: Synthesis and numerical modeling. Computational Mechanics 54 (2014), 71-83

[4] S.C. Lynch, R.M. McMeeking. Ferroelectric/ferroelastic interactions and a polarization switching model. Acta Metallurgica et Materialia 43 (1995), 71-83

[5] M. Etier, V.V. Shvartsman, Y. Gao, J. Landers, H. Wende, D.C. Lupascu. Magnetoelectric effect in (0-3) $\mathrm{CoFe}_{2} \mathrm{O}_{4}-\mathrm{BaTiO}_{3}(20 / 80)$ composite ceramics prepared by the organosol route. Ferroelectrics 448 (2013), $77-85$ 


\title{
Electro- and Magneto-active Soft Composites with Periodic and Random Microstructures
}

\author{
Technion $-\stackrel{\text { Stephan Rudykh }}{\text { Israel Institute of Technology }}$
}

We analyze the coupled behavior of dielectric elastomer composites (DEC) and, mathematically analogous, magnetoactive elastomers (MAE) undergoing finite deformations in the presence of external electric or magnetic fields. We specifically focus on the role of microstructures in the coupled response of these active materials. To this end we analyze the composites with (i) periodically and (ii) randomly distributed active particles embedded in soft matrix [1, 2], as well as (iii) layered composites and similar anisotropically structured composites [3]. Through the theoretical analysis and through finite element simulations we identify the key parameters that govern the electro- and magneto- mechanical coupling. We find advantageous microstructures that give rise to significant enhancement of the coupling and performance of the active materials [1]. Moreover, we show that even very similar microstructures, such as periodic composites with hexagonal and rectangular representative volume elements (RVE), exhibit very different behavior both in terms of actuation, and effective properties [2].

Furthermore, we explore the coupled electro- and magneto-elastic instabilities in DEC [4, 5, 6] and MAE [3]. We study the role of the external field (electric or magnetic), microstructure parameters, and material properties on the onset of both microscopic and macroscopic instabilities. We present the multiscale instability analysis for a particular class of periodic microstructures, namely, multilayered active composites. To determine the response of the multilayered structure to an external excitation and mechanical loadings, an analytical solution is derived $[4,3,6]$. The determined from the exact solution local fields are used in the Bloch-Floquet analysis to predict the onset of microscopic instabilities [6]. The onset of macroscopic instabilities is identified by analyzing the homogenized tensor of electro- or magneto-elastic moduli [4, 3]. The results for global bifurcation modes agree with these of the limit of infinite wavelengths in the microscopic instability analysis.

The presentation is based on the joint work with Profs. Kaushik Bhattacharya, Pedro Ponte-Castañeda, Katia Bertoldi, and Gal deBotton.

\section{References}

[1] S. Rudykh, A. Lewinstein, G. Uner and G. deBotton. Analysis of Microstructural Induced Enhancement of Electromechanical Coupling in Soft Dielectrics. App. Phys. Lett. 102 (2013), 151905.

[2] E. Galipaeu, S. Rudykh, G. deBotton and P. Ponte-Castañeda. Magnetoactive elastomers with periodic and random microstructures. Int. J. Solids. Struct. 51 (2014), 3012-3024.

[3] S. Rudykh and K. Bertoldi. Stability of Anisotropic Magnetorheological Elastomers in Finite Deformations: A Micromechanical Approach. J. Mech. Phys. Solids. 61 (2014), 949-967.

[4] S. Rudykh and G. deBotton. Stability of Anisotropic Electroactive Polymers with Application to Layered Media. Z. Angew. Math. Phys.(ZAMP) 62 (2014), 1131-1142.

[5] S. Rudykh, K. Bhattacharya, and G. deBotton. Snap-through Actuation of Thick-wall Electroactive Balloons. Int. J. Nonlinear Mech. 47 (2012), 206-209.

[6] S. Rudykh, K. Bhattacharya, and G. deBotton. Multiscale Instabilities in Soft Heterogeneous Dielectric Elastomers. Proc. Royal Soc. A 470 (2014), 20130618. 


\title{
Multi-scale modeling of beam-like structures: A new boundary condition concept for the RVE
}

\author{
S. Klarmann, F. Gruttmann \\ Technische Universität Darmstadt
}

In this work a coupled two-scale beam model using Timoshenko beam elements [1] with finite displacements on the macro scale and fully non-linear 3D brick elements on the micro scale is proposed. The calculation is carried out with the so-called $\mathrm{FE}^{2}$ concept. To achieve the coupling between the beam and the brick elements, the algorithm from [2] is adapted.

Within the degenerated concept of the Timoshenko beam, the introduction of a pure shear deformation leads to significant problems concerning the equilibrium condition on the micro scale. Applying this deformation mode on the RVE with periodic boundary conditions results in a rigid body rotation. Using linear displacement boundary conditions instead, the wrapping deformation is suppressed on the boundary, leading to a length dependency in the torsional deformation mode. In addition, the shear forces introduce a bending moment, which depends on the length of the RVE and adds spurious normal stresses and a length dependency of the shear stiffness.

To overcome these problems, periodic boundary conditions are applied and the displacement assumptions are modified such that the shear deformation is achieved with force pairs on both ends of the RVE. The resulting model leads to length independent results in tension, bending and torsion and a domain which is able to produce a pure shear stress state. Consequently, only this domain of the model should be homogenized which can be accomplished by modifying the variations in the algorithm [2]. The concept is validated by simple linear and non-linear test problems.

\section{References}

[1] F. Gruttmann, R. Sauer and W. Wagner. Theory and numerics of three-dimensional beams with elastoplastic material behaviour. Int. J. Numer. Meth. Engng. 48 (2000) 1675-1702.

[2] F. Gruttmann, W. Wagner. A coupled two-scale model with applications to layered structures. Int. J. Numer. Meth. Engng. 94 (2013), 1233-1254. 


\title{
Micromechanical modeling of textile materials by means of 1-D structural elements
}

\author{
Markus Mehnert, Sebastian Fillep, Julia Mergheim, Paul Steinmann \\ University of Erlangen-Nuremberg
}

Technical textiles show a nonlinear constitutive behavior that differs from the underlying fiber material and is significantly influenced by heterogeneities on the micro level, e. g. the structural assembly of the fibers and appearing contact zones. To reduce the time and costs for experiments for each practical application case a reliable computational method for the identification of macroscopic material properties in dependency of the micro structure in the sense of a numerical laboratory is needed.

The increased application of textiles in various technical fields is decisively influenced by the recent development of different fibre shapes and the improvement of their properties. Exemplary textiles find application in architecture as constructive structures and design materials, as geo-textiles for erosion defence of embankments or as drainage layers. Due to their adjustable porosity they are well suited as filter material. Their flexibility makes them interesting for usage as carrier material for electronic devices or flexible solar cells. Due to the variety of processable materials even bio-compatible structures for medical applications, e. g. implants, can be found.

In this contribution shell-like textiles are considered. Because of their large area-to-thickness ratio a shell specific formulation for the description of technical textiles on the macroscale is used. On the microscale the fibers that compose the representative volume element are discretized by one dimensional beam elements. Therefore the geometry on the microscale is described by a beam specific formulation. Hence a suitable multiscale method using a specific form of the Hill-Mandel condition that links the shell specific formulation on the macroscale and the beam specific formulation on the microscale is developed.

This contribution deals with the determination of the nonlinear constitutive behaviour of technical textiles. We present selected examples of the macroscopic behaviour of microscopic heterogeneous fibre structured materials. 


\title{
Experimental investigation and approximation of the temperature-dependent stiffness of short-fibre reinforced polymers
}

\author{
L. Kehrer, V. Müller, B. Brylka, T. Böhlke \\ Chair for Continuum Mechanics, Institute of Engineering Mechanics, \\ Karlsruhe Institute of Technology (KIT) \\ Karlsruhe, Germany
}

In the last decades, polymer based composites were increasingly applied as lightweight material in various fields due to their specific material properties. Short-fibre reinforced thermoplastics exhibit advantages concerning fabrication by injection moulding and recycling. The fabrication process causes, however, inhomogeneous and anisotropic mechanical material behaviour due to introduced local fibre orientation distribution, spatial distribution, and varying geometry of the fibres. During the injection process, cooling rates lead to effects on the structure of the composite, cf. e.g. in [2] and [3]. These structures are not balanced and exhibit postcrystallisation effects. An increase of temperature restores the original amorphous structures. Particularly in case of semi-crystalline thermoplastics, the stiffness depends on the degree of crystallisation, which is linked to temperature and time. Hence, the degree of crystallisation causes changes in the mechanical properties. In addition to the anisotropic and inhomogeneous material behaviour, the effective stiffness properties depend further on temperature and strain-rate and are coupled to temperature history.

Material properties of polypropylene as well as fibre reinforced polypropylene are investigated by a tensile test under thermal loading using dynamic mechanical analysis (DMA). Both, post-crystallisation effects and viscoelastic material behaviour of matrix material are detected, while the thermal loading is varied in a range of $-50^{\circ} \mathrm{C}$ and $120^{\circ} \mathrm{C}$. These effects are discussed on the basis of experimental results.

The effective thermoelastic material behaviour of the fibre reinforced composite is modelled using the interaction direct derivative (IDD) estimate developed by [4]. Based on the three-phase model, the IDD estimate takes into account interaction between fibres and the surrounding matrix material and the fibre distribution. The mean field homogenisation with the IDD approach is performed by means of $\mu$-CT data describing the microstructure of the composite [1]. Experimental data of polypropylene matrix obtained by the DMA are used as input parameters for the homogenisation scheme. For various time-temperature loading histories, the effective properties of the composite resulting from numerical homogenisation are compared to experimental results.

\section{References}

[1] V. Müller, B. Brylka, F. Dillenberger, R. Glöckner, S. Kolling, T. Böhlke. Homogenization of Elastic Properties of Short-Fiber reinforced Composites Based on Measured Microstructure Data. (submitted 2014).

[2] E.A. McGonigle, J.H. Daly, S. Gallagher, S.D. Jenkins, J.J. Liggat, I. Olsson, R.A. Pethrick. Physical Ageing in Poly(ethylene terephthalate) - its Influence on cold Crystallization. Polymer 40 (1999), 4977-4982.

[3] S. Meister, A. Jungmeier, D. Drummer. Long-Term Properties of Injection-Molded Micro-Parts: Influence of Part Dimensions and Cooling Conditions on Ageing Behavior. Macromolecular Materials and Engineering 297 (2012), 994-1004.

[4] Q.-S- Zheng, D.-X. Du. An explicit and universally applicable estimate for the effective properties of multiphase composites which accounts for inclusion distribution. Journal of the Mechanics and Physics of Solids 49 (2001), 2765-2788. 


\title{
Coupling atomistic and continuum models with nodes having translational and rotational degrees of freedom
}

\author{
Florian Niederhöfer, Jens Wackerfuß \\ Emmy Noether Research Group MISMO, \\ Institute of Structural Analysis, University of Kassel
}

The coupling between atomistic (discrete) and continuum models is still an active field of research. It is required for multiscale simulations with atomic structures. The presence of ghost forces is one of the main problems by the definition of interfaces. This effect is the result of the coupling of a local (continuum) and a non-local (atomistic) region. The present work deals with this problem on the level of forces (force-based a/c scheme). The presented class of coupling schemes is not restricted to merge an atomistic model with a special kind of continuum element. The atomistic model can consist of bonded, multi-body interactions. In contrast to the most approaches found in literature, the nodes of the continuum models are not restricted to representative atoms with three translational degrees of freedom. The described schemes are able to deal with nodes with rotational degrees of freedom as well. In the Finite Element code mismo [1] the implementation can be done on the element level.

The coupling between an end of a carbon nano tube and a node of a 3D beam (six degrees of freedom) is presented as an application. The dreiding potential [2] is used considering 2-, 3- and 4-body interactions. A defined benchmark without a continuum element enables the analysis of the error resulting from the coupling under different deformations. This example can be used as a part of a multiscale simulation with beam elements and as a key to special kind of boundary conditions related to 3D beams (e.g. bending or torsion).

\section{References}

[1] J. Wackerfuß. Molecular mechanics in the context of the finite element method. International Journal for Numerical Methods in Engineering 77.7 (2009): 969-997.

[2] S. L. Mayo, B. D. Olafson, and W. A. Goddard. DREIDING: a generic force field for molecular simulations. Journal of Physical Chemistry 94.26 (1990): 8897-8909. 


\title{
Representative Volume Element Size Convergence for a Parallel Fiber Bundle Micro-Model
}

\author{
Scott E. Stapleton,Lars Appel, Stefanie Reese \\ Institute for Applied Mechanics, RWTH Aachen University, Germany \\ Institute of Textile Technology, RWTH Aachen University, Germany
}

With the increasing popularity of multi-scale modelling in the field of composites, predicting the microstructure is becoming increasingly important. The local, in-situ microstructure of textile-reinforced composite parts is a result of the earlier manufacturing steps, specifically the infiltration and curing process for the matrix properties and the tow-to-textile and textile-to-preform steps for the fibers. The local fiber orientation is absolutely necessary information for parts which have significant curvatures, since angle changes due to fabric shearing occurring during the draping process can result in large differences in the stiffness and strength properties. To this end, computational models for textiles are being developed to model the draping of fabrics.

Where once only macroscopic models of textiles characterized through extensive experimental programs existed, there is now the possibility of capturing more detailed behaviors at the fiber bundle (tow / roving / yarn) scale. These textile-scale models either create a material model from a Representative Volume Element (RVE), communicate between the textile and macro scales one way or both using homogenization and localization, or only model the textile-scale. These models typically consider a tow as a homogenized continuum, and set the transverse properties of the tow to be a value much smaller than the axial properties. The transverse properties are not explicitly determined, but are estimated.

The present research introduces a fiber-scale model, where the homogenized fiber bundle properties are obtained by an RVE for cases where the fiber radius is magnitudes smaller than the cross-sectional dimensions of the fiber bundle. This RVE is generated using the discrete element method, where each fiber is modelled as a line mass, and elasticity of the fiber is only imitated through the appropriate contact law. The analysis is carried out with an explicit integration scheme where equilibrium is reached at the end of each load step. RVE size convergence is conducted for several different loading scenarios, including shear and compression perpendicular to the fibers and shear in the plane of the fibers. Additionally, the effect of packing pattern and initial pressure on the size convergence is shown. The convergence of stress response and degree of anisotropy will be used to judge convergence. These characteristics are vital to have confidence in the micro-scale response, which is then passed to the next larger scale in the simulation. 


\title{
Comparison of the Interface Orientation Distribution Average to RVE simulations
}

\author{
Rainer Glüge, Jan Kalisch \\ University of Magdeburg
}

\begin{abstract}
Analytical estimates of the effective elastic properties of a microstructured material are mostly designed for specific, idealized microstructures. To incorporate the microstructure arrangement, usually higher $n$-point correlation functions are needed, which can be rather complicated. Another possibility to consider arbitrary arrangements is to combine a laminate solution $[1,2]$ with an interface orientation distribution (IOD) average. Since any symmetry of the IOD is transferred to the effective stiffness, the IOD approach captures basic features of the morphology-induced anisotropy. From a mathematical point of view, the approach is similar to second order estimates like the sequential/iterated homogenization approaches [3, 4].

For linear elasticity, one obtains analytical expressions for the effective stiffness of mixtures of two isotropic phases with isotropic, transversely isotropic, and hexahedral IOD. The estimates are in accordance with wellestablished analytic results such as the Hashin-Shtrikman bounds and Hill's findings for phases with equal shear moduli. Further, at extremal volume fractions, the behavior of the IOD approach is converse to that of the self-consistent approach: both approaches coincide up to first order with complementary and mixed Hashin-Shtrikman-bounds.

For hexahedral IOD, the approach is compared to RVE simulations, where different microstructures with equal IOD are considered. We have found a reasonable agreement for phases with shear moduli of similar magnitude and for microstructures in which both phases percolate the material. Within a sequence of similar microstructures, the error attains a local minimum close to the point of equal arrangement of phases, which is at a phase mixture of $50 \% / 50 \%$.
\end{abstract}

\section{References}

[1] G. Francfort, F. Murat. Homogenization and optimal bounds in linear elasticity. Arch. Ration. Mech. Anal. 94 (1986), 307-334.

[2] R. Glüge, J. Kalisch. The effective stiffness and stress concentrations of a multi-layer laminate. Compos. Struct. 111 (2014), 580-586.

[3] A. Braides, D. Lukkassen. Reiterated homogenization of integral functionals. Math. Mod. Meth. Appl. S. 10 (2000), 47-71.

[4] G. deBotton, I. Hariton. High-rank nonlinear sequentially laminated composites and their possible tendency towards isotropic behavior. J. Mech. Phys. Solids 50 (2002), 2577-2595. 


\title{
Continuum modeling of material interfaces and surfaces based on molecular statics computations
}

\author{
Christian Sievers, Jörn Mosler \\ Institute of Materials Research, Materials Mechanics, Helmholtz-Zentrum Geesthacht
}

The effective macroscopic properties of a broad variety of different materials are defined by the properties of the involved material interfaces and surfaces. However, in contrast to classical bulk materials, the mechanical properties of material interfaces can usually only be determined indirectly. For that purpose, two different approaches are presented which allow to compute macroscopic properties of material interfaces based on molecular statics computations. While the first of those is based on the principle of energy equivalence, the second one relies on the principle of stress equivalence. The advantages and disadvantages of both frameworks are analyzed. 


\title{
A multiscale contact homogenization approach for hysteresis friction of rubber on rough surfaces
}

\author{
Paul Wagner $^{1}$, Peter Wriggers ${ }^{1}$, Corinna Klapproth ${ }^{2}$, Corinna Prange ${ }^{2}$ \\ ${ }^{1}$ Institute of Continuum Mechanics, Hannover \\ email: wagner@ikm.uni-hannover.de,wriggers@ikm.uni-hannover.de \\ ${ }^{2}$ Continental Reifen Deutschland GmbH, Hannover \\ email: corinna.klapproth@conti.de,corinna.prange@conti.de
}

\begin{abstract}
Understanding the frictional behaviour of elastomers on rough surfaces is of high practical importance in many industrial applications. For example the traction of a tire is directly linked to the material properties of the considered elastomer and the surface conditions of the road track [1]. One goal of our studies is to gain a deeper understanding of the underlying contact physics at all length scales. Another aim is to determine a macroscopic coefficient of friction for varying material and surface properties and to validate the results with experimental data.

One of the main physical aspects of rubber friction on rough surfaces is the internal energy dissipation due to cyclic loading and unloading, called hysteresis. This effect is mainly influenced by the micro roughness of the surface [2]. To capture all details and information down to microscale at acceptable computational costs it becomes necessary to incorporate all physical aspects into a multiscale framework. Some multiscale approaches for sliding rubber samples are presented in $[3,4]$. In this study a new FEM multiscale approach for rubber friction on rough rigid road surfaces is suggested focusing on hysteresis effects.

For modelling rubber hysteresis a finite linear viscoelastic material model containing a series of maxwell elements is used. With a spectral analysis of the considered road surface a decomposition into a micro- and macro-roughness is applied. The pressure- and velocity-dependent friction law gained from homogenized micro calculations is then incorporated at the macro scale in the FEM formulation.

We will present the main features of the multiscale framework. Furthermore a numerical validation of the method will be shown for an artificial example. The method is also applied to a measured rough surface showing the agreement of measured and calculated coefficients of friction for two different rubber materials.
\end{abstract}

\section{References}

[1] K.A. Grosch. The relation between the friction and viscoelastic properties of rubber. Proceedings of the Royal Society of London A. 274 (1963), 21-39.

[2] B.N.J. Persson. Theory of rubber friction and contact mechanics. Journal of Chemical Physics 115 (2001), $3840-3861$.

[3] P. Wriggers, J. Reinelt. Multi-scale approach for frictional contact of elastomers on rough rigid surfaces. Comput. Methods Appl. Mech. Engrg. 198 (2009), 1996-2008.

[4] I. Temizer, P. Wriggers. A multiscale contact homogenization technique for the modeling of third bodies in the contact interface. Comput. Methods Appl. Mech. Engrg. 198 (2008) 377-396. 


\title{
Reduced order nonlinear homogenization of composites with cohesive interfaces
}

\author{
Matthias Leuschner and Felix Fritzen \\ YIG Computer Aided Material Modeling, Karlsruhe Institute of Technology \\ transition to: Efficient Methods for Mechanical Analysis (EMMA) Research Group, \\ University of Stuttgart
}

The mechanical behavior of composite materials is often strongly influenced by the constitutive properties of the interface between the individual phases. For example, brittle or ductile decohesion of particle or fiber reinforcements can occur which may (i) influence the effective material response directly and (ii) trigger secondary deformation processes in the surrounding phases (fiber breakage, formation of localization bands). Therefore, the consideration of the interfaces of the materials is important for the homogenization of nonlinear materials.

In order to homogenize the mechanical behavior of solids with nonlinear interfaces we propose a reduced order model based on the pRBMOR for nonlinear solids [1,2]. A low-dimensional basis of global ansatz functions is used to parameterize the displacement jump on the cohesive interface. Micromechanical considerations help to condense out the mechanical equilibrium equations in the bulk material. However, the cohesive tractions on the interface have to satisfy the local constitutive model which is assumed to belong to the class of SD-CZ [3]. A variational principle is used to derive a minimization problem delivering necessary conditions that can be interpreted in terms of a constitutive equilibrium in the weak sense. The resulting method is computationally efficient and has low memory requirements.

The reduced order model is applied to three-dimensional microstructures with imperfect interfaces. The accuracy of the predictions is examined and the influence of the choice of the training data for the basis identification is quantified. A comparison to kernel-based interpolation methods is carried out [4]. Future developments are briefly sketched in order to illustrate possible extensions and their significance for realistic multiscale simulations.

\section{References}

[1] F. Fritzen, M. Leuschner. Reduced basis hybrid computational homogenization based on a mixed incremental formulation. Comput. Methods in Appl. Mech. Eng. 260 (2013), 143-154.

[2] F. Fritzen, M. Hodapp, M. Leuschner. GPU accelerated computational homogenization based on a variational approach in a reduced basis framework. Computs in Method. Appl. Mech. Eng. 278 (2014),186-217.

[3] M. Leuschner, F. Fritzen, J.A.W. van Dommelen, J.P.M. Hoefnagels. Potential-based constitutive models for cohesive interfaces: Theory, implementation and examples. Composites Part B: Engineering 68 (2015), $38-50$.

[4] F. Fritzen, M. Leuschner. Nonlinear reduced order homogenization of materials including cohesive interfaces. submitted manuscript (2015). 


\title{
Use of Gurtin-Murdoch model in predicting effective properties of nano-composites with application to nanoporous gold
}

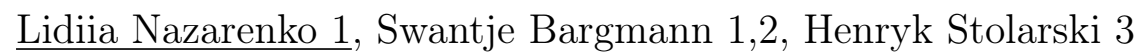 \\ Institute of Continuum Mechanics and Material Mechanics, TU Hamburg, Germany 1 \\ Institute of Materials Research, Helmholtz-Zentrum Geesthacht, Germany 2 \\ Department of Civil, Environmental and Geo- Engineering, University of Minnesota, USA 3
}

In this contribution a mathematical model accounting for matrix/inclusion interface effects and used in evaluation of the effective bulk modulus of random nanocomposites [1] is modified and applied to evaluation of the effective shear modulus. To this end the method of conditional moments [2] is combined with a new notion of the energy-equivalent inhomogeneity and used to predict the effective properties of a nanocomposite with randomly distributed nanoparticles. The interface effect is described via complete Gurtin-Murdoch equations [3], which requires proper identification of the energy associated with the surface gradient of displacements present in those equations. This issue constitutes the main focus in the proposed presentation.

With the help of interface energy accommodating all terms of Gurtin-Murdoch equations the real system, consisting of the inhomogeneities and their surfaces possessing different properties and residual stresses, is replaced by energy-equivalent inhomogeneities with modified bulk properties which incorporate the interface effects. Such an approach replaces the problem with interface effects by the problem without such effects, but with modified properties of the inhomogeneities. The modified properties of the energy-equivalent inhomogeneity are obtained assuming that - for the uniform state of strains within the inhomogeneity - its energy is equal to the sum of the energies of the unmodified inhomogeneity and the energy of the interface. This approach is particularly suitable in the context of the method of conditional moments used here for determination of the effective stiffness tensor of the material, as it assumes that the state of deformations within the inhomogeneities is uniform. Closed-form expressions for the effective moduli of a composite consisting of a matrix and randomly distributed spherical nanoinhomogeneities are derived for both the bulk and the shear moduli. Dependence of those moduli on the radius of nanoparticles is included in these expressions.

The presented approach is adopted to the investigation of porous open cell structures and applied for prediction of effective properties of nanoporous gold accounting for surface effects. The effective Young's moduli of nanoporous gold are analyzed for varying volume content of the nanocavities and for two different values of the stiffness tensor of the reference medium (needed in method of conditional moments). The respectable degree of agreement between the experimental results and those obtained by the method of conditional moments in combination with energy-equivalent inhomogeneity approach illustrates versatility of the proposed method and its viability in analysis of nanomaterials of various structures, including materials with porous open cell structure.

\section{References}

[1] L. Nazarenko, S. Bargmann, H. Stolarski. Influence of interfaces on effective properties of nanomaterials with stochastically distributed spherical inclusions. Int. J. Solids Struct. 51 (2014), 985-997.

[2] L. Nazarenko, L. Khoroshun, W.H. Müller, R. Wille. Effective thermoelastic properties of discrete-fiber reinforced materials with transversally-isotropic components. Continuum Mech. Thermodyn. 20 (2009), 429-458.

[3] M.E. Gurtin, A.I. Murdoch. A continuum theory of elastic material surfaces. Arch. Rational Mech. 4 (1975), $291-323$. 


\section{S09: Flows and transition}

This section will focus on the analysis and modeling of transition from laminar to turbulent flow using DNS, LES, RANS equations, and experiments.

Contributions are expected in, but not limited to, the following topics: stability of incompressible and compressible flows, fundamental study of the dynamics of transition, influence of the wall roughness on transition, transition modelling for LES and RANS equations, transition in flows with complex geometries, subcritical transition. 


\title{
Stability analysis of the flow past miniature vortex generators in a Blasius boundary layer
}

\author{
L. Siconolfi ${ }^{1}$, S. Camarri ${ }^{1}$ and J. H. M. Fransson ${ }^{2}$ \\ ${ }^{1}$ University of Pisa \\ ${ }^{2}$ Linne Flow Centre, KTH Mechanics
}

Transition delay from laminar to turbulent regime in a boundary layer (BL) is a subject of fundamental importance for its direct connection with the reduction of friction drag. Considering a Blasius BL, for external disturbances with low intensity, the transition scenario is dominated by exponentially growing plane waves, known as Tollmien-Schlichting (TS) waves. In the literature, it has been shown that TS waves can be damped and transition delayed by means of a proper modulation of the streamwise velocity in the BL, commonly called streaks (see, for instance, [1] and [2]). The streaks are low- and high-speed regions inside the BL, typically obtained with surface mounted vortex generators in order to activate the lift-up mechanism.

Among various configurations for obtaining streaks in a BL, the use of small winglets, commonly referred to as miniature vortex generators (MVGs), has shown to lead to the formation of streaks with good characteristics, as documented in [3]. This type of BL control is experimentally investigated in [4] (see also [5]), where a spanwise array of MVGs is installed on a flat plate in order to quantify its stabilizing effect on the evolution of the TS waves, generated upstream of the MVGs. For all the experimental configurations documented in [4], the TS waves undergo an amplification in the near wake of the MVGs but, moving further downstream, the TS wave amplitude shows different behaviours depending on the experimental set-up. For some configurations (e.g. C01 and C11 in [4]), the experiments show a monotonic decrease of the TS wave amplitude along the flat plate. For other configurations (e.g. C09 and C05 in [4]), the damping of the TS waves ends at a given distance from the MVGs and, further downstream, the amplitude of the TS wave starts to grow again. The aim of this work is to propose an interpretation of these different behaviours based on the investigation of the stability properties of the controlled BL.

Some representative experimental set-up are selected among all the configurations documented in [4] and simulated by Direct Numerical Simulations (DNSs) in order to have highly resolved velocity fields in space. The DNS results, validated against the experimental data, are then used in the Bi-Global stability analysis in order to estimate the neutral stability curves for the controlled cases. The stability curves are discussed in comparison with the one of the uncontrolled Blasius BL and they are shown to be in good agreement with the available experimental observations, thus providing an interpretation for the behaviour of TS waves. Finally, the results from the stability analysis are used to characterize further the stabilization mechanism induced by the MVGs.

\section{References}

[1] C. Cossu, L. Brandt. On Tollmien-Schlichting-like waves in streaky boundary layers. Eur. J. Mech./B Fluids 23 (2004), 815-833.

[2] J. H. M. Fransson, L. Brandt, A. Talamelli, C. Cossu. Experimental study of the stabilization of Tollmien-Schlichting waves by finite amplitude streaks. Phys. Fluids 17, 054110, (2005).

[3] J. H. M. Fransson, A. Talamelli. On the generation of steady streamwise streaks in flat-plate boundary layers. J. Fluid Mech 698 (2012), 221-234.

[4] S. Shahinifar, J. H. M. Fransson, S. Sattarzadeh, A. Talamelli. Scaling of streamwise boundary layer streaks and their ability to reduce skin-friction drag. J. Fluid Mech 733 (2013), 1-32.

[5] S. Shahinifar, S. S. Sattarzadeh, J. H. M. Fransson, A. Talamelli. Revival of Classical Vortex Generators Now for Transition Delay. Phys. Rev. Lett. 109, 074501, (2012). 


\title{
P-norm optimal 3D perturbations in the Poiseuille flow
}

\author{
Mirko Farano $^{1,2}$, Stefania Cherubini ${ }^{2}$, Jean-Christophe Robinet ${ }^{2}$ and Pietro De Palma ${ }^{1}$ \\ ${ }^{1}$ DMMM, Politecnico di Bari, Via Re David 200, 70125 Bari, Italy \\ ${ }^{2}$ DynFluid Laboratory, Arts et Metiers ParisTech, \\ 151 Boulevard de l'Hopital, 75013 Paris, France
}

The fastest route to turbulence in a subcritical $\left(R e=4000 \leq R e_{c}=5772.2\right)$ plane Poiseuille flow is investigated by means of a Lagrange-multiplier direct-adjoint optimization process in order to find the threedimensional perturbative velocity field that provides the largest perturbation growth at a given time horizon $\left(T_{o p t}\right)$. In particular, spatially localized three-dimensional optimal perturbations are sought, like the ones that can be found including non-linear terms in the optimization process $[1,2]$. In this work, we aim at obtaining a similar localization also in a linear framework by a suitable choice of the objective function, as proposed in [3] for the same flow case in a two-dimensional framework. Instead of the classical $L^{1}$ norm of the perturbative energy density, we use as objective function a general $L^{p}$ norm that provides localization of the optimal perturbation even in a linear framework.

We focus our attention on the small time scale (typical of the Orr mechanism), choosing a short optimization time, and on moderate initial energies $E_{0}$, in order to avoid the triggering of strong non-linear effects, and thus allow a comparison between the non-linear 1-norm optimisation and the linear p-norm one. For $T_{\text {opt }}=10$ and $E_{0}<2 \cdot 10^{-6}$ the 1-norm non-linear optimal shows a weaker localization than the corresponding linear p-norm one. However, the linear optimal vortices are streamwise-aligned, in contrast with the non-linear ones [1]. For a given initial energy, the linear p-norm optimization at $T_{o p t}=10$ provides a disturbance with higher values of the three components of the velocity, which is thus more efficient than the non-linear optimal in inducing transition.

Increasing the target time, the effects of non-linearity on the shape of the optimal perturbations, in terms of streamwise/spanwise modulation and spatial localization of the vortices, begin to be more and more relevant, at high enough energy levels. On the other hand, the p-norm linear optimal appears to be not affected by an increase of the target time. Therefore, for higher target times $\left(T_{\text {opt }} \geq 20\right)$, the 1-norm non-linear optimal provides the most efficient route to transition in terms of time to transition and initial energy.

Finally, the transition mechanisms of the optimal perturbations discussed above are studied by direct numerical simulations. In particular, the p-norm optimal follows a transition path similar to the oblique transition scenario [4], with slightly oscillating streaks, which saturate and then experience secondary instability. Concerning the non-linear optimal, at short times, it is also characterized by a modal composition resembling an oblique wave. However, its time evolution differs from an oblique wave one, since it rapidly forms bent streaks with large amplitude instead of streamwise aligned ones. Non-linear optimals are thus able to provide a faster route to transition by skipping the phase of streak saturation. This study thus provides an analysis of the potential and the limits of p-norm optimisations for obtaining localized optimal structures able to lead to transition even in a linear framework, avoiding the hard computational and storage costs typical of a non-linear optimization approach.

\section{References}

[1] S. Cherubini, P. De Palma, J. C. Robinet, A. Bottaro. The minimal seed of turbulent transition in the boundary layer. Journal of Fluid Mechanics 689:221-253, 2011.

[2] S. Cherubini, P. De Palma. Nonlinear optimal perturbations in a couette flow: bursting and transition. Journal of Fluid Mechanics 716:251-279, 2013.

[3] D. P. G.Foures, C. P. Caulfield, P. J. Schmid. Localization of flow structures using $\infty$-norm optimization. Journal of Fluid Mechanics 729:672-7011, 2013.

[4] P. J. Schmid, D. S. Henningson. Stability and transition in shear flows. Springer, Vol. 142, 2001. 


\title{
Unified description of bifurcation processes associated with laminar boundary-layer separation
}

\author{
Alfred Kluwick, Stefan Braun \\ Vienna University of Technology, \\ Institute of Fluid Mechanics and Heat Transfer, \\ Getreidemarkt 9, 1040 Vienna
}

\begin{abstract}
The presentation will concentrate on flows where no steady state exists if an appropriately defined controlling parameter exceeds a critical value while non-uniqueness is observed for subcritical values of this parameter. Special attention is placed on flow phenomena which are associated with the passage through criticality. Based on a triple deck analysis it found that they can be decribed as solutions of differential equations of Fisher type which are better known from evolution studies of gene populations. Special examples which will be discussed include 2D marginally separated flows, weakly 3D transonic flows in slender channels and fully 3D subsonic flow past expansion ramps.
\end{abstract}




\title{
Investigation of the roughness-induced transition: linear and non-linear optimal perturbations
}

\author{
J.-Ch. Loiseau ${ }^{1,2}, \underline{\text { S. Cherubini }}^{2}$, P. De Palma ${ }^{1}$ and J.-Ch. Robinet ${ }^{2}$ \\ ${ }^{1}$ Politecnico di Bari, Bari, Italy \\ ${ }^{2}$ DynFluid, Arts et Métiers ParisTech, Paris, France
}

For small amplitude disturbances and supercritical Reynolds numbers, linear stability theory predicts the slow transition of flat plate boundary layer flows as a result of the generation, amplification and secondary instability of Tollmien-Schlichting (TS) waves. Fransson et al. [1] have shown that the streaks induced by a three-dimensional roughness element placed on the flat plate can stabilize these TS waves. Unfortunately, beyond a given amplitude of the roughness element, the resulting flow can transition right downstream the roughness element. Such transition past cylindrical roughness elements has been investigated by Loiseau et al. [2] in the framework of global stability where it has been shown that transition could be explained by a global instability of the flow. Despite the plausible explanation it provides, this study does not rule out the possibility for the flow to experience bypass transition wherein the flow transitions to turbulence despite all the modes of the linearised Navier-Stokes operator being linearly stable. Such transition is related to the nonnormality of the linearised operator: small disturbances can experience a large transient amplification due to constructive interferences of non-orthogonal stable modes. If the growth is sufficiently large, such disturbances can trigger non-linear effects allowing them to self-sustain, resulting in bypass transition to turbulence. It is the computation and investigation of such perturbations that we are addressing in the present work using the framework of linear and non-linear optimal perturbation analysis.

Provided a linearly stable base flow $\mathbf{U}_{b}$, the aim of this work is to compute the linear and non-linear velocity perturbations $\mathbf{u}$ optimizing the objective functional $\mathcal{J}(\mathbf{u})=\frac{1}{2} \int_{V} \mathbf{u}(T) \cdot \mathbf{u}(T) \mathrm{dV}$. That is, we look for the perturbation at time $t=0$ which provides the maximum value of the objective functional at a given target time $t=T$. The optimization problem is subject to a set of partial differential constraints, i.e. the perturbative (linear or non-linear) Navier-Stokes equations, that must be verified at each time and each point of the computational domain. A constraint on the initial value of the perturbation's kinetic energy is also imposed, i.e. $E(0)=E_{0}$. These optimal perturbations are computed using a Lagrange multipliers technique which involves the adjoint Navier-Stokes equations. For the particular flow configuration considered, once spatially discretised, the optimisation problem involves almost 100 millions of degrees of freedom. To solve it, a rotation-update gradient-based method [3] is used.

The linearly stable flow investigated is the same as in [1] and [2]. The base flow consists in a horseshoe vortex wrapped around the cylindrical roughness element, whose legs give birth further downstream to streamwise velocity streaks. A central low-speed region is also generated in the wake of the roughness element due to the blockage it induces. Linear optimal perturbation analyses have shown that, despite the flow being linearly stable, infinitesimal perturbations can have their energy amplified by a factor $10^{6}$ over a time interval $T \simeq 55$ before decaying due to the linearly stable nature of the flow. While the optimal perturbation consists in alternated patches of velocity oriented against the base flow shear and localized in the vicinity of the roughness element, the optimal response is a wavepacket of velocity patches, now oriented along the base flow shear, that has travelled further downstream. The underlying amplification mechanisms have been investigated using the Reynolds-Orr equation which governs the transfer of energy between the base flow $\mathbf{U}_{b}$ and the perturbation $\mathbf{u}$. This analysis has revealed that the perturbation bases its transient amplification on two different mechanisms: ( $i)$ the Orr mechanism at short times $(t<40)$, and $(i i)$ the lift-up effect at larger times $(t>40)$. How nonlinearity influences the optimal perturbation and associated response, as well as the energy extraction process, is currently under investigation with the computation of the corresponding non-linear optimal perturbations.

\section{References}

[1] J.H. Fransson, L. Brandt, A. Talamelli and C. Cossu. Experimental study of the stabilization of Tollmien-Schlichting waves by finite amplitude streaks. Phys. Fluids 17 (2005), 05110.

[2] J.-Ch. Loiseau, J.-Ch. Robinet, S. Cherubini and E. Leriche. Investigation of the roughness-induced transition: global stability analyses and direct numerical simulations. J. Fluid Mech. 760 (2014), 175 -211.

[3] D.P.G. Fourès, C. Caulfield and P. Schmid. Localization of flow structures using $\infty$-norm optimization. J. Fluid Mech. 729 (2013), $672-701$. 


\title{
Extreme event detection in near-wall turbulence using reflection-encoded readout of micropillar arrays
}

\author{
F. Hegner1, V. Mikulich1, B. Axtmann2, U. Rist2, Ch. Brücker1 \\ Technical University Freiberg, 1 \\ University Stuttgart, 2
}

This contribution describes a method to detect specific events in the near-wall turbulence with micropillar arrays. Such events are e.g. the recently discovered rare backflow events, which have been so far not been detected using state-of-the art techniques to measure the velocity close to the wall such as hot-wire or LDA probes [1]. This is because the sensing volume needs to be located away from the wall, and the frequency of occurrence drastically reduces with distance from the wall. Second, in order to capture the strong velocity gradients within the viscous sublayer, the diameter of the measurement volumes has to be as small as the viscous length scale, which brings along a drastic reduction of the sampling rate [1]. In comparison, flexible micropillar structures tailored for the specific flow conditions can provide distributed quantitative measurements of the WSS in sufficient spatial and temporal resolution [2]. So far, the recordings are interpreted in a post-processing routine to obtain the WSS field and no direct read-out is possible. The present paper uses a new technique to detect these events in a quasi online manner. Therefore, the pillars are tilted in streamwise direction and have a rectangular front-wall which is reflective coated. If the array is illuminated with parallel light from the side, the reflected light spots are of maximum intensity at certain bending loads. The recording camera can be set in such a position that these reflections disappear at zero load which signals the appearance of critical points in the flow [3]. A camera records these dark spots on-line and allows to record these rare backflow events. Since those events are correlated with strong vortices in the log-layer the sensors can be used for flow control purposes. The calibration of the system is done in a planar Couette flow facility at different shear rates. Measurements are shown for well defined disturbance patterns in the flow and are compared with theoretical response behavior in unsteady flow conditions.

\section{References}

[1] P. Lenaers, Q. Li, G. Brethouwer, P. Schlatter, R. Örlü. Rare backflow and extrem wall-normal velocity fluctuations in near-wall turbulence. Physics of Fluids 24 (2012), 035110.

[2] Ch. Brücker. Signature of varicose wave packets in the viscous sublayer. Phys. Fluids 20 (2008), 061701

[3] J. Cardesa, J. Monty, J. Soria, M. Chong. Skin-friction critical points in wall-bounded flows. 1st Multiflow Summer Workshop, IOP Publishing, Journal of Physics: Conference Series 506 (2014), 01200 


\title{
Spreading of Linear Disturbances in Boundary Layers at Mach Number Five and the Effect of Wall Cooling
}

\author{
Andreas Jocksch ${ }^{1}$, Leonhard Kleiser \\ Institute of Fluid Dynamics, ETH Zurich, 8092 Zurich, Switzerland; ${ }^{1}$ now with \\ CSCS - Swiss National Supercomputing Centre, Via Trevano 131, 6900 Lugano, Switzerland
}

In this contribution we focus on inviscid linear stability eigensolutions of zero pressure gradient flat plate boundary layers at Mach number $M=5$ without and with wall cooling. The spreading of linear disturbances in these boundary layers is investigated and its relation to the transition process is discussed.

The undisturbed stationary, two-dimensional base flow is obtained from the boundary layer equations for a perfect gas with a ratio of specific heats $\gamma=1.4$ and a constant Prandtl number of $\operatorname{Pr}=0.72$. Sutherland's law for viscosity with a constant $S u=S u^{*} / T_{\infty}^{*}=110 \mathrm{~K} / 293 \mathrm{~K}$ is applied where $T_{\infty}^{*}$ is the freestream temperature and $*$ denotes dimensional values. The Dorodnitsyn-Howarth transformation is applied to the boundary layer equations and the resulting ordinary differential equation system is solved with a shooting method.

At $M=5$ inviscid instabilities are present in the boundary layer [1] which we compute with a local eigenvalue solver based on a shooting method. For the singularity (inflection point) a contour deformation in the complex plane is made. The contour deformation is also applied to the baseflow where the same shooting method is employed.

The resulting dispersion relation $\omega(\alpha)$, where $\omega$ is the frequency and $\alpha$ is the wavelength, is evaluated at $M=5$. The first mode - equivalent to the Tollmien-Schlichting mode in incompressible flow - shows its largest growth in the oblique direction. Higher two-dimensional modes are present as well. Two-dimensional second-mode instabilities show the largest growth rates. Wall cooling damps the first mode and amplifies higher modes. For a wall temperature $T_{w}$ that equals the freestream temperature, the first mode is no longer present and higher modes have merged [1].

We use the method of steepest descent [2] to determine the spreading of local disturbances. The solutions are wave packets described by rays $x / t=\partial \omega / \partial \alpha\left(\partial^{2} \omega / \partial \alpha^{2} \neq 0\right)$ of constant growth $\alpha_{i} x / t-\omega_{i}$ in the limit of time $t \rightarrow \infty$. Since the method provides the solution for this limit and inviscid instabilities are eigensolutions far downstream in the boundary layer (also in the limit $t \rightarrow \infty$ ) the method appears to be well suited for the problem.

In the case of the adiabatic wall we analyse modes 1 and 2 separately with the method of steepest descent. For every ray of the resulting wave packet we take the solution with the largest growth. For the streamwise direction, the solution of mode 2 shows higher growth rates throughout than the packet of mode 1 . Thus mode 1 is negligible compared to mode 2 , and mode 2 dominates the wave packet.

The amplification of higher modes by wall cooling with $T_{w}=T_{\infty}$ results in wave packets of larger extent. The wave packet has the special property of very high wave numbers at the trailing edge which propagates slowly.

Although the results clearly show that the boundary layers are convectively unstable, we interpret the slow trailing edge of the packet for wall cooling as a trend towards absolute instability. It appears that for the cooled wall the transition properties become close to those resulting from an absolute instability. This can be seen in turbulent spots which become highly elongated in the streamwise direction for cooled walls [3]. Since the propagation velocity of the turbulence is always larger or equal to the spreading of linear disturbances [2], we see the strong elongation of the spots as a result of the linear stability properties and the large streamwise spreading rates. Long elongated structures are known from absolutely unstable flows like the rotating disc boundary layer where stationary streamwise vortical structures are observed in the transitional zone.

\section{References}

[1] L. M. Mack. Boundary-layer linear stability theory. Technical report AGARD No. 709, AGARD, Neuilly sur Seine, France (1984).

[2] W. van Saarlos. Front propagation into unstable states. Phys. Rep. 386 (2003), 29-222.

[3] A. Jocksch, L. Kleiser. Growth of turbulent spots in high-speed boundary layers on a flat plate. Int. J. Heat Fluid Fl. 29 (2008), 1543-1557. 


\title{
Receptivity and non-uniqueness of turbulent boundary layer flows
}

\author{
Bernhard Scheichl \\ Institute of Fluid Mechanics and Heat Transfer, Vienna University of Technology, Austria \\ $\mathrm{AC}^{2} \mathrm{~T} \mathrm{GmbH}$ (Austrian Center of Competence for Tribology), Wiener Neustadt, Austria
}

This contribution ties in with the established asymptotic theory of boundary layer stability in the limit of large globally formed Reynolds numbers, Re, surveyed e.g. in [1]. In particular, the asymptotically correct description of the propagation and excitation of Tollmien-Schlichting (TS) waves in a (two-dimensional) laminar boundary layer by spatially isolated events, as typically an oscillating surface protuberances, and/or acoustic and vortex waves in the exterior flow lays the foundation of what has been referred to as receptivity theory.

As an important result of the analysis, the boundary layer is highly susceptible to downstream-growing instabilities that originate in weakly unstable modes referring to the vicinity of the lower branch of the neutral curve in the $R e$-wavenumber plane. Hence, these modes finally trigger the classical scenario of laminar-turbulent transition. Upstream of this event, the aforementioned rigorous stability and receptivity theory applies in its currently available, advanced form, which could explain many interesting phenomena with great success [1].

Interestingly, the initiation of TS waves and finally their impact on transition by distributed rather than localised disturbances has not gained much awareness, probably due to the associated mathematical difficulties. Here surface roughness forms the, from an engineering point of view, probably most important and typical example. By the same token, one may ask how a more-or-less developed and nominally two-dimensional and steady turbulent boundary layer reacts on such perturbations. This question is even more interesting as it has been shown quite recently that a fully developed turbulent boundary layer manifests itself in two distinctly different structures, depending on the driving pressure gradient and upstream conditions, and the transition between those is an issue of their stability against small disturbances. In [2] the associated question of non-uniqueness was clarified for the specific case of self-preserving flows under the strongest adverse pressure gradients found possible; a preliminary study sheds some light on their temporal evolution for relatively long times and associated stability. The asymptotic approach requires to extend the classical Reynolds-averaging to a conditional sampling in an asymptotic sense, i.e. by starting with a time filter of a width depending on the global reference quantities and then suitably straining the non-dimensional time, $t$, in dependence on Re. Let $x$ denote the accordingly non-dimensional streamwise coordinate and $D O(1)=O(1)$ be a measure for the streamwise velocity deficit in the core of the boundary layer. The asymptotic analysis of the accordingly averaged Navier-Stokes equation then allows to express non-unique states of the base flow and their stability and reorganisation by common secular-term elimination for correspondingly long fractions of time in form of the evolution equation

$$
D \partial D / \partial \theta=1-m(\theta) D^{2}+n D^{3}, \quad D=D(\theta, \xi), \quad \theta:=\ln t /(\ln R e)^{2 / 3}, \quad \xi:=x-t^{3 / 4} .
$$

Here the function $m$ accounts for the imposed variation of the external driving pressure and the coefficient $n$ for nonlinearities due to inertia; it might depend on $x$ if surface curvature is taken into account. The distortion of the time is necessitated by the self-similar structure of the base flow. For constant and positive values of $m$, the notations upper/lower branch now refer to the curve in the $(m, D)$-plane that describes unstable/stable steadystate solutions of (1) for accordingly large/small velocity deficits. Near its turning point, including effects of weak spanwise variations of the flow modifies (1) to the well-known Fisher's equation having exciting properties.

We extend the analysis by considering perturbations with wavelengths much smaller than the reference length of the $x$-direction, like the representative TS waves in laminar flow. We then study the receptivity of a turbulent boundary layer not only against pressure disturbances but also small-scale surface waviness. Due to the predominantly inviscid nature of turbulent flow on the scales considered, the analysis differs distinctly from its counterpart applying to laminar boundary layers. Most important, no specific turbulence model is needed.

\section{References}

[1] R. J. Bodonyi. Boundary layer stability - asymptotic approaches. In: Recent Advances in Boundary Layer Theory (ed. A. Kluwick), CISM Courses and Lectures 390, Springer (1998), pp. 49-74.

[2] B. Scheichl, A. Kluwick. Non-unique turbulent boundary layer flows having a moderately large velocity defect: a rational extension of the classical asymptotic theory. Theor. Comput. Fluid Dyn. 27(6) (2013), $735-766$. 


\title{
Axisymmetric flow over a sudden expansion in an annular pipe
}

\author{
Behnaz Beladi, Hendrik C. Kuhlmann \\ Vienna University of Technology, Tower BA/E322, Getreidemarkt 9, 1060 Vienna, \\ behnaz.beladi@tuwien.ac.at
}

Many investigations of the flow over a backward-facing step have been carried out for plane channel flows, see e.g. $[1,2,3]$. Here, we investigate the flow over a sudden expansion in an annular pipe in which the radius of the inner cylinder $R_{i}^{\text {in }}$ decreases suddenly to $R_{i}^{\text {out }}$, whereas the radius of the outer cylinder $R_{o}$ remains constant. The steady axisymmetric flow is computed using primitive variables discretized by a second-order finite-volume method. The resulting algebraic equations are solved by Newton-Raphson iteration, as in [3]. The problem under consideration is an extension of the plane-channel sudden-expansion problem which is recovered in the limit of small radius ratios, i.e. for $\eta^{\text {out }}=R_{i}^{\text {out }} / R_{o} \rightarrow 1$ with a fixed non-zero step height $R_{i}^{\text {in }}-R_{i}^{\text {out }}>0$.

Streamline patterns and properties of the separation zones are presented as functions of the outlet radius ratio $\eta^{\text {out }}$ for a fixed expansion ratio $H / h=2$ and Reynolds numbers in the range $10^{-2} \leq \mathrm{Re} \leq 800$. The Reynolds number at which the flow starts to separate from the outer cylinder decreases as the outlet radius ratio $\eta^{\text {out }}$ decreases. This indicates the flow separation on the outer cylinder is stronger the smaller inner radius $R_{i}^{\text {out }}$ of the outlet is. In fact, for $\eta^{\text {out }}<0.3$ the jet emerging from the narrow upstream annulus into the wider downstream annulus remains attached to the inner cylinder for a long distance downstream of the step. This is accompanied with the separation zone on the outer cylinder being elongated in downstream direction. As result further separation zones arise nested inside the separation zone defined by the streamline separating first from the outer cylinder. Moreover, owing to the intense flow inside the stretched separation zone on the outer wall co-rotating vortices resembling Kelvin's cat's eyes arise inside the separation zone.

\section{References}

[1] D. Barkley, M. G. M. Gomes, R. D. Henderson. Three-dimensional instability in flow over a backward-facing step. J. Fluid Mech. 47 (2002), 167-190.

[2] F. Durst, M. Breuer, G. Biswas. Backward-Facing Step Flows for Various Expansion Ratios at Low and Moderate Reynolds Numbers. Journal of Fluids Engineering. 126 (2004), 362-374.

[3] D. Lanzerstorfer, H. C. Kuhlmann. Global stability of the two-dimensional flow over a backward-facing step. Journal of Fluid Mechanics. 693 (2012), 1-27. 


\title{
The Clebsch transformation and its capabilities towards fluid and solid mechanics
}

\author{
Markus Scholle $^{1}$, Florian Marner ${ }^{1}$ \\ ${ }^{1}$ Heilbronn University, Institute for Automotive Technology and Mechatronics, \\ D-74081 Heilbronn, Germany.
}

In fluid dynamics, Clebsch made use of the representation for the velocity field $\vec{u}$ in terms of three potentials $\Phi, \alpha, \beta$ as

$$
\vec{u}=\nabla \Phi+\alpha \nabla \beta
$$

in order to construct a first integral of the equations of motion in case of an inviscid flow with vortices [1]. Apart from this, he recieved a self-adjoint form of the equations allowing for deriving them from a variational formulation. In later times the Clebsch transformation has been extended towards more general cases, for instance to baroclinic flow [2] by including thermal degrees of freedom. The capability of this method, however, is not restricted to fluid dynamics: Wagner [3] applied this methodical approach to the Maxwell equations in classical electrodynamics and Schoenberg [4] made use of it in quantum theory within the context of a quantization of vortex tubes. He also gave a detailed description of the symmetry group of gauge transformations of the Clebsch potentials. For Calkin [5] the use of Clebsch potential was requiered in order to formulate an variational principle in Magnetohydrodynamics and, finally, by Zuckerwar and Ash [5] Clebsch potentials have been used for the analysis of compressible flow with volume viscosity. Shear viscosity, however, has not been part of their investigations and has, to our best knowledge, not yet been formulated in terms of Clebsch variables.

In his paper [6] Scholle found general rules for the construction of Lagrangians in continuums theory based on symmetry consideration. In this context the mathematical structure of the Clebsch transformations appears as a natural outcome.

It is the aim of this paper to demonstrate how Clebsch variables can be applied to viscous flow on the one hand, leading to a first integral of Navier-Stokes equations as a first example.

As a second example, solid mechanics is considered: by making use of an analogy between vortices in fluid flow on the one hand and dislocations in crystals on the other hand, a dynamic theory of dislocations can be established by using a certain modification of the Clebsch transformation.

Examples are given and further perpectives of the method are discussed.

\section{References}

[1] H. Lamb. Hydrodynamics. Cambridge University Press (1974).

[2] R. Seliger, G.B. Witham. Variational principles in continuum mechanics. Proc. Roy. Soc. A 305. (1968), $1-25$.

[3] H.-J. Wagner. On the use of Clebsch potentials in the Lagrangian formulation of classical electrodynamics. Physics Letters A 292. (2002), 246-250.

[4] M. Schoenberg. Vortex Motions of the Madelung Fluid. Nuovo Cimento 1, (1955), 543-580.

[5] M.G. Calkin. An action principle for magnetohydrodynamics. Can. J. Phys. 41. (1963), 2241-2251.

[5] A. J. Zuckerwar, R. L. Ash. Volume viscosity in fluids with multiple dissipative processes. Phys. Fluids 21. (2009), 033105.

[6] M. Scholle. Construction of Lagrangians in continuum theories. Proc. Roy. Soc. A 460. (2004), 3241-3260. 


\title{
Potential theory application to superfluidic multiphase flows
}

\author{
Iu. V. Brazaluk \\ Dniepropetrovsk National University named after O.Honchar, Dniepropetrovsk, Ukraine
}

Last decades superfluidic flows attract constantly growing interest of investigators in the field of fluid mechanics and cryogenic technologies. Mathematical models of superfluidic flows are relatively simple and belong to well-known and good-investigated ideal fluid flow theory. However there is a sufficient difference between traditional ideal fluid flow and superfluidic flow in plane case, concerning circulation and correspondent lifting force. According to L. Prandtl viscous forces, acting during long time in boundary layer, generate vortices that are moved by flow on enough far distance. At last, flow with zero separated vortex sheet is realized asymptotically in time. However there is not any viscous force in superfluidic flow and, consequently, there is not any mechanism of circulation generation. Thus zero-circulation condition must be prescribed for any plane superfluidic flow instead additional conditions for determination of circulation. Most of usual mathematical models of multiphase flows belong to Eulerian models and are based on some homogenization principle for phase interaction. In contrast to them, only hydrodynamic interaction forces and possibly dynamical forces in non-stationary flows may take place in the considered case. Dynamical forces cannot be so sufficient due to specific constrictions to superfluidic flow exists. Hydrodynamic interaction forces can be analyzed only by Lagrangian approach. Stationary multiphase superfluidic flow is unique case completely driven by hydrodynamic interaction forces. The problem is formulated using velocity potential as Neumann problem for Laplace equation. Due to extremely complex shape of solution domain only boundary element method can be effectively used in this case. Dependences of hydrodynamic interaction force on shape of bodies, their sizes and distances between them are calculated numerically in plane and space cases. The most interesting was problem about superfluidic flow around regular grid of identical bodies. Stability of this system is investigated numerically for the plane case of regularly situated circles. The considered system is stable in longitudinal direction and unstable in transversal direction. 


\section{S10: Turbulence and reactive flows}

The topic of this session is the analysis and modeling of turbulent non-reactive and reactive flows based on DNS, LES, RANS, and experiments.

A special focus is on fundamentals in turbulence, turbulent reactive flows, turbulent multi-phase flows, modeling and simulation of complex turbulent flows, the interface of numerical algorithms, chemical and physical modeling, as well as high-performance computing with its application to turbulence. 


\title{
On the universality of small-scale statistics in turbulence
}

\author{
Jörg Schumacher \\ Technische Universität Ilmenau, D-98684 Ilmenau, Germany
}

The universality of statistical fluctuations of the velocity in different statistically stationary turbulent flows is one of the central questions of turbulence research which goes all the way back to the classical works of Kolmogorov, Obukhov, Taylor and Corrsin. We demonstrated universality recently for three different flow configurations using very high-resolution direct numerical simulations based on spectral methods. It was shown that Reynolds number dependence of normalized second, third and fourth order moments of the kinetic dissipation rate is the same for all three flows and given by a power law

$$
\left\langle\epsilon^{n}\right\rangle=A_{n} R e^{d_{n}} \quad \text { for } \quad n=2,3,4 .
$$

The kinetic energy dissipation rate field contains the velocity gradients and is defined as

$$
\epsilon(\mathbf{x}, t)=\frac{\nu}{2}\left(\frac{\partial u_{i}}{\partial x_{j}}+\frac{\partial u_{j}}{\partial x_{i}}\right)^{2}
$$

Small-scale universality means that the scaling coefficients $d_{n}$ are found to be the same for different flows while the prefactors $A_{n}$ contain all the flow specifics and are not universal. The exponents were predicted for the case of homogeneous isotropic turbulence in Ref. [1]. The scaling of the moments has been obtained in simulations of homogeneous isotropic box turbulence, of a turbulent channel flow and a turbulent convection flow in a closed cylindrical vessel, respectively. The unique scaling behavior of the dissipation rate moments, which is obtained in these flows, is established beyond Reynolds numbers $R e=u_{r m s} L / \nu \sim 100$ with $u_{r m s}$ being the root-mean-square velocity and $L$ the outer system scale. It reflects small-scale universality of the turbulence in the bulk. These relatively small Reynolds numbers of a few hundred mark also the threshold beyond which the statistics of the velocity gradients crosses over from sub-Gaussian or Gaussian to intermittent statistics [2].

The studies can be considered as a change of paradigms. Rather than seeking universality of turbulence in the velocity statistics at large Reynolds numbers, i.e., for an extended inertial cascade range, a detailed analysis of the velocity gradient statistics at the much smaller Reynolds number can unravel important properties of turbulence. The comparison of the scalings was performed in the bulks of the channel and the convection cell. One central point of the future work is to extend this analysis towards the walls and to define new criteria for the determination of the possible breakdown of small-scale universality due to the strong local shear. Our studies showed that an optimal removal of the large-scale flow patterns is essential to investigate the transition to intermittency better and to narrow the range of Reynolds numbers at which the velocity gradients start to follow an intermittent behavior.

\section{References}

[1] V. Yakhot. Probability densities in strong turbulence. Physica D 216 (2006) 166-174.

[2] J. Schumacher, D. Scheel, D. Krasnov, A. Donzis, V. Yakhot, and K. R. Sreenivasan. Small-scale universality in turbulence. Proc Natl. Acad. Sci. USA 111 (2014), 10961-10965. 


\title{
Scaling in turbulent canonical flows - a ubiquitous property of all moments?
}

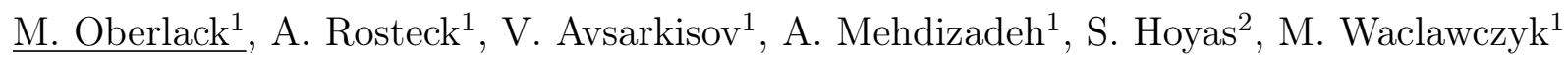 \\ ${ }^{1}$ Chair of Fluid Dynamics, Technische Universität Darmstadt, Germany \\ ${ }^{2}$ CMT Motores Termicos, Universitat Politecnica de Valencia, Valencia, Spain
}

Text-book knowledge proclaims that Lie symmetries such as Galilean transformation lie at the heart of fluid dynamics. These important properties also carry over to the statistical description of turbulence, i.e. to the Reynolds stress transport equations and its generalization, the multi-point correlation equations (MPCE). Interesting enough, the MPCE admit a much larger set of symmetries, in fact infinite dimensional, subsequently named statistical symmetries.

Most important, theses new symmetries have important consequences for our understanding of turbulent scaling laws. The symmetries form the essential foundation to construct exact solutions to the infinite set of MPCE, which in turn are identified as classical and new turbulent scaling laws. Examples on various classical and new shear flow scaling laws including higher order moments will be presented. Even new scaling have been forecasted from these symmetries and in turn validated by DNS.

Turbulence modelers have implicitly recognized at least one of the statistical symmetries as this is the basis for the usual log-law which has been employed for calibrating essentially all engineering turbulence models. An obvious conclusion is to generally make turbulence models consistent with the new statistical symmetries. 


\title{
$\mathrm{NO}_{x}$ formation in premixed flames: a direct numerical simulation study
}

\author{
P. Trisjono, A. Gibelhaus, $\underline{\text { H. Pitsch }}$ \\ Institute for Combustion Technology, RWTH Aachen University, 52056 Aachen, Germany
}

The development of high-fidelity modeling strategies for pollutant formation in turbulent premixed flames is often severely impeded by the lack of reliable data. In this work, a new direct numerical simulation of a lean turbulent methane-air flame at a jet Reynolds number of $\mathrm{Re}=9000$ including complex $\mathrm{NO}_{x}$ and $\mathrm{CO}$ pollutant formation chemistry is presented and used for mathematical and physical analysis and improvement of $\mathrm{NO}_{x}$ models.

In the first part of this talk, two existing $\mathrm{NO}_{x}$ models due to Ihme and Pitsch [1] and Pecquery et al. [2], which have originally been developed for nonpremixed flames, have been reformulated in the context of premixed combustion and evaluated against the DNS database. The concept of optimal estimators [3], which is a powerful mathematical tool for the selection of the set of model parameters and the evaluation of the quality of a model's functional form, is then employed for a systematic error analysis and possibilities to improve both models are discussed. Finally, the implications of the optimal estimator analysis are investigated in a physical analysis of the $\mathrm{NO}_{x}$ chemistry. It is shown that the so called $\mathrm{NNH}$ pathway is significantly affected by turbulence, which in turn can be related to the behavior of the hydrogen radical in the flame.

\section{References}

[1] M. Ihme, H. Pitsch. Modeling of radiation and nitric oxide formation in turbulent nonpremixed Flames using a Flamelet/progress variable formulation. Phys. Fluids. 20 (2008), Art. No. 055110.

[2] F. Pecquery, V. Moureau, G. Lartigue, L. Vervisch, A. Roux. Modelling nitrogen oxide emissions in turbulent flames with air dilution: Application to LES of a non-premixed jet-flame. Combust. Flame 161. (2014), 496-509.

[3] A. Moreau, O. Teytaud, J.-P. Bertoglio. Optimal estimation for large-eddy simulation of turbulence and application to the analysis of subgrid models. Phys. Fluids. 18 (2006), Art. No. 105101. 


\title{
High-resolution LES of the Cambridge stratified burner
}

\author{
Fabian Proch ${ }^{1}$, Pascale Domingo ${ }^{2}$, Luc Vervisch ${ }^{2}$, Andreas M. Kempf ${ }^{1}$ \\ ${ }^{1}$ Institute for Combustion and Gasdynamics (IVG), Chair for Fluid Dynamics, \\ University of Duisburg-Essen, 47048 Duisburg, Germany \\ ${ }^{2}$ CORIA - CNRS, Normandie Université, INSA de Rouen, Technopole du Madrillet, \\ BP 8, 76801 Saint-Etienne-du-Rouvray, France
}

Most state-of-the-art combustion devices feature regions with premixed or partially premixed flames. To understand and optimize such devices, large eddy simulation (LES) has become the best available approach. The modeling of the thin flame front is still challenging though, as its thickness cannot be resolved on practical meshes. A common approach for resolving the flame on the computational grid is by thickening or filtering the flame itself, often combined with tabulated chemistry based on the flamelet assumption [1]-[3].

The presented work shows results from a LES where the grid was strongly refined in order to resolve the flame. This way, a detailed analysis of the flame behavior is possible. Furthermore, the validity of the modeling approaches can be checked. The computed configuration is a turbulent stratified methane/air burner, which has been investigated at the University of Cambridge and the Sandia National Labs. The burner consists of a central bluff body surrounded by two co-annular premixed slots and a co-flow of air. Detailed measurements for velocities, temperature and species concentrations are available [4]-[5]. In the investigated setup, the equivalence ratio takes a value of 0.75 in both slots, the Reynold number is 5,960 in the inner and 11,500 in the outer slot.

Turbulent combustion is modeled with the flamelet generated manifolds approach (FGM), where the chemical state is determined from a lookup-table depending on the local value of the mixture fraction and the progress variable. The table is based on freely propagating one-dimensional flames, which were computed for different initial composition with the reaction kinetics library Cantera. More details on the model can be found in [2]. The grid resolution is 100 microns, which is sufficient to resolve the progress variable field. The computational domain has a size of $112 \times 120 \times 120 \mathrm{~mm}$, leading to a total number of 1.6 billion cells. Computations have been carried out on 64,000 cores on the Blue Gene/Q supercomputer JUQUEEN at Juelich, Germany.

To give a first impression of the behavior of the flame and the flow field, instantaneous contour plots of velocities, temperature, equivalence ratio and species concentrations are presented and analyzed. Afterwards, mean and rms radial profiles are compared against the experimental data to validate the simulation. In the next step, the combustion process and the turbulence-flame interaction is analyzed further: we present PDFs for quantities like flame displacement speed, tangential and normal strain rate and the curvature of the flame, all conditional on the progress variable. To find further potential correlations and dependencies between the relevant quantities, additional JPDFs are generated and discussed.

The authors gratefully acknowledge the computational time on JUQUEEN awarded by the John vonNeumann Institute for Computing, Juelich.

\section{References}

[1] S. Nambully, P. Domingo, V. Moureau, L. Vervisch. A filtered-laminar-flame PDF sub-grid scale closure for LES of premixed turbulent flames. Part I: Formalism and application to a bluff-body burner with differential diffusion. Combust. Flame 161 (2014), 1756-1774.

[2] F. Proch, A. M. Kempf. Numerical analysis of the Cambridge stratified flame series using artificial thickened flame LES with tabulated premixed flame chemistry. Combust. Flame 161 (2014), 2627-2646

[3] R. Mercier, T. Schmitt, D. Veynante, B. Fiorina. The influence of combustion SGS submodels on the resolved flame propagation. Application to the LES of the Cambridge stratified flames. Proc. Combust. Inst. 35 (2015), 1259-1267,

[4] M.S. Sweeney, S. Hochgreb, M.J. Dunn, R.S. Barlow. The structure of turbulent stratified and premixed methane/air flames I: Non-swirling flows. Combust. Flame 159 (2012), 2896-2911.

[5] R. Zhou, S. Balusamy, M.S. Sweeney, R.S. Barlow, S. Hochgreb. Flow field measurements of a series of turbulent premixed and stratified methane/air flames. Combust. Flame 160 (2013), 2017-2028. 


\title{
A Flamelet Progress Variable model for compressible reacting flows
}

\author{
$\underline{\text { A. } \text { Coclite }^{1,2}}$, G. Pascazio ${ }^{1,2}$, P. De Palma ${ }^{1,2}$ \\ ${ }^{1}$ Dipartimento di Meccanica, Matematica e Management \\ ${ }^{2}$ Centro di Eccellenza di Meccanica Computazionale \\ Politecnico di Bari, Via Re David 200, 70125, Bari, Italy
}

\begin{abstract}
The industrial and scientific communities are devoting major research efforts to develop and assess innovative technologies for advanced propulsion systems. Among such technologies, supersonic propulsion systems or cryogenic combustion, based on hydro-carbon oxidation, are considered as a key issue to achieve better propulsive performance, more stable flames and lower environmental impact. Therefore, it is necessary to enhance the knowledge of the combustion phenomena. In this context, the simulation of turbulent reacting flows is very useful to cut down experimental costs and to achieve a thorough comprehension of the physical mechanisms involved. The aim of the present work is to investigate the capabilities of a particular modelling of the presumed Probability Density Function (PDF) needed to evaluate Favre averages in the Flamelet Progress Variable (FPV) approach, analysing its role in the simulation of compressible reacting flows.
\end{abstract}

The model used to study such phenomena is an extension of the standard FPV turbulent combustion model [1], combined with a Reynolds Averaged Navier-Stokes (RANS) equation solver. In the FPV model, all of the thermo-chemical quantities are evaluated by solving transport equations for the mixture fraction $Z$ and a progress parameter $\Lambda$. When using a turbulence model in conjunction with FPV model, a PDF is required to compute statistical averages (e.g., Favre average) of chemical quantities. The choice of such PDF must be a compromise between computational costs and accuracy. State-of-the-art FPV models are built presuming the functional shape of the joint PDF of $Z$ and $\Lambda$. The mixture fraction is widely accepted to behave as a passive scalar with a mono-modal behaviour modelled by a $\beta$-distribution. Moreover, $Z$ and $\Lambda$ are assumed statistically independent so that the joint PDF coincides with the product of the two marginal PDFs. The model employed in this work discards these two constitutive hypotheses and evaluates the most probable joint distribution of $Z$ and $\Lambda$ without any assumption on their behaviour using the Statistically Most Likely Distribution approach [2]. Two flames will be simulated in which the compressible effects are due, to the supersonic inlet velocity of the fuel and oxidizer and, to the real gas effects present in the cryogenic oxidation of hydrogen.

Preliminary results have been obtained for the supersonic $H_{2}$-air combustion proposed by Cheng et al. [3] at the NASA Langley Research Center and for the $\mathrm{LO}-\mathrm{H}_{2}$ cryogenic combustor proposed by ONERA in its test facility MASCOTTE [4]. The latter was developed by ONERA to study fundamental processes which are involved in the combustion of cryogenic propellants, in particular dealing with the super-critical $L O x$ - $H_{2}$ combustion problem. Moreover, the flow through the NASA Langley Center supersonic burner shows a complex shock-expansion structure that is crucial for the stability and efficiency of the combustion process, representing a reference combustor model for the design of modern supersonic burners.

\section{References}

[1] C. D. Pierce, P. Moin, Progress-variable approach for large-eddy simulation of non-premixed turbulent combustion, J. Fluid Mech. 504 (2004) 73-97.

[2] S. Pope, PDF methods for turbulent reactive flows, Prog. Energy Combust. Sci. 11 (1985) 119-192.

[3] T. S. Cheng, J. A. Wehrmeyer, R. W. Pitz, Simultaneous temperature and multispecies measurement in a lifted hydrogen diffusion flame, Combust. Flame 91 (3) (1992) 323-345.

[4] J. Thomas, S. Zurbach, Test case rcm-3: supercritical spray combustion at 60 bar at mascotte, Lampoldshausen, Germany (March 25-27 2001). 


\title{
Assessment of subgrid-scale models for large-eddy simulation of complex flows
}

\author{
Hubert Baya Toda, Karine Truffin, Gilles Bruneaux, Olivier Cabrit, Franck Nicoud \\ IFP Energies nouvelles, 92852 Rueil-Malmaison, France \\ University of Melbourne, Victoria 3010, Australia and CERFACS, Toulouse, France \\ University of Montpellier, UMR CNRS 5149, 34095 Montpellier, France
}

Large-Eddy Simulation (LES) in complex geometries and industrial applications like piston engines, gas turbines, or aircraft engines requires the use of advanced subgrid-scale (SGS) models able to take into account the main flow features and the turbulence anisotropy. Keeping this goal in mind, we report computations of a LESdedicated experiment of a pulsatile hot-jet impinging a flat-plate in the presence of a cold turbulent cross-flow [1]. Unlike commonly used academic test cases, this configuration involves different flow features encountered in complex configurations: shear/rotating regions, stagnation point, wall-turbulence, and the propagation of a vortex ring along the wall. This experiment was designed with the aim to use quantitative and nonintrusive optical diagnostics such as Particle Image Velocimetry, and to easily perform a LES involving a relatively simple geometry and well-controlled boundary conditions. Hence, two eddy-viscosity-based SGS models were investigated: the dynamic Smagorinsky model [2] and the $\sigma$-model [3]. The latter is a static, eddy-viscosity based subgrid-scale model derived from the analysis of the singular values of the filtered velocity gradient tensor $g_{i j}=\partial u_{i} / \partial x_{j}$. It has by construction the property to automatically vanish as soon as the resolved field is either two-dimensional or two-component, including the pure shear and solid rotation cases. In addition, the $\sigma$-model generates no subgrid-scale viscosity when the resolved scales are in pure axisymmetric or isotropic contraction/expansion and has the proper cubic behavior near solid boundaries. It reads [1]:

$$
\nu_{\mathrm{SGS}}=\left(C_{\sigma} \Delta\right)^{2} \frac{\sigma_{3}\left(\sigma_{1}-\sigma_{2}\right)\left(\sigma_{2}-\sigma_{3}\right)}{\sigma_{1}^{2}}
$$

where $\sigma_{1} \geq \sigma_{2} \geq \sigma_{3} \geq 0$ are the three singular values of the filtered velocity gradient tensor $g_{i j}$.

Both models give similar results during the first phase of the experiment (vortex generation and early propagation). However, it was found that the dynamic Smagorinsky model could not accurately predict the vortex-ring propagation after its impingment on the wall, while the $\sigma$-model provides a better agreement with the experimental measurements. Setting aside the implementation of the dynamic procedure (implemented here in its simplest form, i.e., without averaging over homogeneous directions and with clipping of negative values to ensure numerical stability), it is suggested that the mitigated predictions of the dynamic Smagorinsky model are due to the dynamic constant, which strongly depends on the mesh resolution. Indeed, the shear-stress near the wall increases during the vortex-ring impingement leading to a less refined mesh in terms of wall units, $\mathrm{y}+$. This loss of resolution induces a poor damping of the dynamic constant, which is no longer able to adjust itself to ensure the expected y3-behavior near the wall. It is shown that the dynamic constant is never small enough to properly balance the large values of the squared magnitude of the strain-rate tensor, $2 S_{i j} S_{i j}$.

The experimental database is made available to the community upon request to the authors.

\section{References}

[1] H. Baya Toda, O. Cabrit, K.Truffin, G. Bruneaux and F. Nicoud. Assessment of subgrid-scale models with a large-eddy simulation-dedicated experimental database: The pulsatile impinging jet in turbulent cross-flow. Phys. Fluids 26, 075108 (2014)

[2] M. Germano, U. Piomelli, P. Moin, and W. Cabot. A dynamic subgrid-scale eddy viscosity model. Phys. Fluids A 3(7), 1760-1765 (1991).

[3] F. Nicoud, H. Baya Toda, O. Cabrit, S. Bose, and J. Lee. Using singular values to build a subgrid-scale model for large eddy simulations. Phys. Fluids 23(8), 085106 (2011). 


\title{
Assessment of non-linear $k-\epsilon$ turbulence models for the prediction of wind flow around isolated buildings and multiple obstacles
}

\author{
Marc Moonens, Alessandro Parente \\ Aero-Thermo-Mechanics Department, Université Libre de Bruxelles, Bruxelles, Belgium
}

Computational Fluid Dynamics (CFD) is widely used to study flow phenomena in the lower part of the atmospheric boundary layer (ABL), with application to pollutant dispersion, risk analysis, optimization and siting of windmills and wind farms, and microclimate studies. Numerical simulations of ABL flows are often carried out using Reynolds-averaged Navier-Stokes (RANS) in combination with the $k-\epsilon$ turbulence model. The present paper investigates the potential improvement of two-equation turbulence models in the simulation of the flow around bluff bodies, by means of the addition of non-linear terms in the Reynolds stresses closure. The investigation is based on a zonal approach for the interaction between the boundary layer and an obstacle, introduced in [1] and [2]. In particular, the turbulence model formulation is gradually modified from the unperturbed ABL to the wake region: the approaching wind profiles are described using a consistent formulation for neutral ABL flows, whereas a non-linear turbulence model is applied to the regions affected by the presence of the obstacles. Three different variants of non-linear $k-\epsilon$ turbulence models are considered here, two cubic models, hereafter indicated as Craft [3] and Erhard [4], and a quadratic one [5].

Two test cases are considered, the flow around an isolated building and within a regular array of obstacles. Experimental data are obtained from the online repository at the Meteorological Institute of Hamburg University (CEDVAL A1-1, http://www.mi.uni-hamburg.de/Data-Sets.432.0.html and CEDVAL B1-1, http://www.mi.uni-hamburg.de/Category-B.627.0.html). An initial sensitivity study was performed to assess the improvement that can be obtained using the non-linear models and to determine the optimal values of the zonal modeling parameters and of the turbulence model constant $C_{\mu}$. Results on both test cases show that non-linear models can improve the predictions of velocity and turbulent kinetic energy in the wake region, although the typical overestimation of the reattachment length, associated with $k-\epsilon$ turbulence models, is still present. The improvement is more important for the more complex geometrical configurations, while the single building case still presents unsolved challenges. Further investigation are being conducted to determine the relative importance on the predictions of the non-linear terms with respect to the turbulence model parameter, $C_{\mu}$, and to elucidate the effect of the geometry on the modeling approach.

\section{References}

[1] A. Parente, C. Gorlé, J. van Beeck, C. Benocci. Improved k- $\varepsilon$ model and wall function formulation for the RANS simulation of ABL flows. J. Wind Eng. Ind. Aerodyn. (2011a), 99:267-278.

[2] A. Parente, C. Gorlé, J. van Beeck, C. Benocci. A comprehensive modelling approach for the neutral atmospheric boundary layer: consistent inflow conditions, wall function and turbulence model closure. BoundaryLayer Meteoro. (2011b), 140(3):411-428.

[3] T. J. Craft, B. E. Launder, K. Suga. Development and application of a cubic eddy-viscosity model of turbulence. Int J Heat Fluid Flow. (1996), 17(2):108-115.

[4] J. Ehrhard, N. Moussiopoulos. On a new nonlinear turbulence model for simulating flows around buildingshaped structures. J. Wind Eng. Ind. Aerodyn. (2000), 88(1):91-99.

[5] T. H. Shih, J. Zhu, J. L. Lumley. A realizable Reynolds stress algebraic equation model. NASA Tech Memo 105993. 1993. 


\title{
Simulation of wake effects in windfarms using an Actuator Disk implementation
}

\author{
C. Hornung, A. G. Class, M. Viellieber, \\ Karlsruhe Institute of Technology (KIT) \\ Institute for Nuclear and Energy Technologies (IKET) \\ Hermann-von-Helmholtz-Platz 1 \\ 76344 Eggenstein-Leopoldshafen \\ Germany
}

Wind turbines extract energy from the approaching flow field resulting in reduced wind speeds, increased turbulence and a wake downstream of the wind turbine. Wakes extending up to $20 \mathrm{~km}$ downstream of a windfarm are reported [1]. In [2] a multi-scale numerical method is proposed that addresses length scales of the boundary layer, windfarm, and meso-scale by individual representative simulations and two-way coupling. Within this framework the implementation of an Actuator Disk (AD) model into OpenFOAM including a proper atmospheric boundary layer (ABL) condition is presented. For a meso-scale area of open sea, the standard ABL conditions of OpenFOAM can not be used. The pre implemented ABL boundary condition for OpenFOAM is restricted to roughness lengths greater than $0.001 \mathrm{~m}$. The conditions are modified in order to achieve adequate homogeneous flow properties throughout the considered domain, that is $30 \mathrm{~km}$ in length, enabling almost constant velocity profiles. A new AD class is implemented in OpenFOAM to establish an arbitrary number and location of windturbines. The class uses standard $\mathrm{k}-\epsilon$ turbulence model. We investigate the wake effect of a windfarm composed of windturbines with different heights and different lateral spacing. Specifically the windfarm Trianell Borkum in the eastern sea of Germany is simulated.

The discussed simulations are part of the validation of the advanced multi scale approach that intends to simulate complete wind farms in an computational effective way [2]. Within this multi scale approach it is planed to replace the $\mathrm{AD}$ model with a two way coupling scheme that dynamically interpolates between the mentioned length scales. Sub grid forces similar to those of an AD model are implemented within both the wind turbine region and its wake. The multi scale approach and its anticipated advantages are presented.

\section{References}

[1] M. Bruun Christiansen. Wake effects of large offshore wind farms identified from satellite SAR. Remote Sensing of Environment 98 (2014) 251-268.

[2] A. G. Class, M. Viellieber, N. Moussiopoulos, F. Barmpas. Two Way Coupled Micro/Meso Scale Method for Wind Farms. Proceedings in Appl. Maths. Mechs. 14 (2014). 


\title{
Numerical Simulation of Continuous and Pulsed Film Cooling at the leading edge of a symmetrical turbine blade
}

\author{
Nemdili Fadéla, Azzi Abbès and Dellil Ahmed Zineddine \\ Laboratoire Aero Hydro dynamique Navale, LAHN \\ Université des Scienences et de la Technologie d'Oran, USTO, Algeria
}

This paper investigates the effect of the pulsating film cooling at the leading edge of a symmetrical turbine blade model on the film cooling effectiveness. The blade used in the present study is symmetrical with a length of $515 \mathrm{~mm}$ and a maximum width of $72 \mathrm{~mm}$, as seen in Haslinger and Hennecke [1]. The leading edge of the model has one row of holes on each side $(\mathrm{D}=4 \mathrm{~mm})$ with a lateral spacing of $5 \mathrm{D}$. In the streamwise direction, the holes are inclined $110 \mathrm{deg}$. to the surface and located so that the trailing edge of the hole is at s/D $=3.1$, where $\mathrm{s}$ is the length along the blade from the stagnation point and $\mathrm{s} / \mathrm{D}=0$ is the blade stagnation line. In the present investigation, calculations were carried out only for the streamwise injection (without inclination) and an approach velocity of $30 \mathrm{~m} / \mathrm{s}$. The length to diameter ratio of the film holes is 4 and the density ratio is 1 . The present simulations were conducted using the finite-volume code ANSYS-CFX. The numerical finite volume method is used to resolve the Unsteady Reynolds Averaged Navier Stokes Equations coupled at turbulence model. Detailed numerical film cooling effectiveness has been made for both steady (continuous) and pulsating flows. The jet pulsing frequencies of $5 \mathrm{~Hz}, 10 \mathrm{~Hz}$, and $20 \mathrm{~Hz}$ have been studied. The computational domain is discretized using a highly refined multi-bloc structured grid including the plenum area. The Reynolds-stress tensor is approximated within the context of the turbulence model, coupled with a one equation model resolving the near-wall viscosity affected regions. The numerical simulations are conducted for different blowing ratios, ranging from 0.3, to 2.0. Film cooling effectiveness contours on the blade surface and lateral averaged adiabatic film cooling effectiveness are presented, and only the continuous jet results are compared with the available measurements. In addition to the validation data, several longitudinal, transversal contours and vector planes are reproduced. The computations with the standard model reproduce the well-known underpredicted lateral spreading of the jet, and consequently, lower values of the lateral averaged adiabatic film cooling effectiveness has been obtained. It was found mainly that pulsating for low blowing ratios, cold jet decreases the film cooling effectiveness, and for higher blowing ratios 1.5 and 2.0, pulsing increases the film cooling effectiveness, which is enhanced, with no effect of frequency levels.

\section{References}

[1] Haslinger, W., and Hennecke, D. K., High Resolved Distribution of Adiabatic Film Cooling Effectiveness forTurbine Leading Edge Film Cooling, Proc. 13th Int. Symposium Air Breathing Engines, Chattanooga, Tenn., Sept. ISABE 97â7113, 1997, pp 797-806. 


\title{
Coarse-Grid-CFD for the Thermal Hydraulic Investigation of Rod-Bundles
}

\author{
M. Viellieber, A. G. Class \\ Institute for Nuclear and Energy Technologies (IKET) \\ Hermann-von-Helmholtz-Platz 1 \\ 76344 Eggenstein-Leopoldshafen \\ Germany
}

A nuclear reactor core, that is a few meters in height and diameter is composed of hundreds of fuel assemblies which are again composed of tenth of fuel rods with a diameter of about $10 \mathrm{~mm}$. The relevant length scales for a Computational fluid dynamics (CFD) simulations range therefore from the sub- millimetre range, relevant for the sub channels up to several meters. Describing such a multi-scale situation with CFD is extremely challenging and the traditional approach is to use integral methods. These are sub channel and sub assembly analyses codes requiring closure by empirical and experimental correlations. A CFD simulation of a complete nuclear reactor set up resolving all relevant scales requires exceedingly large computational resources. However, in many cases there are repetitive geometrical assemblies and flow patterns. Based on this observation the general approach of creating a parametrized model for a single segment and composing many of these reduced models to obtain the entire reactor simulation, becomes feasible.

With our method, the Coarse-Grid-CFD (CGCFD) ([1] [2]), we propose to replace the experimental or empirical input with proper CFD data. In an application the methodology starts with a detailed, well-resolved, and verified CFD simulation of a single representative segment. From this simulation we extract in tabular form so-called volumetric forces which upon parametrization is assigned to all coarse cells. Repeating the fine simulation for multiple flow conditions parametrized data can be obtained or interpolated for all occurring conditions to the desired degree of accuracy . Note, that parametrized data is used to close an otherwise strongly under-resolved, coarsely meshed model of a complete reactor set up. Implementation of volumetric forces are the method of choice to account for effects as long as dominant transport is still distinguishable on the coarse mesh. In cases where smaller scale effects become relevant the Anisotrop Porosity Formulation (APF) allows capturing transport phenomena occurring on the same or slightly smaller scale compared to the coarse mesh resolution. The typiccal application of the APF are local flow obstructions, flow guiders, spacers, wire wraps, etc.

Within this work we present results of several fuel assemblies, that were investigated with our methodology. In particular, we show thermal hydraulic Coarse-Grid-CFD simulations including a 127 pin LBE cooled wire wrapped fuel assembly. General guidelines for the proper application of CGCFD, limits of its application and potential numerical cost saving are discussed.

\section{References}

[1] M. Viellieber, A. G. Class. ANISOTROPIC POROSITY FORMULATION OF THE COARSE-GRID-CFD (CGCFD). Proceedings of the 2012 20th International Confernece on Nuclear Engineering collocated with the ASME 2012 Power Conference ICONE20-POWER2012.

[2] Viellieber, M and Dietrich,P. and Class, Investigation of a Wire Wrapped Fuel Assembly with the Anisotropic Coarse-Grid-CFD International Journal for Nuclear Power, 2013. 


\title{
Particles in Turbulence: Macro- Consequences from Micro- Interactions
}

\author{
Alfredo Soldati \\ Università degli Studi di Udine
}

Turbulent fluids and small particles or droplets or bubbles are common to a number of key processes in energy production, product industry and environmental phenomena. In modelling these processes, the dispersed phase is usually assumed uniformly distributed. Indeed, it is not. Dispersed phases can be focused by turbulence structures and can have a time-space distribution which barely resembles prediction of simplified averaged modelling.

Preferential distribution controls the rate at which sedimentation and re-entrainment occur, reaction rates in burners or reactors and can also determine raindrop formation and, through plankton, bubble and droplet dynamics, the rate of oxygen-carbon dioxide exchange at the ocean-atmosphere interface.

In this talk, we will review a number of physical phenomena in which particle segregation in turbulence is a crucial effect describing the physics by means of Direct Numerical Simulation of turbulence.

We will elucidate concepts and modeling ideas derived from a systematic numerical study of the turbulent flow field coupled with Lagrangian tracking of particles under different modeling assumptions. We will underline the presence of the strong shear which flavors wall turbulence with a unique multiscale aspect and adds intricacy to the role of inertia, gravity and buoyancy in influencing particle motion.

Through a number of physical examples of practical interest such as boundary layers, free-surface and stratified flows, we will show that a sound rendering of turbulence mechanisms is required to produce a physical understanding of particle trapping, segregation and ultimately macroscopic flows such as surfacing, settling and re-entrainment. 


\title{
An efficient numerical method for fully resolved particle simulations on high-performance computers
}

\author{
Lennart Schneiders, Jerry H. Grimmen, Matthias Meinke, Wolfgang Schröder \\ Institute of Aerodynamics, RWTH Aachen University, \\ Wüllnerstr. 5a, 52062 Aachen, Germany
}

Turbulent particle-laden flows are encountered in many technical and natural environments such as pulverized coal combustors, in atmospheric precipitation, or the transport of pollutants through the human airways. For large-scale simulations of these systems often Lagrangian particle tracking methods are used, in which the particles are traced assuming a point-mass behavior. To validate and improve these models and to enhance the understanding of the multiphase characteristics of these flows so-called fully resolved simulations are applicable. In these simulations, the discrete solution is resolved up to the particle boundary layers such that an accurate formulation of the fluid-particle momentum exchange driving the particle motion is obtained and turbulence modulation effects are captured. For particle sizes comparable to or smaller than the Kolmogorov microscale, this results in a vast increase of the computational costs which are beyond that of a regular direct numerical simulation. To this end, flexible solution schemes are required to efficiently resolve the particles along their trajectories. Numerical methods based on hierarchical Cartesian meshes provide an ideal framework by facilitating dynamic mesh adaptation near the particle surfaces and dynamic load-balancing routines.

In this contribution, a finite-volume method for fully resolved particle simulations on adaptively refined Cartesian meshes is presented. The fluid phase is discretized by a finite-volume scheme with cut-cells at the boundaries [1]. This yields a fully conservative description of the fluid-particle momentum and heat exchange which is essential to obtain accurate and stable results [1]. The particle surfaces are efficiently represented using a level-set formulation by which multiple independently moving and intersecting boundaries can be resolved [2]. The initial mesh generation and the domain decomposition are performed automatically based on Hilbert spacefilling curves [3]. During the simulation, the solution-adaptive mesh refinement strategy described in [1, 4] is used to dynamically resolve the particle boundary layers and wakes. Since the frequent changes to the computational mesh inherently create a strong imbalance of the individual process loads, a new dynamic loadbalancing strategy has been developed to automatically regenerate the domain decomposition and redistribute cells among processes.

\section{References}

[1] L. Schneiders, D. Hartmann, M. Meinke, W. Schröder, An accurate moving boundary formulation in cut-cell methods, J. Comput. Phys. 235 (2013), 786-809.

[2] C. Günther, M. Meinke, W. Schröder, A flexible level-set approach for tracking multiple interacting interfaces in embedded boundary methods, Comput. Fluids. 102 (2014), 182-202.

[3] A. Lintermann, S. Schlimpert, J. H. Grimmen, C. Günther, M. Meinke, W. Schröder, Massively parallel grid generation on HPC systems, Comput. Meth. Appl. Mech. Eng. 277 (2014), 131-153.

[4] D. Hartmann, M. Meinke, W. Schröder, An adaptive multilevel multigrid formulation for Cartesian hierarchical grid methods, Comput. Fluids. 37 (2008), 1103-1125. 


\title{
A stochastic SGS model for Lagrangian particle tracking in large-eddy simulation velocity fields
}

\author{
A. Innocenti ${ }^{1}$, S. Chibbaro ${ }^{2}$, C. Marchioli ${ }^{3}$, A. Soldati ${ }^{3}$ and M.V. Salvetti ${ }^{1}$ \\ University of Pisa, Italy \\ Pierre and Marie Curie University and CNRS, France \\ University of Udine, Italy
}

The dispersion of small inertial particles in inhomogeneous turbulent flows is important in a number of industrial applications and environmental phenomena, such as, for instance, mixing, combustion, depulveration, spray dynamics, pollutant dispersion or cloud dynamics. Direct Numerical Simulations (DNS) of turbulence coupled with Lagrangian Particle Tracking (LPT) have demonstrated their capability to capture the mechanisms characterizing particle dynamics in turbulent flows. Due to the computational requirements of DNS, however, analysis of problems characterized by complex geometries and high Reynolds numbers demands alternative approaches; Large-Eddy Simulation (LES) is increasingly gaining in popularity, especially for cases where the large flow scales control particle motion. LES is based on a filtering approach of the fluid phase governing equations; thus, only the filtered fluid velocity is available for particle tracking and particles are prevented from interacting with the small (unresolved) Sub-Grid Scales (SGS) of turbulence. This may strongly influence clustering of inertial particles and leads to significant underestimation of particle preferential concentration and deposition rates ( see e.g. [1]). Hence, there is currently a general consensus about the need to model the effect of SGS turbulence on particle dynamics.

To address this issue, a new stochastic model for subgrid scales of large eddy simulation of turbulent polydispersed two-phase flows is presented. The model is based on the formalism for the filtered density function (FDF) approach in LES simulations. Contrary to the FDF used for turbulent reactive single-phase flows, the present formalism is based on Lagrangian quantities and, in particular, on the Lagrangian filtered mass density function (LFMDF) as the central concept.

A first example of Langevin model constructed within the above formalism is proposed considering isotropic sub-grid fluctuations, but taking into account crossing-trajectory effects and paying attention to the consistency of the model with the fluid limit case. The results of LES simulations of particle-laden channel flow, carried out with the proposed SGS stochastic model, will be compared against DNS data.

\section{References}

[1] C. Marchioli, M.V. Salvetti, and A. Soldati. Some issues concerning large-eddy simulation of inertial particle dispersion in turbulent bounded flows. Phys. Fluids 20 (2008), 040603. 


\title{
Budget analysis of the kinetic energy for bubbly flows
}

\author{
Claudio Santarelli, Jean Roussel, Jochen Fröhlich \\ Technische Universität Dresden
}

Bubbly flows are a crucial part of many industrial and environmental applications like bubble column reactors and power plants. One of the most important issues in the framework of such flows is the modification of the turbulence of the liquid phase induced by the bubbles. To address this topic a rigorous mathematical formulation was developed and the conservation equations of mass, momentum and kinetic energy of the two-phase mixture were provided to account for the interaction of the two phases [1]. The conservation equation of the kinetic energy $K$ reads

$$
\bar{f} \frac{D K}{D t}=\Pi+\epsilon+C+I,
$$

where $\bar{f}$ is the averaged liquid indicator function, $\Pi$ is the production term accounting for the energy transfer between mean and fluctuating flow and $\epsilon$ the dissipation rate responsible for the conversion of the kinetic energy into thermal internal heat at small scales. The term $C$ collects the transport processes of the energy due to the fluctuating pressure field, the velocity fluctuations (triple correlation) and due to the molecular diffusion. The first three terms of the right hand side of Eq. (1) are commonly referred to as the single-phase terms since they appear also in the conservation equation of $K$ in single-phase flows. The term $I$ is the interfacial term and is characteristic of two-phase flow. It accounts for the energy transfer between the bubbles and the fluid and is a function of the forces at the phase boundary.

In the present work numerical data are employed for the evaluation of Eq. (1) to provide valuable insight into the modification of the fluid flow induced by the bubbles. The data were generated by means of Direct Numerical Simulations where the bubbles are accounted for with an Immersed Boundary Method [2]. Such simulations address the flow of disperse bubbles in turbulent vertical channel and statistical quantities of both phases have already been provided [3,4]. For the investigated regime $K$ is enhanced by the presence of the bubbles with respect to the single-phase flow, and the analysis of Eq. (1) allows a precise quantification of the mechanisms that induce such modification. It is shown that the interfacial term and the production term provide a positive contribution for the transport of $K$ while the dissipation term and the transport term have a negative contribution.

\section{References}

[1] I. Kataoka, A. Serizawa. Basic equations of turbulence in gas-liquid two-phase flow. Int. J. Multiphase Flow 15 (1989), 843-855.

[2] T. Kempe, J. Fröhlich. An improved immersed boudary method with direct forcing for the simulation of particle laden flow. Int. J. Multiphase Flow 231 (2012), 3663-3684.

[3] C. Santarelli, J. Fröhlich, J., Simulation of bubbly flow in a vertical turbulent channel. Proc. Appl. Math. Mech. (2012), 503-504.

[4] C. Santarelli, J. Fröhlich, J., Characterisation of bubble clusters in simulations of upward turbulent channel flow. Proc. Appl. Math. Mech. (2013), 19-22. 


\title{
Asymptotic solutions of an extended Korteweg-de Vries equation describing solitary waves with weak and strong downstream decay in turbulent open-channel flow
}

\author{
Markus Müllner \& Wilhelm Schneider \\ Vienna University of Technology
}

Solitary waves in two-dimensional, turbulent open-channel flow over a bottom with varying roughness are considered. An asymptotic analysis of this problem was first given in [1]. In its steady-state version, the following extended KdV equation for the surface elevation of the wave, subject to homogeneous boundary conditions far up and downstream, respectively, was derived:

$$
H_{1, X X X}+\left(H_{1}-1\right) H_{1, X}=\beta\left[H_{1}-\Gamma(X)\right], \quad H_{1} \rightarrow 0 \text { for } \quad X \rightarrow \pm \infty .
$$

The bottom roughness is varied according to $\Gamma(X)=\Gamma_{L} \varphi(X)$, with the constant $\Gamma_{L}=\mathcal{O}(1)$ and the step function $\varphi(X)$ being 1 in the region of enlarged bottom roughness $0<X<L$, otherwise zero. The dissipation parameter is assumed to be weak, i. e. $\beta \ll 1$. In order to obtain a classical soliton solution, eigensolutions with $\Gamma_{L}=\lambda$ were studied in [1], and "conservation" equations have been used to determine the eigenvalue $\lambda$ and the leading-order inviscid solution. In [2] the problem was solved numerically, and the results obtained for the surface elevations and the eigenvalues are in very good agreement with the asymptotic solutions.

In the present work, the asymptotic analysis is extended to the second order in $\beta$. In addition to the solitons of the classical type, the cases are considered when the roughness parameter differs from the eigenvalue, i. e. $\Gamma_{L} \neq \lambda$, as proposed recently in [3]. In this case the expansions require distinguishing between inner and outer regions. The solution in the outer region far downstream is characterized by a long, but shallow, tail attached to the solitary wave. In the talk, the solutions for the surface elevation, the location of the wave crest, and the minimum value of $\Gamma_{L}$ are discussed. A comparison with the numerical results is also presented.

\section{References}

[1] W. Schneider. Solitary waves in turbulent open-channel flow, JFM 726, 137-159 (2013)

[2] R. Jurisits. Numerical solutions of an extended Korteweg-de Vries equation describing solitary waves in turbulent open-channel flow, submitted to PAMM (2014)

[3] W. Schneider. Analysis of stationary solitary waves in turbulent open-channel flow, submitted to J. Hydr. Eng. (2014) 


\title{
Recent results in isotropic turbulence decay theory
}

\author{
Pierre Sagaut, Vincent Mons, Marcello Meldi \\ M2P2, Aix-Marseille University, France \\ d'Alembert Institute, UPMC, France \\ PPrime, University of Poitiers, France
}

The aim of the present contribution is to summarize recent results obtained by the authors $[1,2,3,4,5,6,7]$ dealing with isotropic turbulence theory, more more precisely with the isotropic turbulence/grid turbulence decay. While classical theories commonly found in usual textbooks bridge between the large-scale asymptotic behavior of turbulence and the features of the algebraic decay regime, several lack of consistency with recent findings have been reported during the last decade.

First, one should notice the discrepancies between experimental and DNS data on the one hand, and most theoretical results on the other hand. This point was emphasized in [3], and no satisfactory explanation is available at present time. A very recent study shows that the departure from exact isotropy that exists in all grid turbulence experiments cannot explain the dispersion of the experimental results [5].

The idea that the decay features are related to asymptotic behavior of the initial solution at very large scales seems to be among the main flaws of most existing theories. As a matter of fact, very recent advances clearly show that turbulence decay is governed by features of scales that are slightly larger than the integral scale of turbulence. This point was illustrated by the possibility of observing non-self-similar solutions over arbitrary long time. But a clear, mathematically grounded demonstration was given in [7], in which a variational data assimilation method based on EDQNM adjoint solution is used to identify governing scales and features of the initial condition.

A second point to be discussed during the presentation is related to the existence of self-similar solutions. While they are mathematically friendly, the physical meaning and the existence of such solutions are more problematic. An EDQNM analysis was conducted, whose main results is that self-similar solutions may exist, but only under very stringent conditions on the initial condition.

\section{References}

[1] Meldi, M., Sagaut, P. (2012) On non-self similar regimes in homogeneous isotropic turbulence decay $J$. Fluid Mech. 711, 364-393

[2] Meldi, M., Sagaut, P. (2013) Pressure statistics in self-similar freely decaying isotropic turbulence J. Fluid Mech. 717, R2, 1-12

[3] Meldi, M., Sagaut, P. (2013) Further insights into self-similarity and self-preservation in freely decaying isotropic turbulence, Journal of Turbulence 14(8), 24-53

[4] Meldi, M., Sagaut, P. (2014) An adaptive numerical method for solving EDQNM equations for the analysis of long-time decay of isotropic turbulence, J. Comput. Phys. 262, 72-85

[5] Mons, V., Meldi, M., Sagaut, P. (2014) Numerical investigation on the partial return to isotropy of freely decaying homogeneous axisymmetric turbulence, Phys. Fluids 26, 025110

[6] Meldi, M., Lejemble, H., Sagaut, P. (2014) On the emergence of non-classical decay regimes in multi-scale/ fractal generated isotropic turbulence J. Fluid Mech. 756, 816-843

[7] Mons, V., Chassaing, J.C., Gomez, Th., Sagaut, P. (2014) Is isotropic turbulence decay governed by asymptotic behavior of large scales ? An EDQNM-based Data Assimilation study, Phys. Fluids 26, 115105 


\title{
Numerical simulation of Dense Gas Compressible Homogeneous Isotropic Turbulence
}

\author{
L. Sciacovelli, P. Cinnella \\ DynFluid Laboratory, Arts et Métiers-ParisTech, Paris, France \\ Dip. di Meccanica, Matematica e Management, Politecnico di Bari, Italy \\ Dip. d'Ingeneria dell'innovazione, Università del Salento, Lecce, Italy
}

Turbulent flows of real gases represent a research field of utmost importance for the wide range of applications. Among them, flows of supercritical fluids, i.e., fluids whose temperature and pressure conditions are higher than the critical ones, are of interest for several industrial and technological purposes, such as chemical extraction/fractionation of natural products, aerospace propulsion or energy production [1]. An interesting family of real gases is represented by the so-called Bethe-Zel'dovich-Thompson (BZT) fluids, heavy polyatomic compounds characterized by a region of negative values of the Fundamental Derivative of Gas Dynamics $\Gamma$ [2], in which non-classical phenomena such as rarefaction shock-waves, mixed shock/fan waves and shock splitting can occur [3].

In this work, the decay of Compressible Homogeneous Isotropic Turbulence (CHIT) for dense gas flows in BZT conditions is analysed. The impact of real gas effects on the evolution of turbulent structures is investigated and compared with CHIT of perfect gas (PFG) flows. In order to perform simulations of high Reynolds number turbulent flows, Implicit Large-Eddy Simulations (ILES) and DNS are performed. ILES approach consists in capturing the energy-containing and inertial ranges of turbulent flows, and relying on the intrinsic or added dissipation of the discretization scheme to drain energy at subgrid scales. A tenth-order accurate centered scheme is used for the discretization of the convective fluxes. The scheme is supplemented by a high-order nonlinear artificial viscosity term, inspired from [5], that is 9th-order accurate in smooth flow regions and becomes 1st-order accurate near flow discontinuities. A Ducros-type sensor [6] is used to make sure to minimize the impact of artificial viscosity on the resolved vortical flow structures. In order to take into account dense gas effects, the simple polytropic Van der Waals (VDW) equation of state (EoS) is used. This is computationally inexpensive compared to more complex thermodynamic models, and provides a reasonable qualitative description of the main effects of interest. The fluid under investigation is a heavy fluorocarbon, namely PP11 $\left(\mathrm{C}_{14} \mathrm{~F}_{24}\right)$, which exhibits BZT effects for thermodynamic conditions near (but outside of) the critical region. The CFD code has been validated against literature results for both inviscid [7] and viscous [8] cases. To initialize the isotropic turbulence field, divergence-free initial conditions with no density fluctuations were assumed. A Passot-Pouquet-type initial spectrum is considered and the peak wavenumber is fixed to $k_{0}=2$. Different turbulent Mach numbers are considered and mesh resolutions ranging from $64^{3}$ to $512^{3}$ are analysed. Since the initialization is quasi-incompressible, for relatively high-Mach numbers an initial numerical transient is observed, through which the compressible components of the turbulent structures increase and a physical state of fully developed turbulence is reached. Inviscid simulations were performed both with PFG and VDW equations of state for different initial turbulent Mach numbers. For the chosen initial thermodynamic conditions, relatively close to the critical region, the compressibility factor exhibits large variations throughout the flow. This leads to large density and speed of sound fluctuations and to larger local values of the Mach number, and subsequently to remarkable differences in turbulence decay. High turbulent velocity fluctuations deriving from high turbulent Mach numbers lead to the occurrence of eddy shocklets [9], which strongly modify turbulence. In the zone of eddy shocklets, indeed, the pressure is highly correlated with dilatation, and the production of dilatational dissipation increases, leading to a conversion of kinetic energy into internal energy. Furthermore, for BZT fluids working in regions where $\Gamma<0$, the second law of thermodynamics requires that compression shocks cannot form; hence, locally, occurrence of compressive eddy shocklets is physically not admissible, whereas expansion shocks are allowed. In the VDW case, extremely strong expansion regions are present, whereas compressions are shown to be weaker than in the PFG case. This makes us argue the presence of expansion shocklets, whose presence will be investigated and a detailed analysis will be presented in the final paper. 


\section{References}

[1] G. Brunner. Applications of supercritical fluids. Annual Review of chemical and biomolecular engineering (2010), 321-342.

[2] P.A. Thompson. A Fundamental Derivative in Gas Dynamics. Physics of Fluids 14 (1971) $1843-1849$.

[3] P. Cinnella, P.M. Congedo. Inviscid and viscous aerodynamics of dense gases. Journal of Fluid Mechanics 580 (2007), 179-217.

[5] J.W. Kim, D.J. Lee. Adaptive Nonlinear Artificial Dissipation Model for Computational Aeroacoustics. AIAA Journal 39 (2001), 810-818.

[6] F. Ducros, V. Ferrand, F. Nicoud, C. Weber, D. Darracq, C. Gacherieu, T. Poinsot. Large-eddy simulation of the shock/turbulence interaction. Journal of Computational Physics 152 (1999), 517-549.

[7] E. Garnier, M. Mossi, P. Sagaut, P. Comte, M. Deville. On the use of shock-capturing schemes for large-eddy simulation. Journal of Computational Physics 153 (1999), 273-311.

[8] R. Samtaney, D.I. Pullin, B. Kosovic. Direct numerical simulation of decaying compresible turbulence and shocklet statistics. Physics of Fluids 13, 5 (2001), 1415-1430.

[9] S. Lee, S.K. Lele, P. Moin. Eddy shocklets in decaying compressible turbulence. Physics of Fluids A 3 (1991). 


\title{
High Reynolds number effects on turbulent scalings in compressible channel flow
}

\author{
Davide Modesti ${ }^{1}$ Sergio Pirozzoli ${ }^{1}$ Matteo Bernardini ${ }^{1}$ \\ ${ }^{1}$ Dipartimento di Ingegneria Meccanica e Aerospaziale, Università di Roma 'La Sapienza'
}

In this work the effect of the Reynolds number in a compressible isothermal channel flow is investigated through a series of direct numerical simulations (DNS). The bulk Mach number based on the wall temperature is kept fixed at 1.5, and the bulk Reynolds number is increased up to reach $R e_{\tau} \approx 1000$. Previous works [1]-[2] at lower Reynolds number have confirmed the validity of Morkovin's hypothesis but have questioned the use of Van Driest velocity transformation in the presence of cold walls. For this reason alternative transformations of the velocity profile and turbulence statistics have been proposed, as, for instance, the local scaling. We show that Van Driest transformation recover its efficacy as the Reynolds number is increased, while the local scaling diverges. The same holds true for the density scaled turbulent stresses $\bar{\rho} / \bar{\rho}_{w} R_{i j}$, which tend to collapse on their incompressible counterparts at sufficiently high Reynolds number. Excellent agreement with the incompressible stresses is found in the outer layer region, while substantial differences emerge approaching the wall. A further analisys of the outer layer reveals other analogies with incompressible wall bounded flows. It has recently been shown [3]-[4] in fact, that the incompressible velocity defect in the outer region follows very closely a parabolic law. We show that, under equivalent hypotheses, it is possible to obtain the same law for Van Driest transformed velocity.

\section{References}

[1] G.N. Coleman, J. Kim, R.D. Moser. A numerical study of turbulent supersonic isothermal-wall channel flow. J. Fluid Mech. 305(1995), 159-183.

[2] Y. Morinishi, S. Tamano,K. Nakabayashi. Direct numerical simulation of compressible turbulent channel flow between adiabatic and isothermal walls. J. Fluid Mech. 502(1995), 273-308.

[3] S. Pirozzoli. Revisiting the mixing-length hypothesis in the outer part of turbulent wall layer: mean flow and wall friction. J. Fluid Mech. 702(1995), 273-308.

[4] M. Bernardini S. Pirozzoli P. Orlandi. Velocity statistics in turbulent channel flow up to $R e_{\tau}=4000$. J. Fluid Mech. 702(1995), 273-308. 


\title{
Effect of outer stratification inside the inner region of a convective boundary layer
}

\author{
Juan Pedro Mellado, Jade Rachele Garcia, Chiel van Heerwaarden \\ Max Planck Institute for Meteorology, Bundesstr. 53, 20146 Hamburg, Germany
}

The near-wall region of a free convective boundary layer (CBL) that is forced by a homogeneous, constant surface buoyancy flux and that penetrates into a fluid with a constant buoyancy gradient $N^{2} \geq 0$ is investigated by means of direct numerical simulation.

Atmospheric and laboratory measurements of the vertical profiles of the mean temperature and of the r.m.s. of the temperature fluctuation show deviations from the predictions made according to the classical similarity theory $[4,1]$. The reason for these deviations is attributed to the formation of large-scale circulations that strongly interact with the flow inside the near-wall region. This interaction invalidates the basic assumption made in the classical similarity theory, which neglects the influence of outer scales inside the near-wall region. This result prompts the following question: Does an effect of the outer stratification strength $N^{2}$ on the largescale circulations imply an effect of $N^{2}$ on the near-wall region? We address this question by comparing two configurations: a neutral stratification case, $N^{2}=0$, which is representative of a CBL that grows into a residual layer [3], and a strong stratification case, $N^{2}>0$, which is representative of a CBL in the equilibrium (quasi-steady) entrainment regime [2].

We find that, near the wall, the vertical profiles of the mean buoyancy and buoyancy r.m.s. are approximately independent of $N^{2}$. As observed in atmospheric measurements and Rayleigh-Bénard convection, these profiles show some deviations from the scaling laws derived according to classical similarity theory: whereas the mean buoyancy gradient varies as $z^{-4 / 3}$ with respect to the distance to the surface, $z$, the r.m.s of the buoyancy fluctuation varies as $z^{-1 / 2}$, instead of $z^{-1 / 3}$. The vertical extent over which the previous scaling laws are observed depends on $N^{2}$. This result can be interpreted as a dependence of the inner-layer thickness on $N^{2}$ : For a given CBL thickness, $h$, the inner-layer thickness in the stably stratified is $\simeq 0.25 h$, which is more than three times the value in the neutrally stratified case. One possible physical explanation for this result is that the entrainment zone in the neutrally stratified case penetrates deeper inside the CBL, modifying thereby the flow structure closer to the wall.

\section{References}

[1] F. Chillà, J. Schumacher. New perspectives in turbulent Rayleigh-Bénard convection. Eur. Phys. J. E 35 (58) (2012), 1-25.

[2] J. R. Garcia, J. P. Mellado. The two-layer structure of the entrainment zone in the convective boundary layer. J. Atmos. Sci. 71 (2014), 1935-1955.

[3] J. P. Mellado. Direct numerical simulation of free convection over a heated plate. J. Fluid Mech. 712 (2012), $418-450$.

[4] S. S. Zilitinkevich \& co authors. The influence of large convective eddies on the surface-layer turbulence. Q. J. Roy. Meteorol. Soc. 132 (2006), 1423-1455. 


\title{
Reynolds-analogy factor and new formulations for the temperature defect law for turbulent boundary layers on a plate
}

\author{
Igor Vigdorovich \\ Institute of Mechanics, Lomonosov Moscow State University, Moscow, Russia
}

The well-known temperature defect law, valid in the outer region of the turbulent boundary layer, see e.g. [1], uses as a characteristic scale the so-called friction temperature, which is calculated from the skinfriction coefficient and Stanton number for which measurements of both wall shear stress and heat flux are needed. The same applies to the universal heat-transfer law [1], which relates three quantities: the Stanton number, the skin-friction coefficient, and a Reynolds number. For the turbulent boundary layer on a flat plate, such measurements, as far as we know, were performed in a single work [2].

When deriving the similarity laws [1], only dimensional analysis is used and a single physical assumption, according to which at high Reynolds numbers, molecular viscosity and heat conductivity are not essential outside the viscous sublayer while the outer scale (layer thickness) exerts no influence on the wall region.

The present paper suggests another approach to the classical problem under consideration, which is based on solving the momentum and heat-transfer equations, closure conditions for which are formulated (under the same physical assumption [1]) in terms of functional dependences of the turbulent shear stress and turbulent heat flux upon velocity and temperature gradients. The existence of these functional relations is a consequence of the fact that the considered turbulent flow in whole depends only on a limited number of governing parameters. The idea of such a closure method was first formulated in [3] and then used in subsequent works.

Another essential element of the investigation is a special change of variables in the boundary-layer equations [4], which allows us to seek the solution to the problem in the form of asymptotic expansions in high values of the logarithm of the Reynolds number based on the boundary-layer thickness. As a result, along with the known similarity laws for the outer and wall regions of the boundary layer, the expression for the Reynoldsanalogy factor is obtained which implies two new formulations for the temperature defect law. The first one is completely similar to the velocity defect law, i. e. contains neither the Stanton number nor the turbulent Prandtl number and the second one uses only thermal quantities, i. e. does not contain the skin-friction coefficient. A heat-transfer law is obtained that relates only thermal quantities. These relations can already be compared with a wider set of experimental data. Thus, we use the work [5], in which the temperature profiles and skin friction are measured but there is no data on heat flux, and the paper [6], which reports Stanton numbers but contains no values of skin-friction coefficients.

\section{References}

[1] B.A. Kader, A.M. Yaglom. Heat and mass transfer laws for fully turbulent wall flows. Int. J. Heat Mass Transfer 15 (1972), 2329-2353.

[2] P.H. Hoffmann, A.E. Perry. The development of turbulent thermal layers on flat plates. Int. J. Heat Mass Transfer 22 (1979), 39-46.

[3] I.I. Vigdorovich. Similarity laws for the velocity and temperature profiles in the wall region of a turbulent boundary layer with injection and suction. Doklady Phys. 48 (9) (2003), 528-532.

[4] I.I. Vigdorovich. Asymptotic analysis of turbulent boundary layer flow on a flat plate at high Reynolds numbers. Fluid Dynamics 28 (4) (1993), 514-523.

[5] J.B. Bell. Heat transfer to turbulent boundary layers in pressure gradients. M.S. Thesis. Univ. Melbourne (1966).

[6] D.G. Whitten, W.M. Kays, R.J. Moffat. The turbulent boundary layer on a porous plate: Experimental heat transfer with variable suction, blowing and surface temperature. Rep. HMT-3. Stanford Univ. 1967. $162 \mathrm{p}$. 


\title{
Experimental and numerical studies of active and passive control of combustion instabilities
}

\author{
M. Bauerheim, L. Gicquel, G. Staffelbach and T. Poinsot \\ IMF Toulouse, 31400 Toulouse
}

\begin{abstract}
The mitigation of combustion instabilities is a major issue for many combustion programs $[1,2]$. This can be achieved by adding active control systems (usually closed-loop real-time controllers) to existing combustion chambers or by building new chambers which are intrinsically stable. Active control techniques for combustion have been a major research theme between 1980 and 2000: multiple demonstrations of their efficiency to control unstable acoustic modes in laminar and turbulent configurations have been produced and the talk will present some of these results. Building chambers which never exhibit unstable modes is a much more difficult task, which relies heavily on our understanding of the fundamental mechanisms leading to instability. Here Large Eddy Simulations [3] have become the basic tool to predict unsteady combustion and coupling between flames and acoustics which are a major contributor to most instabilities. But theory remains a necessary approach because LES is not able to reveal why a given chamber becomes unstable or what should be changed to control the instability in most cases. The presentation will describe recent progress in LES [3], experiment [7] and theory $[4,5,6]$, applied to the case of annular combustion chambers which are used for helicopter or aircraft
\end{abstract} engines.

\section{References}

[1] T. Poinsot and D. Veynante "Theoretical and numerical combustion". Editor: T. Poinsot. www.cerfacs.fr/elearning. 2011.

[2] T. Lieuwen and V. Yang "Combustion Instabilities in Gas Turbine Engines. Operational Experience, Fundamental Mechanisms and Modeling", Prog. Aero. Astr. AIAA Vol 210. 2005

[3] L. Gicquel, G. Staffelbach and T. Poinsot. Large Eddy Simulations of gaseous flames in gas turbine combustion chambers. Prog. Energ. Comb. Sci. (2012) 38, 6, 782-817.

[4] M. Bauerheim, F. Nicoud and T. Poinsot. Theoretical analysis of the mass balance equation through a flame at zero and non-zero Mach numbers. Combustion and Flame. 1, 60-67, 162, 2015.

[5] M. Bauerheim, J.F. Parmentier, P. Salas, F. Nicoud and T. Poinsot. An analytical model for azimuthal thermoacoustic modes in an annular chamber fed by an annular plenum. Combustion and Flame. 5, 13741389, 161, 2014.

[6] M. Bauerheim, M. Cazalens and T. Poinsot. A theoretical study of mean azimuthal flow and asymmetry effects on thermo-acoustic modes in annular combustors. Proc. Comb. Inst. 35, 3, 3219-3227.

[7] M. Bauerheim, G. Staffelbach, N.A. Worth, J.R. Dawson, L.Y.M. Gicquel and T. Poinsot Sensitivity of LESbased harmonic flame response model for turbulent swirled flames and impact on the stability of azimuthal modes. Proc. Comb. Inst. 35, 3, 3355-3363. 


\title{
LES of high-frequency transverse combustion instabilities in complex combustion chambers
}

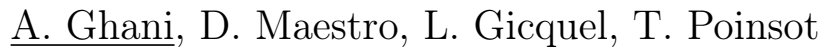 \\ CERFACS, 42 av. Coriolis, 31057 Toulouse \\ IMF Toulouse, 31400 Toulouse
}

Combustion instabilities have been the topic of numerous studies in the last ten years because these phenomena represent a major danger for many combustion programs as well as a challenging scientific problem [1]. Large Eddy Simulation (LES) have provided a unique tool to understand the mechanisms driving combustion oscillations [2] but most studies have focused on longitudinal low-frequency modes because models are easier to develop for these cases where acoustic waves are planar and longitudinal and simulations are less demanding because long wavelength perturbations and large vortices are involved. However, in many cases (rockets, gas turbines, post combustion), transverse high-frequency modes also appear and are more difficult to predict and seldom computed. Since they are also much more destructive and can cause a destruction of the combustion chamber in a few seconds, investigating these transverse modes has become a new field of application of LES.

In the present work, a generic experiment performed by Volvo [4, 5] is studied using LES of compressible reacting flows because this experiment exhibits both low-frequency $(150 \mathrm{~Hz})$ and high-frequency transverse modes (typically $1300 \mathrm{~Hz}$ ). The flame is stabilized on a wedge in a constant section duct and fed with premixed gas. Turbulent combustion is modeled using a thickened flame approach with a two-step chemical scheme. NSCBC boundary conditions are imposed at inlet and outlet to control acoustic reflections [3]. A Helmholtz solver is used to determine all acoustic modes of the combustor and check which ones appear when combustion instabilities begin. The mechanism which leads to transverse oscillations is discussed and a simple model is proposed to capture these modes.

A second characteristic of the Volvo experiment is that, while most LES codes capture the cold flow with precision in this setup, the accuracy of LES for the reacting case is much lower [4]. The present work discusses this issue too and shows that reacting flow results depend on multiple phenomena which are often neglected by LES users: inlet turbulence, temperature of the flame holder, existence of combustion instabilities.

\section{References}

[1] T. Poinsot and D. Veynante "Theoretical and numerical combustion". Editor: T. Poinsot. www.cerfacs.fr/elearning. 2011.

[2] L. Gicquel, G. Staffelbach and T. Poinsot. Large Eddy Simulations of gaseous flames in gas turbine combustion chambers. Prog. Energ. Comb. Sci. (2012) 38, 6, 782-817.

[3] V. Granet, O. Vermorel, T. Leonard, L. Gicquel and T. Poinsot Comparison of Nonreflecting Outlet Boundary Conditions for Compressible Solvers on Unstructured Grids. AIAA Journal (2010) 48(10).

[4] P. Cocks, V. Sankaran and M. Soteriou. Is LES of reacting flows predictive? Part 1: Impact of numerics. 20130170. 51st AIAA Aerospace Sciences Meeting including the New Horizons Forum and Aerospace Exposition.

[5] R.R. Erickson and M.C. Soteriou, The influence of reactant temperature on the dynamics of bluff body stabilized premixed flames, Combustion and Flame, Volume 158, Issue 12, December 2011, Pages 24412457 , 


\title{
Ignition and mixing in shock-bubble interaction with chemical reactions
}

\author{
F.R. Diegelmann, V.K. Tritschler, S. Hickel, N.A. Adams \\ Institute for Aerodynamics and Fluid Mechanics, Technische Universität München.
}

We present results for two-dimensional simulations of a reacting shock-bubble interaction (RSBI) including detailed chemistry. The configuration serves to investigate the interaction of two shock-induced phenomena: The Richtmyer-Meshkov instability (RMI) and the ignition of a $\mathrm{H}_{2}-\mathrm{O}_{2}$ gas mixture by the sudden increase of temperature and pressure. The pressure sensitivity of the $\mathrm{H}_{2}-\mathrm{O}_{2}$ combustion is used to trigger different reaction wave characteristics. Initial pressures between $p_{\text {init }}=0.25$ and $0.75 \mathrm{~atm}$ cover low pressure reactions and high pressure chemistry, leading to two types of reaction waves: subsonic deflagration and supersonic detonation. The different propagation velocities of these reaction waves significantly affect the temporal and spatial evolution as well as the mixing of the bubble gas. The latter plays a crucial role in many reactive shock-accelerated inhomogeneous flows, such as supersonic combustion engines [1].

The Richtmyer-Meshkov instability [2,3] is a shock-induced hydrodynamic instability occurring at the interface, separating two fluids of different densities. RMI occurs on a wide range of scales, from the largest in astrophysics [4] to intermediate scales in combustion [5] down to very small scales in inertial confinement fusion [6]. In reactive fluids, such as supersonic combustion, the rapid and efficient mixing of fuel and oxidizer is crucial as the detention time of the fuel-oxidizer mixture in the combustion chamber is only a few milliseconds [1]. RMI promotes mixing by baroclinic vorticity production and can promote the burning efficiency of supersonic combustion engines [7]. However, investigations of RMI in reactive gas mixtures imply a second essential effect: The shock wave can lead to shock-induced ignition in terms of deflagration or detonation.

In our work the full set of compressible, reacting, multicomponent Navier-Stokes equations is solved. A $2^{\text {nd }}$ order Strang time splitting [8] is used to separate the stiff source term from the Navier-Stokes equations. This results in a system of partial differential equations and a system of stiff ordinary differential equations. The latter is solved with the $5^{\text {th }}$ order backward differentiation formula [9]. The Navier-Stokes flux is discretized by an adaptive central-upwind $6^{\text {th }}$ order weighted essentially non-oscillatory (WENO-CU6) scheme [10]. Time integration is realized by $3^{\text {rd }}$ order strongly stable Runge-Kutta scheme [11]. The detailed Ó Conaire reaction mechanism [12], containing nine species and 19 intermediate reactions is used to calculate the chemical reaction rates.

The setup consists of a gas bubble filled with a stoichiometric mixture of $H_{2}, \mathrm{O}_{2}$ and $X e$, surrounded by $\mathrm{N}_{2}$. A planar shock wave $(\mathrm{Ma}=2.3)$ propagates through the domain and compresses the bubble gas. The convex shape of the bubble (Atwood number $\mathrm{A}<1$ ) leads to shock focusing at the downstream pole. The sudden rise in pressure and temperature increases the reaction rates until ignition. A deflagration wave is observed at low initial pressure, characterized by an increase of $H, O$ and $O H$ with the corresponding constant pressure distribution over the subsonic reaction wave. Higher initial pressure opens a different branch of reactions with a distinct increase of $\mathrm{HO}_{2}$ and $\mathrm{H}_{2} \mathrm{O}_{2}$, resulting in a detonation wave. The subsonic deflagration wave leads to a flow field dominated by hydrodynamic effects (Damköhler number $\mathrm{Da}<1$ ), whereas the supersonic detonation wave forces a chemical reaction driven evolution $(\mathrm{Da}>1)$.

Mixing is highly affected by different reaction waves. Tomkins et al. [13] identified three main mixing regions in a shock-bubble interaction: The vortex cores, the outer interface and the bridge region connecting the two main vortex cores. The latter contributes up to $40 \%$ to the mixing. Due to the subsonic propagation velocity of the deflagration wave only the vortex cores and the long-term evolution of the outer interface are disturbed in the low pressure simulation, whereas the bridge region remains unaffected. This leads to a slight reduction of the mixing over time compared to the non-reacting simulations. Higher pressure accompanied by a detonation of the gas mixture shows a different behavior. The detonation wave acts as a second shock wave inducing additional vorticity at the interface, interfering with the growth of the secondary instabilities and eliminating the important bridge region. As a result, the mixing is reduced by up to $50 \%$.

\section{References}

[1] J. Yang, T. Kubota, E. Zukoski, Applications of shock-induced mixing to supersonic combustion, AIAA Journal 31 (1993) 854-862. 
[2] R. D. Richtmyer, Taylor instability in shock acceleration of compressible fluids, Communications on Pure and Applied Mathematics 13 (2) (1960) 297-319.

[3] E. Meshkov, Instability of the interface of two gases accelerated by a shock wave, Fluid Dynamics 4 (5) (1969) 101-104.

[4] D. Arnett, The Role of Mixing in Astrophysics, The Astrophysical Journal Supplement Series 127 (2000) $213-217$.

[5] A. Khokhlov, E. Oran, G. Thomas, Numerical simulation of deflagration-to-detonation transition: the role of shock-flame interactions in turbulent flames, Combustion and Flame 117 (1-2) (1999) 323-339.

[6] J. D. Lindl, R. L. McCrory, E. M. Campbell, Progress toward ignition and burn propagation in inertial confinement fusion, Physics Today 45 (9) (1992) 32-40.

[7] F. Marble, E. Zukoski, J. Jacobs, G. Hendricks, I. Waitz, Shock Enhancement and Control of Hypersonic Mixing and Combustion, in: AIAA 26th Joint Propulsion Conference, Orlando, FL, (1990).

[8] G. Strang, On the Construction and Comparison of Difference Schemes, SIAM Journal on Numerical Analysis 5 (3) (1968) 506-517.

[9] P. N. Brown, G. D. Byrne, A. C. Hindmarsh, Vode: A variable-coefficient ode solver, SIAM Journal on Scientific and Statistical Computing 10 (5) (1989) 1038-1051.

[10] X. Y. Hu, Q. Wang, N. A. Adams, An adaptive central-upwind weighted essentially non-oscillatory scheme, Journal of Computational Physics 229 (23) (2010) 8952-8965.

[11] S. Gottlieb, C.-W. Shu, Total variation diminishing Runge-Kutta schemes, Mathematics of Computation 67 (1998) 73-85.

[12] M. Ó Conaire, H. Curran, J. Simmie, W. Pitz, C. Westbrook, A comprehensive modeling study of Hydrogen oxidation, International Journal of Chemical Kinetics 36 (2004) 603-622.

[13] C. Tomkins, S. Kumar, G. Orlicz, K. Prestridge, An experimental investigation of mixing mechanisms in shock-accelerated flow, Journal of Fluid Mechanics 611 (2008) 131-150. 


\title{
Large-eddy simulations of controlled wake flows using a penalty model of synthetic micro-jet
}

\author{
Noele Peres $^{1}$ and Richard Pasquetti ${ }^{1}$ \\ ${ }^{1}$ Laboratoire J.A. Dieudonné, UMR CNRS $n^{\circ} 7351$ \\ Université de Nice - Sophia Antipolis, 06108 Nice Cedex 02, France
}

The present work is motivated by the need of the automotive industry to manufacture vehicles that progressively reduce both their negative environmental impact and their dependence upon oil. An attractive perspective is then using synthetic (zero net mass flux) micro-jets in view of reducing the drag coefficient, but an efficient control strategy has not yet emerged. This is why it is of interest to study the influence of micro-jets on complex wake flows, as e.g. generated by cars. Simplified models of micro-jets are required to address such multiscale problems with existing CFD codes. We describe an approach based on a modelling of each micro-jet by an adhoc source term of momentum and its implementation in an existing spectral LES solver of the incompressible Navier-Stokes equations (SVVLES code, see e.g. [1]).

The numerical approximation is based on a multidomain Chebyshev-Fourier parallelized method. The time scheme makes use of a fractional step method, involving an explicit treatment of the advection terms, using an operator integration factor (OIF) semi-Lagrangian method, an implicit treatment of the diffusion terms and a projection step. To address turbulent flows, this numerical approximation is associated to a Spectral Vanishing Viscosity (SVV) stabilization technique. The obstacle is modeled using a pseudopenalization technique, that consists of a modification of the time scheme in order to approximately cancel the velocity field inside the volume of the obstacle. Complex geometries may be then considered with simple meshes, so that very efficient solvers can be used. The model developed for the synthetic micro-jets makes use of a penalty like term in the momentum equation, with a variable penalty coefficient that culminates during the expulsion phase [2].

Results are presented for the backward facing step turbulent flow, for the D-shaped body and for the square back Ahmed body. For the backward facing step, at Reynolds number $R e=33000$, the goal is to reduce the recirculation length. To this end, a synthetic micro-jet is located close to the end side of the step with an inclination angle of $45^{\circ}$. Our results are compared with the experimental results of [3], showing a similar trend with a decrease of the recirculation length at the lowest frequencies of the micro-jet. The two others cases are concerned with the reduction of the drag coefficient of motor vehicles. First we consider a D-shaped body, at $R e=20000$, equipped with synthetic micro-jets at its two end sides. Finally, for the square back Ahmed body [4], a strategy of drag reduction based on a perturbation of the near wake flow is investigated, see e.g. [5]. The results of several simulations at $R e=512000$ are presented, considering different numbers, orientations, flowrates and frequencies of synthetic microjets located at the four edges of the rear of the Ahmed body with drag reduction up to $4 \%$.

This work is supported by the French National Research Agency (ANR), in the frame of the project Limitation of the impact of vehicles on the environment by means of aerodynamic control using synthetic micro-jets (LIVE-CAMS).

\section{References}

[1] M. Minguez, R. Pasquetti, E. Serre. High-order large-eddy simulation of flow over the Ahmed body car model. Phys. Fluids. 20 (2008), 095-101.

[2] R. Pasquetti, N. Peres. A penalty model of synthetic micro-jet actuator with application to the control of wake flows. Submitted.

[3] K. B. Chun and H. J. Sung. Control of turbulent separated flow over a backward-facing step by local forcing. Experiments in fluids. 21 (1996), 417-426.

[4] S.R. Ahmed, G. Ramm. Salient features of the time-averaged ground vehicle wake, SAE Paper No. 840300. (1984).

[5] M. Rouméas, P. Gilliéron, A. Kourta. Analysis and control of the near-wake flow over a square-back geometry. Comput. Fluids. 38 (2009), 60-70. 


\title{
Computational Aeroacoustics of Subsonic and Supersonic Impinging Jets
}

\author{
Wilke, R., Sesterhenn, J. \\ Technische Universität Berlin, Fachgebiet Numerische Fluiddynamik, \\ Müller-Breslau-Str. 11, 10623 Berlin, Germany
}

Impinging jets have a high relevance in practical cooling applications e.g. of aircraft turbine blades since they feature a very high local heat transfer. In addition, impinging jets are used for short take-off and vertical landing (STOVL) aircraft. Impinging jets are characterised by extremely high noise load, which can cause material fatigue and deafness. Additionally to the three noise sources of free jets (turbulent mixing noise, shock induced noise and "screech"), impinging jets feature an additional source, called impinging tones. Those tones can exceed the mean noise by more than $10 \mathrm{~dB}$ and its physical origin is not yet completely understood. [1].

In order to find out the physical origin of the impinging tones, direct numerical simulations (DNS) of subsonic as well as supersonic impinging jet are carried out. The present investigation concentrates on simulations with Reynolds number of $R e=3300$ and $R e=8000$ and pressure ratios of $p_{t} / p_{\infty}=1.5$ and $p_{t} / p_{\infty}=2.15$. Grids with $512^{3}$ respectively $1024^{3}$ points are used. The latter one $(R e=8000)$ represents the real occurring Reynolds Number in turbine blades.

The subsonic jet features multiple discrete frequencies. A dynamic mode decomposition approach, described in [2], is used to identify the dynamic behaviour of the jet. One discrete tone and its first sub-harmonic correspond to the primary vortices developing in the shear layer of the jet. A second discrete tone and its first sub-harmonic also exists. The origin of this tone is not yet clarified.

The simulation of the supersonic impinging jet features a standoff shock. A detailled analysis of the strong influence of this shock on the sound spectrum will be given.

\section{Acknowledgments.}

The simulations were performed on the national supercomputers Cray XE6 and Cray XC40 at the High Performance Computing Center Stuttgart (HLRS) under the grant number GCS-ARSI/44027.

\section{References}

[1] G. Sinibaldi, L. Marino, G.P. Romano. Aeroacoustics of Free and Impinging Jets. 9th European Fluid Mechanics Conference (2012).

[2] P. J. Schmid. Dynamic mode decomposition of numerical and experimental data. Journal of Fluid Mechanics (2010), 5-28. 


\section{S11: Interfacial flows}

Understanding and controlling of interfacial phenomena in multiphase flows is one of the main challenges in fluid mechanics, at the crossroads of scientific disciplines like Mathematics, Physics, Chemistry and Engineering.

Examples are particle-laden flows, bubble columns, fuel atomization, casting, oil recovery, film flows, spreading and dewetting of (complex) liquids including suspensions, polymer solutions, liquid crystals, colloids and biofluids. All these systems are central for technological advances in the chemical, pharmaceutical, environmental and food industries and crucial for the development of Microfluidics and Nanostructuring. Goal of this Section is to provide a representative overview of the latest developments in this area, covering models, numerical methods and experimental techniques but also surveying new physical insights and recent technical advancements. 


\title{
Simulating Free-Surface Flows with Moving Boundaries: A Combination of an Interface-Tracking and an Interface-Capturing Approach
}

\author{
Markus Frings, Marek Behr, and Stefanie Elgeti \\ Chair for Computational Analysis of Technical Systems, \\ CCES, RWTH Aachen University, Germany
}

\begin{abstract}
A method is presented to simulate free-surface flows driven by a moving boundary. This kind of flow occurs for example in the numerical simulation of the high-pressure die casting process. In this simulation, a moving plunger (the moving boundary) pushes molten metal from a partially filled gating chamber (free-surface) into the die. Since the simulation is afterwards used in an automatic optimization loop, there is a need for an efficient and robust simulation model.

Both, the free-surface and the moving boundary can be treated as interfaces. In simulations with such interfaces, two main types of methods can be distinguished: (1) interface tracking (IT), and (2) interface capturing (IC). The difference lies in the description of the interface explicitly by a boundary conforming mesh in IT and implicitly by an additional scalar field in IC.

In IT, the interface movement can be directly applied to the boundary nodes. Thereby, the computational domain is deformed based on the node movement. In order to maintain a valid mesh in the interior, the mesh node positions must be adjusted. In contrast, in the IC the computational mesh is fixed and the interface position is determained based on an additional scalar field. As the interface evolves, its movement is described by a modification of the scalar field. The IT has its strengths in the modelling of uniform and limited interface distortion. In the case of large surface deformation, a remeshing might become necessary, which is a hindrance in an automatic optimization loop. Also, the merging of two interfaces is an issue in IT. IC is suitable for the modelling of free-surface flows with large surface deformations. It allows for nearly arbitrary surface deformation and the contact of the surface with a domain wall (e.g., the top wall of the gating chamber) is not an issue.

In the approach at hand, both types of interface description are combined. The IT is applied to the moving boundary, while the IC is applied to the free surface. For the plunger movement we use a Deformable-SpatialDomain/Stabilized-Space-Time (DSD/SST) finite element formulation in combination with a mesh update method for the interior node positions [1]. This method moves the boundary nodes according to the prescribed plunger movement. The mesh itself (containing both phases) is treated like an elastic material. As a result, the interior node positions are updated in a way that keeps the mesh valid. The free-surface flow is modelled by the level-set method [2]. The scalar field in the level-set method is the signed distance to the interface. Thus, the zero level-set describes the interface position. The sign of the function can be used to determine the density and viscosity for the flow field computations. The level-set field (and therefore also the interface) is transported with the fluid velocity.

Both methods are implemented in the in-house flow solver XNS, which employs P1P1 finite element with a Galerkin/Least-Sqares stabilization technique. Basis for the flow simulation are the incompressible and isothermal Navier-Stokes equations.

The combined approach represents an efficient and robust method appropriate for the numerical optimization of the high-pressure die casting process. Furthermore, it is possible to apply the presented approach to other applications, where a combination of a two-phase or free-surface flow with moving boundaries occurs.
\end{abstract}

\section{References}

[1] A.R. Johnson and T. Tezduyar. Mesh update strategies in parallel finite element computations of flow problems with moving boundaries and interfaces. Computer Methods in Applied Mechanics and Engineering, 119 (1994), 73-94.

[2] M. Sussman, P. Smereka and S. Osher. A level set approach for computing solutions to incompressible two-phase flow. Journal of Computational Physics, 114 (1994), 146-159. 


\title{
Towards higher-order XFEM for interfacial flows
}

\author{
Thomas-Peter Fries \\ Institute of Structural Analysis, Graz University of Technology, \\ Lessingsstr. 25/II, 8010 Graz, Austria \\ fries@tugraz.at, www.ifb.tugraz.at
}

The simulation of two-phase flows is characterized by the presence of moving interfaces in space and time. The interfaces separate the involved fluids each having individual properties such as density and viscosity. As a consequence, the resulting physical fields such as pressure and velocity feature jumps and kinks at the interface. The accurate treatment of the discontinuous parameters and fields renders the simulation of two-phase flows a challenging task and a large number of numerical methods has evolved.

Recently, the XFEM is used increasingly for two-phase flows because it enables accurate approximations of discontinuous solutions within elements $[1,2,4]$. This is achieved by enriching the classical finite element approximation space through the partition of unity concept $[5,6]$. The XFEM in combination with the level-set method enables a consistent treatment of problems with moving interfaces. Herein, the aim is to use the XFEM for higher-order accurate approximations of two-phase flows [3].

A number of issues has to be considered to achieve this aim:

- An accurate enrichment for jumps and kinks is needed in the XFEM that enables higher-order approxiomations of jumps and kinks within elements.

- A higher-order accurate time integration scheme is needed for the incompressible Navier-Stokes equations and the level-set transport equation. The discontinuous Galerkin method in time is a natural choice, i.e. higher-order space-time finite elements are realized.

- An accurate numerical integration is needed for the evaluation of the weak forms. A quadrature is proposed that captures curved interfaces within elements.

- A higher-order accurate reinitialization of the level-set function is needed.

The emphasis of this work is on the higher-order accurate integration of the weak form. The level-set function is higher-order and the resulting zero level set is curved within the elements. A consistent integration scheme is proposed that decomposes the background (space-time) elements into special sub-elements with curved faces. This integration also enables the evaluation of surface tension on the interface, which is one dimension lower than the spatial dimension of the problem.

\section{References}

[1] H. Sauerland, T.P. Fries. The extended finite element method for two-phase and free-surface flows: A systematic study. J. Comput. Phys. 230 (2011), 3369-3390.

[2] H. Sauerland, T.P. Fries. The stable XFEM for two-phase flows. Computers \& Fluids 87 (2013), 41-49.

[3] K.W. Cheng, T.P. Fries. Higher-order XFEM for curved strong and weak discontinuities. J. Comput. Phys. $82(2010), 564-590$.

[4] K.W. Cheng, T.P. Fries. XFEM with hanging nodes for two-phase incompressible flow. Comp. Methods Appl. Mech. Engrg. 245 (2012), 290-312.

[5] N. Moës and J. Dolbow and T. Belytschko. A finite element method for crack growth without remeshing. Internat. J. Numer. Methods Engrg. 46 (1999), 131-150.

[6] T.P. Fries and T. Belytschko. The extended/generalized finite element method: An overview of the method and its applications. Internat. J. Numer. Methods Engrg. 84 (2010), 253-304. 


\title{
Numerical Modelling of Laser-Induced Cavitation Bubbles with a Finite Volume Method
}

\author{
Max Koch ${ }^{1}$, Christiane Lechner ${ }^{2}$, Fabian Reuter ${ }^{1}$, Karsten Köhler ${ }^{1}$, \\ Robert Mettin ${ }^{1}$, Werner Lauterborn ${ }^{1}$ \\ ${ }^{1}$ CDLCME, Georg-August-University Göttingen \\ ${ }^{2}$ Vienna University of Technology
}

We report on the numerical modelling of laser-induced cavitation bubbles with the finite volume method using the open source software package OpenFOAM [1, 2]. The goal is to correctly predict the dynamics of a laser generated bubble, including collapse and rebound, as well as the velocity and pressure field in the surrounding liquid for asymmetric situations like bubbles close to a rigid boundary or next to a second bubble.

We consider the bubble to be filled with an adiabatic van der Waals gas. Compressibility of the liquid is included via the Tait equation of state, which allows shock waves to develop upon bubble generation and collapse. At the present stage of our work evaporation and condensation is not included in the model.

The pressure-based volume of fluid solver compressibleInterFoam has been extended for our purpose. We show that the slow convergence of the bubble volume with grid resolution stems from a large numerical error in the mass that the bubble aquires during collapse. The violation of mass conservation for a very similar numerical scheme previously has been reported in the literature [3]. A comparativley simple correction of the bubble mass at every time step solves that problem and allows for an accurate prediction of collapse and rebound of strongly collapsing bubbles with a reasonably sized grid in axial symmetry. A further improvement of the original solver concerns the stabilization of the algorithm for the case of strong pressure waves impinging on the numerically smeared liquid/gas interface.

To validate our approach we consider spherically symmetric and axially symmetric collapse scenarios. For a spherical bubble collapsing in an unbounded liquid the bubble evolution is in very good agreement with predictions of the Gilmore model up to the second collapse as well as with the experimental data given in [4]. The pressure field in the surrounding liquid is validated by comparing form and location of the shock waves to numerical and experimental data available in the literature. Furthermore by comparing with experimental data it is shown, that the finite volume simulation can very well predict the shape of a bubble that collapses near a solid boundary.

\section{References}

[1] OpenFOAM, http://www.openfoam.com.

[2] The OpenFOAM-extend project, http://www.extend-project.de/.

[3] S.T. Miller, H. Jasak, D.A. Boger, E.G. Paterson, and A. Nedungadi. A pressure-based, compressible, two-phase flow finite volume method for underwater explosions. Computers and Fluids, 87 (2013), $132-143$.

[4] S. Müller, M. Bachmann, D. Kröninger, T. Kurz, and P. Helluy. Comparison and validation of compressible flow simulations of laser-induced cavitation bubbles. Computers and Fluids, 38 (2009), 1850 - 1862. 


\title{
A comparison of viscoelastic and empirical rheological models in context of squeeze flows
}

\author{
Stefan Descher, Olaf Wünsch \\ Chair of Fluid Mechanics, Institute of Mechanics, University of Kassel
}

For polymer melts a wide range of measurable rheological phenomena can be modelled by nonlinear differential constitutive equations. Typical phenomena are stress-relaxation, shear-thinning, normal stress differences and strain-hardening. But often empirical models which are only capable to describe the shear behaviour correctly are used. For non-newtonian squeeze flows several publications regarding slip and no-slip boundary conditions are available. A review and enumeration of publications is given by Engelmann in [1].

In this contribution an isothermal, constant velocity, no-slip squeeze flow is discussed. It is inspired by technical squeezing flows during polymer processing and validation experiments, that can be done with commercial rotational rheometers. There is a relation to investigations presented in [2], where the flow type in squeeze flows between square plates was analysed. In contrast, here the cross section of the plates is circular and the temporal progress of the averaged normal stress on the plates is evaluated. This plays a significant role in the design of presses in industrial applications. The rheological models that are used are the generalized Maxwell model with the nonlinear extension from Giesekus and the empirical Carreau-Yasuda model. The simulations are performed with the Finite-Volume-Method using OpenFOAM. In its framework a solver based on pimpleFoam (PISO and SIMPLE merged) was developed to calculate viscoelastic flows. The main difference to the standard solver viscoelasticFluidFoam [3] is the sequence of steps to couple pressure, velocity and stress in order to improve the convergence behaviour. The implementation of the problem domain is done by using axisymmetric boundary conditions, thus it is reduced to a wedge. Since the height of the domain is reduced due to squeezing a dynamic mesh is used.

\section{References}

[1] J. Engelmann, C. Servais, A. S. Burbige: Squeeze flow theory and applications to rheometry: A review. J. Non-Newtonian Fluid Mech, 132 (2005) 1-27.

[2] A. Al-Baldawi, O. Wünsch: Simulating of a pressing process of a viscoelastic polymer melt. Proc. Appl. Math. Mech., 12 (2012) 471-472.

[3] J. L. Favero, A. R. Secchi, N. S. M. Cordozo, H. Jasak: Viscoelastic flow analysis using the software OpenFOAM and differential constitutive equations. J. Non-Newtonian Fluid Mech, 165 (2010) 1625-1636. 


\title{
On the implementation of free-slip interfaces for the immersed boundary method
}

\author{
Tobias Kempe, Jochen Fröhlich \\ TU Dresden, Institute of Fluid Mechanics, 01062 Dresden, Germany
}

The numerical simulation of spherical and ellipsoidal bubbles in purified fluids requires the application of the free-slip boundary condition at the bubble surface [1]. This talk presents a numerical method for the implementation of free-slip boundary conditions for immersed boundary methods as proposed and enhanced in various papers $[2,3,4]$.

In contrast to other numerical approaches for multiphase flows, the realization of free-slip interfaces for immersed boundary methods is not straightforward and is not available in literature up to now. The reason is, that the method treats the liquid as well as the gas phase as a field of constant density and viscosity with a fictitious fluid inside the bubble. The motion of the disperse phase is computed explicitly by solving its momentum balance. It is coupled to the continuous phase via additional source terms in the Navier-Stokes equations. The outer fluid in the vicinity of the interface is substantially affected by the inner fluid. As a consequence, an intrinsic realization of free-slip interfaces, like for schemes with variable viscosity and density, is not possible. Several approaches to achieve such a condition were conceived and implemented. Using various tests the respective performance was assessed and compared. It turns out that an appropriate forcing is not immediate and requires to overcome certain difficulties. The new method is first described for spherical objects and then extended to general curved interfaces. The scheme is applied to spherical and ellipsoidal particles without or with prescribed constant angular velocity. It is shown that the proposed method yields the same order of convergence as the method for no-slip boundaries and does not degrade computational efficiency. The results are compared to analytical solutions for creeping flow around a sphere with free-slip surface and to numerical reference data obtained on a body-fitted grid. The numerical tests confirm the excellent performance of the new method.

\section{References}

[1] R. Clift, J. R. Grace, and M. E. Weber. Bubbles, Drops, and Particles. Dover Publications (1978).

[2] M. Uhlmann. An immersed boundary method with direct forcing for the simulation of particulate flows. Journal of Computational Physics, 209 (2005), 448-476.

[3] T. Kempe, J. Fröhlich. An improved immersed boundary method with direct forcing for the simulation of particle laden flows, Journal of Computational Physics, 231 (2012), 3663-3684.

[4] T. Kempe, J. Fröhlich. Collision modelling for the interface-resolved simulation of spherical particles in viscous fluids. Journal of Fluid Mechanics, 709 (2012), 445-489. 


\title{
Linear stability of a thin non-isothermal droplet spreading on a rotating disk
}

\author{
$\underline{\text { K. Boettcher, T. Externbrink }}$ \\ Fluid Mechanics, Bio- and Biochemical Engineering, TU Dortmund
}

In many industrial applications solids are coated by using centrifugal forces, e.g. in spin-coating used for coating wafers. In the first step of the process a liquid droplet is placed on the disk and spreads over the solid due to gravity, centrifugal forces and wetting. During the spreading the contact line may get corrugated, giving rise to the so-called fingering instability. In a preceded work the linear stability of the contact-line was investigated for a thin isothermal droplet, using lubrication approximation and Tanners law to cover the behavior of the dynamic contact angle [1]. The results show that the stability depends on the base-state curvature of the free interface at the contact line. As the Marangoni effect can be used to alter the curvature [2], this presentation investigates the base state of the spreading including thermal gradients between the plate and the surrounding gas or in the plate itself. A linear stability analysis tries to answer the question, how those contact-line instabilities may be influenced.

\section{References}

[1] K.E.R Boettcher, P. Ehrhard. Contact-line instability of liquids spreading on top of rotating substrates. European Journal of Mechanics - B/Fluids 43 (2014), 33-44.

[2] K. Boettcher, J. Hötzel, P. Ehrhard. Temperature effects in thin droplets. Proc. Appl. Math. Meth. 12 (2012), 519-520. 


\title{
A circulating gravity wave in a cylindrical tank
}

\author{
Herbert Steinrück, \\ Vienna University of Technology, Institute of Fluid Mechanics and Heat Transfer
}

Circulating gravity wave in a cylindrical tank A cylindrical vertical tank, partially filled with water is considered. The upper lid of the tank rotates around the cylinder axis inducing a rotational flow in the air gap between the water surface and the lid. It has been observed that above a critical angular velocity of the lid a circulating gravity wave in the water develops with an angular wave speed almost independent of the driving speed of the lid. The wave form is well known and can be described as first approximation by a potential flow. We are interested in the excitation mechanism and want to determine the critical speed of the lid by an asymptotic analysis with respect to small wave amplitudes. The key idea is to study the mechanical energy budget of the flow. Moreover, to simplify the analysis we assume that the air flow above the water given as a rotating flow with an Ekman-layer at the bottom. For small amplitudes the core flow in the water can be considered as the superposition of time periodic potential flow and a solid body rotation. The angular frequency of the gravity wave can be determined by an eigenvalue problem. By considering the boundary layers at the interface to the water and the cylinder walls the momentum an energy budget will be determined. Analytical results for the angular frequency in dependence of the driving angular speed of the lid will be given and compared to experimental results. Moreover, the minimal angular speed of the lid where the gravity wave can be observed will be given. 


\title{
Experiments beyond the limits of Nusselt theory: The linear stability of gravity-driven films over undulated inclines
}

\author{
M. Schörner, D. Reck and N. Aksel \\ Dept. Applied Mechanics and Fluid Dynamics, University of Bayreuth, Germany
}

We present experimental results on the linear stability of gravity-driven films over strongly undulated inclines. Within our parameter study we vary the Reynolds number, the liquid's viscosity and the inclination angle of the substrate. Furthermore, we change the frequency of the linear disturbances which we impose to the basic flow. That way, we obtain stability charts which show a rich variety of phenomena induced by the undulation of the incline: We found a slight destabilization as well as a very significant stabilization of the flow compared to the corresponding Nusselt flow. Moreover, we report on transmutations from long-wave to short-wave type instabilities. Even a fragmentation of the stability chart, which leads to stable and unstable isles, is observed.

Our study extends former experimental [1,2] and numerical [3] approaches and explores the above-mentioned complex stability behavior of gravity-driven films over strongly undulated inclines in-depth. We are now able to classify the stabilizing and the destabilizing effects. Concerning the stabilizing effects, we identified a significantly increased mean film thickness of the flow due to the undulation compared to the corresponding Nusselt flow. Consequently, this leads to a decreased free surface velocity and hence the flow is stabilized. Beyond this, we proved the destabilizing effect of hydraulic jumps and a strongly curved free surface of the liquid. This enabled us to explain the fragmentation of the stability charts and thus the existence of stable and unstable isles phenomena which clearly exceed the limits of Nusselt's theory.

\section{References}

[1] T. Pollak, N. Aksel. Crucial flow stabilization and multiple instability branches of gravity-driven films over topography. Phys. Fluids 25 (2013), 024103.

[2] Z. Cao, M. Vlachogiannis, V. Bontozoglou. Experimental evidence for a short-wave global mode in film flow along periodic corrugations. J. Fluid Mech. 718 (2013), 304-320.

[3] Y.Y. Trifonov. Stability of a film flowing down an inclined corrugated plate: The direct Navier-Stokes computations and Floquet theory. Phys. Fluids 26 (2014), 114101. 


\title{
Continuous size-dependent separation of blood components from human whole blood samples in microfluidic spirals
}

\author{
L. Sprenger ${ }^{1,2}$, S. Dutz ${ }^{3}$, T. Schneider ${ }^{1}$, S. Odenbach ${ }^{2}$, U. O. Häfeli ${ }^{1}$ \\ ${ }^{1}$ Faculty of Pharmaceutical Sciences, University of British Columbia, Vancouver, BC V6T 1Z3, Canada \\ 2 TU Dresden, Chair of Magnetofluiddynamics, Measuring and Automation Technology, 01062 Dresden, Germany \\ ${ }^{3}$ Institut für Biomedizinische Technik und Informatik, Technische Universität Ilmenau, 98693 Ilmenau, Germany
}

Parasites, like those causing leishmaniasis [1], appear in very small concentrations in the blood of infected humans. This makes a diagnosis challenging based solely on visual proof of the whole blood sample. In this context our work aims at developing a microfluidic point-of-care device with the focus on a simplified continuous separation process which requires no pretreatment of the blood.

The separation process is based on the so-called Dean effect [2], which denotes a double vortex structure perpendicular to the primary flow along a curved duct. The effect is characterized by the Dean number $D e=\rho v D_{h} / \eta\left(D_{h} /(2 R)\right)^{0.5}$, involving the primary flow velocity $v$, the fluid's parameters density $\rho$ and viscosity $\eta$, and the geometric parameters hydraulic diameter $D_{h}=4 A / P(A$ : cross-sectional area, $P$ : wetted perimeter) and the duct's radius $R$. This flow phenomenon can be applied to focus solid components dispersed in a liquid phase. Depending on their size and presumably also their shape and concentration they migrate across the primary flow to an equilibrium position near the inner wall, where they are trapped by a balance of Dean force, inertial lift force, and wall lift force [4].

Experiments are conducted aiming at designing a microfluidic spiral capable of separating spherical particles, used as simplified model system, of the diameter of $2-18 \mu \mathrm{m}$ by symmetrically splitting the spirals outlet. It is expected that the presence of red blood cells in the flow decreases the efficacy of the process while increasing the channel's fluidic resistance. Experiments therefore also shed light on the conflict of diluting the blood sample which decreases the particles' low inlet concentration while separation in the duct is enhanced with a positive effect on the particles' outlet concentration.

The separation efficacy is described by the ratio between the particle concentration at the inner outlet and inlet (separation ratio $s_{r}$ ). It must be taken into account that the symmetrical splitter can lead to a theoretical maximum of a factor of $s_{r}=2$. Inlet samples are diluted with a buffer, and experiments are carried out containing more than $20 \%$ of blood $\left(c_{b}>0.2\right)$, exactly $20 \%$ of blood $\left(c_{b}=0.2\right)$, and containing no blood at all $\left(c_{b}=0\right)$. If the system's parameters lead to $D e>1$ and a ratio of particle size and hydraulic diameter of $d_{p} / D_{h}>0.12$ the separation efficacy results in: $c_{b}>0.2 \rightarrow s_{r}=1.25 \pm 0.23 ; c_{b}=0.2 \rightarrow s_{r}=1.6 \pm 0.28 ; c_{b}=0 \rightarrow s_{r}=1.95 \pm 0.23$. The use of an asymmetric splitter results in a reduction of the volumetric flow rate in the inner outlet by $25 \%$. This leads to $c_{b}=0 \rightarrow s_{r}=2.25 \pm 0.16$, and therefore enhances the mean efficacy by roughly $15 \%$. A two-step separation process with two spirals connected in a row results in $c_{b}=0 \rightarrow s_{r}=3.69 \pm 0.32$. Limits for two-step separation and asymmetric splitters will be stressed more in further experiments.

The experimental investigations are accompanied by a numerical evaluation of the macroscopic fluid flow of whole blood in a microfluidic channel. The blood flow is implemented by two shear-thinning models for the viscosity, namely Quemada [5] and Carreau-Yasuda [6]. The simulation reveals that by using experimentally relevant inlet velocities in a curved duct of a cross-section of $45 \times 55 \mu^{2}$ the flow leads to such high shear gradients in the liquid that the viscosity of the blood in the duct's cross-section can be approximated by a constant viscosity of 3-4 mPas depending on the model in use. Further simulations address the particles' trajectories in the duct considering liquid-solid interactions.

Experimental and numerical investigations show that the presented microsystem can be applied to separate rare components from highly concentrated samples such as whole blood.

Acknowledgements: Financial supports by a fellowship within the Postdoc-Program of the German Academic Exchange Service (DAAD) and by a discovery grant from the Natural Sciences and Engineering Research Council (NSERC) in Canada are gratefully acknowledged.

\section{References}

[1] World Health Organization. World Health statistics (2014).

[2] W. R. Dean. Philos. Mag. (Ser. 7$) 4$ (1927), 208-223.

[4] D. Di Carlo et al. Proc. Natl. Acad. Sci. USA 104 (2007), 18892-18897.

[5] A. Marcinkowska-Gapińska et al. Med. Bio. Eng. Comput. 45 (2007), 837-844.

[6] J. Boyd et al. Phys. Fluids 19 (2007), 093703. 


\title{
Modeling and simulation of mobile jet agitator for a biogas plant
}

\author{
Aria Alimi ${ }^{1}$, Olaf Wünsch ${ }^{1}$ \\ ${ }^{1}$ Chair of Fluid Mechanics, Institute of Mechanics, University of Kassel
}

Nowadays, the biogas plants are one of the major natural energy sources in the world. The mixture inside these plants, which consists of different types of slurry, has to be stirred due to the sedimentation of highviscous component after a certain amount of time. Currently, there are submersible motor agitators in use for this purpose, which need a long time to stir the whole component and also need opening the cover of biogas plant every time. The goal of this work is to investigate a new concept to mix the content of a biogas plant having none of the above restrictions based on a mobile stirring system that works in a closed pumping process. It works on a similar principle as a jet pump of a watercraft. The content of the plant will be pumped out and then will be pumped inside with a high inlet velocity on different positions already defined on the plant body.

In order to design such a mixing process, we simulate it numerically using Finite-Volume-Method for different types of meshed cylinder geometries. The problem involves an unsteady-state flow with two unmixable phases and will be calculated using Volume of Fluid (VOF) solver. The material properties of the contents -the most important one are the density and viscosity- have to be analyzed using rheometer device. The model consists of two different phases: the high-viscous component which has a viscosity of about 40 Pas, and the low-viscous component with a viscosity of about 0.003 Pas. The mixing process will be analyzed through the study of the flow field. Furthermore, the parameter study such as different inlet velocities or inlet directions will be performed.

Regarding the validation of simulations, different experiments are carried out on a labor scale biogas plant with transparent walls. Considering the similitude theory, appropriate materials come in use for both high and low viscous components and the mixing process will be monitored closely. The mixing process in both numerical simulations and the experiments will be compared and the results would be discussed.

\section{References}

[1] M. Gerber. Ganzheitliche stoffliche und energetische Modellierung des Biogasbildungsprozeses. Dissertation, Faculty of Mechanical Engineering, Ruhr-Universität, Bochum (2009)

[2] P. Mähnert. Kinetik der Biogasproduktion aus nachwachsenden Rohstoffen und Gülle. Dissertation, Landwirtschaftlich-Gärtnerische Fakultät, Humboldt-Universität, Berlin (2007) 


\title{
Interaction of a finite-size particle with a wall
}

\author{
Francesco Romanò, Hendrik C. Kuhlmann \\ Vienna University of Technology, Tower BA/E322, Getreidemarkt 9, 1060 Vienna
}

The motion of a particle moving near a domain boundary is investigated. If the tangential velocity along the boundary is large the streamlines are dense and the finite size of a particle can have a profound effect on its trajectory. Despite of Stokes numbers as small as $10^{-5}$, this effect has been shown to be important in certain free-surface flows $[1,2,3]$.

Here we consider the steady two-dimensional flow in a lid-driven cavity. We examine the particle motion using different particle motion models: perfect tracers, inertial point particles (Maxey-Riley equation) one-way coupled to the flow, and finite-size particles the flow around which is fully resolved (two-way coupling). We discuss and quantify the finite-size effect and compare the results with the particle-surface interaction model originally introduced by [1].

As a results of the simulation of the fully resolved motion of finite-size particles we directly obtain the forces and torques on the particle. This information is used to derive a particle-motion model which can be employed to simulate the transport of a large number of particles and which represents an improvement of the inelastic particle-surface interaction model originally introduced by [1].

The numerical technique used to simulate the phenomenon belongs to the hp-methods (DG-FEM, in particular) and it is coupled with the so-called "smoothed-profile method" (SPM). This approach was developed in [4] for low and moderate Reynolds numbers. More recently, it has been further extended to high-Reynolds-number flows. The strengths of this approach are (a) the absence of moving grids as are required for, e.g., the ALE technique and (b) an accurately and efficient computation of the particle motion. The latter is ensured thanks to the spectral convergence and use of high-order methods of the DG-FEM technique.

\section{References}

[1] Hofmann, E., Kuhlmann, H. C. Particle accumulation on periodic orbits by repeated free surface collisions Phys. Fluids 23 (2011), 0721106-1-0721106-14.

[2] Muldoon, F. H., Kuhlmann, H. C. Coherent particulate structures by boundary interaction of small particles in confined periodic flows Physica D 253 (2013), 40-65.

[3] Mukin, R. V., Kuhlmann, H. C. Topology of hydrothermal waves in liquid bridges and dissipative structures of transported particles Phys. Rev. E 88 (2013), 053016-1-053016-20.

[4] X. Luo, M. R. Maxey, G. E. Karniadakis, Smoothed profile method for particulate flows: Error analysis and simulations, J. Comput. Phys. 228 (2009), 1750-1769. 


\title{
The magnetoviscous effect of a biocompatible ferrofluid diluted in sheep blood.
}

\author{
J. Nowak, S. Odenbach \\ Technische Universität Dresden, Institute of Fluid Mechanics, \\ Chair of Magnetofluiddynamics, 01062 Dresden, Germany
}

Ferrofluids, suspensions of magnetic nanoparticles in suitable carrier liquids, are receiving a growing importance in biomedical applications. While being used in magnetic resonance imaging with great success for several years, new approaches like e.g. magnetic drug targeting are of great interest [1]. This approach focuses on binding chemotherapeutic agents to the magnetic particles suspended in the fluid. These ferrofluids are arterially injected and concentrated in the tumor by the application of external magnetic fields in the respective region. This application being in the focus of current research has the potential to decrease side effects of conventional chemotherapy and to increase its effectiveness.

To guarantee a safe and effective application of the ferrofluids the knowledge of the flow characteristics is important. For ferrofluids used in the engineering context the magnetoviscous effect (MVE), a rise in viscosity due to the formation of particle-structures caused by the application of an external magnetic field, is well known and investigated in some detail [2]. This effect was detected for bicompatible ferrofluids as well [3]. Nevertheless up to now no extends were made to characterize the flow behavior of biocompatible ferrofluids if a dilution in blood occurs.

This experimental study focuses on the rheological investigation of a biocompatible ferrofluid mixed with sheep blood. Results measured using a specially designed shear rate-controlled rheometer [4] are compared to results measured with a novel capillary rheometer designed for the special needs of the measurement conditions existing.

The relative increase of viscosity, the MVE, if an external magnetic field is applied for pure ferrofluids is compared for both rheometers, revealing an effect above $1000 \%$ for low shear rates. For higher shear, which have to be expected during a potential biomedical application, the MVE is still measurable using the capillary viscosimeter. If the ferrofluid is diluted with sheep blood an increasing viscosity caused by an external magnetic field can be measured as well, being significantly different if compared to water as diluting agent, Therefore particle-particle interactions of the ferrofluids nanoparticles and the cells found in the sheep blood have to be assumed.

Financial support by the Deutsche Forschungsgesellschaft under grant no. OD18/23-1 is gratefully acknowledged.

\section{References}

[1] C. Alexiou et al. Cancer therapy with drug loaded magnetic nanoparticles - magnetic drug targeting. J. Magn. Magn. Mater. 323, 1404-1407 (2011)

[2] J. Nowak et. al. The influence of hydrodynamic diameter and core composition on the magnetoviscous effect of biocompatible ferrofluids. J. Phys.: Condens. Matter 26, 176004 (2014)

[3] S. Odenbach. Magnetoviscous Effects in Ferrofluids. LNP 71, Springer, Berlin (2002)

[4] S. Odenbach, H.Störk. Shear dependence of field-induced contributions to the viscosity of magnetic Fluids at low shear rates. J. Magn. Magn. Mater. 202, 155-158 (1998) 


\title{
The anisotropy of the magnetoviscous effect in a magnetite ferrofluid with weak interparticle interactions
}

\author{
J.M. Linke, S. Odenbach \\ TU Dresden, Chair of Magnetofluiddyanmics, 01062 Dresden, Germany
}

Ferrofluids are colloidal suspensions of magnetic nanoparticles in a carrier liquid. If the magnetic particles are large enough to interact by dipole-dipole interactions in an applied magnetic field, chain-like microstructures of associated particles are formed in the direction of the applied field. These microstructures can rupture if the ferrofluid is sheared. Both, the formation and the disintegration of the microstructures are accompanied by a change in the viscous properties of the ferrofluid. The change in viscosity, called magnetoviscous effect MVE [1], depends on the intensity of the applied field and the shear, and also on their relative orientation. While this anisotropy is known from theoretical and experimental studies, measurements of the magnetoviscous properties are generally conducted in rotational or capillary flow rheometers [2], which allow only one distinct orientation of the magnetic field with respect to the fluid flow.

In the present work, a four-coil magnet system and a specially designed slit die viscometer [3] have been used to study the anisotropy of the MVE in a magnetite ferrofluid for three different directions of the magnetic field: in the direction of the flow, in the direction of the velocity gradient, and in neutral direction parallel to the vorticity. The measurement of the three corresponding Miesowicz viscosity coefficients allows a comprehensive magnetorheological characterization of the magnetite ferrofluid. Effects like the field-induced increase of the MVE, the shear thinning and the shear stability for field directions perpendicular to the vorticity have been confirmed. A negative MVE related to the crystal anisotropy of magnetite as observed by Grants [4] for constant shear rates in neutral direction could not be confirmed.

The highest MVE has been measured in a magnetic field perpendicular to the flow and to the vorticity. Elongated microstructures oriented in this direction have the highest moment of inertia and therefore offer the highest resistance to the flow resulting in the highest change in viscosity. On the other hand, elongated structures oriented parallel to the direction of the flow offer less resistance to the flow. Hence at low shear rates the lowest MVE has been observed in a field parallel to the flow. However, if the shear rate is increased, the lowest MVE is found in a field parallel to the vorticity. This is in agreement with Shliomis' theory of non-interacting particles [5] which predicts that for individual spherical particles the viscosity increase is zero for the magnetic field parallel to the vorticity. Hence the observed anisotropy of the MVE corroborates the field-driven formation and shear-induced disintegration of elongated chain-like microstructures in the ferrofluid. Nonetheless, future experiments will have to be complemented by molecular dynamics simulations to relate microstructural changes and rheological behaviour in ferrofluids.

We gratefully acknowledge the financial support by the Deutsche Forschungsgemeinschaft (DFG grant Od18-18).

\section{References}

[1] S. Odenbach. Magnetoviscous Effects in Ferrofluids. Lecture Notes in Physics M71 (2002), Springer, Berlin.

[2] M. Gerth-Noritzsch, D.Y. Borin, S. Odenbach. Anisotropy of the magnetoviscous effect in ferrofluids containing nanoparticles exhibiting magnetic dipole interaction. J. Phys.: Condens. Matt. 23 (2011), 346002.

[3] J.M. Linke, S. Odenbach. Anisotropy of the magnetoviscous effect in a ferrofluid with weakly interacting magnetite nanoparticles. Submitted to J. Phys.-Condens. Mat.

[4] A. Grants, A. Irbitis, G. Kronkalns, M.M. Maiorov. Rheological properties of magnetite magnetic fluid. J. Magn. Magn. Mater. 85 (1990), 129.

[5] M.I. Shliomis in Ferrofluids: Magnetically Controllable Fluids and Their Applications. Lecture Notes in Physics 594, p. 85-111, S. Odenbach ed. (2002), Springer, Berlin. 


\title{
An innovative phase transition modeling for reproducing cavitation through a five-equation and seven-equation models and complex equation of state
}

\author{
M.G. Rodio ${ }^{+}$, P.M. Congedo ${ }^{+}$, R. Abgrall* \\ $\left.{ }^{+}\right)$INRIA Bordeaux-Sud-Ouest, France

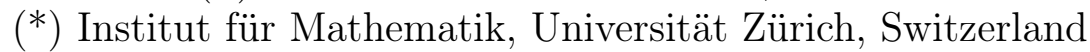

This work is devoted to model the heat and mass transfer through a liquid/vapor interface in compressible two-phase models. Modeling the heat and mass transfer between the phases is of utmost importance when simulating flows in fuel injectors, turbo-pumps, nozzles, etc. Usually, in some of these applications, cavitation could appear because of a strong liquid acceleration, yielding a strong rarefaction wave and hence with vapor/gas bubbles creation. The consequences of cavitation, such as noise, performance device reduction and wall corrosion, are extremely negative. For these reasons, this phenomenon requires a good theoretical comprehension and, as consequence, an accurate predictive physical model is required.

The most important reference about the treatment of heat and mass transfer terms, for the Interface Diffusive models, is the work of Saurel et al. [1]. The authors proposed a phase transition modeling for a single velocity and single pressure model (five equations model) relying on a thermo-chemical relaxation. In fact, by obtaining the equality of temperature and chemical potential for the two phases, this relaxation assures the evolution of liquid phase from a metastable state to an equilibrium state on the saturation curve. During this evolution, the liquid phase releases mass in order to evaporate before assuming a new state on the saturation curve. Then, the authors proposed an original procedure to identify the liquid/vapor interface. In recent years, few works have been proposed for treating phase transition modeling in Interface Diffusive models $[2,1,3,5,4,6]$.

The first idea of the present contribution is to couple the treatment of heat and mass transfer terms proposed in [1] for a five equation model, with the procedure for solution admissibility of [5], thus preserving the positivity of the solution and reducing consistently the computational cost. The second innovative point of this work is the extension of the proposed model to six and seven-equation models, thus showing its flexibility.

For closing the compressible seven-equation two-phase model, an equation of state (EOS) is necessary to define the thermodynamic behavior of each single phase and of the liquid-vapor mixture, under the saturation curve. When complex fluids are considered, such as cryogenic, molecularly complex ones, the use of simple EOS can produce imprecise estimation of the thermodynamic properties, thus leading to the deterioration of the accuracy of the prediction. Increasing the complexity of the model and calibrating the additional parameters with respect to the available experimental data are certainly valid options for improving the model prediction. Nevertheless, this is very challenging because of the numerical difficulties for the implementation of more complex mathematical model and because of the large uncertainties that generally affect the experimental data. Actually, the most used EOS for reproducing the liquid/vapor behavior under the saturation curve is the Stiffened Gas (SG) EOS [1,5]. The reason is that this EOS allows an explicit mathematical calculations of important flow relation. Moreover, in mass transfer problems it assures the positivity of speed of sound in the two-phase region, under the saturation curve.

So, finally, the third contribution of this work is to show how the numerical solver based on a Discrete equation Method (DEM) formulation [7] can be modified to include a more complex equations of state than the SG for the vapor region.

\section{References}

[1] R. Saurel and F. Petitpas and R. Abgrall. Modelling phase transition in metastable liquids. Application to cavitating and flashing flows. Journal of Fluids Mechanics, 607 (2008), 313-350.

[2] E. Goncalves. Modeling for non isothermal cavitation using 4-equation models. International Journal of Heat and Mass Transfer, 76 (2014), 247-262.

[3] A. Zein and M. Hantke and G. Warnecke. Modeling phase transition for compressible two-phase flows applied to metastable liquids. Journal of Computational Physics 229 (2010), 2964-2998. 
[4] F. Daude and P. Galon and Z. Gao and E. Blaud. Numerical experiments using a HLLC-type scheme with $\{$ ALE $\}$ formulation for compressible two-phase flows five-equation models with phase transition. Computers \& Fluids 94 (2014), 112-138.

[5] M. Pelanti and K. M. Shyue. A mixture-energy-consistent six-equation two-phase numerical model for fluids with interfaces, cavitation and evaporation waves. Journal of Computational Physics 259 (2014), 331-357.

[6] Y. Wang, L. Qiu, R. D. Reitz and R. Diwakar. Simulating cavitating liquid jets using a compressible and equilibrium two-phase flow solver. International Journal of Multiphase Flow 63 (2014), 52-67.

[7] R. Abgrall and R. Saurel. Discrete Equations for Physical and Numerical Compressible Multiphase Mixtures. Journal of Computational Physics, 186 (2003), 361-396. 


\title{
Numerical investigation of a liquid displacing a gas in thin porous layers
}

\author{
Tim Neumann, Konrad Boettcher, Lutz Gödeke, Peter Ehrhard \\ Strömungsmechanik, Bio-und Chemieingenieurwesen \\ TU Dortmund \\ Emil-Figge-Str. 68, D-44227 Dortmund \\ Email: Tim.Neumann@bci.tu-dortmund.de
}

Liquid flow through a porous media is of great importance in many engineering problems, e.g. calculating the heat and mass transfer in fixed bed catalytic reactors or estimating the potential environmental risks of fracking. The cells of an lithium-ion battery may be described as thin porous layers sintered on the electrodes, with a thin and permeable layer separating these two porous domains, so that one elementary cell is composed of three porous layers with heterogeneous characteristics of the porosity. The whole battery consists of many elementary three-layer cells.

This presentation deals with a liquid displacing a gas in such an elementary three-layer cell, which occurs during the filling of the battery with the electrolyte. The investigation is based upon the volume-averaged Navier-Stokes equations, using a volume of fluid method to cover the multiphase flow. The flow is investigated with respect to the wall-effect and capillary action in porous media. The Ergun equation is used to estimate the permeability, depending on the distance to the wall. Since the layers are thin and the characteristic size of the particles is comparatively large, friction of the electrode is taken into account with respect to the mobility of the contact line. The implemented models are validated against analytical results, showing only small deviation. Thereafter, results of the displacement flow in the elementary cell are presented and discussed, showing a not negligible influence of the modeled effects onto the flow characteristics. 


\title{
Mechanisms for wave generation in countercurrent air-water turbulent flows
}

\author{
Francesco Zonta $^{1}$, Miguel Onorato ${ }^{2}$, Alfredo Soldati ${ }^{1}$ \\ ${ }^{1}$ Department of Elec., Manag. and Mechanical Enginerring, University of Udine, \\ 33100 Udine, Italy. \\ ${ }^{2}$ Department of Physics, University of Torino, 10125 Torino, Italy.
}

Mass, momentum and energy transport phenomena through a deformable air-water interface are important in many geophysical processes and industrial applications. In this study, we use Direct Numerical Simulation (DNS) of the Navier Stokes equations to analyze the dynamics of the interface between air and water when they are driven by opposite pressure gradients (countercurrent configuration). The Reynolds number $\left(R e_{\tau}\right)$, the Weber number $(W e)$ and the Froude number $(F r)$ fully describe the physical problem. We examine the problem of the transient growth of interface waves for different combinations of physical parameters. Keeping $R e_{\tau}$ constant and varying $W e$ and $F r$, we show that, in the initial stages of the wave generation process, the amplitude of the interface elevation $\eta$ grows in time as $\eta \propto t^{2 / 5}$. Later in time, the waves growth rate differs depending on the value of $\mathrm{Fr}$,We: for small capillary waves, we do not observe substantial changes from $t^{2 / 5}$ law; for longer gravity waves we observe a faster growth rate. From the single wave mode analysis, we observe a similar behavior for the growth rate of the most significant modes. Simple phenomenological models have been derived to explain our results. At steady state, our data confirm previous results: wavenumber spectra, $E\left(k_{x}\right)$, of the surface elevation in the capillary range are in good agreement with the prediction of the Wave Turbulence Theory. Finally, we also evaluate wave-induced modifications of the average wind and current velocity profiles. 


\title{
Diffuse interface models for locally inextensible vesicles
}

\author{
Sebastian Aland, John Lowengrub, Axel Voigt \\ Institut für wissenschaftliches Rechnen, TU Dresden, Germany \\ Department of Mathematics, UC Irvine, California, USA
}

The presence of lipid molecules at the membrane of lipid vesicles makes the membrane itself incompressible. This property is also termed inextensibility and implies that the surface divergence of the velocity field vanishes at the vesicle surface:

$$
\nabla_{\Gamma} \cdot \mathbf{v}=0 \quad \text { on } \Gamma \text {. }
$$

We present new diffuse interface models for the dynamics of such inextensible vesicles in a viscous fluid [1]. A new feature of this work is the implementation of the local inextensibility condition by using a local Lagrange multiplier harmonically extended off the interface. To make the method even more robust, we develop a local relaxation scheme that dynamically corrects local stretching/compression errors, thereby preventing their accumulation. We present numerical results that confirm the effectiveness of the proposed models in a test case scenario of vesicles in shear flow. Finally we apply the model to a problem of clathrin-mediated endocytosis. Clathrin proteins attach to the membrane and alter locally its bending stiffness and spontaneous curvature. This process can lead to budding and pinch-off of small vesicles. First numerical simulation results will be shown.

\section{References}

[1] S. Aland, S. Egerer, J. Lowengrub, A. Voigt Diffuse interface models of locally inextensible vesicles in a viscous fluid. J. Comp. Phys. 277 (2014), 32-47. 


\title{
Polymer Devolatilization in a Rotating Apparatus
}

\author{
$\underline{\text { Stefan Hirschfeld }}^{1}$, Lutz Hermann ${ }^{1}$, Olaf Wünsch ${ }^{1}$ \\ ${ }^{1}$ Chair of Fluid Mechanics, Institute of Mechanics, University of Kassel
}

Devolatilization processes are of great importance in polymer processing. This unit operation is carried out several times within the production process of plastics. Devolatilization or degassing is the controlled removal of volatile compounds which are often harmful to health and environment. Furthermore, these substances downgrade the properties of the polymer products. There are two different mechanisms of devolatilization for polymer melts: film degassing and bubble degassing. Film degassing takes place on continiuous free surfaces between the liquid polymer phase and the gas phase. Mass transfer proceeds due to diffusion. Bubble degassing involves a more complex mechanism, where foaming is introduced as the solution is supersaturated. This means the ambient pressure is below the equilibrium partial pressure. Similar processes as by film degassing occur inside each bubble in the foam. Futhermore, the devolatilization equipment is classified into two main categories: rotating and nonrotating devolatilizers. Rotating devices are usually used for devolatilization of high-viscous polymers. $[1,2]$

This contribution presents the experimental study of film degassing in a partial filled agitator vessel. A laminar fluid flow with a distinct free surface for the degassing process occurs. High-viscous polydimethylsiloxane with a viscosity of about 100 Pas enriched with 1,1,2-trichloro-1,2,2-trifluoroethane is used as model substance system. Degassing is introduced by purging with a nitrogen gas flow. Concentration analysis of the liquid phase is realized by thermogravimetric measurements. The exiting gas stream of the test apparatus passes through two cold-traps in series to precipitate the volatile compound. The results of the weighted condensate are compared with the concentration analysis. All experiments were performed at ambient temperature. Data for the vapor-liquid equilibrium at the phase interface are obtained by measurement of pressure, temperatur and volume flow in the gas stream. The experiments are evaluated and discussed based on the surface renewal theory or penetration theory by Higbie and Danckwerts [3]. This model assumes that the diffusion in the polymer is the rate-controlling step of devolatilization. Mass transfer is modeled as an locally unsteady process within an exposure time of liquid at the free surface. Afterwards, this degassed fluid elements are assumed to be complete mixed into the bulk of the liquid phase.

\section{References}

[1] VDI-Gesellschaft Kunststofftechnik: Aufbereitungstechnik 2006. Entgasungsprozesse in der Aufbereitungstechnik. VDI-Verlag, Düsseldorf, 2006.

[2] R.J. Albalak. Polymer Devolatilization. Hanser Publishers, München, 1996.

[3] P.V. Danckwerts. Significance of liquid-film coefficients in gas absorption. Ind. Eng. Chem. 43 (1951) 6, 1460-1467. 


\title{
Gas Bubbles in Micro-Capillaries - Hydrodynamics and Mass Transfer
}

\author{
Peter Lakshmanan, Peter Ehrhard \\ TU Dortmund University - BCI - Fluid Mechanics
}

Process design of multi-phase unit operations on the micro-scale asks for detailed knowledge of the hydrodynamics and mass transfer behavior of discrete disperse fluid particles in confined geometries. In the present work, the focus is on single gas bubbles in vertical capillaries. Hydrodynamics and mass transfer are examined using numerical methods.

The hydrodynamic behavior of a single bubble in a capillary including its deformation is examined numerically using a modified level-set method [1]. Additionally, the influence of surface-active contaminations ('surfactants') and the resulting Marangoni effects are taken into account [2]. This enables the differentiation of bubbles with a mobile, i.e. clean, interface and those with a immobile, i.e. contaminated, interface. Based on the hydrodynamics, the mass transfer is examined, again employing numerical methods. In the present work, mass transfer is from the bubble into the bulk fluid. The system is assumed to be dilute, i.e. the concentrations are small and do not influence other fluid properties ('one-way coupling'). By using either asymptotic approximations or very high mesh resolutions, the local concentration field in and around the gas bubble can be resolved, which gives access to the local mass transfer in terms of a local Sherwood number.

Two modes of operation are examined for the cases of a mobile and immobile gas-liquid-interface: (i) A gas bubble rising in a vertical capillary filled with a stagnant liquid; (ii) A gas bubble levitated in a vertical capillary.

Results are in good agreement to experimental data and available correlations from literature. Based on the knowledge of the drag coefficients and local and also integral Sherwood numbers over a wide field of parameters

$\left(R e, S c, d_{\text {bubble }} / d_{\text {capillary }}\right)$ and mode of operation allows for the analysis and optimization of unit operations employing multiphase micro-capillaries.

\section{References}

[1] E. Olsson, G. Kreiss. A conservative level set method for two phase flow. J. Comp. Sc. 210 (2005), 225-246.

[2] P. Lakshmanan, P. Ehrhard. Maragoni effects caused by contaminants adsorbed on bubble surfaces. J. Fluid Mech. 647 (2010), $143-161$. 


\title{
Simulation of mass transfer at free liquid/liquid interfaces
}

\author{
Christian Heckmann, Peter Ehrhard, Peter Lakshmanan \\ Strömungsmechanik, Bio- und Chemieingenieurwesen \\ TU Dortmund \\ Emil-Figge-Str. 68, D-44227 Dortmund \\ Email: christian.heckmann@bci.tu-dortmund.de
}

\begin{abstract}
Mass transfer in multiphase systems finds extensive application in chemical-engineering problems, like chemical reactions, extraction, or adsorption. In liquid-liquid systems, usually, the mass transfer resistance in both phases appears to be relevant - this is a conjugated problem. Hence, a detailed knowledge of the mechanisms and characteristics of the mass-transfer process in conjunction with the geometrical properties of the multiphase system appears to be crucial for the process design. Numerical simulations are certainly an ideal tool for a local examination of such mass-transfer processes, since experiments usually remain restricted to integral measurements.

We present a concept for the numerical simulation of the mass transfer at a free liquid/liquid interface, based on the open-source software package openFOAM. Starting point for the hydrodynamics of the examined twophase system is an imported steady-state interface. On both sides of this interface (separate) computational domains for both liquids are arranged, whereas a kinematic coupling at this interface is in place. Hence, the flow can be computed within both computational domains. The mass transfer is examined, based on this steady-state hydrodynamics, by solving a transport equation of a passive (scalar) concentration at both sides of the interface. At the liquid/liquid interface, transition conditions arise from both the interfacial dissolution equilibrium and mass-flux continuity. Our numerical approach is validated against analytical limiting cases.
\end{abstract}




\title{
Three-phase contact line pinning at structured surfaces by molecular dynamics simulation
}

\author{
Stefan Becker, Maximilian Kohns, Martin Horsch, and Hans Hasse \\ Laboratory of Engineering Thermodynamics, University of Kaiserslautern, Germany
}

The wetting behaviour of a fluid in contact with a solid substrate is important for many applications and processes. Molecular simulation allows to investigate adsorption and wetting with atomistic resolution. In the present study, the massively-parallel molecular dynamics code ls1 mardyn [1] is employed to simulate adsorption and wetting in dependence on the fluid-solid interaction and the structure of the solid substrate. In this way, the influence of the surface morphology on the contact line and the contact angle is captured.

For a perfectly planar surface and a sessile droplet, both interacting by the truncated and shifted LennardJones potential, the contat angle is determined from density profiles and correlated in terms of the magnitude of the fluid-wall dispersion energy [2]. The entire range between complete wetting and dewetting is covered, as well as the temperature range from the triple point up to the critical point. Contact line pinning is considered for the boundary between planar and hydrophilic rough regions in the Wenzel state, and for the epitaxial Cassie state, where a contact line of an advancing droplet is stuck at an edge.

The validity of the Gibbs inequality, which describes the range of contact angles at which pinning occurs, is confirmed down to the nanometre length scale. The precise position of the equimolar contact line, however, deviates from the edge of the substrate by a characteristic length of about $5 \AA$, due to the influence of the adsorbed precursor layer on the three-phase contact region.

\section{References}

[1] C. Niethammer, S. Becker, M. Bernreuther, M. Buchholz, W. Eckhardt, A. Heinecke, S. Werth, H. J. Bungartz, C. W. Glass, H. Hasse, J. Vrabec, M. Horsch, J. Chem. Theory Comput. 10(10), 4455 (2014)

[2] S. Becker, H. M. Urbassek, M. Horsch, H. Hasse, Langmuir 30(45), 13606 (2014) 


\title{
New density based OpenFOAM solver with a stochastic fields approach for two phase flow
}

\author{
Michael Ranft $^{1}$, Andreas G. Class ${ }^{2}$, Thomas Jordan ${ }^{1}$ \\ ${ }^{1}$ Institute for Nuclear and Energy Technologies, Karlsruhe Institute of Technology \\ ${ }^{2}$ Areva Nuclear Professional School, Karlsruhe Institute of Technology
}

A new density based solver is implemented in the $\mathrm{C}++$ library OpenFOAM, applying the finite volume method. For a second order accurate space discretization the provided interpolation schemes and limiters are used. The time discretization a Dual Time Stepping technique is used, whereby at each physical time step the flow is treated as a pseudo steady state problem. The equations are integrated over a so-called pseudo time $\tau$ until steady state is reached, where $\tau$ is only a relaxation factor with no physical meaning. The previous flow variables in physical time are treated as explicit source terms in the pseudo time integration. With Dual Time Stepping the physical time steps aren't limited by the Courant-Friedrichs-Lewy condition and the von Neumann criterion.

The convective fluxes through the cell faces are provided by new implemented standard flux schemes. Additional a preconditioning technique is adopted for removing the stiffness from the system of flow equations at low Mach numbers. The preconditioning scales the acoustic eigenvalues of the system until they are of the same order as the convective eigenvalues.

For time integration in pseudo time SSP Runge-Kutta time schemes are used. Whereby schemes with more stages than order extend the CFL coefficient and lead to greater pseudo time steps. Further accelerating of time integration in pseudo time without influencing the accuracy of the transient problem in the physical time is done with the following techniques. A local time step, depending on the local CFL and von Neumann criterion, allows greater time steps where local velocity is small or grid space is large. An implicit residual smoothing is done extending the stability limits of the scheme. The residuals for the Runge-Kutta stage are exchanged with a weighted average residual of neighboring cells. A bulk viscosity damping technique is added, damping error waves in the computational flow domain by adding a small value of viscosity to the momentum and optional to the energy equation. A multigrid algorithm is applied, using a series of successive coarser grids. For the interaction between the different grids a Full Approximation Storage (FAS) is used, whereby a Full Multigrid (FMG) initialize the solutions at the different meshes, beginning at the coarsest grid.

The effective turbulence viscosity and effective thermal diffusivity are provided by the k- $\omega$ SST turbulence model for the diffusion fluxes. And the stochastic field method by Valiño [1] is used for simulating the two phase flow. This approach was extended by Dumond et al. [2] for simulating a cavitating flow. Their procedure is integrated into the new density based flow solver, where the two phase flow is treated as single fluid and the stochastic fields provide the mass fractions of the two phases.

The main differences between the new solver and the published density based solver in the framework of OpenFOAM are the use of SSP Runge-Kutta schemes, instead of standard low-storage, the new integration of a FMG-FAS multigrid scheme and the extension with a stochastic field method. Solving all flow equations including stochastic field and turbulence model at each Runge-Kutta stage is another advantage.

\section{References}

[1] L. Valiño. A Field Monte Carlo Formulation for Calculating the Probability Density Function of a Single Scalar in a Turbulent Flow. Flow, Turbulence and Combustion, 60 (1998), 157-172

[2] J. Dumond, F. Magagnato, A. Class. A. Stochastic-field cavitation model. Physics of Fluids, 25 (2013). 


\section{S12: Waves and acoustics}

Waves are a ubiquitous natural phenomenon and acoustics are, besides surface water waves, the most obvious representatives, familiar to anybody and quantitatively known to any student of mathematics, physics or a technical subject. To this corresponds a long mathematical tradition, continuing today in the accurate numerical computation of linear and nonlinear wave phenomena.

Our session is devoted to the simulation and understanding of waves and wave interactions. The range of applications is thus very broad, while the focus is meant to be on the unifying physical phenomenon. In the past years we had numerous contributions from solid mechanics, porous media flow, turbulence and aeroacoustics, from crack detection to explosions. 


\title{
Propagation of acoustic waves in a turbulent boundary layer
}

\author{
Xavier Gloerfelt \\ DynFluid Lab, Arts et Metiers ParisTech, Paris, France
}

Excitation of the walls of a vehicle by turbulent boundary layers constitutes a major source of interior noise, and can be dominant for cruising trips. The random character of the pressure distribution induced by the boundary layer has a major effect on the noise transmitted through the fuselage and its cross-spectral density plays a major role in determining the effective force causing motion to the structure. In the present study, flat-plate turbulent boundary layers with zero pressure gradient are computed using direct noise computation, i.e. solving the compressible Navier-Stokes equations. These numerical experiments allows the determination of the direct aeroacoustic contribution, of low intensity but high efficiency, together with the hydrodynamic pressure due to the turbulence, which can excite structural modes [1].

Noise intensities radiated in the freestream indicate that the sources are turbulence quadrupoles, explaining the low efficiency. In particular, a $U^{8}$ power-law dependence is found confirming the quadrupolar nature of wall turbulence, in accordance with Powell's reflection principle [2]. For Mach numbers ranging between 0.3 and 0.9 , we have seen that the acoustic wavefronts have a large wavelength and an upward-oriented directivity. The acoustic domain for the frequency-wavenumber representation of the wall pressure, which is the imprint of acoustic waves on the wall, is analysed. At the low frequencies of interest, it can not be unambiguously separated from the convective ridge due to the relatively high Mach numbers considered, but the acoustic activity is clearly visible. Unlike the far-field noise, the high frequency range is dominated by acoustic waves propagating in the downstream direction, whereas counterflow waves are almost absent.

To explain this paradox, ray tracing [3] is used to characterize propagation effects due to the mean boundary layer profile. By neglecting the slow thickening of the TBL in the flow direction, the effect of the mean flow on the acoustic propagation is well-described by the Pridmore-Brown operator for a simple shear flow [4]. A shadow zone is formed upstream of the source because of the refraction effects, and for sufficiently high frequencies, the refraction of acoustic rays lead channeled waves trapped inside the boundary layer downstream of the source. The latter phenomenon, commonly observed in ocean acoustics, is referred to as channeling or wave guide effect. According to [5], the multiple trapped channeled waves can form caustics, leading to localised regions of intense pressure. They argue that the caustics can thus participate to the mechanisms sustaining coherent structures in wall turbulence. The range of acoustic frequencies must be compatible with the turbulent characteristic frequencies such as the bursting and ejection events.

The ray tracing is performed for the different Mach numbers of the large-eddy simulation databases. As the freestream Mach number decreases, the critical angle for the formation of a shadow zone becomes higher. A large amount of acoustic energy is focused near the critical angle for higher Mach numbers. Channelling effect is also reinforced. As a consequence, in the high-frequency limit, the wall acoustics is essentially in the downstream direction due to the channeled waves, and the acoustic peak in the rearward direction is hard to observe because of refraction effect. Only turbulent scattering can provide acoustic energy in this direction.

\section{References}

[1] Gloerfelt, X. and Berland, J., Turbulent boundary-layer noise: direct radiation at Mach number 0.5, J. Fluid Mech., Vol. 723, 2013, pp. 318-351.

[2] Gloerfelt, X. and Margnat, F., Effect of Mach number on boundary layer noise, 20th AIAA/CEAS AeroAcoustics Conference, 16-20 June, Atlanta, Georgia, AIAA Paper 2014-3291, 2014.

[3] Candel, S., Numerical solution of conservation equations arising in linear wave theory: application to aeroacoustics, J. Fluid Mech., Vol. 83, No. 3, 1977, pp. 465-493.

[4] Suzuki, T. and Lele, S., Green's functions for a source in a boundary layer: direct waves, channelled waves and diffracted waves, J. Fluid Mech., Vol. 447, 2003, pp. 129-173.

[5] Abrahams, I., Kriegsmann, G., and Reiss, E., Sound radiation and caustic formation from a point source in wall shear layer, AIAA Journal, Vol. 32, No. 6, 1994, pp. 1135-1144. 


\title{
Non-destructive testing of porous media by means of sound wave analysis
}

\author{
Bettina Albers \\ Technische Universität Berlin \\ Department of Soil Mechanics and Geotechnical Engineering
}

The theoretical investigation of sound waves in porous materials is motivated by the possible construction of non-destructive testing methods. Here, geotechnical applications are of interest where low frequencies from zero to $100 \mathrm{~Hz}$ occur but also systems with higher frequency ranges may be tested non-invasively. Examples are concrete and other construction materials, road surfaces and pavements (some MHz), biological materials like bones and soft tissues, e.g. the surface of the heart (up to $3 \mathrm{MHz}$ ) right up to surface coatings by nanomaterials (approximately $100 \mathrm{MHz}$ ).

In the geotechnical area the wave propagation analysis may lead to the construction of several non-destructive testing methods.

The analysis of surface waves (see e.g. [1] or [2]) may help to develop a method for soil characterization. By use of the SASW-technique conclusions about building grounds can be drawn from the measurement of sound wave speeds. I.e. no expensive and invasive acoustical measurements in boreholes or laboratory tests are necessary to characterize the soil prior to a building project.

In problems like seepage processes in road and dam constructions or tunneling in rocks different permeability in different directions plays an important practical role. The analysis of monochromatic waves in two-component poroelastic materials described by a model with anisotropic permeability yields two different shear modes [3]. Due to their appearance one can construct a device for measuring the anisotropy of the permeability. It would have to induce shear waves of different polarization and different directions of propagation. Then, one could measure principal values and directions of the tortuosity by comparing the amplitudes of arrivals for different polarization of signals.

Also the analysis of sound waves in partially saturated porous media [4] provides the hope for the construction of non-destructive testing methods. Namely, the velocity of the P1-wave which is the fastest wave and thus the first arrival on an oscillogram, increases abruptly to nearly the double of its value for a high degree of saturation. This provides the hope for the development of a monitoring method to warn against land slides.

\section{References}

[1] B. Albers, K. Wilmanski. Monochromatic surface waves on impermeable boundaries in two-component poroelastic media, Cont. Mech. Thermodyn. 17 (2005), 269-285.

[2] B. Albers. Monochromatic Surface Waves at the Interface between Poroelastic and Fluid Halfspaces. Proc. Royal Soc. A, 462 (2006), 701-723.

[3] B. Albers, K. Wilmanski. Acoustics of two-component porous materials with anisotropic tortuosity. Cont. Mech. Thermodyn. 24 (2012), 403-416.

[4] B. Albers. Modeling and Numerical Analysis of Wave Propagation in Saturated and Partially Saturated Porous Media (2010) habilitation thesis, Shaker, Aachen. 


\title{
Calculation of the effective speed of sound in corrugated pipes by multiple scales
}

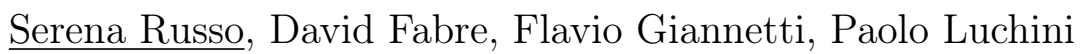 \\ Università degli Studi di Salerno, Italy \\ IMFT, University of Toulouse, France
}

Corrugated pipes are commonly used in many engineering and industrial applications because they combine local rigidity with global flexibility. In many cases, the flow of air through such pipes can cause a loud whistling at specific frequencies. In most industrial cases, such whistling is an undesired effect as it may result in severe noise and structural vibration problems (see [1] for a recent review, covering industrial applications and available work on this problem). On the other hand, the ability of such ducts to generate clear and tonal sound is also used in a musical toy called the "hummer" or "Voice of the dragon" [2].

The mechanism responsible to whistling is known to combine two ingredients. First, vortex shedding occurs at the upstream edge of the cavities forming the wall of the pipe. Small disturbances are amplified along the shear layer of the cavities by the Kelvin-Helmholtz instability and impinge on the downstream edges of the cavities, producing a fluctuating axial force which behaves like an acoustic source. Secondly, this synchronized vortex shedding leads to a global resonance if the shedding frequency coincides with the natural acoustical frequencies of the tube.

In the case of a corrugated pipe, the presence of cavities at the walls modifies the effective speed of sound $c_{e f f}$ with respect to the case of straight pipe $\left(c_{0}\right)$, so the resonance frequencies are given by $f_{m}=m f_{1}=m c_{e f f} / 2 L$, where $m=1,2,3, \ldots$ and $L$ is the length of the pipe. The knowledge of $c_{e f f}$ is thus crucial to predict the whistling frequencies of such devices.

The purpose of this paper is to accurately compute the effective velocity of sound based on a numerical solution of the Helmholtz equation in a periodic domain. It is derived on the assumption that the acoustical wavelength is large compared to the periodicity of the pipe which is verified in most applications. The results will be compared with those derived from a simpler model proposed by Cummings and Elliott [3], that gives an estimation of the effective speed of sound as function of the geometry of the duct

$$
\left(\frac{c_{e f f}}{c_{0}}\right)^{2}=\frac{V_{i n}}{V_{t o t}}
$$

where $V_{\text {in }}$ is the volume of the inner part of the duct (excluding the cavities) and $V_{\text {out }}$ is the total volume (including the cavities). The results are presented for a variety of geometries used in the literature, considering both axisymmetric and 2D cases: the Cummings-Elliott model reproduces well the results derived with our approach for short cavities but significant disagreement is found for longer cavities.

\section{References}

[1] B. Rajavel, M. G. Prasad. Acoustics of corrugated pipes: a review. Applied Mechanics Review, Vol. 65, pp.050801 (2012).

[2] G. Nakiboğlu,S. P. C. Belfroid, J. Golliard, A. Hirschberg. On the whistling of corrugated pipes: effect of pipe length and flow profile. J. Fluid Mech. 672 (2010), 78-108.

[3] J. M. Elliott. Corrugated Pipe Flow: Lecture notes on the mathematics of acoustics. Imperial college press, London (2005). 


\title{
Numerical Calculation of Acoustic Sources for the Landing Gear of Aeroplane during Take-off and Landing
}

\author{
Bosko Rasuo, Vladimir Jazarevic \\ University of Belgrade, Faculty of Mechanical Engineering
}

The sound which is generated from the aircraft during the take-off and landing is one of the main problems for the people who live in the areas near the airport. It is very important to allocate and accurately calculate acoustic sources generated from turbulent flow produced by the aerodynamics components of the aircraft. This is done in order to calculate inhomogeneous term of Helmholtz equation which serves as a prediction tool of sound propagation in the domain. It is used subgrid-scale stabilized (SGS) finite element method for solving incompressible Navier-Stokes equation which simulate turbulent flow. Afterwards is done double divergence of Litghill's tensor in order to calculate acoustics sources. Further, the transformation from time domain to frequency domain is used with Direct Fourier Transform which leads to smaller memory usage and computational cost.

The aim of the article is to show that previously mention method lead to better and richer representation of the spectrum of frequencies obtained from turbulent flow. Good representation of spectrum will give better inhomogeneous term of Helmholtz equation. Better prediction and calculation of acoustics sources will lead to reduction of sound generation through design of aerodynamics components on the aircraft. 


\title{
Forward and Inverse Viscoacoustic Modelling in a Tunnel Environment
}

\author{
Khayal Musayev $^{1}$, Klaus Hackl ${ }^{1}$, and Matthias Baitsch ${ }^{2}$ \\ ${ }^{1}$ Ruhr University Bochum \\ ${ }^{2}$ Bochum University of Applied Sciences
}

In this work, the goal is to model forward acoustic waves in a tunnel environment with attenuation and to do full waveform inversion. In reality, there is no material without attenuation. Some materials, such as rocks, have so low attenuation that, in a small domain, the waves are almost not damped at all. At the same time, there are materials with high attenuation. In an environment with such materials, the attenuation has to be taken into account in order to model the waves properly. In this study, attenuation effect is integrated into acoustic equation by using Kolsky-Futterman model ([1], [2])which only replaces velocity field with a complexvalued field in frequency domain. Apart from attenuation, another objective is to consider an inhomogeneous density field. Mainly, acoustic equation with a constant density field is referred to in many studies. In many cases, it may suffice to model waves appropriately. However, in reality, the density field of ground can be highly inhomogeneous. The objective is to investigate the effect of the inhomogeneity in waves, and to search for density field $\rho$ and attenuation parameter $Q$ as well as pressure wave velocity $c$ using full waveform inversion.

\section{References}

[1] Kolsky, H. The propagation of stress pulses in viscoelastic solids. Philosophical Magazine 1 (1956), 693-710.

[2] Futterman,W. Dispersive body waves. Journal of Geophysics Research 67 (1962), 5279 -5291. 


\title{
On numerical simulation of tsunami run-up on shore
}

\author{
Nina Shokina \\ Rechenzentrum der Albert-Ludwigs-Universität Freiburg, Freiburg im Breisgau, Germany
}

The numerical modelling of tsunami run-up on shore is done using the allocation of waterfront (adaptive grid method), i.e. in the domain with moving boundary. The domain is covered with a moving grid, and one of the boundary coordinate lines coincides with waterfront. The waterfront position is obtained using the exact analytical solutions of the shallow water equations in the vicinity of waterfront within the time period, which is equal to the time step of the used finite difference predictor-corrector scheme $[1,2]$. The dynamically adaptive grid is constructed on each time step, thus, the time expenses can become larger than those for the solution of difference equations. Two grids are used to avoid that. The basic grid is generated before the solution of the problem and covers the water area and the part of adjoining land. The boundary coordinate line of this grid is located on the land and well spaced from the initial waterfront position, guaranteeing that the wave will never reach this position. The computational grid is generated on each time step and adapted to the numerical solution and moving waterfront. This grid is " $1 \mathrm{D}$ ", because its nodes move along one family of coordinate lines of the basic grid only. The grid nodes are defined by 1D equidistribution method for plane curves.

The computation of the inundation zones after tsunami run-up on the shore with weakly curved waterfront line is done. For a plane slope the results are compared with the known analytical solutions and experimental data. A good correspondence of results and analytical solutions is obtained for the range of parameters, for which those solutions are valid. The numerical experiments have shown that the substitution of irregular slope with plane one can lead to the large errors in estimation of inundation zones. Also, the pictures of wave interaction with shore differ greatly. After the wave runs up to a plane slope, it runs down with the generation of the principal reflected wave. For an irregular slope, the reflected wave is generated in the moment when the run-up still continues on the shore; and, while the water is present on low gradient irregular slope, several reflected waves are generated, which is not so for a flat slope.

The method does not work for a highly irregular shoreline, in particular, when simply connected water domain becomes multiply connected due to the formation of separate islands. Then a combined method [3] is used, based on two approximations of the shallow water model: 1D and 2D. First, the 2D model with the reflecting boundary condition on the shore is used for modelling of wave motion from its source to the shore. The continuous record of wave parameters on a given isobathic line is done. This isobathic line is located well spaced from the shore, sufficient for the minimization of the influence of reflected waves on the form of the main part of in-going wave. The continuous record of wave parameters on a given isobathic line is done during the computation. This isobathic line is located at a certain distance from a shore, sufficient for the minimization of the influence of reflected waves on the form of the main part of in-going wave. Then, this data is used as boundary conditions for 1D computations along cross-sections, drawn from this given isobathic line in the shore direction. On the basis of these 1D computations the vertical and horizontal run-ups are computed along the chosen cross-sections. The procedure is developed for the reconstruction of inundation zone boundary using the detailed digital land relief. The method of allocation of moving waterfront point (adaptive grid method) is used for run-up computation in $1 \mathrm{D}$ cross-sections. The modelling of waterfront point motion is done on the basis of the analytically obtained motion law for this point. The developed methodology is applied for computation of inundation zones and has shown [3] the satisfactory correlation of the numerical results and field data.

\section{References}

[1] N.Yu. Shokina. To the problem of construction of difference schemes on movable grids. Rus. J. Numer. Anal. Math. Model. 27(6) (2012), 603-626.

[2] N. Shokina. Some notes on monotonicity preserving schemes on adaptive grids. Proc. Appl. Math. Mech., Special Issue: 84th Annual Meeting of the International Association of Applied Mathematics and Mechanics (GAMM), Erlangen 2014. 14 (1) (2014), 853-854.

[3] S.A. Beisel, N.Yu. Shokina, G.S. Khakimzyanov, L.B. Chubarov, O.A. Kovyrkina, V.V. Ostapenko. On some numerical algorithms for computation of tsunami runup in the framework of shallow water model. I. Comput. Techolog. 19(1) (2014), 40-62. 


\title{
Potential flow simulations of Peregrine-type deep water surface gravity wave packets
}

\author{
Arne Wenzel , Dennis Bünte, Norbert P. Hoffmann \\ Hamburg University of Technology, Dynamics Group, Germany \\ Hamburg University of Technology, Institute of Biomechanics, Germany \\ Imperial College London, Department of Mechanical Engineering, United Kingdom
}

Applying a higher order spectral method [1] for potential flow we numerically determine the time evolution of non-linear deep water wave packets originating from initial conditions derived from the Peregrine breather [2] solution of the weakly non-linear Schrödinger equation (NLS) [3]. A number of experimental studies on this NLS solution confirm its role for deep water oceanic rogue waves, see e.g. [4, 5].

In this contribution we modulate the non-linear Stokes wave solution [6] according to early stages of the Peregrine breather solution of the NLS and then follow the evolution of the resulting wave packet within the potential flow approximation.

The spatio-temporal evolution of the wave packets qualitatively agrees well with what would be expected from the NLS solution. However some quantitative discrepancies do exist: the propagation speed of the packets depends on the wave steepness of the background waves, and the maximum wave envelope amplification occurs slightly later than predicted from the NLS. The amplification factor itself exceeds slightly the factor known from the NLS solution.

\section{References}

[1] D. G. Dommermuth and D. K. P. Yue. A high-order spectral method for the study of nonlinear gravity waves Journal of Fluid Mechanics 184 (1987), 267-288

[2] D. H., Peregrine. Water waves, nonlinear Schrödinger equations and their solutions. The Journal of the Australian Mathematical Society. Series B. Applied Mathematics 25 (1983), 16-43.

[3] V. E. Zakharov. Stability of periodic waves of finite amplitude on the surface of a deep fluid. Journal of Applied Mechanics and Technical Physics 9 (1972) 190-194.

[4] A. Chabchoub, N. P. Hoffmann and N, Akhmediev. Rogue wave observation in a water wave tank Physical Review Letters 106 (2011), 204502.

[5] A. Chabchoub, N. Akhmediev and N. P. Hoffmann. Experimental study of spatiotemporally localized surface gravity water waves. Physical Review 86(2012), 016311.

[6] J. D. Fenton. The numerical solution of steady water wave problems. Computers \& Geosciences 14 (1988), $357-368$. 


\title{
Application of variational-asymptotic method to modulation theory
}

\author{
K. C. Le, L. T. K. Nguyen \\ Lehrstuhl für Mechanik - Materialtheorie, Ruhr-Universität Bochum
}

This work presents the application of the variational-asymptotic method to the Whitham's modulation theory for differential equations of nonlinear dispersive waves. The Whitham averaging method is based on two important assumptions: the differential equation possesses the solution of steady profile and the existence of a number of associated local conservation laws [1]. Shortly later he developed an equivalent method based on an average variational problem [2]. Since it requires the knowledge about the uniform solution or its first integrals of the corresponding differential equation in one wavelength, it cannot be extended to systems where such solutions are not known in advance. In contrary, the variational-asymptotic method develops the multiscale approach which enables one to reduce systematically variational problems containing a small parameter to the average variational problems via the solution of the so-called strip problems [3, 4, 5]. By applying the variational-asymptotic method, we obtain the modulation theory for several differential equations such as the Korteweg-de Vries (KdV) equation [6], the scalar Boussinesq equation [7, 8], the one- and two-dimensional sineGordon equations $[9,10]$. Depending on the the type of the differential equations we may derive the theories of either amplitude or slope modulation. In the limit of trains of solitons or positons, the asymptotic solutions of the modulation equations can be obtained.

\section{References}

[1] G. B. Whitham. Nonlinear dispersive waves. Proceedings of the Royal Society A 283 (1965), 238-261.

[2] G. B. Whitham. Linear and nonlinear waves. Wiley-Interscience, New York (1974).

[3] V. L. Berdichevsky. Variational-asymptotic method of constructing a theory of shells. Appl. Math. Mech. 43 (1979), 664-687.

[4] V. L. Berdichevsky. Variational principles of continuum mechanics. Springer, Berlin (2009).

[5] K. C. Le, L. T. K. Nguyen. Energy methods in dynamics. Springer, Berlin (2014).

[6] K. C. Le, L. T. K. Nguyen. Amplitude modulation of waves governed by Korteweg-de Vries equation, Int. J. Eng. Sci. 83 (2014), 117-123.

[7] K. C. Le, L. T. K. Nguyen. Amplitude modulation of water waves governed by Boussinesq's equation, Nonlinear Dynamics (submitted).

[8] L. T. K. Nguyen. Wronskian formulation and Ansatz methtod for bad Boussinesq equation. Vietnam J. Math. (accepted).

[9] K. C. Le, L. T. K. Nguyen. Slope modulation of waves governed by sine-Gordon equation. Communications in Nonlinear Science and Numerical Simulation, 18 (2013), 1563-1567.

[10] K. C. Le, L. T. K. Nguyen. Slope modulation of ring waves governed by two-dimensional sine-Gordon equation, Wave Motion (submitted). 


\title{
A novel approach to mode - tracing in SBFEM - Simulations: Higher-order Taylor- and Padeapproximation
}

\author{
Fabian Krome, Hauke Gravenkamp \\ Federal Institute for Materials Research and Testing Division 8.4, Berlin
}

This contribution presents the study of strange phenomena in wave mode representations of waveguides. For this study the waveguides are computed by means of Scaled Boundary Finite Element Methods (SBFEM). Different approaches of mode tracing are used to identify the characteristics of the resulting wave modes. Higher order differentials of the underlying eigenvalue problem are the basis for these approaches.

The main idea behind mode tracing approaches is to reduce the cubic computation time to solve the eigenvalue problem for each frequency of interest. There exist several algorithms for mode tracing from semi-analytical approaches [1] to approximation methods [2]. Although these algorithms show great performances for a variety of cases, under certain unfavorable circumstances these methods run into problems - mainly expressed in the loss of solutions or producing a small number of wrong solutions. Luckily these problems are reproducible and occur at certain frequencies only.

This contributions goal is to present and discuss the underlying cause for these problems. The fascinating effects at critical frequencies are displayed and a suggestion for a stabilization for the solution process is made. This study bases its conclusion on a numerical viewpoint. A different way of identifying the problem and phenomena through an analytical viewpoint can be found in [3].

Using the model developed by Hauke Gravenkamp [4] the modeling of waveguides is based on a second order eigenvalue problem:

$$
\left(k^{2} \mathbf{E}_{\mathbf{0}}-i k\left(\mathbf{E}_{\mathbf{1}}^{\top}-\mathbf{E}_{\mathbf{1}}\right)-\mathbf{E}_{\mathbf{2}}\right) \boldsymbol{\Psi}=\mathbf{M}_{\mathbf{0}} \boldsymbol{\Psi} \boldsymbol{\Omega}
$$

with $\mathbf{E}_{\mathbf{0}}, \mathbf{E}_{\mathbf{1}}, \mathbf{E}_{\mathbf{2}}, \mathbf{M}_{\mathbf{0}} \in \mathbb{C}, k$ being the wave number and $\boldsymbol{\Psi}, \boldsymbol{\Omega}$ corresponding frequencies and displacements. The main focus lies on frequencies $\Omega$ at which certain modes show strongly coupled behavior.

Main aspects in this study include high order differentials of the eigenvalue problem and the corresponding Taylor and Pade approximations for the eigenvalue problem as a whole.

\section{References}

[1] B.N. Pavlakovic, M.J.S. Lowe, D.N. Alleyne, Disperse: a general purpose program for creating dispersion curves.in: D. Thompson, D. Chimenti (Eds.), Review of Progress in Quantitative NDE, Plenum Press (1997), 185-503.

[2] H. Gravenkamp, H. Man, C. Song, J. Prager. The computation of dispersion relations for three-dimensional elastic waveguides using the Scaled Boundary Finite Element Method. J. of Sound and Vibration. 332 (2013), $3756-503$.

[3] B.R. Mace, E. Manconi. Wave motions and dispersion phenomena: Veering, locking and strong coupling effects. J. Acoust. Soc. Mechs. 131 (2012), 1015-503.

[4] H. Gravenkamp, C. Song, J. Prager. A numerical approach for the computation of dispersion relations for plate structures using the Scaled Boundary Finite Element Method. J. of Sound and Vibration. 331 (2012), 2545-503. 


\title{
Numerical modeling of a sub-sonic roll-over load along a rods skin
}

\author{
Wolfgang Weber, Bernd W. Zastrau, David Löpitz, Daniel Balzani \\ Technische Universität Dresden, Institut für Mechanik und Flächentragwerke, \\ George-Bähr-Straße 1, 01069 Dresden, Germany
}

\begin{abstract}
To adequately describe the wave propagation in rod-like structures is a matter of enormous practical relevance. Practical applications involve, but are not restricted to, pile-driving for off-shore wind turbines and dynamic pull-out of reinforcement elements in composite materials, for example [1, 2, 3]. As can readily be seen these problems give significant challenges for both the analytical modeling and the numerical implementation. These challenges arise e.g. from the interaction of the traveling wave with the host in which the rod is embedded in. For example, the dynamic pull-out of a reinforcement element is characterized by a relative slip between the reinforcement and the embedding matrix. But before relative slip at a differential element of the reinforcement can occur, the bond of the respective element (of differential length) to the surrounding matrix has to be completely damaged which equals a crack. Thus, a crack front will propagate along the skin of the rod. Whereas the rod initially had a perfect bond to the surrounding matrix it is now subdivided into two parts: one with a completely damaged bond and another with a perfect bond. However, the boundary between these two parts will travel with the speed of the crack front which itself follows the stress wave propagating through

To cope with such and other phenomena in a first step a rather simple example will be investigated. It consists of a semi-infinite rod at whose skin a constant longitudinal load is traveling from the free end to infinity. Herein, the speed $c_{q}$ this load propagates with is lower than the longitudinal wave speed $c_{L}$ within the rod so that a sub-sonic problem is at hand. The material behaviour of the homogeneous rod is characterized as linearly elastic and isotropic. Within this contribution firstly an analytical model describing the wave propagation is presented. Afterwards, the results of this analytical approach are compared to the results obtained by standard FEM using 8-node-solid-elements. Afterwards, the results of this analytical approach are further analyzed by numerical calculations. The adequate description of the specific boundary conditions is obtained by special surface elements that allow for the roll-over load.
\end{abstract} the rod.

\section{References}

[1] C. Gan, Y. Wei, S. Yang. Longitudinal wave propagation in a rod with variable cross-section. Journal of Sound and Vibration 333 (2014), 434-445, doi: 10.1016/j.jsv.2013.09.010.

[2] N. Sridhar, Q.D. Yang, B.N. Cox. Slip, stick, and reverse slip characteristics during dynamic fibre pullout, Journal of the Mechanics and Physics of Solids 51 (2003), 1215-1241, doi: 10.1016/S0022-5096(03)00035-8.

[3] Q.D. Yang, A. Rosakis, B.N. Cox. Dynamic fibre sliding along debonded, frictional interfaces. Proceedings of the Royal Society A: Mathematical, Physical and Engineering Science 462 (2006), 1081-1106, doi: 10.1098/rspa.2005.1602. 


\section{S14: Applied analysis}

This Session is devoted to the mathematical analysis of natural phenomena and engineering problems. In this area PDEs play a basic role. Therefore lectures discussing analytical aspects of PDE problems as well as problems in the Calculus of Variations are welcome. On account of the importance of applications in Materials Science, the Session will have a special focus on the following topics:

Shape memory polymers

Liquid crystals

Bio-materials

Ferromagnetic materials

Two-phase fluids

Gas storage in alloys and polymers

Phase transitions in smart materials

Damage and other dissipative processes in solids

The interest in such issues is witnessed by an intense research which requires a delicate interplay between experiments, modeling, theoretical analysis of PDEs and their numerical approximation. This Session is intended to be an occasion to discuss the various aspects of this interdisciplinary subject, taking advantage of the contributions of young researchers in the field. 


\title{
Weak solution to certain problem in fluid mechanics
}

\author{
Eduard Feireisl
}

Czech Acad. Sci. Prague, Czech Republic

We discuss several concepts of weak, dissipative, very weak and/or measure valued solutions to various problems in fluid mechanics. We show that many equations modeling inviscid fluids may posses infinitely many weak solutions and even infinitely many dissipative weak solutions. Suitable admissibility criteria will be proposed. 


\title{
A low volume-fraction limit for martensic microstructures in shape-memory alloys
}

\author{
$\underline{\text { Johannes Diermeier }}$ \\ University of Bonn
}

Shape-memory materials have the property that they recover their shapes under heating if they have been deformed at a low temperature. The reason for this behaviour is a solid-solid phase transition between different lattice structures. One can observe the occurence of microstructure around interfaces between different phases of the material.

We consider a variational model of the energy of a deformation and restrict ourselves to a scalar valued, two dimensional, geometrically linearized case with two variants of martensite, the low temperature state, in which one of the variants has a much smaller volume fraction than the other one. The energy is given as the sum of an elastic term and a surface term

$$
E^{\varepsilon, \theta}(v)=\int_{(0,1)^{2}} \partial_{x} v^{2}+\min \left\{\left|\partial_{y} v+\theta\right|^{2},\left|\partial_{y} v-1\right|^{2}\right\} \mathrm{d} \mathcal{L}^{2}+\varepsilon\left\|D^{2} v\right\|\left((0,1)^{2}\right)
$$

where $\theta \ll 1$ is the small volume fraction. The deformation $v \in W^{1,2}\left((0,1)^{2}\right)$ satisfies $D v \in B V\left((0,1)^{2}\right)$ and zero-boundary values $v(0, y)=0$ which force the microstructure to occur.

It is known that the energy follows the scaling law $E^{\varepsilon, \theta} \sim \varepsilon^{2 / 3} \theta^{2 / 3}$ if $\varepsilon \leq \theta^{2}$ and $E^{\varepsilon, \theta} \sim \theta^{2}$ otherwise. In the first case the upper bound is achieved by a self-similar twinning construction whereas one uses a single phase of martensite in the second case.

We are interested in a deeper analysis for $\varepsilon \sim \theta^{2}$. We set $\varepsilon=\sigma \theta^{2}$ for some $\sigma \in \mathbb{R}^{+}$and determine the reduced model in the limt $\theta \rightarrow 0$ by means of $\Gamma$-convergence. 


\title{
Homogenization of layered structures with rigid components in single-slip finite elastoplasticity
}

\author{
Fabian Christowiak, Carolin Kreisbeck \\ Faktultät für Mathematik, Universität Regensburg
}

In a first step towards a better understanding of the macroscopic response of elastoplastic composites, we consider materials with a bilayered structure. While one material component is rigid in the sense that it does not allow any plastic deformation, the other one is softer and has one active slip system with linear hardening. The model in two spatial dimensions is based on a time-discrete variational approach to single-slip finite plasticity, and is inspired by models for homogeneous single crystalline materials as studied for instance in $[1,2]$.

In this presentation, we characterize the asymptotic behavior of the energy in the first time increment as the layer thickness tends to zero in terms of $\Gamma$-convergence. In fact, due to the anisotropy of the setup, the resulting $\Gamma$-limit depends critically on the orientation of the slip system relative to the layers. This effect is reflected in the following two special cases, for which we provide explicit formulas. If the slip direction is parallel to the layers, the limit energy functional admits only deformations that are combinations of a global rotation and shear in slip direction. In contrast, for a slip direction orthogonal to the layers, one observes a complete blocking of the slip system, so that macroscopically the material sample is so rigid that it can only be rotated globally.

The main technical difficulty is to capture the rigidity introduced by the layered geometry in order to obtain a suitable lower bound. This requires a new rigidity result combining the Rešetnjak theorem [3] applied to each rigid layer with the process of decreasing layer thickness. For the upper bound a careful analysis of admissible microstructures is needed, which on a technical level means solving the differential inclusion induced by the variational models. Furthermore, we compare our findings to results of non-linear homogenization theory, in particular the common cell and multi-cell formulas [4].

\section{References}

[1] S. Conti, F. Theil. Single-slip elastoplastic microstructures. Arch. Ration. Mech. Anal. 178 (2005), no.1, 125-148.

[2] S. Conti, G. Dolzmann, C. Kreisbeck. Asymptotic behavior of crystal plasticity with one slip system in the limit of rigid elasticity. SIAM J. Math. Anal. 43 (2011), no. 5, 2337-2353.

[3] J. G. Rešetnjak, Liouville's conformal mapping theorem under minimal regularity hypotheses. Sibirsk. Mat. Ž. 8 (1967), 835-840

[4] S. Müller. Homogenization of nonconvex integral functionals and cellular elastic materials. Arch. Ration. Mech. Anal. 99 (1987), no. 3, 189-212 


\title{
Homogenization for dislocation based gradient visco-plasticity
}

\author{
Sergiy Nesenenko \\ Universität Duisburg-Essen, Germany
}

In this work we study the homogenization for infinitesimal dislocation based gradient viscoplasticity with linear kinematic hardening and general non-associative monotone plastic flows. The constitutive equations in the models we study are assumed to be only of monotone type. Based on the generalized version of Korn's inequality for incompatible tensor fields (the non-symmetric plastic distortion) due to Neff/Pauly/Witch, we derive uniform estimates for the solutions of quasistatic initial-boundary value problems under consideration and then using a modified unfolding operator technique and a monotone operator method we obtain the homogenized system of equations. A new unfolding result for the Curl Curl-operator is presented in this work as well. The proof of the last result is based on the Helmholtz-Weyl decomposition for vector fields in general $L^{q}$-spaces. 


\title{
On the use of potential fields in fluid mechanics
}

\author{
$\underline{\text { Florian Marner }}^{1}$, Markus Scholle $^{1}$ \\ ${ }^{1}$ Heilbronn University, Institute for Automotive Technology and Mechatronics, \\ D-74081 Heilbronn, Germany.
}

For many systems of differential equations arising in classical physics the introduction of auxiliary potential fields turned out to be beneficial in various respects. For instance in classical fluid mechanics, potential fields have been employed to enable the integration of the equations of motion. As is well known, Bernoulli's equation is obtained as a first integral of Euler's equations in the absence of vorticity and viscosity if the velocity vector $\vec{u}=\nabla \Phi$ is perceived as the gradient of a scalar potential. The so-called Clebsch transformation

$$
\vec{u}=\nabla \varphi+\alpha \nabla \beta
$$

involving three scalar potentials allows for a further extension to flows with non-vanishing vorticity; the resulting set of equations together with the continuity equation turn out to be self-adjoint, allowing for a variational formulation. A generalization of the Clebsch transformation in the case of baroclinic flows has been formulated e.g. by [3]. All attempts in classic literature, however, are restricted to inviscid flows. The finding of a potential representation which makes possible the integration of the Navier-Stokes equations is therefore a very desirable aim.

Progress on this topic was reported by [1] who constructed a first integral of the two-dimensional incompressible Navier-Stokes equations by making use of an auxiliary potential field and a representation of the fields in terms of complex coordinates. Based on the alternative first integral formulation, a convenient representation of the dynamic boundary conditions has been established and an efficient finite element method for solving twodimensional free surface flows developed [2]. Moreover, using a tensor potential rather then a scalar potential, the theory can be successfully generalized to encompass three-dimensional Navier-Stokes flow.

The search for potential fields allowing for the integration of the full Navier-Stokes equations is closely related to the construction of a Navier-Stokes Lagrangian. Such a Lagrangian can be extracted from the aforementioned tensor potential representation. However, a viable alternative results if the considerations are founded on and the formulation is derived from the basic physics directly. Since viscosity leads to dissipation and therefore to the irreversible transfer of mechanical energy to heat, thermal degrees of freedom have to be taken into account. Following this idea, a generalization of the Clebsch transformation to baroclinic flow can be found, as shown by [3]. The authors propose a further extension to viscous flow, which can be shown to fulfill the relevant symmetry criteria given in [4]. The resulting Lagrangian reproduces the Navier-Stokes equations except for an additional term, which may be controlled by an appropriate relaxation method.

\section{References}

[1] M. Scholle, A. Haas, P.H. Gaskell. A first integral of Navier-Stokes equations and its applications. Proc. Roy. Soc. A 467. (2011), 127-143.

[2] F. Marner, P.H. Gaskell, M. Scholle. On a potential-velocity formulation of Navier-Stokes equations. Phys. Mesomech. 17(4). (2014), 341-348.

[3] R. Seliger, G.B. Witham. Variational principles in continuum mechanics. Proc. Roy. Soc. A 305. (1968), $1-25$.

[4] M. Scholle. Construction of Lagrangians in continuum theories. Proc. Roy. Soc. A 460. (2004), 3241-3260. 


\title{
Well-Posedness and Stability for some Volume-Preserving Curvature Flows with Boundary Contact or Triple Junctions
}

\author{
Helmut Abels, Nasrin Arab, Harald Garcke, Lars Müller \\ Faculty of Mathematics, University of Regensburg
}

We will consider the dynamic stability of a spherical cap on a flat plane in three space dimensions with a dynamic boundary condition which is associated to an energy of the contact line between the spherical cap and the plane. Using a suitable parametrization we can apply results on maximal $L^{p}$-regularity for parabolic equations with dynamic boundary conditions and a generalized principle of linearized stability. To this end a careful analysis of the spectral properties of the linearized operator is needed. Moreover, we will briefly discuss the dynamic stability of a symmetric planar network evolving under curve diffusion.

\section{References}

[1] H. Abels, H. Garcke, L. Müller. Local well-posedness for volume-preserving mean curvature and Willmore flows with line Tension. Preprint, arXiv:/1403.1132

[2] H. Abels, H. Garcke, L. Müller. Stability of spherical caps under the volume-preserving mean curvature flow with line tension. Preprint, arXiv:/1403.1139, to appear in Nonlinear Anal.

[3] H. Abels, N. Arab, H. Garcke. On convergence of solutions to equilibria for fully nonlinear parabolic problems with nonlinear boundary conditions. Preprint, arXiv:/1403.4526 


\title{
Nonlocal Cahn-Hilliard equation with a reaction term
}

\author{
E. Rocca ${ }^{1}, \underline{\text { S. Melchionna }}{ }^{2}$ \\ ${ }^{1}$ University of Vienna, Faculty of Mathematics \\ ${ }^{2}$ Weierstrass Institute for Applied Analysis and Stochastics, Berlin
}

We prove existence, uniqueness, regularity and separation properties for a nonlocal Cahn-Hilliard equation with a reaction term. We deal here with the case of logarithmic potential and degenerate mobility as well as uniformly lipschitz in $u$ reaction term $g(x, t, u)$.

\section{References}

[1] Frigeri, S., Grasselli, M., Rocca, E., A diffuse interface model for two-phase incompressible flows with nonlocal interactions and noncostant mobility, preprint azx: 13036446v2 (2014), 1-44

[2] Londen, S., Petzeltovà, H., Regularity and separation from potential barriers for a non-local phase-field system, J. Math. Anal. Appl. 379 (2011) 724-735 


\title{
Well-posedness and optimal control of Allen-Cahn type equations with singular potentials and dynamic boundary conditions
}

\author{
Luca Calatroni, Pierluigi Colli \\ Cambridge Centre for Analysis, Univeristy of Cambridge, UK \\ Dipartimento di Matematica "F. Casorati", Universitá degli studi di Pavia, Italy.
}

We prove well-posedness results for the solution to an initial and boundary value problem for an Allen-Cahn type equation describing the phenomenon of phase transitions for a material contained in a bounded and regular domain. The dynamic boundary conditions for the order parameter account for interactions with the walls. Our results are showed by using regularisations of the nonlinearities of the problem and performing some a priori estimates which allow us to pass to the limit thanks to compactness and monotonicity arguments [1]. Moreover, some results on the optimal control problem will be reviewed for a class of singular potentials including the derivatives of logarithmic potentials confined in $[-1,1]$ and subdifferentials of the indicator function of the interval $[-1,+1]$ up to concave perturbations $[2,3]$.

\section{References}

[1] L. Calatroni, P. Colli. Global solution to the Allen-Cahn equation with singular potentials and dynamic boundary conditions. Nonlinear Anal. 79 (2013), 12-27.

[2] P. Colli, J. Sprekels. Optimal control of an Allen-Cahn equation with singular potentials and dynamic boundary condition. to appear in SIAM J. Control. Optim.

[3] P. Colli, M.H. Farshbaf-Shaker, J. Sprekels. A deep quench approach to the optimal control of an Allen-Cahn equation with dynamic boundary conditions and double obstacles. to appear in Appl. Math. Optim. 


\title{
Relaxation in nonlinear elasticity with constraints on the determinant
}

\author{
Sergio Conti, Georg Dolzmann \\ Universität Bonn \\ Universität Regensburg
}

Consider the variational problem in nonlinear elasticity of the form $E[u]=\int W(D u) d x$. Here $u: \mathbb{R}^{n} \rightarrow \mathbb{R}^{n}$ the elastic deformation and $W$ represents the free energy density which is defined on $n \times n$-matrices with positive determinant and and satisfies $W\left(F_{n}\right) \rightarrow \infty$ along sequences $\left(F_{n}\right)_{n \in \mathbb{N}}$ of matrices with det $F_{n}>0$ and $\operatorname{det} F_{n} \rightarrow 0$ for $n \rightarrow \infty$. It is shown that under suitable growth assumptions the functional $\int W^{\mathrm{qc}}(D u) d x$ is an upper bound for the relaxation of $E$, and that it coincides with the relaxation if the quasiconvex envelope $W^{\mathrm{qc}}$ of $W$ is polyconvex and has $p$-growth from below with $p \geq n$. This includes several physically relevant examples.

\section{References}

[1] S. Conti, G. Dolzmann. On the theory of relaxation in nonlinear elasticity with constraints on the determinant. Arch. Ration. Anal. Mech. (to appear). 


\title{
A Linear Scale-Space Theory for Continuous Nonlocal Evolutions
}

\author{
Giovanno Marcelo Cárdenas, Joachim Weickert, Sarah Schäffer \\ Mathematical Image Analysis Group \\ Dept. of Mathematics and Computer Science, Campus E1.1 \\ Saarland University, 66041 Saarbrücken, Germany
}

\begin{abstract}
Most scale-space evolutions are described in terms of partial differential equations. In recent years, however, nonlocal processes have become an important research topic in image analysis. The goal of our paper is to establish well-posedness and scale-space properties for a class of nonlocal evolutions. They are given by linear integro-differential equations with measures. In analogy to Weickert's diffusion theory (1998), we prove existence and uniqueness, preservation of the average grey value, a maximum-minimum principle, image simplification properties in terms of Lyapunov functionals, and we establish convergence to a constant steady state. We show that our nonlocal scale-space theory covers nonlocal variants of linear diffusion. Moreover, by choosing specific discrete measures, the classical semidiscrete diffusion framework is identified as a special case of our continuous theory. Last but not least, we introduce two modifications of bilateral filtering. In contrast to previous bilateral filters, our variants create nonlocal scale-spaces that preserve the average grey value and that can be highly robust under noise. While these filters are linear, they can achieve a similar performance as nonlinear and even anisotropic diffusion equations.
\end{abstract}




\title{
Rate-independent systems with viscosity and inertia - Evolutionary Gamma-convergence
}

\author{
Riccarda Rossi, Marita Thomas \\ Università di Brescia \\ Weierstrass Institute, Berlin
}

This talk is the second part of two contributions dealing with the analysis of rate-independent processes coupled with rate-dependent ones and inertial effects in a general framework. It focuses on Evolutionary Gammaconvergence results based on variational convergence in the spirit generalized gradient flows. The abstract results are substantiated with problems from applications, such as damage, delamination, and plasticity. 


\title{
Analysis of a gradient enhanced damage model
}

\author{
Christian Meyer, Livia Susu \\ TU Dortmund
}

The talk is concerned with a damage model including two damage variables, a local and a non-local one, which are coupled through a penalty term in the free energy functional in the spirit of [1]. After introducing the precise model, we prove existence and uniqueness for the viscous regularization thereof. Moreover, we rigorously study the limit for penalization parameter tending to infinity. It turns out that in the limit both damage variables coincide and satisfy the classical viscous damage model discussed in [2].

\section{References}

[1] B. J. Dimitrijevic, K. Hackl. A method for gradient enhancement of continuum damage models. Technische Mechanik. 28 (2008), 43-52.

[2] D. Knees, R. Rossi, C. Zanini. A vanishing viscosity approach to a rate-independent damage model. Mathematical Models and Methods in Applied Sciences. 23 (2013), 565-616. 


\title{
Mixed-growth plasticity and generalized rigidity
}

\author{
$\underline{\text { Janusz Ginster }}$ \\ University of Bonn
}

Under large stresses metals generate dislocations which are local defects of the crystalline lattice and can be interpreted as line-singularities of the elastic strain field. Straight parallel edge dislocations can be described by a variational model in the orthogonal plane of the singularities. The dislocations are then point singularities of the strain field $\beta$, i. e.

$$
\operatorname{curl} \beta=\sum_{i}^{N} b_{i} \delta_{x_{i}}
$$

for some dislocation points $x_{i} \in \mathbb{R}^{2}$ and Burgers' vectors $b_{i} \in \mathbb{R}^{2}$. The set of feasible dislocation points and Burgers' vectors is determined by the interatomic spacing $\varepsilon>0$.

The energy for a strain $\beta: \mathbb{R}^{2} \supseteq \Omega \rightarrow \mathbb{R}^{2 \times 2}$ and a corresponding singularity distribution $\mu=\sum_{i}^{N} b_{i} \delta_{x_{i}}$ connected to $\beta$ through (1) is of the form

$$
E_{\varepsilon}(\mu, \beta)=\int_{\Omega} W(\beta) d x .
$$

Near singularities the strain field typically diverges as $\frac{1}{|x|}$, so in order to obtain a finite energy we consider energy densities $W$ with quadratic growth near $S O(2)$ and subquadratic growth at infinity.

We will identify the limit as $\varepsilon \rightarrow 0$ of a suitably scaled version of the energy $E_{\varepsilon}$ by means of $\Gamma$-convergence. As a key ingredient we will present a rigidity estimate for fields whose curl is a bounded measure. 


\title{
New results on Cahn-Hilliard-Navier-Stokes systems with nonlocal interactions
}

\author{
Sergio Frigeri \\ Weierstrass Institute for Analysis and Stochastics, WIAS, Berlin
}

In this contribution we shall consider the nonlocal Cahn/Hilliard-Navier-Stokes system describing flow and phase separation of a binary mixture of incompressible viscous isothermal fluids. We shall first briefly recall some basic mathematical results concerning well-posedness, regularity and asymptotic behavior under different assumptions on mobility and double-well potentials (cf. $[1,2,3,4,5,6])$. Then, we shall turn to the new results concerning optimal control (cf. [7]) and concerning the existence of weak solutions for the case of the nonlocal Cahn-Hilliard/Navier-Stokes system with unmatched densities (nonlocal Abels-Garcke-Grün model).

\section{References}

[1] P. Colli, S. Frigeri, M. Grasselli, Global existence of weak solutions to a nonlocal Cahn-Hilliard-NavierStokes system, J. Math. Anal. Appl. 386 (2012), 428-444.

[2] S. Frigeri, C.G. Gal, M. Grasselli, On nonlocal Cahn-Hilliard-Navier-Stokes systems in two dimensions, submitted. Wias Preprint No. 1923 (2014).

[3] S. Frigeri, M. Grasselli, Global and trajectories attractors for a nonlocal Cahn-Hilliard-Navier-Stokes system, J. Dynam. Differential Equations 24 (2012), 827-856.

[4] S. Frigeri, M. Grasselli, Nonlocal Cahn-Hilliard-Navier-Stokes systems with singular potentials, Dyn. Partial Differ. Equ. 9 (2012), 273-304.

[5] S. Frigeri, M. Grasselli, P. Krejčí, Strong solutions for two-dimensional nonlocal Cahn-Hilliard-NavierStokes systems, J. Differential Equations 255 (2013), 2587-2614.

[6] S. Frigeri, M. Grasselli, E. Rocca, A diffuse interface model for two-phase incompressible flows with nonlocal interactions and nonconstant mobility, Wias Preprint No. 1941 (2014).

[7] S. Frigeri, E. Rocca, J. Sprekels, Optimal distributed control of a nonlocal Cahn-Hilliard/Navier-Stokes system in 2D, Wias Preprint No, 2036 (2014) 


\title{
Wulff shape and isoperimetric characterization of crystals
}

\author{
Paolo Piovano \\ Department of Mathematics, University of Vienna, Austria
}

In this talk the problem of analytically explaining why particles at low temperature arrange in periodic lattices will be considered and the emergence of the Wulff shape of crystals will be investigated. Ground states of phenomenological energies accounting for two-body (and three-body) short-ranged interactions will be shown to be connected subsets of the reference lattice, and their energies will be exactly quantified.

As the energy favors particle bonding and 'boundary' particles have in general less bonds, ground states are intuitively expected to have minimal 'perimeter', or maximal 'area'. This intuition will be verified by introducing a suitable notion of perimeter and area of configurations, and by showing that ground states are characterized as those configurations which realize equality in a discrete isoperimetric inequality.

In view of this characterization the emergence of a macroscopic Wulff shape as the number of particles grows will be established, and ground states will be shown to deviate from the asymptotic Wulff shape at most by $\mathrm{O}\left(n^{3 / 4}\right)$ particles. This result nicely reflects the inherent multiscale nature of the crystallization phenomenon. 


\title{
Effective Maxwell equations in a geometry with flat rings of arbitrary shape
}

\author{
Ben Schweizer, Agnes Lamacz \\ Technische Universität Dortmund, Fakultät für Mathematik
}

Propagation of light in heterogeneous media is a complex subject of research. It has received renewed interest in recent years, since technical progress demands smaller devices and offers new possibilities. At the same time, theoretical ideas inspired further research. Key research areas are photonic crystals, negative index metamaterials, perfect imaging, and cloaking. In the field of negative index materials, which we want to focus on in this contribution, two very influential works are the theoretical study by Veselago [3] and the approach of Pendry and others to the actual construction of such materials; see, e.g., [1].

The mathematical analysis of negative index materials is connected to a study of singular limits in Maxwell's equations. In this contribution, see [2], we present a result on homogenization of the time harmonic Maxwell's equations in a complex geometry. The homogenization process is performed in the case that many (order $\eta^{-3}$ ) small (order $\eta^{1}$ ), thin (order $\eta^{2}$ ), and highly conductive (order $\eta^{-3}$ ) metallic objects are distributed in a domain $\Omega \subset \mathbb{R}^{3}$. We determine the effective behavior of this meta-material in the limit $\eta \searrow 0$. For $\eta>0$, each single conductor occupies a simply connected domain, but the conductor closes to a ring in the limit $\eta \searrow 0$. This change of topology allows for an extra dimension in the solution space of the corresponding cell-problem. Even though both original materials (metal and void) have the same positive magnetic permeability $\mu_{0}>0$, we show that the effective Maxwell system exhibits, depending on the frequency, a negative magnetic response. This magnetic activity is the key feature of a negative index meta-material.

\section{References}

[1] J. Pendry, A. Holden, D. Robbins, W. Stewart. Magnetism from conductors and enhanced nonlinear phenomena. IEEE Trans. Microwave Theory Tech. 47 (1999), 2075-2084.

[2] B. Schweizer, A. Lamacz. Effective Maxwell equations in a geometry with flat rings of arbitrary shape. SIAM J. Math. Anal. 45 (2013), 1460-1494.

[3] M. V.Veselago. The electrodynamics of substances with simultaneously negative values of $\varepsilon$ and $\mu$. Soviet Physics Uspekhi 10 (1968), 509-514. 


\title{
A line-tension energy for dislocation networks on several slip planes
}

\author{
$\underline{\text { Peter Gladbach }}$
}

\author{
Universität Bonn
}

We study dislocation networks contained in finitely many parallel slip planes starting from the model by Koslowski, Cuiti no, and Ortiz, which couples a nonconvex Peierls potential acting on the slip fields with long-range elastic stress.

In the limit for small lattice spacing, in the sense of $\Gamma$-convergence, we obtain a line-tension functional depending on the Burger's vector and direction of the dislocation lines. For certain configurations we observe that oscillatory and splitting microstructure achieves a lower energy than straight dislocations.

As both the lattice parameter and separation of the slip planes tend to zero simultaneously, parallel dislocation lines in different planes interact. For certain configurations a coupling is favorable. In intermediate scaling regimes we show that a two-scale microstructure produces a lower energy than any single-scale one and that some two-scale microstructure is in fact optimal.

\section{References}

[1] Koslowski, M., Cuitiño, A.M., Ortiz, M. A phase-field theory of dislocation dynamics, strain hardening and hysteresis in ductile single crystal. J. Mech. Phys. Solids 50, 2597-2635, 2002.

[2] Conti, S., Garroni, A., Müller, S. Singular Kernels, Multiscale Decomposition of Microstructure, and Dislocation Models. Arch. Rational Mech. Anal. 199, 779-819, 2011.

[3] Conti, S., Gladbach, P. A line-tension model of dislocation networks on several slip planes. Submitted. 


\section{S15: Applied stochastics}

The session welcomes contributions on the following topics: uncertainty quantification; risk analysis and assessment; Bayesian methods in engineering; decision analysis; probabilistic modelling; stochastic mechanics. Applications to large scale engineering problems involving the use of computer models, like fluid mechanics and structural mechanics, are encouraged. 


\title{
Efficient Bayesian analysis of rare events in numerical models
}

\author{
Daniel Straub, Wolfgang Betz, Iason Papaioannou \\ Engineering Risk Analysis Group, Technische Universität München
}

Bayesian analysis is a consistent and effective framework for combining new information with existing models, in which prior probabilistic models are updated with new data and observations. For engineering models, Bayesian analysis can be interpreted as a stochastic inverse analysis. The Bayesian framework allows the engineer to combine uncertain and incomplete information with models from different sources. Moreover, it provides probabilistic information on the accuracy of the updated model.

When performing Bayesian analysis of engineering models, numerical methods such as Markov Chain Monte Carlo (MCMC) are necessary to evaluate the posterior distribution of the model parameters and to make predictions with the posterior model. Recently, the authors proposed a novel approach to Bayesian analysis, which is based on structural reliability concepts, termed BUS [1, 2]. By exploiting analogies between Bayesian analysis and the estimation of the probability of rare events (i.e. classical structural reliability problems), BUS offers a plethora of possible solution strategies. In particular, the authors have applied Subset Simulation [3], which has shown to enable efficient and robust Bayesian analysis in high dimensions [4]. However, other structural reliability methods can be applied and are more efficient for certain types of (lower-dimensional) problems. For example, the First-Order Reliability Method (FORM) or importance sampling methods such as line sampling are employed in [1].

Because BUS is based on structural reliability methods, it is particularly effective for updating the probability of rare events. Other approaches to Bayesian updating of rare events rely on a two-step approach, by first computing samples from the posterior distribution, and then estimating the probability of the event based on these samples, e.g. [5]. For rare events, the probability of interest is low, and a two-step approach is computationally expensive. On the other hand, the BUS approach allows to solve the Bayesian updating problem in a single step, by formulating the Bayesian updating as a system reliability problem. This allows using sampling approaches that are optimized towards solving such a system problem, but it also enables the use of non-sampling based approaches, in particular FORM/SORM methods.

In this contribution, the application of FORM-based BUS approaches to solving the Bayesian updating of rare events is investigated in detail. The factors influencing the performance of the algorithm are studied and recommendations on the use of the method are made. The algorithms are applied to illustrative examples as well as to a realistic large-scale example.

\section{References}

[1] D. Straub. Reliability updating with equality information. Probabilistic Engineering Mechanics, 26(2) (2011), $254-258$.

[2] D. Straub, I. Papaioannou. Bayesian Updating with Structural Reliability Methods. Journal of Engineering Mechanics, Trans. ASCE, in print (2014).

[3] S.-K. Au, J. L. Beck. Estimation of small failure probabilities in high dimensions by subset simulation. Probabilistic Engineering Mechanics, 16(4) (2014), 263-277.

[4] W. Betz, I. Papaioannou, D. Straub. Adaptive variant of the BUS approach to Bayesian updating. Proc. Eurodyn (2014), Porto, Portugal.

[5] H. Jensen, C. Vergara, C. Papadimitriou, E. Millas. The use of updated robust reliability measures in stochastic dynamical systems. Computer Methods in Applied Mechanics, 267 (2013), 293-317. 


\title{
Polynomial chaos approach for tuned bladed rotors with geometric modification
}

\author{
M. Koebelé-Cousquer, C. Proppe \\ Institute of Engineering Mechanics (ITM), Karlsruhe Institute of Technology
}

Bladed rotors are often considered in theory as periodic systems, but in fact they feature inevitable small imperfections caused by material defects and manufacturing tolerances which break the cyclic periodicity. This is called mistuning. The loss of the periodicity changes drastically the dynamic behavior of a rotor. Typically the forced response level of the mistuned bladed rotor is larger than the tuned design. Because of the random nature of mistuning, the determination of this amplitude magnification has to be considered as a stochastic problem. One of the classical ways to analyse mistuning is then to perform a Monte Carlo simulation.

In order to minimize the computational costs, a reduced order model has to be generated from the finite element model of the rotors. The way in which mistuning is implemented depends on the used reduction method. Recently Sinha $[1,2]$ developed a method to consider geometric mistuning via random variations in geometries of blades. This method has been called modified modal domain approach (MMDA). In the same time Mbaye et al. [3] developed another reduction method for intentionally geometric mistuning. This method is based on the use of the cyclic modes of the different sectors, which can be obtained from a usual cyclic symmetry modal analysis.

We search to create a new method inspired of this both methods. The key idea is to use a polynomial chaos approach to appoximate the cyclic modes of the different sectors with random geometry modifications. Let us consider the rotor with all blades having the same random geometry. The cyclic modes are solution of the following stochastic problem:

$$
\left[-\omega_{k}^{2}(\boldsymbol{\xi}) \mathbf{M}(\boldsymbol{\xi})+\mathbf{K}(\boldsymbol{\xi})\right] \boldsymbol{\psi}_{k}(\boldsymbol{\xi})=\mathbf{0}
$$

$\mathbf{M}$ and $\mathbf{K}$ are the mass and the stiffness matrices and $\boldsymbol{\xi}$ is the standard normal random vector of geometric variations. The eigenvalue $\omega_{k}^{2}$ and the associated eigenvector $\boldsymbol{\psi}_{k}$ are considered respectively as a second-order random variable and a second-order random vector. They can then be aproximated by a truncated polynomial chaos expansion. When the input random vector $\boldsymbol{\xi}$ is standard normal, the adapted decomposition basis may be the family $\left\{H_{\boldsymbol{\alpha}}\right\}_{|\boldsymbol{\alpha}|=0}^{r}$ of the $p$-dimensional Hermite polynomials of degree not exceeding $r$, which are orthogonal with respect to the Gaussian measure. Eigenvalues and eigenvectors are then approximated as follows:

$$
\omega_{k}^{2}(\boldsymbol{\xi}) \simeq \sum_{\boldsymbol{\alpha},|\boldsymbol{\alpha}|=0}^{r} f_{\boldsymbol{\alpha}, k} H_{\boldsymbol{\alpha}}(\boldsymbol{\xi}), \quad \boldsymbol{\psi}_{k}(\boldsymbol{\xi}) \simeq \sum_{\boldsymbol{\alpha},|\boldsymbol{\alpha}|=0}^{r} \mathrm{~g}_{\boldsymbol{\alpha}, k} H_{\boldsymbol{\alpha}}(\boldsymbol{\xi})
$$

The computation of the chaos coefficients $f_{\boldsymbol{\alpha}, k}$ and $\mathbf{g}_{\boldsymbol{\alpha}, k}$ can be performed using a projection method based on Gaussian quadrature scheme.

This approximation might make it possible to built an appropriate projection basis for any random geometric mistuned bladed disk. The purpose of this contribution is to examine the accuracy of such approximation.

\section{References}

[1] A. Sinha. Reduced-order model of a bladed rotor with geometric mistuning. ASME J. Turbomach 131 (2009), 031007.

[2] Y. Bhartiya, A. Sinha. Reduced order modeling of a bladed rotor with geometric mistuning via estimated deviations in mass and stiffness matrices. J. Eng. Gas Turbines Power 135(5) (2013), 052501.

[3] M. Mbaye, C. Soize, J-P. Ousty. A reduced-order model of detuned cyclic dynamical systems with geometric modifications using a basis of cyclic modes. J. Eng. Gas Turbines Power 132(11) (2010), 112502. 


\title{
Damage models described by uncertain parameters
}

\author{
Muhammad Sadiq Sarfaraz, Hermann G. Matthies \\ Institute of Scientific Computing \\ Technische Universität Braunschweig 38106 Braunschweig Germany
}

\begin{abstract}
Heterogeneities appearing on fine scales and incomplete knowledge of parameters governing the behaviour of continuum materials necessitate to consider the parameters defining their constitutive model as uncertain. Thus, the material parameters describing both the elastic/reversible behavior as well as inelastic/irreversible behavior are modelled as random variables/fields. To be able to simulate inhomogeneous and anisotropic behavior, the parameters of the model are random tensor fields with appropriate properties, like e.g. positive definitiveness.

In this work the focus is on the quantification of the uncertainty in the response of coupled elasto-plastic damage model described by material uncertainty. The uncertainties will be represented by Karhunen-Loève and polynomial chaos expansions, where particular care has to be taken to preserve the afore mentioned properties. The solution is computed with a non-intrusive stochastic Galerkin method.

Results are shown which show the influence of the modelling choices.
\end{abstract}




\title{
Uncertainty quantification for linear elastic bodies with fluctuating input parameters
}

\author{
A. Dridger, I. Caylak, R. Mahnken \\ Chair of Engineering Mechanics, University of Paderborn, Paderborn, Germany
}

In many physical and engineering applications deterministic solvers like the finite element method (FEM) are commonly used to treat problems which are described by partial differential equations (PDEs). Technically, many system parameters are stochastic. Thus, the deterministic PDE becomes a stochastic partial differential equation (SPDE). One possible approach to solve SPDEs is the stochastic finite element method (SFEM).

The governing equations of elasticity including the variational formulation of the SPDE are introduced. For the numerical application it is necessary to discretize the equation in the spatial and the stochastic domain. The spatial discretization is performed by ordinary finite element methods whereas the stochastic discretization uses WiEneR's polynomial chaos [2] (with so-called Hermite polynomials [1],[2] as basis functions) to expand the coefficients in deterministic and stochastic parts.

In this work a linear elastic body with two independent stochastic material parameters based on experimental data is studied. The key idea is the split of the elasticity matrix $\underline{C}(\omega)$ into a deviatoric part $\underline{C}_{\text {dev }}(\omega)$ and a volumetric part $\underline{C}_{v o l}(\omega)$.

In order to compare the appropriate choice of fluctuating input parameters two different approaches are presented: The first one presupposes the knowledge of the distribution of the random variables Young's modulus $E(\omega)$ and Poisson's ratio $\nu(\omega)$. The second one assumes the distribution of shear modulus $G(\omega)$ instead of $\nu(\omega)$ to be known. Computational approaches involving polynomial chaos are used to expand these variables. Therefore, GALERKIN projection [1] can be applied to reduce the stochastic PDE into a system of deterministic PDEs. In this work normally distributed random variables are considered. Thus, the number of stochastic dimensions is equal to the number of independent input parameters. Subsequently, the resulting equation system is solved iteratively. Finally, our method is applied in a numerical example for a plate with a ring hole.

\section{References}

[1] H. G. Matthies, A. Keese. Galerkin methods for linear and nonlinear elliptic stochastic partial differential equations. Comput. Methods Appl. Mech. Engrg., 194:1295-1331 (2005).

[2] R. G. Ghanem, P. D. Spanos. Stochastic Finite Elements: A Spectral Approach. Springer, New York (1991). 


\title{
Probabilistic Sensitivities for Fatigue Using Adjoint Methods
}

\author{
Mohamed Saadi \\ University of Wuppertal
}

We present a new approach to develop methods and design tools to determine the sensitivity of the probability of failure of mechanically and thermally loaded hot gas component $\Omega$ under the variation of design parameters and production-related deviations. Based on the local and probabilistic model described in [1] and [2], we compute the sensitivity of the probabilistic cost functional $J_{\text {sur }}(\Omega, u)$ :

$$
J_{\text {sur }}(\Omega, u)=\int_{\partial \Omega}\left(\frac{1}{N_{\text {det }}(T, \epsilon(\nabla u(x)))}\right)^{m} d A
$$

where $u$ is the displacement, $m$ is the shape parameter of the Weibull distribution and $N_{\text {det }}$ is the Weibull scale parameter which is supposed to be depended on the elastic strain tensor $\epsilon$ and the temperature $T$. This approach applied the adjoint methods to reduce the computational cost by computing these sensitivities.

$$
\left\{\begin{array}{c}
\text { solve } \overbrace{\left(\frac{\partial R_{M}}{\partial u}\right)^{T} \lambda_{1}=\left(\frac{\partial J}{\partial u}\right)^{T}}^{\text {purely mechanical adjoint equation }} \\
\text { and } \overbrace{\left(\frac{\partial R_{T}}{\partial T}\right)^{T} \lambda_{2}=\left(\lambda_{1}^{T} \frac{\partial R_{M}}{\partial T}-\frac{\partial J}{\partial T}\right)^{T}}^{\text {thermal-mechanical adjoint equation }} \\
\text { and compute } \underbrace{\frac{d J}{d X}=\lambda_{2}^{T} \frac{\partial R_{T}}{\partial X}-\lambda_{1}^{T} \frac{\partial R_{M}}{\partial X}+\frac{\partial J}{\partial X}}_{\text {global sensitivity equation }}
\end{array}\right.
$$

\section{References}

[1] S. Schmitz, G. Rollmann, H. Gottschalk, R. Krause. Risk estimation for LCF crack initiation. Journal-ref: Proc. ASME-Turbo Expo 2013, GT2013-94899.

[2] S. Schmitz, G. Rollmann, H. Gottschalk, R. Krause. Probabilistic Analysis of LCF Crack Initiation Life of a Turbine Blade under Thermomechanical Loading. arXiv:1310.0629v1, February 2013. Submitted to the Proceedings of the Seventh International Conference on Low Cycle Fatigue. 


\title{
Irreversible material behaviour under presence of uncertainty
}

\author{
Bojana Rosic and Hermann G. Matthies \\ Institute of Scientific Computing, TU Braunschweig, Braunschweig, Germany
}

Within the framework of small and large strain analysis the focus is set on the rate-independent evolutionary problem with general hardening whose material characteristics are assumed to be uncertain. By introducing the material properties in the form of random fields the irreversible and work-dissipating process is described via a stochastic convex energy function and evolution equations for internal variables. This allows the reformulation of the problem into the stochastic minimisation of a smooth convex energy functional on discrete tensor product subspaces whose unique minimizer is obtained via a stochastic closest point projection algorithm. Its numerical computation is performed with the help of methods of functional approximation, i.e. a direct, purely algebraic way of computing the response in each iteration of Newton-like methods. Furthermore, the method is contrasted to the less efficient but more accurate non-intrusive variant which evaluates the residuum in each iteration via high-dimensional integration rules based on random or deterministic sampling - Monte Carlo and related techniques. In addition to these, the problem is solved with the help of the stochastic collocation method. Finally, the methods are validated on a series of test examples whose reference solution is computed via direct integration methods. 


\title{
Jump Phenomena and Bifurcations in Stochastic Vehicle-Road Dynamics
}

\author{
Walter V. Wedig \\ KIT - Karlsruhe Institute of Technology, Germany \\ E-Mail: wwedig@t-online.de
}

Vehicles riding on uneven rough road surfaces [1] are excited to vertical and horizontal vibrations. The describing system equations are linear when the vehicle velocity is constant and there is a control providing a stationary driving force process needed to maintain an exactly constant vehicle speed.

More important is the inverse problem of a constant driving force meanwhile the velocity process is stationary fluctuating around a mean velocity as the consequence of the up and down of roads. The paper investigates second order road profiles modelled by linear filter equations [2] under white noise which include the limiting profile of harmonic wave roads.

Because of the forced velocity fluctuations, the describing equations of motion are now highly non-linear effecting velocity jumps when the driving force reaches critical values. These phenomena are similar to the amplitude jumps of the stochastic Duffing oscillator which are possible for narrow-banded base excitations by roads.

The applied simulation and statistical evaluation [3] are explained in detail by means of the extended Kramers oscillator under white noise. The stationary solution of its Fokker-Planck equation can explicitly be derived showing typical jump phenomena in form of two-modular density distributions which are numerically estimated by means of a separation technique.

\section{References}

[1] K. Sobczyk, D.B. MacVean, J.D. Robson. Response to Profile-Imposed Excitation With Randomly Varying Transient Velocity. Journal of Sound and Vibration, 52 (1977), 37-49.

[2] W. Wedig. Dynamics of Cars Driving on Stochastic Roads. Ed. by Spanos \& Deodatis in Proceedings of Computational Stochastic Mechanics. Milpress, Rotterdam (2003), 647-654.

[3] W.Wedig. Simulation of Road-Vehicle Systems. Prob. Eng. Mechanics, 27 (2012), 82-87. 


\title{
Regional Frequency Analysis of extreme rainfall events in Tuscany (Italy)
}

\author{
$\underline{\text { Valentina Chiarello }}^{1}$, Enrica Caporali ${ }^{1}$, and Hermann G. Matthies ${ }^{2}$ \\ ${ }^{1}$ Dep. of Civil and Environmental Engineering, University of Florence, Via di Santa Marta 3, \\ 50139 Florence, Italy \\ ${ }^{2}$ Institute of Scientific Computing, Technische Universität Braunschweig, Hans-Sommer-Str. \\ 65, D-38092 Braunschweig, Germany
}

\begin{abstract}
The assessment of extreme hydrological events at sites characterized by short time series or where no data record exists, has been mainly obtained by regional models. Regional frequency analysis based on the index variable procedure is implemented to describe the annual maximum of rainfall depth of short durations in Tuscany region. The probability distribution TCEV - Two Component Extreme Value is used in the frame of the procedure for the parameters estimation based on a three levels hierarchical approach [1]. This methodology deal with the delineation of homogeneous regions, the identification of a robust regional frequency distribution, the assessment of the scale factor, i.e. the index rainfall. The data set includes the annual maximum of daily rainfall of 351 gauge stations with at least 30 years of records, in the period 1916-2012, and the extreme rainfalls of short duration, 1 hour and 3, 6, 12, 24 hours. Different subdivisions hypotheses have been verified. A four regions subdivision, coincident with four subregions, which takes into account the orography, the geomorphological and climatic peculiarities of the Tuscany region, has been adopted. Particularly, for testing the regional homogeneity, the cumulate frequency distributions of the observed skewness and variation coefficients of the recorded times series, are compared with the theoretical frequency distribution obtained through a Monte Carlo technique. The related L-skewness and L-variation coefficients are also examined [2]. The application of the Student $t$-test and the Wilcoxon test for the mean, as well as the $\chi^{2}$ was also performed. Further tests of subdivision hypotheses have been made through the application of discordancy $D$ and heterogeneity $H$ tests and the analysis of the observed and theoretical TCEV model growth curves. For each region the daily rainfall growth curve has been estimated. The growth curves for the hourly duration have been estimated when the daily rainfall growth curve is not able to describe the sample cumulate frequency distribution. In order to define the index rainfall for each homogenous region and each duration, a multivariate model that combines the Mean Annual Precipitation, the elevation, the aspect and the sample mean of the annual maxima time series of the gauge sites has been used.
\end{abstract}

\section{References}

[1] M. Fiorentino, S. Gabriele, F. Rossi, P. Versace. Hierarchical approach for regional flood frequency analysis. Regional Flood Frequency Analysis, Singh, V. P. (Ed.), Reidel Publishing Company,(1987), 35-49.

[2] J. R. M. Hosking, J. R. Wallis. Regional frequency analysis, an approach based on L-moments. Cambridge University press, Cambridge, UK. 224 pp, (1997). 


\title{
On Gaussian approximation of the strength of Daniels' bundle with brittle Weibull fibers
}

\author{
Václav Sadílek, Miroslav Vořechovský \\ Brno University of Technology, Faculty of Civil Engineering, Institute of Structural Mechanics
}

The paper deals with the classical fiber bundle model with equal load sharing (load of the broken filament is distributed uniformly non-broken filaments), sometimes referred to as the Daniels bundle [1] or the democratic bundle [4]. This model is significant for strength of quasi-brittle structures and reliability of many parallel systems. Daniels formulated a multidimensional integral and also recursive formula for evaluation of the strength distribution function: In the same paper, he showed the strength of the bundle, $G_{n}$, (the peak force) tends to Gaussian distribution under quite broad conditions and he gave closed formulas for the mean value and standard deviation of the Gaussian distribution. Sornette later confirmed this result using a Kolmogorov theorem. The convergence of a random peak force to Gaussian distribution is very slow in terms of the number of fibers and therefore, Smith [3] proposed a correction term for the mean value that improves the original Daniels formula for small bundles.

In the present paper, the authors exploit their own implementation of the recursive formula for evaluation of the distribution function for strength of the fiber bundle. The implementation was made in high-level programming language Python using scientific packages - NumPy (scientific computing with arrays) and mpmath (library for real and complex floating-point arithmetic with arbitrary precision). This implementation enables to calculate values of cumulative distribution funciton (CDF) for large numbers (thousands) of fibers in bundle including values deep in the left tail of the distribution (probabilities $10^{-600}$ ). This computer program has been used to accurately calculate the distribution functions $G_{n}$ :

$$
\begin{aligned}
& G_{n}(x)=\mathrm{P}\left(Q_{n}^{*} \leq x\right)=\sum_{k=1}^{n}(-1)^{k+1}\left(\begin{array}{l}
n \\
k
\end{array}\right)[F(x)]^{k} G_{n-k}\left(\frac{n x}{n-k}\right) \\
& \text { where } G_{1}(x) \equiv F(x), G_{0}(x) \equiv 1 \text { and }\left(\begin{array}{l}
n \\
k
\end{array}\right)=\frac{n !}{(n-k) ! k !}
\end{aligned}
$$

for bundles with Weibull fibers $F(x)=1-e^{-(x / s)^{m}}$ with scale parameter $s=1$ and varied number of filaments $n$ and varied shape parameter $m$ and results were stored in database. This database has been used to calculate the mean values and standard deviations of the peak force. These values are compared to the available formulas $[1,2,3]$. We have found that for small bundles ( $n$ between 2 and 100 ) the formulas are not accurate. Therefore the authors propose improved closed-form predictions for the mean and standard deviation.

The main motivation of the work is to formulate an improved analytical formula for the distribution function $G_{n}$ that will be valid deep to the left tail where the real distribution strongly deviates from the Gaussian approximation.

Acknowledgment: The work was supported by the project CZ.1.07/2.3.00/30.0005 of Brno University of Technology and by project no. FAST-S-13-1889.

\section{References}

[1] H.E. Daniels.The Statistical Theory of the Strength of Bundles of Threads. Proc. Royal Soc. (London). 183A (1945), 405-435.

[2] H.E. Daniels. The Maximum of a Gaussian Process Whose Mean Path Has a Maximum, with an Application to the Strength of Bundles of Fibres. Advances in Applied Probability. 21 (2) (1989), 315-333.

[3] R.L. Smith, S.L. Phoenix. Asymptotic Distributions for the Failure of Fibrous Materials Under Series-Parallel Structure and Equal Load-Sharing. Journal of Applied Mechanics. 48 (1981), 75-82.

[4] D. Sornette. Elasticity and failure of a set of elements loaded in parallel. J. Phys. A: Math. Gen. 22 (1989), L243-L250. (Letter to the Editor) 


\title{
Best practice in metamodeling for data derived from civil engineering applications
}

\author{
Maria Steiner, Tom Lahmer \\ Research Training Group 1462, Bauhaus-Universität Weimar, Germany \\ Institute of Structural Mechanics, Bauhaus-Universität Weimar, Germany
}

In many fields of civil engineering, problems frequently exist in which a high computation time for determining the optimal deployment of construction materials is necessary. To reduce the costs in these situations and to keep the computation time low without losing model quality, the application of mathematical models is useful. With response surface methods, which are also called metamodels, calculations and forecasts can be completed faster because of a reduced amount of numerical or experimental data. In this contribution, the applicability of metamodels to civil engineering problems are first analyzed. Furthermore, it seeks to answer the question of which model is the most suitable for specific application fields.

The available types of metamodels can be divided into deterministic and stochastic models, whereby these groups differ significantly in their properties and thus in their applicability. For the deterministic methods, Polynomial Regression [2], Radial Basis Function Interpolation [2] and Moving Least Squares [5] are specific examples. In the case of the stochastic methods the Stochastic Process Model (Kriging) and its variations [1] are to be emphasized. In addition, all of the existing models differ greatly from each other regarding computational effort, flexibility, predictive ability and robustness. Moreover, there is a differentiation between interpolation and regression possible. Regressive methods are often more appropriate for noisy measurement data because a smoothing occurs.

In order to investigate when an application is useful, a specific example is considered, namely a variance based sensitivity analysis [6] of a model representing a triaxial soil test, in which various finite element methods are used. To achieve sufficiently accurate and robust values, a large amount of data is necessary, which magnifies the computation time with increasing number of input parameters. This can lead already for a comparatively simple model, Mohr Coulomb, with five parameters to a computation time of at least 22 hours.

This duration shows clearly that an application of metmodels could be extremely rewarding. By using for example linear regression, the computation time is reduced extremely and the solution shows a similar trend. However, there are also some questions that occur:

- How much data is necessary to produce the various metamodels?

- Will a less complex model be preferred despite a lower accuracy?

- And, according to what criteria is the decision made, which model is optimal?

At that, it is always a trade-off between expense and accuracy by which the decision gets more complicated and may vary depending on the situation. Because of that, there are different error calculations to compare the various metamodels. It must be distinguished whether an untrained additional data set is available to test the quality of the response surfaces and the prediction ability or whether the existing data is completely used for the model construction. The complexity of the model, which depends on the number of unknown model parameters, can also be included in this analysis. Different kinds of error values and comparison criteria are described for example in [3] and [4].

The aim for the analysis of response surface methods with several examples, like the mentioned sensitivity analysis, is to infer to the general applicability for models used in civil engineering. In this context it is necessary to classify the engineering models based on their underlying partial differential equation, which can be elliptic, parabolic and hyperbolic. This separation is related to the different engineering fields of the problems. For example, static systems and problems related to the heat equation differ extremely. Because of that, it is probable that different metamodels are optimal for each application field. In each of these partial differential equation categories, fundamental solutions exist, which provide in the simplified one dimensional case explicit and simple to evaluate functions that can be used for tests.

A case that can also be important is one in which no single metamodel has emerged as the optimal method, so that it is necessary to discuss a combination of several response surface methods. In conclusion, it is possible to increase the use of metamodels by providing an overview of the relation between these models and the corresponding classes of civil engineering problems and thereby improve the construction of engineering models. 


\section{References}

[1] N.A.C. Cressie. Statistics for spatial data. Wiley series in probability and mathematical statistics: Appli ed probability and statistics (1993), $105-210$

[2] A. Forrester, A. Sobester, A. Keane. Engineering design via surrogate modelling: A practical guide. Progress in Aerospace Sciences, Wiley (2008), 40-50

[3] D.C. Montgomery, G.C. Runger. Applied statistics and probability for engineers. John Wiley \& Sons, fifth edition (2010), 401-512

[4] T. Most, J. Will. Sensitivity analysis using the Metamodel of Optimal Prognosis. Tagesband der Weimarer Optimierungs- und Stochastiktage 8.0 (2011)

[5] D. Roos, T. Most, J. Unger, J. Will. Advanced surrogate models within the robustness evaluation. Tagesband der Weimarer Optimierungs- und Stochastiktage 4.0 (2007)

[6] A. Saltelli, M. Ratto, T. Andres, F. Campolongo, J. Cariboni, D. Gatelli, Mi. Saisana, S. Tarantola. Global Sensitivity Analysis: The Primer. John Wiley \& Sons (2008), 155-167. 


\title{
Asymptotic sampling - a tool for efficient reliability computation in high dimensions
}

\author{
Christian Bucher \\ Faculty of Civil Engineering, Vienna University of Technology
}

\begin{abstract}
Advanced Monte Carlo methods developed over the past years allow the computation of very small exceedance probabilities such as those required for structural reliability analysis with high precision. Typically these method focus on a particular fixed threshold value for which the exceedance probability is computed. It may, however, sometimes be more convenient to have information about the entire tail of the distribution rather than only one specific quantile. This is particularly useful in the context of reliability-based structural optimization. This contribution presents an extension of the asymptotic sampling method to compute the failure probability using a small number of samples with artificially increased standard deviations. Numerical examples demonstrate the applicability and efficacy of the suggested approach.
\end{abstract}

Generally, the probability of failure $P_{F}$ in an $n$-dimensional space of random variables $X_{1}, \ldots X_{n}$ can be computed as [3]

$$
P_{F}=\int \underset{D_{F}}{\ldots} \int f_{X_{1}, \ldots X_{n}}\left(x_{1}, \ldots x_{n}\right) \mathrm{d} x_{1} \ldots \mathrm{d} x_{n}
$$

In this equation, $f_{X_{1}, \ldots X_{n}}\left(x_{1}, \ldots x_{n}\right)$ denotes the joint probability function of the random variables $X_{1}, \ldots X_{n}$ and $D_{F}$ denotes the failure domain, i.e. the region of the $n$-dimensional random variable space in which failure occurs. Typically, this is denoted in terms of a scalar limit state function $g($.$) attaining negative values, i.e.$ $D_{F}=\left\{\left(X_{1}, \ldots X_{n}\right) \mid g\left(X_{1}, \ldots X_{n}\right) \leq 0\right\}$.

Since the failure probabilities to be computed are usually very small, say of the order of $P_{F}=10^{-5}$, it is not feasible to evaluate the integral in Eq. (1) using standard numerical integration procedures. This is due to the fact that the number of integration points required to perform e.g. Gaussian integration in dimension $n$ grows exponentially with $n$. Monte Carlo methods do not have this dependence on the dimension, however, crude or plain Monte Carlo simulation requires a number of samples roughly larger effort. Hence, specific methods have been developed which utilize the fact that in many cases only a small region in $n$-dimensional space actually contributes significantly to the failure probability. Sampling methods based on this idea are called importance sampling [4]. For good efficiency, these importance sampling methods require some level of detailed knowledge about the problem, and in many cases this knowledge is primarily based on the most probable failure point or design point. If such information is not available, or can be made available only at high computational expense, alternative methods such as Asymptotic sampling [2] may be advantageous. This method does not rely on the knowledge of the relevant failure region, but rather on the scaling of the failure probability (or reliability index $\beta$ ) with a scaling of the standard deviations in uncorrelated Gaussian space. Asymptotic properties of this relation [1] are exploited.

The contribution will review this method in the light of its applicability to systems reliability analysis and provide new insights into the optimal choice of algorithmic parameters.

\section{References}

[1] K. W. Breitung. Asympotic approximations for multinormal integrals. Journal of Engineering Mechanics, 110(3):357-366, 1984.

[2] C. Bucher. Asymptotic sampling for high-dimensional reliability analysis. Probabilistic Engineering Mechanics, 24:504-510, 2009.

[3] C. Bucher. Computational analysis of randomness in structural mechanics. Structures and Infrastructures Book Series, Vol. 3. Taylor \& Francis, London, 2009.

[4] R. Y. Rubinstein and D. P. Kroese. Simulation and the Monte Carlo Method. Wiley, 2nd edition, 2008. 


\title{
Polynomial Chaos and the Heave Motion of a Cylinder in Random Seas
}

\author{
Edwin Kreuzer, Eugen Solowjow \\ Institute of Mechanics and Ocean Engineering, Hamburg University of Technology, 21071 Hamburg, Germany
}

In this contribution, the random heave motion of a floating cylinder subject to stochastic wave forcing is investigated numerically. In particular, we obtain numerical solutions for the response distribution with the generalized Polynomial Chaos (gPC) framework for irregular wave excitation described by the JONSWAP spectrum.

Floating offshore systems are generally subject to irregular sea waves, which can be described by sea spectra such as the JONSWAP or the Pierson-Moskowitz spectrum. Due to the stochastic forcing, the responses of the considered systems are stochastic processes as well. Within the framework of stochastic dynamics we are dealing with stochastic equations of motion excited by colored noise. In offshore engineering, the frequency domain approach is usually the method of choice for obtaining solutions when the considered systems are assumed to be linear. However, the analyses of transients and nonlinear systems require time domain methods.

The direct Monte Carlo method is the simplest method for treating stochastic ordinary differential equations in time domain and is widely applied throughout the ocean engineering community. It is well known that the accuracy of the solution obtained with the direct Monte Carlo method scales inversely to the square root of the number of samples, e. g. in general the Monte Carlo method is computationally expensive if a high accuracy is desired. Xiu and Karniadakis [1] present a method for solving stochastic differential equations, popularly known as the generalized Polynomial Chaos framework. Numerical examples show a substantial speed-up compared to the direct Monte Carlo method. Although many parts of the scientific computing community have taken great interest in the gPC framework in the recent years, there are not many applications to problems in offshore engineering so far.

The analysis of the heave motion excited by irregular waves requires two steps within the gPC framework. First, the random forcing has to be represented as a truncated Karhunen-Loève (K-L) expansion. This representation of the stochastic waves in terms of a few uncorrelated random variables can be obtained by solving a Fredholm integral equation of the second kind. Thereby, the kernel can be obtained from the power spectral density of the sea state, which is generally known (see above). The solution of the Fredholm integral equation allows to state the stochastic process as a linear superposition of eigenfunctions with a minimum number of stochastic dimensions. For narrow banded sea states only very few terms in the K-L representation are required indeed. Despite its importance, the implementation of the K-L is considered as a complex task, because of the difficulty in solving the Fredholm integral equation. However, Sclavounos presents in a recent work [2] a new representation of stochastic ocean waves based on the K-L decomposition.

The second and final step in order to determine the response of a stochastically excited offshore structure is to transform the stochastic equation of motion in deterministic ordinary differential equations. The unknown stochastic solution of the equation of motion is stated with a polynomial chaos expansion (PCE) ansatz. The theoretical justification derives from the Cameron-Martin theorem, which states that the PCE of a stochastic process converges in $L_{2}$ to the stochastic process itself. This solution ansatz is substituted into the equation of motion. We take a Galerkin projection of the equation of motion onto previously chosen basis polynomials. The procedure ensures that the stochastic equation of motion reduces to a system of coupled deterministic differential equations for the coefficients of the PCE. Once the PCE coefficients have been computed with ordinary time integration schemes, the approximate moments of the distribution of interest can be obtained.

We present the application of the method to the example of a heaving cylinder in irregular waves.

\section{References}

[1] D. Xiu and G.E. Karniadakis. The Wiener-Askey Polynomial Chaos for Stochastic Differential Equations. SIAM Journal on Scientific Computing, 24(2): 619-644, 2002.

[2] P.D. Sclavounos. Karhunen-Loeve representation of stochastic ocean waves. Proceedings of the Royal Society A, 468(2145): 2574-2594, 2012. 


\title{
Stochastic quantification of the impact of uncertainty in inlet conditions on the aerodynamics of a 5:1 rectangular cylinder
}

\author{
M.V. Salvetti ${ }^{1}$, P. Shoeibi Omrani, ${ }^{2}, \underline{\text { A. Mariotti }}^{1}$ and J.A.S. Witteveen ${ }^{3}$ \\ ${ }^{1}$ University of Pisa, Italy \\ ${ }^{2}$ Netherlands Organization for Applied Scientific Research (TNO), The Netherlands \\ ${ }^{3}$ Center for Mathematics and Computer Science (CWI), The Netherlands
}

\begin{abstract}
The Benchmark on the Aerodynamics of a Rectangular Cylinder (BARC) collects the results of wind-tunnel experiments and of numerical simulations of the flow around a rectangular cylinder with chord-to-depth ratio equal to 5 and infinite spanwise size. The considered flow configuration is of practical interest, e.g. in civil engineering, and, in spite of the simple geometry, the related flow dynamics and topology is complex. The results of about 70 experimental and numerical realizations of the BARC flow configuration were collected and reviewed in [1]. It was observed that some of the flow quantities, as the standard deviation of the lift coefficient, the distribution of mean and fluctuating pressure on the cylinder sides are affected by significant dispersion, both in experiments and in simulations. The observed dispersion can be explained by the extreme sensitivities of some flow features to uncertainties which can be hardly avoided or controlled in experiments or simulations. In particular, besides modeling uncertainties and numerical errors, in numerical simulations it is difficult to exactly reproduce the experimental conditions due to uncertainties in the experimental set-up parameters. We investigate herein the impact of some parameter uncertainties on the numerical predictions of the BARC test case using probabilistic methods. The following uncertain set-up parameters are investigated in the subsequent uniform ranges: the angle of incidence $\alpha\left(-1^{\circ}-1^{\circ}\right)$, the longitudinal turbulence intensity $I_{\mathrm{x}}(0.001-0.03)$, and the turbulence length scale $L(0.1 D-5 D), D$ being the cylinder depth. These are quantities which are difficult to exactly control and measure in experiments. Numerical simulations are carried out by means of a URANS approach. The Stochastic Collocation (SC) method [2] is employed to perform the probabilistic uncertainty propagation of the three set-up parameters. This results in 25 URANS simulations based on the Smolyak sparse grid extension of the level-2 Clenshaw-Curtis quadrature points. The propagation of the considered uncertainties on the quantities of interest for the BARC test case [1] is then characterized in a probabilistic manner. The numerical error is estimated by carrying out the stochastic analysis on two different grids. The impact of turbulence modeling is similarly investigated by repeating the analysis for two RANS closure models. This additional uncertainty quantification information can lead to new insights into the unresolved issues of the BARC flow test case.
\end{abstract}

\section{References}

[1] Bruno, L., Salvetti, M.V. and Ricciardelli, F. : Benchmark on the aerodynamics of a rectangular 5:1 cylinder: and overview after the first four years of activity, J. Wind Eng. Ind. Aerod., 126, 87-106 (2014).

[2] Xiu, D. and Hesthaven, J.S. : High-order collocation methods for differential equations with random inputs, SIAM J. Scient. Comp., 27, 1118-1139 (2005). 


\title{
A Bayesian approach for the quantification of parametric and model-form uncertainties in dense-gas flow models
}

\author{
$\underline{\text { Xavier Merle }}^{1}$, Paola Cinnella ${ }^{1,2}$ \\ ${ }^{1}$ Laboratoire DynFluid, Arts et Métiers Paristech \\ ${ }^{1,2}$ Universitá del Salento
}

In this work, we adopt a Bayesian approach to quantify uncertainties associated to the thermodynamic models used for the simulation of dense gas flows, i.e. flows of gases characterized by complex molecules in thermodynamic conditions of the general order of magnitude of the liquid/vapor critical point. Thermodynamic data about the working fluid in use are used for calibrating the material-dependent coefficients of equations of state (EOS) used to model the thermodynamic behavior of the working fluid. It has been shown that dense gas flow simulations can be extremely sensitive to the model used to describe the fluid thermodynamic behavior [1]. EOS give raise to two kinds of uncertainties: the first one concerns the mathematical form of the EOS to be used for a given fluid; on the other hand, the material-dependent coefficients associated to the EOS are imperfectly known. Previous work [1] has shown that for some particularly complex gases the model-form uncertainty can be even overwhelming with respect to the parametric uncertainty. This is why we look here for a methodology allowing to account simultaneously for both forms of uncertainty.

As a first step, the available thermodynamic data on the fluid of interest are used for the Bayesian calibration of the material-dependent coefficients of a EOS. This results in posterior distributions of the coefficients than can be propagated through the flow solver to quantify the uncertainty on the output quantities of interest (QoI) and compared to aerodynamic data available for validation purposes. Different popular EOS for dense gases, like the cubic Soave-Redlich-Kwong [2] and Peng-Robinson-Strjyek-Vera [3] EOS and the 5th-order virial Martn-Hou EOS [4], are calibrated using either pressure-volume-temperature data and/or speed of sound data. The statistical model includes both an experimental error term and a model-inadequacy term. The results are then propagated through a numerical dense gas flow solver [5] to quantify the resulting uncertainty on output quantities of interest like the pressure distribution at the arifoil wall or the aerodynamic coefficients. For this purpose we use a response-surface method which reconstructs the code response to the thermodynamic model coefficients as an analytical function and reduce the computational cost (see, e.g. [6]. The flow configuration considered here is a transonic flow over an airfoil. The simulations are carried out for different choices of the operating conditions, and namely of the thermodynamic state, Mach number and angle of attack of the incoming flow, to investigate the predictive capabilities of the calibrated models.

Secondly, to account for the availability of several alternative models, a Bayesian-model-average of the three EOS under study is constructed. The averaged equation, which accounts simultaneously for parametric and model-form uncertainty, is also propagated through the dense gas code and the improvements over the standard approach based on a single model structure are investigated.

Full descriptions of the statistical methodology and of the results will be provided at the conference.

\section{References}

[1] P. Cinnella et al.. Sensitivity analysis of dense gaz flow simulations to thermodynamic uncertainties. Phys. Fluids 23 (2011), 116101-20.

[2] G. Soave. Equilibrium constants from a modified Redlich-Kwong equation of state'. Chem. Eng. Sci. 27 (1972), 1197.

[3] R. Stryjek and J. H. Vera. An improved Peng-Robinson equation of state for pure compounds and mixtures. Can. J. Chem. Eng. 64 (1986) 323.

[4] J. J. Martin, Y. C. Hou. Development of an equation of state for gases'. AIChE J. 1 (1955) 142.

[5] P. Cinnella, P. Congedo. Numerical solver for dense gas flows. AIAA J. 43(2005), 2458-2460.

[6] X. Merle, P. Cnnella. Bayesian quantification of thermodynamic uncertainties in dense gas flows. Reliability Engineering \& System Safety 134(2015), 305-323. 


\title{
Investigation of cavitation and turbulence models uncertainties for cavitating flows
}

\author{
$\underline{\text { P.M. Congedo }}^{+}$, M.G. Rodio $^{+}$, E. Goncalves* \\ $\left.{ }^{+}\right)$INRIA Bordeaux-Sud-Ouest, France \\ (*) Department of Aeronautical Engineering, Laboratory Pprime, Poitiers, France
}

The collapse of vapour bubbles in high-pressure region can produce dramatic effects, such as failure, erosion and other undesirable effects. An open question is still related to the interaction between cavitation and turbulence. In particular, an accurate description of interactions between the vapour and liquid phases requires accurate physical models and a way to take into account the dynamics of the interface, multiscale effects, turbulence and thermodynamics.

Several numerical approaches have been proposed to reproduce cavitating flows in external and internal configurations. Principally the models can be regrouped in two major categories: interface models and twophase models. In the first case, the liquid and the vapour phase are separated by an interface, then the systematic reconstruction of interface and the applicability to complex geometries are the most challenging issues. Concerning two-phase models, the two phases are treated as a mixture. Difficulties of these models are related to the mixture's properties estimation based on the liquid-vapour mixture ratios [1]. Differences between the various models in the second category mostly come from the relation that defines the density field. For more details concerning the various modelling approaches, Refs. [3, 4, 2] are strongly recommended.

A large literature exist on the use of RANS model for performing this kind of computation. Nevertheless, the assumption of some empirical coefficients remains someway arbitrary, and sometimes tuned for reducing the distance between experiments and numerical solution. For these reasons, it is crucial to consider this epistemic (since it is due to a lack of knowledge) uncertainty in the problem, thus providing a measure of the variability of the numerical solution, i.e. assessing the quality of the numerical prediction. Recently, Rodio \& Congedo [5] have proposed a study about the impact of various sources of uncertainty (on the cavitation model and on the inlet conditions) on the prediction of cavitating flows by coupling a non-intrusive Polynomial Chaos stochastic method with a cavitating CFD solver.

This work is focused on the assessment of different sources of uncertainties for a Venturi configuration, which is one of the most studied for cavitating flows. In particular, contributions are related to the propagation of uncertainties for some turbulence model parameters and their calibration with respect to the experimental data.

\section{References}

[1] A. Hosangadi and V. Ahuja. A numerical study of cavitation in cryogenic fluids, part ii: New unsteady model for dense cloud formation. In Proceedings of the 6th International Symposium on Cavitation, Wageningen, The Netherlands, 2006.

[2] E. Goncalves. Modeling for non isothermal cavitation using 4-equation models. International Journal of Heat and Mass Transfer, 76 (2014), 247-262.

[3] J. P. Franc and C. Pellone. Analysis of thermal effects in a cavitating inducer using rayleigh equation. Journal of Fluids Engineering, 129:974-983, 2007.

[4] Y. Utturkar, J. Wu, G. Wang, and W. Shyy. Recent progress in modeling of cryogenic cavitation for liquid rocket propulsion. Prog. Aerospace Sci., 41(7):558-608, 2005.

[5] M.G. Rodio and P.M. Congedo. Robust analysis of cavitating flows in the venturi tube. European Journal of Mechanics - B/Fluids, 44(0):88-99, 2014. 


\title{
Influence of Uncertainties of the Aerodynamical Parameters on the Simulation Model for a Civil Aircraft with Active High Lift System
}

\author{
$\underline{\text { Noemi Friedman }}^{1}$, Jobst Henning Diekmann ${ }^{2}$, Hermann G. Matthies ${ }^{1}$ \\ ${ }^{1}$ Institute of Scientific Computing, TU Braunschweig, Germany \\ ${ }^{2}$ Institute of Flight Systems, DLR, Braunschweig, Germany
}

This contribution presents the propagation of uncertainties of the eigenmotions of an active high lift civil aircraft model. These uncertainties are due to incertitude of the aerodynamical parameters of the model.

With the aim to extend the usage of already existing small airports in the close vicinity of bigger cities, a new medium range civil aircraft design, that can take off and land on relatively short runways, was proposed [1]. The short take off and land distance is achieved by an active high lift system based on an internally blown flap system. However, among other design difficulties, extreme high lift along with low dynamic pressure puts high demands on the trim and controllability of such an aircraft. In addition there is a strong cross-coupling between lift and thrust resulting in the need for active control to achieve acceptable handling qualities [2]. In order to be able to test meneuveribility of the aircraft a stochastic aerodynamic analysis was carried out. First, a nonlinear deterministic model was developed by the Institute for Flight System at DLR, simulating the longitudinal motion of the active high lift supported aircraft [2].

The main focus of this contribution is to enhance the reliability of this simulation model by quantifying its uncertainties, that is to analyse the aircraft behavior under the influence of uncertainties of its aerodynamical coefficients. This quantification was carried out in a non-intrusive manner, meaning that the original model was treated in a black box fashion, while the constraints and input parameters were changed to random variables instead of deterministic ones. The probability distribution function of the uncertain parameters were defined by the professionals. Besides analysis of the aircraft model the contribution targets to present different non-intrusive approaches, with which such sensitivity analysis and uncertainty quantification can be carried out without significantly changing the original, deterministic simulation model. The non-intrusive uncertainty quantification was first developed for a basic aircraft simulation model [3] and further extended in this contribution for the targeted updated aircraft simulation model, that already includes the active high-lift system.

Acknowledgements

Financial support has been provided by the German Research Foundation (Deutsche Forschungs-gemeinschaft - DFG) in the framework of the Sonderforschungsbereich 880.

\section{References}

[1] R. Radespiel, H. Wolfgang. SFB 880 - Fundamentals of high-lift for future commercial aircraft. CEAS Aeronautical Journal (2014), doi:10.1007/s13272-014-0103-6

[2] J.H. Diekmann. Analysis of trimmable conditions for a civil aircraft with active high-lift system. CEAS Aeronautical Journal, Springer Verlag, Wien, (2014), doi:10.1007/s13272-014-0132-1

[3] B. Rosić, J.H. Diekmann. Methods for the Uncertainty Quantification of Aircraft Simulation Models, Journal of Aircraft, (2014), doi: 10.2514/1.C032856 


\title{
Stochastic quantification of the effects of inlet velocity conditions on the dynamics of spatially evolving mixing layers
}

\author{
M. Meldi, M.V. Salvetti and P. Sagaut \\ University of Poitiers, France \\ University of Pisa, Italy \\ University of Aix-Marseille, France
}

\begin{abstract}
Mixing layers are present in many technological applications or environmental flows and they have been extensively studied in the literature. Mixing layers are characterized by the evolution and the interaction of coherent vortex structures, which form at the interface between two parallel coflowing streams with different velocities and play a major role in the bulk mixing of the fluids and in the growth of the mixing layer. It is well known that the inlet conditions, which may be not exactly known in practical applications, significantly affect the dynamics of mixing layers. The most common assumption in studying this flow configuration is that the two coflowing streams are parallel, i.e. the two inlet bulk flow velocities have the same direction; in this case, the coherent vortex structures form due to the effect of the gradient of velocity occurring in the normal direction. However, it may happen in practice that this condition is not verified and that the two streams are actually tilted of an angle $\theta$. Previous experimental studies [1] have shown that the flow evolution is extremely sensitive to the three-dimensionality of the inlet velocity field. A second parameter influencing the flow transition from the laminar regime to the fully developed turbulent one, as well as the dynamics of the generated eddies [2], is $\alpha=\left(U_{1}-U_{2}\right) /\left(U_{1}+U_{2}\right)$, where $U_{1}$ and $U_{2}$ are the two asymptotic stream velocities.
\end{abstract}

In the present work, we propose to quantify the impact on flow evolution of the angle between the two streams, $\theta$ and of $\alpha$ through direct numerical simulations and by adopting a stochastic approach, in which $\theta$ and $\alpha$ are considered as aleatory variables and the uncertainty is propagated over the output quantities of interest. To reduce the required computational resources, a continuous representation of the investigated flow quantities over the random variable space (response surface) is obtained through a simple, inexpensive model. The response surface is reconstructed starting from a limited number of samples taken at known discrete points on the uncertainty space, i.e., single deterministic numerical simulations. In particular, as already done for classical mixing-layers in [3], the response surface is built through generalized Polynomial Chaos (gPC).

The deterministic simulations needed to obtain the response surface are direct numerical simulations carried out through the open-source code OpenFOAM. The Reynolds number is $R e=\frac{\left(U_{1}-U_{2}\right) \delta_{0}}{2 \nu}$ is set equal to 100, in which $\delta_{0}$ is the inlet vorticity thickness and $\nu$ the kinematic viscosity. The following ranges of variation of the random variables have been chosen: $0 \leq \theta \leq \pi / 12$ and $0.2 \leq \alpha \leq 0.7$. As previously mentioned, in our approach, each deterministic simulation corresponds to a discrete point in the two dimensional uncertainty space. These points are the quadrature nodes of a two dimensional Gauss-Legendre quadrature of the uncertainty space. This quadrature rule has been chosen as it accurately fits with the enforced probability density function of the random variables, which has been chosen as uniform. The gPC expansion for the two random variables is truncated at the third order, which means that the minimum number of simulations required for a full convergence of the response surface model is $4^{2}$. The flow quantities of interest, for which the uncertainty propagation is quantified through the response surface, are the three components of the mean velocity vector, $U$ and of its quadratic function $M=(U 1-U)\left(U-U_{2}\right)$, which is related to the momentum thickness. The spatial evolution of the two quantities is analyzed for each component as well as for the vector magnitude. The results are as well compared with the information which could be obtained by deterministic DNS simulations, carried out for different values of the input parameters.

\section{References}

[1] Hackett J. E., Cox D. K. The three-dimensional mixing layer between two grazing perpendicular streams. J. Fluid Mech. 43 (1970), $77-96$.

[2] Ko J., Lucor D., Sagaut P. Sensitivity of two-dimensional spatially developing mixing layer with respect to uncertain inflow conditions. Phys. Fluids 20 (2008), 077102.

[3] Meldi M., Salvetti M. V., Sagaut P. Quantification of errors in large-eddy simulations of a spatially evolving mixing layer using polynomial chaos. Physics of Fluids 24 (2012), 035101. 


\title{
Solution of the First passage problem by Path Integration for normal, Poissonian, and alpha-stable white noise
}

\author{
$\underline{\text { Mario Di Paola }}^{1}$, Antonina Pirrotta ${ }^{1}$, Christian Bucher $^{2}$ \\ ${ }^{1}$ DICAM, Università degli Studi di Palermo \\ ${ }^{2}$ Faculty of Civil Engineering, Vienna University of Technology
}

In this contribution, the first passage problem is examined for linear and nonlinear systems driven by Poissonian and normal white noise input. The problem is handled step-by-step accounting for the Markov properties of the response process and then by Chapman-Kolmogorov equation. the final formulation consists just of a sequence of matrix-vector multiplications giving the reliability density function at any time instant. Comparison with Monte Carlo simulation reveals the excellent accuracy of the proposed method.

The first passage problem has been investigated in many publications over almost a century because of its relationship to the safety of structural systems under random excitations. The exact solution to the first passage problem is not available because even in the case of a normal white noise process the Fokker-Planck equation with associated boundary conditions is in general unknown [11]. Approximate methods have been proposed $([2,8,1,12,10])$, however, the analytical approximation methods are available only for light damping for the stochastic averaging, weak nonlinearity and Gaussian approximations.

In order to determine the probability distribution of the first passage time, efficient solution of the FokkerPlanck equation is necessary. Moreover, we need a solution of the problem step-by-step in order to cancel the trajectories that for the first time leave the safe domain (absorbing barrier problem). In order to have such a control on the path of the trajectories the only way is using the so-called Path Integration (PI) method. It mainly consists of using the Chapman-Kolmogorov (CK) equation giving the probability density at a certain time instant as weighted sum of the contributions of the various trajectories that in a previous time instant start with deterministic initial condition. As the interval between the two time instants becomes small, then the so-called short time Gaussian approximation [9] remains still valid and the step-by-step solution technique of the CK equation reverts to the PI method. Many papers have been devoted to this subject for normal $[5,13,3]$ and Poissonian white noise [6, 7].

The PI method is versatile and in [4] it has been used for solving the first passage problem. It mainly consists in defining the so-called reliability function which is a function giving the probability that the various trajectories will remain inside the safe barrier conditioned by the fact that each of them never crosses the barrier up to the observation time.

In this contribution, by using the concepts exploited in [4] the first passage problem is revisited in the light of the cell mapping method and extended to the case of Poissonian and alpha-stable input. It is shown that the reliability function by discretization of the Chapman-Kolmogorov equation may be easily implemented in a computer program as just a sequence of matrix-vector multiplications whose sizes depend on the threshold barriers and the spatial discretization steps. Moreover, as the input is stationary the reliability function is ruled by a transition matrix that does not explicitly depend on time so that is can be computed once beforehand.

\section{References}

[1] H. H. Gray. First passage time in a random vibrational system. Journal of Applied Mechanics, 33:187-191, 1966.

[2] S. H.Crandall, K. L. Chandrimani, and R. G. Cook. Some first passage problems in random vibration. Journal of Applied Mechanics, 33:532-538, 1966.

[3] D. V. Iourtchenko, E. Mo, and A. Naess. Response probability density functions of strongly non-linear systems by the path integration method. Int. J. Non-Linear Mechanics, 41:693-705, 2006.

[4] D. V. Iourtchenko, E. Mo, and A. Naess. Reliability of strongly nonlinear single degree of freedom dynamic systems by the path integration method. Journal of Applied Mechanics, 75(6):061016-1-061016-8, 2008.

[5] A. Naess and M. Johansen. Response statistics of nonlinear, compliant offshore structures by path integral solution method. Probabilistic Engineering Mechanics, 8:91-106, 1993. 
[6] M. D. Paola and R. Santoro. Path integral solution for non-linear system enforced by poisson white noise. Probabilistic Engineering Mechanics, 23:164-169, 2008.

[7] A. Pirrotta and R. Santoro. Probabilistic response of nonlinear systems under combined normal and poisson white noise via path integral method. Probabilistic Engineering Mechanics, 26:26-32, 2011.

[8] J. R. Rice and F. P. Beer. First occurrence time of high-level crossings in a continous random process. Journal of the Acoustical Society of America, 39:323-335, 1965.

[9] H. Risken. The Fokker-Planck Equation - Methods of Solution and Application. Springer, Berlin Heidelberg NewYork Tokyo, 1984.

[10] P. D. Spanos. Survival probability of non linear oscillators subjected to broad band random disturbances. Int. J. Non-Linear Mechanics, 17:303-317, 1982.

[11] J. N. Yang and M. Shinozuka. First passage time problem. Journal of the Acoustical Society of America, 47:393-394, 1970.

[12] J. N. Yang and M. Shinozuka. On the first excursion probability in statonary narrow-band random vibration. Journal of Applied Mechanics, 39:733-738, 1972.

[13] J. S. Yu, G. Q. Cai, and Y. K. Lin. A new path integration procedure based on guass-legendre scheme. Int. J. Non-Linear Mechanics, 32:759-768, 1997. 


\title{
Adaptation and Enhancement of Generalized Polynomial Chaos for Industrial Applications
}

\author{
Philipp Glaser $^{\mathrm{a}}$, Kosmas Petridis ${ }^{\mathrm{a}}$, Vincent Heuveline ${ }^{\mathrm{b}}$

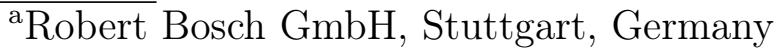 \\ ${ }^{\mathrm{b}}$ Engineering Mathematics and Computing Lab (EMCL), University Heidelberg, Germany
}

Many industrial applications involve uncertain data. Quantifying them is a big challenge. For example, unknown disturbances can influence the parameters of the system, and thereby the result of the simulation. Measurements have to be performed when comparing the model with the reality, but sensors are also noisy. It is, in some cases, not even possible to state an exact model because of insufficient knowledge of the system. Addressing all those uncertainties simulation models need to be expanded by stochastic elements. Which leads us to the requirement that we should be able to numerical compute a stochastic solution.

This contribution presents ideas, how to adapt stochastic elements to industrial applications and reduce the number of simulations in comparison to Monte Carlo. Especially when the mathematical model equations are not known and just an input/output interface is given. For this so called black box model which can be generated by many simulation tools the generalized polynomial chaos based on the idea of Wiener [2], and extended by Xiu and Karniadakis [3] is a suitable technique. One main advantage of this concept can be seen in the ability to produce functional representations of stochastic variability. This yields in a reduction of the simulation number compared to classical methods and can intensified by using a sparse grids approach [1].

Looking at industrial applications the concept can be applied to a huge amount of systems. For example, components including electronic drives, fuel injection valves, and mechanical parts. As an example of use a simplified model of a DC drive with six uncertain parameters is taken into account.

\section{References}

[1] M. Eldred, C. Webster, P. Constantine. Evaluation of non-intrusive approaches for Wiener-Askey generalized polynomial chaos. 12th AIAA/ISSMO Multidisciplinary Analysis and Optimization Conference. (2008), $1-22$.

[2] N. Wiener. The homogeneous chaos. Amer. J. Math. 60 (1938), 897-936.

[3] D. Xiu, G. E. Karniadakis. The Wiener-Askey polynominal chaos for stochastic differential equations. SIAM J. Sci. Comput. 24 (2002), 619-644. 


\section{S16: Optimization}

Optimization is the next natural step after simulation with increasing importance in the future. The aim of this session is to provide the basis of a holistic overview of all areas of optimization. Thus abstracts from both a theoretical as well as an applied perspective are welcome. 


\title{
Construction of a Magnet as an Absolute Positioning Scale
}

\author{
Aleksandar Mojsic, Joanna Sokoł, Marina Ludszuweit, Armin Fügenschuh \\ Helmut Schmidt University
}

We consider the problem of measuring distances to a high precision, that is, on a micrometer resolution over a length of several meters. Several techniques are currently in use, such as optical, electronic, mechanical. We focus on magnetic scales. Hereto, a ferromagnetic stripe is magnetized by a certain pattern. Then a sensor head, consisting of a number of Hall cell elements, is reading the field strength. Based on this information, the goal is to determine the position on the scale. Two different principles are in use, the incremental and the absolute positioning systems. For an incremental measurement of a position, the sensor must start in a defined initial position, and from there it moves to the desired position, while the sensor logic counts the number of polarization changes on the stripe. Such systems are easy to construct, but have the disadvantage that the sensor always needs to start from its initial position, which makes such systems slow and inflexible for certain applications. Here, absolute positioning systems are favorable, which are able to determine the correct position from the local magnetic information. In the present state-of-the-art, such system come, for example, with two parallel magnetic stripes and rather large sensor heads, which makes the production of such systems more expensive and also limits their range of potential applications.

To overcome the current difficulties of absolute positioning systems, we develop a new magnetic encoding scheme together with a corresponding position computing logic. In this new approach, the angles between the individual poles on the stripe are no longer only perpendicular (as they are in the currently used rulers), but deviate from this within some bounds. Hence we can use a larger variety of angles to encode further information on the absolute position. The magnetic poles are thus not squares or rectangles, but trapezoids in general. A specially constructed sensor, developed by our industrial partner, is used to read the magnetic field signal. On this sensor, a number of Hall cell elements is reading the magnetic ruler. Compared to current absolute positioning systems, this sensor head is much smaller, since we have to read from the same magnetic pole several signals. From these signals, we now reconstruct the absolute position on the ruler.

This engineering problem leads to several mathematical and algorithmic problems. The first issue is the calibration of a hall sensors. Due to small differences during the production process, two Hall cell elements typically measure slightly different values from the same underlying magnetic signal. We overcome this obstacle by solving an overdetermined linear system on the measured data for each Hall cell.

The second issue is the numerical approximation of a magnetic signal on the scale. Finite element codes turned out to be too slow, too time consuming, and too coarse with respect to the solution quality. We achieve the approximation as a linear combination of a certain number of basis polynomial functions of order around 10. This results in an algorithm that is such resource efficient in terms of memory and time, that it is suitable for an implementation an a DSP or FPGA. (This is a research project of its own, conducted by our academic partners at TU Berlin.) We then compute the inverse to the signals, that is, we estimate the position on the scale that is the best-fit to the measured signal data.

The third issue is to construct the scale such that it allows for a unique inverse on each individual position (on a micrometer scale, there are several million positions to be encoded). Hereto we use mixed-integer linear programming, and we use IBM ILOG CPLEX as solving tool. The structure of the scale can be represented by a sequence of trapezoids of the same height that can be glued to create a stripe, therefore we will be looking for such sequence. Magnets as trapezoids, are represented by the lengths of upper and lower bases. Each magnet will be assigned a position-index in the sequence of the trapezoids where it occurs. We use binary variable for every triple (uplen; lowlen; pos) from a given set. The variable is equal to 1 iff a magnet with upper base length uplen and lower base length lowlen is in the position pos. To optimization goal is to find a scale of maximum length that still allows for a unique position determination. The constraints of this optimization problem state that for every magnet the angle between the leg of trapezoid and its base is near $\frac{\Pi}{2}$ and that for the obtained scale we will have unique positions. The first condition can be easily written as a linear constraint. We meet the second condition by dividing the main problem into the problems of creating a few short scales that compose into the final scale. In each short scale we avoid using two magnets consecutively if they were already used in such a way in previous parts. With properly chosen magnet lengths this condition is sufficient (but not necessary) to give the uniqueness in readings of magnetic field. The general optimization task is how to construct the longest possible ruler and to keep the uniqueness of the magnetic pole pattern. At the same time the second best fitting norm should be on a stable distance from zero which provide the stability of the absolute positioning algorithm. 


\section{References}

[1] L. A. Wolsey, G. L. Nemhauser. Integer and Combinatorial Optimization. John Wiley \& Sons, New York, 1999. 


\title{
Control of water reservoirs aeration process
}

\author{
Mohamed Abdelwahed \\ Department of Mathematics, College of Science, King Saud University, Riyadh, KSA
}

The mechanical aeration process in water reservoirs is one of the most used techniques to combat eutrophication. It consists of pumping a source of compressed air in the reservoir bottom via injectors in order to create a dynamic and aerate the water by bringing it in contact with the surface air. We focus in this work in the first hand to the direct problem. It concerns the numerical simulation of the resulting two phase water air-bubbles flow. Different models can be used to describe this problem. Using the fact that the water phase is dominant, we use a simplified model in which the water phase is governed by the Navier-Stokes equations and the aeration effects are taken into account through a source term representing the air effect. Our discretization method is based on three dimensional mixed finite element method [1].

In the other hand, we look at the inverse problem: find the optimal injectors location generating the best motion in the fluid with respect to the aeration purpose. Each injector $\operatorname{Inj}_{k}, 1 \leq k \leq m$, is modeled as a small hole $\omega_{z_{k}, \varepsilon}=z_{k}+\varepsilon \omega^{k}$ around a point $z_{k}$ and having an injection velocity $u_{i n j}^{k}$, where $\omega^{k} \subset \mathbb{R}^{3}$ are bounded and smooth domains containing the origin. The optimal injectors location is characterized as the solution to a topological optimization problem. The topological sensitivity analysis is used to solve this problem. The main idea is to compute the asymptotic topological expansion with respect to the insertion of an injector.

To present this method, we consider the case in which $\Omega$ contains a single injector having the form $\omega_{z, \varepsilon}=$ $z+\varepsilon \omega$. The topological sensitivity method provides an asymptotic expansion of an optimal design function $j$ of the form:

$$
j\left(\Omega \backslash \overline{\omega_{z, \varepsilon}}\right)=j(\Omega)+f(\varepsilon) \delta j(z)+o(f(\varepsilon)), \quad \forall z \in \Omega,
$$

where $f(\varepsilon)$ is a scalar positive function going to zero with $\varepsilon$. This expression is called the topological asymptotic expansion and $\delta j$ the topological gradient. In order to minimize the cost function, the best location to insert a small hole in $\Omega$ is where $\delta j$ is the most negative. In fact if $\delta j(z)<0$, we have $j\left(\Omega \backslash \overline{\omega_{\varepsilon}}\right)<j(\Omega)$ for small $\varepsilon$. The function $\delta j$ can be used as a descent direction in the domain optimization process.

In order to obtain a topological sensitivity analysis of the used model, which is the Navier-Stokes equations, with respect to the insertion of an injector, we first discretize the model in time using the characteristics method. We obtain at each time step a generalized Stokes problem. In this work, we derive in the first hand a simple topological sensitivity analysis for the generalized Stokes equations [2]. Our analysis is based on the asymptotic behavior of the velocity perturbation caused by the presence of the injector $\omega_{z, \varepsilon}$. This result is then used to the study of the topological sensitivity analysis for the Navier-Stokes problem [3].

The obtained numerical results show the efficiency of the numerical algorithm [2]. Only few iterations are needed to obtain the optimal injectors location. At each iteration we only need to solve the direct and the adjoint problems on a fixed grid.

\section{References}

[1] M. Abdelwahed, M. Amara, Numerical analysis of a two-phase flow model, International Journal of Computational Methods. 9(3) (2012), 28 pages.

[2] M. Abdelwahed, Optimization of Injectors Location in a Water Reservoirs Aeration Problem. Mathematical Problems in Engineering, Article ID 875979 (2013), 10 pages.

[3] M. Abdelwahed, M. Hassine, Topology optimization of time dependent viscous incompressible flows, Abstract and Applied Analysis. Article ID 923016 (2014), 9 pages. 


\title{
Virtual Process Design in Combined Electromagnetic-Classical Forming Processes: Optimization of Current Parameters
}

\author{
Marco Rozgic̀ $^{1}$, Marcus Stiemer ${ }^{1}$, Yalin Kiliclar $^{2}$, Stefanie Reese $^{2}$ \\ ${ }^{1}$ Theory of Electrical Engineering, Helmut Schmidt University Hamburg, Germany \\ ${ }^{2}$ Institute of Applied Mechanics, RWTH Aachen University, Germany
}

Preceding research showed that the combination of quasi-static deep drawing and high speed electromagnetic impulse forming, as described in [1], can lead to an extension of classical, purely quasi-static forming limits, if the process parameters are carefully adjusted [2]. Moreover, it was indicated that in round cup deep drawing sharper bottom edge radii can be achieved by combining this manufacturing process with electromagnetic impulse forming. In this case, not only the deep drawing parameters, like punch radius and blankholder force, have to be considered, but the parameters describing the triggering current in the electromagnetic part of the combined process have also to be adjusted carefully to avoid material failure.

The pulses usually used in electromagnetic forming are given by decaying sinus waves, where the forming process is only governed by the first half wave. The field produced by the subsequent half waves mainly interacts with the tool, which has two major effects: on the one hand, the temperature increase of the polymer matrix where the tool coil is embedded in causes thermal wear, as recent research [3] shows, on the other hand the stresses resulting from the Lorentz force between the coil windings leads to mechanical wear of the tool. To avoid fast tool deterioration, methods to cut away or damp the subsequent half waves are of major interest [4].

Following the ideas from [5], in this work virtual process design comprising finite element simulation and mathematical optimization is utilized to study in how far sharp pulsed triggering currents can be controlled and adjusted to lead to sharper bottom edge radii in round cup forming. Particularly, an optimum double exponential current pulse is identified. This class of pulses is parametrized as an example for pulses with mono-directed current employed to reduce the wear of the tool coil.

A viscoplastic material model based on the multiplicative decomposition of the deformation gradient in the context of hyperelasticity has been used to model the material behaviour [6] both during deep drawing and in the electromagnetic forming step. It includes all important characteristics as the nonlinear kinematic and isotropic hardening, anisotropy, and ductile damage in the context of continuum damage mechanics. The model is incorporated into the commercial simulation software LSDYNA and, hence, used within the mathematical optimization procedure.

\section{References}

[1] Y. Kiliclar, O. K. Demir, I. N. Vladimirov, L. Kwiatkowski, S. Reese, and A. E. Tekkaya, "Simulation of electromagnetic forming of a cross-shaped cup by means of a viscoplasticity model coupled with damage at finite strains," Key Engineering Materials, vol. 554 - 557, pp. 2363-2368, 2013.

[2] V. S. Balanethiram and G. S. Daehn, "Hyperplasticity: Increased forming limits at high workpiece velocity," Scripta Metallurgica et Materialia, vol. 30, no. 4, pp. 515-520, 1994.

[3] S. Gies, C. Löbbe, C. Weddeling, and A. Tekkaya, "Thermal loads of working coils in electromagnetic sheet metal forming," Journal of Materials Processing Technology, vol. 214, no. 11, pp. 2553 - 2565, 2014.

[4] H. Bodenseher, H. Holtschmit, and G. Siewerth, "Einrichtung zum elektromagnetischen Umformen von Werkstuecken," German Patent DE 2205981 A1, Aug. 16, 1973, dE Patent App. DE19,722,205,981.

[5] F. Taebi, O. Demir, M. Stiemer, V. Psyk, L. Kwiatkowski, A. Brosius, H. Blum, and A. Tekkaya, "Dynamic forming limits and numerical optimization of combined quasi-static and impulse metal forming," Computational Materials Science, vol. 54, pp. 293 - 302, 2012.

[6] I. N. Vladimirov, M. P. Pietryga, Y. Kiliclar, V. Tini, and S. Reese, "Failure modelling in metal forming by means of an anisotropic hyperelastic-plasticity model with damage," International Journal of Damage Mechanics, vol. 23, pp. 1096-1132, 2014. 


\title{
Methods with Successive and Parallel Approximations of Inverse Operators for the Nonlinear Least Squares Problem
}

\author{
Roman Iakymchuk and Stepan Shakhno \\ Sorbonne Universités, UPMC Univ Paris 06, ICS, F-75005 Paris, France \\ Sorbonne Universités, UPMC Univ Paris 06, UMR 7606, LIP6, F-75005 Paris, France \\ roman.iakymchuk@lip6.fr, Phone: +33(0)1442787 73 \\ Ivan Franko National University of Lviv, Universitetska Str. 1, 79000, Lviv, Ukraine \\ s_shakhno@franko.lviv.ua, Phone: +380322394304, Fax: +380322616048
}

In this paper, we are concerned with finding the approximate solution of the nonlinear least squares problem [1]:

$$
\min f(x):=\frac{1}{2} F(x)^{T} F(x),
$$

where $F$ is a Fréchet differentiable operator defined on $\mathbb{R}^{n}$ with values on $\mathbb{R}^{m}, m \geq n$.

For solving the problem (1), we propose a Gauss-Newton type method with the successive approximation of the inverse operator

$$
\begin{aligned}
x_{k+1} & =x_{k}-A_{k} J\left(x_{k}\right)^{T} F\left(x_{k}\right), \\
A_{k+1} & =A_{k}\left[2 E-J\left(x_{k+1}\right)^{T} J\left(x_{k+1}\right) A_{k}\right], k=0,1,2, \ldots
\end{aligned}
$$

where $J(x)=F^{\prime}(x) ; E$ is an identity matrix; $x_{0}$ and $A_{0}$ are given initial approximations to the exact solution $x^{*}$ and to the inverse operator $A^{*}=\left[J\left(x^{*}\right)^{T} J\left(x^{*}\right)\right]^{-1}$, respectively. The method (2)-(3) consists of two branches, meaning computations of $x_{k+1}$ and $A_{k+1}$ that are executed by turn. The idea and the advantage of the successive approximation of the inverse operator is to construct the approximate solution of the problem (1) without solving a linear system of algebraic equations.

We derive parallel variants of the Gauss-Newton type method (2)-(3) with both synchronous and asynchronous approximations of the inverse operator. Our study is focused on analyzing the convergence of the derived methods as well as on parallel implementations of them. We investigate the local convergence of the sequential and two parallel methods with approximations of the inverse operator under classic Lipschitz conditions. In addition, we examine the convergence order of the proposed methods. As numerical experiments, we carry out a set of standard tests, comparing the studied methods against the well-known Gauss-Newton method. Furthermore, we develop parallel implementations of these methods and present the results of performance scaling on recent shared-memory architectures.

We foresee to apply parallelization techniques - such as the parallel approximation of inverse operator- to the other methods. In particular, we plan to study the two-step Gauss-Newton method [2] as it uses only one inverse over two function evaluations.

\section{References}

[1] J. M. Dennis and R. B. Schnabel. Numerical Methods for Unconstrained Optimization and Nonlinear Equations. Prentice-Hall, New York, 1983.

[2] R. Iakymchuk and S. Shakhno. On the Convergence Analysis of a Two-Step Modification of the GaussNewton Method. Book of Abstracts of the 85th Annual Meeting of the International Association of Applied Mathematics and Mechanics (GAMM), Erlangen, Germany, March 10-14, 2014. P. 623. 


\title{
Geometry Optimization of Branched Sheet Metal Structures with a Globalization Strategy by Adaptive Cubic Regularization
}

\author{
Thea Göllner, Stefan Ulbrich \\ TU Darmstadt
}

We consider the geometry optimization of sheet metal structures with webs for increased stiffness. The structures as well as the webs may exhibit curvature and can be produced using the technologies linear flow splitting and linear bend splitting, developed within the Collaborative Research Centre (CRC) 666, and possible additional forming operations like hydroforming.

To describe the mechanical behaviour of the structures the three-dimensional linear elasticity equations are used which leads to a PDE-constrained optimization problem. The geometry of the considered parts is parameterized by means of free form surfaces. We present an algorithm using adaptive cubic regularization as globalization strategy and exact geometry constraints as well as corresponding convergence results. Further, inexactness is introduced in both, the objective function and its gradient. The proposed algorithm is applied to geometry optimization problems for branched sheet metal parts and numerical results for an engineering application example from CRC 666 are given. 


\title{
Multidimensional parametrization of microcells in two scale optimization with sparse grid interpolation
}

\author{
D. Hübner, M. Stingl \\ Friedrich-Alexander University Erlangen-Nürnberg
}

We consider structural optimization problems in linear elasticity with locally periodic porous materials consisting of a matrix and soft inclusions. The method of homogenization has proven to be capable of solving such types of problems [1]. To avoid unnecessary computational effort we split this setting into an offline and an online phase. In the former we calculate a catalogue of effective material properties by solving linear elastic problems on a microcell for given sets of parameters. The parameters may for instance describe the shape of a structure inside a cell at the microscopic level. In the optimization process (online phase) at the macroscopic level these precalculated material properties have to be interpolated. However the generation of the material catalogue is very expensive for more than just a few parameters. To overcome this so-called curse of dimensionality we consider an interpolation method using sparse grids [2, 3]. We present numerical results for compliance minimization.

\section{References}

[1] M. P. Bendsoe, N. Kikuchi. Generating optimal topologies in structural design using a homogenization method. Computer methods in applied mechanics and engineering 71 (1988), 197-224.

[2] H.-J. Bungartz, M. Griebel. Sparse grids. Acta numerica 13 (2004): 147-269.

[3] J. Valentin. Hierarchische Optimierung mit Gradientenverfahren auf Dünngitterfunktionen. Universität Stuttgart, Fakultät Informatik, Elektrotechnik und Informationstechnik, Masterarbeit Nr. 3629 (2014) 


\title{
A staggered approach to structural shape and topology optimization
}

\author{
Stefan Riehl, Paul Steinmann \\ Chair of Applied Mechanics, University of Erlangen-Nürnberg
}

This contribution is concerned with the formulation and evaluation of a staggered optimization routine using results from shape and topological sensitivity analysis. To this extent, we interrelate a parameter-free approach to shape optimization and an evolutionary-type element removal procedure that relies on the topological sensitivity $[1,2]$ as a rejection criterion. As opposed to classical evolutionary-type algorithms in which elements may be removed from the domain boundary as well as from the interior of the domain at the same time, we specify an advancing-front algorithm that is only allowed to remove elements from the existing design boundary of the domain. This process is repeated until the minimum topological sensitivity is no longer encountered at the design boundary but in the interior of the domain. Subsequently, we invoke the traction method [3] for shape variation in order to achieve a more accurate approximation of the true optimal shape for the newly established design boundary. Having obtained the desired layout by shape variation, we create a hole in the interior of the domain by removing all cells that are adjacent to the nodal point that exhibits the minimum topological sensitivity and resume the advancing front algorithm to expand the newly established hole.

To allow for a successful coupling of the element removal procedure and the shape variation phase within a staggered optimization routine the overall outline is subject to geometrical constraints and is accompanied by a boundary smoothing technique.

\section{References}

[1] S. Garreau, P. Guillaume, M. Masmoudi. The topological asymptotic for PDE systems: the elasticity case. Journal on Control and Optimization 39 (2001), 1756-1778.

[2] A. Novotny, R. Feijo, E. Taroco, C. Padra. Topological sensitivity analysis for three-dimensional linear elasticity problem. Computer Methods in Applied Mechanics and Engineering 196 (2007), 4354-4364.

[3] H. Azegami, S. Fukumoto, T. Aoyama. Shape optimization of continua using NURBS as basis functions. Structural and Multidisciplinary Optimization 47 (2013), 247-258. 


\title{
On curvature control in node-based shape optimization
}

\author{
Oliver Schmitt, Paul Steinmann \\ Chair of Applied Mechanics \\ University of Erlangen-Nuremberg
}

In this work we present and compare three approaches to control of the curvature of the domain boundary in two-dimensional shape optimization. This is of interest with regard to manufacturing constraints [1], for instance in milling processes. In node-based shape optimization the coordinates of the FE nodes are used as design variables. Hence objective and constraint functions have to be formulated in terms of the nodal coordinates. Therefore, we investigate various formulations for the curvature of the domain boundary in terms of the coordinates of the boundary nodes. In addition, the sensitivities of these expressions with respect to the design nodes are required for the application of gradient-based optimization algorithms.

In the first approach, we consider a local representation of the boundary by a smooth curve in arc-length parametrization. After expanding the curve in a Taylor series up to second order a curvature can be assigned to each node depending on the coordinates of its direct neighbors [2]. In the second approach, three neighboring nodes are interpolated by a quadratic polynomial. To ensure definiteness of the polynomial the triple of considered nodes is translated and rotated into a reference system. In the last approach, a NURBS (nonuniform rational B-spline) curve [3] is defined using the FE nodes as control nodes. In contrast to the previous approaches the control nodes are in general not interpolated. Furthermore, the NURBS weights can be used to influence the pathway of the curve towards or away from the control nodes.

All three approaches result in a curvature value for each design node based on the evaluation of the second derivative of the corresponding local approximation. In the context of a constrained shape optimization problem, a constraint aggregation formulation [1] such as the Kreisselmeier-Steinhauser function may be applied.

\section{References}

[1] O. Schmitt, J. Friederich, P. Steinmann. Manufacturing constraints in parameter-free sensitivity-based shape optimization. In Proceedings of the 1st International Conference on Engineering and Applied Sciences Optimization. (2014), 2532 - 2542, Kos Island, Greece

[2] G. Taubin. Estimating the tensor of curvature of a surface from a polyhedral approximation. Proceedings of IEEE International Conference on Computer Vision. (1995), 902-907

[3] L. Piegl, W. Tiller. The NURBS Book. Springer, 1995 


\title{
Optimizing Extrusion Dies with Profile Shape as an Objective Function
}

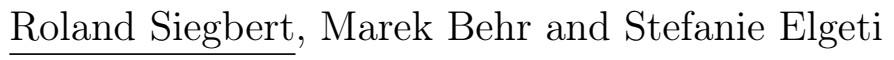 \\ Chair for Computational Analysis of Technical Systems (CATS) \\ RWTH Aachen University
}

In the application of plastics profile extrusion, die design is complicated by the rather unintuitive and nonlinear behavior of plastics melts. Traditionally, this obstacle is overcome by the experience of the die designer during an arduous cut-and-try process $[1,2]$. It has been successfully demonstrated $[3,4]$, that this design process can be supported by solving an inverse problem with the product shape as input and the die geometry as design vector of an optimization problem. In this optimization problem, the Navier-Stokes-Equations, which govern the flow of the plastics melt through the die, serve as constraints.

Optimization with the aim of balancing the extrusion die, meaning that the objective is a homogeneous outflow velocity distribution, has already been investigated extensively $[3,4,5,6]$. In the second phase of the Cluster of Excellence "Integrative Production Technologies for High-Wage Countries" at the RWTH Aachen University, an effort is underway to take die swell into account and thus to extend the scope of the numerical shape optimization. The optimization is performed utilizing the in-house flow solver XNS, which uses the finite element method with Galerkin/Least-Squares stabilization, can utilize various parallel machines (IBM Blue Gene, etc.), and is able to exploit the common communication interfaces for distributed-memory systems (SHMEM and MPI). Recently, XNS has been coupled with a nonlinear optimization library [7] in an optimization framework with a geometry kernel utilizing T-Splines, PB-Splines and NURBS.

This talk focuses on die swell and its incorporation into an objective function in the design of extrusion dies. It is investigated how die swell influences the optimization strategy, and first applications using industrial dies are shown.

\section{References}

[1] W. Michaeli. Extrusion Dies for Plastics and Rubber. 3rd ed. Hanser Gardner Publications (2009).

[2] M. M. Kostic, L. G. Reifschneider. Design of Extrusion Dies, Encyclopedia of Chemical Processing (2006), 633-649.

[3] S. Elgeti, M. Probst, M. Nicolai, L. Pauli, M. Behr. and Ch. Hopmann. Design Objectives in Shape Optimization of Profile Extrusion Dies. Conference Proceedings of the First ECCOMAS Young Investigators Conference (2012).

[4] R. Siegbert, S. Elgeti, M. Behr, K. Kurth, C. Windeck and Ch. Hopmann. Design Criteria in the Numerical Design of Profile Extrusion Dies. Key Engineering Materials Journal (2013), 794-800.

[5] O. S. Carneiro, J. M. Nobrega, F. T. Pinho. Flow balancing in extrusion dies for thermoplastic profiles. International Polymer Processing (2004), 1-11.

[6] N. D. Gonalves, O. S. Carneiro, and J. M. Nobrega. Design of complex profile extrusion dies through numerical modeling. Journal of Non-Newtonian Fluid Mechanics (2013), 103-110.

[7] S. G. Johnson. The NLopt Nonlinear-Optimization Package. http://ab-initio.mit.edu/nlopt 


\title{
Robust optimization of trusses under dynamic loads via nonlinear semidefinite programming
}

\author{
Anja Kuttich, Stefan Ulbrich \\ TU Darmstadt
}

We consider the problem of optimal truss topology design with respect to uncertainty in time-dependent loads via a robust optimization approach. To evaluate the stability and stiffness of truss structures we use the mean square displacement as a suitable objective function, which allows for an efficient calculation of a worst-case scenario. In the dynamic case the static equilibrium conditions must be replaced by a system of ordinary differential equations taking into account Newton's law of motion. Using the $\mathrm{H}_{\infty}$ norm of the transferfunction for the worst-case objective function and the Bounded-Real Lemma the optimal dynamic truss topology design problem can be expressed via nonlinear semidefinite programming. We apply a sequential semidefinite programming method to solve the resulting nonlinear semidefinite programming problem and present numerical results. 


\title{
About the design of morphing airfoils under uncertainties
}

\author{
F. Fusi *, G. Quaranta *, A. Guardone*, P.M. Congedo ${ }^{+}$ \\ (*) Department of Aerospace Science and Technology, Politecnico di Milano \\ $\left.{ }^{+}\right)$INRIA Bordeaux-Sud-Ouest, France
}

\begin{abstract}
Shape optimization has been widely used in aerospace vehicles design to define the design which provides the best performance given a set of objectives and constraints [1]. In aerodynamics, it has proved effective in the application to fixed-wing aerodynamic design, as well as in the case of helicopter rotor blades. However, in the latter case, many obstacles still refrain a wide employment of optimization techniques, mainly because rotor blades feature a complex aerodynamics, whose prediction requires accurate yet expensive analysis based on Computational Fluid Dynamics. In addition, the flow conditions of each section of the blade are largely variable for two main reasons. First, blades are required to operate with satisfactory performance in extremely different conditions: in hover approximately steady and azimuthally symmetric flow is encountered, whereas forward flight features an unsteady periodically changing flow. In the latter case, the advancing side of the rotor encounters transonic flow condition, while the retreating side is close to stall conditions. Secondly, significant unsteady effects due to the blade dynamics and flexibility, as well as the interaction with vortices and wakes trailed from preceding blades, affect the blade operating conditions. As a result, even when optimization is employed, the aerodynamic performance in the flight envelope may be often sub-optimal. A very attractive solution to this problem is the employment of a morphing blade, that is a blade capable of changing its shape during flight. Morphing rotor design is a challenging and active area of research: recent studies have focused on blades with adaptable span, chord or twist, as well as conformable airfoils [2]. With regard to the latter, airfoil adjustment has been mainly concerned with camber variation for vibration reduction [3] or dynamic stall delay [4].

In such a context the work presents an optimization framework for dealing with morphing airfoils for helicopter rotor blades. With regard to the morphing strategy, a conformable camber line is considered to deal with variable flow conditions encountered in forward flight with the objective of increasing the aerodynamic performance on the advancing and retreating sides of the rotor. The morphing airfoil consists of a conformable camber line which changes over the period of rotation of the blade, while the thickness distribution is held fixed. Another peculiarity of this approach is that the optimal shape is sought to be robust. This means that the optimal design should be designed to cope with the variation in the operating conditions arising both from the assumptions and simplifications introduced in the models at the design stage, and from the numerous sources of uncertainty present in the realistic world. The optimization algorithm is built to seek an optimal design which is less sensitive with respect to changes of the uncertain parameters defining the problem. Taking into account the uncertainty of the modeling variables in the optimization procedure results in a more reliable optimal design, providing satisfactory performance also in off-design conditions. In particular, the optimization method includes a robustness measure of the optimal design, based on the computation of low-order statistics of the fitness function when variations in the operating conditions (arising not only from the assumptions introduced in the models employed in the design stage, but also from the numerous sources of uncertainty present in the realistic world) are considered. Some preliminary studies on this work have tackled this optimization problem without constraints on the evolving airfoil shape. The present contribution is focused on the introduction of a constraint on the thickness distribution, and on the impact of this geometric constraint on the optimization loop. Furthermore, a complete discussion on the airfoil parameterization and its influence on the optimization process will be addressed.
\end{abstract}

\section{References}

[1] W. Yao, X. Chen, W. Luo, M. van Tooren, J. Guo. Review of uncertainty-based multidisciplinary design optimization methods for aerospace vehicles. Progress in Aerospace Sciences. 47 (2011), 450-479.

[2] S. Barbarino, O. Bilgen, R. M. Ajaj, M. I. Friswell, D. J. Inman. A Review of Morphing Aircraft. Journal of Intelligent Material Systems and Structures. 22 (2011), 823-877.

[3] F. Gandhi, M. Frecker, A. Nissly. Design Optimization of a Controllable Camber Rotor Airfoil. AIAA Journal. 46 (2008), 142-153. 
[4] M. F. Kerho. Adaptive Airfoil Dynamic Stall Control. Journal of Aircraft. 44 (2007), 1350-1360. 


\title{
Robust Design using classical optimization
}

\author{
Stefan Erschen, Markus Zimmermann \\ BMW Group Research and Innovation Center, Knorrstrasse 147, 80937, Munich, Germany \\ e-mail: stefan.erschen@mytum.de
}

One challenge of developing complex products is to account for a high number of interactions between many subsystems, components and requirements. This leads to uncertainties which can often not be quantified. One example is the lack of knowledge, which arises due distributed development processes over several departments or even companies, changing environmental conditions or non-avoidable changes in influencing parameters during the development phase. This contribution shows, how to use classical optimization approaches to deal with these uncertainties. Classical approaches such as Performance Optimization, Robust Design Optimization and Reliability Assessment are often used for developing products, but reveal some major drawbacks if one deals with uncertainties in a very early phase.

For the purpose of developing robust design, Zimmermann and Hoessle [1], see also [2], describe a stochastic approach for finding a box within the solution space, each edge representing an independent parameter interval in the n-dimensional space. This provides space for unavoidable variations due to uncertainties. For maximum robustness the box is required to be as large as possible. The proposed algorithm seeks for intervals of each design parameter, which guarantee that the system reaches the targets specified, i.e. the design constraints. The algorithm is based on Monte Carlo sampling and can handle any non-linear, high dimensional and noisy problem. Although the algorithm can deal with all kinds of input-output-mappings the computational cost is quite high for complex models. Fender [3], describes a different approach for finding a hyper-box within a solution space. His approach is based on simplified physical models for crash applications, simple enough to derive analytical constraints, i.e. the limit state function for feasible designs. Thus, the problem of finding a box within the feasible domain is solved by classical optimization under constraints. Therefore the edges are moved within the feasible region such that the minimum of all parameter intervals is maximized. The approach is advantageous in terms of the computational burden but requires simplified physical models.

The presented approach is based on finding the largest box within the feasible region by using the requirements of the product as constraints. Focus is on describing the solution space mathematically, e.g. by linear regression. The problem of finding the largest box under constraints is solved by an interior point method algorithm. The method is applied to a real world problem for chassis design and the advantages compared to the stochastic approach are shown.

\section{References}

[1] M. Zimmermann, J. Edler von Hoessle. Computing solution spaces for robust design. International Journal for Numerical Methods in Engineering, 94:290-307, 2013.

[2] L. Graff. A stochastic method for the identification of solution spaces in high-dimensional design spaces. PhD Thesis, Philosophisch-Naturwissenschaftliche Fakultät, Universität Basel, Switzerland, 2013.

[3] J.H.W. Fender. Solution Spaces for Vehicle Crash Design. PhD Thesis, Chair of Computational Mechanics, Technische Universität München, Germany, 2014. 


\title{
Optimization of the double sided spiral groove thrust bearing: A comparison to approximate analytical solutions
}

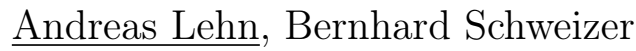 \\ TU Darmstadt
}

Reliable high performance bearings are essential in rotordynamics. As speeds and loads are continuously increased the demands on bearing technology grow simultaneously. Optimization of bearing characteristics becomes more important under these circumstances. For hydrodynamic thrust bearings, recently a special design has been presented [1,2], which is supposed to enhance the load carrying capacity by $60 \%$ compared to the best known thrust bearing type [3]. The bearing consists of two spiral grooved surfaces and is therefore called a double sided spiral groove bearing. Early calculations of the bearing performance base on approximate analytical methods.

In order to verify those results, a detailed numerical model of the bearing is presented by the authors. With the help of a particle swarm optimization method, optimal bearing parameters are determined and a comparison is drawn to other thrust bearing designs. It is shown that previously published results overestimate the performance of the double sided spiral groove bearing severly. Performance charts for optimized configurations with respect to load carrying capacity and friction coefficient are delivered.

\section{References}

[1] A. Yemelyanov, I. Yemelyanov. Physical models, theory and fundamental improvement to self-acting spiralgrooved gas bearings and visco-seals. Proc. Instn Mech Engrs, 213, 1999

[2] A. Emel'yanov, I. Emel'yanov. Theory of binary spiral-grooved gas bearings. Fluid Dynamics, 35:351-360, 2000.

[3] E. Muijderman. Spiral Groove Bearings. PhD thesis, University Delft, 1964 


\title{
Multi-criteria optimisation of the vibro-isolation properties
}

\author{
Igor Maciejewski, Tomasz Krzyzynski \\ Departament of Mechatronics and Applied Mechanics \\ Faculty of Technology and Education \\ Koszalin University of Technology \\ Sniadeckich St. 2, Koszalin, 75-453 PL
}

The paper deals with a methodology of shaping the vibro-isolation properties of suspensions applied in automotive systems. The developed optimization procedure allows to find a Pareto-optimal [1] system configuration for the conflicting vibro-isolating criteria: the isolated body acceleration and the relative displacement of the suspension system. In order to optimize both of the conflicting vibro-isolation criteria, a minimization of the isolated body acceleration (primary criterion) is proposed taking into account the relative displacement of suspension system that is transferred to a nonlinear inequality constraint. The correctness of the proposed methodology is evaluated using experimental research of the best solutions of seat suspension. This research is performed using the semi active and active systems with optimal controller settings. The optimization is performed for the various excitation signals.

\section{References}

[1] R.T. Marler, J.S. Arora. Survey of multi-objective optimization methods for engineering. Structural and Multidisciplinary Optimization 26 (2004), 369-395. 


\section{S17: Applied and numerical linear algebra}

The aim of this section is to bring together experts in the field of applied and numerical linear algebra, discussing recent theoretical and algorithmic developments. 


\title{
Designing rational filter functions for solving eigenvalue problems by contour integration
}

\author{
Marc Van Barel \\ Department of Computer Science, KU Leuven, Belgium
}

In this talk, the following eigenvalue problem is considered. Given an integer $m \geq 1$, a domain $\Omega \subset \mathbb{C}$ and a matrix-valued function $T: \Omega \rightarrow \mathbb{C}^{m \times m}$ analytic in $\Omega$, we want to compute the values $\lambda \in \Omega$ (eigenvalues) and $v \in \mathbb{C}^{m}, v \neq 0$ (eigenvectors) such that

$$
T(\lambda) v=0 .
$$

Note that this formulation reduces to the linear eigenvalue problem in case $T(z)=A-z B$, and to the polynomial eigenvalue problem when $T(z)$ is a polynomial matrix. If the problem size $m$ is equal to 1 , then the problem reduces to that of computing all the zeros $\lambda$ of the analytic scalar function $T$ inside the domain $\Omega$.

The number of eigenvalues could be large, e.g., when $m$ is large, or in case of a polynomial eigenvalue problem when the degree of the polynomial matrix is large. In several applications, one is not interested in all eigenvalues but only in those lying in a certain region(s) of the complex plane. Therefore, we can reduce the original problem of finding all eigenvalues into one where we are only interested in those eigenvalues (and corresponding eigenvectors) lying within (or in the neighborhood) of a given closed contour $\Gamma \subset \Omega$.

The approach discussed in this talk is based on (numerical approximations of) contour integrals of the resolvent operator $T(z)^{-1}$ applied to a rectangular matrix $\hat{V}$ :

$$
\frac{1}{2 \pi i} \int_{\Gamma} f(z) T(z)^{-1} \hat{V} d z \quad \in \mathbb{C}^{m \times q}
$$

where $f: \Omega \rightarrow \mathbb{C}$ is analytic in $\Omega$ and $\hat{V} \in \mathbb{C}^{m \times q}$ is a matrix chosen randomly or in another specified way, with $q \leq m$.

The contour integral is approximated by a quadrature rule with nodes $t_{j}$ and corresponding weights $u_{j}$, i.e.,

$$
\int_{\Gamma} f(z) d z \approx \sum_{j=1}^{N} u_{j} f\left(t_{j}\right) .
$$

As was explained in [1], from Keldysh' theorem, we know that the resolvent function $T(z)^{-1}$ can be written (for simple eigenvalues $\lambda$ ) as

$$
T(z)^{-1}=\sum_{k} v_{k} w_{k}^{H} \frac{1}{z-\lambda_{k}}+R(z)
$$

with $R(z)$ an analytic function where $T(z)$ is analytic. Note that if $T^{-1}$ is a matrix-valued strictly proper rational function, the analytic function $R$ is equal to zero. This is the case, for example, if $T(z)=A-z B$ with $B$ nonsingular or if $T(z)$ is a matrix polynomial in $z$ with nonsingular highest degree coefficient.

Hence, applying the quadrature rule on the moments of the resolvent function $z^{l} T(z)^{-1}$ gives us

$$
\begin{aligned}
\int_{\Gamma} z^{l} T(z)^{-1} d z & \approx \sum_{j=1}^{N} u_{j} t_{j}^{l} T\left(t_{j}\right)^{-1} \\
& =\sum_{k} v_{k} w_{k}^{H} \sum_{j=1}^{N} \frac{u_{j} t_{j}^{l}}{t_{j}-\lambda_{k}}+\sum_{j=1}^{N} u_{j} t_{j}^{l} R\left(t_{j}\right) .
\end{aligned}
$$

The filter functions $b_{l}(z)$ are defined as the rational functions of degree $\delta$ corresponding to the quadrature rule as follows

$$
b_{l}(z)=\sum_{j=1}^{N} \frac{u_{j} t_{j}^{l}}{t_{j}-z} .
$$

In [2], we argued that it is important to have robust and cheap ways to design good filter functions. This design consists in finding the weights $u_{j}$ and the nodes $t_{j}$ such that the following conditions are satisfied: 
1. $b_{l}(z)=b_{0}(z) z^{l}$

2. $\left|b_{l}(z)\right|$ is large inside $\Gamma$ and small outside $\Gamma$;

3. $\left|\sum_{j=1}^{N} u_{j} t_{j}^{l} R\left(t_{j}\right)\right|$ is small.

We call the function $b_{0}(z)$ "the filter function" and denote it as $b(z)$. It is easy to see that as long as $l+\nu<N$ with $\nu$ the degree of the numerator of the filter function $b(z)$, the first condition is satisfied. To satisfy the second and third condition, we will solve an optimization problem looking for the variables $u_{j}$ and $t_{j}$ such that $\left|b_{l}(z)\right|$ is large inside $\Gamma$ and small outside $\Gamma$. The validity of the approach will be illustrated by some numerical experiments.

\section{References}

[1] W.-J. Beyn. An integral method for solving nonlinear eigenvalue problems. Linear Algebra and its Applications, 436:3839-3863, 2012.

[2] M. Van Barel and P. Kravanja. Nonlinear eigenvalue problems and contour integrals. Technical Report TW656, Department of Computer Science, KU Leuven, October 2014. 


\title{
Parallel Bidiagonal SVD via the Method of Multiple Relatively Robust Representations
}

\author{
Jan Winkelmann, Paolo Bientinesi \\ RWTH Aachen
}

One of the central problems in numerical linear algebra is the computation of the singular value decomposition (SVD) of a real bidiagonal matrix. One way to calculate the bidiagonal SVD is by reducing the problem to a real symmetric tridiagonal eigenproblem. The state of the art eigensolver-based SVD method is the Divide and Conquer SVD algorithm, with a run-time complexity of $\mathcal{O}\left(n^{3}\right)$ in the worst case, and $\mathcal{O}\left(n^{2}\right)$ in the best case. A more modern eigensolver is the method of Multiple Relatively Robust Representations $\left(\mathrm{MR}^{3}\right)$, which calculates $k$ eigenpairs with $\mathcal{O}(n k)$ operations in the worst case. A bidiagonal SVD solver based on $\mathrm{MR}^{3}$ promises to be an improvement over the current state of the art by providing lower asymptotic runtime and the ability to calculate a subset of singular triplets at reduced cost. Currently, no implementation is readily available that uses $\mathrm{MR}^{3}$ as an SVD solver. We provide such a solver, adapted from of an existing $\mathrm{MR}^{3}$ implementation.

Using $\mathrm{MR}^{3}$ as a bidiagonal SVD solver in a numerically stable way is not a trivial task. Willems and Lang [1] have presented a modification of $\mathrm{MR}^{3}$ that can be used to obtain bidiagonal SVDs in a numerically stable manner. The approach uses $\mathrm{MR}^{3}$ on Golub-Kahan matrices. Unfortunately, this approach does not work if $\mathrm{MR}^{3}$ is used in a black-box manner. The reason lies in the way the input matrix is represented internally by the algorithm. $\mathrm{MR}^{3}$ uses $L D L^{*}$ factorizations to ensure accuracy of the results, and $L D L^{*}$ factorizations of definite matrices provably guarantee high accuracy. As high accuracy is always desirable, black-box $\mathrm{MR}^{3}$ shifts the eigenvalue spectrum of the input matrix to be definite. However, this shift causes problems with the quality of the extracted SVD later on. Willems and Lang solved this problem by avoiding the initial shifting of the spectrum, thus guaranteeing the quality of the obtained SVD.

Indeed, shifting the eigenvalue spectrum of the input matrix is something $\mathrm{MR}^{3}$ does multiple times. Roughly speaking, $\mathrm{MR}^{3}$ is similar to bisection and inverse iteration in a manner that guarantees the orthogonality of the eigenvectors. This cannot be accomplished if some of the eigenvalues are clustered, that is, if they have small relative distances. In $\mathrm{MR}^{3}$, clusters are broken by shifting the spectrum close to the clustered eigenvalues, as this enlarges their relative distances. After the shift, the eigenpairs for the newly "unclustered" eigenvalues can be calculated with high accuracy.

Our contribution consists of the following elements: We extended an existing $\mathrm{MR}^{3}$ eigensolver implementation (incidentally also by Willems) to be a bidiagonal SVD solver. We equipped our implementation with shared-memory parallelism. Previous work has shown that $\mathrm{MR}^{3}$ can achieve a high degree of parallelism [2]. We adopted the same task parallel approach. This entails the decomposition into three distinct tasks, (1) shifting the spectrum to break clusters, (2) refinement and classification of the eigenvalues after a shift, and (3) calculating the eigenpair for eigenvalues with large relative distances. In this task we discuss the implementation of our shared-memory parallel SVD solver and present results regarding runtime and the quality of the resulting decomposition.

\section{References}

[1] P.R. Willems, B. Lang. The $\mathrm{MR}^{3}-\mathrm{GK}$ Algorithm for the Bidiagonal SVD. Electronic Transactions on Numerical Analysis. (2012) Volume 39, 1-21.

[2] M. Petschow, E. Peise, P. Bientinesi. High-Performance Solvers for Dense Hermitian Eigenproblems. SIAM Journal on Scientific Computing. (2013) Volume 35(1), pp. C1-C22. 


\title{
A Hessenberg reduction algorithm for diagonal plus low rank matrices
}

\author{
Dario A. Bini, Leonardo Robol \\ University of Pisa \\ Scuola Normale Superiore, Pisa
}

We present an algorithm for Hessenberg reduction of matrices of the form $A=D+U V^{*}$ where $D$ is real diagonal and $U, V$ are in $\mathbb{C}^{n \times k}$. Examples of such problems may be encountered as a first step in the solution of structured eigenvalue problems. Some classes of block companion matrices (see for example [1]) do have this particular structure and thus may benefit from an efficient method for the computation of the Hessenberg form.

The algorithm uses a rank $k$ generalization of the quasiseparable techniques developed in [4], obtaining an asymptotic cost for the reduction of $O\left(n^{2} k\right)$ floating point operations. Other algorithms have been presented in the literature, for example in [3] and [2]. Some of them do have an asymptotic cost in the rank that is cubic, thus making them not effective when the rank $k$ is bigger than $n^{\frac{1}{3}}$. This can be an issue when dealing with linearizations of matrix polynomials that often have a quasiseparable rank not negligible with respect to the size of the problem. For this reason we focused our attention at obtaining a linear complexity in $k$.

We introduce some theoretical results on rank conservation that allow to sharply bound the quasiseparable ranks of the matrices obtained at each step of the reduction and explicitly represent every matrix without redundancy in the rank. More precisely, we provide an explicit representation of the trailing principal submatrix of $Q_{j} A Q_{j}^{*}$, the matrix obtained at the $j$-th step of the reduction. This representation can be used to compute the unitary matrix $Q_{j+1}$. Moreover, we discuss different representations for these intermediate matrices and analyze the numerical stability of the approach. Some strategies are proposed in order to avoid cancellation in the process with the purpose of increase its stability.

\section{References}

[1] Bini, Dario A., and Leonardo Robol. On a Class of Matrix Pencils Equivalent to a Given Matrix Polynomial. arXiv preprint arXiv:1406.1025 (2014).

[2] Delvaux, Steven, and Marc Van Barel. A Hessenberg reduction algorithm for rank structured matrices. SIAM Journal on Matrix Analysis and Applications 29.3 (2007): 895-926.

[3] Eidelman, Yuli, Israel Gohberg, and Luca Gemignani. On the fast reduction of a quasiseparable matrix to Hessenberg and tridiagonal forms. Linear algebra and its applications 420.1 (2007): 86-101.

[4] Vandebril, Raf, Marc Van Barel, and Nicola Mastronardi. Matrix computations and semiseparable matrices: linear systems. Vol. 1. JHU Press, 2010. 


\title{
An extended Hessenberg form for Hamiltonian matrices
}

\author{
$\underline{\text { Micol Ferranti }}^{1}$, Bruno Iannazzo ${ }^{2}$, Thomas Mach ${ }^{1}$, Raf Vandebril ${ }^{1}$ \\ ${ }^{1}$ Dept. of Computer Science, KU Leuven, Belgium \\ Dep ${ }^{2}$ t. of Mathematics and Computer Science, Università degli studi di Perugia, Italy
}

The QR method is one of the most used algorithms to compute eigenvalues of medium sized matrices. It is a two step method: the matrix is first transformed via unitary similarity transformations to a convenient condensed form, whose eigenvalues are then computed via a suitable iterative method.

The typical generic condensed form is the well known Hessenberg form, see, e.g., [4]. However, when the original matrix exhibits particular properties, the preservation of the structure through the whole procedure is advisable. For this reason, some special formulations of the QR method have been developed for specific classes of matrices (i.e., symmetric, unitary, etc.).

On the other hand, it has been shown in [3] that the classic Hessenberg form is not the only possible choice as an intermediate condensed form. A much wider family of matrices can be used within a QR-like algorithm, in order to achieve the same results. This family extends classes such as Hessenberg and Hessenberg-like, and will be named extended Hessenberg form.

A Hamiltonian matrix is represented by

$$
H=\left[\begin{array}{cc}
A & G \\
F & -A^{H}
\end{array}\right] \in \mathbb{C}^{2 n \times 2 n},
$$

where $F=F^{H}$ and $G=G^{H}$. The eigenvalues of a Hamiltonian matrix are symmetric with respect to the imaginary axis. To preserve this symmetry within a QR algorithm, it would be desirable to work exclusively with Hamiltonian matrices during the iterative process.

The generic Hessenberg form does not retain the Hamiltonian structure. Thus many attempts have been made to design a suitable Hamiltonian condensed form, see, for example, [1, 2]. Until now, a Hamiltonian QR algorithm has been found for matrices $H$ whose bottom-left block $F$ has rank 1 [1]. This algorithm makes use of the so called Hamiltonian Hessenberg form.

In this talk we will show how the arguments presented in [3] can be adapted to the Hamiltonian context, in order to derive a new Hamiltonian condensed form, which extends the classic Hamiltonian Hessenberg form.

\section{References}

[1] R. Byers. A Hamiltonian QR-algorithm. SIAM Journal on Scientific and Statistical Computation, 7(1):212229, 1986.

[2] C. C. Paige and C. F. Van Loan. A Schur decomposition for Hamiltonian matrices. Linear Algebra and its applications, 41:11-32, 1981.

[3] R. Vandebril. Chasing bulges or rotations? A metamorphosis of the QR-algorithm. SIAM Journal on Matrix Analysis and Applications, 32:217-247, 2011.

[4] D. S. Watkins. Francis's algorithm. American Mathematical Monthly, 118:387-403,2011. 


\title{
Rank-revealing decomposition via block anti-triangular factorization
}

\author{
Nicola Mastronardi, Paul Van Dooren \\ Istituto per le Applicazioni del Calcolo "M. Picone", sede di Bari \\ Consiglio Nazionale delle Ricerche \\ Via G. Amendola, 122/D, I-70126 Bari, Italy \\ Catholic University of Louvain Department of Mathematical Engineering \\ Avenue Georges Lemaitre 4 \\ B-1348 Louvain-la-Neuve, Belgium
}

An algorithm for computing the block anti-triangular (BAT) form of a symmetric matrix has been recently introduced [1]. In particular, given a symmetric matrix $A \in \mathbb{R}^{n \times n}$, an orthogonal matrix $Q \in \mathbb{R}^{n \times n}$ is sought such that

$$
A=Q M Q^{T}=\left[\begin{array}{cccc}
\mathbf{0} & \mathbf{0} & \mathbf{0} & \mathbf{0} \\
\mathbf{0} & \mathbf{0} & \mathbf{0} & Y^{T} \\
\mathbf{0} & \mathbf{0} & X & Z^{T} \\
\mathbf{0} & Y & Z & W
\end{array}\right] \begin{aligned}
& \} n_{0} \\
& \} n_{1} \\
& \} n_{2} \\
& \} n_{1}
\end{aligned}
$$

with $n_{0}, n_{1}$ and $n_{2}$ depending on the inertia of $A$. Moreover, $Q \in \mathbb{R}^{n \times n}$ is an orthogonal matrix,$Z \in \mathbb{R}^{n_{1} \times n_{2}}$, $W \in \mathbb{R}^{n_{1} \times n_{1}}$ are symmetric, $Y \in \mathbb{R}^{n_{1} \times n_{1}}$ is a nonsingular lower anti-triangular matrix and $X \in \mathbb{R}^{n_{2} \times n_{2}}$ is symmetric definite.

It has been shown that the latter BAT factorization of a symmetric matrix can be efficiently updated/downdated by a symmetric rank-one modification. Moreover, the updating of a matrix obtained appending to a BAT matrix one more row and, symmetrically, a column, represents the kernel step of the algorithm described in [1] and can be done in $O\left(n^{2}\right)$ floating point operations.

In this talk we will describe an algorithm that factorizes a symmetric matrix as the product $Q M Q^{T}$, with $\mathrm{M}$ in a rank-revealing BAT form, i.e., denoted by $\sigma_{i}, i=1, \ldots, n$, the singular values of $M$ in a decreasing order, and supposed $\sigma_{k} \gg \sigma_{k+1}$,

$$
M=\left[\begin{array}{ll}
M_{11} & M_{12} \\
M_{12}^{T} & M_{22}
\end{array}\right], \quad M_{11} \in \mathbb{R}^{(n-k) \times(n-k)}, M_{11} \in \mathbb{R}^{(k \times k}, M_{12} \in \mathbb{R}^{k \times(n-k)},
$$

with $0 \leq k \leq n$, we have

$$
\operatorname{cond}\left(M_{11}\right) \simeq \sigma_{1} / \sigma_{k} \text { and }\left\|M_{11}\right\|_{F}^{2}+\left\|M_{12}\right\|_{F}^{2} \simeq \sigma_{k+1}+\cdots+\sigma_{n} .
$$

Numerical results will be shown together with comparisons with existing algorithms for symmetric rankrevealing factrorizations available in the literature [2].

\section{References}

[1] N. Mastronardi, P. Van Dooren. The antitriangular factorization of symmetric matrices, SIAM J. Matrix Anal. Appl., 34 (2013), 173-196.

[2] P.C. Hansen, P.Y.Yalamov, Computing Symmetric Rank-Revealing Decompositions via Triangular Factorization, SIAM Journal on Matrix Analysis and Applications, 23 (2001), 443-458. 


\title{
On complex $J$-symmetric eigenproblems
}

\author{
Peter Benner ${ }^{1}$, $\underline{\text { Heike Faßbender }}^{2}$, Chao Yang ${ }^{3}$
}

${ }^{1}$ Max Planck Institute for Dynamics of Complex Technical Systems, Magdeburg, Germany

${ }^{2} \mathrm{TU}$ Braunschweig, Institut Computational Mathematics,

AG Numerik, Braunschweig, Germany

${ }^{3}$ Lawrence Berkeley National Laboratory, Computational Research Division, Berkely, USA

The eigenproblem $H_{C} x=\lambda x$ for matrices

$$
H_{C}=\left[\begin{array}{cc}
A & C \\
D & -A^{T}
\end{array}\right] \in \mathbb{C}^{2 n \times 2 n}, \quad A, C=C^{T}, D=D^{T} \in \mathbb{C}^{n \times n} .
$$

will be considered. Please note, that here $X^{T}$ denotes transposition, $Y=X^{T}, y_{i j}=x_{j i}$, no matter whether $X$ is real or complex, while $X^{H}$ denotes conjugate transposition, $Y=X^{H}, y_{i j}=\overline{x_{j i}}$.

For

$$
J_{n}=\left[\begin{array}{cc}
0 & I_{n} \\
-I_{n} & 0
\end{array}\right] \in \mathbb{R}^{2 n \times 2 n}, \quad I_{n} \in \mathbb{R}^{n \times n}
$$

we have

$$
\left(H_{C} J\right)^{T}=H_{C} J
$$

Matrices $H_{C}$ are called complex- $J$-symmetric. The eigenvalues of $H_{C}$ display a symmetry: they appear in pairs $(\lambda,-\lambda)$. If $x$ is the right eigenvector corresponding to $\lambda, H_{C} x=\lambda x$, than $J x$ is the left eigenvector corresponding to the eigenvalue $-\lambda$ of $H_{C},(J x)^{T} H_{C}=-\lambda(J x)$.

Any complex $J$-symmetric matrix $X$ is said to be in structured Schur form if

$$
X=\left[\begin{array}{cc}
R & B \\
0 & -R^{T}
\end{array}\right], \quad R, B=B^{T} \in \mathbb{C}^{n \times n},
$$

where the nonzero eigenvalues of $R$ either have positive real part or zero real part and positive imaginary part. We will prove that for any complex $J$-symmetric matrix $H_{C}$ there exists a complex symplectic and unitary matrix $W \in \mathbb{C}^{2 n \times 2 n}$

$$
W^{T} J W=J \quad W^{H} W=I,
$$

such that $W^{H} H_{C} W$ is in structured Schur form.

The most popular way to compute the standard Schur form of a general matrix is the $Q R$ algorithm. It is tempting to derive a structured QR algorithm for transforming $H_{C}$ iteratively into structured Schur form. We will discuss why this is not possible and suggest other methods to compute eigenvalues and eigenvectors of $H_{C}$. 


\title{
Nonlinear eigenvalue problem expressed in Hermite basis
}

\author{
Heike Fassbender, Nikta Shayanfar \\ AG Numerik, Institut Computational Mathematics, Technische Universität Braunschweig
}

Polynomial eigenvalue problems have been used extensively in different areas of mechanics, such as machine foundations, electronic model of metal strip, simplified nuclear power plant problem, acoustic wave problem, Whipple bicycle model, etc. A comprehensive collection of the eigenvalue problems from models of real life as well as structured ones is studied in [3]. The standard form of a polynomial eigenvalue problem is to find scalars $\lambda$ and nonzero vectors $x$ such that they satisfy

$$
P(\lambda) x=0
$$

where $P: \mathbb{C} \rightarrow \mathbb{C}^{n \times n}$ is a given matrix-valued polynomial of degree $s$ as

$$
P(\lambda)=\sum_{i=0}^{s} P_{i} \lambda^{i}, \quad P_{i} \in \mathbb{C}^{n \times n}, \quad P_{s} \neq 0 .
$$

The variable $\lambda \in \mathbb{C}$ and the nonzero vector $x \in \mathbb{C}^{n \times 1}$ are the sought eigenvalue and eigenvector, respectively.

The growing attention to the polynomial eigenvalue problems has created the necessity of studying linearization, which is the standard computational approach to solving eigenvalue problems. One of the first papers discussing the main idea is [4]. It is based on finding a linear matrix polynomial $L(\lambda)$ such that the spectral structure of this particular linearization reproduces that of a given matrix polynomial $P(\lambda)$. More precisely, the above equation is converted to $L(\lambda) y=0$, where $L(\lambda)$ is a larger size matrix polynomial with the same spectral properties, specially same eigenvalues. Then it can be solved by standard techniques developed for linear eigenvalue problems.

On checking the history of linearization, we find that linearization has been done in different bases, see [1] among others. Actually, matrix polynomials expressed in other bases other than monomial, occur in many applications. For instance, Bernstein basis appears in computer-aided geometric design. Legendre basis helps solving the problems in partial differential equations with symmetries in the boundary conditions. Lagrange polynomial interpolation is traditionally viewed as a tool for theoretical analysis, and [2] demonstrates several advantages to computation in the Lagrange basis. Generalization of the Lagrange basis leads to the Hermite interpolation problem. To satisfy functional or aesthetic criteria, designed objects often have to exactly match prescribed data, such as a series of points and derivatives. This idea leads to the presentation of the Hermite basis, for which the linearization has not been comprehensively studied in literature. This contribution presents ideas of solving nonlinear eigenvalue problems in Hermite basis. We introduce a new linearization for an eigenvalue problem in which the matrix polynomial is expressed in Hermite basis, and the property of strong linearization is to be investigated. This linearization shows a reliable behavior and an acceptable accuracy in comparison to the recent results in the literature.

\section{References}

[1] A. Amiraslani, R. Corless, P. Lancaster. Linearization of matrix polynomials expressed in polynomial bases. IMA J. Numer. Anal. 29 (2009), 141-157.

[2] J.P. Berrut, L.N. Trefethen, Barycentric Lagrange interpolation. SIAM Rev. 46 (2004), 501-517.

[3] T. Betcke, N.J. Higham, V. Mehrmann, C. Schröder, F. Tisseur. NLEVP: a collection of nonlinear eigenvalue problems. ACM Trans. Math. Software 39 (2013), 1-28.

[4] I. Gohberg, M.A. Kaashoek, P. Lancaster. General theory of regular matrix polynomials and band Toeplitz operators. Integral Equations Operator Theory 11 (1988), 776-882. 


\title{
Backward error of polynomial eigenvalue problems solved by linearization
}

\author{
$\underline{\text { Piers W. Lawrence }}^{1}$, Marc Van Barel $^{2}$, Paul Van Dooren ${ }^{1}$ \\ ${ }^{1}$ Université Catholique de Louvain, Department of Mathematical Engineering \\ ${ }^{2} \mathrm{KU}$ Leuven, Department of Computer Science
}

It is commonplace in many application domains to utilize polynomial eigenvalue problems to model the behaviour of physical systems. Many techniques exist to compute solutions of these polynomial eigenvalue problems. One of the most frequently used techniques is linearization, in which the polynomial eigenvalue problem is turned into an equivalent linear eigenvalue problem with the same eigenvalues, and with easily recoverable eigenvectors. The eigenvalues and eigenvectors of the linearization are usually computed using a backward stable solver such as the QZ algorithm. Such backward stable algorithms ensure that the computed eigenvalues and eigenvectors of the linearization are exactly those of a nearby linear pencil, where the perturbations are bounded in terms of the machine precision and the norms of the matrices defining the linearization. With respect to the linearization, we may have solved a nearby problem, but we would also like to know if our computed solution is the exact solution of a nearby polynomial eigenvalue problem.

Furthermore, there has recently been a steady increase in the number of distinct linearizations proposed in the literature, depending mainly on the basis in which the polynomial eigenvalue problems are represented. Certainly, the choice of basis can have a dramatic effect on the backward errors, as can the particular choice of linearization. One of the objectives of this work is to develop a framework for analyzing different polynomial bases and linearizations in a uniform way. Thus, we investigate a particular class of linearizations where the polynomial coefficients are separated from the recurrence relations of the polynomial basis employed.

We use one-sided factorization to relate the linearization to the original polynomial in a very particular way. Given a linearization $\mathcal{L}(\lambda)$ of a polynomial matrix $P(\lambda)$, we find a one-sided factorization $\Phi(\lambda)$, such that $\mathcal{L}(\lambda) \Phi(\lambda)=P(\lambda) \otimes e_{1}$, where $e_{1}$ is the first unit vector. Since the QZ algorithm computes the exact solution of a slightly perturbed linearization, we investigate

$$
(\mathcal{L}(\lambda)+\Delta \mathcal{L}(\lambda))(\Phi(\lambda)+\Delta \Phi(\lambda))=\left[\begin{array}{c}
P(\lambda)+\Delta P(\lambda) \\
0
\end{array}\right]
$$

to first order. The perturbation $\Delta \Phi(\lambda)$ to the one-sided factorization is chosen in order to maintain the structure in the bottom of (1). For a given specific basis, we utilize the appropriate convolution matrices in order to obtain upper bounds for the norm of the coefficients of the perturbation $\Delta P(\lambda)$.

For some specific polynomial bases and linearizations, we are able to formulate these upper bounds in a simple way. Thus, we obtain the conditions under which the backward error of the solution of the polynomial eigenvalue problems are small. 


\title{
IgA vs. FEA in the Spectral Approximation: Symbol-Based Analysis
}

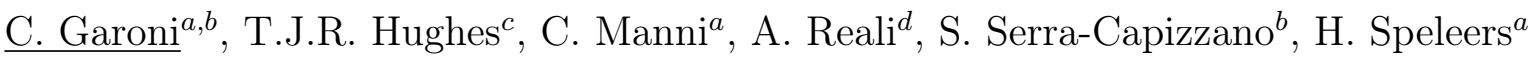 \\ ${ }^{a}$ Department of Mathematics, University of Roma 'Tor Vergata' \\ ${ }^{b}$ Department of Science and High Technology, University of Insubria (Como, Italy) \\ ${ }^{c}$ Institute for Computational Engineering and Sciences, The University of Texas at Austin \\ ${ }^{d}$ Department of Civil Engineering and Architecture, University of Pavia (Italy)
}

Isogeometric Analysis (IgA) was recently introduced by T.J.R. Hughes and his research team in order to reduce the gap between Computer-Aided Design (CAD) and Finite Element Analysis (FEA). The main idea in IgA is to use the same basis functions provided by CAD systems - usually, B-splines or NURBS - both in the approximation of differential problems and in the description of the geometry of the related physical domains.

After its birth, IgA has gained a lot of attention because of its remarkable spectral approximation properties, which make it superior to FEA. Considering for simplicity the 1D Laplacian eigenvalue problem

$$
\left\{\begin{array}{l}
-u_{\ell}^{\prime \prime}=\lambda_{\ell} u_{\ell}, \quad \text { in }(0,1) \\
u_{\ell}(0)=u_{\ell}(1)=0
\end{array}\right.
$$

whose solutions are the pairs $\left(\lambda_{\ell}, u_{\ell}\right)$ with $\lambda_{\ell}=\omega_{\ell}^{2}=(\ell \pi)^{2}$ and $u_{\ell}(x)=\sin (\ell \pi x), \ell=1,2, \ldots$, what has been observed in the literature $[8,1,7,6]$ is the following.

- The spectrum $\left\{\omega_{\ell, \operatorname{IgA}}^{2}, \ell=1, \ldots, N\right\}$ of $p$-degree IgA discretization matrices consists of a unique branch, the so-called 'acoustical branch', which provides a good (and convergent) approximation of practically all the exact eigenfrequencies $\left\{\omega_{\ell}^{2}, \ell=1, \ldots, N\right\}$, except for few outliers.

- The spectrum $\left\{\omega_{\ell, \mathrm{FEA}}^{2}, \ell=1, \ldots, N\right\}$ of $p$-degree FEA discretization matrices consists of $p$ branches, one 'acoustical' and $p-1$ 'optical'; only the acoustical branch provides a good approximation of the first $N / p$ eigenfrequencies $\left\{\omega_{\ell}^{2}, \ell=1, \ldots, N / p\right\}$, whereas the other discrete eigenfrequencies $\omega_{\ell, \mathrm{FEA}}^{2}, \ell>N / p$, related to the optical branches, are spurious and diverge with $p$.

It is then clear that the approximation of the spectrum of the underalying continuous operator - in this case, the (negative) 1D Laplacian - is much better in the IgA case than in the FEA case. Similar observations also holds for differential problems different from (1); see, e.g., [8].

In this contribution, we analyze the above phenomena through the theory of Generalized Locally Toeplitz (GLT) sequences [9, 10] and the related notion of 'spectral symbol' [2] (see also [3, 4, 5]). The identification of the symbol for both IgA and FEA matrices allows us to give a compact description of the asymptotic spectrum of these matrices, and leads to a clean explanation of:

- the presence of a unique spectral branch, when the eigenvalue problem (1) is approximated by $\operatorname{IgA}$;

- the appearance of $p$ spectral branches when $p$-FEA is used instead of IgA.

More generally, the symbol-based analysis allows one to predict the existence of $p-k$ branches when $C^{k}$ continuous basis functions with any intermediate regularity $0 \leq k \leq p-1$, are used for the approximation of (1). The latter prediction is confirmed by numerical experiments, an can be extended to the $d$-dimensional setting through tensorization arguments $[2,4,5]$.

\section{References}

[1] J.A. Cottrell, A. Reali, Y. Bazilevs, T.J.R. Hughes. Isogeometric Analysis of structural vibrations. Comput. Methods Appl. Mech. Engrg. 195 (2006), 5257-5296.

[2] C. Garoni, T.J.R. Hughes, A. Reali, S. Serra-Capizzano, H. Speleers. Smoothness versus polynomial degree: why IgA outperforms FEA in the spectral approximation. In preparation.

[3] C. Garoni, C. Manni, F. Pelosi, S. Serra-Capizzano, H. Speleers. On the spectrum of stiffness matrices arising from Isogeometric Analysis. Numer. Math. 127 (2014), 751-799. 
[4] C. Garoni, C. Manni, S. Serra-Capizzano, D. Sesana, H. Speleers. Spectral analysis and spectral symbol of matrices in isogeometric Galerkin methods. In preparation.

[5] C. Garoni, S. Serra-Capizzano, D. Sesana. Spectral analysis and spectral symbol of $d$-variate $\mathbb{Q}_{p}$ Lagrangian FEM stiffness matrices. Tech. Report 2014-021, Dept. Information Technology, Uppsala University, Sweden.

[6] T.J.R. Hughes, J.A. Evans, A. Reali. Finite Element and NURBS approximations of eigenvalue, boundaryvalue, and initial-value problems. Comput. Methods Appl. Mech. Engrg. 272 (2014), 290-320.

[7] T.J.R. Hughes, A. Reali, G. Sangalli. Duality and unified analysis of discrete approximations in structural dynamics and wave propagation: comparison of $p$-method Finite Elements with $k$-method NURBS. Comput. Methods Appl. Mech. Engrg. 197 (2008), 4104-4124.

[8] A. Reali. An Isogeometric Analysis approach for the study of structural vibrations. Journal of Earthquake Engineering 10 (2006), 1-30.

[9] S. Serra-Capizzano. Generalized locally Toeplitz sequences: spectral analysis and applications to discretized partial differential equations. Linear Algebra Appl. 366 (2003), 371-402.

[10] S. Serra-Capizzano. The GLT class as a generalized Fourier analysis and applications. Linear Algebra Appl. 419 (2006), 180-233. 


\title{
How to compute efficiently the Markovian Joint Spectral Radius?
}

\author{
A. Cicone, N. Guglielmi, V. Y. Protasov \\ Università degli Studi dell'Aquila \\ Moscow State University
}

Given a finite set of matrices $\mathcal{F}=\left\{A_{i}\right\}_{i=1}^{N}$, with $A_{i} \in \mathbb{C}^{d \times d}$, the Joint Spectral Radius (JSR) of $\mathcal{F}$ is given by the generalization of the Gelfand's formula for the spectral radius of a matrix i.e. $\lim _{k \rightarrow \infty} \sup _{P \in \mathcal{P}_{k}(\mathcal{F})}\|P\|^{1 / k}$, $k \in \mathbb{N}$, where $\mathcal{P}_{k}(\mathcal{F})$ is the set of all possible products of length $k$ of matrices in $\mathcal{F}$. In recent works it has been proved that the JSR can be computed exactly, under suitable and general conditions, using polytope norms, see e.g. [1].

In some cases, however, not all the products are allowed, because the matrices in $\mathcal{F}$ are multiplied each other following some Markovian law. Recently Kozyakin [2] showed that it is still possibile to compute Joint Spectral Radius in the Markovian case as the classical JSR of a significantly higher dimensional set of matrices $\widehat{\mathcal{F}}=\left\{\widehat{A}_{i}\right\}_{i=1}^{N}$, with $\widehat{A}_{i} \in \mathbb{C}^{N d \times N d}$. This implies that the exact evaluation of the Markovian JSR can be achieved in general using a polytope norm in $\mathbb{C}^{N d}$, which is a challenge task if $N$ is large.

In this talk we address the question whether it is possible to reduce the computational complexity for the calculation of the Markovian JSR showing that it is possible to transform the problem into the evaluation of $N$ polytope multinorms in $\mathbb{C}^{d}$.

As an illustrative application we shall consider the zero-stability of variable stepsize 3-step BDF formulas.

$2000 M S C: 15 \mathrm{~A} 18,15 \mathrm{~A} 60,65 \mathrm{~F} 15,65 \mathrm{~F} 35$

\section{References}

[1] N. Guglielmi, F. Wirth, and M. Zennaro. Complex polytope extremality results for families of matrices. SIAM journal on matrix analysis and applications. 27.3 (2005), 721-743.

[2] V. Kozyakin. The Berger-Wang formula for the Markovian joint spectral radius. Linear Algebra and its Applications. 448 (2014), 315-328. 


\title{
On Krylov subspace methods for the time-fractional Schrödinger equation
}

\author{
Roberto Garrappa, Igor Moret, Marina Popolizio \\ Università degli Studi di Bari \\ Università degli Studi di Trieste \\ Università del Salento, Dipartimento di Matematica e Fisica "Ennio De Giorgi", Italy
}

\begin{abstract}
Among the various applications of the numerical evaluation of matrix functions we recently addressed the numerical solution of the time-fractional Schrödinger equation. This model is a fundamental topic in physics and it differs from the classic Schrödinger equation since the time derivative is replaced by a fractional one, according to the basic definition by Caputo [1]. Its numerical solution is far more difficult than the standard case and it is still an open problem. The approach we discuss grounds on the possibility to express its solution by means of the Mittag-Leffler (ML) function. Once a discretization is used for the spatial derivative, one faces with the problem to compute this ML function with matrix arguments, usually of large dimension. An additional difficulty is represented by the spectrum of the resulting operators since eigenvalues can belong to the imaginary axis. We analyze the standard Krylov projection method and the Shift-and-Invert approach to approximate this function; their convergence properties are discussed, together with related issues. Numerical tests are presented to confirm the strength of the approach under investigation.
\end{abstract}

\section{References}

[1] K. Diethelm. The analysis of fractional differential equations. Lecture Notes in Mathematics, Springer-Verlag (2010).

[2] R. Garrappa, I. Moret, M. Popolizio. Solving the time-fractional Schrödinger equation by Krylov projection methods. Journal of Computational Physics (2014), in press. 


\title{
Stability-Preserving Parametric Model Reduction by Matrix Interpolation using Invariance Properties of Krylov Subspaces
}

\author{
Andreas Barthlen, Patrick Lang \\ Fraunhofer ITWM
}

With this contribution we present an idea, how one can guarantee preservation of asymptotic stability for parametric model reduction by matrix interpolation for a linear time-invariant system $\Sigma(p)=\left(I_{n}, A(p), B(p), C(p)\right.$, based on a procedure proposed in [1]. Parametric model order reduction in general has become a popular research field over the past years, as using regular model reduction for every parameter choice is impractical, at the very least though inefficient. For that purpose methods based on matrix interpolation in particular, introduced e.g. in [2], have proven themselves to be quite useful. The basic idea is to choose a set of sampling points $p^{(i)}$ over the parameter space and to use regular model reduction methods to locally reduce the system for the chosen sampling points. The reduced parametric system can then be obtained by interpolating the matrices of the reduced systems.

Unfortunately, without modification such an interpolation will in general yield meaningless results, let alone an asymptotically stable system. A method to ensure stability was introduced in [1], where one computes matrices $P_{i}$ such that the original system $\Sigma_{i}=\Sigma\left(p^{(i)}\right)$ satisfies

$$
P E>0, \quad P A+(P A)^{T}<0
$$

which is a sufficient criteria for asymptotic stability. This criteria is preserved under one-sided model reduction and interpolation with nonnegative weights and has been used e.g. in [3], where it is applied to the local reduced systems. We show, that one can use the invariance of the input Krylov subspace under a nonsingular transformation $\mathrm{P}$ to directly compute reduced systems $\Sigma_{i}=\left(E_{r, i}, A_{r, i}, B_{r, i}, C_{r, i}\right)$ that satisfy the asymptotic stability criteria

$$
E_{r, i}>0, \quad A_{r, i}+A_{r, i}^{T}<0
$$

without explicitly computing the matrices $P_{i}$. This is not only less computationally expensive than explicitly computing the transformation matrices $P_{i}$ and subsequently transforming the original system, but additionally gives us more freedom regarding the local reduced systems.

\section{References}

[1] R. Eid, R. Castañé-Selga, H. Panzer, T. Wolf and B. Lohmann. Stability-preserving parametric model reduction by matrix interpolation. Mathematical and Computer Modelling of Dynamical Systems Vol. 17 No. 4 (2011), 319-335.

[2] H. Panzer, J. Mohring, R. Eid, B. Lohmann. Parametric Model Order Reduction by Matrix Interpolation. Automatisierungstechnik Vol. 58 No. 8 (2010), 475-484.

[3] M. Geuß, H. Panzer, T. Wolf, B. Lohmann. Stability Preservation for Parametric Model Order Reduction by Matrix Interpolation. Proceedings of the European Control Conference, Strasbourg, France (2014), 1098-1103. 


\title{
Comparison of polynomial Krylov methods with limited memory consumption for approximating Stieltjes matrix functions
}

\author{
Stefan Güttel* ${ }^{*}$ Marcel Schweitzer ${ }^{\dagger}$ \\ *The University of Manchester \\ ${ }^{\dagger}$ Bergische Universität Wuppertal
}

Approximating $f(A) \mathbf{b}$, the action of the matrix function $f(A)$ on a vector $\mathbf{b}$, is an important task in many areas of scientific computing. Especially when $A$ is large and sparse, iterative methods which extract their approximations from a polynomial Krylov subspace $\mathcal{K}_{m}(A, \mathbf{b})$ are the methods of choice in many applications. One of the drawbacks of these methods is that the amount of reuired memory grows with the number of iterations which are performed, as a full basis of $\mathcal{K}_{m}(A, \mathbf{b})$ has to be stored. This often limits the feasible number of iterations, in particular in large scale computations. In recent years, different approaches have been employed for circumventing this problem, namely restarting approaches (which allow to only ever store a small, fixed number of vectors at a time) and rational Krylov methods (which typically need far less iterations to find an approximation of a certain accuracy than polynomial methods, but require solving a linear system in each iteration).

In some applications, solving linear systems with $A$ (or shifted versions of it) by direct methods is not feasible, or $A$ is only implicitly available via a routine, which, given a vector v, returns the matrix vector product $A \mathbf{v}$. In these cases, the linear system solves in each iteration of a rational Krylov method can only be performed approximately by another iterative method. In case that $A$ is Hermitian positive definite, a straight-forward choice is the conjugate gradient method which only ever requires storing a fixed number of vectors throughout all iterations. When the number of outer (rational) Krylov iterations needed for reaching the desired accuracy is small, such inner-outer Krylov methods can also be interpreted as Krylov methods with limited memory consumption, independent of the number of inner iterations.

Given these possible different approaches for approximating $f(A) \mathbf{b}$ in presence of limited available storage, the natural question that arises is the following:

Given a limited amount of memory (storage of at most $m$ vectors of length $N$ ), what is the most efficent method to approximate $f(A) \mathbf{b}$ ?

While "most efficient" can of course have several meanings, we will focus on finding the method which requires the minimal number of matrix-vector products. For the case that $f$ is a Stieltjes function, one can estimate the asymptotic convergence factors of the different methods. These can then be used to give guidelines for judging how well-suited which method is in a given situation. In addition, we compare these guidelines to the performance observed in actual numerical computations for the different methods. 


\title{
Orthogonal projection vs. Oblique projection in Krylov subspace recycling
}

\author{
Matthias Bolten, Nemanja Božović, Andreas Frommer \\ Bergische Universität Wuppertal
}

Many problems in engineering, numerical simulations in physics etc. require the solution of long sequences of slowly changing linear systems. The paper [1] proposes an algorithm that reduces the cost of solving subsequent systems by recycling selected subspaces generated for the previous systems. The subspace which is to be recycled is chosen in a way that the eigenvalues of smallest magnitude are approximately deflated. This can substantially improve the convergence of the method. Their algorithm, called GCRO-DR, uses an orthogonal projection for deflation. What we propose is an algorithm that uses an oblique projection in the spirit of [2] for deflating the eigenvalues of smallest magnitude. This oblique projection requires approximations to right and left eigenvectors, and we propose a way of getting the approximations to the left eigenvectors, without having to build a Krylov subspace with respect to $A^{H}$, which saves work. We will also show some numerical comparisons.

\section{References}

[1] Michael L. Parks, Eric de Sturler, Greg Mackey, Duane D. Johnson and Spandan Maiti, Recycling Krylov subspaces for sequences of linear systems, SIAM Journal on Scientific Computing, 28 (2006), 1651-1674.

[2] Martin H. Gutknecht, Spectral deflation in Krylov solvers: A theory of coordinate space based methods, ETNA Journal, 39 (2012), 156-185. 


\title{
A new approach for preconditioning discontinuous Galerkin discretizations
}

\author{
Martin J. Gander, Soheil Hajian \\ University of Geneva
}

Domain decomposition preconditioners and in particular the additive Schwarz method are favorite preconditioners for classical finite element methods (FEM). There is a huge effort in designing similar preconditioners for discontinuous Galerkin (DG) discretizations. It has been shown that additive Schwarz methods use different mechanism for convergence when applied to a DG discretization compared to the classical FEM. More precisely, additive Schwarz methods, when applied to DG, use a non-overlapping Robin transmission condition for the communication between subdomains. This is exactly the same transmission condition that optimized Schwarz methods (OSM) use to obtain fast convergence. In this talk we present an OSM preconditioner for a particular DG discretization along with theoretical convergence estimates.

\section{References}

[1] Martin J. Gander, Soheil Hajian. Analysis of Schwarz Algorithms for a Discontinuous Galerkin Method. arXiv: 1404.4518 [math.NA] 


\section{Rational least squares approximation via RKFIT \\ Mario Berljafa and Stefan Güttel The University of Manchester}

For given matrices $\{A, F\} \subset \mathbb{C}^{N \times N}$ and a vector $\mathbf{v} \in \mathbb{C}^{N}$, we consider the problem of finding a rational function $R_{m}^{*}$ of type $(m, m)$ such that

$$
\left\|F \mathbf{v}-R_{m}(A) \mathbf{v}\right\|_{2}^{2} \text { is small. }
$$

We propose an iterative algorithm called RKFIT for its solution. At each iteration RKFIT constructs a rational Krylov space and manipulates an associated Arnoldi decomposition to find better approximations to the poles of $R_{m}^{*}$. In the special case when $A$ and $F$ are diagonal matrices, we can compare RKFIT to the popular vector fitting algorithm by Gustavsen and Semlyen (1999).

RKFIT is part of a MATLAB Rational Krylov Toolbox available for download from

http://www.guettel.com/rktoolbox 


\title{
A fast nonstationary preconditioning strategy for ill-posed problems, with application to image deblurring
}

\author{
Marco Donatelli \\ Dipartimento di Scienza e Alta Tecnologia, Università dell'Insubria, 22100 Como, Italy.
}

We consider the iterative solution of ill-posed equations

$$
T x=y,
$$

where $T: \mathcal{X} \rightarrow \mathcal{Y}$ is a linear operator between two Hilbert spaces $\mathcal{X}$ and $\mathcal{Y}$ with unbounded (Moore-Penrose) generalized inverse. Hence, problem (1) has to be regularized for a numerical solution. As usually often in the applications, we assume that, instead of the exact data $y \in \mathcal{Y}$ of (1), we are only given approximate data $y^{\delta} \in \mathcal{Y}$ with $\left\|y^{\delta}-y\right\| \leq \delta$, where $\delta \geq 0$ is the corresponding noise level.

When linear equations with $T^{*} T+\alpha I$ or $T T^{*}+\alpha I$, with $\alpha>0$, are computationally expensive to solve, taking an appropriate approximation $C$ of $T$ the following algorithm has been proposed in [4]. Starting with an initial guess $x_{0}$ we compute, for $n=0,1,2, \ldots$,

$$
h_{n}=C^{*}\left(C C^{*}+\alpha_{n} I\right)^{-1} r_{n}, \quad r_{n}=y^{\delta}-T x_{n}
$$

and set

$$
x_{n+1}=x_{n}+h_{n} .
$$

Note that the linear equation $(2 \mathrm{a})$ is equivalent to minimizing the Tikhonov functional

$$
\left\|C h_{n}-r_{n}\right\|^{2}+\alpha_{n}\left\|h_{n}\right\|^{2} \longrightarrow \min .
$$

over $h_{n} \in \mathcal{X}$, where $C$ is the aforementioned approximation of $T$, and $\alpha_{n}$ is the associated regularization parameter.

In the literature on iterative solvers for (usually well-posed) problems of the form (1) the operator

$$
P=C^{*}\left(C C^{*}+\alpha_{n} I\right)^{-1}
$$

in (2a) would be called preconditioner. As we will select different regularization parameters $\alpha_{n}$ in each iteration, it is therefore appropriate to call the new scheme (2) a nonstationary preconditioned iteration. We provide a theoretical analysis of the new scheme, using regularization parameters that are chosen by a certain adaptive strategy. On the other hand, the parameter sequence $\left(\alpha_{n}\right)_{n}$ could be also simply defined by the geometric sequence

$$
\alpha_{n}=\alpha q^{n}, \quad n=0,1,2, \ldots,
$$

where $\alpha>0$ and $0<q \leq 1$.

For image deblurring problems the matrix $T$ has a structure depending on the boundary conditions and the approximation $C$ can be a discrete convolution operator that operates entirely in the Fourier domain. Hence the matrix vector product with the preconditioner $P$ in (4) can be computed by two fast Fourier transforms (FFTs). The numerical performance of this method turns out to be superior to state of the art iterative methods for least square problems, including the conjugate gradient iteration for the normal equation, with and without additional preconditioning.

We discuss also possible improvements of the iteration (2). For instance the nonnegative constraint can be easily enforced, preserving the convergence of the method, by simply projecting every iteration into the nonnegative cone. Furthermore, other weighted norms could be used to define $h_{n}$ in $(2 \mathrm{a})$ in order to preserve special features of the computed solution [1].

Regularization preconditioners can be used also inside recent thresholding iterative methods for image deblurring problems. We discuss the synthesis approach, but our proposal can be applied also to the analysis approach. Thanks to the well-known property that every image has a sparse representation in the wavelet domain, some recent deblurring models are based on a regularization term that promotes the sparsity of the wavelets coefficients

$$
\hat{x}=W x,
$$


where $W$ defines the wavelet transform. For instance the regularized problem can be formulated in terms of wavelets coefficients as

$$
\min _{\hat{x}}\left\{\mu\|\hat{x}\|_{1}+\|\hat{x}\|_{2}^{2}: T W^{T} \hat{x}=y^{\delta}\right\}
$$

where $\|\cdot\|_{p}$ denotes the $p$-norm, $p \geq 1$, and $\mu>0$ is a regularization parameter to be chosen. The solution of (6) can be computed by the linearized Bregman splitting that converges very slowly. Hence a preconditioning strategy is usually employed, obtaining the modified linearized Bregman iteration based on a regularizing preconditioner diagonalized by FFTs [3]. We show that this approach can provide low quality restored images when appropriate boundary conditions are imposed to reduce possible boundary artifacts. Accordingly we propose a new modified linearized Bregman iteration based on the preconditioner (4) that improves the quality of the restoration and save some computational cost at the same time [2]. Similarly to (2) our simple iteration is

$$
\left\{\begin{array}{l}
z_{n+1}=z_{n}+W C^{T}\left(C C^{T}+\alpha_{n} I\right)^{-1}\left(y^{\delta}-T W^{T} \hat{x}_{n}\right), \\
\hat{x}_{n+1}=S_{\mu}\left(z_{n+1}\right),
\end{array}\right.
$$

where $S_{\mu}$ is the soft-thresholding function defined component-wise as

$$
S_{\mu}(\xi)=\operatorname{sgn}(\xi)(|\xi|-\mu)_{+}
$$

Some numerical experiments show that our proposed preconditioners provide accurate and fast restorations when compared with the state of the art methods with sparsity constraints or total variation regularization.

\section{References}

[1] A. Buccini, M. Donatelli. Approximated projected generalized iterated Tikhonov. Manuscript 2015.

[2] Y. Cai, M. Donatelli, T. Z. Huang. Regularization preconditioners for frame-based image deblurring without boundary artifacts. Submitted 2014.

[3] J. F. Cai, S. Osher, Z. Shen. Linearized Bregman iterations for frame-based image deblurring. SIAM J. Imaging Sci. 2-1 (2009), 226-252.

[4] M. Donatelli, M. Hanke. Fast nonstationary preconditioned iterative methods for ill-posed problems, with application to image deblurring. Inverse Problems. 29 (2013), 095008. 


\title{
Iterated fractional Tikhonov regularization
}

\author{
Davide Bianchi, Alessandro Buccini, Marco Donatelli, Stefano Serra-Capizzano \\ Dipartimento di Scienza e Alta Tecnologia, Università dell'Insubria, 22100 Como, Italy
}

We consider linear operator equations of the form

$$
K x=y,
$$

where $K: \mathcal{X} \rightarrow \mathcal{Y}$ is a compact linear operator between Hilbert spaces $\mathcal{X}$ and $\mathcal{Y}$. We assume $y$ to be attainable, i.e., that problem (1) has a solution $x^{\dagger}=K^{\dagger} y$ of minimal norm. Here $K^{\dagger}$ denotes the (Moore-Penrose) generalized inverse operator of $K$, which is unbounded when $K$ is compact, with infinite dimensional range. Hence problem (1) is ill-posed and has to be regularized in order to compute a numerical solution. We want to approximate the solution $x^{\dagger}$ of the equation (1), when only an approximation $y^{\delta}$ of $y$ is available with

$$
\left\|y^{\delta}-y\right\| \leq \delta
$$

where $\delta$ is called the noise level. Since $K^{\dagger} y^{\delta}$ is not a good approximation of $x^{\dagger}$, we approximate $x^{\dagger}$ with $x_{\alpha}^{\delta}:=R_{\alpha} y^{\delta}$ where $\left\{R_{\alpha}\right\}$ is a family of continuous operators depending on a parameter $\alpha$. A classical example is the Tikhonov regularization defined by $R_{\alpha}=\left(K^{*} K+\alpha I\right)^{-1} K^{*}$, where $I$ denotes the identity and $K^{*}$ the adjoint of $K$.

Recently, new Tikhonov based regularization methods have been proposed in [1], [2] and [3], under the name of fractional Tikhonov, to reduce the oversmoothing property of the Tikhonov regularization in standard form, in order to preserve the details of the approximated solution. Their regularization and convergence properties have been previously investigated showing that they are of optimal order.

In this talk, we firstly provide saturation results similar to the well-known saturation result for Tikhonov regularization: let $R(K)$ be the range of $K$ and let $Q$ be the orthogonal projector onto $\overline{R(K)}$, if

$$
\sup \left\{\left\|x_{\alpha}^{\delta}-x^{\dagger}\right\|:\left\|Q\left(y-y^{\delta}\right)\right\| \leq \delta\right\}=o\left(\delta^{\frac{2}{3}}\right)
$$

then $x^{\dagger}=0$, as long as $\overline{R(K)}$ is not closed. Such results motivated us to introduce the iterated versions of fractional Tikhonov methods in the same spirit of the iterated Tikhonov method. We prove that those iterated methods can overcome the afore-mentioned saturation results.

Afterwards, inspired by the works $[4,5]$ we introduce the nonstationary variants of our iterated methods. Differently from the nonstationary iterated Tikhonov, we have two nonstationary sequences of parameters. In the noise free case, we give sufficient conditions on these sequences to guarantee the convergence providing also the corresponding convergence rates. In the noise case, we show the stability of the proposed iterative schemes proving that they are regularization methods. Finally, few selected examples confirm the previous theoretical analysis, showing that a proper choice of the nonstationary sequences of parameters can provide better restorations compared to the classical iterated Tikhonov.

\section{References}

[1] E. Klann, R. Ramlau. Regularization by fractional filter methods and data smoothing. Inverse Problems 24 (2) (2008), 025018.

[2] E. M. Hochstenbach, L. Reichel. Fractional Tikhonov regularization for linear discrete ill-posed problems. BIT, 51 (1) (2011), 197-215.

[3] D. Gert, E. Klann, R. Ramlau, L. Reichel. On fractional Tikhonov regularization. Private notes (2014).

[4] M. Hanke, C. W. Groetsch. Nonstationary iterated Tikhonov regularization. J. Optim. Theory Appl. 98 (1) (1998), 37-53.

[5] M. Brill, E. Schock. Iterative solution of ill-posed problems-a survey. Model optimization in exploration geophysics, ed. A. Vogel, Vieweg, Braunschweig (1987). 


\title{
Spectral behavior of preconditioned non-Hermitian multilevel block Toeplitz matrices with matrix-valued symbol
}

\author{
M. Donatelli, C. Garoni, M. Mazza, S. Serra-Capizzano, D. Sesana \\ University of Insubria, \\ Department of Science and High Technology, Via Valleggio 11, 22100 Como, Italy
}

\begin{abstract}
This contribution is devoted to preconditioning strategies for non-Hermitian multilevel block Toeplitz linear systems associated with a multivariate Lebesgue integrable matrix-valued symbol. In particular, we consider special preconditioned matrices, where the preconditioner has a band multilevel block Toeplitz structure, and we complement known results on the localization of the spectrum with global distribution results for the eigenvalues of the preconditioned matrices. In this respect, our main result is as follows. Let $I_{k}:=(-\pi, \pi)^{k}$, let $\mathcal{M}_{s}$ be the linear space of complex $s \times s$ matrices, and let $f, g: I_{k} \rightarrow \mathcal{M}_{s}$ be functions whose components $f_{i j}, g_{i j}: I_{k} \rightarrow \mathbb{C}, i, j=1, \ldots, s$, belong to $L^{\infty}$. Consider the matrices $T_{n}^{-1}(g) T_{n}(f)$, where $n:=\left(n_{1}, \ldots, n_{k}\right)$ varies in $\mathbb{N}^{k}$ and $T_{n}(f), T_{n}(g)$ are the multilevel block Toeplitz matrices of size $n_{1} \cdots n_{k} s$ generated by $f, g$. Then $\left\{T_{n}^{-1}(g) T_{n}(f)\right\}_{n \in \mathbb{N}^{k}} \sim_{\lambda} g^{-1} f$, i.e. the family of matrices $\left\{T_{n}^{-1}(g) T_{n}(f)\right\}_{n \in \mathbb{N}^{k}}$ has a global (asymptotic) spectral distribution described by the function $g^{-1} f$, provided $g$ possesses certain properties (which ensure in particular the invertibility of $T_{n}(g)$ for all $n$ ) and the following topological conditions are met: the essential range of $g^{-1} f$, defined as the union of the essential ranges of the eigenvalue functions $\lambda_{j}\left(g^{-1} f\right), j=1, \ldots, s$, does not disconnect the complex plane and has empty interior. This result generalizes the one obtained in [1], concerning the non-preconditioned case $g=1$.

Numerical experiments confirm the theoretical analysis and suggest the choice of optimal GMRES preconditioning techniques to be used for the considered linear systems. Moreover, the obtained results can be used for the spectral analysis of the Preconditioned Hermitian/Skew-Hermitian Splitting (PHSS) method applied to multilevel block Toeplitz linear systems with a Hermitian positive definite multilevel block Toeplitz matrix as a preconditioner [3].
\end{abstract}

\section{References}

[1] M. Donatelli, M. Neytcheva, S. Serra-Capizzano. Canonical eigenvalue distribution of multilevel block Toeplitz sequences with non-Hermitian symbols. Oper. Theory Adv. Appl. 221 (2012), 269-291.

[2] M. Donatelli, C. Garoni, M. Mazza, S. Serra-Capizzano, D. Sesana. Spectral behavior of preconditioned non-Hermitian multilevel block Toeplitz matrices with matrix-valued symbol. Appl. Math. Comput. 245 (2014), 158-173.

[3] M. Donatelli, C. Garoni, M. Mazza, S. Serra-Capizzano, D. Sesana. Preconditioned HSS method for large multilevel block Toeplitz linear systems via the notion of matrix-valued symbol. Numer. Linear Algebra Appl., submitted. 


\title{
Local Fourier Analysis of Pattern Structured Operators
}

\author{
M. Bolten, K. Kahl, $\underline{\text { H. Rittich }}$ \\ Applied Computer Science Group University of Wuppertal
}

Multigrid methods [5] are used to compute the solution $u$ of the system of equations

$$
L u=f
$$

where $L$ is typically a discretization of a partial different equations (PDE) and $f$ a corresponding, given right hand side. Local Fourier Analysis (LFA) [2, 5, 6] is well known to provide quantitative estimates for the speed of convergence of multigrid methods, by analyzing the involved operators in the frequency domain.

For the initial formulation of LFA [1] it was crucial to assume that all involved operators have constant coefficients. For many PDE operators the coefficients vary continuously in space. Thus if the grid is fine enough the discrete operator $L$ will only vary slightly between neighboring grid points and hence can be well approximated by an operator with locally constant coefficients. Thus constant coefficient are often reasonable assumption.

However, when analyzing more complex problems or even the multigrid method as a whole this assumption is too restrictive. Interpolation and restriction operators typically act differently on variables that have a coarse grid representative and those who do not have one. Another example are patter relaxation schemes like the Red-Black Gauß-Seidel method where red points of the grid are treated differently from the black ones.

It is possible to analyze these cases $[3,4]$ when allowing for interaction of certain frequencies (see also [5, 6]). Even more, it turns out that when we allow for more frequencies to interact we can analyze operators given by increasingly complex patterns. In our talk we will illustrate a general framework for analyzing pattern structured operators, i.e., operators whose action is invariant under certain shifts of the input function. Furthermore, we discuss different applications.

\section{References}

[1] A. Brandt. Multi-level adaptive solutions to boundary-value problems. Math. Comput., 31(138):333-390, 1977.

[2] A. Brandt. Rigorous quantitative analysis of multigrid, I: Constant coefficients two-level cycle with $L_{2}$-norm. SIAM J. Numer. Anal., 31(6):1695-1730, 1994.

[3] K. Stüben and U. Trottenberg. Multigrid methods: fundamental algorithms, model problem analysis and applications. In Multigrid methods (Cologne, 1981), volume 960 of Lecture Notes in Math., pages 1-176. Springer, Berlin-New York, 1982.

[4] C.-A. Thole and U. Trottenberg. Basic smoothing procedures for the multigrid treatment of elliptic 3D operators. Appl. Math. Comput., 19(1-4):333-345, 1986.

[5] U. Trottenberg, C. W. Oosterlee, and A. Schüller. Multigrid. Academic Press, 2001.

[6] R. Wienands and W. Joppich. Practical Fourier Analysis For Multigrid Methods. Chapman Hall/CRC Press, 2005. 


\title{
Fast Recovery and Approximation of Hidden Cauchy Structure
}

\author{
Jörg Liesen, Robert Luce \\ TU Berlin
}

A Cauchy matrix $C(s, t) \in \mathbb{C}^{m, n}$ is defined by $n+m$ data points $s \in \mathbb{C}^{m}, t \in \mathbb{C}^{n}$ satisfying $s_{i} \neq t_{j}$ for all $i, j$, the Cauchy points, via the relation

$$
C(s, t)=\left[c_{i j}\right]:=\left[\frac{1}{s_{i}-t_{j}}\right] .
$$

One can take advantage of the peculiar structure of Cauchy matrices, for example when computing matrix vector products ("the fast multipole method") or computing an LU factorization, resulting in algorithms that may be faster than general purpose algorithms by an order of magnitude. These algorithms require that the Cauchy points $s, t$ are explicitly given.

In this contribution we first study the problem of recovering the Cauchy points $s, t$, if only the entries of $C(s, t)$ are known. We give a simple $\mathcal{O}(m+n)$ algorithm for that task. Hence it can be checked in $\mathcal{O}(m n)$, whether any given matrix is a Cauchy matrix. The second problem we study in this work is approximating a given matrix $A \in \mathbb{C}^{m, n}$ with a Cauchy matrix. For this one would ideally like to solve the nonlinear problem

$$
\min _{s \in \mathbb{C}^{m}, t \in \mathbb{C}^{n}}\|C(s, t)-A\|_{F}
$$

We assume $a_{i j} \neq 0$ for all $i, j$, which is guaranteed when $A$ is a Cauchy matrix.

Instead of solving the presumably difficult problem (1), we solve the ordinary linear least squares problem

$$
\min _{s \in \mathbb{C}^{m}, t \in \mathbb{C}^{n}}\left\|D(s, t)-A^{[-1]}\right\|_{F}
$$

where $D(s, t):=\left[s_{i}-t_{j}\right]$, and the superscript $[-1]$ denotes the elementwise inverse of a matrix. We derive an $\mathcal{O}(m n)$ algorithm for the optimal solution of (2). Note that the complexity of our algorithm is linear in the input, and is thus of optimal complexity. Further we prove approximation bounds that relate the solutions of (1) and (2). We pay special attention to the problem of approximating Cauchy points $s, t$ from a given noisy Cauchy matrix, i.e. where $A=C(s, t)+N$, and the matrix $N$ represents a source of data uncertainty. 


\title{
Parallel Tensor Sampling
}

\author{
Lars Grasedyck, Christian Löbbert \\ Institut für Geometrie und Praktische Mathematik, RWTH Aachen
}

We consider the problem to reconstruct a tensor in the class of hierarchical low rank tensors from a small number of samples. The number of samples is proportional to the number of degrees of freedom in the respective tensor format [1], but there is in general no guarantee that the tensor can be reconstructed unless a certain restricted isometry property is assumed. Practical examples provide evidence that the reconstruction works in general pretty good, e.g. in the case of a parametric elliptic PDE where many parameters may enter into the diffusion coefficient, the right-hand side, the boundary conditions or the domain itself [2]. In such a case the method is competitive with, e.g. adaptive sparse grids or quasi Monte Carlo. Our aim is to parallelize the sampling process. Unlike for Monte Carlo methods, our sampling is sequential in nature and we modify it to allow several levels of parallelism: We can either apply parallelism for a small number of samples $(\sim 100)$ without any additional overhead, for a medium number of samples $(\sim 10000)$ with a mild overhead, or finally a complete parallelism for all samples $(\gg 10000)$ albeit with a considerable overhead [3]. The overhead does not influence the runtime behaviour but rather the total energy consumption. We thus have three gears for three levels of parallelism ranging from energy efficient (first gear) to high speed (third gear).

\section{References}

[1] J. Ballani, L. Grasedyck, M. Kluge. Black Box Approximation of Tensors in Hierarchical Tucker Format. Num. Lin. Alg. Appl. 438(2): 639-657 (2013).

[2] J. Ballani, L. Grasedyck: Hierarchical tensor approximation of output quantities of parameter-dependent PDEs. Preprint 385, IGPM, RWTH Aachen, http://www.igpm.rwth-aachen.de/preprint.

[3] L. Grasedyck, C. Löbbert: Parallel Tensor Sampling in the Hierarchical Tucker Format. In preparation. 


\title{
Multigrid methods for tensor structured problems
}

\author{
Matthias Bolten*, Karsten Kahl*, Sonja Sokolovic* \\ *Bergische Universität Wuppertal
}

We look at linear systems whose matrix is related to a tensor structure of the kind $A=\sum_{i} \bigotimes_{j} E_{i}^{j}$. These problems are found, e.g., in stochastic Markov chain models or in the solution of high-dimensional Poisson equations. Due to the tensor structure of the models, the dimension of the operator $A$ grows rapidly for larger models. So the tensor structure has to be exploited for solving these systems efficiently. Multigrid methods have proven to be well-suited for these kind of problems, e.g., the Poisson equation. To be able to also use multigrid methods for high-dimensional models we build a multigrid method which keeps the tensor structure intact to guarantee computational savings on all grids. We investigate the question what kind of smoothing and coarsening have to be chosen to guarantee a nice convergence in this case and present an approach to adapt the algebraic multigrid framework to this tensor setting using tensor truncation techniques. 


\title{
Hierarchical tensor approximation of parameter-dependent PDEs
}

\author{
$\underline{\text { Jonas Ballani }}$ \\ EPF Lausanne
}

Parametric PDEs appear in a large number of applications, as e.g. in uncertainty quantification or optimisation. Typically, the amount of data to approximate and represent the solution scales exponentially in the parameter dimension. Therefore, a crucial task is to develop special numerical techniques that rely on data-sparsity in order to cope even with high parameter dimensions. In this talk, we will discuss low-rank tensor techniques that allow to reduce the complexity to a linear dependence on the parameter dimension. In particular, our aim is to adaptively construct an approximation of the solution in the hierarchical tensor format from a relatively small set of data samples. Once this approximation from an offline computation is available, the evaluation of quantities of interest becomes a cheap online task. Moreover, the explicit tensor representation can be used to compute stochastic properties of the solution in a straightforward way. The potential of this approach is illustrated by numerical examples.

This is joint work with Lars Grasedyck (RWTH Aachen) and Daniel Kressner (EPF Lausanne). 


\title{
On the block and global methods for linear systems with multiple right hand sides
}

\author{
A. Frommer, S. Rashedi, g. Ebadi \\ Bergische Universität Wuppertal Germany, \\ Tabriz University, Iran
}

We present new block and global methods for solving large nonsymmetric linear systems of equations with multiple right-hand. Dealing with many right hand sides at the same time allows to use multiplications of a sparse matrix times with block-vectors which are particularly efficient on current processor architectures.

The block version of BiCGSTAB introduced in [1] may suffer from numerical instability when the number of right-hand sides is large and we improve numerical stability by using a QR-factorization of the descent and/or the residual matrix. We also compare these new implementations with the global BiCGSTAB method, presented in[1]. Moreover, as observed in [2], for problems arising from partial differential equations and having complex eigenvalues, the block BiCGSTAB and global BiCGSTAB methods have a tendancy to not work well. We therefore introduce a new version of block BiCGSTAB and global BiCGSTAB in the spirit of the BICGSTAB2 method known for single right hand sides.

Several numerical examples demonstrate that the different variants of the global methods can achieve a smoothed residual and may be more competitive than the block solvers.

\section{References}

[1] A. El Guennouni, K. Jbilou, H. Sadok, A block version of BiCGSTAB for linear systems with multiple right-hand sides, Electron. Trans. Numer. Anal. 16 (2003), 129-142.

[2] M.H. Gutknecht, Variants of BiCGStab for matrices with complex spectrum, IPS Research Report No.(1991), $91-14$.

[1] J. Zhang, H. Dai, Global CGS algorithm for linear systems with multiple right-hand sides, Numer. Math. J. Chin. Univ. 30 (2008) 390-399 (in Chinese). 


\section{S18: Numerical methods for differential equations}

For all fields of applications the mathematical models are primarily based on differential equations. Hence, their numerical solution plays a fundamental role in numerical mathematics. This section covers mainly the construction and the behavior of numerical methods for differential equations including those of ordinary as well as of partial differential type. 


\title{
Well-Balanced, Entropy Stable Discontinuous Galerkin Spectral Element Method for the Shallow Water Equations
}

\author{
Andrew R. Winters, Gregor J. Gassner \\ Mathematical Institute, University of Cologne
}

This contribution presents an arbitrary high-order accurate nodal discontinuous Galerkin spectral element type method for the one dimensional shallow water equations with a non-constant bottom topography

$$
\begin{gathered}
\frac{\partial h}{\partial t}+\frac{\partial(v h)}{\partial x}=0, \\
\frac{\partial(v h)}{\partial t}+\frac{\partial\left(v^{2} h+g h^{2} / 2\right)}{\partial x}=-g h \frac{\partial b}{\partial x} .
\end{gathered}
$$

Here, $h$ is the height of the water and $v$ is the velocity. The constant $g$ is the acceleration due to gravity and the function $b=b(x)$ represents the bottom topography of the surface over which the fluid flows. The method uses a skew-symmetric formulation of the continuous problem in combination with a special numerical flux at element interfaces to exactly preserve the entropy, which is also the total energy for the shallow water equations $[1]$.

A problem with entropy conservative formulations is they may suffer breakdown if used without dissipation to capture shocks. Physically, entropy must be dissipated at a shock. However, an isentropic algorithm does not allow the capture of this physical process, which results in the generation of large amplitude oscillations around the shock [2]. Another dire issue is that entropy conservative formulations can not converge to the weak solution as there is no mechanism to admit the dissipation physically required at the shock. We use entropy analysis techniques in the context of DG approximations to remedy any stability issues of entropy conservative schemes. We derive a minimal dissipation term to add at grid cell interfaces and stabilize the approximation. This dissipation term is the minimum required for stability, it does not and is not designed to eliminate overshoots near shocks.

Of particular importance for numerical approximations of the shallow water equations is the well-balanced property. The well-balanced property is an attribute that a numerical approximation can preserve a steady-state solution known colloquially as the "lake at rest" condition:

$$
h+b=\text { constant }, \quad v=0 .
$$

This steady state solution is important because relevant waves in a flow may be viewed as small perturbations of the "lake at rest," see [3]. A good numerical method for the shallow water equations should accurately capture both the steady states and their small perturbations (quasi-steady flows). Such a property diminishes the appearance of unphysical waves with magnitude proportional to the grid size (a so-called "numerical storm"), which are normally present for numerical schemes that cannot preserve the steady-state.

Numerical tests are performed to demonstrate the theoretical findings, the high-order accuracy of the method, and the capability of the scheme to compute the solution of a rapidly varying flow over a smooth bed, and the perturbation of a stationary state.

\section{References}

[1] G.J. Gassner, A.R. Winters, D.A. Kopriva, A well balanced and entropy conservative discontinuous Galerkin spectral element method for the shallow water equations, Appl. Math. Comput. (under review).

[2] M. Carpenter, T. Fisher, E. Nielsen, S. Frankel, Entropy stable spectral collocation schemes for the Navier-Stokes equations: Discontinuous interfaces, SIAM J. Sci. Comp. 36 (5) (2014) B835-B867.

[3] E. Audusse, F. Bouchut, M.-O. Bristeau, R. Klein, B. Perthame, A fast and stable well-balanced scheme with hydrostatic reconstruction for shallow water flows, SIAM J. Sci. Comp. 25 (6) (2004) 2050-2065. 


\title{
An Enhanced Time-Discontinuous Galerkin Method for Rotating Geometries such as Artificial Blood Pumps
}

\author{
Stefan Haßler, Lutz Pauli, Marek Behr \\ Chair for Computational Analysis of Technical Systems (CATS) \\ CCES, RWTH Aachen University, 52056 Aachen, Germany
}

Heart failure is the main cause of death in developed countries and a heart transplant is often the only permanent medical treatment. However, the number of donor hearts is significantly lower than the number of patients in need of a transplant. Therefore, artificial blood pumps, such as Ventricular Assist Devices (VADs), are often the only alternative. State-of-the-art devices can support the failing heart for up to 5 years and can therefore function as a bridge to transplant or even as a bridge to recovery.

Computational Fluid Dynamics (CFD) has become an important development step for new VADs. It is possible to predict the hydraulic performance sufficiently with computational simulations. A change in the pump geometry can be applied easily within the simulations and its change in performance can be predicted. Therefore, CFD simulations can reduce the number of costly prototypes and experiments. The biocompatibility including hemolytic characteristics can also be estimated based on new modeling approaches [1].

Axial or centrifugal blood pumps always contain a rotating part that drives the blood flow. For timedependent pump simulations, one has to take the mesh deformation into account, which can be done with the shear-slip mesh update method (SSMUM) [2]. With this method, we insert an update layer in the pump geometry; this layer is one element thick and created in a structured way. The update layer connects the non-moving part of the mesh with the rotating part. The elements in this layer deform with the rotation. After a certain degree of rotation, the connectivity of the mesh in the update layer is changed. This method is implemented in our in-house flow solver XNS, which is based on the time-discontinuous Galerkin finite element method. XNS is also capable of massively parallel distributed-memory simulations, which makes it suitable for the complex geometries of VADs and the high Reynolds number flows that require fine meshes.

What remained unaccounted for in the previous version of the method is that a change in connectivity between two adjacent time slabs influences the jump term that results from the discontinuous Galerkin method. The crucial step towards improved accuracy of integration is the consideration of the spatial overlap of elements from the previous and the current time slab. We will discuss the methods we use to track the elements, to compute their spatial overlap, and to handle the communication. Blood pump simulations, which are calculated using these methods, will be shown. Our results will be compared with particle image velocimetry (PIV) experimental data.

\section{References}

[1] L. Pauli, J. Nam, M. Pasquali, M. Behr. Transient Stress-Based and Strain-Based Hemolysis Estimation in a Simplified Blood Pump. International Journal for Numerical Methods in Biomedical Engineering. 29 (2013), 1148-1160.

[2] M. Behr, D. Arora. Shear-Slip Mesh Update Method: Implementation and Applications. Computer Methods in Biomechanics and Biomedical Engineering. 6 (2003), 113-123. 


\title{
A posteriori error estimates for IMEX schemes
}

\author{
Jan Giesselmann, \\ Institute of Applied Analysis and Numerical Simulation, University of Stuttgart, Germany
}

In many engineering applications physical processes acting on a wide variety of time scales need to be accounted for. One way to avoid severe time step restrictions while also (hopefully) avoiding the solution of poorly conditioned nonlinear problems in each time step is to discretize the processes acting on small time scales implicitly while those acting on slow time scales are treated explicitly. This leads to the construction of IMEX, i.e., implicit-explicit, schemes. Examples include, but are not restricted to, atmospheric flows [4] and reaction-diffusion problems [5].

While the a posteriori error analysis of implicit Runge-Kutta and discontinuous Galerkin temporal discretisations is rather well-developed, see [1] and references therein, little is known for explicit and IMEX schemes. We will use an ordinary differential equation (ODE) setting (which can be understood as a semi-discretisation of a partial differential equation) to illustrate how reconstruction techniques can be employed to obtain rigorous a posteriori error estimators for certain IMEX schemes. In the past this strategy has been used successfully for implicit temporal discretisations [1], elliptic and parabolic partial differential equations [2], and hyperbolic conservation laws [3].

The fundamental idea consists in computing a reconstruction $\hat{u}$ of the numerical solution $u_{h}$ and splitting the error between $u_{h}$ and the exact solution $u$ into an error between $u$ and $\hat{u}$ and an error between $\hat{u}$ and $u_{h}$. In the example at hand the reconstruction $\hat{u}$ is explicitly computable from $u_{h}$ so that it is straightforward to account for the difference $\hat{u}-u_{h}$. The main reason why splitting the error as described above offers an advantage is that while $u_{h}$ might be defined at a finite number of time instances only, the reconstruction $\hat{u}$ is defined in such a way that it can be inserted into the ODE. Then the appropriate stability theory of the ODE can be used to bound the difference between the exact solution $u$ and the reconstruction $\hat{u}$ in terms of the residual occurring when $\hat{u}$ is inserted into the ODE. We will derive an explicit bound for the norm of the residual and show that the error estimator obtained in this way is of the same order as the error of the numerical method itself.

This is joint work with Tristan Pryer (Reading).

\section{References}

[1] G. Akrivis, Ch. Makridakis, R. Nochetto. Galerkin and Runge-Kutta methods: unified formulation, a posteriori error estimates and nodal superconvergence. Numer. Math. 118 (2011), 429-456.

[2] O. Lakkis, Ch. Makridakis. Elliptic reconstruction and a posteriori error estimates for fully discrete linear parabolic problems. Math. Comp. 75 (2006), 1627-1658.

[3] J. Giesselmann, Ch. Makridakis, T. Pryer. A posteriori analysis of discontinuous Galerkin schemes for systems of hyperbolic conservation laws. ArXiV Preprint 2014, http://arxiv.org/abs/1405.7616

[4] M. A. Kopera, F. X. Giraldo. Analysis of adaptive mesh refinement for IMEX discontinuous Galerkin solutions of the compressible Euler equations. J. Comput. Phys. 275 (2014), 92-117.

[5] T. Koto. IMEX Runge-Kutta schemes for reaction-diffusion equations J. Comput. Appl. Math. 215 (2008), $182-195$. 


\title{
Well balanced ALE: on time dependent mesh adaptation for Shallow Water flow
}

\author{
L. Arpaia M. Ricchiuto \\ INRIA Bordeaux, team CARDAMOM
}

\begin{abstract}
The interest in the numerical simulatation of water waves on moving grids lies in the accurate calculation of wave heights during the inundation stage and consequentely the correct prediction of the inundated regions. Static grids are inherently not efficient for this kind of phenomena where wave fronts crosses all the domain, steepen near the coastlines and spread into the land. In this paper, with the moving mesh refinement algorithm, developed among the others by [1], we redistribute clevery the points during the computation: it is possible to follow advancing fronts through gathering nodes in the region where the wave steepens, thus avoiding excessive numerical dissipation while remaining efficient in terms of CPU time. The sharp front is followed during all the inundation stage in order to compute accurately also the inundation region which is not known a priori and for which a static refinement results, in general, less robust, see [2]. An elegant formulation to deal with balance laws on moving grids consists in writing them respect to an arbitrary moving reference system, called Arbitrary Eulerian Lagrangian framework (ALE). Shallow Water equations are discretized by means of a Residual Distribution (RD) method in an ALE framework which follows the grid distortion. The resulting scheme is interpolation free. We show that, while RD schemes on fixed grids satisfy very naturally the WellBalanced property, with a naives extension of the same RD scheme in ALE form this property is lost. However a simple change of variable permits to recover it. With numerous benchmarks we assess the impact of the dynamic adaptation, and more in general the adaptive ALE algorithm, in terms of level of error/CPU time, proper resolution of hydaulic jumps and ability to handle complex flows where different kind of waves interact.
\end{abstract}

\section{References}

[1] H. Tang and T. Tang. Adaptive mesh methods for one and two-dimensional hyperbolic conservation laws. SIAM J. Numer. Anal. 41(2):487-515, 2003.

[2] F. Zhou, G. Chen, Y. Huang, J.Z. Yang, H.Feng. An adaptive moving finite volume scheme for modeling flood inundation over dry and complex topographhy. Water Resour. Res. 49:1914-1928, 2013. 


\title{
Computation of state variables of one-dimensional non-stationary pipe flow at orifices on pipe ends.
}

\author{
Herbert Niessner \\ NiMa, Baden-Rütihof, Switzerland
}

\begin{abstract}
Boundary conditions of orifices in conjunction with the graphical method of characteristics given in [1] are reformulated and made ready to be used with finite difference methods. Orifice flow to the apparent narrowest cross-section is assumed conserving entropy and energy, thereafter not recovering pressure without exceeding sound speed. These conditions allow to eliminate state variables at the narrowest cross-section. Additional equations stem from mass conservation and a balance of the Riemann variable over the outgoing cross-characteristic and either further energy conservation or a balance of entropy over the outgoing particle path. They permit to determine the state at the boundary of the computational grid for usual finite difference methods. A single nonlinear equation has to be solved only in case of outflow.
\end{abstract}

\section{References}

[1] E. Jenny. Berechnungen und Modellversuche über Druckwellen grosser Amplituden in Auspuff-Leitungen. Thesis ETH Zürich 1949 


\title{
An adaptive space-time discontinuous Galerkin method for Maxwell's equations
}

\author{
Christian Wieners, Stefan Findeisen, Willy Dörfler \\ Karlsruhe Institute of Technology
}

We consider a variational Petrov-Galerkin space-time discretization of liner wave equations (acoustic, elastic or electro-magnetic waves) using discontinuous Galerkin elements with upwind flux in space and continuous Galerkin elements in time. The discretization is adaptive with independent choice of polynomial degrees $\mathrm{p}$ in space and q in time for every space-time cell. The discretization is fully implicit, and the overall linear problem is solved with a parallel Krylov method using a multigrid preconditioner based on a subspace hierarchy with lower polynomial degrees. The adaptivity is controlled by a dual weighted residual error estimator with respect to a given linear error functional.

We present numerical examples for 2D Maxwell's equations demonstrating the efficiency of the error estimation, the adaptive strategy, and the parallel solution process. 


\title{
Tracking-type Finite-Volume Schemes for Phase Transition Problems
}

\author{
Maria Wiebe \\ University of Stuttgart, Institute for Applied Analysis and Numerical Simulation
}

\begin{abstract}
Systems of conservation laws can be used to model phase transitions of different kinds. One approach to solve these equations is using classical finite volume schemes. A problem encountered when using finite volume schemes for phase transition problems is the appearance of values for which the system is not hyperbolic any more due to integral averaging. Additionally, classical finite volume schemes do not resolve undercompressive phase transitions, which are non-Laxian shock waves.

This contribution shows the generalization of the scheme from [1] to the system case as well as to multiple space dimensions. The algorithm works with a sharp-interface approach, which means that the position of the phase transition is tracked with a time dependent mesh. Therefore, averaged values from two distinct phases are avoided and one can treat phase transitions separately. To solve the Riemann problem at the phase boundary, we use Riemann solvers, which allow undercompressive shock waves. Numerical examples calculated within this framework as well as properties of the scheme, such as wellposedness and conservation of the integral will be presented.
\end{abstract}

\section{References}

[1] Ch. Chalons, P. Engel, and Ch. Rohde. A conservative and convergent scheme for undercompressive shock waves. SIAM J. Numer. Anal. 52 (2014), no. 1, 554-579. 


\title{
Coupling Surface and Subsurface Flows
}

\author{
Jim Magiera \\ University of Stuttgart, Institute of Applied Analysis and Numerical Simulation
}

Coupled surface-subsurface flows appear in many environmental and industrial applications, such as spreading of a contaminant by rivers and aquifers, or transport processes in fuel cells. Therefore, appropriate models that integrate free fluid flow and porous medium flow, efficient numerical methods for such models, and analysis is needed.

In this contribution coupled surface-subsurface models, consisting of the Stokes equations or shallow-water-like equations in the free flow domain, and Darcy's law or Brinkman's equation in the porous medium, are presented. Both flow regimes are coupled at a sharp interface via physically motivated coupling conditions.

To solve the coupled model efficiently, splitting schemes are applied, which allow to use already available algorithms for each flow domain and reduce the overall computational complexity.

Numerical simulation results are presented. 


\title{
Multigrid preconditioning for time-periodic Navier-Stokes problems
}

\author{
Daniel Hupp ${ }^{1}$, Dominik Obrist ${ }^{2}$, Peter Arbenz ${ }^{1}$ \\ ${ }^{1}$ Computer Science Department, ETH Zürich \\ ${ }^{2}$ ARTORG Center, University of Bern
}

In science and engineering, many problems are driven by time-periodic forcing. In fluid dynamics, this occurs for example in turbines or in human blood flows. In the absence of turbulence, if a fluid is excited long enough, a time-periodic steady state establishes. In numerical models of such flows, the steady state is computed by simulating this transient phase with a time-stepping method until the periodic state is reached. In this work, we present an alternative approach by solving directly for the steady state solution, introducing periodic boundary conditions in time. This allows us to use a multi-harmonic ansatz in time for the solution of the Navier-Stokes equation. problem formulation.

To obtain the steady state solution, a big system of nonlinear equations has to be solved, which we do by Picard iteration. We compute the Picard correction by solving a linear system with GMRES. This linear system can be written as

$$
\left(\begin{array}{cc}
F & G \\
D & 0
\end{array}\right)\left[\begin{array}{l}
\delta \mathbf{u} \\
\delta p
\end{array}\right]=\left[\begin{array}{l}
\mathbf{r}_{\mathbf{u}} \\
r_{p}
\end{array}\right]
$$

Here, $D, G$, and $F$ denote the divergence, the gradient operator, and the convection-diffusion operator, respectively. $\delta \mathbf{u}$, and $\delta p$ are the velocity and pressure Picard correction containing all Fourier modes. $\mathbf{r}_{\mathbf{u}}$ and $r_{p}$ are the residual vectors of the nonlinear problem. Note, that the different Fourier modes are only coupled in $F$, due to the nonlinearity of the convective term of the Navier-Stokes equation.

In previous work [1], a generic preconditioner was used for solving (1). In the present work, we present a new problem-specific preconditioner: a block triangular preconditioner

$$
\left(\begin{array}{cc}
M_{F} & G \\
0 & M_{S}
\end{array}\right),
$$

where $M_{S}$ is a least squares commutator [2]. Solving with $M_{S}$ amounts solving two Laplace problems of the form $D G$. Because the Fourier modes are not coupled in the Laplace problem, the modes can be solved in parallel.

$M_{F}$ is the block diagonal of $F$, such that the Fourier modes are not coupled in $M_{F}$ either. Thus, we have to solve a convection-diffusion problem for every Fourier mode, which can also be done concurrently. Our implementation employs the discretization and the parallelization capabilities of the well established IMPACT code [3].

We solve the convection-diffusion problems with GMRES preconditioned by a geometric multigrid using standard prolongation and restriction. Gauss-Seidel or damped Jaccobi are used as smoother. The performance of the Gauss-Seidel smoother is enhanced for matrices that are close to lower triangular. This occurs, when an upwinding discretization for the convective terms is used, and the unkowns are ordered according to the convection direction. We observe sensitivity of the smoothing quality of Gauss-Seidel with regard to the numbering of unkowns depending on the convection direction.

Finally, we measure the complexity and convergence rate of our multigrid implementation, and investigate the performance of the preconditioner (2) by solving various time-periodic flow problems.

\section{References}

[1] P. Arbenz, D. Hupp, D. Obrist A parallel solver for the time-periodic Navier-Stokes equations. Parallel Process. Appl. Math. (2013), 291-300.

[2] H. Elman, V. E. Howle, J. Shadid, R. Shuttleworth, R. Tuminaro. Block preconditioners based on approximate commutators. SIAM J. Sci. Comput. 27 (2006), 1651-1668

[3] R. Henniger, D. Obrist, L. Kleiser. High-order accurate solution of the incompressible Navier-Stokes equations on massively parallel computers. J. Comput. Phys. 229 (2010), 3543-3572. 


\title{
Online Parameter Identification for Traffic Simulation via Lagrangian Sensing
}

\author{
Elvira Thonhofer, Stefan Jakubek \\ Institute of Mechanics and Mechatronics, Vienna University of Technology, \\ Getreidemarkt 9, A-1060 Vienna
}

Macroscopic traffic simulations are based on the transport equation with a flux term governed by the speed of the flow, treating traffic as a compressible fluid. An auto-regressive model is used to solve the transport equation. We present a method to estimate model parameters based on Lagrangian measurement data that can be used for online traffic simulation. Identifiability is investigated using the Fisher Information Matrix.

In order to identify model parameters online measurement data must be collected. Two approaches are described by several authors. Commonly, Eulerian traffic data are collected by stationary loop detectors [1]. While this approach works well on highways with long sections between on- and offramps it is not ideal in urban traffic networks. The number of required stationary detectors causes relatively high installation and maintenance costs. Hence, the utilization of Lagrangian data, where the sensor moves with the flow, has recently become an area of intensive research [2]. Vehicles equipped with sensors record and transmit so-called floating car data (speed, headway, position) that can be utilized both online and offline.

The relation between driving speed and local traffic density is described by a Fundamental Diagram (FD) [3]. For simplicity the FD is generally assumed to be a convex, (linear) function with constant parameters both spatially and temporally. However, in reality these parameters may vary with environmental conditions such as weather (fog, snow, rain) and particular traffic situations (accidents, blocked lanes). For high-resolution urban traffic simulations it is therefore necessary to take the corresponding parameter changes into account.

We propose a generic FD based on a piecewise linear function, [4] that is fully defined by six parameters, which are estimated based on Lagrangian measurement data. An individual vehicle in traffic is described by an auto-regressive model where the driving speed during a time step is governed by the measured headway and the parameters of the FD. An optimization problem is formulated, where the parameters of the FD represent the decision variables, the optimization goal is to minimize the prediction error of the model. The prediction error is defined as the (mean squared) difference between the one-step-ahead-prediction and the true (measured) position of a vehicle.

Additionally, identifiability of the parameters is evaluated. The quality of the estimated parameters depends on the quality of the underlying measurement data. We use the concept of parameter sensitivity to investigate identifiability. The Fischer Information Matrix (FIM) is computed for the current set of parameters and the measurement data at hand. The singular value decomposition of the FIM provides sufficient information on identifiability. Ill conditioned singular values indicate that some parameters can not be estimated well from the data set at hand, hence, the data quality is insufficient. However, parameters that can be identified from a certain measurement data set may still be used. Utilizing both the estimated parameters found based on Lagrangian and Eulerian sensing allows for high accuracy while at the same time keeping sensor-costs low. In both cases the FIM can be utilized do determine which parameters are estimated reliably. Note here that blending algorithms must take spatial and temporal relevance into account as well.

\section{References}

[1] L. Muñoz, et al. Traffic density estimation with the cell transmission model. IEEE American Control Conference, Vol. 5. (2003).

[2] Y. Yuan, J.W.C. Van Lint, R.E. Wilson, F. van Wageningen-Kessels, S.P. Hoogendoorn. Real-time Lagrangian traffic state estimator for freeways. IEEE Transactions on Intelligent Transportation Systems (2012), 13(1), 59-70.

[3] D.C. Gazis. Traffic Theory. International Series in Operations Research \& Management Science, Springer (2002).

[4] E. Thonhofer, E.Luchini, A. Kuhn, S. Jakubek. Online Parameter Estimation for a Flexible, Adaptive Traffic Network Simulation. International Conference on Connected Vehicles, Vienna. (2014). 


\title{
Robust Identification of Parametric Radiation Force Models via Impulse Response Fitting
}

\author{
Hannes Hatecke, Stefan Krüger \\ Hamburg University of Technology, Institute of Ship Design and Ship Safety
}

\begin{abstract}
Hydrodynamic time-domain simulations typically use frequency-domain data in order to model radiation forces. The transformation into time-domain is based on the impulse response function method also known as Cummins Equation [1]. Due to the convolution terms in this method, the equations of motion are integrodifferential equations, which are expensive to compute and unsuitable for general control purposes. In order to avoid the convolution terms, Cummins Equation can be substituted by a system of first order ordinary differential equations, a so-called state-space model. Several identification methods have been developed to obtain the state-space parameters that represent the same dynamics as the Cummins Equation [2, 3, 4]. Nevertheless, their entire application is limited to a few academic cases of low order state-space systems which is mostly due to the lacking robustness of the identification methods.

This article describes a new, straightforward and robust method to obtain state-space representations for radiation forces. In short, our method uses a prony analysis to fit the impulse response function to a state-space system of diagonal form. Particularly with regard to the robustness, this method has been found to be superior to all existing frequency-domain data identification methods.
\end{abstract}

\section{References}

[1] W. Cummins. The impulse response function and ship motions. Schiffstechnik 9 (1962), 101-109.

[2] M. Schmiechen. On state space models and their application to hydrodynamic systems. NAUT Report, Department of Naval Architecture, University of Tokyo (1973).

[3] Z. Yu, J. Falnes. State-space-modelling of a vertical cylinder in heave. Applied Ocean Research 17 (1995), $265-275$.

[4] R. Taghipour, T. Perez, T. Moan. Hybrid frequency-time domain models for dynamic response analysis of marine structures. Ocean Engineering 35 (2008), 685-705. 


\title{
Numerical simulation for wine fermentation based on IDEs
}

\author{
Volker Schulz, Christina Schenk \\ Trier University, Germany
}

In the literature, many models, i.e. kinetic models based on ordinary differential equations and metabolic models, already exist to describe the process of wine fermentation. However, the dynamics due to yeast cell growth plays an important role in this fermentation process. This is why we take a closer look at the mass structure of yeast cells by introducing a nonlinear integro-partial differential equation for the population balance of yeast and integro-ordinary differential equations for the other substrates such as sugar, nitrogen, oxygen and the product, i.e. ethanol.

The derived model is solved numerically using a finite volume scheme for the discretization of the mass domain and BDF methods for the resulting system of time dependent ordinary differential equations.

In this context different flux approximation schemes and boundary conditions are studied. 


\title{
Improving real-time capability of linearly-implicit solvers using structural information
}

\author{
Thomas Loderer $^{\mathrm{a}}$, Christian Bertsch ${ }^{\mathrm{a}}$, Vincent Heuveline ${ }^{\mathrm{b}}$

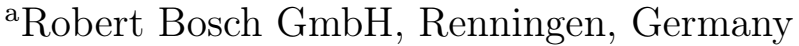 \\ ${ }^{\mathrm{b}}$ Engineering Mathematics and Computing Lab (EMCL), University Heidelberg, Germany
}

There is a growing demand for realtime simulation in automotive industry [1]. A significant challenge in this context is an efficient and stable solution of stiff ordinary differential equations [2]. The target of this work is to improve the real-time capability for a given benchmark model, i. e., to increase efficiency while keeping the stability and accuracy of the simulation within an appropriate limit. As linearly implicit methods provide adequate results for the benchmark problem, the approach of this work is to reduce the computational effort of the simulation by savings for the solution of the arising system of linear equations.

For this purpose, direct methods using structural information such as Schur complement are studied [3]. Beforehand the structure of the model equations is analysed and the findings are used for a preprocessing of the system. The direct solution by Schur complement is deployed to further profit by the knowledge about the model's structure. Furthermore the Schur complement is utilized to enable parallelization of the solution of the system of linear equations. The combination of structural preprocessing and direct solution by Schur complement delivers significant savings in computational cost. The results are verfied by runtime measurements of implementations on a realtime simulation system.

\section{References}

[1] K. Popovici and P. J. Mosterman, Real-Time Simulation Technologies: Principles, Methodologies, and Applications, Computational analysis, synthesis, and design of dynamic models series, Taylor \& Francis Group, 2012.

[2] M. Arnold, B. Burgermeister, C. Führer, G. Hippmann, and G. Rill, Numerical methods in vehicle system dynamics: State of the art and current developments, Vehicle System Dynamics, 49 (2011), pp. 1159-1207.

[3] Y. Saad and M. H. Schultz, Gmres: A generalized minimal residual algorithm for solving nonsymmetric linear systems, SIAM Journal on scientific and statistical computing, 7 (1986), pp. 856-869. 


\title{
Interpolatory Model Reduction for Quadratic Bilinear Descriptor Systems
}

\author{
Mian Ilyas Ahmad, Lihong Feng, Peter Benner \\ Max Planck Institute of Complex Technical Systems, Magdeburg, Germany.
}

\begin{abstract}
A large class of nonlinear systems can be written as quadratic bilinear systems by using exact transformations [1], in addition to systems that naturally appear in the quadratic bilinear form. To simulate or control such nonlinear systems, we need to utilize model reduction techniques that construct a reduced system with a response comparable to the original system. We propose interpolation based projection techniques for model reduction of quadratic bilinear descriptor systems. The approach identifies projection matrices from the bilinear approximation of the original quadratic bilinear descriptor system and applies Petrov-Galerkin projection to the original system to construct the required reduced quadratic bilinear descriptor system. Two different techniques of computing the projection matrices from the bilinear approximation are proposed. One is based on linear parametric model reduction [2,3] and the other is the bilinear rational Krylov method [4]. These methods can be used for model reduction of bilinear systems, however here we are considering their use for model reduction of descriptor systems with quadratic bilinear nonlinearities. The results are compared with the standard quadratic bilinear projection method [1] and it is observed that the approximations through the proposed methods are comparable to the standard method. An advantage of the proposed method is that the computations associated with the quadratic term in the construction of the projection matrices can be saved.
\end{abstract}

\section{References}

[1] C. Gu. QLMOR: a projection-based nonlinear model order reduction approach using quadratic-linear representation of nonlinear systems. IEEE Transactions on Computer-Aided Design of Integrated Circuits and Systems. 30-9 (2011), 1307-1320.

[2] P. Benner and L. Feng. A robust algorithm for parametric model order reduction based on implicit moment matching. In: A. Quarteroni, G. Rozza (ed.) Springer MS\&A series, Vol. 8: Reduced Order Methods for modeling and computational reduction, Springer International Publishing Switzerland, (2014), 159-185.

[3] P. Benner and T. Breiten. On $\mathcal{H}_{2}$-model reduction of linear parameter-varying systems. Proceedings in Applied Mathematics and Mechanics. 11-1 (2011), 805-806.

[4] T. Breiten and T. Damm. Krylov subspace methods for model order reduction of bilinear control systems. SCL. 59-10 (2010), 443-450. 


\title{
A joint IMEX-MOR approach for Water Networks
}

\author{
Sara Grundel, Lennart Jansen \\ Max-Planck Institute for Dynamics of Complex Technical Systems, Magdeburg, Germany. \\ Heinrich-Heine-Universität, Düsseldorf, Germany
}

\section{Introduction}

The simulation of fluids within a large network of pipes poses several mathematical challenges. Typically after spatial discretization the resulting mathematical system is a nonlinear differential algebraic system. Standard techniques are often slow due to the stiffness of the equation. We will show a several step process on how to improve on the timing. A first and major step in order to achieve stable and fast simulators for these problems is what we call the decoupling step. In that step we are able to model the system as a discrete index 1 DAE. This step is only possible due to the chosen discretization we use. Next we use a combination of Model Order Reduction (MOR) methods in order to create a smaller scale index 1 Differential Algebraic Equation. And last but not least we use an implicit-explicit (IMEX) integration method to reduce the time-step for the stiff nonlinear differential equation. We will only present a simplified network here which includes pipes, reservoirs and so called demand nodes. This system will actually result in an ODE which simplifies the discussion.

\section{Modeling}

It is common to define a connected and directed simple graph $G=G(V, E)$ representing the pipe network. This allows a more compact representation of the model equations. The set $V$ are the nodes and $E$ are the edges and we will describe the different node and edge elements in the following. Reservoirs are water sources with unlimited capacity. Thus, we assume that they have a constant pressure $p=p_{s}$. Furthermore no balance equation holds at a reservoir, since an arbitrary amount of water may leave or enter the reservoir. In contrast to reservoirs, tanks have limited capacity. Never the less, pressure can in- or decrease even though the tank is full or empty respectively. We will not talk about tanks in more details here. A demand node has a given demand $q_{\mathrm{s}}: I \longrightarrow \mathbb{R}_{+}$. Thus, the difference between the amount of water flowing towards a node, and the amount of water flowing away from the node has to be $q_{\mathrm{s}}: \sum q_{\text {in }}^{i}-\sum q_{\text {out }}^{i}=q_{\mathrm{s}}(t)$ with $q_{\text {in }}^{i}$ and $q_{\text {out }}^{i}$ being the incoming and outgoing flow of edges connected to the demand node, respectively. It is possible that $q_{\mathrm{s}}(t)=0$ for all $t \in I$.

Now we will discuss the pipe model. The behavior of a pipe is described by a continuity equation and an equation describing the movement inside a pipe. We consider circular pipes with diameter $D$, cross-section $A=\frac{\pi}{4} D^{2}$ and length $\ell$. The independent variables are space $x \in[0, \ell]:=\Omega \subset \mathbb{R}$ and time $t \in\left[t_{0}, T\right]:=I \subset \mathbb{R}$. The time dependent variables are the mass flow $m: \Omega \times I \longrightarrow \mathbb{R}$ and the pressure $p: \Omega \times I \longrightarrow \mathbb{R}$. The parameters $a, \rho$ and $c$ depend on material properties of the pipe and the gas. $\alpha$ is the angle of the pipe and $g$ is the gravitational constant. With this, we get the following partial differential equation, which describes the behavior in pipes

$$
\begin{aligned}
\frac{\partial p}{\partial t}(x, t)+\frac{a^{2}}{A} \frac{\partial m}{\partial x}(x, t) & =0 \\
\frac{\partial m}{\partial t}(x, t)+A \frac{\partial p}{\partial x}(x, t)+\rho A g \sin \alpha+c|m(x, t)| m(x, t) & =0
\end{aligned}
$$

Further edge elements are valves and pumps, which we also omit in this abstract.

From now on we consider a network with $n_{p}$ many pipes and $n_{d}$ many demand nodes and $n_{r s}$ many reservoirs. For each pipe $i$, we get a flow $m_{i}: \Omega_{i} \times I \longrightarrow \mathbb{R}, \Omega_{i}=\left[x_{L_{i}}, x_{R_{i}}\right] \subset \mathbb{R}, I=\left[t_{0}, T\right]$ and a pressure $p_{i}: \Omega_{i} \times I \longrightarrow \mathbb{R}$ both depending on space $x \in \Omega_{i}$ and time $t \in I$. The node variables are $p_{d}: I \longrightarrow \mathbb{R}^{n_{d}}$ and $p_{r s}: I \longrightarrow \mathbb{R}^{n_{r s}}$, the pressures at demand nodes and reservoirs. We define

$m_{L}(t)=\left(m_{i}\left(x_{L_{i}}, t\right)\right)_{i \in \mathcal{A}_{p i}}, \quad m_{R}(t)=\left(m_{i}\left(x_{R_{i}}, t\right)\right)_{i \in \mathcal{A}_{p i}}, \quad p_{L}(t)=\left(p_{i}\left(x_{L_{i}}, t\right)\right)_{i \in \mathcal{A}_{p i}}, \quad p_{R}(t)=\left(p_{i}\left(x_{R_{i}}, t\right)\right)_{i \in \mathcal{A}_{p i}}$.

$m_{L}$ being the vector with all pipe flows at their tail-node and $m_{R}$ the flow vector at their head-nodes and similarly for $p_{L}$ and $p_{R}$. Note, that the node pressures coincide with the head- and tail pressures of the pipes. We call the vector of demand and reservoir pressures by $p$. 
Last we define the incidence matrices $A_{R} \in \mathbb{R}^{n_{p} \times n_{d}}$ and $A_{L} \in \mathbb{R}^{n_{p} \times n_{d}}$ for the demand nodes with $\left(A_{R}\right)_{i j}=\left\{\begin{array}{ll}1 & \text { if demand node } j \text { is head of pipe } i \\ 0 & \text { else }\end{array}\right.$ and $\left(A_{L}\right)_{i j}=\left\{\begin{aligned}-1 & \text { if demand node } j \text { is tail of flow } i \\ 0 & \text { else }\end{aligned}\right.$ and $A_{R}^{r s}$ and $A_{L}^{r s}$ analogously for the reservoirs. We can combine them all and get the full incidence matrix $A:=\left(\begin{array}{ll}A_{R}^{r s}+A_{L}^{r s} & A_{R}+A_{L}\end{array}\right)$. With the help of these matrices we can write the spatial discretized system as

$$
\begin{aligned}
\dot{p}_{R}+D_{\alpha}\left(m_{L}-m_{R}\right) & =0 \\
\dot{m}_{L}+D_{\beta} A^{T} p+\gamma+G\left(m_{L}\right) m_{L} & =0 \\
A_{R} m_{R}+A_{L} m_{L} & =q_{\text {set }} \\
A_{R} p_{d}=p_{R} \quad A_{L} p_{d}=p_{L} \quad A_{R}^{r s} p_{r s}=p_{R} \quad A_{L}^{r s} p_{r s}=p_{L} \quad p_{r s} & =p_{\text {set }}
\end{aligned}
$$

To obtain these equation it is crucial to chose a suitable spatial discretization. $D_{\alpha}$ is a diagonal matrix containing $\alpha_{i}=a_{i}^{2} / A_{i} / \ell_{i}$ on the diagonal and $D_{\beta}$ similar with $\beta_{i}=A_{i} / \ell_{i} . \gamma$ is a vector with $\gamma_{i}=\rho_{i} A_{i} g \sin \alpha_{i}$ and $G\left(m_{L}\right)$ is a diagonal matrix function such that $G\left(m_{L}\right)_{i}=c_{i} m_{L}^{i}$. The vector $q_{\text {set }}$ has an entry for every demand node showing the given demand at that particular node given by $q_{s}(t)$ and similar is $p_{\text {set }}$ the vector of the given pressures $p_{s}$ at the reservoirs.

By selecting a matrix $A_{\text {select }}$ which picks one pipe for each node that has a right end in that given node we can rewrite the system of equation in the variables $p_{d}$ and $m_{L}$.

$$
\dot{p}_{d}+A_{\text {select }} D_{\alpha}\left(-C q_{\text {set }}+C A m_{L}\right)=0, \quad \dot{m}_{L}+D_{\beta} A^{T}\left(\begin{array}{c}
p_{d} \\
p_{\text {set }}
\end{array}\right)+\gamma+G\left(m_{L}\right) m_{L}=0
$$

We will explain in detail how we create the matrix $C$ which is the crucial part in this decoupling process. This resulting ODE is of size $n_{d}+n_{p}$ and has the general structure

$$
\dot{x}=T x+g(x, t)=f(x, t) \quad \text { with } \quad T=\left(\begin{array}{cc}
0 & A_{\text {select }} D_{\text {alpha }} C A \\
D_{\beta}\left(A_{R}+A_{L}\right)^{T} & 0
\end{array}\right)
$$

and the vector $x$ is combined by $p_{d}$ and $m_{L}$.

\section{IMEX}

In order to solve this stiff and nonlinear ODE we make use of implicit-explicit (IMEX) integration methods. This allow us to deal with the stiffness in an efficient way while not having to solve large-scale nonlinear problems. First order methods are of the flavor

$$
\frac{x_{n+1}-x_{n}}{h}=(1-\gamma) T x_{n}+\gamma T x_{n+1}+g\left(x_{n}, t\right)
$$

for $\gamma \in[0,1]$ and time step $h$. We study convergence properties of that by analyzing the matrix $T$ and the function $g$ as well as the differences for several values of $\gamma$. If $\gamma=0$ we get explicit Euler and if $\gamma=1$ we get a combination of implicit Euler for the linear part an explicit Euler for the nonlinear part. We will also show the differences within this class of methods as well as the difference to second order methods following [1].

\section{Model Order Reduction}

On the resulting ODE (2) we use the Model Order Reduction techniques Proper Orthogonal Decomposition (POD) together with Discrete Empirical Interpolation (DEIM) by [2]. POD is a projection-based method where we find a projection matrix $W$ such that the solution of $(2) x \approx W \hat{x}$ for $\hat{x}$ in a lower dimensional space. The resulting low-dimensional ODE is then given by

$$
\dot{\hat{x}}=W^{T} T W \hat{x}+W^{T} g(W \hat{x}, t) .
$$

DEIM is then used to create a truly low-dimensional function approximating $W^{T} g(W \hat{x}, t)$.

The combination of POD-DEIM with the IMEX integration results in a significant speedup of simulation time.

\section{References}

[1] Ascher, Uri M and Ruuth, Steven J and Wetton, Brian TR. Implicit-explicit methods for time-dependent partial differential equations. SIAM Journal on Numerical Analysis. 32 (1995) 797- 823.

[2] Chaturantabut, Saifon and Sorensen, Danny C.Nonlinear model reduction via discrete empirical interpolation. SIAM JSIAM Journal on Scientific Computing. 32 (2010), 2737-2764. 


\title{
Upwind Based Parameter Uniform Convergence Analysis for Two Parametric Parabolic Convection Diffusion Problems by Moving Mesh Methods
}

\author{
Pratibhamoy Das and Volker Mehrmann \\ pratibha@math.tu-berlin.de and mehrmann@math.tu-berlin.de \\ Institut für Mathematik, Technische Universität Berlin, Germany
}

In this contribution, we consider the adaptive mesh generation of a two parametric parabolic convectiondiffusion-reaction partial differential differential equation in singular perturbation context. These problems exhibit boundary layers for which one needs to generate layer adapted meshes through adaptive procedure (see [1]). The adaptive moving mesh algorithm which starts with a fixed number of mesh points and moves it in a way which can automatically detect the accurate locations and widths of the layers, is used for our analysis.

The following singularly perturbed two parametric parabolic initial-boundary-value problem (IBVP) is considered on a domain $\Omega=\Omega_{x} \times(0, T]$ with $\Omega_{x}=(0,1)$,

$$
\begin{gathered}
\frac{\partial u}{\partial t}-\epsilon \frac{\partial^{2} u}{\partial x^{2}}-\mu b(x, t) \frac{\partial u}{\partial x}+c(x, t) u=f(x, t),(x, t) \in \Omega, \\
u(x, 0)=u_{0}(x), x \in \bar{\Omega}_{x}, u(0, t)=u(1, t)=0, t \in[0, T] .
\end{gathered}
$$

with sufficiently smooth $\beta_{1}>b(x, t)>\beta_{2}>0$ and $c(x, t) \geq 1$. Here, $\beta_{k}, k=1,2$ are constants. The parameters $0<\epsilon \ll 1$ and $0<\mu \ll 1$ are known as the singular perturbation parameters. The presence of these parameters ensure the existence of the boundary layers.

The present error analysis is based on the mesh equidistribution principle [2] which starts with a error monitor function and distributes the error in a way so that each subinterval have same error measurement. In this work, we consider a curvature based monitor function $\alpha^{n+1}+\left|\frac{\partial^{2} w\left(x, t_{n+1}\right)}{\partial x^{2}}\right|$ (defined at each time step $\left.t_{n+1}\right)$, which involves the singular component $w(x, t)$ of the solution $u(x, t)$ by decomposing the solution $u(x, t)=$ $v(x, t)+w(x, t)$. Here $\alpha^{n+1}$ is constant. The derivatives of $v(x, t)$ are bounded independent of perturbation parameters.

In this context, we want to note that the rigorous error analysis for time dependent problems are not available for moving mesh methods with singular perturbation framework. This work considered the discrete problem with upwind discretization for convection dominated term. A rigorous error analysis at each time interval is provided. The present analysis will generalize the adaptive method proposed in [3] (which was only for convection-diffusion problems) to reaction-diffusion as well. We have shown that the proposed method converges uniformly with respect to the perturbation parameters $\epsilon$ and $\mu$. It converges with first-order accuracy with respect to space and time. The numerical experiments validate the theoretical findings.

\section{References}

[3] P. Das and S. Natesan. Numerical solution of a system of singularly perturbed convection diffusion boundary value problems using mesh equidistribution technique. Aust. J. Math. Anal. Appl., 10(1) (2013). 1-17.

[1] P.A. Farrell, A.F. Hegarty, J.J.H. Miller, E. O’Riordan, G.I. Shishkin. Robust Computational Techniques for Boundary Layers. (2000). Chapman-Hall/CRC. New York.

[2] W. Huang and R. D. Russell. Adaptive Moving Mesh Methods. (2011). Springer. 


\title{
Adaptive FEM with goal-oriented error estimation and an approximation of the dual problem for inelastic problems
}

\author{
Kai-Uwe Widany, Rolf Mahnken \\ Chair of Engineering Mechanics, University of Paderborn, Germany
}

Goal-oriented error estimation allows to refine meshes in space and time with respect to arbitrary quantities.[1] This approcach is attractive because the quantity may be somethin which is actually important for the problem to be solved. The required dual problems that need to be solved usually require weak formulations and the Galerkin method in space and time to be established.[2] Unfortunately this is in conflict with structures of current finite element implementations for solid mechanics.[3] Therefore, we propose an approach to approximate the dual problem while maintaining these structures. Here, the dual problem is constructed based on the discretized primal problem. For the error estimation we apply a higher-order recovery procedure.[4] An application to elasto-plasticity is given. Numerical examples demonstrate the effectiveness of the procedure.

\section{References}

[1] R. Becker, R. Rannacher, A Feed-back approach to error control in finite element methods: basic analysis and examples, East-West J. Numer. Math 4 (1996), 237-64.

[2] M. Schmich, B. Vexler, Adaptivity with dynamic meshes for space-time finite element discretizations of parabolic equations, SIAM J. Sci. Comput. 30 (2008), 369-393.

[3] P. Wriggers, Nonlinear Finite Element Methods, Springer-Verlag (2008).

[4] K.-U. Widany, R. Mahnken, Adaptivity for parameter identification of incompressible hyperelastic materials using stabilized tetrahedral elements, Comput. Methods Appl. Mech. Engrg. 245-246 (2012) 117-131. 


\title{
An Adaptive Local Basis for Elliptic Problems with Complicated Discontinuous Coefficients
}

\author{
Stefan A. Sauter, Monika Weymuth \\ Universität Zürich
}

We will present a generalized finite element method for the discretization of elliptic partial differential equations in heterogenous media. In [1] a method has been introduced to set up an adaptive local finite element basis (AL basis) on a coarse mesh with mesh size $H$ which, typically, does not resolve the matrix of the media while the textbook finite element convergence rates are preserved. This method requires $O\left(\log (1 / H)^{d+1}\right)$ basis functions per mesh point where $d$ denotes the spatial dimension of the computational domain. Since the continuous differential operator is involved in the construction, the method presented in [1] is only semidiscrete. In our talk, we will present a fully discrete version of the method, where the AL basis is constructed by solving finite dimensional localized problems. A key tool for the discretization of the differential operator is the theory developed in $[2,3]$. We will prove that the optimal convergence rates are preserved and give some complexity estimates.

\section{References}

[1] L. Grasedyck, I. Greff, and S. Sauter. The AL Basis for the Solution of Elliptic Problems in Heterogeneous Media. SIAM J. Multiscale Model. Simul. 10 (2012), 245-258.

[2] D. Peterseim and S. Sauter. Finite elements for elliptic problems with highly varying, nonperiodic diffusion matrix. Multiscale Model. Simul. 10 (2012), 665-695.

[3] M. Weymuth. Fully Discrete Version of the AL Basis for Elliptic Problems with General $L^{\infty}$-Coefficient. Technical Report (2013), Institut für Mathematik, Universität Zürich, https://www.math.uzh.ch/compmath/index.php?id=reports. 


\title{
Error measurement and enhanced FEM for phase field modeling
}

\author{
Ingo Münch

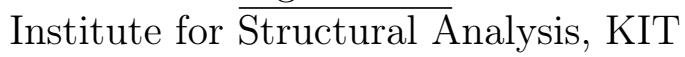

The analytical solution for the spatial evolution of a field variable governed by the Allen-Cahn equation

$$
\lambda \frac{\partial \varphi}{\partial t}=\nabla \cdot \mu-\eta
$$

serves as basis for a thorough investigation of finite element techniques on phase field modeling. A non-conserved phase field variable $\varphi \in \mathbb{R}$, free Landau energy $\psi_{w}(\varphi)$, and gradient energy $\psi_{g}(\nabla \varphi)$ constitutes the GinzburgLandau free energy density

$$
\psi=\psi_{w}(\varphi)+\psi_{g}(\nabla \varphi)
$$

Internal microforces $\eta$ and microstresses $\mu$ balance the process of phase evolution and transition [1], defined by

$$
\eta:=\frac{\partial \psi_{w}}{\partial \varphi}, \quad \mu:=\frac{\partial \psi_{g}}{\partial(\nabla \varphi)}
$$

We convert the Allen-Cahn equation to a discrete problem with help of Ritz-Galerkin's method

$$
G:=\int_{\mathcal{B}} \mu \cdot \nabla \delta \varphi+(\eta+\lambda \dot{\varphi}) \delta \varphi \mathrm{d} V-\int_{\partial \mathcal{B}} \mu \cdot n \delta \varphi \mathrm{d} A=0
$$

Neumann boundary conditions $\mu \cdot n=0$ apply on all surfaces $\partial \mathcal{B}$. Thus, a numerical method has to fulfill Dirichlet boundary conditions and the left integral of Eq. 3. Linearizing $G$ with respect to $\varphi$ prepares the Newton-Raphson algorithm for numerical solution. Incremental values of the phase parameter $\varphi$ are denoted with $\Delta \varphi$, thus

$$
\operatorname{Lin} G:=G^{\star}+\int_{\mathcal{B}}\left(\frac{\partial \mu}{\partial \nabla \varphi} \cdot \nabla \Delta \varphi\right) \cdot \nabla \delta \varphi+\frac{\partial \eta}{\partial \varphi} \Delta \varphi \delta \varphi+\lambda \Delta \dot{\varphi} \delta \varphi \mathrm{d} V=0
$$

Additionally, the backward Euler scheme is used for time integration. At time step, $t$ and iteration, $i$ within the Newton-Raphson method, it holds:

$$
\dot{\varphi}=\frac{\varphi_{t}^{i+1}-\varphi_{t}^{0}}{\Delta t}, \quad \varphi_{t}^{i+1}=\varphi_{t}^{i}+\Delta \varphi^{i}, \quad \Delta \varphi^{0}=0, \quad \Delta \dot{\varphi}=\frac{\Delta \varphi^{i}}{\Delta t}
$$

Time increments $\Delta t>0$ enter the numerical formulation as regularization parameter for free energy states above the minimum. In the numerical formulation it interacts with $\lambda$ via

$$
\lambda \Delta \dot{\varphi}=\frac{\lambda}{\Delta t} \Delta \varphi^{i}
$$

Falk [2] elaborated the analytical solution of a one dimensional phase transition ${ }^{1}$ reading

$$
\varphi(x)=\sqrt{\frac{\beta}{\alpha}} \frac{\varphi_{0} \sinh (\lambda x / \xi)}{\sqrt{A+\sinh ^{2}(\lambda x / \xi)}}
$$

An adaptive p-method is proposed, which is generally applicable for finite elements. The method preserves continuity of the shape functions and is particularly advantageous in the context of parallelized computing environment. Using internal wave function, the FEM shows a significant decrease of the error, $e$ defined with help of Eq. 7.

\section{References}

[1] M. E. Gurtin. Generalized Ginzburg-Landau and Cahn-Hilliard equations based on a microforce balance. Physica D 92 (1996), 178-192.

[2] F. Falk. Ginzburg-Landau Theory of Static Domain Walls in Shape-Memory Alloys. Z. Phys. B 51 (1983), $177-185$.

\footnotetext{
${ }^{1}$ Parameters $\alpha, \beta, \varphi_{0}, A, \lambda, \xi$ given byn own constitution in Eq. 1
} 


\title{
Interior penalty finite element methods for high-order local boundary conditions
}

\author{
$\underline{\text { Kersten Schmidt }}^{1}$, Julian Diaz ${ }^{2}$ and Christian Heier ${ }^{1}$ \\ ${ }^{1}$ Technische Universität Berlin, ${ }^{2}$ Université de Pau et des Pays de l'Adour
}

Local absorbing boundary conditions are used to mimick the solution in presence of an infinite exterior in diffusion problems or time-harmonic scattering problems, in highly conducting bodies or thin layers. We consider Dirichlet-to-Neumann boundary conditions involving higher tangential derivatives. (see an analysis in [1]). If only second derivatives are present, i.e., for the Neumann, Robin and Wenttzel conditions, and the boundary is smooth enough, we can incorporate the condition in usual piecewise continuous finite element methods. For higher derivatives trial and test functions with higher continuity (at least) along the boundary or auxilliary unknowns may be used. We propose as an alternative nonconforming interior penalty finite element methods for usual continuous finite element spaces in additional terms on the nodes of the boundary appear. For fourth order PDEs a similar approach has been introduced by Brenner and Sung [2]. We will present well-posedness results and a-priori $h$-convergence error estimates for uniform polynomial degrees. The theoretical convergence results are validated by a series of numerical experiments.

\section{References}

[1] K. Schmidt, C. Heier. An analysis of Feng's and other symmetric local absorbing boundary conditions. Accepted in ESAIM: Math. Model. Numer. Anal.

[2] S. Brenner, L.-Y. Sung. $C^{0}$ interior penalty methods for fourth order elliptic boundary value problems on polygonal domains. J. Sci. Comput. 22-23(2005), 84-118. 


\title{
Generalized approximated regular boundary element method
}

\author{
D.V. Yevdokymov \\ Dniepropetrovsk National University named after O.Honchar, Dniepropetrovsk, Ukraine
}

Last years, numerical modeling had become universal tool and extremely important part of modern sciences, technologies and other fields of human activity. Nevertheless a number of computers and their computational opportunity are sufficiently increased, different requirements to numerical algorithms remain very serious. For example, there are an effectiveness requirement, accuracy requirement, simplicity of coding and so on. To satisfy the mentioned requirements, new numerical algorithms are developed and existing algorithms are constantly improved. Such improvement of boundary element algorithms is an aim of the present work, which continues a series of publications by the author devoted to regular boundary element algorithms. The regular boundary element method (Kupradze's functional equation method) is similar to usual collocation technique with only difference: the collocation points are situated inside or outside the solution domain. Thus well-known singularities are excluded from the algorithm. The second case of collocation point situated inside the solution domain is considered here. In contrast to other cases, exact boundary integral equation cannot be obtained in this algorithm. However, the formulated approximated boundary integral equations are always second kind and they matrices of linear algebraic equation system with better properties than in other cases. Since the collocation point is situated inside the domain, but outside the boundary, the desirable solution in this point can be formally expanded into Taylor's series. The main idea of the present work is to increase an approximation order due to using more terms of the mentioned Taylor's series. Correspondent algorithms are developed and realized as computer codes for boundary-value problems for Laplace equation and initial-boundary-value problems for heat conduction equation. Calculations are made for special sets of test problems with known analytical solutions to illustrate workability and effectiveness of the proposed approach and to determine optimal parameters of the algorithms such as collocation point positions and number of terms of the Taylor's series. As a result of computational experiments, it is shown that for rational algorithm parameters an accuracy of the calculation can be sufficiently improved due to "better properties of matrix of the linear algebraic equations'. Beside of that, explicit regular boundary element algorithms are proposed for initial-boundary-value problems for heat conduction equation. The last kind of algorithms does not require a solution of linear algebraic equation, in general, saving, in the same time, enough high accuracy of whole calculations.

\section{References}

[3] A.A. Griffith. The phenomena of rupture and flow in solids. Philos. Trans. Roy. Soc. London 221 (1921), 163-198. 


\title{
Boundary element application to non-linear boundary integral equations
}

\author{
M. V. Poliakov \\ Dniepropetrovsk National University named after O.Honchar, Dniepropetrovsk, Ukraine
}

Last decades, boundary element method has become a powerful tool of numerical modeling. However the boundary element method, first of all, is directed to linear boundary-value problems, for which it provides extremely high accuracy and effectiveness. There are a lot of specific difficulties, which arise under boundary element method is being applied to non-linear problem. The present work is devoted to determination and analysis of cases, when boundary element method is effective in non-linear problem application. The most important boundary element advantage is possibility of analysis boundary alone. If pure boundary formulation is not obtained, an effectiveness of boundary element algorithms is sufficiently less, than effectiveness in the first case. An evident conclusion can be made that boundary alone formulation of non-linear problems are high effective too, similar to linear problems. Thus it is necessary to find such classes of non-linear problems. First of all, boundary-value problems with linear differential operators and non-linear boundary conditions are considered. Moving boundary problem and unknown boundary problems are something like of previous class, if they have linear differential operators too. The third group of problems is the most difficult for determination and analysis, these problems have non-linear differential operators, which permit some linearization transformation (for example, substitution) giving a problem with linear differential operator, that is, the obtained problem belongs to the first class. As it is well-known, non-linear problems can have non-unique solution. The nonlinear problems of all above mentioned classes arising in practical applications are usually solved using an iterative process to handle nonlinearity of boundary integral equations. It's a pity, but such approach doesn't give any opportunity to find several solutions, if the solution is not unique. The computation result in this case are completely depends on initial approximation (first step of iteration process). To make some conclusion about uniqueness of solutions, it is necessary to solve a lot of problem with great variety of initial approximations, what were made in the present work for several problems. However, there are other possible approaches to non-linear boundary integral equations based on boundary element technique, for example, Newton's method. The last direction is not developed properly yet. To illustrate the considered cases several examples of non-linear boundary integral equations are solved numerically. 


\title{
Boltzmann collision operator: How to model rotational invariance
}

\author{
Hans Babovsky \\ Technische Universitaet Ilmenau
}

Due to the availability of affordable computer power (CPU, GPU), discrete kinetic schemes for the simulation of the Boltzmann equation gain increasing interest. In contrast to Monte Carlo schemes, they are free of fluctuations and can resolve situations hard to calculate with stochastic methods.

Considering kinetic models on integer grids with small grid parameter $h$ as a numerical integration scheme, their order of consistency has been investigated in a couple of papers. However, the number of grid points to be relevant for such kinds of estimates is very high - too high for efficient numerical schemes. Thus for practical purposes one has to consider models for which the grid parameter and the truncation errors when cutting the grid to a finite size are not negligible. Thus one has to adjust the collision parameters obtained as numerical weights in such a way that the overall performance in the regime of interest is acceptable. One particular point of interest is the validity of physical invariances (translational, rotational), since these are in conflict with a finite-size integer grid if the considered Mach numbers are not very small.

In recent years kinetic models on integer grids have been investigated systematically $[1,2]$. The present contribution reports on the continuation of these studies following a new approach based on so-called multilayer systems. These are composed of single layers each of which considers only collisions $(v+p, v+q) \leftrightarrow(v, v+p+q)$ with fixed vectors $p$ and $q$. This point of view reveals a couple of interesting properties of a large class of discrete kinetic models. In particular, it allows to derive without too much effort the correct numerical weights by considering each layer as a specific integration point of the numerical quadrature rule.

Furthermore, it is possible to apply the results of previous works $[4,3]$ concerning the structure of the solutions to the Milne and Kramers problems. This allows to constitute a relation between the weights attached to each of the layers and the flow parameters (viscosity, heat conduction) in the regime close to the Navier-Stokes domain. The knowledge of these relations allows to fit flow properties in order to cut down truncation effects and to restore invariance properties like rotational symmetry of solutions.

\section{References}

[1] H. Babovsky. A numerical model for the Boltzmann equation with applications to micro flows. Computers and Mathematics with Applications. 58 (2009), 791-804.

[2] H. Babovsky. Discrete kinetic models in the fluid dynamic limit. Computers and Mathematics with Applications. 67 (2014), 256-271.

[3] H. Babovsky and T. Płatkowski. Kinetic boundary layers for the Boltzmann equation on discrete velocity lattices. Arch. Mech. 60 (2008), 87-116.

[4] C. Bardos, R. Caflish und B. Nicolaenko. The Milne and Kramers problems for the Boltzmann equation of a hard sphere gas. Comm. Pure Appl. Math. 39 (1986), 323-352. 


\title{
Fully-Implicit Log-Conformation Formulation of Viscoelastic Constitutive Laws
}

\author{
Philipp Knechtges, Marek Behr and Stefanie Elgeti \\ Chair for Computational Analysis of Technical Systems (CATS) \\ CCES, RWTH Aachen University, Germany
}

The robust and stable simulation of viscoelastic flows has been and still is a struggle of computational rheology. This fact, which is usually referred to as the High Weissenberg Number Problem, is not fully understood and the origin of the problem, i.e., whether it is an insufficiency of the numerical discretization or the model itself, is yet to be determined. One approach to tackle the problem from the numerical side is the so-called log-conformation formulation. The latter, which has first been proposed in 2004 by Fattal and Kupferman [1], owes much of its popularity to the circumstance that it was found to be crucial for numerical discretizations to resemble the analytical solution in the preservation of the positive-definiteness of the conformation tensor. The $\log$-conformation formulation simply achieves this by using $\boldsymbol{\Psi}=\log \boldsymbol{\sigma}$ as the primal variable of the simulation instead of the conformation tensor $\boldsymbol{\sigma}$. However, the corresponding set of constitutive equations derived in [1] carry the small intricacy that the formulation of the constitutive equation requires an algebraic decomposition of the velocity gradient field. In addition to hindering the closed formulation of the full system of partial differential equations in combination with the Navier-Stokes equations, this also complicates the application of further analytical tools. One of these tools with particular importance for numerical simulations is the Newton-Raphson algorithm, which promises quadratic convergence for the solution of the whole system.

In this talk we will present our recent development of a fully-implicit log-conformation formulation [2]. The key concept is the same as in the original log-conformation formulation: We substitute the polymeric stress or the conformation tensor as our primal variables by the logarithm of the conformation tensor. As such we also inherit the property that the conformation tensor is positive-definite by design. The substantial difference to the original method is that we can formulate a constitutive equation in the new variable that completely avoids the algebraic decomposition of the velocity gradient and thus paves the way for the Newton-Raphson algorithm. Following the theoretical description of the method, the talk will subsequently cover results of an implementation in our in-house FEM flow solver, ranging from benchmark problems to die swell simulations [3].

\section{References}

[1] R. Fattal, R. Kupferman. Constitutive laws for the matrix-logarithm of the conformation tensor. Journal of Non-Newtonian Fluid Mechanics 123 (2) (2004), 281-285.

[2] P. Knechtges, M. Behr, S. Elgeti. Fully-implicit log-conformation formulation of constitutive laws. Journal of Non-Newtonian Fluid Mechanics 214 (2014), 78-87. arXiv: 1406.6988 [math.NA].

[3] L. Pauli, M. Behr, S. Elgeti. Towards shape optimization of profile extrusion dies with respect to homogeneous die swell. Journal of Non-Newtonian Fluid Mechanics 200 (2013), 79-87. 


\title{
Approximate exponential time integration for diffusion problems
}

\author{
Tianshi Sun, Michael Manhart \\ Fachgebiet Hydromechanik, Technische Universität München
}

We develop a class of numerical methods for the time integration of the unsteady diffusion equation, based on an approximation of the Exponential Time Integration (ETI). ETI has gained importance following the work of Cox and Matthews [1] and with recent developments in efficient methods for computing the matrix exponential [2]. The formulation of this Approximate Exponential Time Integration (AETI) makes use of a combination of the Von Neumann analysis and the Taylor series expansion. We describe schemes with second and higher order of accuracy, and extend the method to show how it can be transformed into a new algorithmic structure. We benchmark the method with respect to classical explicit numerical schemes that treat time integration and spatial approximation separately. Our analysis and numerical tests demonstrate that the new formulation is more accurate and efficient than combinations of Explicit Euler and Runge-Kutta (RK) methods of second, third and fourth order with explicit differencing schemes up to sixths order and allows for considerably larger time integration steps.

\section{References}

[1] S. M. Cox and P. C. Matthews. Exponential time differencing for stiff systems. J. Computational Physics. 176 (2002).

[2] M. Eiermann and O. Ernst. A restarted Krylov subspace method for the evaluation of matrix functions. SIAM J. Numer. Anal. 44 (2006), 2481-2504. 


\title{
On multi-dimensional discrete equations of convolution type
}

\author{
Alexander V. Vasilyev, Vladimir B. Vasilyev \\ National Research Belgorod State University, Belgorod, Russia \\ Lipetsk State Technical University, Lipetsk, Russia
}

We consider a special class of integral operators of convolution type, namely a simplest variant of Calderon - Zygmund operators. Such operators arise in a lot of applied problems, and the studying their properties is of a special interest. We start from the Calderon-Zygmund operator [1]

$$
K: u(x) \longmapsto v \cdot p \cdot \int_{D} K(x-y) u(y), \quad x \in D,
$$

and its discrete analogue

$$
K_{d}: u_{d}(\tilde{x}) \longmapsto \sum_{\tilde{y} \in D \cap \mathbf{Z}^{m}} K(\tilde{x}-\tilde{y}) u_{d}(\tilde{y}), \quad \tilde{x} \in D \cap \mathbf{Z}^{m}
$$

where $D$ is one of canonical domains $\mathbf{R}^{m}, \mathbf{R}_{+}^{m}=\left\{x \in \mathbf{R}^{m}: x_{m}>0\right\}, C_{+}^{a}=\left\{x \in \mathbf{R}^{m}: x_{m}>a\left|x^{\prime}\right|, a>\right.$ $\left.0, x^{\prime}=\left(x_{1}, \ldots, x_{m-1}\right)\right\}, \mathbf{Z}^{m}$ is integer lattice in the space $\mathbf{R}^{m}$, and study the invertibility properties for such operators. These considerations are based on symbol properties of Calderon - Zygmund operators, discrete Fourier transform, and periodic analogue of classical Riemann boundary problem $[2,3,4,5]$.

According to classical symbol of the Calderon - Zygmund operator $K[1]$

$$
\sigma(\xi)=v \cdot p \cdot \int_{\mathbf{R}^{m}} K(x) e^{-i x \cdot \xi} d x, \xi \in \mathbf{R}^{m} \backslash\{0\},
$$

we define a symbol for the discrete operator $K_{d}$ by multi-dimensional Fourier series

$$
\sigma_{d}(\xi)=\lim _{N \rightarrow+\infty} \sum_{\tilde{x} \in Q_{N} \cap \mathbf{Z}^{m}} K(\tilde{x}) e^{-i \tilde{x} \cdot \xi}, \xi \in \mathbf{T}^{m},
$$

where $Q_{N}=\left\{x \in \mathbf{R}^{m}: \max _{1 \leq k \leq m}\left|x_{k}\right| \leq N\right\}, \mathbf{T}^{m}=[-\pi, \pi]^{m}$ is basic cube of periods.

A correlation between the symbols $\sigma(\xi)$ and $\sigma_{d}(\xi)$ for the case $\mathbf{R}^{m}, \mathbf{R}_{+}^{m}$ was considered in papers [3, 4, 5]. For studying convolution equations with the operator $K_{d}$ for the case $D=C_{+}^{a}$ authors develop the theory of multi-dimensional Riemann boundary problem which has its continual analogue [2]. It was found that the existence of a wave factorization for the symbol $\sigma(\xi)$ with respect to the cone $C_{+}^{a}$ implies the existence of required factorization for the symbol $\sigma_{d}(\xi)$. For this purpose the authors consider a certain class of holomorphic functions in special tube domains over cones.

The reported study was partially supported by RFBR, research project No. 14-41-03595 a.

\section{References}

[1] S.G. Mikhlin, S. Prössdorf. Singular integral operators. Akademie-Verlag, Berlin, 1986.

[2] V.B. Vasil'ev. Regularization of multidimensional singular integral equations in non-smooth domains. Trans. Moscow Math. Soc. 59 (1998), 65-93.

[3] V.B. Vasilyev. Elliptic equations and boundary value problems in non-smooth domains. In: Operator Theory: Advances and Applications, 2011, V.213. Birkhäuser, Basel. P.105-121.

[4] A.V. Vasilyev, V.B. Vasilyev. Discrete singular operators and equations in a half-space. Azerb. J. Math. 3:1 (2013), 84-93.

[5] A.V. Vasilyev, V.B. Vasilyev. Discrete singular integrals in a half-space. arXiv:1410.1049. 


\section{S19: Optimization of differential equations}

In this session novel developments devoted to optimization and optimal control problems governed by ordinary or partial differential equations will be discussed. The focus is on theoretical investigations, numerical analysis, algorithnmic issues as well as on application. 


\title{
Two Types of Globally Convergent Numerical Methods for Coefficient Inverse Problems
}

\author{
Michael V. Klibanov \\ Department of Mathematics and Statistics \\ University of North Carolina at Charlotte \\ Charlotte, NC 28223, USA \\ E-mail: mklibanv@uncc.edu
}

\begin{abstract}
The goal of this talk is to present two existing types of globally convergent numerical methods for Coefficient Inverse Problems (CIPs). CIPs are nonlinear. Conventional least squares Tikhonov functionals for CIPs suffer from the phenomenon of multiple local minima and ravines. This means that any optimization method should have its starting point in a sufficiently small neighborhood of the exact solution. Therefore, conventional Tikhonov functional for a CIP inevitably leads to a locally convergent numerical method. However, such a neighborhood is rarely known in applications. Although there are many publications devoted to numerical solutions of CIPs, almost all of them still have the same fundamental drawback: local convergence. Naturally, there are counter examples showing that locally convergent numerical methods for CIPs are inherently unstable, since their results heavily depend on the starting points of iterations, see, e.g. [3, 10, 13].

Thus, both the most challenging and the most important question in a numerical treatment of a CIP is "How to rigorously obtain at least one point in a small neighborhood of the exact solution without any advanced knowledge of this neighborhood?" We call a numerical method, which positively addresses this question, globally convergent.

There are currently two types of globally convergent numerical methods for CIPs with single measurement data. The first type is the Beilina-Klibanov method, which was initiated in their work [2]. Results of many publications up to 2012 were summarized in the book [3]. The global convergence theory was fully developed $[3,4]$. Besides, the method is completely tested on both transmitted $[3,5,10]$ and backscattering $[6,7,8,14,15]$ experimental data.

The second type is based on constructions of globally strictly convex cost functionals for CIPs. The key element of such a functional, which makes it globally strictly convex, is the presence of a Carleman Weight Function $(\mathrm{CWF})$, i.e. the function involved in the Carleman estimate for a corresponding Partial Differential Operator. The development of this type of method was started by Klibanov in 1997 [11, 12]. Now is a renewed interest in it [9, 13]. First numerical results can be found in the work of Klibanov and Thánh [13]. Besides, another close idea, which was developed recently is the one of Baudoin, de Buhan and Ervedosa [1].
\end{abstract}

\section{References}

[1] L. Baudouin, M. de Buhan and S. Ervedoza, Global Carleman estimates for waves and applications, Communications in Partial Differential Equations, 38, 823-859, 2013.

[2] L. Beilina and M.V. Klibanov, A globally convergent numerical method for a coefficient inverse problem, SIAM J. Sci. Comp., 31, 478-509, 2008.

[3] L. Beilina and M.V. Klibanov, Approximate Global Convergence and Adaptivity for Coefficient Inverse Problems, Springer, New York, 2012.

[4] L. Beilina and M.V. Klibanov, A new approximate mathematical model for global convergence for a coefficient inverse problem with backscattering data, J. Inverse and Ill-Posed Problems, 20, 513-565, 2012.

[5] L. Beilina and M.V.Klibanov, Reconstruction of dielectrics from experimental data via a hybrid globally convergent/adaptive inverse algorithm, Inverse Problems, 26, 125009, 2010.

[6] L. Beilina, N. T. Thành, M. V. Klibanov and M. A. Fiddy, Reconstruction from blind experimental data for an inverse problem for a hyperbolic equation, Inverse Problems, 30, 025002, 2014.

[7] L. Beilina, N.T. Thánh, M.V. Klibanov and J.B. Malmberg, Reconstruction of shapes and refractive indices from backscattering experimental data using the adaptivity, Inverse Problems, 30, 105007, 2014. 
[8] L. Beilina, N.T. Thành, M. V. Klibanov and J.B. Malmberg, Globally convergent and adaptive finite element methods in imaging of buried objects from experimental backscattering radar measurements, arxiv, $1409.1167 \mathrm{v} 1,2014$.

[9] L. Beilina and M.V. Klibanov, Globally strongly convex cost functional for a coefficient inverse problem, Nonlinear Analysis: Real World Applications, 22, 272-278, 2015.

[10] M. V. Klibanov, M. A. Fiddy, L. Beilina, N. Pantong and J. Schenk, Picosecond scale experimental verification of a globally convergent numerical method for a coefficient inverse problem, Inverse Problems, 26, 045003, 2010.

[11] M.V. Klibanov, Global convexity in a three-dimensional inverse acoustic problem, SIAM J. Math. Analysis, 28, 1371-1388, 1997.

[12] M.V. Klibanov, Global convexity in diffusion tomography, Nonlinear World, 4, 247-265, 1997.

[13] M.V. Klibanov and N.T. Thành, Recovering of dielectric constants of explosives via a globally strictly convex cost functional, arxiv: 1408.0583, 2014.

[14] N.T. Thành, L. Beilina, M. V. Klibanov and M. A. Fiddy, Reconstruction of the refractive index from experimental backscattering data using a globally convergent inverse method, SIAM J. Sci. Comp., 36, B273-B293, 2014.

[15] N.T. Thành, L. Beilina, M. V. Klibanov and M. A. Fiddy, Imaging of buried objects from experimental backscatetring time dependent measurements using a globally convergent inverse algorithm, arxiv, $1406.3500 \mathrm{v} 1,2014$. 


\title{
A Riemannian SQP method for PDE constrained shape optimization
}

\author{
Volker Schulz, Martin Siebenborn, Kathrin Welker \\ University of Trier, FB IV, Department of Mathematics \\ Universitätsring 15, 54296 Trier, Germany
}

e-mail: volker.schulz@uni-trier.de, siebenborn@uni-trier.de, welker@uni-trier.de

\begin{abstract}
Shape optimization problems arise frequently in technological processes which are modeled in the form of partial differential equations. In many practical circumstances, the shape under investigation is parameterized by finitely many parameters, which on the one hand allows the application of standard optimization approaches, but on the other hand limits the space of reachable shapes unnecessarily. Shape calculus, which has been the subject of several monographs $[1,3,7]$ presents a way out of that dilemma. However, so far it is mainly applied in the form of gradient descent methods, which can be shown to converge. The major difference between shape optimization and the standard PDE constrained optimization framework is the lack of the linear space structure in shape spaces. If one cannot use a linear space structure, then the next best structure is the Riemannian manifold structure as discussed for shape spaces for example in [2]. The publication [4] makes a link between shape calculus and shape manifolds and thus enables the usage of optimization techniques on manifolds in the context of shape optimization. This talk presents a vector bundle framework [5] based on the Riemannian framework established in [4], which enables the discussion of Lagrange-Newton methods within the shape calculus framework for PDE constrained shape optimization. This novel Riemannian vector bundle framework will be discussed for a specific academic example. Like in [6] the representation of the shape derivative expressed as a boundary integral which is needed for a numerical implementation is deduced from its represantation as a domain integral.
\end{abstract}

\section{References}

[1] M. C. Delfour and J.-P. Zolésio. Shapes and Geometries: Analysis, Differential Calculus, and Optimization. Advances in Design and Control. SIAM Philadelphia, 2001.

[2] P. W. Michor and D. Mumford. Riemannian geometries on spaces of plane curves. J. Eur. Math. Soc. (JEMS), 8:1-48, 2006.

[3] B. Mohammadi and O. Pironneau. Applied Shape Optimization for Fluids. Numerical Mathematics and Scientific Computation. Clarendon Press Oxford, 2001.

[4] V. H. Schulz. A Riemannian view on shape optimization. Foundations of Computational Mathematics, 14:483-501, 2014.

[5] V. H. Schulz, M. Siebenborn, K. Welker. Towards a Lagrange-Newton Approach for PDE constrained shape optimization. Trends in PDE Constrained Optimization, volume 165 of International Series of Numerical Mathematics, Springer, 2014.

[6] V. H. Schulz, M. Siebenborn, and K. Welker. Structured inverse modeling in parabolic diffusion problems. submitted to SICON, arXiv:1409.3464, 2014.

[7] J. Sokolowski and J.-P. Zolésio. Introduction to Shape Optimization: Shape Sensitivity Analysis. Springer, 1992. 


\title{
A second order convergent trial method for free boundary problems in three dimensions
}

\author{
Monica Bugeanu, Helmut Harbrecht \\ University of Basel
}

This talk is concerned with the solution of a generalized Bernoulli free boundary problem by means of a trial method. At the free boundary, we prescribe the Neumann boundary condition and update the free boundary with the help of the remaining Dirichlet boundary condition. An inexact Newton update is used which results in a second order convergent iterative method. Numerical examples show the feasibility of the present approach. 


\title{
Primal-Dual Active Set Strategy for Singular Optimal Control Problems
}

\author{
Simon Bechmann, Julia Fischer \\ Department of Mathematics in Engineering Sciences, University of Bayreuth, Germany
}

Our focus is on singular optimal control problems including partial differential equations which are not described by a control-to-state operator. In such case it is not justified to consider a reduced problem with a single optimization variable. Despite not having a control-to-state operator the proving of existence of optima, the derivation of first order optimality conditions as well as the numerical solving of these problems is possible. The existence of solutions for optimal control problems including singular systems with multiple states and the derivation of first order optimality conditions by a penalization method is shown in [2].

Using Lagrangian techniques in [1] the existence of Lagrangian multipliers satisfying an unqualified optimality system is shown. For some cases the validity of the qualified form is proven.

We prove that the regularity condition of Robinson resp. Kurcyusz-Zowe holds and obtain a qualified system of optimality conditions for a broad class of linear PDEs.

Some specifics of the coupled optimality system are presented. Applicability and efficiency of the primal-dual active set strategy for this class of problems is investigated. We apply results of [3] and propose assumptions which guarantee superlinear local convergence known from classical problems.

\section{References}

[1] Fursikov, A.V.. Optimal Control of Distributed Systems. Theory and Applications. Translations of Mathematical Monographs. 187. Providence, RI: AMS, American Mathematical Society., (2000).

[2] J.L. Lions. Control of Distributed Singular Systems., Bordas, (1985).

[3] Anton Schiela. A Simplified Approach to Semismooth Newton Methods in Function Space. SIAM J. Optim., 19:1417-1432, (2008). 


\title{
Adaptive Optimal Control of the Obstacle Problem
}

\author{
Christian Meyer ${ }^{1}$, Andreas Rademacher ${ }^{1}, \underline{\text { Winnifried Wollner }}^{2}$ \\ ${ }^{1}$ Technische Universität Dortmund \\ ${ }^{2}$ Universität Hamburg
}

The talk is concernded with the adaptive optimal control of the obstacle problem, as described in [1]. To this end, a posteriori error estimates for optimization problems subject to an obstacle problem are derived. To circumvent the nondifferentiability inherent to this type of problem, a sequence of penalized but differentiable problems is introduced. Differentiability of the central path is shown and separate a posteriori dual weighted residual estimates for the errors due to penalization, discretization, and iterative solution of the discrete problems are provided. The effectivity of the derived estimates is demonstrated on two numerical examples.

\section{References}

[1] Ch. Meyer, A. Rademacher, W. Wollner. Adaptive optimal control of the obstacle problem Hamburger Beiträge zur angewandten Mathematik 2014-10 (2014). 


\title{
Optimal Control of a linear unsteady Fluid-Structure Interaction Problem
}

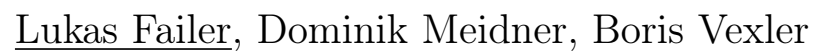 \\ Technische Universität München \\ Faculty of Mathematics \\ Garching by Munich, Germany
}

Fluid-Structure Interaction (FSI) problems have been extensively studied from theoretical and numerical point of view in the last decade. More and more applications for shape- and parameter- estimation of FSI are regarded recently, for example in [3] and [4]. But especially about gradient based algorithms for optimal control of the unsteady FSI problem with a distributed control, little is known. If the control is distributed in space and time it is necessary to compute the gradient of the reduced cost functional accurately to obtain a fast and converging algorithm. Thereby the difficulty in Fluid-Structure Interaction occurs due to the coupling conditions. Only if the adjoint coupling conditions are fulfilled accurately in the numerical simulation, a correct transport of information across the interface can be guaranteed.

To analyze the coupling conditions in the adjoint equation we consider a minimization problem subjected to a linear Fluid-Structure interaction problem. The linear FSI problem was already analyzed in [1] and [2]. We proof the existence of a minimizer for the optimal control problem and we derive systematically necessary optimality conditions using a novel symmetric monolithic formulation. The presented formulation enables an easy numerical implementation as the resulting equations can be solved using methods already developed for FSI. We analyze the properties of the resulting optimality system and present results on higher regularity for the optimal control.

\section{References}

[1] V. Barbu, Z. Grujic, I. Lasiecka, A. Tuffaha. Existence of the energy-level weak solutions for a nonlinear Fluid-Structure Interaction model. Recent Trends in Applied Analysis. 440 (2007), 55-82.

[2] Q. Du, M. D. Gunzburger, L.S. Hou, J. Lee. Analysis of a linear Fluid-Structure Interaction problem. Disc. Cont. Dyn. Syst. 9 (2003) 633-650.

[3] M. Perego, A. Veneziani, C. Vergara. A variational approach for estimating the compliance of the cardiovascular tissue: An inverse Fluid-Structure Interaction problem. SIAM J. Sci. Comput. 33 (2011), 1181-1211.

[4] T. Richter, T. Wick. Optimal control and parameter estimation for stationary Fluid-Structure Interaction problems. SIAM J. Sci. Comput. 35 (2013), B1085-B1104. 


\title{
Parabolic optimal control problems with pointwise controls
}

\author{
Boris Vexler, Dmitriy Leykekhman \\ TU Munchen \\ University of Connecticut
}

We consider a parabolic optimal control problem:

$$
\min _{q, u} J(q, u):=\frac{1}{2} \int_{0}^{T}\|u(t)-\widehat{u}(t)\|_{L^{2}(\Omega)}^{2} d t+\frac{\alpha}{2} \int_{0}^{T}|q(t)|^{2} d t
$$

subject to the second order parabolic equation

$$
\begin{aligned}
& u_{t}(t, x)-\Delta u(t, x)=q(t) \delta_{x_{0}}, \\
& (t, x) \in I \times \Omega, \\
& u(t, x)=0 \text {, } \\
& u(0, x)=0, \\
& (t, x) \in I \times \partial \Omega,
\end{aligned}
$$

and subject to pointwise control constraints

$$
q_{a} \leq q(t) \leq q_{b} \quad \text { a.e. in } I .
$$

Here $I=[0, T], \Omega$ is a convex polygonal or polyhedral domain, $x_{0} \in \Omega$ fixed, and $\delta_{x_{0}}$ is the Dirac delta function. The parameter $\alpha$ is assumed to be positive and the desired state $\widehat{u}$ fulfills $\widehat{u} \in L^{2}\left(I ; L^{\infty}(\Omega)\right)$. The control bounds $q_{a}, q_{b} \in \mathbb{R} \cup\{ \pm \infty\}$ fulfill $q_{a}<q_{b}$.

This problem can be classified as optimal control problem with a pointwise control. To approximate the problem numerically we use the standard continuous piecewise linear approximation in space and the first order discontinuous Galerkin method in time. Despite low regularity of the state equation, we establish almost optimal $h^{2}+k$ convergence rate in $2 \mathrm{D}$ and $h+\sqrt{k}$ in $3 \mathrm{D}$ for the control in $L^{2}$ norm. I will explain the key regularity estimate and new a priori fully discrete global and local error estimates in $L^{2}\left([0, T] ; L^{\infty}(\Omega)\right)$ norms for parabolic problems. These new error estimates are essential in our analysis and using we improve almost twice the previously obtained error estimates in [1]. The 2D result were published in [2], but 3D results are new and still a work in progress.

\section{References}

[1] W. Gong, M. Hinze, Z. Zhou. A priori error analysis for finite element approximation of parabolic optimal control problems with pointwise control. SIAM J. Control Optim. 52 (2014), 97-119.

[2] D. Leykekhman, B. Vexler. SOptimal a priori error estimates of parabolic optimal control problems with pointwise control. SIAM J. Numer. Anal. 51 (2013), 2797-2821. 


\title{
Optimal Control of Signorini Contact Problems
}

\author{
Thomas Betz, Christian Meyer \\ TU Dortmund
}

The talk is concerned with an optimal control problem governed by the Signorini contact problem, which is modelled by a variational inequality (VI) of the first kind. The solution operator associated with this VI is known to be not Gâteaux-differentiable. However, based on a technique introduced in [1], one can prove that it is directionally differentiable with a directional derivative, which is a VI of first kind itself. This allows to derive strong stationarity conditions as necessary conditions for local optimality under rather restrictive assumptions. Moreover, we employ this directional derivative for the design of an efficient trust-region optimization algorithm.

\section{References}

[1] F. Mignot. Contrôle dans les Inéquations Variationelles Elliptiques. J. Func. Anal. 22 (1976), 130-185. 


\title{
Frequency-sparse Control of Bilinear Quantum Systems
}

\author{
Gero Frisecke $^{1}$, Felix Henneke ${ }^{1}$, Karl Kunisch ${ }^{2}$ \\ ${ }^{1}$ Technische Universtät München \\ ${ }^{2}$ Karl-Franzens-Universität Graz
}

An interesting phenomenon in bilinear quantum control is the oscillatory nature of the one dimensional control field. Those oscillations pose serious problems for the interpretation and experimental realization of the field. To overcome these difficulties we propose the use of time-frequency controls in a space of function-valued measures. This leads to an optimization problem of the form

$$
\begin{gathered}
\min _{\psi, u} \frac{1}{2}\langle\psi, \mathcal{O} \psi\rangle+\alpha\|u\|_{\mathcal{M}\left(\mathbf{R} ; H_{0}^{1}(0, T)\right)} \\
i \psi(t)=\left(H_{0}+(B u)(t) H_{1}\right) \psi(t)
\end{gathered}
$$

where $u$ is an element of the space $\mathcal{M}\left(\mathbf{R} ; H_{0}^{1}(0, T)\right)$ of $H_{0}^{1}$-valued measures on the real line and the control operator $B$ reconstructs the control field from the time-frequency information. The operators $H_{0}$ and $H_{1}$ are determined by the quantum system at hand and the control objective is to minimize the expectation value of the observable $\mathcal{O}$. This optimal control problem is non-convex and non-smooth. Optimal control with function-valued measures was already studied for a convex parabolic problem [1].

Previous attempts to deal with the oscillatory structure of control fields in quantum control are mostly based on frequency representations [2]. However, additional frequency localization comes at the cost of less time structure. Time-frequency representations were used only in combination with derivative free algorithms [3]. Our proposed systematic optimal control approach is based on sparsity enhancing functionals and timefrequency representations. We do not control the field itself but use a time-frequency control space that promotes sparsity in the frequency component and smoothness in time. In numerical experiments this leads to control fields which consist of a small number of pure frequencies each modulated with a smooth envelope function. The frequencies as well as the envelopes are obtained automatically without prior knowledge of the quantum system.

In this talk we will present a general framework for sparse quantum control, analyze the resulting optimality conditions and study different numerical examples.

\section{References}

[1] K. Kunisch, K. Pieper, B. Vexler. Measure valued directional sparsity for parabolic optimal control problems. SIAM J. Control Optim. 52 (2014), 3078-3108.

[2] Q. Ren, G.G. Balint-Kurti, F.R. Manby, M. Artamonov, T.S. Ho, H. Rabitz. Quantum control of molecular vibrational and rotational excitations in a homonuclear diatomic molecule: A full three-dimensional treatment with polarization forces. J. Chem. Phys. 124, 014111 (2006).

[3] S. Ruetzel, C. Stolzenberger, F. Dimler, D.J. Tannor, T. Brixner. Adaptive coherent control using the von Neumann basis. Phys. Chem. Chem. Phys. 13 (2011), 8627-8636. 


\title{
Looking for strictly positive solutions of elliptic PDEs
}

\author{
Anton Schiela \\ Universität Bayreuth, Germany
}

Consider, under the usual assumptions, an elliptic PDE in weak form:

$$
\int_{\Omega} \nabla v \cdot A(x) \nabla u+c(x) u v d x+\int_{\Gamma} r(x) v u d s=\int_{\Omega} f v d x+\int_{\Gamma} g v d s \quad \forall v \in V .
$$

It is easy to prove that for non-negative right-hand sides $g$ and $f$ we obtain non-negative solutions. Moreover, it is easy to believe that $f$ and $g$ can be chosen in a way that $y$ is strictly positive (even if one of $f$ and $g$ is required to be 0). This and similar issues are of interest in optimal control, when it comes to verifying Slater conditions for a class of concrete problems. However, a rigorous proof of this conjecture is much harder to obtain, in particular, if one wants keep the regularity assumptions on the domain and the data as weak as possible. The aim of this talk is to give an account on achieved and possible solutions to this surprisingly deep issue. 


\title{
Time optimal control for the monodomain equations - a monolithic approach
}

\author{
Karl Kunisch ${ }^{1}, \frac{\text { Konstantin Pieper }^{2}}{{ }^{1} \text { University of Graz }}$ Armin Rund $^{1}$ \\ ${ }^{2}$ Technische Universität München
}

In this talk we present and analyze a time optimal control formulation for a reaction diffusion system arising in cardiac electrophysiology. Specifically, we consider the monodomain equations, which consist of a parabolic equation coupled to an ordinary differential equation (in each point in space), and provide a simplified model describing the electric activity of the heart. They allow for challenging wave phenomena (e.g., reentry waves), which physiologically correspond to undesired arrhythmia. Since the required time frame for a successful termination of such waves depends heavily on the data, we propose an optimal control formulation with a free final time. We show that, under certain conditions on the parameters, the optimal solutions of this problem steer the system into an appropriate stable neighborhood of the resting state. To this end, we derive some new regularity results and asymptotic properties for the monodomain equations in combination with the RogersMcCulloch gating model.

For the numerical realization we consider a monolithic optimization approach, which simultaneously optimizes for the optimal times and optimal controls. Its practical realization is based on a semismooth Newton method. We prove local superlinear convergence in function space (under a second order condition) and present a globalization strategy. We present numerical examples which show the efficiency of the proposed approach and underline the theoretical results. 


\title{
The optimal shape of a pipe
}

\author{
Andreas Schulz \\ Karlsruher Institute of Technology, Institute of Applied and Numerical Mathematics
}

A recent question in fluid dynamical shape optimization is, whether or not one can optimize the shape of a pipe to reduce frictional losses in fluid transportation compared to a cylindrical shape. Under reasonable assumptions on the model, including a non-slip condition on the shell, an incompressible and stationary fluid flow, and a volume constraint, an optimality proof for the cylindrical shape will be presented. The topic was addressed in [1], whereas the presented optimality proof can be found in [2].

\section{References}

[1] A. Henrot, J. Privat. What is the optimal shape of a pipe?. Arch. Rational Mech. Anal. 196/1, (2010), 281-302.

[2] A. Schulz. The optimal shape of a pipe. Journal of Applied Mathematics and Physics. 64/4, (2013), $1177-1185$. 


\title{
Topological derivative for nonlinear magnetostatics
}

\author{
Samuel Amstutz, Peter Gangl, Ulrich Langer \\ Département de Mathématique, Université d'Avignon, Avignon, France \\ Doctoral Program "Computational Mathematics", Johannes Kepler University, Linz, Austria \\ Institute of Computational Mathematics, Johannes Kepler University, Linz, Austria
}

In topology optimization we are looking for the optimal distribution of material in a design domain in order to minimize a given domain-dependent objective function. One way to find such optimal designs is to use toplogical sensitivity information. The topological derivative of a domain-dependent functional $\mathcal{J}=\mathcal{J}(\Omega)$ represents its sensitivity with respect to a perturbation of the domain. At a spatial point $x_{0}$, the topological derivative is defined as the quantity $G\left(x_{0}\right)$ satisfying a topological asymptotic expansion of the form

$$
\mathcal{J}\left(\Omega_{\varepsilon}\right)-\mathcal{J}(\Omega)=\rho(\varepsilon) G\left(x_{0}\right)+o(\rho(\varepsilon)) .
$$

Here $\Omega_{\varepsilon}$ denotes the perturbed configuration where a hole of radius $\varepsilon$ around the point $x_{0}$ has been introduced in the domain $\Omega$, and $\rho(\varepsilon)$ is a positive function with $\lim _{\varepsilon \rightarrow 0} \rho(\varepsilon)=0$. The sign of $G\left(x_{0}\right)$ indicates whether introducing a hole around $x_{0}$ would increase or decrease the objective function.

Topological derivatives for optimization problems constrained by linear partial differential equations (PDEs) are well-understood. However, little is known about topological derivatives in combination with nonlinear PDE constraints. We will derive the formula of the topological derivative for the topology optimization of an electric motor where the state equation is the nonlinear equation of two-dimensional magnetostatics. Furthermore, we will address implementational issues and present numerical results for the optimization of an electric motor. 


\title{
Multiple state optimal design problems with random perturbation
}

\author{
Marko Vrdoljak \\ University of Zagreb, Croatia
}

A multiple state optimal design problem with presence of uncertainty on the right-hand side is considered, in context of stationary diffusion with two isotropic phases. Perturbation term is given by a random function on a probability space. Similar problem with one state equation is considered in [2]. We shall address the question of relaxation by the homogenization method and necessary conditions of optimality. The case of discrete probability space leads to another multiple state problem (possibly with infinite number of states), which could be treated by similar techniques to those presented in [1,3]. Relaxation can be expressed in a simpler form for problems with spherical symmetry in case of minimization (or maximization) of convex combination of compliances.

\section{References}

[1] G. Allaire. Shape optimization by the homogenization method. Springer-Verlag, 2002.

[2] G. Buttazzo, F. Maestre. Optimal shape for elliptic problems with random perturbations. Discrete Contin. Dyn. Syst. 31 (2011), 1115-1128.

[3] M. Vrdoljak. On Hashin-Shtrikman bounds for mixtures of two isotropic materials. Nonlinear Anal. Real World Appl. 11 (2010) 4597-4606. 


\title{
Robust methods and adaptivity for the numerical solution of variational inequalities for phase-field-based fracture problems
}

\author{
Thomas Wick \\ Johann Radon Institute for Computational and Applied Mathematics (RICAM) \\ Austrian Academy of Sciences
}

In this presentation, we consider phase-field-based fracture propagation in elastic media. The main purpose is the development of a robust and efficient numerical scheme. To enforce crack irreversibility as a constraint, we use a primal-dual active set strategy, which can be identified as a semi-smooth Newton's method. The active set iteration is merged with the Newton iteration for solving the fully-coupled nonlinear partial differential equation discretized using finite elements, resulting in a single, rapidly converging nonlinear scheme. It is well-known that phase-field models ask for the resolution of a length-scale regularization parameter in terms of the local mesh size parameter. To achieve this, a predictor-corrector scheme for local mesh adaptivity is presented. Finally, steps towards a posteriori error estimation and convergence results are undertaken. Numerical examples are consulted to illustrate our findings. 


\title{
A Bridge from State-constrained ODE Optimal Control to State-constrained elliptic PDE Optimal Control: New Necessary Condtions, New Optimization Problems, and New Numerical Methods
}

\author{
Michael Wrensch ${ }^{1}$, Simon Bechmann ${ }^{2}$, $\underline{\text { Hans Josef Pesch }}^{2}$, Armin Rund ${ }^{3}$ \\ ${ }^{1}$ Brose, Coburg \\ ${ }^{2}$ University of Bayreuth \\ ${ }^{3}$ University of Graz
}

Based on different reformulations of state-constrained elliptic optimal control problems with distributed control and a hypothesis on the structure of the active set, new necessary conditions are obtained which exhibit higher regularity of the multiplier associated with the state constraint. Moreover, we obtain also new jump and sign conditions. Measures are no longer an issue, so that regularization techniques become superfluous. Finally, the method can be fully described in function spaces which is an essential element to obtain a mesh-independent numerical method.

The new approach mimics the well-known Bryson-Denham-Dreyfus indirect adjoining method which is the preferred ansatz in solving state-constrained optimal control problems with ordinary differential equations numerically. However, in the context of PDE constrained optimization this approach turns out to be extremely involved.

Mathematically the reformulations lead to a new kind of set optimal control problem, where the active set of the state constraint, resp. the interface between the inactive and the active set are to be determined as part of the solution as the switching points in multipoint boundary value problems based on first-order necessary conditions for ODE constrained optimal control problems. Various formulations of this type of PDE optimization problem as shape and/or topology as well as bilevel optimization problem or, concerning the numerical solution, as free boundary value problem are discussed.

Moreover, parallels can be drawn to optimization on vector bundels which seems to be essential for the design of an appropriate Newton-type method, since the optimization over sets of admissible active sets have a nonlineare structure. This requires an answer to the question how does a Newton method looks on a nonlinear manifold where no Banach space structure is present.

Numerical results will demonstrate the performance of the new method.

This presentation will open new research directions in PDE constrained optimal control as indicated in the final outlook. 


\title{
Optimal control for lithium-ion batteries
}

\author{
Georg Vossen ${ }^{1}$, Dirk Roos ${ }^{1}$, Alexander Struck ${ }^{2}$ \\ ${ }^{1}$ Niederrhein University of Applied Sciences, Krefeld, Germany \\ ${ }^{2}$ Rhein-Waal University of Applied Sciences, Kleve, Germany
}

The decreasing of fossil energy sources makes energy storage systems to one of the central components of electric systems, particularly, for purely battery-operated vehicles. A central challenge is the development of efficient battery management systems for the storage, delivery and recovery of electric energy. Nowadays, lithium-ion batteries are very common for portable electronics and furthermore, of growing importance for electric vehicles.

Modelling the electro-chemical phenomena occurring in re- and discharging batteries leads to very complicated systems of nonlinearly coupled partial differential, algebraic and integral equations for the ion concentrations, fluxes and potentials. If further thermal and mechanical processes or the coupling of multiple batteries are considered, the models become even more complex. Numerical simulation and, in particular, (optimal) control of such systems can therefore be a very difficult and time-consuming issue.

In this talk we will discuss a specific model for a lithium-ion battery. The model involves ionic concentrations, ionic currents and potentials in and between the positive and negative electrode together with the battery temperature as state variables. The resulting system is a nonlinear PDAE system with 10 partial and 4 algebraic equations involving the famous nonlinear Butler-Volmer kinetics for describing the interaction of ionic currents and potentials. The numerical simulation is implemented based on a finite difference approach.

Optimal control tasks for charging the system subject to control and state constraints are discussed. For the numerical treatment of the optimal control problems, a first-discretize-then-optimize approach is firstly chosen. This leads to a high-dimensional nonlinear optimization problem which is solved by an efficient nonlinear optimization solver. Necessary optimality conditions can be derived and numerically verified.

Further numerical techniques to solve the optimal control problems are presented. For the occurring state constraint of order 1, a feedback control law is computed to formulate and solve the corresponding so-called induced optimization problem. This method provides not only the state and control variables in an efficient way but also the values of switching times with high accuracy. Furthermore, the method can be used to investigate second order sufficient conditions and sensitivity analysis.

\section{References}

[1] N.A. Chaturvedi, R. Klein, J. Christensen, J. Ahmed, A. Kojic. Algorithms for Advanced BatteryManagement Systems. Control Systems, IEEE 30 (2010), 49-68.

[2] V. Subramanian, V. Boovaragavan, V. Ramadesigan, M. Arabandi. Mathematical model reformulation for lithium-ion battery simulations: Galvanostatic boundary conditions. J. Electrochem. Soc. 156 (2009), A260-A271.

[3] R. Klein, N.A. Chaturvedi, J. Christensen, J. Ahmed, R. Findeisen, A. Kojic. Optimal Charging Strategies in Lithium-Ion Battery. American Control Conference (2011), 382-387.

[4] G. Vossen. Switching time optimization for bang-bang and singular controls. J. Opt. Theory and Appl. 144 (2010), 409-429. 


\title{
Numerical approximation of a quasistatic evolution problem in cohesive fracture
}

\author{
M. Artina ${ }^{1}$, F. Cagnetti $^{1}$, M. Fornasier $^{2}$, F. Solombrino $^{1}$ \\ ${ }^{1} \mathrm{TU}$ Munich \\ ${ }^{2}$ University of Sussex
}

Given two Euclidean spaces $\mathcal{E}^{n}$ and $\mathcal{E}^{m}$ of dimension $n$ and $m$, respectively, with $n>m$, a (nonconvex, nonsmooth) energy functional $J: \mathcal{E}^{n} \rightarrow[0,+\infty)$, a linear operator $A: \mathcal{E}^{n} \rightarrow \mathcal{E}^{m}$, and a time dependent forcing $f:[0, T] \rightarrow \mathcal{E}^{m}$, we discuss under very general assumptions on $J$ and $A$ the convergence of a simple numerical procedure providing a discrete quasistatic evolution relative to the energy function $J$ and the linear constraint pair $(A, f)$. This one is defined as a measurable mapping $u:[0, T] \rightarrow \mathcal{E}^{n}$ satisfying the stationarity condition

$$
u(t) \text { is a critical point of } J \text { on the affine space }\{A v=f(t)\} \text { for almost every } t \in[0, T],
$$

as well as the following energy inequality: there exists a bounded measurable function $q:[0, T] \rightarrow \mathcal{E}^{m}$ such that $A^{*} q(t) \in \partial J(u(t))$ (the subdifferential of $J$ at $u(t)$ ) and

$$
J(u(t)) \leq J(u(0))+\int_{0}^{t}\langle q(s), \dot{f}(s)\rangle d s, \text { for almost every } t \in[0, T],
$$

where the scalar product $\langle\cdot, \cdot\rangle$ is the Euclidean one. The requiring of an energy inequality to be satisfied is motivated by the applications in continuum mechanics.

As a detailed case study, we indeed consider the functional $J$ coming from the finite element discretization in space, with mesh size $h>0$, of the cohesive fracture energy analysed in [2]

$$
\frac{1}{2} \int_{\Omega \backslash \Gamma}|\nabla u|^{2} d x+\int_{\Gamma} g\left(\left|u^{+}-u^{-}\right|\right) d \mathcal{H}^{d-1},
$$

with $\Gamma$ being a prescribed crack path, $u$ the displacement, $u^{+}$and $u^{-}$the traces of $u$ on the two sides of $\Gamma$, and $g:[0, \infty) \rightarrow[0, \infty)$ is, in general, a $C^{1}$, nondecreasing, bounded, concave function with $g(0)=0$ and $\sigma:=g^{\prime}\left(0^{+}\right) \in(0,+\infty)$. This energy is usually coupled with a linear constraint in the form of a time-dependent Dirichlet datum. In a simplified situation, that is when $d=2$ and $\Gamma$ is a rectilinear crack, we also show that the discrete evolution given by our constructive numerical procedure converges, for a mesh size $h \rightarrow 0$, to a continuum quasistatic evolution in the sense of [2], where existence had instead been shown by means of a vanishing viscosity approach.

\section{References}

[1] M. Artina, F. Cagnetti, M. Fornasier, F. Solombrino, in preparation.

[2] F. Cagnetti. A vanishing viscosity approach to fracture growth in a cohesive zone model with prescribed crack path. Math. Models Methods Appl. Sci. 18 (2008), no. 7, 1027-1071. 


\title{
Sparse optimal control of the KDV equation
}

\author{
Anne-Celine Boulanger, Philip Trautmann, Boris Vexler \\ Technische Universitaet Muenchen, Chair of Optimal Control
}

We focus in this work on optimal control problems of the following form

$$
\min _{q \in \mathcal{M}\left(\Omega, L^{2}(I)\right)} J(y)=\frac{1}{2}\left\|y-y_{d}\right\|_{L^{2}\left(\Omega, L^{2}(I)\right)}^{2}+\alpha\|q\|_{\mathcal{M}_{I}}
$$

where $y$ is the solution of the nonlinear Burgers-Korteweg de Vries equation with a time dependent measure valued source term acting as control

$$
\left\{\begin{array}{l}
\partial_{t} y+\partial_{x} y+\partial_{x x x} y-\gamma \partial_{x x} y+y \partial_{x} y=q \text { in } \Omega \\
y(., 0)=y(., L)=\partial_{x} y(., L)=0 \text { in } \Gamma \\
y(0, .)=0 \text { on } \Omega .
\end{array}\right.
$$

which is known to have traveling wave solutions. The control space $\mathcal{M}_{I}$ is either the Bochner space $L^{2}(I, \mathcal{M}(\Omega))$ or the space of vector measures $\mathcal{M}\left(\Omega \cdot L^{2}(I)\right)$ with values in $L^{2}(I)$. For both choices the controls are sparse in space and distributed in time. But the first space allows for moving dirac measures and the second not. We will tackle the following theoretical questions: well posedness of the KdV equation with a non-smooth source term, existence and characterization of an optimal control, algorithmic treatment of the problem by a semismooth Newton method in function space. In the end, we present some numerical examples that motivate our work: sparse stabilization of the KDV equation and sparse inverse source problems for the KDV equation (reconstruction of the topography and/or topography changes). 


\title{
Traffic flow control: avoiding shocks via variable speed limit
}

\author{
Maria Laura Delle Monache, Benedetto Piccoli, Francesco Rossi \\ Dept. of Mathematical Sciences, Rutgers University - Camden, Camden, NJ, USA, \\ Dept. of Mathematical Sciences and CCIB, Rutgers University - Camden, Camden, NJ, USA, \\ Aix Marseille Université, CNRS, ENSAM, Université de Toulon, Marseille, France
}

In this contribution, we propose a control strategy for a traffic flow problem. We concentrate on the variable speed limit (VSL) problem. This means that the control variable, i.e. the maximal velocity for the VSL problem, varies in space and can be used to control traffic flow in order to reduce traffic congestion. From a mathematical point of view, this leads to conservation laws, i.e. hyperbolic partial differential equations, with space-dependent flux, [4].

This kind of problems have been considered usually in a discrete setting due to the difficulties of applying optimal control theory in a continuous setting. We propose to actuate a control strategy that could be used in the continuous setting at least for the scalar conservation law. In fact, for many applications, not only the numerical simulation of the nonlinear dynamics is of interest but also optimization or control issues.

We consider the classical Lighthill-Whitham-Richards (LWR) model for traffic flow on a single infinite road $[2,3]$, with a space dependent flux function:

$$
\left\{\begin{array}{l}
\partial_{t} \rho(t, x)+\partial_{x} f(x, \rho(t, x))=0 \quad(t, x) \in \mathbb{R}^{+} \times \mathbb{R} \\
\rho(0, x)=\rho_{0}(x)
\end{array}\right.
$$

where $\rho \in\left[0, \rho_{\max }\right]$ is the mean traffic density and $\rho_{\max }$ the maximal density allowed on the road. We suppose that the flux function is space-dependent and it is given by $f(x, \rho)=v(x, \rho) \rho$ where $v(x, \rho)=V^{\max }(x) \rho(1-$ $\left.\frac{\rho}{\rho_{\max }}\right)$ is the mean traffic speed.

The maximal speed $V^{\max }(x)$ is the control parameter. We propose a theoretical approach and a numerical one to show how to reduce traffic congestion, i.e., avoiding shocks formation by modifying the maximal speed in space. In particular, we will show numerical simulations obtained with the Runge-Kutta discontinuous Galerkin method in the spirit of [1].

\section{References}

[1] S. Canic, B. Piccoli, J.-M. Qiu, T. Ren. Runge-Kutta discontinuous Galerkin method for traffic flow model on networks. pre-print. (2014).

[2] M. J. Lighthill, G. B. Whitham. On kinematic waves II. A theory of traffic flow on long crowded roads. Proc. Roy. Soc. London Ser. A 229 (1955), 317-346.

[3] P. I. Richards. Shock waves on the highway. Operations Research. 4 (1956), 42-51.

[4] P. Zhang, R.-X. Liu. Hyperbolic conservation laws with space-dependent flux: I. Characteristics theory and Riemann problem. Journ. of Comp. and Appl. Math. 156 (2003), 1-21. 


\title{
Luré dynamical systems - existence and stability results
}

\author{
J. Gwinner \\ Institute of Mathematics, Department of Aerospace Engineering, \\ Universität der Bundeswehr München, D - 85577 Neubiberg, Germany
}

In this contribution we study multivalued Luré dynamical systems that can be formulated as

$$
\left\{\begin{array}{l}
\dot{x}(t)=A x(t)+B u(t)+f(t) ; x(0)=x_{0} \\
u(t) \in \mathcal{M}[C x(t)+D u(t)]
\end{array}\right.
$$

where $\mathcal{M}$ is the subdifferential of a convex closed and proper function or more general monotone operator. Such dynamical systems were treated in finite dimensions in [1] with applications in nonsmooth electronics. Moreover, they arise in feedback control [2]. Here we extend recent existence and stability results [3, 4] to this class of dynamical systems.

\section{References}

[1] B. Brogliato , D. Goeleven. Well-posedness, stability and invariance results for a class of multivalued Luré dynamical systems. Nonlinear Analysis 74 (2011), $195-212$.

[2] P. Grabowski, F.M. Callier. Lur'e feedback systems with both unbounded control and observation: wellposedness and stability using nonlinear semigroups. Nonlinear Anal. 74 (2011), 3065 -3085.

[3] J. Gwinner. A note on linear differential variational inequalities in Hilbert space. t System modeling and optimization, IFIP Advances in Information and Communication Technology 391. (2013), 85-91.

[4] J. Gwinner, On a new class of differential variational inequalities and a stability result. Math. Programming 139 (2013), $205-221$. 


\title{
A smooth and localized version of the Hughes model for pedestrian flow
}

\author{
J. A. Carrillo, S. Martin and M.-T. Wolfram \\ Imperial College London \\ RWTH Aachen University \\ Radon Institute for Computational and Applied Mathematics
}

In the classical model of Hughes for crowd motion [1] pedestrians seek to minimize their travel time to a-priori known destinations/exits, but try to avoid regions of high density. This is achieved by coupling the continuity equation of the density $\rho(x, t)$ to an Eikonal equation, which determines the optimal walking paths. One of the basic assumptions is that the overall density of the crowd is known to every agent at every point in time. We present results on a modification that includes localizing effects such as limited vision to a Hughestype equation. The basic mechanism permits agents to perceive information on the current crowd density only in a local neighborhood, while taking assumptions on the density outside that region. Furthermore, we suggest a smoothing operator for the velocity field and discuss detailed boundary conditions. The model is presented on both a microscopic and macroscopic perspective. Our main object of study is the effect of localised path optimisation on the overall performance of the crowd in e. g. an evacuation scenario. We first analyse the domain of dependence for the planning problem of each pedestrians, and then quantify and illustrate the ability of the crowd to evacuate effectively with numerical experiments. Surprisingly, it will turn out that evacuation times can even improve in some situations. The question we investigate is hence complementary to mean-field game approaches [2] to crowd dynamics, where pedestrians anticipate future crowd states and therefore are more capable than in the original Hughes' model.

\section{References}

[1] R.L.Hughes. A continuum theory for the flow of pedestrians. TransportationResearch Part B: Methodological, 36(6). (2002), 507-535.

[2] M. Burger, M. Di Francesco, P. Markowich, and M.-T. Wolfram. Mean field games with nonlinear mobilities in pedestrian dynamics. DCDS B, 19(5). (2014), 1311-1333. 


\title{
On Modelling, Simulation and Optimisation of Solar Updraft Towers with Sloped Collectors
}

\author{
Ingenuin Gasser ${ }^{1}$, Muhammad Junaid Kamboh ${ }^{2}$ \\ ${ }^{1}$ Fachbereich Mathematik, Universität Hamburg, \\ Bundesstrasse 55, 20146 Hamburg, Germany \\ ${ }^{2}$ Technische Universität Hamburg Harburg, \\ Eißendorfer Straße 42, D-21073 Hamburg, Germany
}

This contribution is concerned with the modelling, simulation and optimization of so called solar updraft towers, a special form of a solar thermal power plant. The classical solar updraft towers have flat collectors $[1,2,3]$. Here we focus on a generalization of this idea including sloped collectors, designed in particular for higher latitudes on earth [4].

The main idea is to study how the reduction of the solar power (per aerea) at higher latitudes on earth can be compensated by a special non-planar collector of the power plant. Our model is again a generalization of a model proposed for flat collectors [5]. We focus mainly on gas dynamic issues and use a simple model for the energy transfer. Using an appropriate one dimensional model combined with asymptotic low Mach number techniques we are able to derive a simplified model which allows fast simulations and therefore optimization with respect to system parameters. We show simulations and optimize with respect to various parameters which are important when planning such a power plant.

\section{References}

[1] I. Cabanyes. Proyecto de motor solar. La Energia Electrica, 5(4) (1903).

[2] J. Schlaich, W. Schiel. Solar Chimneys. Encyclopedia of Physical Science and Technology, 3rd Edition, Academic Press, London (2001).

[3] G. Weinrebe. Das Aufwindkraftwerk Wasserkraftwerk der Wüste. Nova Acta Leopoldina NF 91, 339 (2004), $117-141$.

[4] E. Bilgen, J. Rheault. Solar chimney power plants for high latitudes. Solar Energy 79 (2005), 449-458.

[5] I. Gasser. Modelling and Simulation of a Solar Updraft Tower. American Institute of Mathematical Sciences 2 (1) (2009), 191-204. 


\title{
The identifiability approach for time-dependent full waveform inversion
}

\author{
Drosos Kourounis, Olaf Schenk \\ Institute of Computational Science \\ Università della Svizzera italiana
}

Full-waveform inversion (FWI) has long been considered the next logical step in deriving detailed velocity models. Despite recent advances in high-performance computing which make three dimensional acoustic FWI feasible today, it still remains a computationally very demanding inverse problem due to the huge number of geological parameters that need to be identified. A mathematically sound method is described for selecting the part of the parameter space that is best identifiable from the seismic acquisition geometry. This is combined with either interior-point or sequential quadratic programming methods for performing FWI for the subset of parameters that have been characterized as identifiable. The suggested approach results in a significant reduction of the parameter space. Local minima inherent to the original larger optimization space are avoided and the computational complexity of the inversion process, especially if second-order gradient information are desired, is therefore significantly decreased. 


\title{
Local minimization algorithms for dynamic programming equations
}

\author{
Dante Kalise, Axel Kröner, Karl Kunisch \\ Johann Radon Institute for Computational and Applied Mathematics \\ INRIA Saclay and CMAP, Ecole Polytechnique \\ Institute of Mathematics and Scientific Computing, University of Graz
}

To solve Hamilton-Jacobi Bellman (HJB) equations numerically often for evaluating the Hamiltonian the minimum over a set of admissible controls has to be determined. In particular, when considering control problems with nonsmooth cost functionals this is a challenging subject. In this talk we demonstrate the importance of redoing these minimization problems accurately and propose algorithms by which this can be achieved effectively. We focus the presentation on semi-Lagrangian (SL) schemes for first order HJB equations. The considered class of equations includes control problems with $L^{1}$-control term.

Starting with an infinite horizon control problem we introduce a SL-scheme and consider a first-order primaldual method (also known as Chambolle-Pock algorithm [1]) and semi-smooth Newton methods (see, e.g., [2]) for the minimization within the SL-scheme. In contrast to the broadly used approach, to compare the values for a finite subset of controls and to compute the minimum over this set by comparison, the proposed algorithms lead for the same cpu time to more accurate solutions and for specific settings convergence can be shown. For nonsmooth optimal control problems with $L^{1}$-control term sparse controls are observed.

\section{References}

[1] A. Chambolle and T. Pock, A first-order primal-dual algorithm for convex problems with applications to imaging, J. Math. Imaging Vis. 40 (2011), no. 1, 120-145.

[2] Ito, K., Kunisch, K.: Lagrange Multiplier Approach to Variational Problems and Applications, Advances in design and control, vol. 15. Society for Industrial Mathematics (2008). 


\title{
Optimization of Multirate Partial Differential Algebraic Equations
}

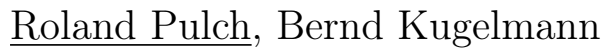 \\ Department of Mathematics and Computer Science, University of Greifswald, Germany
}

The mathematical modeling of electric circuits yields systems of differential algebraic equations (DAEs). We consider systems of the form

$$
\frac{\mathrm{d} \mathbf{q}(\mathbf{x})}{\mathrm{d} t}=\mathbf{f}(\mathbf{b}(t), \mathbf{x}(t))
$$

with the solution $\mathbf{x}:\left[t_{0}, t_{\text {end }}\right] \rightarrow \mathbb{R}^{k}$ and predetermined input signals $\mathbf{b}:\left[t_{0}, t_{\text {end }}\right] \rightarrow \mathbb{R}^{\ell}$. It holds that $\mathbf{q}: \mathbb{R}^{k} \rightarrow \mathbb{R}^{k}$ and $\mathbf{f}: \mathbb{R}^{\ell} \times \mathbb{R}^{k} \rightarrow \mathbb{R}^{k}$. We assume that the solution represents a high-frequency oscillation, whose amplitude as well as frequency changes slowly in time due to the input signals. It follows that a time integration of the system (1) becomes costly, since each fast oscillation has to be resolved. Alternatively, a multidimensional signal model changes the system of DAEs (1) into a system of multirate partial differential algebraic equations (MPDAEs), see $[1,2]$. We obtain the system

$$
\frac{\partial \mathbf{q}(\hat{\mathbf{x}})}{\partial t_{1}}+u\left(t_{1}\right) \frac{\partial \mathbf{q}(\hat{\mathbf{x}})}{\partial t_{2}}=\mathbf{f}\left(\mathbf{b}\left(t_{1}\right), \hat{\mathbf{x}}\left(t_{1}, t_{2}\right)\right)
$$

with the solution $\hat{\mathbf{x}}:\left[0, T_{1}\right] \times\left[0, T_{2}\right] \rightarrow \mathbb{R}^{k}$ and a local frequency function $u:\left[0, T_{1}\right] \rightarrow \mathbb{R}$. Both initial-boundary value problems (IBVPs) and biperiodic boundary value problems (BBVPs) are considered for the system (2).

The local frequency function represents a degree of freedom in the multidimensional model. The aim is to identify appropriate solutions $\hat{\mathbf{x}}$, which exhibit a low amount of variation such that the solution can be resolved on a relatively coarse grid. For this purpose, we investigate minimization problems based on either the functional

$$
J(\hat{\mathbf{x}}):=\int_{0}^{T_{1}} \int_{0}^{T_{2}}\left\|\frac{\partial \hat{\mathbf{x}}}{\partial t_{1}}\right\|_{2}^{2} \mathrm{~d} t_{2} \mathrm{~d} t_{1} \quad \text { or } \quad I\left(t_{1}, \hat{\mathbf{x}}\right):=\int_{0}^{T_{2}}\left\|\frac{\partial \hat{\mathbf{x}}}{\partial t_{1}}\right\|_{2}^{2} \mathrm{~d} t_{2} \quad \text { for each } t_{1} \in\left[0, T_{1}\right] .
$$

The functional $J$ is defined for the global solution, whereas the functional $I$ is specified pointwise. Necessary conditions for optimality were derived for IBVPs in [3] and for BBVPs in [4] by assuming the existence and uniqueness of an optimal solution. Numerical methods including these necessary conditions yield the optimal solution.

In our contribution, we prove the existence and uniqueness of optimal solutions with respect to each functional in (3), which satisfy either IBVPs or BBVPs of the MPDAE system (2). Moreover, it can be shown that the pointwise minimization based on the functional $I$ is equivalent to the global minimization using the functional $J$. This property allows for solving IBVPs by a method of lines, where the pointwise minimization is done successively. Furthermore, we interpret the minimization problems in the context of optimal control. It follows that the direct approach in optimal control is inefficient for this problem class, whereas the indirect approach in optimal control represents the method of choice. The numerical solution of BBVPs can be done by a method of characteristics including the necessary conditions for an optimal solution, since the MPDAEs (2) exhibit a hyperbolic structure. We present the numerical simulation of a test example modeling an electric circuit, whose solution features both amplitude modulation and frequency modulation.

\section{References}

[1] H.G. Brachtendorf, G. Welsch, R. Laur, A. Bunse-Gerstner: Numerical steady state analysis of electronic circuits driven by multi-tone signals. Electr. Eng. 79 (1996), 103-112.

[2] O. Narayan, J. Roychowdhury: Analyzing oscillators using multitime PDEs. IEEE Trans. CAS I 50 (2003), 894-903.

[3] R. Pulch: Initial-boundary value problems of warped MPDAEs including minimisation criteria. Math. Comput. Simulat. 79 (2008), 117-132.

[4] R. Pulch: Variational methods for solving warped multirate partial differential algebraic equations. SIAM J. Sci. Comput. 31 (2008), 1016-1034. 


\title{
A priori error estimates for nonstationary optimal control problems with gradient state constraints
}

\author{
Francesco Ludovici ${ }^{1}$, Ira Neitzel ${ }^{2}$, Winnifried Wollner ${ }^{1}$ \\ ${ }^{1}$ Hamburg Universität \\ ${ }^{2}$ Technische Universität München
}

This talk deals with error estimates for space-time finite element discretization of semilinear parabolic optimal control problems subject to inequality constraints on the gradient of the state variable.

In particular, we will focus on pointwise in time and averaged in space gradient state constraints of the form

$$
\int_{\Omega}|\nabla u(x, t)|^{2} \omega(x) d x, \forall t \in[0, T],
$$

where $u$ denotes the state variable and $\omega(x)$ is a weighting function.

Consideration of these constraints is motivated by industrial applications in the steel and glass production, where stress averages are often considered in order to avoid material failure.

Making use of the discontinuous Galerkin method for the time discretization and of standard conforming finite elements for the space discretization, we derive convergence rates as temporal and spatial mesh size tends to zero. 


\title{
Direct and indirect multiple shooting for parabolic optimal control problems
}

\author{
Thomas Carraro, Michael Geiger \\ Institute for Applied Mathematics, Heidelberg University
}

In the context of ordinary differential equations, shooting techniques have become a state-of-the-art solver component, whereas their application with partial differential equations (PDE) is still in an early phase of development. We present two multiple shooting approaches for optimal control problems governed by parabolic PDE. We derive both direct and indirect shooting for PDE optimal control from the same extended problem formulation. This approach shows that they are algebraically equivalent on an abstract function space level. However, discussing their algorithmic realizations, we underline the differences between direct and indirect multiple shooting. In the presented numerical examples we cover both linear and nonlinear parabolic side conditions.

\section{References}

[1] T. Carraro, M. Geiger, R. Rannacher. Indirect Multiple Shooting for Nonlinear Parabolic Optimal Control Problems with Control Constraints. SIAM J. Scientific Computing, Vol. 36(2), pp. A495-A521(2014).

[2] T. Carraro, M. Geiger. Direct and Indirect Multiple Shooting for Parabolic Optimal Control Problems. Multiple Shooting and Time Domain Decomposition Methods, Springer (to appear in 2015). 


\title{
Functional a posteriori estimates for cost functionals of elliptic optimal control problems
}

\author{
Johann Radon Institute for Computational and Applied Mathematics \\ Austrian Academy of Sciences
}

In this talk, new results on functional type a posteriori estimates for elliptic optimal control problems with control constraints are presented, see [1]. More precisely, we derive new, sharp, guaranteed and fully computable lower bounds for the cost functional in addition to the already existing upper bounds, see [2]. Using both, the lower and the upper bounds, we arrive at two-sided estimates for the cost functional. These bounds finally lead to sharp, guaranteed and fully computable upper estimates for the discretization error in the state and the control of the optimal control problem.

\section{References}

[1] M. Wolfmayr, A note on functional a posteriori estimates for elliptic optimal control problems, Ricam-Report 2014-39, 2014.

[2] A. Gaevskaya, R. H. W. Hoppe, and S. Repin, A posteriori estimates for cost functionals of optimal control problems, Numerical Mathematics and Advanced Applications, Proceedings of the ENUMATH 2005, (2006), pp. 308-316. 


\title{
Multiobjective Optimization of the Flow Around a Cylinder Using Model Order Reduction
}

\author{
$\underline{\text { Sebastian Peitz, Michael Dellnitz }}$ \\ Insititute for Industrial Mathematics and Chair for Applied Mathematics \\ University of Paderborn \\ Warburger Str. 100 \\ 33098 Paderborn, Germany
}

In many applications the optimization of multiple, potentially conflicting objectives is desired. In this case, one is interested in calculating the set of optimal compromises between these goals, the so-called Pareto set [1], instead of searching for one specific optimum. This is expressed as a multiobjective optimization problem:

$$
\begin{gathered}
\min _{x \in \mathbb{R}^{n}}\{F(x)\}, \\
F: \mathbb{R}^{n} \rightarrow \mathbb{R}^{k}, F(x)=\left(f_{1}(x), \ldots, f_{k}(x)\right) .
\end{gathered}
$$

There exist various approaches to address (MOP) such as scalarization techniques (weighted sum) [2], continuation methods [2] or evolutionary algorithms [3]. Additionally, set oriented algorithms have proven to be very efficient for the computation of Pareto sets, e.g. the subdivision algorithm presented in [4], where the set is approximated by a nested sequence of increasingly fine box coverings. This method is capable of calculating the entire, globally optimal Pareto set, also in the situation where the set is disconnected.

All algorithms computing a set of optima have in common a high number of function evaluations. Consequently, when the evaluation of the system under consideration is costly, as is the case for dynamical systems described by partial differential equations, the computation quickly becomes infeasible. To avoid this problem, model order reduction such as Proper Orthogonal Decomposition (POD) [5] or Dynamic Mode Decomposition (DMD) [6] can be a useful tool to reduce the computational effort.

In this presentation, we apply the subdivision algorithm described above to the two-dimensional flow around a rotating cylinder in order to calculate the Pareto set for the competing objectives lift $\left(C_{L}\right)$ an drag $\left(C_{D}\right)$ :

$$
\min _{u \in U} J(y(u))=\min _{u \in U}\left(\begin{array}{c}
-C_{L}(y(u)) \\
C_{D}(y(u))
\end{array}\right) \text { s.t. } e(y(u), u)=0,
$$

where the state $y=\left(v_{x}, v_{y}, p\right)^{T}$ consists of the velocity and pressure field of the fluid, $u=\left(v_{\partial \Omega_{i n}}, \omega_{c y l}\right)^{T} \in U$ is the control vector consisting of the inflow velocity and the angular velocity of the cylinder and $e$ is an equality constraint induced by the 2D incompressible Navier-Stokes equations. We then approximate the dynamical system by a reduced order model using DMD and a Galerkin Projection and solve (P1) based on the reduced model. Finally, we analyze the tradeoff between computational effort and quality of the approximation given by this method.

\section{References}

[1] K. Miettinen, Nonlinear Multiobjective Optimization. International series in operations research \& management science (1999).

[2] C. Hillermeier. Nonlinear Multiobjective Optimization: A Generalized Homotopy Approach. Springer (2001).

[3] O. Schütze, S. Mostaghim, M. Dellnitz, J. Teich. Covering Pareto sets by multilevel evolutionary subdivision techniques. Evolutionary multi-criterion optimization. Springer Berlin Heidelberg (2003).

[4] M. Dellnitz, O. Schütze, T. Hestermeyer. Covering Pareto Sets by Multilevel Subdivision Techniques. Journal of Optimization Theory and Applications 124(1) (2005), 113-136.

[5] P. Holmes, J. L. Lumley, G. Berkooz. Turbulence, coherent structures, dynamical systems and symmetry. Cambridge university press (1998).

[6] P. J. Schmid. Dynamic mode decomposition of numerical and experimental data. Journal of Fluid Mechanics 656 (2010), 5-28. 


\title{
A Certified Reduced Basis Approach for Parametrized Linear-Quadratic Optimal Control Problems with Control Constraints
}

\author{
Eduard Bader*, Mark Kärcher*, Martin Grepl ${ }^{\dagger}$, Karen Veroy-Grepl* \\ * Aachen Institute for Advanced Study in Computational Engineering Science, \\ RWTH Aachen University, Schinkelstraße 2, 52062 Aachen, Germany \\ (e-mail: bader,kaercher,veroy@aices.rwth-aachen.de); \\ † Numerical Mathematics, RWTH Aachen University, Templergraben 55, \\ 52056 Aachen, Germany (e-mail: grepl@igpm.rwth-aachen.de)
}

\begin{abstract}
Many problems in science and engineering can be modeled in terms of optimal control problems governed by parametrized partial differential equations (PDEs). While the PDE describes the underlying system or component behavior, the parameters often serve to identify a particular configuration of the component — such as boundary and initial conditions, material properties, and geometry. In such cases - in addition to solving the optimal control problem itself — one is often interested in exploring many different parameter configurations and thus in speeding up the solution of the optimal control problem. However, using classical discretization techniques such as finite elements or finite volumes even a single solution is often computationally expensive and time-consuming, a parameter-space exploration thus prohibitive. One way to decrease the computational burden is the surrogate model approach, where the original high-dimensional model is replaced by a reduced order approximation. These ideas have received a lot of attention in the past and various model order reduction techniques have been used in this context. However, the solution of the reduced order optimal control problem is generally sub-optimal and reliable error estimation is thus crucial. Besides serving as a certificate of fidelity for the sub-optimal solution, our a posteriori error bounds are also a crucial ingredient in generating the reduced basis with greedy algorithms.

A new approach for efficient computation of error bounds for unconstrained distributed control problems was proposed in [2]. This approach, however, and all other existing approaches in the literature, see e.g. [3, 4, 5], are not directly applicable to the important case with additional constraints on the control.

In this work we extend the methodology presented in [6] to consider PDE-constrained optimal control problems. The authors in [6] develop a certified Reduced Basis (RB) method that provides sharp and inexpensive a posteriori error bounds for variational inequalities. In particular, the approach has advantages compared to prior work on variational inequalities with the RB method [1]. The methodology in [6] not only $(i)$ provides sharper error bounds that mimic the convergence rate of the RB approximation, but also ( $i i)$ does so at an online cost that is independent of the high dimension of the original problem.

In particular we use the approach presented in [6] $(i)$ to construct a feasible RB approximation of the control and ( $i$ i) to derive efficiently computable a posteriori error bounds. The main idea is to generate two RB-systems. The first one is "standard" and is used to construct low dimensional approximations for the state and the Lagrange multiplier. In the second one we construct nonnegative slack variables for the control and so can generate feasible low dimensional surrogates for the control. Finally, we extend the proof of the $a$ posteriori error bounds from [2] to derive efficient a posteriori bounds for the control error in the constraint case.
\end{abstract}

\section{References}

[1] B. Haasdonk, J. Salomon, and B. Wohlmuth. A reduced basis method for parametrized variational inequalities. SIAM J. Numer. Anal., 50(5), (2012), 2656-2676.

[2] M. Kärcher, M. Grepl, and K. Veroy. Certified reduced basis methods for parametrized distributed optimal control problems, submitted to SIAM Journal on Control and Optimization, (2014).

[3] F. Negri, G. Rozza, A. Manzoni, and A. Quarteroni. Reduced basis method for parametrized elliptic optimal control problems. SIAM J. Sci. Comput., (2013). Accepted for publication.

[4] F. Negri. Reduced basis method for parametrized optimal control problems governed by PDEs. Master's thesis, Politecnico di Milano, (2011). 
[5] G. Rozza, A. Manzoni, and F. Negri. Reduction strategies for pde-constrained optimization problems in haemodynamics. In Proceedings ECCOMAS Congress, Vienna Austria, (2012).

[6] Z. Zhang, E. Bader, and K. Veroy. A duality approach to error estimation for variational inequalities, submitted to SIAM Journal on Scientific Computing, (2014), URL http://arxiv.org/abs/1410.2095. 


\title{
Wavelet-based lossy trajectory compression for optimal control of parabolic PDEs
}

\author{
Sebastian Götschel, Martin Weiser \\ Zuse Institute Berlin \\ \{goetschel,weiser\}@zib.de
}

For the solution of optimal control problems governed by parabolic PDEs, methods working on the reduced objective functional are often employed to avoid a full spatio-temporal discretization of the problem. The evaluation of the reduced gradient requires one solve of the state equation forward in time, and one backward solve of the adjoint equation. As the state enters into the adjoint equation, the storage of a full $4 \mathrm{D}$ data set is needed. If Newton-CG methods are used, two additional trajectories have to be stored. To get accurate numerical results, in many cases very fine discretizations in time and space are necessary, which leads to a significant amount of data to be stored and transmitted to mass storage.

To overcome these storage problems, we developed and analyzed a lossy compression method, using the hierarchic basis representation of the finite element solution combined with quantization of coefficients. Due to a pointwise control of the compression error, the error transport of the adjoint equation is not taken into account $[1,2,3]$.

In this talk, we present a more advanced compression scheme, based on a lifted wavelet transform, which allows to control the error in the $L^{2}$ - and $H^{-1}$-norm instead of $L^{\infty}$. We discuss the adaptive choice of quantization tolerances and give numerical examples.

Keywords: optimal control, parabolic PDEs, trajectory storage, adaptive lossy compression MSC 2000: 35K55, 49M15, 65M60, 68P30, 94A29

\section{References}

[1] S. Götschel, N. Chamakuri, K. Kunisch, M. Weiser. Lossy compression in optimal control of cardiac defibrillation. J. Sci. Comput. 60(1) (2014), 35-59.

[2] S. Götschel, M. Weiser. Lossy compression for PDE-constrained optimization: adaptive error control. Comput. Optim. Appl. (2014), doi:10.1007/s10589-014-9712-6.

[3] M. Weiser, S. Götschel. State trajectory compression for optimal control with parabolic PDEs. SIAM J. Sci. Comput. 34(1) (2012), A161-A184. 


\section{S20: Dynamics and control}

Dynamics and control is an interdisciplinary section which in particular adresses mathematical systems theory and control engineering. The contributions to this section are also concerned with the mathematical understanding of the dynamics of controllers which appear in actual applications. 


\title{
Control design of the vibration reduction systems
}

\author{
Igor Maciejewski, Tomasz Krzyzynski \\ Departament of Mechatronics and Applied Mechanics \\ Faculty of Technology and Education \\ Koszalin University of Technology \\ Sniadeckich St. 2, Koszalin, 75-453 PL
}

The paper deals with an original control algorithm for semi-active and active suspension that are often used in automotive systems. The developed procedure assists in finding the structure of control system, which can predict the desired active force, is able to adapt to various working conditions and is robust in response to varying mass loading of the suspension system. The control system consists of the primary controller that calculates the desired active force. Then the desired force has to be approximately achieved by the semi-active or active element with calculated input signal using the reverse model [1]. The PD predictor speeds up the semi-active or active system control because the actuating time of the force actuator is partly eliminated.

\section{References}

[1] M. Maslanka, B. Sapinski. Experimental study of vibration control of a cable with an attached MR damper. Journal of Theoritical and Applied Mechanics 45(4) (2007), 893-917. 


\title{
Coordination of guidance and stabilization tasks for bicycle rider modelling
}

\author{
Johannes Edelmann, Martin Haudum, Manfred Plöchl \\ Institut für Mechanik und Mechatronik \\ Technische Universität Wien
}

Based on a nonlinear mathematical bicycle-rider model, this study aims to contribute to understanding the steering behaviour of a bicycle rider. In contrast to automobile drivers at regular driving conditions, the bicycle rider has to stabilize the motion of the bicycle at low velocities to prevent toppling over, see e.g. [1], in addition to guiding the vehicle along the desired trajectory. The human bicycle rider may accomplish these tasks by applying steering torque, roll torque or movement of his/her knees, [2]. As the dynamic response of the yaw rate and steering angle to a steering torque input, see e.g. [3], or roll torque input shows in general non-minimum phase characteristics, the control bandwidth is limited to low frequencies. Thus, the guiding capabilities of the bicycle rider are restricted by this bandwidth limitation. Considering the stability properties of the system at low velocities, in addition, the rider has to stabilize the motion of the bicycle. Hence, the control requirements of the bicycle-rider system become even more demanding.

In this study, the controllability of the bicycle-rider system is investigated by applying either steering or roll torques, and consequences of the characteristic dynamic behaviour of the system on the bicycle rider control demands are addressed. Based on findings on the control behaviour of aircraft pilots and automobile drivers, a three-layer bicycle rider model is presented that aims to mimic the human rider control behaviour. The steering behaviour is represented by an anticipatory feed-forward control layer and a compensatory feedback control layer to guide the bicycle along a desired trajectory, and a stabilizing feedback control layer to stabilize the motion of the bicycle within its unstable velocity range. While the feed-forward control layer is based on the 'ideal driver', [4], different approaches to represent both the compensatory and stabilizing feedback control layer are discussed considering human cognition and motor capabilities.

\section{References}

[1] R. Hess, J.K. Moore, M. Hubbard. Modeling the manually controlled bicycle. IEEE Transactions on Systems, Man, and Cybernetics - Part A: Systems and Humans 42 (2012), 545-557.

[2] J.D.G. Kooijman, A.L. Schwab, J.K. Moore. Some observations on human control of a bicycle, Proc. of ASME 2009 International Design Engineering Technical Conferences \& Computers and Information in Engineering Conference, DECT2009-86959, August 30 - September 2, 2009, San Diego, CA.

[3] D.J.N. Limebeer, R.S. Sharp. Bicycles, motorcycles, and models. IEEE Control Systems Magazine 26 (2006), $34-61$.

[4] M. Mitschke. Driver-vehicle-lateraldynamics under regular driving conditions. Vehicle System Dynamics 22 (1993), 483-492. 


\title{
Parameter identification of a scaled experimental running gear
}

\author{
Alexander Keck*, Christoph Schwarz* \\ *German Aerospace Center, Institute of System Dynamics and Control, Oberpfaffenhofen
}

For the development of a mechatronic track guidance system in the DLR project "Next Generation Train" steerable, individually powered, independently rotating wheels (IRW) are favored [2]. This configuration offers optimal radial adjustment between the rolling and moving direction. Thus unnecessary creep in the wheel rail contact is avoided and a significantly reduced wear is expected especially in tight curves [1]. To test the system behavior and develop appropriate control algorithms for the mechatronic track guidance system, an experimental running gear for the existing 1:5 roller rig was designed and manufactured at DLR. The running gear is equipped with several measurement devices such as laser position and force/torque sensors. In addition, the DLR RailwayDynamics Library implemented in the simulation environment Modelica/Dymola is used to model and simulate the behavior of the scaled running gear [3].

In order to receive a simulation model, that coincides with the real running gear in the frequency range of interest, it is necessary to quantify the characteristic parameters of the system. To this aim several methods have been applied in this work: CAD data were utilized, specific parameters of the system were measured individually and the system behavior in simulation and experiment were compared by exploiting the comprehensive sensor data of the test rig. Individual measurements were preferred to identify the stiffness of the virtual rotational spring, which acts around the z-axis between the wheel-carrier and the frame. This effect is not only based on the vertical primary suspension of the running gear but also influenced by the parasitic friction of the motor cables going from the frame to the wheel-modules.

In order to explore the technical boundaries of the wheel hub motors a pseudo random binary sequence (PRBS) is used to determine their dynamic properties. On the basis of these measurements a transfer function for each of the four wheel hub motors is built and introduced in the simulation model. Finally an optimization task was performed in which experimental results of the running gear on the test rig were compared with simulation results in order to adjust additional parameters. The presentation of the initial control design of the running gear and its behavior in simulation and experiment will conclude the presentation.

\section{References}

[1] B. Kurzeck, A. Heckmann, C. Wesseler, M. Rapp. Mechatronic track guidance on disturbed track: The trade-off between actuator performance and wheel wear. Vehicle System Dynamics 52 (2014), 109-124.

[2] B. Kurzeck, A. Heckmann, I. Kaiser, C. Wesseler, A. Keck. Potenziale aktiv geregelter Losradfahrwerke im Hochgeschwindigkeitsverkehr - Ergebnisse aus dem DLR Projekt "Next-Generation-Train". ZEVrail Zeitschrift fÃCEr das gesamte System Bahn 138 (2014), 304-312.

[3] A. Heckmann, A. Keck, I. Kaiser, B. Kurzeck. The Foundation of the DLR RailwayDynamics Library: the Wheel-Rail-Contact. Proceedings of the 10th International ModelicaConference (2014). 


\title{
Nonlinear control of a variable displacement vane pump
}

\author{
Marius Köster, Alexander Fidlin \\ Institut für Technische Mechanik, Karlsruher Institut für Technologie
}

\begin{abstract}
Variable displacement vane pumps are used for mid-pressure applications in a variety of systems. Due to their potential for power loss reduction they are most prominently featured in automotive applications. Variable displacement vane pumps provide a hydraulic consumer with varying levels of volume flow, depending on the eccentricity of the pump. Eccentricity control is typically achieved by means of hydraulic actuation. For some applications, hydraulic actuation is a result of an appropriate choice and design of self-regulating valves. For more advanced applications, servo valves can be utilized - allowing for a more sophisticated control of the systems. As hydraulic systems are inherently nonlinear due to the physics governing the processes involving hydraulic elements, adequate control strategies should take these nonlinearities into account. In practice, most control strategies in hydraulic systems have remained linear despite the advances in nonlinear control during the past decades, resulting in suboptimal performance of the controled systems. However, successfull application of nonlinear control strategies in the context of hydraulic systems has been reported [1], [2]. Therefore, in this contribution a volume flow control strategy based on input-output linearization [4] of a variable displacement vane pump system is suggested.
\end{abstract}

The system at hand consists of a variable displacement vane pump, a load modeled by a hydraulic resistance and a servo valve. Assuming an ideal valve with no dynamic behavior, the system can be represented by a four state system model. Because the pump is actuated by volume flow provided by the pump itself, the pump system dynamics are intricate and feature a switching behavior in correspondence with desired increases or decreases in volume flow. To describe the switching behavior, two sub systems are introduced to the system description. In order to compensate the system's switching behavior, an input-output linearization is performed for each of the sub-systems. The relative degrees of the sub-systems are identical with $r=3$, leaving stable zero dynamics in the form of volume flow balances that are subject to the switching behavior of the system. A linear controller is then applied to the partially linearized system, yielding asymptotic trajectory tracking behavior in accordance with the requirements.

Based on the Byrnes-Isidori normal form, a normal form observer following Roebenack [3] then is constructed in order to obtain the states required for the nonlinearity-compensating feedback. The validity of both control and observation strategy are shown in simulations.

\section{References}

[1] J. Chiriboga et al. Input-Output feedback linearization control of a load-sensing hydraulic system. Control Applications, Proceedings of the 4th IEEE Conference on Control Applications. IEEE, (1995).

[2] H. Hahn et al. Input/Output linearization control of an electro servo-hydraulic actuator. Control Applications, Proceedings of the Third IEEE Conference on Control and Applications. IEEE, (1994).

[3] K. Röbenack. Zum High-Gain Beobachterentwurf für eingangs-/ausgangslinearisierbare SISO-Systeme. Automatisierungstechnik 10/(2004) 481-488.

[4] S. Sastry. Nonlinear Systems. Springer. (1999). 


\title{
A kinematic approach based on an equivalent track for a skid-steering robot
}

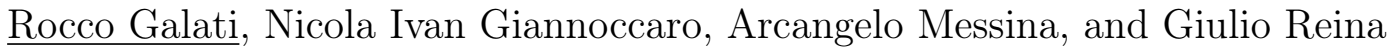 \\ Università del Salento, Dipartimento di Ingegneria dell'Innovazione \\ Via per Monteroni 73100, Lecce, Italy \\ e-mail: rocco.galati@unisalento.it
}

The skid steering principle is based on controlling the relative velocities of both the tracks as differential drive wheeled vehicles. However, control of tracked locomotion poses a more complex problem because variation of the relative velocity of the two tracks results often in slippage of the vehicle. This contribution propose a kinematic approach for improving the motion control and dead-reckoning of a skid-steering robot, considering the effects of the slippage but without introducing the complexity of dynamics computation. This problem has been recently tackled by researchers and some experimental results for some types of tracked robots have reported in [1], [2], [3], [4].

The equivalent track approach has been here applied to a skid-steering robot (Husky A200), characterized by an high maximum payload $(75 \mathrm{~kg}$ ) and by a high power than makes it able to move in very difficult conditions (snow, iced terrains, etc.), where the slippage effects become predominant. The equivalent track of the kinematic model has been identified by means of several experimental tests with different velocities of the robot wheels measured by integrated encoders. The equivalent track model has then been used for outdoor tests in closed loops; the odometric estimation results are encouraging showing very low errors in the reconstruction of the trajectory. Details about the robots characteristics, the approach algorithm and the experimental results will be given in the presentation and in the full paper.

\section{References}

[1] J. Martinez, A. Mandow, J. Morales, S. Pedraza, A. Garcia-Cerezo, The Int. J. of Robotic Research 24, 867-878 (2005).

[2] M. Berenguel, Proceedings Int. Conf. on Mechatronics, (2009), pp. 1-6.

[3] G. Longoria, Proceedings Am. Contr. Conf., (2012), pp. 6816-6821.

[4] M. Burke, Proceedings 2012 IEEE Int. Conf. on Rob. And Automation, (2012), pp. 97-102. 


\title{
Harmonic Mistuning of Blisks
}

\author{
Linus Pohle, Lars Panning-von Scheidt, Jörg Wallaschek \\ Institute of Dynamics and Vibration Research \\ Leibniz Universität Hannover \\ Appelstr. 11, 30167 Hannover, Germany
}

\begin{abstract}
Manufacturing tolerances and wear cause small deviations of the turbine blades' geometry. Such damage to the cyclic symmetry can effect a significant amplification of the vibration amplitudes. To estimate the maximum vibration amplitude, two common methods are useable. On the one hand, the limit for the overall maximum vibration amplitude can be calculated using the WHITEHEAD factor [1]. This factor overestimates the amplitude of a random mistuning pattern. On the other hand, Monte-Carlo simulations are used to estimate the maximum amplitude of the given blade tolerances [2]. This method is very time-consuming and requires high computational effort.

The full length paper describes a new method to calculate the maximum amplification of a given manufacturing tolerance. Two effects of mistuned blisks are analyzed in this paper. The separation of two orthogonal nodal diameters could be shown and described in detail. Furthermore, the excitation of more than one nodal diameter by only one engine order could be found. If the eigenfrequencies of these nodal diameters are close together, the vibration amplitude of some blades increases. Based on a lamp-mass model, it is shown which maximum amplitude can be achieved. The idea is to use a harmonic mistuning pattern to get the highest influence at small tolerances for one single nodal diameter. This influence cannot be beaten by a randomly mistuned system and limits the maximum amplitude. The given analysis is validated by a common Monte-Carlo simulation. Based on this analysis, the benefits of an intentional mistuning are shown. Due to the separation of the nodal diameter, the vibration amplitude is robust towards small mistuning tolerances. Furthermore, it is possible to split off close nodal diameters to prevent mode interaction.
\end{abstract}

\section{References}

[1] D. S. Whitehead: Effect of Mistuning of the Vibration of Turbomachine Blades Induced by Wakes. J. Mechanical Engineering Science 8 15-21. (1966).

[2] P. Castanier, C. Pierre: Investigation of the Combined Effects of Intentional and Random Mistuning on the Forced Response of Bladed Disks. AIAA-98-3720. (1998). 


\title{
Stability analysis of implicit difference equations
}

\author{
V. Mehrmann ${ }^{1}$, D.D. Thuan ${ }^{2}$ \\ ${ }^{1} \mathrm{TU}$ Berlin, ${ }^{2}$ Hanoi University of Science and Technology
}

The stability analysis for linear implicit $m$-th order difference equations in descriptor form is discussed. We allow the leading coefficient coefficient to be singular, i.e., we include the situation that the system does not generate an explicit recursion. A spectral condition for the characterization of asymptotic stability is presented and computable formulas are derived for the real and complex stability radii in the case that the coefficient matrices are subjected to structured perturbations.

\section{References}

[1] V. Mehrmann and D.D. Thuan, Stability analysis of implicit difference equations under restricted perturbations. PREPRINT 16/2014, Institut für Mathematik, TU Berlin, 2014. url: http://www.math.tuberlin.de/preprints/Submitted for publication, 2014. 


\title{
Controllability characterization for switched DAEs
}

\author{
Ferdinand Küsters, Stephan Trenn \\ University of Kaiserslautern, Germany
}

Switched differential algebraic equations (switched DAEs) of the form

$$
E_{\sigma} \dot{x}=A_{\sigma} x+B_{\sigma} u
$$

with switching signal $\sigma: \mathbb{R} \rightarrow\{1,2, \ldots, N\}$ play an important role when modeling systems with algebraic constraints subject to sudden structural changes, for example electrical circuits with switches. Controllability is a fundamental systems property and is concerned about the ability to reach any final feasible state from any initial feasible state. Based on the single switch result presented at the previous GAMM Annual Meeting [1] the controllability characterization will be generalized to the general case. Furthermore, several examples are presented which highlight special features of controllability for switched DAEs, for example, the dependence of the switching times as well as the ability for instantaneous control. Some pitfalls leading to some incorrect statements in [1] are also highlighted.

\section{References}

[1] M. G.-M. Ruppert and S. Trenn (2014), Controllability of switched DAEs: The single switch case, PAMM 14 (Special Issue: 85th Annual Meeting of the GAMM), to appear 


\title{
The Kalman-Yakubovich-Popov Inequality for Differential-Algebraic Equations
}

\author{
Timo Reis ${ }^{1}$, Olaf Rendel ${ }^{1}$, Matthias Voigt ${ }^{2}$ \\ ${ }^{1}$ Universität Hamburg, Fachbereich Mathematik \\ ${ }^{2}$ Technische Universität Berlin, Institut für Mathematik
}

The Kalman-Yakubovich-Popov lemma is one of the most famous results in systems and control theory. Loosely speaking, it states equivalent conditions for the positive semi-definiteness of a so-called Popov function on the imaginary axis in terms of the solvability of a certain linear matrix inequality, namely the KalmanYakubovich-Popov (KYP) inequality. In applications, this lemma plays an important role in assessing feasibility of linear-quadratic optimal control problems or characterizing dissipativity of linear control systems.

In the literature, there exist manifold attempts to generalize this lemma to differential-algebraic equations. However, most of these approaches make certain restrictive assumptions such as a bounded index or impulse controllability. In this talk we show how to drop these restrictions by considering the KYP inequality on the system space, i.e., the subspace in which the solution trajectories of the system evolve. Moreover, we present results on the solution structure of this inequality. In particular, we consider rank-minimizing, stabilizing, and extremal solutions. These results can then be interpreted as a generalization of the algebraic Riccati equation to a very general class of differential-algebraic control systems, see [1,2] for details.

\section{References}

[1] T. Reis, O. Rendel, M. Voigt. The Kalman-Yakubovich-Popov inequality for differential-algebraic equations. Hamburger Beiträge zur angewandten Mathematik 2014-27 (2014).

[2] M. Voigt. On Linear-Quadratic Optimal Control and Robustness of Differential-Algebraic Systems, Dissertation, Otto-von-Guericke-Universität Magdeburg (2015). In preparation. 


\title{
Controlled invariance for DAEs
}

\author{
Thomas Berger \\ Universität Hamburg, Germany
}

We study the concept of locally controlled invariant submanifolds for nonlinear descriptor systems. In contrast to classical approaches, we define controlled invariance as the property of solution trajectories to evolve in a given submanifold whenever they start in it. It is then shown that this concept is equivalent to the existence of a feedback which renders the closed-loop vector field invariant in the descriptor sense. This result is motivated by a preliminary consideration of the linear case.

Local controlled invariance leads to the concept of output zeroing submanifolds. We show that the outcome of the differential-algebraic version of the zero dynamics algorithm yields a maximal output zeroing submanifold. The latter is then used to characterize the zero dynamics of the system. In order to guarantee that the zero dynamics are locally autonomous (i.e., locally resemble the behavior of an autonomous dynamical system), sufficient conditions involving the locally maximal output zeroing submanifold are presented. 


\title{
Control of underactuated systems with coupling input forces
}

\author{
Sebastian Korczak \\ Warsaw University of Technology
}

The dynamic system is called underactuated if unlimited control inputs cannot produce any system accelerations. The most popular reason of that is a lesser number of inputs than the number of degrees of freedom. Current review of underactuated systems and their control is presented in [1]. Trajectory tracking and stabilization problems are usually solved with backstepping technique [2], sliding mode [3] or optimization methods. In these problems, the partial feedback linearization method and system flatness property are useful.

This contribution is focused on problem of interacting inputs, which occur e.g. in VTOL aircraft, copter, surface vessel or underactuated mobile platform models. Most researchers simplify analyzed models to obtain noncoupling inputs. A few articles [4] present control of systems with coupled inputs by using control law only for noncoupled state variables and verifying behavior of remaining variables. We propose a method for control of all system state variables with coupled inputs based on the computed torque technique combined with stability analysis [5].

\section{References}

[1] Y. Liu, H. Yu. A survey of underactuated mechanical systems. IET Control Theory and Applications 7.7 (2013), 921-935.

[2] H. Sira-Ramirez. Dynamic second order sliding mode control of the hovercraft vessel. IEEE Transactions on control systems technology 10.6 (2002): 860-865.

[3] Z. Hui, S. Jihong. Path following control of underactuated ship based on nonlinear backstepping. IEEE International Conference on Information and Automation 10.6 (2013): 860-865.

[4] R. Olfati-Saber. Global configuration stabilization for the VTOL aircraft with strong input coupling. Proc. of IEEE Transactions on Automatic Control 47.11 (2002), 1949-1952.

[5] S. Korczak. Tracking control of an underactuated rigid body with a coupling input force. Archives of Control Sciences 24.3 (2014): 321-332. 


\title{
Stabilization using discounted optimal control problems
}

\author{
Vladimir Gaitsgory ${ }^{1}$, Lars Grüne $^{2}$, Neil Thatcher $^{3}$
}

Department of Mathematics, Macquarie University, NSW 2109, Australia ${ }^{1}$

Mathematisches Institut, Universität Bayreuth, 95440 Bayreuth, Germany²

Weapons and Combat Systems Division, Defence Science and Technology Organisation Edinburgh SA 5111, Australia ${ }^{3}$

Since the invention of the linear-quadratic regulator it is known that optimal control can be used in order to obtain stabilizing feedback controllers for control systems. A generalization of this approach to nonlinear systems leads to an optimization criterion of the form

$$
\underset{u \in \mathcal{U}}{\operatorname{minimize}} \int_{0}^{\infty} \ell(x(t), u(t)) d t
$$

subject to

$$
\dot{x}(t)=f(x(t), u(t)), x(0)=x_{0}
$$

and potentially further state and/or control constraints, with $\mathcal{U}$ denoting the set of measurable control functions. Unlike the linear quadratic case, however, this nonlinear optimal control problem is in general very difficult to solve, both analytically and numerically.

In this talk we show that in addition to well known other approaches like model predictive (receding horizon) control or Zubov's method, discounted optimal control

$$
\underset{u \in \mathcal{U}}{\operatorname{minimize}} \int_{0}^{\infty} e^{-\delta t} \ell(x(t), u(t)) d t
$$

for sufficiently small $\delta>0$ may also yield stabilizing controls. Since discounted optimal control problems are easier to solve than undiscounted ones (at least numerically), this simplifies the task of constructing stabilizing controls.

We present sufficient conditions, similar to those for model predictive control, which guarantee that discounted optimal trajectories are asymptotically stable at a desired equilibrium. We also discuss the effect of numerical errors when solving the discounted problem. The performance of the approach will be illustrated by a numerical example. 


\title{
Economic Model Predictive Control under Bounded Disturbances
}

\author{
Florian A. Bayer, Frank Allgöwer \\ Institute for Systems Theory and Automatic Control, \\ University of Stuttgart
}

In the last years, economic model predictive control did arise as a new approach in the field of model predictive control (MPC). In economic MPC, more general cost functions can be considered, especially functions which are related to the "economic" performance of the system, e.g., maximizing the profit of a plant or minimizing the used energy - hence the name. This induces that the stage cost may not be positive definite with respect to a given set point. This is in contrast to the well known concept of stabilizing MPC, where a desired steady-state is tracked by employing a cost function which is positive definite with respect to this steady-state. Due to the non-definiteness, the optimal closed-loop behavior is not necessarily convergent but can be cyclic or even chaotic. Stability, if desired, can in general not be derived by the usual definiteness condition of the cost but is usually depending on the notion of dissipativity (see, e.g., [1]).

A difficulty in many practical applications of both stabilizing and economic MPC are disturbances. In previous publications in the field of robust economic MPC, the general idea was to design the MPC controller with respect to the undisturbed system and to track the resulting nominal closed-loop behavior with approaches from robust MPC. However, as the disturbances can directly affect the closed-loop performance of the system (in a negative as well as in a beneficial way), taking the influence of the disturbances into consideration within the design of the control input seems to be essential when working in the described "economic" framework. In this talk, we provide an introduction to and an overview of an approach for robust economic MPC where the influence of the disturbances is explicitly taken into account within the optimization. We propose to employ a specifically modified cost function which is based on an integration over all possible disturbances and can thus be seen as an averaging (see [2]). By using this specific cost function and suitable tube-based methods from robust MPC, reasonable approximations of the influence of the disturbance onto the behavior of the closed-loop system are given for the considered nonlinear discrete-time systems under bounded disturbances. Not only can recursive feasibility be guaranteed, but also bounds on the closed-loop average performance can be derived. In addition, stability as well as optimal operation regimes will be discussed. These can be guaranteed by the system property of dissipativity for the nominal closed-loop system. However, we will indicate that for some specific setups additional constraints are needed to enforce this dissipative behavior. We will show for some examples how taking the disturbances into account within the open-loop optimization results in a better average closed-loop performance.

\section{References}

[1] M.A. Müller, D. Angeli, and F. Allgöwer. On necessity and robustness of dissipativity in economic model predictive control. IEEE Trans. Autom. Control (2014), to appear, DOI: 10.1109/TAC.2014.2361193.

[2] F.A. Bayer, M.A. Müller, and F. Allgöwer. Tube-based Robust Economic Model Predictive Control. J. Proc. Contr. 24(8) (2014), 1237-1246. 


\title{
Approximate linear programming for optimal control design: a solution based on function approximation and randomization
}

\author{
Alessandro Falsone and Maria Prandini \\ Dipartimento di Elettronica, Informazione e Bioingegneria, Politecnico di Milano, Italy \\ \{alessandro.falsone,maria.prandini\}@polimi.it
}

The goal of this work is developing computationally effective control design methods for large scale systems where continuous dynamics, discrete dynamics, and uncertainty are tightly coupled, [1]. We adopt the quite comprehensive modeling framework of Markov Decision Processes (MDPs) with a hybrid (i.e. with both a continuous and a discrete component) state space, and explore the joint use of function approximation and randomized methods to defeat the curse of dimensionality, which hampers the use of standard control design techniques.

We focus on the problem of designing a state feedback control policy that maximizes an average infinitehorizon discounted reward function for an MDP with a hybrid state space and a discrete input space. The considered stochastic optimal control problem can be solved -in principle- through dynamic programming by determining the fixed-point of the Bellman equation so as to determine the so-called optimal value function and then the optimal control policy (see e.g. [2]). The optimal value function and policy can be efficiently computed when the state and control spaces are finite and not too large compared with the memory storage capacity. If the state space has a continuous component of small dimension, computations can be performed by gridding the space, approximating the original MDP with a finite state MDP, and determining the (approximate) optimal value function and control policy on the grid points. As a result, the practical use of dynamic programming is limited by the exponential growth with the problem dimensions of the computation and storage requirements.

Inspired by [3] and [4], we develop an approximate dynamic programming method resorting to random sampling of the state space instead of gridding in order to overcome these limits and avoid that complexity scales exponentially with the state space dimension.

As in [5] dealing with finite state MDP, we start by rephrasing the Bellman equation as a constrained Linear Program (LP) where the unknown quantity to be determined via optimization is the optimal value function, and it is subject to a set of constraints, one per each state-control input admissible value. When the state space has a continuous component, the resulting LP is infinite dimensional in both the decision variables and the constraints. By expressing the optimal value function to be determined as a linear combination of a finite number of basis functions, then, the infinite LP is transformed into a semi-infinite LP, i.e., an LP with finite decision variables but infinite constraints, which, in turn, can be tackled via the so-called scenario approach to robust convex optimization, $[6,7]$. The idea of the scenario approach is to consider a finite number $N$ of constraints only, by extracting at random $N$ state-input pairs, and to solve the resulting finite LP. If $N$ is appropriately chosen, then, the originally infinite constraints are guaranteed to be satisfied probabilistically, with a certain confidence. Quality of the obtained approximation of the value function (and, hence, of the resulting policy) is strongly affected by the choice of the basis functions. This is indeed a key issue in function approximation as well, where data samples in the form of input and output of an unknown (static) function are available and an approximation of the function through a linear combination of basis functions is looked for. Families of universal approximators have been studied in this contest (see e.g. $[8,9,10]$ ), but an effective method to select a finite number of functions out of a given family is still to be developed. The present paper represents a first step in this direction within the more challenging contest of approximate dynamic programming, the additional challenge being that input/output data samples are not available for the unknown optimal value function.

An iterative randomized scheme is proposed to compute an approximate solution to the infinite LP reformulation of Bellman equation. At each iteration, the basis functions are given and the resulting semi-infinite LP can be solved via constraint sampling. Through the iterations, the number of basis functions is increased by adding a suitably chosen basis function at each iteration. The iterative procedure is halted when some performance goal is reached or some upper bound on the number of basis functions is hit.

From an implementation point of view, the solution to each scenario LP involves integral calculations, which may hamper the computational efficiency of the method. However, in certain cases, depending on the stochastic kernel governing the MDP evolution and the chosen basis functions, integrals admit an analytic solution, which makes the overall approach particularly appealing. The approach is tested on a multi-room heating benchmark example. 


\section{References}

[1] J. Lygeros and M. Prandini. Stochastic hybrid systems: a powerful framework for complex, large scale applications. European Journal of Control vol. 16, no. 6 (2010), 583-594.

[2] L. Busoniu, R. Babuska, B. De Schutter, and D. Ernst. Reinforcement Learning and Dynamic Programming Using Function Approximators (2010).

[3] A. Petretti and M. Prandini. An approximate linear programming solution to the probabilistic invariance problem for stochastic hybrid systems. Proc. of the 53rd IEEE Conference on Decision and Control, Los Angeles, USA (2014).

[4] N. Kariotoglou, S. Summers, T. Summers, M. Kamgarpour, and J. Lygeros. Approximate dynamic programming for stochastic reachability. Proceedings of the European Control Conference, Zurich, Switzerland (2013).

[5] D. de Farias and B. V. Roy, The linear programming approach to approximate dynamic programming. Oper. Res., vol. 51, no. 6 (2003), 850-856.

[6] G. Calafiore and M. Campi. The scenario approach to robust control design. IEEE Transactions on Automatic Control, vol. 51, no. 5 (2006), 742-753.

[7] M. Campi, S. Garatti, and M. Prandini. The scenario approach for systems and control design. Annual Reviews in Control, vol. 33, no. 2 (2009), 149-157.

[8] G. Cybenko. Approximation by superpositions of a sigmoidal function. Mathematics of Control, Signals and Systems, vol. 2, no. 4 (1989), 303-314.

[9] J. Park and I. W. Sandberg. Universal approximation using radial-basis-function networks. Neural Comput., vol. 3, no. 2 (1991), 246-257.

[10] L. Breiman. Hinging hyperplanes for regression, classification, and function approximation. IEEE Transactions on Information Theory, vol. 39, no. 3 (1993), 999-1013. 


\title{
Multi-objective optimal control of fluid mixing
}

\author{
Sina Ober-Blöbaum, Kathrin Padberg-Gehle \\ Mathematics Institute, Freie Universität Berlin \\ Department of Mathematics, Technische Universität Dresden
}

Different aspects of controlling transport and mixing in fluid flows have received considerable scientific interest in the last few years. One particular focus is on controlling the evolution of advected density fields in a manner that is optimal for a predefined objective, for example such that a maximal degree of mixedness is achieved, or such that a given target density is reached as good as possible. However, many applications require the optimization of not only one but several conflicting objectives at the same time leading to multiobjective optimal control problems.

In this contribution we present a multi-objective optimal control framework for the optimization of advective processes with respect to certain transport or mixing properties.

Our work is based on the problem of the optimal control of mixing in Stokes fluid flow presented in [1], where a passive material is advected by the flow. To derive the underlying differential equation, we assume that the velocity field $\mathbf{u}(\mathbf{x}, t)$ is induced by a fine set of force fields $\mathbf{F}(\mathbf{x}, t)=\sum_{i=1}^{n} \alpha_{i}(t) \mathbf{F}_{i}(\mathbf{x})$, which can be modulated arbitrarily with time. Then $\mathbf{u}$ can be represented as

$$
\mathbf{u}(\mathbf{x}, t)=\sum_{i_{1}}^{n} \alpha_{i}(t) \mathbf{u}_{i}(\mathbf{x})
$$

where $\mathbf{u}_{i}$ is the velocity field induced by the force field $\mathbf{F}_{i}$. The advection of a density field $c(\mathbf{x}, t)$ by the velocity field (1) is then described by

$$
c_{t}(\mathbf{x}, t)=-\mathbf{u}(\mathbf{x}, t) \cdot \nabla c(\mathbf{x}, t)=-\sum_{i_{1}}^{n} \alpha_{i}(t) \mathbf{u}_{i}(\mathbf{x}) \cdot \nabla c(\mathbf{x}, t), \quad c(\mathbf{x}, 0)=c_{0}(\mathbf{x}) .
$$

Here, $\mathbf{c}(\cdot, t)$ is the state and $\alpha(t)$ is the control input of the control system (2).

Whereas in [1] the modulation of the force fields is searched for that achieves the best mixing for a fixed value of the action (which is the time integral of the kinetic energy per unit mass of the fluid body), in this contribution, we are interested in determining the time-dependent control $\alpha$ such that best mixing with minimal action is achieved. This is an multi-objective optimal control problem with the two conflicting objectives maximal degree of mixedness, which is quantified in terms of a mix-variance described in [1], and minimal action.

For the solution of such a multi-objective optimal control problem, the set of optimal compromises, the so-called Pareto set, has to be determined. To numerically approximate the Pareto set we combine numerical optimal control methods with reference point techniques described in [2]. By choosing appropriate reference points in the objective space, the multi-objective optimal control problem is replaced by a sequence of distance minimizing optimal control problems whose solutions are known to be points of the Pareto set. For each optimal control problem we derive the adjoint systems that provide first-order necessary optimality conditions. An adjoint based optimization together with a gradient descent method is used to solve the single-valued optimal control problems such that the Pareto set is approximated successively.

We demonstrate the flexibility of the methodology in a number of example systems, which may serve as simple models of microfluidic devices.

\section{References}

[1] G. Mathew, I. Mezić, S. Grivopoulos, U. Vaidya, L. Petzold. Optimal control of mixing in Stokes fluid flows. J. Fluid Mech. 580 (2007), 261-281.

[2] S. Ober-Blöbaum, M. Ringkamp, and G. zum Felde. Solving multiobjective optimal control problems in space mission design using discrete mechanics and reference point techniques. In 51st IEEE International Conference on Decision and Control, pages 5711-5716, Maui, HI, USA, 10-13 December 2012. 


\title{
An optimal junction solver for traffic flow
}

\author{
F. Ancona ${ }^{1}$, A. Cesaroni ${ }^{1}$, G.M. Coclite ${ }^{2}, \underline{\text { Mauro Garavello }}^{3}$ \\ ${ }^{1}$ University of Padova \\ ${ }^{2}$ University of Bari \\ ${ }^{3}$ University of Milano Bicocca
}

We consider a junction composed by $m$ incoming roads (modeled by the real interval $I_{i}=(-\infty, 0)$ ) and $n$ outgoing ones (modeled by the real interval $I_{i}=(0,+\infty)$ ). On each road $I_{i}$ we consider the Lighthill-WhithamRichards model for traffic

$$
\partial_{t} u_{i}+\partial_{x} f\left(u_{i}\right)=0
$$

where $u_{i}=u_{i}(t, x)$ denotes the density of traffic at time $t$ and position $x$ in the $i$-th road $I_{i}$ and $f: \mathbb{R} \rightarrow \mathbb{R}$ is the flux. Given an initial datum $\bar{u}_{i}$ on each road, we consider the Cauchy problem at the junction

$$
\begin{cases}\partial_{t} u_{1}+\partial_{x} f\left(u_{1}\right)=0 & x \in I_{1}, t>0 \\ \vdots & \\ \partial_{t} u_{n+m}+\partial_{x} f\left(u_{n+m}\right)=0 & x \in I_{n+m}, t>0 \\ u_{1}(0, x)=\bar{u}_{1}(x) & x \in I_{1} \\ \vdots & \\ u_{n+m}(0, x)=\bar{u}_{n+m}(x) & x \in I_{n+m} .\end{cases}
$$

We address our attention to an optimal control problem for system (2). More precisely, given $T>0$ and a function $\mathcal{J}: \mathbb{R}^{m} \rightarrow \mathbb{R}$, we want to select a solution to (2) which maximizes the functional

$$
\int_{0}^{T} \mathcal{J}\left(f\left(u_{1}(t, 0)\right), \ldots, f\left(u_{m}(t, 0)\right)\right) d t
$$

and which respects additional constraints describing the preferences of drivers. The functional (3) depends on the traces of the fluxes of the solution to (2) at the junction.

We show that, under suitable assumptions, this maximization problem admits solutions. In general there are infinitely many solutions to the Cauchy problem (2), which maximize (3). Moreover we present some additional criteria in order to select an optimal solution. 


\title{
Vanishing dielectric constant regime for the Navier Stokes Maxwell equations
}

\author{
Donatella Donatelli, Stefano Spirito \\ University of L'Aquila \\ GSSI - Gran Sasso Science Institute
}

In this talk the limit of vanishing electric permittivity in the context of Magnetohydrodynamic (MHD) equations is discussed. The classical MHD equations model the evolution of the velocity and the magnetic field in a plasma fluid. The equations are derived from the Navier-Stokes equations and the Maxwell equations by using the classical continuos mechanics theory. In particular, since in a typical conductor the characteristic velocity is much smaller than the velocity of the light the displacement of the current in the Maxwell equations is usually neglected. When one does not make this assumption in the place of the MHD equations the so called Navier-Stokes-Maxwell system are obtained. In this talk we discuss how to recover in a rigorous way the classical MHD equations from the Navier-Stokes-Maxwell system in the limit of small electric permittivity.

\section{References}

[1] D. Donatelli, S. Spirito. Vanishing dielectric constant regime for the Navier Stokes Maxwell equations. Preprint 2014, arXiv:1407.6147. 


\title{
Schaeffer's regularity theorem and the case of systems
}

\author{
L. Caravenna, L. V. Spinolo \\ Dipartimento di Matematica, Università di Padova \\ IMATI-CNR Pavia
}

Several regularity results hold for the Cauchy problem involving one scalar conservation law having convex flux. Among these, Schaeffer's theorem guarantees that for smooth initial data which are generic, in the Baire sense, the entropy admissible solution develops at most finitely many shocks, locally, and stays smooth out of them. My talk will focus on showing that Schaeffer's regularity result cannot be extended to strictly hyperbolic systems of conservation laws, in one space variable, satisfying the assumption analogous to the convexity of a single equation (genuinely nonlinearity): there is a set of initial data which is open in the Schwartz space and which give rise to countably many shock discontinuities, locally in space-time. This suggests that the $\mathrm{SBV}$-regularity is the suitable notion of regularity for $1 d$-systems of conservation laws.

\section{References}

[1] L. Ambrosio, C De Lellis. A note on admissible solutions of 1D scalar conservation laws and 2D HamiltonJacobi equations. J. Hyperbolic Differ. Equ., 1(2004,4), 813-826.

[2] P. Baiti, H. K. Jenssen. Blowup in $L^{\infty}$ for a class of genuinely nonlinear hyperbolic systems of conservation laws. Discrete Contin. Dynam. Systems, 7(2001,4):837-853.

[3] S. Bianchini, L. Caravenna. SBV regularity for genuinely nonlinear, strictly hyperbolic systems of conservation laws in one space dimension. Comm. Math. Phys., 313(2012,1), 1-33.

[4] L. Caravenna, L. V. Spinolo. Schaeffer's regularity theorem for scalar conservation laws does not extend to genuinely nonlinear, strictly hyperbolic systems. Manuscript, 2014.

[5] C. M. Dafermos. Regularity and large time behaviour of solutions of a conservation law without convexity. Proc. Roy. Soc. Edinburgh Sect. A, 99(1985,3-4):201-239, .

[6] D. G. Schaeffer. A regularity theorem for conservation laws. Advances in Math., 11(1973), 368-386. 


\title{
Minimal data rates and entropy in digitally networked systems
}

\author{
Christoph Kawan \\ University of Passau \\ Faculty of Computer Science and Mathematics
}

In digitally networked control systems the assumption of classical control theory that information within feedback loops can be transmitted instantaneously, lossless and with arbitrary precision is violated. This raises the question about the smallest data rate above which a given control task can be solved. For the problem to render a subset $Q$ of the state space invariant, the minimal data rate can be described by means of an entropy-like quantity, the so-called invariance entropy (cf. [1, 4]). If one considers a single feedback loop and assumes that the system is completely controllable and uniformly hyperbolic on $Q$, the invariance entropy can be expressed in terms of Lyapunov exponents (cf. [2]). Furthermore, one can show that there are no cheaper strategies (i.e., such with smaller data rates) to solve the problem than by stabilization at periodic trajectories within $Q$. The proof of this result combines techniques from the hyperbolic theory of dynamical systems with methods from nonlinear control theory. For networks with several subsystems, which can all communicate with each other, there are different possibilities to formulate the question about the smallest data rate for the invariance problem, but also in this setting entropy-like quantities can be introduced to solve the problem (cf. [3]).

\section{References}

[1] F. Colonius, C. Kawan. Invariance entropy for control systems. SIAM J. Control Optim., 48, 3(2009), $1701-1721$.

[2] A. Da Silva, C. Kawan. Invariance Entropy of Hyperbolic Control Sets. Submitted. Preprint available on arXiv:1408.2416 [math.OC]

[3] J.-Ch. Delvenne, C. Kawan. Network entropy and data rates required for networked control. Submitted. Preprint available on arXiv:1409.6037 [math.OC]

[4] C. Kawan. Invariance Entropy for Deterministic Control Systems - An Introduction. Lecture Notes in Mathematics 2089. Berlin: Springer (2013). 


\title{
Reachable states of a quasilinear hyperbolic control system: an application to particulate processes
}

\author{
Max Planck Institute for Dynamics of Complex Technical Systems, Magdeburg, Germany
}

In this presentation, we consider a class of quasilinear systems governed by the following differential equations

$$
\frac{\partial}{\partial t} w(x, t)+\frac{\partial}{\partial x} F(w(x, t))=0, \quad x \in(0, l), t \geq 0,
$$

where $w(x, t)=\left(w_{1}(x, t), w_{2}(x, t), \ldots, w_{n}(x, t)\right)^{T} \in \mathbb{R}^{n}$ describes the state of the system at a point $x$ and time $t$, and the flux $F: \mathbb{R}^{n} \rightarrow \mathbb{R}^{n}$ is assumed to be of class $C^{1}$. System (1) describes a mathematical model of a moving bed chromatographic process by using the material balance of the solutes in both liquid and solid phases. The goal of this work is to study the reachable sets for such a model by using the volumetric flow rate ratio of both phases and concentrations at the inlet of the column as control parameters $u$. The importance of this investigation is underpinned by the practical requirement to estimate the quality of separation depending on parameters of the operating envelope of a chromatographic process.

To study the steering problem and estimate the set of reachable states, we represent system (1) with boundary controls as an abstract differential equation in a suitable Hilbert space $H$ :

$$
\frac{d}{d t} \xi(t)=A \xi(t)+\Phi \xi(t)+B u, \quad \xi(t) \in H, u \in \mathbb{R}^{m} .
$$

We assume that $A: D(A) \rightarrow H$ is the infinitesimal generator of a $C_{0}$-semigroup of bounded linear operators $\left\{e^{t A}\right\}_{t \geq 0}$ on $H$, and that the nonlinear operator $\Phi: H \rightarrow H$ satisfies the Lipschitz condition. In addition to control system (2), we also consider its linear approximation:

$$
\frac{d}{d t} \xi(t)=A \xi(t)+B u, \quad \xi(t) \in H, u \in \mathbb{R}^{m} .
$$

The reachable sets of system (3) may be estimated by using the approach described in the book [1].

Theorem $1\left[1\right.$, p. 98] Let $\left\{Q_{N}\right\}_{N=1}^{\infty}$ be a family of bounded linear operators on $H$ such that $e^{t A}$ and $Q_{N}$ commute, and let

$$
\lim _{N \rightarrow \infty}\left\|Q_{N} \xi\right\|=0 \quad \text { for all } \xi \in H .
$$

Assume that, for $\xi^{0}, \xi^{1} \in H$ and any $N \geq 1$, there is a control $u^{N} \in L^{2}(0, \tau)$ such that

$$
P_{N}\left(\xi\left(\tau ; \xi^{0}, u^{N}\right)-\xi^{1}\right)=0, P_{N}=I-Q_{N},
$$

and

$$
\lim _{N \rightarrow \infty}\left(\left\|Q_{N} B\right\| \cdot\left\|u^{N}\right\|_{L^{2}(0, \tau)}\right)=0 .
$$

Then, for any $\varepsilon>0$, there exists an $N_{0}(\varepsilon)$ such that

$$
\left\|\xi\left(\tau ; \xi^{0}, u^{N}\right)-\xi^{1}\right\|<\varepsilon \quad \text { for each } N \geq N_{0}(\varepsilon) .
$$

Here $\xi\left(t ; \xi^{0}, u\right)$ denotes the mild solution of system (3) with the initial data $\left.\xi\right|_{t=0}=\xi^{0} \in H$ and control $u \in L^{2}(0, \tau)$.

In this work, we extend Theorem 1 for the case of quasilinear control systems. By exploiting this extension, we derive sufficient controllability conditions and construct a family of open-loop controls that solves the steering problem for system (2).

\section{References}

[1] A. Zuyev. Partial Stabilization and Control of Distributed Parameter Systems with Elastic Elements, Springer (2015). 


\title{
Automated generation of a dynamic feedforward control law using local model networks with disturbance inputs
}

\author{
Nikolaus Euler-Rolle, Christoph Hametner, Stefan Jakubek \\ Christian Doppler Laboratory for Model Based Calibration Methodologies at the \\ Institute of Mechanics and Mechatronics, Vienna University of Technology
}

\begin{abstract}
An approach is presented, which automatically generates a dynamic feedforward control law for nonlinear dynamic systems represented by discrete-time local model networks (LMN). By exploiting the generic model structure of LMN, a feedback linearizing input transformation, which subsequently is used to dynamically feedforward control the nonlinear process efficiently, can be found in a general and automated way from the data-driven dynamic process model directly. This model is represented by an LMN (e.g. [1]), which is a well-established multiple-model approach for data-driven modelling of nonlinear systems. As an interpolation between different local models, each valid in a certain operating regime, the LMN offers a versatile structure for the identification of nonlinear dynamic systems. Each operating regime represents a simple model, e.g. a linear regression model [2], whose parameters are found by identification.
\end{abstract}

When control tasks are considered, nonlinear model structures such as LMN can also be used to determine control laws and their parameters, e.g. [3]. This contribution proposes and discusses such an approach for the class of LMN where no internal dynamics are involved, i.e. the LMN has full relative degree with respect to the control signal input. Although the complexity of LMN increases with the amount of local linear models to form a sophisticated nonlinear model, the model structure still remains generic. This fact can be beneficially exploited in the application of feedback linearization (e.g. [4]) to this structure. Basically, a nonlinear system is linearized exactly by using a nonlinear coordinate transformation such that the resulting transformed system consists of an input transformation, linear external dynamics and unobservable internal dynamics. To determine the required feedforward input signal for the desired reference trajectory, the input transformation is utilized. In addition, several disturbance inputs are considered in the feedforward control law without restrictions on their relative degree. This is a common situation when control tasks are tackled which involve one controlled input and various known disturbances or inputs not used for control.

Occasionally, the required time and resources for a detailed physical modelling of a sophisticated nonlinear process are not available. In such a situation, data-driven modelling can deploy its full potential as merely input-output data are required to obtain a dynamic process model. Hence, the proposed approach supersedes the need for an in-depth knowledge of the underlying nonlinear process as the generic model structure of LMN allows for an automated generation of a feedforward control law. Both, LMN and the concept of feedback linearization are by themselves well established concepts in academia as well as in the industry. However, combining both ideas offers the opportunity to provide a substantial tool to dynamically feedforward control any arbitrary nonlinear process with measureable disturbances and with knowledge of measured input-output data only. The application of the proposed approach to the airpath of a diesel engine, where the intake manifold pressure is controlled by a variable-geometry turbocharger, yields good tracking performance.

\section{References}

[1] O. Nelles. Nonlinear System Identification. Springer-Verlag Berlin Heidelberg (2001).

[2] R. Murray-Smith, T.A. Johansen. Multiple Model Approaches to Modelling and Control. Taylor \& Francis (1997).

[3] C. Hametner, C. Mayr, M. Kozek, S. Jakubek. PID controller design for nonlinear systems represented by discrete-time local model networks. Int. J. Cont. 86 (2013), 1453-1466.

[4] A. Isidori. Nonlinear Control Systems. Springer-Verlag Berlin Heidelberg New York (1995). 


\title{
Structure-preserving discrete-time LQR problems
}

\author{
Kathrin Flaßkamp, Todd D. Murphey \\ Northwestern University, USA
}

The optimal solution of a linear quadratic regulator (LQR) problem in continuous time, i.e. the state and adjoint trajectories, solves a linear Hamiltonian two-point boundary value problem. Often, the optimal solution is determined by the corresponding Riccati matrix differential equation. When approximating the true solution by numerical integration, it is desirable to preserve the problem's original symplectic structure given by the Hamiltonian dynamics.

We first study the discretization approach based on Euler integration, which is commonly used in control applications. As a corollary of Hager's result in [1], this leads to a discretization of the state-adjoint system by a symplectic Euler method. In the discrete-time LQR case, we link this to the well known discrete-time Riccati equations. In this contribution, we focus on optimal control of mechanical systems, for which the symplectic structure due to Hamiltonian dynamics can be preserved by using variational integrator discretizations (cf. [2]), also known as discrete mechanics models. Using a result from [3], we complement the symplectic state discretization with a discrete adjoint scheme to obtain a symplectic state-adjoint scheme. Then we derive modified discrete-time Riccati equations.

The symplectic discretization of the Hamiltonian state-adjoint system allows for backward error analysis (cf. [2]). In particular, the existence of a modified Hamiltonian of the optimal control problem is guaranteed. Applications of discrete-time LQR control go beyond linear systems, as, for instance, projection-based optimization methods for nonlinear systems use local linearizations and subsequently solve linear quadratic problems (cf. [4]).

\section{References}

[1] W.W. Hager. Runge-Kutta Methods in Optimal Control and the Transformed Adjoint System. Numerische Mathematik 87 (2000), 247-282.

[2] E. Hairer, C. Lubich, G. Wanner. Geometric Numerical Integration: Structure-Preserving Algorithms for Ordinary Differential Equations. Springer (2006).

[3] S. Ober-Blöbaum, O. Junge, and J.E. Marsden. Discrete mechanics and optimal control: an analysis. Control, Optimisation and Calculus of Variations 17(2) (2011), 322-352.

[4] J. Schultz, E. Johnson, T.D. Murphey. Trajectory Optimization in Discrete Mechanics. A. Müller, Z. Terze (Eds.): Differential-Geometric Methods in Computational Multibody System Dynamics. Springer International Publishing (In Press) 


\title{
Reconstruction of independent sub-domains for a class of Hamilton-Jacobi equations and application to parallel computing
}

\author{
Adriano Festa, \\ RICAM - Johann Radon Institute \\ for Computational and Applied Mathematics \\ Austrian Academy of Sciences
}

A previous knowledge of the domains of dependence of a Hamilton-Jacobi equation can be useful in its study and approximation. Information of this nature is, in general, difficult to obtain directly from the data of the problem. In this talk we introduce formally the concept of independent sub-domain discussing its main properties and we provide a constructive implicit representation formula. Using such results we propose an algorithm for the approximation of these sets that is shown to be relevant in the numerical resolution via parallel computing.

\section{References}

[1] M. Bardi and I. Capuzzo-Dolcetta, Optimal Control and Viscosity Solutions of Hamilton-Jacobi-Bellman Equations. Birkhäuser, Boston 1997.

[2] S. Cacace, E. Cristiani, M. Falcone and Athena Picarelli, A patchy dynamic programming scheme for a class of Hamilton-Jacobi-Bellman equation, SIAM J. Scientific Computing, vol. 34 (2012) no. 5, pp. 2625-2649.

[3] A. Festa, R.B.Vinter, Decomposition of Differential Games, Preprint (2014). 


\section{S21: Mathematical image processing}

Over the last decade mathematics has become the cornerstone in Signal and Image processing ranging from various methods for signal reconstruction to modelling of imaging modalities over its classical disciplines compression, denoising, segmentation, and registration to feature extraction. The used methodologies include such diverse fields as harmonic analysis, inverse problems, variational analysis, mathematical statistics, partial differential equations, optimization, approximation theory and sampling theory. The aim of this section is to foster interdisciplinary collaboration and the development of new directions in mathematical signal and image processing spawned from the interaction of various mathematical communities. 


\title{
On learning reaction diffusion models
}

\author{
Yunjin Chen, Thomas Pock \\ Institute for Computer Graphics and Vision, Graz Univeristy of Technology
}

In this work, we study the propblem of learning nonlinear reaction-diffusion models for image restoration. We extend conventional nonlinear reaction diffusion models by several parametrized linear filters as well as several parametrized influence functions. We propose to train the parameters of the filters and the influence functions through a loss based approach. Experiments show that our trained nonlinear reaction-diffusion models largely benefit from the training of the parameters and finally lead to the best reported performance on common test datasets for image restoration. 


\title{
Adaptive Total Variation Regularization
}

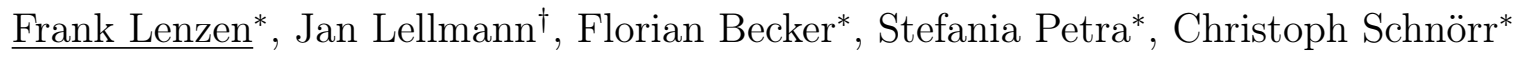 \\ * HCI \& IPA, Heidelberg University, Germany \\ $\dagger$ DAMTP, Centre for Mathematical Sciences, Cambridge, UK
}

Due to its property to encourage piecewise constant solutions, Total Variation (TV) plays an important role as regularizer for various image restoration tasks. In order to also deal with piecewise affine structures, higher order variants such as Total Generalized Variation (TGV) [1] have been proposed.

To even further improve the restoration quality of TV regularization, various adaptive methods have been proposed in literature. Among these, we find the class of direction-dependent TV methods, which we refer to as anisotropic TV methods.

The main focus of the adaptive methods are e.g. the preservation of contrast, edges, corners, slopes and fine image structures. To steer the adaptivity, additional information is required. We therefore can distinguish between data-driven approaches, where this information is taken from the input image or additional data, and solution-driven approaches, where the adaptivity is depending on the solution of the problem.

We also remark that while the classical TV regularization is convex some of the proposed approaches in literature yield non-convex problems. While in view of the restoration quality these non-convex methods show advantages, their theoretical and numerical treatment comes with some difficulties.

In our talk we will first give an overview over TV variants lately proposed in literature and then discuss a novel method we have proposed recently $[2,3]$. This strategy combines advantages of convex and non-convex approaches. It is based on a fixed-point problem, where the unknown on the one hand is the sought solution to the problem and on the other hand steers the adaptivity. Solving this problem numerically results in a sequence of convex problems, while the regularization asymptotically mimics a non-convex regularization. Reformulating the fixed-point problem as a Quasi-Variational Inequality Problem (QVIP) enables us to provide theoretical results for existence and uniqueness of the fixed point.

Finally, we exemplarily consider the applications of denoising, (non-blind) deblurring and inpainting.

\section{References}

[1] K. Bredies, K. Kunisch, and T. Pock. Total Generalized Variation. SIAM J. Imaging Sciences, 3(3):492-526, 2010 .

[2] F. Lenzen, F. Becker, J. Lellmann, S. Petra, and C. Schnörr. A class of quasi-variational inequalities for adaptive image denoising and decomposition. Computational Optimization and Applications, 54(2):371-398, 2013.

[3] F. Lenzen, J. Lellmann, F. Becker, and C. Schnörr. Solving quasi-variational inequalities for image restoration with adaptive constraint sets. SIAM Journal on Imaging Sciences (SIIMS), 7(4):2139-2174, 2014. 


\title{
Joint Motion Estimation and Image Reconstruction
}

\author{
Hendrik Dirks, Martin Burger, Carola-Bibiane Schönlieb
}

Modern microscopes are able to visualize even the smallest biological processes within cells. One example is intracellular flow where the flow dynamics within a single cell are visualised by a sequence of microscopic images over time. Extracting accurate information on very short timescales, however, comes with a lack of spatial quality of the images in terms of resolution and noise.

One possibility to counteract this and enhance the image sequence is to use the fact that the recorded images are connected by motion of objects over time. In this talk we present a model that couples flow information into an image inpainting/denoising problem and simultaneously calculates the flow pattern between timesteps. Inspired by the ROF model [1] for image reconstruction

$$
\min _{u} \frac{1}{2}\|K u-f\|_{2}^{2}+\alpha\|\nabla u\|_{1}
$$

with a general linear operator $K$ that might represent for example a convolution, and a $T V-L^{1}$ model for motion estimation [2]

$$
\min _{\boldsymbol{v}}\left\|u_{t}+\nabla u \cdot \boldsymbol{v}\right\|_{1}+\beta\|\nabla \boldsymbol{v}\|_{1}
$$

we want to combine these models now, to similarly reconstruct an image sequence $u$ and the underlying motion field $\boldsymbol{v}$. Therefore we use a constrained variational model

$$
\begin{aligned}
\min _{u, \boldsymbol{v}} \int_{0}^{T} \frac{1}{2}\|K u-f\|_{2}^{2}+\alpha\|\nabla u\|_{1}+\beta\|\nabla \boldsymbol{v}\|_{1} d t \\
\text { s.t. } u_{t}+\nabla u \cdot \boldsymbol{v}=0 .
\end{aligned}
$$

In the analytical part necessary conditions for the existence of minimizers for (1) are presented. In the end we introduce a primal-dual [3] minimization scheme and show some of our results.

\section{References}

[1] Leonid I Rudin, Stanley Osher, and Emad Fatemi. Nonlinear total variation based noise removal algorithms. Physica D: Nonlinear Phenomena, 60(1):259-268, 1992.

[2] Christopher Zach, Thomas Pock, and Horst Bischof. A duality based approach for realtime tv-l 1 optical flow. In Pattern Recognition, pages 214-223. Springer, 2007.

[3] Antonin Chambolle and Thomas Pock. A first-order primal-dual algorithm for convex problems with applications to imaging. Journal of Mathematical Imaging and Vision, 40(1):120-145, 2011. 


\title{
Regularization methods for flow fields with smooth transitions and sharp edges
}

\author{
Lena Frerking $^{1,4}$, Martin Burger ${ }^{1,4}$, Dietmar Vestweber ${ }^{2,4}$, Christoph Brune ${ }^{3,4}$ \\ Applied Mathematics Münster, University of Münster ${ }^{1}$ \\ Max Planck Institute for Molecular Biomedicine ${ }^{2}$ \\ Department of Applied Mathematics and MIRA - Institute for Biomedical Technology and \\ Technical Medicine, University of Twente ${ }^{3}$ \\ Cells in Motion - Cluster of Excellence, University of Münster ${ }^{4}$
}

With this contribution we present ideas for variational flow models that combine the abilities to recover smooth transitions as well as sharp edges inside of a flow field. Our aim is to estimate the intracellular and extracellular flows of migrating cells, which are expected to be sharp at the cell boundaries, but at least partially smooth inside of a cell. We study regularizers that are composed of total variation and of terms that allow for smooth transitions, i.e. we want to minimize functionals of the following type:

$$
J(u)=\lambda\left\|f_{t}+(\nabla f)^{T} \cdot u\right\|+\min _{v}\left\{\alpha_{0}\|\nabla u-v\|+\alpha_{1} R(v)\right\},
$$

where $f$ denotes the brightness of two consecutive images and $u$ the vector field we are looking for. The first term of the functional $\mathrm{J}(\mathrm{u})$ is the optical flow constraint and the second term depicts the total variation part of the regularization. Possible choices for $R(v)$ that we want to focus on are $\frac{1}{2}\|v\|_{2}^{2}$ or $\|\nabla v\|_{1}$. The latter leads us to total generalized variation regularization, which is up to now mainly used for static models like denoising and reconstruction. To enhance the exactness of the recovery, we analyze the effect of Bregman iteration on the results and the influence of different norms for the data fidelity.

\section{References}

[1] K. Bredies, K. Kunisch, T. Pock Total generalized variation. SIAM Journal on Imaging Sciences 3 (2010), 492-526.

[2] A. Chambolle, T. Pock. A first order primal-dual algorithm for convex problems south applications to imaging. Journal of Mathematical Imaging and Vision 40 (2011), 120-145.

[3] R. Ranftl, S. Gehrig, T. Pock, H. Bischof. Pushing the limits of stereo using variational stereo estimation. Intelligent Vehicles Symposium (IV), IEEE (2012), 402-407.

[4] M. Benning, C. Brune, M. Burger, J. Müller. Higher-order TV methods - enhancement via Bregman iteration. Journal of Scientific Computing 54 (2013), 269-310. 


\title{
Regularisation by Circular Hough Transform
}

\author{
$\underline{\text { Joana Grah }^{1}}$, Martin Burger ${ }^{2}$, Carola-Bibiane Schönlieb ${ }^{1}$ \\ ${ }^{1}$ Department of Applied Mathematics and Theoretical Physics, University of Cambridge, UK \\ ${ }^{2}$ Institute for Computational and Applied Mathematics, University of Münster, Germany
}

In 1962, Paul Hough patented a method to recognise straight lines in images [3]. The Hough transform, named after its inventor, was then further developed and generalised by Duda and Hart in 1972 [2]. They extended it to other parametrised curves and first applied it to circle detection. Nowadays, the circular Hough transform (CHT) is a well-established and widely-used shape detection method. In its continuous formulation, it can be written as a path integral along a circle for a function $f\left(x_{1}, x_{2}\right), x_{1}, x_{2} \in \mathbb{R}$ :

$$
\iint f\left(x_{1}, x_{2}\right) \delta\left(r-\sqrt{\left(x_{1}-c_{1}\right)^{2}+\left(x_{2}-c_{2}\right)^{2}}\right) d x_{1} d x_{2}
$$

where $\left(c_{1}, c_{2}\right)$ are the centre coordinates and $r$ is the radius.

This contribution makes use of the CHT in a different context. In particular, it is utilised in regularisation terms occurring in connection with variational methods for image processing tasks such as denoising or segmentation. The idea arose while performing automatic detection of circularly shaped mitotic cells in phase contrast microscopy images by recognising maxima of the CHT, which had been applied to an edge image, as well as cell tracking, where the CHT might also replace TV regularisation terms in the segmentation functionals. Since in most applications we are only looking for a small number of circular objects, the CHT image should accordingly contain a few peaks and hence the sparser it is, the clearer becomes the circular shape in the original image. This observation motivates integrating the CHT in regularisers for images containing circular objects.

In recent years, total variation regularisation has proven to be a convenient choice in many applications. In image denoising, the most popular example is the ROF model [4], which can be written in the following discrete form:

$$
\min _{u} \frac{\lambda}{2}\|u-f\|_{2}^{2}+\|\nabla u\|_{1}
$$

where $f$ is a given noisy image and $u$ denotes the optimal denoised image to be obtained. Inspired by the fact that edge detection is performed before applying the CHT in the context of circle recognition and also due to the natural sparsity of edge images, we penalise the $\ell_{1}$-norm of the CHT of the gradient image. The corresponding model reads

$$
\min _{u, d} \frac{\lambda}{2}\|u-f\|_{2}^{2}+\|H d\|_{1}+\chi_{0}(\nabla u-d),
$$

where $H$ denotes the CHT written as a discrete linear operator and $\chi_{0}$ is the characteristic function of the set $\{0\}$. Note that the operator $H \nabla$ was split using the auxiliary variable $d$. In order to minimise this energy numerically, we use the first-order primal-dual algorithm proposed by Chambolle and Pock [1].

We present several results using synthetic as well as real-world examples. Although in most cases TV denoising can obtain better reconstructions with respect to PSNR or SSIM, it is remarkable that it also performs well using the proposed regularisation and it can be observed that the staircasing effect typical for TV denoising seems to be attenuated. Moreover, circular objects are smoothed and their histograms tend to be equalised, which can be advantageous for the task of cell mitosis detection and tracking as mentioned above. In addition, we discuss possible enhancements, e.g. obtained by combining TV and CHT regularisation or by using non-linear regularisation terms such as $\|H(|\nabla u|)\|_{1}$.

\section{References}

[1] A. Chambolle, T. Pock. A first-order primal-dual algorithm for convex problems with applications to imaging. Journal of Mathematical Imaging and Vision (2011), 40(1):120-145.

[2] R.O. Duda, P.E. Hart. Use of the Hough transformation to detect lines and curves in pictures. Communications of the ACM (1972), 15(1):11-15.

[3] P.V.C. Hough. Method and means for recognizing complex patterns, December 18 1962. US Patent 3,069,654.

[4] L.I. Rudin, S. Osher, E. Fatemi. Nonlinear total variation based noise removal algorithms. Physica D: Nonlinear Phenomena (1992), 60(1):259-268. 


\title{
Artifact-free variational MPEG decompression
}

\author{
Kristian Bredies, Martin Holler \\ University of Graz
}

The MPEG (Motion Picture Experts Groups) video compression standard is one of the most well-known and widely-used methods to compress and store digital video data. The underlying concepts of MPEG compression, namely motion compensation and Block Discrete Cosine Transform (BDCT) encoding, are at the heart of almost any modern video compression method. MPEG achieves high compression rates which come, however, with the cost of loss of data due to quantization (rounding) of BDCT coefficients. This loss of data is responsible for disturbing artifacts in the decompressed movie.

In this talk, we present a variational method for improved MPEG decompression which is able to reduce compression-induced artifacts. By utilizing the information provided by the compressed MPEG file, our approach relies on the extraction of quantization intervals associated with the compressed data. These intervals must contain the "original data", i.e., the data prior to compression, and are used to describe the convex set $D$ of all possible reconstructions. We then variationally decompress the video by minimizing a spatio-temporal regularization functional $\mathcal{R}$ subject to the data being contained in $D$.

As the decoding process, i.e., the mapping which takes the stored coefficients to the spatio-temporal video data, constitutes a linear operator $A$, the variational problem can be phrased as

$$
\min _{d \in D} \mathcal{R}(A d) .
$$

Our method conceptually works for all versions of MPEG compression, but is implemented for MPEG-2 compressed video data, as this version is still one of the standard encoding formats and can be seen as a realistic but still tractable blueprint for most video compression methods.

For regularization, we use the Infimal Convolution of Total Generalized Variation (ICTGV) functionals as introduced in [3]. The introduction of this functional is motivated by the observation that, for spatio-temporal regularization, the ratio between the spatial and temporal step-size - say $\lambda$ - is not given a-priori and a combination of different ratios can be utilized to further improve reconstruction quality. Consider for instance the spatio-temporal second order Total Generalized Variation (TGV) [1] functional which is given as

$$
\operatorname{TGV}_{\lambda, \alpha}^{2}(u)=\min _{v} \alpha_{1}\left\|\nabla_{\lambda} u-v\right\|_{1}+\alpha_{0}\left\|\mathcal{E}_{\lambda} v\right\|_{1},
$$

where $\lambda>0$ can be interpreted as a weight of the temporal derivatives appearing in the spatio-temporal gradient $\nabla_{\lambda}$ and symmetrized gradient $\mathcal{E}_{\lambda}$. This additional degree of freedom, which in fact defines a trade-off between spatial and temporal regularization, can be exploited to further improve reconstruction quality by optimally balancing between two choices of $\lambda$. This is realized by the infimal convolution of two second order TGV functional which is given as

$$
\operatorname{ICTGV}_{\lambda, \beta, \alpha}^{2}(u)=\min _{v} \operatorname{TGV}_{\lambda_{1}, \alpha}^{2}(u-v)+\beta \operatorname{TGV}_{\lambda_{2}, \alpha}^{2}(v) .
$$

and used for regularization in the present work.

For the numerical solution of (1) we use the primal dual algorithm of [2] and present an implementation that covers all orders of spatio-temporal TGV regularization and infimal convolutions thereof. Our numerical experiments confirm that, using ICTGV regularization, we are able to significantly reduce compression artifacts in MPEG compressed videos and obtain a good reconstruction quality even at relatively high compression rates.

\section{References}

[1] K. Bredies, K. Kunisch, T. Pock. Total generalized variation. SIAM J. Imag. Sci. 3(3) (2010), 492-526.

[2] A. Chambolle, T. Pock. A first-order primal-dual algorithm for convex problems with applications to imaging. J. Math. Imaging Vision 40 (2011), 120-145.

[3] M. Holler, K. Kunisch. On Infimal Convolution of TV-Type Functionals and Applications to Video and Image Reconstruction. SIAM J. Imag. Sci. 7(4) (2014), 2258-2300. 


\title{
Cartoon-Texture-Noise Decomposition with Transport Norms
}

\author{
Christoph Brauer, Dirk Lorenz \\ TU Braunschweig
}

We investigate the problem of decomposing an image into three parts, namely a cartoon part, a texture part and a noise part. We argue that norms originating in the theory of optimal transport should have the ability to distinguish certain noise types from textures. Hence, we present a brief introduction to optimal transport metrics and show their relation to previously proposed texture norms $[1,2,3,4]$. We propose different variational models of the form

$$
\min _{u, v} \alpha F_{u}(u)+\beta F_{v}(v)+\gamma F_{w}\left(u^{0}-u-v\right)
$$

and investigate their performance. These models yield a decomposition of an observed image $u^{0}$ into cartoon $u$, texture $v$ and noise $w=u^{0}-u-v$. Our algorithmic approach exploits recent advances in non-smooth large-scale optimization [5,6]. Moreover, inspired by [7] we illustrate the relations of the proposed models for cartoon-texture-noise decomposition to the theory of optimal transport.

\section{References}

[1] Rudin, L.I., Osher, S., Fatemi, E.: Nonlinear total variation based noise removal algorithms. Physica D 60 (1992) 259-268

[2] Meyer, Y.: Oscillating Patterns in Image Processing and Nonlinear Evolution Equations. Volume 22 of University Lecture Series. AMS (2001)

[3] Vese, L.A., Osher, S.J.: Modeling textures with total variation minimization and oscillating patterns in image processing. Journal of Scientific Computing 19(1-3) (December 2003)

[4] Osher, S., Solé, A., Vese, L.: Image decomposition and restoration using total variation minimization and the $H^{-1}$ norm. SIAM Multiscale Modeling and Simulation 1(3) (July 2003) 349-370

[5] Chambolle, A., Pock, T.: A first-order primal-dual algorithm for convex problems with applications to imaging. Journal of Mathematical Imaging and Vision 40 (2011) 120-145

[6] Lorenz, D., Pock, T.: An inertial forward-backward algorithm for monotone inclusions. Journal of Mathematical Imaging and Vision (2014)

[7] Lellmann, J., Lorenz, D.A., Schönlieb, C.B., Valkonen, T.: Imaging with Kantorovich-Rubinstein discrepancy. to appear in SIAM Journal on Imaging Sciences (July 2014) [http://arxiv.org/abs/1407.0221]. 


\title{
Asymptotics of Spatial Sparsity Priors
}

\author{
Pia Heins, Martin Burger \\ University of Muenster
}

Sparsity regularization in inverse problems based on minimizing the $\ell^{1}$-norm is an important and versatile tool and can even be extended to realize more advanced a-priori information such as differently structured sparsity for unknowns being matrices. Regularization functionals, which promote sparse solutions, are indeed usually finite-dimensional. However, in many applications an infinite-dimensional modeling especially in the pre-image space seems to be more reasonable. Nevertheless, due to computational rather than applicationrelated reasons, spatial sparsity is usually imposed on some spatial grid. Therefore, the establishment of an appropriate asymptotic theory behind these discrete approaches is of particular importance. An underlying asymptotic theory moreover permits an analysis independent of discretization and thus yields robustness.

This talk is devoted to the asymptotics of regularization methods applicable for instance to spatial sparsity regularization. We are interested in recovering continuum limits such as total variation regularization or recent approaches in the space of Radon measures, cf. for instance [1], [2] and [3]. Thereby, we will also obtain continuum limits, which were not understood up to now. In order to study the asymptotics of regularization functionals, we utilize $\Gamma$-convergence, which is a useful and reasonable framework for this purpose.

In so doing, we are able to compute some $\Gamma$-limits. We not only consider usual $\ell^{p}$-norms for $p \geq 1$, but also analyze the asymptotics of the $\ell^{0}$-"norm". On the basis of these insights, we moreover deduce some $\Gamma$ limits for certain types of mixed norms. In order to verify our results numerically, we furthermore consider the deconvolution of a sparse spike pattern for different discretizations as the step size of the grid becomes smaller.

\section{References}

[1] O. Scherzer and B. Walch. Sparsity Regularization for Radon Measures. Scale Space and Variational Methods in Computer Vision. Springer Berlin Heidelberg (2009), 452-463.

[2] K. Bredies and H. K. Pikkarainen. Inverse problems in spaces of measures. ESAIM: Control, Optimization and Calculus of Variations 19 (2013), 190-218.

[3] V. Duval and G. Peyré. Exact Support Recovery for Sparse Spikes Deconvolution. CNRS and Université Paris-Dauphine, July (2013). 


\title{
Image Warping via Optimal Transport with Sources
}

\author{
Jan Maas ${ }^{1}$, Martin Rumpf ${ }^{2},{\text { Stefan } \text { Simon }^{2}}^{2}$ \\ ${ }^{1}$ IST Austria \\ ${ }^{2}$ University of Bonn
}

In this contribution a new optimal transport approach for image warping is presented. The warp is obtained via the minimization of an action functional, which takes into account the transport costs and a source term. The source term locally measures the variation of the image density in $L^{1}$, which includes not only absolutely continuous sources with respect to the Lebesgue measure, but also singular measures. Existence of minimizing paths in the space of Radon measures is demonstrated. Furthermore, a robust and effective discretization is derived following the approach by Benamou and Brenier. Characteristic test cases underline the qualitative properties of this approach and selected applications show the potential for image matching, distance computation on the space of image, and weighted averaging of textures. 


\title{
Non-rigid registration for pre-operative 3D surfaces and intra-operative $2.5 \mathrm{D}$ surfaces
}

\author{
Rosalia Tatano, Benjamin Berkels, \\ Aachen Institute for Advanced Study in Computational Engineering Science (AICES), \\ RWTH Aachen University, Schinkelstr. 2, 52062 Aachen, Germany
}

Image Guided Surgery (IGS) benefits from the fusion of different image data sources. In particular, the fusion of pre-operative and intra-operative information is helpful to the physicians. Due to the different image acquisition devices that are available before and during a surgery, registration of images of different dimensions is called for. For instance, [1] illustrates such benefits in the context of cranial neuronavigation by matching 2D intra-operative photographs with pre-operative MRI data sets.

In this talk, a novel non-rigid registration algorithm that matches pre-operative 3D surfaces and intra-operative $2.5 \mathrm{D}$ surfaces is presented, building on ideas proposed in [2] to register intra-oprative time-of-flight surfaces to pre-operative surfaces extracted from computed tomography for radiation therapy.

The goal of the proposed method is to find a non-rigid deformation that matches an intra-operative surface to a pre-operative one. Here, it is important that the deformation is defined on the intra-operative surface due to its lower dimension compared to the pre-operative surface. The registration problem is formulated as an energy minimization problem, in which the key roles are played by a matching energy term that involves the signed distance of the 3D surface to estimate the mismatch between the two surfaces and a regularization term for the deformation. As is usual for image registration approaches [3], the minimization of the energy is achieved through a multilevel coarse-to-fine strategy. On each level, a derivative based optimization approach is used for the numerical solution of the problem.

\section{References}

[1] B. Berkels, I. Cabrilo, S. Haller, M. Rumpf, C. Schaller. Co-registration of intra-operative brain surface photographs and pre-operative MR images. Int. J. Comput. Assist. Radiol. Surg. 9 (2014), 387-400.

[2] S. Bauer, B. Berkels, J. Hornegger, M. Rumpf. Joint ToF image denoising and registration with a CT surface in radiation therapy. Third International Conference on Scale Space Methods and Variational Methods in Computer Vision, Lecture Notes in Computer Science, Springer (2011), 98-109.

[3] J. Modersitzki. Numerical methods for image registration. OUP Oxford (2004). 


\title{
Active-contours for image segmentation relying on non-stationary subdivision schemes
}

\author{
Paola Novara ${ }^{1}$, Lucia Romani ${ }^{2}$, Daniel Schmitter ${ }^{3}$, Virginie Uhlmann ${ }^{3}$ and Michael Unser ${ }^{3}$ \\ ${ }^{1}$ University of Insubria, Italy \\ ${ }^{2}$ University of Milano-Bicocca, Italy \\ ${ }^{3}$ EPFL Lausanne, Switzerland
}

Image segmentation is one of the primary steps in image analysis and visual pattern recognition. Active contours (also known as snakes) are well-known tools for image segmentation, belonging to the wider class of deformable models. An active-contour is essentially a curve or a surface that evolves within a $2 \mathrm{D}$ or a $3 \mathrm{D}$ image from some initial position, in order to detect the boundary of the object of interest. Active contours have become popular because they facilitate user interaction, not only during the initialization phase, but also during the minimization process of the energy function that governs the evolution of the curve/surface towards the boundary of the region to be segmented. The extensive research activity carried out in this field has resulted in many active-contour variants which differ both in the type of representation and in the choice of the energy function to be minimized. In this talk we present a new class of active-contours, never investigated so far, which describes the curve/surface in a discrete fashion by using a sufficiently refined polyline/polyhedral mesh obtained by the iterative application of non-stationary univariate/bivariate subdivision schemes. The subdivision schemes at the basis of our deformable models are non-stationary subdivision schemes at least $C^{1}$-continuous, with the property of translation invariance and the capability of perfectly outlining ellipses/ellipsoidal objects. A selection of illustrative examples in the context of biomedical images segmentation will demonstrate the excellent tradeoff between flexibility and efficiency that can be achieved by the newly derived active-contours. 


\title{
Deterministic Sparse FFT Algorithms
}

\author{
Gerlind Plonka, Katrin Wannenwetsch \\ Institute for Numerical and Applied Mathematics \\ University of Göttingen
}

We propose deterministic stable FFT algorithms to compute a sparse vector $\mathbf{x} \in \mathbb{C}^{N}$ from its Fourier transformed vector $\widehat{\mathbf{x}}=\mathbf{F}_{N} \mathbf{x}$, where $\mathbf{F}_{N}$ denotes the Fourier matrix of order $N$. In case of nonnegative vectors being $M$-sparse, we need at most $\min \{M \log (N), N\}$ Fourier values in order to recover $\mathbf{x}$ and (for $M^{2}<N$ ) at most $\mathcal{O}\left(M^{2} \log N\right)$ arithmetical operations. Here, the sparsity $M$ needs not to be known in advance but will be determined during the algorithm. If the considered vector $\mathbf{x}$ is not sparse, we obtain a usual FFT algorithm requiring $\mathcal{O}(N \log N)$ arithmetical operations.

In case of vectors $\mathrm{x} \in \mathbb{C}^{N}$ with small support $M$, we present a new sparse FFT using only $\mathcal{O}(M \log N)$ arithmetical operations if the support length $M$ of $\mathbf{x}$ is a priori known. If the support length is not known in advance, the idea can be modified using Prony kind methods such that we need $\mathcal{O}(M \log M \log N)$ arithmetical operations.

The algorithms work iteratively, where each iteration step only involves the solution of a linear system of size at most $M$. We develop adaptive strategies to ensure that the coefficient matrix in the linear system is well-conditioned. For this purpose, we have to study Vandermonde matrices with knots on the unit circle. 


\title{
Reconstruction of Multivariate Exponential Functions from Measurements
}

\author{
Thomas Peter \\ Institut für Mathematik und Informatik, Universität Osnabrück
}

Recovering an $M$-sparse sum of complex exponentials from equidistant measurements can be accomplished e.g. by using Prony's method or one of its numerical variants [1]. We want to reconstruct a $d$-dimensional signal

$$
f(x)=\sum_{j=1}^{M} c_{j} \mathrm{e}^{\left\langle x, f_{j}\right\rangle}, \quad x, f_{j} \in \mathbb{C}^{d}, \quad c_{j} \in \mathbb{C}
$$

by generalising those ideas to higher dimensions. In $[2,3]$ methods are introduced that show, how important subclasses of this multi-dimensional problem can be solved by reducing them to several one-dimensional problems. Only recently, another approach called superresolution was introduced in [4] that can also be generalised to more than one dimension.

Here we want to present a different approach based on Prony's method that involves the analysis of zero sets of $d$-variate polynomials. All the mentioned ideas share a significant amount of similarities that we would like to address.

\section{References}

[1] D. Potts, M. Tasche. Parameter estimation for nonincreasing exponential sums by Prony- like methods. Linear Algebra and its Applications, (2012).

[2] G. Plonka and M. Wischerhoff. How many Fourier samples are needed for real function reconstruction? Journal of Applied Mathematics and Computing, 42 no. 1-2, (2013).

[3] D. Potts, M. Tasche. Parameter estimation for multivariate exponential sums. Technical University Chemnitz, (2012).

[4] E. Candès, C. Fernandes-Grands. Towards a Mathematical Theory of Super-resolution. Communications on Pure and Applied Mathematics 67.6 (2014). 


\title{
Ambiguities in one-dimensional discrete phase retrieval from Fourier magnitudes
}

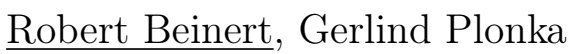 \\ University of Goettingen
}

The phase retrieval problem has many applications in physics. For example, it occurs in crystallography, electron microscopy, astronomy, and optics. We consider the reconstruction of an unknown complex-valued signal $\mathbf{x}$ with finite support from the moduli of its Fourier transform $|\widehat{x}(\omega)|$. It is well known that the considered phase retrieval problem always has multiple solutions. For example, we can construct further solutions by rotating, shifting, or reflecting and conjugating a given solution of the problem. Besides these trivial ambiguities, there are also problems with further non-trivial ambiguities.

If we can describe the signal $\mathbf{x}$ as a convolution of elementary factors, we can determine all possible solutions of a phase retrieval problem - trivial or non-trivial. Furthermore, such a factorization always exists for signals of finite length.

There have been many different approaches to enforce uniqueness of the one-dimensional, real phase retrieval problem, especially the interference with known and unknown reference signals or the additional consideration of end points of the unknown signal. Most of these ideas can also be applied to the complex phase retrieval problem. In some applications, besides the moduli of the Fourier transform, the moduli of the unknown complex-valued signal are known.

With the aid of the representation of the ambiguities as convolution of elementary factors, we can show that this additional information about the unknown signal reduces the set of ambiguities such that every or almost every signal can be uniquely recovered up to some trivial ambiguities.

\section{References}

[1] R. Beinert, G. Plonka. Ambiguities in one-dimensional discrete phase retrieval from Fourier magnitudes. University of Goettingen (2014) Preprint. 


\title{
Optimal mollifiers for the reconstruction of spherical images from circular means
}

\author{
Ralf Hielscher, Michael Quellmalz \\ Technical University of Chemnitz, Germany
}

We are concerned with the reconstruction of spherical images from mean values along great circles or small circles. More precisely, we consider for circles $\mathcal{C}(\boldsymbol{\eta}, t)=\left\{\boldsymbol{\xi} \in \mathbb{S}^{2} \mid \boldsymbol{\xi} \cdot \boldsymbol{\eta}=t\right\}$ on the two-sphere $\mathbb{S}^{2}$ the inversion of the operator

$$
\mathcal{M}: C\left(\mathbb{S}^{2}\right) \rightarrow C\left(\mathbb{S}^{2} \times[-1,1]\right), \quad \mathcal{M} f(\boldsymbol{\eta}, t)=\int_{\mathcal{C}(\boldsymbol{\eta}, t)} f(\boldsymbol{\xi}) \mathrm{d} \boldsymbol{\xi}
$$

given discrete, noisy data $g_{m}=\mathcal{M} f\left(\boldsymbol{\eta}_{m}, t_{m}\right)+\varepsilon_{m}, m=1, \ldots, M$, at points $\left(\boldsymbol{\eta}_{m}, t_{m}\right) \in \mathbb{S}^{2} \times[-1,1]$ corrupted by white noise $\varepsilon_{m}$. Our approach combines the mollifier idea with a quadrature rule to derive an estimator of the form

$$
f * \psi \approx f_{\psi}(\boldsymbol{\xi})=\sum_{m=1}^{M} \omega_{m} g_{m} \Psi\left(\boldsymbol{\xi} \cdot \boldsymbol{\xi}_{m}, t_{m}\right),
$$

where $\omega_{m} \in \mathbb{R}_{+}, m=1, \ldots, M$ are some quadrature weights and the radial function $\psi \in L^{2}(\mathbb{S})$ with $\psi=\mathcal{M}^{*} \Psi$ is the mollifier. In our talk we will give optimal minimax rates

$$
\inf _{\psi} \sup _{\substack{f \in H_{s}\left(\mathbb{S}^{2}\right) \\\|f\|_{s}<S}} \mathbb{E}\left\|f-f_{\psi}\right\|_{2}^{2}
$$

together with asymptotically optimal mollifiers $\psi$ as $M \rightarrow \infty$ for the different sampling schemata $\left(\boldsymbol{\eta}_{m}, t_{m}\right) \in$ $\mathbb{S}^{2} \times[-1,1], m=1, \ldots, M$ provided that $f$ belongs to a Sobolev ball. Finally, we illustrate our findings by numerical experiments and discuss fast algorithms for the forward as well as for the inverse transform that make use on the nonequispaced fast Fourier transform on the sphere. 


\title{
A robust estimation method for camera calibration with known rotation.
}

\author{
Amir Egozi ${ }^{1}$, Peter Maass ${ }^{1}$, Chen Sagiv $^{2}$ \\ ${ }^{1}$ the University of Bremen \\ ${ }^{2}$ SagivTech Ltd.
}

Extrinsic camera calibration is the process that yields the camera pose, i.e., its location and orientation with respect to a world reference frame. Usually its been calculated by estimating the epipolar geometry between two images and aggregating the results.

Epipolar geometry describes the relative geometry of two images depicting the same scene. It is encoded in a $3 \times 3$ singular matrix known as the fundamental matrix [3]. Estimating the fundamental matrix and thus the epipolar geometry, is a core ingredient for many of computer vision algorithm such as structure-from-motion [5], vision-based robot navigation [4] and even for intra-operative guidance [2].

A common practice is to use matching invariant features, e.g. SIFT, SURF etc. followed by a robust modelfitting algorithm. Typically, a RANSAC [1] like algorithm that samples a set of putative correspondences until an outliers-free set is found. The main weakness of RANSAC-like scheme is the requirement of sampling a valid set, where all are inliers, with a high probability. The number of sampling subsets that are needed is exponential with the subset's cardinality.

In this paper, we present an algorithm for relative pose estimation that exploited measurements data from inertial sensors, such as accelerometer, magnetometer and gyroscope which yield the camera orientation. We demonstrate that in case of known orientation the problem reduced to a 3-dimensional least-squares optimization problem, which can be readily implemented into a 3-points RANSAC scheme.

Experimental results on synthetic and real data assessed the accuracy and efficiency of our algorithm.

\section{References}

[1] M.A. Fischler and R.C. Bolles. Random sample consensus: A paradigm for model fitting with applications to image analysis and automated cartography. Communications of the ACM, 24(6):381-395, June 1981.

[2] S. Giannarou, Zhiqiang Zhang, and Guang-Zhong Yang. Deformable structure from motion by fusing visual and inertial measurement data. In Intelligent Robots and Systems (IROS), 2012 IEEE/RSJ International Conference on, pages 4816-4821, Oct 2012.

[3] Richard Hartley and Andrew Zisserman. Multiple view geometry in computer vision. Cambridge university press, 2003.

[4] Kurt Konolige, Motilal Agrawal, and Joan Solà. Large-scale visual odometry for rough terrain. In Makoto Kaneko and Yoshihiko Nakamura, editors, Robotics Research, volume 66 of Springer Tracts in Advanced Robotics, pages 201-212. Springer Berlin Heidelberg, 2011.

[5] Noah Snavely, Steven M. Seitz, and Richard Szeliski. Photo tourism: Exploring photo collections in 3d. In $A C M$ SIGGRAPH, pages 835-846. ACM, 2006. 


\title{
Harmonic analysis of projectors
}

\author{
Martin Ehler, Manuel Gräf \\ University of Vienna, Faculty of Mathematics
}

The analysis of complicated data, say high-dimensional data, usually requires some sort of dimension reduction step. We shall develop a general framework for choosing suitable projectors in finite dimensional Euclidean space.

Indeed, the Grassmannian can be considered as the set of orthogonal projectors of fixed rank in the $d$ dimensional Euclidean space. Cubatures and designs on the Grassmannian have been well-studied in the recent literature. On the other hand, particular sets of projectors with potentially varying ranks have been used in signal processing under the name fusion frames. The relations between cubatures, designs, and fusion frames have already been investigated in the literature when the rank was held fixed. Here, we introduce cubatures and designs in unions of Grassmannians and discuss the relations towards fusion frames with varying ranks. We characterize cubatures and designs in unions of Grassmannians by means of the fusion frame potential matching a certain lower bound, and we present parametric families of symmetric designs in unions of Grassmannians.

\section{References}

[1] M. Ehler, M. Gräf. Cubatures and designs in unions of Grassmannians, arXiv:1411.5865 (2014). 


\title{
Perturbations of frame sequences and the effect on their duals
}

\author{
Gitta Kutyniok $^{1}$, Victoria Paternostro ${ }^{2}$, Friedrich Philipp ${ }^{1}$ \\ ${ }^{1}$ Technische Universität Berlin \\ ${ }^{2}$ University of Buenos Aires
}

We study small perturbations of (operator-valued) frame sequences and examine the canonical and alternate duals of original and perturbed sequence. It is proved that to each dual of the unperturbed frame sequence there is a dual of the perturbed one such that also the duals are "close to each other". Roughly speaking, the duals are stable under small perturbations.

We also study the perturbation effect on fusion frame duals - a concept which was recently introduced by Heineken et al. in [1]. It turns out that this kind of duality is much harder to tackle. However, we obtain a corresponding stability result in the finite-dimensional case.

\section{References}

[1] S. Heineken, P.M. Morillas, A.M. Benavente, M.I. Zakowicz. Dual fusion frames. Arch. Math. 103 (2014), $355-365$. 


\title{
Construction of Multichannel Wavelets via Full Rank Subdivision Schemes
}

\author{
Costanza Conti ${ }^{1}$, Mariantonia Cotronei ${ }^{2}$, Tomas Sauer $^{3}$ \\ ${ }^{1}$ DIEF, Università di Firenze, Italy \\ ${ }^{2}$ DIIES, Università Mediterranea di Reggio Calabria, Italy \\ ${ }^{3}$ Lehrstuhl für Math. mit Schwerpunkt Dig. Bild., University of Passau, Germany
}

Full rank vector subdivision schemes, in particular those which have an interpolatory nature, are connected to matrix refinable functions generating orthogonal multiresolution analyses for the space of vector-valued signals. Corresponding matrix wavelets (here called multichannel wavelets) lead to a quite natural "wavelet" tool for the analysis of vector-valued signals. Such kind of signals, which arise in several real world situations, like image processing (color images), finance, seismology, or biomedical applications (e.g. electroencephalographic data), cannot be processed using classical tools operating on vector-valued data component by component ignoring the possible relation between the components. In this talk, we discuss the properties of full rank interpolatory vector subdivision schemes, the connection with orthogonal matrix scaling functions, the concept of multichannel multiresolution analysis, and an algorithm for the explicit construction of orthonormal multichannel wavelets, together with some examples of applications. 


\title{
Shearlet-Based Edge Detection: Flame Fronts and Tidal Flats
}

\author{
Georg Heygster ${ }^{1}$, Johannes Kiefer ${ }^{2}$, Emily J. King ${ }^{3}$, Zhen Li $^{1}$, Wang-Q Lim ${ }^{4}$, Rafael Reisenhofer ${ }^{3}$ \\ ${ }^{1}$ Institut für Umweltphysik, Universität Bremen \\ ${ }^{2}$ Produktionstechnik, Universität Bremen \\ ${ }^{3}$ Zentrum für Technomathematik, Universität Bremen \\ ${ }^{4}$ Institut für Mathematik, TU Berlin
}

\begin{abstract}
Shearlets are wavelet-like systems which are better suited for handling geometric features in multi-dimensional data than traditional wavelet systems [1]. As such, it seems natural to use shearlets for edge detection. A novel method for edge detection which is in the spirit of phase congruency [2] but is based on a complex shearlet transform will be presented. Pixels are assigned values between 0 (not an edge) and 1 (sharp transition). Intermediate values indicate the presence of a smoother transition between different areas. Additionally, this approach to edge detection yields an approximate tangent direction of detected edges as a byproduct of the edge measure computation.

Two applications of the edge detection method will be discussed. The tracking and classification of flame fronts is a critical component of research in technical thermodynamics. Quite often, the flame fronts are transient or weak and the images are noisy. The standard methods used in the field for the detection of flame fronts do not handle such data well. Fortunately, using the shearlet-based edge measure yields dramatically better results.

The Wadden tidal flats are a biodiverse region along the North Sea coasts of the Netherlands, Germany, and Denmark. They are a UNESCO World Heritage site due to their ecological importance. Thus, surveying the delicate region and tracking the topographical changes are of utmost importance. One approach to mapping the area is to use pre-existing Synthetic Aperture Radar (SAR) images, supplemented with other information like water level [3]. Unfortunately, SAR data suffers from multiplicative noise as well as sensitivity to environmental factors, like how much wind was blowing across the surface of the water. The first large-scale mapping project of that type showed good results but only with a tremendous amount of manual interaction on top of the computerized image processing methods. In addition to weaknesses inherent in the methods used, there are many edges in the data which are not boundaries of the tidal flats but are edges of features like fields or islands. A method using a combination of the shearlet-based edge measure with a diffusion-based image segmentation will be presented.
\end{abstract}

\section{References}

[1] K. Guo, D. Labate. Optimally Sparse Multidimensional Representation using Shearlets. SIAM J. Math Anal. 39 (2007), 298-318.

[2] P. Kovesi. Phase congruency: A low-level image invariant. Psychological Research, 64 (2000), 136-148.

[3] G. Heygster, J. Dannenberg, J. Notholt. Topographic Mapping of the German Tidal Flats Analyzing SAR Images With the Waterline Method. IEEE Trans. Geosci. Remote Sens 48.3 (March 2010), 1019-503. 


\title{
Classification of Edges Using Compactly Supported Shearlets
}

\author{
Gitta Kutyniok, Philipp Petersen \\ Technische Universität Berlin
}

We analyze the detection and classification of singularities of functions $f=\chi_{B}$, where $B \subset \mathbb{R}^{d}$ and $d=2,3$. It will be shown how the set $\partial B$ can be extracted by a continuous shearlet transform associated with compactly supported shearlets. Furthermore, if $\partial S$ is a $d-1$ dimensional piecewise smooth manifold with $d=2$ or 3 , we will classify smooth and non-smooth components of $\partial S$. This improves previous results given for shearlet systems with a certain band-limited generator, since the estimates we derive are uniform. Moreover, we will show that our bounds are optimal. Along the way, we also obtain novel results on the characterization of wavefront sets in 3 dimensions by compactly supported shearlets. Finally, geometric properties of $\partial S$ such as curvature are described in terms of the continuous shearlet transform of $f$. 


\title{
Approximation in variation for nonlinear Mellin integral operators
}

\author{
Laura Angeloni, Gianluca Vinti \\ Dipartimento di Matematica e Informatica, Università degli Studi di Perugia, \\ Via Vanvitelli 1, 06123, Perugia (Italy)
}

Mellin analysis is well-known in approximation theory and Mellin operators are widely studied in the literature (see [13, 11] for an extensive theory, and, for other approximation results, $[9,8,7,4,5])$. The importance of Mellin operators is not only from a mathematical point of view, but also because of their applications in several fields. For example, they are connected with some problems of Signal Processing: indeed, Mellin analysis is very useful in situations in which, in order to reconstruct a signal, the samples are not uniformly spaced, as in the classical Shannon Sampling Theorem, but exponentially spaced (see, e.g., [10, 12]).

In this direction, we present the theory and some recent applications of Mellin integral operators: we will show how they can be used to approximate multivariate functions of bounded variation and we will also discuss some possible applications of such operators, together with other similar families of operators, to problems of image reconstruction.

In particular, we will study a family of nonlinear Mellin integral operators defined as

$$
\left(T_{w} f\right)(\mathbf{s})=\int_{\mathbb{R}_{+}^{N}} K_{w}(\mathrm{t}, f(\mathrm{st})) \frac{d \mathrm{t}}{\langle\mathrm{t}\rangle}, \mathbf{s} \in \mathbb{R}_{+}^{N}, w>0,
$$

where $\left\{K_{w}\right\}_{w>0}$ is a family of kernel functions, $\langle\mathrm{t}\rangle:=\prod_{i=1}^{N} t_{i}, \mathrm{t}=\left(t_{1}, \ldots, t_{N}\right) \in \mathbb{R}_{+}^{N}$, and $f: \mathbb{R}_{+}^{N} \rightarrow \mathbb{R}^{\text {is a }}$ function of bounded variation.

As pointed out before, our interest about approximation results in $B V$-spaces is due also to the important applications of such results in some problems of image reconstruction: indeed, the setting of $B V$-spaces is suitable in order to describe jumps of grey-levels of the image that correspond, from a mathematical point of view, to discontinuities. In this direction it is essential to consider the case of functions of several variables. For this reason we will use a new multidimensional concept of variation and, in order to treat Mellin integral operators, the most natural approach is to frame our study on $\mathbb{R}_{+}^{N}$ equipped with the $\log$-Haar measure $\mu(A):=\int_{A}\langle\mathrm{t}\rangle^{-1} d \mathrm{t}$, where $A$ is a Borel subset of $\mathbb{R}_{+}^{N}$. For references about approximation results in $B V$-spaces in multidimensional frame, see e.g. $[6,2,1,3]$. The new multidimensional concept of variation that we will use was introduced in [5] and it was adapted to the setting of $\mathbb{R}_{+}^{N}$ from the multidimensional variation by Tonelli.

By means of this concept of variation, we first obtain several estimates for the family of integral operators $\left\{T_{w} f\right\}_{w>0}$, proving, for example, that they are well-defined and that they map $B V\left(\mathbb{R}_{+}^{N}\right)$ into itself. Then we prove the main convergence theorem, which states that

$$
\lim _{w \rightarrow+\infty} V\left[T_{w} f-f\right]=0
$$

whenever $f \in A C\left(\mathbb{R}_{+}^{N}\right.$ ) (the space of absolutely continuous functions on $\mathbb{R}_{+}^{N}$ ). Introducing suitable Lipschitz classes, we also study the problem of the rate of approximation. Moreover, in the particular case of Fejér-type kernels, we obtain that all the assumptions of our results are implied by the classical condition that the absolute moments of order $\alpha$ of the kernels are finite.

\section{References}

[1] L. Angeloni. Approximation results with respect to multidimensional $\varphi$-variation for nonlinear integral operators. Z. Anal. Anwendungen. 32(1) (2013), 103-128.

[2] L. Angeloni, G. Vinti. Convergence and rate of approximation for linear integral operators in $B V^{\varphi}-$ spaces in multidimensional setting. J. Math. Anal. Appl. 349 (2009) 317-334.

[3] L. Angeloni, G. Vinti. Approximation in variation by homothetic operators in multidimensional setting. Differential Integral Equations 26(5-6) (2013), 655-674. 
[4] L. Angeloni, G. Vinti. Convergence and rate of approximation in $B V^{\varphi}\left(\mathbb{R}_{+}^{N}\right)$ for a class of Mellin integral operators. Atti Accad. Naz. Lincei Cl. Sci. Fis. Mat. Natur. Rend. Lincei (9) Mat. Appl. 25 (2014), $217-232$.

[5] L. Angeloni, G. Vinti. Variation and approximation in multidimensional setting for Mellin operators. New Perspectives on Approximation and Sampling Theory-Festschrift in honor of Paul Butzer's 85th birthday, Birkhauser, in print, 2014.

[6] C. Bardaro, P.L. Butzer, R.L. Stens, G. Vinti. Convergence in variation and rates of approximation for Bernstein-type polynomials and singular convolution integrals. Analysis 23 (2003), 299-340.

[7] C. Bardaro, I. Mantellini. On Mellin convolution operators: a direct approach to the asymptotic formulae. Integral Transforms Spec. Funct. 25(3) (2014), 182-195.

[8] C. Bardaro, J. Musielak, G. Vinti. Nonlinear Integral Operators and Applications. De Gruyter Series in Nonlinear Analysis and Applications, New York, Berlin, 9, 2003.

[9] C. Bardaro, S. Sciamannini, G. Vinti. Convergence in $B V_{\varphi}$ by nonlinear Mellin-type convolution operators. Funct. Approx. Comment. Math. 29 (2001), 17-28.

[10] M. Bertero, E.R. Pike. Exponential-sampling method for Laplace and other dilationally invariant transforms: I. Singular-system analysis. II. Examples in photon correlation spectroscopy and Fraunhofer diffraction. Inverse Problems 7 (1991), 1-20, 21-41.

[11] P.L. Butzer, S. Jansche. A direct approach to the Mellin Transform. J. Fourier Anal. Appl. 3 (1997), 325-376.

[12] P.L. Butzer, S. Jansche. The Exponential Sampling Theorem of Signal Analysis. Atti Sem. Mat. Fis. Univ. Modena, Suppl. Vol. 46, a special issue of the International Conference in Honour of Prof. Calogero Vinti (1998), 99-122.

[13] R.G. Mamedov. The Mellin transform and approximation theory. "Elm", Baku, 1991 (in Russian). 


\title{
Multivariate sampling Kantorovich operators: from the theory to the Digital Image Processing algorithm
}

\author{
Danilo Costarelli, Gianluca Vinti \\ Department of Mathematics and Computer Science, University of Perugia, Italy \\ danilo.costarelli@gmail.com, gianluca.vinti@unipg.it
}

Applications to Approximation and to Signal Theory by means of family of sampling operators have been largely studied since $1980 \mathrm{~s}$, in order to investigate possible extensions or generalization of the classical WittakerKotelnokov-Shannon (WKS) sampling theorem, see e.g. [5, 1, 11]. The WKS sampling theorem provides an exact reconstruction formula for band limited, finite energy functions. In order to weaken the above assumptions on the signal being reconstructed, the German mathematician P.L. Butzer introduced the generalized sampling operators, see e.g., $[4,3]$. These operators revealed to be very suitable to approximate (in some sense) continuous signals $f$ by means of sampling series involving sample values $f(k / w), k \in \mathbb{Z}, w>0$. However, in real word cases, signals (such as images) are often discontinuous. For instance, gray scale images are characterized by jumps of gray levels in the areas close to the edges of images, and they can be modeled by multivariate discontinuous signals. Here, we present the theory and some applications to Digital Image Processing (D.I.P.) of the multivariate sampling Kantorovich operators (see e.g., $[2,7,6]$ ), defined by:

$$
\left(S_{w} f\right)(\underline{x}):=\sum_{\underline{k} \in \mathbb{Z}^{n}} \chi\left(w \underline{x}-t_{\underline{k}}\right)\left[\frac{w^{n}}{A_{\underline{k}}} \int_{R_{\underline{k}}^{w}} f(\underline{u}) d \underline{u}\right], \quad \underline{x} \in \mathbb{R}^{n},
$$

where $f: \mathbb{R}^{n} \rightarrow \mathbb{R}$ is a locally integrable function such that the above series is convergent for every $\underline{x} \in \mathbb{R}^{n}$. The function $\chi: \mathbb{R}^{n} \rightarrow \mathbb{R}$ is called a kernel, and it satisfies suitable properties. Moreover, $t_{\underline{k}}=\left(t_{k_{1}}, \ldots, t_{k_{n}}\right)$ denotes vectors where each $\left(t_{k_{i}}\right)_{k_{i} \in \mathbb{Z}}, i=1, \ldots, n$ is a certain strictly increasing sequence of real numbers with $\Delta_{k_{i}}=t_{k_{i+1}}-t_{k_{i}}>0$. Note that the sequences $\left(t_{k_{i}}\right)_{k_{i} \in \mathbb{Z}}$ are not necessary equally spaced (irregular sampling scheme). The symbols $R_{\underline{k}}^{w}$ denote the sets of the form:

$$
R_{\underline{k}}^{w}:=\left[\frac{t_{k_{1}}}{w}, \frac{t_{k_{1}+1}}{w}\right] \times\left[\frac{t_{k_{2}}}{w}, \frac{t_{k_{2}+1}}{w}\right] \times \ldots \times\left[\frac{t_{k_{n}}}{w}, \frac{t_{k_{n}+1}}{w}\right]
$$

$w>0$ and $A_{\underline{k}}=\Delta_{k_{1}} \cdot \Delta_{k_{2}} \cdot \ldots \cdot \Delta_{k_{n}}, \underline{k} \in \mathbb{Z}^{n}$. The sampling Kantorovich operators $S_{w}$ (see also [12, 8, 13]) represent an $L^{1}$-version of the generalized sampling operators and they are very suitable to reconstruct not necessarily continuous signals, thus applications to image reconstruction can be deduced.

The main approximation results for the above operators are respectively a pointwise and uniform convergence theorem for bounded continuous and bounded uniformly continuous functions on $\mathbb{R}^{n}$. In this case, a result concerning the order of approximation can be obtained considering signals in suitable Lipschitz classes of the Zygmund-type (see $[9,10]$ ). In order to obtain a convergence result for not necessarily continuous signals, the theory of multivariate sampling Kantorovich operators has been studied in the general setting of Orlicz spaces $L^{\varphi}\left(\mathbb{R}^{n}\right)$, where $\varphi$ is a convex $\varphi$-function, see e.g. [4]. These spaces are very general and they include as noteworthy special cases the well-known $L^{p}$-spaces. Other important and useful examples of Orlicz spaces, for which the above theory can be applied, are provided by the interpolation and the exponential spaces.

In $L^{\varphi}\left(\mathbb{R}^{n}\right)$, the modular convergence of the family $\left(S_{w} f\right)_{w>0}$ to $f$ is proved, being $f \in L^{\varphi}\left(\mathbb{R}^{n}\right)$. In the latter setting, in order to study the rate of approximation for the sampling Kantorovich operators, a suitable definition of Lipschitz classes can be introduced by using the modular functional of the space $L^{\varphi}\left(\mathbb{R}^{n}\right)$.

Based on the above approximation results, from the definition of the operators in (I) and for a suitable choice of the kernel function $\chi$, an algorithm for image reconstruction and enhancement can be implemented.

A great importance in the above D.I.P. algorithm is the choice of the multivariate kernel function $\chi$, which in general influence the rate of approximation and the performance of the sampling Kantorovich operators. A procedure that allow us to construct multivariate kernels by the product of univariate kernels satisfying the assumptions of the above theory can be showed. Examples of one-dimensional kernels are the well-known Fejér's kernel, the central B-spline of order $k \in \mathbb{N}$, the Jackson-type kernels and many others, see e.g., $[2,7]$. 


\section{References}

[1] C. Bardaro, P.L. Butzer, R.L. Stens, G. Vinti. Approximation of the Whittaker sampling series in terms of an average modulus of smoothness covering discontinuous signals. J. Math. Anal. Appl. 316 (2006), $269-306$.

[2] C. Bardaro, P.L. Butzer, R.L. Stens, G. Vinti. Kantorovich-type generalized sampling series in the setting of Orlicz spaces. Sampl. Theory Signal Image Proces. 6 (1) (2007), 29-52.

[3] C. Bardaro, P.L. Butzer, R.L. Stens, G. Vinti. Prediction by samples from the past with error estimates covering discontinuous signals. IEEE, Trans. Inform. Theory 56 (1) (2010), 614-633.

[4] C. Bardaro, J. Musielak, G. Vinti. Nonlinear Integral Operators and Applications, De Gruyter Series in Nonlinear Analysis and Applications, New York, Berlin 9 (2003).

[5] P.L. Butzer, R.L. Stens. Linear prediction by samples from the past. Advanced topics in Shannon sampling and interpolation theory, 157-183, Springer Texts Electrical Eng., Springer, New York (1993).

[6] F. Cluni, D. Costarelli, A.M. Minotti, G. Vinti. Enhancement of thermographic images as tool for structural analysis in earthquake engineering. NDT \& E (2014) DOI: 10.1016/j.ndteint.2014.10.001, in print.

[7] D. Costarelli, G. Vinti. Approximation by multivariate generalized sampling Kantorovich operators in the setting of Orlicz spaces. Bollettino U.M.I. 9 (IV) (2011), 445-468.

[8] D. Costarelli, G. Vinti. Approximation by nonlinear multivariate sampling Kantorovich type operators and applications to Image Processing, Num. Funct. Anal. Optim. 34 (8) (2013), 819-844.

[9] D. Costarelli, G. Vinti. Order of approximation for sampling Kantorovich operators. J. Integ. Equations Appl. 26 (3) (2014) 345-368.

[10] D. Costarelli, G. Vinti. Rate of approximation for multivariate sampling Kantorovich operators on some functions spaces. J. Integ. Equations Appl. 26 (4) (2014), in print.

[11] G. Vinti. A general approximation result for nonlinear integral operators and applications to signal processing. Applicable Anal. 79 (2001), 217-238.

[12] G. Vinti, L. Zampogni. Approximation by means of nonlinear Kantorovich sampling type operators in Orlicz spaces. J. Approx. Theory 161 (2009), 511-528.

[13] G. Vinti, L. Zampogni. Approximation results for a general class of Kantorovich type operators. Adv. Nonlin. Studies 14 (2014), 991-1011. 


\title{
Stockwell Transform and Applications to Image Analysis
}

\author{
Ubertino Battisti, Salvatore Pirro, Luigi Riba, Luigi Sambuelli \\ Università degli Studi di Torino \\ ITABC-CNR Roma \\ Université de Pau et des Pays de l'Adour \\ Politecnico di Torino
}

Let $f$ be a signal of finite energy, then

$$
(\mathrm{S} f)(b, \xi)=(2 \pi)^{-1 / 2} \int_{\mathbb{R}} e^{-2 \pi \mathrm{i} t \xi} f(t)|\xi| e^{-(t-b)^{2} \xi^{2} / 2} d t, \quad b, \xi \in \mathbb{R}
$$

is called the Stockwell transform (S-transform) of the signal $f$. It was first introduced in 1996 by R. G. Stockwell et al. in connection to seismic problems, see [2]. Roughly speaking, we can say that $|(\mathrm{S} f)(b, \xi)|$ represents the energy of the signal $f$ at a given time $b$ and at a given frequency $\xi$. Clearly, this statement is not really precise due to the Heisenberg Uncertainty Principle.

The S-transform lies in between the Short Time Fourier Transform and the Wavelet Transform. It shares with the Short Time Fourier Transform a clear time-frequency representation. In fact, it can be seen as the Fourier transform of the signal $f$ cut by an analyzing window, in this case a Gaussian. The main difference is that the window is frequency dependent. This change implies that, as the frequency increases, the width of the analyzing window shrinks. This idea is consistent with the Nyquist Theorem. On the other hand, this feature shows a clear link with the Wavelet Transform, but in this case we have not a time-scale domain, rather a real time-frequency plot. The computational cost of the S-transform of a signal of length $N$ is $\mathcal{O}\left(N^{2} \log N\right)$, the same of the Short Time Fourier Transform and of the of Continuous Wavelet Transform.

In collaboration with L. Riba [1], we have studied a modified S-transform, called DOST, introduced again by Stockwell in [3]. This transform gained interest after the fast algorithm introduced by Y. Wang and J. Orchard [4], which reduced drastically the complexity to $\mathcal{O}(N \log N)$. This improvement enlarged the applications of the DOST to problems in image processing as, for example, denoising and compression. The DOST decomposes a signal $f$ of length $N$ into $N$-coefficients $f_{p, \tau}$. Each coefficient $f_{p, \tau}$ represents the time-frequency content of the signal in a certain time frequency box. The properties of the transform imply that, for low frequencies, we have very high precision in frequency, and low precision in time; while, for high frequencies, we have high precision in time and low precision in frequency. Translated into image analysis, this means that the DOST is able to detect with high precision edges.

We will finally present some results obtained in collaboration with L. Riba, L. Sambuelli and S. Pirro. We applied the DOST to noise reduction in the framework of GPR (Ground Penetrating Radar) data. In particular to GPR analysis of Archaeological sites. This type of data is usually affected by a large number of noises, therefore the post processing plays a central role. The traditional tools in this field are based on Fourier Analysis, while time-frequency methods are not usually considered.

\section{References}

[1] Ubertino Battisti, Luigi Riba Window-dependent Bases for Efficient Representations of the Stockwell Transform. preprint on http://arxiv.org/abs/1406.0513.

[2] R. G. Stockwell, L. Mansinha, and Lowe R. P. Localization of the complex spectrum: the S transform. IEEE Transactions on Signal Processing, 44:998-1001, 1996.

[3] R. G. Stockwell. A basis for efficient representation of the S-transform. Digital Signal Processing, 17:371$393,2007$.

[4] Y. Wang and J. Orchard. Fast discrete orthonormal Stockwell transform. SISC, 31:4000-4012, 2009. 


\title{
Applications of Approximation Theory to thermographic images in earthquake engineering
}

\author{
Federico Cluni*,Danilo Costarelli, Anna Maria Minotti,Gianluca Vinti \\ *Departement of Civil and Environmental Engineering, University of Perugia, Italy \\ federico.cluni@unipg.it \\ Department of Mathematics and Computer Science, University of Perugia, Italy \\ danilo.costarelli@gmail.com \\ annamaria.minotti@dmi.unipg.it \\ gianluca.vinti@unipg. it
}

In this talk we present some applications of an approximation process by means of a family of sampling type operators to thermographic images, (see e.g., $[2,1,3,4]$ ).

The mathematical theory of these operators shows how it is possible to reconstruct and to enhance multivariate signals, such as images. In particular, we are able to reconstruct images taken from thermographic survey of masonry walls, and to enhance their quality. Thermographic images are largely used to make diagnosis and monitoring buildings. Moreover these images are used both to assess effective dimensions of structural elements and for masonry texture, i.e. the mutual arrangement of the blocks (made of stones and/or bricks) and mortar joints inside the wall portion analyzed.

For the image texture we use digital images algorithms that perform as follow: first of all we apply a median filter to the image using a suitable mask, then the image is converted into a black and white image by means of a suitable thresholding, morphological operators are used to enhance the quality of the separation of the phase such as the closure of the area to eliminate salt-and-pepper noise and erosion and dilation operators to smooth the contours of the inclusions.

We will show that the image texture obtained by the application of our algorithms is more realistic from an engineering point of view and more fitting to the real structure and therefore allows us to make an accurate analysis of the building.

The reconstruction methods are used to estimate the mechanical characteristics of the masonry walls of a real-world case-study. These mechanical properties allows us to analyze the response of a masonry structure under seismic actions in terms of modal analysis.

Our interest is in the applicability of the proposed procedure and the advantages that can be achieved in comparison with more traditional approaches. In particular our model suggests a method to overcome some difficulties that arise when dealing with the vulnerability analysis of existing structures and allows us to estimate the mechanical characteristics of the masonries using non-destructive methods, (see e.g., $[5,6]$ ).

\section{References}

[1] C. Bardaro, P.L. Butzer, R.L. Stens, G. Vinti. Kantorovich-type generalized sampling series in the setting of Orlicz spaces. Sampl. Theory Signal Image Proces. 6(1) (2007), 29-52.

[2] C. Bardaro, J. Musielak, G. Vinti. Nonlinear Integral Operators and Applications. De Gruyter Series in Nonlinear Analysis and Applications, New York, Berlin 9 (2003).

[3] D. Costarelli, G. Vinti. Approximation by multivariate generalized sampling Kantorovich operators in the setting of Orlicz spaces. Bollettino U.M.I. 9 (IV) (2011), 445-468.

[4] D. Costarelli, G. Vinti. Approximation by nonlinear multivariate sampling Kantorovich type operators and applications to Image Processing. Num. Funct. Anal. Optim. 34(8) (2013), 819-844.

[5] F. Cluni, D. Costarelli, A.M. Minotti, G. Vinti. Enhancement of thermographic images as tool for structural analysis in earthquake engineering. NDT \& E (2014) DOI: 10.1016/j.ndteint.2014.10.001, in print.

[6] F.Cluni, D.Costarelli, A.M.Minotti, G.Vinti. Applications of sampling Kantorovich operators to thermographic images for seismic engineering. J. Comput. Anal. Appl. 19(4) (2015), 602-617. 


\title{
A generalization of the Zak transform with applications in sampling theory and physics
}

\author{
Dominik Jüstel \\ Technische Universität München
}

The Zak transform on $\mathbb{R}^{d}, d \in \mathbb{N}$, has many applications in signal processing and quantum mechanics it provides a time-frequency decomposition of a signal [1] and serves as a tool to study quantum mechanical systems with lattice symmetries [2]. Generalizations of the Zak transform to locally compact abelian groups and beyond have been studied in [3, 4].

In this contribution, a framework will be presented that includes these generalizations, while also being applicable to the action of unimodular automorphism groups on a quite general class of locally compact groups, for example. This generalized Zak transform is based on a Weil formula for quasi-invariant measures on double coset spaces. It naturally has applications for the sampling of signals at orbits of groups that act on the underlying space and provides complete sets of functions to study physical systems with respect to their symmetries. In particular, an application in radiation design will be presented.

\section{References}

[1] A. J. E. M. Janssen. The Zak Transform: A Signal Transform for Sampled Time-Continuous Signals. Philips J. Res. 43 (1988), 23-69.

[2] J. Zak. Finite Translations in Solid-State Physics. Phys. Rev. Lett. 19 (1967), 1385-1387.

[3] E. Kaniuth, G. Kutyniok. Zeros of the Zak Transform on Locally Compact Abelian Groups. Proc. Amer. Math. Soc. 126 (1998), 3561-3569.

[4] G. Kutyniok. The Zak Transform on Certain Locally Compact Groups. J. of Math. Sciences 1 (2002), 62-85. 


\title{
Multivariate Generalized Sampling Type Series: estimates of pointwise convergence
}

\author{
Carlo Bardaro, Ilaria Mantellini \\ Department of Mathematics and Informatics University of Perugia
}

This contribution presents a Kantorovich multivariate version of the generalized sampling series of a function $f: \mathbb{R}^{N} \rightarrow \mathbb{R}$, namely

$$
\left(K_{p(n)} f\right)(x)=\sum_{k \in \boldsymbol{Z}}^{N} \varphi(p(n) x-k)\left[\langle p(n)\rangle \int_{Q_{k, n}} f(u) d u\right], \quad x \in \mathbb{R}^{N},
$$

where $p: \mathbb{N} \rightarrow \mathbb{R}^{N}$ is a vector valued function satisfying suitable assumptions, $\varphi$ is a suitable kernel and

$$
Q_{k, n}=\prod_{j=1}^{N}\left[\frac{k_{j}}{p_{j}(n)}, \frac{k_{j}+1}{p_{j}(n)}\right]
$$

with $p=\left(p_{1}, \ldots, p_{N}\right), k=\left(k_{1}, \ldots, k_{N}\right)$ and for any vector $a=\left(a_{1}, \ldots, a_{n}\right)$, we put $\langle a\rangle=\prod_{j=1}^{N} a_{j}$. We study some asymptotic formulae for the pointwise convergence of the operators $K_{p(n)} f$ to $f$. Then we introduce certain suitable linear combinations of these operators in order to obtain a better order of the pointwise convergence. Finally we apply the theory to the multivariate Bochner-Riesz sampling series. These results were obtained in [2]. Related operators were also studied in [4] and [5], in the frame of Orlicz type spaces in which extensions of the one-dimensional theory introduced in [1] are outlined.

More recent generalized versions are also introduced in [3], in which a Durrmeyer type approach is used.

These studies have direct applications to signal processing, in particular to image reconstruction.

\section{References}

[1] C. Bardaro, P.L. Butzer, G. Vinti. Kantorovich-type generalized Sampling series in the setting of Orlicz spaces. Sampl. Theory Signal Image Process. 6 (1) (2007), 29-52.

[2] C. Bardaro, I. Mantellini. On linear combinations of multivariate generalized sampling type series. Mediterr. J. Math. 10 (2013), 1833-1852.

[3] C. Bardaro, I. Mantellini. Asymptotic expansion of generalized Durrmeyer sampling type series. Jaen J. Approx. 62 (2014), 143-165.

[4] D. Costarelli, G. Vinti. Approximation by multivariate generalized sampling Kantorovich operators in the setting of Orlicz Spaces. Boll. Unione Mat. Ital, special volume dedicated to Prof. Giovanni Prodi, 9 (IV) (2011), 445-468.

[5] G. Vinti, L. Zampogni. Approximation by means of nonlinear Kantorovich sampling type operators in Orlicz Spaces. J. Approx. Theory 161 (2009), 511-528. 


\title{
A New Blind Source Separation Numerical Technique
}

\author{
Ivan Gerace, Maurizio Scognamiglio \\ Universitá degli Studi di Perugia \\ Dipartimento di Matematica e Informatica \\ Via Vanvitelli, 1 \\ I-06123 Perugia (Italy)
}

Digital Document Restoration (DDR) consists of a set of processes finalized to the visual and aesthetic improvement of a virtual reconstruction of the document. By DDR a document can be analyzed without deteriorating it. Main degradation types are: Bleed-through, that is a physical phenomenon due to the ink infiltration from the opposite side of a page; show-through, that is an effect due to the scanning process and the paper transparency. We deal here with the problem of estimating the original sources from two data mixtures of these sources produced by the bleed through or the show through effect. This problem belongs to the Blind Source Separation (BSS) class of problems.

We assume that the mixture model is linear. This assumption is not always verified in the real cases, anyway it is essential to construct the basement of more complex models. The data images that we consider are the front and back of a degraded document. The inverse problem consists in estimating both the source images and the mixture matrix, given the observed data. This problem is a well known ill-posed problem [2], that is, in some cases, the solution neither exists, nor is unique, nor can be stable in presence of noise.

With the aim of restored the well-position of the problem, different techniques are proposed in the literature; in particular many of them that imposed the orthogonality of the results $[1,3]$. We present here a new technique that try to find the best factorization of the covariance matrix of the data. The experimental results confirm the goodness of such a technique.

\section{References}

[1] A. Hyvarinen. Fast and robust fixed-point algorithms for independent component analysis. IEEE Transactions on Neural Networks 10 (1999), 626-634.

[2] A. Tonazzini, I. Gerace, F. Martinelli. Multichannel blind separation and deconvolution of images for document analysis. IEEE Trans. Image Processing, 19 (2010), 912-925.

[3] A. Tonazzini, E. Salerno, L. Bedini. Fast correction of bleed-through distortion in grayscale documents by a blind source separation technique. International Journal of Document Analysis 10 (2007), 17-25. 


\title{
Geometric Means of Toeplitz Matrices by Positive Parametrizations
}

\author{
Dario A. Bini, Bruno Iannazzo, Stefano Serra Capizzano \\ Università di Pisa, Italy \\ Università di Perugia, Italy \\ Università dell'Insubria, Italy
}

We consider the problem of finding means of geometric type of Hermitian positive definite matrices which are also Toeplitz, i.e., constant along their diagonals.

One might be tempted to use the geometric mean of positive definite matrices, the Karcher mean [3], which is obtained as the barycenter in a suitable Riemannian geometry on the positive definite matrices. Unfortunately, the geometric mean is not well suited for averaging Toeplitz matrices, since the mean of Toeplitz matrices may not be Toeplitz.

To overcome this difficulty, two different approaches have been introduced:

- an axiomatic approach, which produces a structured geometric mean, satisfying most of the properties which are desirable for a geometric mean, but giving up some of them, in order to enforce the structure [4];

- a constructive approach, which defines a new metric on Toeplitz matrices and choose as the geometric mean the barycenter there, said to be the Kähler mean [1].

The latter mean can be obtained using a parametrization of the $n \times n$ positive definite Toeplitz matrices through $n$ complex numbers with positive real part, moreover it can be uncoupled considering just $n$ scalar barycenters [4].

By using different parametrizations of the Toeplitz matrices, we generalize this approach obtaining different definitions of Toeplitz means with the same features as the Kähler mean but with better computational properties. We investigate further the asymptotic behaviour of these means with respect to the functional interpretation of sequences of Toeplitz matrices of increasing size [5].

The problem under investigation arises in radar signal processing, where the use of differential geometry in mathematical statistics (information geometry) has given recently important updates [2]. In particular, in the design of radar systems in the presence of a randomly moving background (clutter), such as the see, a way to proceed is to do repeated measures of a suitable autocorrelation matrix relative to the noised signal. These matrices are Toeplitz and positive definite and need to be averaged, and their average should verify some properties of the geometric mean.

\section{References}

[1] M. Arnaudon, M., F. Barbaresco and L. Yang. Riemannian Medians and Means With Applications to Radar Signal Processing. IEEE Journal of Selected Topics in Signal Processing, 7-4 (2011), 595-604.

[2] F. Barbaresco. Information geometry manifold of Toeplitz Hermitian positive definite covariance matrices: Mostow/Berger fibration and Berezin quantization of Cartan-Siegel domains. International Journal of Emerging Trends in Signal Processing, 1 (2013), 1-11.

[3] D. A. Bini and B. Iannazzo. Computing the Karcher mean of symmetric positive definite matrices. Linear Algebra Appl., 438-4 (2013), 1700-1710.

[4] D.A. Bini, B. Iannazzo, B. Jeuris and R.Vandebril. Geometric means of structured matrices. BIT, 54-1 (2014), 55-83.

[5] A. Böttcher, B. Silbermann. Introduction to large truncated Toeplitz matrices. Springer-Verlag, New York, NY, 1999. 


\title{
A unifying theory for convergence of linear sampling operators in Orlicz spaces
}

\author{
Gianluca Vinti, Luca Zampogni \\ University of Perugia, Italy.
}

Sampling operators play a crucial role in Signal and Image Processing. The generalized sampling series [1]

$$
S_{w} f(x)=\sum_{k \in \mathbb{Z}} \chi(w x-k) f\left(\frac{k}{w}\right)
$$

where $\chi$ is a continuous kernel with compact support over $\mathbb{R}$ is just an example of a discrete operator which can be used to reconstruct a function (signal) from its sampling values $f\left(\frac{k}{w}\right)$. However, the series (1) fails to be appropriate when $f$ is no longer continuous. Obvious practical reasons led to the introduction of a sampling series of Kantorovich type [2], namely

$$
K_{w} f(x)=\sum_{k \in \mathbb{Z}} \chi(w x-k) w \int_{\frac{k}{w}}^{\frac{k+1}{w}} f(u) d u .
$$

In $(2)$, the values $f\left(\frac{k}{w}\right)$ are replaced by the averages

$$
w \int_{\frac{k}{w}}^{\frac{k+1}{w}} f(u) d u
$$

with the result of reducing the so-called time-jitter errors and obtaining the convergence for a wider class of functions (e.g., for functions which lie in an Orlicz space $L^{\varphi}(\mathbb{R})$ ). However, other sampling operators have been defined to treat signals when other types of errors appear, for instance the round-off errors.

In order to manage simultaneously the theories of discrete linear sampling operators, we introduce the following general form of the sampling series

$$
V_{w} f(x)=\sum_{k \in \mathbb{Z}} \chi\left(w x-t_{k}\right) L_{t_{k} / w} f
$$

where $\left(L_{t_{k} / w}\right): M(\mathbb{R}) \rightarrow \mathbb{R}$ is a family of continuous functionals having as domain the set $M(\mathbb{R})$ of measurable real functions [6]. The values $t_{k}$ are introduced to consider nonuniform sampling.

We discuss the construction and analyze the convergence of the series (3), including the general setting of Orlicz spaces $L^{\varphi}(\mathbb{R})$ (i.e., for not necessarily continuous functions).

Motivated by the developments in [7], the construction can be further generalized to the setting of topological groups, by introducing the operators

$$
T_{w} f(z)=\int_{t \in H} \chi\left(z-h_{w}(t)\right) L_{h_{w}(t)} f d \mu(t),
$$

where $H, G$ are locally compact topological groups, $\left(h_{w}\right): H \rightarrow G$ is a family of homeomorphisms, $\left(L_{h_{w}(t)}\right)$ : $M(G) \rightarrow \mathbb{R}$ is a family of continuous operators having as domain the set $M(G)$ of measurable functions defined on the topological group $G$ and $\mu(t)$ is the Haar measure on $H$. This approach allows to include integral operators, to generalize convolution and Mellin operators and to discuss multidimensional signals as well (see, e.g., $[3,4])$.

\section{References}

[1] P. L. Butzer. A survey of the Whittaker-Shannon sampling theorem and some of its extensions. J. Math. Res. Expositions 3 (1983), 185-212. 
[2] C. Bardaro, P. L. Butzer, R.L. Stens and G. Vinti. Kantorovich-type generalized sampling series in the setting of Orlicz spaces. Sampling Theory in Signal and Image Processing 6 (2007), 29-52.

[3] D. Costarelli and G. Vinti. Approximation by multivariate generalized sampling Kantorovich operators in the setting of Orlicz spaces. Boll. Unione Mat. Ital. 9 (2011), 445-468.

[4] D. Costarelli and G. Vinti. Approximation by nonlinear multivariate sampling Kantorovich type operators and applications to image processing. Numer. Funct. Anal. Optim. 34 (2013), 819-844.

[5] G. Vinti and L. Zampogni. Approximation by means of nonlinear Kantorovich sampling type operators in Orlicz spaces. Journal of Approx. Theory 161 (2009), 511-528.

[6] G. Vinti and L. Zampogni. A unifying approach to convergence of linear sampling type operators in Orlicz spaces. Advances in Differential Equations 16 (2011), 573-600.

[7] G. Vinti and L. Zampogni. Approximation results for a general class of Kantorovich type operators. Adv. Nonlin. Stud. 14 (2014), 991-1012. 


\title{
Digital image processing algorithms for diagnosis in arterial diseases
}

\author{
Danilo Costarelli, Marco Seracini, Gianluca Vinti \\ Department of Mathematics and Computer Science, University of Perugia, Italy
}

Computed Tomography images (C.T.) are currently part of the routine procedure in medical diagnostic techniques. The use of CT images can be focused on the evaluation of occlusion rate of arterial vessels in presence of atheromas, atherosclerosis, and thrombi's formation process. The correct individuation of the morphology of these arterial anomalies allows specialists to diagnose the risk rate for the health of patients and to decide for surgical stent implants [3]. Due to no radio opacity of the blood, the CT image acquisition procedure often does not allow the doctors to identify by visual analysis the anatomy of the occlusive structures in the arterial vessels: in these cases the use of radio-opaque contrast medium becomes necessary. On the other hand the introduction of radio-opaque contrast medium is critical in patients affected by kidney dysfunctions and is anyway not healthy in any case.

Multivariate sampling Kantorovich operators $[2,1,9,5,4,10]$ and subsequent wavelet analysis allow to emphasize the pathological arterial vessel morphology improving the visual diagnosis even without contrast medium introduction. The previous family of operators are suitable for studying not necessarily continuos signals/images as the ones involved in the medical field [7]. Increasing the sampling rate and choosing an appropriate kernel we are able to enhance the images under consideration [6].

The standard in medical digital images (Digital Imaging Communication in Medicine, D.I.CO.M.) preserves linear and area measurements even using differently compression algorithms.

CT images are gray-scale bidimensional matrices and it's possible to use the discrete two dimensional wavelet transform (2D D.W.T.) [8], deriving it from the continuos wavelet transform (C.W.T.) defined as:

$$
\gamma(s, \tau)=\int f(t) \psi_{\tau, s}^{*}(t) d t \quad, \quad t \in \mathbb{R}
$$

where $s$ is the scale factor, $\tau$ is the translation and $\psi^{*}$ is the complex conjugate of the mother wavelet function, defined as:

$$
\psi(t)=\frac{1}{\sqrt{s}} \psi\left(\frac{t-\tau}{s}\right) \quad, \quad \tau, s \in \mathbb{R}
$$

and assuming in the DWT case the form:

$$
\psi_{j k}(t)=\frac{1}{\sqrt{s_{0}^{j}}} \psi\left(\frac{t-k \tau_{0} s_{0}^{j}}{s_{0}^{j}}\right) \quad, \quad j, k \in \mathbb{N}
$$

The 2D DWT together with the enhancement performed on CT images allow to extract more precisely the contours of the pathological occlusive structures while DICOM standard preserves the real geometrical dimensions, all resulting in a more precise medical diagnosis.

\section{References}

[1] C. Bardaro, P.L. Butzer, R.L. Stens, G. Vinti. Kantorovich-type generalized sampling series in the setting of Orlicz spaces. Sampl. Theory Signal Image Proces. 6 (1) (2007), 29-52.

[2] C. Bardaro, J. Musielak, G. Vinti. Nonlinear Integral Operators and Applications, De Gruyter Series in Nonlinear Analysis and Applications, New York, Berlin 9 (2003).

[3] P. Cao, E. Ceri, A. Ciucci, P. De Rango, G. Isernia, G. Parlani, F. Verzini. Effect of Stentgraft Model on Aneurysm Shrinkage in 1,450 Endovascular Aortic Repairs. Eur J Vasc Endovasc Surg. 2013 Aug; 46 (2):192-200. doi: 10.1016/j.ejvs.2013.05.012. Epub 2013 Jun 19.

[4] F. Cluni, D. Costarelli, A.M. Minotti, G. Vinti. Enhancement of thermographic images as tool for structural analysis in earthquake engineering. NDT \& E (2014) DOI: 10.1016/j.ndteint.2014.10.001, in print. 
[5] D. Costarelli, G. Vinti. Approximation by multivariate generalized sampling Kantorovich operators in the setting of Orlicz spaces. Bollettino U.M.I. 9 (IV) (2011), 445-468.

[6] D. Costarelli, G. Vinti. Approximation by nonlinear multivariate sampling Kantorovich type operators and applications to Image Processing, Num. Funct. Anal. Optim. 34 (8) (2013), 819-844.

[7] R. C. Gonzales, R. E. Woods. Digital Image Processing, Prentice Hall, (2008).

[8] Oleg Kobylin, Vyacheslav Lyashenko. Comparison of standard image edge detection tecniques and of method based on wavelet transform. International Journal of Advanced Research 8 (2) (2014), 572-580

[9] G. Vinti, L. Zampogni. Approximation by means of nonlinear Kantorovich sampling type operators in Orlicz spaces. J. Approx. Theory 161 (2009), 511-528.

[10] G. Vinti, L. Zampogni. Approximation results for a general class of Kantorovich type operators. Adv. Nonlin. Studies 14 (2014), 991-1011. 


\title{
Nonnegative Tensor Grid Decomposition
}

\author{
Bruno Iannazzo $^{1}$, $\underline{\text { Ben Jeuris }}^{2}$, Filippo Pompili $^{1}$ \\ ${ }^{1}$ Università degli Studi di Perugia, Italy \\ ${ }^{2} \mathrm{KU}$ Leuven, Belgium
}

The topic of Nonnegative Matrix Factorization (NMF) consists in finding two nonnegative matrices $U \in$ $\mathbb{R}^{m \times k}$ and $V \in \mathbb{R}^{k \times n}$ such that the product $U V$ is the best approximation of a given nonnegative matrix $M \in \mathbb{R}^{m \times n}$ for some criterium. In most applications, it is desirable to obtain a factorization where $k$ is much smaller than $n$, since this allows the approximation of the columns of $M$ as linear combinations of the fewer columns of $U$. In terms of segmentation, the nonnegativity of the elements in these matrices provides an interesting interpretation of the factors $U$ and $V$ : the columns of $U$ can be interpreted as the templates in the segmentation, while the entries in $V$ hold the probability that a certain column of $M$ belongs to a such a template.

A generalization of this problem replaces the matrices $M$ and $U$ by a tensor grid in which each element $M_{i j}$ and $U_{i \ell}$ is now a positive definite matrix of size $\mu$, with $i=1, \ldots, m, j=1, \ldots, n$, and $\ell=1, \ldots, k$. The factorization itself has recently been generalized by Xie et al. [2] by minimizing the cost function

$$
\mathcal{E}(U, V)=\sum_{i=1}^{m} \sum_{j=1}^{n}\left\|M_{i j}-\sum_{\ell=1}^{k} U_{i \ell} v_{\ell j}\right\|_{F}^{2},
$$

where $\|\cdot\|_{F}$ is the Euclidean (Frobenius) norm.

The above model for factorizing the tensor grid $M$ implicitly assumes Euclidean geometry for the combination of $U$ and $V$ and the distance between $M$ and this combination. However, the set of positive definite matrices has a natural intrinsic geometry which has proven advantageous in the modelling of application data [3]. Applying this geometry to the method for combining $U$ and $V$ and to the distance function between $M$ and the combination results in our new cost function

$$
\mathcal{R}(U, V)=\sum_{i=1}^{m} \sum_{j=1}^{n} \delta^{2}\left(M_{i j}, K\left(v_{1 j}, \ldots, v_{k j} ; U_{i 1}, \ldots, U_{i k}\right)\right),
$$

where $\delta$ is the intrinsic distance of the positive definite matrices and $K$ represents the weighted Karcher mean [1], for which $K\left(v_{1 j}, \ldots, v_{k j} ; U_{i 1}, \ldots, U_{i k}\right)$ is defined as the unique minimizer over the set of positive definite matrices of the function $f(X)=\sum_{\ell=1}^{k} v_{\ell j} \delta^{2}\left(X, U_{i \ell}\right)$.

Finally, as a compromise between the geometry of the positive definite matrices and the speed of the Euclidean model, we discuss the Log-Euclidean setting. The main idea of this setting is to retract positive definite matrices to the set of symmetric matrices using the matrix logarithm, and afterwards proceeding as in the Euclidean case. Doing so results in a third cost function

$$
\mathcal{L}(U, V)=\sum_{i=1}^{m} \sum_{j=1}^{n}\left\|\log \left(M_{i j}\right)-\sum_{\ell=1}^{k} v_{\ell j} \log \left(U_{i \ell}\right)\right\|_{F}^{2} .
$$

All three models are applied to test data where both low rank $(k)$ image reconstruction and data segmentation are investigated, in terms of both accuracy and computational time.

\section{References}

[1] J. Lawson, Y. Lim. Weighted means and Karcher equations of positive operators. Proceedings of the National Academy of Sciences of the United States of America. 110(2013), 15626-15632.

[2] Y. Xie, J. Ho, B.C. Vemuri. Nonnegative Factorization of Diffusion Tensor Images and Its Applications. Lecture Notes in Computer Science. 6801 (2011), 550-561.

[3] P. Zakeri, B. Jeuris, R. Vandebril, Y. Moreau. Protein fold recognition using geometric kernel data fusion. Bioinformatics. 30 (2014), 1850-1857. 


\title{
Poisson Noise Removal from High-Resolution Electron Micrographs based on periodic Block-matching
}

\author{
N. Mevenkamp ${ }^{1}$, P. Binev ${ }^{2}$, W. Dahmen ${ }^{3}$, P. M. Voyles ${ }^{4}$, A. B. Yankovich ${ }^{4}$, B. Berkels ${ }^{1}$ \\ ${ }^{1}$ AICES Graduate School, RWTH Aachen University \\ ${ }^{2}$ Department of Mathematics, University of South Carolina \\ ${ }^{3}$ Institute for Geometry and Practical Mathematics, RWTH Aachen University \\ ${ }^{4}$ Materials Science and Engineering, University of Wisconsin-Madison
}

In this talk, we propose a denoising technique optimized for images from Scanning Transmission Electron Microscopy (STEM). This is an imaging technique that provides sub-ångstrom, atomic resolution images of crystalline structures by moving a focused electron probe over a very thin material along a regular grid and measuring the electrons exiting on the other side of the material within a certain annulus. In many applications, the ability to extract valuable information such as atom positions, from the acquired images, is severely obstructed by low signal-to-noise ratios due to limitations to the electron dose during acquisition. These limitations result from noticeable damage inflicted on the material by the electron beam.

The presented denoising strategy is based on the Block-matching and 3D filtering algorithm (BM3D) [1] with improvements tailored to the special features of atomic-resolution electron micrographs of crystals limited by Poisson noise. The major improvement consists of an optimized block-matching strategy that predicts the periodic structure of the observed crystal and distributes adaptively refined non-local search windows throughout the image [2]. Furthermore, it is shown that such a true non-local block-matching strategy can lead to a highly non-uniform distribution of the number of aggregated estimates available in each pixel, resulting in poor estimation in image regions where the distribution of block estimates is sparse. Possible remedies for this deficiency are discussed.

Numerical experiments were conducted with simulated low dose STEM images of crystals with different grid structures. The results show an increase in peak signal-to-noise ratio (PSNR) of $2-4 \mathrm{~dB}$ when using our method instead of the original BM3D algorithm. Our method also improves electron microscopy related quantitative measures, such as detection fraction and precision (standard deviation of detected inter-atomic distances). The precision in the estimates of simulated low-dose electron micrographs of perfect crystals produced by our proposed algorithm are within $7-15 \mathrm{pm}$. These results are competitive with the best reported precisions in the literature for single shot STEM images [3]. Furthermore, a new measure, called fidelity, is introduced, which evaluates the discrepancy between the atom centers detected in the estimate and the ones detected in the ground truth. Our experiments indicate that this error can be significantly reduced by denoising electron micrographs prior to atom detection.

\section{References}

[1] K. Dabov, A. Foi, V. Katkovnik, K. Egiazarian. Image Denoising by Sparse 3-D Transform-Domain Collaborative Filtering. Image Processing, IEEE Transactions on, 16 (2007), 2080-2095.

[2] N. Mevenkamp, A. B. Yankovich, P. M. Voyles, B. Berkels. Non-local Means for Scanning Transmission Electron Microscopy Images and Poisson Noise based on Adaptive Periodic Similarity Search and Patch Regularization. VMV 2014: Vision, Modeling \& Visualization, Eurographics Association, (2014), 63-70

[3] K. Kimoto, T. Asaka, X. Yu, T. Nagai, Y. Matsui, K. Ishizuka. Local crystal structure analysis with several picometer precision using scanning transmission electron microscopy. Ultramicroscopy, Elsevier, 110 (2010), $778-782$. 


\title{
Application of learning algorithms for colour recognition on underwater images
}

\author{
Julian Hoth, Wojciech Kowalczyk \\ Chair of Mechanics and Robotics, University of Duisburg-Essen, Lotharstr. 1, \\ 47057 Duisburg, Germany
}

Objects look very different in the underwater environment compared to their appearance in sunlight. The main reason is that the penetration of light through seawater is highly dependent on the wavelength of the light [4]. Suspended particles in the water can further decrease the overall quality of underwater images [1, 2].

High quality images with correct colouring simplify the detection of underwater objects and may allow the use of visual SLAM algorithms developed for land-based robots underwater. Hence, image processing is required to obtain images of high quality and correct colouring. Over the last decade significant progress has been made in this direction.

Current algorithms focus on the colour reconstruction of scenery at diving depth [2, 5]. This has the advantage that a significant part of sunlight is still present and different colours can still be distinguished although they may be tainted due to light filtered through seawater. Other approaches require a known geometric relations between the camera and the object [6], assume a simple relation between colours under different lighting conditions using Beer's law [4], or require manual work [3].

Unfortunately, at greater depth the filtering is much stronger such that Beer's law is not sufficient. Also different colours are strongly tainted and can no longer be distinguished. To solve this the main factor of interest for the influence on colour is the penetration depth $d_{p}$ which is a strong function of the wavelength $\lambda$.

The approach suggested here uses a special light source with a defined wavelength to illuminate the environment. The defined wavelength is characterised by a high penetration depth in water. Particularly, wavelengths of $450-550 \mathrm{~nm}$ are of interest in ocean water [8,7]. It is also possible to use a light source emitting white light as distant objects will also have a colour change due to absorbtion in seawater that will result in a similar appearance of the surroundings as with the defined wavelength source.

Within this study the image taken under these special lighting conditions is fed into a learning algorithm which was calibrated using images of objects with known colour and structure. The colours in the image are then transformed by the algorithm to obtain an image as seen under white light in air.

The new procedure currently uses the original image without any additional filtering. In the next step prefilters will be included into the algorithm to remove reflection effects and to smooth the brightness in the image. Additionally, the pre-processing after Bazeille et al. [1] will be tested. It may also be of interest to include the information of neighbouring pixels for the calculation of the colour to remove single pixels which are not transformed correctly.

The developed algorithm and procedure can be applied also for other applications, where other ranges of wavelengths are used, to obtain the image as seen under white light from data under different lighting conditions.

\section{References}

[1] S. Bazeille, I. Quidu, L. Jaulin, J.-P. Malkasse. Automatic underwater image pre-processing. In Proceedings of the Caracterisation du Milieu Marin, Brest, France (2006).

[2] A.T. Celebi, S. Ertürk. Visual enhancement of underwater images using empirical mode decomposition. Expert Systems with Applications 39 (2012), 800-805.

[3] Z. Chen, H. Wang, J. Shen, X. Li, L. Xu. Region-specialized underwater image restoration in inhomogeneous optical environments. Optik - International Journal for Light and Electron Optics 125(9) (2014), $2090-2098$.

[4] J. Åhlén. Colour Correction of Underwater Images Using Spectral Data. PhD thesis, Uppsala Universitet (2005).

[5] K. Iqbal, R.A. Salam, A. Osman, A.Z. Talib. Underwater image enhancement using an integrated colour model. International Journal of Computer Science 34(2) (2007), 529-534. 
[6] D. Lee, G. Kim, D. Kim, H. Myung, H.-T. Choi. Vision-based object detection and tracking for autonomous navigation of underwater robots. Ocean Engineering 48 (2012), 59-68.

[7] C.D. Mobley. Light and Water: Radiative Transfer in Natural Waters. Office of Naval Research, United States of America, 2nd edition (2004).

[8] R.C. Smith, K.S. Baker. Optical properties of the clearest natural water (200-800 nm). Applied Optics, 20(2) (1981), 177-184. 


\title{
Edge detection based on fractional order differentiation and its application to railway track images
}

\author{
Christian Telke, Michael Beitelschmidt \\ Technische Universität Dresden, Faculty of Mechanical Science and Engineering, \\ Department of Solid Mechanics, Chair of Dynamics and Mechanism Design \\ 01062 Dresden, Germany \\ e-mail: Christian.Telke@tu-dresden.de, Michael.Beitelschmidt@tu-dresden.de
}

Recurrent standard measurement tasks are often necessary and scheduled within the development cycle of railway vehicles. Many of these measurement tasks need to be done in a very early stage of the project or in the offer period of a light rail vehicle. Thereby, a main focus is the recognition of the track geometry and its disturbances without significant influence on the daily scheduled operation. By the means of this measured data it is possible to calculate the structural stress within significant assembly parts of the train [1]. This track geometry detection process is realized by a novel contactless modular measurement system. Within the development process of this system, many challenges according to the camera based railway recognition occurred. In this context, a eCRONE (extended Contour Robuste d'Ordre Non Entier) operator - an extended version of the non-local CRONE edge detection operator introduced by [2] - based on GRÜNWALD-LETNIKOV definition of fractional order differentiation is presented. In recent years, fractional calculus is getting more and more important to various applications such as [3], [4] and [5]. Equation (1) shows the one-dimensional CRONE operator ${ }^{\mathrm{CR}} \mathrm{D}_{x}^{\nu} f(x)$, which is based on a superposition of a causal and a non-causal derivative of a function $f(x)$ of fractional order $\nu \in \mathbb{R}$.

$$
{ }^{\mathrm{CR}} \stackrel{\mathrm{D}}{\leftrightarrow}_{x}^{\nu} f(x)=\left(\delta_{N} x\right)^{-\nu} \sum_{j=0}^{N-1} \underbrace{\frac{\Gamma(j-\nu)}{\Gamma(-\nu) \Gamma(j+1)}}_{g_{j}}\left[f\left(x-j \delta_{N} x\right)-f\left(x+j \delta_{N} x\right)\right] .
$$

Within (1), the gamma function is denoted by $\Gamma$ and the GRÜNwALD coefficients are given by $g_{j}$. A reformulation of (1) yields the following filter kernel matrix as

$$
{ }^{\mathrm{CR}} \stackrel{\mathrm{D}}{\hookrightarrow}_{x}^{\nu}=\left(\begin{array}{ccccccccc}
\vdots & \vdots & & \vdots & \vdots & \vdots & & \vdots & \vdots \\
0 & 0 & & 0 & 0 & 0 & & 0 & 0 \\
g_{m} & g_{m-1} & \cdots & g_{1} & 0 & -g_{1} & \cdots & -g_{m-1} & -g_{m} \\
0 & 0 & & 0 & 0 & 0 & & 0 & 0 \\
\vdots & \vdots & & \vdots & \vdots & \vdots & & \vdots & \vdots
\end{array}\right) .
$$

Based on (1) and (2), a two-dimensional filter kernel, which takes the local neighborhood of the actual processed pixel into account, is presented in the paper. This significantly improves the detection performance and the stability to noise related to classical operators like Sobel, PrewitT etc. Furthermore, a description how the eCRONE detector response leads by the means of the discrete RADON transform to a mathematical description of parametric geometries like lines or circles within the responses is given.

Additionally, some first results of a practical application of the above mentioned algorithms within a real measurement campaign are presented respectively.

\section{References}

[1] Harter M., Durchgängige Simulation von Strukturspannungen in Straßenbahnen unter Berücksichtigung gemessener Gleislage. PhD thesis, Institut für Bahnfahrzeuge und Bahntechnik, Professur für Fahrzeugmodellierung und Simulation, Technische Universität Dresden, 2010.

[2] Mathieu B., Melchior P., Oustaloup A., and Ceyral Ch.. Fractional differentiation for edge detection. Signal Processing, 83(11):2421 -2432. Fractional Signal Processing and Applications, 2003. 
[3] Oldham K., Spanier J., The fractional calculus / theory and applications of differentiation and integration to arbitrary order. Acad. Pr., 1974.

[4] Pu Y., Zhou J., Yuan X., Fractional differential mask: A fractional differential-based approach for multiscale texture enhancement. Image Processing, IEEE Transactions on, 19(2):491-511, Feb 2010.

[5] Schmidt A. Finite-Elemente-Formulierungen viskoelastischer Werkstoffe mit fraktionalen Zeitableitungen. $\mathrm{PhD}$ thesis, Universität Stuttgart, 2003/01. 


\section{S22: Scientific computing}

Scientific Computing is concerned with the efficient numerical solution of mathematical models from both science and engineering. The field covers a wide range of topics: from mathematical modeling over the development, analysis and efficient implementation of numerical methods and algorithms to software and finally application for the solution of complex real-world problems on modern computing systems. This interdisciplinary field combines approaches from applied mathematics, computer science and a wide range of applications in which in-silico experiments play an increasingly important role. 


\title{
Micro-macro parareal methods for multiscale problems
}

\author{
Giovanni Samaey \\ Numerical Analysis and Applied Mathematics, Department of Computer Science, KU Leuven
}

We introduce a micro-macro parareal algorithm for the time-parallel integration of multiscale-in-time systems. The algorithm first computes a cheap, but inaccurate, solution using a coarse propagator (simulating an approximate slow macroscopic model), which is iteratively corrected using a fine-scale propagator (accurately simulating the full microscopic dynamics). This correction is done in parallel over many subintervals, thereby reducing the wall-clock time needed to obtain the solution, compared to the integration of the full microscopic model over the complete time interval. We provide a numerical analysis of the algorithm for a prototypical example of a micro-macro model [1], namely singularly perturbed ordinary differential equations, and illustrate the method for problems arising in climate modeling and molecular dynamics.

\section{References}

[1] F. Legoll, T. Lelievre and G. Samaey. A micro/macro parareal algorithm for singularly perturbed ordinary differential equations, SIAM Journal on Scientific Computing 35:A1951-A1986, 2013. 


\title{
Adaptive space time finite element methods for dynamic nonlinear thermomechanical coupled problems
}

\author{
Andreas Rademacher \\ Chair of Scientific Computing, Technische Universität Dortmund \\ Vogelpothsweg 87, 44227 Dortmund, Germany, Andreas.Rademacher@tu-dortmund.de
}

In this contribution, we consider nonlinear thermomechanical coupled problems of the following type: Find a displacement field $u$ and a temperature distribution $\theta$, which fulfill the equation of motion with a temperature dependent stress field and the heat equation including a heat source depending on the displacement:

$$
\begin{aligned}
\rho \ddot{u}-\operatorname{div}(\sigma(u, \theta)) & =f \\
\rho c_{p} \dot{\theta}-\operatorname{div}(\kappa(\theta) \nabla \theta) & =g(u)
\end{aligned}
$$

Additionally, appropriate intial and boundary conditions have to be specified. The continuous model is discretized with a space time Petrov Galerkin method using continuous and piecewise d-linear basis functions in space and time, which can be reduced to a time stepping scheme due to the discontinuous piecewise constant temporal test functions, see for instance [1]. The resulting coupled system in each time step is solved by a staggered scheme. The aim is to control the discretization error as well as the error in the solution scheme in a nonlinear goal functional. To this end, we use the dual weighted residual (DWR) method. In detail, we combine the ideas from [1] for goal oriented error estimation in nonlinear time dependent problems and from [2] for balancing discretization and numerical error in static problems. The discretization error is measured by the usual DWR techniques discussed for time dependent problems, e.g., in [1], while the numerical error is estimated by testing the residuum with the discrete dual solution, cf. [2]. However, the error estimator can only be evaluated after solving the complete primal and dual problem. Thus, we have to design a special solution algorithm. The main idea is to solve the primal problem doing only one step of the staggered solution scheme in each time step. Then the discretization and the numerical error is estimated. If the numerical error is dominant, an additional loop over all time steps including one step of the staggered scheme is executed. Otherwise, the mesh is adaptively refined. By this approach, we can reduce the numerical effort for the accurate solving of thermomechanical problems by adopting the solution scheme to the special needs of the underlying problem and the chosen goal functional.

\section{References}

[1] A. Rademacher. Adaptive Finite Element Methods for Nonlinear Hyperbolic Problems of Second Order. Dissertation, TU Dortmund, (2009).

[2] R. Rannacher, J. Vihharev. Adaptive finite element analysis of nonlinear problems: balancing of discretization and iteration errors. J. Num. Math. 21 (2013), 23-61. 


\title{
Parallel simulation of large scale multibody systems
}

\author{
Michael Klöppel ${ }^{1}$, Andreas Naumann ${ }^{1}$, Volker Waurich ${ }^{2}$, Marcus Walther ${ }^{3}$, Jörg Wensch ${ }^{1}$ \\ ${ }^{1} \mathrm{TU}$ Dresden, Institute of Scientific Computing \\ ${ }^{2} \mathrm{TU}$ Dresden, Institute of Processing Machines and Mobile Machinery \\ ${ }^{3}$ TU Dresden, Center for Information Services and High Performance Computing
}

\begin{abstract}
Virtual prototyping plays an ever increasing role in the engineering disciplines. Nowadays, engineers can rely on powerful tools like object oriented modeling languages, e.g., Modelica. Models written in this language can be simulated by open source software as well as commercial tools. The advantage of this approach is that the engineers can concentrate themselves on modeling, whereas the numerical intricacies of the simulation are handled by the software. On the other hand the simulations are usually slower than implementations which are parallelized and optimized by hand. This can lead to computation times which are infeasible in practice, e.g., when a real time simulation is necessary for a hardware-in-the-loop simulation.

In this contribution we are concerned with speeding up such automated simulations by parallelization (on desktop hardware as well as HPC systems). We examine the three classical approaches, i.e., parallelism across the system, parallelism across the method and parallelism across the steps. Additionally, the influence of the problem formulation on the simulation time is discussed. We demonstrate what kind of information can be gained from the automatically generated equations and how this information can be used to efficiently speed up the computation. The implemented methods are demonstrated on engineering examples. Furthermore, successful method will be made available in the open source simulation software OpenModelica.
\end{abstract}




\title{
Simulation of coupled machine components on long time scales
}

\author{
Andreas Naumann, Michael Klöppel, Jörg Wensch \\ TU Dresden, Institute of Scientific Computing
}

The accuracy requirements in recent mass production processes demand new compensation techniques and structures of tool machines. To achieve these requirements, one has to know the thermo-elastic behavior and interaction of the coupled components during the design phase.

We discretize the machine components using finite elements. The heat exchange of the components couples the temperature fields, which leads to additional constraints on parallel FEM. The discretization in space leads to a system of coupled ODEs, which are of stiff parabolic type with fast time-varying source terms. We concentrate on numerical methods to solve those equations on large timescales and reduce the timestep restriction to the fast coupling. This includes averaging techniques, as well as parallel in time methods. 


\title{
Boris-SDC: A high-order Boris integrator
}

\author{
Mathias Winkel, Robert Speck ${ }^{\dagger}$ Daniel Ruprecht*

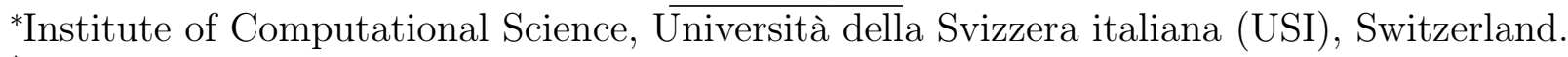 \\ ${ }^{\dagger} J$ ülich Supercomputing Centre, Forschungszentrum Jülich, Germany.
}

This talk will introduce and investigate the high-order Boris-SDC method [1] for integrating the equation of motion for electrically charged particles in an electric and magnetic field.

The well-known and widely used Boris scheme $[2,3]$ is a second-order accurate Lorentz force integrator. Based on the standard velocity-Verlet scheme for molecular dynamics simulations, this approach resolves the seemingly implicit velocity dependence in the equations of motion by exploiting the rotational character of the magnetic field contribution. In doing so, it provides a fully explicit, cheap particle pusher and has become the de-facto standard integration method for this kind of problems. Today, the Boris method is applied in essentially all particle-based plasma physics simulations involving magnetic fields, e.g. in laser-plasma physics algorithms as well as space weather and fusion-related simulations [4].

We extend the second-order method to arbitrary orders of accuracy by coupling the Boris approach with spectral deferred corrections (SDC, [5]). SDC can be considered as preconditioned Picard iteration for computing the solution of a collocation method. In this interpretation, inverting the preconditioner corresponds to a sweep with a low-order method. While common implementations make use of implicit or explicit Euler integrators, we employ velocity-Verlet as base sweeper [6] and incorporate the Boris method as solver for the implicit velocity dependency.

Boris-SDC provides a generic way to extend the classical Boris integrator to a high-order method. Stability, convergence order and conservation properties of the method are demonstrated for different simulation setups. The method reproduces the expected high order of convergence for a single particle and for the center-of-mass of a particle cloud in a Penning trap and shows good long-term energy stability. Besides its high formal order, its attractive properties and ease-of-implementation provide the foundation of a considerably improved approach to particle trajectory integration in electric and magnetic fields. Furthermore, the formal methodology presented here can be understood as a blueprint for the adaptation of other specialized integration methods to SDC.

This method also pioneers upcoming time-parallel integration for this problem. Using SDC-based integrators like the one presented here, the 'parallel full approximation scheme in space and time' (PFASST, [7]) allows to integrate multiple time-steps simultaneously using sweeps of SDC on different levels of a space-time hierarchy. The presented results constitute an important step towards a parallel-in-time Boris integrator.

\section{References}

[1] M. Winkel, R. Speck, D. Ruprecht, A high-order Boris integrator, arXiv:1409.5677 [math.NA], 2014.

[2] J. Boris, Relativistic plasma simulation-optimization of a hybrid code, in: Proceedings of the Fourth Conference on Numerical Simulation of Plasmas, Naval Research Laboratory, Washington, D.C., 1970, pp. 3-67.

[3] C. K. Birdsall, A. B. Langdon, Plasma physics via computer simulation, McGraw-Hill, New York, 1985.

[4] J. P. Verboncoeur, Particle simulation of plasmas: review and advances, Plasma Physics and Controlled Fusion 47 (5A) (2005) A231-A260.

[5] A. Dutt, L. Greengard, V. Rokhlin, Spectral deferred correction methods for ordinary differential equations, BIT Numerical Mathematics 40 (2) (2000) 241-266.

[6] M. Minion, Spectral deferred correction methods for second-order problems, in preparation.

[7] M. Emmett, M. Minion, Toward an efficient parallel in time method for partial differential equations, Communications in Applied Mathematics and Computational Science 7-(1) (2012) 105-132. 


\title{
Efficient Nested Chebyshev Smoothing
}

\author{
Patrick Sanan, Olaf Schenk \\ Università della Svizzera italiana
}

Chebyshev semi-iteration, as a polynomial smoother, has been shown to be an effective component of a multigrid process, particularly in parallel settings where an estimate of the largest eigenvalue can be obtained efficiently [2]. As a preconditioner, the approach has been shown to be superior even to nested Kyrlov methods as a preconditioner in some cases (namely those when good spectral estimates are available a priori), and can be more effectively analyzed due to its linearity, for fixed spectral estimates [4]. Related to this property is a corresponding benefit in the context of contemporary hybrid HPC architectures: local Chebyshev smoothing can be applied with a single kernel launch on an accelerator device, and computed using recently-developed blocking techniques which increase cache reuse and utilize more of the available arithmetic intensity on modern accelerators; further, these can be used to reduce global communication with a multigrid cycle by performing more aggressive coarsening [1].

We investigate efficiency gains using local Chebyshev smoothing in the context of preconditioning challenging Stokes problems from mantle convection, on a large system with hybrid CPU/GPU nodes. Our recent comparative studies [3] within the PASC GeoPC Project ${ }^{1}$ have demonstrated that in practical simulations, "heavy smoothing" can be employed to reduce the amount of communication required, mitigating a bottleneck at extreme scale. We investigate the effects of varying the accuracy of automatic eigenvalue estimates, and examine the effects of combining Chebyshev smoothers hierarchically, as global smoothers, preconditioned by local smoothers working on GPUs.

\section{References}

[1] P Ghysels, P Klosiewicz, and W Vanroose. Improving the arithmetic intensity of multigrid with the help of polynomial smoothers. Numerical Linear Algebra with Applications, pages 1-15, 2011.

[2] Jonathan Hu, Ray Tuminaro, Mark F. Adams, and Marian Brezina. Parallel Multigrid Smoothing: Polynomial versus Gauss-Seidel. Journal of Computational Physics, 188(2):593-610, 2003.

[3] Patrick Sanan, Sascha M. Schnepp, Dave A. May, and Olaf Schenk. Exploring solver space for stokes flow with highly heterogeneous viscosity structure [poster]. AGU Fall Meeting 2014, San Francisco, California, United States, December 15-19,2014.

[4] Andrew J. Wathen and Tyrone Rees. Chebyshev semi-iteration in preconditioning for problems including the mass matrix. Electronic Transactions on Numerical Analysis, 34:125-135, 2009.

\footnotetext{
${ }^{1}$ http://www.pasc-ch.org/projects/projects/geopc/
} 


\title{
Rayleigh Quotient Inverse Iteration with Adaptive Algebraic Multigrid for Lattice QCD
}

\author{
Matthias Rottmann \\ University of Wuppertal, Germany
}

In lattice Quantum Chromodynamics (lattice QCD) computations, the calculation of physical observables oftentimes requires approximations to the full inverse of very large sparse matrices, i.e., inverses of the lattice Wilson Dirac operator $D$. Numerical methods used in the Physics community highly demand singular vectors corresponding to smallest singular values of $D$. In this talk we present a Rayleigh quotient inverse iteration approach in combination with an adaptive algebraic multigrid solver. We show numerical results for medium scale parallelizations of up to 4,192 cores and problem sizes of up to 200,000,000 unknowns. 


\title{
An Auxiliary Space Type Preconditioner for Simulations in Lattice QCD
}

\author{
James Brannick ${ }^{1}$, Andreas Frommer ${ }^{2}$, Karsten Kahl ${ }^{2}$, Björn Leder ${ }^{2}$, \\ Matthias Rottmann ${ }^{2}$, Artur Strebel ${ }^{2}$ \\ ${ }^{1}$ Penn State University \\ ${ }^{2}$ Bergische Universität Wuppertal
}

In this talk we motivate, analyze and experimentally validate a new preconditioner for the overlap operator of lattice QCD. Lattice QCD simulations are among today's most demanding supercomputer applications, and substantial resources are spent in these computations. Most frequently, these simulations are based on repeatedly solving systems with the Wilson-Dirac operator $D_{W}(\kappa)$, a nearest-neighbor discretization of the respective Dirac equation.

From a theoretical point of view, the overlap operator

$$
D_{o v l}=\rho I+\Gamma_{5} \operatorname{sign}\left(\Gamma_{5} D_{W}\left(\kappa_{o v l}\right)\right)
$$

is particularly attractive since it respects an important physical property, chiral symmetry, which is violated by other lattice discretizations. From a practical point of view, the overlap operator has the disadvantage that its computational cost can be two orders of magnitude larger than when using standard discretizations due to the occurence of the matrix sign function.

The basic idea of the preconditioner we propose is to use the Wilson-Dirac $D_{W}\left(\kappa_{W}\right)$ operator to form a preconditioner for the overlap operator. This may be regarded as a variant of the fictitious (or auxiliary) space preconditioning technique that has been used for developing and analyzing multilevel preconditioners for various nonconforming finite element approximations of PDEs. One works with a mapping from the original space to a fictitious space, yielding an equivalent problem that is easier to solve. Preconditioning is then done by (approximately) solving this equivalent problem. The convergence properties of auxiliary space preconditioning depend on the choice of the fictitious space, and its computational efficiency depends, in addition, on the efficiency of the solver used in that space.

For the overlap operator in lattice QCD, choosing its kernel - the Wilson-Dirac operator - as the auxiliary space preconditioner is facilitated by the fact that both operators are defined on the same Hilbert space. In this way, the preconditioner for the former can be constructed using an adaptive algebraic multigrid solver for the latter on the same finite dimensional lattice.

A crucial issue is to establish an optimal connection between the parameters $\rho, \kappa_{\text {ovl }}$ and $\kappa_{W}$. We do so in a theoretical analysis for the case that $D_{W}$ is normal. Although this is not exactly the case in realistic settings, we show that current smearing techniques indeed drive the Wilson-Dirac operator towards normality, thus providing a motivation why our preconditioner works well in computational practice. We demonstrate that our technique is able to reduce the computational cost for solving systems with the overlap operator substantially, reaching speed-ups of a factor of 10 or more in realistic settings, involving large lattices and state-of-the-art implementations on parallel computers, particularly the JUROPA cluster system at FZ Jülich. 


\title{
A GPU implementation of the Factored Sparse Approximate Inverse preconditioner for the iterative solution of SPD linear systems
}

\author{
Massimo Bernaschi*, Mauro Bisson*, Carlo Fantozzi ${ }^{\dagger}$, Carlo Janna $^{\ddagger}$ \\ *Institute for Computing Applications, CNR \\ ${ }^{\dagger}$ Dept. of Information Engineering, University of Padova \\ ${ }^{\ddagger}$ Dept. of Civil, Architectural and Environmental Engineering, University of Padova
}

It is well known that GPUs exhibit significantly higher peak performance than conventional CPUs. However, due to their programming model and microarchitecture, only highly parallel algorithms can exploit their full potential. In this context, the development of effective algorithms for the solution of large and sparse linear systems of equations

$$
A \mathbf{x}=\mathbf{b},
$$

where $A$ is the system matrix, $\mathbf{x}$ is the solution vector and $\mathbf{b}$ is right-hand-side, is still an open problem. Conjugate gradient-like algorithms are good candidates for this task as they require only sparse matrix-byvector products, dot products and vector updates, which have all already been implemented successfully on GPUs $[4,5]$. However, the performance of any iterative solver is strictly related to the availability of an effective preconditioner, that is, an approximation of $A^{-1}$ which has to be relatively cheap to compute and apply to a vector. The development of effective preconditioners on GPUs is not straightforward and several attempts have already been made (see, e.g., [1, 3]). In the present contribution, we propose the Factored Sparse Approximate Inverse (FSAI) [2], which, with its inherent parallelism, is an optimal preconditioner for the conjugate gradient solver. FSAI gives an explicit approximation of $A^{-1}$ in factored form, i.e. $G G^{T} \simeq A^{-1}$, and is computed by minimizing the matrix function

$$
\|I-G L\| \rightarrow \min
$$

over all the matrices $G$ having a prescribed lower triangular sparsity pattern. In (1) $L$ is the exact lower triangular factor of $A$, which is not required to be explicitly known during the computation of $G$. While the FSAI application to a vector involves only sparse matrix-by-vector products, a GPU-based implementation requires a nontrivial recasting of multiple computational steps. One of the main issues is to find a suitable data mapping between threads and data in order to exploit the massive GPU parallelism. To that purpose, we resorted to a solution that we successfully exploited in a pretty different context [6]. The other issue is the need of carrying out the Cholesky decomposition on large batches of small systems. We experimented with different strategies and obtained the best performances by using a block of threads per system and performing the factorization exclusively in register memory. We illustrate our experience with the full porting of a parallel FSAI preconditioner on NVIDIA GPUs; experiments show a significant speedup over the CPU-only, OpenMPbased implementation.

\section{References}

[1] M.M. Dehnavi, D.M. Fernandez, J.-L. Gaudiot, D.D. Giannacopoulos. Parallel Sparse Approximate Inverse Preconditioning on Graphic Processing Units. IEEE Trans. Par. Distr. Syst. 24:9 (2013), 1852-1862.

[2] C. Janna, M. Ferronato, F. Sartoretto, G. Gambolati. FSAIPACK: a software package for high performance FSAI preconditioning, ACM Trans. Math. Softw. 41 (2015).

[3] R. Li, Y. Saad. GPU-accelerated preconditioned iterative linear solvers. J. Supercomput. 63 (2013), $443-466$.

[4] G. Oyarzun, R. Borrell, A. Gorobets, A. Oliva. MPI-CUDA sparse matrix-vector multiplication for the conjugate gradient method with an approximate inverse preconditioner. Computers \& Fluids 92 (2014), $244-252$.

[5] M. Verschoor, A. C. Jalba. Analysis and performance estimation of the Conjugate Gradient method on multiple GPUs. Parallel Comput. 38. (2012), 552-575.

[6] M. Bernaschi, E. Mastrostefano,"Efficient breadth first search on multi-GPU systems", Journal of Parallel and Distributed Computing, 73, 1292 (2013). 


\title{
Finite Volume Methods for Sound-Advection-Buoyancy Systems
}

\author{
Jörg Wensch ${ }^{1}$, Michael Klöppel ${ }^{1}$, Oswald Knoth ${ }^{2}$, Andreas Naumann ${ }^{1}$ \\ ${ }^{1}$ TU Dresden, Institute of Scientific Computing \\ ${ }^{2}$ Institute of Tropospheric Research, Leipzig
}

\begin{abstract}
The simulation of atmospheric dynamics relies on the numerical solution of the Euler equations. We consider here buoyancy-sound-advection systems for stratified fluids. These systems exhibit wave type solutions which impose CFL-based restrictions on the time step size. Buoyancy, sound and advection cause seperate restrictions, each on their own scale. We develop Finite Volume Methods where a splitting approach allows different treatment of fast and slow waves. The methods are optimized with respect to order and maximum stable CFL numbers. Different scenarios for the scales of sound, advection and buoyancy are discussed.
\end{abstract}




\title{
HPC methods for structured inverse modeling in diffusive processes
}

\author{
Volker Schulz, Martin Siebenborn \\ Trier University, Mathematics Department, 54296 Trier, Germany
}

In many applications, which are modeled by diffusion processes, there is a small number of different materials involved with distinct boundaries. It is thus reasonable not only to estimate the permeability parameter itself but also the contour of the spatial distribution by methods of shape optimization. Depending on the complexity of the interfaces, this requires the solution of several very large systems of equations resulting from fine discretizations of PDE systems.

It is thus obligatory to develop efficient software for supercomputers that guarantees scalability also for problems where most degrees of freedom are located on interfaces. Here it turns out that the efficiency of the optimization algorithm strongly depends on the applied load balancing technique since there are many costly operations which are only computed on the surfaces to be optimized. It is thus discussed how load balancing can be adapted such that surfaces in the FEM mesh are also evenly distributed among processors.

This talk introduces a limited memory BFGS approach for shape optimization in diffusive flow processes. It is shown that superlinear convergence can be achieved which is a significant speedup against optimizations based only on shape gradients. These techniques are utilized in order to fit a model of the human skin to data measurements and not only estimate the permeability coefficients but also the shape of the cells.

\section{References}

[1] V. Schulz and M. Siebenborn and K. Welker. Structured inverse modeling in parabolic diffusion problems. arXiv:1409.3464. 2014

[2] V. Schulz and M. Siebenborn and K. Welker. Towards a Lagrange-Newton approach for PDE constrained shape optimization. arXiv:1405.3266. 2014 


\title{
Using Automatic Differentiation to Create Sparse Jacobians for the Solution of Nonlinear Partial Differential Equations
}

\author{
Florian Zwicke, Philipp Knechtges, Marek Behr and Stefanie Elgeti \\ Chair for Computational Analysis of Technical Systems, CCES, RWTH Aachen University, Germany
}

During the development of numerical simulation software, a number of situations arise, where it becomes necessary to differentiate either mathematical terms or the output of a program function. One very important example is the solution of nonlinear partial differential equations (PDE). During the linearization step needed to solve the discretized form of the equations, e.g., based on the Newton-Raphson algorithm, the Jacobian of the residual with respect to the unknown field variables is needed:

$$
\frac{\partial \mathbf{F}\left(\mathbf{u}_{k}\right)}{\partial \mathbf{u}} \cdot \Delta \mathbf{u}_{k+1}=-\mathbf{F}\left(\mathbf{u}_{k}\right)
$$

Although the manual calculation of the Jacobian (or a close approximation thereof) is a viable choice, the resulting code will always be specific to a single PDE and discretization scheme, which may render it difficult to adapt the implementation to a new simulation model. In addition, the program code to calculate the derivative will often be difficult to read, a circumstance, which can invite errors in the implementation. A simple method of validating the analytical derivation as well as the implementation is often lacking. While, e.g., the successful minimization of the residual can be taken as an indication that the derivative is correct, it does not conclusively prove it.

In recent years, a lot of research has been carried out in the field of automatic differentiation (AD). Such methods allow the mathematical differentiation of program functions, which may or may not have a closed analytical representation. In order to take a first step towards utilizing $\mathrm{AD}$ in our in-house finite element software XNS, which is written in Fortran, we automatized the creation of the Jacobian for a Newton-Raphson iteration with the software Tapenade [1]. As a result, the task of implementing a new set of equations is reduced to the effort of calculating the residual of the discretized weak form.

As a test case we used the viscoelastic fluid equations [2], which were chosen due to their significant nonlinearities. The equations are discretized in XNS using the GLS-stabilized finite element method. We will discuss the various problems that we had to solve on our path towards a useful result. Most importantly, we will present the different approaches that we explored as a means of exploiting the sparsity of the Jacobian matrix in an efficient manner. We will conclude by showing that we were able to create a method, which is already reliable and flexible, and opens up possibilities to either quickly and easily implement new equations or to validate an existing manual implementation of the derivative.

\section{References}

[1] L. Hascoët, V. Pascual. The Tapenade Automatic Differentiation tool: Principles, Model, and Specification. ACM Transactions on Mathematical Software 39 (2013), 20:1-20:43.

[2] P. Knechtges, M. Behr and S. Elgeti. Fully-implicit log-conformation formulation of constitutive laws. Journal of Non-Newtonian Fluid Mechanics 214 (2014) 78-87. arXiv:1406.6988 [math.NA]. 


\title{
Multiple-output variable fidelity modeling of vehicle aerodynamics under geometric shape variations
}

\author{
A. Sauerbrei, R. Zimmermann \\ Numerics Group, Institute 'Computational Mathematics', TU Braunschweig
}

Variable-fidelity modeling (VFM), sometimes also termed multi-fidelity modeling, refers to the exploitation of two or more data layers of variable accuracy in order to construct an inexpensive emulator of a given highorder numerical model. In practical applications, this situation arises, when simulators of different accuracy for the same physical process are given and it is assumed that many low-fidelity sample points are affordable, but the high-fidelity model is extremely costly to assess.

More precisely, the VFM objective is to construct a predictor function based on the variable-fidelity sample data sets, which interpolates the primary sample data driven by the trend indicated by the secondary data, where it is tried to use as few high-fidelity sample points as possible to achieve a desired level of accurancy.

While previous work on VFM has focused on scalar-valued multiple-input-single-output models $[1,2]$, in this work, we extend the approach to large-scale nonlinear multiple-input-multiple-output models. To this end, we combine the VFM with the method of proper orthogonal decomposition. In particular, we are interested in the approximation of complete field solutions of dimensions ranging from several hundreds of thousands up to several millions as they arise from the spatial discretization of partial differential equations in industrial applications.

The method is illustrated on an automotive computational fluid dynamics problem, where the objective is to predict the complete flow-field corresponding to a given spatial discretization under multi-parameter variations of the shape of the rear of the car. Fully and partly converged flow solutions act as data layers of high- and low fidelity, respectively. The approach is non-intrusive in the sense that the underlying flow solver acts as a black box function and no intrinsic modifications are required.

\section{References}

[1] M. C. Kennedy, A. O'Hagan, Predicting the output from a complex computer code when fast approximations are available. Biometrika, Vol. 87 No. 1 (2000), 1-13.

[2] Z.-H. Han, S. Görtz. A Hierarchical Kriging Model for Variable-Fidelity Surrogate Modelling. AIAA Journal Vol. 50 No. 9 (2012), 1885-1896. 


\title{
Sensitivities calculations for unsteady flow problems
}

\author{
Marius Stoia-Djeska, Florin Frunzulica \\ Politehnica University of Bucharest \\ Faculty of Aerospace Engineering, Bucharest, Romania
}

In this work we present an efficient approach for the calculation of the sensitivities in the case of unsteady problems like the compressible transient high-speed flows around a wing-section. The sensitivities are of the lift and pitching moment dimensionless coefficients and are determined with respect to the time-dependent boundary conditions. These sensitivities are necessary for the solution of inverse problems or for the synthesis of an active control law. If the number of design parameters is large, the adjoint method is typically used because the cost of computing the sensitivities does not depend on the number of design parameters. However, the underlying partial differential equations of the optimization problems are usually for a steady state, even their solution is obtained using a time-dependent numerical algorithm. The so-called one-shot approach introduced by Ta?asan (1991) the optimization problem is solved simultaneously with the governing equations. An iterative process called piggy-back allows the calculation of the state and adjoint variable at each iteration, followed by the upgrade of the design parameters. The extension of the one-shot method to unsteady problems is of crucial importance, due to the efficiency of the computational process. This has been conceptually done by Gauger and all (2013). In this work, we first derive the adjoint equations for the Euler model. The emphasis is on the correct formulation of the adjoint boundary conditions, in the framework of the imposition of the unsteady boundary conditions. Finally, we propose an algorithm for the efficient solution of the forward and adjoint problem simultaneously, by using a piggy-back iteration process inside an one-shot method to advance in time.

\section{References}

[1] S. Ta'asan. One-shot methods for optimal control of distributed parameter systems I: Finite dimensional control. ICASE 91-2 66 (1991).

[2] S. Gunther, N.R. Gauger, Q. Wang. Extension of the One-Shot Method for Optimal Control with Unsteady PDEs. EUROGEN 2013. (2013). 


\title{
EWE - A coupled electro-mechanical heart model in the general purpose FEM framework MOOSE
}

\author{
Daniel Ruprecht, Mathias Winkel, Rolf Krause \\ Università della Svizzera italiana \\ Center for Computational Medicine in Cardiology and Institute of Computational Science \\ Lugano, Switzerland
}

The talk will present ongoing efforts on the development of a coupled electro-mechanical heart model called EWE, which are part of the Center for Computational Medicine in Cardiology in Lugano. The model is implemented using the open source general purpose $\mathrm{C}++$ finite element framework MOOSE [1], which is based on the libMesh library [2] and has been used for a number of different applications in a broad range of sicentific fields [3].

So far, EWE comprises a nonlinear mechanical model coupled with a monodomain electrocardiac model. Cellular ion channels are represented through the model by Bernus et al. [4]. Using the MOOSE framework resulted in a major acceleration of software development: Although only a few months old, coupled simulations of electrocardiology and cardiomechanics are now possible with EWE and the talk will show some preliminary results. For parallelization, the code relies on the widely used and well developed solver library PETSc [5]: Scaling benchmarks on a Linux cluster and a state-of-the-art Cray supercomputer will be presented and discussed and the further directions of development for EWE will be outlined.

\section{References}

[1] Multiphysics Object-Oriented Simulation Environment (MOOSE), http://mooseframework.org/.

[2] B. S. Kirk and J. W. Peterson and R. H. Stogner and G. F. Carey, libMesh: A C ++ Library for Parallel Adaptive Mesh Refinement/Coarsening Simulations, Engineering with Computers, 2006.

[3] D. Gaston, C. Newman, G. Hansen, and D. Lebrun-Grandie, MOOSE: A parallel computational framework for coupled systems of nonlinear equations, Nuclear Engineering and Design, 2009.

[4] O. Bernus, R. Wilders, C. W. Zemlin, H. Verschelde, A. V. Panfilov, A computationally efficient electrophysiological model of human ventricular cells, American journal of physiology. Heart and circulatory physiology, 2002.

[5] S. Balay et al., http://www.mcs.anl.gov/petsc, 2014. 


\title{
Enriching Finite Elements with meshless nodes in structural mechanics
}

\author{
Massimiliano Ferronato, Carlo Janna, Andrea Zanette \\ Department ICEA, University of Padova, Italy
}

The automatic generation of meshes for the Finite Element method can be an expensive computational burden, especially in structural problems with localized stress peaks. The use of meshless methods can address such an issue, as these techniques do not require the existence of an underlying connection among the nodes selected in a general domain. However, a thoroughly meshfree technique can be computationally quite expensive. Usually, the most expensive tasks rely in identifying the nodal contacts and computing the Galerkin integrals.

In this communication we advance a novel hybrid technique that blends Finite Elements with the Meshless Local Petrov-Galerkin method (MLPG, [1]) with the aim at exploiting the most attractive properties of each procedure. The idea is based on the original proposal by Ferronato et al. [2] and relies on the use of the Finite Element Method to compute a background solution that is locally improved by enriching the approximating space with the basis functions associated to a few meshless nodes, thus taking advantage of the flexibility ensured by the use of particles disconnected from an underlying grid. Adding the meshless particles only where needed avoids the cost of mesh refining, or even of re-meshing, without the prohibitive burden of a thoroughly meshfree approach. In particular, two enriching methods are introduced and discussed, with applications in structural mechanics.

\section{References}

[1] S.N. Atluri, T.L. Zhu. A new Meshless Local Petrov-Galerkin (MLPG) approach in computational mechanics. Comp. Mech. 22 (1998), 117-127.

[2] M. Ferronato, A. Mazzia, G. Pini. A Finite Element enrichment technique by the Meshless Local PetrovGalerkin method. CMES-Comp. Model. Eng. Sci. 62 (2010), 205-222. 


\title{
Simulations of turbulent Rayleigh-Bénard convection with a spectral element method
}

\author{
Gijs L. Kooij ${ }^{1}$, Mike A. Botchev ${ }^{2}$, Bernard J. Geurts ${ }^{1,3}$ \\ ${ }^{1}$ Multiscale Modeling and Simulation, Faculty of EEMCS, University of Twente \\ ${ }^{2}$ Mathematics of Computational Science, Faculty of EEMCS, University of Twente \\ ${ }^{3}$ Faculty of Applied Physics, Fluid Dynamics Laboratory, TU Eindhoven
}

In this study, we present direct numerical simulations of Rayleigh-Bénard convection in a rotating cylinder, comparing a finite volume approach with a spectral element method. The skew-symmetric finite volume method by Verzicco and Orlandi [4] is widely used for simulations of Rayleigh-Bénard convection in cylindrical domains. This method is second-order accurate in space. The spectral-element method offers higher-order discretizations by increasing the polynomial order of the basis functions. In theory, higher-order discretizations should produce better resolved solutions for the same number of grid points. A recent study by Scheel et al. [3] suggests that the spectral element method is indeed effective in resolving the fine scales of turbulent convection. Through a direct comparison of turbulent high Rayleigh number flows, results at a range of spatial resolutions and polynomial orders will be compared for accuracy and efficiency.

As a specific application, we address the theory by Grossmann and Lohse [2], which describes how heat transfer scales with the Rayleigh number in Rayleigh-Bénard convection. These scaling laws do not apply for the rotating case. The purpose of our simulations is to measure the heat transfer of Rayleigh-Bénard convection in a rotating cylinder, and to analyze the scaling of heat transfer under influence of rotation. We use the spectral element method implemented in the open-source code Nek5000, which has been developed originally by Fischer [1].

\section{References}

[1] P.F. Fischer. An overlapping Schwarz method for spectral element solution of the incompressible NavierStokes equations. J. Comp. Phys. (1997), 133, 84-101.

[2] S. Grossmann, D. Lohse. Scaling in thermal convection: a unifying theory. J. Fluid Mech. (2000), 407, 27-56.

[3] J.D. Scheel, M.S. Emran, J. Schumacher. Resolving the fine-scale structures in turbulent Rayleigh-Bénard convection. New J. Phys. 15 (2013), 113036.

[4] R. Verzicco, P. Orlandi. A finite-difference scheme for three-dimensional incompressible flows in cylindrical coordinates. J. Comp. Phys. 123 (1996), 402-414. 


\title{
A fully meshless method for 'gas - evaporating droplet' flow modelling
}

\author{
Oyuna Rybdylova $^{1}$, Alexander N. Osiptsov ${ }^{2}$, Sergei S. Sazhin ${ }^{1}$, Steven Begg ${ }^{1}$, Morgan Heikal $^{1}$ \\ ${ }^{1}$ Sir Harry Ricardo Laboratories, Centre for Automotive Engineering, \\ School of Computing, Engineering and Mathematics, Faculty of Science and Engineering, \\ University of Brighton, Brighton BN2 4GJ, UK \\ ${ }^{2}$ Laboratory of Multiphase Flows, Institute of Mechanics, \\ Lomonosov Moscow State University, Moscow 119192, Russia
}

Dilute two-phase flows are usually modelled using the Eulerian-Lagrangian approach. However, meshless Lagrangian techniques for fluid flow (gas or liquid without admixture) simulations have been actively developed during the last years [1]. These methods make it possible to develop fully meshless methods for two-phase flows as well. In [2] a method combining the viscous-vortex and the Fully Lagrangian [3] approaches was suggested to simulate particle-laden flows for which accurate calculations of the particle number density are required.

In the present study, the viscous-vortex method is developed to model two-phase flows with phase transitions. The method is based on consideration of three systems: viscous-vortex blobs, thermal-blobs and droplets; and can be applied for numerical simulation of 2D non-isothermal flows of 'gas-evaporating droplets' in the framework of the one-way coupled two-fluid approach. The carrier phase is viscous incompressible gas. The dispersed phase is presented by a cloud of identical spherical droplets. Due to evaporation, radii and masses of droplets are time dependent. The carrier phase parameters are calculated using the viscous-vortex and thermal-blob method; the dispersed phase parameters are calculated using the Lagrangian approach. The developed method retains the advantages of the Lagrangian approaches (time efficient, meshless simulations) and takes into account phase transitions in two-phase flows. A simplified model of phase transition is used, more advanced evaporation models are discussed in e.g. [4] and will be included in further studies. Two applications have been considered: (i) a standard benchmark - Lamb vortex; (ii) a cold spray injected into a hot quiescent gas. In the latter problem three cases corresponding to three droplet sizes were investigated. The smallest droplets (of the three cases considered) are shown to be easier entrained by the carrier phase and form ring-like structures. Larger droplets are shown to evaporate slowly as expected. The medium sized droplets tend to concentrate in two narrow bands stretched along the jet axis. The largest droplets form a two-phase jet, which remains close to the jet axis.

\section{References}

[1] G.-H. Cottet, P. Koumoutsakos, Vortex methods: Theory and practice, Cambridge University Press, 2000.

[2] N. A. Lebedeva, A. N. Osiptsov, S. S. Sazhin, A combined fully lagrangian approach to mesh-free modelling of transient two-phase flows, Atomization and Sprays 23 (1) (2013) 47-69.

[3] A. Osiptsov, Lagrangian modelling of dust admixture in gas 245 flows, Astrophysics and Space Science 274 (1-2) (2000) 377-386.

[4] S. Sazhin, Droplets and Sprays, Springer, 2014. 


\title{
The Qualitative Analysis and the Critical Hypersurfaces of Stationary Elliptic PDEs
}

\author{
$\underline{\text { A. Nastase }}$ \\ Aerodynamics of Flight, RWTH, Aachen University, Germany
}

\begin{abstract}
Many boundary value problems of PDEs of the applied mathematics lead to the solving of equivalent stationary elliptic quadratic algebraic equations (QAEs) with variable coefficients. The qualitative analysis of such a QAE is started here by the determination of its behavior by systematical variation of its free and linear terms, from $-\infty$ to $+\infty$ and by its visualization. It comes out that, for these variations of their coefficients, the QAE has a critical hypersurface, which is obtained by cancellation of its great determinant as in $[1,2]$. This critical hypersurface can be considered as a limit of existence of real solutions of the stationary elliptic QAEs.
\end{abstract}

\section{References}

[1] A. Nastase. Computation of Supersonic Flow Over Flying Configurations, Elsevier, Oxford, UK, (2008), $224-250$.

[2] A. Nastase. Hybrid Navier-Stokes Solutions for Aerodynamical Global Optimal Design, Proc. of EngOpt 2008, Paper 750, Ed. J. Herskovits, Rio de Janeiro, Brazil, (2008). 


\section{S23: Applied operator theory}

The session welcomes talks in operator theory, including: differential operators, operator semigroups, spectral theory, operators in indefinite inner product spaces, function spaces and mathematical physics. 


\title{
Squeezing of arbitrary order
}

\author{
Franciszek Hugon Szafraniec
}

Uniwerytet Jagielloński Kraków, Poland

Let $A^{(k)}$ be an operator defined formally as $a_{+}^{k}+a_{-}^{(k)}$, where $a_{+}$and $a_{-}$are the creation and annihilation operators of the quantum harmonic oscillator, resp.; $A^{(1)}$ generates the displacement operator while $A^{(2)}$ does the squeeze one. My intention is to discuss how essential selfadjointness of $A^{(k)}$ depends on $k=1,2, \ldots$, the problem which in quantum optics has been waiting for long time to be settled on rigorously.

The talk is based on [1].

\section{References}

[1] K. Górska, A. Horzela, F.H. Szafraniec, Squeezing of arbitrary order: the ups and downs, Proc. Royal Soc. Ser. A Math. Phys. Eng. Sci. 20140205. DOI 10.1098/rspa.2014.0205. 


\title{
Lebesgue type decompositions and Radon-Nikodym derivates for unbounded linear operators and relations
}

\author{
$\underline{\text { Henk de Snoo }}$ \\ Johann Bernoulli Institute for Mathematics and Computer Science \\ University of Groningen \\ P.O. Box 407, 9700 AK Groningen \\ Nederland
}

This is an overview of work in progress with Seppo Hassi (Vaasa) and Zoltán Sebestyén (Budapest) about Lebesgue decompositions. Recall the Lebesgue decomposition of a measure in an absolutely continuous part and a singular part. Ando [1] and Simon [7] have given similar decompositions for pairs of nonnegative operators and for forms, respectively. Following Ando and Simon there have been many contributions to this topic, including recent work by Sebestyén and coworkers.

It will be recalled how linear operators and relations in a Hilbert space allow a Lebesgue decomposition in a closable part and a singular part, see [3], where the original work of P.E.T. Jorgensen [5] and S. Ôta [6] concerning decompositions of linear operators was continued and extended. More general Lebesgue type decompositions will be discussed, including the uniqueness of such decompositions and the corresponding Radon-Nikodym derivatives. It will be shown how the Lebesgue type decompositions of relations gives rise to similar decompositions for pairs of bounded operators and pairs of (nonnegative) sesquilinear forms. A different treatment involving parallel sums and differences was given in [2] for the case of pairs of nonnegative forms. Finally some attention will be paid to an orthogonal operator decomposition which does not belong to the above types; see [4].

\section{References}

[1] T. Ando. Lebesgue-type decomposition of positive operators. Acta Sci. Math. (Szeged) 38 (1976), 253-260.

[2] S. Hassi, Z. Sebestyén, H.S.V. de Snoo. Lebesgue type decompositions for nonnegative forms. J. Functional Analysis 257 (2009), 3858-3894.

[3] S. Hassi, Z. Sebestyén, H.S.V. de Snoo, F.H. Szafraniec. A canonical decomposition for linear operators and linear relations. Acta Math. Hungarica 115 (2007), 281-307.

[4] S. Hassi, H.S.V. de Snoo, F.H. Szafraniec, Componentwise and canonical decompositions of linear relations. Dissertationes Mathematicae 465 (2009) (59 pages).

[5] P.E.T. Jorgensen. Unbounded operators, perturbations and commutativity problems. J. Functional Analysis 39 (1980), 281-307.

[6] S. Ôta. On a singular part of an unbounded operator. Zeitschrift für Analysis und ihre Anwendungen 7 (1987), 15-18.

[7] B. Simon. A canonical decomposition for quadratic forms with applications to monotone convergence theorems. J. Functional Analysis 28 (1978), 377-385. 


\title{
Remarks on the convergence of pseudospectra
}

\author{
Sabine Bögli, Petr Siegl \\ Mathematical Institute, University of Bern, Switzerland
}

We establish the convergence of pseudospectra for closed operators acting in different Hilbert spaces and converging in the generalised norm resolvent sense. As an assumption, we exclude the case that the limiting operator has constant resolvent norm on an open set. We extend the class of operators for which it is known that the latter cannot happen by showing that if the resolvent norm is constant on an open set, then this constant is the global minimum. We present examples exhibiting various resolvent norm behaviours and illustrating the applicability of our results.

\section{References}

[1] S. Bögli, P. Siegl. Remarks on the convergence of pseudospectra. Integral Equation Operator Theory 80 (2014), 303-321. 


\title{
Recent results on functional calculus for Tadmor-Ritt operators
}

\author{
Felix L. Schwenninger, Hans Zwart \\ Department of Applied Mathematics, University of Twente, The Netherlands
}

An operator $T$ on a Banach space $X$ is called Tadmor-Ritt if its spectrum is contained in the closed unit disc of the complex plane and its resolvent satisfies

$$
\left\|(z I-T)^{-1}\right\| \leq \frac{C(T)}{|z-1|}, \quad \text { for }|z|>1,
$$

for some positive constant $C(T)$. Such operators appear in studying stability of numerical schemes. The question whether $T$ is power-bounded, i.e. $\sup _{n \in \mathbb{N}}\left\|T^{n}\right\|<\infty$, was answered positively by Lyubich [1] and independently by Nagy, Zemanek [2] in 1999. This result can be seen as a consequence of a more general result about boundedness of a functional calculus for $T$. We will give an introduction to such a calculus and discuss recent results concerning its bound. This generalizes work by Vitse [3].

\section{References}

[1] Yu. Lyubich. Spectral localization, power boundedness and invariant subspaces under Ritt's type condition. Studia Math. 134 vol. 2 (1999), 153-167.

[2] Béla Nagy and Jaroslav Zemánek. A resolvent condition implying power boundedness. Studia Math. 134 vol. 2 (1999), 143-151.

[3] P. Vitse. A band limited and Besov class functional calculus for Tadmor-Ritt operators. Arch. Math. (Basel) 485 vol. 4 (2005), 374-385. 


\title{
A functional analytic look upon Remling's oracle theorem
}

\author{
Christian Seifert, Hendrik Vogt \\ TU Harburg \\ Universität Bremen
}

\begin{abstract}
Remling's oracle theorem [1] is a corner stone in the study of the absolutely continuous spectrum of onedimensional Jacobi operators and Schrödinger operators. Roughly speaking, it says that one-dimensional operators with absolutely continuous spectrum always are 'somewhat periodic'.

The proof given by Remling relies on limit properties of the asymptotic value distribution of the TitchmarchWeyl $m$-function belonging to the operator. We indicate a different approach based on the Poisson transform on the half plane, and we explain how the method can be applied to general Sturm-Liouville operators.
\end{abstract}

\section{References}

[1] Christian Remling. The absolutely continuous spectrum of Jacobi matrices. Ann. of Math. 174 (2011), $125-171$. 


\title{
On Abstract grad-div Systems
}

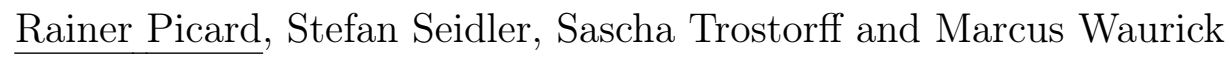 \\ TU Dresden
}

In the framework of a particular class of dynamical systems based on the investigation of typical systems of partial differential equations, skew-selfadjointness of the spatial operator $A$ involved plays an important role as a key reference case for modeling physical phenomena, see e.g. [4]. Typically, skew-selfadjointness hinges on the particular form of $A$ as

$$
A=\left(\begin{array}{cc}
0 & -G^{*} \\
G & 0
\end{array}\right)
$$

where $G: D(G) \subseteq H_{0} \rightarrow H_{1}$ is a closed densely defined linear operator between the Hilbert spaces $H_{0}, H_{1}$. A typical case is where $G=\operatorname{grad}=\left(\begin{array}{c}\partial_{1} \\ \partial_{2} \\ \partial_{3}\end{array}\right)$ realized as the operator of weak differentiation from $L^{2}(\Omega)$ to $L^{2}(\Omega)^{3}$, where $\Omega$ is a non-empty open set in 3 -dimensional Euclidean space. In this case $-G^{*}$ is the weak divergence operator with containment in $D\left(G^{*}\right)$ generating a generalization of vector fields being "tangential" even though a normal may not exist. The particular class of operators $A$ under consideration is an abstraction of this situation, hence the term "abstract grad-div systems", where the role of the partial derivatives $\partial_{k}, k=1,2,3$, is replaced by general Hilbert space operators. It is demonstrated by three examples how this concept can be utilized in approaching evolutionary problems. As an application we consider first the so-called Guyer-Krumhansl model of thermodynamics, [2]. We conclude with a discussion of (dynamic) surface dissipation boundary conditions in acoustics, $[1,5]$, and an impedance type boundary condition in electrodynamics (Leontovich boundary condition), [3], under this new perspective.

\section{References}

[1] Angelo Favini, Gisèle Ruiz Goldstein, Jerome A. Goldstein, and Silvia Romanelli. The heat equation with generalized Wentzell boundary condition. J. Evol. Equ. 2 (2002), 1-19.

[2] R. A. Guyer and J. A. Krumhansl. Dispersion relation for second sound in solids. Phys. Rev. 133 (1964), A1411-A1417.

[3] M. Leontovich. A new method to solve problems of EM wave propagation over the earth surface. (Russian). Izv. Akad. Nauk SSSR, Ser. Fiz. 8 (1944), 16-22.

[4] R. Picard and D. F. McGhee. Partial Differential Equations: A unified Hilbert Space Approach, Volume 55 of De Gruyter Expositions in Mathematics. De Gruyter. Berlin, New York. 518 p., 2011.

[5] A. Venttsel'. On boundary conditions for multidimensional diffusion processes. Theory Probab. Appl. 4 (1959), 164-177. 


\title{
Eigenfunction expansions associated with the one-dimensional Schrödinger operator
}

\author{
Daphne J. Gilbert \\ Dublin Institute of Technology, Ireland
}

We consider the one-dimensional Schrödinger operator $H$ on $L_{2}(-\infty, \infty)$ associated with

$$
L u:=-u^{\prime \prime}+q(r) u=\lambda u, \quad-\infty<r<\infty,
$$

where $q(r): \mathbb{R} \rightarrow \mathbb{R}$ is locally integrable, $\lambda \in \mathbb{R}$ is the spectral parameter, and the differential expression $L$ is in Weyl's limit point case at both infinite endpoints. In this case the unique selfadjoint operator $H$ is defined by

$$
H f=L f, \quad f \in D(H),
$$

where $f \in D(H)$ when $f, L f \in L_{2}(\mathbb{R})$, and $f, f^{\prime}$ are locally absolutely continuous on $\mathbb{R}$. We refer to $H$ as the Schrödinger operator on the line.

Starting from the well known Weyl-Kodaira formulation [1], an alternative form of the expansion is derived in such a way that the principal features contributing to it, namely a scalar spectral density function, the multiplicity properties of the spectrum and the generalised eigenfunctions, are explicitly exhibited in the expansion [2], [3]. Moreover, the spectral types of the generalised eigenfunctions, which may be absolutely continuous, singular continuous or pure point, can be determined from the asymptotic behaviour of the generalised eigenfunctions at both endpoints using the theory of subordinacy [4]. The main steps of the proof will be briefly outlined and the results illustrated by one or more simple examples.

\section{References}

[1] K. Kodaira. The eigenvalue problem for ordinary differential equations and Heisenberg's theory of S-matrices. Amer. J. Math. 71 (1949), 921-945.

[2] I.S. Kac. On the multiplicity of the spectrum of a second-order differential operator and the associated eigenfunction expansion. Izv. Akad. Nauk SSSR Ser. Mat. 27 (1963), 1081-1129 (in Russian).

[3] D.J. Gilbert. Eigenfunction expansions associated with the one dimensional Schrödinger operator. Oper. Theory: Adv. Appl. 227 (2013), 89-105.

[4] D.J. Gilbert. On subordinacy and analysis of the spectrum of one-dimensional Schrödinger operators with two singular endpoints, Proc. Roy. Soc. Edinburgh Sect. A 128 (1989), 213-229. 


\title{
Collocation-quadrature methods and fast summation for Cauchy singular integral equations with fixed singularities
}

\author{
P. Junghanns, Robert Kaiser, D. Potts, \\ Chemnitz University of Technology, Germany,
}

This contribution deals with the notched half plane problem of two-dimensional elasticity theory, which considers a straight crack perpendicular to and ending at the boundary of the elastic half plane. The problem can be modeled by a hypersingular integral equation, the solution of which is the crack opening displacement. For the numerical solution of this equation we propose a collocation-quadrature method, which looks for an approximation of the derivative of the crack opening displacement. This derivative is the solution of a Cauchy singular integral equation with additional fixed singularities,

$$
\frac{1}{\pi} \int_{-1}^{1}\left[\frac{1}{y-x}-\frac{1}{2+y+x}+\frac{6(1+x)}{(2+y+x)^{2}}-\frac{4(1+x)^{2}}{(2+y+x)^{3}}\right] u(y) d y=f(x), \quad-1<x<1 .
$$

A first numerical approach to this equation, which uses collocation methods, was described in [1]. This paper pays mainly attention to the computational aspects by using special structural properties of the matrices of the respective discretized equations. Basing on a recent result [2] on the stability of collocation methods applied to such integral equations we give, using $\mathrm{C}^{*}$ algebra techniques, necessary and sufficient conditions for the stability of collocation-quadrature methods. These methods have the advantage that the respective system of equations has, in contrast to [1], a very simple structure and allows the application of fast summation methods which results in a fast algorithm with $\mathcal{O}(n \log n)$ complexity.

\section{References}

[1] M. R. Capobianco, G. Criscuolo and P. Junghanns. On the numerical solution of a hypersingular integral equation with fixed singularities. Oper. Theory Adv. Appl. (2008), 95-116

[2] P. Junghanns, R. Kaiser and G.Mastroianni. Collocation for singular integral equations with fixed singularities of particular Mellin type. Electr. Trans. Numer. Analysis (2014) 


\title{
Exponential stability of a second order integro-differential equation with delay
}

\author{
Sascha Trostorff \\ Faculty of Mathematics and Sciences, Institute for Analysis, TU Dresden
}

Motivated by a recent article [1], we study a wave equation of the form

$$
u^{\prime \prime}(t)-\Delta u(t)-\int_{-\infty}^{t} k(t-s) \Delta u(s) \mathrm{d} s+\kappa u^{\prime}(t-h)=f(t) \quad(t \in \mathbb{R})
$$

on a domain $\Omega \subseteq \mathbb{R}^{n}$ with homogeneous Dirichlet boundary conditions. Here, $k$ is a suitable operator-valued kernel, $h>0, \kappa \in \mathbb{R}$ and $f$ is a given source term. We provide a way to rewrite this equation as an abstract first-order evolutionary problem and we discuss sufficient conditions on the coefficients involved, which yield the exponential stability of the second order problem.

\section{References}

[1] F. Alabau-Boussouira, S. Nicaise, C. Pignotti. Exponential stability of the wave equation with memory and time delay. Technical report. arXiv:1404.4456. 


\title{
The div M grad Without Ellipticity
}

\author{
Amru Hussein ${ }^{1}$, Vadim Kostrykin ${ }^{1}$, David Krejčiřik ${ }^{2}$, \\ Konstantin A. Makarov ${ }^{3}$, Stephan Schmitz ${ }^{1}$ \\ ${ }^{1}$ Institut für Mathematik, Johannes Gutenberg-Universität Mainz, Germany \\ ${ }^{2}$ Department of Theoretical Physics, Nuclear Physics Institute ASCR, Řž, Czech Republic \\ ${ }^{3}$ Department of Mathematics, University of Missouri, Columbia, USA
}

The talk discusses some recent results [1] on $\operatorname{div} M$ grad-operator on a bounded domain for sign-indefinite coefficient matrices $M$. A simplest example of such kind is $\mathcal{L}=-\frac{d}{d x} \operatorname{sign}(x) \frac{d}{d x}$ on a bounded interval. We prove several new representation theorems for indefinite quadratic forms, generalizing results of [2], [3], [4], [5]. Using these theorems, for a wide class of coefficient matrices we prove the existence of a unique self-adjoint operator $\mathcal{L}$ associated with the form $\langle\operatorname{grad} u, M \operatorname{grad} u\rangle$. We discuss several examples when the operator $\mathcal{L}$ turns out to have a nontrivial essential spectrum.

\section{References}

[1] A. Hussein, V. Kostrykin, D. Krejčiřik, K. A. Makarov, S. Schmitz. The divMgrad without ellipticity: A quadratic form approach. In preparation.

[2] A. McIntosh. Bilinear forms in Hilbert space. J. Math. Mech. 19 (1970), 1027-1045.

[3] A. Fleige. Non-semibounded sesquilinear forms and left-indefinite Sturm-Liouville problems. Integ. Equ. Oper. Theory 33 (1999), 20-33.

[4] A. Fleige, S. Hassi, H. de Snoo. A Krein space approach to representation theorems and generalized Friedrichs extensions. Act Sci. Math. (Szeged) 66 (2000), 633-650.

[5] L. Grubišić, V. Kostrykin, K. A. Makarov, K. Veselić. Representation theorems for indefinite quadratic forms revisited. Mathematika 59 (2013), 169-189. 


\title{
Rational matrix solutions of a Bezout type equation on the half plane
}

\author{
A.E. Frazho, M.A. Kaashoek, A.C.M. Ran \\ Purdue University, West Lafayette, USA \\ VU university Amsterdam, The Netherlands
}

VU university Amsterdam, The Netherlands and North West University, South Africa

A state space description is given of all stable rational matrix solutions of a general rational Bezout type equation on the right half plane. To be precise, let $G$ be a stable and proper rational $m \times p$ matrix function. We shall be interested in stable rational $p \times m$ matrix-valued solutions $X$ of the Bezout type equation

$$
G(s) X(s)=I_{m}, \quad \Re s \geq 0 .
$$

Throughout we shall assume that $G$ admits a state space realization of the form

$$
G(s)=C\left(s I_{n}-A\right)^{-1} B+D
$$

with $A$ a stable matrix.

A state space formula for a particular solution $X$ satisfying a certain $H^{2}$ minimality condition is presented, as well as a state space formula for the inner function describing the null space of the multiplication operator corresponding to the Bezout equation, and a parameterization of all solutions using the particular solution and this inner function. A state space version of the related Tolokonnikov lemma is also presented.

The talk will present the main results of [1]. The analogues for the unit disc case can be found in $[2,3]$.

\section{References}

[1] A.E. Frazho, M.A. Kaashoek, A.C.M. Ran. Rational matrix solutions of a Bezout type equation on the half plane. Oper. Theory Adv. Appl. 237 (2013), 145-160.

[2] A.E. Frazho, M.A. Kaashoek, A.C.M. Ran. Right invertible multiplication operators and stable rational matrix solutions to an associate Bezout equation, I. the least squares solution. Integral Equations Operator Theory 70 (2011), 395-418.

[3] A.E. Frazho, M.A. Kaashoek, A.C.M. Ran. Right invertible multiplication operators and stable rational matrix solutions to an associate Bezout equation, II: Description of all solutions. Oper. Matrices 6 (2012), $833-857$. 


\title{
On the trace class property of the resolvent regularization of a Dirac-type operator on $\mathbb{R}^{3}$
}

\author{
Fritz Gesztesy, Marcus Waurick \\ University of Missouri \\ TU Dresden
}

In [1], C. Callias discusses the perturbed Dirac operator $L=\sum_{j=1}^{n} \gamma_{j} \partial_{j}+\Phi$, where $n \in \mathbb{N}$ odd, $\gamma_{j}$ are the elements of the Euclidean Dirac algebra realized as $2^{\hat{n}} \times 2^{\hat{n}}$-matrices with $n=2 \hat{n}+1$ and $\Phi$ is a multiplication operator of multiplying with a bounded $C^{\infty}$-function with values in the selfadjoint $m \times m$-matrices. Assuming suitable decay conditions on the derivatives of $\Phi$, it can be shown that $L$ is a Fredholm operator as an operator on $L^{2}\left(\mathbb{R}^{n}\right)^{2^{n}} m$. In [1] the index, ind $L$, of $L$ is obtained with the help of the resolvent regularization

$$
\text { ind } L=\lim _{z \rightarrow 0+} z \operatorname{trtr}_{\text {int }}\left(\left(L^{*} L+z\right)^{-1}-\left(L L^{*}+z\right)^{-1}\right)
$$

provided $\operatorname{tr}_{\text {int }}\left(\left(L^{*} L+z\right)^{-1}-\left(L L^{*}+z\right)^{-1}\right)$ is a trace class operator in $L^{2}\left(\mathbb{R}^{n}\right)$, where trint denotes the operator mapping any bounded linear operator considered as a block operator matrix on $L^{2}\left(\mathbb{R}^{n}\right)^{2^{\hat{n}} m}$ to the sum of the diagonal entries. In the talk, for $n=3, m=2$, we give an example for a potential $\Phi$ satisfying the decay and differentiability conditions assumed in [1], where the trace class property of $\operatorname{tr}_{\text {int }}\left(\left(L^{*} L+z\right)^{-1}-\left(L L^{*}+z\right)^{-1}\right)$ is violated.

\section{References}

[1] C. Callias. Axial anomalies and index theorems on open spaces. Commun. Math. Phys. 62 (1978), $213-234$. 


\title{
On the spectrum of non-selfadjoint operators over dynamical systems
}

\author{
Christian Seifert \\ Technische Universität Hamburg-Harburg \\ Institut für Mathematik \\ Schwarzenbergstraße $95 \mathrm{E}$ \\ 21073 Hamburg, Germany
}

We consider equivariant continuous families of discrete one-dimensional operators over arbitrary dynamical systems. We introduce the concept of a pseudo-ergodic element of a dynamical system. We then show that all operators associated to pseudo-ergodic elements have the same spectrum and that this spectrum agrees with their essential spectrum. As a consequence we obtain that the spectrum is constant and agrees with the essential spectrum for all elements in the dynamical system if minimality holds.

This is joint work with Siegfried Beckus (FSU Jena, Germany), Daniel Lenz (FSU Jena, Germany) and Marko Lindner (TU Hamburg-Harburg, Germany). 


\title{
Uniform mean ergodicity of $C_{0}$-semigroups in a class of Fréchet
} spaces

\author{
Angela A. Albanese, José Bonet, Werner J. Ricker \\ Dipartimento di Matematica e Fisica "E.De Giorgi" \\ Università del Salento, I-73100 Lecce, Italy \\ Instituto Universitario de Matemática Pura y Aplicada IUMPA \\ Universidad Politècnica de Valencia, E-46071 Valencia, Spain \\ Math.-Geogr. FakultÃt, Katholische Universität \\ Eichstätt-Ingolstadt, D-85072 Eichstätt, Germany
}

Let $(T(t))_{t>0}$ be a strongly continuous $C_{0}$-semigroup of bounded linear operators on a Banach space $X$ such that $\lim _{t \rightarrow \infty}\|T(t) / t\|=0$. Characterizations of when $(T(t))_{t>0}$ is uniformly mean ergodic, i.e., of when its Cesàro means $r^{-1} \int_{0}^{r} T(s) d s$ converge in operator norm as $r \rightarrow \infty$, are known. For istance, this is so if and only if the infinitesimal generator $A$ has closed range in $X$ if and only if $\lim _{\lambda \downarrow 0^{+}} \lambda R(\lambda, A)$ exists in the operator norm topology $(R(\lambda, A)$ is the resolvent operator of $A$ at $\lambda)$. These characterizations, and others, are shown to remain valid in the class of quojection Fréchet spaces, which includes all Banach spaces, countable products of Banach spaces, and many more. An example is presented which shows that the extension fails to hold for all Fréchet spaces. 


\title{
On deformations of classical Jacobi matrices
}

\author{
Michał Wojtylak \\ Jagiellonian University, Cracow
}

Let

$$
A=\left(\begin{array}{cccc}
a_{0} & b_{0} & & \\
b_{0} & a_{1} & b_{1} & \\
& b_{1} & a_{2} & \ddots \\
& & \ddots & \ddots
\end{array}\right), \quad a_{i} \in \mathbb{R}, b_{i}>0 .
$$

be a classical Jacobi matrix. We will try to reveal the spectrum of the product $H A$, where $H=\operatorname{diag}(-1,1,1, \ldots)$. We will start with a short motivation, lying in the fields of signal analysis (extracting information from a highly noisy signal) and numerical analysis. The main problem will be to locate the (unique) nonpositive eigenvalue of $H A$, that is the unique eigenvalue with the eigenvector $x$ satisfying $\langle H x, x\rangle \leq 0$. This eigenvalue can be approximated by the eigenvalue of nonpositive type of the finite truncation of the matrix $H A$, the properties of this approximation will be studied in detail.

Joint work with M. Derevyagin, L. Perotti, D. Vrinceanu.

\section{References}

[1] M. Derevyagin, L. Perotti, D. Vrinceanu, On the convergence of poles of the Padé approximants of a generalised Nevanlinna function, in preparation.

[2] D. Bessis, R. Perotti, Universal analytic properties of noise: introducing the J-matrix formalism, J. Phys. A: Math. Theor. 42 (2009) 365-202. 


\section{S24: History of mechanics}

This section will provide a forum for the presentation of historical and/or speculative studies on mechanics focusing on the relations between concepts from antiquity up to now, which could be of interest to historians of mechanics and physics as well as researchers in mechanics.

The contributions of those authors who have benefited from the study of old theories for developing current models in the field of applied mechanics and mathematics will be also considered. This in order to highlight the importance of historical and epistemological perspective setting for the current and future developments in science.

With these aims in mind, topics of applications will include (but are not limited to):

Virtual work laws

Variational methods and conservation principles

Molecular foundation for continuum mechanics

Coarse graining processes and the use of Cauchy-Born rule

Continuum and statistical thermodynamics

Material deformation theories and experimental results in solid mechanics

Inelastic deformations, damage and fracture

Development and use of the Ricci's tensor calculus in deformation theories of solids, shells and fluids

Mechanical models for ancient constructions 


\title{
The change of perspective with the advent of Quantum Mechanics
}

\author{
S. Esposito \\ I.N.F.N. - Naples' Unit \\ Complesso Universitario di M. S. Angelo, Via Cinthia, 80126 Naples, Italy \\ (Salvatore.Esposito@na.infn.it)
}

Differently from other previous generalizations, such as Einstein's Theory of Relativity for example, Quantum Mechanics has introduced a radical change of physical perspective in the study of the microscopic world with respect to Classical Mechanics. The Newtonian determinism, centered about the initial position and velocity (or momentum), has abdicated in favor of quantum determinism, based on Heisenberg's uncertainty relations and centered about the initial wave-function of the system.

Also, the recovery of the "classical limit" showed to be not at all obvious, even just from a philosophical point of view: the mathematical limit of vanishing Planck constant $h \rightarrow 0$ requires an interpretation that is completely dissimilar, for instance, from that natural of small velocities $v \ll c$ of the Theory of Relativity, or any other generalization handled by Physics. The mathematical formalism has changed accordingly, and assumed a foundational character that cannot be disjointed by the physical interpretation.

In this talk I will review the main conceptual steps that have changed our view of the mechanical world in the XX century, by focussing on the issues mentioned above, as well as on applications of Quantum Mechanics that have had a direct consequence also on the macroscopic world. The strict relationship between micro- and macro-world, indeed, is a peculiar feature of the modern technological applications, which have affected at a large extent - not at all limited to philosophical issues - the applied mechanics behind daily life.

\section{References}

[1] A. Messiah. Quantum Mechanics. Dover, 1999.

[2] R.P. Feynman, R.B. Leighton, M. Sands. The Feynman Lectures on Physics, Volume III - Quantum Mechanics. Addison Wesley, 2005.

[3] A. Drago and S. Esposito. Following Weyl on Quantum Mechanics - The contribution of Ettore Majorana. Found. Phys. 34 (2004), 871-887.

[4] S. Esposito. Majorana and the path-integral approach to Quantum Mechanics. Ann. Fond. De Broglie 31 (2006), 1-19. 


\title{
Walter Noll and the Bourbakization of Mechanics
}

\author{
Gianpietro Del Piero \\ Dipartimento di Ingegneria, Università di Ferrara, Italy \\ International Research Center M\&MoCS, Cisterna di Latina, Italy
}

There was a moment, in the second half of the 19th century, in which a strong need for axiomatization was felt by the mathematical community. This goal was pursued by a number of eminent scientists, and continued in the subsequent century, up to the monumental work of the Bourbaki group. Thanks to this, many basic definitions, concepts and notations of mathematics were fixed in the way they are now universally known and accepted. The same did not happen for mechanics, for which a systematic apparatus was developed only for discrete systems. For continuum mechanics, the pioneering work of the fathers was not followed by an adequate axiomatic settling. So it can be claimed that, as far as continuum mechanics is concerned, Hilbert's sixth problem on the axiomatization of physical sciences is still open.

Since long time, there has been a diffuse feeling that the giants of the past did the whole job, that what they did is definitive, and that further investigation on the principles would be rewardless. And yet, just after the half of the 20th century, a live interest in the fundamentals of continuum mechanics unexpectedly arose. Roughly, it went along three main directions. The first, stimulated by the many technical problems raised during the 2nd wold war, was addressed to theories of inelastic behavior, such as plasticity, damage, fracture, and fatigue. The second was a renewed interest in generalized continua, resuscitated after the oblivion which followed the appearing of the Cosserat's book in 1909. And, finally, there was a reborn attention the foundations. Initially, this was carried on by a single man, W. Noll. In the years between the end of the 1950's and the half of the 1970's he produced a series of papers, in which the whole corpus of the foundations was revisited. The list of his main achievements includes

- a neat distinction between general field equations and constitutive equations valid for specific classes of materials (1958),

- the principle of indifference of the constitutive equations to changes of observers (1958),

- the principle of local action, discriminating between local and non-local material behavior (1958),

- a mathematical definition of the material symmetries (1958),

- the proof of of Cauchy's conjecture on the dependence of the contact force on the normal to the contact surface (1959),

- the theory of materials with fading memory (with B.D. Coleman, 1960),

- the deduction of Newton and Euler's general laws from the principle of indifference of power (1963),

- the new theory of simple materials, explicitly designed to include plasticity and damage in a general scheme for material behavior (1972),

- some basic statements on the level of regularity to be required to the regions occupied by a continuous body (with E.G. Virga, 1988),

- the removal of inertia, of linear and angular momentum, and of kinetic energy from the basic concepts of mechanics (1995).

Though most of these items are today common heritage, some did not receive adequate attention. The subsequent research activity involved a number of researchers, and split into several directions, not all of great moment. But, surprisingly enough, very little progress was made in some strategic esearch lines, such as multiscale kinematics or the mechanics of fractal bodies. A remarkable result, coming from an idea of M.E. Gurtin and L.C. Martins (1976), subsequently developed by M. Šilhavỳ (1985, 1991), is that Cauchy's tetrahedron theorem, rather than a consequence of the balance of the linear momentum, is a regularity property of the system of the contact actions. The enormous potential impact of this result on the axiomatization of continuum mechanics, and especially of the mechanics of generalized continua, has not yet been realized. In the proposed communication, following in part the contents of my paper [1], I will try to give an idea of the relevance of this result, of some immediate consequences, and of possible future developments.

\section{References}

[1] G. Del Piero. Non-classical continua, pseudobalance, and the law of action and reaction. Math. Mech. of Complex Systems 2 (2014), 71-107. 


\title{
Beltrami and mathematical physics in non-Euclidean spaces
}

\author{
Danilo Capecchi, Giuseppe Ruta \\ "Sapienza" università di Roma, Dipartimento di ingegneria strutturale e geotecnica
}

\begin{abstract}
Eugenio Beltrami (Cremona 1836 - Roma 1900) in some of his works investigated the behaviour of elastic continua embedded in non-Euclidean spaces. In these papers, Beltrami used his great skills in pure mathematics with the aim of understanding the geometrical nature of physical space, where natural phenomena occur. In particular, starting from his thorough knowledge of geometry and his mastery in continuum mechanics, he asked himself if the actual space, where elastic, electric, thermal, and magnetic phenomena occur, were Euclidean or not. Such a theme was well present among the mathematical scientific community at the end of the 19th century, especially after the contributions by the Russian Nikolaj I. Lobacevskij (1793-1856), the Hungarian Janos Bolyai (1802-1860), the German Bernhard Riemann (1826-1866), and Beltrami himself in the development of non-Euclidean geometry. The first two, in the first decades of the 19th century, searched for a geometry which could be built independent of Euclid's fifth postulate, hence worked on a purely mathematical basis. Beltrami and Riemann, who were long in touch in Pisa, on the other hand were interested not only on the basics of non-Euclidean geometry, but also on how non-Euclidean geometry could affect the field equations of the great problems of mathematical physics of their time, that is, elasticity and electro-magnetism.

Beltrami's works on the field opened for sure the path for a new way of intending mathematical physics. Some of Beltrami's pupils followed him in this enterprise, among them Ernesto Cesaro, who devoted some investigation to elasticity in hyper-spaces. In this contribution, we sketch Beltrami's original results, basing on his papers, and propose a critical discussion on them and on their influence.
\end{abstract}

\section{References}

[1] Capecchi D, Ruta G (2014) Strength of materials and theory of elasticity in 19th century Italy. Springer, Dordrecht

[2] Beltrami E (1902-1920) Opere matematiche (4 vol), Hoepli, Milan

[3] Beltrami E (1868) Saggio di interpretazione della geometria non euclidea. Giornale di matematiche, vol 6, pp. 284-312. In: Beltrami E (1902-1920) Opere matematiche (4 vol), Hoepli, Milan, vol 1, pp. 374-405

[4] Beltrami E (1880-82) Sulle equazioni generali della elasticità. Annali di Matematica pura e applicata, s. 2, vol X, pp. 46-63. In: Beltrami E (1902-1920) Opere matematiche (4 vol), Hoepli, Milan, vol 3, pp. 383-407

[5] Beltrami E (1884) Sull'uso delle coordinate curvilinee nelle teorie del potenziale e dell'elasticità. In: Beltrami E (1902-1920) Opere matematiche (4 vol), Hoepli, Milan, vol 4, pp. 136-179

[6] Beltrami E (1886) Sull'interpretazione meccanica delle formole di Maxwell. In: Beltrami E (1902-1920) Opere matematiche (4 vol), Hoepli, Milan, vol 4, pp. 190-223

[7] Cesaro E (1894) Sulle equazioni dell'elasticità negli iperspazi. Rendiconti della Regia Accademia dei Lincei, s. 5 , t. 3 , pp. $290-294$ 


\title{
Gustav R. Kirchhoff and the dynamics of tapered beams
}

\author{
Antonio M. Cazzani, \\ University of Cagliari
}

The dynamics of straight tapered beams (within the framework of Euler-Bernoulli beam theory) was first studied by Gustav Kirchhoff in a seminal paper dating 1879[1]. The importance of this contribution was already recognized by Todhunter and Pearson who cited it in their monumental work[3].

Kirchhoff applied for the first time an approach based on what is now known as Forbenius method to the solution of the fourth order partial differential equation which is now generally written in the form:

$$
E \frac{\partial^{2}}{\partial x^{2}}\left(J(x) \frac{\partial^{2} y}{\partial x^{2}}\right)+A(x) \rho \frac{\partial^{2} y}{\partial t^{2}},
$$

where $x$ is the beam axis, $y$ its deflection, $t$ represents time and $E, \rho, A, J$ are respectively the Young's modulus, the density, the cross-section area and the corresponding area moment of inertia.

He showed the general solution and then take explicitly into account the two cases of a tapered beam having a wedge and a cone/pyramid shape with a linear taper.

This result was subsequently generalized in the following decades by many authors to other kind of taper law and also to truncated cones, pyramids etc. [3].

The present contribution is concerned with an analysis of the original paper and of the solution method applied there and only roughly described by the author, which allowed him (see [3]) to reduce the solution of the fourth order equation to that of two second order equations.

\section{References}

[1] G.R. Kirchhoff. Über die Transversalschwingungen eines Stabes von veränderlichem Querschnitt. Monatsberichte der könig. Preuss. Akad. Wissenschaften zu Berlin (1879), 815-828.

[3] I. Todhunter, K. Pearson. A History of the Theory of Elasticiti and of the Strength of Materials. Cambridge University Press, 1893, Vol. 2, Part 2, 92-98.

[3] H.-C. Wang. Generalized Hypergeometric Function Solutions of the Transverse Vibration of a Class of Nonuniform Beams. ASME J. Applied Mechanics 34 (1967), 702-708.

[4] J. Vdovič. Über die Transversalschwingungen eines Stabes von veränderlichem Querschnitt, ZAMM 53 (1973), 148-151. 


\title{
On the Derivation of the Equations of Hydrodynamics 65 years after Irving\&Kirkwood
}

\author{
Paolo Podio-Guidugli \\ Accademia Nazionale dei Lincei \\ and \\ Department of Mathematics, University of Rome TorVergata
}

In their landmark paper [1], Irving and Kirkwood demonstrated that certain basic equations central to all of continuum mechanics (in their terminology, 'the equations of hydrodynamic') can be derived from a statistical setting of the Newtonian motion problem for a system of mass points. The equations they derived are the standard balances of mass, momentum, and energy. Certain mathematical shortcut in [1] were later corrected by Noll [2], who returned to the subject much later [3], when his 1955 paper was translated into English. Noll's main contribution is a constructive formula for the stress tensor that corresponds to the multifold particle interactions one deals with in a discrete framework; important recent contributions to the subject are, among others, those in $[4,5]$.

In this talk, I plan to discuss along the path of [1] other concepts and laws of importance in continuum mechanics, such as the entropy concept and the balance of moment of momentum.

\section{References}

[1] J.H. Irving and J.G. Kirkwood, The statistical mechanical theory of transport processes. IV. The equations of hydrodynamics. J. Chem. Phys. 18 (6), 817-829 (1950).

[2] W. Noll, Die Herleitung der Grundgleichungen der Thermomechanik der Kontinua aus der statistischen Mechanik. Indiana Univ. Math. J. 4, 627-646 (1955).

English translation: Derivation of the fundamental equations of continuum thermodynamics from statistical mechanics. J. Elasticity 100, 5-24 (2010).

[3] W. Noll, Thoughts on the concept of stress. J. Elasticity 100, 25-32 (2010).

[4] E.B. Tadmor and R.E. Miller, Modeling Materials. Continuum, Atomistic and Multiscale Techniques. Cambridge University Press, 2011.

[5] N.C. Admal and E.B. Tadmor, Stress and heat flux for arbitrary multibody potentials: A unified framework. J. Chem. Phys. 134, 184106 (2011). 


\title{
How does dynamic complexity contribute to the advancement of mechanics
}

\author{
Giuseppe Rega \\ Department of Structural and Geotechnical Engineering, \\ Sapienza University of Rome, Italy, \\ giuseppe.rega@uniroma1.it
}

Starting with the pioneering studies of Henry Poincaré, and being nurtured by the outstanding contributions of a number of giants in modern science, the dynamics of complex systems has become a revolutionary area of research in the last few decades of the 20th century and around the turn of the millennium [1]. The theoretical achievements earlier obtained within the applied mathematics and physics communities have later on entailed meaningful outcomes also within the mechanics community, as soon as the importance of nonlinear phenomena in view of technical applications has been realized. Based on sophisticated analytical, geometrical, and computational techniques employing powerful concepts and tools of dynamical systems, bifurcation, and chaos theory, a wide variety of applications to mechanical and structural systems has flourished, properly updating and complementing them with meaningful experimental verifications and with a view to engineering aims.

Without attempting at prematurely interpret the underlying, and still unsteady, process of mutual influence between different scientific areas, it appears fully legitimate to wonder about what dynamic complexity has contributed to the advancement of mechanics and, even more, about how can further contribute to its future. For the sake of an accessible introductory treatment of a difficult matter, one can schematically distinguish between the involved phenomenological and methodological aspects, with also possible technological consequences, though being all of them somehow interconnected.

From a phenomenological viewpoint, the unquestionable enrichment brought to mechanics by the knowledge of dynamical systems stands in the detection, description and understanding of a multitude of nonlinear events of theoretical and practical interest (e.g., [2]). To name just a few concepts and ensuing practical tools, one can think of the local and, mostly, global bifurcations determining strong, and often sharp, changes of system response, of the highly non-regular, and seemingly exoteric, features which characterize chaotic behaviors, of the structural stability issues providing a well-founded theoretical framework for the interpretation of still unexplained engineering phenomena (such as flutter or galloping).

From the methodological viewpoint, it is worth distinguishing between general and specific aspects. General aspects are concerned with making the need of a cross-disciplinary approach to science definitely clear. Indeed, in this respect, complexity and nonlinear dynamics represent updated paradigms which allow to consciously address a variety of disciplines sharing common, though technically distinct, phenomenological aspects [1]. They govern a huge amount of events in both hard and soft scientific areas, which encompass physics, chemistry, biology, and engineering, with all of their varied branches, as well as medicine, economy, arts, and architecture, with even possibly undue significance and misleading interpretations. At the same time, modern mechanics is fruitfully contaminated by a variety of other disciplines, which include not only nonlinear dynamics and complexity but also, e.g., control and optimization.

More specific methodological aspects are concerned with an expected change of perspective in the analysis and control of engineering systems, and in the ensuing assessment of their safe design [3]. Consideration of global nonlinear phenomena entails revisiting the classical concept of theoretical stability, as earlier formulated (Euler) and later on enriched under different perspectives (Lyapunov, Koiter, and others), and accounting for the potentially dramatic effects of the small - yet finite - dynamical perturbations always occurring in real systems, which have to be properly framed within a practical stability perspective capable to guarantee the achievement of the needed load carrying capacity. Global phenomena govern the robustness of static/dynamic solutions against variations of initial conditions and/or control parameters, and affect the dynamic integrity of mechanical/structural systems in applications ranging from the macro- to the micro/nano-scale. Dynamic integrity concepts and tools allow to reliably analyse and control global dynamics, to effectively interpret and predict unexpected experimental behaviors, to provide important hints towards safe and aware engineering design. Pursuing practical stability means abandoning the merely local perspective traditionally assumed in the analysis and design of systems and structures, and moving to a global one where the whole dynamic behaviour of the system is considered, even if being actually interested in only a small (but finite) neighbourhood of a 
given solution. In spite of its conceptual simplicity, this is a paramount enhancement, full of theoretical and practical implications.

As a matter of fact, the great potential of nonlinear dynamics to significantly enhance performance, effectiveness, reliability and safety of systems has not yet been exploited to the aim of conceiving/developing novel design criteria. Yet, fundamental understanding of various nonlinear physical phenomena producing bifurcations and complex response has now reached such a critical mass that it's definitely time to develop a novel design philosophy, as well as the basic technologies, capable to take full advantage of the natural richness of behavior offered by the inherently nonlinear systems of mechanics.

\section{References}

[1] T. Hikihara, P. Holmes, T. Kambe, G. Rega. Introduction to the focus issue: Fifty years of chaos: Applied and theoretical. Chaos, 22:047501-1-4, 2012.

[2] A.H. Nayfeh, B. Balachandran. Applied Nonlinear Dynamics, Wiley, New York, 1995.

[3] G. Rega, S. Lenci. A global dynamics perspective for system safety from macro- to nano-mechanics: Analysis, control and design engineering. Submitted. 


\title{
On the role of virtual work in Levi-Civita's parallel transport
}

\author{
Giuseppe Iurato ${ }^{1}$, Giuseppe Ruta ${ }^{2}$ \\ ${ }^{1}$ University of Palermo, IT, ${ }^{2}$ Sapienza University, Rome, IT
}

In this contribution we point out the role of the virtual work principle in the origin of the notion of parallel transport by Tullio Levi-Civita. For a history of the virtual work principle, we may quote [1].

The current historical literature usually reports that parallel transport was motivated by Levi-Civita's attempt to give a geometrical interpretation to the covariant derivative of absolute differential calculus. A careful reading of the Introduction to [2], however, shows that Levi-Civita searched to simplify the computation of the curvature of a manifold. In Section 1 of [2] he considers a metric on a finite-dimensional manifold $V_{n}$

$$
d s^{2}=\sum_{i k} a_{i k} d x_{i} d x_{k}
$$

and embeds $V_{n}$ into an ordinary Euclidean space $S_{N}$ with a sufficiently great dimension, so that it may be described by the system (corresponding to equation (1) of [2], Section 1)

$$
y_{\nu}=y_{\nu}\left(x_{1}, \ldots, x_{n}\right) \quad \nu=1,2, \ldots, N
$$

formally coinciding with a smooth holonomic system subjected to (invertible) virtual displacements. In Section 2 Levi-Civita considers an arbitrary direction of $S_{N}$ with unit vector $\vec{f}$ of direction cosines $f_{\nu}$, and another arbitrary direction at a point $P$ of $V_{n}$. This last has unit vector $\vec{\alpha}$ with direction cosines $\alpha_{\nu}$ with respect to $S_{N}$. The point $P$ may be thought varying on a given smooth curve $\mathcal{C}$ lying on $V_{n}$ and naturally parameterized by the abscissa $s$ in equation (1), thus $\alpha_{\nu}$ depend on $s$.

Considering an infinitesimal variation $d s$ of $s$, the cosine of the angle between $\vec{f}$ and $\vec{\alpha}$, i.e., $\sum_{\nu=1}^{N} \alpha_{\nu}(s) f_{\nu}$, undergoes the variation $d s \sum_{\nu=1}^{N} \alpha_{\nu}^{\prime}(s) f_{\nu}$. Then, ordinary parallelism between $\vec{\alpha}$ and $\vec{f}$ would require this variation to vanish as $\vec{f}$ varies, thus implying $\alpha_{\nu}$ to be constant. Levi-Civita, however, imposes the weaker condition that the angle between $\vec{\alpha}$ and $\vec{f}$ is constant when $\vec{f}$ varies on $V_{n}$. That is, he supposes the variation $d s \sum_{\nu=1}^{N} \alpha_{\nu}^{\prime}(s) f_{\nu}$ to vanish only for the directions tangent to $V_{n}$ as $P$ varies along the curve $\mathcal{C}$. Hence, LeviCivita claims that these directions are compatible with the constraints (2), so that, replacing $f_{\nu}$ with quantities proportional to them, his definition of parallelism implies

$$
\sum_{\nu=1}^{N} \alpha_{\nu}^{\prime}(s) \delta y_{\nu}=0
$$

for any displacement $\delta y_{\nu}$ compatible with the constraints (2). With a suitable interpretation of $\vec{\alpha}$ in the first formulas of Section 3 in [2], equation (3) is but a formulation of the virtual work principle for the smooth bilateral holonomic system of equation (2). Hence, Levi-Civita defines his notion of parallel transport and applies it to a Riemannian manifold, in particular to the computation of its curvature. Precisely, he deduces equation (8) of [2], Section 2

$$
\sum_{\nu=1}^{N} \alpha_{\nu}^{\prime}(s) \frac{\partial y_{\nu}}{\partial x_{k}}=0 \quad(k=1,2, \ldots, n)
$$

then equation $\left(1_{a}\right)$ of [2], Section 3, where he provides the exact formal intrinsic conditions characterizing the parallel transport of the direction $\vec{\alpha}$ along $\mathcal{C}$ as a function of its directional parameters $\xi_{1}, \ldots, \xi_{n}$ with respect to $V_{n}$, within the framework of absolute differential calculus. Furthermore, in the following sections of [2], Levi-Civita does not make any explicit mention to covariant derivatives, except for a hint to Ricci's rotation coefficients in Section 13.

In conclusion, from a historical standpoint, the virtual work principle played a key role in the origin of the idea of connection in Riemannian geometry.

\section{References}

[1] D. Capecchi. History of Virtual Work Laws. A History of Mechanics Prospective. Birkhäuser, Boston, 2012.

[2] T. Levi-Civita. Nozione di parallelismo in una varietà qualunque e conseguente specificazione geometrica della curvatura riemanniana. Rendiconti del Circolo Matematico di Palermo. XLII (1917), 173-215. 


\title{
Nineteenth century molecular models with a glance at modern discrete-continuum theories
}

\author{
$\underline{\text { Patrizia Trovalusci }}$ \\ Department of Structural and Geotechnical Engineering, \\ Sapienza University of Rome, Italy, \\ patrizia.trovalusci@uniroma1.it
}

This contribution focuses on the genesis of constitutive theories for continuous models originated from discontinuous descriptions of materials which in turn historically coincides with the genesis of continuum mechanics. The molecular theory of elasticity, as developed by Navier, Cauchy and Poisson $[4,3,6]$ in the $19^{\text {th }}$ century, represents an attempt to give explanations 'per causas' of elasticity, which were presumed to stem from the natural attractive or repulsive properties of elementary particles ('molecules'), as in the original idea of Newton, specified later by Boscovich, Coulomb and others. In these mechanistic descriptions the molecules, or atoms, are perceived as ultimate particles without extension inside which no forces are accounted for. These particles interact in pairs through forces, depending on their mutual distance, directed along the line connecting their centres ('central-force' scheme). Macroscopic quantities, like stress, elastic moduli, etc., were then derived as averages of molecular material quantities over a convenient volume element, called 'molecular sphere of action', outside which intermolecular forces are negligible.

However, this scheme led to experimental discrepancies concerning the number of elastic constants needed to represent material symmetry classes. Successively, Voigt and Poincaré introduced mixed energetic/mechanistic approaches $[9,10,5]$ providing a refined descriptions of the classical molecular model that solve the problem of the underestimation of the number of the material constants related to the central-force scheme. In particular, Voigt introduced potentials of force and moment interactions exerted between pairs of rigid bodies, while Poincaré proposed a multibody potential description $[8,1,2]$. Even if both Voigt and Poincaré, removing the centralforce scheme, offered a good solution to the controversy about the elastic constants, the mechanistic-molecular approach, which was longer supported also by Saint Venant, was abandoned in favour of the energetic-continuum approach by Green, and their works have been neglected for long time.

Now these ideas found a renewed interest with reference to the problem of constitutive modelling of complex materials. Current researches in solid state physics, as well as in mechanics of materials, show that energyequivalent continua obtained by defining direct links with lattice systems are still among the most promising approaches in material science. The mechanical behaviour of complex materials, characterised at finer scales by the presence of heterogeneities of significant size and texture, strongly depends in fact on their microstructural discrete nature. By lacking in material internal scale parameters, moreover, the classical continuum does not always seem appropriate to describe the macroscopic behaviour of such materials taking into account the size, the orientation and the disposition of the micro heterogeneities. This calls for the need of non-classical continuum descriptions obtained through multiscale approaches aimed at deducing properties and relations by bridging information at proper underlying discrete micro-levels via energy equivalence criteria. The circumstances in which the inadequacy of the classical hypothesis of lattice mechanics (lattice homogeneous deformations and central-force scheme), not very differently than in the past, still call for the need of improved constitutive models will be discussed and the suitability of adopting discrete-continuous Voigt-like models, based on a generalization of the so-called Cauchy-Born rule used in crystal elasticity as in classical molecular theory of elasticity, will be pointed out. It will be shown that this approach allow us to identify continua with additional degrees of freedom (micromorphic, multifield, etc.), which are essentially non-local models with internal lengths and dispersive properties [7].

Acknowledgements: This research has been partially supported by the Italian 'Ministero dell'Università e della Ricerca Scientifica' (Research fund: MIUR Prin 2010-11; Sapienza 2013).

\section{References}

[1] D. Capecchi, G. Ruta, and P. Trovalusci. From classical to Voigt's molecular models in elasticity. Archive for History of Exact Sciences, 64:525-559, 2010. 
[2] D. Capecchi, G. Ruta, and P. Trovalusci. Voigt and Poincaré's mechanistic-energetic approaches to linear elasticity and suggestions for multiscale modelling. Archive of Applied Mechanics, 81(11):1573-1584, 2011.

[3] A.-L. Cauchy. Sur l'équilibre et le mouvement d'un système de points matériels sollicités par des forces d'attraction ou de répulsion mutuelle. Exercices de Mathématiques, 3, 1822, 1827:188-213, 1828. (Euvres 2 (8): $227-252$.

[4] C.-L.-M.-H. Navier. Mémoire sur le lois de l'équilibre et du mouvement des corps solides élastiques (1821). In Mémoires de l'Academie des Sciences de l'Institut de France, volume 7 of II, pages 375-393, 1827.

[5] H. Poincaré. Leçons sur la théorie de l'élasticité. Georges Carré, Paris, 1892.

[6] S.-D. Poisson. Mémoire sur l'équilibre et le mouvement des corps élastiques. In Mémoires de l'Académie des Sciences de l'Institut de France, volume 8, pages 357-405, 1829. Lu à l'Académie 1828.

[7] P. Trovalusci. Molecular approaches for multifield continua: origins and current developments. In T. Sadowsky and P. Trovalusci, editors, Multiscale Modelling of Complex Materials: phenomenological, theoretical and computational aspects, number 556 in CISM Courses and Lectures, pages 211-278. Springer, Berlin, 2014.

[8] P. Trovalusci, D. Capecchi, and G. Ruta. Genesis of the multiscale approach for materials with microstructure. Archive of Applied Mechanics, 79:981-997, 2009.

[9] W. Voigt. Theoretische Studien über die Elasticitätsverhältnisse der Kristalle. In Abhandlungen der Gesellschaft der Wissenschaften zu Göttingen, XXXIV, 1887.

[10] W. Voigt. Lehrbuch der Kristallphysik. B. G. Teubner, Leipzig, 1910. 


\title{
The discovery of the vector representation of moments and angular velocity $(1750-1830)$
}

\author{
Sandro Caparrini, \\ Dipartimento di Matematica, Università di Torino
}

The introduction of vectors in mechanics falls naturally into two distinct periods. In fact, force and velocity belong to the elements of point mechanics, while moment of forces and angular velocity are mainly used to describe the motion of rigid bodies. Therefore, the vector representation of the latter kind of quantities must be traced in papers written after 1750 .

The first statement and proof that the infinitesimal rotations about concurrent axes can be composed according to the parallelogram law are due to Paolo Frisi (1759). Contemporarily with Frisi, another Italian mathematician, Tommaso Perelli, obtained the same theorem, but did not publish his work.

Lagrange gave a purely analytical formulation of this theorem in the first edition of his Méchanique analitique (1788). He came close to establishing the vectorial character of small rotations, yet he failed to do so. In the second edition (1811) of his treatise, whose title was now changed to Mécanique analytique, Lagrange added a completely new analysis of the decomposition of a given infinitesimal rotation along the coordinate axes of two different systems of rectangular Cartesian coordinates, demonstrating that the partial rotations around the coordinate axes transform as the components of a displacement. Thus he succeeded at last in formulating the vectorial representation of small rotations.

After 1811, this result was taken up by Jacques Frédéric Français (1812), Jacques Philippe Marie Binet (1814) and Poisson (1833). A detailed study of the angular velocity vector appeared in Poinsot's Théorie nouvelle de la rotation des corps (1834). Poinsot made full use of the vectorial properties of the angular velocity in his classic theory of the motion of a rigid body.

The discovery that moments of forces are vectors was made by Euler and can be found in two papers written in1780 but published only in 1793 . He obtained this result as a corollary to a formula for the moment of force about a point, expressed in terms of components. Euler saw that his formula indicates that moments of forces can be represented by a directed line segment and can be decomposed by means of the parallelogram law.

Unaware of Euler's results, a few years later Laplace published two articles based essentially on the law of transformation of moments in passing from one system of coordinates to another (1798). His discovery of the invariable plane is roughly equivalent to the vectorial representation of moment of momentum. The connection between Laplace's invariable plane and Euler's formula was established by Gaspard de Prony in his Mécanique philosophique (1800).

A purely geometrical approach to the theory of moments of forces was first obtained by Poinsot in his Éléments de statique (1803). Here he created the concept of a couple of forces, and demonstrated that if we represent a couple with a segment perpendicular to its plane we can compound two couples by means of the parallelogram law. In 1808 Poisson represented the moment of a force by means of a parallelogram.

These new discoveries were further developed. In 1814 Binet wrote the law of moment of momentum in a form which takes into account the geometric representation of moments; in 1818 he created the concept of areal velocity and derived the principle of moment of momentum for a system of mass points from the law $\mathrm{F}=$ ma and the equality of action and reaction (this result is usually called "Poisson's theorem" in modern textbooks). Antonio Bordoni generalized Euler's formula to non-orthogonal Cartesian axes (1820).

In 1826 Cauchy wrote a sequence of five papers in which he brought the theory of moments to its final form. Cauchy's treatment does not differ in essence from the theory that we find today - in vector formulation - in every textbook.

\section{References}

[1] S. Caparrini. The Discovery of the Vector Representation of Moments and Angular Velocity. Archive for History of Exact Sciences 56 (2002), 151-181. 


\title{
Domenico Fontana and the entrance of mechanics in architecture
}

\author{
R. Masiani, C. Tocci \\ University of Rome La Sapienza, Department of Structural and Geotechnical Engineering
}

Compared to that of his contemporaries, the work of Domenico Fontana takes its place in the late sixteenth century architecture with connotations of significant originality. Not so much for the quality of the outcomes, "constantly poised between elegance and obviousness" according to the sharp synthesis of Paolo Portoghesi [1], but for the greater importance attached to the structural and constructional problems of architecture that seems to reveal, in some cases, a not superficial knowledge of the basic concepts of mechanics and the awareness of the possibility to use them in practice.

Beginning from the reading of archive's documents relating to the static restoration of the abacus of Marcus Aurelius column made in 1589 [3], and, for comparison with the description of the translation of the Vatican obelisk contained in the famous book published the year after [2], the contribution attempts to outline as a preliminary the relationship of Domenico Fontana with the new technical literature of the second half of the sixteenth century (Federico Commandino, Guidobaldo del Monte, Bernardino Baldi).

The analysis of the building site processes, concisely described for the Marcus Aurelius column and meticulously illustrated for the Vatican obelisk, allows illustrating - not by chance on two occasions in which structural and constructional tasks are prevailing, if not exclusive, compared to architectural concerns - not only the complete mastery that Fontana exhibits about the statics of elementary machines (superbly described, for example, in Guidobaldo del Monte's Mechanicorum Liber) but also, and above all, the ability to exploit such a mastery as a concrete support in the phase of structural invention.

From this point of view, Domenico Fontana unequivocally heralds an attitude that would become shared approach more than a century later, representing one of the first examples in history in which mechanics is consciously employed for the structural control of architecture.

\section{References}

[1] G. Curcio, N. Navone, S. Villari (eds.). Studi su Domenico Fontana. Mendisio Academy Press/Silvana Editoriale (2011).

[2] D. Fontana. Della trasportatione dell'obelisco vaticano e delle fabbriche di Nostro Signore Papa Sisto V. Roma (1590).

[3] R. Masiani, C. Tocci. Ancient and modern restoration for the Column of Marcus Aurelius in Rome. International Journal of Architectural Heritage. 6:5 (2012), 542-561. 


\title{
On the graphic statics for the analysis of masonry domes
}

\author{
N. Cavalagli, V. Gusella \\ Department of Civil and Environmental Engineering, University of Perugia, Perugia, Italy
}

During the second half of the 19th-century the graphic statics widely disseminated for the analysis of structures, from the academic to the architectural and engineering circles ([1],[2]). In the studying of masonry domes, the crucial aspect of the graphic statics is the analysis of a three-dimensional structural behaviour in a bi-dimensional space. The "calculus problem" is well exposed by Camillo Guidi in his treatise, in which he wants to underline that «staticamente indeterminato è il tracciamento della curva delle pressioni; né la teoria dell'elasticità fornisce di questo problema una soluzione semplice e pratica; conviene perciò accontentarsi di una soluzione approssimata, o piuttosto di una specie di calcolo di verifica» [3]. Eddy [4] was the first to propose a graphical method for the study of a slice of dome interacting with neighbours, by considering, in the balance of a block, both the meridian and hoop forces. In addition to Eddy's method, very complex for a practical application, other solutions can be found in literature, among them the contributions of Schwedler [5], Lévy [6], Wolfe [7], Guidi [3] and another one shown in an italian practical manual for architects [8]. Owing to the indeterminateness of the problem, all these methods differ in the initial hypotheses made on the position of the thrust surface.

The aim of this paper is to compare the results obtained by the application of several methods to an hemispherical dome with the analytical and numerical results given by the membrane theory and the Finite Element Method respectively.

In addition, a specific case study will be analysed, the dome of the Basilica of Santa Maria degli Angeli in Assisi [9]. This case consists of a single shell masonry dome with a lantern at the top, which generates a concentrated external load on the dome slice analysed by means of graphic statics. The results obtained by the application of several graphical methods will be discussed, taking also into account the numerical solution obtained by a finite element model.

\section{References}

[1] E. Benvenuto. An Introduction to the History of Structural Mechanics. Springer, New York. (1991).

[2] D. Capecchi, G. Ruta. La Scienza delle Costruzioni in Italia nell'Ottocento. Springer, New York. (2011).

[3] C. Guidi. Lezioni sulla Scienza delle Costruzioni. Vol. III. Vincenzo Bona, Torino. (1928).

[4] H.T. Eddy. New constructions in graphical statics. D.Van Nostrand, New York (1877).

[5] J.W. Schwdler. Die Construction der Kuppeldächer. Zeitschrift für Bauwesen. 16 (1866), 7-34.

[6] M. Lévy. Cupoles en maçonnerie, Chapter II, La statique graphique esesapplications aux constructions, IV Partie. Gauthier-Villars Imprimeur-Librarie, Paris. (1888).

[7] W.S. Wolfe. Graphical Analysis: A text book on graphic statics. McGrow-Hill Book Co, New York. (1921).

[8] CNR. Manuale dell'architetto, Consiglio Nazionale delle Ricerche, Roma. (1953).

[9] N. Cavalagli, V. Gusella. Dome of the Basilica of Santa Maria degli Angeli in Assisi: Static and Dynamic Assessment. International Journal of Architectural Heritage. 9(2)(2015), 157-175. 


\title{
G. W. Leibniz's "Machina Deciphratoria", the first described cypher machine from the $17^{\text {th }}$ century, constructed and built in 2013/14 for the Leibniz Exhibition of the Leibniz Universität Hannover
}

\author{
Erwin Stein \\ Professor of Mechanics and Computational Mechanics, Leibniz Universität Hannover (LUH), \\ Curator of the Leibniz Exhibition of the LUH
}

According to Leibniz's major postulate Theoria cum praxi, his plan of general progress in the $2^{\text {nd }}$ half of the $17^{\text {th }}$ century, the beginning of the age of enlightenment, was to combine his new Scientia generalis with the Ars inveniendi and the Ars combinatoria, both in connection with his Calculus logicus.

Important technical outputs were his famous Machina arithmetica, the first Four-species-calculating-machine with a uniform logic for the gear mechanisms (especially the decimal carries), and moreover, the description of a Machina deciphratoria, a cypher machine for six-fold coding and decoding of messages via monoalphabetic substitutions of letters in $2 \times 6=12$ alphabet strips, each with 26 letters. This gear-driven machine was designed according to Leibniz's proposals by Prof. Nicholas Rescher, University of Pittsburgh, PA, constructed in detail and mounted by Klaus Badur and built by Gerald Rottstedt, both Garbsen near Hannover, Germany, in 2013/14. This machine became part of the Leibniz Exhibition of our University as a permanent loan of the Fritz Behrens Stiftung Hannover.

It is remarkable that in both very different machines the so-called stepped drum, a cogwheel with linearly decreasing tooth lengths in five planes (from 12 to 2 cogs), plays an important role. In the cypher machine it is attached at the left end of the transport drum which can be fixed in six axial positions of the stepped drum. Coding (substituting) of a letter is realized by pressing the related key, by which the transport drum is rotated by $60^{\circ}$. The teeth of the stepped drum are rotating the cogwheel of the display drum (beneath the transport drum) on which the coded letter is visible on a strip of 26 alphabet letters. It is obvious that the six possible axial positions of stepped drum enormously increase the number of possible encryptions.

Leibniz's invention of very different new products - using the same key components - shows his modern holistic and system-oriented (not product-oriented) thinking within his Ars inveniendi, because a single general method and its solutions yields very many single inventions (from a letter by Leibniz to Duke Ernst August of Hannover in 1685/87).

The lecture also touches the exciting history of cryptography from ancient times until today with undeclared electronic cypher wars. 